


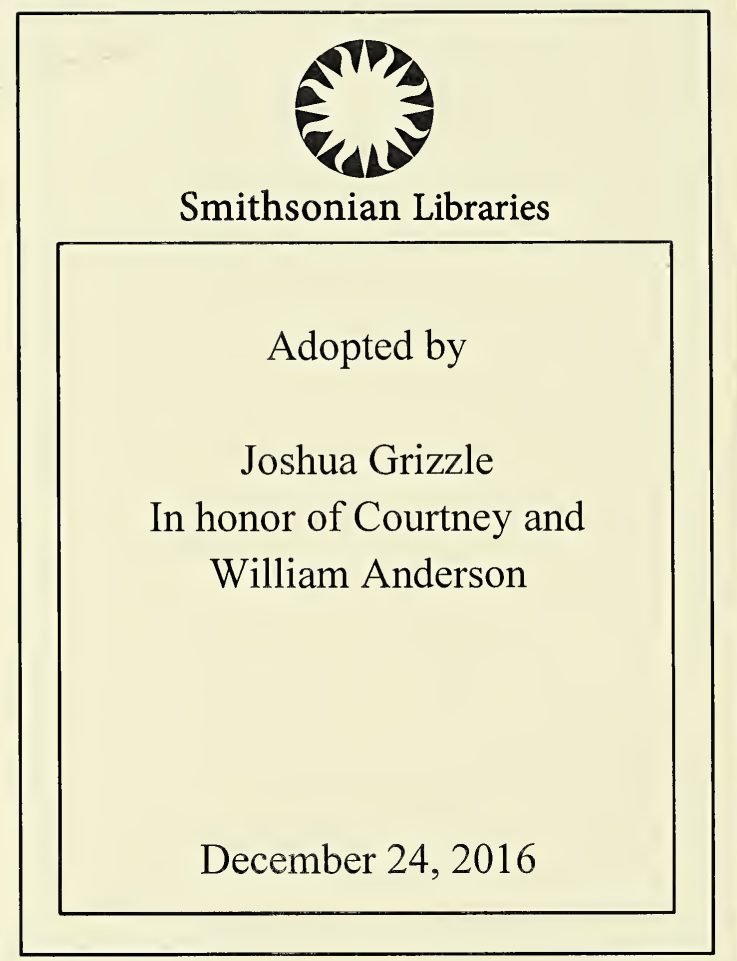









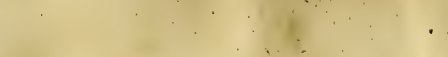


$10=24,45,4 m$ enmyentan th

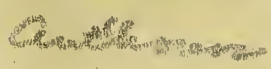

30 



NEW CYCLOP AEDIA

oF

\section{DOMESTIC · ECONOMY, AND}

\section{PRACTICAL HOUSEKEEPER.}

ADAPTED TO ALL CLASSES OF SOCIETY,

AND COMPRISING

SUBJECTS CONNECTED WITH THE INTERESTS OF EVERY FAMILY;

SUCH AS

DOMESTIC EDUCATION, HOUSES, FURNITURE, DUTIES OF MISTRESS,

DUTIES OF DOMESTICS, THE STOREROOM, MARKETING, TABLE

AND ATTENDANCE, CARE AND TRAINING OF CHILDREN,

CARE OF THE SICK, PREPARATION OF FOOD FOR

CHILDREN AND INVALIDS, PRESERVATION OF

HEALTH, DOMESTIC MEDICINE, THE ART

OF COOKERY, PERFUMERY, THE

TOILET, COSMETICS,

AND

FIVE THOUSAND PRACTICAL RECEIPTS AND MAXIMS.

FROM THE BEST ENGLISH, FRENCH, GERMAN, AND AMERICAN SOURCES.

\section{ILLUSTRATED WITH}

OVER TWO HUNDRED ENGRAVINGS.

EDITED BY MRS. E. F. ELLET, AdTHOR OF "THE WOMEN OF THE REVOLUTION," ETC, ETC.

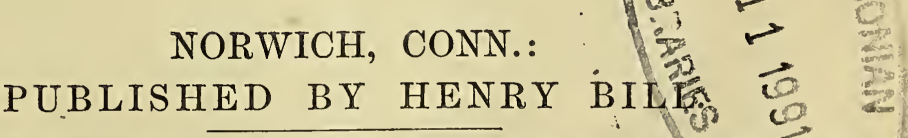

O. A. BROWNING, Toledo, O.; C. C. HASKELL, Lewiston, Me.; IRA A. SMITH, MLfFoRd, Mass.; ANd HUGH HERON, Chicago, ILt., Generat Agents. 1872. 
Entered, according to Act of Congress, in the year 187 , BY HENRY BILL,

In the Office of the Librarian of Congress, at Washington.

Rand, Avery, \& Co., Printers,

3 Cornhill, Boston. 


\section{PREFA C .}

THE importance of the subjects introduced, and their influence upon the happiness of the household, constitute a sufficient claim for a favorable reception of this volume; for, though many books have been written on domestic affairs, yet no treatise so complete in all its parts, within the limits of a convenient manual, has been given to the public before the appearance of this work. In many matters beside cookery does the inexperienced housewife need instruction and guidance. It has been a study, in this volume, to reduce to practical rules the best theories concerning an extensive and varied range of household duties, and to furnish simple and useful directions in each branch of this most interesting of sciences, that the work might be safely consulted in all matters relating to the manifold responsibilities of the housekeeper.

The young housekeeper will appreciate the advantages of a compendium which embraces rules and advice pertaining to all the duties expected of her; advice as to early house training, the taking and furnishing of a house; the different 
departments filled by servants, and the superintendence of the mistress; the laving in of stores and purchases in market; the art of making and arranging things used every day, and of setting out a table to advantage; the care of children, and the best food for them; the preparations most useful for invalids; the attendance at meals, and various matters in household management which no book before this has ever taught in detail, but which are commonly left to the slow teaching of individual experience. While endeavoring to make this manual or cyclopedia so complete as to meet all the wants of those who lack knowledge, care has been taken to arrange the various departments with clearness and method. A carefully prepared and copious index will at once direct the reader to any thing wanted.

In the department of Cookery, arranged under forty-five heads, an unusually large variety of recipes is given for the styles in every-day living-soups, meats, sauces, \&c., because it was desirable to include the latest improvements, and because the want of variety in such preparations is usually complained of in.American cookery. The French having so much the advantage of us, it is as well to learn something more of their boasted art, that those who choose may avail themselves of the knowledge. A number of choice recipes, therefore, from very recent French and English works, have been added to those contributed by American housekeepers 
of long experience and tried skill. Several valuable recipes, never before made public, have been furnished for this work by Delmonico, Taylor, Wagner, Sneckner, and other proprietors of celebrated establishments in New York.

The Toilet Department, and Perfumery, Cosmetics, \&c., do not strictly belong to housekeeping; but every lady must desire some knowledge of them, and it is convenient to have a manual containing instructions as to the compounding of articles required for the hair and complexion. The fair reader will not object to the space and attention devoted to these matters.

The Family Medical Guide is not designed to interfere with the province of the physician, but to furnish simple and approved recipes for use when medical advice cannot be procured, and palliatives to promote the comfort of the sick. All these recipes have been submitted to a prominent physician, and approved by him.

The section appropriated to the sick is unusually eomprehensive; containing many new recipes. The Miscellaneous Department is also enriched with several tried and excellent ones, never before published. The numerous illustrative cuts, which are found in no other volume, will help to explain the method and use of various housekeeping articles.

E. F. E. 



\section{CONTENTS.}

PART I.

CHAPTER I.

Thoughts and Maxims on Housekeeping, 15

CHAPTER II.

The Dwelling House, \&c., . 18

CHAPTER III.

Furnishing a House, . • • • . 21

CHAPTER IV.

Plate, Cutlery, House-linen, \&c., 24

CHAPTER V.

Servants, • . . . . . . 26

CHAPTER VI.

Duties of the Housewife. The Dinner, . 31

Duties of the Servants, _ . . . 41

CHAPTER VII.

CHAPTER VIII.

The Care of Children, . • . . 46

CHAPTER IX.

The Care of the Sick, : . - 50

CHAPTER X.

The Store-room and Marketing,

CHAPTER XI.

Domestic Manipulation, • • • 59

CHAPTER XII.

Decanting, Straining and Filtering Liquids, 65 CHAPTER XIII.

The Manufacture and Use of Cements, 69 CHAPTER XIV.

Powdering, Grinding, \&c., . 72
CHAPTER XV.

Knots, Parcels, \&c.

CHAPTER XVI.

Adulteration of Food and Purity of Water, 78 CHAPTER XVII.

Boiling, Stewing, \&c., $\quad$ • $\quad$. $\quad$ • $\quad$. 81

CHAPTER XVIII.

Economy of Heat, • • • • • 84

CHAPTER XIX.

Cleaning and Disinfecting, • . 88

CHAPTER XX.

Fermenting and Distilling, . . . 91

CHAPTER XXI.

Laying out Tables and Folding Napkins, . 94 CHAPTER XXII.

Trussing and Carving, . $\quad . \quad .103$

CHAPTER XXIII.

Culinary Otensils, . . . . . 116

CHAPTER XXIV.

Cookery as an Art, . . . . . . 124

CHAPTER XXV.

Foreign Terms used in Cookery, . 126

CHAPTER XXVI.

Condiments, .

CHAPTER XXVII.

Rudiments of Cookery, • • • . 136

CHAPTER XXVIII.

Hints and Maxims, • • • • 143

CHAPTER XXIX.

Articles in Season for each Month, . 149 


\section{PART II.}

Receipts for boups,

Meat Soups,

Soups of Gan.e, Poultry, \&c.,

Vegetables,

Fish Soups,

Fish,

Shell Fish,

Sauces for Fish,

Gravies, Sauces, \&c.

Ketchups,

Farces and Stuffings,

Receipts for Dressing Beef

Receipts for Dressing Veal,

Receipts for Dressing Mutton, .

Receipts for Dressing Lamb, .

Receipts for Dressing Pork,

Sausages and Forcemeat, .

Curing Meat, Potting and Collaring,

Poultry and Game,

Venison,

Vegetables,

Salads,

Pickles and Store Room Sauces,

Paste, Meat, Game and Fish Pies,

Fruit Pies, Puffs, \&c.,

Puddings,

Sweet Puddings,

Italian Pastes, .

Rice,

Cheese,

Pancakes, Fritters

Various Modes of Cooking Eggs,

To Make Bread,

Biscuits and Warm Cakes,

Butter,
PAGE

Cakes, \&c. PAGT

Custards, Creams, Jellies, \&c., . . . . 486

. 155

Coffee, Tea and Chocolate, . . . 494

156 Preserves, \&c., • • • • • . 498

. 171

. 179

.185

. 188

. 204

. 214

.218

- 242

. 243

. 249

- 276

. 296

- 311

. 318

. 337

. 341

. 356

- 374

- 376

. 390

- 393

- 406

- 425

- 436

. 439

. 450

- 452

. 454

- 457

. 459

. 464

- 467

Beverages,

C • • • • 514

Food and Cookery for Children,

Savory Dishes for Breakfast, . . . 529

Bills of Fare, . $\quad$. $\quad$ • $\quad$. $\quad$ • 532

\section{PART III.}

Perfumery, . . . . . . .537

Essences and Extracts, . . . . 538

Compound Odors, or Bouquets, . . 542

Spirituous Infusions, . . . . 543

Oils for the Hair, . . . , . 545

Cosmetics, . $\quad$ • $\quad$ • $\quad$ • $\quad .546$

Powders, . . . . . . . 546

Soaps, • • • $. \quad . \quad . \quad .547$

Cold Creams, . $\quad . \quad$.. .547

Pommades, . . . . . . 548

Salves and Balsams, . . . . . 549

Vinegars, . . . . . . . 549

Salts, • • • • $. \quad . \quad . \quad .551$

Cassolettes, . $\quad . \quad . \quad . \quad .551$

Sachets, . . . . . . . 552

Hair Washes, . . . . . . 553

Fumigating Paper, . . . . . 553

Pastilles for Necklaces, Bracelets, \&c., . 554

Hair Dyes, . . . • • . 554

Depilatory, $\quad \bullet \quad \cdot \quad \cdot \quad \cdot \quad \cdot \quad .555$

Shaving Pastes, . . . . . . 555

The Family Medical Guide, . . . 556

Miscellaneous Receipts, . . . . 567

. 583 


\section{PART FIRST.}

THOUGHTS AND MAXIMS ON HOUSEKEEPING. 



\section{PRACTICAL HOUSEKEEPER.}

\section{PART I.}

\section{THOUGHTS AND MAXIMS ON HOUSEKEEPING.}

\section{CHAPTER I.}

Dr. Stark says, "The only test of the utility of knowledge is its promotion of the happiness of mankind." Viewed thus, the subject we are about to treat presents claims superior to most others, and is eminently worthy of study. The superintendence of a house, and the management, forethought, domestic economy, and good sterling sense requisite for the discharge of this duty, demand application and perseverance, and ought to receive as much, at least, as is bestowed on the acquisition of music, painting, or any of the ornamental accomplishments. Young ladies are educated to shine in society; would it not be well if they were also sedulously taught-by a system of training - to perform the homely duties which make home the abode of comfort?

It has been said that Americans in general have little attachment to home. Often, indeed, is the domestic comfort, so prized in England, absent from the abodes even of the wealthy, in our land. May not this undeniable fact, and the roving propensity of young people in this country, be attributable to the circumstance that girls, whose condition exempts them from servitude, are brought up wholly without reference to home duties? Even those who may depend on their own labor for a subsistence, are taught some trade, or superficially qualified as teachers, or instructed in various branches of needlework; while they know little or nothing of household matters; though such knowledge would enable them to command an independence. It is not alone the wife and mother who should be skilled in domestic affairs; every girl who has emerged from childhood, is liable to be called on to take charge of a house. If the mother is bedridden, or deceased, why should the father of daughters sixteen or eighteen years old be compelled to look elsewhere for a housekeeper, and intrust the management to the hands of a stranger?

The general cultivation of this valuable knowledge, too, would make the occupation of a "help," or servant, more acceptable to thousands who now prefer starvation in a garret, or the ruin of health in sedentary employments. The more attention is turned to this branch of learning, the more will its importance be recognized, and the higher place will it assume in the list of useful arts; and a degree of respect being accorded to those who excel, more will be found ready to engage in it as a profession. What an improvement would be made, by such a result, in our social and domestic life!

We would not be understood to say 
that we would have a woman merely an upper servant in the house it is her province to guide, or that we undervalue intellectual attainments and elegant cccupations. But it will not detract from the charm of these. nor from the dignity of the well-bred lady-to be familiar with the routine of domestic duties, well acquainted with the minutiæ of household economy, and competent to direct, or if need be, teach her servants; ay, even, to do things herself in cases of necessity. On the other hand, will it not enhance admiration and strengthen regard, to see that she possesses these acquirements, and is willing to exercise them to promote the comfort of others? Who can tell that she may not, at some period of life, be thrown into circumstances where the mere fine lady would be utterly helpless, or where intellectual refinement and distinguished position may prove less available than industry, ingenuity, and practical common sense?

What is a kingdom, a merchant's counting-house, or a mechanic's workshop without a head? We do not mean a mere master, but a presiding intellect to plan, contrive, direct, and guide. Not less absolute or important is the rule of a sensible woman in her own home. She has full scope for the exercise of good taste, prudence, and refinement. She is invested with an amount of responsibility she perhaps never dreamed of, for her influence over the temper, habits, actions, and dispositions of those about her is very great, and great in proportion should be her self-government, for with self the command must begin, if it is to be worth having.

There is much talk, nowadays, about the "rights" and "mission" of woman. Without entering into the merits of the subject, we would only say, that if women, from the highest to the lowest, were systematically educated to wield properly the great power they indukitably possess -a power which can be made to move the secret springs of action and the machinery of business - they would have little reason to complain of the want of influence; and were they so trained to enter actively and energetically into domestic employments and affairs, that none could deem it a pursuit unworthy of them, they would find ample scope for the exercise of their faculties, and the acquisition of means to live.

There is a medium, however, in all things. A woman who worries all within her reach by her ultra-housewifery, who damps one down with soap and water, poisons one with furniture polish, takes away one's appetite by the trouble there is about cooking the simplest thing, and fidgets one by over-done tidiness and cleanliness, is almost as much to be avoided as a downright slut, or the veriest simpleton who ever took counsel with her stupid servant as to how long a potato ought to be boiled; she exercises a pernicious influence on all, and is a misery to herself and others.

Neither would we have domestic economy and home duties vaunted, or made the constant theme of conversation; they are the private employments of a woman; she must study other things in order to entertain her relatives and frienids. Those who talk most of their duties are generally those who perform them most imperfectly. When a man returns to his home, or enters his sittingroom, fatigued and perhaps disappointed by the business of the day, he does not want to be annoyed by the detail of domestic accidents, the misdemeanors of servants, and the cheating of tradespeople; he has had his worries during the day, too, and, with that pride, or reserve, or want of confidence which is peculiar to most men, he perhaps keeps them to himself. Let his example be followed in all cases where advice, or support, o. assistance is not absolutely necessary, 
and pleasant, cheerful themes be chosen, or some amusement selected which shall render the evening and leisure hours those of relaxation and enjoyment, and tend to give a charm and zest to home which no other place can possess. Men are free to come and go as they list, they have so much liberty of action, so many out-door resources if wearied with indoors, that it is good policy, if nothing else, to make home attractive as well as comfortable.

An education in household matters should be complete-comprising the knowledge requisite for use in all departments.

Many a girl can make good pastry, jellies, \&c., for an evening party, and being much complimented for her labors by those who relish the produce, forthwith fancies herself a capital housewife, while, in all probabiiity, she scarcely knows how vegetables are cooked, is profoundly ignorant of the prices of the commonest articles of daily consumption, and could not tell of what material a housecloth onght to be made. And how few there are who could, in case of need, make a cup of good gruel, or a glass of white-wine whey, or even a little broth or barley-water! We do not say that they could not manage to produce something resembling these things, but the capricious appetite of the invalid would reject the tasteless messes. Many have suffered martyrdom from this one neglected branch of female education.

Perhaps few branches of female education are more useful than great readiness in figures. Accounts should be regularly kept, and not the smallest article omitted to be entered. If balanccd every week or month, the income and outgoings will be ascertained with facility, and their proportions to each oth. er duly observed. Some people fix on stated sums to be appropriated to each different article, as house, clothes, pocket, education of children, \&c. Whatever may be the amount of household expenditure, a certain mode should be adopted and strictly adhered to. Besides the regular account-book in which the receipt of money and every payment should be regularly entered, a common-place book should be always at hand for the entry of observations regarding agreements with servants, tradesmen, and various other subjects, so as to enable the lady at once to ascertain the exact state of the affairs under her immediate management.

A minute account of the annual income and the times of payment should be taken in writing; likewise an estimate of the supposed amount of each article of expense ; and those who are early accustomed to calculations on domestic articles will acquire so accurate a knowledge of what their establishment requires, as will enable them to keep the happy medium between prodigality and parsimony.

Some aver that they have no capacity for this matter, no taste for that. But if the things referred to are duties, cultivate a taste-persevere in endeavoring to improve a capacity for them. This world is a beautiful one, spite of what grumblers say, and thousands would find it a much happier one if they studied more what they ought to do, and sought their pleasure or indulged their fancies less. Every human being exercises some influence on the character, happiness and destinies of others, and is accountable for opportunities wasted, and blessings neglected or transformed. This is especially true of women. Every sensible, high-minded, right-hearted woman, be she peeress or peasant-is, or may be, a blessing to many.; if not by great deeds or achievements the world calls heroic, by a simple, quiet, straightforward performance of the duties which lie before her, and are therefore given her to do. 
As it is the business of man to provide the means of living comfortably, so it is the province of woman to dispose judiciously of those means, and maintain order and harmony in all things. On her due performance of her part rest the comfort and social peace of home; while misery and ruin follow her neglect. Some women, it is true, are placed in an unnatural position by having the burden of supporting the family thrown upon them. They have double duties, and a harder part to sustain; but their toils may be materially lightened by care and method in what they have to do.

So much by way of exordium. We shall now proceed through the various matters appertaining to a house, endeavoring to present a clear chart of the necessary business, pointing out hidden rocks, and showing how these may be avoided, and the vessel sent to float in calm waters. With much labor we have collected information on all subjects that fall within the proper scope of housekeeping; and multifarious as these are, it is our belief that she who consults our index will be sure to find whatever she can wish to know.

\section{CHAPTER II.}

That sensible and oft-quoted old lady, Mrs. Glasse, begins one of her recipes thus: "First catch your hare." Following so good an example, we will first take a house and furnish it, before laying down axioms for its management.

Before any steps are taken, the income or pecuniary means of the parties about to commence housekeeping, should be well considered. It is not well to rush into matrimony without due attention to such sublunary matters as dollars and cents; for the notion that when once a couple is married, all will go right, is a foolish one. A young man with an income of four or five hundred dollars, every cent of which has been annually swallowed up by his own expenses, falls in love with a young lady who can sing and play well, speak French and produce marvels in the way of crochet or ornamental work, who loves sentimental poetry and romance, and can trim herself a neat bonnet; but knows little of the realities of life. Having never known what it is to want any thing-she has no idea that any thing can be wanted. The young man feels certain in his own mind that a wife will be an actual saving to him; and makes an eloquent declaration of his affection. The prospect for the future is but slender; for he ignores the fact that he has been accustomed to spend half his income on clothes and amusements, which he does not think of doing without; and she forgets how much she is in the habit of spending on gloves, ribbons, perfumes, et cetera. When they are married - the bridal dress and entertainment not being reckoned among their expenses-they find themselves fettered by a thousand inconveniences, and obliged to deny themselves travelling and many other kinds of recreation; more than that, they discover that the etiquette of this enlightened age, imperiously demands reckless expenditure, when common sense would advise more than usual economy; and without losing the social position they aim to preserve, they can do nothing but submit. What an effect on the success of after-life must be such a beginning!

However, we do not consider it our mission to enter on Quixotic quarrels with the ways of the world. It is, as our young people soon find-

"A very good world to live in,

To lend, to spend, or to give in;

But to beg, or borrow, or get one's own,

'Tis the very worst world that ever was known."

In taking a house, the first matters to 
be considered, are the rent we can afford to give, or the money that can be devoted to its purchase, and the locality that suits us best.

A dwelling in town affords many opportunities for social intercourse, amusement, and the acquisition of general knowledge; a suburban residence offers some advantages in healthiness of location and convenience. The remote country has a distinct class of enjoyments, though want of society often makes time pass slowly. Where locality is not specified, always choose one as open and airy as may be, and where the soil, or at any rate the subsoil, is not clay, where the drainage is good, and there is an ample supply of water, and no neighboring factories giving out noxious gases and poisonous smoke and vapor. Too close a vicinity to a churchyard is likewise to be avoided. Of course, the house must be capable of accommodating the family who are to occupy it, and there should always be a spare room or two which can be used for bed-rooms, or other purposes in case of emergency. There should be closets, cellars, \&c.. and good ventilation front and back. A fee to a well-qualified surveyor is often well bestowed; for he may detect serious faults in a house which, to an ordinary observer, seems well built and comfortable.

The agreement with the landlord should be clearly understood, and all liabilities as to taxes, local rates, house repairs, with charges for fixtures, \&c., inquired into, and definitely arranged, before the agreement is signed.

It will be well for every house to have some shelter at its entrance; a porch or portico, in a style regulated by that of the rest of the building, will be found useful. A hall, vestibule, or entry, is essential, and the size and location of the staircases considerably affect the convenience of the dwelling. The diningroom should be so placed that the way to it from the kitchen is easy, and yet so that the noise or odors do not prove annoying. The general style of the drawing-room should be light and cheerful; that of the library plain and quiet. The bed-chambers should be as lofty and spacious as possible, and so contrived that a thorough draught can be obtained, to change the air completely. Each should have a chimney fireplace. Small closets and recesses are to be avoided as sleeping apartments. T'he nursery should be near the chamber of the mistress.

Dressing and bath-rooms should be as uniformly attached to bed-rooms as the size of the house and means of the owner will permit; the bath is an indispensable convenience. Every house should be provided with two water-closets at least. In large establishments, a breakfast-room looking eastward and with glass doors opening on a garden or lawn, a billiardroom for exercise within doors, a rooni for hunting and fishing tackle, a gallery for music, paintings, or statuary, a lady's boudoir or sitting-room tastefully ornamented, a school-room, and domestic laboratory, with a conservatory, are convenient additions. A number of rooms for domestic offices are connected with the house, and various cellars, as well as separate buildings, appropriated to many different purposes-which we shall not describe particularly. The kitchen will be examined under another head.

In contriving the mode of warming a house, attention should be paid not merely to economy of fuel, but to the preservation of a salubrious atmosphere. A chimney fireplace or grate is preferable to a stove, which is apt to give the air a close or disagreeable smell, and produce headache and stupor. Count Rumford imagined that the hot iron roasted the dust that settled on it, which dust was composed of all sorts of animal and vegetable matters; others complain of 
the extreme dryness of the air. Stoves of brick or earthenware, used in various parts of Europe, are said to be better than iro but all close stoves are liable to the objection, that in using them it is difficult to change the air continually, or procure proper ventilation. The same difficulties prevail, to some extent, in warming a house by a furnace, and the dryness of the air is often productive of discomfort. Steam might afford an agreeable and convenient method of warming apartments. Of all the modes usually adopted, the advantage seems to lie with the open fire. The temperature should be steady and not too high: say $60^{\circ}$ or $62^{\circ}$. Apartments in our cities are generally kept at a dangerous degree of heat. Bed-rooms should not be warmed, more than to have the "outside chill" taken off.

Ventilation is a very important consideration. The atmospheric air in breathing loses the constituent that sustains life, and must be got rid of, and replaced by air that is fresh and pure. This change of air should be continually going on in our apartments. Windows that open at the top are useful, the vitiated air ascending to the ceiling. The practice of sleeping in rooms not sufficiently ventilated, is utterly destructive to health. It should be remembered also that the vital part of air is exhausted by a burning light. It would be well to have a ventilator in the centre of the ceiling, which can be concealed by ornamental work in plaster; and in the admission of fresh air, care should be taken that it does not come in a direct stream, so as to produce unpleasant draughts.

It is often necessary to fumigate or disinfect the air contaminated by noxious effluvia. Muriatic acid and nitric acid fumes have been employed for this purpose, and chlorine, a most effectual agent in destroying noxious qualities in the air, is generally employed. Chloride of lime and chloride of soda are used as the most convenient preparations. The latter is called Labarraque's Disinfecting Liquid. Vinegar is used in sick rooms, and quick lime, alone or mixed with ashes, in sinks, sewers, \&c.

Having taken our house, it generally wants a thorough cleaning and airing. In spring, autumn, and winter, fires should be kept for three or four days, according to the time the house has been empty, and to the repairs it has undergone during that interval; for of course nobody ought to enter a house in the state of dirt and disrepair in which it is usually left by an out-going tenant, or if they do so under the notion that the landlord will set it all to rights after they are in, they will find out their mistake, and repent their confidence.

It sometimes happens that the chief rooms are not papered and painted until the house is let. In such case the in-coming tenant generally has the power of choosing the papers, or panellings, and paint. He will, of course, select such as will best harmonize with the color which the furniture and hangings should have.

We will now suppose the house taken, cleaned thoroughly, and well aired, and will proceed to furnish it. But first we must pause to observe that young people will do well carefully to consider matters before they take upon themselves the troubles and responsibilities of housekeepers. Where their joint savings, or some sum especially bestowed for the purpose by friends or parents, will enable them to make the necessary outlay for furniture, linen, \&c., and yet have something left to put by for "a wet day," and the rent and taxes can be afforded by the income of the husband, it is all well and good. But if money must be borrowed, or debts incurred, begin life in the quietest way, rather than with these incumbrances. Take board or apartments 
for a time, until you see your way clear. chairs or hall seats. If there is a closet From $\$ 150$ to $\$ 400$ a year will pay for for hanging up hats; cloaks, \&c. it should part of a house in a city, and much less be near the door. Door scrapers shculd in a village. Board can be obtained in respectable houses at four or five dollars a week and upwards in our largest towns. There are some disadvantages in this mode of living. It is customary to say that boarders are victimized; one is sometimes brought into contact with disagreeable individuals, who become enemies if they find they are not received in a sociable or friendly manner; and on the whole, it is wiser to keep house with three or four rooms. There are always respectable families to be found, who will let a set of apartments. Now to the business of furnishing a house. always be placed at the entrario.

\section{Pictures on the Wall.}

Pictures, if well chosen, add much to the appearance of a room, and impart to it an air of completeness, and a home look, which many people know how to appreciate. To produce this effect, the subjects of the pictures must be such as we can truly sympathize with, something to awaken our admiration, reverence, or love. All the feelings of our nature may be illustrated by pictures. There are some which we seem to make bosom companions of ; others have a moral effect, and at times prevent our going astray by their silent monitions. It is, therefore, worth while to take pains and choose good subjects, whether in engravings or paintings, and to frame and hang them suitably when chosen. Gilt frames are most suitable for rather dark paintings, and on a deep colored wall; while prints look well in a frame of composition, oak, rose-wood, or bird's-eye maple, finished with a gilt moulding. Care should be taken to hang them in a proper light, so as best to bring out all the effects of the pictures, and to place them so that the light shall fall from the same side as represented by the painter. In picture galleries and great houses, brass rods are fixed all around the room close to the ceiling, from which the pictures are hung, but in small rooms it is often best not to show the lines or wires by which the pictures hang. This is done by nailing a strong cord across the back, about two inches below the top, and then suspending it from two nails standing out but a little way from the wall. When there are several pictures in a room, the ordinary rule is, to have either the upper or lower edge of the frames in a line, on whichever side they may be hung. 
For bed-room furniture, mahogany, maple-wood, and oak, are the best and prettiest; there are also very serviceable, well-polished, stained wood imitations of all these three; and there are, too, very common and trumpery imitations, which turn shabby in a few months, and are generally badly put together, and do no service; two good chairs are worth a dozen of such rubbish.

As a general rule we should advise avoidance of all cheap, showy furnishing establishments: likewise, unless you are wealthy, of all fashionable upholsterers. Patronize good, old-established houses of business.

Never buy second-hand bedsteads, bedding, or hangings, unless you are well convinced that no more than you bargain for is included in the purchase.

Iron and brass bedsteads, which can now be had of every size, form, and price, are far preferable, both as regards health, cleanliness, and lightness, to any others.

It can scarcely be necessary to repeat how injurious to health are very small bed-rooms, and the same rule will apply to curtains which prevent the change of air, compelling us to breathe over again a portion of the air we have expired. The curtains should never enclose the bed. In low chambers, the bed should be near the floor, and the best place for it is at the middle of the side of the room, not touching the wall.

Chintz or dimity are better for bedfurniture than damask, moreen, or any fabric containing wool; they harbor less dust and are less liable to hide vermin.

Three-ply carpets are best adapted for bed-rooms. Never place carpet under a bed, or you provide a resting-place for all the dust and flock which daily falls from the mattresses, and establish a nice hot-bed for fleas. Let the carpet be made in about three pieces, in order that it may be frequently taken up and beaten or shaken, and the floor scrubbed clean.
Soft feather beds cause an undue warmth that weakens the action of the skin, and makes one susceptible to cold. A well stuffed feather bed, or a mattress, should be used. Good mattresses of wool, and wool and horsehair. iron bedsteads, and as little bed-furniture, curtains, \&c., as may be, with a light quilt, are the best preventives against rising languid, inert, and unfit in the morning for the duties of the day. The covering should be light. A wide bed affords the luxury Franklin recommends, of moving from side to side, and a bed should have but one occupant.

Bed-room and dressing-room chairs should be light. Couches, tables, dressing-glasses, wash-stands, \&c., are necessary articles, and the couches may be made of cheap materials, covered with chintz or brown holland. Bureaus and wardrobes will not be forgotten. Fireguards of painted wire are a security against accidents. Of the smaller articles in use a host might be enumerated ; but everyday need will suggest them.

Never crowd a bed-room with furniture; have that which is really useful and requisite, and no more ; and in fitting it up, always remember that illness often comes when we least expect it, and take care that your room shall possess such articles as will then be needful for comfort and ease.

A dining-room requires little furniture, but that little should be good and handsome, and of mahogany.

About furnishing drawing-rooms we can give no directions, so much depends upon taste. We would only reiterate our warning to beware of showy, veneered, vamped-up furniture, or, when the room has had a fire in it some dozen times, you will be startled occasionally by reports as if small cannon were discharged, and on rising to investigate such alarming noises, you will find, perhaps, a crack across one door of the beautiful rosewood 
cabinet, or a gaping chasm in that lovely centre table, or a piece of carved work flown off the card table, showing only pine beneath!

Here, again, a little furniture tastefully arranged is far better than a crowd of articles ; besides, in one's course through life, furniture accumulates gradually, and if it is necessary to sell one thing in order to make way for another, that is a very losing business.

We now come to the kitchens, where the wants are multifarious ; for here must be accumulated means of feeding, and cleaning, and keeping in order the whole house. Of course we can give no detailed account of what will be required, as all depends upon the extent and style of the household; all we can do, therefore, is to make one or two general remarks on the durability of different wares.

As few copper cooking utensils as possible should be had, and those few should be most thoroughly tinned in the inside, and always carefully cleaned and dried before being put away. We prefer block tin to anything else for saucepans, pots, and kettles generally. Iron does not so quickly or plainly tell any tale of dirt or neglect; cast iron is very brittle, and cannot be repaired when broken; and copper is likely to harbor verdigris. A good double block tin saucepan should always have the cover, the handle, and the back, kept bright as silver; and the top, spout, front, and handle of the kettle, should also be kept bright; for besides that a polished surface maintains heat better than an uneven, blackened one, it looks wonderfully better; and if the smoke is never allowed to gather on, these parts, it is easy to keep the utensils as bright as they were at first.

For stewpans, iron tinned on the inside is most useful.

The ancients seem to have used lamps of various forms; an improvement on torches, certainly, but a more simple con- trivance than candles, which in the twelfth century and afterwards, came into use throughout Europe. Wax, spermaceti, and tallow, with different kinds of oil for lamps of an improved fashion, are still in use. Spirit gas and camphene are cleanly substitutes, but extremely dangerous. The lighting of apartments by inflammable gas is one of the most useful results of the investigations of modern science. It is said that Murdoch, an engineer, was the first to make this discovery available on an extensive scale. He commenced his experiments in 1792.

Candlesticks for common house or kitchen use should be of tin or brass, and large enough to save grease spots. There is no wear in japan.

Wooden bowls for washing glass and china, and block tin or zinc hand bowls will be fuund most serviceable.

All utensils for the conveyance of water about a house should be of metal, as water-cans of different sizes, hot-water ewers with covers, shaving mugs, \&c., as thereby much breakage will be saved, and these, if bought good at first, will, with ordinary care, last a very long time. The same remark applies to foot-baths. Very pretty toilet sets for the wash-stand are also now made in zinc, and beautifully painted or japanned.

Sarcophagus and other extraordinarily shaped coal-scuttles, are to be avoided as most troublesome and awkward affairs, out of which it is next to impossible to extract coal conveniently.

Soyer gives the following list of kitchen articles for a family of six.

Eight copper stewpans, two larger ones, holding one gallon and a half, and the next one gallon, the others smaller by degrees to one pint; one oval fish-kettle, holding about one gallon and a half; one middle-sized braising-pan; one preserving-pan; one round bowl for beating whites of eggs ; two sauté-pans ; one omelette-pan; one frying-pan; one bain-ma- 
rie ; six saucepans for sauces ; one middlesized tin pie-mould; two tinjelly-moulds; one tin flanc mould for fruit ; one freezingpot, with every requisite; two bakingsheets ; one gridiron ; one small salamander; one colander-spoon; one bottle-jack ; two spits ; one dripping-pan ; one screen; one sugar-pan; two soup-ladles; eight copper spoons, two of them colanders; two wire baskets; one wire sieve; two hair sieves; twenty-four tartlet-pans; two tammies; one jelly-bag; twelve wooden spoons; two paste-brushes; one pair of scissors; two kitchen knives; six larding-needles; one packing-needle; one box of vegetable-cutters; one box of paste-cutters; one meat-saw; one cutlet chopper; one meat chopper; six meathooks, tinned; one rolling-pin ; eight kitchen basins; six china pie-dishes; six earthen bowls for soups and gravies; four kitchen table-cloths; eighteen rubbers; twelve fish-napkins; six pudding-cloths; four round towels.

\section{CHAPTER IV.}

WiтH regard to all those articles which fall under the general denomination of "plate," we should advise that all imitations be avoided; let those who cannot afford silver be content to use simple metal, which does not pretend to be more than it really is. All the imitations of silver will, even with the utmost care, betray themselves in a very short time, and have a would-be-genteelif-I-could sort of air, which is ten thousand times more ridiculous than the plainest of all materials; besides, the money they cost would purchase a few real articles, which are always worth their weight in silver, whereas the imitations have only a nominal value, and lose even that as they become discolored and dull.

Metal tea and coffee pots may be had very good, and in handsome patterns, and are far more durable than china, drawing better, and retaining heat longer.

The following is a list of the usual articles in silver required to furnish the table.

$\begin{array}{ll}\text { Dishes and covers. } & \text { Asparagus tongs. } \\ \text { Table knives and forks. } & \text { Cheese scoops. } \\ \text { Dessert knives and forks. } & \text { Knife rests. } \\ \text { Table spoons. } & \text { Nut crackers. } \\ \text { Dessert spoons. } & \text { Grape scissors. } \\ \text { Gravy spoons. } & \text { Tea urns. } \\ \text { Soup ladles. } & \text { Coffee urns. } \\ \text { Sauce ladles. } & \text { Tea pots. } \\ \text { Salt spoons, with gilt } & \text { Coffee filterers. } \\ \text { bowls. } & \text { Sugar basin. } \\ \text { Fish slice. } & \text { Cream ewers. } \\ \text { Trays and waiters. } & \text { Sugar tongs. } \\ \text { Bread baskets. } & \text { Tea spoons. } \\ \text { Cake baskets. } & \text { Toast racks. } \\ \text { Decanter stands. } & \text { Butter conlers. } \\ \text { Dt canter labels. } & \text { Snuffer trays. } \\ \text { Liqueur and buttle stands. Snuffers. } \\ \text { Cruet frames. } & \text { Candlesticks. } \\ \text { Egg frames. } & \end{array}$

Cheap cutlery is mistaken economy. Good knives and forks will, with ordinary care, last for years; common ones have no wear in them, and never can be made to cut well.

Crockery, china, and glass, we need say little about, for they may be had at all prices and of all qualities. The mould ed or cast glass looks as well as cut glass, if not placed in contrast with it, and wears as long, and costs considerably less. For dishes, pitchers, butter-coolers, \&c., we should always use it; decanters, wineglasses, and tumblers, do not look so well in it.

As regards ornamental china, or glass, or what not, little can be said, these things being so much matters of taste; but it is better to have but one, and that one really handsome and good, than a crowd of cheap, showy trifles; besides, these are things which gradually accumulate, and therefore it is always better to devote the money in hand to necessary articles, and leave the more ornamental ones for after consideration. A good clock for the kitchen, and a handsome one for the drawing-room, are useful and necessary things, especially the former.

In household linen, again, it is false 
economy to buy common or cheap materials. For sheets, linen, cotton, and Swiss twilled calico are used; these substances are now woven wide enough to render a seam unnecessary, and all we have to do is to measure the width of the beds and allow an extra half yard; the ordinary length of a sheet is three yards and a half. The pillow cases must be of the same material as the sheets. Marseilles quilts are too heavy to be beneficial to health. Any industrious housewife may knit very serviceable and pretty counterpanes in squares or shellshaped pieces, during those periods when she is chatting, or between the lights, or at hours when she would otherwise most probably be doing nothing. It is for such useful purposes as these we value knitting, crochet, \&c., for they can be made the means of economy and usefulness, instead of being, as they too often are, employed on useless expensive trifles.

The most durable sort of linen for sheeting is the Russian, German, or Irish fabric, a good stock of which should be laid in to avoid the necessity of frequent or irregular washing.

An inventory of furniture is given by Soyer as follows:-

Twelve pairs of sheets; ten ditto pillow-cases; three dozen napkins; two dozen and a half various-sized tablecloths, including breakfast, dinner, \&c.; six servants' table-cloths; three dozen towels; six round towels; three dozen kitchen rubbers; two dozen napkins for fish, vegetables, and fruits; six puddingcloths; two dozen damask d'oylies; one. dozen Berlin wool ditto. I, also have occasionally in the wash the cover of the carpet. the anti-macassars, which I have knitted at my leisure, and the netted window curtains. Of glass and china, I have the following; they are counted every month, and the broken ones replaced:-three dozen wine-glass- es ; two dozen champagne ditto: two dozen claret ditto; three dozen goblets; six water caraffes ; six decanters ; one liqueurstand; twelve liqueur-glasses; two glass pitchers; one celery-glass; one triflebowl; eight dessert-dishes. China : one full dinner service; one common set for kitchen; one common tea service for kitchen; one good tea service; une breakfast service; one good dessert service.

The following is my list of plate:three dozen prongs; two ditto tablespoons; one and a half ditto dessertspoons; one and a half ditto dessertforks; two ditto tea-spoons; six saltspoons; one cheese-knife; four butterknives ; one asparagus-tongs ; two sugartongs; two soup-ladles; four sauce-ladles; two gravy-spoons; two sugar-ladles; two salvers; one bread-basket; four candlesticks ; one hot-water dish for haunch of mutton.

Table-cloths, tray-cloths, and dinner napkins will of course come under the category of "linen," and can be obtained at very reasonable prices compared with what they were twenty years since. Towels, too, are included in this list. In the case of chamber towels, again, comes diversity of opinion; some preferring a soft, others a hard, some a rough, and some a smooth towel; damask and diaper are not soft enough for some delicate skins. For our own part we like towels which administer a certain amount of friction to the skin, and all medical men agree that this is requisite to health. For the kitchen, round towels, tea-cloths, and glass-cloths, will be required, as well as dusters, puddingcloths, knife-cloths, house-cloths, and flannels for cleaning. These will have to be purchased at the same time by young housekeepers newly furnishing, who " have no rags-poor things," and therefore may as well be mentioned here. Old sheets make good glass cloths; old table-cloths make nice soft towels; all 
dresses of cotton, or old dress-linings, will serve for dusters, and old blankets for house-flannels.

There are also needed toilet-covers for chamber tables, and chests of drawers, \&c., carpet covers, muslin for chamber window-curtains, muslin for drapery for the toilet-table, coarse sheeting for dusting-sheets to cover the beds or drawingroom furniture when sweeping and cleaning; a yet coarser sheet to lay down in front of the stoves when they are being cleaned; chamois leathers for cleaning the plate, brass, steel, and windows ; and bags for the best brooms.

Then we come to brushes, and their name is legion. Oh, this furnishing a house is a serious affair! a carpet-broom. a short-handled one for the stair carpets, a hair-broom for the bed-rooms, and another for the passages and kitchens; feather-brushes, dusting-brushes, stovebrushes, hearth-brushes, shoe-brushes, plate-brushes, paste-brushes, clothesbrushes, a hat-brush, and a table-brush to remove the crumbs from the tablecloth, are all needed; and these should be bought at a good warehouse, and of good quality, if we would have them do us service, and not fall to pieces, or lose their hair, as soon as they are fairly brought into use.

There are many items yet unmentioned, but it will not be requisite for us to waste our time, or that of our readers, by enumerating them all seriatim; we will therefore proceed to other matters.

\section{CHAPTER $\nabla$}

Supposing now that we have our house, and it is furnished, the next thing to determine is how many servants can be afforded. Must we be content with one, a "general house-servant;" or can we afford a cook and housemaid, or even aspire to the gentility of a man-servant or a butler?

The expenses must be determined by the sum which can, without incurring debts or living too closely up to one's income, be devoted to " housekeeping," under which head we include rent, taxes, wages, and every outlay appertaining to the house. Now, in reckoning the expenses of a servant, the question of wages is not the only one to be considered; there is the board and washing; and $\$ 100$, exclusive of wages, is the lowest at which the keep of each servant can be estimated.

In hiring servants always be particular in inquiring their character, and, if possible, learn something of the people with whom they have lived; let all stipulations as to wages, extras, holidays, and such matters, be clearly specified and rightly understood.

The greatest trouble in housekeeping is the difficulty of procuring and retaining good servants. In some parts of the world this difficulty is not realized; their servants having been trained for the labor allotted to them, and being contented and happy in their condition without aspirations after change. But in America, both in the city and country, the case is different; too often men and women look upon service as degrading to them, and will prefer any hardship or privation to engaging in it as a business. Those who do so for a time, are usually tormented with jealous fears that their dignity will be infringed on, or are found neither qualified nor trustworthy. Housekeepers are mainly dependent on the Irish and German emigrants, who as a rule are utterly ignorant of household service, and have to be taught every thing; often receiving wages for months before they begin to make themselves useful. By the time they can be trusted to do the work, they are corrupted by intercourse with other ser- 
vants, or persons who prompt them to make exactions on your time for visiting their numerous relatives from the old country, as well as to fill your kitchen with strangers, till the annoyance becomes intolerable. A complaint on this score from the employers is followed by an outbreak of insolence, and the abrupt departure of the servant you have taken such pains to teach;-and so unusual is it to find one who knows any thingthat she will readily get another place, perhaps with one of your neighbors who has envied you her possession.

An English lady gave this account of the progress of a favorite- "The first year she was a good servant; the second an indulgent mistress; the third an intolerable tyrant."

A good maxim is to select servants not younger than thirty; they are, as a rule, less fond of change, and better satisfied when really comfortable. But change is the order of things in the United States.

Respecting servants, there are a few things which cannot be too strongly urged; one is, never to retain a cook who is not fond 'of her occupation; for unless she take pleasure in her art, she cannot be depended upon for accuracy in the preparation of dishes with which she is well acquainted, and will not easily be induced to acquire any thing new. She also must possess a natural regard for cleanliness, or all the pains in the world will never render her cleanly: where dirty habits are manifested, dismissal should follow, for in almost every instance they will be found incurable. Another point of main importance is her temper; for if that be not good, she will be disinclined to receive instructions, and, if found fault with, may, out of pique, spoil a dinner; whereas a goodhumored, intelligent servant, when made acquainted with the habits of the house, and equal to her common duties, will hardly fail of success when called upon by her mistress to try any of those receipts which she has not already used.

The low character of servants heard of at common intelligence offices has caused such places to be held in small esteem; for their recommendations can in no case be depended on. There are institutions in Great Britain which have for their object the security of housekeepers from the evils of disreputable and dishonest inmates. The officials are so minute in their inquiries into character, as to preclude the necessity of those who hire from their office taking any trouble themselves in the business. Servants whose characters will not bear the strictest investigation will scarcely apply to such institutions. They are sorely needed in this country.

It may be curious to see the list of servants which form the household of a British nobleman of high rank, or a wealthy citizen, who keeps from twenty to thirty domestics.

$$
\text { Women. }
$$

Housekeeper.

A lady's maid for each grown lady of the family. Cook.

Upper housemaid.

Laundry maid.

Under housemaid.

Under laundry maid.

Still room maid.

Kitchen maid. Scullion,

(To this establishment that of the nursery is added.) Men.

House steward.

Groom of the chamber.

Valet to each gentleman in the family. Man cook. Butler.

Gentleman's footman.

Lady's footman.

Under butler.

Gentleman's coachman.

Lady's coachman.

Couriers. Outriders.

Grooms, in number according to the stud. Under servants. Errand boys. Steward's boy.

In the United States so many servants are rarely kept in one family, even among millionnaires, or in the Southern States; 
where formerly it was customary for each member of the family to have a separate attendant. An extensive establishment would perhaps number a coachman, groom, porter, footman, gardener, butler, and perhaps a valet and French cook; the female servants consisting of a housekeeper, cook, scullion, lady's maid, chambermaid, laundrymaid, nurse, and one or more maids-of-all-work. Indeed the most wealthy families keep less than half this number; and the average not more than three women and one manservant. Those of slender income are content with one for "general housework."

In all large establishments in England the men and women servants, in the intervals of their employments, are never allowed to sit in the same room, but have their separate places of resort, assembling together only at dinner and supper. It is the duty of the steward and housekeeper to see this regulation observed.

In servants generally we look for the essential qualities of integrity, sobriety, cleanliness, and general propriety in manner, with knowledge of their duties in the departments they profess to understand. A glance at some reprehensible practices among them may be useful, as every instance of pilfering and trickery accumulates odium on the whole class. One of the chief anxieties of housekeeping is the apprehension of the dishonesty of those who are under our roof and receiving bread from our hands; and suspicion, for which there is often just cause, injures both the employer and the employed. Cooks have been found to dispose of provisions in other ways than for the use of the family they serve. Presents are sometius demanded by servants from the tradespeople dealt with by the master. The so-called "honor," which prevents a servant from exposing the frauds and mis- conduct going on among others, is but another name for deception and dishonesty. The servant who knows of frauds and is silent, becomes an accomplice.

Though it is very disagreeable to suspect any one's honesty, it is yet prudent to weigh meat, sugar, \&c., when brought in, and to compare the weight with the charge. Scales should be placed in the kitchen, near the door used by the tradespeople. The knowledge of such things as weights and scales being in use, will operate as a check to any petty fraud which might otherwise be contemplated.

In large establishments abroad the servants have sometimes an allowance of food, or are kept on board wages. The former system prevails on plantations at the South, among field laborers only; the servants in families, as in the Northern States, taking their meals immediately after, and on the remains of the family meals.

The perquisites of servants are, in many cases, so many encroachments on the property of their employers, who tacitly allow, while they in principle condemn the practice. There is no doubt that perquisites tend to corrupt the morals of domestics, placing their own interests in opposition to those of others, and offering temptation against which their integrity is not always proof. Among these objectionable customs, one that particularly calls for attention as an odious kind of taxation, is the practice of servants receiving vails, or presents in money, from visitors. It is a species of bribery for services which ought to be performed without it, and tends to make servants less attentive to those who cannot give them great pecuniary rewards. This custom has grown into disuse in England, and most highly respectable families make it a condition in hiring their servants, that they shall accept no such gifts, but when they are offered 
shall inform the visitor that it is contrary to the rule of the house to take them. It was formerly so usual in hotels in the United States for the attendants to expect gifts from the guests, that one could not obtain any service without it. I knew a party of travellers visiting Niagara, to leave the diningroom, unable to obtain any thing to eat, the waiters not attending to them. One who had been longer in the house, informed them they would not be served unless each person gave fifty cents to one of the waiters-who, in fact, were paid no wages, but allowed to extract all they could from travellers! This is downright swindling on the part of hotel proprietors, and no respectable landlord now permits such impositions. In all the first-class hotels in our cities, persons should be particularly requested to give no fees to servants. The charges per day at hotels certainly ought to cover ordinary attendance; extra services may be paid for. But as the custom still prevails more or less among many travellers to give gratuities to servants, proprietors of public houses ought to be the more resolute in abolishing a practice tending to produce murmuring, discontent, and neglect of duties among their domestics. The caution should be inserted among the printed rules of the establishment, and the servant discharged who is known to receive any thing in this way.

Still more onerous and odious is the custom that inflicts a tax upon visitors in private families, in violation of the sacred obligations of hospitality. Such a burden is this felt to be, that many are compelled to refuse invitations to the houses of their friends on account of it. A lady friend of ours informed us that the expense of a short visit to one of her neighbors was made, by this necessity, to exceed what she would have paid at the highest rate of hotel fare. We found this the case on spending a day or so in a very large establishment at the invitation of the owner; each servant on the premises expecting gratuities. They learn, of course, to estimate the worth or standing of a guest by the amount bestowed on them, and frequently to treat with insolence or contempt those who cannot give so much. A "help" in New Jersey, was highly indignant at receiving present of a mousseline de laine dress instead of a silk one, and declared she would not have it made up. We knew of another lady who emptied her purse to give five dollars each to several flaunting girls in the house where she was staying; though she was obliged to deny herself many things for want of the money. "If you do not give them something very elegant. they will make fun of you ; nothing simple will please them;" said another lady when consulted on the subject. What a motive for liberality!

There are very few who have moral courage enough to be independent in such things, even though their charities have to be stinted in consequence; especially when it is known that the mistress fre quently asks her servants what they received from her guests, and even draws conclusions founded on their information! So at the risk of inconvenience or impoverishment, the tax must be paid; though as a rule it is always paid with secret dissatisfaction. What an insult to the name of hospitality is this!

The prevailing motive for this kind of liberality is not the charity which delighteth in giving-but a selfish fear of being thought penurious by one's friends, or of being ridiculed by saucy servants. We do not remember a single instance in which the custom, considered imperative, when mentioned at all, has not been mentioned with condemnation. It should be utterly and for ever abolished. The mistress, in hiring a domestic, should make known her invariable rule that such 
things are not to be allowed, and should let it be known among all her friends.

We do not object to the largest kind of liberality in giving. The poor and needy have claims that meet us at every turn; and the most rigid self-denial to satisfy their just demands, is commendable. It is an excellent rule-"My superfluity must give way to my neighbor's convenience; my convenience to his necessity ; my necessity to his destitution." In instances where our regard is attracted by a kind and faithful domestic in a friend's family, or where circumstances would render a gift peculiarly acceptable, it is pleasant to give and right to receive. We object to the system which makes present-giving compulsory without regard to the feelings or means of the donor, or the necessities of the receiver. And what well-bred lady who invites her friends for the pleasure of their society, would willingly have them feel under the necessity of putting themselves to inconvenience to give large fees to her servants, already well paid for the trifling services they render?

The custom which we have understood is actually prevalent in some places, of visitors "making up in presents" the expense incurred by their friends in hospitably entertaining them,-is certainly "better honored in the breach than the observance." What hospitality can there be, when an equivalent is offered and received? It would be more fair and open to make the bargain regularly in dollars and cents. The indirect exaction of compensation in this way, frequently beyond what could reasonably be charged, appears to us to be speculation without the $s$. Yet we occasionally hear of this as expected from visitors. A lady in the States once said to her guest: "I know you will want to make me some nice present before you go away; I will tell you what I would like: \&c." A lady from the country who staid a fortnight with a city friend, left money when she went away to purchase "some sort of a present." All we have to say of this and every other practice tending to make gifts (which should be free as the love that ought alone to prompt them) in the slightest degree compulsory, is, that it is wrong, and entirely subversive of true friendship.

To return to our subject. It is in vain that societies are formed for the encouragement of faithful domestics, by giving premiums and high testimonials to those who serve a reasonable time in the same family. These last are not valued, where a choice of places can be had without them, and the roving disposition is fostered by the notion of independence and the certainty of being well paid for doing as one pleases.

The only protection to housekeepers from this endless source of discomfort, is to be found in correcting the mistaken notion among American girls that a place in domestic service is less honorable than the severer toils of seamstresses, binders, shopkeepers, or milliners' workwomen. If they could be persuaded, instead of wasting away their lives in health-destroying needlework, miserably paid for by speculators in female servitude,- to engage in the active and varied duties of domestics, secure of a good home and abundant wages-a new era would commence for American housewives.

Something might be done towards this end by regulating the hours devoted to household employments, in such a way as to leave a portion of the day for the girl's own time, which she is at liberty to employ as she pleases; and by encouraging her to use it in the acquirement of useful knowledge. "It requires a refined mind to dust properly ;" and the cultivation of intellect will not be thrown away in any department. Let the humiliation of servitude be thus taken away, and persons who have seen better days will engage in the occupa- 
tion. We would recommend the establishment of an Association in our large cities-to find suitable places for persons of a respectable class, stipulate for suitable privileges, and persuade them to this easy mode of earning a livelihood. The gratitude of the community would be due to such an institution.

\section{CHAP'TER VI.}

As a country cannot be governed without laws, neither can a household; and the mistress should be as absolute in her own house as a sovereign in her dominions. Order and regularity are the key-stones to comfort, and our housewife must carefully arrange and digest her scheme of government in the first place, and be always alive to any modifications which emergencies, or prudence, or circumstances, may call for. And she must understand what she is about, or her scheme will be worthless; she must be able to teach, nay, to demonstrate upon occasions; she must be regular in her own habits if she would have those about her regular, neat in all that concerns herself, attentive to the details of housekeeping, economical, just, active, and considerate. She must neither hold the reins of government loosely and negligently, nor too sternly, but must quietly exercise a general and regular surveillance over every part of her house and household; and this can be done without tyranny, witlout vexatious interference, or exhibitions of temper. Let the servants once feel that this is her habit, and they will act accordingly; and if the place is good, conduct themselves so as to endeavor to please and keep it. And it is the interest, as well as duty, of every mistress, to make her servants comfortable; to see that they have a sufficiency of good food, that they are well lodged; that they have time to mend and wash their own clothes, nay, that they know how to do so, and do it.

"Do not send your clothes home to your mother to wash and mend," said a friend of ours to a new servant; "you will have plenty of time to arrange them yourself."

"Please, mum, I don't know how!" replied the girl.

"Well, Mary, you shall be taught, then, for it is time you did know how!"

"But please, mum, I don't want to learn!" said the girl; and learn she would not, for the foolish induigence which leads mothers to spare their children all occupations they do not like, is as prevalent among the poorer as among other classes.

To return: a mistress need never forget herself, nor weaken her authority, nor show any false indulgence; but in numerous ways she will have the opportunity of endeavoring to guide, to advise, and to benefit those dependent upon her; but she must be patient, if she would really do good. She must remember what may have been the early education, the trials and temptations, the experiences of those girls, and must not expect too much from them. As we have before said, she must hold the reins of government with a firm hand; she must not overlook neglect of duties, irregularities of conduct, want of order or cleanliness, or inattention to her commands; but she can notice these things quietly, without loss of temper, and when alone with the offender; and she can also notice and praise neatness, attention, obedience, and such like, and not accept the good as mere matters of course, and only mark the evil. She should likewise endeavor to induce her servant, by example and precept, to be regular in attendance on religious worship, and make Sunday to them in some degree a day of rest, instead of one of extra cooking and 
work, and should have a supply of those excellent little works to lend them, which are published by the religious societies. While she inculcates economy in things relating to herself, she should try to induce them to save; to put by regularly a certain portion of their wages, and not be extravagant in clothes, but make and mend their things properly.

There is little saved by giving paltry wages; a useful servant will not accept them, and those who do, cannot clothe themselves respectably, and will too often eke out their means by peculation. It is well to begin with moderate wages, and promise an annual increase, which promise both induces a wish to please, and takes away one great excuse for leaving, viz., a desire of "bettering herself." Even in the present "degenerate age," attached servants are to be found, and may be created. We are all more or less human, and human affections and home ties act upon us; we learn to love those who are kind, courteous, and considerate; we value the notice and regard of our superiors, and we take a certain pride in deserving their good opinion. The fault lies in that antagonism of classes, that want of understanding each other, that forgetfulness that all are fellow-creatures, that tendency to regard each other as mutual enemies to be conquered or circumvented.

But we will pass to another topic. The amount of the income will determine what sum can be allowed per annum for housekeeping; for besides, there will be clothing expenses to be provided for, sundries of various kinds, expenses of illness, on which we must all reckon, and there ought to be a reserve fund regularly laid by to provide for any unforeseen emergency, or form the "nest egg" of a provision for a rising family. Well, suppose the sum determined! the next question is, how to apportion it so as to combine economy with comfort, and secure a regular and uniform style of living; not luxuries to-day and parsimnny to-morrow. Now, how can our young housekeeper do this, if she knows little or nothing of the prices of provisions; if she scarcely remembers when things are in season and may be purchased at a reasonable rate, and when they are actual extravagances; if she has no idea what quantity of this or that ought to be consumed, by a family of a certain size, per week or per month; and, above all, if she has little aptitude for domestic management, and considerable contempt for all such vulgar details? Few who have read that truthful sketch of Dickens, the "child-wife," will forget the pretty helplessness of Dora; but, although this reads well in a novel, very few such girls, and there are many of them, will meet with husbands as indulgent; for men like to see their horne well ordered, and to feel the comforts of good management. Every girl, or almost every girl, looks upon marriage as the great aim and end of her existence; but unfortunately, she regards it, as it is treated in novels, as the concluding chapter, the entrance to that "and-lived-happily-everafter" state of bliss which we read of in fairy tales. And certainly it is the entrance to a new life, though not one so rose-colored as she dreamed; it takes her from the home where she has been nurtured, cared for, provided for, perhaps petted and indulged, and places her where she will have to be the presiding intelligence; where all will look to her for guidance and instruction; where her happiness and comfort, and that of her husband, will depend upon how she is qualified to fulfil the duties she has undertaken. Mothers, wishing to have your daughters well married, how have you prepared them for the duties you well know they will have to perform? Perhaps you were domestically educated, for our grandmothers were more careful 
in their generation, and thought it no degradation to be good housewives! Perhaps you have had to fight your way through a host of painful lessons, of annoyances, losses, and domestic worries. Do you never look back upon that miserable time, and feel anxious to spare your children a similar probation? Life, even at its best, has enough of painful experiences, without our doubling them by neglecting to teach each individual, in proper time, the things she will be expected to know when she reaches woman's estate.

Every housekeeper should keep a strict account of all her expenditures; should see that each bill is receipted when paid; should file all receipts, and keep them for a year at least-we should rather say two or three. All housekeeping bills should be paid every week, for it is easier to pay small sums than large ones; and besides, the correctness of the bills can then be ascertained. The mistress should look over each one herself, and thus she will detect, and can check, any inaccuracy on the part of the tradesmen, or extravagance on the part of her servants. Should she be her own housekeeper, let her deal regularly with respectable tradesmen, for they will rarely risk losing a good customer by sending bad goods. Bargain-hunting is always perilous, even to good julges. Cheap tea, coffee, sugar, \&c., are all adulterated; cheap vegetables and fruit are generally stale ; cheap meat is that which has been sent ready killed to the market, and therefore is by no means as fresh âs might be wished; and cheap poultry and fish are to be regarded with very great suspicion.

Those who have store-closets will find their advantage in purchasing some things wholesale. Candles should be bought in the latter part of summer, when they are usually cheaper, and a store laid in, for they improve by keeping. So does a single maid-of-all-work, or general soap. Coal, too, should be ordered in July or August, and if there is cellarage, a stock for the winter laid in. Many groceries may be purchased in quantities at considerable saving. The same remark applies to bacon, butter, and cheese ; but unless there are good dry storerooms, these latter. cannot be kept. It is useless to make pickles or preserves unless the house is dry-in damp localities these things mildew and spoil; nor are we sure that in small families it is economical to make them at all, they can be bought so reasonably. All stores should be kept by the mistress, and given out as required.

All good homekeepers will provide themselves with weights and scales, and thus be prepared to check the quantities of stores sent them by their tradespeople, who are as liable to make errors in weighing as in casting their bills. We cannot too particularly impress this upon the attention of our readers, as an essential means of protecting themselves against errors in weight, whether arising from accident or design. Many heads of families are exceedingly particular about the price of their purchases, who are utterly regardless whether or not they have the weight they paid for. Tradesmen are aware of this trust reposed in them, and too often take advantage of it. Unlike the symbol of Justice, who, as she holds the scales in her hands, is blind to all partialities, some tradesmen (some especially amongst the class who are purveyors to the poor) are too open-eyed to see that they descend in their own favor.

In the realm which our housewife is to govern, order, cleanliness, punctuality, and economy must be the fundamental principles which, by precept and practice, she endeavors to enforce. It matters little whether her subjects are limited to 


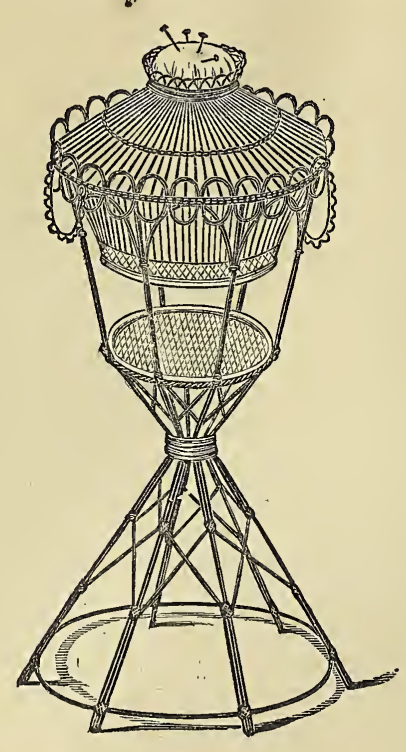

Work Table.

servant, as it is now the fashion to call this domestic, or whether she reign over two, three, or more "helps," still the principle must be the same; a certain routine of daily and weekly duties must be laid down, and it lies with the mistress to see that not only these are performed properly, but that all incidental matters, are likewise attended to. However wealthy a family may be, they are not willing that their houses and furniture should be injured by neglect or mismanagement. Money can enable a man to hire more domestics, but it cannot provide that these persons shall be cleanly, diligent, trustworthy, and painstaking; it cannot secure him from the consequences of their ignorance, their carelessness, their extravagance. Nothing but the supervision of the mistress, or a good housekeeper, can do this. If, then, a rich man, who can pay the best wages, and hire the most experienced servants, finds still that he lacks something, how much more will one of moderate income or of limited means suffer, should he not find in his wife a housewife?

What I am now about to say, some of my readers may think perhaps out of place; nevertheless I shall say it. A woman cannot really do her duty as a wife, mother, or mistress of a family, unless she is fully sensible of the importance of health, and gives to all sanitary measures their due attention. With loss of health come diminished powers of usefulness. Languor and delicacy in a wife may call forth the sympathies, but do not increase the comforts or happiness of a professional or business man; neither do they render a woman more inclined for, or equal to, the performance of her part in domestic life. And too many of our young girls render themselves languid, feeble, and delicate, by inattention to the commonest requirements of human nature. The crying evil of small towns is usually the want of baths attached to houses, and the small size of bed-rooms; now these are in general accepted as things which must be endured, and little or no attempt is made to palliate them. All medical men, however, agree that plentiful ablutions of the body with cold or tepid water, and a good supply of fresh air in every sleeping and sitting-room, do more to preserve health than all the drugs in the pharmacopœia. And next to these come early rising, avoidance of late hours and crowded assemblies, regular exercise in the open air, attention to diet, and abstinence from pernicious viands, as pastry, sweetmeats, rich gravies, unripe fruit, \&c. Pork, veal, and various kinds of vegetables can only be eaten sparingly and occasionally by some persons. Spirits should only be used medicinally, that is to say, at times when common sense tells us they might be of benefit. To take them habitually is equivalent to slow poison.

Besides the benefit a woman derives in 
her health and person from attention to all matters relative to personal care, she will gain another in the effect of her example upon her dependants; for we are all, to a certain extent, creatures of imitation, and prone to follow example, be it good or bad. Servants who see before them one who consistently practises the virtues of economy, regularity, personal cleanliness, and general neatness, will never run diametrically counter to all this, but will in some degree shape their conduct accordingly, while all the precepts in the world, without practice, will but go in at one ear and out at the other.

Where only one or two servants are kept, the mistress will do well not to leave her chamber before she has opened her windows, and laid the bed-clothes back over two chairs so as to insure the sheets and blankets, heated by contact with the body all night, being well aired and cooled. No bed should be made, or night-dress folded up, until it has been aired, and suffered thoroughly to cool for at least two hours. Nurseries should be aired while the children are at breakfast, and while they are taking their morning walk. Dining and drawing-rooms require a current of fresh air passed through them at least once every day, to dislodge all the stale atmosphere tainted by the smell of food, flowers, \&c., and by having been inhaled by those using the rooms. Many of our readers have doubtless been struck, on entering some houses, by the close, faint, unwholesome smell they, coming from the fresh air, at once perceive. Those who dwell in it habitually are not conscious of it. They dread the chill of fresh air, or the dust it will bring with it into their rooms; and therefore shut it carefully out, and cherish in its stead a species of slow poison-a heavy atmosphere loaded with all sorts of pernicious gases.

Light, too, is another forbidden luxury in some houses. Heavy Venetian blinds jealously protect the delicate hues of the curtains and carpets from its influence, and the inmates consequently fade instead of their upholstery; for a human being can no more do without light than can a flower, and we only need place this latter in a cellar for a few days, and we shall see how it will look. It must not, however, be supposed that we would recklessly suffer the noonday sun to shine on our damask curtains or tapestried carpets, or that we should open our windows when rain, hail, or snow beat full upon them; all we wish to do is, to advise such a use of the choicest gifts of Providence, as health requires and common sense would dictate.

Nor is it only with a view to exercising a salutary influence upon her domestics and strengthening herself, that we would counsel our housewife to pay strict attention to all matters of sanitary importance. A female writer of some celebrity has said, "If, before marriage, a woman has been deluded into the notion that a multiplicity of small ailments invested her character with an interesting kind of delicacy, the sooner she becomes well after marriage, the better for herself and all around her."

Now we do not intend to assert that there are not many men who are unwearied in their tenderness in time of illness; but this we must say, that there are thousands more who "vote sickness a bore;" who have little sympathy with, little tolerance for it; who married to have a cheerful companion, not a drooping, languid invalid, to come home to; and who soon begin to seek elsewhere that companionship and cheerfulness they have failed to find at home. And alas! when a man's love has once been dimmed, or alienated from his wife, it never wholly recovers its lost lustre, but remains a mere mechanical matter of duty or honor, and too often not even 
that. Matrintony may bind a man to his wife legally, but herself only can retain her empire over his heart; and to do this, she will need even more than her former charms and attractions and fascinations, besides a vast variety of other attributes which her new position will require of her.

Women little dream what they peril when, after marriage, they neglect the accomplishments, the tasteful dress and adornment, the charming coquetlerie of manner, which enthralled the lover. They not only risk their happiness, for it depends on him, but they neglect what ought to be their highest ambition, that of proving how much dearer is the wife than the mistress, and of rendering his home a refuge from cares, a scene of tranquil happiness, of social enjoyment, and of real comfort. Milton is not uttering a merely poetical exclamation, but a great truth, when he says-

"For nothing lovelier can be found

In woman than to study household good,

And good works in her husband to promote;"

for a woman who is true to herself will inevitably be the better angel of her husband. Contact with her true heart, her gentle pursuance of all her duties, and with her cheerful, rational, and earnest spirit, will restore the tone of his mind, defiled, saddened, rendered morbid, it may be, by contact with the outer world and all its chicanery, its worry and its debasing influences. Wisely are all things ordered, if we would not, by our follies, our impatience and self-seeking, derange them. Beautiful is the mission of woman, if she would but see it in all its holiness and brightness.

Those who have a home which they can make happy, will not sigh for contact with the outer world, to be permitted to wrestle and contend among its fierce trials and the fiercer spirits that struggle there for daily bread; or despise the peaceful path of domestic duty, which, although it has its trials, is yet in a great degree sheltered; or reject the gentle ties of wife, mother, sister, to study some learned profession, and rush into those haunts and paths already too crowded with the sterner sex. Such must be the lot, nevertheless, of many women, whom necessitous circumstances have forced into an unnatural position.

Our "Inodel housewife" believes with us that we should endeavor a

"Well ordered home, man's best delight, to make; And by submissive wisdom, modest skill, With every gentle, care-eluding art,

To raise the virtues, animate the bliss, And sweeten all the toils of human life; This is true female dignity and praise."

So turn we now again to more practical matters.

There are few things more perplexing at first to young housewives than the momentous question of dinner. A social dinner party, and a quiet family dinner, require equal consideration. We once heard of an old bachelor who, to save himself the daily trouble of stating what he would have for dinner, drew up a programme of dinners for every day in the year, and handed it over to his housekeeper; and a lady has lately published a pamphlet, entitled, "What shall we have for dinner, in order to save idle folks the trouble of thinking."

Now, a good cookery book, a short walk round the region where the marketing is done, and a knowledge of family likes and dislikes, will generally enable even a novice to arrange this important matter, at least so far as the ordering goes, the cooking being another consideration. Joints should always, when weather permits, be purchased fresh, and then hung as long as is deemed requisito to fit them for eating. A knowledge of the sauces and condiments appropriate to every dish, is a subject well worth attention. The cooking of regetables is an 
important point, and one in which we mcal of the tradesman oughtnot to emumay derive much useful instruction from late the entertainments of the higher the French. Vegetables cannot be too classes; but if merely two or three dishfresh; in large towns we can form no idea how little we know of the real flavor and delicacy of green vegetables, accustomed as we are to have them at least a day after they are cut.

Fish should be chosen by touch and look. If it feels flabby, and looks pale about the gills, and dull about the eyes, it is to be avoided; firmness of body, brightness and fulness of eyes, and ruddiness of the gills, are signs of freshness. Urabs and lobsters must be selected by weight, not size, and the olfactory organs employed to test their sweetness.

We have already spoken of the desirableness of dealing regularly with respectable people, but no rule is without its exception; and those who are pretty good judges of articles of provision, may often obtain some variety by looking about them. Practice and habitual attention and observation, and the wholesome annoyance of one or two blunders, will soon give experience.

We would advise that our housewife should see occasionally that all the cooking utensils are kept clean; that there is no waste or extravagance, that the ends of loaves, spare bits of cut bread, bones of meat, cold vegetables, \&c., are not recklessly thrown about. There are in every neighburhood only too many poor to be found, who will most gratefully receive the scraps from the richer man's table; and a little inquiry will always find some family on whom such odds and ends may well be bestowed. The tealeaves should be regularly put by in some vessel, to be used in sweeping.

Perhaps there are few things in which the respectability of a man is more immediately felt, than the style of dinner to which he may accidentally bring home a visitor. Every one ought to live according to his circumstances, and the es be well served, with the proper accompaniments, the table-linen clean, the small sideboard neatly laid, and all that is necessary be at hand, the expectation of both the husband and friend will be gratified, because no interruption of the domestic arrangements will disturb their social intercourse.

Hence the direction of a table is no inconsiderable branch of a lady's concern, as it involves judgment in expenditure, respectability of appearance, and the comfort of her husband, as well as of those who partake of their hospitality. Inattention to it is always inexcusable, and should be avoided for the lady's own sake, as it occasions a disagreeable degree of bustle and evident annoyance to herself, which is never observable in a wellregulated establishment. In doing the honors of her table, the mode of carving is also of importance, and will be treated of in a future chapter.

The mode of covering the table differs in taste. It is not the multiplicity of things, but the choice, the dressing, and the neat, pleasing look of the whole, which give respectability to her who presides. The table should be furnished with more than the necessary quantity of plate, or plated ware, and glass, to afford a certain appearance of elegance; and if accompanied by a clean cloth and a neatly dressed attendant, it will show that the habits of the family are those of gentility. For a small party, or a títe-àtite, a dumb waiter is a convenient contrivance, as it partly saves the attendance of servants. The cruets should be looked to and filled every day an hour before dinner ; and much trouble and irregularity are saved when there is company, if servants are accustomed to prepare the table and sideboard in similar order daily: Too many or too few dishes are extremes 
not uncommon: the former encumbering | room well lighted, of comfortable temthe dinner with a superfluity which par- perature, and well rentilated. The pleastakes of vulgarity, whilst the latter has ure of eating a good dinner is greatly enthe appearance of poverty or penurious- hanced when comfort is studied, and ness.

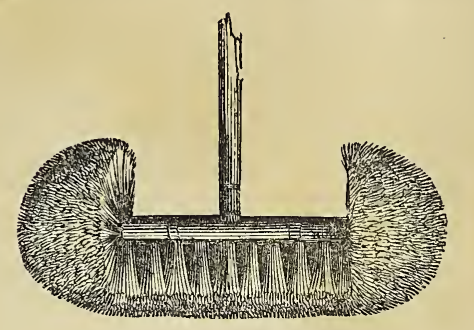

Servants should always be accustomed to lay the cloth and serve dinner as neatly when the family is alone as when company is expected; they should likewise be taught to bring up and place on the table or sideboard everything likely to be required during the meal, and not have to leave the room repeatedly on trifling errands. The mistress should glance around to see that all is there; and if she perceives omissions, mention them before dinner commences. Servants should also be taught to wait at table without bustle or noise ; to remove plates, $\&$ c., without rattling them; to open and close the doors gently; to lift covers from dishes so as not to let the drops of condensed steam fall on the table or those seated at it. If these things are ordinarily insisted upon, the mistress of the house will not, when she gives a dinner party, sit on thorns, trembling lest some gaucherie be committed.

Those who would give dinner parties, must, generally speaking, if their niénage is small, hire a professed cook. A small, well cooked, well chosen dinner, is far preferable to a table crowded with dishes. Symmetrical arrangement of the dinner table, too, is a powerful adjunct. The silver should be bright, the glass sparktaste gratified.

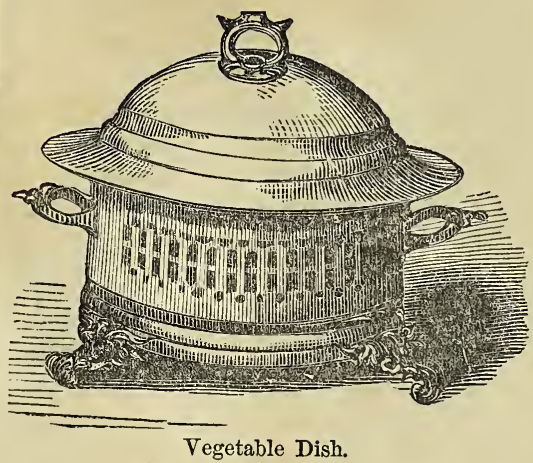

The wines, if you give any, should be good; it is better to give only one or two kinds, and let those be good, even though they be only old-fashioned port and sher-

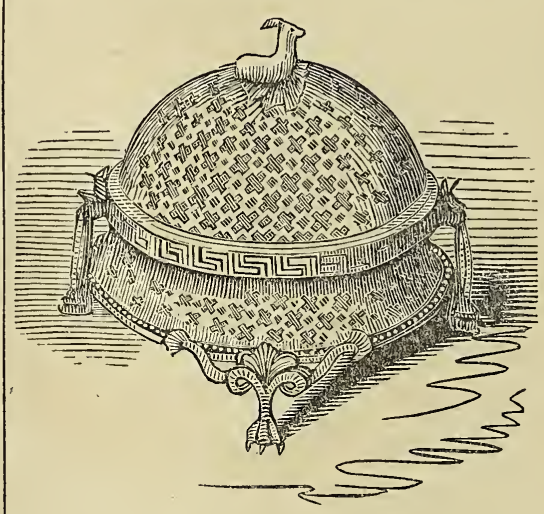

ry, than to aim at greater things, and set before the guest those "cheap and nasty" imitations of other vintages.

Choose the company and arrange them as carefully as the dishes. Ill assorted guests are difficult to please, while persons who assimilate find additional zest ling, the table-linen pure and snowy, the in their social enjoyment. 


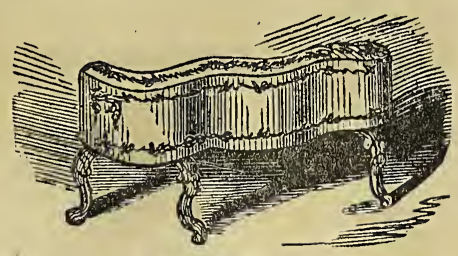

Vegetable Warmer.

The dessert should be well selected, and more choice than plentiful. By choice,

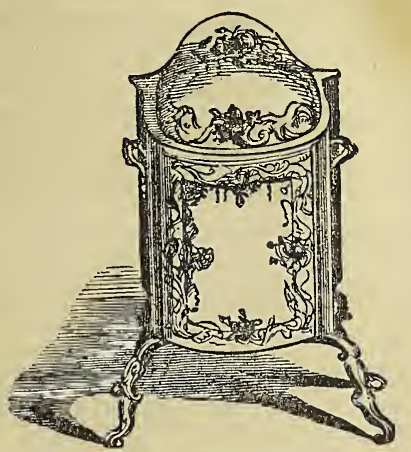

Plate Warmer.

do not let us be understood to mean extravagant, consisting of fruits not yet in

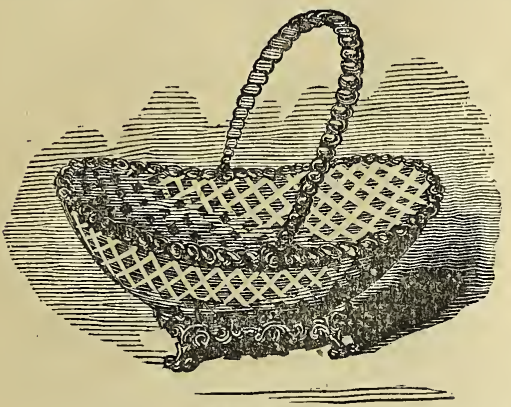

Cake Basket.

season nor having their proper flavor, or of preserved fruits, or fancy confectionery. All these are prejudicial to health, and we cannot understand why people who dine out should be tempted to eat indigestible things; why what ought to be a means of social enjoyment, should be made a matter of form, ostentation, and discomfort. Let the dessert consist of fine specimens of the fruits in season, backed in winter by a few dried fruits and biscuits.

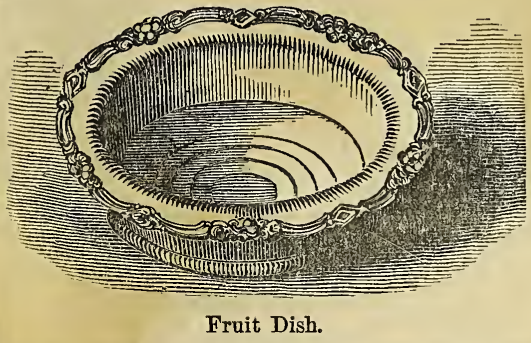

Soyer's housewife thus describes her dinner:-The first thing to be looked to is the lights ; these ought to be so placed as not to intercept the view of any person at the table, but at the same time they ought to be enough to show everything off to advantage. I prefer removing some of the lights from the table to the sideboard when the cloth is removed, as the light after dinner ought to be more subdued. In laying the cloth, we place it over the baize and remove it after dinner, as Mr. B. says he likes to see the mahogany; for when he asks a city friend to come and put his feet under his mahogany, it looks rather foolish if he never sees it. I have, as you know, my table rather wide, that is, six feet. and I generally place a vase of flowers in the centre, as I think their freshness and odor add greatly to the appearance of the table, and admit a flanc on each side. We prefer the

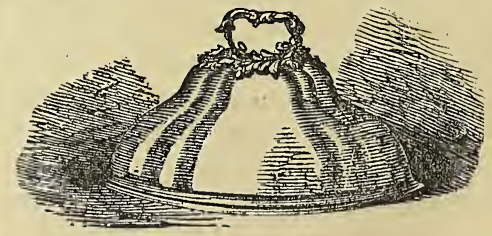

Dish Cover.

old English plan of taking the top and bottom of the table, instead of me and Mr. B. together at the side. 
"The cloth being laid with its proper side uppermost, I order a napkin, two knives, two prongs, two tablespoons, and two wineglasses to be placed for each person, a saltcellar between every other; that being a condiment which every one nses, though often wrongly; the cruetframes and other requisites are kept on the sideboards. I then have the fish and soup served together; the potatoes and sauce on the sideboard; I serving the soup, and Mr. B. the fish ; and often a little dish of fried fish, such as smelts, \&c., to remove the soups. This gives me an opportunity of seeing that my guests are properly attended to, and also leisure to take wine with any gentleman who challenges me. During the time this course has been progressing, the cook has had time to dish up the removes nice and hot, and get all up close to the door, as I like as little time as possible to intervene in changing the dishes; and these consist generally of variously dressed chickens, which I have before me, as this gives an opportunity for the gentleman on my right to display his gallantry; Mr. B., who is a capital carver, either has a saddle or a haunch of mutton, or a quarter of lamb before him. the rest of the dishes consisting of a tongue and entrées. I select those most easy to carve, and also easy for the cook to prepare. This is a period of dinner where a great deal depends upon the attendants; they should know almost by the look what this lady or that gentleman requires, and what kind of regetables to hand them; a first-rate butler should be able to judge by the physiognomy to whom he should offer mint sauce with the lamb, and who prefers cayenne; on their attention and hot plates depends the success of the substantial part of the dinner.

"As soon as I see that all are served, and words are few in consequence of the organ which utters them being employed in another way, I give a look to the two

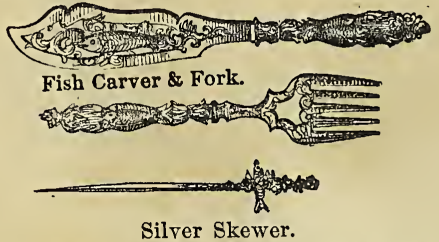

servants which they understand, and immediately two reports are heard-they are from two bottles of champagne, opened at the same time by the attendants, who have each a salver with six glasses on it; this takes but a short time to serve, and prepares the palate for the entrées, which generally get praised; indeed, my cook would think something was wrong if two of the dishes did not go down empty. By having the champagne thus, I find it goes much further than if only one bottle was opened at the time, there being sufficient left in the bottles for a gentleman to challenge a lady to take champagne with him. If I

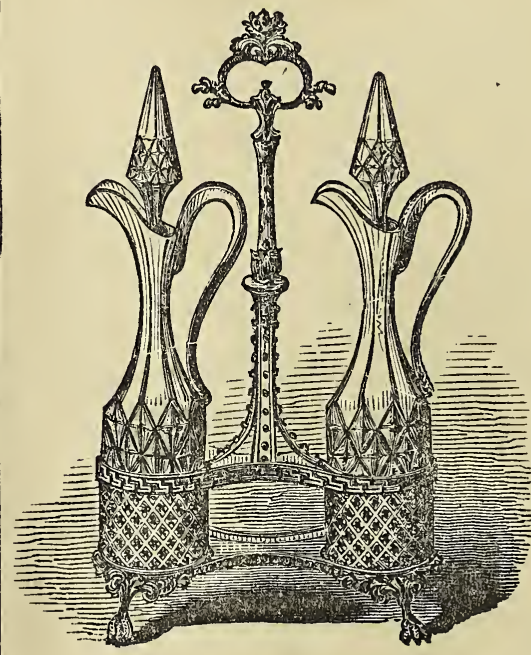

have game I remove the top and bottom dishes with them, and make the sweets a separate course, taking care to have cold plates for the jelly, and having the 
liquors handed round when the sweets are on the table; one cheese I place opposite Mr. B., and macaroni opposite myself. In the dessert I generally introduce some new importation, such as bananas, sugar-cane, American lady apples, prickly pears, \&c. ; these also give a subject for the gentlemen to talk about when the ladies have left, as free trade, colonial policy, \&c. About half an hour after the dessert is on the table, and when I see that the conversation is becoming less general, I retire to the drawing-room; the servants then remove the used glass and plates, and Mr. B. introduces some of his choice claret or Burgundy in ice coolers."

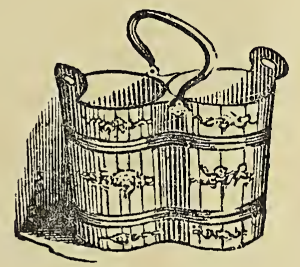

Wine Cooler.

There are so many varieties of evening parties that no directions can be given respecting them. As a general rule, we would advise that they should be as simple, unostentatious, and sociable, as possible. It is the extravagance which has been introduced into these matters, the insane desire of outvying each other felt by individuals, which is the bar to real social enjoyment, and prevents us from being as lively a people as the French. Why cannot we meet to converse', have music, dance, or amuse ourselves in any rational way, and be content with light, simple refreshments, and a sincere welcome? Surely such réunions are more enjoyable than crowds, grand suppers, superb toilettes which are scarcely seen in the crush, and suffocating heat or currents of cold air. Such assemblies upset the house of the party-giver for a week at least, weary and worry her, and are criticised most severely by all her "dear friends" who did not enjoy themselves, nor receive the attention they expected. In them all is most certainly "vanity and vexation of spirit;" there is no pleasant converse, no comfort, no intellectual enjoyment; weariness, lassitude, headache, and expense, are the only concomitants of such parties. May our "model housewife" have courage to reject them altogether.

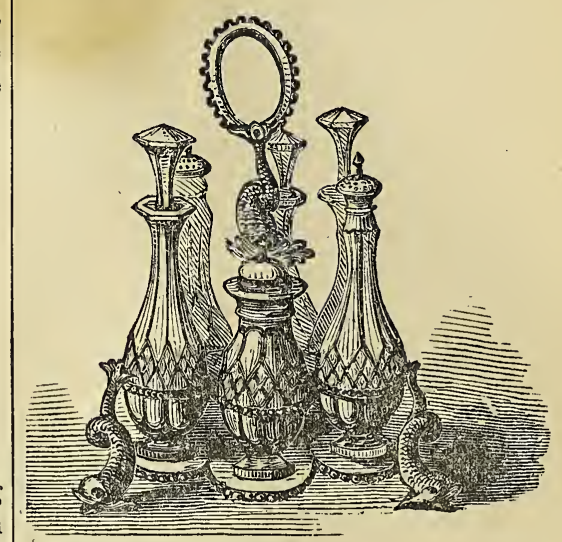

\section{CHAPTER VII.}

Few persons would believe what a waste of money and property arises simply from want of care. We cannot, of course, expect this carefulness from ser' vants, from those who are often thought less, inexperienced girls, coming from a home where there was little worthy of care, perhaps from a place, where there was no time to bestow due attention on the various duties. Few servants stay long enough in a situation to become at. tached to, and, as it were, identified with 
it and its belongings; they are ever striving to "better themselves," or, in other words, obtain higher wages. It matters not that they are comfortable. that they like their employers; that they are welltreated, and not over-worked;-should some acquaintance or relative be receiving higher wages than they are getting, they will sacrifice all to "go and do like. wise;" and too often not in order that they may contribute more to lay up a fund in case of illness or misfortune, but to be able to dress more smartly, or indulge in some favorite pleasure. All this is much to be regretted, as it too often entails loss of health, comfort, and respectability on the young woman. But it is not this point from which we now have to regard it. It is the mistress, not the servant, whom we are addressing, and whom we would guard from the troubles which must befall her, if she, too, be careless, if she does not know and see that her property is well managed and regularly and properly attended to.

We have-before said that no household knowledge can be deemed superfluous, or ought to come amiss to a woman, who is to be at the head of a house. Surely she is not the less a lady because she understands how her house should be managed and cleaned, and kept tidy?-because she can teach her servants when they are ignorant, put method into their proceedings when they are careless, and quietly but attentively look on and superintend when they are tolerably efficient? We are all macie of the same clay, though difierently tempered by education and position; we are all subject to vicissitudes, and cannot tell what a day, or even an hour, may bring forth; is it not then, wiser to be armed at all points, prepared for all circumstances, equal to any emergency?

These preliminary remarks have been made to preface the directions we are about to give for the daily routine of a servant's duties, which else might have seemed out of place here, had it not been our opinion that, in order to manage servants well, a mistress should fully understand what their actual duties are.

Early rising is indispensable if a servant would do her duty; it is not possible that the rooms can be dusted, the fires lit, the breakfast got ready, and all the little incidental jobs done, unless a servant is down stairs at least two hours before breakfast-time. We should fix six o'clock as the proper hour at which work should begin all the year round; for in winter even more has to be done than in summer, and few things are more unpleasant than to have servants pottering about their work" all day, instead of getting through the chief and dirtiest part of it in the morning hours. If we make a call, which gives us the most favorable impression of the family, - to see a tidylooking, clean servant, answer the door; or to have it opened by one who looks as if she had deemed it necessary when blacking the stoves to black her own per son also?

A servant should be trained to rise about half-past five, throw open her bed, and her window, too, when the weather permits; unclose the shutters of the staircase and dining-room, open the windows of this latter to air it; pass into the kitchen, and open the shutters and windows there; light the kitchen fire; well rinse the kettle, and fill it with fresh water; see that the boiler is well supplied with water, and then proceed to prepare the room required for breakfast.

The rug must be folded up and remored, and the proper cloth laid down in front of the fire-place before the grate is touched (we are supposing that it is winter); the box containing the black-lead and brushes for a black stove, or the emery paste, and leather, brushes, cloth, \&c., for a polished stove, and the scuttle containing coal, wood, \&c., must be brought up. 
Once a week, at least, the soot should be gently swept down from the chimney into a shovel, as it otherwise gathers all round the lower ledges, and is very apt to take fire. The stove must be polished with a brush, or a cloth, according to its nature, every morning, and thoroughly cleaned at least once a week. The fire may then be laid and lighted, and in doing this there is some art. Where one girl will light and re-light the same fire three or four times over, consuming in each attempt a quantity of wood, and paper, another will, with less wood, kindle a brisk fire at once. The mystery lies in obtaining a perfect draught of air through the pile of materials, and placing those in juxtaposition which are most combustible in nature. Where this is properly done there will be little smoke, and great saving of fuel ; but fire-lighting requires the use of one's senses and some skill, commonplace a matter as we may think it.

This much having been done, the sweeping comes next. Now, it will not be requisite thoroughly to sweep the carpet all over above once a week; on the other six days those parts of the room chiefly used, alone require sweeping, and this may be done with a dust-pan and a soft hand-brush. Then comes the dusting, and in doing this a feather brush should be used for the frames and chair covers, and a duster for the furniture. On the cleaning day the carpet must be well swept with a carpet-broom and tealeaves, the furniture well cleaned and rubbed, the mantel-piece and ledges washed, the inside of the windows cleaned, and every ornament well dusted.

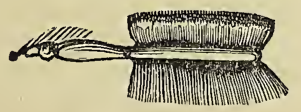

Window Brush.

The street-door steps should be clean- ed, the mats shaken, the passage swept, and the brasses polished before the family come down.

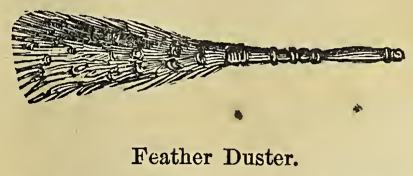

The breakfast is then to be prepared; the cloth laid, the breakfast service properly arranged, the ham or eggs, or whatever it may be, cooked, the toast made, the butter set in clear, fresh water, the coffee prepared and the milk boiled.

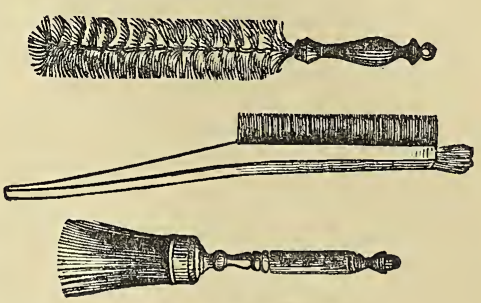

Brushes for Cleaning.

The servant next proceeds to the bedrooms, opens the windows, lays the bedclothes back to air, and turns up the mattresses or feather-beds in each room; then empties the slops, cleanses and rinses all basins, ewers, bottles, \&c., wipes up all slops, and brings fresh water to supply all wants in each room. The beds are then to be made, and the rooms dusted. On cleaning day, which should come for each room once a-week, the chamber utensils must all be well washed in warm water, the carpets taken up and shaken, the floor scrubbed, the curtains shaken, and the furniture cleaned. During the summer the floor under the bed should be washed over three times a-week at least, to remove all dust and flue.

Before all this can be finished, the breakfast will have had to be removed, and this should be methodically done; the china being gathered on a tray with- 
out either fuss or rattle, the crumbs brushed from the cloth, and this latter doubled up in its original folds, and any crumbs which may have fallen on the flowr swept up into a dust-pan.

The servant will now get her own breakfast, and then wash up and put away all the breakfast things, having first set aside all the eatables, giving the scraps to the cat, and taking care that nothing is wasted.

The up-stairs work having. been done, the candlesticks and lamps should be cleaned and trimmed; and then the knives cleaned. Where only one servant is kept, she will ere this have had to think about dinner, and manage so as to make the earlier preparations for that important matter between whiles. A mistress should always inform her domestic charged with such duties, what she intends having for dinner, when the girl is removing the breakfast things, otherwise she cannot possibly arrange her daily duties so as to attend to the cooking without neglecting them; and the mistress should, as far as possible, endeavor to arrange her dinner so as to suit the household duties of the particular day for which she is catering. For each day should have its special duties; as, Monday, the wash for towels, dusters, servants' clothes, \&c., and looking up the clothes for the laundress; Tuesday, cleaning the attics; Wednesday, the best bed-rooms; Thursday, the drawing-room ; Friday, the dining-room and plate; and Saturday, the hall, staircase, and kitchen, covers, \&c. Such regulations once laid down, the servant will know what duties each day requires of her; and the mistrcss, being aware what has to be done, will be able to give her orders accordingly,-for nothing can be done without good management in a small household. Both mistress and servant must exercise forethought. or the whole day will be one sccne of hurry, scurry, and discomfort.
The next thing, then, is dinner, and this meal having been served and removed, the servant must, if the family are in the habit of retiring to the drawing-room, run up and see that all is right there; the fires burning and curtains let down, if in winter, or any little customary summer arrangements made. Then comes washing up the dinner things, and preparing for tea, and serving that at the proper hour.

While the family are at tea is the best time for the servant to go up to the bedrooms, turn down the beds, close windows, light fires, or perforin any other customary or necessary duty.

The tray has to be removed, and cups used at that meal, washed and put away. The tea leaves should be squeezed tolerably dry. and put away in an old dish or basin, for sweeping. All window shutters should be closed and doors bolted at dusk, both in winter and summer. If supper is taken. it is usually carried up on a tray covered with a cloth; after that comes the putting out of all fires and lamps, the fastening the hall door, and then to rest.

Any one who takes the troulle to reflect upon the multifarious occupations of a female domestic, in a family where but one is kept, cannot but perceive the absolute necessity of a regular plan of proceedings, and also of the need there is for consideration, patience, and kindness. With all the activity and good-will in the world, it is physically impossible that a girl can be in two plices at once; and yet she may be just performing some nice operation in cookery while a visitor may be knocking at the door, and her mistress ringing impatiently to have something done towards tidying herself or the apartment. How is this to be managed? It can only be settled quietly by the mistress bestirring herself, and aiding in smoothing the difficulty. Where two or more servants are kept, one should always 
be tidy and ready to answer the door, and the lady of the house and her apartments should always be mis à quatre épingles, or, in other words, in such or-, der that a visit from royalty itself would scarcely create any bustle. Indeed, the test of a well-arranged establishment is the absence of all fuss. The advent of relatives from the country bent on finding a home and a bed there, the arrival of unexpected guests to dinner, or any such unexpected events, will, of course, slightly derange the economy of a household, especially if it be a small one; but our housewife will not allow this to be visible. Quietly and without confusion she will take opportunities of giving the necessary orders; and if her servants are regularly trained, they will comprehend and second her at once. In every house spare sheets, blankets, and bed-clothes should always be kept aired, in case of their being required on any sudden emergency, and this can be done by laying them between the palliasse and mattress of any bed in regular use. We have before spoken of the advantage of always having the store-closet well supplied. It is a great mistake to fancy that anything is saved by purchasing articles that will keep, in small quantities, and certainly the loss of time incurred by having constantly to send out for trifles, is both a great waste and a great inconvenience. No servant can get through her work properly if she has repeatedly to run out on errands, or if she is called off frequently on frivolous pretexts. A nistress is quite right in exacting from her servants a regular and proper fulfilment of all their duties; but on her part she must give them time and opportunity to do this. If she sees they want method, she must try to teach them; and through all must remember how defective their early education has too often been, how little preparation they have received for the path of life they have to follow, and be patient with them. The great social evil of the present age is, that females are rarely trained with reference to their probable duties. This evil pervades all classes, from "the lowest to the highest. The daughter of the millionnaire is seldom taught to consider how great are the social responsibilities her wealth and position impose upon her,-to regard herself as a steward of the Almighty, a medium through which His blessings may flow to those poor and unfortunate ones, whose lot is cast so far apart from hers. If she learns at all that "to whom much is given, much will he required," she probably imagines that much to be in the way of style, dress, and accomplishments; which, to a certain extent, is not far wrong, for the circulation of money is a commercial advantage, and those elevated in position should also be elevated in mental and moral attributes; but this is not all required of them. There is a duty to the poor, the duty of kindness, charity, and mercy. If all who have it in their power would but attend to this a little, how much good might be done! and what sits so gracefully on woman as such gentle deeds and holy virtues?

Then, as we have before said, the daughters of the working class are hardly prepared for their position as wives, mothers, and housewives; and the children of the poor have too often a still worse bringing up, being reared amid misery, poverty, dirt, and vice, if not in their own fanilies, at least in many of those around them.

Vast as has been the general spirit of progression in this age, it is marvellous that these every-day matters have been, practically speaking, so totally overlooked. There is much theory, but the greater part of it is speculative and utopian. We are not advocates of systems, but would 
recommend good common sense instead; for systems can only be applied to certain parties and in certain ways, whereas commion sense will cull the best parts of many systems, and adapt them to suit the peculiar position or character it has to deal with,-and thus give to that character a firmness and freedom from conventional prejudices which will, when strengthened by right principles, make it good and useful.

\section{CHAP'TER VIII.}

Arrong the many duties a woman is called upon to fulfil, surely none can be deemed of such paramount importance as those she owes to her children. Now it is by no means our intention to write a chapter of "advice to mothers," or enter upon the subject of the education and general management of little people; all we have to do with the matter here is to notice it as forming a branch of our subject; for the nursery is an important part of a house, and upon its good management much of the comfort, as well as the health and well-being of every member of the family, will depend.

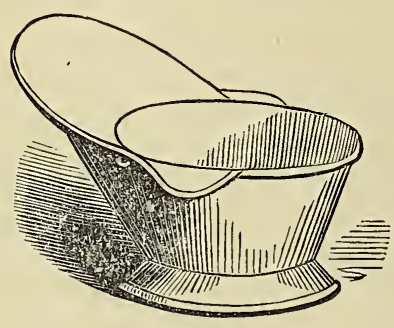

Sitz Bath.

If ever a woman has need of thought, care, and patience, it is when she becomes a mother. Who can look upon that most helpless of all created beings-a new-born infant-and not feel pity for it, interest in it; and surely no mother can bestow her first kiss on the little unconscious innocent without feeling some sense of the responsibilities which now rest upon her-for it is not only a human being, but an immortal soul which is committed to her charge.

Wherever such an arrangement can be made, a good-sized airy room should be set apart for the nursery, and always kept scrupulously clean, well ventilated, and of an even wholesome temperature. Warmth is highly necessary to the health of infants and young children-but not the warmth of a close, over-heated room, for that oppresses their lungs, and makes them delicate and very susceptible of cold. Let the child be comfortably clad, and a natural temperature of body thus maintained. The health of very many infants is impaired by the foolish custom of exposing their little chests and arms by finely-worked low bodies and very short sleeves, tied up so as effectually to prevent their being of any use in the way of covering. Very young children have so little natural heat, that they absolutely require that the temperature of their bodies should be kept up, by means of clothing, to a proper standard.

The furniture of the nursery ought to be very simple, and to consist of no more things than are absolutely necessary. The "bassinet," a wicker basket stuffed very soft and lined within, two and a-half or three feet long, and one and - a-half wide, has been used in England for very young infants, and may be carried about. Cradles and swinging cots are condemned by medical men, and are now less used than cribs. Children's chairs of various sizes, a washstand, shower bath, a child's bath, or a large earthernware basin fixed in a stand of wood, various vessels of earthenware, a nursery lamp for warming liquids when there is no fire. sponges and linen baskets-comprise the requisite articles of furniture. A wire guard for the fire, and 
bars for the windows should never be omitted.

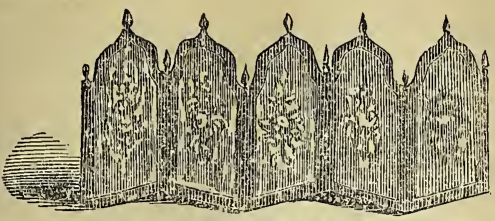

French Folding Fender.

Next to warmth, in the category of matters indispensable to health is cleanliness. Morning and night, an infant or a child under three years of age, should be bathed in tepid water in winter, and cold water in summer. This operation

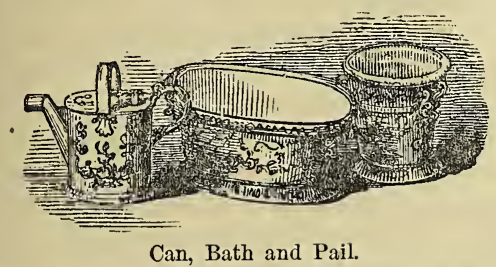

should be performed both thoroughly and quickly, and then the whole body wiped quite dry with a soft towel, and the limbs and back gently rubbed with the hand. The skin of an infant is so delicate that a little dirt, a slight accumulation of powder, any dampness, any rough handling

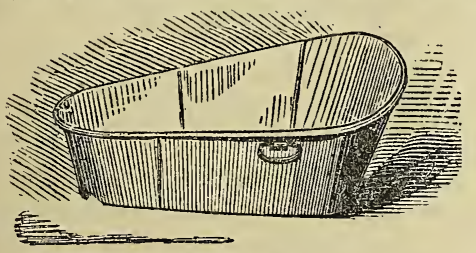

Infants' Bath

or friction with a coarse cloth, will break ur crack it, and often produce a humor which is not easily got rid of. The $c$ othes of an infant, and especially those in immediate contact with its body, should be soft and clean. Its bed-clothes must likewise be kept well aired, and frce from damp and unpleasant smells.

Very young chilären should be kept, as much as possible, in a recumbent posture; the less a child is allowed to sit upright for the first six montlis, the stronger will be its back afterwards. Too strong a light is prejudicial to young children; neither within nor out of doors should their eyes be exposed to anything like glare; they ought likewise to be shielded from high winds. Sleep is a great strengthener of children, when it is

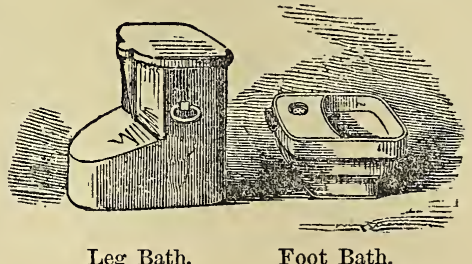

natural, and care should be taken that they lie comfortably as regards position, are warm, and sheltercd from all draughts and from light, though allowed sufficient air. In all things endeavor to establish regular habits from the very first; regular hours for feeding, sleeping, dressing, and undressing, should be observed. Few people can form an idea how much their own comfort and the child's well-being will be promoted by this. Of course, every now and then illness, or some unforeseen event, will interfere with this system, but it must be resumed again as soon as possible. We are aware that many reople will dcem this regularity unnecessary, but from the cradle to the grave, we are all more or less the creatures of habit; and from the time a child first begins to notice anytling, its habits may be said to commence.

When a child is fed, it should be from a proper bottle, and with light, thin food, not given too hot or in large quantities. Farinaceous substances of rarious kinds are best adapted for children of tender age. For the first twelvemonth these may be given in a fluid, and semi-fluid state, and afterwards in the shape of ruddings either 
baked or boiled. Many children will not take meat, and they do not appear to thrive less than others; nature at this early age is generally the best pilot, and we shall seldom err if we follow her lead.

The period of teething is always one of anxiety, and requires additional care and watchfulness; but plenty of fresh air, good and sufficient, but not over-feeding, and an occasional warm bath or gentle aperient when there appears to be tendency to fever, will in most cases ward off serious evils. The less physic a clild takes the better in a general way-not that we would advise mothers ever to slight any actual premonitory symptoms of illness; infantile diseases being fiequently very rapid in their course.

There is no occasion to teach children to walk; when they are strong enough, they will invariably find out the proper use of their feet; let them lie on the floor, and then sit, and then crawl ; and by-andby they will stand up, and then walk along, holding by something; and then, when they appear capable of doing yet more, encourage them to venture alone, but watch carefully that they do not fall, or they may be frightened, and thrown back some weeks. After a child is a year old, it must cease to be regarded as an irrational being by those about it; a quick, lively baby is always taking notice; its intelligence is becoming daily and hourly developed;-in a word, the first rudiments of education are entering its little mind. This may sound extravagant and fanciful, but what says proverbial philosophy? "With his mother's milk the child drinketh education;" and let any one take three children of from a year-and-a-half to two years old, one of the three shall have been brought up by a quiet or ignorant nurse, one by an affectionate and sensible mother, and the other among a family of children; the first, unless it be a prodigy, will be far behind the second in quickness, and the third will be as much before the second. We would punish any one who mispronounced words before babies, or taught them to call things or animals by ridiculous names; and we would doubly punisl any one who instilled far into the hearts of young children by threats of "bogies," "black men," and such 'like nursery monsters. Many a grown man and woman has found it difficult to overcome the dislike to darkness implanted in them when young children by some nursery bug-bears. Teach a clilid rather to believe that angels watch over it, induce it to be good from a fear of grieving its parents, but never destroy or sully that sweet confidence which is the most blessed part of childhood. Never tell a child a falsehood either; if you promise a thing, do it; if you say a thing, act up to it. Empty threats and vain promises made to quiet a child, teach it afterwards, in its turn, to disregard truth, and make it distrust those whom, next to God, it should love and honor; whereas "example is a constant monitor."

We are not advocates for "cramming" children too early with crude, dry learning; but much may be taught without effort. "Line upon line" may be instilled by such simple stories, such pleasant gossip as children love to listen to, such bright pictures as they delight to look upon and have explained to them. There is in the present age a royal road to all the fundamental instruction needed by children for the first eight or ten years of their lives; the dull, uninteresting paths we had to tread are now decked with so many flowers that they have become attractive and pleasant. Even the very toys and games are made means of improvement; and history, arithmetic, and geography may now be learned as actual amusements.

The physical wants of growing children are a sufficiency of good, plain food at regular hours, exercise in the open air, 
well-ventilated rooms, clothing warm success, we plant it in a fitting soil, we enough to promote health, and sufficient- water it at proper times, we give to it its ly easy in fit not to impede growth or a due proportion of air and sun; shall we free use of the limbs: strong shoes or boots to protect the feet from damp, and a proper allowance of sleep taken at regular hours, - no child under six years of age being suffered to sit up after eight o'clock, and all under twelve being safely in bed by nine.

Late hours, hot, crowded places, as theatres, \&c., and children's parties, are all very destructive of health ; irregularities of all kinds are to be avoided, such as too long walks, any great excitement or over-fatigue; for growing children require all their strength and energies for natural purposes; and being more excitable than grown persons, exhaust themselves the sooner.

It may be thought by some that we are diverging from our subject, but "our housewife," if she be now single, may have younger sisters and brothers, and may probably one day have children of her own, when she will learn

\footnotetext{
" How infinite the wealth of love and hope, Garnerer in th.ose same tiny treasure houses! And oh! what bankrupts in the world we feel, When death, like some remorseless creditor, Seizes on all we fondly thought our own."
}

At all events, the care of children is one of woman's most solemn responsibilities-one of the most sacred of all the trusts committed to her care; for has not a clild an immortal soul ?

Those who live much among children should carefully purify their every thought, word, and action,-for the ductile and impressionable nature of a child, chameleon-like, takes its hue from that of the characters which surround it. "The seeds of first instructions are dropped into the deepest furrows ;" therefore we cannot omit this chapter of our "Thoughts and Maxims."

If we would cultivate a flower with not equally study the constitution and physical requirements of a child, in order to bestor upon it the requisite nurture? for children, like flowers, require studyall are not equally hardy, equally vigorous, equally beautiful. Some resemble hot-house plants, and are frail and delicate; others, like the hardy evergreen, can bear and brave change of temperature and hardship. The system which would be suitable for one of these natures would be injurious to the other-hence, judgment is needful.

Again, if we would train a vine so as to render it luxuriant and productive of fine fruit, we bestow much pains on the arrangement of each branch; we do not neglect the smallest shoot, or wantonly injure or twist the least branch; if we prune, we do so but to remove that which might militate against the health or produce of the tree. Shall we bestow less culture upon the moral education of a child? Shall we not, with equal care, endeavor to remove each evil propensity, and foster each good disposition?. Is not the fruit of tenfold more importance? Pure and innocent is the mind of a child; let no care be spared to preserve it so. Those who would govern children by harshness greatly err; a child never loves the person it fears; besides, all fear is more or less slavish and degrading. Rather teach them to act rightly from a sense of what is right and what is wrong; found their obedience on affection; gire tiem principles of action, and teach them to reason and think, and, above all, make religion their guiaing star.

A child who fears God and honors his parents is armed for the world's warfare with a breast-plate, which, if not invulnerable, at least will turn aside many an 
arrow. Our favorite Tupper quaintly but truly says:-" When his reason yieldeth fruit, make thy child thy friend, for a filial friend is double gain, a diamond set in gold. As an infant thy mandate was enough; as he grows in years, let him hear thy reasons." Believe me, we wantonly trifle not only with our own happiness, but with that of those little ones committed to our charge, when we neglect to watch over the treasures we ought so dearly to prize, when we trifle with the hearts, minds, and souls of our children, ignoring their value, our responsibility, and the awful reckoning which will be required of us. We are all too apt to treat children as dolls, to dress and caress them one hour, and send them out of our way the next, not deeming that beneath the seeming thoughtless gaiety of those little ones there lurks a world of feeling and sensibility. Kindness is as necessary to them as daily food. We do not mean false indulgence, but considerate kindness. An unjust, a cold or harsh word or action, especially if undeserved, penetrates the heart of a child with as keen a sting as it does ours; and who shall say how many have grown up callous and reckless from having their first affections blunted, their feelings and wishes disregarded? Consistent practice is worth a whole workd of precept, and example will influence while words or coercion are fruitless.

Once more, then, we would beg "our housewife" to study what are her duties towards children. We would entreat mothers so to train their daughters that they may never have cause to blush and tremble for their grandchildren. It is no figure of speech to say that " the child is father to the man;" study diligently, therefore, how best

" - to rear the tender thought,

To teach the young idea how to shoot;

To pour fresh instruction o'er the mind,
To breathe the enlivening spirit, and to fix The generous purpose in the glowing breast."

Nor let children, who should be ous blessings, become our torments

"Wbile young our folly, and when old our fear."

Lastly, but not of the least importance in the good training of children it should be rigidly observed by parents, never to show any difference of opinion in their treatment before them. Nothing can be more pernicious to their moral culture, engendering in them habits of disobedience, for they cannot obey one parent without disobeying the other. We earnestly recommend attention to this observance; for, besides the evil of disobedience that is almost sure to follow a difference in opinion from those in authority over the child, the latter is sometimes prone to disregard the instructions and admonitions of both, and set up principles of his own, more in accordance with his inclinations, however erroneous they may be.

A chapter is devoted to cookery for children.

\section{CHAPTER IX.}

Another duty, of great importance, devolves on woman; namely - the care of the sick. From the highest to the lowest none are, properly speaking, exempt from this charge. 'Tis true that those who are rich can hire experienced nurses, but still the responsibility, the anxiety, rests with the mistress, for she cannot hire affection, thoughtful care, and all those little attentions which make the sole comfurt of an invalid; she can merely secure a species of human machine which mechanically performs its duties, and between whiles eats, drinks, sleeps, and comforts itself. There are many excellent and kind-hearted profes- 
sional nurses, but there are also more who become, as it were, petrified by the habitual contemplation of suffering, and who merely regard the patient with an eye to business.

In a sick room the kindness and attention of the nurse often work far greater marvels than the skill of the doctor, for she is there every hour, she sees every change, and can minister to many little wants. Those trifles which make up the events of an invalid, those minor details, so unimportant to a person in health, those whims, and desires, and nervous susceptibilities which appear almost childish to lookers-on, will be studied by a good, conscientious nurse, and overlooked or disregarded by one who either does not feel interested in the patient, or has not sufficient sympathy to induce her to study these matters. In the former case, the invalid will be soothed and cheered; in the latter, irritated and depressed. Surely it is not difficult to conceive which influence must act most beneficially upon the system.

Gentleness, watchfulness, firmness, judgment, some delicacy of feeling and savoir faire, and a truly Chrisțian spirit, are the distinguishing characteristics which will best adapt a woman for fulfilling this phase of her duties. There are many who, from intense solicitude or romantic devotion, will give way to those impulses of feeling which lead them to devote themselves so entirely to the nursing of some beloved relative, that all other duties, and even self, are wholly forgotten. What is the consequence of this error of judgment? Their own health succumbs, and they become not only useless, but render themselves sources of trouble and anxiety. How much better to have husbanded their strength so as to be able to remain useful! That despised article, common sense, would teach us all so much if we would but stop to listen; if we would not mount, each one, our own peculiar hobby. and gallop off at a tremendous rate, heedless of all sign-posts, and often regardless of even hedges and ditches. Affection! feeling! sentiment! nerves! how much has been done and left undone in these names! while poor dear common sense has been decried as a most unfeeling person, and rudely ejected when she strove to make herself heard.

Yet in few places is common sense a more valuable assistant than in the sick room; aided by self-command and good feeling, she will transform the most uninitiated person into an excellent and efficient nurse. Let us hear a few of her fundamental principles.

Speak in a low but perfectly distinct voice. both to the invalid and to any one who enters the room, in order that, al though no unnecessary sound may be heard, the patient's ear may not be fatigued by striving to catch the words, or excited by mutterings or whispers addressed to some one else.

Let your countenance be calm ' and cheerful, your manners soothing and hopeful, and your words such as may cheer or comfort the mind.

Avoid all fuss, all hasty movements, all noises that may startle or disturb; let even your dress and shoes be chosen with reference to quiet.

Keep everything in its place, so that in an instant you can put your hand on it when required; have hot water, clean cups, spoons, glasses, and well-aired body and bed linen always handy; let the air of the room be changed frequently; avoid all bad smells, or remuve them as soon as possible; pay strict attention to the temperature of the chamber, and keep it as even as may be; and regulate the light with equal care.

The furniture of a sick room should be adapted to the wants of the invalid. A reclining-chair will be found convenient; and a rocking-chair for exercise, with a 
movable one for those who cannot walk. The bedstead should be contrived to afford ease by change of position. Dr. Arnott's hydrostatic bed has been mentioned as particularly useful for invalids.

All food offered to invalids should be daintily prepared, and presented in the most careful manner. How often, when one has been longing for food, has he turned from it with disgust, because he has seen the nurse cool it with her breath, or taste it, and then drop the spoon back into it, and present it. Nor is it well to inquire of invalids what shall be got for them. If they express a wish for some particular thing; well and good, let it be got for them, if reasonable; but a trifling delicacy unexpectedly brought will often tempt the appetite ; besides, a sick person, or even a convalescent, is often too languid to be at the trouble of thinking about eating, and would sooner go without; or if he or she chooses something, it may be the very thing which would be improper or prejudicial, and then comes disappointment, and a species of disgust for all else, for in illness the appetite is ever capricious.

Nowhere is cleanliness of such paramount importance as in the sick room. Do not let us be misunderstood here. We do not mean that an immensity of sweeping, scouring, and dusting is to be done, but simply that the chamber must be kept clean and ventilated, that the bed and body linen must be changed often enough to refresh without fatiguing the patient, and that the air must be purified by means of vinegar, or other disinfectants.

As there are so many kinds of illness, no general rules can be laid down, and our friend, common sense, must be allowed to be the special adviser. In one case body and mind may be paralyzed by weakness or languor; in another the body may be agonized, and yet the mind clear and active; while in a third the body may be sane, the mind insane. Hence no one who is a mere machine, guided by certain rules, can be a good and efficient nurse; no one who does not study how to minister to the mind as well as the body, who will not endeavor, to a certain extent, to identify herself with the tastes, feelings, and even prejudices of the patient, can be really useful.

What we have hitherto said applies chiefly to adult patients; to nurse a sick child may seem a far easier task, but is not so. What gentleness, firmness. playfulness, and, above all, what patience is needed in the sick chamber of a child !for in time of illness, a child is doubly a child, almost a baby again.

Every housekeeper should always have a store of old linen, cambric, and calico rags, and old pieces of flannel ; these are easily obtained by saving worn-out linen, flannel, and other garments, or at least the useful portions of them, and treasuring old silk and cambric handkerchiefs. Such things are invaluable in time of sickness for poultices, fomentations, leeches, \&c. Those who have them not will do well to visit a pawnshop, or the emporium of one of those purchasers of wardrobes; and having there found one or two articles of no value but to be torn up, to buy these, bring them home, have them thoroughly washed, and then put them away for use.

It may seem to some that we dwell too long on a sad and painful subject, but it is good for us at times to be serious, to turn from the contemplation of life's pleasures and enjoyments, and look upon its darker pages; for it has been beautifully said by an eminent writer, that "suffering is not a slender, dark thread, winding every now and then through a warp of dazzling brightness, but it is interwoven with the whole texture. It is not incidental, but designed for us; it 
enters into God's purposes ; it has a great | and pomades, or pickles and confectionwork to do, and we know nothing of life until we comprehend its purposes." Again: "Suffering nourishes the tenderest sympathies of our nature; it raises us to energy and a consciousness of our own powers, and at the same time infuses the meekest dependence on God; it stimulates us to cherish and prize the blessings of this world, and at the same time weans us from and lifts us above mere earthly things."

There is no home into which sickness may not come at any hour; and as it is to woman that the office of nurse is in-variably delegated, surely every woman ought to learn betimes all that will best qualify her to become the ministering angel, whose presence shall bless the long hours of pain and confinement. False delicacy, foolish weakness, and all that can militate against usefulness, should be early overcome. We have seen a daughter scream, and weep, and wring her hands, while her mother lay fainting before her; we have seen a mother shriek and fall on her knees, and utter words of agony, when some accident happened to her child. But how did all this unavailing grief benefit the sufferers? How much better she does, who, controlling her feelings, thinks only how she can administer relief. Some might deem her callous, but others know what real feeling is concealed behind the pale face, white compressed lips, and quivering eyelids; to utter it might weaken her, and incapacitate her from rendering that prompt assistance which may even be requisite to preserve life. It is our private opinion, that every woman should have a general knowledge of the anatomy of the human frame, and of the functions of its various organs; also, that she should be acquainted with chemistry to a sufficient extent to prevent her from committing absurd errors in cookery, or ery; and to this we would add, that she will find it useful to understand the qualities and action of the drugs and medicaments in most common use, in order that she may not administer them to herself, or any members of her family "promiscuously," but with some slight notion of what she is about. We do not, however, mean to advise "our housewife" to practise quackery on herself or those belonging to her; for many a constitution has been ruined by injudicious physicking; but there are cases in which some household remedy, promptly applied, will often prevent a long illness, and then every one should know how to act.

Women should understand the making and applying of poultices, leeches, fomentations, warm baths, and similar things; but how few do! They may fancy themselves clever in such matters. We have known a young person who rushed headlong on in everything of the kind she undertook; made a poultice in the kitchen, and wondered that it was cold as ice by the time she had carried it up to the second flocr; applied fomentations, and damped the bed and night-clothes, so that a chill, instead of heat, was produced.

In another portion of our book is given a large list of articles of food for invalids, with directions for cooking; and also a chapter containing remarks on diseases common in families.

\section{CHAPTER X.}

THE STORE-ROOM AND MARKETING.

IT must be borne in mind that an in. ventory of furniture, linen, and china should be kept, and the things examined by it twice a year, or oftener if there be a change of servants; the articles used in the making of perfumes, cosmetics, by servants should be intrusted to their 
care, with a list, as is done with plate. In articles not in common use, such as spare bedding, tickets of parchment, numbered and specifying to what they belong, should be sewed on each; and minor articles in daily use, such as household cloths and kituhen requisites, should be occasionally locked to. The best means to preserve blankets from moths is to fold and lay them under the feather-beds that are in use, and shake them occasionally; when soiled, they should be washed, not scoured.

A proper quantity of household articles should always be allowed for daily use. Each should also be kept in its proper place, and applied to its proper use. Let all repairs be done as soon as wanted, remembering the old adage of "a stitch in time ;" and never if possible, defer any necessary household duty a moment beyond the time when it ought to be attended to.

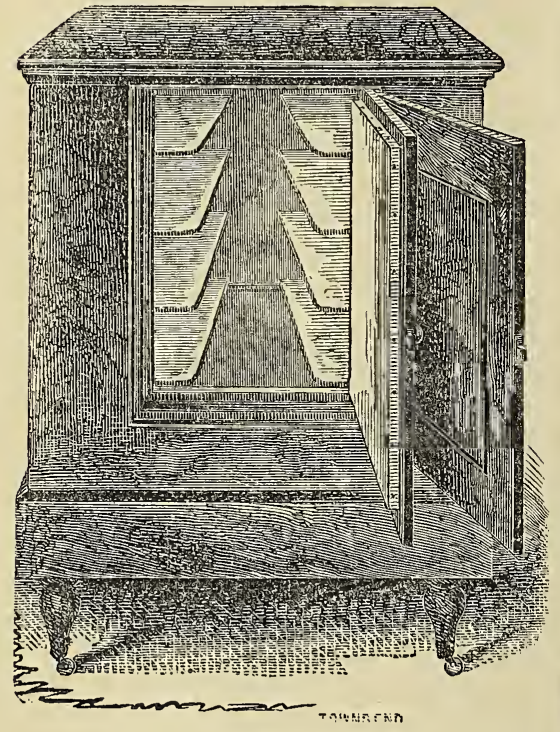

Refrigerator.

A Store-room is essential for the custody of articles in constant use, as well as for others which are only occasionally called for. These should be at hand when wanted, each in separate drawers, or on shelves and pegs, all under the lock and key of the mistress, and never given out to the servants but under her inspection.

Pickles and preserves, prepared and purchased sauces, and all sorts of groceries, should be there stored; the spices pounded and corked up in small bottles, sugar broken, and everything in readiness for use. Lemon-peel, thyme, parsley, and all sorts of sweet herbs, should be dried and grated for use in seasons of plenty; the tops of tongues saved, and dried for grating into omelets, \&c.; and care taken that nothing be wasted that can be turned to good account.

Bread is so heavy an article of expense that all waste should be guarded against, and having it cut in the room will tend much to prevent it; but, for company, small rolls, placed in or on the napkin of each guest, are the most convenient, as well as the most elegant. Bread should be kept in earthern pans with covers.

Sugar being also an article of considerable expense in all families, the purchase demands particular attention. The cheapest does not go so far as that better refined, and there is a difierence even in the degree of sweetness. The close, heavy, and shining white, or loaf-sugar, should be chosen. The best sort of brown has a bright, crystalline appearance, as if mixed with salt, and if feeling coarse when rubbed between the fingers, is better than when more powdery. East India sugars are finer for the price, but not so strong; consequently, unfit for wines and sweetmeats, they do well for common purposes. To pound white sugar, rolling it with a bottle, and sifting, wastes it less than a mortar.

Both soap and tallow candles are improved by keeping, and are best when made in cool weather; at which time it will be prudent to lay in a stock of both. They are, indeed, better for being kept 
eight or ten months, nor will they spoil for two years if kept in a cool place; and there are few articles that better deserve care in buying, and allowing a regulated quantity of, according to the size of the family.

Wax, spermaceti, and composite candles ought to be always purchased in quantities of not less than a dozen pounds, on which an allowance is usually made.

Soap should be cut in pieces that will make a long square, when first brought in, and kept out of the air two or three weeks; for, if it dries quickly, it will crack, and, when wet, break. Put it on a shelf, leaving a space between each piece, and let it grow hard gradually; thus it will save a full third in the consumption; but, for coarse washing, soft soap will go further than the hard.

Soda, by softening the water, saves a great deal of soap. It should be melted in a large ewer of water, some of which pour into the tubs and boiler; and when the latter becomes weak add more.

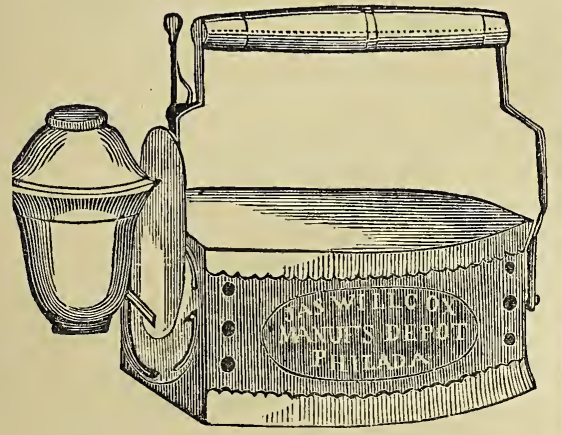

Self-Heating Flat Iron.

Many good laundresses advise soaping linen in warm water the night previous to washing, as facilitating the operation with less friction. This should always be done with fine muslins and laces.

The price of starch depends upon that of flour; the best will keep good in a dry warm room for some years.
Everything should be kept in the place best suited to it, as much waste may thereby be avoided.

Great care should be taken of jellybags, tapes for collared things, \&c., which, if not perfectly scalded and kept dry, give an unpleasant flavor when next used.

$V$ egetables will keep best on a stone floor, if the air be excluded; meat in a cold dry place, where the air is freely admitted; sugar and sweetmeats require a dry place, so does salt; candles, cold, but not damp; dried meats, hams, bacon, and tongues, the same. All sorts of seed for puddings, such as rice, \&c., should be covered close to preserve them from insects; but if kept long that will not be sufficient, unless they be occasionally sifted. Apples and pears should be laid upon very clean and dry straw, to prevent a musty taste; nor should they be exposed to either light or air. The floor of a dark garret is a good place in which to deposit them; or, which is still better, shelves made by strips of wood about two inches wide, placed an inch and a half apart, and the apples laid between them. They should be ranged singly in rows, without touching each other. and should be often inspected, both to wipe them, if damp, and to reject those which may appear to be getting rotten; but the larger sort of pears should be tied up by the stalk. Apples may also be preserved in excellent condition for a long period by being packed in large barrels with dry sand, but require to be used immediately when they are taken out.

Coarse nets suspended in the storeroom are very useful in preserving the finer kinds of fruit, lemons, \&c., which are spoiled if allowed to touch. When lemons and oranges are cheap, a proper quantity should be bought and prepared, both for preserving the juice, and keeping the peel for sweetmeats and grating; especially by those who live in the country, where they cannot always be had. 
They are perpetually wanted in cookery.

The best way of scalding fruits, or boiling vinegar, is in a stone jar, on a hot iron hearth, or by putting the vessel into a saucepan of boiling water, called a water-bath, after its having been closely corked, but not quite filled, as the heat may occasion the fruits to swell; but if they diminish after this is done, the vessel must then be filled.

Onions, shallots, and garlic should be hung up for winter use in ropes from the ceiling; as should dried pursley, basil, savory, and knotted-marjoram, or London-thyme, and tarragon, to be used when herbs are ordered, but with discretion, as they are very pungent.

Hams should be well covered in paper bags, and put in a chest or barrel, with layers of charcoal or ashes between. When you take one out to cut for use, put it away in a dark place, well covered.

Herbs should be kept from the air. Herb tea, to do any good, should be made very strong. Herbs must be gathered while in blossom. Those who have a little ground will do well to raise the most useful herbs; apothecaries make large profits on them.

Suet and lard keep better in tin than earthen-ware. Suet keeps good a year, if chopped, packed in a stone jar, and covered with molasses. Or, pick suet free from veins and skin, melt it in water before a moderate fire, let it cool till it forms a hard cake, then wipe it dry and put it in clean paper, in linen bags.

\section{MARKETING.}

Although respectable butchers may, in most cases, be relied upon for the goodness of the meat which they sell to regùlar customers, yet many persons (both ladies and gentlemen) go to market and choose for themselves, when, if not competent judges, the inferior sorts generally fall to their lot. In country towns, also, there are frequently but one or two market-days in the week; and the meat being brought from a distance, it is an essential point of good housekeeping to be so well acquainted with the quality as not to be easily imposed upon.

BeEF.-Well-fed beef may be known by the texture and color; the lean will exhibit an open grain of deep coral-red, and the fat will appear of a healthy, oily smoothness, rather inclining to white than yellow. The suet firm and white. Yellow fat is a test of meat of an inferior quality. Heifer beef is but little inferior to ox beef; the lean is of a closer grain, the red paler, and the fat whiter. Cow beef may be detected by the same signs, save that the older the beast the texture of the meat will appear closer, and the flesh coarser to the sight, as well as harder to the touch. The grain of bull beef is coarser and closer still, the fat hard and skinny, the lean of a deep red, and it has a stronger scent. $O x$ beef is the richest and largest; but in small families, and to some tastes, heifer beef is preferred, if finely fed. In old meat, a streak of horn runs between the fat and lean of the sirloin and ribs; the harder this is, the older, and the flesh is not finely flavored; that is to say, the horn has become so firm as to appear like bone; but oxen are always the better if kept until five or six years old.

Ox Tongue. - To choose a neat's tongue, it should be of moderate size, plump, firm, and smooth to the touch; for if the skin appears rough and shrivelled, it will be found hard and flavorless.

Veat.-When you observe the kidney well surrounded with fat, you may be sure the meat is of good quality. The whitest is not the best veal; but the flesh of the bull-calf is of a brighter color than that of the cow-calf. The fillet of the latter is generally preferred on account of the udder. There is a vein in 
the shoulder very perceptible; and its color indicates the freshness of the meat; if a bright red or blue, it is recently killed ; if any green or yellow spots are visible, it is stale. The suet will be flabby, and the kidney will smell. The other parts should be dry and white; if clammy or spotted, the meat is stale and bad. If more than eight or ten weeks old, the flesh becomes coarse. You will, of course, get it somewhat cheaper, and it may, to some palates, be found equally good in flavor, but not so delicate in either the color or the texture of the flesh.

Mutron.-The best is of a fine grain, a bright color, the fat firm and white. It is better for being full-grown. The ram mutton may be known by the redness of the flesh, and the sponginess of the fat. The flesh of the ewe is paler, and the texture finer, but neither the meat nor gravy so well flavored, though a "maiden ewe," when it can be got, is considered particularly fine. If you wish to have a good haunch, choose one of small size and moderately fat, looking at the same time to the closeness of the grain and the whiteness of the fat; but more particularly see that the flesh is dark-colored, that being a proof of age, and the older the better. In boiled mutton this is not of so much importance as in roasted.

Lam B should be eaten very fresh. In the fore-quarter, the vein in the neck being any other color than blue betrays it to be stale. In the hind-quarter, try the kidney with your nose; the faintness of its smell will prove it to be stale. It loses much of its nicety of flavor after it reaches four months old. It ought, therefore, to be small and white in appearance, and well covered with fat; the younger the more delicate.

Pork.-The rind should be thin, the fat very firm, and the lean white; the principal point to be observed is the firm- ness of the fat. If the rind is tough, thick, and cannot easily be impressed by the finger, it is old. Pigs that are short in the legs and bodies, and have thick necks, and not long heads and ears, are always to be preferred. When fresh, the flesh will be smooth and dry; if clammy, it is tainted. What is called "measly pork" is very unwholesome, and may be known by the fat being full of kernels. Pork fed at still-houses does not answer for curing in any way, the fat being spongy. Dairy pork is the best.

Porkers from three to four months old are the most delicate, and six months is the latest age at which they should be killed as such.

Bacon.-If the rind be thin, the fat firm and of a reddish tinge, the lean tender, of a good color, and adhering to the bone, you may conclude it is good and not old. If there are yellow streaks in it, it is rusty.

For hams, stick a sharp knife under the bone, and also up to the knuckle. If it comes out with a pleasant smell, the ham is good; but do not buy it if the knife has a bad scent. Hams short in the hock are best; nor should long-legged pigs be chosen for any purpose.

Brawn.-The horny part of the young brawn will feel moderately tender, and the flavor will be better than the old, the rind of which will be hard.

Venison.-The fat should be clear, bright, and thick; and if the cleft of the haunch be smooth and close, it is younm; but if the cleft is close and tough, it is old. To judge of its sweetness, run a very sharp narrow knife into the shoulder or haunch, and you will know by the scent. Few people like it when it has much of the haut-gout ; but it bears keeping better than any sort of meat, and if eaten fresh killed it is not so good as mutton. Observe the neck of a fore-quarter; if the 
vein be bluish, it is fresh; if it have a green or yellow cast, it is stale. In the hind-quarter, if there is a faint smell under the kidney, and the knuckle is limp, the meat is stale. If the eyes be sunk, the head is not fresh. When you can learn where it has been reared, choose forest-venison, as that is the best; for some of the dealers stall-feed the deer, and fatten them with oil-cake. It should be full five years old.

In every sort of provisions the best of the kind goes furthest, cuts up to greatest advantage, and affords most nourishment. Round of beef, fillet of veal, and leg of mutton, are joints that bear a higher price; but as they have more solid meat, they deserve the preference. It is worth notice, however, that those joints which are inferior may be dressed as palatably, and, being cheaper, they ought to be bought in turn.

In loins of meat, the long pipe that runs by the bone should be taken out, as it is apt to taint; as also the kernels of beef. Rumps and aitchbones of beef are often bruised by the blows the drovers give the beasts, and the part that has been struck always taints; therefore, do not purchase these joints if bruised.

All meat should be carefully examined. and wiped with a dry cloth as soon as it comes in; and if flies have blown upon it, the part must be cut off. This should be daily observed until it is dressed, as it not only tends to preserve the meat long in perfection, but prevents that musty flavor too often perceived in the outer slice when brought to table.

In the country, where meat is often carried a great distance, it should be well covered up with a cloth, over which fresh cabbage-leaves would keep it cool. These cautions are more needful, as in some families great loss is sustained by the spoiling of meat. The fly may in some measure be prevented by dusting upon the parts most likely to be attacked, pep- per and ginger mixed, after wiping, which should never be omitted; but a more easy and effectual mode is to exclude the fly by using a wire meat-safe, or to cover the joints with a long loose gauze or thin cloth, and hang them up from the ceiling of an airy chamber.

In summer meat should be wiped every day, or sprinkled with pepper to keep off the flies; and should it wear any appearance of becoming tainted, it should be brushed over with pyroligneous acid; or even if already slightly infected, either the acid or roughly-pounded charcoal, if well rubbed into the meat, will restore it. The meat should also be brought in early in the morning, as, if much exposed to the sun, the heat will render it flabby. In frosty weather, meat is sometimes in a congealed state, and should be thawed by soaking it in cold water before putting it to the fire.

Meats become more tender, and consequently more digestible, as well as better favored, by hanging; but veal and lamb will not bear it so long as the flesh of older animals. In summer, two days is long enough for lamb and veal, and from three to four for beef and mutton; in cold weather, these latter may be kept for more than double that time without risk of being tainted.

\section{POULTRY AND GAME.}

Turkey.-The cock bird when young has a smooth black leg with a short spur. The eyes bright and full, and moist supple feet when fresh; the absence of these signs denotes age and staleness; the hen may be judged by the same rules.

Fowls like a turkey; the young cock has a smooth leg and a short spur; when fresh the vent is close and dark. Hens when young have smooth legs and combs; when old these will be rough.

Geese.-In young geese the feet and bills will be yellow and free from hair 
When fresh the feet are pliable; they are stiff when stale.

Ducks may be selected by the same rule.

Pigeons, when fresh, have supple feet, and the vent will be firm; if it be discolored and supple they are stale.

Plovers, when fat, have hard vents; but, like almost all other birds, may be chosen by the same rules.

Other game birds may be chosen by the above rules.

\section{TO CHOOSE FISH.}

Fish.-In every sort of fish, stiffness, redness of the gills, and brightness of the eyes, are invariable signs of freshness; thickness of the flesh generally marks the good condition of all fish, and its firmness is an essential requisite. Fish, when quite fresh, curl round, but are particularly elastic, rising immediately upon the pressure of the fingers; and their staleness or freshness may be measured by the possibility of making an impression. If stale, the impression will remain ; but, if fresh, it will rise again on removal of the pressure.

Salmon.-The fish stiff, the scales very bright, the belly thick, the gills a brilliant color, and the flesh when cut, a beautiful red, will prove it to be a fine fresh fish. It cannot be too fresh.

Cod.-The best fish are thick at the neck, very red gills, firm white flesh, bright and blood-shot eyes, and small head. The liver should be of an opaque white; the whiter, the better the fish; when out of season the liver becomes red. It is improved by being crimped, as this increases its firmness, and then it requires keeping one day before using.

Whitings.-A clear color and firm bodies, indicate a superior quality.

Mackerel.-Bright eyes, thick bodies, stripes black on the back, the prismatic colors very predominant on the belly, denote freshness, and goodness. The male is the best; his stripes are straight; those of the female, wavy.

Pike, Carp, I'erch, Smelts, Gudgeons, \&c., may be judged by the above rules.

Eels. - The bright silver-hued belly and thickness of back, are the guides in their selection.

Lobsters.-To be had in perfection should be boiled at home; choose the heariest. When they are boiled the tail should have a gond spring; the cock lobster has a narrow tail in which the two uppermost fins are stiff and hard; the hen has a broad tail, and these fins are softer. The male has the best flavor; the flesh is firmer; and the color when boiled is brighter than the hen.

Oysters.-There are many sorts of oysters; when the oyster is alive the shell will close upon the knife; the common oyster should be used for sauce, and the natives, of which there are several kinds, should be sent to table.

\section{CHAPTER XI}

UNDER the head of Domestic Manipulation, we propose giving a series of articles on the numerous and essential manual operations that are constantly required in every family, and which, whether they are well or ill done, must of necessity be performed. The term Domestic Manipulation, employed in the widest sense, would include all the manual operations required in a house, but we propose to limit it to such as partake in a slight degree of a scientific character ; thus the operations of Filtering, Decanting, Weighing, Measuring, Bottling, Corking, Unstoppering, Pounding, Heating, Boiling, Distilling, Cementing, \&c. \&c. will be included; whilst Dusting, Washing, and Scrubbing, though no less, 
in strictness, manipulations, will be passed over in silence.

We propose treating of the manipulations connected with Botrues avd Decanters, \&c., under the following heads: Cleaning, Drying, Corking, Tying down, Stoppering, and Unstoppering.

Cleaning.--Perhaps no more effectual and easy mode of cleaning wine and beer bottles can be recommended than that commonly adopted, viz., the use of small shot and water; in the case of old port wine bottles, howerer, it often occurs that the mechanical action of the shot does not remove the hardened crust from the interior; a small quantity of pearlash or soda, or still better, washing liquids, added to the water, will soften the crust sufficiently to permit its easy removal. There is, however, one objection to the use of shot for the purpose of cleaning bottles; unless due care be taken, by the violence of the shaking it often happens that several become firmly. wedged between the bottom and sides of the bottles, and are not removed by the subsequent rinsings of clean water, and if the bottles are used for acid wines or other liquids, (almost all home-made wines contain a considerable portion of free acid,) the shots are slowly dissolved; and from the metallic arsenic which they contain, as well as from the lead itself, the liquid is rendered poisonous. This effect may be readily guarded against by removing any shots which may have become fixed, by a stiff wire slightly hooked at the end.

Decanters are formed of flint glass, which is much softer and more readily scratched than the common kinds; they require therefore a less rough treatment; in general, warm (not boiling) water, with the addition of a few pieces of coarse brown paper, and if requisite a little soda, will be found effectual; should greater force be required, a small portion of tow wrapped round the notched end of a moderately stiff wire, and used with a little strong soda, will be found sufficient. Sand or ashes should never be employed in cleaning decanters, as they roughen and totally disfigure the brilliant surface of the glass.

Drying.-It is scarcely necessary to speak of the advantages of being able to dry thoroughly both decanters and common bottles; if the former, after having been cleaned, are put away wet, they become musty; and many liquids are much injured by being put into wet bottles. Some of our readers have doubtless experienced the inefficiency of the ordinary means of drying decanters, \&c.; after draining for some days they still remain damp, and if placed near a fire the warmth merely drives the vapor to the colder part of the vessel ; they may, however, be readily and quickly dried after draining, by making them slightly warm and blowing in fresh air with a pair of bellows, which rapidly carries out the damp vapor, and leaves the vessel perfectly dry. If bellows are not at hand, the damp air may be drawn out (not blown) with the mouth, assisted by a tube sufficiently long to reach nearly to the bottom of the decanter; in the laboratory a piece of glass tube is usually taken, being always at hand, but for domestic use a piece of paper may be rolled up so as to form an extemporaneous and effectual substitute.

Corking.-Little can be said with regard to the corking of bottles, beyond stating the fact that cheap bad corks are always dear; the best corks are soft, velrety, and free from large pores; if squeezed they become more elastic and fit more closely. If good corks are used of sufficiently large size to be extracted without the corkscrew, they may be employed many times in succession, especially if they are soaked in boiling water 
afterwards, which restores them to their original shape, and renews their elasticity.

Tying doun.-The operation of tying down corks merits a longer notice, as without it many effervescent liquids could not be preserved. The most common mode of fastening down corks, is with the ginger-beer knot, which is thus made : First the loop is formed as in Fig. 1,

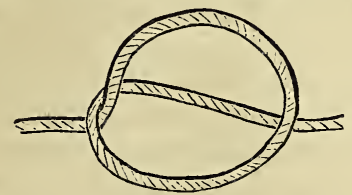

Fig. 1.

then that part of the string which passes across the loop is placed on the top of the cork, and the loop itself passed down around the neck of the bottle, and by pulling the ends of the cord is made tight beneath the rim; the ends of the string are finally brought up, and tied either in a double knot, or in a bow on

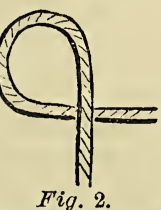
the top of the cork. When ginger-beer is made at home, it will be found most advantageous to use the best corks, and to tie them down with a bow, when both corks and strings may be made use of repeatedly.

For effervescent wines, such as champagne, gooseberry, \&c., which require to be kept a longer time, and are more valuable, a securer knot is desirable. which may be made thus:-A loop as in Fig.

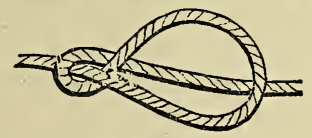

Fig. 3.

2 is first former, and the lower end is then turned upwards and carried behind the loop as shown at Fig. 3 ; it is then pulled through the loop as in Fiq. 4, and in this state is put over the neck of the bottle; the part $a$ being on one side, and the two parts of the loop on the other;

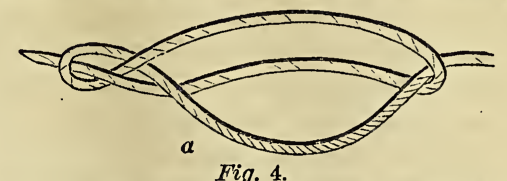

on pulling the two ends the whole becomes tight round the neck, and the ends, which should be quite opposite, are to be brought up over the cork, twice twist-

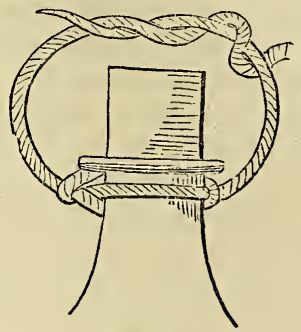

Fig. 5.

ed, as in Fig. 5, and then tied in a single knot.

Stoppering.-The stoppering of bottles is an operation usually performed by the makers; it may, however, be useful to know that badly fitting stoppers may be readily fitted by regrinding; this is done by dipping the stopper in a mixture of fine sand, or still better, emery and water, replacing it, and turning it backwards and forwards with a slight pressure; fresh sand must be applied from time to time. When the fitting is exact, so that the stopper turns freely without shaking, the whole may be finished off by using a little fine emery and oil.

Unstoppering.-This operation is much more likely to be required than the last one described, for the stoppers of decanters, smelling-bottles, \&c., from various causes, frequently become fixed, and many are the fractures both of bottles and stoppers, caused by the misdirested efforts to remove them. In treating of the various means that may lue employed 
we will mention them in the order in which they should be tried, beginning with the simpler and more easy, and passing on to those which are more effectual, and at the same time, unfortunately, more dangerous. The first method, then, that should be tried, is to press the stopper upwards with the fore-finger and thumb of the left hand (the other fingers holding the neck of the bottle), and at the same time giving the stopper a succession of short, sharp, light taps, with the wooden handle of a chisel, knife, or small hammer; care must be taken not to strike the stopper with sufficient force to break it, and it should be borne in mind that it is not the force of the blow, but the vibration, or jar, which is effectual in loosening it; should this plan be found ineffectual after a short trial, it may probably be from the stopper being cemented by some substance, such as the dried sugar of a sweet wine. In such cases we should endeavor to dissolve the cement by a suitable solvent, which should be placed in the groove between the stopper and the bottle; thus if the stopper is cemented with sugar, gum, or salt. water may be used ; in many circumstances, oil is advantageous, or spirit, or even strong acid may be used; whatever liquid is employed it should be allowed to remain some days, being renewed if requisite, and the tapping, \&c., should be again had recourse to.

Should these methods fail, a piece of cloth may be dipped in very hot water and wrapped round the neck of the bottle, when the heat causes the expansion of the glass, and if the stopper be tapped or twisted before the heat has had time to enlarge it, its removal may be effected; this operation must necessarily be a quick one, for if the stopper is heated and enlarged, as well as the bottle, it is obvious that no benefit will result. In the laboratory it is often customary to heat the bottle, not by a strip of cloth dipped in hot water, but by turning it rapidly over the flame of a lamp; in this way there is more danger of cracking the bottle, and the plan is not to be recommended in general, although employed with considerable success by thuse who, like operative chemists, are constantly in the habit of applying heat to glass vessels. It will at once be seen that the plan is fraught with great danger if applied to bottles containing inflammable liquids, as spirits, \&c.

The most effectual mode of removing stoppers, especially those of small bottles, such as smelling-bottles, remains to be described. Take a piece of strong cord, about a yard or four feet in length, double it at the middle, and tie a knot (Fig. $6, b)$ so as to form a loop $(a)$ of about $b$

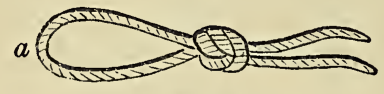

Fig. 6.

four inches in length at the doubled end, bring the knot close to one side of the stopper, and tie the ends tightly together on the opposite side, as at Fig. 7, e, so as to

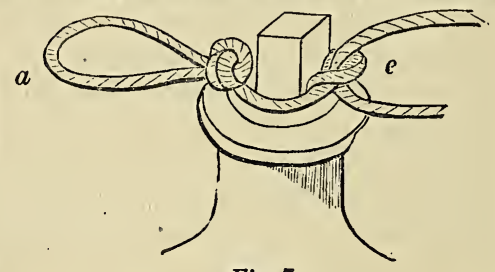

Fig. 7.

fasten the string securely round the neck of the stopper; now pass one of the ends through the loop $(a)$, and then tie it firmly to the other end; tis doubled cord is then to be placed over a bar or other support, then if the bottle is surrounded by a cloth to prevent accidents in case of fracture, and pulled downwards with a jerk, the force of which is gradually increased, it will be found that in a short time the stopper is liberated. Two pre- 
cautions are requisite; one is, that the course to, pastiles are prepared for the strain on both sides of the stopper is purpose, being superior to a heated iron equal; the other, that care be taken that rod, as they continue to burn and retain when the stopper is liberated, it is not their heat, whilst the latter requires to be dashed by the rebound against any hard substance, which would cause its fracture.

\section{CUTTING, GRINDING, AND WRITING ON} GLASS.

We have described the most advantageous modes of extracting fixed stoppers from decanters, \&c. It is possible that some of our readers may have followed our advice sufficiently well to have succeeded, in cracking the necks of their decanters. In case any should have been so unfortunate, or rather we would say, if we were quite sure we were not addressing ladies-so clumsy, let them not despair; dexterity in manipulation comes by practice; and as no evil is without a remedy, we will next consider what can be done with the broken decanter. Unless it is cracked down to the bottom, it may be cut off and converted into a handsome sugar basin; or if not high enough for that purpose, will serve for a pickledish, or a flower-stand, \&c.; and in the same way, a tumbler broken at the upper part will furnish an elegant salt-cellar, or serviceable soap-dish; and even common bottles, if sufficiently stout, may be made into useful jars, instead of being consigned to the dust-heap.

The operation of cutting glass, consists in leading a crack in the required direction; this is readily done by a hot iron rod, a piece of pointed burning charcoal, or, what is still better, a burning pastile-which is somewhat similar in its composition to those used for fumigation; and which latter, although rather expensive, and inconvenient from their shape, may be applied for the purpose. When the operation of cutting up glass vessels into useful forms is much had re- re-heated, if the crack has to be led any considerable distance. Pastiles are readily made by rubbing up half an ounce of powdered gum tragacanth with water, so as to form a mucilage about as thick as ordinary starch; this should be allowed to remain a few hours, and then mixed with a quarter of an ounce of benzoin, previously dissolved in the smallest possible quantity of proof spirit; after mixing them together in a mortar, as much powdered charcoal should be added as will form a stiff paste, and the whole well worked together, rolled into sticks the size of a common black-lead pencil, and dried. As thus prepared, they should be free from cracks, and solid throughout; and on being ignited at the end, they will burn steadily away to a point. If an iron rod is used, it should be nearly as stout as the little finger, and taper at the end for an inch and a half to a blunt point. Before commencing the line along which it is wished to divide the glass, it should be marked with a pen and ink, and allowed to dry, when the iron, heated to dull redness, on the lighted extremity of the pastile, should be brought to the end of a crack, being held in a slanting direction with regard to the glass, as shown in the cut, and slowly moved in an oblique direction towards the line; the crack will be found to follow the heated point, and may thus be led as required, even passing over parts varying very considerably in thickness, as in the case of the flutings on a cut decanter; but it cannot, with certainty, be made to pass suddenly from a very thin to a very stout part, or the reverse: thus it may be led around the sides of a tumbler, but could hardly be made to pass down one side, across 
the bottom and up the other. The rapidity with which the operation is performed, depends upon the heat of the iron or pastile ; if the former is very hot, or the latter made to burn more vividly by blowing upon it, the operation is quickened, but it is not performed with so much certainty, as the crack may pass on further than is desirable: care should be taken not to lead the crack too near

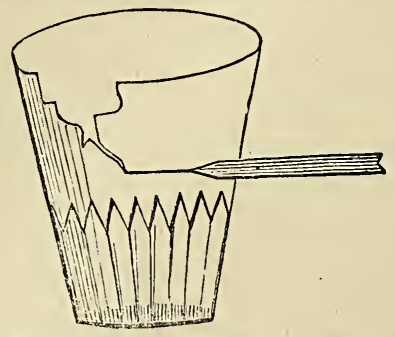

the edge of the vessel, or to another crack, as in that case it is apt to leave the proper course, and fly suddenly to the edge, to which an inexperienced operator should not attempt to go nearer than half an inch.

It sometimes occurs that a piece is broken out of a glass, without leaving any crack to commence from; in this case, one must be made, by heating the edge (one formed by the fracture, if possible) with the iron or pastile, and instantly applying the moistened finger. When a crack is formed, which may be used as described above, care must be taken not to cause an extensive fracture, which may run across the intended line of division ; this may be avoided by comniencing the crack at some distance from the line, and by applying the heated point for a very short time, preferring to make two or three unsuccessful attempts rather than to hasten the operation, and risk the destruction of the glass. When a glass vessel has been thus divided, the edges are sufficiently sharp to cut the fingers in handling; and are usually must be filled up with some cement; it wavy; it is therefore necessary to make them smooth and even. The most ready way of doing this is, by grinding them down on a flat sandstone, or ordinary paving-stone, with a little sharp sand or emery, and water, taking care to move the glass in a circular direction, and not merely backwards and forwards; the smoothness of the whole will depend entirely on that of the stone, and on the fineness of the sand or emery employed. If, from any irregularity, there is much glass to grind away, it is preferable to commence with sand, and finish with emery on a smooth stone; if the edges are not thus ground down, they should have the sharp angles, which are really dangerous, removed by a fine file, which should be moistened with oil of turpentine or camphene, as this liquid has an extraordinary effect in increasing the action of the file upon the glass, and at the same time protecting the steel instrument from wear.

Advantageous as cracks are in glass vessels whenever we wish to separate them into two parts, they are by no means desirable under other circumstances; and it is as important to know how to stop their progress, as to lead them forward. This is readily done in stout glass, by drilling a hole about half an inch in advance of the crack, which gradually passes on into it, and then its farther progress is arrested. Holes may be drilled in glass with a common drill and bow, the place being first marked with a file or flint, and the drill point kept wet with oil of turpentine. It is hardly necessary to state, that a crack existing in the neck of a decanter, and liable to be forced apart with the stopper, could not be arrested in its progress by powder may be used with oil of turpentine; and after the operation, the hole such means. If necessary, a little emery 
the vessel is to be used for holding liquids, a little fresh slacked lime, moistened with equal parts of white of egg and water, may be used for this purpose.

The grinding of glass on a flat stone with sand or emery, and water, is often useful in making a bottle stand steadily; and by its means a wineglass with a broken foot may be turned to good account; for if as much of the stem as possible is knocked off, by striking it with the back of a knife, the remainder may be ground away so that the vessel will stand.

One of the most important Dome:tic Manipulations, although one of the most simple and easy, is the labelling of glass vessels. It is not too much to affirm, that scores of lives might have been saved if this had been attended to. In cases of accidental poisoning, we usually find that the victim has drunk from some bottle which has been put away without a label ; and thus some corrosive liquid used for cleaning, or some poisonous lotion, has been inadvertently swallowed. One of the most ready modes of labelling glass, and other objects, consists in having at hand a sheet of paper, which has had spread on one side some gum water, mixed with half its weight of coarse brown sugar, and allowed to dry; this may be cut into labels, written on, and readily attached to glass by moistening with the tongue; the white margin of a sheet of postage stamps answers the purpose very well. If, however, acid liquids are used, or the vessel is placed in a damp situation, as a cellar, other means must be had recourse to. With a little practice, it is easy to write in a legible, though not very conspicuous manner, on glass, with a gun-flint, or with the sharp-edged fragments of common flint. In the laboratory what is called a writing diamond is used for this purpose ; this should not be confounded with a glazier's diamond, which is used for dividing, and not scratching glass. We would here caution our readers against writing on glass with a diamond ring, $\& c$., as the practice injures the jewel considerably; in the glazier's diamond, the natural edges of the crystal are used, which are not liable to injury as are the cut angles of a brilliant.

When glass vessels are exposed to damp, the best mode of writing on them is to prepare an ink for the purpose, by mixing the common cheap varnish. called Brunswick black, with half its weight of oil of turpentine, or what is the same thing, in a purer state, camphene; this should be kept in a closely corked bottle, and used with a broad nibbed quill pen; it soon dries, and though pale, is very distinct, and almost imperishable. If it is required much darker, about a quarter of an hour after it has been done a little lampblack should be rubbed over it, with cotton or wadding, when it imme diately becomes as black as common ink, and resists dainp, and rubbing or wiping with either wet or dry cloths for a very long time. The same ink is equally advantageous for use with white earthenware; and although we have never had occasion to use such a mixture, there is no doubt that a little whiting mixed thin with aly common varnish, would furnish an equally useful ink for writing on black botties.

\section{CHAPTER XII.}

\section{DECANTING, STRAINING, AND FILTER-} ING OF LIQUIDS.

The decanting of liquids is, under ordinary circumstances, an operation sufficiently simple to require no explanation; but the ease and certainty with which it can be performed, depend entirely upon the form of the vessel from which the liquid is poured; the adhesion existing between liquids and solids giving rise to 
a tendency in the former to run down the outside of the ressel; and if the latter is nearly full, or very large in circumference, or the sides approach the perpendicular direction, this accident almost always occurs. The difficulty of returning a glass of wine to the decanter, or of pouring from one full tumbler into another, are well known examples of this inconvenience.

Advantage may, however, be taken of the adhesion of liquids to solids, and by it the former may be led into the required direction. This cannot be better illustrated than by a description of the means by which a glass of wine may be returned, without spilling, to the decanter. If a teaspoon is dipped into the wine, so as to become wetted with it, and then held perpendicularly with the bowl downwards, and the point over, but not touching, the entrance into the decanter, and the edge of the glass be made to touch the back of the spoon, it will be found, on inclining the former, that the wine, having a perpendicular solid body to adhere to and run down, will do so in preference to trickling along the oblique outer surface of the wineglass; and in this mode a liquid may be poured steadily out of any similar vessel with so little disturbance as not to agitate any sediment that may exist in it. In the laboratory of the chemist, a piece of glass rod is usually employed for this purpose; but a spoon, or pencil, or any similar substance having a surface capable of being wetted by the liquid, answers equally well.

If, however, the vessel out of which it is wished to decant is large, very full, or the sides, on pouring, are nearly perpendicular, the plan is not successful ; thus, it could not be employed in aiding the transfer of the liquid from one full tumbler to another. Even this may be accomplished without the aid of a funnel, or without spilling, by preventing the adhesion of the liquid to the edge or side of the vessel out of which it is poured, which may be readily done by greasing the rim, when it will be found quite practicable to pour out of a nearly full tumbler without spilling.

In many instances, the employment of a syphon in decanting will be found very advantageous, particularly when the containing vessel is large, and cannot be readily moved, or when there is any sediment which it is desirable not to disturb. The most simple form of this instrument consists of a tube, bent as in Fig. 1, with one leg shorter than the other; this may be made of glass, pewter, or, in fact, of any kind of stiff tubing that will retain its form-a piece of gutta percha pipe, carefully bent by a moderate warmth, whilst a piece of stout cord is in the interior to prevent the sides closing together, answers very well. Before use, the syphon must be filled with liquor; this is best accomplished by turning it upside down, with the opening to the short leg raised on a level with that of the long one, when the liquid should be poured into the former. When both legs are filled, they should be closed with the fingers; the shorter leg introduced into the liquid it is wished to draw off; and the opening of the longer leg brought to a lower level than that of the shorter, and

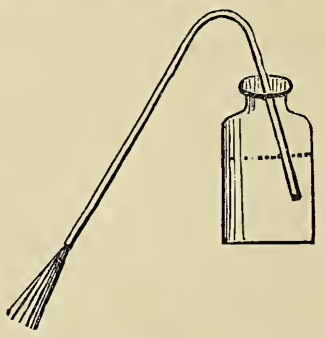

Fig. 1.

on removing the fingers the liquid will flow as in Fig. 1, until it is below the level of the short ley. If the syphon is made of small tubing, or is lessened at 
the opening so as not to exceed one quarter of an inch in diameter, there will be no occasion to close the end of more than one leg with the finger, as the liquid will not flow when it is brought to the proper position unless both orifices are open; and thus the necessity of plunging the finger into the liquid is obviated, and the syphon can also be used with a narrownecked bottle, into which the hand could not be passed.

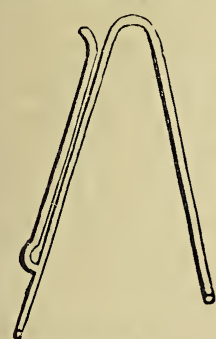

To do away with the necessity of filling the syphon before use, the instrument is usually made with a sucking tube, as in Fig. 2; in this case, all that is requisite is, to introduce the short leg, close the opening to

Fig. 2. the long one, and by the action of the mouth, draw up the liquid until both legs are full, when on removing the finger, the stream will flow. A very ingenious syphon of this kind is described by the German chemist Mohr; it is thus constructed:-Take a long Eau de Cologne bottle, and, with a file and turpentine, make a deep notch across, about an inch and a half frorn the bottom; then, with a charcoal point or pastile, or hot iron. produce a crack, and cut off the bottom, grinding it smoothly; then take a tube bent at an angle of forty-five degrees, and, by means of a good cork, perforated with a rat-tail rasp, fit it tightly in the bottom of the bottle, and add also

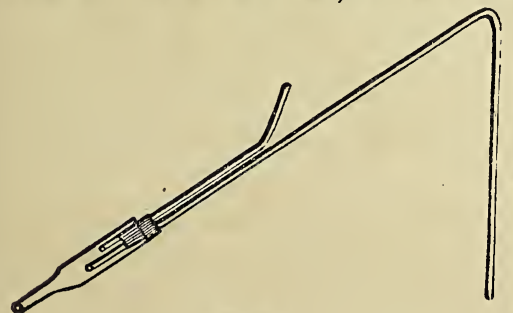

Fig. 8. another piece of tubing for a suction tube ; the whole will then have the appearance represented in Fig. 3, and will form an exceedingly useful, and very convenient syphon.

In emptying large stone bottles or carboys, the following plan may be had recourse to:-Perforate a sound cork with two openings by a rat-tail rasp, and

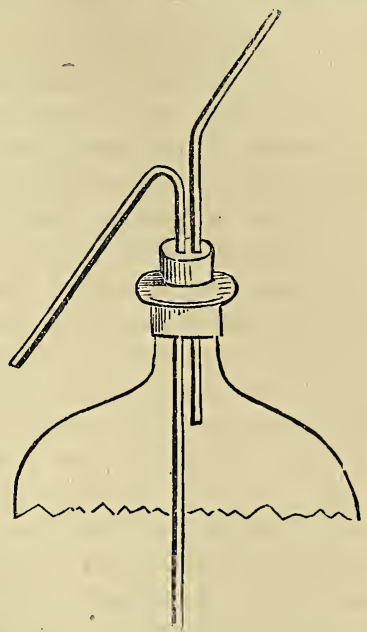

Fig. 4.

fit, air-tight, two tubes bent as in Fig. 4 . On blowing through the upper, the liquid will be forced to ascend and run over the bend of the other, which will then act as a syphon. This plan is exceedingly useful in emptying carboys of corrosive liquids, as oil of vitriol, \&c. ; and if all the joints are-as they should be-air-tight, the flow may be arrested by closing the upper tube with the finger. In the figure the outer leg of the syphon is shortened to save space; in practice it must be of sufficient length to be lower than the inner leg witlin the vessel.

If a syphon is required frequently for decanting the same kind of liquid, it is found troublesome to be constantly filling: it before each time of using; this trouble is obviated by the use of an instrument 
formed with legs of equal length, which are turned up at the ends, as in Fig. 5 ; this having been filled, may be hung up in the erect position, and the liquid will

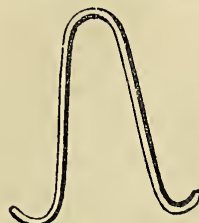

Fig. 5. not escape, but on plunging one end into a liquid, it will be found immediately to flow from the other, provided that the latter is below the level of the surface of the liquid.

The operations of straining and filtering are frequently required in domestic manipulations, and the apparatus employed usually consists of sieves and a jelly-bag. As, in many other instances, it will be found advantageous to import several contrivances from the laboratory to the kitchen, one of the most useful (because most simple) strainers consists of a square frame, formed of four pieces of wood nailed together at the corners, with a piece of calico, linen, or canvas, of suitable fineness, tacked to the four sides; this strainer is particularly useful in separating any solid substance -as the residue in making wines-or if grated potatoes are put on one made of coarse cloth, the starch can be readily washed through, leaving the useless portion on the strainer; the cloth should not be tacked very loosely, as it bags down when any substance is put on it, and the liquid runs away below from the centre. This strainer is a most useful one ; it is readily made, of any degree of fineness, and of any size ; and it also possesses the great advantage, that, if necessary, the tacks fastening the cloth can easily be withdrawn, when the substance remaining can be rolled up in the cloth, and tightly squeezed, to express the last portions of liquid.

In cases where a finer filtration is required than can be obtained by means of a cloth, as in cleaning turbid wine or spirit, the use of filtering-paper is recom- mended. This paper is merely a stouter kind of blotting-paper, thick varieties of which answer very well for domestic purposes; it is most simply used by taking a square piece, folding it into half-by bringing the two opposite edges together -and then folding the oblong so obtained across its length; by this means a small square is obtained, one quarter the original size, which may be opened into a hollow cup, having three thicknesses of paper on one side, and one on the other; this is to be placed with the point downwards, in a funnel, and the liquid poured in; and as soon as the pores of the paper are expanded by the moisture, it will be found to flow through perfectly clear; care must be taken in making the filter, not to finger it much where the two foldings cross each other, as a hole is readily made at that part, and the filter spoiled. The objection to this simple contrivance is, that from its flat sides applying themselves closely to those of the funnel, the flow of the liquid is impeded, and is. therefore, slow. This effect may be obviated by the use of the plaited filter, the construction of which we will endeavor to describe. A square piece of filtering, or stout blotting-paper is to be doubled, and the oblong so obtained is to be again folded in half, when if the last fold is opened, it will have the appearance of Fig. 6. From the corners $b b$, folds are to be creased in the direction towards $a$, but not reaching it for half an inch; these are indicated by the dotted lines, which

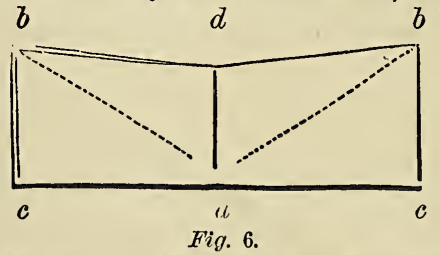

divide the double paper into four triangles, each of which is to be again folded into eighths, and care must be taken that 
all the folds are made the same way, that is, projecting to the same side of the paper. When complete, the doubled and creased paper will appear as Fig. 7. Now

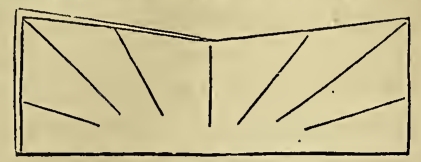

Fig. 7.

divide each eighth into half, by a fold in the opposite dircction to those previously made, when it will be found that the whole will readily fold up like a paper fan; the projecting loose ends which are formed by the corners $b$, should be cut off, and the double sides separated for the first time by blowing them apart, when the whole may be readily opened out as

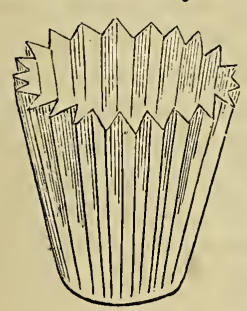

Fig. 8. in Fig. 8. In making this filter, which takes a much less time than to follow the description, two precautions are requisite. The folds should be made at once with one firm pressure, and not with a series of rubbings; and all the creases should stop short of the middle, otherwise a hole will be made at that point, long before the filter is completed. The advantages of this filter are that it exposes a large surface for' the liquid to pass through; and from its only being in contact with the funnel where the angles project, the current flows away readily.

The best means for filtration of water, and the construction of water filters, will be treated of when we speak of the "domestic manipulation" connected with that liquid.

\section{CHAPTER XIII.}

THE MANUFACTURE AND USE OF CEMENTS.

The term cement, includes all those substances employed for the purpose of causing the adhesion of two or more bodies, whether originally separate, or divided by an accidental fracture. As the substances that are required to be connected together are exceedingly various, and differ very much in their properties as to texture, \&c., \&c., and as the conditions under which they are placed, with regard to heat and moisture, are also exceedingly variable, a number of cements, possessed of very different properties, are required; for a cement that answers admirably under one set of circumstances, may be perfectly useless in others. A vast number of cements are known and used in the various arts; but they may all be referred to a few classes, and our object in this paper will be to describe the manufacture and use of the best of each class, and also to state what are the general principles upon which the success or failure of cementing usually depends.

The different parts of a solid are held together by an attraction between their several particles, which is termed the attraction of cohesion, or cohesive attraction. The amount of this varies with the substances; thus, the cohesion of the particles of iron to one another is enormously great, whilst that between those of chalk is but small. This attraction acts only when the particles are in the closest possible contact; even air must not be between them. The attraction of cohesion which takes place between the parts of the same substance, must not be confounded with that of adhesion, which is the attraction of different substances to one another; for example, the particles of a piece of wood are united by cohesive attraction, whilst the union of glue and wood to each other depends on adhesive attraction. And it is important that this distinction be borne in mind, for, in almost all cases, the cohesion between the particles of the cement is very much less than the adhesion of tho 
cement to other bodies; and if torn apart, the connected joint gives waynot by the loosening of the adhesionbut by the layer of cement splitting down the centre. Hence the important rule, that the less cement in a joint, the stronger it is. Domestic manipulators usually reverse this, by letting as much cement as possible remain in the joint, which is, therefore, necessarily a weak one. A thick, nearly solid cement, which cannot be pressed out of the joint, is always inferior to a thinner one, of which merely a connecting film remains between the united surfaces.

Having thus mentioned the general principles that ought always to be borne in mind, we will now proceed to describe the manufacture of some of the more useful cements, and their mode of use.

Mouth Glue affords a very convenient means of uniting papers, and other small light objects; it is made by dissolving by the aid of heat, pure glue, or gelatine, with about one quarter or one-third of its weight of coarse brown sugar, in as small a quantity of boiling water as possible; this, when perfectly liquid, should be cast into thin cakes on a flat suriace very slightly oiled, and as it cools cut up into pieces of a convenient size. When required for use one end may be moistened by the mouth, and is then ready to be rubbed on any substances it may be wished to join ; a piece kept in the desk or work-box is very convenient.

Paste is usually made by rubbing up flour with cold water and boiling; if a little alum is mixed before boiling it is much improved, being less clammy, working more freely in the brush and thinner; a less quantity is required, and it is therefore stronger. If required in large quantity, as for papering rooms, it may be made by mixing one quartern of flour, one quarter pound of alum and a little warm water; when mixed, the re- quisite quantity of boiling water should be poured on whilst the mixture is being stirred. Paste is only adapted to cementing paper; when used it should be spread. on one side of the paper, which should then be folded with the pasted side inwards, and allowed to remain a few minutes before being opened and used; this swells the paper, and permits its being more smoothly and securely attached. Kept for a few days, paste becomes mouldy, and after a short time putrid; this inconvenience may be obviated by the use of-

Permanent Paste, made by adding to each half-pint of flour paste without alum, fifteen grains of corrosive sublimate, previously rubbed to powder in a mortar, the whole to be well mixed ; this, if prevented from drying, by being kept in a covered pot, remains good any length of time, and is therefore convenient; but unfortunately it is extremely poisonous, though its excessively nauseous taste would prevent its being swallowed accidentally; it possesses the great advantage of not being liable to the attacks of insects.

Liquid Glue is made by dissolving shell-lac in water, by boiling it with borax, which possesses the peculiar property of causing the solution of the resinous lac. This preparation is convenient for its cheapness and freedom from smell, but it gives way if exposed to long-continued damp, which that made with naphtha resists.

Of the use of common glue, very little need be said; it should also be prepared in a glue-pot or double vessel to prevent its being burned, which injures it very materially. The objection to the use of this contrivance is, that it renders it impossible to heat the glue in the inner vessel to the boiling point; this inconvenience can be obviated by employing in the outer vessel some liquid, which 
boils at a higher temperature than pure water, such as saturated solution of salt. This boils at $224^{\circ}$ Fahr., $12^{\circ}$ above. the heat of boiling water, and enables the glue in the inner vessel to be heated to a much higher temperature than when pure water is employed. If a saturated solution of nitre is used, the temperature rises still higher.

Waterproof Cements are very numerous; a very good one for uniting china and glass will be found elsewhere. It should be stated, however, that the gum ammoniac should be also dissolved in a small quantity of spirit. Mastic, used instead of ammoniac, makes a clearer cement. This mixture, under various fanciful titles, is usually sold at a most exorbitant rate.

Lime and Egg Cement is frequently made by moistening the edges to be united, with white of egg, dusting on some lime from a piece of muslin, and bringing the edges into contact. A much better mode is to slake some freshly burned lime with a small quantity of boiling water; this occasions it to fall into a very fine dry powder, if excess of water has not been added. The white of egg used should be intimately and thoroughly mixed, by beating, with an equal bulk of water, and the slaked lime added to the mixture, so as to form a thin paste which should be used speedily, as it soon sets. This is a valuable cement, possessed of great strength, and capable of withstanding boiling water. Cements made with lime and blood, scraped cheese, or curd, may be regarded as inferior varieties of it. Cracked vessels, of earthenware and glass, may often be usefully, though not ornamentally repaired by white lead spread on strips of calico, and secured with bands of twine. But in point of strength, all ordinary cements yield the palm to Jeffery's Patented Marine Glue, a compound of India-rubber, shell-lac, and coal-tar naphtha. When applied to china or glass, the substances should be cautiously made hot enough to melt the glue, which should then be rubbed on the edges so as to become fluid, and the parts brought into contact immediately. When well applied, the mended stem of a common tobacco-pipe will break at any other part, in preference to the junction. The color of the glue unfortunately prevents its being used.

- The Red Cement, which is employed by instrument-makers for cementing glass to metals, and which is very cheap, and exceedingly useful for a variety of purposes, is made by melting five parts of black resin, one part of yellow wax, and then stirring in, gradually, one part of red ochre or Venetian red, in fine powder, and previously well dried This cement requires to be melted before use, and it adheres better if the objects to which it is applied are warmed. A soft cement, of a somewhat similar character, may be found useful for covering the corks of preserved fruit, and other bottles, and it is made by melting yellow wax with an equal quantity of resin, or of common turpentine, (not oil of turpentine, but the resin), using the latter for a very soft cement, and stirring in, as before, some dried Venetian red. Bearing in mind our introductory remarks, it will be seen that the uniting broken substances with a thick cement is disadvantageous, the object being to bring the surfaces as closely together as possible. As an illustration of a right and a wrong way of mending, we will suppose a plaster of Paris figure broken; the wrong way to mend it is by a thick paste of plaster, which makes, not a joint, but a botch. The right way to mend it, is by means of some well-made carpenter's glue, which, being absorbed into the porous plaster, leaves merely a film covering the two 
surfaces. If well done, the figure is stronger there than elsewhere.

One useful substance is termed mastic cement, which is used for making a superior coating to inside walls, and which must not be confounded with the resin mastic. It is made by mixing twenty parts of well-washed and sifted sharp sand, with two parts of litharge, and one of freshly burned and slaked quick-lime, in fine dry powder. This is made into a putty, by mixing with linseed oil ; it sets in a few hours, having the appearance of light stone; and we mention it as it may be frequently employed with advantage in repairing broken stone-work (as stairs) by filling up the missing parts. The employment of Roman cement, plaster, \&c., for masonry work, hardly comes within the limits of Domestic Manipulation.

sิ.

\section{CHAPTER XIV.}

DIVIDING, POWDERING, GRINDING, ETO.

The operations of chopping, powdering, grinding, \&c., are so frequently required in cooking, and the other branches of domestic economy, as to render any description of their utility wholly unnecessary; and we may therefore confine ourselves to describing the best means of accomplishing the object desired. Powdering is usually performed by the aid of the pestle and mortar. Most of the works on Cookery recommend the use of a marble mortar; this material is about one of the worst that could be selected for the purpose. In the first place, it is expensive; secondly, it is rapidly corroded, even by the weak acids used for food; thirdly, it is readily stained by oily substances; fourthly, it is absorptive of strong flavors, imparting them readily to the next substance pounded; and lastly, it is brittle, and even if not broken, is not calculated to withstand much wear. By far the best material for the purpose is the Wedgewood ware; mortars made of it are cheaper, cleaner in use, and stronger than those of marble, and are not corroded by acids or alkalies-their pre-eminence is so great, that they are invariably used by druggists.

The act of powdering requires great tact and practice to perform it neatly and rapidly. After the object has been broken into small pieces by blows from the pestle, a grinding action is required; this should at first be given by striking the fragments, not in the centre of the mortar, but towards the side furthest from the operator; the pestle, by this means, grinds over them in its descent to the centre, and much more rapidly accomplishes their division than if mere blows are given. After the object has been divided to a certain extent, blows are entirely useless, and a grinding in circles becomes requisite; if the circle is confined to one part of the mortar, the same portions get rubbed over and over again, the others escaping; this is aroided by constantly and regularly altering the size of the circles. If they are commenced in the centre, they should gradually increase in size until the sides are reached, and then contract again, and so on. By this means the whole of the

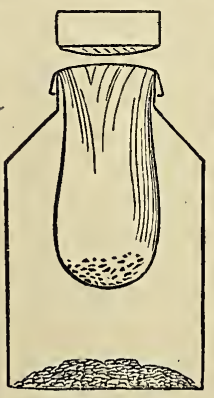

Fig. 9. powder is brought under the action of the pestle, and the operation is much quicker than if performed at random. One great fault usually committed in powdering, is the endeavor to operate on too large a quantity of material at one time. The operation is much more rapidly conducted if small portions are ta- 
ken; and if the material is tough, and contains much fibrous matter, the process may be very much shortened by removing those parts which are sufficiently powdered, by sifting from time to time through a sieve. This may be objectionable, however, from the fine powder escaping into the air ; in this case, the following contrivance will be found useful: A cylindrical tea-canister of the requisite size is taken, with a loosely fitting lid (or if tight, the lid may be enlarged by four slits being made partly up the sides ); a bag of lawn is dropped into the canister, the top being turned over the edge ; the powder to be sifted is put in a bag, the lid put on, and, by tapping and shaking, the finest portions pass into the canister without any escaping into the air-a point of very considerable importance where the powder is irritating or expensive.

All vegetable, and many mineral substances, are much more readily powdered after having been thoroughly dried; so far is this process carried, that many drugs are dried so as to lose fifteen per cent. of their weight before powdering. After drying, substances should not be exposed to the air, but, unless they are of sucb a nature as to be softened by heat, are better operated on whilst still warm. Flints are more readily powdered by being heated to redness and quenched in cold water; charcoal, for tooth powder, whilst still warm from drying. Gum can only be powdered whilst perfectly dry. Camphor, which is with great difficulty powdered alone, yields readily if a drop or two of spirit is poured on it. Substances which clog together and cake under the pestle, are not uncommon; to these it is sometimes requisite to add sand, which may afterwards be separated-this prevents the clogging; but its use is often impracticable. Lime, if required in very fine pow- der, for dusting over plants to kill slugs, \&c., is readily obtained by slaking it, when fresh burned, with boiling water; when, if too much water is not used, it falls into an exceedingly fine powder.

Sal-ammoniac, and some other saline bodies, are most readily powdered by dissolving them in as small a quantity of boiling water as possible, and stirring the solution rapidly as the water is boiled away, or as the solution cools. Before dismissing the pestle and mortar, we may allude to its use in mixing powders together, although a much more ready mode of doing this is with a sieve. Two or more powders stirred together and passed two or three times through a sieve, are much more intimately mixed, than if rubbed for a long time in a mortar. Metals cannot be divided in the mortar; the most convenient mode of proceeding: if they are fusible under a white heat, is to melt them, and pour them whilst liquid into a pail of water, which should be full to avoid any spluttering, and the hotter the metal, the more filmy the particles. It is scarcely requisite to state, that the metal should be poured in a circle, so as not to collect at one place.

Chopping is usually performed in the kitchen, with a large cornmon knife; but is more speedily done by some of the improved contrivances similar to the following:-The chopping-board should be

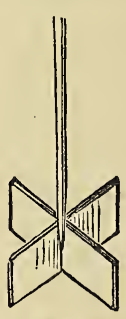

Fig. 10.

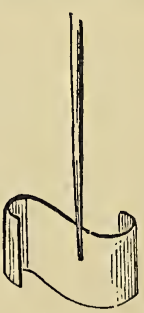

Fig. 11. made of hard wood, with the grain at right angles to the surface of the board, 
by which it is rendered much more durable, than if they are parallel to it. The chopping-knives should be fixed at right angles to the handles, and may be either of the preceding patterns. If a large quantity of material has to be acted on, we would recommend a board as above, not less than three inches thick, and smooth on both sides, so that either may be used, of the requisite size-say eighteen inches or two feet in diameter. On this should stand a loose bottomless tub, to confine the materials, and the whole resting on the floor, should be used with a knife, sufficiently long in the handle to be employed by a person standing erect, and it should have a small cross-bar for the hands, as shown in Fig. 12.

Small chopping-knives are sold, consisting of three blades riveted together; and a very convenient one is made by fastening, at convenient distances, a number of flat circular disks, sharpened at the edges. to a central axis with a handle at each end.

Many substances, such as stale bread, dried herbs, \&c., may be very conveniently powdered by rubbing them through

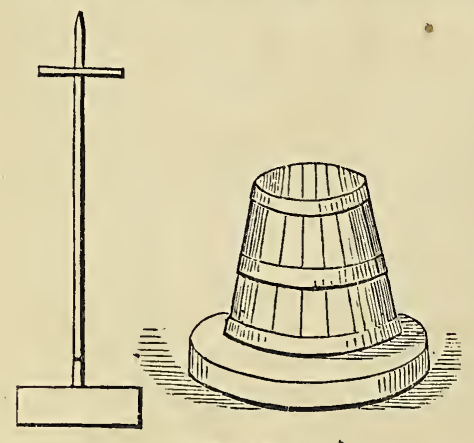

Fig. 12.

a wire sieve, of the requisite degree of fineness. Herbs intended for use in this way, should be dried as rapidly as possible, without being scorched, in small heaps, before the fire; parsley and others done in this way, may be powdered, re- taining their bright green color and flavor, both of which are preserved if they are corked tightly in bottles, and kept in a dry, dark cupboard. The use of waxed paper to preserve dried powders in, or for tying them down in jars, or generally as a very good substitute for bladder, will often be found convenient. It is readily made by laying a sheet of smooth stout paper on a warm iron plate, as the top of a kitchen oren; on this place the thin tissue or other paper to be waxed; put a piece of wax on it, and as it melts, rub it over, spreading it evenly. One end of a cork, covered with two thicknesses of linen, answers very well for a rubber. If a hot plate is not at hand, the sheet of paper may be held before the fire, and rubbed over, as it warms, with the cut edge of a cake of white wax; but this requires the co-operation of two persons.

\section{CHAPTER XV.}

KNOTS, PACKAGES, PARCELS, ETC.

The poet Crabbe, speaking of the mriting of the rustics, signing his parish registers, says-

"'Tis strange that men

Who guide the plough should fail to guide the pen! For half a mile the furrows even lie;

For balf an inch the letters stand awry."

A parallel remark might with equal justice be made on the gentler sex, who, after exercising a degree of tact, neatness, and tasteful invention, that the selfstyled "lords of the creation" might in vain hope to rival, in the formation of a piece of needlework, knittifig, netting, or crochet, are, for the most part, totally unable, when it is finished, to tie it up so as to make a decent parcel ; ladies' packages are, in fact, the opprobrium of the sex-the annoyance of all carriers, who 
have any thing to do with their conveyance, and the torment of their owners ; the cords are certain to become loose, the knots are sure to slip, except when a slip-knot is requisite, and then it is a fixture! It is in the hope that we may be instrumental in improving this state of things, that we are induced to devote this chapter to Knots, Packages, Parcels, \&c., and we shall at once lay before our fair readers a method of tying a parcel neatly and securely, and at the same time affording facilities for releasing the contents without destroying the string by cutting it away-a too ordinary practice, especially where time is an object.

The most simple purpose for which a knot is required, is the fastening togeth-

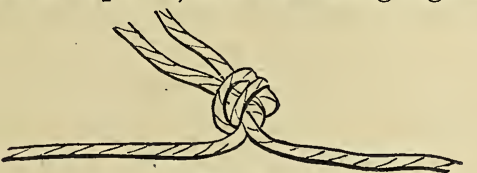

Fig. 13.

er of two pieces of string or cord: the knot selected for this purpose should possess two important properties ;-it should be secure from slipping, and of small size. Nothing is more common than to see two cords attached together in a manner similar to that shown in Fig. 13. It is scarcely possible to imagine a worse knot; it is large and clumsy, and as the cords do not mutually press each other, it is certain to slip if pulled with any great "force. In striking contrast to this-the worst of all, we place one of the best; namely, the knot usually employed by netters, and which is called by sailors "the sheet-bend." It is readily made by bending one of the pieces of cord into a loop ( $a$ b, Fig. 14), which is to be held between the finger and thumb of the left hand; the other cord $c$ is passed through the loop from the farther side, then round behind the two legs of the loop, and lastly, under itself, the loose end coming out at $d$. In the smallness of its size, and the firmness with which the various parts grip together, this knot

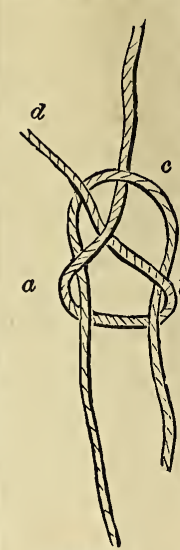

Fig. 14. surpasses every other: it can, moreover, be tied readily when one of the pieces, viz. $a b$, is exceedingly short; in common stout twine, less than an inch being sufficient to form the loop. The above method of forming it is the simplest to describe, although not the most rapid in practice; as it may be made in much less time by crossing the two ends of cord ( $a$ b, Fig. 15) on the tip of the fore-finger of the left firmly by the left thumb, which covers the crossing; then the part $c$ is to be

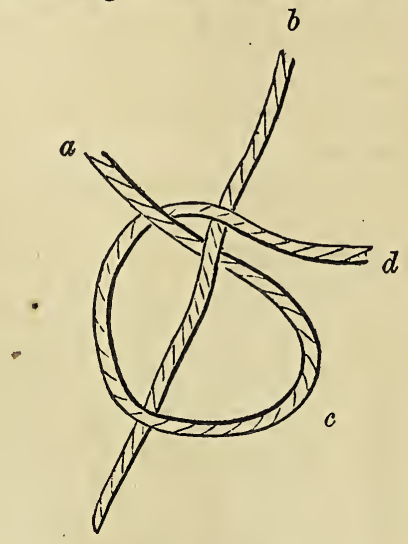

Fig. 15.

wound round the thumb in a loop as shown in the figure, and passed between the two ends, behind $a$ and before $b$; the knot is completed by turning the end $b$ downwards in front of $d$, passing it through the loop, securing it under the left thumb, and tightening the whole by pulling $d$. As formed in this mode, it is more rapidly made than almost any other knot; and, as before stated, it excels all in security 
and compactness, so firmly do the various turns grip each other, that after having been tightly pulled, it is very difficult to untie ; this the only drawback to its usefulness, and in this respect it is inferior to the reef-knot, Fig. 16, which is made in precisely the same manner that a shoestring is tied, only pulling out the ends instead of leaving them as bows. The only precaution necessary in making a reefknot is, to observe that the two parts of each string are on the same side of the loop; if they are not, the ends (and the bows if any are formed) are at right angles to the cords; the knot is less secure, and is termed by sailors a granny-knot. Other knots are occasionally used to connect two cords, but it is unnecessary to describe them, as every useful purpose may be answered by those abovementioned.

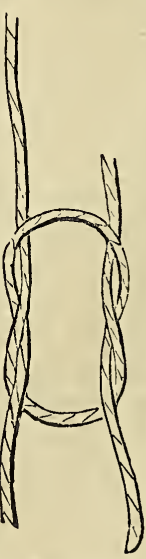

The binding knot (Figs. 17 and 18) is
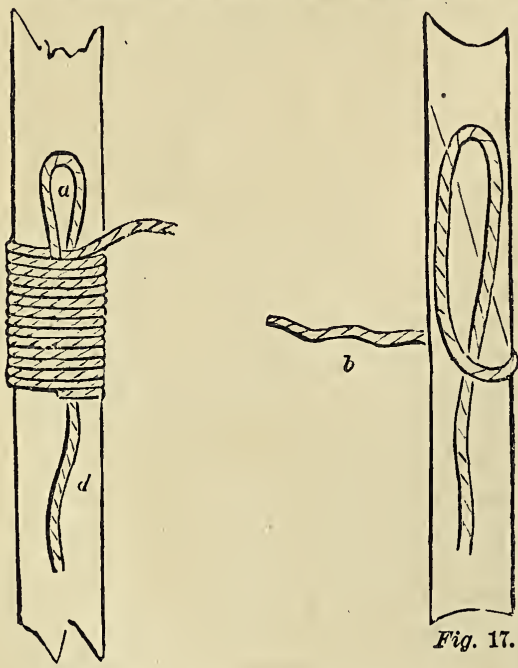

Fig. 18. exceedingly useful in connecting broken sticks, rods, \&c., but some difficulty is often experienced in fastening it at the finish; if, however, the string is placed over the part to be united, as shown in Fig. 17, and the long end $b$, used to bind around the rod, and finally passed through the loop a, as shown in Fig. 18, it is readily secured by pulling $d$, when the loop is drawn in, and fastens the end of the cord.

For fastening a cord to any cylindrical object, one of the most useful knots is the clove hitch, which, although exceedingly simple and most easily made, is one of the most puzzling knots to the uninitiated. There are several modes of forming it, the most simple being perhaps as follows:-make two loops, precisely sini-

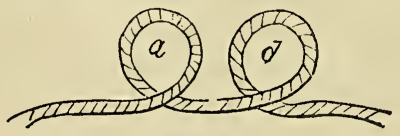

Fig. 19.

lar in every respect as $a$ and b, Fig. 19, then bring $b$ in front of $a$, so as to make both loops correspond, and pass them over the object to be tied, tightening the ends; if this is properly done, the knot will not slip, although surrounding a tolerably smooth cylindrical object, as a pillar, pole, \&c. This knot is employed by surgeons in reducing dislocations of the last joint of the thumb, and by sailors in great part of the standing rigging. The loop which is formed when a cable is passed around a post or tree to secure a vessel near shore, is fastened by what sailors term two half hitches, which is simply a clove hitch made by the end of the rope which is passed around the post or tree, and then made to describe the clove hitch around that part of itself which is tightly strained.

From the tying of knots we may pass on to the tying over of bottles, preserves, jars, \&c.; the object with which this 
operation is performed is either to prevent the excess of air or the escape or entrance of moisture ; the act itself is so very simple as to require no explanation; but a few words may be said on the choice of material, which should be varied so as to suit the exigencies of each particular case. When a vessel of spirit is to be tied over, leather is frequently selected-a very erroneous practice, as the vapor of spirit passes readily through that substance, but cannot penetrate bladder, which should be invariably used for the purpose. So effectually is spirit confined' by bladder, that when weak spirits are put into bladders or into vessels tied over with bladder, and allowed to remain some time, they are strengthened, as the vapor of the water passes away, that of the spirit being retained.

Bladder or other animal membranes of the same nature, in a moist and flaccid state, are usually selected for tying over preserves and jams, for which they are well adapted. Many persons place a thin piece of brandied paper in the jar resting on the jam, in addition to tying it down; this assists in excluding air and preventing mouldiness, but we have found a piece of very thin paper moistened with white of egg much more efficacious. The thin sheet-lead used for lining the interior of tea-chests, or stout tin-foil, is very advantageously used in tying down vessels containing specimens of natural history preserved in spirits, as they effectually prevent the escape of the latter for a long series of years. The plan usually pursued is to tie the cork over first with a single bladder, then with the metal, and finally with a second piece of bladder, which is afterwards covered with a coat of black paint.

The tying up of parcels in paper is an operation which is seldom neatly performed by persons whose occupations have not given them great facilities for constant practice. Whether the paper be wrapped round the objects, as is the case usually when it is much larger than sufficient to enclose them, or merely folded over itself, as is done by druggists, who cut the paper to the required size, it is important that the breadth of the paper should be no longer than sufficient to enable it to be folded over the ends of the object enclosed, without passing over the opposite side; it is impossible to make a neat or close parcel with paper which is too broad; excess in length may be readily disposed of by wrapping it round; but excess of breadth should be cut away. With regard to turning in the ends the mode adopted by grocers is the best. The most common cause of failure in parcels is their being badly corded; we will, therefore; (however unnecessary the description of so simple a performance may appear to those already acquainted with it), describe the most readily acquired mode of cording.

Let a single knot be made in the end of the cord, which is then passed round the box or parcel. This knotted end is now tied by a single hitch round the middle of the cord (Fig. 20), and the whole pulled tight. The cord itself is then carried at right angles round the end of the parcel, and where it crosses the transverse cord on the bottom of the box (Fig. 21), it should (if the parcel is heavy, and requires to be firmly secured) be passed over the cross cord, then back underneath it, and pulled tightly, then over itself; lastly, under the cross cord, and on around the other end of the box. When

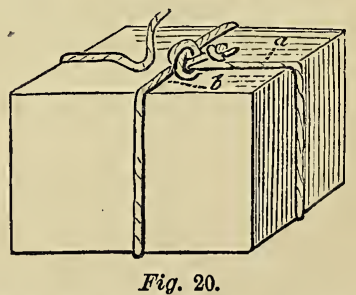

it reaches the top it must be secured by 
passing it under at that part of the cord which runs lengthways ( $a$, Fig. 20), pulling it very tight, and fastening it by two half hitches round itself. The great cause

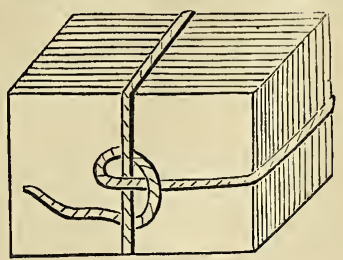

Fig. 21.

of parcels becoming loose is the fact of the cord being often fastened to one of the transverse parts (as $b, F i g .20$ ), instead of the piece running lengthways, and in this case it invariably becomes loose. The description may perhaps be rendered clearer by the aid of the figures, which exhibit the top and bottom of a box corded as described. The cords, however, are shown in a loose state to allow their arrangements to be perceived more easily.

\section{CHAPTER XVI.}

IN spite of our receipts and our philosophy, the briskness of the fire, the skill of our cook, the excellence of the oven, the bright array of pots, kettles, pans, moulds, griddles and gridirons, and the presiding genius of even a half Fortunatus sort of a purse, or the most rigid scale and measure of economy, one grand puzzle besets alike all kitchensthe difficulty of really getting the ingredients on which the mystery of food manufacture is to be exercised.

The very water we have to cook with, is crowded with millions of monsters things with two heads and no heads, with countless legs and no legs, with jaws and pincers and claws, and most wonderful springy tails; in some water well nigh enough of them to make a sort of soup, to say nothing of the chalk. lime, iron, and a host of other impuri ties.

The sugar, if it be brown, without taking note of such items as a little lead, a good deal of sand, some clay and flour, is pretty nearly as thick as it can hold of chips of cane and swarms of mites.

Our tea, if green, is painted and polished with Prussian blue, turmeric powder, and China clay, and is a mixture of all the leaves that the wonderful industry and ingenuity of the Chinese, can accomplish; we have old tea-leaves dried and twisted up, and colored and glazed, and sold for black and green; we have even gunpowder made up of dust and sand, and gum, faced as they call it with plumbago.

Coffee, fragrant, and refreshing, has almost become a myth; we may have pneumatic coffee-pots that will not let the finest dust pass through their strainers, French coffee-pots, German coffeepots, and all kinds of traditional directions for the manufacture : but not one of them can help us to make coffee, unless as good old Mrs. Glass would say, "we have first got our coffee ;" and what with foreign roguery and home roguery the chances are twenty to one against us, that the brown powder we are at so much pains with, once flourished at the end of a blue flower, on a long stalk under our own hedges, being known where it grew under the name of wild endive, christened in trade chicory, and being in reality a tall and aristocratic sort of dandelion, possessing too the medicinal properties of dandelion, and none whatever of the properties of coffee. But even if people be taken with a liking for this dandelion tea instead of coffee, they cannot have it pure. The chicory itself is far too costly to content the avaricious roguery of a number of deal- 
ers, and so it is adulterated with roasted corn, parsnips, manglewurzel, beans, Egyptian lupin seed, Kiscuit powder, burnt sugar, roasted carrots, oak bark tan, acorns, mahogany sawdust, and no little sand, the result of the original dirt judiciously left as a make-weight upon the root of the chicory itself.

Mustard can scarcely be said to have even the color of mustard, for it is colored with turmeric, and what passes for mustard is in many a case little more than mere husks and flour.

Pepper is messed up with wheat flour, mustard-seed husks, sago-meal, pea-flour, and ware-house sweepings; nor does it fare better with food for invalids, oatmeal being mingled with far less digestive barley-meal at half the price. Arrow-root (which it should be understood is the produce of under-ground branches or bulbs of the maranta plant, growing in the West and East Indies, having gained its name of arrow-root from the belief that it was a remedy against poisoned arrows,) is to the utmost economized; and though its purity is often of great importance to the invalid, there is for the most part sold instead, sago-flour, tapioca-flour, and most commonly of all, potato-starch.

Milk and bread are not so much adulterated. But the milk, partly by the kind of keep of the cows, partly by a little careful skimming, and in a multitude of cases by the liberal aid of the pump, is duly thinned. Flour and bread, of old mixed with plaster of Paris, ground bones, and potato-starch-thanks to the cheapening of pure materials, has come to content itself with alum only. But this running account of roguery, except for its curiousness, would be of little use without a few hints, as to partial detection and prevention.

As to tea, it is best to be content with black tea alone.

For sugar, the best advice is-if you like to pay for dirt, and to mix it with your preserves, puddings, and pastry, and choose to believe that sugar which moistens even the thick paper they place it in, and which looks dark, smells strong, and sticks to your fingers, is richer in sweetening than clear sparkling white sugar, out of which none of the sweetening but all of the dirt has been washed-then buy brown sugar.

"Please tell the people over the way," said a gentleman, "that I would take it as a particular faror, if in future they will send me the cow's hairs on one plate, and the butter on another, and I can mix them myself as I want them." Such is our advice as to coffee. It seems beyond the reach of average human honesty to sell it pure. The chicory is so fragrant-so wholesome-such an improvement on the flavor of the Arabian berry, and withal so much cheaper, that mixed it must be. We say, therefore, Buy your coffee in the berry, raw; your chances are at all events fifty to one better of having coffee only. Roast and grind it for yourselves, and, if you like chicory or dandelion, endive, or any other weed with it, why, buy the roots, scorch. them and grate them, and, like the man with the hairs in his butter, mix them to your taste. But do not, unless you choose to cheat your stomachs, buy ground coffee. A mill will soon pay for itself; and at all events never purchase canistered or bottled coffee, for in ninetynine cases out of a hundred an additional dose of dust is made to pay for the tin or glass.*

* It may not be amiss to show how tea is made in China, and coffee amongst the Turks.

The art of making tea consists in pouring the water on and off immediately, so as to get the fiavor.

Coffee-making is a more intricate affair, and cannot be fully conveyed in a receipt. The coffee must be slowly roasted, not burnt, and brought only to an amber brown; it must be roasted day by day, and reduced by pounding to an impalpable powder. In making it, two opposite and apparently incompati- 
As to water-every one knows that plumbers make the bottoms of the cisterns thicker than the sides, because the water eats the lead away; hard water does so more than soft, and water from the same source more at some times than others. Lead, as the phrase is, accumulates in the system, so that ever so little, taken day by day, at length sums up to a poisonous dose sufficient to mar the health. The remedy for this mischief is simply to have the pipes made of gutta percha. Many spring waters, though of course free from the animal impurities, abound still more in the medicinal. To render such waters fit for healthful use, some process of purification is absolutely essential, and such purification very perceptively improves both their cooking and washing properties.

Ordinary filters certainly free water from a considerable quantity of dirt, but not from the medicinal ingredients, nor even from all the animalculæ, some of which, though quite visible as monsters with a microscope, nevertheless find their way through the filter. One of the simplest processes of purification, if people will only take the trouble to perform it-and it is surely worth it for the increase of comfort and the advantage to health-is-for every forty gallons that the cistern holds to pour in one gallon of lime-water ; this has the effect of throw-

ble ends are to be secured-strength and flavor. To obtain the first, it must be boiled; by boiling, the second is lost. The difficulty is surmounted by a double process,-one thorough cooking, one slight one; by the first a strong infusion is obtained, by the second that infusion is flavored. Thns a large pot with coffee-lees stands simmering by the fire; this is the sherbet. When a cup is wanted, the pounded coffee is put in the little tin or copper pan, and placed on the embers ; it fumes for a moment, then the sherbet is poured on; in a few seconds the soth (caïmah) rises; presently an indication that it is about to boil is made manifest, when the coffee is instantiy taken from the fire, carried into the apartment, turned into the cup, and drunk."- $U r$ guhurt's Pillars of Hercules. ing down from the water a large proportion of the chemical ingredients, and no small multitude of the animalculæ. Another method of purification is by long slow boiling, then allowing the water to cool, and filtering it. Some trouble no doubt there is in any such course ; but pure water, like pure air, is essential to a life of health, and those who will not be at the trouble, must make up their minds to some degree of infirmity and unhappiness.

The subject of the Water-supply to large towns is one of the highest importance to the well-being of the community.

The quality of water for domestic purposes depends mainly upon its degree of hardness or softness; and this in its turn depends almost entirely upon the quantity of lime dissolved in some form or other in the water. It is found, upon experiment, that one gallon (weighing 70,000 grains) of pure water will not dissolve more than two grains of chalk, and so acquire two degrees of hardness; and that whenever more is contained in water, the excess is always owing to the presence of carbonic acid gas, which enables it to dissolve a much larger quantity. The practical part of our subject depends on this fact; for if by any means we can get rid of the carbonic acid, the dissolved chalk is necessarily precipitated, and the hard water, unfit for culinary and domestic purposes, becomes soft, and well adapted to both these uses. Carbonic acid is in part expelled from water by heating it to the boiling point: a still larger quantity is got rid of after boiling for some few minutes, and nearly every trace disappears at the end of half an hour ; and just in proportion as the carbonic acid gas is expelled, so does the chalk fall, rendering the water in the first instance turbid, and becoming deposited on the interior surface of kettles, 
where it forms the well-known rock of fur.

It has been found that water of 14 degrees of hardness lost two degrees when merely made to boil; boiling for five minutes reduced the hardness to 6 degrees; and for a quarter of an hour, to little more than 4 degrees. The practical application of this knowledge needs scarcely to be pointed out. Whenever a soft water is required, boil for several minutes before using. In making tea, for instance, the economy and general superiority of a soft water is well known. How many a young gentleman, with a smattering of science just enough to inform him that water gets no hotter however long or violently it is boiled, has laughed at his grandmother's antiquated notions, because she requested that the water might be made to boil thoroughly before the tea was made: the old lady could give no very satisfactory explanation of her prejudice, yet it was not the less a correct one.

Before going further in this matter, it may be stated that there are some waters in which the lime is dissolved in the form of gypsum (sulphate of lime): in these, the hardness is of a permanent character, and cannot be lessened by boiling. Tea made under such circumstances may be improved, either by the addition of a very small quantity of carbonate of soda, or the tea should be kept soaking for half an hour, under such circumstances as will retain the heat.

In washing, the use of hard water is, as is well known, extremely prejudicial. The explanation is exceedingly simple: every degree of hardness in a gallon of water destroys 10 grains of soap.

There is one practical matter of great importance, to which we wish to draw the attention of all concerned: it is the effect of boiling linen in hard water. If clothes are put into cold water, and then boiled the precipitation of chalk takes place on the clothes, and whatever coloring matter exists in the water goes down with the chalk, and also becomes attached to the linen, rendering it of that disagreeable and unremovable dirty hue which is so characteristic of certain laundries. If boiling is absolutely requisite for white fabrics, it should be done in water which has been boiled half an hour, allowed to stand, and then poured off from the sediment; otherwise, from the immediate precipitation of the chalk, the dirt is boiled in and thoroughly fixed to the fabric. A moment's consideration will convince any one, that a deposit similar to the fur in a tea-kettle cannot be expected to improve the appearance of white linen. Where clean rain water can be obtained, there is no objection to the boiling of clothes in it; as, being absolutely free from lime, no precipitation can take place. The use of soda in softening water employed in washing, is well known; but the remedy is not without its own evil: it weakens the fibre of the cloth, and unless it is much more thoroughly removed by rinsing than is usually the case, it occasions a very permanent yellow tinge when the cloth is heated.

\section{CHAPTER XVII.}

BoIliNG, STEWING, ETC.

From considering the properties of hard and soft water, we pass by a natural transition to the employment of that liquid in culinary operations. In practice, nothing can at first sight appear more simple than the operation of boiling, whether it be confined to the mere heating of a liquid, or extended to the preparation of an article of food; yet it is one which involves chemical principles of a very high order, and which is by no 
means so simple a matter as it may be regarded at a cursory glance.

To trace the steps of the process from its commencement, let us imagine a vessel of water placed over the fire, and receiving constantly a supply of heat from that source ; the effect is, that its temperature gradually rises from about $50^{\circ}$ or $60^{\circ}$, the usual warmth of ordinary water, to $212^{\circ}$, the point at which boiling takes place; but before it reaçes that height, a number of bubbles may be observed foaming on the sides of the vessel; these gradually increase in size, and when they become sufficiently buoyant, quit their position, rise to the surface, and escape; they consist of air previously dissolved in the water, and which is expelled by the increased heat. Water which has been boiled and allowed to become cold, without much exposure to the air, fails to reabsorb the quantity it previously contained, and consequently has its character somewhat altered. Thus, it freezes more readily than water which has not been boiled, in consequence of the air not having to be expelled in the act of solidifying, as is usually the case : hence, the ice from boiled water is free from those numerous air bubbles which are always to be observed in common ice. It possesses also a mawkish, unpleasant taste, and is totally unable to preserve the life of any aquatic animal. The presence of this minute quantity of air in ordinary water, is very essential to its utility. Faraday found that water, totally destitute of air, does not boil in the usual mode; but when heated to the boiling point, it at once, with an instantaneous and violent explosion, passes into the form of steam. This strange fact, which shows upon what small, and, apparently, trivial circnmstances, the comfort-nay, we may truly say-the existence of man depends, is strikingly shown by a very ingenious experiment, devised by that celebrated chemist. He took a piece of Wenham Lake ice, (which, from peculiar local causes, such as being formed from spring water, is totally destitute of air,) and melted it under a covering of sweet oil; this prevented the absorption of any air during the liquefaction; on continuing the heat, the water rose in temperature, and on reaching the boiling point, suddenly burst into steam, with an explosive power sufficiently great to scatter the glass vessel in which the experiment was made into fragments; and had it not been for a protecting covering of wire gauze, very serious effects might have ensued.

From the precipitation of the dissolved chalk present in most kinds of water, a cloudiness or slight turbidity is always to be observed in boiled water.

After the escape of the air, bubbles of steam, at first very small in size, are formed at the bottom of the vessel, those formed at first are at once cooled from the whole water not being of an equal temperature, and are condensed before they reach the surface: this very rapid and successive condensation of numerous small bubbles gives rise to that peculiar vibration which occasions what is termed the singing of the teakettle, and which, as is well known, is indicative of its approach to the boiling point; when the whole water is uniformly heated, this effect no longer occurs, but the bubbles of steam rise to the surface and escape. After hav. ing been heated to $212^{\circ}$, the temperature of water no longer rises ; it is not possible, under ordinary circumstances, to increase the temperature in the slightest degree; for all the extra heat that is given to boiling water merely produces increased quantity of steam, by which it is carried off, without affecting the heat of the remaining water. 'This is a matter of considerable practical importance in cookery; and it should be always borne in mind, that the most gentle simmer is as efficacious in cooking as the most violent boil- 
ing; for the degree of heat in both cases is precisely the same, so that after having once raised the water to the boiling point, the most moderate fire is sufficient in ordinary cases to keep it there; by attention to this point, a most enormous saving may often be effected in the consumption of fuel, although this is a consideration that will be more fully entered into in a subsequent chapter.

Thick liquids, which do not readily permit the escape of steam or the rapid motion between the particles of fluid, may, however, be readily heated at the part exposed to the fire to a much higher degree, while those portions not immediately in contact with the heat are much colder ; from this cause they are very apt to be charred, and if articles of food, they are totally spoiled. To avoid this effect, recourse may be had to the bain marie, which is simply the same contrivance that may be observed in a carpenter's glue-pot, applied to the preparation of articles of food,-being merely an inner vessel to contain the substance to be heated; this is placed in an outer one, the space between the two containing water. On placing this contrivance on the fire, it is obvious that the substance in the inner ressel being heated solely by the boiling water, cannot possibly become burnt. This most useful contrivance is adopted in all first-class kitchens, and is equally indispensable in the chemist's laboratory; by its aid, soups, gravies, \&c., can be kept hot any length of time without risk, preserves made without burning, \&c. The chief precautions required in its use are, that the inner vessel should be thin and formed of metal, so as to allow the rapid transmission of heat from boiling water, and care should be taken that the outer vessel does not boil dry. One serious disadvantage attends its use as ordinarily employed: it is, that it is impossible to heat substances in it to the boiling point, for the water itself is only at that temperature and the substance in the inner vessel is always a few degrees below. This evil, however, may be entirely obviated, by using a solution in the outer ressel, which boils at a higher temperature than $212^{\circ}$, and which will therefore raise the inner vessel and its contents to that point; thus, if the water be made to dissolve as much common salt as it is capable of doing, it will not boil until it is heated to $224^{\circ}$; or if it is saturated with sal-ammoniac or nitre, the heat will rise $12^{\circ}$ or $14^{\circ}$ higher. We need scarcely say that the first of these substances will be found a very useful and economical addition to the bain marue. When chemists require a still higher temperature, they have recourse to a bath of olive oil, which is capable of bearing a degree of heat as high as $500^{\circ}$; but its extreme danger over an open fire entirely precludes its use in any culinary operation.

The mode of conducting the operation of boiling should not be uniform, but vary with the different purposes required. Thus, in the case of meat, a temperature of $212^{\circ}$ hardens, instead of softening, two of the substances which it contains; namely, the fibrine, or material forming the chief part of the muscular fibre, and the albumen, or portion which is analogous to white of egg; if, on the contrary, meat is cooked by means of water at a lower temperature, the most nutritious parts are dissolved out, and the solid food left comparatirely innutritious. The celebrated German chemist, Liebig, proposes the following plan:-he recommends that a piece of meat of considerable size should be taken and plunged into perfectly boiling water, over a good fire; that the water should be kept boiling for a few minutes, and then a portion of cold water, equal in quantity to about one-half of the boiling water, should be thrown in: this will reduce the temperature to about $160^{\circ}$, at which point the meat 
should be kept until thoroughly done; for man; uncooked starch not being which, however, takes a much longer readily or easily digested. And even in time than the ordinary mode.

The object of this mode of proceeding is, in the first instance, to harden the exterior of the meat, converting it into a sort of crust, which prevents the escape of the nutritious juices into the water, while the long continuance of the gentle heat afterwards cooks the interior without hardening either the albumen or the fibrine. Of course, where the object of boiling is to make soup, the opposite plan must be had recourse to; the meat should be in small instead of large pieces, put on in cold water and very slowly heated, so that all the soluble parts may be dissolved before the fibre is hardened by the action of boiling water.

In boiling eggs, the effect of heat in hardening the albumen is well known; by being suddenly plunged into boiling water, the outside is hardened to the greatest degree of which it is capable, and is thereby rendered exceedingly difficult of digestion, while the inside is barely warmed; if, on the contrary, they are placed in cold water, which is then raised to the boiling point, removed from the fire, and allowed to stand about a minute (or two, if required to be well done,) it will be found that, instead of having an almost leathery consistence, the white will be unifurmly partially hardened, and will furnish a much more pleasant and digestible article of diet; the improvement, in fact, is so great, that common eggs cooked in this manner very nearly approach new laid ones in quality.

If the operation of boiling has to be performed on any substance containing starchy matters-as potatoes, rice, flour, \&c., then the heat must, on the contrary, be raised to a sufficient degree to burst the little grains of which the starch consists, and liberate the interior nutritious portions, before it can become fit food the case of those vegetable-feeding animals whose power of digesting such substances surpasses that of man, there is the greatest advantage to be derived from the use of cooked food, as the most intelligent and scientific farmers at the present day well know; and we would strongly urge on those of our readers who keep pigs to try the experiment of baking the potatoes they give them; for this process, like boiling, has the effect of bursting the starch grains; they will find the effect to be, that the food will go much further, all of it being digested, and that the quality of the flesh will be very materially improved.

\section{- \\ CHAPTER XVIII. \\ ECONOMY OF HEAT.}

Perhaps few of our readers are aware of the extraordinary wastefulness of our usual processes for obtaining artificial heat; at the most moderate computation, seven-eighths of the warmth produced by an open fire, pass up the chimney, and are entirely useless; and according to other estimates, which we regard as being nearer the truth: fourteen parts out of every fifteen are uselessly wasted. It may be asked what are the defects of a common fireplace that render it so wasteful, and in what way is the heat carried off? In reply it may be stated, that one half the heat produced passes away with the smoke and heated air arising from the fire, a quarter is carried up by the draught of cold air from the room, which, flowing around the fire and between it and the mantel-piece, rises with the smoke. Again, the soot which passes away is unburned fuel, and is therefore useless; and a large portion of 
heat is thrown downwards upon the ash- door, it is converted into a close stoves es and is wasted; while the iron of which the ranges are generally made, conveys away a considerable quantity. On the continent of Europe the production of heat is more economically managed-stoves of very admirable construction being constantly had recourse to, both for the purpose of producing warmth and for cookery. It is to the latter application of heat that we must mainly confine ourselves, having been at some considerable pains in examining the various stoves and ranges now to be obtained.

The cooking-stove common in Europe, consists of an enclosed fire-pan, with a grating below and a lid at the top for the supply of fuel; this is enclosed in an oren. supported on the floor of the room by feet, and which is heated by the warmth thrown nut by the sides of the fire-pan, and also by a flue spreading over the top, which is thus heated; while the upper surface of the flue forms a hot plate, on which many saucepans, \&c., can be kept boiling, and any vessel can also be placed over the fire by the removal of the lid. As the draught is under perfect control, the fuel is slowly consumed, and the stove affords means of baking, boiling, frying, and stewing, at a very small expense.

Those of our readers who visited Prince Albert's Model Cottages opposite the Exhibition in London, may have noticed a stove looking very much like a long oblong box, standing on four legs, having two doors in the front, one opening into a large sized oven, the other disclosing the fire-grate, which was fed by the removal of a lid at the top; - the draught from the fire passed over the oven, heating it, and the hot plate above. This stove combines all the advantages of the continental cooking-stove, with the cheerful appearance of an open fire; at the same time, by closing up the fire-place with an excessively small consumption of fuel. It bakes admirably, either large joints, or bread, at the same, time it boils a saucepan and steamer over the fire-hole, and also four large, or six smaller saucepans on the top of the hot plate. It fries well, and broils before the fire, and this with less than one half the fuel that was employed to do a portion of the work in the range. The Bain Marie, which is so excellenta means of keeping soups, gravies, sauces, \&c., hot without burning or drying them up, is readily used with it; and the sauté pan. or deep frying-pan, which is employed as a preliminary operation in most French made dishes, is conreniently used; this contrivance derives its name from the verb sauter, to jump-the meat being rapidly turned over and browned previous to stewing. The only disadvantage attending the use of this stove is, that it is not calculated for roasting, but every other operation in cooking it performs infinitely better than a common fire. This stove, which is termed the Cottager's Stove, is made by Messrs. Benham, Wigmore Street, London.

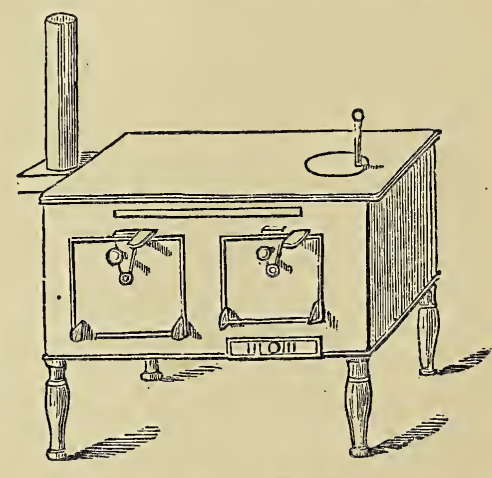

Cottager's Stove.

Those who object to a stove, preferring a range, even at a greater sacrifice of economy, should still be somewhat guided by correct principles in their selection: 6 
a range surrounded by iron is an absurdity, as the metal conducts away the heat rapidly; it should be backed and lined with fire-brick, or Welsh lumps, which throw out the heat with great power. In an open fire-place, the active combustion is wanted in front for roasting, and there only should air enter the fire; in most ranges the air enters below, causing the greatest heat to be thrown upon the ashes. It may be thought that closing up the bottom would produce the same effect as allowing it to be choked up with ashes in a common grate, deadening the fire; this is not the case with a properly constructed range. Backed with a slanting back of fire clay, the ashes can be readily removed at the bottom, and all the draught being in front, there is a bright fire at the place where it is required.

In situations where gas is to be obtained, it forms a ready, and for some purposes, very economical means of obtaining heat; its economy does not arise

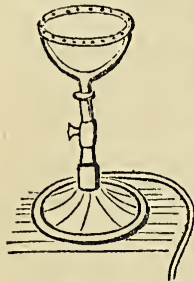

Fig. 2. from its cheapness compared with other means, but from the fact that it need not be lighted till the instant it is required, and can be as quickly extinguished when it has done its required duty; for heating any vessels containing liquids, especially if the heat is required to be only of short continuance, gas will be found extremeiy advantageous. A ring burner, constructed as shown in Fig: 2, less than three inches in diameter, will quickly boil a gallon of water in a metallic vessel; burners of this description are usually used in the laboratory, surrounded by a case made. of sheet iron or tinned plate, as in Fig. 3 ; this serves to support the vessel to be heated, to steady the jets of flame, and to conduct every portion of hot air against the bottom; the door also gives a ready access to the burner for the purposes of lighting the gas.
For the domestic use of gas in heating we believe there is no contrivance so useful as the following:-A circular hole, from two to four or more inches in diameter, is cut in the dresser, through which is passed a sheet-iron tube, supported by three little elbows; this tube projects a few inches above the table, and about a foot and a half below; its lower end is open, and into it projects a gas-pipe, furnished with a stop-cock; the upper extremity is covered with a sheet of wire gauze, similar to that used for blinds, on which, as shown in Fig. 4, may be placed some pieces of pumice-stone, surrounded

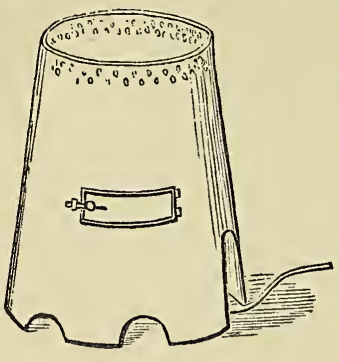

Fig. 3.

and kept together by a broad ring;-neither the pumice-stone nor the ring, however, are essential parts of the contrivance. The action of this arrangement is as follows:-When the gas is turned on

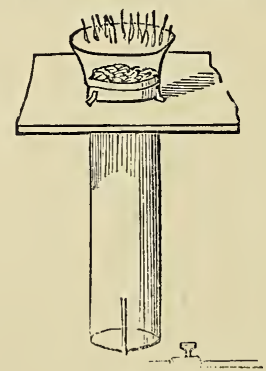

Fig. 4.

it escapes from the pipe, rising through the tube, and mixing with the air contained within it; this mixture then escapes through the wire gauze, and naay be lighted on its upper side, without pass- 
ing through it to the gas below; the flame should be perfectly free from smoke, which indicates too much gasshould be pale, colorless, and not soil any bright metal placed in it; if the flame is in the slightest degree yellow it will do this, and then the gas should be partly turned off-on the contrary, if there is not enough gas, the flame will be extinguished. When lighted, the pumice becomes red-hot, and throws out a great heat; when used in boiling, the vessel should be supported a short distance over the flame by a trivet; if it is made to rest on the top of the ring, and is sufficiently large to close it entirely, the current is stopped and the flame extinguished, whilst the unburned gas still escapes below. This contrivance is most useful ; it is lighted in an instant, is perfectly free from smoke, no unburned gas escapes, it throws out great heat, and may be employed to heat bright tools with much more convenience than a charcoal fire. The objections to its use are, that in burning it produces, as all gas does, a quantity of carbonic acid gas, deteriorating the air, and that the flame cannot be very much enlarged or diminished, so that if fires of different power are required, two or more of the contrivances must be put in order. Otherwise, the instantaneous action, small cost, great heating power, and cleanliness of the plan, strongly recommend it. In summer weather, in many small families, it can be made to dispense altogether with the use of a fire. By a little variation, the whole contrivance may be made to stand on the table, like Fig. 2 ; in this and other cases, vulcanized India-rubber will be found to form by far the best kind of flexible tube, being quite impervious, very durable, and excessively pliant. Those who wish to try the experiment of heating on this plan, may readily do so by covering the top of the glass chimney of any common burner with a piece of wire gauze, folding it over the sides; the gas may then be turned on, and lighted above the gauze, after it has mingled with the air in the chimney: a small burner, however, daes not afford sufficient gas for the purpose, and there is consequently too much air, and the flame is weak and liable to go out.

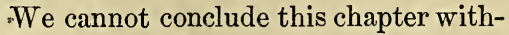
out entering our most earnest protest against all those injurious cortrivances for burning charcoal without a flue. The use of charcoal-braziers in a large kitchen is not to be recommended, but a charcoal stove in a dwelling-room is most objectionable. Charcoal, in burning, produces carbonic acid gas, an invisible and therefore insidious poison, which is so deadly in its effects, that if the air of a room contains but one-tenth of its bulk, the breathing of it for any time is fatal. Every pound of charcoal, in burning, produces more than three pounds and a half of this deadly gas.

In Paris, in the years 1834 and $\mathbf{1 8 3 5}$, there were 360 cases resulting from the fumes of charcoal, of which more than 260 were fatal. In order to test the effects of these stoves, Mr. Coathupe, of Wraxall, shut himself up in a close room containing eighty cubic yards of air, with one of them in action. In four hours he was seized with giddiness, which, in an hour's time, became most intense; he then had the desire to vomit, but not the power; this was followed by an utter loss of strength, throbbing at the temples, and agonizing headache, but no sense of suffocation; finding that the experiment was becoming dangerous, he essayed to open the window, but had the greatest difficulty in so doing; and when his wife came into the room, he was found in a speechless state, in which he remained for some time.

In a fatal case, which happened in St. 
John's Wood, where two girls were killed by the use of these stoves, it was found, that the quantity of carbonic acid produced was capable of rendering poisonous the air of a room ten times the size of the one in which it was used. Men who, knowing the poisonous effects of these stoves, still sell them, recommending them as wholesome, with the deliberate assertion that their prepared fuel, which is merely charcoal disguised, is not deleterious, evince a much more lively interest in the state of their own pockets, than in the lives of their customers.

Let it not be imagined that the case of Mr. Coathupe is but a solitary one; the action of burning charcoal is of the same deleterious nature on all persons. In January 1836, seventy people suffered the same symptoms, though in a milder degree, in the church at Downham, in Norfolk, England, where two of these dangerous contrivances had been introduced: and in the Annales d'Hygéine, tom. xi., will be found an account of the suicides in the department of the Seine in ten years; these were 4595 in number, of which 1426 deaths were produced by burning charcoal. We would again recommend our readers under no circumstances, however much they may be recommended by false assertions, to armit stoves without flues into their houses.

\section{CHAPTER XIX.}

\section{CLEANING AND DISINFECTING.}

WE have elsewhere given directions as to the best mode of cleaning various articles, such as bottles, glass, \&c. What remains, therefore, under this head, is to furnish hints for cleaning miscellaneous articles, which have not been included under previous accounts; and the very important process of disinfecting, which may be regarded as a medical cleaning, falls, naturally, into the same chapter. As the substances to be submitted to the process of cleaning vary greatly from one another, we shall find it more convenient to throw our remarks into the form of miscellaneous hints, than to arrange them. in a chapter for consecutive reading.

Iron-work which is exposed to wet, rusts rapidly; it is usually preserved from the action of moisture either by covering it with two or three coatings of paint, as is customary in large out-door works, or by brushing it over with a varnish termed Brunswick black; this plan is usually followed in the case of smaller substances used in-doors. A very superior plan for protecting small iron goods from the injurious action of wet, is to heat them a little below redness, and whilst hot to brush them over with common linseed oil, which is decomposed by the heat, and forms a thin, very firm coating of varnish, which is quite impervious to water, and unlike paint or Japan-black, does not chip off. It is evident that this plan can only be adopted in few cases, but where it is available we very strongly recommend it. Floor Boarding and other wood work is exceedingly apt to be stained by various substances spilt upon it. Ink stains, for instance, are extremely obstinate; they withstand washing many times, and at last turn to a rusty iron color, from the application of the alkali of the soap. But the black stain of recent ink and the rusty iron-mould may be removed by the action of oxalic or muriatic acid. As wood is not likely to be injured like cloth or linen, the latter may be used, being the cheaper, and it should be diluted with two or three times its bulk of water, and applied until the stain is removed. Grease which has been trodden 
in, or has remained a long time, should be first softened by the application of a little turpentine, and then it will be found to yield much more readily than it otherwise would to the action of fuller's earth and pearlash or soda. Fruit stains are quickly removed by the action of a little chloride of lime, mixed with water, and applied until the desired effect is produced. It should be borne in mind that all vegetable colors are utterly destroyed beyond any restoration, by the energetic action of this agent.

Paint, when soiled, is readily cleaned by soap and water; soda and pearlash are frequently employed, but they act by removing a portion of the paint, and if not thoroughly washed off with clean water afterwards, they will be found to soften the whole. Caustic alkalies, such as the solution for washing on the new plan, will rapidly dissolve paint, and are therefore inapplicable for cleaning; they may, however, be usefully employed in removing paint from wood, where such an operation is requisite.

I late may be cleaned by rouge, or if this is not readily obtained, by washed whiting; this is readily made by stirring some whiting up with water, then allowing the larger particles and the grit to subside, and pouring off the water charged with the finer powder, which is allowed to settle, and dried for use. When plate is very much stained, it may be cleaned with putty powder, but this preparation would soon wear away the silver if used frequently or unnecessarily. It may be mentioned, that this substance is not made from putty, as its name might seem to imply, but is a rust or oxide of tin, obtained by heating the metal.

Japanned Goods, such as tea-boards, should not have boiling water poured upon them, but should be washed with warm water, and polished with a piece of wash-leather and fine flour.

Knives-The common wooden knife- board wears out the knives very rapidly, it is therefore much better to employ a piece of buff-leather to cover the board; for very superior cutlery emery powder should be used instead of Bath-brick. Whatever the material of the board, it will be rapidly spoiled by cleaning the backs of knives upon the edge of the board; to prevent this evil, a small piece of leather should be fixed on one end to clean the backs upon.

Steel Forks are readily cleaned by having a pot of damp moss or hay, with some sand intermixed, into which they may be repeatedly thrust. If knives or forks get an unpleasant taint which cannot be removed readily, they may be plunged into the mould of the garden, which has much absorptive power, and rapidly removes such odors.

Bedsteads may be freed from vermin by brushing them over in the cracks with a mixture formed of one ounce of corro sive sublimate, dissolved in half a pint of oil of turpentine, and the same quantity of any spirit, such as strong gin or whiskey; this effectually prevents their harboring. But when first applied, it has a disagreeable odor from the turpentine, and great care must be taken with it, as it is excessively poisonous. It has been found that the presence of the odor of creosote has effectually driven away these enemies to our nocturnal peace. On the whole, constant and unremitting cleanliness, and the employment of iron bedsteads, which are now manufactured of the most elegant forms, are the best means of getting rid of these pests.

We pass on to the important operations of disinfecting. Various means have been proposed of lessening or utterly destroying the infectious emanations that proceed from persons in certain diseases, and which frequently have the power of attaching themselves with greater or less tenacity to articles of wearing 
apparel, furniture, \&c. Generally speaking, a good system of ventilation is sufficient to prevent infection. When rooms are properly aired, a disease can seldom be caught more than a few feet from the patient; or even in the case of those most infectious disorders, scarlet-fever, and small-pox, it seldom spreads more than a few yards; but if the air of a room is confined, the infection is concentrated, and becomes much more certain in its action.

Downy and fibrous materials readily receive infection; it may, in fact, in many instances, be folded up in them, and so retained almost any length of time; but if they are thoroughly exposed to a free current of air, it is dissipated in a short time. It should be mentioned, that infectious diseases are more readily received in certain states of the body; thus, fear, timidity, niental anxiety, and such states of mind, by lowering the general tone of the system, render it much more liable to contract infectipus or contagious diseases; a state of exhaustion from bodily fatigue, or from hunger, has the same tendency. Infection is also more readily received through the lungs than through the skin; therefore, it is important never to receive the breath of a patient, and, as a sailor would say, always to keep to the windward side of him. Amongst the domestic disinfectants, vinegar has a great reputation, but undeservedly so ; its only action is to overpower, by its odor, the smell of a sick room-as a destroyer of the peculiar influences that engender disease, it has no power. Burning substances act in the same manner. Burnt brown paper, fumigating pastiles, tobacco, only act by substituting one smell for another. The ridiculous practice of carrying about a piece of camphor is very common, and is perfectly inefficacious. If it has any action at all, it must be an injurious one ; for camphor is a stimulant, and its constant inhalation must tend to lower the system, and so produce the very evil it is supposed to remedy.

The best means of preventing infection, are ventilation and cleanliness in every particular. The best means of destroying it are those powerful chemical agents which have the power of uniting with the hydrogen which is supposed to form part of the infectious substances.

The most powerful, easily controlled, and in every sense the best disinfectant, is chlorine gas. This agent at once destroys every trace of infection in all substances submitted to its action. Its formation is perfectly under control, and goes on in a gentle manner for days together, without requiring care or attendance. We consider that the slow liberation of chlorine is far superior to the employment of chloride of lime, which gives forth the gas in a modified form.

In one case of a school where scarletfever had returned after several attempts at purification, chlorine effected the complete removal of every trace of the disease. Various modes of liberating chlorine are known to chemists ; but, for such purposes as the present, where a slow, uniform, and constant action is required, there are none equal to the following plan:-One pound of common table-salt is to be intimately mixed by stirring with an equal weight of a substance called manganese, which may be readily obtained from any good chemist. Small portions of this mixture should be placed in shallow pans (the saucers of common flower-pots answer the purpose); and upon them should be poured a mixture of oil of vitriol (sulphuric acid) and water, the quantity required for the above weights, viz., for one pound of each ingredient, being two pounds of oil of vitriol and one of water, both by weight. These should have been previously mixed in a wooden vessel, being stirred by a wooden lath, and allowed to become cool before being poured on the salt and manganese. 
as the mixing of the acid and water generates great heat. Too much care cannot be taken with the acid, as it is excessively corrosive in its nature, and destroys most substances with which it comes in contact. When these materials are all mixed, chlorine is slowly evolved for a period of three or four days, and in so gentle a manner, that not the slightest irritating or unpleasant effect is produced. If it is wished to cause the more rapid production of gas, the saucer may be placed over a basin of boiling water, or upon a hot brick; but the slow generation for a considerable length of time is what should be more especially aimed at. It is needless to say, that all substances supposed to have been contaminated, should be spread out so as to receive the influence of the gas; the bed-linen, and all woollen garments, being exposed by being spread out on chairs, lines, \&c.; the drawers and cupboards opened; and while the disinfecting is in actual operation, the windows and doors should be kept shut, to prevent the dissipation of the chlorine. It is found that two ounces of manganese, with a proportionate quantity of the other materials, is sufficient for a room twenty feet wide, forty feet long, and twelve feet high, which contains 20 $\times 40 \times 12=9600$ cubic feet.

It may be mentioned, that breathing chlorine in this highly diluted state, is not injurious to the general health; although, in a concentrated form, or unless very much diluted with air, it is irritating in the highest degree. In the very dilute state, it is occasionally prescribed for the inhalation of consumptive patients.

For the satisfaction of those who rely on the authority of a great name, it may be stated, that chlorine used in the manner here recommended, was employed by one of the most illustrious of English chemists, in the case of the Penitentiary, when a violent and fatal disease broke out there, and with complete success. The operator in this case was FARADAY.

It may occasionally be found more convenient to use another mixture for the liberation of chlorine gas, in which case the following may be employed:One part of manganese is to be drenched with four parts by weight of muriatic acid (spirits of salts), mixed with one part of water. The gas is evolved slowly in the cold, and rapidly if assisted by a gentle heat. This process is rather more expensive, and possesses no advantage over the one previously described.

\section{CHAPTER XX.}

\section{FERMENTING AND DISTILLING.}

Is ordinary language, the term fermentation is employed to signify the peculiar changes which take place when a solution of sugar, or any vegetable substance containing saccharine matter, is converted into spirit-this, however, is only one of many of such kinds of action, which are well known to chemists. The most important fermentations are the saccharine fermentation, when sugar is formed by a change taking place in starch; the vinous, when spirit is formed from sugar; and the acetous, in which vinegar or acetic acid is formed from spirit.

The saccharine fermentation, or the formation of sugar from starch, is interesting, though it does not influence such operations as are included in our Domestic Manipulations. If starch is dissolved in water, a little wheat flour added. and the whole exposed to a moderately warm temperature, it will be found that after a few days, varying in number with the degree of warmth, the starch has disappeared, and the liquid has become sweet from the formation of sugar. The same change takes place with much greater 
rapidity if starch is boiled with a solution of malt, which contains an active principle called diastuse, capable of bringing about this fermentation in a short time. The formation of sugar from starch is an operation which constantly occurs in all growing seeds, the effect being to change an insoluble substance, such as starch, into one which is capable of being dissolved in the juices of the young plant, and nourishing it during the early stages of its growth. In the operation of malting barley, the change is induced artificially, for the purpose of producing sugar in the malt, which is afterwards made to undergo the second kind of fermentation, namely, the vinous, or that in which spirit is produced.

When sugar, either that which naturally exists, in many plants, or as formed from starch as just mentioned, is dissolved in water, so as to form a moderately weak solution, and the whole exposed to a degree of warmth varying from seventy to eighty degrees, it rapidly undergoes a remarkable change, provided a small quantity of any vegetable ferment is present-such as yeast, or the juice of the grape, or of many other fruits. The sugar wholly disappears, and is resolred into two substances-one a gas, termed carbonic acid, which escapes, giving rise to a slow effervescence; and the other, a portion of spirit, which remains in the liquid. This lind of fermentation is inuch more difficult to prevent than to establish; in making syrups, it is found especially annoying; for if the quanity of sugar used is too small, the syrup is certain to ferment and spoil ; and if too much is added, it crystallizes out in the solid form; as a general rule, however, it is found that two parts, by weight, of sugar, to one part, by weight. of water, or other liquid-such as the juice of fruits, made into a syrup by boiling for a short time-neither ferments nor crystallizes.
In the act of fermentation the spirit produced by the process last described is changed into acetic acid, or vinegar. Here, also, the presence of some substance capable of commencing the fermentation is requisite, for pure spirit and water will not undergo the change. The ferment employed may be the vinegarplant-or it may be a little vinegar, which may have been previously formed.

For the rapid progress of the acetous fermentation, a higl temperature is requisite-even as great as about eightysix degrees-and free exposure to air is essential. The best vinegar is that made from weak wine, at Orleans; the plan followed is to introduce a portion of vinegar into the ressels, adding the wine at intervals, and never quite emptying tliem. In this country, a weak beer is brewed, without hops, for the purpose of making vinegar, and a small quantity of dilute oil of vitriol is added, after the vinegar is formed, to destroy the mouldiness that is otherwise apt to be present. Vinegar may be formed from any weak spirituous liquid; but it should te borne in mind that two circumstances are essential to success-namely, a high summer temperature, either natural or artificial, and free exposure to air.

The process of distillation is one which is used for separating liquids from each other that boil at different degrees of heat. In domestic economy, it is most frequently employed to obtain spirit, more or less flavored, or scented, with some volatile essential oil. The apparatus commonly used is the Still, for boiling the liquid to generate the vapor, and a long spirally twisted tube termed the worm, which is placed in a tub of cold water, and through which the steam passes to be condensed. The worm is the most objectionable part of the modern still; its great evil is the difficulty with which it is cleaned, so as to prevent one strong-flavored substance spoiling 
those which are distilled afterwards. If the coils of the worm are not very numerous, a bullet, with a string attached, may be passed through it, and a sponge or small bottle-brush, fastened to the string, may be worked backwards and forwards; but if there are several coils, it will be found impossible to do this, from the resistance caused by friction. In this case, the only plan is to close one end of the

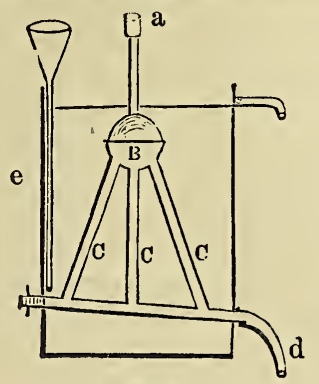

Fig. 1.

worm with a cork, and fill it with a solution of caustic alkali, allowing it to remain for some hours, and repeating the application with fresh liquid, if it be required.

In Germany, the worm is being superseded by an excellent condenser, which is so superior that we are induced to give a sketch of it, hoping that it may lead to its adoption in this country. The vapors from the still pass into the tube A (Fig 1), by which they are conducted into $\mathrm{B}$, a hollow globe, made to unscrew at its centre. The vapors, passing along the tubes C. are condensed, and the distilled liquid drops from $\mathrm{D}$. The pipe $\mathrm{E}$ should convey a constant stream of cold water to the bottom of the tub, and this. rising as it is warmed by abstracting heat from the tubes and globe, should escape by F. All the tubes being straight, it is obvious that they can be readily cleaned from their ends.

In the laboratory, distilling is most frequently performed with vessels term- ed retorts, or even from flasks; but as these are not very applicable to domestic purposes, we pass them over.

In domestic practice, the still is usually employed to obtain some water or spirit flavored with essential oil, or the oil itself, and the process should be slight y modified so as to suit each case. The vegetable substance should not be placed on the bottom of the still itself, as in that case it might become burnt, and so give an unpleasant flavor to the whole; but a bottom of wicker-work should be placed in the still in the first instance for it to rest upon, or a perforated board. The substance to be distilled should be placed in the still, covered with water, for some hours before the fire is lighted; no more water being ad.ded than sufficient to cover it, if the preparation of oil is the object.

Herbs, for distilling, should be collected on a dry day, and-unless the oil resides in the seeds, as in the case of caraway, anise, \&c., or in the flowers, as in the rose, lavender, \&c.-just before the flowers have opened, as at that period there is the greatest quantity of essential oil in the plant. All plants cultirated for distillation, should be grown in a situation where they can receive a full amount of sun-light, as shade or darkness very much tends to prevent the formation of essential oil.

The liquid which comes out of the worm, is a mixture of water highly flavored with the substance, and some undissolved oil-this latter is sometimes 67 heavier and sometimes lighter than water, either sinking or floating; in the latter case the oil may be readily separated by filling a bottle with the nixture, and when the oil has collected at the top. carrying it off by a few threads of cotton Fig. 2. placed as in the figure (Fig. 2) 
taking care that they are moistened with oil before arranging them; the cotton acts as a syphon, and removes the whole of the oil. If the object of the operation is to obtain the oil and not the distilled water, the latter should be preserved, and used again and again with fresh herbs, because having in the first operation dissolved as much oil as it is capable of doing, it causes no loss in the subsequent distillations.

\section{CHAPTER XXI.}

\section{LAYING OUT TABLES AND FOLDING NAPKINS.}

The art of laying out a table, whether for breakfast, luncheon, dinner, tea or supper, consists in arranging the various dishes, plate, glass, \&c., methodically, and adhering to the rules we are about to make known.

Much trouble, irregularity, and confusion will be avoided in a house when there is company, if servants are instructed to prepare the table, sideboard, or dinner-wagon, in a similar manner and order daily.

All tables are usually laid out according to the following rules, yet there are local peculiarities which will necessarily present themselves, and should be adopted or rejected, as may appear proper to the good housewife:-

Breakfasts. - The table should be covered with a clean white cloth; the cups and saucers arranged at one end, on a tray if desirable; or the coffee-cups and saucers may be arranged at the right-hand side of one end of the table, and the tea-cups and saucers at the left: the tea-pot and coffee-pot occupying the space between in front, and the urn that at the back. Some persons substitute cocoa or chocolate for coffee, in which case they are to be placed the same. The slop-basin and milk-pitcher should be placed to the left; and the cream, and hot milk-pitchers, with the sugar basin, to the right.

The remainder of the table should be occupied in the centre by the various dishes to be partaken of ; while at the sides must be ranged a large plate for meat, eggs, \&c., and a small one for toast, rolls, \&c., with a small knife and fork for each person; the carving knife and fork being placed point to handle; the butter and bread knives to the right of their respective dishes, which occupy the centre part, and spoons in front of the hot dishes with gravy. Salt-cellars should occupy the four corners, and, if required, the cruets should be placed in the centre of the table.

Dry toast should never be prepared longer than five minutes before serving, as it becomes tough, and the buttered, soppy and greasy, if too long prepared. Hot rolls should be brought to table covered with a napkin.

Every dish should be garnished appropriately, either with sippets, ornamental butter, water-cresses, parsley, or some one of the garnishes we shall point out in a future page.

The dishes usually set upon the table are selected from hot, cold, and cured meats ; hot, cold, cured, and potted fish ; game; poultry, cold or devilled; fruit, ripe, preserved, or candied; dressed and undressed vegetables; meat-pies and patties, cold; eggs ; honey-comb ; and savory morsels - as grilled kidneys, ham-toast, devils, \&c.

Déjeuners à la fourchette are laid the same as suppers, except that tea and coffee are introduced; but in sporting circles not until the solids are removed.

When laid for a marriage or christening breakfast, a bride's or christening cake 
should occupy the centre instead of the épergne or plateau.

Luncheons, or Noonings.-The luncheon is laid in two ways; one way is to bring in a butler's tray with let-down sides, on which it is previously arranged upon a tray cloth, and letting down the sides and spreading the cloth upon the dining table, to distribute the things as required. The other is to lay the cloth as for dinner. with the pickle-stand and cruets opposite each other; and, if in season, a small vase of flowers in the eentre ; if not, a water-pitcher and tumblers, which may be placed on a side-table at other times. The sides of the table are occupied by the requisites for each guest, viz., two plates, a large and small fork and knives, and dessert-spoon. . A folded napkin, and the bread under, is placed upon the plate of each guest.

Carafes, with the tumblers belonging to and placed over them, are laid at the four corners, with the salt-cellars in frout of them, between two table-spoons laid bowl to handle.

If French or light wines are served, they may be placed in the original bottles in ornamental wine vases, between the top and bottom dishes and the vase of flowers, with the corks drawn and partially replaced.

The dishes generally served for luncheons are the remains of cold meat neatly trimmed and garnished: cold game hashed or plain; hashes of all descriptions ; curries ; minced meats ; cold pies, savory, fruit, or plain; plainly cooked cutlets, steaks, and chops ; omelettes; bacon; eggs; devils and grilled bones; potatoes; swectmeats ; butter; cheese; salad and pickles. In fact almost anything does for lunch. whether of fish, flesh, fowl, pastry, vegetables: or fruit.

Ale and porter are generally served, but occasionally sherry, marsalla, port, or home-made wines, are introduced, with biscuit and ripe fruit.

A good housewife should always have something in the house ready to convert into a neat little luncheon, in case a few friends drop in, and it is astonishing how a really nice looking affair may be made out of the remains of the dinner served the day before: some glass, a sprinkle of plate a few flowers, some good ale. or a little wine, and above all, a hearty welcome.

NAPKins.-Dinner napkins should be about twenty-eight inches broad, and thirty inches long. They may be folded in a variety of was, which impart a style to a table, without adding much to the expense, and may be readily accomplished with a little practice and attention to the following directions and diagrams.

\section{1.-THE MITRE.-(Fig. 1.)}

Fold the napkin into three parts longways, then turn down the right-hand corner, and turn up the left-hand one. as in Fig. 2, A and B. Turn back the point $A$ towards the right, so that it shall lie behind $\mathrm{C}$; and $\mathrm{B}$ to the left, so as to be behind $D$. Double the napkin back at the line $\mathrm{E}$, then turn up $\mathrm{F}$ from before and $G$ from behind, when they will appear as in Fig 3. Bend the corner II towards the right, and tuck it behind I, and turn back the corner K towards the left, at the dotted line, and tuck it into a corresponding part at the back. The bread is placed under the mitre, or in the centre at the top.

\section{2.-THE EXQUISITE.-(Fig. 4.)}

Fold the napkin into three parts longways, then fold down two-fifths of the length from each side, as in Fig. 5, at A; roll up the part $B$ towards the back, repeat on the other side, then turn up the corner towards the corner $\mathrm{A}$. and it will appear as $\mathrm{D}$. The centre part $\mathrm{E}$ is now 
to be turned up at the bottom, and down ' at the top, and the two rolls brought under the centre piece, as in Fig. 4. The bread is placed under the centre band, $K$, Fig. 4.

\section{3.-THe Collegian.-(Fig. 6.)}

Fold the napkin into three parts longways, then turn down the two sides towards you, so that they shall appear as in Fig. 7 ; then roll up the part A underneath until it looks like. B, Fig. 8. Now take the corner $B$ and turn it up towards $\mathrm{C}$, so that the edge of the rolled part shall be even with the central line; repeat the same on the other side, and turn the whole over, when it will appear as in Fig. 6. The bread is placed underneath the part $K$.

\section{4.-The Cinderella.-(Fig. 9.)}

Fold the napkin into three parts longways, then turn down the two sides as in Fig. 7 ; turn the napkin over, and roll up the lower part as in Fig. 10, A, B. Now turn the corner $B$ upwards towards $\mathrm{C}$, so that it shall appear as in D ; repeat on the other side, and then bring the two parts $\mathrm{E}$ together so that they shall bend at the dotted line; and the appearance will now be as Fig. 9. The bread is placed under the apron part, $K$, Fig. 9.

$$
\text { 5.-THE FLIRT.-(Fig. 11.) }
$$

Fold the napkin into three parts longways, then fold across the breadth, co'nmencing at one extremity, and continuing to fold from and to yourself in folds about two inches broad, until the whole is done; then place in a tumbler, and it will appear as in the illustration.

$$
\text { 6.-THe neapolitan.-(Fig. 12.) }
$$

Fold the napkin into three parts longways, then fold one of the upper parts upon itself from you ; turn over the cloth with the part having four folds from you, and fold down the two sides so as to appear as in Fig. 7 ; then roll up the part A underneath, until it appears as in the dotted lines in Fig. 13, at B. Now turn up the corner B towards C, so thai the edge of the rolled part shall be even with the central line: repeat the same upon the opposite side, and turn the whole over, when it will appear as in Fig. 12: the bread being placed underneath the part $\mathrm{K}$, as represented in the illustration.

$$
\begin{gathered}
\text { 7.-THE "FAVORITE," OR OUR OWN. } \\
\text { (Fig. 14.) }
\end{gathered}
$$

Fold the napkin into three parts longways, then turn down the two edges as in Fig. 7, and roll up the part A on both sides, until as represented on the righthand side in Fig. 14; then turn it backwards (as A B) on both sides; now fold down the point $C$ towards you, turn over the napkin, and fold the two other parts from you so that they shall appear as in Fig. 15. Turn the napkin over, thus folded, and raising the centre part with the two thumbs, draw the two ends (A and $B$ ) together, and pull out the parts (C and D) until they appear as in Fig. 14. The bread is to be placed as repre sented in K, Fig. 14.

\section{DINNERS.}

Dinners.-The appearance a dinnertable presents does not depend so much upon a profuseness of viands, as upon the neatness, cleanliness, and well-studied arrangement of the whole. Taste, if well directed, may produce a handsome dinner; whereas three times the amount of money may be expended upon another, and yet not make even a respectable appearance.

We cannot too strongly urge the necessity of having things done in the same manner every day as when there is company. The servants become accustomed 


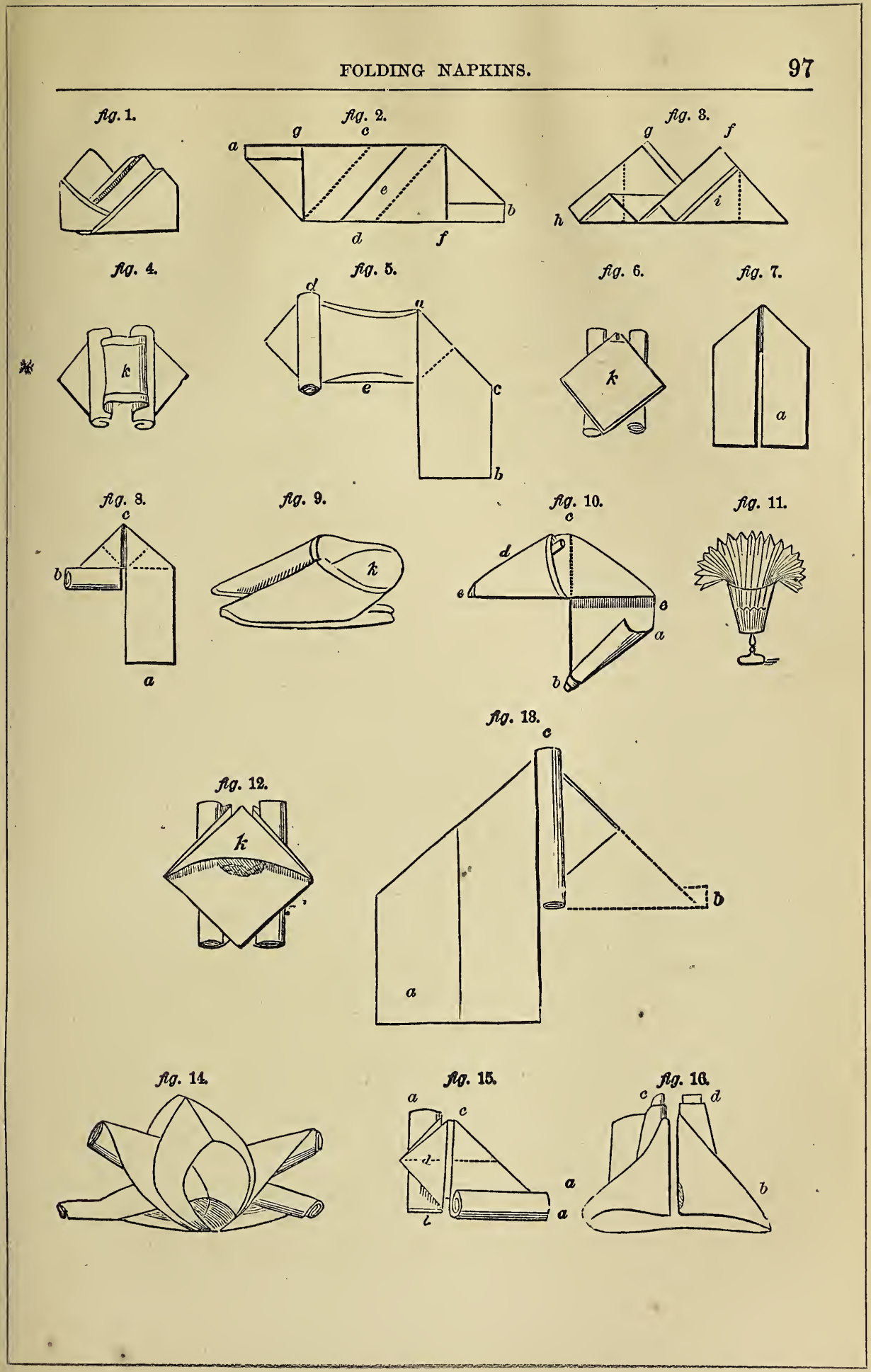


to waiting properly, things are always at hand, and they do not appear awkward when visitors drop in; then every thing is regular, and goes on smoothly.

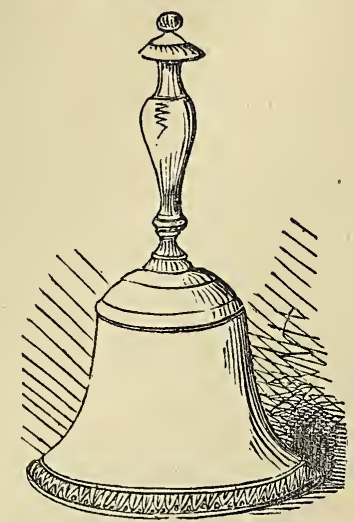

To Lay the Cloth.-The table should he well polished, and then covered with a cloth, over which a fine white damask one should be spread. If the white cloth is to be kept on after dinner, it is customary to spread a small cloth at either end of the table where the large dishes are placed, to protect the long cloth from accidental spots arising from gravy, \&c.; these slips are removed after dinner, and the cloth cleaned with crumb-brushes. In some houses an entire upper cloth is placed upon the table instead of slips, and this being removed after dinner, does not require the tedious process of brushing the table-cloth.

When the cloth has been spread, place carafes, with the tumblers belonging to and placed over them, between every four persons, a salt-cellar between every third person, and a large and small knife, fork, and spoon, to each guest, with two wine-glasses, a champagne-glass, and a tumbler, to the right of each, and the bread placed in or under folded napkins between the knives, forks, and spoons; and at large entertainments or public dinners, the name of each guest neatly written on a card in front of the napkin, so as to prevent confusion. The centre ornament, usually a candelabrum, plateau, an épergne, or a vase of artificial flowers, must now be set on, and the mats for the various dishes arranged; then the winecoolers or ornamental vases placed between the centre piece and the top and bottom dishes, with the wines in the original bottles, loosely corked; the spoons for helping the various dishes, asparagus tongs, fish knife and fork or slice, and carving knives and forks, are placed in front of the respective dishes to which they belong; and knife-rests opposite to those who have to carve; with a bill of fare, and a pile of soupplates before those that have to help the soup.

In arranging or laying out a table. several things require particular attention, and especially the following:-

Plate should be well cleaned, and have a bright polish; few things look worse than to see a greasy-looking épergne and streaky spoons. Glass should be well rubbed with a wash-leather, dipped in a solution of fine whiting and stone-blue, and then dried; afterwards it should be polished with an old silk handkerchief. Plates and dishes should be hot, otherwise the guests will be disgusted by seeing flakes of fat floating about in the gravy. Bread should be cut in pieces about an inch thick, and each round of a loaf into six parts, or if for a dinner party, dinner rolls should be ordered. The bread is placed under the napkins, or on the left of each guest, if dinner napkins are not used; some of the bread being placed in a bread-tray covered with a crochet cloth upon the sideboard. Lights, either at or after the dinner, should be subdued, and above the guests, if possible, so as to be shed upon the table, without intercepting the view. Sauces, either bottle, sweet, or boatvegetables, and sliced cucumber, or glazed 
onions for stubble goose, should be placed | above the others, point to handle. The upon the sideboard; a plate basket for removing the soiled plates is usually placed under the sideboard, or some other convenient part of the room; and two knife-trays, covered with napkins, are placed upon a butler's tray; these are used for removing soiled carvers and forks, and the soiled silver. It is useful to hare a large-sized brad-awl, a corkscrew, and funnel, with strainer; the former to break the wire of the champagne bottles, and the latter to strain port wine, if required to be opened during dinner.

To lay out the sideboard or tray.-Little requires to be done, except to arrange the silver, knives, cruets, and various dishes to be placed there. The silver should be arranged on one end of the sideboard, as in Figs. 1 and 2, the gravy spoons being placed bowl to handle, and the cheese-scoop, marrow-spoon, and salad spoons or scissors, where most convenient. The knives are placed as in Fig. 3, for the convenience of removal, because by this means a single knife can be ab-

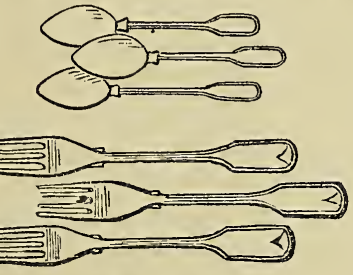

Figs. $1 \& 2$.

stracted without disturbing the others;

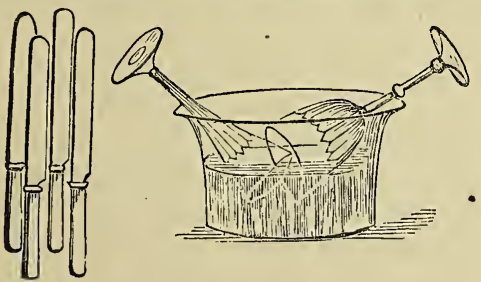

Figs. $3 \& 4$.

carving knives and forks should be placed wine-glasses, tumblers, and finger-glasses, for dessert, are placed where most convenient, but usually in the centre of the back, with ice-plates near to them, and the wine-glasses placed in the fingerglasses. as in Fig. 4 ; but when only one glass is used, that is placed in the centre, mouth downwards. At very large or fashionable dinners, the finger-glasses are sometimes placed on the dinner-table with the plain and colored wine-glasses in them, and the same, refilled, are placed on again at dessert. The cruets, sauces, $\& c$, are placed at one end, and the vegetables, \&c., in the centre front of the sideboard.

To place the dishes on the table.-Each servant should be provided, at large dinders, with a bill of fare, and instructed at small ones, where the dishes are to be placed. No two dishes resembling each other should be near the same part of the table. Soups or broth should always be placed at the head of the table; if there are two, top and bottom; if four, top, bottom, and two sides, opposite each other, or alternately with fish. Fish should be placed at the head of the table; if there are two sorts, have fried at the bottom and boiled at the top; if four, arrange the same as the soup. We may observe, that a white and a brown, or a mild and a high-seasoned soup, should occupy either side of the centre piece, and that it looks handsomer to have fried and boiled fish opposite each other, but they should never be placed upon the same dish. Fish is generally served upon a napkin, the corners of "which are either turned in or thrown over the fish, or upon a piece of simple netting, which is turned in all round; but we recommend our readers to use the elegant serviette, as being more stylish.

The first course generally consists of soups and fish, which are removed by the roasts, stcws, \&c., of the second course. 
The second course, when there are three, consists of roasts and stews for the top and bottom; turkey or fowls, ham garnished, tongue, or fricandeau, for the sides; with small made dishes for corners, served in covered dishes, as curries, ragoûts, fricassees, stews, \&c.

When there are two roasts, one should be white, and the other brown. Removes are generally placed upon large dishes, for, as they supply the place of the fish and soups, they constitute the principal part of the dinner. What are termed flancs are not so large as the removes, nor so small as the entrées, or made dishes, and are generally served in a differently formed dish. They are seldom used except when there are eighteen or twenty persons.

Entrées, or made dishes, require great care in placing them upon the table, otherwise the gravy slops over and soils the dish ; they are, therefore, usually served with a wall of mashed potatoes, rice, or other vegetables, to keep them in their proper place. They should also be served as hot as possible.

When there is but one principal dish, it should be placed at the head of the table. If three dishes, the principal to the head, and the others opposite each other, near the bottom; if four, the largest to the head, the next size to the foot, and the other two at the sides; if five, place the same as for four, with the smallest in the centre; if six, place the same as for four, with two small dishes on each side; if seven, put three dishes down the centre of the table, and two on each side; if eight, four dishes down the middle, and two on each side, at equal distances; if nine, place them in three equal lines, but with the proper dishes at the top and bottom of the table; if ten, put four down the centre, one at each corner, and one on each side, opposite the vacancy between the two central dishes; or four down the middle and three on each side, opposite the vacancies of the centre dishes; if twelve, place them in three rows of four each, or six down the middle, and three at equal distances on each side. If more than twelve, they must be arranged on the same principles, but varying according to number.

Oval or circular dining-tables require to have the dishes arranged in a shape corresponding to the table.

The third course consists of game, delicate vegetables, dressed in the French style, then puddings, creams, jellies, \&c.

When there are only two courses, the first generally consists of soups and fish, removed by boiled poultry, ham, tongue, stews, roasts, ragoûts, curries, or made dishes generally, with vegetables. The second consists of roasted poultry or game at the top and bottom, with dressed vegetables, maccaroni, \&c., succeeded by jellies, creams, preserved fruit, pastry and general confectionery. It is generally contrived to give as great a variety as possible in these dinners: thus-a jelly, a cream, a compôte, an ornamental cake, a dish of preserved fruit, fritters, a blancmange, a pudding, \&c.

Salads and celery are usually served before the puddings and pastry are introduced. In Great Britain, after the third course, cheese, ornamented butter, salad, radishes, celery in a glass bowl or on a dish; sliced cucumber (and at small parties, marrow-bones), are served. A marrow-spoon, cheese-scoop, and butterknife, being required upon the table, are to be placed near the dishes, a knife and fork near the celery, and a pair of salad-scissors or a fork and spoon in the bowl with the salad.

The cheese may be served in a glass bowl, and handed round from right to left; or surrounded with the elegant serviette, and placed upon the cheesecloth. The bread may be served as usual, piled up on a crochet cloth in a plated bread-basket placed in the centre. 
Waiting at Table.-Much confusion is avoided by having an attendant upon each side of the table; or, if the party is large, more than one, according to the number. The usual number required for parties is given below ; and if the income admit of it, the scale may be increased according to the second column, which will materially add to the comfort of the guests.

$$
\begin{aligned}
& \text { Guests. Servants. } \\
& 6 . . . .12 \\
& 12 \div: \circ: \circ: 22 \\
& 15 \text {. . . . . } 34 \\
& 20 \text {. . . . } 46 \\
& 30 \text {. . . } 68 \\
& 40 \text {. . . . } 912 \\
& 50 \text {. . . . } 1220 \text { \&c. }
\end{aligned}
$$

Every attendant should be neatly attired, have a white neckcloth and white gloves on, should know where all the articles reqired are, where the dishes are to be placed, and, in fact, be acquainted with the whole routine of the party; and therefore it is better to provide each one with a bill of fare.

When every guest is seated, a servant appointed for that purpose should stand by the side of each dish, with the right hand upon the cover; and as soon as grace is said, the cover is to be removed, and placed in some convenient part of the room. The plates for soup should then be taken singly from the pile opposite the person serving it, and carried to those guests who desire that particular soup, observing that ladies are to be attended to before gentlemen, and that these should commence from the head of the table, continuing until both sides are helped.

Soon after the soup has been served, the servants may pass down each side of the table, and ask each guest what they will take, helping them to the dish desired as soon as it can be procured. When champagne is given, it is handed round upon a waiter or salver at small parties, commencing at the right-hand side of the table from the top and bottom simultaneously, without any distinction as regards ladies or gentlemen. In large parties-and we prefer the arrangement ourselves even in small ones-the bottle being enveloped as far as the neck with a clean dinner-napkin, the wine is helped in the same order as before; but instead of being handed round on a salver, the servants pour the wine into the glass, at the right-hand side of each guest. By these means there is less danger of the glasses being broken by any awkward collision. The champagne is generally iced in summer, and cool in winter, and is served as soon as the soup is finished, or just after the guests have been helped to the second course of removes.

Liqueurs are handed round when sweets are on the table. Sauces are handed round in the sance-boat, and when served, placed on the side-board or dinner

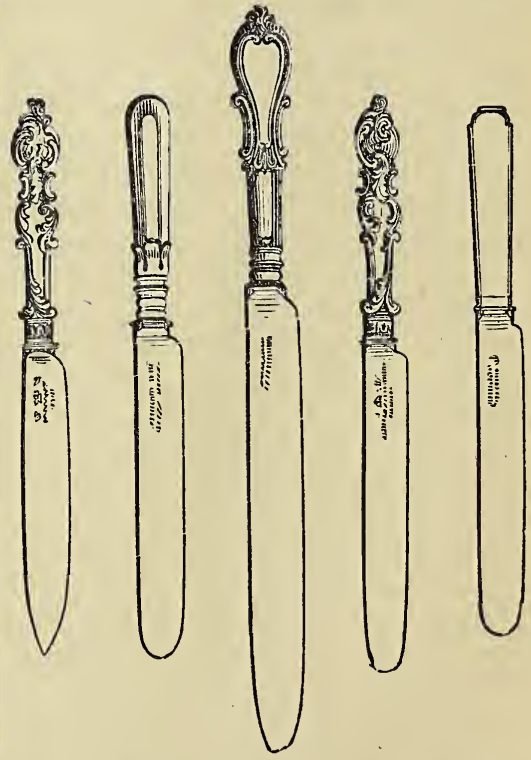

wagon; if only a family party, they are 
returned to the table. Sweet sauces are handed round in glass dishes, and bottle sauces in a stand or basket made for that purpose.

In removing the dinner things, one servant goes round the table with a butler's tray, and the other removes and places the things upon it. The cloth is then brushed with a crumb-brush; or the two sides are turned in, and then the cloth dexterously jerked off the table, the lights replaced, and the dessert set on.

When knives, forks, and spoons are removed from dishes or plates, they should be placed in proper trays covered with napkins ; one being used for the silver, the other for the steel articles.

When plates or dishes are removed from the table, great care is to be observed with respect to holding them horizontally, otherwise the gravy; syrup, or liquid, may injure the dresses of the guests.

In some circles the fashion prevails of placing finger-glasses on table immediately preceding dessert; but in others, cut-glass bowls, partially filled with rose or orange flower water, iced in summer and lukewarm in winter, are handed down each side of the table, upon salvers; into these the guest dips the corner of the dinner napkin, and just touches the lips and the tips of the fingers.

\section{Desserts, Teas, and Suppers.}

The Desser t.-The dessert may consist of merely two dishes of fruit for the top and bottom; dried fruits, biscuits, filberts, \&c., for the sides and corners; and a cake for the centre.

When the party is large, and ices are served, the ice-plates are placed round the table, the ice-pails at both ends of the table, and dishes with wafer-biscuits, at the sides. Some persons have the ices served in glass dishes, which, together with the wafer biscuits, are handed round before the usual dessert.
When there is preserved ginger, it follows the ices, as it serves to stimulate the palate, so that the delicious coolness of the wines may be better appreciated.

The side and corner dishes usually put on for dessert, consist of :-Comp tes in glass dishes; frosted fruit served on lace-paper, in small glass dishes; preserved and dried fruits, in glass dishes; biscuits, plain and fancy; fresh fruit, served in dishes surrounded with leaves or moss ; olives, wafer biscuits, brandyscrolls, \&c.

The centre dish may consist either of a savoy or an ornamental cake, on an elevated stand-a group of waxen fruit, surrounded with moss-a melon-a pine apple-grapes-or a vase of flowers.

Each plate should contain a knife, fork, and spoon, with two wine-glasses, arranged upon a d'oyley, as in Fig. 5 . These are to be placed before each guest, and a finger-glass, with cold water in

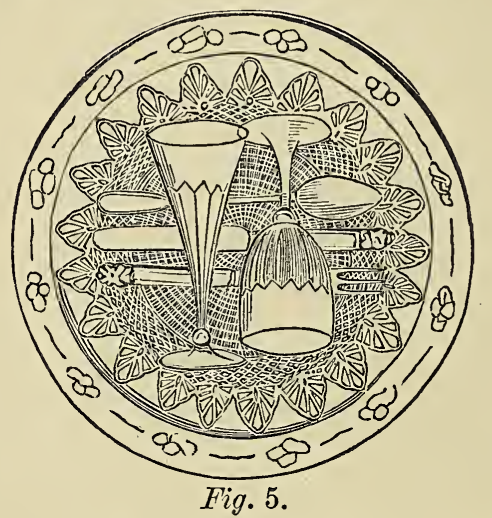

summer, and luke-warm water in winter, on the right of each plate; with grapescissors, and melon knife and fork, before their respective dishes. Glass bowls containing sifted sugar. with pierced ladles, or others filled with cream, are to be placed near the centre dish, if they are required. A cut glass pitcher with a tumbler on either side should be 
placed in a convenient part of the centre of the table.

The wine, either cooled or not, should be placed at, both ends of the table, or at the bottom, if only a small party, the decanters being placed in castors, though this fashion is now nearly abolished.

Zests are put down after the dessert is removed, and consist chiefly of anchovy toasts, devilled poultry and game, and biscuits, gravy toast, grills, \&c.

Coffee is the last thing served, and is generally handed round upon a salver; after this, the gentlemen withdraw to the drawing-room.

Tea.-After a dinner party, the tea is generally handed round by two servants, the one having tea and coffee, with hot milk, cream, and sugar upon one tray; the other having thinly cut and rolled bread and butter, biscuits and cake, upon another tray.

If served at an evening party or dance, a servant helps the guests to tea or coffee, which is arranged upon a side-table in a small room. The tea and coffee occupy the two ends of the table, on either side of the urn, which is placed in the centre and back. In front of the urn are ranged the sugar-candy for coffee, sugar, hot milk, cream, bread and butter, cake, and biscuits.

Tea, when only for a small party, may be brought in upon a tray, the tea and coffee-pots occupying the centre of the tray; the cups and saucers the front; and the hot milk, cream, slop-basin, and sugar, the ends. The urn is placed at the back of the tray; and the bread and butter, cut or not, with cake, biscuits, muffins, crumpets, or toast, at the sides.

Suppers.-The great secret of laying out a supper consists in arranging the china, glass, silver, linen, lights, confectionery, substantials, trifles, flowers, and other articles, with a due regard to form, color, size, and material.
A supper table should neither be too much crowded, nor too scanty, nor scattered and broken up with small dishes. Two dishes of the same description should not be placed near each other: dishes should not be heaped up as if for a ploughman's repast, but contain sufficient to make them look well, without being over or under-done as regards quantity.

Hot suppers are now seldom served; for people dine later than they did formerly; and besides being more expensive than cold ones, they also give more trouble.

The centre of the table is generally occupied by an épergne, vase of flowers, globe of fish upon an elevated stand, a plateau, or small fountain ; around which are arranged :-Dried, preserved, frosted, or candied fruits; custards, jellies, and trifles, in glasses; and small biscuits. The top and bottom of the table are furnished with game, fowls, or meat; the sides have dishes of ham sliced; tongue; collared, potted, hung, and grated beef; brawn, mock or real; savory pies; lobsters ; oysters ; dressed crab or cray-fish ; prawn pyramids; sandwiches of ham, beef, tongue, anchovy, or other savory morsels ; tarts, tartlets ; cake, biscuits ; whipped and other creams ; jellies, blancmange; caramel baskets; patties, \&c.

\section{CHAPTER XXII.}

\section{TRUSSING AND OARVING.}

Poultry.-The feathers must be plucked as soon as possible after killing-and the down singed off with lighted paper, the crop emptied by a slit cut in the back of the neck, the vent opened to clean out the entrails, the gall-bag carefully removed, and the liver and gizzard cleansed in cold water. The necks are to be cut off close to the body, the skin being pushed 
up above the part where it is cut, and / who like it ; and the carcass is preferred afterwards drawn down and wrapped by some to other parts. over the end of the neck. Before fastening it down, pour cold water through the body of the fowl. The back-bone and two bones leading to the pinions may be broken. and then the bird is to be trussed.

Carving.-The carving-knife for poultry and game is smaller and lighter than that for meat; the point is more peaked, and the handle longer.

In cutting up wild-fowl, duck, goose, or turkey, more prime pieces may be obtained by carving slices from pinion to pinion without making wings, which is a material advantage in distributing the bird when the party is large.

To Truss a Goos.-Pick and stub it ar clean, cut the feet off at the joint, and the pinion off at the first joint. Then cut off the neck close to the back, leaving the skin of the neck long enough to turn over the back. Pull out the throat, and tie a knot at the end. Loosen the liver and other matters at the breast end with the middle finger, and cut it open between the rent and the rump. Draw out the entrails, wipe the body out clean with a cloth, beat the breast-bone flat with a rolling pin, put a skewer into the wing, and draw the legs up close; put the skewer through the middle of the leg, and through the body, and the same on the other side. Put another skewer in the small of the leg, tuck it close down to the sidesman, run it through, and do the same on the other side. Cut off the end of the vent, and make a hole large enough for the passage of the rump, as by that means it will keep in the seasoning much better. The best parts are the breast slices; the fleshy part of the wing, which may be divided from the pinion; the thigh-bone, which may be casily divided in the joint from the legbone; the pinion; and next, the sidebones. The rump is a nice piece to those

To Truss a Turkey.-When the bird is picked carefully, break the leg bone close to the foot, hang on a hook, and draw out the strings from the thigh; cut the neck close off to the back, taking care to leave the crop-skin long enough to turn over the back. Remove the crop, and loosen the liver and gut at the throat end with the middle finger. Cut off the vent, remove the gut, pull out the gizzard with a crooked wire, and the liver will soon follow; but be careful not to break the gall. Wipe the inside perfectly clean with a wet cloth, then cut the breast-bone through on each side close to the back, and draw the legs close to the crop, then put a cloth on the breast, and beat the thigh bone down with a rolling-pin till it lies flat.

If the turkey is to be trussed for boiling, cut the first joint of the legs off; pass the middle finger into the inside, raise the skin of the legs and put them under the apron of the bird. Put a skewer into the joint of the wing and the middle joint of the leg; and run it through the body and the other leg and wing. The liver and gizzard must be put in the pinions, care being taken to open and previously remove the contents of the latter; the gall bladder must also be detached from the liver. Then turn the small end of the pinion on the back, and tie a packthread over the ends of the legs to keep them in their places.

If the turkey is to be roasted, leave the legs on, put a skewer in the joint of the wing, tuck the legs close up, and put the skewer through the middle of the legs and body; on the other side put another skewer in at the small part of the leg. Put it close on the outside of the sidesman, and push the skewer through. and the same on the other side. Put the liver and gizzard between the pinions. 
and turn the point of the pinion on the back. Then put, close above the pinions, another skewer through the body of the bird.

To Truss Fowls.-Fowls must be picked very clean, and the neck cut off close to the back. Take out the crop, and, with the middle finger, loosen the liver and other parts. Cut off the vent, draw it clean, and beat the breast-bone flat with a rolling pin.

If the fowl is to be boiled, cut off the nails of the feet, and tuck them down close to the legs. Put your finger into the inside, and raise the skin of the legs; then cut a hole in the top of the skin, and put the legs under. Put a skewer In the first joint of the pinion, and bring the middle of the leg close to it; put a skewer through the middle of the leg, and through the body, and then do the same on the other side. Open the gizzard, remove the contents, and wash it well; remove the gall-bladder from the liver. Put the gizzard and the liver in the pinions, turn the points on the back, and tie a string over the tops of the legs, to keep them in their proper places.

If the fowl is to be roasted, put a skewer in the first joint of the pinion, and bring the middle of the leg close to it. Put the skewer through the middle of the leg, and through the body, and do the same on the other side. Put another skewer in the small of the leg, and through the sidesman; do the same on the other side, and then put another through the skin of the feet, which should have the nails cut off.

The prime parts of a fowl, whether roasted or boiled, are the wings, breast and merry-thought; and next to these. the neck-bones and side-bones; the legs are rather coarse- of a boiled fowl, however, the legs are rather more tender than a roasted one; of the leg of a fowl the thigh is the better part, and therefore when given to any one should be separated from the drum-stick, which is done by passing the knife underneath, in the hollow, and turning the thigh-bone back from the leg-bone.

To Truss Chickens.-Pick and draw them in the same manner as you would fowls ; but, as their skins are very tender, plunge them into scalding water, and remove, when the feathers will come off readily.

If they are to be boiled, cut off the nails, notch the sinews on each side of the joint, put the feet in at the vent, and then peel the rump. Draw the skin tight over the legs, put a skewer in the first joint of the pinion, and bring the middle of the legs close. Put the skewer through the middle of the legs, and through the body; and do the same on the other side. Clean the gizzard, and remove the gall from the liver; put them into the pinions, and turn the points on the back.

If for roasting, cut off the feet, put a skewer in the first joint of the pinions, and bring the middle of the legs close. Run the skewer through the middle of the legs and body, and do the same on the otlier side. Put another skewer into the sidesman, put the legs between the apron and the sidesman, and run the skewer through. Having cleaned the liver and gizzard, put them under the pinions, turn the points on the back, and pull the breastskin over the neck.

To Truss Pheasants.-Pick them clean, cut a slit at the back of the neck, take out the crop, loosen the liver, and gut the breast with the fore-finger; then cut off the vent, and draw them. Cut off the pinion at the first joint, and wipe out the inside with the pinion. Beat the breastbone flat with a rolling-pin, put a skewer in the pinion, and bring the middle of the legs close. Then run the skewer through the legs, body, and the other pinion, twist the head, and put it on the 
end of the skewer, with the bill fronting the breast. Put another skewer into the sidesman, and put the legs close on each side of the apron, and then run the skewer through all. If you wish the cock-pheasant tolook well, leave the beautiful feathers on the head, and cover with paper to protect them from the fire. Save the long feathers from the tail; and when cooked, stick them into the rump before sending to table.

If the pheasants are for boiling; put the legs in the same manner as in trussing a fowl.

To Truss Pigeons.-Pick clean, take off the neck close to the back; then remove the crop, cut off the vent, and draw out the entrails and gizzard, but leave the liver, as a pigeon has no gall-bladder.

If for roasting, cut off the toes, cut a slit in one of the legs, and put the other through it. Draw the leg tight to the pinion, put a skewer through the pinion, legs, and body, and with the handle of the knife break the breast flat. Clean the gizzard, and put it under one of the pinions, and turn the points on the back.

If for boiling or stewing, cut the feet off at the joint, turn the legs, and stick them in the sides, close to the pinions. If for a pie, they must be done in the same manner.

To Truss Wild Fowl.-Pick clean, cut off the neck close to the back, and, with the middle finger, loosen the liver and other parts. Cut off the pinions at the first joint; then cut a slit between the vent and the rump, and draw them clean. Clean them properly with the long feathers on the wing, cut off the nails, and turn the feet close to the legs. Put a skewer in the pinion, pull the legs close to the breast, and run the skewer through the legs, body, and the other pinion. Cut off the end of the vent, and put the rump through it.
All kinds of wild-fowl are to be truss. ed thus:

To Truss Woodcock, Plover and Snipe. -If these birds are not very fresh, great care must be taken in picking them, as they are very tender to pick at any time; for even the heat of the hand will sometimes take off the skin, which will destroy the beauty of the bird. When picked clean, cut the pinions in the first joint, and with the handle of a knife beat the breastbone flat. Turn the legs close to the thighs, and tie them together at the joints. Put the thighs close to the pinions, put a skewer into the pinions, and run it through the thighs. body, and other pinions. Skin the head, turn it, take out the eyes, and put the head on the point of the skewer, with the bill close to the breast. These birds must never be drawn.

To Truss small Birds.-Pick well, cut off their heads, and the pinions of the first joint. Beat the breast-bone flat, and turn the feet close to the legs, and put one into the other. Draw out the gizzard, and run a skewer through the middle of the bodies. Tie the skewer fast to the spit when you put them down to roast.

To Truss Hare or Rabbit.-A hare or rabbit should be paunched, or cleaned, as soon as it is killed. The inside should be kept dry and peppered. When trussed, the sinews of the hind-legs must be cut, and the legs turned towards the head and fastened to the sides. The fore-legs must be turned to meet the hind-legs, and fastened. with slight skewers to the body. The head is thrown back and kept in place by a skewer passed through it. The stuffing is put in, and the skin sewed up. A string fastens all compactly together. The ears of a rabbit are to be cut off when roasted or boiled. 


\section{CARVING.}

Ladies ought especially to make carving a study; at their own houses, they grace the table, and should be enabled to perform the task allotted to them with sufficient skill to prevent remark or the calling forth of eager proffers of assistance from good-natured visitors near, who probably would not present any better claim to a neat performance.

Carving presents no difficulties ; it requires simply knowledge. All display of exertion or violence are in very bad taste; for, if not an evidence of the want of ability on the part of the carver, they present a very strong testimony of the toughness of a joint or the more than full age of a bird: in both cases they should be avoided. A good knife of moderate size, sufficient length of handle, and very sharp, is requisite ; for a lady it should be light, and smaller than that used by gentlemen. Fowls are very easily carved, and joints, such as loins, breasts, fore-quarters, \&c. The butcher should have strict injunctions to separate the joints well.

The dish upon which the article to be carved is placed, should be conveniently near to the carver, so that he has full control over it; for if far off, nothing can prevent an ungraceful appearance, nor a difficulty in performing that which in its proper place could be achieved with ease.

In serving fish, some nicety and care must be exercised; here lightness of hand and dexterity of management is necessary, and can only be acquired by practice. The flakes which, in such fish as salmon and cod are large, should not be broken in serving, for the beauty of the fish is then destroyed, and the appetite for it injured. In addition to the skill in the use of the knife, there is also required another description of knowledge, and that is an acquaintanee with the best parts of the joint, fowl, or fish being carved. Thus, in a haunch of venison, the fat, which is a favorite, must be served with each slice; in the shoulder of mutton there are some delicate cuts in the under part. The breast and wings are the best parts of a fowl, the trail of a woodcock on a toast is the choicest part of the bird. In fish a part of the roe, melt, or liver should accompany the piece of fish served; the list. however, is too numerous to mention here; and, indeed, the knowledge can only be acquired by experience. In large establishments the gross dishes are carved at the buffet by the butler, but in general they are placed upon the table.

Fish is served with a fish-slice, or the new fish-knife and fork, and requires very little carving, care being required, however, not to break the flakes, which, from their size, add much to the beauty of cod and salmon. Serve part of the roe, melt, or liver, to each person. The heads of carp, part of those of cod and salmon, sounds of cod, and fins of turbot, are likewise considered delicacies.

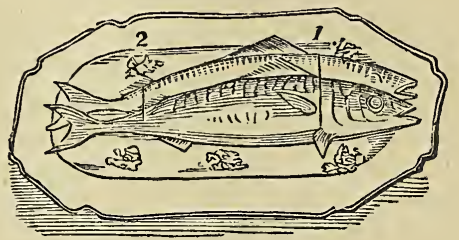

Mackerel

Should be deprived of the head and tail by passing the slice across in the direction of lines 1 and 2 ; they should then be divided down the back, so as to assist each person to a slice; but if less is required, the thicker end should be given, as it is more esteemed. If the roe is asked for, it will be found between 1 and 2.

Barbel, Carp, Haddock, Herring, Perch, Whiting, \&c., should be helped the same 
as Mackerel; remembering that the head of the Carp is esteemed a delicacy.

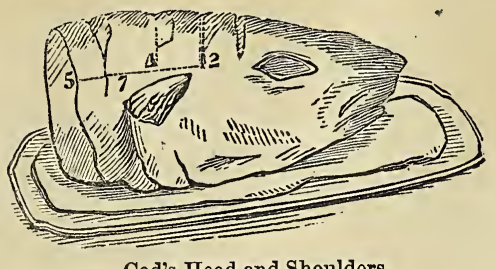

Cod's Head and Shoulders.

Pass the fish-slice or knife from 1 to 6 down to the bone; then help pieces from between $1-2$, and $3-4$, and with each slice give a piece of the sound, which lies under the back-bone, and is procured by passing the knife in the direction 45. There are many delicate parts about the head, particularly the oyster, which is the cheek, below the eye ; and a great deal of the jelly kind, which lies about the jaws. The tongue aud palate are considered delicacies, and are obtained by passing the slice or a spoon into the mouth.

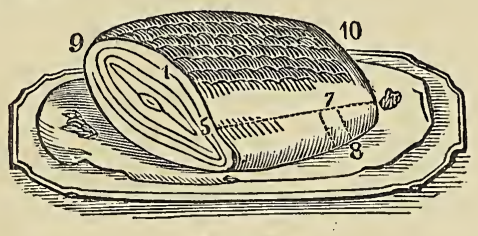

Salmon.

Give a portion of the back and belly to each person, or as desired. If a whole salmon is served, remember that the choice parts are next the head, the thin part is the next best, and the tail the least esteemed. Make an incision along the back, 9 to 10 , and another from 1 to 2 , and 3 to 4 ; cut the thickest part, between $5-6,10-2$, for the lean; and $7-8$ for the fat. When the fish is very thick, do not help too near the bone, as the flavor and color are not so good.

Although carving with ease and elegance is a very necessary accomplishment, yet most people are lamentably deficient not only in the art of dissecting winged game and poultry, but also in the important point of knowing the parts most generally esteemed. Practice only can make good carvers ; but the directions here given, with accompanying plates, will enable any one to disjoint a fowl, and avoid the awkwardness of disfiguring a joint.

In the first place, whatever is to be carved should be set in a dish sufficiently large for turning it if necessary; but the dish itself should not be moved from its position, which should be so close before the carver as only to leave room for the plates. The carving-knife should be light, sharp, well-tempered, and of a size proportioned to the joint, strength being less required than address in the manner of using it. Large solid joints, such as ham, fillet of veal, and salt beef, cannot be cut too thin; but mutton, roast pork, and the other joints of veal, should never be served in very slender slices.

A Round (buttock) or Aitch-bone of Beef.-Pare off from the upper part, of

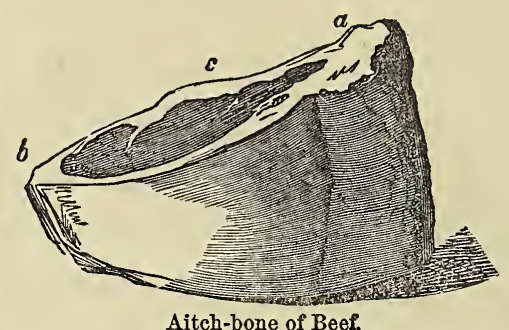

either, a slice from the whole surface, of about half an inch thick, and put it aside; then cut thin slices of both lean and fat, in the direction from $a$ to $b^{\circ}$. The soft fat, which resembles marrow, lies at the back of the aitch-bone, below $c$, but the firm fat must be cut in slender horizontal slices at $a$, and is much better than the soft when eaten cold.

Ribs of Beef.-Cut along the whole length of the bone, from end to end, $a$ to 
$b$, either commencing in the centre or at one side, having the thin end towards you ; but if cut from the bone and formed into a round, with the fat end doubled into the centre, it must then be cut in the same manner as the round of beef.

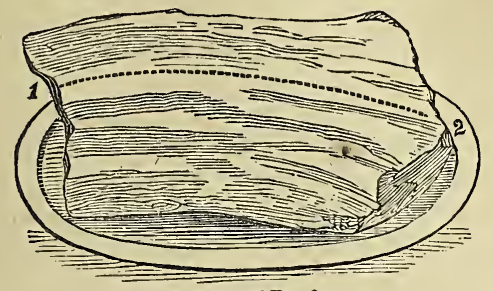

Brisket of Beef.

Brisket of Beef must be carved in the direction 1 and 2, quite down to the bone, after cutting off the outside, which should be about three-quarters of an inch thick.

Sirloin of Beef.-Cut in the same man-

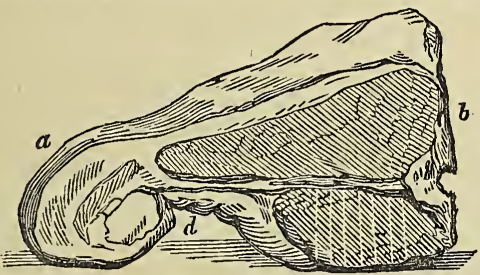

Sirloin of Beef.

ner as the ribs, commencing either at the centre or the side, as from $a$ to $b$. The under part should be cut across the bone, as at $c$ for the lean, and $d$ for the rich fat; many persons prefer the under to the upper part, the meat being more tender.

Fillet of Veal.-Carve it in the same

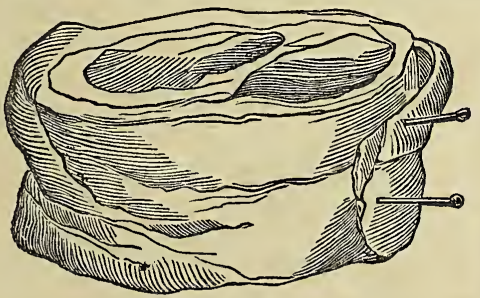

Fillet of Veal.

manner as the round of beef; but the up- per slice should be cut somewhat thinner, as most persons like a little of the brown, and a portion of it should be served along with each slice, together with a slice of the fat and stuffing, which is skewered within the flap.

Neck of Veal.-Cut across the ribs, as

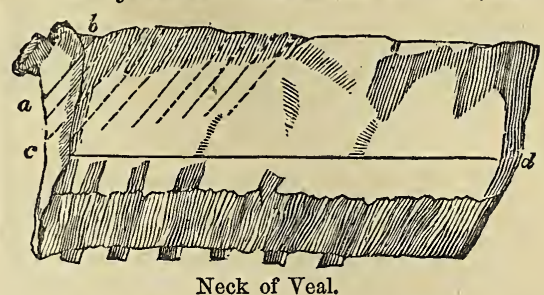

at $a$ to $b$ : the small bones, as at $c$ to $d$, being cut off, divided, and served separately, for it is not only a tedious, but a vulgar operation to attempt to disjoint the ribs.

Loin of Veal.-The joint is placed in the dish in the same manner as a sirloin of beef, but should be turnsd up, and the whole of the kidney and fat cut out; the fat being usually put upon a dry toast and served as marrow. The loin is then returned to its former position, and the meat should be cut across the ribs as in the neck, serving it with a slice of kid. ney.

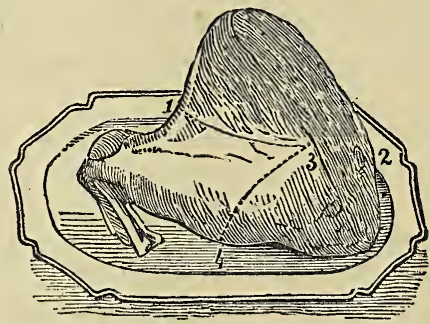

Knuckle of Veal.

Knuckle of Veal is to be carved in the direction 1-2. The most delicate fat lies about the part 4 , and if cut in the line $3-4$, the two bones, between which the marrowy fat lies, will be divided.

Breast of Veal, being very gristly, is 
not easily divided into pieces. In order, therefore, to avoid this difficulty, put your knife at $a$ about four inches from

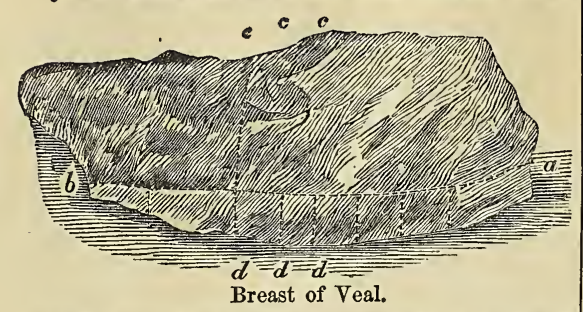

the edge of the thickest part (which is called "the brisket"), and cut through it to $b$, to separate it from the long ribs: cut the short bones across, as at $d, d, d$, and the long ones as at $c, c, c$; ask which is chosen, and help accordingly. The remaining scrag part is seldom served at table, but forms an excellent stew when dressed in the French mode.

Shoulder of Veal.-Cut in the same manner as a shoulder of mutton, beginning on the under side.

Calf's Head.-Cut slices from $a$ to $b$ in

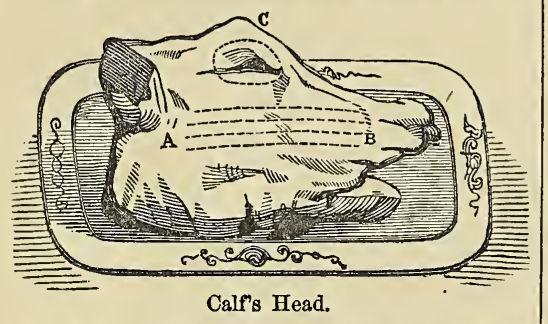

the figure, which describes only half the head, letting the knife go close to the bone. Many like the eye at $c$, which you rnust cut out with the point of your knife, and divide in two, along with some of the glutinous bits which surround it. If the jaw-bone be taken off, there will be found some fine lean, and under the head is the palate, which is reckoned a delicacy.

The tongue and brains are dished separately, but served in small portions along with the head.
Leg of Mutton.-The best part of a leg

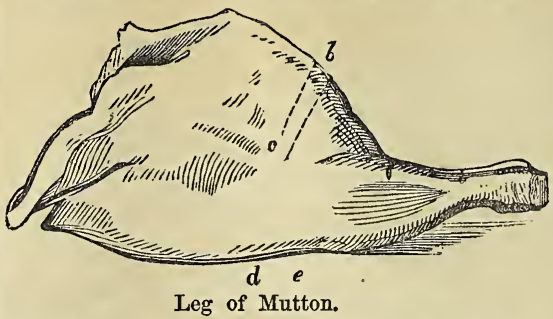

of mutton, whether boiled or roasted, is midway between the knuckle and the broad end. Begin to help there from the roundest and thickest part, by cutting slices, not too thin, from $b$ down to $c$. This part is the most juicy; but many prefer the knuckle, which, in fine mutton, will be very tender, though dry. There are very fine slices in the back of the leg; therefore, if the party be large, turn it up and cut the broad end; not across in the direction you did the other side, but longwise, from the thick end to the knucklebone. To cut out the cramp-bone, which some persons look upon as a deliacy, pass your knife under in the direction of $e$, and it will be found between that and $d$. Shoulder of Mutton, though commonly looked upon as a very homely joint, is by many preferred to the leg, as there is much variety of flaror, as well as texture, in both the upper and under parts.

The figure represents it laid in the dish as always served, with its back uppermost. Cut through it from $a$ down to

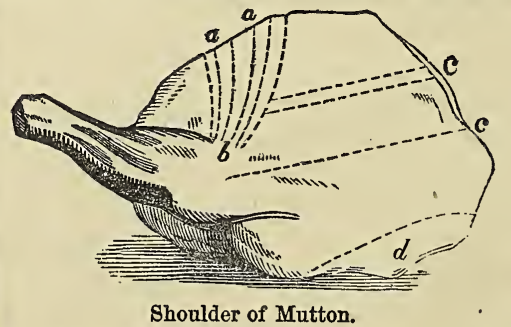

the blade-bone at $b$; afterwards slice it along each side of the blade-bone from $c$ 
$c b$. The prime part of the fat lies on back-bone, from $a$ to $b$, with slices of fat the outer edge, and is to be cut in thin slices in the direction of $d$.

The under part, as here represented,

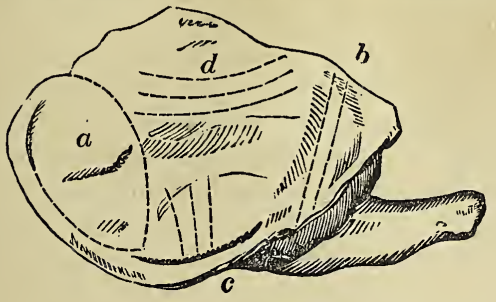

contains many favorite pieces of different sorts, as, crosswise, in slices, near the shank-bone at $b$; and, lengthwise, in broad pieces, at the further end $a$; as well as in the middle and sides in the manner designated at $c$ and $d$.

Should it be intended to reserve a portion of the joint to be eaten cold, all this undermost part should be first cut away and served separately, both as it eats better hot than cold, and as the upper remaining part will appear more sightly in the table.

Loin of Mutton.-Cut the joints into chops and serve them separately; or cut slices the whole length of the loin; or run the knife along the chine-bone, and then slice it, the fat and lean together, as shown in the cut of the saddle, in the next column.

Neck of Mutton.-It should be prepared for table as follows:-Cut off the scrag; have the chine-bone carefully sawn off, and also the top of the long bones (about an inch and a half,) and the thin part turned under; carre in the direction of the bones.

The scrag of mutton, when roasted, is very frequently separated from the ribs of the neck, and in that case the meat and bones may be helped together.

Sadale of Mutton.-Cut in long and rather thin slices from the tail to the end, beginning at each side close to the

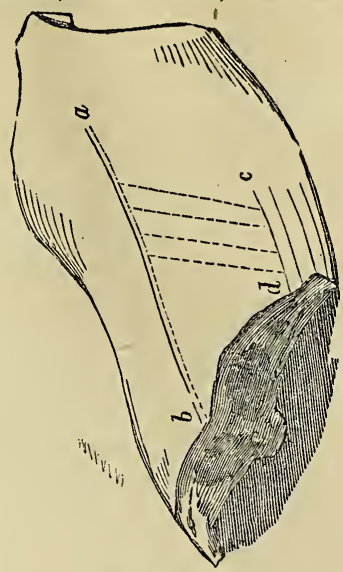

Saddle of Mutton.

from $c$ to $d$; or along the bone which divides the two loins, so as to loosen from it the whole of the meat from that side which you then cut crosswise, thus giving with each slice both fat and lean. The tail end is usually divided and partly turned up. Some butchers also skewer the kidneys across the incision, but it is not usual at genteel tables, and the inci sion is better omitted.

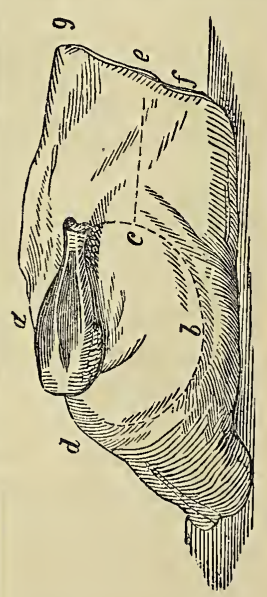

Fore-Quarter of Lamb.

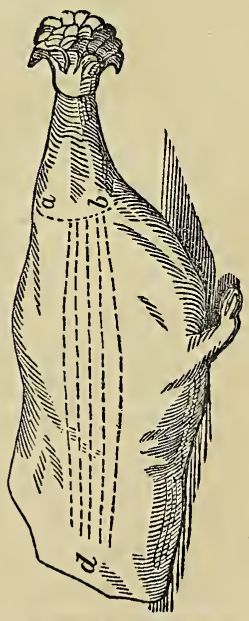

Haunch of Venison. 
Haunch of Mutton.-A haunch is the leg and part of the loin, and is cut in the same manner as a haunch of venison.

Fore-Quarter of Lamb.-Pass the knife under the shoulder in the direction of $a, c, b, d$, so as to separate it from the ribs without cutting the meat too much off the bones. A Seville vrange or lemon should then be divided, the halves sprinkled with salt and pepper, and the juice squeezed over the under part. A little cold or melted butter is then put between both parts, after which the shoulder is placed in a separate dish to be helped by some other person. Iivide the ribs from $d$ to $e$, and then serve the neck $f$, and breast $g$, as may be chosen.

Haunch of Venison.-Have the joint lengthwise before you, the knuckle being the farthest point. Cut from $a$ to $b$, but be careful not to let out the gravy; then cut along the whole length from $a$ down to $d$. The knife should slope in making the first cut, and then the whole of the gravy will be received in the well. The greater part of the fat, which is the favorite portion, will be found at the left side, and care must be taken to serve some with each slice.

Neck of Venison.-Cut across the ribs diagonally; or it may be cut in slices the whole length of the neck. The first method is equally good, and much more economical.

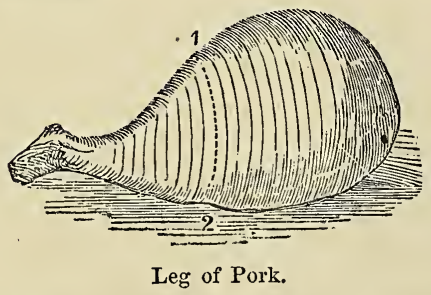

A leg of Pork, whether boiled or roasted, is carred the same. Commence about midway, between the knuckle and the thick end, and cut thin deep slices from either side of the line 1 to 2 .
Pork.-In helping the roast loin and leg, your knife must follow the direction of the scores cut by the ooo' upon the skin which forms the crackling, as it is too crisp for being conveniently divided, and cannot therefore be cut across the bones of the ribs, as in loin of mutton. The scores upon the roasted leg are, however, always marked too broadly for single cuts ; the crackling must therefore be lifted up from the back to allow of thin slices being cut from the meat; the seasoning is under the skin at the larger end.

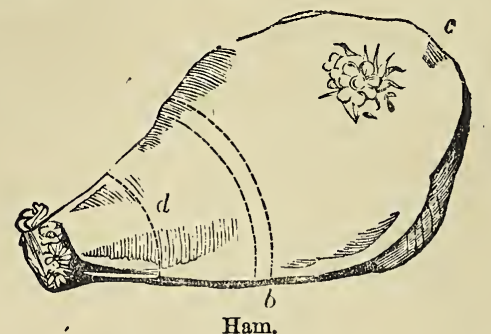

Ham.-Serve it with the back upwards, sometimes ornamented, and generally having, as in France, the shank-bone covered with cut paper. Begin in the middle by cutting long and very thin slices from $a$ to $b$, continuing down to the thick fat at the broad end. The first slice should be wedge-shaped, that all the others may be cut slanting, which gires a handsome appearance to them. Many persons, however, prefer the hock at $d$ as having more flavor; it is then carved lengthwise from $c$ to $d$.

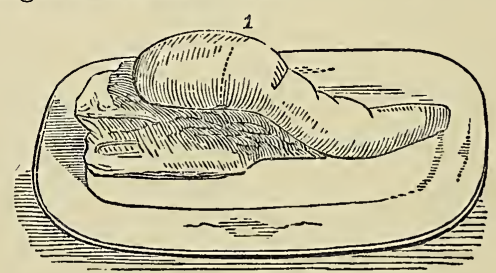

A Tongue.

A Tongue should be cut across, nearly 
through the middle, at the line 1 , and thin slices taken from each side; a portion of the fat, which is situated at the root of the tongue, being helped with each.

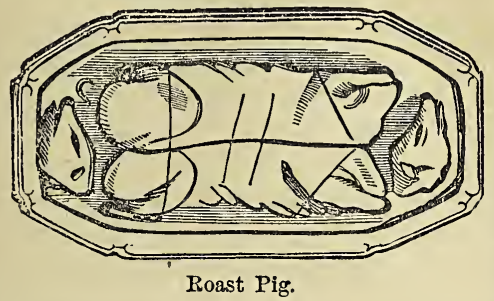

Sucking-pig.-T'The cook usually divides the body before it is sent to table-as thus-and garnishes the dish with the jaws and ears.

Separate a shoulder from one side, and then the leg, according to the direction given by the line along the carcass. The ribs are then to be dividel; and an ear or jaw presented with them, and plenty of sauce and stuffing. The joints may either be divided into two each, or pieces may be cut from them. The ribs are reckoned the finest part; but some people prefer the neck end, between the shoulders.

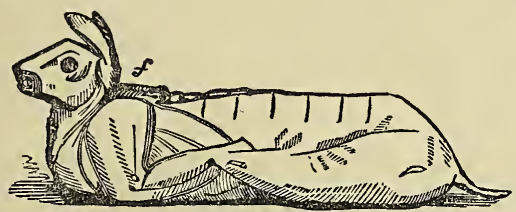

Rabbit.

Rabbits.-Put the point of the knife under the shoulder at $b$, and so cut all the way down to the rump, along the sides of the backbone, in the $\operatorname{limb} b, a$, cuttıng it in moderately thick slices; or, after removing the shoulders and leys, cut the back crosswise in four or five pieces; but this can only be done when the rabbit is very young, or when it is boned. To separate the legs and shoulders, put the knife between the leg and back, and give it a little turn inwards at the joint, which you must endeavor to hit, and not to break by force. The shoulders may be removed by a circular cut around them. The back is the most delicate part. and next to that the thighs. A portion of the stuffing should be served with each slice. The brains and ears of sucking-pig and rabbit are also considered epicurean titbits, which must not be neglected; wherefore, when every one is helped, cut off the head, put your knife between the upper and lower jaw and divide them, which will enable you to lay the upper flat on your plate; then put the point of the knife into the centre, and cut the head into two.

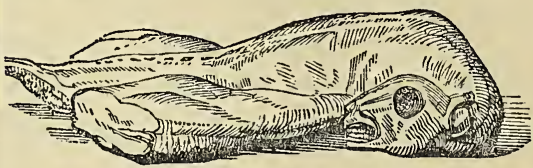

Boiled Rabbit.

Boiled Rabbits.-The legs and shoulders should be first taken off, and then the back cut across into two parts, which is easily done by a bend of the knife in the joint underneath, about the middle of the back. The back is the best, and some of the liver should always accompany it.

The carving of both WINGED GAME and POULTRY requires more delicacy of hand and nicety in hitting the joints than the cutting of large pieces of meat, and, to be neatly done, requires considerable practice.

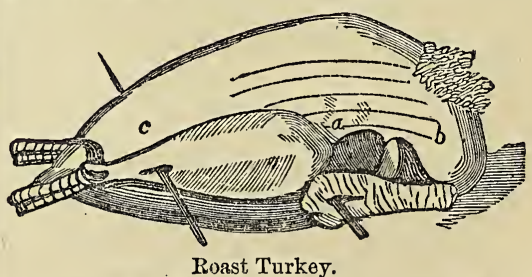

Roast Turkey.-Cut long slices from 
each side of the breast down to the ribs, beginning at $a b$ from the wing to the breastbone. Then turn the turkey upnn the side nearest you, and cut off the leg and wing; when the knife is passed between the limbs and the body, and pressed outward, the joint will be easily perceived. 'Then turn the turkey on the other side, and cut off the leg and wing. Separate the drumsticks from the leg bones, and the pinions from the wings; it is hardly possible to mistake the joint. Cut the stuffing in thin slices, lengthwise. Take off the neck-bones, which are two triangular bones on each side of the breast ; this is done by passing the knife from the back under the blade part of each neck-bone, until it reaches the end: by raising the knife the other branch will easily crack off. Separate the carcass from the back by passing the knife lengthwise from the neck downward. Turn the back upwards and lay the edge of the knife across the backbone about midway between the legs and wings; at the same moment, place the fork within the lower part of the turkey, and lift it up; this will make the back-bone crack at the knife. The croup, or lower part of the back, being cut off, put it on the plate with the rump from you, and split off the side-bones by forcing the knife through from the rump to the other end.

The choicest parts of a turkey are the side-bones, the breast and the thigh-bones. The breast and wings are called light meat; the thigh-bones and side-bones dark meat. When a person declines expressing a preference, it is polite to help to both kinds.

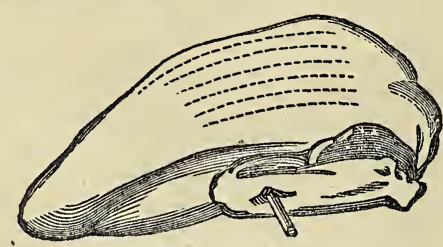

Boiled Turkey.
Boiled Turkey is carved in the same way as the roast, the only difference being in the trussing; the legs in the boiled being, as here shown, drawn into the body, and in the roast skewered.

Roast Fowl.-Slip the knife between the leg and body, and cut to the bone; then with the fork turn the leg back, and the joint will give way if the bird is not old. Take the wing off in the direction of $a$ to $b$, only dividing the joint with your knife. When the four quarters are thus removed, take off the merry-thought from $c$, and the neck bones; these last, by putting in the knife at $d$, and pressing it, will break off from the part that sticks to the breast. The next thing is to divide the breast from the carcass, by

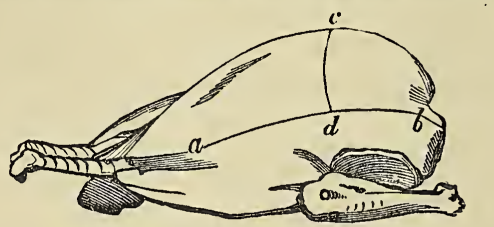

Roast Fowl.

cutting through the tender ribs close to the breast, quite down to the tail. Then

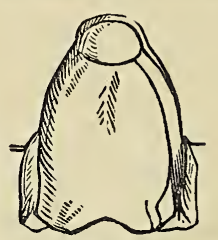

Boiled Fowl, breast.

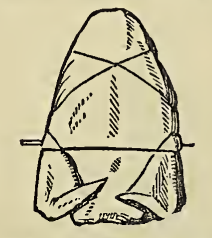

Boiled Fowl, back.

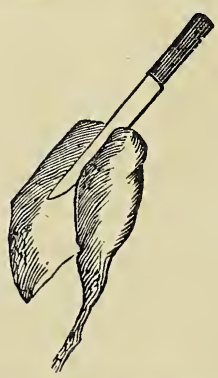

lay the back upwards, put your knife into the bone half way from the neck to the 
rump, and on raising the lower end it will separate readily. Turn the rump from you, take off the two sidesmen, and the whole will be done. To separate the thigh from the drumstick of the leg insert the knife into the joint as above. It requires practice to hit the joint at the first trial. The breast and wings are considered the best parts.

If the bird be a capon, or large, and roasted, the breast may be cut into slices in the same way as a pheasant.

The difference in the carving of boiled and roast fowls consists only in the breast of the latter being always served

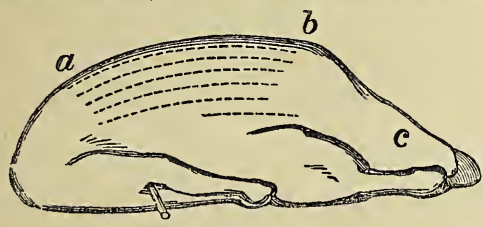

Roast Goose.

whole, and the thigh-bone being generally preferred to the wing.

Geese.-Cut thin slices from the breast at $a$ to $b$; the wing is generally separated as in turkeys, but the leg is almost constantly reserved for broiling. Serve a little of the seasoning from the inside by cutting a circular slice in the apron at $c$.

Pheasant.-Slip the knife between the

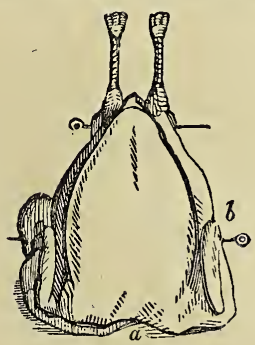

Pheasant.

leg and the breast: cut off a wing small from $a$ to $b$; then slice the breast, and you will have two or three handsome cuts. Cut off the merry-thought by rassing the knife under it towards the neck, and cut all the other parts as in a fowl. The breast, wings, and merrythought are the most esteemed: but the thigh has a high flavor.

Partridge.-It may be cut up in the

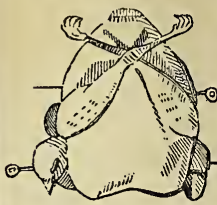

Partridge. same manner as a fowl; but the bird being small, it is unusual to divide it into more than three portions - the leg and wing being left together, and the breast helped entire; the back, being only served along with some of the other parts. If the birds are very young, and the party not over large, the whole body is not unfrequently only separated into two pieces, by one cut of the knife from head to tail.

Quaits.-Generally helped whole.

Grouse, Snipe, and Woodcock.-Proceed as for partridge, except that the trail or entrails of the two latter is served up on toast. As regards these different sorts of game, the thigh of the pheasant and the woodcock is the best, and the breast and wing of the partridge and grouse ; but the most epicurean morsel of all is the trail of the woodcock served up on toast. Smaller birds should always be helped as they are roasted, whole.

Wild-Duck, Widgeon, and most sorts of water-fowl.-Make two or three incisions, as long slices, into the breast, on which a glass of hot port-wine is poured; have ready a lemon cut in half, on one side covered with salt, and on the other

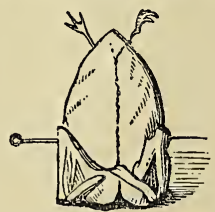

Pigeon, back.

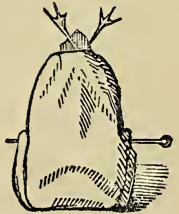

Pigeon, breast. with cayenne pepper. Put both together, then squeeze the juice over the breast; 
after which the slices and limbs may be served round.

Pigeons.-Cut them in half, through both back and breast; the lower part is generally thought the best.

Fish requires very little carving; it should be carefully helped with a fishslice, which, not being sharp, prevents the flakes from being broken, and in salmon and cod these are large and add much to their beauty.

\section{NAMES OF THE VARIOUS JOINTS IN ANIMALS.}

\section{1.-Beef.}

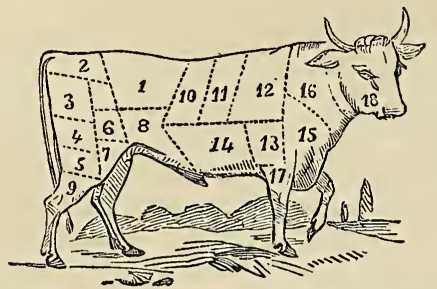

\section{Find Quarter.}

1. Sirloin.

2. Rump.

3. Aitch-Bone.

4. Buttock.

5. Mouse-Buttock.

6. Veiny Piece.

7. Thick Flank.

8. Thin Flank.

9. Leg.

10. Fore-ribs; 5 ribs.

\section{Fore Quarter.}

11. Middle-rib; 4 ribs 12. Chuck; 3 ribs.

13. Shoulder, or Leg of Mutton Piece.

14. Brisket.

15. Clod

16. Neck or Sticking Piece.

17. Shin,

\section{2.-Mutton or Lamb.}

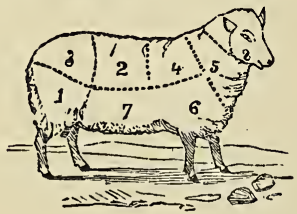

1. Leg.

2. Loin, best end.

3. Loin, chump end.

4. Neck, best end.

5. Neck, scrag end.
6. Shoulder.

7. Breast.

8. Head.

A Chine is two Necks.

A Saddle is two Loins.

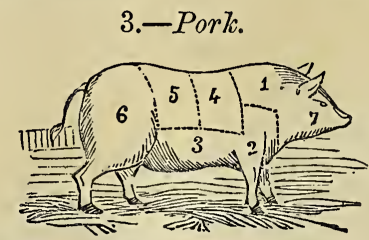

1. The Spare-rib

2. The Hand.

\begin{tabular}{l|l} 
2. The Hand. & 6. Leg. \\
3. The Belly or Spring. & 7. Head.
\end{tabular}

4. Fore-loin.

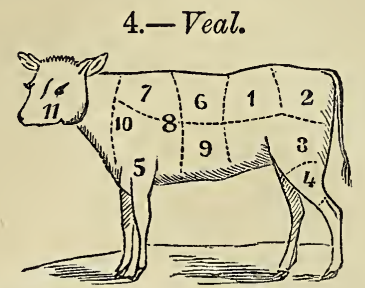

1. Loin, best end.

2. Loin, chump end.

3 , Fillet.

4. Hind-knuckle.

5. Fore-knuckle.

6. Neck, best end.
5. Hind-loin.

\section{5.-Venison.}

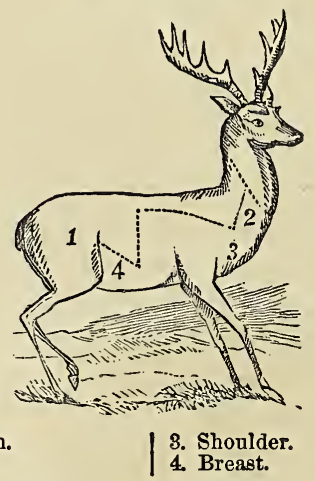

\section{CHAPTER XXIII.}

\section{OULINARY UTENSILS.*}

Tre various utensils used for the pre paration and keeping of food are made.

* For the cuts in this chapter, and for the engrav. ings of house-keeping utensils throughout the book 
either of metal, glass, pottery ware, or wood; each of which is better suited to some particular purposes than the others. Metallic utensils are quite unfit for many uses, and the knowledge of this is necessary to the preservation of health in general, and sometimes to the prevention of immediate dangerous consequences.

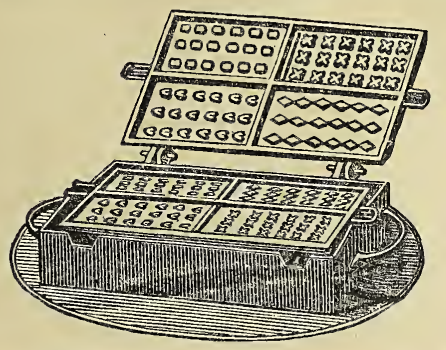

Waffle Iron to revolve over hole in Range or Stove.

The meta's commonly used in the construction of these ressels are silver, copper, brass, tin, iron, and lead. Silver is preferable to all others, because it cannot be dissolved by any of the substances used as food. Brimstone unites with silver, and forms a thin brittle crust over it that gives it the appearance of being tarnished. The discoloring of silver spoons used with eggs arises from the brimstone contained in eggs. Nitre or saltpetre has also a slight effect upon

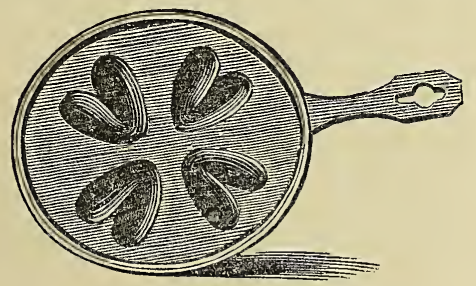

Egg Frier or Fancy Cake Baker.

silver, but nitre and silver seldom remain

the Publisher is indebted to the courtesy of several dealers in New York, whose kind approval of the work has greatly encouraged its issue. long enough together in domestic uses to require any particular caution.

Copper and brass are both liable to be dissolved by vinegar, acid fruits, and pearlash. Such solutions are highly poisonous, and great caution should be used to prevent accidents of the kind. Vessels made of these metals are generally tinned, that is, lined with a thin coating of a mixed metal, containing both tin and lead. Neither acids, nor any thing containing pearlash, should ever be suffered to remain above an hour in vessels of this kind, as the tinning is dissolvable by acids, and the coating is seldom perfect over the surface of the copper or brass.

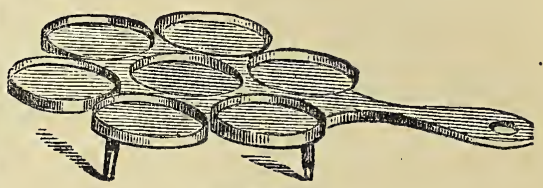

Muffin Baker.

The utensils made of what is called block tin are constructed of iron plates coated with tin. This is as liable to be dissolved as the tinning of copper or brass vessels, but iron is not an unwholesome substance, if even a portion of it should be dissolved and mixed in the food. Iron is therefore one of the safest metals for the construction of culinary utensils; and the objection to its more extensive use only rests upon its liability to rust, so that it requires more cleaning and soon decays. Some articles of food, such as quinces, orange-peel, artichokes, \&c., are blackened by remaining in iron vessels, which therefore must not be used for them.

Leaden vessels are very unwholesome, and should never be used for milk and cream if it be ever likely to stand till it become sour. They are unsafe also for the purpose of keeping salted meats.

The best kind of pottery ware is oriental china, because the glazing is a per- 
fect glass, which cannot be dissolved, and the whole substance is so compact that liquid cannot penetrate it. Many of the English pottery wares are badiy glazed, and as the glazing is made principally of lead, it is necessary to avoid putting vinegar and other acids into them. Acids and greasy substances penetrate into unglazed wares, excepting the strong stone ware; or into those of which the glazing is cracked, and hence give a bad flavor to any thing they are used for afterwards. They are quite unfit, therefore, for keeping pickles or salted meats. Glass ressels are infinitely preferable to any pottery ware but oriental china, and should be used whenever the occasion admits of it.

Wooden vessels are very proper for keeping many articles of food, and should always be preferred to those lined with lead. If any substance has fermented or become putrid in a wooden cask or tub, it is sure to taint the vessel so as to produce a similar effect upon any thing that may be put into it in future. It is useful to char the insides of these wooden vessels before they are used, by burning wooden shavings, so as to coat the insides with a crust of charcoal.

As whatever contaminates food in any way must be sure, from the repetition of its baneful effects, to injure the health, a due precaution with respect to all culinary vessels is necessary for its more certain preservation. There is a kind of hollow iron ware lined with enamel, which is superior to every other utensil for sauces or preserves ; indeed it is preferable for every purpose.

A kitchen should always be well furnished; there is no necessity that it should be profusely so, but there should be a sufficiency of every thing which can aid in producing the dishes preparing, with the success which is so essential to the gratification of the palate. A good workman cannot work well with bad tools, neither can good cooks do justice to their proficiency, if they possess not the necessary utensils suitable to the various modes of cooking. And when this important point has been realized, cleanliness in every article used should be scrupulously observed; no utensil should be suffered to be put away dirty ; it not only injures the article itself materially, to say nothing of the impropriety of the habit, but prevents its readiness for use on any sudden occasion. No good cook or servant would be guilty of such an act; those who are. do so either from laziness or want of system, or a nature naturally dirty; if a very strong hint will not suffice, it is of little use speaking out. A servant who is inherently dirty or slovenly, should never be retained; it is better and easier to change frequently until the mistress is suited, however unpleasant frequent changes may prove, than Quixotically attempt to cure a person of this description. Cleanliness is the most essential ingredient in the art of cooking, and at any personal sacrifice should be maintained in the kitchen.

The fixtures or fittings of a kitchen depend upon the builder, and in modern houses sufficient attention is paid to the situations of the range, dresser, larder, \&c., to embody convenience.

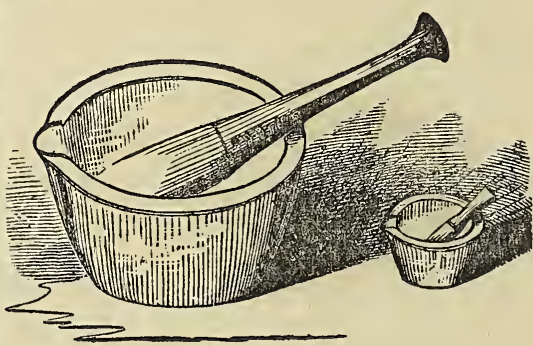

In furnishing a kitchen, there should be every thing likely to be required, but not one article more than is wanted. Unnecessary profusion creates a litter; 
and a deficiency too often sacrifices the perfection of a dish. The following articles, of which we give engravings, are requisite.

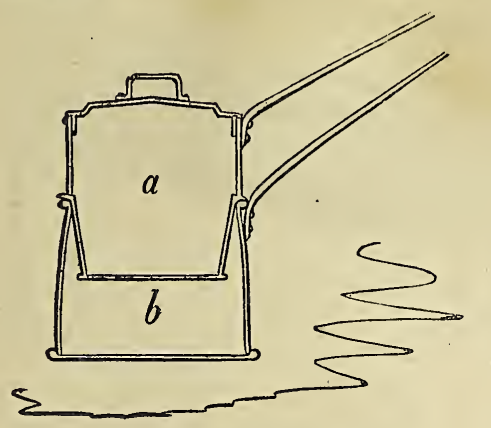

The Nursery Milk Warmer.

This is a very useful apparatus, on the principle of the Bain Marie, or Water Bath.

It consists of a block-tin saucepan to hold water, and a smaller one, with cover of the same material to fit within, but not to reach to the bottom of the external saucepan. This effectually prevents the common accident of burning what is intended to be boiled; for the reason that the heat cannot be increased beyond boiling or $212^{\circ}$; $a$ represents the inner, and $b$ the external saucepan; the cover seen at the top will fit both saucepans.

A Double Floored Griddle, the best
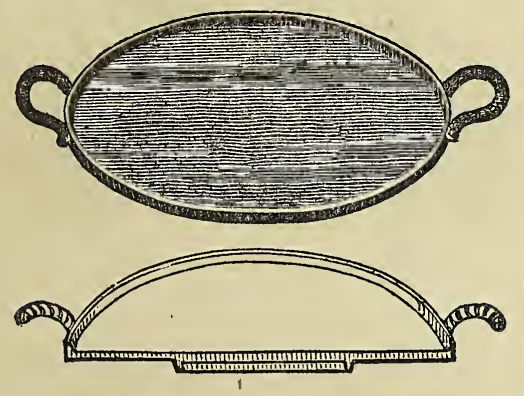

Double Floored Griddlo.

iron Griddle in use. Very thick; therefore it gets hot or cold gradually, retains the heat longer, cooks better, and smokes less than ordinary griddles.

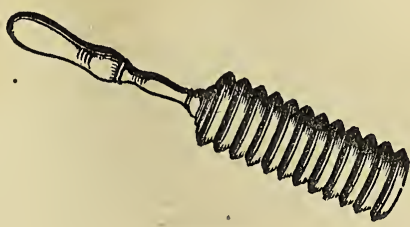

Beefsteak Maul.

A Beefsteak Pounder for making beefsteak tender.

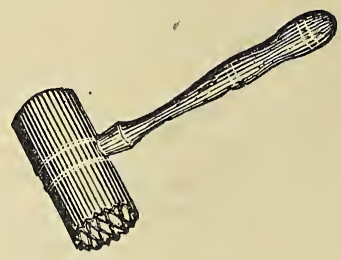

Beefsteak Pounder.

Wooden Moulds for moulding butter.

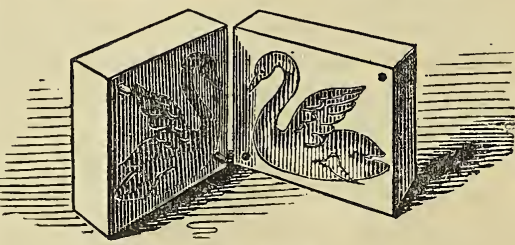

Butter Moulds.

For working butter into form, similar to Butter Hands, the

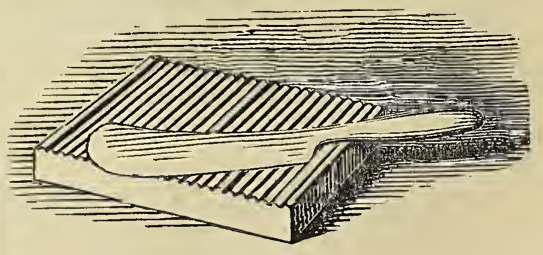

Butter Board and Knifo. 


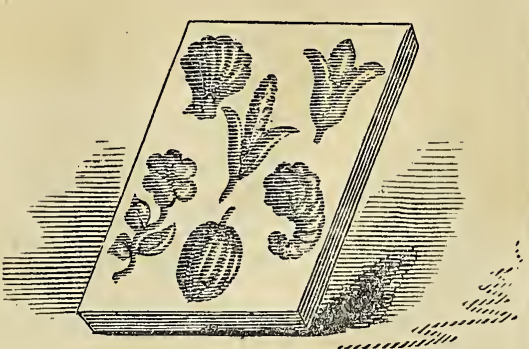

Biscuit Mould or Block.

The pastry is rolled into this and forms half the biscuit; others are made and fastened together, either before or after being baked.

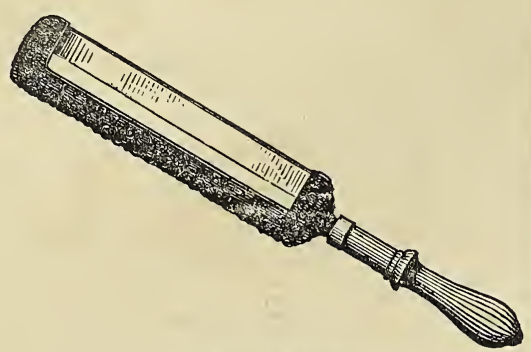

Tea Bread Knife.

For cutting bread; it can be regulated by screws at the ends to cut any thickness.

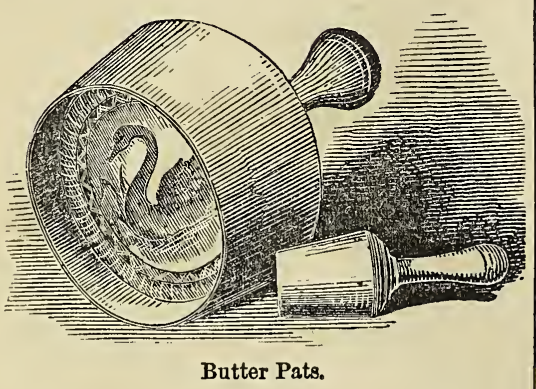

A mould and stamp for butter by which you can have the full size of the stamp, and the butter any thickness.

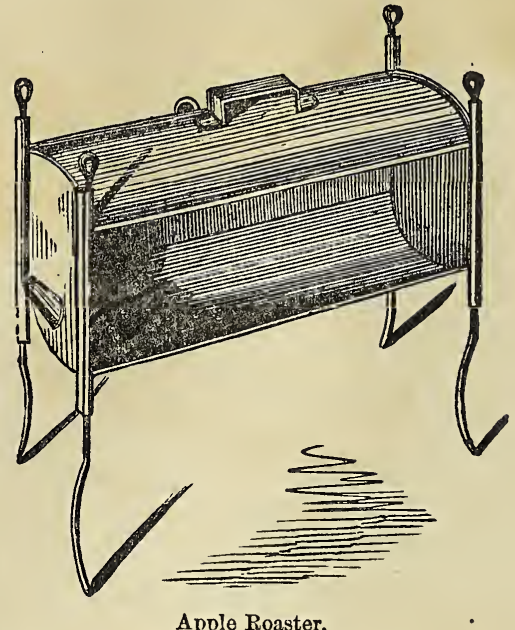

Tin Apple Roasters are often made with a shelf, making two divisions, ronsting double the quantity.

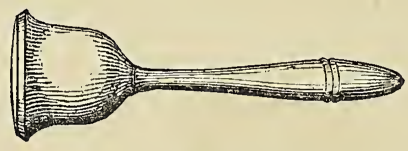

Potato Masher

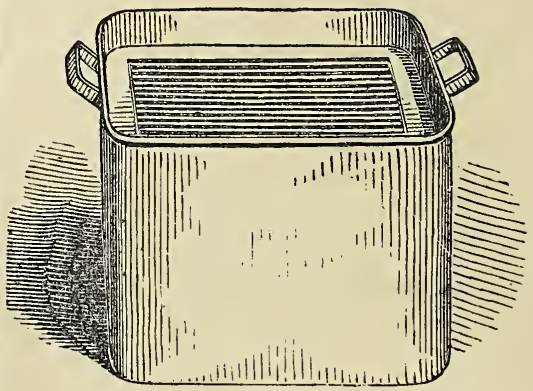

Knife Washer.

To wash Ivory, Pearl, or other handled knives thoroughly, without allowing the greasy water to come in contact with and thereby soil or stain the handles. 


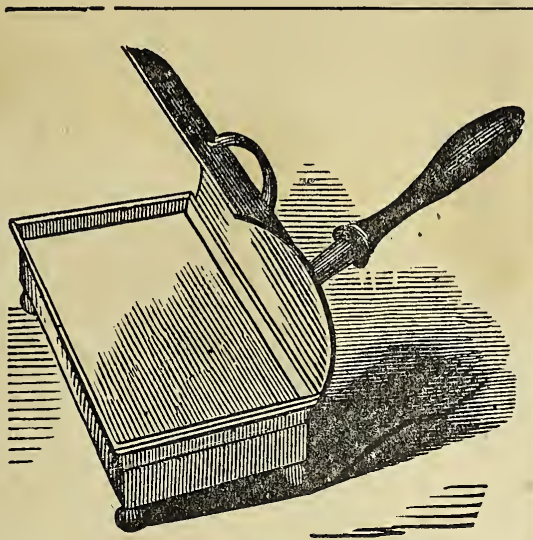

Cheese Toaster.

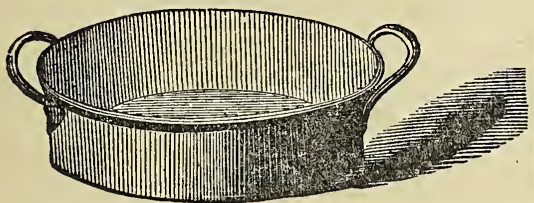

Preservo Pan Enamelled.

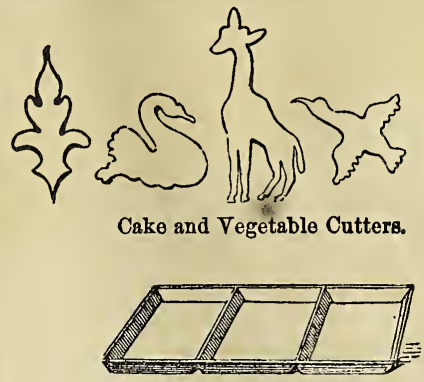

Sponge Cake Pans.

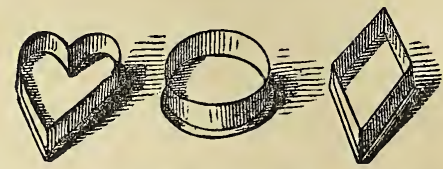

Queen Cake, or Pound Cake Pans.
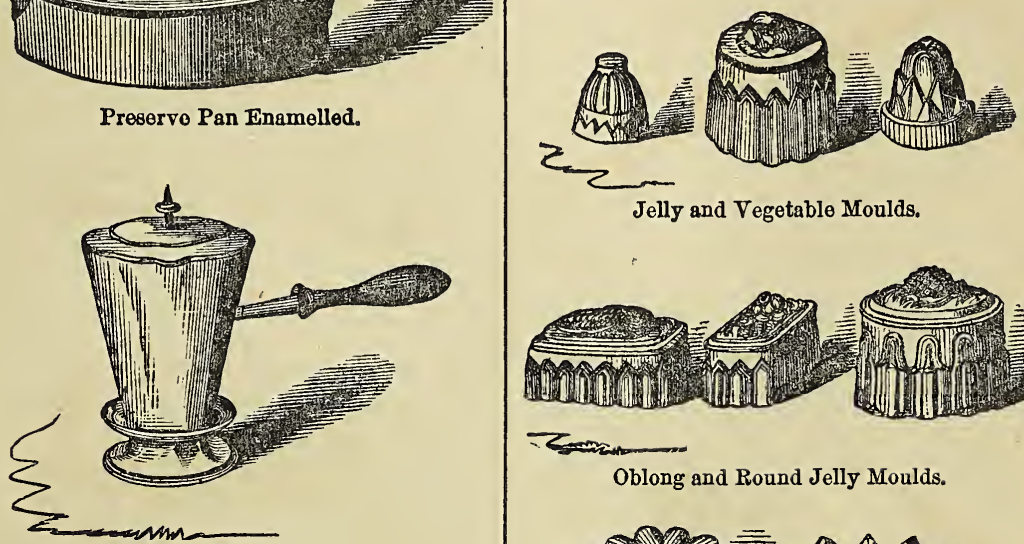

Etna.

It will boil a pint. of water in three minutes.
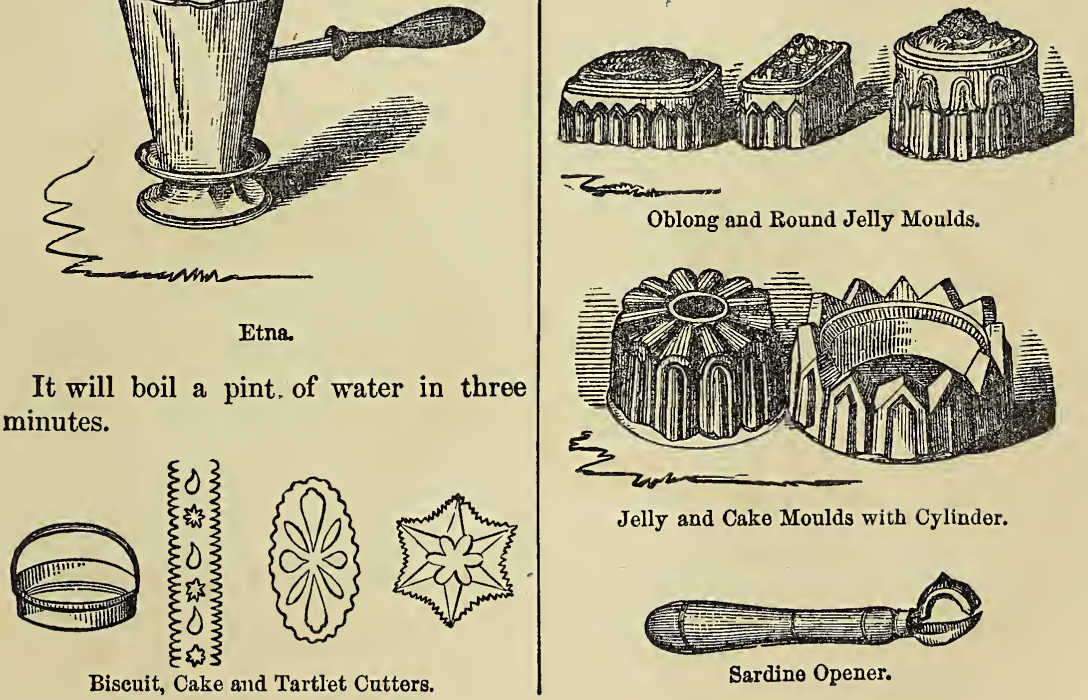

Jelly and Cake Moulds with Cylinder.

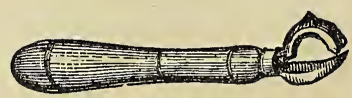

Sardine Opener. 


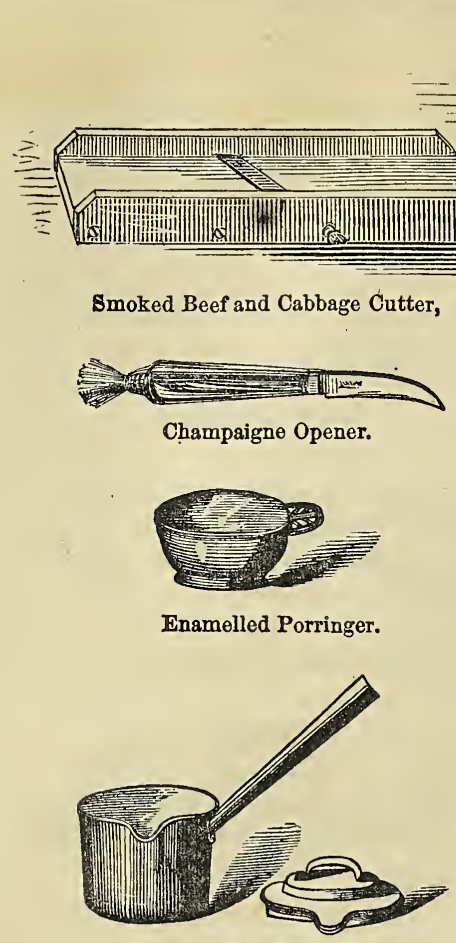

Enamelled Saucepan.

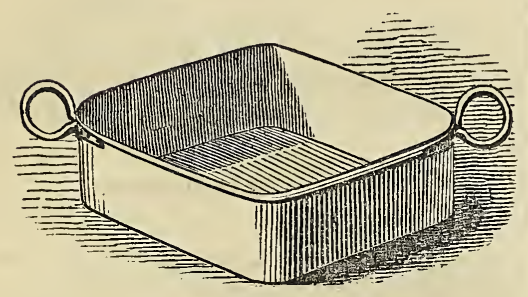

Bain Marie.

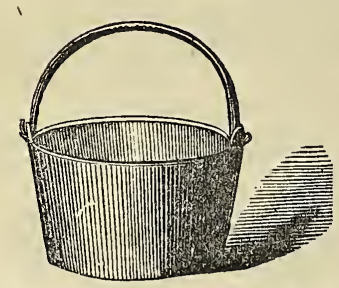

Enamelled Preserving Kettle.

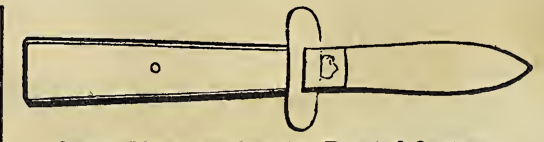

Oyster Knife, for Opening Roasted Oysters.
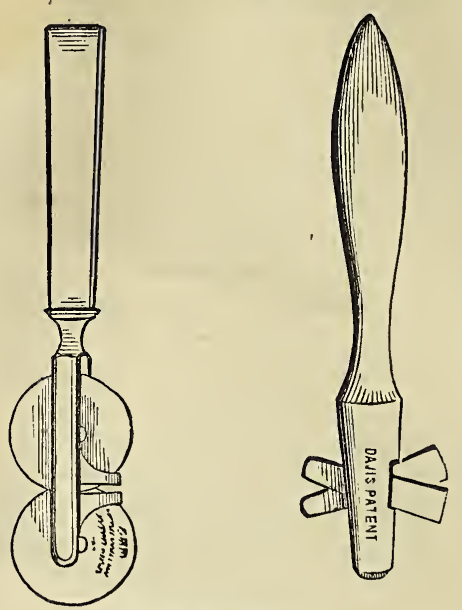

Knife Sharpeners.

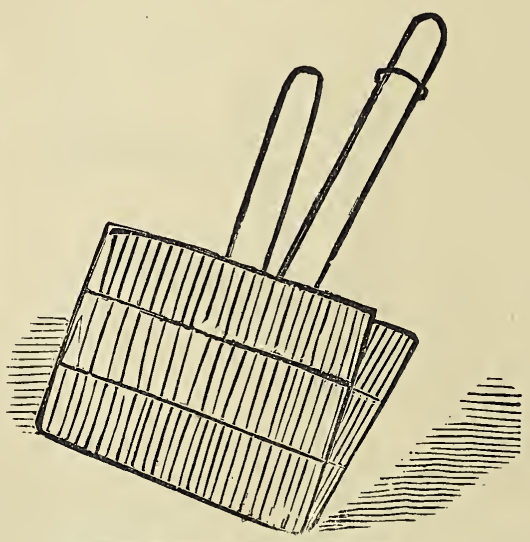

Oyster Broiler, or Toast Gridiron.

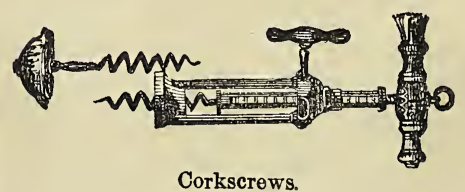




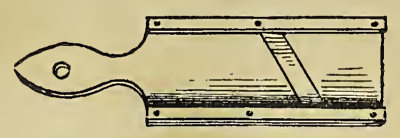

Cucumber and Vegetable Slicer.

Saucepan Digester.-The great importance of the digester, not only to poor families, but to the public in general, in producing a larger quantity of wholesome and nourishing food, by a much cheaper method than has ever been hitherto obtained, is a matter of such serious and interesting consideration, that it cannot be too earnestly recommended to those who make economy 'in the support of their families an object of their attention. The chief, and indeed the only thing necessary to be done, is to direct a proper mode of using it to advantage; and this mode is both simple and easy. Care must be taken in filling the digester, to leave room enough for the steam to pass off through the valve at the top of the cover. This may be done by filling the digester only three parts full of water and bruised bones or meat, which it is to be noticed are all to be put in together. It must then be placed near a slow fire, so as only to simmer, and this it must do for the space of eight or ten hours. After this has been done, the soup is to be strained through a hair sieve or cullender, in order to separate any bits of bones. The soup is then to be put into the digester again, and after whatever vegetables, spices, \&c., are thought necessary are added, the whole is to be well boiled together for an hour or two, and it will then be fit for immediate use.

Any thing that is to be warmed and sent to table a second time should be put into a basin or jar, placed in hot water, which is not permitted to come to the boiling point. If allowed to boil, the meat will harden, or the sauce will be reduced and become thick: by avoiding these chances the flavor will be preserved, and the viands may be warmed up more than once without injury. The steamapparatus now employed in most kitchens is. admirably adapted to this purpose, since the heat can be regulated to the required temperature.

A spacious movable screen, large enough to completely cover the fire, lined throughout with tin, and having shelves for the warming of plates and dishes, should also be an appendage ; and there should be an abundance of kitchen utensils of the best kind, kept in their proper places and strictly clean. Cleanliness is, indeed, of the first importance, and no kitchen-maid should ever put away a metal saucepan which has been used for any other purpose than merely boiling pure water, without scalding it thoroughly and then drying it.

Every kitchen should be provided with a clock to keep the cook to her time; also with a large and a small marble mortar for the pounding of meat, with chopper, meat-saw, various-sized scoops for vegetables, when required for haricos \&c., paste-cutters, steak-tongs, \&c., and those insignificant, though useful little articles, minute-glasses, to regulate the boiling of an egg; nor should a spicebox, containing whole pepper, mace, nutmegs, and cinnamon, be forgotten ; together with various dried sweet herbs. Scales, with weights from $\frac{1}{4} \mathrm{oz}$. to $2 \mathrm{lbs}$., should be placed on the dresser, and the weights carefully kept in regular order. A set of tin measures with small spouts or lips, and with the contents distinctly marked upon them, from a gallon down to half a gill, will also be found very convenient. It is likewise well to have a set of wooden measures, from a bushel to a quarter of a peck.

Let it be remembered, that, of liquid measure- 
T'wo gills are half a pint.

Two pints are one quart.

Four quarts are one gallon.

\section{Of dry measure-}

Half a gallon is a quarter of a peck.

- One gallon is half a peck.

Two gallons are neck.

Four gallons are half a bushel.

Eight gallons are one bushel.

About twenty-five drops of any thin liquid will fill a common-sized teaspoon.

A common tumbler holds half a pint.

Four tablespoonfuls, or half a gill, will fill a common wine-glass.

Four wine-glasses will fill a half-pint or common tumbler, or a large coffee-cup.

A quart black bottle holds in reality about a pint and a half.

Of flour, butter, sugar, and most articles used in cakes and pastry, a quart is generally about equal in quantity to a pound avoirdupois (sixteen ounces). Avoirdupois is the weight designated throughout this book.

Ten eggs generally weigh one pound before they are broken.

A tablespoonful of salt or brown sugar is generally about one ounce.*

Sieves, of various descriptions, are very essential. Every utensil for cookery should be of various sizes, so as to suit the quantity of which the dishes may be composed; and each should be kept in a fixed place, as well as washed and dried immediately after using. The cook should also be charged to take care of jelly-bags, tapes for the collared

* Mrs. Hale gives the following table, by which persons not having scales and weights at hand may readily measure the articles wanted to form any receipt, without the trouble of weighing. Allowance to be made for an extraordinary dryness or moisture of the article weighed or measured.

WEIGHT AND MEASURE.

Wheat flour . . . 1 pound is . . 1 quart. Indian meal . . . . 1 pound, $2 \mathrm{oz}$., is 1 quart. Butter, when soft . . 1 pound is . . 1 quart. Loaf sugar broken . . 1 pound is . . . 1 quart. White sugar, powdered 1 pound, 1 oz., is 1 quart. Best brown sugar . . 1 pound, 2 oz.. is 1 quart. Eggs . . . . . 10 eggs are . . 1 pound Flour . . . . 8 quarts are . 1 peck. Flour . . . 4 pecks are .. 1 bushel. things, \&c., which, if not perfectly scalded and kept dry, give an unpleasant $/$ flavor when next used.

\section{CHAPTER XXIV.}

Liebig, in his work on "The Cliemistry of Food," says: "Among all the arts known to man, there is none that enjoys a juster appreciation, and the products of which are more universally admired, than that concerned in the preparation of our food. Led by an instinct which has almost reached the dignity of conscious knowledge, and by the sense of taste which protects the health, the experienced cook, with respect to the choice. admixture, and preparation of food, has made acquisitions surpassing all that chemical and physiological science has done in regard to the doctrine or theory of nutrition."

"I do not yet despair," says another writer. "of seeing the day when the culinary science, like others, will have its qualified professor:." "The art of cookery," another observes, "is the analeptic part of the art of physic." Dr. Mandeville says, "Physicians should be good cooks, at least in theory."

Dr. Arbuthnot says - "The choice and measure of the materials of which our body is composed-of what we take daily by pounds, is, at least, of as much importance as what we take seldom and only by grains and spoonfuls. Count Rumford remarks: "In what art or science could improvements be made that would more powerfully contribute to increase the comforts and enjoyments of mankind?"

A quaint writer says truly: "The stomach is every man's master;" and Armstrong attributes to the good cook the useful knowledge: 
"How best the fickle fabric to support Of mortal man; in healthful body, how A healthful mind the longest to maintain."

To prevent diseases is surely better than to cure them. The French enjoy a happy equilibrium of spirits more constantly than any other nation; Dr. Kitchiner says, it is because "their elastic stomachs, unimpaired by spirituous liquors, digest vigorously the food they render easily assimilable by cooking it sufficiently; doing half the work of digestion by fire and water, till

"The tender morsels on the palate melt, And all the force of cookery is felt."

The cardinal virtues of cookery are cleanliness, frugality, nourishment, and palatableness.

The term "gourmand" or "epicure," says a distinguished writer on the subject, is not synonymous with "glutton," who eats as long as he can sit, like the great eater of Kent, whom Fuller places among his worthies; telling us "he ate thirty dozen pigeons at one meal ; at another, four score rabbits, and eighteen yards of black pudding. London measure :" nor does the term epicure suit a fastidious appetite only excited by dainties, such as the brains of peacocks or parrots, the tongues of thrushes or nightingales, \&c. It means one who has good sense and good taste enough to relish food cooked according to scientific principles; so prepared that the palate be not offended, and that it be renderes easy of digestion. Thus the temperate man is the greatest epicure; for the perfection of enjoyment depends on the perfection of the faculties of mind and body.

The philosopher, Descartes, when a cavilling Marquis said: "What, do you philosophers eat dainties?" replied, "Do you think Providence made good things only for fools!" Bosweli says, Dr. Johnson had nice discernment in the science of cookery, and talked of good eating with uncommon satisfaction.
Boileau says, the Norman conqueror William, bestowed portions of land on his favorite chief cook. The Doomsday Book records a grant to Robert Argyllon, for the service of "making in an earthen pot, in the kitchen of our lord the king, a mess called "De la groute,", $\rightarrow$ a kind of plum-porridge - on the day of the coronation. This dish was served to king George IV., at his coronation.

The luxury of different ages has furnished many curiosities in cookery. $\Lambda$ mong the ancients, a porpus and wild boar were highly esteemed. and the swan was a dis of state; the crane was a dainty in 1. ilam the Conqueror's time; and seals, curlews, hcrons, bitterns, and the peacock-this last, "the food of lovers and the meat of lords "-were fashionable at baronial entertainments. The peacock was stuffed with spices and sweet herbs, roasted and served whole; after it was dressed, being covered with the skin and feathers, the tail spread, and the beak and comb gilt. "Some were covered with leaf-gold. These birds adorned English tables till the beginning of the seventeenth century.

The pie, full of living birds, was a favorite dish, and a common joke at old English feasts. The dwarf, Jeffrey Hudson, was served up in a cold pie about 1630, before King Charles, at the Duke of Buckingham's table. The baron of beef was a favorite of old English hospitality. Don Anthony, of Guevara, chronicler to Charles V., says. he saw at a feast, "a horse roasted, a cat in jelly, lyzard in hot broth, and frogges fried, \&c."

The Roman sauces described by Coelus Apicius, a celebrated Roman epicure, who wrote a curious cookery book in Latin, and invented a soup made of the livers of a peculiar fish which sometimes sold at the rate of sixty dollars the pound-would be hardly more palatable to us than the black broth of the Spartans which caused the citizens of Sybaris 
to wonder no longer why the Spartans were so fearless of death. Yet one of the receipts of Apicius required the death of three or four dozen animals. The Esquimaux eat raw fish, and melted fat is a delicacy among Arctic natives. Many of the northern Indians eat the intestines and blood. Tartars feed on dogs, and some of the natives of Australia, New Holland, and other countries, were wont to regale themselves upon ants and worms, while mice were an African delicacy. Norwegians mixed the powdered bark of trees with meal to bake in cakes.

La Chapelle's Nouveau Cuisinier, published in 1748, mentions the cooking of a turkey in the shape of a football or a hedgehog; a shoulder of mutton in that of a beehive; an entrée of pigeons in the form of a spider or a frog. Such whimsical harlequinade has now become oldfashioned, though the taste for decoration remains.

By a strange misconception. cookery has in all ages been intrusted to ignorant persons, who practise it without reference to those general principles which connect it with chemistry as much as other arts are allied to that science. Yet in the days of the patriarchs, the province of the cook, it seems, was not a servile one. A princess would not disdain to dress the lamb her lord had killed and brought from the flock. In Homer's time kings and princes killed their own cattle and cooked the flesh.

Perhaps the march of improvement may induce professors of gastronomy to elevate their calling by connecting its practice with the principles of science. The brewers and distillers of the present day are philosophical chemists, understanding the "principles on which they act, and their processes are more certain, economical, and manageable than formerly; why may not cooks be philosophers? Why may not equal advantages be derived from the application of sci- ence to those arts which relate to the management of solid sustenance?

It has been said that the best books on cookery have been written by medical men.

\section{CHAPTER XXV.}

\section{A GLOSSARY OF FOREIGN TERMS USED IN COOKERY.}

French cookery is of so diverse a nature that many volumes have already been written upon the subject; and new modes of dressing the same things are so constantly being invented, that we must content ourselves with merely giving a few explanations of the terms adopted by most of our professed cooks.

Atelets. Small silver skewers.

$B a b a$. A French sweet yeast cake.

Bain Marie. A flat vessel containing boiling water, intended to hold also other saucepans for the purpose either of cooking or keeping their contents hot. The Bain Marie is called in the English kitchen Beau Méré Pan. This term is 'old, having its origin with the alchymists, who, finding that sea-water boiled at a high temperature, and did not evaporate so quickly, used a pan containing sea-water ; hence the term: Bain Marie; or, SeaWater Bath.

Bard.-A slice of thin bacon fat, used for covering the breasts of birds, the back of a hare, or any substance that requires the assistance of fat where larding is not preferred.

Beignet, or Fritter.-Anything that is enveloped in a casing of batter or egg, and fried. Thus we have fritters of fruit, vegetables, cream, \&c.

Blanc.-A white broth used to improve the color of chickens, lamb, \&c.

Blanch.-To set anything on the fire in 
cold water, and when it boils strain it off and plunge it into cold water.

Blanquettes. - A kind of fricassée, made of slices of white meat cut thin, and warmed in white sauce thickened with the yolk of eggs.

Bouilli.-Beef very much boiled and served with sauce.

Bouillon. - The common soup of France.

Bouquet.-A bunch of parsley and scallions tied up to put in soups, \&c.

Bouquet garni, or Assaisonné. The same, with the addition of cloves or aromatic herbs.

Bourguignote. - A ragout of truffles.

Braise.-This is a method of dressing meat, poultry, \&c., without evaporation. It is done by lining a braising pan with thin slices of bacon, beef, or veal ; upon which place whatever you may intend to braise; and also add carrots, onions, lemons, lay leaf, herbs, pepper, and salt.

Brioche.-A French yeast cake.

Buisson (en).-A fanciful mode of dressing up pastry, \&c.

Callipash.-The glutinous meat of the upper shell of a turtle.

Callipee.-The glutinous meat of the under shell of a turtle.

Caramel.-Sugar boiled down until the water is evaporated, and then formed in ornamental devices for decorating gâteaux-baskets, \&c. It is also used for coloring gravies when reduced almost to the burning point, and then dissolved in water. Its use is not much to the credit of the cook.

Capilotade.-A hash of poultry.

Civet.-A hash of game or wild fowl.

Compeigne.-A French sweet yeast cake, with fruit, \&c.

Compotier.-A dish in the dessert service purposely for the compote.

Couronne (en).-To serve any prescribed articles on a dish in the form of a crown.
Court or short, to stew.-The reduction of a sauce until it becomes very thick.

Croutons.-Bread cut in various shapes and fried lightly in butter or oil.

Casserole.-A rice-crust moulded in the form of a pie; when baked, filled with a mince or purée of garne, or with a blanquette of white meat. Also a stewpan.

Compote--Stewed fruits served with syrup. There are also compotes of pigeons and other small birds.

Consommé is a strong clear gravy, drawn from the long stewing of any kind of meat. to be either used as broth, or made into soups and sauces.

Coutis, or cullis, is a rich brown gravy commonly used for the purpose of coloring as well as thickening and flavoring many sorts of soups and sauces. It is made in various ways, chiefly upon a foundation of ham and veal, slices of which are put into a closely covered stewpan, with only a small quantity of water, and boiled over a brisk fire until the whole becomes brown and thick. See Sauces.

Croquettes.-A mince of either fish, meat, or poultry, made very savory, with a small quantity of sauce, formed into shapes of any kind, rolled in egg and bread-crumbs, and fried crisp.

Croustades, or Dresden patties, made of paste or bread baked in small moulds, and filled with mince of any kind.

Dorez.-To wash pastry, \&c., with yolk of egg well beaten.

Dorure.-Yolks of eggs well beaten.

"En papillote." - White paper is greased with oil or butter. and then folded over a cutlet or small fish, fastening it by screwing the paper at the edges.

Entremets.-Small ornamental dishes served in the second and third courses.

Entrée.-A corner dish for the first course. In large dinners the side dishes are called flancs. 
Financière. - An expensive, highly flavored, mixed ragoutt.

Flan.-A French custard.

Farce.-Forcemeat.

Faggot.-A small bunch of parsley and thyme and a bay-leaf tied up.

Fricandeaux - may be made of any boned piece of veal, in pieces of not more than two or three lbs. weight, chiefly cut from that portion of the fillet which we have described as the thick part. It is a frequent dish as an entrée at good tables, and requires great care to serve in perfection.

Giteau. - A cake or pudding.

Glaze (to).-To reduce sauces to a jelly, that they will adhere to the meat.

Glace or Glaze.-Stock boiled down to a consistency, and used to improve the appearance of all braised dishes; it should be warmed in the bain-marie, and applied with a brush.

Glaze, Glace Ice, is composed of white of $\mathrm{egg}$ beaten with powdered sugar.

Godiveau.-A common veal forcemeat.

Gras $(a u)$, - means that the article is dressed with meat gravy.

Gratin.-A layer of any article intended for this purpose is spread over a dish that will bear the fire. and is placed on a stove or hot ashes until it burns.

Hors d'œurre.-A small dish served during the first course.

Lard (to).-To stick bacon, or whatever meat may be named, into poultry, meat, \&c. It is accomplished with a larding pin, one end of which is square and one hollow. The lardon is put into this hollow, the point is then inserted in the meat, and on being drawn through leaves the bacon or lardon standing in its proper place. It requires practice to do this well.

Lardon.-The pieces into which bacon or other meats are cut for the purpose of larding.

Liaison.-A finish with yolks of eggs and cream for ragouts and sauces.
Madeleines._- Cakes made of the samo composition as pound cakes.

Maigre (au).-Soups, \&c., dressed without meat.

Mask.-To cover completely.

Marinade. - A liquor prepared for boiling or stewing fish or meat in: it is sometimes used cold.

Meringue:-A very light preparation, made of sugar and whites of eggs beaten to snow.

Matelote.-A rich stew of fish with wine.

Miroton consists of small slices of meat cut thin, and not larger than a crownpiece, and made into various sorts of ragouts; and dished up in a circular form.

Nouilles.--An Italian paste resembling macaroni, but flat instead of being in pipes. Panada.-Bread soaked in milk, used principally for quenelles and fine farces.

Passer.-To fry lightly.

Pìté.-A raised crust pie.

Poélée.-A light braise for white meats; the difference between this and the braise is that in the former, the meat, or whatever it may be, need not be so much done as the latter.

Potage.-A term for soup.

The Pot-au-feu is truly the foundation of all good cookery, and of that we shall treat rather largely in our chapters on soups and sauces.

Purée.-A culinary operation which should be carefully attended to, and which, although known for preparing potted meats and fish, is seldom employed by common cooks in any thing but pea-soup. It consists in either pounding the meat or fish in a mortar until it becomes a paste. or in stewing roots or pulse in boiling water until they are softened into a thick pulp, when they are passed, first through a colander, or through a purée presser, and afterwards through a wire or hair sieve, and being thinned with broth, may be formed into soups of various sorts. 
Quenelles and Godiveaux are different sorts of forcemeat, composed of meat or fish, with bread and yolk of egg, and fat of some kind; seasoned in various ways, and either spread upon rolled meat, or formed into balls and fried as garnish to entrées, or served separately as entrées.

To make Quenelles.-When the forcemeat is made, take two tablespoons, fill one with the meat, dip a knife in hot water, with which smooth it over; then dip the other spoon into boiling water, and with it remove the meat from the first spoon, and slip it into a buttered "stewpan; so on until you have the number you want: then cover them with stock, and boil them about ten minutes, or until firm, and they are fit for use. For small quenelles use teaspoons.

- Rissole. - A mince of meat or fish, enclosed in paste, or made up into shapes or balls in the manner of minced collops, and used either as side-dishes, or as a garnish for stews or hashes, now more elegantly termed fricassées and rago ûts.

Roux.-A thickening for white soups or gravy, made of flour and butter.

Salmis.-A hash made of game, which is cut up when only half roasted; whereas a hash is made from dressed game.

Sauter. - To fry very lightly.

Sabotière or Saubeti re.-A pewter or tin vessel in which are placed the moulds containing whatever is intended to be frozen.

Tourner or turn.-To stir a sauce; also to pare and cut roots, vegetables, and fruits very neatly.

Tamis, or "tammy," a strainer of thin woollen canvas, or silk, used for straining soups and sauces.

Tourte.-A kind of tart baked in a shallow tin.

Vanner.-To take up sauce or any other liquid in a spoon and turn it over very quickly.

Velouté.-For heightening the flavors of soups, made dishes, \&c.
Vol-au-vent.-An extremely light puff paste, in which are enclosed minces of sweetbread, poultry, game, \&c.

\section{CHAPTER XXVI.}

\section{CONDIMENTS.}

There is one class of materials in constant use in the kitchen:-the condiments ; without these, soups, sauces, and all made dishes, would be insipid; and the judicious application of them is essential.

If all kinds of condiments were of the same quality - that is, if all salt, pepper, and mustard, \&c., were alike, receipts might be given as in a Pharmacopoeia, with distinct measures for each; but as nothing differs so much as these simple condiments, everything must be left to the palate of the cook, and on him or her alone depends the flavor of the alimentary substances partaken of.

Of all condiments, that in most general use is SALT ; the health of every individual depends upon it, and it is as much required as food or drink. Even on those continents far away from the shores washed by the briny ocean, we find it in springs, and in crystal globules encrusting the earth.

It is chemically known as chloride of sodium, being a combination of chlorine and sodium. Its use as an antiseptic, and as a condiment, is too well known to be repeated here.

Rock Salt is the unpurified salt, as dug from the mines. This is purified by boiling, \&c., and crystallized by heat.

Bay Salt is the coarse large crystal salt, taking its name from the salt that formerly was made in pits by the overflow or letting in of the sea at the head of Bays, and which was evaporated by the heat of the sun. Almost all the fish 
cured in France at the present day is done by this kind of salt, the duty upon foreign salt being so high.

Pepper, so extensively used at present in Europe, may be said to be a modern invention. Before the introduction of pepper, many plants, such as saffron, \&c., were used, until this West Indian fruit became more general and easy to obtain.* The plant is a climbing one (piper nigrum,) and exceedingly pretty when in fruit. The berry is gathered with the skin or pulp around the stone, and that which is intended for black pepper is allowed to remain thus, while for white pepper it is blanched and rubbed until the skin comes off. In all dark sauces use the black pepper.

Long Pepper, the fruit of the piper longum, is a plant very similar to the former, and, if possible, it has a more aromatic flavor than the black pepper.

I doubt if pepper was known to the Romans. If so, it would have been known in Egypt, where we find no trace of it, although some authors have considered it as the ammonium of the ancients.

Allspice, or, as it was formerly called, Pimenta, is another of those productions of the climes near the Equator, which has come much into use by Europeans. It is the fruit of the pimenta vulgaris. That which is the freshest is the best; as the aromatic oil which it contains is not dissipated by keeping. The essential oil it contains can be used in very small quantities in cookery, but should not be subjected to long boiling, or it loses its flavor.

* It is a singular circumstance, that this very small fruit should be the cause of England's possessing one of the largest portions of her dominions. In the reign of Elizabeth, and during the war with Spain, pepper, which had then become an article of necessity, rose to an extravagant price, and to obtain it cheaper, the Queen granted Letters Patent to certain merchants to trade to the East Indies, which was the origin of the present East India Company.
Ginger is the root of a plant, a native of the East Indies and Brazils, and also naturalized in the West India Islands. The plant grows in moist places, and is of a reed-like form, bearing a yellow flower. The root, when fresh, should be of a light green, solid and heavy. A spongy or woolly root should be avoided. The virtues of ginger chiefly consist in a volatile oil which it possesses. This is best obtained by bruising the root, and making an infusion in spirits of wine or boiling water in a closed bottle; or, if thinly sliced and infused in vinegar, it is pleasant in a salad. It is usually employed in cookery by having the root desiccated by the heat of the sun until perfectly dry and hard; and then powdered till its aromatic oil is lost. It should be more frequently used in cook- . ery, and as a condiment as well as for dessert.

Cloves are the buds of a flower, not arrived at maturity, of an East Indian tree, called caryophillus aromaticus. This, in common with many other buds of flowers of various kinds, contains a large quantity of essential oil. It has a very pungent, aromatic smell. Its nature is stimulating. It should not be boiled too long, or its flavor is lost in evaporation.

Nutmeg is the interior of a fruit belonging to a tree, a native of the lslands of the Eastern Archipelago, called Banda, and known in botany as the myristica moschata. It is strongly aromatic, and very volatile, and considered agreeable. It is used in powder, and not subjected to much heat, which would cause it to lose its qualities.

MACE.-This is the skin which covers the shell containing the nutmeg. It is cut into small pieces and dried. This is more frequently used in cookery than the nutmeg, as its essential oil and aromatic flavor are not so soon evaporated.

CaYenne is the dried pod of a well- 
known plant, the capsicum, now so often reared in hot-houses. It is a native of the East and West Indies, and South America. It is of a very pungent nature, and a strongly acrid flavor. The best is of an orange color. There are several kinds of capsicums; the capsicum anruum or annual capsicum; the capsicum grossum, or hell pepper; and the capsicum cerasiforme, or cherry pepper. The pods of these are frequently pickled. The essence of cayenne, by infusing in alcohol, is a very excellent seasoning.

Cinnamon is the under-bark of a tree, the laurus cinnamomum, a species of bay, with leaves like laurel, found in many East India Islands. but chiefly in Ceylon. It possesses a very fine aromatic flavor and smell. The essential oil, when extracted, is better to use than the bark itself.

This tree bears a fruit similar in shape to the acorn, but smaller. It has neither smell nor taste; but, boiled in water, yields an oil which, on becoming cold, is as hard as tallow : it is used as an ointment, and also to burn. The outside of the root of this tree yields camphor and oil of camphor.

C ASsia is also a bark of a similar kind of tree to the cinnamon; the laurus cassia. It does not contain so much flavor as cinnamon; but the aromatic qualities are very similar. Its oil is often sold for that of cinnamon.

Cassia Buds are the pods of the şeed of the cinnamon tree. They contain an oil equal in flavor and pungency to the cinnamon. They are not often used in cookery, their value not being generally known.

Mustard is the powder of the seed of the plant, the sinapis nigra. It grows wild in many places, and can be cultivated in almost any soil. It is of a strong, pungent nature, and is difficult to concentrate. It should be mixed with cold water and salt. It derives its name from the French moutarde, who derive it from the Latin of multum ardet, which became corrupted into moult arde. In France they prepare it in a variety of ways. Mustard is a condiment easily adulterated; and particular care should be used in its selection.

Onions, a bulbous root, so much used in cookery, is a native of this country. By some it is eaten in the raw state; and if partaken of freely, its effects pervade the whole system, and the person who uses them may be known at some distance, from the peculiar odor which belongs to the root. Its moderate use is wholesome. It is also good against many diseases of the skin; but should never be eaten too freely by weak stomachs. There are several sorts. The common. called the allium cepa; the GaRLIC, allium stavium; the SHALOT, allium ascalonicum ; the LEEK, the allium porrum; the RocayboLe, the allium scordoprasum; the Chive, the allium chœnoprasum. The manner of using them is described briefly in the various receipts. Onions have been used in cookery from a very early period; and in Spain and Portugal form the principal food of the lower classes-being, from the nature of the climate, much milder than in this country.

The next articles among the condiments are the herbs; among these, MaRJORAM is that most in use; it is an herb which contains a large quantity of what may be called essential oil, which is extracted in cooking, and imparts its flavor to the viands in which it may be used. There are several kinds of marjoram in use, but that known as the pot marjoram, origanum onites, when well cultivated, is the best for general use; there is the sweet marjoram, origanum marjorana ; the winter sweet marjoram, origanum heracloticum; and the common marjoram; origanum vulgare. The essence of this herb may be used in 
cookery; the more common plan is to dry it for use, but it should be used fresh when it can be procured.

SAvORY.-There are two kinds, the winter and summer; the latter contains the most flavor-it can be easily dried and bottled for keeping.

BASIL is also an herb in great use; it grows to great perfection if well cultivated. Its aromatic smell is very similar to cloves: there are two kinds, the sweet basil, ocymum basilicum; and the least basil, ocymum minimum. The first has the best flavor and smell.

Тнхме.-There are two, the lemon, thymus citriodorus, and the common thyme, thymus vulgaris; the first is the best to use in all dishes wherever this herb is required.

Pennyroyal is not much in use in cookery, but can be used where mint cannot be had.

Mint - Spearmint or common mint is that most in use; it should never be used in large quantities. Some of it infused in vinegar, and the vinegar afterwards used with a salad, gives a fine flavor and a novelty to that dish, and it is used in vinegar with lamb.

SAGE is an important herb, and by many considered an agreeable condiment; there are several kinds; that whose leaves have a purple tinge is considered, best for culinary use.

PARsLex, although at present so prominent in use in our kitchens, was unknown to our forefathers; it is only within a century that it has become so general : it was inţroduced into England from Italy. There are three varieties, the common or plain-leaved, the curled or double-leaved, and the broad-leaved; the curled is the best-looking, but has not so much flavor as the common; the broad-leaved is used for its root, which is excellent cut up thinly in soup, \&c. It may be obtained all the year. To keep it well, it should be dried and cut up, and placed in well corked bottles, but should only be used when fresh cannot be procured.

TARRAgon is not so much used as it ought to be; if used more in soup, instead of the stronger herbs, it would be liked; but great care must be observed, as its aromatic qualities are quickly dissipated by heat. It is a native of the north of Europe, and known in botany as the artemisia dracunculus.

ClaRY is a plant of the sage kind; its leaves are sometimes used to flavor soups, and it is liked by a few.

Fennel may be used often with advantage; it is, however, rarely used but with fish.

Celery, now grown to such perfection, is the cultivated wild plant known as smallage. Celery is a great deal used in cookery, but it should not be subjected to too long boiling; if its flavor is required, and no root to be obtained, the seed of the smallage can be used instead. It is generally supposed that the roots and leaves of smallage are poisonous; we would not recommend them to be used, as the seed can always be obtained, and the flavor is finer. The salts of celery is a modern improvement, and a great addition to our culinary condiments.

BORAGE is not much used in cookery; its flavor is slightly bitter, and it is sometimes used to flavor ale, cider, \&c., for a summer drink.

HoRSE-RADISH is the root of a plant; the aroma which it contains is quickly dissipated by heat-it is therefore used in a raw state by being grated; an essence may be obtained by infusion with spirits of wine, which, added to mustard after it is made; gives it an agreeable flavor.

Chervil is getting more into use every day; its flavor is exceedingly pleas- 
ant, and can be employed in soups and salads, especially in made dishes, with the greatest advantage.

CAPERS are the buds of a small trailing shrub, a native of the south of Europe ; those grown on the Rock of Gibraltar are considered the finest in the world. They are very excellent with almost all boiled meats, fish, and salads.

Broom Buds, when pickled, sometimes replace capers.

Nasturtiums are sometimes used in the place of capers, being pickled in the same way, but they-have not the least resemblance; they may be used for salads. The flower of the nasturtium is a very pretty decoration for boiled fish and salads.

BARBERRIES are the fruit of a plant; some centuries since, they appear to have been often used, but more modern importations have superseded them; they have a very acid flavor, and, if pickled, could enter into the composition of many dishes, and also make a good preserve.

GHERKINs are the young cucumber, or often the fruit of the uncultivated plant; they are almost always pickled, and are an elegant addition to all salads and many entrées.

BEET-Rоoт is a root of a plant which of late years has been extensively cultivated for the purpose of feeding cattle; the color which it possesses is easily extracted, for the purpose of tinting various articles employed in cookery. When boiled, it is a beautiful addition to every salad.

Mushrooms are greatly used in domestic cookery, and are a general favorite; their liquor, when extracted with salt, becomes a condiment known under the name of Ketchup, very extensively employed in cookery ; this may be considered wholesome, as it is known to facilitate the digestion. The largest are the best to produce the ketchup, and the small button ones to use in dishes; they should be nicely trimmed, and put into a jar with lemon-juice to blanch them; should be submitted to very little heat, or their delicacy will be lost, and nothing but a tough substance left to eat.

Truffle is, without doubt, a modern introduction. So late as the year 1720 , in the regency of Louis $X V$., we first hear of its being used, when Dubois, the minister, introduced it at his dinners, the Duke of Orleans gave it to his mistresses, and the gallants of the day brought it into notice in their nocturnal repasts.

It was at this period that the science of cookery made such rapid improvement in France, and when extravagance in the preparation of the repasts was carried to the highest point; this was a period when "non ex pane solo vivit homo." Many of the sauces first discovered at that period have descended to the present day-such as Soubise and Bechamel, named after the noblemen who first partook of them. Then it was that the celebrated "Dinde aux Truffes" (turkey with truffles) first made its appearance. Since this period, the truffle has been considered the "to kalon" of condiments, and as the indispensable ornament of all banquets; or, as Brillat Savarin says, "La truffe est le diamant de la cuisine."

Within the last half century, the place (Perigord) which produces the finest of these roots, produced the first of diplomatists-namely, Talleyrand, who was at the same time a great gourmet. The locality may have had its influence; certain it is he would never give a decision upon any momentous question until after dinner, and having partaken of his favorite dish, Truffe aux Vin de Champagne. They are a species of fungus, tube, or root, generally found within a few inches of the surface of the earth, and under oak trees; known also as the subterranean "puff-ball," tuba cibarium. The best are supposed to come from "Peri- 
gord,' the next from Angoulême-these are black; the white come from Piedmont, and the Carolinas; they ought to be eaten fresh. On the European continent, they are found by means of dogs and pigs, trained on purpose.

They should not be subjected to too long boiling, as the aroma is very volatile.

$\mathrm{B}_{\mathrm{AY}}$ is the leaf of a species of laurel, -but in using it care must be taken that the leaf of the bay-laurel, or cherrylaurel, (prunus laurocerasus,) is not used for it, as it contains prussic acid. This is a common evergreen in the southern States. The bay or sweet bay, (laurus nobilis,) an evergreen of the South of Europe, may be used to great advantage in cookery, as it imparts an agreeable and aromatic flavor, differing very widely from any spices that can be used for the same purposes. It is cultivated extensively in gardens, and should be used according to the directions given in the receipts.

SAFFRON was formerly-that is, in the fourteenth century-much used in cookery in Europe. It has bulbous roots, like the onion and flowers; the fibres grow from the botton of the bulb, having roots with coats or skin one over the other, and bears naked flowers without stem. At present it is only used in the kitchen for the purpose of coloring. In Portugal, Spain, and Turkey, it is used at the present day to a great extent.

Angelica, a condiment much used in the confectionery department of the kitchen, is the stalk of a plant like celery, which grows in gardens, and is also found wild. When gathered, it is dried, preserved, and candied. Rouen, in France, is very celebrated for it.

VANilla is used chiefly to impart flavor to various articles in confectionery; it has also a very agreeable smell, which has caused it to be used in scenting different perfumes, and thus diminished its use in the kitchen. It is the produce of a climbing plant like ivy, having a large leaf and black flower, which leaves a pod containing black peas; the form of the pod is like a scimitar, from which it takes its name, being in Spanish vargairita.

When covered with tin-foil, and kept in a moist place, it will become covered with flakes of silver. It denotes the best quality, and is fit for use ; it will do to be infused several times.

SUGAR is the concentrated juice of a plant which grows in hot climates, and is also to be obtained from other trees and vegetables, especially from beet-root. It is used very extensively in cookery, imparting a mellow flavor to all dishes; it is of an antiseptic nature, and ought to be employed more abundantly in preserv ing animal substances than it is. This condiment was unknown to the ancients, who employed honey in place of it.

ORANGE is the fruit of a tree originally a native of China, but now grown extensively in all warm climates. The juice of this fruit is a sweet acid, differing greatly in flavor from the lemon; its peel is of use in cookery. The Seville and Malta oranges are a variety. of this fruit.

ORANGE-FLOWER, a distillation from the flower, is used a great deal in cookery.

VERJUICE is the juice of a grape of that name, and on the European continent was used to a great extent, until the introduction of the lemon. The grape is gathered unripe, pressed, allowed to ferment, and then put into large vats to mature; the older it is, the better the flavor. It enters into the composition of several of the sauces in use at the present day; if pure, it may be preferable for several dishes to vinegar.

OLIVE is a fruit not much used in cookery; it grows on a low tree with long narrow leaves, rough underneath; the fruit contains a long and small kernel; the fruit, when pressed, yields the 
oil known as olive oil. This is a most valuable article in cookery; it ought to be tasteless and inodorous, but when long exposed to the air becomes rank and unfit for use.

ARRow-ROOT is the powder of the root of a tree growing in very warm and moist climates, of about two to three feet in height, known in botany as the maranta arundinarea. In some countries, the flour of this root is mixed with that of Indian corn. and makes excellent bread. It is produced in the same way as the starch from potatoes.

TAPIOCA is also a starch, produced from the roots of a Brazilian plant called Cassava, a very elegant but poisonous plant, with beautiful white and pink flowers. It is now in general use, as it is found, when mixed with a little wine or spirits, to be an excellent stimulant to an invalid, without possessing any heat. ing qualities. It should be well washed in water, and boiled until it becomes transparent, when it should be well stirred, and whatever is required to be mixed with it should be added. It will, when mixed with other flour, make very good bread.

Salsop is another root of a plant called orchis morio, a native of the East, where it is used as an article of food to a great extent; it is reduced to powder, and readily dissolves in boiling water. It is a light, pleasant drink, and, to persons of a feverish constitution, is exceedingly wholesome.

SAGO is another of these starches, similar to Tapioca, made from the pith of the palm-tree, a native of the Indies. It is prepared precisely in the same way as Tapioca.

Soy is obtained from the seed or fruit of an Indian plant, called dotichos soja, or soya; it is made like ketchup; it enters greatly into use in the variety of sauces that are made for the table, being of a very strong; sweet-bitter taste; it should be used in moderation in cookery.

Chetney is a production of the East Indies, which, of late years, has come considerably into use; it is made by mixing together a variety of fruits, and allowing them to ferment until they become acid: some spices are then mixed with them-and it is bottled for use ; the older it is, the better it becomes. That made in the Vale of Cashmere is considered the best.

CURRY-POwDER is another of those Indian condiments which have latterly come much into use, and it is only to be regretted that it is not cheaper, to allow it to be more generally used, as it is one of those stimulating condiments which would be invaluable to the poor; its use would prevent the habit of taking other stimulants which produce intoxication.

Lemon is the fruit of a tree, a native of the South of Europe and the Indies; it is a most valuable production, not only for cookery, but for the health of the human race. Its juice, when diluted with water, and sweetened, makes a most refreshing and wholesome drink. The peel contains an essential oil, which imparts a strong pungent flavor to those dishes to which it is added. The true flavor of this fruit can only be had when fresh and ripe. In the time of Henry VIII., this fruit was a great rarity, and was partaken of at dessert with a quantity of sugar mixed with it.

The LrME is a species of lemon, the juice of which is more acid.

The Citron is also another species; the juice is sweeter.

Vinegar derives its name from the French vin aigre, or sour wine, and is known in chemistry as acetic acid. This antiseptic liquor can be produced from all herbs, plants, regetables, and fruits, which produce sugar, and even that singular production of nature known as honey, and of which the juices will go 
into fermentation. These juices, when first expressed, are called wine, or syrup; they undergo a fermentation known as the vinous, after which, when exposed to a certain degree of heat, they enter into the acetous fermentation, and produce that acid pungent liquor known as vinegar. Of late years, it has also been produced by the distillation of wood, more particularly oak, and known as pyroligneous acid, which is intensely acid, and highly stimulating. When employed in cookery, it should be used judiciously, and with great care. The best vinegar is that which contains the least vegetable matter, such as coloring, \&c., and which has the strongest acid taste, without being hot.

The different flavored vinegars may be made by infusion, without the aid of heat, of the different substances from which the flavor is required.

\section{CHAPTER XXVII.}

\section{RUDIMENTS OF COOKERY.}

A FEW simple general directions, on a subject of so much importance to family and individual comfort as cooking, will be found useful, even though there be some repetition in subsequent instructions.

The kitchen should be furnished with long and round tables; a dresser with shelves.serving as a plate rack; closets or cupboards, common chairs, benches, wooden stools and sinks. Towel rollers, plate warmers, a weighing machine, speaking tubes, a dumb waiter, a refrigerator, hot water dishes, and dish covers of wire gauze, are also among the indispensables. The fireplace should have plenty of light.

The chief object of cookery is to make the meat tender and easy of digestion, without depriving it of its juices. Solid food, especially plain roast or boiled meat, requires to be well adapted to the digestive powers ; for it is trying to many constitutions, and health is often injured by dining upon improperly cooked "plain joints." Butcher's meat, when brought from market, should be examined, trimmed, and wiped clean; sprinkled in summer with a little salt and pepper, and hung up. Mutton and veal must be taken down and wiped each day. Venison is hung some time before cooking, and should be wiped dry and dredged with ginger or pepper, to prevent the fly from settling on it; the dredging wiped off before cooking. We will take up the simple culinary processes in turn.

Boiling.-This is seldom done properly ; the error most common among American cooks is that of boiling meat fast over a strong fire, which renders it hard and nearly tasteless; whereas, a gentle simmering for nearly double the time, with half the quantity of fuel and water, will better accomplish the purpose.

For instance: the most common, and almost universal dish throughout France, is a large piece of plainly boiled fresh beef, from which the soup-or "potage," as it is there called-has been partly made, and which is separately served up as "bouilli" accompanied by strong gravy and minced vegetables, or stewed cabbage. Now this, as constantly dressed in the French mode, is ever delicate both in fibre and flavor; while, in the English manner of boiling it, it is always hard and insipid. The reason of which, as explained by that celebrated cook, Carême, who superintended the kitchen of His Majesty George IV., is this :- "The meat, instead of being put down to boil, as in the English method, is in France put in the pot with the usual quantity of cold water, and placed at the corner of the fireplace, where, slowly becoming hot, the heat gradually swells the muscular fibres of the beef, dissolving the gelatinous substances therein contained, and disengaging that portion 
which chemists term 'osmazome,' and which imparts savor to the flesh-thus both rendering the meat tender and palatable, and the broth relishing and nutritive; while, on the contrary, if the pot be inconsiderately put upon too quick a fire, the boiling is precipitated, the fibre coagulates and hardens, the osmazome is hindered from disengaging itself, and thus nothing is obtained but a piece of tough meat, and a broth without taste or succulence."

Meat, salted or fresh, should be put first into cold water just sufficient to cover it; to be warmed gradually and renewed, as it wastes away, with hot water. The scum must be skimmed off as fast as it rises. As soon as the water boils, the kettle should be taken off the fire, but placed near enough to keep it simmering, which it will do with little heat. The allowance of time in the summer is eighteen or twenty minutes to each pound; in winter rather more. The time of reckoning must begin when the water boils. Salt meats require very slow simmering, and must have more time. Allowance must also be made for age.

The nice look of every thing boiled depends on skimming the pot well. If let alone, the scum boils down and sticks to the meat, giving it a coarse and uncleanly appearance. A little cold water and salt thrown in will help to bring up the scum. It is not necessary to wrap the meat in a cloth. Less heat is required to keep liquids boiling in copper and iron, than in tin saucepans.

Dried or salted fish and meats require soaking in cold water before boiling.

Meat and poultry will lose their flavor and firmness, if left in the water after they are done; as will also fish, which will break to pieces.

The water in which fish, meat, or poultry has been boiled, should be saved; this pot-liquor, as it is called, may be made into soup.
Remember to heat the water containing the meat, very slowly; a joint of ten pounds' weight should not boil in less than forty minutes. The more slowly it simmers afterwards, the tenderer, plumper, and whiter it will be.

An experienced cook placed a thermometer in water in that state which cooks call gently simmering; the heat was $212^{\circ}$ viz., equal to the strongest boiling. Two mutton chops were covered with cold water; one was boiled on a gallop, the other simmering gently for three-quarters of an hour; the flavor of the simmered chop was decidedly superior; the liquor was more savory, and when cold had more fat on its surface. The chop boiled quickly was hard and insipid.

Take care that the covers of your boiling pots fit closely, and only remove them for skimming. If exposed to the smoke the meat will have a bad taste.

The less water the better, so that tho joint is covered; a quart of water is generally allowed to a pound. As the water is diminished by evaporation, fresh boiling water may be poured into tho saucepan.

Beef and mutton may be a little underdone; but lamb, pork, and veal, are uneatable unless thoroughly boiled.

A fishdrainer, four skewers, or a sour plate, wrong side upwards, laid on the bottom of the boiling pot, will prevent the lower side of the meat from being overdone, or burnt by sticking.

The broth of every joint may be made into savory soup.

Meat loses in cooking from one-fifth to one-third of its weight. Boiling is the more economical way of cooking, if the broth be used. One pound a day for each person in the family is a liberal allowance for dinner.

The sauces sent to table with boiled meat must be served in boats, not poured over it.

Roasting.-The first preparation must 
be to see that the spit is cleaned with sand and water. It should be wiped while hot, as soon as meat is drawn from it, and scoured always before use. Joint the meat properly for the convenience of the carrer, and balance it evenly on the spit, that its motion may be regular and the fire operate equally on each part of it. Use balance skewers, if necessary, and do not run the spit through the prime part of the joint. Take care that the meat has been kept long enough to be tender. A leg of mutton should never be spitted, as the spit lets out the gravy, and leaves an unsightly perforation just as you are cutting into the pope's eye.

Roast meat by the radiant heat of a clear, glowing fire, otherwise it is baked. The "old English roast beef" was not done in an oven.

The Paschal lamb among the Jews, was roasted whole; among eastern nations sheep are thus brought to table; but it is not done among us, except when pigs, with apples in their mouths, grace the board at country taverns, at some Fourth of July celebration.

Put the joint to roast when the fire is newly made up, that the heat may increase by degrees; proportion the fire to the size of the joint, making it a few inches longer. What would roast a sirloin will burn up a smaller piece. Clear the fire of smoky coals before the joint is put down. The distance from the fire is mentioned by Webster as from nine to thirteen inches; by Kitchiner, from ten to fourteen. It should be put at the furthest point at first. The fat parts should have paper skewered or tied over them, or be covered with a coarse paste of flour and water, fastened on by paper and a string, and taken off in time to brown and froth the meat. Fat meat requires more roasting than lean. In roasting by a string, pass a skewer through each end of the joint, and turn it bottom upwards, that the gravy may flow to each part.
If the joint is thicker at one end than the other, place the spit slanting, the thickest part nearest the fire. Be careful not to put meat too near the fire at first; and the larger the joint the farther off it must be kept; if once scorched, the outside will become hard, and the fire will not penetrate the interior.

Mrs. Hale says : In stirring the fire, be careful to remove the dripping-pan, else dust and ashes may fall in. On no account let the fire get dull and low, as a strong heat is requisite to brown the meat.

A thin joint requires a brisk fire; a large joint, a strong, sound and even fire. When steam rises from the meat, it is done.

Large joints should be put at a moderate distance from the fire, and gradually brought nearer; else the meat will be overdone half way through the joint, and be nearly raw at the bone.

Such meat as is not very fat should have paper placed over it, to prevent it from being scorched.

Do not sprinkle the meat with salt when first put down, as the salt draws out the gravy.

Old meats require more cooking than young. The longer the meat has been killed, the less time it requires to roast it. Very fat meat requires more time than usual.

The general rule is to allow fifteen minutes to a pound for roasting with a good fire, and ten or twenty minutes over, as the family like it well done or not.

Kitchiner says: "Slow roasting is equally important with slow boiling." There should be a meat-screen to guard it from currents of air. Reckon the time to the moment the roast will be eaten-allowing for the previous courses. If a joint stands after roasting, it will not be "done to a turn." Its size and age, the weather, the fire, the degree of attention, and many other circumstances, have to be 
considered, and make this process very difficult to perform in perfection.

When the joint is half done, stir up the fire thoroughly. When it is saturated with heat, steam will begin to rise.

To Baste roast meat, is to moisten it while before the fire, to prevent the surface from being scorched. Meat should be basted at first every ten minutes, and afterwards more frequently. Beef and mutton may be basted with their own dripping. Veal and poultry require an addition of butter, suet, or dripping. Some meats are basted with flavored material: claret, sweet-herbs and butter being used for mutton and lamb. For a pig, cream and melted butter are recommended. For veal and other white meats, a basting is made of the yolk of eggs, grated biscuits, and the juice of an orange. Butter, clarified suet, and drippings, are used commonly for roast meat.

To Brown Meat.-Make the fire brisker when the joint is half done, or add a glaze to improve the appearance.

To Froth it.-Dredge the whole joint lightly with flour, when sufficiently roasted, and immediately baste it with butter or dripping. Stir the fire, and in three minutes take down and dish the meat. Use butter to froth poultry or lamb. The process plumps up the skin, and improves its appearance. Do not use too much flour; the meat should have a light varnish of froth, not a covering of paste.

Dredgings are of flour simply, or flour and grated bread; or sweet herbs dried, powdered and mixed with flour; or powdered sugar mixed with pounded cinnamon and grated breal; or dried and pounded lemon peel mixed with flour; or fennel, or coriander seed, cinnamon and sugar, powdered and mixed with grated bread.

Dripping is the fat which drops from roasting meat, and is used in frying, basting, or making plain pastry. When a joint of beef is roasted, pour the melted fat into a basin of cold, clean water; the heavy particles sink to the buttom; the melted fat floats, and when cold, forms a clear cake of fat, fit for use several days in cool weather. Dripping that has been used for frying (unless fish or pork has been fried in it) may be clarified in this way, for use a second time. Beef suet should be slowly melted, and passed through a sieve into cold water; when caked it may be put into a jar, and kept in a cool place.

The Skimmings of Soups and Gravies - called "top-pot,"-is used by French cooks with flour, to thicken gravies and soups, and by some for frying.

French Cooking.-French cooks subject their meats to a gradual long continued action of heat, making the fibre perfectly tender. The English and Americans cook them too fast; the French would pronounce such food only fit for barbarians. Another peculiarity of French cooking is the variety of flavors imparted to meat. A great number of dishes are prepared from a few original substances, and the addition of a particular sauce gives a name to the dish. In our cooking, the flavor peculiar to each meat is preserved, and no condiment is suffered to overpower it. French dishes are more sightly; light and elegant dishes, not ponderous masses, tempt the appetite. The French cooking is also more economical. Nothing is wasted; and many ingredients are brought into use which we would cast aside as useless.

Braising is an important part of the art of cooking, and, like the sauté, belongs entirely to the French school from which it takes its name, braise being the remains of wood burnt in the oven, or live charcoal: this plan of cookery requires the action of the fire under and over the braising pan, which is air-tight, in order that the aromatic flavor arising 
from its contents may be imbibed by the meat or poultry, and give it that succulence so much esteemed by epicures. Its origin is stated to be owing to a gastronomic society which was formerly in existence in Paris, whose object was to benefit and improve the art of cookery, and who offered a reward of a silver gridiron to any culinary artist $*$ who would discover a new mode of dressing a turkey. Although a gridiron was, no doubt, intended to be used, yet a young artist named La Gacque, warmed by the offer, directed his imagination to quite a different mode, and used the pan instead of the gridiron, and thus composed the braise, which was unanimously approved of by that scientific, gastronomic. and epicurean body, who awarded him the prize. The chief art in braising is to do it slowly, taking care that the ingredients are well proportioned. Put the meat you would braise into a stewpan, and cover it with thick slices of fat bacon; then lay round it six or eight onions, a fagot of sweet herbs, some celery, and, if to be brown, some thick slices of carrots, and trimmings of any fresh meatbones you have, with a pint and a half of water, or the same quantity of stock, according to what the meat is, and add seasoning. Lay on a sheet of paper, cover the pan close, and set it over a slow stove; it will require two or three hours, as its size and quality may direct. Then strain the gravy; keep the meat quite hot; take the fat off by plunging the basin into cold water, which will cause the fat to coagulate; and boil it as quickly as you can till it thickens. If, however, you wish the gravy to adhere to the meat, it must be still further thickened; then with a brush kept for the purpose do over the meat, and if that has been larded, put it into the oven for a few minutes. 'This is called "glazing," and is much in use for madedishes.
A Braising Pan is a sort of obleng kettle, with a bordered lid on which coals or ashes may be put. The lid should closely fit the pan.

Glazing is strong gravy, boiled quickly till thick; and it is put on meat with a brush. Hams, tongues, and stewed beef may be glazed.

Jerked Beef is made by cutting it into thin slices, dipping them into salt or sea water, and drying them in the sun. This is done in the West Indies.

Broiling.-The gridiron must be kept perfectly clean and bright; always wiped when hot after use, and rubbed just before the meat is laid on it. with clean fat or suet. The bars must be thoroughly hot, but not so as to burn. They should be concave, and terminate in a trough to catch the gravy. The fire must be clear and glowing, especially for a beefsteak, for which the passage in Macbeth is a good direction. "When 'tis done, then 'twere well it were done quickly."' Mutton chops do not require quite so brisk a fire. If the article to be broiled is thick, it must be warmed through more gradually. The broiling must be done as quickly as possible, to avoid drying up the juices and hardening the fibre of meat. Beef and mutton require a hotter fire than pork or veal, poultry, or fish. Let the steak be uniformly thick; beefsteaks not more than half an inch thick; mutton a little thicker.

Do not sprinkle the steak with salt before, or while broiling. It draws out the gravy. Pepper may be used. Do not turn the steaks with a fork, but with a small tongs.

A charcoal fire is convenient for broiling. When the chops are placed on the gridiron, set the dish to heat in which they are to be served, and send to table as hot as possible.

Soyer says: "As regards the thickness of the meat to be broiled, that depends in a great measure on the intensity 
of the fire, but the quicker the better, and put them away till wanted. This is and also the sooner it is eaten after taken nuch better than grating bread as it is from the fire the better it will be. I needed, or using oatmeal, \&c.

have latterly, in broiling rump-steaks, added that which, by a great many, is considered an improvement; it is, on turning them the last time, to dredge them out of a dredger with fine holes, in which have been placed four table-spoonfuls of fine biscuit or rusk-powder, one tablespoonful of salt, one teaspoonful of pepper, a saltspoonful of either eschalot-powder or mushroom-powder, or finely pulverized salts of celery, well mixed together, and the steak to be placed in a very hot dish, with a little mushroom-catsup, and a small piece of butter, and served immediately."

Frying.-Though a convenient mode of cooking, this is seldom done well. It is to roast quickly, or scorch meat, \&c., in fat in an iron pan. If little fat is used, it is called sautéing. The pan should be flat and thick-bottomed, with perpendicular sides. Dripping, oil, butter, fresh lard, or clarified suet - perfectly clean, fresh and sweet, must, when melted, cover the bottom of the pan; when this is heated sufficiently, the meat or fish must be put in and turned frequently, from one side to the other. When nearly done, they may be browned by being pressed firmly against the bottom of the pan.

Be careful that the fat or oil is fresh, clean, and free from salt, else what you fry in it will be of bad color and flavor; salt will prevent it from browning.

Fat or oil, to be used again, should be strained through a sieve before it is set aside.

Fat becomes richer from having meat fried in it, and may be used repeatedly; but the fat that has been used for fish cannot be used again for meat.

To prepare crumbs for frying, dry thoroughly in a warm oven, or before the fire, any waste pieces of bread; then pound them in a mortar and sift them,

When you wish fried things to look as well as possible, do them twice over with egg and crumbs.

If eggs be very dear, a little flour and water may be substituted for them in preparłng fish to fry.

In frying use a slice to lift the articles in and out of the pan, and drain them.

To make batter for frying: melt two ounces of butter in a little warm water, and pour it upon half-a-pound of flour; stir it and add water enough to form a batter, thick enough to adhere to whatever is put into it; but it should run freely: add some salt and the beaten whites of two eggs.

A small shallow frying-pan, or sautépan, as it is called, is very useful to fry articles to be stewed: this method differs from common frying, as it only requires butter enough to keep the article from sticking to the pan and burning.

The fire for frying should be free from smoky coals, sharp, and even. Charcoal makes the best frying fire.

The fat should be carefully drained from all fried articles; indeed, they should be so dry as scarcely to soil a cloth. Fish is best drained by wrapping it in soft whitey-brown paper, by which it will so dry as not to soil the napkin upon which it is served.

The great secret in frying is to know when the fat is of a proper heat. Put nothing in till it has done hissing, and is still. Or throw a small bit of bread into the pan; if it fries crisp, the fat is ready; if it burns, too hot. Whatever is fried in fat not heated enough, will be pale and sodden. Fish should be crisp and dry in a few minutes after it is out of the pan; when it is not so, dry it on a cloth before the fire.

Baking.-Baking is a very common and convenient mode of dressing a dinner 
in small families, and much used in these white; put in three pints of boiling United States. The southern cook will water, a handful of salt, a few sprigs of often stand in the midst of a circle of thyme, a bay leaf, an onion stuck with portable ovens, placed on the brick floor cloves, and a bundle of parsley and green of the kitchen, which is usually at some onions. Boil the whole gently till the distance from the dwelling-house.

Legs of mutton and pork, fillets of veal, and many other joints, will bake nicely if inclined to be fat; if lean, they will not taste well, but will shrivel up and lose much in weight.

A pig, geese and ducks, a buttock, leg or shin of beef, a ham, oxcheek, and various kinds of fish, make very nice baked dishes. The oven in preparing all kinds of bread and farinaceous food, is indispensable.

Stewing.-All articles to be stewed should first be boiled gently, then skimmed and set aside in an even heat: on this account, charcoal makes the best fire for stewing.

All stews, or meat dressed a second time, should be only simmered, as the meat should only be made hot through.

A stewpan is the most advantageous vèssel in which stews, hashes, soups, or gravies, can be made ; indeed, for all purposes of boiling, a stewpan is preferable to a deep saucepan, as, in the former, the articles are exposed to more even heat than when they are placed one above another in the saucepan, and are likely to be broken in stirring.

The best stewpans are made of copper or iron; they should be kept covered as much as possible, unless you wish to reduce the gravy.

Be careful not to fry in a stewpan; or if so, with great care, and sufficient butter to save the tinning from melting.

Most of the directions for making soups and gravies apply also to this branch of cookery.

Poëling.-Put into a stewpan with two pounds of veal, a pound of beef suet, one of butter, one of very fat bacon, all onion is done, strain it through a sieve, and set it by for use. I öle will keep for a week, and will give relish and whiteness to everything boiled in it. Do not boil any lean of bacon; it will give a red color from the saltpetre. Meat in poëling must be under-done.

Larding.-Have ready larding-pins of different sizes, according to the article to be done; cut slices of bacon into bits of a proper length, quite smooth, and put on a larding-needle to suit it, with which pierce the skin and a very little of the meat, leaving the bacon in, and the two ends of equal length outwards. Lard in rows the size you think fit.

The same effect with regard to flavor, may be produced by raising the skin and laying a slice of fat bacon beneath it.

Doubing consists in passing bacon through meat, while larding is on the surface only.

Glazing is done by brushing melted glaze or jelly over the article, and letting it cool; in some cases it is requisite to cover the articles with two or three coats of glaze, allowing each to cool as it is laid on. The glaze should be of a clear yellow brown, and as thick as good treacle.

If you have not the glaze ready, sift a little sugar over the article to be glazed, and finish in the oven, with a salamander, or red hot shovel.

Blanching makes the article plump and white, and consists in putting it into cold water over the fire, allowing it to boil up, and then plunging it into cold water, where the article should remain until quite cold.

Danger from Copper Saucepans.-The cut up; fry them till the veal becomes saucepans, or stewpans, imperfectly tin- 
ned, is hardly understood. It appears that the acid contained in stews and other made dishes, as lemon-juice, though it does not dissolve copper by being merely boiled in it a few minutes, nevertheless, if allowed to cool and stand in it for some time, will acquire poisonous matter, as verdigris, in the form of a green band, or crust, inside the vessel. It has likewise been proved that weak solutions of common salt, such as are daily made by adding a little salt to boiling vegetables, fish, or meat, act powerfully on copper vessels, although strong solutions or brine would not affect them.

It is, however, in vain to hope that cooks will attend to the nice distinctions by which copper stewpans may be rendered safe ; the general advice given by prudent physicians is, therefore, against their use at all.

The kettles in which the soups are made should be well tinnedd, and kept particularly clean, by being washed in hot water and rubbed dry before they are put away. If they are not kept well tinned, the taste as well as the color of the soup will be liable to be affected by the iron; and if the soup-kettle be made of copper, and the tinning not quite perfect, everything cooked in it will be more or less poisonous, as everything which is sweet, salt, or sour, extracts verdigris from copper.

Boning, besides its other advantages, is particularly recommended, on account of its economy: the bones of turkeys, fowls, hares, \&c., assisting to make gravy, while they are nearly useless when left in the bodies of the animals a a boned fowl also, rendered solid by stuffing, will go much further than when dressed in the common way. Hares should always be boned, for the sake of the improvement in their appearance, and being so much more easily carved. In the hiring of a cook by persons resident in the country, it is therefore essential to ascer- tain whether she is mistress of that art; for as to teaching her by printed cirections, it would be time thrown away, and she can only acquire it by practice; but it may be learned by a few lessons from the poulterer.

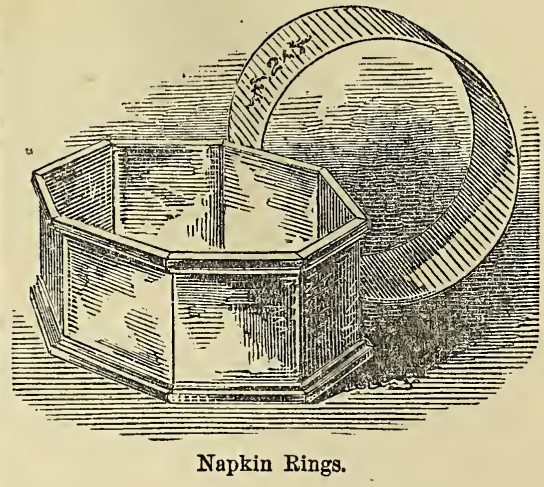

\section{CHAPTER XXVIII.}

\section{HINTS AND MAXIMS.}

If you chance to occupy the important position of a cook, remember that cleanliness is the first, second and third requisite in point of importance, to be observed.

Keep your flour-box, sugar, salt, and spices always covered, that dust and insects may not get in.

Never put onions or cheese on the same dish with anything else, and never cut anything else with the knife you use for them. Keep a particular pitcher for beer or buttermilk, or you may chance to put milk or water into the remains.

Empty into some outside drain the saucepan in which you have boiled vegetables, as soon as you have done with it; and never put by anything eatable in a saucepan. This is a fearfully common practice with southern cooks. Keep your tin and copper vessels as sweet and clean 
as glass or china. The saucepans are of far more consequence than tumblers or teacups. If glasses be dirty, those who drink from them are disgusted; but if saucepans be foul, they may chance to be poisoned. Many have died from this cause. The sort of rust which forms in copper vessels not kept çlean is a deadly poison. If a housekeeper is careful, she will look into all her saucepans at least once a week, to see if they are well cleaned, or want tinning.

Let the dinner be served quite hot, and have the plates as hot as you can handle them, Cold plates spoil the finest joint of meat; and it is very easy to have plates hot. At breakfast and tea carry in the water quite boiling, and then it will not be your fault if the tea is not good. It is a very common fault in cooks, as soon as the teakettle boils, to set it on one side where the water cools a little, so that it is not quite boiling when poured on the tea.

The true economy of housekeeping is simply the art of gathering up all the fragments, so that nothing be lost. I mean fragments of time, as well as materials. Nothing should be thrown away so long as it is possible to make any use of it, however trifling that use may be; and whatever be the size of a family, every member should be employed either in earning or in saving money.

In early childhood, you lay the foundation of poverty or riches in the habits you give your children. Teach them to save everything; not for their own usefor that would make them selfish-but for some use. Teach them to share everything with their playmates; but never allow them to destroy anything.

As far as possible, have bits of bread eaten before they become hard. Spread the rest out to dry to be pounded for puddings, or soaked for "brewis." This is made of dry crusts and bits of bread soaked in hot milk, mashed, salted and buttered like toast.

An ox's gall will set any color in silk, cotton or woollen. The colors of calico which would fade in one washing will be fixed by it. It is worth while to buy cheap fading goods, and set them in this way. Get out all the liquid from the gall, and cork it up in a large phial. One large spoonful in a gallon of warm water is sufficient. It is also excellent for taking out spots from bombazine and stiff goods, which, washed in it, look as well as new. It must be stirred into the water. After washing cloth in this water, you must wash it if you wish to clear it, in warm suds, not putting soap upon it.

Lamps will have a less disagreeable smell if you dip the wick yarn in strong hot vinegar and dry it.

Do not let coffee and tea stand in tin. Keep tinware dry, and scald woodenware often.

Eggs will keep almost any length of time in lime water properly prepared. One pint of coarse salt, and one pint of unslacked lime, to a common pailful of water. If there be too much lime it will eat the eggs from the shells; and if a single egg be cracked it will spoil the whole. They should be covered with lime water, and kept in a cold place. It is a good plan to lay down eggs in the spring and September for the winter.

Few know how to keep the flavor of sweet marjoram, the best of all herbs for broth and stuffing. It should be gathere d in bud or blossom and dried in a tin kitchen at a moderate distance from the fire; when dry it should be rubbed, sifted, and corked up in a bottle.

Bones from which roasting pieces have been cut may be bought in market for a trifle, and a very rich soup made of them, besides skimming the fat for shortening. Bones from the rump are full of marrow, and will yield a pint of good shortening 
without impairing the richness of the a little ground will do well to raise the soup. The best pieces of beef for soup most useful herbs; apothecaries make are the leg and shin; if boiled very long, the sinews add to the richness of the soup.

The. shoulder of veal is the most economical for roasting or boiling; two dinners may be made from it-the shoulder roasted, and the knuckle cut off to be boiled with pork and greens, or made into soup.

Keep an old blanket and sheet for ironing, and on no account suffer any other to be used. Have plenty of holders, and do not put towiels to such service.

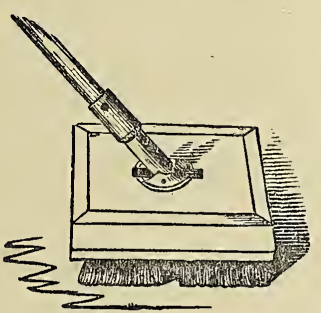

Scrubbing Brush.

Keep a coarse broum for the cellar stairs, wood shed, yard, \&c. Never use a carpet broom for such places.

Never drop knives into hot dishwater. Have a large tin basin to wash them in, just high enough to wash the blades without wetting the handles. Keep your castors covered with blotting paper and green flannel. Keep your salt spoons out of the salt, and clean them often.

Do not wrap knives and forks in woollens, but in strong paper. Steel is injured by lying in woollens. It is a good plan to rub the blades with nice grease, and wrap them up separately, to prevent rust.

Herbs should be kept from the air. Herb tea, to do any good, should be made very strong. Herbs must be gathered while in blossom. Those who have

\section{large profits on them.}

The same pickle used for bacon is good for neats' tongues. Pigs' tongues are nice, prepared in the same way. They are sold in England for reindeers' tongues, as a great luxury.

Buffaloes' tongues should soak a day and a night, and boil six hours.

Molasses used in cooking should be first boiled and skimmed. One or two gallons may be thus prepared at a time. It is a prodigious improvement.

Always have a heavy stone on the top of your pork in pickle. You may keep a bit of fresh meat on this stone, in summer, when you fear it may spoil.

Have plenty of towels in the kitchen, or Biddy will use your white napkins.

Soap dirty clothes, and soak them in water over night. Use hard soap for clothes, and soft for washing floors.

Cutlemon and orange peel, when fresh, into a bottle kept full of brandy. This brandy gives a delicious flavor to pies, cakes, \&c. Roseleaves may be preserved in brandy. Peach leaves steeped in it make an excellent seasoning for custards and puddings.

Grate horseradish when the root is in perfection, put it in bottles, fill it with strong vinegar, and keep it corked tight, for winter use.

Keep a bag for odd pieces of tape and strings, and a bag or box for old buttons.

A little salt sprinkled in starch, while boiling, prevents its sticking; it is also good to stir it with a clean sperm candle.

To separate wax from honeycomb, tie up the comb in a linen or woollen bag; place it in a kettle of cold water, and hang it over the fire. The wax melts and rises to the surface, while all the impurities remain in the bag. A few 
pebbles in the bag will prevent its floating.

Honey may be separated from the comb by placing it in the hot sun or before the fire, with two or three colanders or sieves under it-each finer than the other.

Potatoes boiled and mashed hot, are good in shortcakes and puddings; they save flour and shortening.

To corn meat simply-rub in plenty of salt, and set it in the cellar for a day or two. In summer it is good to corn meat, which will not keep more than a day and a half. If you want to keep it longer, rub in more salt, and secure it from the flies. A little saltpetre rubbed in before you apply the common salt, makes the meat tender; but it is not best to use it in summer.

Legs of mutton are good, aired in the same way as hams-six pounds salt, eight ounces saltpetre, five pints molasses, will make pickle enough for one hundred pounds. Small legs should be kept in pickle twelve or fifteen days; large, four or five weeks; and they should be hung up a day or two before being smoked. Lay them in the oven on crossed sticks, and make a fire at the entrance with cobs or walnut bark, or chips, which impart a sweet taste. .Smoke the smallest pieces forty-eight hours; the large legs four or five days. If hung till thoroughly dry, the mutton may be eaten in thin slices, like hung beef. When legs of meat are put in pickle, the thickest part should be uppermost, as the creature stood while living; also when hung to dry; thus the juices of the meat are kept in. Meat should be turned over once or twice while smoking.

Hams should be well covered in paper bags, and put in a chest or barrel, with layers of charcoal or ashes between. When you take one out to cut for use, put it away in a dark place, well covered.
Let there be a place for every article, and when not in use let every article be in its place.

Keep every utensil ready for immediate use.

The stock pot should never be suffered to be empty, as almost any meats (save salt meats) or fowls make stock; the remnants should never be thrown anywhere but into the stock pot, and should too much stock be already in your possession, boil it down to a glaze: waste is thus avoided.

Keep your meat in a cool, dry place; your fish on ice, and your vegetables on a stone floor free from air.

Cut your soap when it comes in, and let it dry slowly.

Keep your sweet herbs in paper bags, each bag containing only one description of herb. They should be dried in the wind and not in the sun, and when ordered in a receipt should be cautiously used, as a preponderance in any seasoning spoils it.

When oranges or lemons are used for juice, chop down the peel, put them in small pots and tie them down for use.

Apples.-In choosing apples, be guided

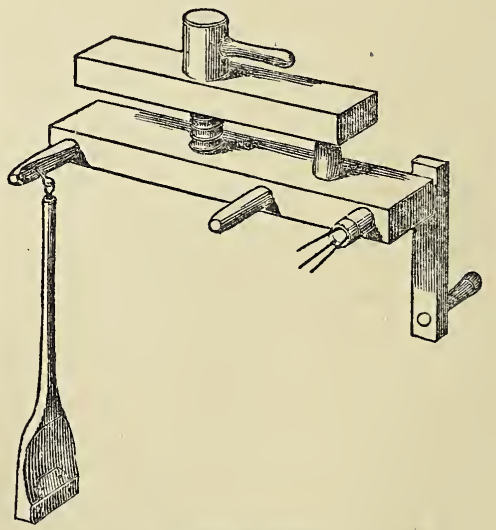

Apple Peeler.

by the weight; the heaviest are the best, 
and those should always be selected which, on being pressed by the thumb, yield with a slight crackling noise. Prefer large apples to small. for waste is saved in peeling and coring.

Apples should be kept on dry straw in a dry place, and pears hung up by the stalk.

Batter for fish, meat, fritters, \&c.Prepare it with fine flour, salt, a little oil, beer, vinegar, or white wine, and the whites of eggs beaten up ; when of a proper thickness, about the size of a nutmeg, it will drop out of the spoon at once. Fry in oil or hog's lard.

CARrots, if young, need only be wiped when boiled-if old, they must be scraped before boiling. Slice them into a dish, and pour over them melted butter.

Cauliflowers. - Cut off the stalks, but leave a little of the green on; boil in spring water with a little salt in it: they must not boil too fast.

Celery.-Very little is sufficient for soups, as the flavor is very predominating. It should be particularly cleanly washed and curled when sent to table. To curl celery, wash well, and take off the outside stalks, cut it to a proper length, split each stalk into three or four divisions with a large needle, then place the head of celery in spring water with the root uppermost, and let it remain for four or five hours-it may then be tastefully arranged on the dish.

GAME may often be made fit for eating when it seems spoiled, by cleaning it and washing with vinegar and water. Birds that are not likely to keep, should be drawn, cropped, and picked, then washed in two or three waters, and rubbed with salt; have in readiness a large saucepan of boiling water, and plunge them into it one by one, drawing them up and down by the legs, so that the water may pass through them. Let them stay for five or six minutes, then hang them up in a cold place; when they are completely drained, well salt and pepper the insides, and thoroughly wash them before roasting.

Gravies.-The skirts of beef and the kidney will make quite as good gravy as any other meat, if prepared in the same manner. The kidney of an $o x$, or the milt, makes excellent gravy, cut to pieces and prepared as other meat, and so with the shank end of mutton that has been dressed, if much gravy is not required. The shank bones of mutton add greatly to the richness of gravies, but they should be first well soaked and scoured clean. The taste of gravies is improved by tarragon, but it should be sparingly used, immediately before serving.

LARD should be carefully melted in a jar put in a kettle of water and boiled, and run into bladders that have been strictly cleaned; the bladders should not be too large, as the lard will becorne rank if the air gets to it. While melting it, put in a sprig of rosemary.

Mustard mixed smooth with new milk, and a little cream added, will keep; it is very soft, and by no means bitter.

SAGo should soak for an hour in water previous to using, to take off the earthy taste.

SUET may be kept for a twelvemonth, thus: choose the firmest and most free from skin or veins, remove all traces of these, put the suet in a saucepan at some distance from the fire, and let it melt gradually; when melted, pour it into a pan of cold spring water; when hard, wipe it dry, fold it in white paper, put it into a linen bag, and keep it in a dry, cool place; when used, it must be scrap ed, and will make an excellent crust, either with or without butter.

Tongue, which has been dried, should be soaked in water three or four hours. One that has not been dried will require but little soaking; put it in cold water, and boil gently till tender. 
Raisin wine may be substituted for sherry, for sweets generally.

Copper vessels, when the tinning is worn off, must never be used, or the poisoning of those who partake of whatever may have been cooked in them is inevitable. They should be 'sent to be re-tinned immediately they require it.

Keep tapes and jelly bags clean, or when again used they will impart an unpleasant flavor.

All soups should be moderately thin and bright.

Meats, such as beef, mutton, and venison, must rather be underdone than overdone, excepting veal and pork, which require to be well done.

Fish should be quite done, but not overdone.

Pastry must be carefully baked; it should be sent to table a pale gold color.

Onions should be kept on ropes in a dry place-a specked one should be removed or it will contaminate the others.

Cold water cracks hot iron infallibly.

Pudding towels should be carefully washed, and kept clean in a dry place. Put a clean round towel on the roller quite as often as necessary.

Be very particular in not letting your stocks and sauces pass over two days without boiling them up, and be careful to stir the thick soups and sauces all the time they are on the fire, and change all your cold meats into fresh clean dishes every morning, wiping down the dressers and shelves, and if allowed larding cloths see that they are clean. Keep your larder door shut, free from dust and damp; do not have your baked paste in the larder, but in your kitchen cupboard, and then see to your game, wiping, and peppering and gingering your venison, arranging the game which requires to be dressed first, and see that all the blood which may have dropped from the game or venison is cleaned from the dresser: and flooring. Then see to the vegetables, removing all stale and what is not wanted, giving it to the poor, either dressed in some way or uncooked; do not be overstocked, but always keep a little reserve. Be sure to look well, every morning to your pickled pork and hams, keep and rub them well, and turn them, marking those to be used first. Your fish must be looked to and well cleaned and washed, and if intended for that day's dinner, kept in water until required; if not, keep it on the marble or stones; your doors should always be shut.

Olean hands, always clean hands.

A dirty kitchen is a disgrace to every one connected with it.

- With these few hints we wind up our remarks, merely adding that many of the receipts given, which are on too large a scale for a small family, may have their proportions equally reduced, and an excellent dish will be the result. In some instances also, the more expensive ingredients may be left out without destroying the integrity of the receipt, discretion and judgment being alone required in these cases.

In conclusion, the mistress of the household will understand that the wellbeing of her establishment depends upon her surveillance; and though her too frequent presence in the kitchen would be

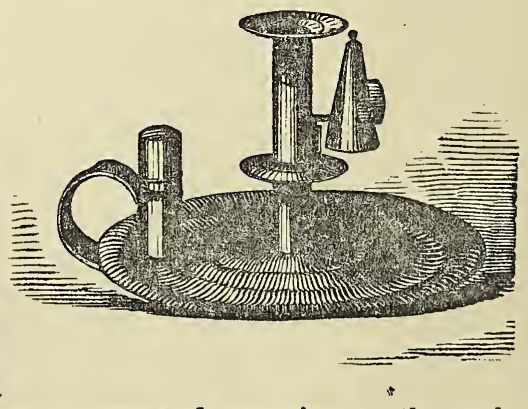

unnecessary and annoying to the cook 
yet she should not be deterred from visiting it by any false delicacy, or deference to an absurd custom which makes it vulgar for a lady to visit her cook in her own domains. If the cook is thrifty and clean, she will be glad to receive the praise to which she is fairly entitled; if dirty and careless, it is very essential that the lady should be acquainted with the fact in order to remedy it by a change.

\section{CHAPTER XXIX.}

ARTICLES IN SEASON FOR EACH MONTH.

\section{JANUARY.}

Fish.-Eels, flounders, haddocks, lampreys, oysters, whitings, clams, muscles, striped bass, salt mackerel, smoked salmon, sardines, anchovies, fish pickled and soused.

Meats.-Beef, mutton, fish, pork, ham, venison, veal, sausages, \&c.

Poultry and game.-Capons, fowls, ducks, geese, Scotch grouse prairie fowls, young rabbits, partridges, pheasants, pigeons, wild birds, turkeys, woodcock, snipe, quails, ducks-canvas back, redhead, broad bill, teal-bear's meat, jugged .hare.

Tegetables.-Winter spinach, turnips, potatoes, sweet potatoes, rice, celery, cabbage, parsnips, carrots, dried white beans, beets, dried herbs, garlic, onions, shalots, leeks, mint, mustard, parsley, sage, rosemary, salsify, thyme, \&c.

Fruits.-Apples, oranges. dried figs, imported grapes, almonds, raisins, dates, filberts, prunes, hard nuts, Brazil and Madeira-nuts, black-walnuts, hickorynuts, peccan-nuts, butternuts, chestnuts.

\section{FEBR UARY。}

Fish.-Codfish, eels, flounders, had- docks, lampreys, oysters, smelts, whitings' clams, muscles, striped bass, smoked salmon, salt mackerel, sardines, anchovies, pickled and soused fish.

Meats.-Beef mutton, fresh pork, sausages, venison, ham.

Poultry and Game.-Fowls, capons, ducks, geese. Scotch grouse, prairie fowls, partridges, rabbits, pheasants, pigeons. turkeys, woodcock, reed birds, quails, snipe, ducks - canvas back, red head, teal, broad bill,-hare, bear's meat.

Vegetables.-Turnips, potatoes, sweet potatoes, winter spinach, rice, celery, cabbage, parsnips, carrots, white beans, beets, garlic, onions, shalots, mint, leeks, mustard, parsley, sage, salsify, thyme, dried herbs.

Fruits.-Apples, oranges, figs, imported grapes, almonds, raisins, filberts, dates, prunes, hazel-nuts, Brazil and Madeiranuts, black walnuts, hickory nuts, peccan-nuts, butternuts, chestnuts.

\section{MARCH.}

Fish.-Codfish, eels, haddocks, flounders, oysters, clams, whitings, striped bass, perch, smelts, lampreys. salt mackerel, smoked salmon, sardines, anchovies, pickled and soused fish.

Meats.-Beef, mutton, pork, ham.

Poultry and Game.-Fowls, capons, tame ducks, geese, Scotch grouse, prairie hens, rabbits, partridges, pheasants, pigeons, turkeys, reed birds, woodcock, snipe, quails, hare, bear's meat.

Vegetables.-Spinach. lettuces, cresses: turnips, potatoes, sweet potatoes, rice, radishes, celery, cabbage, parsnips, carrots, white beans, beets, garlic, onions, shalots, leeks, salsify, parsley, sage, thyme, dried herbs.

Fruits.-Apples, oranges, figs, almonds, raisins, filberts, prunes, hazel-nuts. Brazil and Madeira-nuts, hickory-nuts, peccannuts, chestnuts, dates. 
APRIL.

Fish.-Brook trout, codfish, halibut, shad, striped bass, eels, lobsters, oysters, perch, roach, smelts, clams, shrimps, cray, small fish.

Meats.-Beef, mutton, veal, ham.

Poultry and Game.-Spring chickens, fowls, capon, turkeys, ducks, Scotch grouse, prairie hens, partridges, pheasants, pigeons, quails, woodcock, snipe, bear's meat.

Vegetables.-Lettuces, spinach, turnips, cresses, potatoes, rice, radishes, parsnips, carrots, white beans, beets, garlic, onions, shalots, leeks, mint, mustard, parsley, sage, salsify, thyme, dried herbs, Jerusalem artichokes.

Fruits.-Apples, oranges, figs, almonds, raisins, prunes, dates, \&c.

MAY.

Fish.-Terrapin, fresh salmon, turtle, trout, codfish, halibut, perch, shad, carp, striped bass, eels, English soles, soft crabs, lobsters, salmon, roach, smelts, shrimps, cray fish, prunes, salt and smoked fish.

Meats.-Beef, mutton, veal, ham.

Poultry and Game.-Spring chickens, fowls, capons, Scotch grouse, prairie hens, bear's meat.

Vegetables.-Asparagus, lettuces, cresses, mushrooms, spinach, turnips, potatoes, radishes, rice, parsnips, carrots, white beans, onions, mint, mustard, parsley, sage, thyme, salsify, Jerusalem artichokes, dry herbs.

Fruits.-Apples, oranges, figs, almonds, raisins, prunes, dates.

\section{JUNE.}

Fish.-Terrapin, fresh salmon, turtle, trout, codfish, halibut, perch, pickerel, fresh mackerel, shad, carp, sea-bass, striped bass, eels, fresh herring, king fish, English soles, soft crabs, lobsters, salmon-trout, sturgeon.
Meat.-Beef, mutton, lamb, veal, ham, salt pork.

Poultry and Game.-Spring chickens, fowls, capons, bear's meat, grouse, prairie fowl.

Vegetables. - Asparagus, green peas, Lima beans, white beans, mushrooms, lettuces, cresses, spinach, celery, potatoes, radishes, rice, carrots, onions, garlic, mint, mustard, parsley, sage, thyme, salsify, Jerusalem artichokes, dry herbs.

Fruits.-Strawberries, cherries, currants, oranges, figs, almonds, raisins, prunes, dates.

\section{JULY.}

Fish.-Terrapin, turtle, carp, cod, cray fish, eels, flounders, fresh salmon, herring, lobsters, fresh mackerel, perch, pickerel, salmon-trout, trout, sea-bass, striped bass, halibut, English soles, blue fish, king fish, soft crabs, sturgeon.

Meats.-Beef, lamb, mutton, salt pork.

Poultry and Game.-Chickens, fowls, green geese, pigeons, plovers.

Vegetables. - Artichokes, asparagus, string beans, Lima and white beans, peas of some kinds, carrots, celery, chervil, cucumbers, endive, herbs of all kinds, lettuces, mint, mushrooms, potatoes, purslane, cresses, radishes, salads of all sorts, salsify, spinach, turnips, green corn, tomatoes, succory, squashes, eggplant, cold-slaw.

Fruits. - Apricots, currants, strawberries, raspberries, blackberries, gooseberries, cherries, nectarines, peaches, hothouse grapes, melons of various kinds, oranges, almonds, raisins, prunes, figs, fresh figs, pine apples, damsons, plums.

\section{August.}

Fish.-Terrapin, turtle, perch, codfish, pickerel, carp, eels, fresh salmon, salmontrout, brook-trout, lobster, fresh mackerel, striped bass, sea-bass, English soles, soft crabs, halibut, cray-fish, blue-fish, 
king-fish, sturgeon, smelts, roach, blackbass.

Meats.-Beef, mutton, lamb, veal, salt pork, ham.

Poultry and Game.-Chickens, fowls, ducks, green geese, plovers, bear's meat.

Vegetables.-Artichokes, string-beans, lima and white beans, carrots, celery, chervil, cucumbers, endive, herbs of all kinds, lettuces, mint, potatoes, purslane, radishes, cresses, salads, oyster-plant, spinach, turnips, green corn, tomatoes, succory, squashes, cold slaw, egg plant.

Fruits. - Raspberries, blackberries, peaches, nectarines, green grapes, plums, gooseberries, apricots, harvest apples, mulberries, melons of various kinds, hothouse grapes, pears of different sorts, summer pippins, green gages.

\section{September.}

Fish. - Terrapin, turtle, perch, cod, pickerel, eels, fresh salmon, salmon-trout, trout, lobster, striped luass, sea-bass, black bass, English soles, soft crabs, halibut, cray-fish, blue-fish, king-fish, smelts, sturgeon, roach.

Meats.-Beef, mutton, lamb, veal, ham, salt beef and pork.

Poultry and Game.-Chickens, fowls, ducks, green geese, meadow-larks, bear's meat.

Vegetables. - Artichokes, white and lima beans, carrots, celery, chervil, cucumbers, herbs of all kinds, lettuces. mint, potatoes, purslane, radishes, salads, oyster plant, spinach, turnips, beets, green corn, tomatoes, succory, squashes, cold slaw, egg plant.

Fruits.-Green grapes, magnum bonum plum, Palmer and Columbia grapes, egg plums, gooseberries, nectarines, peaches, apples, grapes of different varieties, pears of all kinds.

\section{OCTOBER.}

Fish.-Terrapin, salmon, striped bass, sea-bass, blue-fish, salmon-trout, lobsters, codfish, halibut, black-fish, pickerel, carp, eels, soft crabs, perch, trout, oysters.

Meats.-Beef, mutton, pork, veal, venison, lamb.

Poultry and Game.-Meadow-larks, chickens, capons, ducks, turkeys, partridges, wild pigeon, English snipe, grouse or prairie-hens, pheasants, quails, wild ducks, hares, rabbits, bear's meat.

Vegetables. - Artichokes, cauliflower, broccoli, succory, carrots, celery, leeks, onions, parsnips, potatoes, spinach, turnips, egg-plant, lettuce, tomatoes, carrots, cabbage, rice, cauliflower, artichokes, celery, leeks, onions, parsnips, sweet potatoes. potatoes, radishes, salads, savoys, winter spinach, tomatoes, turnips, succory, beets, oyster-plant, egg-plant, dry herbs.

Fruits.-Late peaches, apples, filberts. almonds, hazel-nuts, grapes, quinces, walnuts, hickory-nuts, chestnuts, chincopins, peccan-nuts.

\section{November.}

Fish.-Eels, oysters, clams, codfish, haddock, lampreys, whitings, muscles, striped bass, salt mackerel, smoked salmon, sardines, anchovies, \&c.

Meats. - Beef, mutton, lamb, pork, venison.

Poultry and Game.-Chickens, fowls, capons, ducks, geese, Scotch grouse, prairie-hens, hares, rabbits, partridges, pheasants, pigeons, turkeys, reed-birds, woodcock, snipe, quails, canvas-back, red-head, broadbill, teal-ducks, meadowlarks, bear's meat.

Vegetables.-Celery, cauliflower, cabbage, radishes, winter-spinach, turnips, potatoes, sweet potatoes, rice, parsnips, carrots, dry white beans, beets, dried herbs, garlic, onions, shalots, leeks, mint, mustard, parsley, sage, thyme, salsify.

Fruits.-Late peaches, apples, oranges. 
almonds, raisins, filberts, prunes, figs, / rie-hens, hares, rabbits, partridges, hazelnuts, Brazil and Madeira nuts, dates, pheasants, pigeons, reed-birds, woodcock, walnuts, hickory-nuts and chestnuts. $\quad$ snipe, quails, ducks, canvas back, redhead, broadbill, teal,-bear's meat.

\section{December。}

Fish.-Oysters, clams, eels, haddock, lampreys, whitings, muscles, stripedbass, salt mackerel, smoked salmon, sardines, anchovies, \&c.

Meats. - Beef, mutton, pork, veal, venison.

Poultry and Game.-Fowls, turkeys,

Vegetables.-Cauliflower, celery, cabbage, winter spinach, turnips, potatoes, sweet potatoes, rice, parsnips, carrots, dry beans, beets, dried herbs, garlic, onions, shalots, leeks, mint, mustard, parsley, sage, thyme, salsify.

Fruits.-Apples, oranges, bananas, almonds, raisins, filberts, prunes, figs, hazelnuts, Brazil and Madeira nuts, dates, ranons, ducks, geese, Scotch grouse, prai- walnuts, hickory-nuts, chestnuts. 
PART SECOND.

$\mathbb{R} \mathrm{E} \mathbb{E} \mathbb{P} \mathrm{S}$ 



\section{RECEIPTS.}

\section{S OUPS.}

There is no dish, perhaps, that comes soup utensil is technically terrned the to table which gives such general satis- stock-pot.

faction as well prepared soup; let the When fat remains on any soup, a teaappetite be vigorous or delicate, an ex- $\mid$ cupful of flour and water mixed quite cellent soup will always prove grateful smooth, and boiled in it, will make it to it; and as this is beyond contradiction, it should be the province of the cook to be always in a position to produce it at a short notice.

There should always be plenty of dried herbs in the store closet, these may be purchased chiefly in quantities at the beginning of the autumn, of any marketgardener. Herbs may be very well kept, as indeed they are usually, in paper bags; they should be all labelled. When time is an important object, the necessity for this is obvious-they are always to be had when wanted, and the bag should be immediately replaced after using.

There should be a saucepan, or a kettle of iron well tinned, kept for soup only; the lid should fit tightly; one will be found to answer all purposes, being especially useful as a "stock-pot." The inexperienced reader will understand by the term "stock-pot," that soups being of two kinds. brown and white, have different foundations, that of brown being always beef, and that of white, veal; there are many ingredients in each, and it is the various articles which, when put together, are called "stock," hence the rise as scum. when it may be taken off.

If richness or greater consistency be wanted, a good lump of butter mixed with flour, and boiled in the soup, will give either of these qualities.

Long boiling is necessary to give the full flavor of the ingredients, therefore time should be allowed for soups and gravies. Skim frequently and simmer slowly; but do not let the broth cool until it is completely made.

Be sparing in the use of pepper, salt, and spices.

If onions are too strong, boil a turnip with them, and it will render them mild.

Do not keep either soups or gravies in any ressel of tin or copper; and in stirring soup, do it always with a wooden spoon.

Arrow-root, or the mere farina or flour of the potato, is far better for the thickening of soups than wheaten flour.

The basis of all well-made soups, is composed of what English cooks call "Stock," or broth, made from all sorts of meat, bones and the remains of poultry or game; all of which may be put to- 
gether and stewed down in the "Stockpot;" the contents of which are, by the French, termed Consommé. This is chiefly used for the preparation of brown or gravy soups : that intended for white soups being rather differently compounded, though made in nearly the same manner.

To the medley of ingredients, add carrots cut in thin slices, herbs: onions, pepper, and salt; when it has stewed slowly for a short time, pour in the boiling water in proportion to the quantity of meat and soup required; then stew it until it is of a rich consistency, take it from the fire, let it cool, remove the pot.

If required the following day, care should be taken that the deposit or sediment is removed, as also the fat previous to warming; if kept long the pans must be changed; there is as much danger in red glazed earthenware as in metal pans; the latter should never be employed to keep gravies in, if possible. Wherever greater richness is required, it may be obtained by the addition of the jelly of cow-heel, or a lump of butter and flour.

Remember, soup is richer and better for being made the previous day, or even two or three days previously to its being required, if it be warmed each day; to be really good it must be well stewed.

Seasonings for Soups.-Spices should be put whole into soups; allspice is one of the best, though it is not so highly esteemed as it deserves. Seville orangejuice has a finer and milder acid than lemon-juice; but both should be used with caution. Sweet herbs, for soups and broths, consist of knotted marjoram, thyme, and parsley,--a sprig of each tied together. The older and drier onions are, the stronger their flavor; in dry seasons, also, they are very strong: the quantity should be proportioned accordingly. Although celery may be generally obtained for soup throughout the year, it may be useful to know, that leave out the pepper. dried celery-seed is an excellent substitute. It is so strongly flavored, that a drachm of whole seed will enrich half a gallon of soup as much as two heads of celery. Mushrooms are much used, and when they cannot be obtained, fresh mushroom ketchup will answer the purpose, but it should be used very sparingly, as nothing is more difficult to remove than the over-flavoring of ketchup. A piece of butter, in proportion to the liquid, mixed with flour, and added to the soup, when boiling, will enrich and thicken it. The finer flavoring articles, as ketchup, spices, wines, juice, \&c., should not be added till the soup is nearly done. A good proportion of wine is a gill to three pints of soup; this is as much as can be used without the vinous flavor predominating, which is never the case in well made soups. Wine should be added late in the making, as it evaporates very quickly in boiling. Be cautious of over-seasoning soups with pepper, salt, spices, or herbs, for it is a fault that can seldom be remedied; any provision over-salted is spoiled. A teaspoonful of sugar is a good addition in flavoring soups. Vermicelli is added to soups in the proportion of a quarter of a pound for a tureen of soup for eight persons; it should be broken, then blanched in cold water, and is better if stewed in broth before it is put into the soup.

\section{MEAT SOUPS.}

\section{1.- STOCK FOR WHITE SOUPS.}

This is a soup. the foundation of which is veal, - the knuckle, the scrag, or calf's head being the best meat for the purpose, -an old fowl, a little ham, or bacon, mutton, sheep's head, \&c., nearly the same ingredients as for brown soups, save that there must not be much beef, and the proportion of harn and bacon smaller in the latter than former, and when made for white sauce, care must be taken to 


\section{2.-BROWN STOCK.}

Put ten pounds of shin of beef, six pounds of knuckle of veal, and some sheep's-trotters or a cow-heel, in a closely covered stewpan, with very little water to draw out the gravy very gently, and allow it nearly to dry in until it becomes brown. Then pour in sufficient boiling water to entirely cover the meat, and let it boil slowly, skimming it frequently; seasoning it with whole peppers and salt, roots, herbs, and vegetables of any kind. That being done, let it boil gently five or six hours, pour the broth from off the meat, and let it stand during the night to cool. The following morning take off the scum and fat, and put it away in a stone jar for use

Or:-Put into a stewpan a piece of beef, a piece of veal, an old fowl, some slices of ham or bacon, and all the trimmings of meat that can be obtained; add to these materials, where such things are abundant, partridge, grouse, or other game, which may not be sufficiently young and tender for the spit. Put a little water to it, just enough to cover half the meat, and stew very gently over a slow fire or steam apparatus. When the top piece is done through, cover the meat with boiling water or broth; season with spices and vegetables; stew all together for eight or ten hours in an uncovered stewpan; skim off the fat, and strain the liquor through a fine sieve.

Brown stock may be made from an oxcheek, ox-tail, brisket, flank, or shin of beef; which will, either together or separately, make a strong jelly if stewed down with a piece of ham or lean bacon, in the proportion of one-half pound to every seven pounds of meat; but the shin of beef alone will afford a stronger and better flavor.

This stock may also be reduced to a glaze by boiling the skimmed liquor as fast as possible in a newly tinned stew- pan, until it becomes of the desired consistence and of a good brown color ; taking care at the same time to prevent it from burning.

\section{3.-BROWN GRAVY SOUP.}

The meat used for making this soup should be quite fresh, and of the common gravy beef; if the shin be used. break the bones, as the marrow will add to its richness.

Take seven to eight pounds of the meat, cut a small portion of it into thick pieces and put it with three or four large sliced onions into a close stewpan, with a little butter, until fried to a fine brown. That done, add a shank of ham, just cover the meat with cold water-say a couple of quarts-and let it simmer by the fire for at least three hours; during which time it should not be allowed to boil, but, when coming to that point, check it with cold water, and skim it. As the pores of the meat will then be opened, and the gravy drawn, throw in three quarts of warm water; along with a handful or one quarter ounce each of black pepper, allspice, and salt, as well as a bundle of sweet herbs, a few cloves, a couple of shalots, two or three middlingsized carrots and turnips (the latter an hour afterwards), together with a couple of heads of celery, and allow the whole to boil slowly until the meat is done to rags, and the vegetables become tender. Then strain it off, and let it stand during the night. Remove the fat on the following day, set any portion of it on the fire an hour before dinner, and, when thoroughly heated, season it with mushroom or walnut ketchup, and send it up with a plate of toasted bread, cut into small square pieces, without crust.

You will thus form a gallon to five or six quarts of strong soup, according to the quality of the meat; but as it is a winter soup, it will bear keeping, and, if served more than once, the flaror 
may be varied by the addition of different sorts of ingredients, as for instance -first, plain gravy; secondly. gravy and vegetables; and thirdly, vermicelli : or if only one-half or a third part of the quantity be wanted, it may be prepared in the same manner by proportionally reducing the materials. It may be also flavored with red wine.

\section{-4.-SOYER'S STOCK FOR ALL KINDS OF SOUP.}

Procure a knuckle of veal about six pounds in weight, which cut into pieces about the size of an egg, as also half a pound of lean ham or bacon; then rub a quarter of a pound of butter upon the bottom of the stewpan (capable of holding about two gallons), into which put the meat and bacon. with half a pint of water, two ounces of salt, three middlesized onions, with two cloves in each, one turnip, a carrot. half a leek, and half a head of celery: put the cover upon the stewpan, which place over a sharp fire, occasionally stirring round its contents with a wooden spoon, until the bottom of the stewpan is covered with a white thickish glaze, which will lightly adhere to the spoon; fill up the stewpan with cold water, and when upon the point of boiling, draw it to the corner of the fire, where it must gently simmer for three hours, carefully skimming off every particle of grease and scum; pass your stock through a fine hair sieve, and it is ready for use when required.

The above will make a delicious broth for all kinds of clear soups, and of course for thick soups or purées; by boiling it rather faster abou five minutes before passing, you will be better enabled to take off every particle of grease from the surface. In making a stock of beef proceed as above, but allow double the time to simmer; mutton or lamb, if any trimmings, might also be used; if beef, use seven pounds; if mutton, eight; or lamb, seven; of course bones are all included; with care, this broth would be quite clear. To give a little color, as required for all clear soups, use a little brown gravy or browning, but never attempt to brown it by letting it color at the bottom of the stewpan, for in that case you would destroy the greater part of the osmazome.

\section{5.-ANOTHER WAY, MORE ECONOMICAL.}

Instead of cutting up the knuckle of veal so sniall, cut it in four or fire pieces only, and leave the bacon in one piece; then, when the broth is passed, take out the veal, which is very excellent served with a little of the broth for gravy, and the bacon with a few greens upon another dish. This is as I always eat it myself; but some persons may probably prefer a littie parsley and butter sauce or piquant sauce, served with it. Should any of the veal be left until cold, it might be cut into thin slices, and gradually warmed in either of the before-mentioned sauces. Should you make your stock from the leg or shin of beef, stew it double the time, preserve the vegetables boiled in the stock, and serve with beef, or serve the beef with some nice sharp sauce over; the remainder, if cold, may also be hashed in the ordinary way. If of mutton, and you have used the scrags of the neck, the breast, head, or the chump of the loin, keep them in as large pieces as possible; and, when done, serve with a few mashed turnips, and caper sauce, separately; if any remaining until cold, mince it. Lamb would be seldom used for stock, being much too expensive; but in case of an abundance, which may sometimes happen in the country, proceed the same as for mutton.

\section{6. - SOYER'S BROWN GRAVIES.}

Rub an ounce of butter over the bottom of a stewpan capable of holding about three quarts; have ready peeled 
four onions, cut them into thick slices, with which cover the bottom of the stewpan; over these lay about three pounds of beef from the leg or shin, cut into thin slices, with the bone chopped very small; add a small carrot, a turnip cut in slices, and a couple of cloves; set the stewpan upon a gentle fire for ten minutes, shaking it round occasionally to prevent burning; after which let it go upon a slow fire for upwards of an hour, until the bottom is covered with a blackish glaze, but not burnt; when properly done, and ready for filling up, you will perceive the fat that runs from the meat quite clear; fill up the stewpan with cold water, add a teaspoonful of salt; and when upon the point of boiling, set it on a corner of the fire, where let it simmer gently about an hour, skimming off all the fat and scum - which may rise to the surface; when done pass it through a fine sieve into a basin, and put by to use for the following purposes :-For every kind of roast meat, poultry, or game especially; also, to give a good color to soups and sauces. This gravy will keep several days, by boiling it every other day. Although beef is the most proper meat for the above purpose, it may be made of veal, mutton, lamb, or even with fresh pork, rabbits, or poultry.

\section{7.-SOYER'S GLAZE}

Is an almost indispensable article in a cuisine bourgeoise, and should be kept by all persons in the middle classes of life, the advantage being that it will keep for months together, is very simple to make, and is always useful in cookery, however humble; in fact, with it you can dress a very good dinner with very little trouble.

Make a stock omitting the salt, which, when done, pass through a cloth into a basin; then fill the stewpan up a second time with hot water, and let it boil four hours longer to obtain all the succulence from the meat, then pass it through a cloth the same as the first; then pour both stocks in a large stewpan together, set it over the fire, and let it boil as fast as possible, leaving a large spoon in, to stir occasionally and prevent its boiling over; reduced to about three pints, pour it into a smaller stewpan, set again to boil at the corner, skimming well if required; when reduced to a quart, place it quite over the firc, well stirring with a wooden spoon until forming a thickish glaze (which will adhere to the spoon) of a fine yellowish-brown color: pour it into a basin, or, if for keeping any time, into a long bladder, from which cut a slice and use where directed.

\section{8.-BROWNING FOR SOUPS.}

Take two ounces of coarse brown sugar, and pour upon it some thyme water; place it on the fire till it becomes burnt. Or, take two ounces of powdered lump-sugar, and half an ounce of fresh butter; put them together in a fryingpan, and keep on the fire till the mixture becomes a chocolate brown, then add three table-spoonfuls of port wine, and two wine-glassfuls of elder wine, six shalots, half a dram of mace, a dram of allspice, a dram of black pepper, half an ounce of salt, two ounces of ketchup, and an ounce of fresh lemon juice. Boil all together, let the liquor stand to settle, pour off the clear liquor, bottle, and cork tight. Or, take some sugar, white or brown, place it in an iron spoon, heat until liquid, and then drop into half a pint of water; repeat until sufficiently brown.

\section{9.-PORTABLE SOUP.}

There are many advantages connected with this soup, which will present themselves to the lady housekeeper, its constant readiness for use, its forming an excellent stock for gravies, sauces, or 
soups; a few minutss will suffice to make a basin of soup from it.

Take three pounds of beef, a shin of beef, the- bones of which break, a cowheel and two small knuckles of veal, put them in a stewpan and add as much water as will barely cover them, put in three onions and seasoning to taste, stew the meat to ribbons, strain and then put it in the coldest place you can command, when thoroughly cold take off the fat and boil it fast in a stewpan without the lid on a quick fire, let it boil and keep it stirred for at least eight hours, pour it into a pan and let it stand twenty-four hours. then take your largest lip-basin and turn the soup into it, boil sufficient water in the stewpan to reach as high outside the basin which is placed in it as the soup is inside, but do not let any bubble into the basin, keep the water boiling until the water is reduced to a good consistency; it will be then done; it should then be poured into small jelly pots, or in saucers, so as to form cakes when cold, and is best preserved in tin canisters put in dry cool places.

This soup may receive various flavors of herbs or any thing else, by boiling the herbs or other ingredients, and straining the simples noted through water, making it boil. and then melting the soup in it.

\section{0.-WHITE STOCK.}

Take scrag or knuckle of veal, ox-heel, or calf's head, together with an old fowl and the trimmings of any white poultry or game which can be had, and lean ham in the proportion of one pound to every fourteen pounds of meat. Cut it all into pieces (add three or four large unroasted onions and heads of celery, with a few blades of mace; but neither carrots, pepper, nor spice of any kind but mace); put it into a stock-pot with just water enough to cover it : let it boil, and add three onions and a few blades of mace; let it boil for five hours, and it is then fit for use.

$$
\text { 11.-WHITE PORTABLE SOUP. }
$$

Procure as fine a leg of veal as can be obtained, bone it, remove the whole of the skin and fat, chop in pieces two dozen fowls' feet, wash them well, put them into a large iron kettle with three gallons of water, stew until the meat is tender enough to separate, cover down close and stew for eight hours, take a tea-cup and fill it with the soup; set it where it can quickly cool. If when cold it is hard enough to cut with a knife, strain through a sieve and remove all the fat, pour into cups the clear jelly, put them into a stewpan with boiling water until they are like glue; let them cool, and when nearly cold run a ring round them and turn them on to a piece of new flannel ; it will draw all the moisture out of them; turn them in seven hours and continue until they are quite hard. Put them in tin canisters in a dry place.

When any is required, cut a piece about the size of a walnut, pour a pint of boiling water upon it, stir until the soup is dissolved, season with salt, it will make a basin of strong broth ; if for soup steep some vermicelli in water; boil it; then to one cake of the soup pour one pint of water. If two quarts or four pints of soup are required, take four cakes of the soup, and when melted set it over the fire and simmer, pour it into a soup tureen, add thin slices of bread very lightly toasted, and upon them the vermicelli; season to palate.

\section{2.-TRANSPARENT SOUP.}

Cut the meat from a leg of veal in slices as thin as possible, break the bone as small as possible, put the meat into a very large jar and the bones at the top, with a bunch of sweet herbs, a quarter of an ounce of mace, four ounces of blanched garden almonds beat fine; pour upon it a 
gallon of boiling water, let it simmer over a slow fire twelve hours, - all night is best; turn it into a double-bottomed tin saucepan, simmer until reduced to two quarts, remove the scum as it rises, strain it and let it stand two hours, pour into a saucepan, taking care not to let any of the sediment accompany it.

Steep two ounces of vermicelli in water, boil it and put it in the soup before serving up.

\section{3.-SOUP ITALIENNE.}

Cut the meat from a knuckle of veal, break up the bones and make a broth of them, cut half a pound of ham in slices and lay them at the bottom of a stewpan; upon them the meat from the knuckle of veal, with the slices of four carrots, four turnips, a dozen peppercorns, two blades of mace, a large onion, and a head of. celery; cover down close; stew till the gravy is drawn out and the roots are quite tender, pour over them the broth made from the bone of the knuckle until they are covered, add six spoonfuls of rice, stew four hours, work the soup through a sieve, add vermicelli before serving.

\section{4.-POT-AU-FEU.}

This is by far the most wholesome of all soups. Take three pounds of good rump of beef, of any part free from bone and not too fat; put it into an earthen fire-proof pot, with three quarts of water, one large carrot, two turnips, two leeks, a head of celery, and one burnt onion; season, and let the soup boil slowly, skimming it from time to time, for at least five hours; then strain it through a fine sieve, and pour it over thin slices of bread to serve. The meat and regetables make a dish which is afterwards served. Thus cooked, the beef becomes tender and juicy, and is excellent cold.

\section{5-OCHRA SOUP.}

Put on six pounds of fresh beef-allowing a little less than a quart of water to each pound; after it has boiled an hour add two quarts of ochras minced fine as possible. Afterwards a dozen of ripe tomatoes pared and cut up, with two turnips, a few Lima beans, herbs, and other seasoning. The ochras should be dissolved. Strain and serve it with toasted bread cut into slices, put in after it comes out of the pot.

The soup may be made in winter of dried ochras.

\section{6.-PEPPER POT.}

Put four cow's feet and four pounds of tripe to boil with water to cover them and a little salt. When simmered to pieces, take them out, and skim and strain the liquor. Cut up the tripe, put it in the pot, and pour the liquor over it. Add sliced onions and potatoes and herbs, also small dumplings made with flour and butter, and season with pepper and salt. A little butter rolled in flour is an improvement. When done, serve in a tureen.

\section{7.-WHITE SOUP.}

Take a knuckle of veal, separated into three or four pieces, a slice of ham as lean as possible, a few onions, thyme, cloves, and mace, stew twelve or fourteen hours; an old fowl will make it much richer if added. This soup must be made the day before it is required; when removed from the fire, after being sufficiently stewed, let it cool ; and then remove the fat; add to it four ounces of pounded blanched almonds, let it boil slowly, thicken it with half a pint of cream and an egg; it should boil slowly for half an hour, and then be served.

\section{8.-GRAVY SOUP.}

Take a leg of beef and well wash and soak it, break the bone and put it into a 
saucepan with a gallon of water, a large bunch of sweet herbs, two large onions sliced and fried to a nice brown, taking great care they are not burnt, two blades of mace, three cloves, twenty berries of allspice, and forty of black pepper, stew till the soup is as rich as you wish it to be, then take out the meat. When it is cold take off the fat, heat the soup with vermicelli, and the nicest part of a head of celery boiled and cut to pieces, cayenne, and a little salt; carrot may be added with turnip cut into small pieces and boiled with spinach and endive, or the herbs without the vermicelli, or vermicelli only ; add also a large spoonful of soy and one of mushroom ketchup; a French roll should be made hot and put into the soup.

\section{9.-JENNY LIND'S SOUP.}

Soyer says: This is the soup invented and often partaken of by the celebrated cantatrice of the name it bears, who partook of it every day when on a visit to the talented authoress, Mrs. Anna Maria Hall, who was kind enough to forward it to me for this edition.

Make about three quarts of stock, which strain through a fine sieve into a middle-size stewpan; set it to boil; add to it three ounces of sago; boil gently twenty minutes; skim; just previous to serving break four fresh eggs, and place the yolk, entirely free from the white, into a basin, beat them weil with a spoon; add to it a gill of cream; take the pan from the fire, pour in the yolks, stir quickly for one minute, serve immediately; do not let it boil, or it will curdle, and would not be fit to be partaken of. The stock being previously seasoned, it only requires the addition of half a teaspoonful of sugar, a little more salt, pepper, nutmeg; also thyme, parsley, and bay-leaf will agreeably vary the flavor without interfering with the quality.

\section{0.-HARICO SOUP.}

Cut some mutton cutlets from the neck; trim and fry them of a light brown; stew in brown gravy soup till tender. Have ready some carrots, turnips, celery, and onions; fry them in butter for some time, and clear the soup from the fat; then add the vegetables, color it, and thicken it with butter and flour ; season, and add to it a little port wine and ketchup. If the gravy be ready, the soup will require no more time to prepare than may be necessary to render the chops and vegetables tender, and is an excellent family dish. If wanted to be made more highly flavored. put in a little curry-powder.

\section{1.-FRENCH POT-AU-FEU.}

Out of this earthen pot comes the fa. vorite soup and bouilli, which has been everlastingly famed as having been the support of several generations of all classes of society in France; from the opulent to the poorest individuals, all pay tribute to its excellence and worth. In fact, this soup and bouilli is to the French what the roast beef and plumpudding are on a Sunday to the English. No dinner in France is served without soup, and no good soup is supposed to be made without the pot-au-feu. The following is the receipt:-

Put in the pot-au-feu six pounds of beef, four quarts of water, set near the fire, skim; when nearly boiling, add a spoonful and a half of salt, half a pound of liver, two carrots, four turnips, eight young or two old leeks, one head of celery, two onions and one burnt, with a clove in each, and a piece of parsnip; skim again, and let simmer four or five hours, adding a little cold water now and then; take off part of the fat; put slices of bread into the tureen, lay half the vegetables over, and half the broth, and serve the meat separately with the vegetables around. The remainder of the 
broth from the pot-au-feu may be used for any kind of soup instead of the stock. The best part of the beef for the potau-feu is the mouse-buttock, tops of the ribs, clod and stickings.

\section{2.-WHITE SOUP.}

Take a large scrag or a knuckle of veal, and one-half pound of lean ham; some blades of mace and a piece of ungrated nutmeg, sliced onions, and heads of celery, with a little salt and an equal quantity of loaf-sugar. Break the bones, and stew all gently with one gallon to six quarts of water-according to the weight of the meat-until it is done to rags and the soup becomes sufficiently strong: skim it, and strain it through a hair sieve ; or, if allowed to stand during the night, pour it into an earthen vessel, and next morning take off the fat. When preparing it for table, add to the liquor from one-quarter to one-half pound of sweet almonds, blanched and finely pounded; boil a short time and strain again; then put in one-half pint to a pint of good thick cream, and the yolk of an egg; but when the cream and egg are put in, be careful not to let the soup boil, or it will curdle. The safest way to avoid this is to mix the cream and egg in the tureen and pour the soup upon it. This in French cookery is called "Liaison."

\section{3-RICE AND MEAT SOUP.}

Put a pound of rice and a little pepper and broth herbs into two quarts of water ; cover them close, and simmer very softly ; put in a little cinnamon, two pounds of good ox-cheek, and boil the whole till the juices are incorporated into - the liquor.

\section{4.-OX-TAIL SOUP.}

Two ox-tails, if properly stewed, with a couple of pounds of gravy beef and a bone of ham, will make an excellent soup. Cut the tails into joints, and boil very gently for several hours in a sufficient quantity of water, with the beef and ham, carrots, turnips, and celery, two or three onions, a piece of crust of bread, a bunch of sw eet herbs, a clove or two, and some peppercorns. Take out the tails when tender, and let the beef, \&c., boil four hours longer, then strain the liquor, and remove the fat in the same manner as for clear gravy soup. If made without ham bones, or other flavoring ingredients, it will require the addition of a little ketchup, or some of the prepared sauces, and a glass of wine, with a moderate quantity of cayenne. Add the tails and some pieces of carrot and turnip cut into fancy shapes.

When thickened ox-tail soup is preferred, proceed in the same manner as above, and thicken the broth with brown roux.

\section{5.-SOUP A LA FRANCAISE.}

Place in the stewpan six pounds of beef, add a few small veal bones, or one about a pound weight, add a couple of fowls' heads, and a small piece of calf's liver; zover with four quarts of water; when it boils remove the scum, add three or four leeks, a couple of turnips, a head of celery, a burnt onion, a large carrot, salt, and simmer slowly seven hours; let every particle of scum be removed, serve with. sippets of bread in the soup.

\section{6.-TO MAKE FAMILY SOUP.}

Take a shin or leg of beef, that has been newly killed; the fore-leg is best, as there is the most meat on it. Have it cut into three pieces, and wash it well. To each pound allow somewhat less than a quart of water; to ten pounds of the leg, two gallons of water. Put it into a large pot, and add half a tablespoonful of salt. Hang it over a good fire, full eight hours before you dine. When it has come to a hard boil, and the 
scum has risen (which it will do as soon as it has boiled), skim it well; then set it on hot coals in the corner, and keep it simmering steadily, so as to continue a regular heat.

About three hours afterwards, put in a couple of heads of celery, four carrots cut small, and as many onions sliced and fried, with either a very small head of cabbage cut into little pieces, or a large one whole, if to be eaten with the meat, or, if you have any objection to cabbage, substitute a larger proportion of the other vegetables, or else tomatoes instead. Put also in a bunch of herbs, tied up in a thin muslin rag to prevent its floating on the surface. It will require at least eight hour's' cooking; remembering to put in the vegetables three hours after the meat, and the turnips only half an hour before it is done. If you wish to send any portion of the meat to table, take the best part of it out of the soup about two hours before dinner; let the remainder be left in the pot till you send up the soup, as by that time it will be boiled to rags, and have transferred all its flavor to the liquid, and must be strained.

Next day, take what is left of the soup; put it into a pot, and simmer it over hot coals for half an hour; a longer time will weaken the taste. If it has been well made, and kept in a cool place, it will be found better the second day than the first.

If your family is very small. and the leg of beef large, it may furnish soup for several successive days. Half the leg may therefore be sufficient, previously breaking to pieces all the bones with a mallet or kitchen cleaver, which, by causing them to give out their marrow, will greatly enrich the soup.

Or:-When a large quantity of any butcher's meat is brought in for the use of the family, the joints will require trimming: take all the parings, adding a slice or two of bacon, beef or mutton bones, with an old fowl, or a rabbit, if you have one, turnip, and all sorts of vegetables, onions, herbs, a few slices of carrot, ketchup, pepper, salt, \&c. ; put a piece of butter at the bottom of the pan. cover it closely, and put it over a slow fire for a few minutes, shaking the saucepan occasionally. Then pour in boiling water, and let it stew until it is rich; apportioning the water to the quantity of meat. If there be any solid portion of beef, of which the soup is made, let it be taken out previous to the meat being "done to rags," and sent up along with the roots in some of the liquor, thickened and flavored with any piquant sauce: it will form an excellent stew.

These receipts also, are for the standing household dish so well known in France as the pot-au-feu.

Cheap and wholesome potages, in common use among the middling classes in various parts of Europe, are also made in different modes, a few of which are these:-

\section{7.-CUTTAGE SOUP.}

Take two pounds of lean beef, cut into small pieces, with one-fourth of a pound of bacon, two pounds of meally potatoes, three ounces of rice, carrots, turnips, and onions sliced, or leeks and cabbage. Fry the meat, cabbage, and onions in butter or dripping, the latter being the most savory; and put them into a gallon of water, to stew gently over a slow fire for three hours, putting in the carrots at the same time, but the turnips and rice only time enough to allow of their being well done; and mashing the potatoes, which should be then passed through a colander: seasson only with pepper and salt: keep the vessel closely covered. It will make five pints of excellent soup at the cost of about one shilling and eight pence.

28.-SCOTCH KAIL

Is chiefly made of mutton, either fresh 
or salted ; beef is only used when mutton cannot conveniently be had. Three or four pounds of meat should be put into a gallon of cold water, along with a moderate quantity of pearl-barley, with leeks or onions, and allowed to stew unil tender; if salted, put the meat into water over night, changing it once before boiling. Then have ready the hearts of two cabbages cut small, or greens, if cabbages are not in season; put them into the broth, which must be allowed to boil up uncovered until reduced to two quarts. It should only be seasoned with pepper and salt; but will be much improved by the addition of a couple of onions fried in butter ; indeed, both carrots and turnips are also sometimes used, but their addition deprives the soup of the title of "Kail," whieh is derived from the greens which are usually employed.

The meat is served with the soup, and, in like manner as the olla of the Spaniards, or the pot au feu of the French, is the standing household dish among the middle classes of Scotland.

\section{9.-COCK-A-LEEKIE-}

Or as in Scotland called "cocky-leeky" -is there also a very ancient dish, and is recorded to have been a special favorite of James I. It is made thus:-

Stew a large fowl, a marrow-bone, and two or three pounds of beef, with two or three Scotch pints (four to six quarts) of water, and the white ends of two or three dozen of leeks, cut in pieces. Just before serving, add half a pound of prunes, which dish with the soup and the fowl; but not the meat or marrow-bone, which, when put to boil, must be divided, and left uncovered.

Or:- - Put seven pounds of the upper end of a leg of beef, and an old fowl, in a pot, with water enough to cover it, the white parts of two or three dozen of leeks, half-boiled and sliced, and one pound of prunes. Stew till the meat be tender, skimming it well, and, if you choose, the fowl may be disjointed and sent up in the soup. The leeks should be blanched, and as many used as to thicken the soup; but no other seasoning is usually employed than salt and allspice, with a small quantity of mace.

\section{0.-SAGO SOUP.}

Take three pounds of lean beef, a slice of lean ham, and lay them in a stewpan with a lump of butter, draw the gravy gently, add two quarts of water, and a sliced onion which has been browned by frying in fresh butter, add a bunch of sweet herbs, six cloves, a blade of mace, a teaspoonful of allspice, and one of black pepper whole, stew until the soup is rich and brown. then remove the meat and strain the soup clear, put it into a clean stewpan, thicken it to a good consistency with sago.

\section{1.-SHEEP'S HEAD SOUP.}

Have the head carefully cleaned, put it into a stewpan with a little water, and when it is heated through fill up the pot. When it is sufficiently tender, take it up, remove the meat from the bones, and return the bones into the broth, adding onion, sweet herbs, \&c., as before directed. The head and trotters may also be put with some vegetables into an earthen jug, containing half a gallon of water; cover it close up, and bake it; either cut the meat from the bones, when sufficiently tender, and put it in small pieces into the soup, or serve up the head and trotters separately, either whole or with the meat cut off and made into a stew.

In Scotland, the head is usually singed with a red-hot iron to remove the wool, but without burning or otherwise injuring the skin. The head is then soaked during the night, washed, scraped, and split; the brains taken out, and either fried or made into forcemeat balls, and the head stewed in the broth till tender. 


\section{2.-SOYER'S MULLIGATAWNY SOUP.}

Cut up a small knuckle of veal, which put into a stewpan, with a piece of butter, half a pound of lean ham, a carrot, a turnip, three onions, and six apples, add half a pint of water; set the stewpan over a sharp fire, moving the meat round occasionally, let it remain until the bottom of the stewpan is covered with a brownish glaze, then add three tablespoonsful of curry powder, one of curry paste, and half a pound of flour; stir well in, and fill the stewpan with a gallon of water; add a spoonful of salt, the half of one of sugar; when boiling, place it at the corner of the fire, and let it simmer two hours and a half, skimming off all the fat as it rises, then pass it through a tammy into a tureen ; trim some of the pieces of veal, and put it back in the stewpan to boil, and serve with plain boiled rice separate. Ox-tails or pieces of rabbits, chickens, \&c., left from a previous dinner, may be served in it instead of the veal. The veal is exceedingly good to eat. Taste, before serving, if quite palatable.

\section{3.-SCOTCH BROTH.}

Set on the fire four ounces of pearlbarley, with three Scotch pints (or six quarts) of salt water; when it boils skim it, and add what quantity of salt beef or fresh brisket you choose, and a marrowbone or a fowl, with a couple of pounds of either lean beef or mutton, and a good quantity of leeks, cabbages, or savoys; or you may use turnips, onions, and grated carrots. Keep it boiling for at least four or five hours; but if a fowl be used, let it not be put in till just time enough to bring it to table when well done, for it must be served up separately.

Or:-Take the chops from a neck of mutton; cut the remainder up in small pieces, and let it stew the whole day. Take also a breakfast cup of Scotch barley, and boil it in water till it gets dry; then chop fine two large onions and turnips, which put with the barley and chops into a close stewpan, strain the stock into it, let it boil one and a half hours and skim it well, seasoning it only with salt and black pepper. This will make a large tureen of broth, besides preserving the chops for the table.

\section{4.-НОТСН РОТСH.}

Take any quantity of lamb chops, pare off the skin and greater part of the fat, trim the bones, cut the smaller end of the chops into pieces, and lay them along with the chops put in whole in a stewpan in this manner:-A layer of chops at the bottom, covered with every kind of vegetable cut into small pieces-onions, celery, lettuce, carrots, turnips, and green peas; then put on a layer of chops, and so on with the regetables until the whole are added; cover the ingredients with water, and let it stew several hours very gently, until both the meat and regetables become tender, and the soup thick.

Scotch Hotch Potch is made in the same manner, only that both beef and mutton are indiscriminately used, and minced instead of being left in chops.

\section{5.-PEPPER-POT HOTCH POTCH.}

To three quarts of water put vegetables according to the season. In summer, peas, French beans, cauliflowers, lettuce, and spinach ; in winter, beet-root and endive, ${ }^{*}$ carrots, turnips, celery, and onions in both, all cut small; and stew with two pounds of neck of mutton, or a fowl and one pound of pickled pork, in three quarts of water, till quite tender.

On first boiling, skim. Half an hour before serving, add a lobster or crab cleared from the bones. Season with salt and cayenne. A small quantity of rice should be put in with the meat.

* If endive be used, it should, however, be boiled in two or three waters to take off its bitterness. 
Some people choose very small suet dumplings boiled with it. Should any fat rise, skim nicely, and put one-half a cup of water with a little flour.

It may be made of various things, using a due proportion of fish, flesh, fowl, regetables and pulse. In the West Indies it is the universal dish of the colored people; but seasoned so highly with green capsicums and peppers, that it is there called "pepper-pot."

\section{6.-SOUP A LA SAP.}

Divide a pound of beef into thin slices, grate half a pound of potatoes and put them in three quarts of water, add an onion, a pint of grey peas and three ounces of rice; reduce it by boiling to five pints; cut two heads of celery and put them into the stewpan. pour upon them the five pints of soup and pulp the boiled peas into it through a fine tammy or coarse cloth. Stew until it is quite tender, season with pepper and salt, and serve up with fried bread cut in dice.

\section{7.-HESSIAN SOUP.}

Cut into slices three pounds of shin of beef, lay it in a stewpan, put in three onions, five carrots, eight potatoes, a pint and a quarter of split peas, three heads of celery, some whole pepper, salt; pour in by degrees seven quarts of water, stew until reduced to half. If the soup alone be required strain off the vegetables, if not, serve as cooked.

\section{8.-SOUP A LA REINE VICTORIA.}

Take a pound and a half of lean veal, place it in a stewpan with a slice of bacon, which must not be fat, an onion with one clove, a blade of mace, a head of celery, a handful of sweet herbs, four ounces of fresh butter, and some whole white pepper ; set it over a clear fire, move it frequently to prevent burning, or the flavor is ruined. Have some white gravy ready, thicken it, add two quarts to the above ingredients with a few strips of mushrooms; let it boil, and when it reaches that point remove it; skim it clean of all scum or fat. Have ready some vermicelli which has been soaked five minutes in cold water and subsequently stewed in a strong broth; strain on it the soup and serve with blanched chervil leaves in it.

39.-MULLIGATAWNEY SOUP. (ENGLISH.)

A calf's head divided, well cleaned, place with a cow-heel in a well tinned saucepan; boil them till tender, let them cool, cut the meat from the bones in slices, and fry them in butter; stew the bones of the head and heel for some hours; when well stewed, strain, let it get cold and remove the fat. When this is accomplished cut four onions in slices, flour them, fry them in butter until brown, add a tablespoonful and a half of best curry powder obtainable, cayenne pepper, one teaspoonful with a little salt; turmeric powder sufficient to fill a dessert spoon is sometimes added, but the improvement is not manifest to a refined English palate, the curry powder being deemed all that is necessary; add these last ingredients to the soup, boil gently for about an hour and a half, add two dessert spoonfuls of Harvey's sauce; serve.

\section{0.-SPRING SOUP}

May be made of a knuckle of vealallowing a quart of water to each pound -with four calves feet, a little cold ham, or salt and cayenne, simmered slowly for several hours. Add then, two quarts young green peas and a pint of asparagus tops, previously boiled with the juice of spinach and other green herbs or vegetables, and a quarter of a pound of butter rolled in flour.-Boil up together, and serve. 
41.-SUMMER SOUP.

Made of mutton neck or shoulder, simmered as above, in the same proportion of water, with sliced turnips, carrots and onions, a quart of Lima beans, and parsley, sweet marjoram and other herbs; green corn is an excellent addition late in summer, or cauliflower, and a few pickled nasturtions; also small dumplings, mixed with egg and butter; simmer the vegetables till done.

\section{2.-AUTUMN SOUP.}

Take six pounds of lean, cut up, a quart of water to a gallon, add the hock of a ham, boil and skim it, and put in two quarts ochras, cut in small slices, an onion sliced, two quarts ripe tomatoes, cut up, one quart Lima beans; simmer four hours slowly, add the green corn grated off eight ears, season the soup. and boil till the meat is in rags and the soup thoroughly done. Dried ochras may be used in winter.

\section{3.-WINTER SOUP.}

Take a shin or leg of beef, cut in pieces, salt and let it stand all night; next morning put it on with water, a quart to a pound, season with sait, pepper and mace; simmer and skim well. When boiling, put in a head of cabbage cut fine, twelve carrots sliced; a bunch of sweet herbs and parsley; add six turnips and three potatoes, and an onion, all sliced; skim off the fat and simmer slowly till dinner time.

\section{4.-НОTCH РОTCH. (English.)}

Put a pint of peas into a quart of water, boil them until they are so tender as easily to be pulped through a sieve. Take of the leanest end of a loin of mutton three pounds, cut it into chops, put it into a saucepan with a gallon of water, four carrots, four turnips cut in small pieces; season with pepper and salt. Boil until all the vegetables are quite tender, put in the pulped peas, a head of celery, and an onion sliced, boil fifteen minutes and serve.

\section{5.--MUTTON SOUP.}

Cut a neck of mutton into four pieces, put it aside, take a slice of the gammon of bacon and put it in a saucepan with a quart of peas, with enough water to boil them, let the peas boil to a pulp and strain them through a cloth, put them aside, add enough water to that in which is the bacon to boil the mutton, slice three turnips, as many carrots, and boil for an hour slowly; add sweet herbs, onions, cabbage, and lettuce chopped small, stew a quarter of an hour longer, sufficient to cook the mutton, then take it out, take some fresh green peas, add them with some chopped parsley and the peas first boiled, to the soup, put in a lump of butter rolld in flour, and stew till the green peas are done.

\section{1.-LAMB SOUP}

May be cooked as above, save that beef should be substituted for the bacon.

\section{7.-LEG OF BEEF BROTH.}

Take a leg of beef. break the bone in several places, place it in a pan with a gallon of water, remove the scum as it rises and add three blades of mace, a crust of bread, and a small bunch of parsley; boil till the beef is tender; toast some bread, cut it in diamonds, lay it in the bottom of the tureen, put the meat on it, and pour the broth over all.

$$
\text { 48.-VEAL BROTH. }
$$

Stew a knuckle of veal; draw gravy as for stock, add four quarts of water, with celery, parsley, and an onion; simmer till reduced to half, add two or three ounces of rice, but not until the soup is nearly cooked, so that when served the rice may be no more than done. Vermicelli may be used in preference, or for a change. 


\section{9.-MUTTON BROTH.}

Three pounds of the scrag of mutton, put into two quarts of cold water; add onion and turnips, pepper and salt, a few sweet herbs, and a little pearl barley ; skim well, and boil four hours.

These ingredients chiefly depend upon whether this dish is made for an invalid; if so, the omission of any of the ingredients will be regulated according to the advice of the medical attendant.

\section{0.-BAKED SOUPS.}

Take a pound of any lean meat and cut it into dice, place in an earthen jar, or pot, that will hold five quarts of liquid. Slice, and add to it, two onions, two carrots, two ounces of rice washed and previously soaked, a pint of whole or split peas, and some pepper and salt to taste; cover all with a gallon of water, tie a cloth over the top of the jar, or close the lid of the pot down very close, and bake.

This is a cheap and useful soup for poor people, and may be much improved by using the liquor that salt beef, or indeed, any meat has been boiled in, instead of water.

Cheap-for the poor.-Soak a quart of split peas for a day in cold water, and then put them into a boiler with two gallons and a half of water, and two pounds of cold boiled potatoes, well bruised, a faggot of herbs, salt, pepper, and two onions sliced. Cover it very close, and boil very gently for five hours, or until only. two gallons of soup remain.

Another.-Take two pounds of shin of beef, a quarter of a pound of barley, a half-penny worth of parsley, two onions sliced, salt and pepper to taste, and having cut the meat into dice, and broken the bone, place in a gallon pot and fill up with water; boil very gently for five hours. Potatoes, celery tops, cab- bage, or any vegetable left from the day before may be added.

\section{1.-SCOTCH BARLEY BROTH.}

Throw three-quarters of a pound ot Scotch barley into some clean water when thoroughly cleansed place it with a knuckle of veal in a stewpan, cover it with cold water, let it slowly reach a boil, keep it skimmed, add seven onions, and simmer for two hours; skim again and add two heads of celery and two turnips cut in slices, or any shape it pleases the cook; add as much salt as required to make it palatable, let it stew for an hour and a half; it must be well skimmed before the broth is dished; the meat must be previously removed and the broth alone sent to table. If it is intended to send the veal to the table with it, dress it as follows: take two pints of the broth and put it into a stewpan over a clear fire, add two table-spoonfuls of flour to the broth, and keep the broth stirring as you shake it in until it boils; add a little cayenne pepper, two tablespoonfuls of port, boil for two minutes, strain it over the veal and send to table.

\section{2.-GIBLET SOUP.}

Scald and clean thoroughly two sets of goose giblets or twice the number of duck giblets, cut them in pieces, put them in three quarts of stock; if water is used instead of stock add a pound of gravy beef, a bunch of sweet herbs, a couple of onions, half a table-spoonful of whole white pepper, as much salt, and the peel of half a lemon; cover all with water, stew, and when the gizzards are tender, strain the soup.

Now put into a stewpan a paste made of an ounce of butter and a spoonful of flour, stir it over the fire until brown, pour in the soup, let it boil, stirring it well all the while; in ten minutes skim and strain it, add a glass of Madeira, a 
salt-spconful of cayenne, a dessert-spoonful of mushroom ketchup, squeeze in the juice of half a lemon, serve up with the giblets in the soup. It should be sent to table as hot as possible.

\section{3.-POTAGE Å LA REINE}

Is so caller from its having been said to be a favorite soup at the table of Her Majesty.

Stew two or three young fowls for about an hour in good fresh-made veal broth: then take them out, skin them, and pound the breast, or only the white meat, in a mortar until it becomes quite smooth. That done, mash the yolks of three or four hard-boiled eggs with the crumb of a French roll soaked either in the broth or in milk, and mix this with the pounded meat to form a paste, which must be afterwards passed through a sieve. During this operation the bones and skin have been left stewing in the broth, which must then be strained, and the paste put gradually into it: then let it boil briskly for a short time, stirring it all the while to ensure its thorough mixture. When that is done take it from the fire; warm a pint or more of cream, and pour it gently into the soup.

This being a delicate white soup, the broth should only be seasoned with salt and mace, nor should there be any other vegetable used than celery; but the cream may be flavored with almonds.

\section{4.-RICE SOUP.}

Take white stock, season it, and either whole rice boiled till very tender, or the flour of rice may be used; one-half pound will be sufficient for two quarts of broth.

\section{5.-VEAL POTTAGE.}

Take off a knuckle of veal all the meat that can be made into cutlets, \&c., and set the remainder on to stew, with an onion, a bunch of herbs, a blade of mace, some whole pepper, and five pints of water: cover it close; and let it do on a slow fire, four or five hours at least. Strain it, and set it by till next day; then take the fat and sediment from the jelly, and simmer it with either turnips, celery, sea-kale, and Jerusalem artichokes, or some of each, cut into small dice, till tender, seasoning it with salt and pepper. Before serving, rub down half a spoonful of flour with half a pint of good cream and butter the size of a walnut, and boil a few minutes. Let a small roll simmer in the soup, and serve this with it. It should be as thick as middling cream, and, if thus made of the vegetables above mentioned, will make a very delicate white pottage. The pottage may also be thickened with rice and pearlbarley; or the veal may be minced, and served up in the tureen.

\section{6-VEAT BROTH.}

Stew a knuckle of veal of four or five pounds in three quarts of water, with two blades of mace, an onion, a head of celery, and a little parsley, pepper, and salt; let the whole simmer very gently until the liquor is reduced to two quarts; then take out the meat, when the mucilaginous parts are done, and serve it up with parsley and butter. Add to the broth either two ounces of rice separately boiled, or of vermicelli, put in only long enough to be stewed tender. Dish the knuckle separately, and serve it with parsley and butter.

\section{7.-MULLAGATAWNEE.}

Slice some onions and a few shalots, put them in a mortar with half a pound of fresh butter, beat them well, add three or four dessert-spoonfuls of currypowder, a little cayenne pepper and salt; cut up some India pickle, which pound 
well with the other ingredients; add flour sufficient to thicken the soup, and a little cold stock to work the whole into a stiff paste. - When beaten moisten it occasionally with broth made from fresh beef free from fat; when fine enough pass it through a sieve, add to it the gravy that the heart was stewed in, and as much of the beef broth as will make the quantity of soup required. Boil it up, and add more seasoning of cayenne and salt. If not thick enough, add flour and butter, until it becomes of the consistency of good cream. A spoonful of sugar and a little port wine are improvements.

\section{SOUPS OF GAME, POULTRY, \&C.}

\section{8.-VENISON SOUP. (English.)}

Take four pounds of freshly killed venison cut off from the bones, and one pound of ham in small slices. Add an onion minced, and black pepper to your taste. Put only as much water as will cover it, and stew it gently for an hour, keeping the pot closely covered. Then skim it well, and pour in a quart of boiling water. Add a head of celery cut into small pieces, and three blaides of mace. Boil it gently two and a half hours; then put in one fourth of a pound of butter, divided into small pieces, and rolled in flour, and add half a pint of port or Madeira wine. Let it boil a quarter of an hour longer, and send it to table with the meat in it.

Or:-Take a breast of venison, cut it in small pieces, and stew it gently in brown gravy soup. Serve it with roots cut in dice and French beans in diamonds, adding two glasses of port wine when first put on.

The head of the deer chopped in pieces, and the flesh stewed to a jelly, is also an excellent addition to the soup.

59.-VENISON SOUP.

Cut all the meat off a forequarter and shoulder of venison; put it into a pot with two gallons of water, a large onion, a head of celery, and some salt. Simmer it very slowly for forty-eight hours. Break all the bones and put them in an earthen pot just covered with water ; add a little salt, cloves, mace, and red pepper. Place the pot in the oven, set in a larger vessel of water, and let them stew as long as the soup.

Strain the soup clear, and add the juice of the bones. Color the soup with a little flour and a lump of butter as large as a walnut, browned in the fryingpan. Boil it up quickly, and throw in half a pint of port wine.

\section{0.-TURTLE SOUP.*}

Hang up the turtle by the hind fins, cut off the head, and allow it to drain.

Cut off the fore fins; separate the callipash (upper shell) from the callipee (under shell), beginning at the hind fins. Cut off the fat which will be found adhering to the callipash and to the lean meat of the callipee. Then cut off the hind fins. Take off the lean meat from the callipee and from the fins, and cut it into pieces two inches square and put into a stewpan. The callipash, callipee, and fins, must be held in scalding (not boiling) water for a few minutes, which will cause the shell to detach easily.

Cut the callipash and callipee into pieces six inches square, which put into a stock-pot with some light veal stock. Let it boil until the meat is tender, and then take it out into cold water; free the meat from the bones, and cut it into pieces an inch square. Return the bones into the stock and let it boil gently for two hours, strain it off, and it is then fit for use.

Cut the fins across into pieces about

* This receipt is a most excellent one, and is in constant use by the party who favored us with it; and we may add, that some thousands of pounds weight of turtle pass through his hands every year. 
an inch wide, boil them in stock with an onion, two or three cloves, a fagot of parsley and thyme, a sprig of sweet basil and marjoram. When tender take them out and add this stock to the other.

Take the lean meat, put into a stewpan with a pint of Madeira, four tablespoonfuls of chopped green shalots, two lemons sliced, a bunch of thyme, marjoram, and savory (about two tablespoonfuls each when chopped), one and a half tablespoonfuls of sweet basil (chopped), and four tablespoonfuls of parsley. Pound together a nutmeg; one dozen allspice, one blade of mace, five or six cloves, one tablespoonful of pepper and of salt. Mix the whole together with as much currypowder as will lie on a shilling. Put about two-thirds of this to the lean meat, with half a pound of frèsh butter and one quart stock. Let the whole be gently sweated until the meat is done.

Take a large knuckle of ham, cut it into very small dice, put into a stewpan with four large onions sliced, six bay-leaves, three blades of mace, one dozen allspice, three-quarters of a pound of butter; let it sweat until the onions are melted. Shred a small bunch of basil, a larger one of thyme, savory, and marjoram; throw these into the onions, and keep them as green as possible: when sweated sufficiently, add flour according to your judgment sufficient to thicken the soup. Add by degrees the stock in which the callipash and callipee were boiled, and the seasoning stock from the lean meat. Boil for an hour; run through a tammy, and add salt, cayenne, and lemon juice to palate. Then put in the meat; let it all boil gently about half an hour; and if more wine be required, it must be boiled before being added to the sonp. This is for a turtle of from forty to fifty pounds. It should, however, be recollected that the animal is of various weight-from a chicken-turtle of forty pounds to some hundreds-and the condiments must be apportioned accordingly. It should in variably be made the day before it is wanted.

\section{1.-MOCK TURTLE.}

Half a calf's head will be quite sufficient, even if it be small, to provide soup enough for a moderate party, as it will fill a tureen of two quarts; but it must be fresh and unstripped of the skin, which is the most gelatinous part.

Take out the brains; clean the head carefully in hot water, by squeezing it with the hand to press out the blood, and leave it afterwards for an hour in cold water. Then put it into five or six quarts of warm water along with two pounds of veal, two pounds of delicate pickled pork, chiefly fat, a roasted onion or two stuck full of cloves, and the thinly pared rind of a lemon, together with a large bundle of savory pot-herbs, two slices carrots, and a head of celery. Let this boil for two hours; then take up the head and the pork. The head must be stripped of its skin, and the brain, tongue, and eye taken out; let the bones of the head be broken and returned to the soup, and boil two hours longer-the brains being made into forcemeat balls, the tongue skinned and sliced; the black part of the eye should also be taken out, and the remainder minced; the skin being cut into pieces of little more than an inch square. While the stock is boiling, put into a saucepan a small quantity of fresh butter, with some onions sliced thin, a little basil, marjoram, and parsley, a very small quantity of thyme, three bay-leaves, two blades of mace, a few allspice; sweat all these well over the fire; when done, add sufficient flour to thicken the soup. Stir in the boiling stock by degrees to avoid its being lumpy. let it boil gently for an hour, then rub it through a tammy, set it over the fire; when it boils add the meat. About ten minutes before you serve, season the soup 
to your taste with salt, a small quantity of cayenne pepper, a couple of spoonfuls of soy, a good squeeze of lemon-juice, together with nearly a pint of either Madeira or Sherry. Serve with two lemons upon a plate, cut in half, as some people like the soup to be somewhat acid. Mushrooms are sometimes added.

The soup will take at least seven or eight hours in preparation. A calf's head requires half its own weight of neat to make the broth of proper quality.

\section{2.-NEAT'S FEET SOUP.}

Take two neat's feet, cut them as you do a calf's head: take five pints of any sort of broth, the juice and rind of one lemon, some parsley and herbs chopped fine; send these to the oven; when it comès from the oven, put in a pint of strong gravy and a cup of white wine, some hard eggs and forcemeat balls; season with cayenne pepper and salt,

To these an excellent addition will be found in one pound of the belly part of very delicate pickled pork; for it will improve the flavor of the soup, and, if cut neatly into bits. will taste nearly as rich, and quite as savory, as the fat of the head, or the gelatinous parts of the feet.

Any of these receipts for mock turtle may be also male from pig's face and pettitoes; and in most country-houses, as well as at sea-where pigs are generally kept for fresh meat-the head is commonly made into soup in imitation of turtle.

It has become unfashionable among first-rate cooks to put those egg-balls, formerly so common, into mock-turtle ; but as they are still used by those of the old school, we here add their mode of preparation :-

Take out the yolks of some hard-boiled eggs, and beat them in a mortar with a very little salt and cayenne, and make them into a paste with the white of a raw egg. Roll the paste into balls not larger than marbles, put them into the soup, and boil for ten minutes.

\section{3.- XA PORTUE-TURTLE SOUP.}

(Ude's Receipt, most carefully revised.)*

This soup will be made with less difficulty if you cut off the head of the turtle the preceding day.

In the morning open the turtle: which is done by leaning heavily with your knife on the shell of the animal's back, whilst you cut it off all round. Turn it upright on its end, that all the water,.\&c., may run out. Then cut the flesh of along the spine, with your knife sloped towards the bones, for fear of touching the gall, which sometimes escapes the eye. When you have obtained all the flesh which is about the members, wash it clean and let it drain.

Have ready a large vessel full of boiling water on the fire, put in the shells, and when you perceive that they come off easily, take them out of the water, and prick all the shells of the back, belly, fins, head, \&c. Boil the back and belly till you can take off the bones. without, however, allowing the softer parts to be sufficiently done, as they must boil again in the sauce. When these latter come off easily, lay them on earthen dishes singly; for fear they should stick together, and put them to cool. Keep the liquor in which you have blanched the softer parts, and let the bones stew thoroughly in it, as this liquor must be used to moisten the broth. All the flesh of the interior parts, the four legs and head, must be sweated in the following manner:-

Lay a few slices of ham on the bottom

* In perfecting the above receipt for turtle soup, which the author can without vanity assert, is the best if not the only authentic and practical one in print, the author has bestowed his utmost care and attention. When in manuscript he obtained a very high price for it. 
of a very large stewpan. Lay over the ham two or three knuckles of veal, according to the size of the turtle, and over the veal the inside flesh of the turtle, and the members over the whole. Then partly moisten it with the water in which you have boiled the shell, and sweat it thoroughly. You can ascertain if the meat be thorcughly done, by thrusting your knife into the fleshy part of the meat. If no blood issue, moisten it again with the liquor in which the bones, \&c., have been boiling: put in a large bunch of all such sweet herbs as are used in the cooking of a turtle: sweet bacil sweet marjoram, lemon thyme, winter savory, two or three bay-leaves, common thyme, a handful of parsley and green onions, and a large onion stuck with six cloves. Let the whole be thoroughly done. Observe that you must only put in the bunch of parsley and the stalks of the herbs, as you must keep the tender part of the leaves to make a puree of herbs, to introduce in the sauce when finished.

With respect to the members, probe them, to see whether they are done, and when done, drain and send them to the larder, as they are to make their appearance only when the sauce is absolutely completed.

When the flesh is also completely done, drain it on the dish, and make a white thickening, very thin, for turtle soup must not be much thickened; when the flour is sufficiently done on a slow fire, and has a good color, moisten it with the liquor drained through a silk sieve, and turn the sauce over the fire till it boils.

Ascertain that the sauce is neither too thick nor too thin, and then put the stewpan on the side of the stove, to skim off all the white scum, and all the fat and oil, that rise on the surface of the sauce. By this time all the softer parts will be cold enough; cut them about an inch or two square, without waste, throw the whole into the sauce, which must simmer gently. Then try them again; for if done enough, they are not to be kept on the fire any longer. Skim off all the fat and froth. Take all the leaves of the herbs from the stalks, sweet basil, sweet marjoram, lemon thyme, winter savory, common thyme, a handful of parsley and green onions, and a large onion cut in four pieces, with a few leaves of mace; put them in a stewpan, with about a quarter of a pound of fresh butter. Let this simmer on a slow fire, till they are quite melted, then pour in one bottle of good Madeira wine, adding a small bit of sugar, and let this boil gently for one hour. When done, rub this through a tammy, and put it into the sauce. Let this boil till no white scum rises: then take, with a skimmer, all the bits of turtle out of the sauce, and put them in a clean stewpan; when you have all out, pour the sauce over the bits of turtle, through a tammy, and proceed as follows :-

Make some quenelles à tortue (turtle quenelles), which being substitutes for eggs do not require to be very delicate. They are made in the following manner: Take out the fleshy part of a leg of veal, about one pound, scrape off all the meat, without leaving any sinews or fat, and soak in milk about the same quantity of crumbs of bread. When the bread is well soaked, squeeze it, and put it into a mortar with the veal, a small quantity of calf's udder, a little butter, the yolks of four eggs boiled hard, a little cayenne pepper, salt, and spices, and pound the whole very fine. Then thicken the mixture with two whole eggs, and the yolk of another.

Next try this farce or stuffing in boiling hot water, to ascertain its consistency; if you find it too thin. add the yolk of an egg. When the stuffing is perfect- 
ed, take half of it, and put into it some chopped parsley. Let the whole cool, in order to roll it of the size of the yolk of an egg or smaller: poach it in salt and boiling water, and when very hard drain on a sieve, and put it into the turtle. You must make two sorts of quenelles, white and green, those with parsley green, and the other half white.

Before you send up. squeeze the juice of two or three lemons, with a little cayenne pepper, and pour it into the soup. The fins may be served as a plat d'entrée, or side dish, with a little turtle sauce; if not, on the following day you may warm the turtie in the hot-water bath, and serve the members entire with a matelotte sauce, garnished with mushrooms, cocks'-combs, quenelles, \&c. When either lemon-juice or cayenne pepper have been introduced, no boiling must take place. It is necessary to observe, that the turtle prepared a day before it is used is generally preferred, the flavor being more uniform. When lemon juice is used be very cautious that the lemons are good; a musty lemon will spoil all the turtle, and too much will destroy the flavor.

Be particular, when you dress a very large turtle, to preserve the green fat in a separate stewpan, and likewise, when the turtle is entirely done, to have as many tureens as you mean to serve each time. You cannot put the whole in a large vessel, for many reasons: first, it will be long in cooling; secondly, when you take some out, it will break all the rest into rags. If you warm in a hotwater bath, the turtle will always retain the same taste, but if you boil it often, it becomes strong and loses the delicacy of its flavor.

It is not the fashion to serve eggs with turtle, but it may be necessary to inquire whetlier they are preferred.

Some people require besides, fricandeaux, fricassees, \&c., all of which are prepared in the same manner as veal, but made with those parts of flesh that are to be found in the turtle, four in number, two in the legs and two in the shoulders; you may likewise make blanquette, \&c.

\section{4-MOCK TURTLE, ENGLISH FASHION.}

Take a calf's head very white and very fresh, bone the nose part of it; put the head into some warm water to discharge the blood; squeeze the flesh with your hand, to ascertain that it is all out. Mind, the water should never be too hot for you to bear your hand in it; as long as you can bear it the blood will come out, but if you suffer it to be too hot it will turn the head black. This method of disgorging the blood is to give the same heat to the blood as when the animal was alive, and if your hand will bear the heat it shows that the blood may circulate in the veins, but if the water be too hot the blood will curdle, as it does in black pudding. When well disgorged. blanch the head in boiling water; when firm, put it into cold water, and make a blanc to boil the head, as follows :-Cut half a pound of fat bacon, a pound of beef suet, an onion stuck with a clove, and two slices of lemon; add to these slices of carrot, a bunch of parsley, green onions, thyme, bay-leaves, sweet basil, salt and pepper; put all these into a vessel. with water enough to contain the head; tie the head tightly in a clean towel, put it in the blanc, and boil two hours and a half; observe that it be not overdone; let it cool in the liquor, then make the sauce in the following manner:-

Put into a stewpan a pound of ham cut in slices, put over the ham two knuckles of veal, a large onion, and two carrots ; moisten with some of the broth in which you have boiled the head, to half the depth of the meat only: cover the stewpan, and put it over the fire to sweat through; let the broth reduce to 
a very good color, turn up the meat for fear of burning. When you have a very good color, and you find that the glaze is very brown, moisten with the whole of the broth from the head, season with a large bundle of sweet herbs, viz., sweet basil, sweet marjoram, lemon thyme, common thyme, two cloves, a bay-leaf, a few allspice, parsley, green onions, and a few mushrooms; let this boil together for $_{c}$ one hour, then drain it.

Put into a stewpan a quarter of a pound of very fresh butter, let it melt over a slow fire; put to this butter as much flour as it can receive; let it go geritly over a slow fire, till the flour has acquired a very good brown color; moisten this gradually with the broth, which you put through a silk sieve, till you have employed it all; add half a bottle of Madeira; let the sauce boil, that the flour may be well done; take off all the scum and fat, and drain the sauce into a clean stewpan large enough to contain the calf's head; boil the whole till done, cut the calf's head into square pieces of about an inch each; put them to boil in the sauce; season with salt and a little cayenne pepper. The bits of calf's head should always have the skin on one side, but you should leave none of the meat on, which does not adbere to the skin, otherwise the meat will break in the soup, and look unseemly. It is out of fashion now to use eggs, but on this head the taste of the master should be consuited, and you may make quenelles instead.

Observe, that you must not have the quenelles too delicate, for they would break in the soup and spoil the look of it ; the calf's head must not be too much done; thrust your knife into the skin, and if the knife enters and detaches itself easily, the meat is done enough.

Some persons will have their mock turtle green; in which case you must do as follows: Put into a stewpan a quar- ter of a pound of fresh butter, mince one or two onions, add a handful of each of the herbs described above, and some parsley, and sweat it all gently over a slow fire. When the herbs are well done, moisten with some of the sauce; and rub it through a tammy. Mix this with the sauce, and the turtle will be green, without any alteration in the flavor. Cayenne and lemon are always added when you serve up the soup.

65.-THE GARBURE, WITH BROWN BREAD.

Take a knuckle of ham, perfectly sweet, a knuckle of veal, and about six pounds of flank of beef, which put into a pan, with an onion stuck with two cloves, a few carrots, \&c.; pour over the above two ladles of broth, and let the whole sweat over a slow fire. When the meat is done through the middle, cover it entirely with boiling broth, and let the whole stew for three hours. Then take one or more cabbages, which are to be washed clean and blanched. Braise them between layers of bacon, and moisten them with the liquor in which the sweating has ben made, strained through a silk sieve. You must observe that, if the cabbages are not made rich and mellow, they are good for nothing. Add to the above, either sausages, bacon, or stewed legs of geese : mind above all things that the cabbage be not too briny, for the soup then would not be eatable. When the cabbage and broth are stewed enough, cut very thin slices of rye-bread: drain the cabbage in a cloth, so that there be no fat left, then take a large deep silver dish, lay a bed of bread, and over that one of cabbage, and moisten them with a little broth; let them stew on a slow fire. When the cabbage and bread are sufficiently moistened, lay on six or eight beds more of each; and let it simmer on the stove till the bottom of the dish is gratined, as what sticks to the bottom of the dish is most tasty and pal- 
atable. Send up with the ham on the middle; the bacon, the legs of geese, and sausages on the borders, and some broth separately.*

\section{6.-POTAGE A LA REINE-(Queen Soup.) \\ (Ude's New Receipt.)†}

For twelve people take three fat chickens or pullets, which are generally cheaper and better than fowls : skin them, take out the lungs, wash them clean, and put them in a pan with a bunch of parsley only; moisten the whole with good boiling broth: let it stew for an hour, then take out the chickens: soak the crumb of two penny loaves in the broth; take off the flesh of the chickens, and pound it with the yolks of three or four eggs boiled hard. and the crumb of bread which has been sufficiently soaked in the broth. Rub the whole through a tammy; then put a quart of cream on the fire, and keep stirring it continually till it boils. Pour it into the soup. It is not so liable to curdle as when the other method is used, and it tastes more of the chickens. If you think proper to add either barley, rice, or vermicelli, let it be stewed in broth beforehand, and pour it into the soup only when quite done. When you have a great dinner, and fowls are very dear, use the fillets for the firstcourse dishes, and make the soup with the legs only; the soup is as good, but not quite so white, as when mads with the fillets.

\section{7.-RICE CREAM. (Ude.)}

This is flour of rice, which you make yourself in the following manner. Take a pound of rice, well washed in different waters, and drained and wiped with a clean towel. Let it get quite dry, then

* This soup is never seen in this country; it requires a very deep and very large dish.

+ Formerly I used roasted chicken to make this potage, but I have found this new method cheaper, and not so subject to curdle as the other method. pound and shake it through a sieve. Take one or two spoonfuls of this flour, and dilute it with broth, rather cold than hot. All this time have some broth on the fire; throw the flour of rice thus diluted into the broth, and keep stirring till you find the soup is not too thick and may boil without the rice burning. This same kind of rice-flour may serve for soufflés, or puffs, of the second course.

\section{8.-MOCK TURTLE SOUP.}

This soup, if well made, gives general satisfaction. Take a calf's head, thoroughly scraped and cleaned, the skin remaining on; place it in a soup pot; to this add that part of the hand of pickled pork which is free from bones, the fattest end, observing that it is proper to soak it well in water previous to using; put in sweet herbs, a couple of onions, a head of celery, if large, a few truffles and morels, two if small, pounded mace and pepper; add plenty of water, without quite filling the saucepan; boil slowly, until the meat has become tender, then remove it, and cut the meat from the bone into square pieces; break the bones and put them again into the soup; let it simmer for four or five hours, then place it where it can quickly cool, remove the fat and strain the soup; thicken with four and butter; add three table-spoonfuls of Harvey's sauce, four or five glasses of Sherry or Madeira, and squeeze a whole lemon in to it; add the meat of the head and the pork cut into well shaped pieces; conclude with egg balls, or force-meat, or both, warm it and serve; it will be found a delicious soup.

\section{9.-MOCK TURTLE SOUP.}

Blanch half a calf's head sufficiently to draw out the bones; cut off the ear and the tongue; take off the skin of the tongue, lay all separate until cold, and strain off the liquor, and add it to your veal or second stock; cut the meat into 
large square dice, put it into a stewpan with your already prepared stock, and stew it until tender, strain off some of the stock; get another stewpan, cut about one pound of lean York or Westphalia ham, one pound of lean veal, a good fagot of basil and knotted-marjoram, two or three blades of mace, six or seven cloves, two bay-leaves, four onions, the parings of a few mushrooms, half a pound of butter; fry them for some time a nice light brown, dry all up with flour, then add your stock you have previously strained from the cut pieces; if too thick add more stock ; let all boil for some time, keeping it stirred with a wooden spoon; when boiled sufficient, strain it through a tammy or tammysieve into the stewpan that has the cut pieces of the head; boil all together; season with sugar, cayenne pepper, and salt, juice of lemon and white wine; if you wish to preserve the old fashion, by having forcemeat balls, egg, \&c., refer for them to the previous receipt; add them to it when they are blanched. I only put hard-boiled eggs, and, if I have any, a few quenelles.

\section{0.-HARE SOUP.}

An old häre is fitted only for soup or jugging. To render it into soup let it be cleaned, cut into pieces, add a pound and a half or two pounds of beef, to which there is little or no fat; place it at the bottom of the pan; add two or three slices of ham or bacon, or a little of both, a couple of onions, and some sweet herbs; add four quarts of boiling water, let it stew to shreds, strain off the soup and take away the fat; reboil it, add a spoonful of soy or Harvey's sauce, send to the table with a few force-meat balls.

\section{1.-HARE SOUP, OR WILD DUCK SOUP.}

Take a brace of Canadian hares or of wild ducks, and cut them up. Cook one moderately in an earthen pot with as much water as will cover it; some cloves, salt and black pepper; the pot being set in a large vessel full of water, in a close oven.

Simmer the other till it is done completely to rags in a gallon of water, and season it with cloves, salt, and black pepper.

Strain the soup clear and make forcemeat balls of the residue, with the yolk of an egg, some fine herbs, grated lemon peel, and a little stale bread.

The bare or duck cooked in the earthen pot must be cut in pieces two inches square. Color the soup with browning, throw in the forcemeat balls and cut meat; boil it up quick, and add half a pint of port wine.

\section{2.-VELOUTE.}

Take the cuttings and remains of any joints of fowls and veal you may happen to have, weigh four pounds, aud put into a large stewpan, with some onions, carrots, parsley, scallions, three bay leaves, three cloves, and a ladleful of stock; put your stewpan upon a brisk fire, skim well, and be careful the meat does not stick; when enough reduced add as much stock as will nearly fill the stewpan, salt it well, give it a boil, skim, and then put it on the side of the fire to simmer for two hours, after which strain it through a tammy; make a white roux; stir into it for ten minutes a few champignons, then pour on it, a little at a time, the above liquor, let it boil up once, skim, and set it again by the side of the fire for an hour and a half, remove all fat, strain again and then put by for use. The velouté should be colorless, the whiter it is the better.

\section{3.-PIGEON SOUP.}

Take half a dozen of the fattest pigeons you can get, roast them only sufficient to warm them through ; cut the meat from the bones; flour the latter well, and pound them in a mortar; stew them in 
a pint and a half of good gravy, add a piece of butter rolled in flour, a bunch of tarragon, chervil, a few onions, shalots, parsley, and basil, a few turnips, and carrots sliced; season with cayenne and one blade of mace. Boil slowly two hours, pour, and pass through a cullender. Pulp through a tammy, and then with the flesh of the pigeons put them into a saucepan. Let it simmer one hour and serve.

\section{4.-SNAPPING TURTLE SOUP.}

Clean and dissect your snapping turtle; add water according to size, taking care not to put in enough to thin the soup. Stew six or eight hours. Add a teacupful of browned flour, four ounces of butter, six eggs, one ounce of cloves, one ounce of mace, one ounce of allspice, half a pint of sherry wine; boil up and serve.

Soup may be made in the same way of the "couter" of the South. Forcemeat balls made of veal are usually added, with the eggs found in the couter.

\section{5.-SAVORY JELLY.}

Take half a pig's head, boil it for one hour, then cut the meat into small pieces, put it again into the sancepan with half the liquor it was boiled in, add a little seasoning of pepper, salt, and mace, boil another hour ; turn it into a mould to get cold. The above is excellent made from calf's head, which in many countryplaces can be bought for a trifle; but the mould should then be lined with hard boiled eggs, cut into slices, and a little parsley added to the seasoning. This is an economical breakfast or supper dish.

\section{6.-PARTRIDGE SOUP.}

When you have a brace of partridges which prove to be remarkably old, convert them into soup. Skin and cut them up, cut a handsome slice of ham as lean as possible and divide it in four, or cut as many thin slices, put them in the pan, add the partridges with an onion sliced, some celery, and four ounces of butter; brown nicely without burning, put them into the stewpan with one quart and a pint of water, throw in a few white peppers whole: a shank of mutton, salt it to palate, strain, add stewed celery, fried bread, and previous to its boiling skim very clean and serve up.

\section{7.-VEGETABLE SOUPS.}

Although many of the following soups are made purely of vegetables, yet some -and those generally the best-are made on a foundation of some sort of meat-broth in which the roots are stewed.

The broth is therefore the real stock on which these soups are made, though each bears the name of the vegetable which gives it flavor; but when made solely from herbs, roots, and vegetables, and used as the basis for the formation of soupes-maigres, the stock is then composed of every sort in season which can, by long stewing, be reduced to a pulp, in which every species of savory herbssuch as chervil, tarragon, and marjoram - are mixed, together with a few chilis, shalots, and a head of garlic, seasoned with mace, whole peppers, salt, and mushroom ketchup; to which may be added a little curry-powder. The pulp is then strained; and, being thus rather highly seasoned, may be kept for a few days, and used in aid of any other soup. It must be left to simmer during several hours; and should it need browning, some sliced onions, fried in butter, will be all that is necessary.

Thus all the roots and most of such vegetables as can be easily made into purces, and combined with any sort of broth, will, in this manner, make excellent soup of different denominations, 
though all founded upon the same meatstock. The gravy of beef is always preferred for savory soups, and that of veal or fowls for the more delicate white soups: to which from half a pint to a pint of cream, or, if that cannot be had, the same quantity of milk and the yolks of a couple of raw eggs should be added for every two quarts of soup ; remembering, however, that the latter will not impart the richness of cream.

Collect whatever vegetables are in season, take equal quantities, turnips, carrots, cabbage, spinach, celery, parsley, onions, a little mint, \&c., add plenty of herbs, cut them fine, put them into the stewpan, in which has previously been placed some oil; stew gently until the vegetables become tender, then add two quarts of boiling water; stew a quarter of an hour and serve.

Some cooks advocate the introduction of peas, green or white, to this soup: where they are used, they must be boiled until tender in very little water, then mashed into a very loose paste; the vegetables having been scalded are then added, and two hours will suffice for stewing; season it with salt and pepper.

Be careful that it does not burn while cooking, or the whole is spoiled.

\section{8.-TOMATO SOUP.}

Stew half a peck of tomatoes slowly an hour and a half in a pint of water; pass them through a tamis; add half a gallon of veal or mutton broth. Pass through a tamis enough stale bread to thicken the soup. Fry twelve onions brown, and strain them the same way. Add them to the soup with a bunch of fine herbs, and seasoning to taste. Boil up well, and serve.

\section{9.-BEAN SOUP.}

Soak two quarts of dried white beans all night and put on the soup early. To five pounds of beef allow one of bacon cut small. Boil and skim it and put in the beans, drained, and a head of celery cut small. Boil slowly till the beans are melted and the soup is done; strain it, and serve with toasted bread.

\section{0.-ASPARAGUS SOUP WITH GREEN PEAS.}

Make a soup of roots, and when strained, boil a pint of green peas in the liquor. Choose some middling-sized asparagus, cut them in pieces about three inches long, blanch them in boiling water, and then throw them into cold water; drain them and tie them in small bunches, split the tops and boil them with the peas. When done make a purée of them and mix it with the root soup, and garnish with the asparagus. Good meat-broth may be used instead of the root soup.

\section{1.-SOUP DE L'ASPERGE.}

Cut into thin slices half a pound of bacon, lay them in the bottom of the stewpan, cut into lumps six pounds of lean beef and roll it well in flour, cover the pan close, shake occasionally until the gravy is all drawn, then add half a pint of old ale and two quarts of water; throw in some whole peppers and a spoonful of salt, stew gently for an hour, skim the fat, and when an hour has elapsed strain off the soup, then put in it some spinach, two cabbage lettuces, the leaves of white beet, a little mint, powdered sweet aroma and sorrel, boil them, then put in the tops of asparagus cut small; when they are tender the soup is done; serve up hot with a French roll in the middle.

82.-POTAGE $\AA$ LA JULIENNE-(Julien Soup.)

Take some carrots and turnips, and turn them ribbon like, a few heads of celery, some leeks and onions, and cut them all into fillets, thus —. Then take 
about two ounces of butter and lay it at the bottom of the stewpan, with the roots over the butter. Fry them on a slow fire, and keep stirring gently; moisten them with broth and gravy of veal, and let them boil on the corner of the stove; skim all the fat off, and put in a little sugar to take off the bitter taste of the vegetables: you may in summer-time add green peas, asparagus-tops, French beans, some lettuce, or sorrel. In winter-time, the taste of the vegetables being too strong, you must blanch them, and immediately after stew them in the broth: if they were fried in butter their taste would also be too strong. Add bread, as above, in the tureen.

83.-THE JULIENNE WITH CONSOMME(or Broth of Fowl.)

The same as above, only you moisten it with consomm of fowl, and put in the back of a roasted chicken, from the preceding day, which stew with the roots; when boiled for one hour, take it out of the broth, and send up with the bread, as above.

\section{4.-JULIENNE, OR VEGETABLE BROTH.}

Cut various kinds of vegetables in pieces, celery, carrots, turnips, onions, \&c., and having put two ounces of butter in the bottom of a stewpan, put the vegetables on the top of the butter, together with any others that may be in season; stew or fry them over a slow fire, keeping them stirred, and adding a little of the stock occasionally; soak small pieces of crust of bread in the remainder of the broth or stock, and when the vegetables are nearly stewed, add them, and warm the whole up together

\section{5-SOYER'S JULIENNE SOUP.}

This seup is entirely the hereditary property of France, and is supposed to be so called from the months of June and
July, when all vegetables are in full season; and to make it in reality as originally made, a' small quantity of every description of vegetables should be used, including lettuce, sorrel, and tarragon; however. some few sorts of vegetables, mixed together, make a most estimable soup. Weigh half a pound of the vegetables in fair proportions to each other, that is. carrots, turnips, onions, celery, and leeks, which cut into small fillets an inch in length, and of the thickness of a trussing-needle; when done, wash dry, and pass them in butter and sugar as before, ard two quarts of clear soup, adding, just before it is done, a little sorrel, cabbage-lettuce, and chervil or peas, if handy, but it will be excellent without eithor.

\section{6.-A SPRING SOUP.}

The following is recommended for purifying the blood, and consequently clearing the complexion:-Take a handful of fresh sorrel and a handful of water-cresses; cut them up fine, but do not chop them; put them into about two quarts of boiling water, adding a carrot cut up in small pieces, three or four potatoes, cut in quarters, two slices of bread (if of a sixpenny loaf), also cut up; pepper and salt to taste. Boil well till the soup assumes a consistency, which it will do as the vegetables and bread dissolve in it. Stir it frequently, and it will prove a very paiatable soup. With a moderate fire it may be prepared in about two hours. The liquor from any boiled meat may be used instead of plain water.

\section{7.-MACARONI, WITH CONSOMME, OR STOCK-BROTH.}

Take a quarter of a pound of Naples macaroni, and boil it in water, till it is nearly done. Strain well, and put it into a rich consommé to boil. Let it be 
well done; grate some Parmesan cheese, which send up separately in a plate.

\section{8.-CABBAGE SOUP.}

Take four or six pounds of beef, boil with it some black pepper whole for three hours, cut three or four cabbages in quarters, boil them until they are quite tender, turn them into a dish, and serve all together.

\section{9.-MACARONI SOUP.}

Take a quart of gravy soup: break two ounces of Naples macaroni into pieces of little more than an inch long, putting them, by degrees, into a small portion of the boiling soup, to prevent them from sticking together, and let them boil until quite tender, but not soft or pulpy-from fifteen to twenty minutes if quite fresh, but nearly half an hour if at all stale. Vermicelli is used in the same manner. They will improve the consistence of the soup if the quantity above stated be added: but it is useless and does not look well to see, as at some tables, only a few strings of it floating in the tureen. The flavor will also, be much improved if a small quantity of Parmesan cheese be either melted in it, or grated and served up separately.

\section{0.-SOUP § LA CRECI, OR CARROT SOUP.}

Cut half a pound of lean ham in dice, three onions, four turnips, twelve carrots, the outer side red only, a head of celery, a fagot of sweet herbs, two blades of mace, six cloves, a bay-leaf, and half a pound of salt butter; fry all well down in a stewpan until they get a little brown, then add some second stock, and stew until all the roots are quite tender, then rub it through a tammy sieve or tammy cloth with two long spoons; if very thick, add more stock. Season with cayenne and black pepper, and salt, and a good bit of sugar; send up on a napkin some nice fried bread cut in small dice, and not greasy.

\section{1.-GREEN PEA PORRIDGE.}

(A most delicious potage.)

Green peas three pints, milk three pints, butter one-quarter of a pound, flour enough for thickening.

Boil the peas in just water enough to cook them until they are tender; then pour in the milk; when it boils, add the butter and flour rubbed well together; let it boil long enough to cook the flour; season with pepper and salt, and serve hot.

\section{2.-GREEN PEA SOUP.}

Put two quarts of green peas into a stewpan with a quarter of a pound of butter, a quarter of a pound of lean ham, cut into small dice, two onions in slices, and a few sprigs of parsley ; add a quart of cold water, and with the hands rub all well together; then pour off the water, cover the stewpan close, and stand it over a sharp fire, stirring the contents round occasionally; when very tender, add two tablespoonfuls of flour, which mix well in mashing the peas with your spoun against the sides of the stewpan; add two quarts of stock, or broth from the Pot-au-feu, a table-spoonful of sugar, and a little pepper and salt, if required; boil all well together five minutes, when rub it through a tammy or hair sieve; then put it into another stewpan, with a pint of boiling milk; boil five minutes, skim well, and pour it into your tureen. It must not be too thick; serve with croutons of bread.

\section{3.-WINTER PEA SOUP.}

Wash a quart of split peas, which put into a stewpan with half a pound of streaked bacon, two onions in slices, two pounds of veal or beef, cut into small 
pieces, and a little parsley, thyme, and bay-leaf; add a gallon of water, with a little salt and sugar; place it upon the fire, and when boiling, stand it at the side until the peas are boiled to a purée, and the water has reduced to half; then take out the meat, which put upon a dish, to be eaten with the bacon, keeping it hot; rub the soup through a hair sieve or tammy, put it into another stewpan, and when boiling, serve. The meat may also be served in the tureen if approved of. Maigre pea soup may also be made by omitting the meat, adding half a pound of butter, one quart of milk, and omitting a quart of water.

\section{4.-LEEK, OR ONION SOUP.}

The liquor in which a leg of mutton has been boiled will do very well for this broth. Mix a spoonful or two of oatmeal, according to the quantity of broth, in cold water, very smooth, the same as if for gruel; add a little of the broth, by degrees, until the whole is incorporated; then boil the liquor with any quantity of leeks or onions-both or either-until it becomes of the consistence of cream. Or, omit the oatmeal, and substitute flour, stirring the soup very hard for five minutes; and when you are about taking it from the fire, stir in the yolks of two raw eggs beat up with a little more broth, and serve it immediately.

Onions, peeled, pared, and cut into pieces, then shred into a pan and fried in either oil or butter, without any broth, but simply having boiling water poured over them, and some toasted bread, seasoned merely with pepper and salt, are considered very refreshing when thus made into a soup, and much used by ladies throughout Europe after the fatigues of a ball.

95.-POTATO SOUP MAIGRE.

Take some large mealy potatoes; peel and cut them into small slices with an onion; boil them in three pints of water till tender, and then pulp them through a colander; add a small piece of butter, a little cayenne pepper and salt, and, just before the soup is served, two spoonfuls of good cream. The soup must not be allowed to buil after the cream has been put into it.

\section{6.-TOMATO SOUP.}

Wash, scrape, and cut small the red part of three large carrots, three heads of celery, four large onions, and two large turnips; put them into a saucepan with a table-spoonful of butter, and half a pound of lean new ham; let them stew very gently for an hour, then add three quarts of brown gravy soup, and some whole black pepper, with eight or ten ripe tomatoes; let it boil an hour and a half, and pulp it through a sieve; serve it with fried bread cut in dice.

\section{7.-SOUP SORREL-A SUMMER SOUP.}

Take a good quantity of sorrel and mix with it the top leaves of beet-root; boil them thoroughly; press them enough to extract all the water, and chop them until they are almost a paste; when they are quite cold, add the coldest spring water attainable, and mix until rather thicker than cream; cut in thin slices two cucumbers steeped in a mixture of vinegar and a little cayenne; boil three eggs hard, and cut them in very small pieces ; now, having chopped the green ends of young onions small, and added to the paste, pour over cream to your taste, and then add the sliced cucumber and boiled egg; serve up garnished with clean pieces of ice.

\section{8.-TOMATO SOUP.}

Slice two onions and fry them in butter until brown; remove them and fry two dozen tomatoes just sufficient to heat 
them through, then put them into a stewpan with their gravy and the onions, add a head of celery and a carrot sliced; stew gently for half an hour, add three pints of gravy, stew an hour and a half, pulp the whole of the vegetables through a sieve, season with white pepper, salt, and cayenne, serve with sippets of toasted bread cut in shapes.

\section{9.-PEPPER POT. (English.)}

Put in a stewpan three quarts of water; to this add celery, turnips, carrots, lettuces, cut small; add the bones of cold roast meat of any description; half a pound of bacon, the same weight of salted pork; stew gently until the meat is tender, taking care to skim when it first boils.

Boil half a peck of spinach and rub it through a colander; take the bones out of the soup and add the spinach; with it the meat of a lobster or crab minced; season with plenty of cayenne pepper, and salt to taste.

Suet dumplings may be boiled with it, or a fowl, but this is matter of taste. Mutton or beef may be substituted for bacon or pork; this will be obvious when it is understood that a pepper pot is presumed to consist of an equal proportion of flesh, fish, fowl and vegetables.

\section{0.-PUREE OR TURNIP SOUP.}

Get a bunch of turnips, pare them and cut them in thin slices; one head of white celery, one onion; fill up your stewpan with good second white stock, boil them until quite tender, then pass it all through a tammy by rubbing it with wooden spoons, or a tammy sieve; season with sugar, cayenne and salt. Send up fried bread, as for former soups; add half a pint of cream the last thing.

101.-SAUTÉ SOUP. Cut carrots, and turnips, and onions, $/$ broth or water, and put into the tureen and celery, as straws, about one inch long, quite thin; the carrots you will trim, using only the red part, the yellow that is left use for your stock pot; cut your onions in quarters, then cut them the size endways, blanch them for two or three minutes, strain them on the back of a hair sieve to drain; then add them to the quantity of soup required, allowing half a pint to each person; therefore, as you must reduce it to have the flavor ducing it to the quantity you require; season it with lump sugar, cayenne pepper and salt; be sure and not go to the extreme.

\section{2.-SPRING SOUP.}

As saute ; the same roots cut differently, and add, if to be had, spinach, cabbage-lettuce, a very little sorrel, as it turns acid on the stomach, all cut rather small, tarragon, chervil, green asparagus, young peas, cucumbers ; cut the asparagus about one inch long, cut the tarragon and chervil a little, and a few French beans cut; use your consommé stock as before, boiling all your green parts particularly green in water a few minutes, leaving them to be sufficiently done in your stock; if you have a cauliflower boiled, pick a few small pieces and put in the soup-tureen; the boiling soup when poured in will make it hot; season as before.

\section{3.-WINTER VEGETABLE SOUPS.}

Take carrots, turnips, and the heart of a head of celery, cut into dice, with a dozen button onions; half boil them in salt and water, with a little sugar in it; then throw them into the broth; and, when tender, serve up the soup: or use rice, dried peas and lentils, and pulp them into the soup to thicken it.

With many of these soups, small suet dumplings, very lightly made, and not larger than an egg, are boiled either in of your vegetables, allow a pint more, re- 
iust before serving, and are by most persons thought an improvement, but are more usually put into plain gravy-soup than any other, and should be made light enough to swim in it.

Such are the chief soups made of vegetables on a foundation of meat; both those made solely of vegetables, without any intermixture of meat broth, may be made into very palatable soups by using the regetable stock.

\section{FISH SOUPS.}

Notwithstanding the quantity of excellent fish, this nutritious and economical viand is not often introduced at table in the form of soup. Nothing could be more easy than to multiply receipts for an almost endless variety: those that follow will, however, show how much may be done with many kinds of fish which are not in very high estimation.

When not wanted as soupe-maigre, the foundation, or stock, though called " fishsoup," is always better if made of meat, and veal is generally preferred. We, therefore, here add a receipt for its composition, as it may be applied to any kind of fish; though, in those which we shall hereafter specify, it is not mentioned, as they are all intended to be maigre.

\section{4.-STOCK FOR FISH SOUP.}

Take a dozen flounders, or any small flat fish, and the same number of perch; gut and clean them carefully; put them into a stewpan with two quarts of strong veal-broth; add a few slices of lean han, two or three carrots, celery, and onions cut in slices, some sweet herbs and salt, with a little cayenne; stew till the fish will pass through a coarse sieve; then return it into the stewpan, with a good lump of butter and some flour to thicken it; add a couple of large glasses of white wine, and a large spoonful of garlic vinegar. The gravy from potted herrings, anchovies, or a little Oude sauce, will also improve the flavor.

This stock, if once reboiled, will, in cold weather, keep well for a month; or, if served as soup, the quantity may of course be reduced according to the number of the party intended to partake of it, and it will be found excellent. Indeed, any species of fish may be made into soup in the same manner. If meant to be browned, the onions should be fried, and a good spoonful of mushroom ketchup or India soy be added; and red wine will be better than either sherry or Madeira. But if left white, cream should be substituted for ketchup and soy; a glassful of ginger wine will answer the purpose of red wine.

\section{5.-LOBSTER SOUP.}

If prepared fish-stock be not used, the stock of this soup may be made of any fish not of sufficient consequence to be dressed in any other way. Clean and cut them in pieces, take the meat out of one or two lobsters, cut it into small pieces, and lay it aside; break the shell and add it to the fish for the stock, which should be boiled gently for several hours; take the coral of the lobster, pound it in a mortar, with a small piece of the stock. partly pounded, and make it up into forcemeat-balls, with a little chopped parsley, bread-crumbs, and an egg. When the stock is sufficiently done, strain it, thicken it with butter and flour, or cream: warm the lobster in it, and send it up, cut small, with the forcemeat-balls. Season it also delicately, but yet sufficiently to give it flavor, which can be imparted by any of the compound sauces so commonly advertised.

\section{6.-LOBSTER SOUP.}

To one large hen lobster scalded and cleaned, take two quarts of strong calf's 
feet broth; pound the shells, small claws, eggs, fat and coral in a mortar, and simmer three hours in the soup. Cut the tails into square pieces, and fry them brown. Make the claws into forcemeatballs with the yolks of two eggs, grated lemon-peel, parsley, thyme, a very little olives, and grated biscuit, and fry them brown. Boil them up in the soup quick$l y$ in five minutes; add half a pint of port wine, and lemon to taste; with salt, pepper, and a little mace. (This is the best soup on record.)

\section{7.-LOBSTER SOUP. (English.)}

Extract the meat from the shells of four hen lobsters, which have been boiled: put the spawn aside, beat the fins and small claws in a mortar; then place both in a saucepan, with two quarts of water, until the whole goodness of the fish has been drawn; then strain the liquor. Beat in a mortar the spawn, a lump of flour and butter; rub it through a sieve into the soup previously strained; simmer without boiling, that the color may be preserved, ten minutes; squeeze in a piece of a lemon, with a little of the essence of anchovies.

When this dish is sent to table as a feature, forcemeat-balls are served with it; they are made of minced lobster, spawn, crumb of French roll, egg, and mace pounded; roll it in flour, and serve in the soup.

\section{8.-PUREE OF LOBSTER SOUP.}

Get two large hen lobsters, take out all the meat, chop and pound it fine, six anchovies boned, put the shells in some second stock to boil for some time, strain off the liquor into your pounded lobsters, boil all until tender, rub all through a tammy, add one pint of cream; season with cayenne pepper, a little sugar, and salt, and lemon-juice.

109.-CLAM SOUP. To the liquor of fifty clams, allow blade of mace, Harvey sauce and essence three quarts of water, and put it on with a knuckle of veal, the bone chopped. Simmer three hours, put in a bunch of sweet herbs, nutmeg, mace, a table-spoonful of whole pepper, and stew. an hour longer. Then strain and add four ounces of butter rubbed in flour, and the clams chopped in pieces. Boil a quarter of an hour and serve.

\section{0.-OYSTER SOUP.}

Beard four dozen oysters, preserve the liquor in opening them, which must be placed with the beards of the oysters in a stewpan; slice any fish, small freshwater fish will serve excellently well, and adding them, stew for five or six hours; strain and thicken it, add two spoonfiuls of soy or any fish sauce, or omit it, to taste. Add the oysters, and . when they are warm through, serve.

$$
\text { 111.-OYSTER SOUP. }
$$

Get four flounders, or similar portions of any fish, four dozen of large oysters, blanch them slightly, take off the beards and gristle, put the beards and fish into some of your best white stock, boil all together for several hours, add four anchovies washed, strain all off and thicken it with flour and butter, add one pint of cream, put in your oysters you had taken care of, the last thing, just boiling them up in the soup; having passed it through a tammy, season it with cayenne pepper, salt, and a small piece of sugar.

\section{2.-OYSTER SOUP.}

Take fifty oysters, blanch them, but do not let them boil; strain them through a sieve, and save the liquor. Put one-quarter pound of butter into a sterpan; when it is melted, add six ounces of flour ; stir it over the fire for a fow minutes; add the liquor from the oysters, two quarts of real stock, one quart of new milk; season with salt, peppercorns, a little cayenne pepper, a 
of anchovies, a tablespoonful each ; strain it through a tammy; let it boil ten minutes; put the oysters into the tureen with a gill of cream, and pour the boiling soup upon them.

\section{3.-EEL SOUP.}

Take any number of pounds of eels, according to the quantity required; add two-thirds water. If about three or four pounds of eels, add one onion, a small quantity of mace, a little pepper whole, sweet herbs, a crust of the top side of bread; cover down close; stew till the fish separates, strain. Toast slices of bread deep brown, but not to burn; cut into triangular pieces or squares, a piece of carrot two inches long, cut into four slices lengthwise, put into a tureen with the toast, pour the soup on; boiling cream may be added thickened with a little flour, but it should be rich enough without it.

\section{4.-NEW ENGLAND CHOWDER.}

Have a good haddock, cod, or any other solid fish, cut it in pieces three inches square, put a pound of fat salt pork in strips into the pot, set it on hot coals, and fry out the oil. Take out the pork, and put in a layer of fish, over that a layer of onions in slices, then a layer of fish with strips of fat salt pork, then another layer of onions, and so on alternately until your fish is consumed. Mix some flour with as much water as will fill the pot; season with black pepper and salt to your taste, and boil it for half an hour. Have ready some crackers soaked in water till they are a little softened; throw them into your chowder five minutes before you take them up. Serve in a tureen.

\section{5.-NEW ENGLAND CHOWDER.}

Cover the bottom of a pot with slices of boiled salt pork, with a little onions; on this place a layer of fish in large pieces, season with pepper, and cover it with a layer of biscuit soaked in milk, and a layer of sliced potatoes. Put above this another layer of pork, as before, with fish, \&c., the biscuit being on the top of all. Pour in a pint and a half of water, cover, and boil it slowly an hour; then skim and turn it into a deep dish. Thicken the gravy with butter rolled in flour, and parsley.

\section{6. - CLAM SOUP.}

Boil a knuckle of veal in as much water as will cover it well. When it has boiled about half or a quarter of an hour, open half a peck of clams by placing them over the fire, in a very small quantity of water. Pour the juice of the clams into the pot which contains the knuckle of veal. Boil the soup three or four hours, seasoning it with a tablespoonful of pepper. Add no salt, as the clam juice will have made it salt enough. When the veal is quite tender, cut up the clams in small pieces (that is to say, each clam in three or more pieces), and throw them into the soup, with a quarter of a pound of butter chopped into bits. Some persons roll the butter in flour, which they think makes it mix with the clam juice; others use no flour, as they dislike to detect the taste of the flour.

\section{7.-CLAM SOUP, (Prepared by a Dublin Lady.)}

Put forty or fifty clams, in the shells, with as little water as possible. When the liquor has run out from the opened shells, take the clams out and chop them fine, with an onion, a bunch of minced celery, and some mace and pepper. Put all in the soup, and thicken it with two tablespoonfuls of butter rolled in flour, and if you choose, add a little milk. Simmer twenty minutes; stir in the beaten yolks of five eggs; put bits of toasted bread into the tureen and serve. 
118.-LAKE AND POND FISH SOUP.

For every person take a pound each of any fresh-water fish that can be obtained; wash them in salt and water, and stew them with a tomato, carrots, leeks, fried onions, and sweet herbs, in as much water as will cover them; and let them stew until the whole is reduced to a pulp; then strain the liquor, and boil it for another hour until it becomes quite smooth. Then have ready some roots of any sort that may be in season, which have been chopped small, and boiled either in milk or water: add them to the soup, and let it simmer for one-quarter of an hour; season it, if milk has been used, with mace and celery, with a little cayenne; but if made solely with water, then use Chili vinegar, soy, mushroom ketchup, or any of the savory sauces.

\section{FISH.}

Fish should be well washed, scaled, and cleaned, by drawing the entrails through a slit in the under side. The roe and liver should be separated, washed, and cooked with the fish. The roe proves the fish in season. Slimy fish may be scalded in salt and water, but must be dried before cooking.

Fresh fish, when boiled, should be placed in cold, and shell-fish in boiling water.

To keep oysters after washing them, lay them in a tub in a cool cellar, with the deep part of the shell undermost. Sprinkle them with salt and Indian meal, then fill the tub with cold water. Change the water every day. and the oysters will keep fresh a fortnight

Fish should be garnished with horseradish or parsley. The only vegetable served with fish is potatoes.

119.-SALT AND FRESH-WATER FISH. a little vinegar into the water to give the fish firmness. Be careful to let fish be well done, but not to let it break. When very fresh, cod and whiting are very much improved by keeping a day, and rubbing a little salt down the back-bone. Fresh-water fish often have a muddy smell and taste, which is easily got rid of by soaking it. After it has been thoroughly cleansed in strong salt and water, if the fish is not too large, scald it in the same, then dry and dress it.

Fish that is to be boiled must be put on the fire in cold hard water; when it boils, skim with the greatest care; throw in a little cup of cold water to check the extreme of heat, then keep it simmering only, lest the outside break befure the thick and inmer part be done; but "crimped fish" should be put into boiling water, and simmered a few minutes.

When it boils up, some cold water should be put into it to check it, and keep it simmering. The cover should be kept on the kettle to prevent soot falling in and discoloring the fish. Fish should be boiled with a handful of salt, and half a teacupful of vinegar. All fish should be taken out of the water the instant it is done, or it will become woolly. To ascertain when it is done, the fish-plate may be drawn up, and, if done, the meat will leave the bone. To keep it hot. and to prevent it losing its color, the fish-plate should be placed across the fish-kettle. and a clean cloth put over the fish. If left in the water after it is ready, fish loses its firmness. Serve fish on a napkin.

It is impossible to dress fish too fresh. Some kinds will bear keeping beiter than others, but none are improved by it.

Families who purchase a whole salmon, and like it quite fresh, should parboil the portion not required for the day's consumption, and lay it aside in the liquor, boiling up the whole together when wanted. By this means the curd will
set, and the fish be equally good on the 
following day. The custom of serving up rich sauces, such as lobster, is unknown in salmon countries; a little lemon peel or white vinegar being quite sufficient, added to melted butter. Salmon should be garnished with parsley and scraped horseradish.

Small fish may be nicely fried plain, or done with egg and bread crumbs, and then fried. Upon the dish on which the fish is to be served should be placed a damask napkin, folded, and upon this put the fish, with the roe and liver; then garnish the dish with horseradish, parsley, and lemon.

To boil or fry fish nicely, after it is well washed, it should be put in a cloth, and when dry, wetted with egg and bread crumbs. It will be much improved by being wetted with egg and crumbs a second time. Then have your pan with plenty of boiling dripping or lard, put your fish into it, and let it fry rather quickly till it is of a nice brown and appears done. If it is done before being nicely browned, it should be taken from the pan, and placed on a sieve before the fire to drain and brown. If wanted very nice, put a sheet of cap paper to receive the fish. Should you fry your fish in oil, it obtains a much finer color than when done in lard or dripping. Never use butter, as it makes the fish a bad color. Garnish your dish with green or fried parsley.

In broiling fish, be careful that your gridiron is clean; place it on the fire, and when hot, rub it over with suet to hinder the fish from sticking. The fish must be floured and seasoned before broiling. It must be broiled over a clear fire only, and great care must be taken that it does not burn or becnme smoky.

Broiled fish for breakfast should always be skinned, buttered, and peppered.

Fish are broiled, fried, boiled, baked, stewed, in fact cooked in every imaginable fashion; those named are the chief methods. In every kind, the greatest attention and cleanliness must be exercised. A broken, disfigured, or ill-cooked dish of fish presented at table, is quite sufficient to destroy the taste for it for ever; on the contrary, when neatly done it heightens the relish which every one possesses more or less, and imparts an appetite where one may be wanting, while the cook is held in grateful remembrance.

\section{0.-MARINADE}

Is commonly used in France for the purpose of boiling fish. which imbibes from it a more pleasant flavor than it naturally possesses, and has been so generally adopted by professed cooks that we here insert the receipt:-Cut up two carrots, three onions, half a dozen shalots, a single clove of garlic, and put them into a stewpan with a piece of butter, a bunch of parsley, and a bundle of sweet herbs; fry the whole for a few minutes, then add, very gradually. two bottles of any light wine or of cider. Put in a handful of salt, two dozen of peppercorns, the same quantity of allspice, and a couple of cloves. Simmer the whole together for one and a half hours, strain the liquor, and put it by for use.

This marinade, if carefully strained after the fish has been taken out, will serve several times for the same purpose, adding a little water each time. Fish dressed in it should simmer very gently, or rather stew than boil, as it affords to mackerel, fresh herrings, perch, roach; and any of the small river fish, the advantage of dissolving, or so thoroughly softening their bones as to render them more agreeable in eating. For large fish, they should be cut into steaks before being marinaded. Instead of the wine or cider, a quart of table-beer, a glass of 
soy, one of essence of anchovies, and one of ketchup, may be used; or a pint of vinegar and these sauces, fennel, chives, thyme, and bay-leaves, may be added with the wine, cider, \&c. Or, choose a kettle that will suit the size of the fish, into which put two parts water, one of light (not sweet) white wine, a good piece of butter, some stewed onions and carrots, pepper, salt, two or three cloves, and a good bunch of sweet herbs; simmer one-quarter of an hour, let it become cold, then boil the fish therein. Serve with anchovy-sauce and a squeeze of lemon.

\section{1.-COD FISH.}

Fresh cod is good boiled, fried, or made into a chowder. It is too dry a fish to broil. Salt cod should be soaked. in lukewarm water till the skin will wome off easily-then take up the fish, scrape off the skin, and put it in fresh water, and set it on a very moderate fire, where it will keep warm without boiling, as it hardens by boiling. It takes between three and four hours to cook it soft-serve it up with drawn butter.

\section{2.-TO BOIL COD FISH.}

Orimped cod is preferable to the plain; it is likewise better cut in slices than cooked whole; to boil it well, have the water ready boiling with one pound of salt to every six quarts ; put in your fish, draw your fish-kettle to the corner of the fire, where let it simmer slowly from twenty minutes to half an hour; when done, the bone in the centre will draw out easily; if boiled too much, it would eat tough and stringy; should the fish not be crimped, add more salt to the water, it will cause the fish to eat firmer.

\section{3.-COD FISH WITH OYSTER SAUCE.}

Boil three slices of the fish as above, drain and dress them upon a dish without a napkin, blanch three dozen oysters, by putting them into a stewpan, with their juice, upon the fire, move them round occasionally, do not let them boil ; as soon as they become a little firm, place a sieve over a basin, pour in the oysters, beard them, put the liquor again into the stewpan; when boiling, add two cloves, half a blade of mace, six peppercorns, and two ounces of butter, to which you have added a tablespoonful of flour, breaking it into small pieces, stir well together, when boiling, season with a little salt, cayenne pepper, and essence of anchovies; finish with a gill of cream or milk, add the oysters, and sauce over. The remains of the fish may be taken from the bone and placed upon a dish, with a little of the above sauce (to which you have added the yolks of two eggs), then sprinkle over with bread-crumbs, and place it twenty minutes in a hot oven till the breadcrumbs become brown.

\section{4.-COD SOUNDS.-RAGOŨT.}

The sounds should not be much soaked: but thoroughly cleaned. Simmer them for a short time, broil them, having first floured them; when they are just tender, stew them in white gravy which has been well seasoned, add a little cream, a bit of butter, a spoonful of flour, give it a boil, flavor with nutmeg, a small piece of lemon-peel, and a dash of pounded mace; serve.

\section{5.-SLICES OF COD.}

Three slices make a small dish; put them in a baking-dish, cover them over with some good second stock, a little essence of anchovies; when done thicken the stock, and pass it through a tammy, pour it over your fish, season with cayenne pepper, and salt, and lemon juice; if for capers add them, if for maitre d'hôtel, add cream and parsley chopped fine.

\section{6.-CODFISH AU GRATIN.}

This is the best mode of using the re 
mains of a dressed codfish. Put some cold oyster-sauce at the bottom of a piedish, then a layer of the codfish (seasoned with pepper, salt, and an atom of nutmeg), with any of the liver and sound that remains; then repeat the layers of sauce and fish until the dish is full; cover it with bread-crumbs, sprinkle a little butter over, and bake for about half an hour.

\section{7.-COD OMELETTE.}

Break into small pieces the thickest parts of a dressed cod, season it with a little grated nutmeg and a little pounded mace, beat up six eggs well and mix with it, forming it into a paste, fry it as an omelette, and serve as hot as possible.

\section{8.-FISH CAKE.}

Take the meat from the bones of any kind of cold fish, which latter put with the head and fins into a stewpan with a pint of water, a little sa!t, pepper, an onion, and a fagot of sweet herbs to stew for gravy. Mince the meat, and mix it well with crumbs of bread and cold potatoes (equal parts), a little parsley and seasoning. Make into a cake, with the white of an egg, or a little butter or milk; egg it over and cover with breadcrumbs, then fry a little brown. Pour the gravy over, and stew gently fifteen minutes, stirring it carefully twice or thrice. Serve hot, and garnish with slices of lemon, or parsley.

\section{9.-TO C()OK SHEEPSHEAD.}

Rub it with salt and lemon before put-

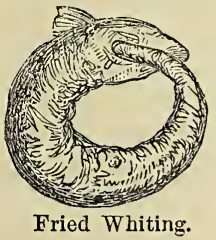

ting it into the water. To every six quarts of water add one pound of salt. Boil a twenty pound fish twenty minutes. Serve on a napkin; garnish with parsley, and eat it with shrimp or lobster sauce.

\section{0.-WHITING AND GRATIN.}

Skin the whitings, and turn their tails into their mouths; put them into a buttered sautépan, with a table-spoonful of chopped onions and four of brown sauce over each; sprinkle over them breadcruntbs and butter, and bake in a moderate oven half an hour. Dress them oll a dish without a napkin. Put twelve table-spoonfuls more brown sauce into the pan, with a tea-spoonful of chopped mushrooms, one of chopped parsley, one of essence of anchovy; pepper, salt, and sugar a little; pour round the dish, and brown it with a salamander.

\section{1.-HALIBUT.}

Halibut should be cut into slices of four pounds each, and may be baked or fried. The skin on the back must be scored. When baked, use a sufficient quantity of butter to keep it moist. If boiled, lay it in the kettle on a strainer, cover it with salted water; boil it slowly half or three-quarters of an hour and skim it well. Garnish it with horseradish, serve it with melted butter. To fry halibut, cut it in slices less than an inch thick; and with this. as with all other fish, take care to have plenty of butter, lard, or oil in the pan, and that it be hot before the fish is put in.

\section{2.-HALIBUT-STEWED.}

Put into a stewpan half a pint of fish broth, a table-spoonful of vinegar, and one of mushroom ketchup; add an anchovy, two good-sized onions cut in quarters, a bunch of sweet herbs, and one clove of garlic; add a pint and a halî of water, let it stew an hour and a quarter, strain 
it off clear, put into it the head and shoulders of a fine halibut and stew until tender; thicken with butter and flour, and serve.

\section{3.-TO COLLOP HALIBUT.}

Cut the fish into nice cutlets of about an inch thick and fry them; then put them into a broth mare of the bones, four onions, a stick of celery, and a bundle of sweet herbs boiled together for half an hour. Strain this broth, thicken it, and stew the fish for half an thour, adding salt. pepper, a grating of nutmeg and pounded mace, a spoonful of soy or fish-sauce, and half that quantity of lemon juice with a little shred lemon peel.

\section{4-HALIBUT A LA CREME.}

Rub the halibut with salt and lemon; put it to boil in a kettle, allowing one ounce of salt to every six quarts of water; simmer over a moderate fire. A halibut of eight pounds should simmer twenty minutes or more. When it begins to crack slightly, lift it with a drainer, and dish it without a napkin, having it first carefully drained, and absorbing the water that runs from the fish with a napkin. Put one pint of cream on the fire in a stewpan, and when near simmering add half a pound of fresh butter; stir it quickly till the butter is melted, but do not let the cream boil ; add three yolks of eggs, season with salt, pepper and lemon juice; pour as much over the halibut as will cover it, and serve the remainder in a boat. Or, if preferred, dish the fish on a napkin, garnish with parsley, and serve the sauce in a boat. This sauce must not be made until the moment it is wanted.

\section{5.-TO DRY HADDOCK}

Choose the finest you can obtain; clean them, remove the eyes, the entrails, and the gills; clear away also all the blood from the backbone. Wipe them as dry as you can with a clean soft cloth, and fill in with salt the spaces which contained the eyes; also rub in a quantity in the inside of the fish; lay them in a cool place on a dry flag-stone, or a piece of board for eighteen or twenty hours, then hang them in a dry place. Four days will be found quite sufficient to prepare them for eating.

136.-TO DRESS DRIED HADDOCK.

They should be skinned, rubbed with egg, and rolled in new bread-crumbs ; lay them in a dish before the fire to brown, baste with butter, and when well browned serve with egg sauce.

\section{7.-TO DRESS HADDOCK}

Clean them very thoroughly, and take off the heads and the skin; put them into boiling water, throw in two moderate-sized handfuls of salt; let them boil as fast as possible, and when they rise to the surface (which they will do, if they have sufficient room), they are done enough. They are sent to table with plain butter for sauce.

\section{8.-BAKED HADDOCK-(Soyer's.)}

Fill the interior of the fish with veal stuffing; sew it up with packthread, and truss it with the tail in its mouth, rub a piece of butter over the back, or egg and bread-crumb it over; set it on a bakingdish, which put in a moderate oven to bake; a common haddock would require but half an hour. The better plan is to run the point of a knife down to the backbone, from which if the flesh parts easily, it is done; dress it upon a dish without a napkin, and serve a sauce round.

\section{9. -TO BAKE HADDOCK}

Cut off the heads, trim and bone them, season with pepper and salt; chop very fine a small quantity of mushroom, onion and parsley; spread it over the fish, lay 
on them small pieces of butter, and place them in a dish with crumbs of bread; bake them from fifty minutes to an hour; skim the gravy, and serve up in the same dish as that in which it was cooked.

$$
\text { 140.-HERRINGS. }
$$

Herrings are dressed in a variety of fashions; they are fried, boiled, broiled, dried, potted, baked, smoked. pickled.

There are three sorts of herrings. fresh, salted, and red herrings; they are cleaned like any other sort of fish; when fresh they are boiled and served with melted butter, white sauce, \&c.; the salted herring should be soaked in cold water before it is cooked; this is broiled. Sometimes it is cut in pieces and eaten raw; the red herring is split down the back, the head and tail taken off, and the fish broiled like the others; they may be also dressed in the following manner: when they have lain in cold water some time, soak them in milk for two hours; then split them down the back; have ready some melted butter in which have been mixed basil and bay leaf minced"small; the yolks of two eggs, pepper and nutmeg; rub the herrings well with this bread, then broil them over a gentle fire, serve with lemon juice; the best red herrings are full of roe, are firm and large, and have a yellow cast; of the fresh herrings the scales are bright; if good, the eye is full and the gill red; the fish should be stiff.

\section{1.-FRESH HERRINGS BAKED.}

Wash the herrings in clear spring water; when they are thoroughly clean drain them. and then, without wiping them, lay them in a dish or baking pan; pepper and salt them; chop finely two or three onions. some parsley, thyme, and strew over them; cover them with equal proportions of vinegar and small beer; tie them over, and let them bake one hour in a slow oven.
They should be kept in the pickle, and make a pleasant dish when cold.

142.-TO POT HERRINGS.

Take from one to two dozen herrings, according to the number you purpose potting; choose them as large, fine, and fresh as you can. Take two ounces of salt, one of saltpetre, two of allspice ; reduce them to an inipalpable powder, and rub them well into the herrings; let them remain with the spice upon them eight hours to drain; wipe off the spice clean, and lay them in a pan on which butter has been rubbed; season with nutmeg, mace, white pepper, salt, and one clove in powder, one ounce each, save the last; lay in two or three bay leaves, cover with butter, and bake gently three hours.

When cool, drain off the liquor, pack the fish in the pots intended for their use, cover to the depth of half an inch with clarified butter, sufficiently melted just to run, but do not permit it to be hot; they will be ready for eating in two days.

\section{3.-POTTED HERRING.}

Take off the heads and tails; clean the fish and shake salt on them to draw out the blood; leave them one night. then rub them with black pepper and allspice. Pack them in a tin pan; put slices of onions and bay leaves between the layers, and pour on vinegar enough to cover them. Lay over them a sheet of buttered paper, or a crust of oatmeal bread; bake them till the bones are dissolved. A little mace, whole pepper, and butter improve them.

\section{4.-TO STEW MACKEREL-(New Way.)}

Take off the heads, the fins, the tails, and, having opened the fish, and taken out all the hard roes, dry them with a cloth, and dredge them lightly with flour; place three or four of them in a 
stewpan, with a lump of butter the size of a walnut, to each fish; put into a small basin a teacupful of water, a tablespoonful of finely chopped onions, the same of chopped parsley, a blade or two of mace, a little pepper and salt, a tablespoonful of anchovy essence, and a small teacupful of ale or porter (if not bitter). Add a tablespoonful of grated bread-crust, not burnt, but a light brown : pour all these ingredients over the fish, and let them stew gently for twenty minutes; have ready the yolks of three eggs, well beaten, and when the fish is sufficiently done, take some of the gravy and mix gradually with the eggs, and, pouring them on the fish, shake the stewpan a little over the fire to thicken the whole, but not to curdle the eggs ; the soft roes added are an improvement: have ready more grated crust, and having placed the fish whole in the dish, shake a little of the grated crust over the whole, so as to make it of a handsome brown. The receipt requires to be carefully followed. If the gravy is too thick, more water may be added; also a glass of sherry, if liked.

\section{5.-MACKEREL}

Are generally served up plain boiled; put them in a kettle containing enough boiling water according to the number, well salted; let simmer nearly half an hour, take them up, drain, and dish them upon a napkin ; serve melted butter in a boat, with which you have mixed a tablespoonful of chopped fennel, boiling it a few minutes.

\section{6.-MACKEREL.}

Cleanse the fish thoroughly inside and out, remove the roe carefully, steep it in vinegar and water, and replace it; place the fish in water from which the chill has been taken, and boil very slowly, from fifteen to twenty minutes; the best criterion is to be found in the starting of fish.) the eyes and splitting of the tail, when that takes place the fish is done; take it out of the water instantly, or you will " not preserve it whole. Garnish with fennel or parsley, and serve up as sauce, either, chopped fine in melted butter. Gooseberry sauzce is occasionally sent to table, but it does not suit every palate.

An English cook says: They should be carefully cleaned both inside and out; then washed in vinegar and water, and left to hang a little to dry before being put into the fish-kettle. A handful of salt should be put into the water, which should be at first cold, and only allowed to boil gently from fifteen to twenty minutes, though some prefer having the water boiling hot. The fish should be watched about that time, as "when the eye starts and the tail splits they are done, and should be immediately taken up ; if left in the water they will break."

The most customary sauce is that of fennel, which has partly superseded the gooseberry; but parsley and butter are still in use.

To B*oil.-Split them down the back, rub the inside with a little vinegar sprinkled with pepper and salt, flour them, broil on a quick fire, and serve, them up with melted butter, parsles, fennel, or lemon sauce. Or, if intended for breakfast, send up the fish plainly broiled without condiments or sauce, and merely rubbed, when done, with a bit of butter.

\section{7.-TO ROAST SHAD-(Sea-shore Receipt.)}

Split your fish down the back after he is cleansed and washed; nail the halves on shingles or shortboard; stick them erect in the sand round a large fire; as soon as they are well browned, serve on whatever you have; eat with cold butter, black pepper, salt, and a good appetite. (This is a delicious way of cooking this 
148.-FOR PRESERVING SHAD.

Place the shad, cleaned and washer, on a layer of salt in the bottom of the vessel, alternating the layers of shad and salt; the salt covering the top ones. Leave them twenty-four hours; then take them out and wipe them dry. For fifty shad, make a pickle of quarter of a pound of saltpetre and a pound of brown sugar mixed with salt enough to cure the fish; put the shad in alternate layers with this dry preparation; and put them away. When a shad is taken out to cook, it should be soaked a few hours before broiling.

\section{9.-TO BROIL A SHAD.}

Shad should be well washed and dried. It may be cut in half and broiled, or you may split it open and lay a small quantity of salt over it, and lay it upon a gridiron well buttered. It will broil in about twenty minutes, and should be thoroughly done. Melted butter may be served in a sauce-boat with it. Shad is even more palatable when baked than broiled. To bake Shad it should be stuffed with mashed potatoes and chopped parsley. Lay it in the oven on a pan which is well covered with butter chopped in pieces; baste the fish with the butter and add fresh quantities as fast as it is absorbed. Half or threequarters of a pound of butter should be used for large-sized shad.

\section{0.-TO ROAST PIKE-(Soycr.)}

This fish, in France, is found daily on the tables of the fish epicures. It is usually baked, when dressed plain. Having cleaned the fish, stuff it, and sew the belly up; butter a saucepan, (a shallow frying-pan,) put the fish into it, and place it in the oven for an hour or more, according to the size; when done, dish it without a napkin, and pour anchovy sauce round it. The fish, before baking, should be trussed with its tail in its mouth, and have four incisions cut in each side, and well buttered over.

\section{1-FISH CAKES.}

Cold boiled fresh fish, or salt codfish is nice minced fine, with potatoes, moistened with a little water, and a little butter put in, done up into cakes of the size of common biscuit, and fried brown in pork-fat or butter.

\section{2.-TO COOK CARP.}

Scour a fresh caught carp, and rub clean with salt and water; but scale him not; open him, and put him, with his blood and liver into a small pot; take a handful of sweet marjoram, thyme, or parsley, a sprig of rosemary and another of savory. Bind them in small bundles, and add them to the carp, with four or five whole onions, twenty pickled oysters, and three anchovies. Pour on claret wine enough to cover the carp, seasoned with salt, cloves, mace and lemon-peel. Cover the pot, and set it on a quick fire. When done, take out the carp, lay it with the broth into a dish. Pour over it a quarter of a pound of butter, melted and beaten with six spoonfuls of the broth, the yolks of two or three eggs, and some of the herbs shred; garnish the dish with lemons, and serve up, and much good to yout.

\section{3.-TU COOK TROUT.}

(Frank Forrester's Method.)

This is the method of the woods, and in the woods I learned it: The trout must be cooked in the open air, by a wood fire on the ground, or a charcoal fire in a small Boston furnace.

Clean and scale your fish; open, clean and wash the inside; for a pound fish take two small skewers of red cedar wood; upon each thread a piece of fat salt pork half an inch square; with 
these fasten the belly of the fish asunder, annex him by the tail to a twig of pliant wood, which suffer to bend over the fire so as to bring the fish opposite to the blaze; place a large biscuit, or a thin slice of dry toast, under the drip of the gravy; cook quickly. For a two pound fish ten minutes will suffice; dish with the biscuit under him, and eat with salt and lemon-juice, or with shrimp or lobster sauce; or a dash of Worcestershire or Harvey sauce; though I think these bad taste.

\section{4.-TO DRESS PICKEREL-(Nobbs.)}

Open and rub the fish within with salt and claret wine; save the milt and a little of the bloody fat ; cut him in two or three pieces, and put him into boiling water, with sweet marjoram, savory, thyme, or fennel, and a good handful of salt. Boil nearly half an hour. For the sauce, take butter, anchovies, horseradish, claret wine, a little of the blood, shalot, or garlic, and lemon sliced; beat all together, and serve.

\section{5.-STEWED CARP.}

Three carps will make a dish; put them in a baking-dish, cut up in thin pieces a carrot, turnip, onion, celery, a fagot of sweet herbs, a bay-leaf, a little mace, six cloves, whole pepper, some good second stock, six anchovies, half a pint of port wine; boil all this together, pour it over the carp while hot, put buttered paper over them, do them in the oven; when done, strain off tbe stock from the fish and thicken it, strain it through a tammy, add a glass more port wine, season it with sugar, lemon-juice, cayenne pepper, and salt.

\section{6.-SOLES, FRIED.}

Have about four pounds of lard or clean fat in a small fish-kettle, which place over a moderate fire; cut off the fins of the sole, and dip it into flour, skake part of the flour off, have an egg well beaten upon a plate, with which brush the fish all over, and cover it with fine bread-crumbs; ascertain if the lard is hot, by throwing in a few breadcrumbs; it will hiss if sufficiently hot; put in the fish, which will require nearly ten minutés' cooking, and ought to be perfectly crisp; drain it on a cloth, dish upon a napkin, garnish with parsley, and serve with shrimp sauce in a boat.

The above quantity of lard or fat, if carefully used and not burnt, would do for several occasions, by straining it off each time after using. All kinds of fish, such as eels, smelts, whitings, flounders, perch, gudgeons, \&c., are fried precisely in the same manner. Regulate the time according to the size of your fish.

157.-PIKE.

Clean and stuff the interior as directed for haddocks, only adding some fillets of anchovies, if handy, and chopped lemonpeel with it; curl round and put in a baking-dish, spread a little butter all over, put in a moderate oven; when about half done egg over with a pastebrush, and sprinkle bread-crumbs upon it ; a middling-sized pike will take about an hour, but that according to the size and the heat of the oven; when done, dress upon a dish without a napkin, and sauce round as directed for baked haddock.

\section{8.-TO FRY SMELTS.}

Dry them slightly in a cloth, and dip them in flour; then have half an ounce of butter or clear fat melted in a basin, into which break the yolk of two eggs, with which rub the smelts over with a brush, dip them in bread-crumbs, fry in very hot lard, dress them on a napkin, garnish with parsley, and serve with shrimp sauce in a boat.

159.-BROILED SMELTS.

When cleansed and wiped dry with a 
cloth, dip them lightly into flour, and put them upon a gridiron over a slow fire, for five or six minutes, turning them carefully when half done; serve plain, or with a little sauce. They are much relished by sick persons. I can highly recommend any kind of white fish cooked in this manner; and it is well known that nothing in the way of food is more digestible than fish.

Water souchet of flounders, soles, and slips may also be served to invalids, by proceeding the same as above.

\section{0.-STURGEON CUTLETS.}

Cut in slices one-quarter inch thick; dry, flour, and egg them; dip them in crumbs, seasoned with pepper, salt, parsley, and thyme; fry them, and serve with Indian pickle, tomato, or piquant sauce.

\section{1.-TO STEW STURGEON.}

Cut the fish in slices one and a half inch thick, djp them in vinegar, dry them well, flour, and broil the slices; then flour and lay them in a stewpan with some good broth, and let them stew gently until perfectly tender; thicken the gravy with butter or cream, add a spoonful of Harrey's sauce, one-half a glass of wine, and serve it up with capers strewed over the top, and garnished with slices of lemon.

\section{2.-ECONOMICAL MODE OF COOKING STURGEON.}

Take a piece of sturgeon about two pounds' weight, and on sending a piece of meat to the baker's to be baked on a stand in a dish, put the sturgeon under it, with a little water, salt, pepper, \&c., and a little chopped eschalot may be used; you can also put potatoes round it. Peas, if in season, are a good accompaniment, with melted butter.

\section{3.-SHRIMP TOAST-CROUTE AUX CRE-} VELLES-(French.)

Boil five pints of shrimps, and as soon as they are cold shell them; take the heads and bruise them in a mortar, put them into barely a quart of water, and let them boil an hour, strain them very clear, and add three parts to half the quantity of good veal stock. Put into a stewpan a lump of butter the size of an egg, and when it has commenced to bubble, stir in one teaspoonful of flour, a little grated nutmeg; a sprinkling of cayenne, and a small quantity of mace, the fourth of a teaspoonful; when this browns, pour in the stock gradually, adding a glass of vin de Bordeaux, and let it boil, then add the shrimps, cut off the bottom crust of a French loaf, hollow out the crumb, and fry the crust in fresh butter until a golden brown; as soon as the shrimps are thoroughly heated, which will be in about three or four minutes, pour them into the hollowed toast.

$$
\text { 164.-SMELTS.-(English). }
$$

This is a very delicate fish, requires delicate handling, and is quickly cooked; draw through the gills and wipe with a soft cloth, but do not wash them; dip them into the yolk of an egg beaten very smooth, and sprinkle them with breadcrumbs as finely as they can be powdered, a little flour may be mixed with the bread-crumbs ; fry them a clear light brown; four minutes will suffice to cook them.

The French method of serving is to skewer six through the gills with a silver skewer, and serve them in sixes. If dished, lay them head and tail alternately, serve with melted butter and garnish with parsley.

\section{5.-TO BAKE SMELTS}

Prepare as above, and instead of strewine forcemeat over them, employ 
only bread crumbs, and moisten with clarified butter; mix in addition to the gravy a glass of Madeira, with a dash of anchovies; this must be added before the smelts are laid in. They will be done in ten minutes.

\section{6.-TROUT.}

Scale, gut, clean, dry, and flour, fry them in butter until they are a rich clear brown, fry some green parsley crisp, and make some plain melted butter, put in one teaspoonful of essence of anchory, and one glass of white wine; garnish when the trout are dished with the crisped parsley and lemon cut in slices; the butter may be poured over the fish, but it is most advisable to send it in a butter tureen.

\section{7.-TO FRY TROUT.}

Scale, gut, and clean them; take out the gills; egg and crumb them; then fry in lard or oil until of a light brown. Serve with anchovy-sauce and sliced lemon.

\section{8.-IN THE FOREIGN MODE.}

Cover the bottom of a small oval paper form with a few very thin slices of fat bacon; cut down the back some nicely-washed small trout, and, having removed the bones, lay the fish open, flat upon the bacon; sprinkle with chopped parsley, pepper, salt, a little mace, and two cloves finely pounded. Bake onehalf hour in a quick oven, and serve in paper.

\section{9-TRUTTE ^ LA GENEVOISE.}

Clean the fish as above, lay them in a stewpan with two glasses of champagne, two glasses of sherry, a fagot of parsley, an onion stuck with cloves, thyme, pepper, and salt, and a piece of the well baked crust of French bread; stew on a quick fire, take out the bread when the fish is done, brown it, mix in butter rolled in flour, and boil up to thicken the sauce; the fish having been taken out when done, pour over them the thickened sauce, serve with lemon sliced and fried bread.

\section{0.-TRUITE À LA PRINCESSE ROYALE.}

Take equal parts of Madeira and water, and let them come to a boil, having emptied, cleansed, washed, and wiped perfectly dry the trout; lay them in; they should only be just covered with the liquor, and will be done in twenty minutes if not boiled too fast; take out the fish and thicken with a piece of butter rolled in flour; add two well beaten eggs with one teaspoonful of cream to the sauce, pouring them from one vessel to another until they are of a creamy consistency; season with salt, pour the sauce upon the fish, and serve.

\section{1.-TROUT A LA TWICKENHAM.}

When you have cleaned your trout, put them into a kettle of boiling water, to which you have added a good handful of salt, and a wine-glassful of vinegar; boil gently about twenty minutes, or according to their size; dress upon a napkin, and serve melted butter, into which you have put a table-spoonful of chopped gherkins, two sprigs of chopped parsley, salt and pepper, in a boat.

The remains of trout, salmon, or mackerel, are excellent pickled:-put three onions in slices in a stewpan, with two ounces of butter, one turnip, a bouquet of parsley, thyme, and bay-leaf, pass them five minutes over the fire, add a pint of water and a pint of vinegar, two teaspoonfuls of salt and one of pepper; boil until the onions are tender, then strain it through a sieve over the fish; it will keep some time if required, and 
then do to pickle more fish by boiling over again.

\section{2. -TROUT STEWED.}

This is a pleasing and delicate fish when nicely stewed. It is dressed very much in the fashion of other small fish stewed, only that it requires perhaps more care in the different processes.

First wash and clean the fish, wipe it perfe:tly dry; put into a stewpan two ounces of butter, dredge in as it melts, flour, and add grated nutmeg, a little mace, and a little cayenne. Stew well, and when fluid and thoroughly mixed, lay in the fish, which, having suffered to slightly brown, cover with a pint of veal gravy; throw in a little salt, a small fagot of parsley, a few rings of lemonpeel; stew slowly forty minutes; take out the fish, strain the gravy clear and pour it over the fish; it may be strained over it; before however, it is poured over; a glass of bucellas may be added to the gravy.

\section{3.-TURBOT.}

Place the turbot, previously to cooking, to soak in salt and water in which a little vinegar has been poured; lay it upon its back in the fish-kettle, fill the latter three parts full with cold water, throw in a handful of salt, a gill of vinegar, let it boil very gradually, and when it boils, add cold water to check; thirty minutes are sufficient to cook it ; serve it upon a cloth as boiled with its back to the dish; garnish tastefully with sprigs of parsley, and horseradish scraped into curls, or with fried smelts, or barberries, and parsley. Lobster sauce.

\section{4.-TURBOT Á LA CRÊME}

Is made from the remains of a turbot left from a previous dinner : pick all the flesh from the bones, which warm in salt and water, and have ready the following sauce: put one ounce of flour into a stew- pan, to which add by degrees a quart of milk, mixing it very smoothly; then add two peeled eschalots, a bouquet of parsley; a bay-leaf and a sprig of thyme tied together; add a little grated nutmeg, a teaspoonful of salt and a quarter ditto of pepper ; place it over the fire, stirring until it forms a thickish sauce; then take it from the fire, stir in a quarter of a pound of fresh butter, and pass it through a tammy; lay a little of it upon the bottom of a convenient-sized dish, then a layer of the fish; season lightly with a little white pepper and salt, then another layer of sauce, proceeding thus until the fish is all used, finishing with sauce; sprinkle a few bread-crumbs over, and put it into a warm oven half an hour; brown with the salamander, and serve upon the dish it is baked on. Any remains of boiled fish may be dressed the same way.

\section{5.-CRIMPED SALMON-A LA CRÉME.}

The salmon, like cod, must be quite fresh or it will not crimp. Cut the body into slices about two inches thick; have ready some salt and water in the proportion of three ounces of salt to a quart of water, with the smallest knob of salt. petre about the size of a nut; dip the salmon slices into this as they are cut, hold them for half a minute, and then rinse them in clear cold spring water, and lay them upon a dish; put a lump of butter well rubbed in flour into a stewpan; while the butter is melting sprinkle in a little salt and cayenne, and when the butter is on the simmer stir in half a pint of cream, keep stirring, and as it boils squeeze in the juice of a quarter of a lemon, and stir in a large teaspoonful of essence of anchovies; add a little more salt to taste.

Having boiled the crimped salmon slices in quick boiling water ten minutes, take them out and let them drain one minute, put them in a clean stewpan, and pour 
over the prepared cream and let it simmer ten minutes; it should not if possible be suffered to boil.

The lemon juice is sometimes deferred until the fish is removed from the cream, a minute's simmer is allowed, and it is then all poured over the salmon and sent very hot to table.

\section{6.-TO BOIL SAIMON.}

(Frank Forrester's own receipt.)

If you are ever so lucky as to catch a salmon, where incontinently you can proceed to cook him-that is to say, in the wilderness, within ten yards of the door of your own shantee, with the fire burning and the pot boiling-good.

Stun him by a heavy blow on the head; crimp him by a succession of cuts on each side, through the muscle, quite down to the back-bone, with a very sharp "knife, in slashes parallel to the gill cover. Then place him for ten minutes in a cold spring, or under the jet of a waterfall. Meanwhile, keep your pot boiling, nay, screeching with intense heat, filled with brine strong enough to bear an egg. Therein immerse him, having cut out the gills, open the belly, and wash the inside; boil him at the rate of seven minutes and a half to the pound; dish him, and serving him with no sauce, save a tureenful of the water in which he has been boiled, proceed to eat him, with no other condiments than a little salt and the slightest squeeze of a lemon. I do not object to cucumber sliced very fine, with a dressing of oil, three tablespoonfuls to one of vinegar, salt and black pepper to taste; but I regard green peas, or any vegetable, with this grand fish, as a Cockney abomination.

\section{7.-SOYER'S RECEIPT-SALMON AU} NATUREL.

Clean and prepare as before; but if he be not fresh enough to crimp, scale him and proceed as follows:
Put your fish in cold water, using a pound of salt to every six quarts ; let it be well covered, and set it over a moderate fire; when it begins to simmer, set it on one side the fire. If the fish weigh four pounds, let it simmer half an hour; if eight, three-quarters, and so on in proportion; dish it on a napkin, and serve lobster or shrimp sauce in a bowl.

\section{8.-TO BOIL SALMON.}

Salmon is dressed in various ways, but chiefly boiled in large pieces of a few pounds' weight. The middle piece is considered, if not the richest, yet the most sightly; then that adjoining the jowl; the tail part, though nearly as good, being usually kept for steaks. It requires great attention, and the boiling must be checked more than once; a piece of four to five pounds, will take nearly an hour, but if double that weight will not require more than twenty minutes beyond that time, and if crimped, still less will be sufficient: let it, however, boil slowly, in the hardest water, on a strainer placed in a large fish-kettle, and be thoroughly done, for nothing is more disgusting than fish that is under cooked; skim it well, or the color will be bad; the moment it is ready, lift up the strainer and rest it across the kettle, that the fish may drain; cover it with a thick cloth.

\section{9.-TO BAKE SALMON.}

Scale it, and take out the bone from the part to be dressed, but fill up the cavity with forcemeat, and bind the piece with tape. Then four it, rub it with yolk of egg, and put it into a deep bakingdish, covering it very thickly with crumbs of bread, chopped parsley, and sweet herbs, together with shrimps, if they can be got, and put into the covering a few small bits of fresh butter; place it in a Dutch oven, or, if already boiled and thus re- 
dressed, heat it only before the fire until browned.

\section{0.-SALMON, PLAIN BOILED-(Soyer.)}

I prefer always dressing this fish in slices from an inch to two inches in thickness, boiling it in plenty of salt and water about twenty minutes; the whole fish may be boiled, or the head and shoulders of a large fish, but they require longer boiling. Salmon eats firmer by not being put into the water until boiling. Dress the fish upon a napkin, and serve with lobster sauce, shrimp ditto, or plain melted butter in a boat with fresh sprigs of parsley boiled a few minutes in it. A salmon weighing about ten pounds will require an hour's gentle boiling; a head and shoulders weighing six pounds, half an hour; the remains may be dressed à la crême, as directed for turbot.

\section{1.-SALMON-TO BOIL.}

This fish cannot be too soon cooked after being caught; it should be put into a kettle with plenty of cold water, and a handful of salt; the addition of a small quantity of vinegar will add to the firmness of the fish; let it boil gently; if four pounds of salmon, fifty minutes will suffice; if thick, a few minutes more may be allowed. The best criterion for ascertaining whether it be done, is to pass a knife between the bone and the fish; if it separates readily, it is done; this should be tried in the thickest part; when cooked lay it on the fish strainer transversely across the kettle, so that the fish while draining may be kept hot. Place a fish plate upon the dish on which the salmon is to be served; fold a clean white napkin, lay it upon the fish plate, and place the salmon upon the napkin. Garnish with parsley.

\section{2.-BROILED SALMON.}

Dip each piece in flour, put it on a gridiron, turn occasionally; fifteen minutes will give it a nice pale yellow color; it should be served with Dutch, or caper sauce.

\section{3.-SALMON BROILED.}

Cut the fish in slices from the best part, each slice should be an inch thick; season well with pepper and salt; wrap each slice in white paper, which has been buttered with fresh butter; fasten each end by twisting or tying; broil over a very clear fire eight minutes. A coke fire, if kept clear and bright is best. Serve with butter, anchovy, or tomato sauce.

\section{4.-DRIED SALMON BROILED.}

Cut and cook as above, save that when it is warmed through, it is enough. Serve plain for breakfast, or with egg sauce if for dinner.

\section{5.-TO DRY SALMON.}

Cut the fish down, take out the inside and roe, rub the whole with common salt after scaling it; let it hang twenty-four hours to drain. Pound three or four ounces of saltpetre, according to the size of the fish, two ounces of bay salt, and two ounces of coarse sugar; rub these, when mixed well, into the salmon, and lay it in a large dish or tray two days; then rub it well with common salt, and in twenty-four hours more it will be fit to dry; wipe it well after draining. Hang it either in a wood chimney or in a dry place, keeping it open with two small sticks. Dried salmon is eaten broiled in paper, and only just warmed through, egg-sauce and mashed potatoes with it; or it may be boiled, especially the part next the head.

\section{6.-TO PICKLE SALMON.-The Newcastle Method.}

Put any quantity of salmon into an earthen jar ; cover it with equal parts of good vinegar and water; add cayenne pepper and salt in proportion to the fish, and bake it in a moderate oven. This 
pickle will keep a long time, with the addition of a little fresh vinegar; and if mace or cloves be added, with a few bayleaves laid in the mouth of the jar, it will be found an improvement. Trout may be preserved the same way. Fish thus pickled, must not be washed previously, but rubbed with a dry cloth.

Though generally eaten cold, yet in Newcastle it is not unfrequently warmed up in its pickle-liquor.

\section{7.-SALMON-TO PICKLE UNDRESSED.}

Scale the fish, rub well with a cloth, and scrape away all the blood about the backbone, but do not wash it; cut off the head, and divide the fish into pieces about six inches long; then boil the pieces in a pickle made of equal parts of vinegar and water, with a few cloves and two or three blades of mace until done. Skim carefully all the time the fish is boiling, and when done remove the fish and pour the liquor into a jar or tub, so that both may become cold; when cold, put the fish into the liquor, with one-third more vinegar, and some whole pepper.

\section{8.-SALMON POTTED.}

Cut a handsome piece from the middle of the salmon; remove the scales, and wipe it with a clean cloth. Rub into it some common salt thoroughly.

Beat up some mace, cloves, and whole pepper; season the salmon with it; place it in a pan with a few bay leaves; cover it with butter, and bake it until thoroughly done; remove it from the gravy, letting it drain thoroughly, then place it in the pots. Clarify sufficient butter to cover all the pots after the salmon has been put into them; put it to cool.

\section{9.-TO PICKLE SALMON.}

Scale, clean, split, and divide into handsome pieces the salmon; place them in the bottom of a stewpan, with just sufficient water to cover them.

Put into three quarts of water one pint of vinegar, a dozen bay leaves, half that quantity of mace, a handful of salt, and a fourth part of an ounce of black pepper.

When the salmon is sufficiently boiled remove it, drain it, place it upon a cloth. Put in the kettle another layer of salmon; pour over it the liquor which you have prepared, and keep it until the salmon is done. Then remove the fish, place it in a deep dish or pan, cover it with the pickle, which, if not sufficiently acid, may receive more vinegar and salt, and be boiled forty minutes. Let the air be kept from the fish, and, if kept for any length of time it will be found necessary to occasionally drain the liquor from the fish ; skim, and boil it.

\section{0.-COLLARED SALMON.}

Cut off the head and shoulders, and the thinnest part of the tail, thus leaving the primest part of the salmon to be collared. Split it, and having washed and wiped it well, make a compound of cayenne pepper, white pepper, a little salt, and some pounded mace. Rub the fish well with this mixture inside and out; roll and bandage with broad tape; lay it in a saucepan; cover it with water and vinegar, one part of the latter to two of the former ; add a table-spoonful of pepper, black and white whole, two bay leaves, and some salt. Keep the lid closed down. Simmer until enough, strain *off the liquor; let it cool; pour over the fish when cold, garnish with fennel.

\section{1.-COLLARED EELS.}

The eels destined to be dressed as above should be the fine $t$ which can be selected: the skin must not be removed, but the bone must be carefully and cleverly extracted. Spiead out the fish, 
and with some finely chopped sage, parsley, and mixed spices, rub the fish well over; then take some broad white tape, bind up the fish tightly; throw a good handful of salt into the water in which it is to be boiled, and a couple of bay leaves. Boil three-quarters of an hour, and if the fish be taken out and hung to dry for twelve hours, it will be the better for it when served. Add to the water in which the fish has been boiled a pint of vinegar, a little whole pepper, some knotted marjoram or thyme. This pickle also should, after boiling about twelve minutes, be suffered to stand as long as the eels are recommended to be hung; previous to serving, the fish must be unrolled so as to abrase the skin as little as possible, and put them into the pickle. Send up in slices or whole, according to taste; garnish with parsley.

\section{2.-EELS BREAD CRUMBED.}

Cut into pieces same length as above, cleaned nicely and well dried; let them be coated with yolk of egg, powdered with bread crumbs; fry them brown; serve with parsley and butter. Garnish with handsome sprigs of parsley.

\section{3.-SPITCHCOCKED EELS.}

There are several ways to spitchcock eels. They are either broiled or stewed. To broil them, see that the gridiron is cleansed and rubbed with suet, to prevent the adhesion of the skin of the fish, which must be suffered to remain on; cut the eels, which should be large, into lengths of six or seven inches, not less, and coat them well with yolk of egg. Pound in a mortar, parsley, nutmeg, mace, cloves, and pepper; this should be rubbed over the fish, and they should be broiled a clear brown; serve with melted butter, fish sauce, according to palate.

$$
\text { 194.-EELS FRIED. }
$$

Cut your eels into pieces three inches heat. long, trim them, dip the pieces into flour, egg over with a paste-brush, and throw them into some bread-crumbs; fry in hot lard as directed for fried soles.

\section{5.-EELS Â LA TARTARE.}

Fry as directed above, and serve on some Tartare sauce; or partly stew first, and, when cold, egg, bread-crumb, and broil gently. This last I much prefer.

\section{6.-STEWED EELS.}

Procure as large eels as possible, which cut into pieces three inches long, and put them into a stewpan, with an onion, a bouquet of two bay-leaves, a sprig of thyme and parsley, six cloves, a blade of mace, a glass of sherry, and two of water ; place the stewpan over a moderate fire, and let simmer about twenty minutes, or according to the size of the eels; when done, drain upon a cloth, dress them in pyramid upon a dish without a napkin, with a matelote sauce over, made as directed for salmon sauce matelote, but using the stock your eels have been cooked in to make the sauce, having previously well boiled it to extract all the fat.

\section{EET PIE.}

Take six skinned eels, remove the heads, and cut them into pieces four inches long; add two dozen oysters, boil them together in a very little water; take out the oysters, and bone the eels by passing them through a sieve.

Take six hard boiled eggs, parsley, fried herbs, lemon-peel, black pepper, salt, mace, nutmeg, and allspice; the whole ground very fine; add these to the eels and oysters with the broth and a bit of fresh butter.

Take a large, deep dish, of yellow earthen ware; put the mass into it, and cover the dish with a fine pastry crust. Bake it in a very hot oven from half an hour to three-quarters according to the 


\section{8.-SHELL FISH.}

Although crabs and lobsters may be bought the whole year round, they are yet only in high season, from the month of July till the close of October. They should be always purchased alive.

If lobsters have not been long taken, the claws will have a strong motion when you put your finger on the eyes and press them. The heaviest, if of good size, are the best, but the largest are not the best. When you buy them ready boiled, try whether their tails are stiff, and pull up with a spring; if otherwise, they are either watery or not fresh. The "cock-lobster," as the male is called is known by the narrow back part of his tail, and the two uppermost fins within it are stiff and hard; but those of the hen are soft, and the tail broader. The male, though generally smaller, has the highest flavor; the flesh is firmer, and the color, when boiled, is a deeper red ; but the female has that fine coral so highly prized by cooks for the improvement of their sauces, which appears with the rudiments of the spawn.

\section{9.-TO BOIL LOBSTERS.}

Put them alive, with their claws tied together, into the water when boiling hot, and keep it so until the fish is done, which, if of a pound weight, will take about a quarter of an hour, and if larger will require not quite the same proportion of time, for if boiled too long the meat will be stringy. Many people are shocked at the apparent cruelty of thus killing them, but death takes place immediately, and life cannot be taken away without pain.

When sent to table to be eaten cold, the tail and body should be split from end to end, the claws cracked, but not unshelled, and the meat may be made into salad, or mixed in such manner as each person pleases, and many persons add a teaspoonful of white powdered sugar, thinking that it gives a mellowness to the whole. It is scarcely necessary to mention that the head" of a lobster, and what are called the "lady-fingers," are not to be eaten.

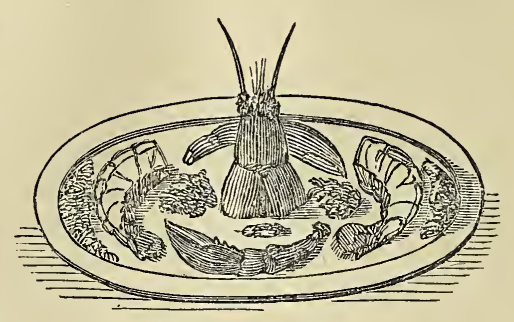

Lobster as served.

200. - TO STEW LOBSTERS.

Pick the meat out of the shell, put it into a stewpan with half a pint of good gravy and a glass of claret; add an onion minced fine, some sweet herbs, and pepper and salt, stew till tender; add a large spoonful of mushroom ketchup, one of essence of anchovy, and a lump of butter rolled in flour. Serve hot, and garnish with bread sippets. If brought on cold as a supper dish, serve in a shape covered with clear jelly.

\section{1.-BOILING LOBSTERS.}

Put the lobsters into boiling water. with a little salt, and boil them till they are cuoked through; the color of the shell is of importance, and is made bright by rubbing the shell with sweet oil after it is wiped. Split the body and tail through, and crack the claws. It is then fit to go to the table, where it must be cut up fine before eating. A dressing, made of salt, mustard, oil, cayenne pepper and vinegar, mixed with the yolk of an egg, is usually prepared for it. The white of an egg (boiled hard, of course,) may be minced fine and strewn over it. 


\section{2.-LOBSTER CURRIED.}

Take the meat of a fine lobster, or two, if they should be small, place in a stewpan two dessert-spoonfuls of curry powder, add of butter two ounces, an onion cut in very fine strips, and three large spoonfuls of fish stock. When they are stewed well, add the lobster, simmer gently for an hour, squeeze in half a lemon, season with a little salt. In the eastern method the expressed iuice of spinach is usually added. This is obtained by simply putting spinach, without any water, into a saucepan, and when done enough, press out the juice, and add it with butter, cayenne, and salt, to the gravy. Prawns may be dressed in this fashion.

\section{3.-CROQUETTES OF LOBSTER.}

Take the meat from the shell, chop it finely, mix it with a little salt, lepper, and pounded mace; take one-fourth part of fine bread crumbs, make it up into balls with melted butter, brush the balls with yolk of egg, and dredge them with bread crumbs, and fry them, serving with or without gravy: if dry, they must be sent up with crisped parsley.

\section{4.-GRATIN OF LOBSTER.}

Take out all the meat from a large lobster, then wash the body, tail, and shells if the lobster is first cut in halves down the back; then dry and butter them, and sprinkle them with bread crumbs, chop up the meat fine, with a little parsley and shalot, a few drops of essence of anchovies, a spoonful of vinegar, cayenne pepper and salt, a little bechamel sance, and boil all well together; then add a yolk of egg, put it to cool, then fill your shells or paper cases, cover it with hread crumbs and some pieces of butter, brown them in the oven, and dish on a napkin.

\section{5.-LOBSTER SALAD.}

Extract the fish from the shell, place it in the centre of the dish in which it is to be served, in the form of a pyramid; arrange the salad round tastefully, and add salad mixture. This dish is not infrequently garnished with the smallest claws of the fish. This is a matter of fancy-or it may be formed into a heap, ornamented with the claws of several lobsters. The first row is formed of cut . cucumbers, the second of eggs boiled hard, and each egg split into four pieces, and the points laid round the salad; the third and bottom row is composed of slices of beet-root and lobster.

206.-LOBSTERS, IN AN ITALIAN SALAD.

Take two lobsters, cut them into pieces by taking off the claws and tail, each of which split in two; the spawn rub through a dry sieve to garnish the salad. made in the following manner : wash two or three cabbage lettuces, cut them in large shreds. slice a beet-root and cucumber, wash, pick, and cut into long shreds four anchovies, chop some tarragon and chervil, two boiled eggs, the yolks and whites chopped separately; if you have any cauliflowers or French beans, boil and put them with the other things to garnish. Having every thing prepared, place the lettuce in the centre of the dish in a heap, and place the lobsters and other things according to your taste, and just before you serve garnish with Italian salad sauce.

$$
\text { 207.-LOBSTER SAIAD.-(Suyer's.) }
$$

Dress a border of hard-boiled eggs, as directed in salad of game, fill the centre with some nice fresh salad, then take the flesh from a middling-sized lobster, which cut into as large slices as possible, which put into a basin, and season with a little peppcr, salt, oi ${ }^{\prime}$, and vinegar, after which dress them pyramidically upon the salad 
and have ready the following sauce: put the yolks of two fresh eggs in a basin, with the yolk of a hard-boiled one rubbed through a sieve ; add half a saltspoonful of salt, and half that quantity of white pepper, and commence stirring round with a wooden spoon with the right hand, holding a bottle of salad oil in the left, dropping it in by degrees and continually stirring; when becoming thickish add a couple of spoonfuls of common vinegar by degrees, still keeping it stirred, then more oil, proceeding thus until you have used three parts of a pint of oil, and a corresponding quantity of vinegar ; by continually working it will form a stiffish cream-looking sauce perfectly smooth; add a little more seasoning if required, and a teaspoonful of chopped parsley, with half that quantity of chopped eschalots; pour over the lobster and serve. Should the sauce curdle in making, the operation must be again performed, putting a yolk of an egg into another basin, working it with a little oil until forming a stiffish paste, then stir in the curdled sauce by degrees until the whole becomes smooth; always choose a cool place to make it in. This requires a little practice.

208.-ANCHOVIES, ESSENCE OF.

A pound of the best anchovies, two quarts of water, two bay-leaves, some whole pepper, a little scraped horseradish, a little thyme, two blades of mace, six shalots chopped small, a gill of port.wine, half the rind of a lemon, a gill of ketchup; boil them together for twenty minutes, then rub them through a tammy with a wooden spoon; when cold put it into pint bottles, cork them close, and keep them in a dry place.

209.-TOAST OF ANCHOVIES.

Prepare toast; fillet some anchovies, ster, is looked upon by many as being pound them in a mortar, add a little butter well pounded into it, a little cayenne pepper, a few drops of lemon-juice; take it out and spread it on the toast.

\section{0. - ANCHOVIES WITH FRIED BREAD.}

Cut some bread thin, then cut out with a plain paste cutter the quantity you require, as you will put one on the other; fry them in lard a very nice brown, then fillet and pound anchovies as before; add a little parsley, and a grain of shalot, rub all through a fine wire or hair sieve, spread one of your toasts rather thick, place another piece of bread on the top; have ready some more filleted anchovies, and garnish each toast, using pickles likewise, or parsley.

\section{1.-TO KNOW GOOD ANCHOVIES.}

The best look red and mellow, and the bones moist and oily, the flesh high flavored, and a fine smell ; if the liquor and fish become dry, add to it a little beef brine.

212.-CHOWDER -A SAILOR'S DISH.

Cut salmon, halibut, or any rich fish, into steaks an inch thick; season them highly with pepper, salt, and cayenne; put a layer of sliced potatoes (raw) in the bottom of the stewpan; next the fish; then a layer of thin sliced ham. Fill the stewpan in this manner; finish with a large piece of butter. Add water enough to moisten the whole; stew slowly two hours, never stirring, but occasionally shaking the pan to prevent it burning to the bottom. If it gets too dry, add a little more water.

$$
\text { 213.-CRAB, }
$$

Though not so well-known as the lobster, is looked upon by many as being a better-flavored fish, and perhaps rather then a layer of broken cabin-biscuit; 
more digestible. The female is considered inferior to the male, and may be known by the claws being smaller, and the tail much wider. The heaviest are usually thought to be the best; but those of a middling size are the sweetest. If light, they are watery; when in perfection, the joints of the legs are stiff, and the body has a very agreeable smell. The eyes look dead and loose when stale, or when the fish have died a natural death. They are boiled in the same manner as lobster, but require rather longer time, and are most usually eaten cold with oil and vinegar, as thus:-Pick out all the fish from the shell, divide it into small pieces, mixing the rich part well with the rest; moisten it with salad dressing, and return it to the shell with an edge all round with sliced lemon.

If hot, pick the fish out as above; then put the meat, with a little nutmeg, salt, pepper, bits of butter, crumbs of bread, and three spounfuls of vinegar, into the shell again, and set it before the fire. You may brown it with a salamander, but it should be always served in the shell. Dry toast should be served to eat with it. Observe to remove "the lady," as it is called.

\section{4.-TO BOIL CRABS.}

Having boiled them twenty minutes, wipe them, crack the claws, rub the shells with oil, and dish them as lobster. 'To cook soft crabs, take away the claws, cut them open, and remove the sand bag and spongy part; then put some butter into a frying-pan, and do them brown on both sides.

\section{5.-TO STEW CRABS.}

Pick the meat carefully out of a large crab and its claws; cut into small pieces, mix it with about a fourth part of breadcrumb:, and a very small quantity of fin ly: shred parsley. Season it well, and return it to the shell with some small bits of butter here and there, enough, when warmed, to keep it moist. Squeeze the juice of a lemon over it, or a spoonful of lemoli-pickle or acid sauce. Put a thick layer of crumbs of bread upon the top with small bits of butter laid all over it, and bake it in the shell before the fire, or in the oven. The shell of one crab will contain the meat of two.

Or:-Boil them, take the meat out of the bodies and the large claws, put it into a stewpan with half a pint of claret, a spoonful of eschalot-vinegar, a little cayenne, some salt, and a piece of butter : let them stew for an hour over a gentle fire, until they are almost dry. Then add a small quantity of fish-stock or gravy, a table-spoonful of essence of anchovy, and a small piece of butter rolled in flour. Serve with sippets of fried bread round the dish.

Another way is to put the meat into a stewpan with half a pint of white wine. a spoonful of eschalot or garlic vinegar, a little parsley and thyme minced fine, the yolks of three eggs boiled hard and minced small, and some salt and cayenne pepper. Let it stew gently till quite tender and almost dryv; then add a piece of butter. stir it about for a few minutes over the fire, wash the shells, butter them, put the stewed meat into them, and serve on a napkin.

\section{6.-SMALL CRABS AND CRAYFISH}

Are made sometimes into soup, but more commonly plain, boiled for about a quarter of an hour and eaten cold. In the lower parts of Germany they are eaten hot, with rye-bread and butter, and are there a favorite supper dish in the heat of summer.

\section{7.-TO STEW CR $\Lambda$ YFISH.}

Boil them in salt and water, pick the meat out of the tails and claws, put them 
into a stewpan with a little butter, some mushrooms, and truffles; moisten them with a little fish-stock, and simmer a short time over a gentle fire. When nearly done, beat the yolks of two or three eggs with a teacupful of cream and a little chopped parsley; let all stew together for a few minutes, stirring it all the time, and serve up in a deep dish.

\section{8.-SOFT SHELLED CRABS.}

Fry the crabs in lard, having taken off the spongy substance, and the sand bag. Crisp parsley in the fat after they come out, and add pepper and salt and rich milk, for the gravy.

\section{9.-TERRAPINS.}

Put them in boiling water to kill them; then skin them and take off the nails, wash and put them on again to boil, adding a teaspoon of salt to every two. When tender, remove the shells, sand bags, and gall, carefully ; cut up the meat, season it with pepper, mace, and nutmeg, and put it in a stewpan with the juice that has come out, and for every two terrapins, four ounces of butter in flour, a glass of white wine and the yolks of two eggs beaten. The wine and eggs to be added after the whole is stewed perfectly tender.

\section{0.-CRABS MINCED.}

Remove the meat, mince small and place in a saucepan with a wineglassful of white wine, pepper and salt, nutmeg, cayenne pepper, and two table-spoonfuls of vinegar. Let it stew for ten minutes; melt a piece of butter the size of a hen's egg, with an anchory and the yolks of two eggs ; beat up and mix well, stir in with the crab, and add sufficient stale bread-crumbs to thicken. Garnish with thin toast cut with a pastry leaf-cutter, or with the claws, and parsley. Lobster may be dressed in the same manner.
221.-CRAYFISH BUTTER.

Take the shells of twenty-four crayfish, clean them well from skin and fibre, put them into an oven to dry, without burning or browning, until they can be beaten to a fine powder; mix the powder very well with three ounces of fresh butter, then put it into a stewpan with a spoonful of hot water, mixing it well together; then squeeze the whole through a tamis over a stewpan of boiling water; skim and butter off into a basin of cold water, that it may set; when cold press it in a napkin.

It forms a beautiful coloring addition to many kinds of dishes, fish especially, and, when mixed with the meat pounded after being taken from the shells, makes fine sauce.

\section{2.-OYSTERS}

Depend very much for goodness upon the beds from which they have been taken. There are several kinds.

They all come into season, according to an old saying, "so long as there is an $\mathrm{R}$ in the month." They are, however, not perfectly good until the beginning of October, and should go out at the end of March.

When the fish is alive and strong, the shell closes on the knife. They should be eaten as opened, the flavor becoming poor if long exposed to the air.

\section{3.-TO SCALLOP OYSTERS.}

Take twelve of the smaller sort, beard them, cut out the hard part which adheres to their shells, and leave them in their liquor; have ready a quantity of crumbs of fresh bread, not too finely grated, and mixed with a little pepper and salt; then grease a scallop-shell, strew upon it some of the crumbs with bits of butter, and lay upon them a layer of the oysters; then crumbs, bits of butter, and oysters, layer upon layer. 
until the shell is filled up; cover it with a thick coating of the crumbs well buttered, and brown it in a Dutch oven. A dozen oysters, with a proper quantity of crumbs, will fill up the largest scallopshell, and take an hour to be thoroughly done.

Some cooks scald the oysters for five minutes, in their own liquor, and mix with them minced shalot, or chives, and jot-herbs; but these, although they may please an epicurean palate, will destroy the natural flavor of the oyster.

\section{4.-TO SCALLOP OYSTERS.}

For one hundred oysters, take four eggs, boiled hard, and chopped very fine ; mix them with a sixpenny loaf crumbled or grated, and add pepper and salt to taste. Put some of the mixture in the bottom of a pie dish, and lay on it lightly a layer of oysters, previously rinsed and drained; add a few bits of butter, then cover the oysters with a layer of bread-crumbs and eggs; another layer of oysters, and so on ; covering the top with bread-crumbs. Bake it three-quarters of an hour.

\section{5.-TO MAKE OYSTER FRITTERS.}

Make a batter with milk, flour, and eggs, beaten light. Have some lard quite hot; dip out a tablespoonful of the batter, put an oyster into it, and let it down into the boiling lard carefully and fry it on both sides. This is a better plan than mixing the oysters and batter together.

\section{6.-TO BROIL OYSTERS.}

Take them from the shells, beard them, and put them with their liquor into tin shapes made to imitate scallops, six in a shell (not more), with a little pepper and butter. Put the shells upon a gridiron over a good fire, and serve them when plump and quite hot. They are delicious this way; but to be eaten in perfection should be cooked in the room where they are eaten. Squeeze a little lemon-juice over them when ther come from the fire.

Or :-They may be put singly in their under shells along with their own liquor, a little minced parsley and spice, and a bit of butter, and thus put upon the gridiron, to be taken off when thoroughly heated.

\section{7.-WITH WIITE SAUCE.}

Beard the oysters, wash them in their own liquor, then strain it, thicken it with melted butter, or white sauce made of cream, not milk, and flour ; season it with a blade of mace and a few whole peppercorns tied in a muslin bag. Simmer the oysters very gently, and serve up with sippets of bread: they will require only a few minutes, and if allowed to boil will become hard.

\section{8.-WITH BPOWN SAUCE.}

Wash the oysters in their liquor, and then strain it, add a glass of wine, two spoonfuls of beef-gravy, some whole pepper, a little salt, a piece of butter, and a spoonful of lemon-juice; boil the whole, stirring it until it is smooth, then put in the oysters, and warm or plump them up without boiling.

\section{9.-OYSTER ROLLS.}

Take about a quart of the largest and finest oysters you can procure, stew them in their own liquor with some pepper, a very little mace, and some green onion chopped fine, thicken them with a little butter and a dust of flour when nearly done enough. Take two French rolls of the square sort baked in tins, cut a piece off the top, and scoop out the greater part of the crumb, fill your roll with the oysters and the liquor, and set them near the fire on a chafing-dish filled with hot coals; as the liquor soaks in fill them with more, or if you have not any left, add a little good gravy boilinghot. 
Or :-Let them first soak; then fasten in the top, cover the entire of the roll in batter, and bake it in the Dutch oven till well browned.

Or:-Mince the oysters, season them only with mace and nutmeg, and fill the rolls up with cream or rich white sauce, and bake them as above: even one large roll will make a nice little dish, and is better than the smaller sort.

\section{0.-OYSTER SAUSAGES}

May also be made of the larger kind, bearded and minced small, together with a moderate quantity of beef suet and bread-crumbs, to which should be added a forcemeat of pounded pork or veal, seasoned with spice and put into the usual skins. If well seasoned, they will keep good for a full week: one pound of beef suet shred fine, and the same quantity of forcemeat, with bread-crumbs, should be put to each pint of oysters.

Or:-Take one-half pound of lean beef or mutton, three-quarters pound of beef suet, two score of oysters bearded and scalded in their own liquor, then dried and all chopped together, adding bread-crumbs and yolks of eggs to bind the materials. Season well with salt, white pepper, mace, and a grate of nutmeg; or if you wish to make it very savory, leave out the mace and nutmeg, but add a little cayenne, with a minced shalot and a spoonful of garlic-vinegar. They may be either made as sausages, or fried into shapes in the usual way.

\section{1.-OYSTERS À L'IMPERIALE.}

Procure, quite fresh, a barrel of oysters, packed as for transmission to the country. Put into a vessel large enough to contain the barrel sufficient water, that when the barrel is in, it may be covered. Heat the water to a boil; when it is boiling, put in the barrel of oysters just as you have received it from the oysterseller; let it boil twelve minutes; take it out, knock off the head, and serve immediately. The flavor of the hot oyster will be found delicious.

\section{2.-OYSTER CURRY.}

Blanch and beard six dozen oysters, leaving them in their own liquor; then cut two middling-sized onions into small dice, and sauté them in a stewpan, with an ounce of butter; when done, mix in two teaspoonfuls of curry powder and one of curry paste, and pass all through a tammy-it ought to be thick; then add the oysters with their liquor, and keep stirring over the fire until the oysters become enveloped in a thick sauce, which they should be in two minutes, when turn them out upon your dish, and serve with rice separately.

\section{3.-ROAST OYSTERS.}

Large oysters not opened, a few minutes before they are wanted, put on a gridiron over a moderate fire. When done they will open; do not lose the liquor that is in the shell with the oyster; send them hot upon a napkin.

\section{4-AN OYSTER PIE, WITH SWEET- BREADS.}

Blanch them and take off the beards, separate them from the liquor, blanch some throat sweetbreads, and when cold cut them in slices, then lay them and the oysters in layers in your dish, season with salt, pepper, a few grains of mace and nutmeg; add some thick sauce, a little cream, and the oyster liquor, and some good veal stock; bake in a slow oven.

\section{5.-BREADED OYSTERS}

Scoop out the crumb from a small loaf, or some small rolls of bread, and put into the cavity oysters stewed with butter and mace, and a little of their liquor. with two or three spoonfuls of rich milk 
added as they are done. Put on the top of the rolls, the pieces sliced off; set them in the oven a few minutes, and serve on a dish, hot.

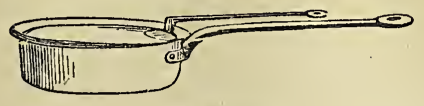

Stewpan.

\section{6.-STEWED OYSTERS.}

The oysters should be bearded and rinsed in their own liquor, which should then be strained and thickened with flour and butter, and placed with the oysters in a stewpan; add mace, lemonpeel cut into threads, some white pepper whole; these ingredients had better be confined in a piece of muslin. The stew must simmer only; if it is suffered to boil, the oysters will become hard; serve with sippets of bread. This may be varied by adding a glass of wine to the liquor, before the oysters are put in and warmed.

\section{7.-SCALLOPED OYSTERS.}

Beard the oysters, wash in their own liquor, steep bread-crumbs in the latter, put them with the oysters into scallop shells, with a bit of butter and seasoning of salt, pepper, and a little grated nutmeg; make a paste of bread-crumbs and butter; cover, and roast them before the fire, or in an oven.

\section{8.-PICKLED OYSTERS IN THE FRENCH} WAY.-(A supper dish.)

Take four dozen oysters, strain the liquor, add six blades of mace. twelve peppercorns, a little grated lemon-peel, and two or three bay-leaves. Put the liquor to boil; when boiling, add the oysters for two minutes. (Some persons put half vinegar, half liquor.)
When cold, strain off the liquor. Place the oysters in a small dish, and garnish with parsley.

\section{9.-PICKLED OYSTERS.}

(By a Lady of New York.)

Scald the oysters in their liquor, or in water with a little salt; take them out with a skimmer, and throw them into cold water. Take whole allspice, black pepper and mace, and boil it up in the liquor in which the oysters were boiled; when it tastes enough of the spices take it off. Let the oysters drain on a sieve. When the liquor is cold add vinegar to taste, and then put in the oysters.

\section{0.-TO DRESS COLD FISH.}

Dip a flat dish in hot water, to prevent cracking; smear it with butter, and sprinkle white pepper on it; then a thick layer of stale bread, grated fine; a layer of the fish, picked from bones, and broken small; a little melted butter -prepared without milk-poured over another layer of bread-then of fish, with butter as before; repeated as often as required for quantity of fish, and size of dish. Smooth the surface with a spoon, and sprinkle slightly fine bread, mixed with white pepper on the top. Place it for twenty or thirty minutes, according to thickness, before a brisk fire, with a tin shade at back of dish, to reflect the heat. Cold washed mutton may be redressed the same way; first wiping the meai, quite free from gravy, in a napkin.

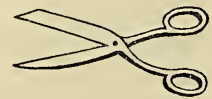

Fish Scissors, to trim pan-fish for frying.

241.-CASSEROLE OF FISH

Is a title given. among others, by 
French cooks, to "poisson rechauffe,", or fish which has been left after being dressed, and is meant to be re-heated. There are almost as many modes of doing this, as of originally dressing the various sorts; but we here only retain a few of the most simple:-

Take any kind of cold fish, and divide it into large flakes; boil two or three eggs hard, and cut them into slices; have also some mashed potatoes; butter a mould, and put in the fish, eggs, and potatoes, with a little delicate seasoning of white pepper; moisten the whole with cream. or thin melted butter, and a spoonful of essence of anchovies; boil the mould and turn it out.

Or:-Take some fish which has been dressed, and rub it through a sieve; to half a pound of fish allow a quarter of a pound of bread-crumbs, two eggs well beaten, one tablespoonful of essence of anchovies, one of Harvey sauce, and a little salt and cayenne pepper ; mix all well together, and put it into a mould; let it boil half an hour, and serve it with a good fish-sauce in the dish.

Or:-Take half a pint of good gravy, with a couple of glasses of wine, two tablespoonfuls of anchovy liquor, and two of vinegar, seasoned high with cayenne, mustard, salt, and shalot; mince the fish, but do not put it in the mortar, and either warm it as a fricassee, and bring it up in a dish with sippets; or, put it into a form, piled up high in the centre, cover it with bread-crumbs and bits of butter, and brown it with a salamander.

\section{2.-FISH TURTLE. (English.)}

For this imitation, sturgeon is the best material, but, if not in season, cut some ling into handsome pieces, and fry it; then boil an equal quantity of skate, also cut in piecos; and having cleaned and soaked two or three cod-sounds, stew them until green, with a little spinach, and cut them into pieces; then have a sufficient quantity of good gravy, into which the Jiquor of two or three dozen of oysters has been strained ; thicken it with cream or butter; put it into a stewpan with the fish already named, a lobster cut in pieces, a spoonful of essence of anchovies, and a glass of Madeira. Warm the whole together, and send it to table with a lemon garnish. The sauce to this dish must be very rich, and of a fine dark color.

\section{3.-FISH PÂTÊ.}

This is a pretty mode of enclosing a fricassee of fish with a potato wall without a crust of pastry. Mash in a mortar as many potatoes as you may want, with a good piece of butter; then, with the bowls of two silver spoons, raise a wall of it two and a half inches high within the rim of the dish to be used. Let the upper part be a little thinner than the lower; smooth it; and, after brushing it all over with egg, put it into the oven to become hot, and a little colored. Before egging it, the outside may be ornamented with flowers, leaves, \&c., by the small tin shapes used to cut paste.

Beat the whole of a crab picked clean from the shell in a marble mortar, with white pepper and salt, nutmeg, and a very few crumbs of bread; warm it with a little gravy thickened with cream or butter and a spoonful of wine, and, when thoroughly warmed, add a little lemonjuice. Pour it into potato walls previously baked, but not covered like a pie, and serve it up hot, either browning it with the salamander, or covering the top with fancifully arranged small claws.

\section{4.-FISH CAKE.}

Cut the meat from the bones, put 
them, the head and fins, over the fire to stew for gravy, with a pint of water, an onion, herbs, pepper, and salt. Mince the meat, put to it one-third part of crumbs of bread, a little minced onion, parsley, pepper, salt, and a very small bit of mace ; mix well, and make it into a cake with white of egg and a little melted butter ; cover it with raspings, and fry it a pale brown, keeping a plate on the top while doing. Then lay it in a stewpan, with the fish gravy, and stew it gently a quarter of an hour ; turn it twice, but with great care not to break it: cover it closely while stewing.

Cake of dressed meat, done in the same way, is remarkably good.

\section{5.-FISH-CUTLETS.}

Chop a considerable quantity of herbs with a small piece of shalot, season it with pepper and salt, and put it into a stewpan with two ounces of butter; as the butter is melting add a teaspoonful of essence of anchovies. Do not allow the butter to more than melt, and mix the whole well' together; then cut any kind of white fish, dressed or raw, into handsome cutlets, and, when the herb seasoning is nearly cold, spread it on the fish thickly with a knife; dredge the fish with bread-crumbs, and cook them on buttered pans in an oven, or before the fire. Stew a few silver button-onions, or a chopped onion, with any green vegetables in season, cut it into dice in a little broth, add nasturtiums and a little of the pickle; keep them in the middle of a dish, and lay the cutlets round.

Or:-Take any fish previously dressed, pull it in pieces, and mix it with a little good stock, and any fish sauce which may have been left from table; spread it on a flat dish, brush it with egg and sprinkle thick with bread-crumbs, cut it out in cutlets, and fry brown.

\section{6.-KEDGEREE FOR BREAKFAST.}

Boil two tablespoonfuls of rice, add any fish previously cooked (salmon or turbot is preferable), and nicely picked; beat up an egg well, and stir it in just before serving. The egg must not boil.

\section{7.-FRICANDELLES OF FISH.}

Take any quantity that may be convenient of either dressed or undressed fish of firm quality; skin and bone it; mince it of the size of dice, with a few anchovies, say two to each pound, seasoned with mace, cayenne, and a grating of nutmeg; soak the crumb of a French roll, one to each pourid, in milk, and beat it up with the yolks of two eggs to each roll, so as to make it into a purée, and put the fish into it; warm the whole gently, and add to it a moderate quantity of cream.

Put the fish thus prepared into a buttered mould, cover it thickly with breadcrumbs, and either bake it in a Dutch oven, or warm it before the fire, and brown it with the salamander.

A few oysters are an excellent addition; and if wine be employed instead of milk, the dish may be dressed in the same manner, but rather more highly seasoned.

\section{8.-A MATELOTE OF FISH. (English.)}

Take carp or tench, or both, together with an eel and any smali fish; cut them into pieces, and put them along with a quantity of button-onions into a stewpan containing just wine or gravy enough of any sort to cover them, and let them stew very gently until nearly done ; then have ready a couple of minced truffles and a good handful of shrimps to mix into the sauce, which may be made of either white or red wine; the red may be made the more savory, but the white wine will be found the most delicate: it should be thickened with yolk of egg, 
and the dish garnished with sippets of fried bread.

\section{9.-RAGOUT OF FISH.}

Take carp, perch, pike, and eels ; clean and scale them well, and cut them into pieces for serving; put in your stewpan a good-sized piece of butter, let it fry to a pale brown, fry some flour in it, and add a quart or two of good bouillon with a glass or two of red wine, and a few cloves and onions. When boiling put your ragoût into it, let it well boil, and add some lemon-juice before serving it up.

\section{0.-A VOL-AU-VENT}

Of fish, of any sort, is an elegant sidedish when prepared either with meat gravy or made up maigre.

Muscles also make an excellent volau-vent.

\section{1.-STEWED FISH, HEBREW FASHION.}

Take three or four parsley-roots, cut them into long thin slices, and two or three onions also sliced, boil them together in a quart of water until quite tender; then flavor it with ground white pepper, nutmeg, mace, and a little saffron, the juice of two lemons, and a spoonful of vinegar. Put in the fish, and let it stew for twenty, or thirty minutes; then take it out, strain the gravy, thicken it with a little flour and butter, have balls made of chopped fish, bread-crumbs, spices, and the yolk of one or two eggs mixed up together, and drop them into the liquor. Let them boil, then put in the fish, and serve it up with the balls and parsley-roots.

\section{2.-SAUCES FOR FISH.}

The stock for fish sauces should be made of the water in which fish has been boiled, adding the bones, fins, \&c., all well stewed down; when fish is filleted, the bones should always be employed in this way: Stew them with an onion and a little white pepper; strain the broth, which will be very rich, thicken it with cream, butter, and flour. or roux, and add whatever the sauce is to be made of. The following may be generally used, according to fancy, for nearly every species of fish:

\section{3.-HORSERADISH SAUCE.}

Stew an onion in a little fish-stock until it will pulp; add a teaspoonful of grated horseradish, and one or two spoonfuls of essence of anchovies. Beat all together over a fire, thicken it with a little butter, and finish with a spoonful of lemon pickle or lemon juice. Vinegar may be substituted, in which case it must be mixed with the horseradish, and boiled with it; while the lemon, or lemon pickle, being of a more delicate flavor, should only be warmed.

Or:-Scrape the horseradish thin and chop it small, or grate it, which is better; warm it in melted butter, adding a spoonful of mushroom ketchup, and one of walnut, or the vinegar from walnut-pickle.

\section{4.-FOR ALL SORTS OF FISH.}

Take a spoonful of vinegar, one of Indian soy, the same of mushroom ketchup aud Harvey's sauce, with a little cayenne. Add three large spoonfuls of melted butter; stir all weli, and heat it over the fire.

Or:-Put equal quantities of water and vinegar into a saucepan, and thicken it with the yolk of an egg to every four spoonfuls of the water and vinegar. Make it quite hot, but do not boil it; stir it or shake the pan all the time; season it to your liking, and add a spoonful of the liquid to every three of melted butter.

\section{5.-WHITE SAUCE.}

Half a pint of cream, two tablespoonfuls 
of mushroom ketchup; one of essence of anchory, with a little cayenne pepper, and an ounce or two of butter rolled in flour; boil all together for five minutes.

\section{6.-BROWN SAUCE.}

Fry an onion in butter and flour until it becomes brown; then simmer it in a glass of port wine, with a tablespoonful of soy and walnut ketchup, seasoned with salt and cayene ; strain it, and thicken it with the necessary quantity of melted butter.

\section{7.-ANCHOVY SAUCE.}

To about half a pint of melted butter put two tablespoonfuls of good essence of anchovies, with the juice of half a lemon. Serve very hot.

\section{8.-FENNEL SAUCE.}

This is a sauce principally used for boiled mackerel. Make the same quantity of melted butter as in the last, to which add a good tablespoonful of chopped fennel; it is usually served in a boat.

\section{9.-BUTTER OF ANCHOVIES.}

To make this butter you must have young anchovies. Take them out of the pickle and wash them well. Take off the bones and head, and then pound them in a mortar with fresh butter, till very fine; rub this through a hair sieve. Put this butter when made, into a pot well covered, to use when wanted; observe, however, that it soon becomes rank.

\section{0.-EGG SAUCE}

Is generally served with salt fish or haddock. Boil six eggs ten minutes; let them get cold; then cut them in pieces about the size of dice; put them into a stewpan with three parts of a pint of melted or drawn butter; add an ounce more fresh butter, with a little pepper and salt; keep the stewpan moving round over the fire until the whole is very hot, and serve in a boat.

\section{1-CAPER SAUCE FOR FISH.}

Take some melted butter, into which throw a small bit of glaze, and when the sauce is in a state of readiness throw into it some choice capers, salt and pepper, and a spoonful of essence of anchovies.

\section{2.-NEW LOBSTER SAUCE.}

If you use the solid flesh for salad, pound the soft part and shell together (in a mortar) very fine, which put into a stewpan, covered with a pint of boiling water; place it over the fire to simmer for ten minutes, then pass the liquor through a hair sieve into a basin; put three ounces of butter into a stewpan. into which rub (cold) a good tablespoonful of flour, add the liquor from the lobster, place it upon the fire, stirring until the point of boiling; season with a little cayenne, and add a piece of anchovy butter, the size of a walnut; or, if any red spawn is in the lobster, mix it with butter, as in the last, and add it, with the juice of half a lemon, just before serving. An anchovy pounded with the lobster-shells would be an improvement, and part of the flesh of the lobster might be served in the sauce.

\section{3.-LOBSTER SAUCE À LA CREME.}

Cut a small lobster into slices the size of half-crown pieces, which put into a stewpan; pound the soft and white parts with an ounce of butter, and rub it through a sieve; pour ten spoonfuls of melted butter, and two of cream, over the slices in the stewpan, add half a blade of mace, a saltspoonful of salt, a quarter ditto of pepper, and a little cayenne; warm gently, and when upon the point of boiling, add the butter and two tablespoonfuls of thick cream; shake 
round over the fire until quite hot, when it is ready to serve.

\section{4.--LOBSTER SAUCE SIMPLIFIED.}

Put the slices of lobster into a stewpan, with ten tablespoonfuls of milk; add a little pepper, salt, cayenne, two cloves, and half a blade of mace; set it upon the fire, and when on the point of boiling, add a piece of butter the size of two walnuts, with which you have mix-

- ed a little flour; shake round over the fire, and when getting rather thick, add two spoonfuls of cream, if at hand, and serve very hot.

\section{5.-LOBSTER SAUCE.-(French receipt.)}

A hen lobster is indispensable for this sauce. Put some of the spawn of the fish into a mortar, to be pounded very fine; add to it a small bit of butter. When very fine, rub it through a hair sieve, and cover till wanted. Break the lobster with great care, cut all the flesh into dice, not too small; dilute some of the red spawn in melted butter, with two spoonfuls of essence of anchovies, a little salt and cayenne pepper, two spoonfuls of thick cream, and mix all well before the meat is added, as that must retain its dice-like form. Do not let this sauce boil. It must be very red. Add to it a teaspoonful of cavice, and observe that the cavice should be very old; two or three years' age renders it excellent.

\section{6.-SOYER'S LOBSTER AND SHRIMP SAUCE FOR SALMON.}

Lobster.-Put twelve spoonfuls of melted butter into a stewpan; cut a middle-sized hen lobster into dice, make one-quarter pound of lobster butter with the spawn by pounding it well in a mortar, adding one-quarter pound fresh butter. and rubbing the mixture through a hair sieve ; add this to the melted butter when just boiling; stir it over the fire till the butter is melted; season with a little essence of anchovy, the juice of half a lemon, and a quarter of a teaspoonful of cayenne; pass it through a tamis (a coarse flannel) into another stewpan; then add the flesh of the lobster. Serve it hot. This sauce must be red; if not red in the lobster, use live spawn.

Shrimp.-Make the melted butter as above, but finish with essence of shrimps, and serve half a pint of pickled shrimps in the boat with it; or the anchovy sauce may be served with shrimps in it as a substitute, if there is no essence of shrimps.

\section{7-SHRIMP SAUCE}

Is also very good as follows: Pound half a pint of shrimps, skins and all, in a mortar, and boil them ten minutes in half a pint of water; pass the liquor through a hair sieve into a stewpan, and add a piece of butter the size of two walnuts, with which you have mixed a good teaspoonful of flour, stir it round over the fire until upon the point of boiling; if too thick, add a little more water; season with a little cayenne and a teaspoonful of essence of anchovies; serve very hot; a few picked shrimps might also be served in it.

\section{8.-LOBSTER SAUCE.}

Put twelve tablespoonfuls of melted butter in a stewpan, cut up a small-sized lobster into dice; make a quarter of a pound of lobster butter with the spawn, as directed; when the melted butter is upon the point of boiling, add the lobster butter, stir the sauce round over the fire until the butter is melted, season with a little essence of anchovies, the juice of half a lemon, and a quarter of a saltspoonful of cayenne pepper; pass it through a tammy into another stewpan, and add the flesh of the lobster. This sauce must be red. 


\section{9.-MATELOTE SAUCE.}

For about a pound slice of salmon make the following quantity of sauce: -Peel thirty button onions, and put half a teaspoonful of sugar in a quartsized stewpan, place it over a sharp fire, and when melted and getting brown, add a piece of butter (the size of two walnuts) and the onions, toss them over now and then until rather brown, then add a glass of sherry; let it boil ; then add half a pint of brown sauce and a gill of broth; simmer at the corner of the fire until the onions are quite tender; skim it well, and add a few mushrooms, if at hand; season with a little salt and sugar, and sauce over any kind of fish where described. The addition of a teaspoonful of essence of anchovies is an improvement. Use where directed.

\section{0.-MATELOTE SAUCE SIMPLIFIED.}

Proceed as above respecting the onions, only add a fourth more butter, and fry them a little browner; then add a glass of sherry and two teaspoonfuls of flour, which stir round gently with a small wooden spoon; add to it about a pint of water, stir now and then till boiling, add three saltspoonfuls of salt, two of sugar, one of pepper, and a bouquet garni ; simmer and skim, add a few drops of coloring to give it a nice brown color; when ready to serve, add a good tablespoonful of anchovy essence; it ought to adhere lightly to the back of the spoon, but not be too thick; sauce over or under, as directed; small pieces of glaze, if thickened with the eggs-(great care must be exercised, for if it should become too hot the eggs would curdle, and render the sauce useless;) then add half a pint of melted butter; stir all together over the fire.

\section{1-OYSTER SAUCE.}

Blanch three dozen oysters, which again put into' the stewpan, with their liquor (after having detached the beards); add six peppercorns and half a blade of mace ; place them over the fire, and when beginning to simmer, add a piece of butter the size of a walnut, with which you have mixed sufficient flour to form a paste, break it in four or five pieces; shake the stewpan round over the fire, and when upon the point of boiling, and becoming thick, add half a gill of milk, or more if required; season with a little cayenne, salt, pepper, and a few drops of essence of anchovies : serve very hot.

\section{2,-MUSSEL SAUCE.}

Proceed exactly the same as for oyster sauce, using only the liquor of the mussels (not the beards) instead of the oysters, and serving the mussels in the sauce; about four dozen would be suffcient.

\section{3.-COD SAUCE.}

Take a bunch of parsley, chervil, two shalots, two cloves, a bay-leaf, some mushrooms, and a bit of butter, soak all together on the fire, adding a small spoonful of flour, and milk or cream sufficient to boil to the consistence of a sauce, and add to it some chopped parsley first scalded.

\section{4.-EEL SAUCE.}

Cut the eels into large pieces and put them into a stewpan with a few slices of bacon, ham, veal, two onions, with all sorts of roots; soak it till it catches. then add a glass of white wine and good broth, a little cullis, three or four tarragon leaves, chervil, a clove of garlic, two of spices, and a bay-leaf; simmer for an hour, skim it very well, and sift it in a sieve for use.

$$
\text { 275.-SAUCE FOR FISH. }
$$

Twenty-four anchovies chopped; ten 
eschalots; two ounces of horseradish, scraped; four blades of mace; one lemon sliced; twelve cloves; quarter of an ounce of black pepper, whole; one gill of the anchovy liquor; one quart of best vinegar; one quart of water. Let the whole simmer on the fire until reduced to one quart, in a covered saucepan; strain and bottle for use. If required for long keeping, add a quarter of an ounce of cayenne pepper.

\section{6.-LOBSTER SAUCE}

Pick the meat from a lobster and cut it into small pieces. Break the shell, and stew it with the legs, \&c., in a pint and a half of water, until reduced to the quantity required; then strain; add flour and water to thicken it. Pound some of the live spawn from the tail, adding a little water to it; when well pounded pour it by degrees into the sauce; let it boil up; add fresh butter to it in the proportion of three-fourths of a pound of butter to a quart of sauce; throw in the lobster: season with a little anchovy, cayenne, salt, and a small quantity of lemon-juice. It should be thick rather than thin.

Or :-Take a cold boiled hen lobster; split the tail, and pound the coral, which is found in the tail and in the body, in a mortar, adding a little sweet oil. Then chop the meat of the body into very small pieces, and rub it, along with the soft parts and coral, through a sieve. That done, cut up the flesh of the claws and tail into dice, and stir the entire mixture gradually into the proper quantity of melted butter, without suffering it to boil; as, if too much heated, the flavor and color of the sauce will be injured. The seasoning should only be a moderate quantity of mace and cayenne, with half a glass of white wine, or a cup of cream, to a pint tureen of siuce.
Crab Sauce is made in the same manner; but crabs, being without coral, and the flesh less firm than that of lobster, form a rather inferior sauce.

\section{7.-SHRIMP SAUCE.}

Pick the heads and skins from some fresh shrimps and stew these offals for half an hour in a small quantity of boiling water to extract their flavor; then strain the liquor and make use of it in melting the butter in which the shelled shrimps are to be dressed; these are then to be put into the butter and liquor to simmer gently for about twenty minutes, in which time they will be sufficiently done, and their flavor is so delicate that neither anchovy sauce, mace, cayenne pepper, nor any other condiment than salt, should be put to them; add a little cream.

A pint of unshelled shrimps will make a tureen of sauce large enough for four or five persons.

\section{GRAVY, SAUCES, ETC.}

There is nothing that requires more attention on the part of the cook than the sauces which are wanted to all made dishes. Where a calf's head or a breast of veal is stewed, nothing more will be required, as both will yield an abundant supply, and it will only be necessary to give it the proper flavor with ham, or beef-bone, and ketchup.

\section{GRAVY}

May be made quite as good of the skirts of beef, kidney, or of the liver of a fat ox, as of any other kind of meat, if cut in pieces, fried with onions, and seasoned with herbs and spices. as other gravies. A clever servant will contrive to supply at a trifling expense, as much gravy as is wanted for the use of a small 
family by stewing down the trimmings of meat and bones. It may even be made of the shank-bones of legs and shoulders of mutton; théy should be thrown into water, and, after a good soaking and brushing, be long boiled. The water in which they are done will add greatly to the richness of gravy, as does the jelly of cow-heels. The latter must lie all night in water, which causes the jelly to be of a good color. When boiled three hours, and become cold, let the fat be carefully taken off; and when apparently quite clear, lay some white paper upon it, rubbing it close with a spoon, which will remove every particle of grease, and it will be as pure as the jelly of a calf's foot.

In preparing meat to stew for gravy, beat it with a mallet or rolling-pin, and score it across in various places, as this will make it give out its juices; season it with pepper and salt, and put it into a stewpan with butter only, heating it gradually until it becomes brown, but shaking the pan frequently to see that it does not burn or stick to the bottom. It will generally be browned sufficiently in half an hour. If kept in a very cool place and covered closely in a stone jar, it will keep good for two or three days in summer, and more than a week in winter, but should not be thickened until it is meant to be used.

Tarragon and knotted marjoram, by some called "London thyme," are a great improvement to gravies, as also all those condiments enumerated in the chapter on soups, but should be added only a short time before serving.

Truffles and morels also thicken and improve the flavor of gravies and soups; half an ounce being carefully washed of each, simmer them in a pint of water, and add the whole.

In the preparation of large dinners for company, it is indispensable to procure strong gravy to color and impart flavor to sauces and ragouts, and this can be done by using coarse pieces of the lean of beef or veal, and the giblets or trimmings of poultry and game, with a small portion of a knuckle of ham; but for this purpose it must be stewed for a long time, skimmed, strained, thickened, and afterwards flavored with whatever condiments are most suited to the dish it is to accompany.

\section{8.-STOCK FOR GRAVY.}

A good mode of making stock for gravies is to cut lean beef thin, put it into a gravy pot without any butter or fat, and set it on a fire covered, but take care it does not burn; let it stay till all the gravy that comes out of the meat is dried up into it again, often shaking it; put as much water as will cover the meat, and let that stew away. Then put to the meat a small quantity of water, herbs, onions, spice, and a bit of lean ham ; simmer till it is rich, and keep it in a closet refrigerator. Do not take off the fall going to be used.

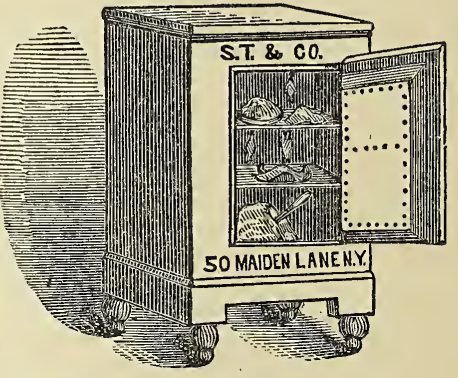

Closet Refrigerator.

Or, an excellent stock may be made in this manner :-Put into a casserole three ounces of butter, four large carrots, six middling-sized onions, three roots of parsley sliced; a small sprig of thyme; three cloves; three bay-leaves; two pounds of small fresh fish cut fine, with salt, pepper, and one-half a bottle of white (but not sweet) wine, and nearly as much broth. Cover close, and simmer until the 
whole be mashed; strain it through a fine sieve. In another casserole stew a pint of mushrooms, a little parsley and herbs, in half a pint of water, till the flavor of all be obtained; strain both liquors, and heat them together.

\section{9.-BEEF GRAVY.}

Cut a piece of the cheek or neck into pieces; strew some flour over it; mix it well with the meat, and put it into the saucepan with as much water as will cover it; an onion, a little allspice, a little pepper, and some salt; cover it cose, and when it boils skim it; then throw in a small crust of bread, or raspings, and stew it till the gravy is rich and good ; strain it off; and pour it into a sauce boat.

280.-GRAVY FOR A HAUNCH OF VENISON.

Cut off the fat from two or three pounds of a loin of old mutton, and set it in steaks on a gridiron for a few minutes, just to brown one side; put them into a saucepan with a quart of water ; cover quite close for an hour, and simmer it gently; then uncover it, and stew till the gravy is reduced to a pint; season with salt only.

\section{1.-VEAL GRAVY.}

When all the meat has been taken from a knuckle of veal, divide the bones, and lay them in a stewpot, with a pound of the scrag of a neck, an ounce of lean bacon, a bunch of parsley, a little thyme, a bit of lemon peel, and a dessert-spoonful of pepper; add as much water as will cover them. Boil and skim it; stop the pot down close, and let it simmer as slowly as possible three hours. Strain ofi, and let it stand till cold; then skim it, and take the jelly from the sediment. Pound some mace fine, and boil it with two spoonfuls of water, and add to the gravy. If cream is to be put to it, do not add the salt until the gravy comes off the fire.

\section{2.-GRAVY TO MAKE MUTTON EAT} LIKE VENISON.

Pick a very stale woodcock or snipe; cut it in pieces (but first take out the bag from the entrails), and simmer with as much unseasoned meat-gravy as you will want. Strain it and serve in the dish; but if the mutton be not long kept, it will not acquire the venison flavor.

\section{3.-VELOUTÉ.}

Take one pound of real, with the remains of a fowl and a dozen full-grown mushrooms, or a smaller number of green truffles; heat these in melted butter, or beef fat, without browning; season with salt, pepper, nutmeg, or mixed spices, to which may be added a couple of carrots and onions, with a table-spoonful or two of flour. When boiled, skim off the fat, and let it simmer for one and a half hours, after which strain it, and keep it closely stopped for further use.

\section{4.-BEEF GRAVY. (French Receipt.)}

Trim with layers of fat bacon the bottom of a thick stewpan; cut four large onions in halves, and lay the flat part over the bacon; take a few pieces of beef, put them in the same manner as in the veal gravy ; moisten with the first broth only. Let this sweat, to get all the gravy out of the beef, and when the broth is reduced, thrust a knife into the meat; let it stew gently on a slow fire till the gravy is a light brown color. Next moisten with some broth; throw in a large bunch of parsley and of green onions; a little salt, and a peppercorn. Let the whoie boil for an hour; rike the fat off, and drain it through a silken sicve, to use when wanted.

\section{5.-GRAVY-CLEAR.}

Slice some beef thinly; broil a part of it over a very clear, quick fire. just enough to give color to the gravy, but 
not to dress it; put that with the raw into a tinned stewpan with a couple of onions, one or two cloves, whole black pepper, berries of allspice, and a bunch of sweet herbs ; cover it with hot water, give it one boil, and skim it two or three times, then cover it, and let it simmer till quite strong.

\section{6.-CURRY POWDERS.}

One ounce of ginger, the same of coriander-seed, one-half ounce of cayenne pepper, and two ounces of fine pale turmeric; these ingredients to be pounded separately to a fine powder, and then warmed by the fire, and mixed together. Put the powder into a wide-mouthed bottle, cork it well down, and put it into a dry place.

Or:-One tablespoonful of corianderseed, one teaspoonful of cumin-seed, the same of turmeric and of cayenne pepper, and one table-spoonful of common flour. When to be used mix all these ingredients in their several proportions, with two table-spoonfuls of lemon-pickle and four of cream adding this mixture to the fried onions and butter, \&c.

Or:-One and a half ounces of mustard-seed scorched and finely powdered, four ounces of coriander seed portdered, four and a half ounces of turmeric, three ounces of black pepper, one and a quarter ounces of cayenne pepper, one ounce of the lesser cardamoms, one-half ounce of ginger, and one of cumin-seed, all finely powdered. The flàvor may be varied by the addition of all or any of the following ingredients: cinnamon, in powder, one ounce ; cloves, ditto, onehalf ounce; mace, ditto, one-half ounce.

\section{7.-CURRY POWDER.}

(Kitchiner's tried Receipt.)

Dry and reduce to a fine powder the following spices:

Coriander-seed, three ounces,

$\begin{array}{ll}\text { Turmeric, } & \text { three ounces, } \\ \text { Black pepper, } & \text { one ounce, } \\ \text { Mustard, } & \text { one ounce, } \\ \text { Ginger, } & \text { one ounce, }\end{array}$

Lesser cardamoms half an ounce, Cayenne pepper, a quarter of an ounce, Cumin-seed, a quarter of an ounce.

Thoroughly pound and mix, and keep them in a closely stopped bottle. Three ounces of the powder steeped ten days in a quart of vinegar or white wine, will impregnate it with the flavor.

\section{8.-DELHI CURRY POWDER.}

Twenty teaspoonfuls of turmeric, eight of pounded chilis or cayenne pepper, and twelve each of cumin, coriander-seed, and dried cassia-leaves.

\section{9.-A PLLAIN CURRY.}

Put into a fryingpan a piece of butter, a small onion cut into pieces, and two cloves of garlic; fry these until brown, put the meat to the above, and add the curry powder, and sufficient cold water to cover the meat, and boil the whole gently until the meat is cooked; then add the juice of a lemon, and a little salt.

$$
\text { 290.-THE ASPIC, A JELLY. }
$$

Take a handful of aromatic herbs, such as burnet, chervil, and tarragon. Boil them in white vinegar; when the vinegar is well scented, pour into the stewpan some consommé of fowl reduced; season well before you clarify. When the aspic is highly seasoned, break the whites of four eggs into an earthen pan, and beat tuem with an osier rod; throw the aspic into the whites of eggs, and put the whole on the fire in a stewpan; keep beating or stirring till the jelly gets white; it is then very near boiling. Put it on the corner of the stove, with a cover over it. and a little fire on the top of it. When quite clear and bright, strain it through a bag, or sieve, or napkin, to be used when wanted. 
N. B. If this is wanted for a mayonaise, or as a jelly in moulds, make sure of its being stiff enough. Then put a knuckle of veal in a small stock-pot, a small part of a knuckle of ham, and two calves' feet, some trimming of fowl or game. Season this with onions, carrots, and a bunch of herbs well seasoned; pour into it half a bottle of white wine. and moisten with good broth; let it boil gently for four hours, then skim away all the fat, and drain it through a silken sieve; put that in a stewpan, with two spoonfuls of tarragon vinegar, and four whites of eggs, salt, and pepper, to clarify; and keep stirring it on the fire till the whole becomes very white, then put this on the side with a little fire over the cover; when you find it clear, drain it in a cloth or jelly-bag, and use it for aspic; if not, do not put in any vinegar: jelly for pie or galantine does not require acid.

\section{1.-EPICUREAN SAUCE.}

Indian soy, two ounces; walnut and mushroom ketchup, of each eight ounces ; port wine, two ounces; white pepper, bruised, half an ounce; shalots, three ounces ; cayenne, a quarter of an ounce ; cloves, half an ounce. Macerate for fourteen days in a warm place; strain, and add sufficient white wine vinegar to make exactly one pint. The above forms a piquant sauce for chops, steaks, \&c. For fish, a little essence of anchovy, and an additional quantity of vinegar, should be added.

\section{2.-CARRACK OR INDIAN SAUCE FOR COLD MEAT.}

Two heads of garlic sliced, five spoonfuls of soy, five spoonfuls of mushroom ketchup, eight spoonfuls of walnut pickle, fifteen anchovies, or five spoonfuls of essence of anchovies, three spoonfuls of mango pickle, one quart of vinegar-mix in a bottle and set it in the chimney corner; shake daily for a month. It is excellent without the mango. [This is an excellent sauce, and without the mango would be mild.]

293-HOT SPICE-A DELICIOUS ADJUNCT TO CHOPS, STEAKS, GRA VIES, SOUPS, \&c.

Three drachms each of ginger, black pepper, and cinnamon; seven cloves; mace half an ounce; cayenne one quarter of an ounce; nutmegs one ounce; white pepper, one ounce and a half; mix. The quantity of cayenne may be increased, should the above not be enough to suit the palate.

\section{4.-A RICH GRAVY.}

Cut beef into thin slices, according to the quantity wanted; slice onions thin, and flour both; fry them of a light pale brown, but do not on any account suffer them to get black : put them into a stewpan, pour boiling water. on the browning in the frying-pan, boil it up, and pour on the meat. Put to it a bunch of parsley, thyme, and savory, a small bit of knotted marjoram, the same of tarragon, some mace, berries of allspice, whole black pepper, a clove or two, and a bit of ham, or gammon of bacon. Simmer till you have extracted all the juices of the meat, and be sure to skim the moment it boils, and often after. If for a hare, or stewed fish, anchovy should be added.

\section{5.-MELTED BUTTER.}

Although it may be presumed that every cook who understands her business knows how to melt butter, it is yet constantly brought to table either too thick or too thin, and not unfrequently filled with lumps of flour or in a state of oil, and requires more care in the management than is generally thought necessary. 
The excellence of melted butter greatly depends upon the pains taken to blend it with the flour before it is put upon the fire, the best plan of doing which is to rub them together with a knife on a wooden trencher. When well mixed, add two table-spoonfuls of hot water, or the same quantity of milk; put it into a small pipkin, shaking it one way until it boils. and not leaving it an instant; it must boil a minute to take off the rawness, and if made of fresh butter add a little salt. Remember that if you set it on the hot coals, or over the fire, it will be oily; if the butter and flour be not well mixed, it will be lumpy; and if you put too much water, it will be thin and poor. By attending to these directions, and only using sufficient flour to prevent the butter from oiling, it will be rich and smooth.

Or:-Mix together by degrees two spoonfuls of flour in cold water; make it smooth and thin; then put on a pint of water, let it boil; stir in the flour and water to make the required thickness, cut half a pound of fresh butter in small pieces, put it into the flour and water, let it boil well; it is then fit for use; a pinch of salt may be required.

When thin melted butter is required to pour over puddings, roast veal, \&c., make it the same way, adding a larger proportion of water or milk, the latter rendering it rather whiter than the water; and if meant to be more rich than common. use cream instead of milk. Indeed the French frequently enrich melted butter by adding the yolk of a raw egg.

\section{6.-MAITTRE D'HÔTEL BUTTER.}

Put one-fourth of a pound of fresh butter upon a plate, the juice of two lemons, and two large table-spoonfuls of chopped parsley, half a teaspoonful of salt, and half that quantity of white pepper; mix all well together, and keep in a cool place for use.

\section{7.-TO BROWN MELTED BUTTER.}

Put a lump of butter into a frying-pan, and toss it round over the fre until it becomes brown; then dredge some flour over it, which has been also browned by putting it either in the oven or before the fire, and stir it round with a spoon until the butter boils.

By adding some of the flavored vinegars and compound sauces to meited butter thus prepared, most of the fish-sauces can be made, and many of those in common use are composed in this simple manner.

298.-PARSLEY AND BUTTER.

Scald a large handful of parsley in boiling water that has some salt in it; when tender chop it fine, and stir it into some rather thick melted butter. There should be sufficient parsley to make the sauce green, and the parsley should not be put to the melted butter until about to be served, otherwise it will burn brown.

\section{9.-FENNEL SAUCE.}

Proceed as for parsley and butter.

The first is used for the various purposes of fish, poultry, and fresh boiled meats; fennel mostly for mackerel.

300.-CAPER SAUCE, WHITE.

Put whole capers into melted butter, adding a little of the vinegar they are pickied in, a pinch of salt, and sufficient cream to make it white. This is used principally for boiled mutton.

\section{1-CAPER SAUCE, BROWN.}

Take some thick brown sauce, adding the vinegar the carers are pickled in; season it highly with cayenne and salt. Put capers to the sauce just before serving ; and they must be used liberally.

Nasturtium buds or seeds are sometimes used as a substitute. 
302.-EGG SAUCE.

Boil the eggs hard, cut them in small dice, and put the pieces into melted butter. The yolk may however be crushed to a powder, and used to thicken the butter. Or, if a more savory sauce is required, boil two eggs hard, mince them very fine, add a third portion of grated ham or tongue, a very little white pepper, and the juice of a lemon; warm it up in melted butter. It is chiefly used for roast fowl and salt codfish; and if the butter be sound, the salted will be found quite as good for all these purposes as the fresh.

\section{3.-SAUCES FOR ROAST BEEF OR MUTTON.}

Grate horseradish on a bread-grater into a basin, then add two table-spoonfuls of cream with a little mustard and salt; mix them well together; then add four table-spoonfuls of the best vinegar, and mix the whole thoroughly. The vinegar and cream are both to be cold. This is a very fine sauce; it may be served in a small tureen.

Or:- Scrape the horseradish thin and chop it small, or grate it, which is better; warm it in melted butter, adding a spoonful of mushroom ketchup, and one of walnut, or the vinegar from walnut pickle.

Or:-Scrape very fine or grate the horseradish; add a little made mustard, and two spoonfuls of pounded white sugar to four of vinegar : mix the whole well together, and place it under the meat, when nearly done, to catch the gravy which drops fron it while roasting. This sauce should be very thick.

\section{4-HORSERADISH SAUCE}

Two teaspoonfuls of mustard, two of white sugar, half a one of salt, and a little more than a wineglass of vinegar- mixed and poured over a stock of grated horseradish. This sauce is good for beef.

305.- QUEEN MARY'S SAUCE.

Take a shoulder of mutton that has hung till it is tender. When three parts roasted, put a soup-plate under it, with three spoonfuls of hot water, the same of port wine, a shalot, an anchovy chopped fine, and a little pepper; baste the meat with this and the gravy that drops from it. When the mutton is taken up, turn the inside upwards, score it various ways, pour the gravy over, and cover it with a quantity of fried crumbs of bread.

\section{6.-MANDRAM,}

Is a sauce commonly used in the West Indies with roast beef and mutton. It is made with a sliced cucumber, a chopped shalot, and a minced green capsicum, mixed up in a couple of table-spoonfuls of Madeira wine and vinegar; and as capsicums can be had green during great part of the autumn, it can be also made elsewhere; but neither chilis nor capsicums, if dried, will impart the same flavor.

\section{7.-MINT SAUCE, FOR ROAST LAMB.}

Pick the leaves off the stalks; wash and dry them carefully; chop them with a sharp knife very quickly to preserve their green color; put it into a boat; add sufficient vinegar to make it liquid, and powdered sugar to take off the acidity of the vinegar.

\section{8.-MUSHROOM SAUCE.}

White.-Put the mushroorns into a stewpan with one ounce of butter, some pepper and salt, and squeeze over them the juice of half a lemon; set them over the fire; when they have given out their liquor, thicken it with flour and butter, 
and add sufficient cream to whiten the sauce. This is a very excellent sauce for fowls, rabbits, and all sorts of white fricassees.

$O r$ :-Wash and pick a pint of young mushrooms, and rub them with salt, to take off the tender skin; put them into a saucepan with a little salt, some nutmeg, a blade of mace, a pint of cream, and a good piece of butter rubbed in flour. Boil them up, and stir them till done; then pour it round the chickens, \&c. Garnish with lemon. If you cannot get fresh mushrooms, use pickled ones, done white, with a little mushroompowder with the cream.

Brown.-It may also be made brown by either putting the buttons into a pipkin, and frying them with a little butter until they become of a delicate brown, and then stewing them in strong gravy of either beef or veal; or by making it of old mushrooms chopped small, fried and stewed in the same manner, and then seasoned according to taste. It is much used for cutlets and many sorts of ragoûts.

\section{9.-CELERY SAUCE.}

Strip the outer parts of the stem, and, after carefully washing the remaining portion, cut it into small pieces; put to it a blade of mace. without any other spice, and stew it in good veal broth until very tender; it will take a good deal of time, more particularly the thick hard end of the root. After this thicken it with melted butter, and flavor it with a small quantity of white wine; or it may be thickened with boiled cream without wine. It is usually served with boiled turkey, but is very delicate with any kind of white poultry or veal.

\section{0.-ONION SAUCE.}

The onions must be peeled, and then boiled till they are tender, then squeeze the water from them, chop them, and add butter that has been melted, rich and smooth, with a little good milk instead of water; give it one boil, serve it with boiled rabbits, partridges, scrag or knuckle of veal, or roast mutton; a turnip boiled with the onions draws out the strengtl.

\section{1.-ONION SAUCE, BROWN.}

Peel and dice the onions; some put an equal quantity of cucumber and of celery, into a quart stewpan, with an ounce of butter; set it over a slow fire, and turn the onion about till it is slightly browned, then gradually stir in half an ounce of flour, add a little broth, and a little pepper and salt, boil up for a few minutes; add a table-spoonful of claret, or port wine, and mushroomketchup; you may add, if you think proper, lemon-juice or vinegar, and rub it through a tammy or fine sieve.

\section{2.-OYSTER SAUCE.}

In opening the oysters, save the liquor, and boil it with the beards, a bit of mace, and lemon-peel; in the mean time throw the oysters into cold water, and drain it off; strain the liquor, and put it into a saucepan with the oysters just drained from the cold water, with sufficient quantity of butter, mixed with as much milk as will make enough sauce. but first rub a little flour with it; set them over the fire, and stir all the while, and when the butter has boiled a few times, take them off, and keep them close to the fire, but not upon it, for if too much done, the oysters will become hard; add a squeeze of lemon-juice, and serve; a little is a great improvement.

\section{3.-SAUCE, HAREVY.}

Chop twelve anchovies, bone and all, very small, with one ounce of cayenne pepper, six spoonfuls of soy, six ditto of 
good walnut pickle, three heads of garlic chopped not very small, a quarter of an ounce of cochineal, two heads of shalots chopped rather large, one gallon of vinegar; let it stand fourteen days, stir it well twice or thrice every day, then pass it through a jelly bag, and repeat this till it is quite clear; then bottle it, and tie a bladder over the cork.

\section{4.-SAUCE (Italian) FOR SALADS.}

Mix together three table-spoonfuls of sauce tournée, one of mustard, some tarragon and chervil shred small, with three table-spoonfuls of Florence oil ; putting in, however, a little at a time ; when perfectly smooth, add also, by degrees, a glass of tarragon vinegar, and a little salt. This sauce cannot be too much mixed.

\section{5.-TARRAGON SAUCE.}

Put two table-spoonfuls of tarragon vinegar into a saucepan, and reduce it to half the quantity, then put to it six spoonfuls of good butter sauce and mix all well together, and if not sufficiently strong put to it a little bit of glaze, and a very little more tarragon vinegar.

\section{6. -TOMATO SAUCE.}

Fresh tomatoes; take out stalk, press them all tightly down in a stewpan, cover them, put them on the fire, strain off the liquor that is drawn from them, add to the tomatoes a slice of raw ham, two shalots, a few spoonfuls of good stock; let it stew for an hour, then rub it through a tammy sieve. Have in another stewpan a little good brown sauce, put your tomato into it, boil all together, season with cayenne, salt, sugar, and lemon-juice.

\section{7.-ORANGE GRAVY SAUCE.}

Put half a pint of veal gravy into a saucepan, add to it half a dozen basil leaves, a small onion, a roll of orange or lemon-peel, and let it boil for a few min- utes, and strain it off; put to the clear gravy, the juice of a Seville orange or lemon, half a teaspoonful of salt, the same quantity of pepper and a glass of red wine, serve it hot; shalot and cayenne may be added.

\section{8.-SAUCE \& LA TARTARE.}

Take the yolk of an egg, and drop into it very slowly a table-spoonful of sweet oil, beating it up by degrees until perfectly mixed; add a table-spoonful of vinegar, a little chopped parsley, a tablespoonful of French mustard, pepper and salt; beat up the whole together: make and keep the sauce in a cold place; put it into a dish, and lay the boiled fowl upon it. This sauce should be beaten till it resembles cream.

\section{9.-MINT SAUCE}

Make mint vinegar by adding a very little cold vinegar to the chopped leaves, with powdered sugar.

\section{0.-FENNEL SAUCE.}

Take as many branches of green fennel as you may require; pick and wash it in the same manner as parsley ; chop it very small, scald and then lay it on a sieve to cool ; put two spoonfuls of velouté, and the same of butter sauce into a saucepan, make them quite hot, take care to stir it well, that they may be properly mixed; rub the fennel in a little butter, and then throw it into the sauce; mix it in thoroughly, and season it with salt, pepper, and nutmeg.

\section{1.-FRESH PORK SAUCE.}

Cut two or three good-sizar, onions into slices, and fry them sytly, then add two spoonfuls of cullis, a little broth, a few mushrooms chopped, a clove of garlic, vinegar, and spice ; let it boil half an hour, reduce to a proper consistence, skim and strain it. 


\section{2.-GLAZE.-(A French Receipt.)}

Glaze is very seldom made on purpose, except on particular occasions. Lay on the fire a stock-pot, with plenty of veal, and a small quantity of beef and ham; moisten with broth; when stewed for a proper time, skim it well. The glaze of sweated broth is not so bright. Season the broth with carrots and onions, a large bunch of parsley, and green onions ; but no turnips or celery, for they give a bitter taste. If you should have a grand dinner, and wish to glaze of a nice color, put more veal into your Espagnole (Spanish sauce.) The moment it comes to a glaze, put part of it into a small stewpan for the purpose of glazing only. The most common glaze is made of remnants of broth, the liquor of braize, or fricandeaux, \&c., which are to be reduced on a brisk fire. If you keep your reduction too long, it will become black and bitter. Always warm your glaze in the hot water bath, that it may not get too brown when you have a grand dinner.

\section{3.-SAUCE ROBERT.-(French Receipt.)}

Cut some onions into small dice, fry them of a fine brown, dust them with flour, and moisten them with some Spanish sauce. Skim it, that the sauce may look bright; put in a little pepper and salt, and just before you send up mix a spoonful of mustard.

\section{4.-SAUCE ROBERT.}

Cut a few onions into dice, which put into a frying-pan with a bit of butter, and fry them lightly; when nicely browned, add a dessert spoonful of flour, a ladleful of stock, the same of vinegar, some salt, and pepper; reduce it to a proper thickness, and when ready for table stir in two dessert-spoonfuls of mustard.

\section{5.-PURÁE OF ONION, OR SOUBISE} SAUCE.

Take a dozen white onions. After having peeled and washed them, cut them into halves, take off the tops and bottoms, mince them as fine as possible, and blanch them to make them taste sweeter, and take off the green color. Then let them melt on a small stove, with a little butter. When they are thoroughly done, and no kind of moisture is left, mix four spoonfuls of béchaiel. Season them well, rub the purée through a tammy, and keep the sauce hot, but without boiling. You must also put a sinall lump of sugar with the sauce if necessary. When you have the oven hot, put the onions hermetically closed in a small stewpan, and let them simmer for one hour, with a small bit of ham. This method gives a better flavor.

\section{6. -BECHAMEL SAUCE.}

Take about half a quarter of a pound of butter, about three pounds of veal cut into small slices, a quarter of a pound of ham, some trimmings of mushrooms, two small white onions, a bunch of parsley and green onions; put the whole into a stewpan, and lay it on the fire till the meat be made firm Then put three spoonfuls of flour; moisten with some boiling-hot thin cream and a ladle of consommé. Keep this sauce rather thin, so that whilst you reduce it the ingredients may have time to be stewed thoroughly. Season it with a little salt, and strain it through a tammy. This sauce should retain no taste of flour, and be very palatable.

\section{7.-DUTCH SAUCE.-(French Receipt.)}

Put into a stewpan a teaspoonful of flour, four spoonfuls of elder vinegar, a quarter of a pound of fresh butter, the yolks of five eggs, and a little salt. Put it on the fire and keep continually stirring it. When it has acquired threkness 
enough, work it well, that you may re-|color. Observe, this is only to be obfine it. If it should not be curdled, you tained by slow degrees. When of a light have no occasion to strain it through a brown pour it into an earthen pan and tammy; season well and serve it up. keep it for use. It will keep a long Some people do not like elder vinegar; time.

in that case use tarragon or plain vinegar. But odorous vinegar is far preferable.

\section{8.-SAUCE BLANCHE-(Or French Melted Butter.)}

Put into a stewpan, a quarter of a pound of fresh butter, a spoonful of flour, a little salt, half a gill or glass of water, half a spoonful of white vinegar, and a little grated nutmeg. . Put it on the fire: let it thicken, but do not allow it to boil, for fear it should taste of the flour. Serve hot.

\section{9.-WHITE ROUX *-(WHITE THICKEN- ING.)-(A French Receipt.)}

Put a good lump of butter into a stewpan, let it melt over a slow fire, and, when melted, drain the butter and take out the buttermilk; then put in the butter two or three spoonfuls of good flour, enough to make a thin paste: keep it on the fire for a quarter of an hour, and take care not to let it color; pour it into an earthen pan to use when wanted.

\section{0.-BROWN THICKENING.}

(A French Receipt.)

Put into a stewpan a piece of butter proportionate to the quantity of thickening intended to be prepared. Melt it gently; take out the buttermilk, then put flour enough to make a paste. Fry it on a slow fire, and then put it again over very red ashes, till it be of a nice

* This is an indispensable article in cookery, and serves to thicken sauces; the brown is for sauces of the same color; and the color must be obtained by slow degrees, otherwise the flour will burn and give a bitter taste, and the sauces become spotted with black.

\section{1.-LEMON SAUCE, WHITE, FOR BOILED FOWLS.}

Put the peel of a small lemon, cut very thin, into a pint of sweet, rich cream, with a sprig of lemon, thyme, and ten white peppercorns. Simmer it gently till it tastes well of the lemon, then strain it and thicken it with a quarter of a pound of butter rubbed in a dessertspoonful of flour; boil it up; then pour the juice of the lemon strained into it, stirring well; dish the fowls, and then mix a little white gravy, quite hot, with the cream, but do not boil them together; add salt according to taste.

\section{2.-LEMON SAUCE FOR BOILED FOWLS.}

Cut small slices of lemon into very small. dice, and put them into melted butter, give it one boil, and pour it over boiled fowls.

\section{3.-BREAD SAUCE.}

Cut in slices the crumb of a French roll, to which add a few peppercorns, one whole onion, a little salt, and boiling milk enough to cover it; let it simmer gently by the side of the fire till the bread soaks up the milk, add a little thick cream, take out the onion, and rub the whole through a sieve; make it very hot, and serve with game or fowls.

\section{4.-BECHAMEL SAUCE.}

Take some veal and ham, cut them into dices; some carrots, cloves, onions, laurel leaves, shalots, parsley, and scallions, all chopped fine; pepper, grated nutmeg, a little salt and butter, a little velouté and consommé, reduce it to half, and then put in some cream; mix it well with your sauce, boil it all together over 
a quick fire, shaking it constantly for an hour; if thick enough, strain it through a sieve.

\section{5.-LIVER SAUCE.}

Take the livers of poultry or game, chop them very small with parsley, scallions, tarragon leaves, and shalots; soak them in a little butter over the fire, and then pound them; add cullis stock, pepper and salt. Give the whole a boil with two glasses of red wine, coriander, cinnamon, and sugar; reduce and strain it, " thicken with a bit of butter rolled in flour; serve it in a sauce-boat.

\section{6.-LIVER SAUCE FOR BOILED CHICKENS.}

Boil the livers till you can bruise them with the back of a spoon; mix them in a little of the liquor they were boiled in, melt some butter very smooth and put to them; add a little grated lemon-peel, and boil up altogether.

\section{7.-TRUFFLE SAUCE.}

Take a pound of truffles; brush and wash them carefully; put them in a stewpan with sume, good gravy, two wineglasses of white wine, a small onion, a faggot of parsley and thyme, and an ounce of bacon fat. Let them stew gently until quite tender; take them out, strain and skim the gravy, thicken it with roux or a lump of flour and butter; peel the truffles, cut them in slices as thick as a penny-piece, warm them in the sauce, and serve.

\section{8.-CHESTNUT SAUCE.}

Scald a score of chestnuts in hot water for ten minutes; skin them; let them stew gently for about half an hour in some good gravy seasoned with a glass of white wine, a little white pepper, salt, and mace or nutmeg; and when quite soft, serve them in the dish.
Or:-Pulp them through a colander to thicken the gravy, making it either brown or white. by using in the former beef-gravy, and in the latter veal-broth, with pounded almonds, and without pepper.

Either of these is equally fit for sauce to guinea-bird or turkey, as well as for stuffing the body of the bird.

\section{9.-SAUCES FOR WHITE POULTRY.} - BOILED.

Lver-sauce.-Take the livers of as many fowls as may be required for the intended quantity of sauce, or, that of a rabbit being much larger, take one liver, boil it with some sprigs of thyme and parsley; dissolve in the water, after taking it out, two anchovies, boned; boil two eggs hard, leave out one white, and shred the rest with the liver, herbs, and anchovies ; pound them together in a mortar, adding a saltspoonful of grated lemon-peel and a little pepper and salt. Put it into the saucepan, squeeze upon it the juice of half a lemon, thicken the liquor with butter and a little flour, add to it the pounded ingredients, and stir it until finished.

Or:-If gravy be used instead of water, and butter be omitted, the above may be properly employed as an excellent sauce for roasted rabbit, or for fullgrown poultry.

\section{0.-WHITE SAUCE.}

Boil a large blade of mace, a féw cloves and peppercorns, in half a pint of soft water, until the flavor be obtained; strain it off, put it into a saucepan with four anchovies chopped fine, a quarter of a pound of butter rolled in flour, and half a pint of cream: boil and stir it well two minutes. Put some in a tureen, and the remainder in the dish.

\section{1.-FOR CHICKENS.}

Take the legs and necks, with a small 
bit of the scrag of veal or mutton; put them into a saucepan with two blades of mace, a few white peppercorns, an anchovy, a head of celery sliced, a bunch of sweet herbs, and a small bit of lemonpeel; boil these in a quart of water to half a pint; strain, and thicken it with a quarter of a pound of butter and some flour; boil it five minutes, then put in two spoonfuls of mushrooms, and, having beaten up the yolks of two eggs with a teacupful of cream, put it intos the sance, and keep shaking it one way over the fire till it is nearly boiling; then put it into a sauce-tureen.

\section{2.-VEGETABLE MARROW SAUCE.}

Few sauces are more delicate as an accompaniment for young chickens, whether roast or boiled, than vegetable marrow when deprived of its seeds, if stewed to a pulp and passed through a fine sieve, so as to form it into a purée, which may be then thinned either with fowl-broth seasuned with mace, or with cream and nutmeg.

\section{3.-SAUCES FOR ROAST FOWLS.}

Stew any moderate quantity of ham, veal, and mushrooms, with sweet herbs, a shalot; a little allspice, and a piece of butter, until all become brown; then let the whole simmer gently for a long time in either weak broth or water, until they form a strong gravy ; strain it. and season it with any additional flavor that may be given by some of the made sauces. Serve hot in a sauce-tureen.

Or :-Put into a small stewpan two slices of ham, a clove of garlic, a laurelleaf, and sliced onion: add a little good gravy, a sprig of knotted marjoram, and a spoonful of tarragon vinegar; simmer slowly an hour, strain off, and put into the dish or a boat.

Or:-Boil some veal-gravy, pepper, salt, the juice of a Seville orange and a lemon, and one-quarter as much of port wine as of gravy; and pour it into the dish or a boat.

Or :-If wanted in a hurry, cut a few slices of calf's liver, or any kind of brown meat; fry them with a little lean bacon and an onion, and, when browned, pour upon them boiling water to what strength you please, and strain it.

\section{4.-WHITE SAUCE.}

It is seldom necessary to buy meat for this favorite sauce, as the proportion of that flavor is but small. The water that has boiled fowls, veal, or rabbit, or a little broth that may be in the house, or the feet and necks of chickens, or raw or dressed veal, will suffice. Stew any of these with a little water, a bit of lemon-peel, some sliced onion, a few white peppercorns, a little pounded mace or nutmeg, and a bunch of sweet herbs, until the flavor be good; then strain it, add a little good cream, a piece of butter, and a little flour: salt to your taste. A squeeze of lemon may be added after the sauce is taken off the fire, shaking it well. Yolk of egg is often used in fricassee; but if you have any cream it is better, as the former is apt to curdle.

Or:-Boil a stick of celery and a bunch of parsley in a pint of milk, adding white pepper and a little salt; then put two ounces of butter into a saucepan, let it melt, add to it an onion sliced thin, dredge in flour until it is a paste, but do not allow it to become brown. Strain the milk, and add it by degrees to the butter and flour, stirring it very well ; then boil the whole together, stirring all the time, and boiling it until it is quite thick and smooth; pass it through a fine sieve or tammy. If wanted to be very rich, let it cool a little, and then add an egg previously beaten, and mix very gradually; warm it over the fire, stirring it well, but do not let it boil, or it will curdle. 
Or:-Mix a teaspoonful of flour with one-quarter pound of butter, a little salt, and ground white pepper; let them be well blended together with a wooden spoon, then add a spoonful of vinegar, and one or two of water; mix the whole together, stirring it one way over the fire.

\section{5.-CULLIS.}

Lay over the bottom of a stewpan as much lean veal as will cover it an inch thick : cover the veal with thin slices of undressed gammon, two or three onions, two or three bay-leaves, some sweet herbs, two blades of mace, and a few cloves. Corer the stewpan, and set it over a slow fire; but when the juices come out, let the fire be a little quicker. When the meat is of a fine brown, fill the pan with good beef broth, boil and skim it, then simmer an hour; add a little water, mixed with as much flour as will make it properly thick; boil it half an hour, and strain it. This will keep a week.

\section{6.-ROUX.}

White--Put two ounces of butter into a stewpan over a slow fire, allow it to melt, then drain off the buttermilk: make it into a paste by dredging flour over it, and keep it on the fire for a quarter of an hour, taking care that it does not lose its color.

Brown is made in the same manner, only allowed to fry of a dark color. French cooks use no other kind of browning.

\section{7.-LIAISON.}

To thicken or enrich White or Fish Soups, use the following:-Pour the soup, boiling hot, on the beaten yolks of two or three fresh eggs, from which the small tough substance found in it, and called "the tread," should be removed, nor should the soup be suffered to boil after the eggs are added, as it is then apt to curdle.

Or:-Break the yolk of an egg with three spoonfuls of cream, beat them up in the tureen and pour the boiling soup upon it. This may be made in the same proportion, and kept in a basin for other dishes.

348.-SAUCE FOR FRICANDEAU, OR ROAST VEAL.

Boil an unwashed anchovy cut small, with a cup of gravy. a glass of port wine, a shalot minced, and the juice of half a small lemon; strain, and mix in the dish with the gravy of the meat. Or use sorrel sauce.

\section{9.-SAUCE À LA MAÎTRE D'HÔTEL.}

Put eight table-spoonfuls of white sauce in a stewpan with four of milk; boil it five minutes, then stir in three ounces of maitre d'hôtel butter; stir it quickly over the fire till the butter is melted, but do not let it boil. This sauce. should be made at the time of serving.

\section{0.-SAUCES FOR GEESE.}

For a Green Goose.-Take half a pint of sorrel-juice, two glasses of white wine, a nutmeg quartered, a cupful of fried. crumbs, and two lumps of sugar; let all boil together, then beat it smooth, adding a piece of fresh butter, and serve it very hot in a tureen, or in the dish with the goose. It should not be made too thick with the bread-crumbs; and if much acid should not be approved, the wine must be equal in quantity to the sorrel-juice.

\section{1.-GOOSEBERRY SAUCE.}

Wash some sorrel, put it into a cloth and press out the juice; melt a piece of butter with flour, using this juice instead of water; let it be very thick. Scald green gooseberries until very tender, and 
add them to the sauce. This is a fine acid sauce if not spoiled with sugar. If not sweetened add a little cayenne pepper.

\section{2.-APPLE SAUCE.}

Pare, core, and slice some apples; and put them in a stone jar, into a saucepan of water, or on a hot hearth. If on a hearth, let a spoonful or two of water be put in to hinder them from burning. When they are done, bruise them to a mash, and put to them a piece of butter the size of a nutmeg, and a little brown sugar if required; but it destroys the slight acid flavor of the apples, and its corrective to goose and pork.

\section{3.-SAVORY APPLE SAUCE.}

Pulp the apples, and, if wanted of a good color, add to them a little juice of beet-root or cochineal : season with cayenne pepper, and a glass of vinegar or lemon-pickle, taking care not to make it too acid. This will prove a substitute for tomato or sorrel sauce, when neither can be obtained; if for the latter, a little spinach-juice will give it a color.

\section{4.-SAUCE FOR DUCKLINGS.}

Take young green onions or chives; chop them very small, then put them into some thick melted butter, with pepper, salt, and a spoonful of lemon-pickle; stir it well together, and, when very hot, put it into the dish with the ducks upon it. Should the flavor of the onions be thought too strong, they may be scalded previously.

Or :-Mix one-quarter pint of sorreljuice, a glass of white wine, some scalded gooseberries, some white sugar, and a bit of butter. Boil them up and serve in a boat.

\section{5.-SAUCE FOR SUCKING-PIG.}

Take the inside of a French roll and boil it to a pulp, along with an onion and a little salt; chop the brains and put them into a teacupful of the gravy that runs from the pig; then take as much butter as will be sufficient for the sauce, and put into it a good squeeze of lemon, with half a glass of white wine.

Some cooks make a stuffing for the pig in nearly the same manner, only making it with less butter and more bread, besides sometimes seasoning it with sage.

\section{6-CURRANT SAUCE.}

Clean an ounce of currants, and boil them in one-half pint of water for a few minutes, pour the whole over a teacupful of bread-crumbs; let it soak, and then add a piece of butter rolled in flour, four or six cloves, and a glass of port wine ; beat it a little, and stir it over the fire until it is quite smooth.

\section{7.-SAUCES FOR WILD-FOWL. (English.)}

A teaspoonful of made mustard, the same of essence of anchovies and red pepper; a tablespoonful of ketchup, and a glass of claret. This receipt is from the Palace, and comes highly recommended.

Or :-Simmer a teacupful of port wine, the same quantity of good meat gravy, a little shalot, a little pepper, salt, a grate of nutmeg, and a bit of mace, for ten minutes: put in a bit of butter and flour, give it all one boil, and pour it through the birds. In general they are not stuffed, but may be done so if liked.

Or:-Mince some shalots very fine, put them into a small saucepan with some peppercorns, two tablespoonfuls of strong, clear gravy, and the sarfe quantity of vinegar; when sufficiently boiled add a little salt, and strain it through a sieve. It may be served either hot or cold.

358.-WHITE SAUCE. (Soyer's.)

Cut and chop a knuckle of veal weigh- 
ing about four pounds, into large dice; also half a pound of lean bacon; butter the bottom of a large stewpan with a quarter of a pound of butter; add two onions, a small carrot, a turnip, three cloves, half a blade of mace, a bouquet of a bay-leaf, a sprig of thyme, and six of parsley; add a gill of water; place over a sharp fire, stirring round occasionally, until the bottom of the stewpan is covered with whitish glaze; then fill up with three quarts of water; add a good teaspoonful of salt, and let it simmer at the corner of the fire an hour and a half, keeping it well skimmed; pass it through a hair sieve into a basin. In another stewpan put a quarter of a pound of butter, with which mix six ounces of flour, stirring over the fire about three minutes; take off, keep stirring until partly cold, when add the stock all at once, continually stirring and boiling for a quarter of an hour; add half a pint of boiling milk; stir a few minutes longer, add a few chopped mushrooms ; if handy, pass through a hair sieve into a basin, until required for use, stirring it round occasionally until cold. The above being a simplified-white sauce, will be referred to very often in receipts.

\section{9.-BROWN SAUCE. (Soyer's.)}

Put two ounces of butter into a stewpan; rub it over the bottom; peel two or three large onions; cut them in thick slices; lay them on the bottom; cut into small pieces about two pounds of knuckle of veal,* all meat, or three pounds if with bone; a quarter of a pound of lean bacon, cut small, two cloves, a few peppercorns, a table-spoonful of salt, two bay-leaves, a gill of water; set it on a brisk fire; let it remain ten minutes, when stir it well round, subdue the fire, let it remain twenty minutes longer,

* Half veal and beef can be used; or if no veal, all beef. and stir now and then until it has a nice brown color; fill your pan with three quarts of water; when boiling, set it on the corner of the stove, with the lid three parts on the saucepan; when boiling; skim fat and all; after one hour. or one hour and a half simmering, pass it through a sieve into a basin. To make the thickening or Roux for it, proceed as follows: -Roux. Put two ounces of butter into a pan, which melt on a slow fire, then add three ounces of flour, stir it with a wooden spoon until getting a thin deep yellow color; this in France is called Roux, being very useful in cookery, and will be often referred to in these receipts. This process will take ten minutes, when remove from the fire for two minutes to cool ; then add at once three pints and a quarter of the above stock; very quickly set it on the fire to boil; remove it to the corner to simmer, and skim. It ought to be entirely free from grease, and of a light chestnut color.

\section{0.-DEMI-GLAZE-THIN BROWN SAUCE FOR MADE DISHES.}

When I have a small dinner-party, I always, as I told you before, make small quantities of white and brown sauce as above, but this is a nice way of clarifying a brown sauce without much trouble, and makes it a beautiful transparent brown color; but although $I$ have made it quite a study that each entrée, or made dish for daily use, should make its own sauce, yet I must impress upon you that this sauce is the real key to cooking a good and ceremonious dinner. Put a pint of brown sauce, in a middle-sized stewpan; add to it half a pint of broth or consommé, put it on the stove, stir with wooden spoon, let it boil as fast as possible, take the scum off which will rise to the surface; reduce it until it adheres lightly to the spoon, pass it through a sieve or tam. my into a basin; stir now and then until cold, to prevent a skin forming on the top; 
put it by until wanted for use. It will keep for a week in winter, by adding half a gill of white broth every other day, and giving it a boil ; the addition of a tablespoonful of tomato sauce gives it a beautiful color; use where indicated.

\section{1.-THIN BROWN SAUCE OF MUSH- ROOMS.}

Put twelve table-spoonfuls of thin brown sauce in a small stewpan to boil, then have six or eight small mushrooms, well cleaned and washed, chop them fine, and place in sauce, and boil for five minutes; taste if it is to your liking; the addition of a little sugar is an improvement; a little cayenne, if liked, may be introduced. The sauce is good for cutlets, broiled fowl, and game, \&c.

\section{2.-ESCHALOT SAUCE.}

Chop fine about a good table-spoonful of eschalot, place it in the corner of a napkin, and pour water over; press until dry, and put in a small stewpan with two table-spoonfuls of vinegar, one clove, a little mace; boil two minutes, add ten table-spoonfuls of demi-glaze, boil a little longer, add a little sugar, and serve.

\section{3.-PIQUANT OR SHARP SAUCE.}

Put two table-spoonfuls of chopped onions, or eschalots, cleaned as above, into a stewpan; put also four table-spoonfuls of vinegar, and a bay-leaf, and boil ; then add ten table-spoonfuls of brown sauce, half a one of chopped parsley, ditto of green gherkins; boil five minutes; skim, add a little sugar, taste if well seasoned, take out bay-leaf and serve.

\section{4.-TARRAGON SAUCE. (Soyer's.)}

Put eight table-spoonfuls of demi-glaze, and four of broth, into a stewpan; boil for a few minutes; add a table-spoonful of vinegar; have ready picked twenty leaves of fresh tarragon; put in to sim- mer two minutes, and serve with any kind of poultry, but especially spring chickens.

\section{5.-BROWN CUCUMBER SAUCE.}

Peel a small fresh cucumber; cut it in neat pieces; put in a stewpan with a little sugar; add half an ounce of butter; set it on a slow fire; stir it now and then; add twelve table-spoonfuls of brown sauce, and eight of broth; let it simmer till tender; skim the butter off; remove the cucumber into another stewpan; pass the sauce, reduce it a little, taste it, pour over, and serve.

\section{6.-MINCE HERB SAUCE.}

Put two table-spoonfuls of finely chopped onions in a stewpan, and a tablespoonful of oil ; place it on the fire; stir a few minutes, add ten table-spoonfuls of demi-glaze, and four of broth or water; boil, skim; if too thick, and the scum should not rise, add half a gill of broth or water; boil, and reduce to ' a proper thickness, and add a table-spoonful of chopped parsley; if at hand, one of mushrooms, and season with a little cayenne; the juice of a quarter of a lemon; serve. I often introduce a little garlic in this.

\section{7.-ROBER'T SAUCE. (Soyer's.)}

Peel and cut up two good-sized onions; put them in a stewpan with an ounce of butter, till they are a nice yellow color; then add eight table-spoonfuls of demiglaze, and two of water or broth; skim, boil quickly; when of a proper thickness add a good table-spoonful of French mustard; season rather high; if no French mustard, use English, but it completely changes the flavor, though still very palatable.

\section{8.-RAVIGOTE SAUCE.}

Put in a stewpan one middle-sized onion sliced, with a little carrot, a little 
thyme, bay-leaf, one clove, a little mace, a little scraped horseradish, a little butter; fry a few minutes, then add three teaspoonfuls of vinegar, ten table-spoonfuls of brown sauce, four of broth; when boiling, skim, add a table-spoonful of currant jelly; when melted, pass all through a tammy, and serve with any kind of meat or poultry; with hare or venison it is excellent.

\section{9.-BROWN MUSHROOM SAUCE.}

Clean and cut twelve small mushrooms in slices; place them in a stewpan with a little butter, salt, pepper, the juice of a quarter of a lemon; set it on a slow fire for a few minutes, then add ten spoonfuls of demi-glaze; boil till they are tender, and serve. A little mushroom ketchup may be introduced.

\section{0.-ORANGE SAUCE FOR GAME.}

Peel half an orange, removing all the pith ; cut it into slices, and then in fillets; put them in a gill of water to boil for two minutes; drain them on a sieve, throwing the water away; place in the stewpan two spoonfuls of demi-glaze, or ten of broth ; and, when boiling, add the urange, a little sugar; simmer ten minutes, skim, and serve. The juice of half an orange is an improvement. This is served with ducklings and water fowl: those that like may add cayenne and mustard.

\section{1.-GARLIC SAUCE.}

'Though many dislike the flavor of this root, yet those that like it ought not to be deprived of it. Put in a stewpan ten table-spoonfuls of demi-glaze, a little tomato sauce, if handy; boil it a few minutes, scrape half a clove of garlic, put it in with a little sugar, and serve.

\section{2.-MINT SAUCE FOR LAMB.}

Take three table-spoonfuls of chopped leaves of green mint, three table-spoon- fuls of brown sugar, and put into a basin, with half a pint of brown vinegar ; stir it well up, add one salt-spoonful of salt, and serve.

\section{3.-LIAISON OF EGGS.}

Break the yolks of two eggs in a basin, with which mix six spoonfuls of milk, or eight of cream; pass it through a fine sieve, and use when directed.

\section{4.-ANCHOVY BUTTER SAUCE.}

Put into a stewpan eight spoonfuls of demi-glaze, or three of broth ; when boiling, add one ounce of anchovy butter ; stir continually till melted; serve where directed.

\section{5.-SOYER'S SAUCE.}

Put six spoonfuls of demi-glaze into a stewpan; when hot, add four spoonfuls of Soyer's Brown Sauce; let boil, and serve with either chop, steak, cutlet, poultry, or game.

\section{6.-PAPILLOTTE SAUCE}

Scrape half an ounce of fat bacon, put it in a pan with four table-spoonfuls of chopped onions; stir over the fire for a few minutes, then add ten table-spoonfuls of brown sauce and boil; then add a tablespoonful of mushrooms chopped, one ditto of parsley, a little nutmeg, a little pepper and sugar, a quarter of a clove of scraped garlic; reduce till rather thickish; put on dish till cold, and use it for any thing you may put up "en Papillotte."

\section{7.-TOMATO SAUCE. (Soyer's.)}

If fresh, put six in a stewpan; having removed the stalk, and squeezed them in the hand to remove pips, \&c., add half an onion, sliced, a sprig of thyme, a bayleaf, half an ounce of celery, one ounce of ham or bacon, same of butter, teaspoonful of sugar, same of salt, a quarter one of pepper, set on fire to stew gently; 
when all tender, add a table-spoonful of flour, moisten with half a pint of broth, boil five minutes; add a little cayenne, taste if highly seasoned, pass it through sieve or tammy, put it back in stewpan, until it adheres rather thick to the back of the spoon, and use it for any kind of meat or poultry. If preserved tomato, proceed as for poivrade sauce, respecting the vegetables, omitting the vinegar, and adding the tomato. instead of the brown sauce, with a table-spoonful of flour and broth to bring it to a proper thickness; pass it through a sieve, and serve as above.

\section{8.-CURRY SAUCE. (Soyer's.)}

Put into a pan four good-sized onions, sliced, and two peeled apples, with a quarter of a pound of butter, the same of lean ham, a blade of mace, fuur peppercorns, two bay-leaves, two sprigs of thyme; stir them over a moderate fire until the onions become brown and tender, then add two table-spoonfuls of the best curry powder, one of vinegar, two of flour, a teaspoonful of salt, one of sugar; moisten it with a quart of broth or milk, or even witer, with the addition of a little glaze; boil till in a pulp, and adhering raiher thickly to the back of the spoon; pass all through a fine sieve or tammy; give it another boil for a few minutes, put it in a basin, and use when required. Any kind of meat, poultry, and fish. or parts of game, are excellent warmed in this sauce, and served with well boiled and dry rice. I have kept this sauce in a cool place in the winter for a month, boiling it now and then. The quantity of powder may be omitted, and a spoonful of curry paste used, or some mangoes.

\section{9.-A VERY GOOD AND USEFUL WHITE SAUCE-(quite new.)}

Put a quart of white sauce in a stewpan of a proper size on a fire; stir con- tinually until reduced to one-third; put two yolks of eggs in a basin, stir them well up, add your sauce gradually, keep stirring, put back in stewpan, set it to boil for a few minutes longer, then add one pint of boiling milk, which will bring it to its proper thickness; that is, when it adheres transparently to the back of a spoon; pass through a tanımy into a basin, stir now and then till cold. If not immediately required, and I have any stock left, I use half of it with half of milk. I also try this way, which is very convenient; when the yolks are in, and well boiled, I put it in a large gallipot. and when cold, cover with pieces of paper; it will keep good in winter for two or three weeks, and above a week in summer: when I want to use a little of it, I only take a spoonful or two and warm it on the fire, and add enough milk or white broth to bring it to a proper thickness, and use when required. This sauce is very smooth, and never turns greasy; it lies beautifully on fowl. or any white made dish; the addition of a drop of cream gives it a very fine white appearance.

\section{0.-ONION PUREE SAUCE.}

Peel and cut six onions in slices; put in a stewpan, with a quarter of a pound of butter, a teaspoonful of salt, one of sugar, a half one of pepper; place on a slow fire to simmer till in a pulp, stirring them now and then to prevent them getting brown, then add one tablespoonful of flour, a pint of milk, and boil till a proper thickness, which should be a little thicker than melted butter; pass through a tammy, warm again, and serve with mutton cutlets, chops, boiled rabbits, or fowl ; by not passing it, it will do for roast mutton and boiled rabbit as onion sauce.

381.-PURÉE OF CAULIFLOWER SAUCE.

Boil a cauliflower well in thrce pints 
of water, in which you have previously put one ounce of butter, one tablespoonful of salt; when done, chop it up, having prepared and slowly cooked in a stewpan an onion sliced, a little celery, half a turnip, one ounce of ham, two of butter, a little bay-leaf, mace, add then the cauliflower, stir round, add a tablespoonful of flour, moisten as above for onions, pass, and finish the same way. It may be made of Jerusalem artichoke or turnip.

\section{2,-BROWN SHARP SAUCE, OR POI- VRADE.-(French Receipt.)}

Put a small bit of butter. a small carrot cut into dice, a few shalots cut in the same way, some parsley roots, six green onions, some parsley, a few slices of ham, a clove, a little thyme, the half. of a bayleaf, a few grains of peppercorn and allspice, with a little mace, in a small stewpan. Let the stewpan be put on a slow fire, till it begins to be of a fine brown all round; then keep stirring with a wooden spoon; pour into the stewpan four spoonfuls of white vinegar and a small bit of sugar. Let this reduce nearly to a glaze; then moisten with some Spanish sauce and a little consommé, that you may be enabled to take the fat off from the sauce; season with cayenne and a little salt. Taste whether it be salt enough, but observe that it should not be too acid; skim off the fat, and strain the sauce through a tammy, and serve up. When this sauce is well made, not too thick, and of good color, it is good with the following entrées:Cotelettes Maintenon, broiled chicken, broiled pigeon, fried clicken, marinades of all sorts, such as calf's feet, lamb's ears, trotters, calf's head, and generally with every thing requiring tart sauces. You may add to it a little preserved tomatoes, to give a good color and sharp taste.

\section{3.-WHITE CUCUMBER PURÉE.}

Peel two, or one large one, cut in slices, put in the stewpan with the same vegetables, \&c. as for the cauliflower; when tender, add a table-spoonful of flour, four gills of milk or broth, boil, finishing as the cauliflower.

\section{4.-SORREL SAUCE, OR PURÉE.}

Wash well six handfuls of sorrel, put it nearly dry into a middle-sized stewpan with a little butter; let it melt, add a table-spoonful of flour, at easpoonful of salt, half one of pepper; moisten to a thick purée, with milk, or broth, or cream; pass it through a sieve, put it back in a stewpan, warm again, add two whole eggs, two ounces of butter, and stir well, and serve where directed.

385.-SPINAGE PURÉE.

Endive purée is often used in France,

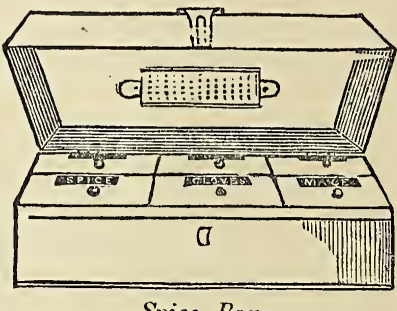

Spice Box.

and called chicorée. This purée may be made like the cauliflower, or only plain boiled, plainly chopped, put into a pan with two ounces of butter, a gill of white sauce, a little grated nutmeg, and a little salt, pepper, and sugar.

\section{6.-INDIA-PICKLE SAUCE.}

Take some gravy, thicken with flour and butter; cut in small dice some India-pickle, add a little of the vinegar, let it boil up, throw in the dice, and serve hot. This is for salmon cutlets; 
it is also good for broiled mackerel or for grilled chicken.

\section{7.-SAUCE PIQUANTE.}

Put into a stewpan the muscles of a knuckle of veal, some slices of ham or bacon, a cupful of gravy, a spoonful or two of strong vinegar, a bay-leaf, a clove of garlic, a little thyme, one clove, and a little salt; skim it, and, when it has stewed a quarter of an hour, pass it through a sieve : then add sorrel, parsley, and mushrooms, all chopped fine, and whole capers ; heat it again, and serve it in the dish.

Or:-Put one or two ounces of butter, with two shred onions, a carrot, a parsnip, a little thyme, laurel, basil, two cloves, two shalots, a clove of garlic, and some parsley, into a stewpan; turn the whole over the fire till it becomes brown, then shake in some flour, and moisten it with some broth and a spoonful of vinegar. Let it boil over a slow fire; skim and strain it; then add pepper and salt; and serve with any dish of which you would wish to have the flavor heightened.

\section{8.-À L'ESPAGNOLE-(or Spanish Sauce.)}

Besides a few slices of ham and veal, put into a stewpan any remains which you niay have of poultry and game, and let them boil for one hour in water more than surisient to cover them; after which skim off the fat from the gravy, add fried onions, sweet herbs, a bay-leaf, spice, mace, and mushrooms, with minced carrots, celery, shalots, and a small clove of garlic, and leave the whole to stew until quite mashed: it will take full two hours. Then strain it, and if the gravy be not strong enough add sufficient coulis or glaze to make it so. If it wants flavor, add soy, and if mushrooms have not been one of the ingredients, put in ketchup : if not thick enough. a little arrowroot, or common flour, with a piece of butter, will have the effect; but if the gravy be truly rich, it will not require the addition.

Such is the foundation of the sauce; but in most cases white wine is added, for the purpose of stewing game: it is needless to say that Madeira and sherry are best, but Cape or ginger wine will be found good substitutes.

\section{9.- SAUCE, À LA PROVENQALE.}

Put into a casserole two spoonfuls of sweet oil, a shalot, a clove of garlic, and some mushrooms, all minced, and placed upon the fire in any sort of good strong broth, seasoned with pepper and salt, together with pot-herbs; leave it there for half an hour; then skim it so far as to remove much of the appearance of the oil ; strain it, and add some white wine to suit the taste.

\section{0.-SAUCE MAIGRE.}

Fry a large quantity of onions and put them along with the above into the casserole, with white wine, until well browned; then strain it; add a table-spoonful of salad-oil with a little flour, and mix it gently, but continually, for a few minutes, until completely mingled.

\section{1.-A L' HOLLANDAISE,}

For meat, fowl, or fish. Put six spoonfuls of water and two of tarragon vinegar, with one ounce of butter, into a stewpan; warm and thicken it with the yolks of two eggs. Make it quite hot, but do not boil it; stir it all the time; squeeze in the juice of half a lemon, and strain it through a sieve. Season with salt and cayenne. It should be quite thick.

\section{2.-SPINACH SAUCE.}

Pick off the stalks of the spinach; wash and drain it ; then, without water, stew till it will beat to a mash. Put in 
a good piece of butter and some milk; simmer, and stir over a slow fire till the sauce be of the consistence of thick melted butter. Add a little pepper and salt while dressing.

\section{3.-PUREE OF CUCUMBERS.}

Slice thin some middling-sized cucumbers, drain the liquor from them, and put them, with four onions, into a stewpan, with a piece of butter; when sufficiently stewed to pulp through a colander, add a large teacupful of cream, a little flour and pepper. Boil twenty minutes and, when going to serve, put in salt.

\section{4.-SAUCE À LA SAINTE MENEHOULD.}

Put some butter into a stewpan, shake over it a little flour; add a cupful of cream, parsley, young onions, one-half a bay-leaf, and a shalot. Put it on the fire, keeping it stirred. Then pass it through a sieve: put it on the fire again with some chopped parsley, a little pepper, and minced mushrooms. Serve it over boiled fowls.

\section{5.-LA MAGNONNAISE.}

Perhaps the most esteemed mode of making sauce for any sort of cold meats or fish is that known in France ly this title.

Put into a round-bottomed basin the yolk of an egg and a pinch of salt; stir in very quickly, drop by drop, two tablespoonfuls of sweet oil, working the spoon very rapidly round, to work in the oil ; when it is thick add a few drops of tarragon vinegar, then more oil, until there is sufficient sauce; thin it with a little nore vinegar. It should be quite thick, and rather acid.

\section{6.-CAR $\Lambda \mathrm{CHI}$.}

Take one head of garlic and cut each clove into two pieces; two teaspoonfuls of cayenne pepper, three tablespoonfuls of soy, three of mushroom ketchup, three of walnut pickle, six anchovies dissolved, two or three spoonfuls of mangoe or India pickle liquor, and a pint of the best vinegar, with sufficient cochineal to color.

Both the above are thus sauces maigres, but when not intended for fast-days, may be much improved by being made into a purée with an admixture of melted calf's-foot jelly.

\section{7.-SAUCE ROYALE}

Is made by cutting a slice of white bread, and stewing it in white broth until it is quite thick; then take it off the fire; pound in a mortar the yolks of two hard-boiled eggs, and half a dozen almonds reduced to paste, with the breast of a cold roast fowl; mix this with the eggs and bread, and pound them all together with a little pepper and salt; add a sufficient quantity of cream to make it of a proper consistence, and simmer the whole over the fire, stirring it all the time without letting it boil.

\section{8.-SAUCE À LA REINE}

Is nearly the same. Bruise the breast of a chicken to a paste, and inoisten it with a consommé of veal or fowl broth; let it stew gently over a slow fire, and then pass it through the tamis and mix with it some béchamel sauce. Then have ready the yolks of two eggs beaten up with a,large spoonful of thick cream, and stir the mixture into the sauce a minute or two before serving it up.

Both the above are served at the most fashionable tables, and may be used with turkey-poults and guinea-birds.

\section{9.-THE ORIGINAL OUDE SAUCE}

Is made with two ounces of butter put into a stewpan; when melted add six onions shred; as soon as they begin 
to take color, put in one and a half dozen of chilis chopped fine; stir it well together for four minutes, then put in a small quantity of dried salt fish, not exceeding two square inches, chopped very fine; keep stirring, and, as the butter dries, add a large cupful of the pulp of fresh tomatoes, a teaspoonful of salt, the juice of a lemon, and a little water; mix the whole very well together, and let it be of the consistence of a paste, though not too dry. It is eaten with cold meat, but will not keep.

\section{0.-CHETNEY AND QUIHI SAUCE.}

Take eight ounces of sharp apples pared and cored, eight ounces of tomatoes, eight ounces of salt, eight ounces of brown sugar, eight ounces of raisins, four ounces of red chilis, four ounces of ginger powdered, two ounces of garlic, and two ounces of shalots. Pound all these ingredients separately in a mortar, using cayenne pepper if chilis are not to be had. Mix the whole together, and add three quarts of vinegar and one quart of lemon-juice. Place the jar containing this composition on a stove, or by the side of a fire in a heat not less than one hundred and thirty degrees Fahrenheit, and stir it twice a-day for a month; then strain, but do not squeeze it dry. Bottle the liquor, which is an excellent sauce for any kind of fish or meat, hot or cold. A teaspoonful will impart a fine flavor to any ragoût, or be sufficient for a pint of broth. The residue, which is the chetney, should be put into pots or jars. It may also be used in sauces and gravies, or eaten like mustard with cold meat, or spread upon grills, being an excellent ingredient for devils, wet or dry.

\section{1.-VEAL GRAVY, OR BLOND OF}

VEAL. (Ude.)

Put a few slices of Westmoreland ham (the lean only) into a pretty thick stew- pan, and lay over them some fleshy pieces of veal. The rump may be used. Pour into the stewpan a sufficient quantity of stock-broth to cover about half the thickness of the meat. Let it sweat on a stove over a brisk fire. Watch the stewpan and the contents for fear of burning. When the broth is reduced, thrust a knife into the meat, that all the gravy may run out; then stew the glaze more gently. When the whole is absolutely glazed, of a good color, let it stew till brown, but take care it does not burn, to prevent which, put it on red-hot ashes. Keep stirring the stewpan over the fire. that the glaze may be all of the same color, and turn the meat upside down. that it may not stick. When the glaze is of a dark-red color, moisten with some hot broth, and let the glaze detach before the stewpan is put on the fire, for it might still burn. Season with mushrooms and a bunch of parsley and green onions. When the gravy has boiled for two hours, it will be done. Take off the fat, and strain it through a silken sieve.

\section{2.-THE CULLIS-(a Brown Jelly.)}

Make the cullis in the same manner as veal gravy, with slices of ham and slices of veal, \&c. When the glaze is of a nice color, moisten it, and let it stew entirely. Season it with a bunch of pars'ey and green onions, mushrooms, \&c. Thell mix some brown thickening with the veal gravy, but do not make it too thick, as the fat could not be got out of the sauce; and a sauce with fat has neither a pleasing appearance nor a good flavor. Let it boil for an hour on the corner of the stove; skim off the fat, and strain it through a tammy, \&c.

\section{3.-SAUCE TOURNÉE.*}

Take some white thickening, dilute it

* Sauce tournée is the sance that the modern cooks call velouté; but velouté, properly so called will be found bereafter. 
with some consommé or broth of fowl; neither too thin nor too thick. A sauce when too thick will never admit of the fat being removed. Let it boil on the corner of the stove. Throw in a few mushrooms, with a bunch of parsley and green onions. Skim it well, and when there is no grease left, strain it through a tammy, to use when wanted.

\section{4.-SAUCE À L' ALLEMANDE.-(German Sauce.)}

This is the same as the last sauce, with the addition of a thickening* of eggs well seasoned. This sauce is always used for the following sauces or ragouts, viz., blanquettes, or white fricassees, of all descriptions, of veal, of fowl, of game, or palates, ragout, loin of veal, with béchamel, \&c., \&c.

\section{5.-THE VELOUTE.}

Take about the same quantity of stockbroth, and sauce tournée, and boil them down over a large fire. When this sauce is very thick, have some thick cream boiling and reduced, which pour into the sauce, and give it a couple of boilings; season with a little salt, and strain through a tammy. If the ham should be too salt, put in a little sugar. Observe, that this sauce is not to be so thick as the béchamel.

\section{6.-VELOUTÉ, OR BÉCHAMEL, A NEW METHOD. (Ude.)}

Put into a stewpan a knuckle of veal, some slices of ham, four or five pounds of beef, the legs and loin of a fowl; all the trimmings of meat or game that you have, and moisten with boiled water sufficient to cover half the meat; make it sweat gently on a slow fire, till the meat is done through; this you may ascertain by thrusting your knife into it; if no

* This thickening is what is called in French, liaison; the yolks of two or four eggs. blood follows, it is time to moisten with boiling water sufficient to cover all the meat. Then season with a bundle of parsley and green onions, a clove, half a bay-leaf, thyme, a little salt, and trimmings of mushrooms. When the sauce has boiled long enough to let the knuckle be well done, skim off all the fat, strain it through a silken sieve, and boil down this consommé till it is nearly a glaze; next take four spoonfuls of very fine flour, dilute it with three pints of very good cream, in a stewpan big enough to contain the cream, consommé, flour, \&c.; boil the flour and cream on a slow fire. When it boils, pour in the consommé, and continue to boil it on a slow fire if the sauce be thick, but on the contrary, if the sauce be thin, on a quick fire, in order to thicken it. Season with salt, but put no pepper. No white sauce admits pepper, except when you introduce into it something chopped fine. Pepper appears like dust, and should therefore be avoided. This sauce should be very thick. Put it into a white basin through a tammy, and keep it in the larder out of the dust.

This sauce is the foundation, if I may so speak, of all sorts of little sauces, where white sauces are preferred. It should always be kept very thick, as you can thin it whenever you please, either with stock-broth or with cream. If too thin, it could not be used for so many purposes.

\section{7.-CAPER SAUCE À LA FRANÇAISE.}

Take some capers, cut them small; put some essence of ham into a small sauce. pan with some pepper; let it boil ; then put in the capers; let them boil two or three times, and they are ready to serve.

\section{8.--CAPER SAUCE, TO IMITATE.}

Boil some parsley very slowly to let it become of a bad color; then cut it up, 
but do not chop it fine; put it into melted butter, with a teaspoonful of salt, and a dessert-spoonful of vinegar; boil up, and then serve.

\section{9.-CAPER SAUCE FOR MEAT.}

Take some capers, chop half of them very fine, and put the rest in whole; then chop some parsley with a little grated bread, and put to it some salt; put them into butter melted very smooth, let them boil up, and then pour them into a sauce-boat.

\section{0.-TOMATO SAUCE FRANÇAISE.}

Cut ten tomatoes into quarters and put them into a saucepan with four onions sliced, a little parsley, thyme, one clove, and a quarter of a pound of butter; set the saucepan on the fire, stirring occasionally, for three-quarters of an hour; strain the sauce through a horsehair sieve, and serve with the directed articles.

\section{1.-TOMATOES, GARNITURE OF.}

Take thirty tomatoes, all as near of a size as possible, and of a good form, cut them in halves, press out the juice, seeds, and pieces by the side of the stalk, but do it with great care, lest the skins. should be injured; make a farce as follows:-take a little ham, garlic, parsley, shalots, champignons shred, the yolks of two hard boiled eggs. crumb of bread, an anchovy, butter, salt, nutmeg, and allspice; give all these a boil, then pound them well, adding at times a little oil, strain it through a quenelle sieve; fill the tomatoes with this farce, place them on a baking tin, cover them with grated bread crumbs and Parmesan cheese, moisten them with a little oil, and bake them in a hot oven; serve the tomatoes as a garnish to a rump of beef, or any other joint you may think proper.

\section{2.-TOMATO SAUCE À L'ITALIENNE.}

Take five or six onions, slice, and put them into a saucepan with a little thyme, bay-leaf, twelve tomatoes, a bit of butter, salt, half a dozen allspice, a little Indian saffron, and a glass of stock; set them on the fire, taking care to stir it frequently, as it is apt to stick; when you perceive the sauce is thick strain it like a purée.

\section{KETCHUPS.}

\section{3.-MUSHROOM KETCHUP.}

Take the full grown flaps of mushrooms, wipe them clean, crnsh them with the hands, throw in a handful of salt with every peck of mushrooms, and let them stand all night; then put them into stewpans, and set them in a quick oven for twelve horrs; strain them through a hair sieve and press out all the juice. To every gallon of liquor put of cloves, Jamaica and black pepper, and of ginger, one ounce each, and half a pound of common salt. Set it on a slow fire and let it boil until half the liquor is wasted, then put it into a clean china vessel, and when cold bottle it.

Another mode of making mushroom ketchup, is to take a stewpan full of the large flap mushrooms that are not worm eaten, and the skins and fringe of those you have pickled; throw a handful of salt among them, and set them by a slow fire. They will produce a great deal of liquor, which you must strain, and put to it four ounces of shalots. two cloves of garlic, an ounce of pepper, a tablespoonful of ginger, mace, and cloves; boil the liquor slowly and skim it well. When cold, bottle and cork it close. In two months boil it again with a little additional spice, and a stick of horseradish,-it will then keep a year; which mushroom ketchup rarely does, if not iboiled a second time. 


\section{4.-WALNUT KETCHUP.}

Take six half-sieves of green walnutshells, put them into a tub, mix them up well with common salt, (from two to three pounds, ) let them stand for six days, frequently beating and mashing them; by this time the shells become soft and pulpy. Then by banking it up on one side of the tub, and at the same time by raising the tub on that side, the liquor will drain clear off to the other; then take that liquor out; the mashing and banking up may be repeated as often as liquor is found. The quantity will be qbnut six quarts. When done let it be simmered in an iron boiler as long as any scum arises; then bruise a quarter of a pound of ginger, a quarter of a pound of allspice; two ounces of long pepper, two ounces of cloves, with the above ingredients; let it slowly boil.for half an hour. When boiled, let an equal quantity of the spice go into each bottle; when corked, let the bottle be filled quite up ; cork them tight, seal them over, and put them into a cool and dry place for one year before they are used.

\section{5.-TOMATO KETCHUP.}

Take six pounds of tomatoes, sprinkle them with salt and let them remain for a day or two, then boil them until the skins will separate easily; pour them into a colander or coarse sieve, and press them through, leaving the skins behind; put into the liquor a pint of Chili vinegar, half a pint of wine, pepper, cloves, ginger and allspice; boil them together until a third part has wasted-bottle them tight. The ketchup must be shaken before it is used. If it is boiled down to one-third, and the corks sealed, it will be much richer, and keep for years.

\section{6.-SAUCE FOR CHOPS AND STEAKS.}

Pound together in a mortar one ounce of black pepper, half an ounce of allspice, one ounce of salt, half an ounce of horse- radish scraped, half an ounce of eschalots. Pour on this half a pint of mushroom ketchup. and half a pint of walnut pickle. Infuse for a fortnight, and strain for use.

\section{7.-NASTURTIAN SAUCE}

Is eaten with boiled mutton. It is made with the green seeds of nasturtians, pickled simply in cold vinegar. Cut about six ounces of butter into small bits, and put them into a, small saucepan. Mix with a wine-g'ass of water sufficient flour to make a thick batter, pour it on the butter, and hold the saucepan over hot coals, shaking it quickly round, till the butter is melted. Let it just boil up, and then take it from the fire. Thicken it with the pickled nasturtians, and send it to table in a boat.

\section{8.-CRANBERRY SAUCE.}

This sauce is very simply made. A quart of cranberries is washed and stewed with sufficient water to cover them; when they burst mix with them a pound of brown sugar, and stir them well. Before you remove them from the fire, all the berries should have burst. When cold they will be jellied, and if thrown into a form while warm, will turn out whole.

\section{9.-CELERY SAUCE.}

Wash and pare a bunch of celery, cut it into pieces, and boil it gently until it is tender; add half a pint of cream, and a small piece of butter rolled in flour; now boil it gently. This is a good sauce * for fowls of all kinds, either roasted or boiled.

\section{FARCES AND STUFFINGS.}

$$
\text { 420. -PANADA. }
$$

Put into a small stewpan one ounce of fresh butter, a table-spoonful of chopped mushrooms, a little chopped thyme and 
parsley, half a blade of mace, pepper and salt ; stew the whole over the fire for a few minutes, then add to it a spoonful of white broth, the crumb of two French rolls previously soaked in milk and squeezed thoroughly dry; stir over the fire until it no longer adheres to the stewpan; * take it off, add the yolks of two eggs, turn it out and leave it to cool ; it is then ready for use.

\section{1.-UDDER.}

Boil a calf's udder in broth or water; when cold trim off the outside: pound and rub through a sieve.

\section{2.-QUENELLE.}

This is one of the most delicate preparations of forcemeat that we have, and requires practice, care and attention to make it in perfection. It is called according to the meat of which it is composed; quenelles of rabbit, chicken, fish, or game.

Take the meat of two rabbits, pound it and rub it through a sieve; take the same quantity of panada, and also of udder or very sweet fresh butter, pound these ingredients for a quarter of an hour, add a table-spoonful of white sauce, two eggs, and two yolks; take a small piece, roll it in a ball, and throw it in boiling water; when done it should cut firm and consistent, yet light and delicate; if more seasoning is required it should be added before it is taken out of the mortar.

To mould them : have two table-spoons, - fill one with the farce, shape with a knife, dipping it in boiling water to make it perfectly smooth; dip the other spoon in hot water, with which take out the quenelłe and lay it in a buttered stewpan; they should be egg-shaped, and perfectly smooth, and boiled for a quarter of an hour.

423.-PANADA FOR FISH QUENELLE, OR FORCEMEAT.

Put two-thirds of half a pint of water into a stewpan holding a quart, with nearly one ounce of butter ; when boiling, stir in a quarter of a pound of flour; keep it moving over the fire until it forms a smooth and tough paste. Take it off the fire, add the yolks of three eggs. When cold, use it where directed.

\section{4.-FORCEMEAT.}

Take equal quantities of cold chicken, veal, and beef, shred very small, and mixed together ; season, at the same time, with a moderate quantity of pepper, salt, sweet herbs, and grated nutmeg - that is to say, if intended for white meat, or for any thing delicately flavored; but if meant for a savory dish, add a little minced ham, and an atom of garlic or a shalot. Put the whole in a stone mortar, and pound it until quite fine, then make it into a paste with a raw egg, some butter, marrow, or fat of some kind. When used, it may either be rolled into round balls and fried for any made dish, or put into any joint of meat or poultry as stuffing; and if kept in a cool place, and well seasoned, it will keep good for several days.

Indeed, by mixing with any potted meat or game an equal proportion of panada, which will always be lighter than breadcrumbs, the cook will have at once a very fine species of farce, to be employed in stuffing olives, fillets of fowl, \&c.

The "farce" is sometimes a delicious preparation of sausage-meat, and is served up alone ; but it enters into the composition of numerous other dishes.

\section{5.-ANOTHER FORCEMEAT.}

Take a pound and a half of lean veal, pound it, and pass it through a sieve; one pound of chopped beef suet, and six ounces of panada, pounded well together; add the veal, season with pepper, salt, and a little nutmeg; mix well; then add the yolks of six eggs and the whites of 
three; when these are thoroughly mixed, | there is thyme and marjoram together; whisk the other three whites of eggs to a very stiff froth, and add it to the forcemeat; when all is well mixed together, it is fit for use.

\section{6.-FARCE CUITE.}

Cut in small pieces some undressed fowl; put them into a stewpan with a piece of butter, a little salt, pepper, and a little nutmeg; shake them over the fire for ten minutes, drain, and let them cool. Put an equal portion of crumbs of bread in the same stewpan with some broth, and a little parsley chopped fine; stir it with a wooden spoon till it becomes quite soft. Let it get cold, then pound the fowl until it will pass through a tamis; pound the bread also, and put it through the sieve; then put equal parts of the meat, butter, and bread together, and pound them with yolks of eggs sufficient to make it into a proper consistence, and keep it in a jar for use.

\section{7.-FARCE OF HAM AND VEAL.}

Mince equal quantities of ham and veal, a bunch of parsley, and some pepper; put it on the fire with a little broth; let it stew very gently, then pound it in a mortar; add to it an equal portion of bread soaked in milk and pounded; pound the whole together with some butter, and mix it with the yolks of eggs.

Bacon and veal which have been used in braising, or any thing highly impregnated with the flavor of herbs and onions, and very rich, may be employed afterwards as a farce, pounded and mixed with panada.*

\section{8.-STUFFING.}

Take equal quantities of beef suet and crumbs of bread; chop the suet very finely; chop together marjoram, thyme, and parsley, having as much parsley as

\section{* Farce for pies.} add to them a salt-spoonfu of grated lemon peel, pepper, salt, and a little grated nutmeg; add eggs sufficient to bind it together.

\section{9.-SEASONING FOR STUFFING.}

One pound of salt dried and sifted, half an ounce of ground white pepper, two ounces of dried thyme, one ounce of dried marjoram, and one ounce of nutmeg. When this seasoning is used, parsley only is required to be chopped in sufficient quantity to make the stuffing green. The proportions are-half a pound of bread-crumbs, three eggs, a quarter of a pound of suet, half an ounce of seasoning, and peel of half a lemon grated.

\section{0.-FORCEMEAT.}

Shred a little ham or gammon, some cold veal or fowl, some beef suet, a small quantity of onion, some parsley, very little lemon-peel, salt, nutmeg, or pounded mace, and either white pepper or cayenne, and bread crumbs. Pound it in a mortar, and bind it with one or two eggs, beaten or strained. For forcemeat patties, the mixture as above.

\section{1.-FOR HARE, OR ANY THING IN IMI. TATION OF IT.}

The scalded liver, an anchovy, some fat bacon, a little suet, some parsley, thyme, knotted marjoram, a little shalot, and either onion or chives, all chopped fine; crumbs of bread, pepper, and nutmeg, beat in a mortar with an egg.

\section{2.-FISH FORCEMEAT.}

Chop, and afterwards pound in a mortar, any kind of fish, adding an anchovy or two, or a tea-spoonful of the essence of anchovies, but do not allow the taste to prevail, and the yolk of a hard-boiled egg. If for maigre, pound butter with it; but otherwise, the fat of bacon pounded separately, and then mixed. Add a 
third portion of bread, prepared by soaking and pounding previously, and mix the whole up with raw eggs. For mackerel, pike, haddock, and soles, take the veal forcemeat, add a little anchovy essence, and use rather less herbs.

\section{3.-OYSTER FORCEMEAT.}

Take a dozen oysters, strain them from the liquor, mince them, and add a quarter of a pound of finely grated bread-crumbs, one ounce and a half of butter broken very small, a dessert-spoonful of parsley, the grated rind of half a lemon; season with a little mace, cayenne, and salt; mix well; then bind together with the yolk of an egg unbeaten, and a little of the oyster liquor. Care must be taken that the oyster flavor predominates.

\section{4.-FORCEMEAT BALLS FOR SOUP MAIGRE.}

Forcemeat balls for maigre soups of any description should be always used to compensate for the savor of which they are deprived by the want of meat-broth ; and may be made of fish and sweet herbs, or only of herbs and crumbs of bread, in the following manner:-

Pound in a mortar the soft parts of a crawfish, or a few shrimps, with an anchovy, and the yolk of a hard-boiled egg, beaten up with crumbs of stale bread, pounded mace, cayenne pepper, salt, and a very little finely minced garlic. Then work it up into a paste with a raw egg to bind it into dough; roll it out, cut it into small balls of the size of marbles, and fry them of a fine brown.

Or:-Leave out the fish, and make them entirely of bread and savory chopped herbs-chervil, tarragon, marjoram, and thyme, with a little garlic and a shalot-and bind them up as above, with egg, butter, and crumbs of bread.

Or:-Chop, and afterwards pound in a mortar, any kind of fish, adding an anchovy or two, or a tea-spoonful of the es- sence of anchovies, but do not allow the taste to prevail, and the yolk of a hardboiled egg: pound butter with it; add a third portion of bread, prepared by soaking and pounding previously, and mix the whole up with raw eggs.

Or:-Beat the flesh and soft parts of a middling-sized lobster, half an anchovy, a large piece of boiled celery, the yolk of a hard egg, a little cayenne, mace, salt, and white pepper, with two table-spoonfuls of bread-crumbs. one ditto of oyster liquor, two ounces of butter warmed, and two eggs long beaten: make into balls; and fry of a fine brown in butter.

Or :-Take a few shrimps, stripped of their shells, an anchovy, and the yolk of a hard-boiled egg, with bread-crumbs and seasoning as above, but adding some finely minced sweet herbs, and omitting the oyster liquor: make all this into a paste with a little butter and a raw egg, made up in balls and fried as before.

Or :- Instead of making the balls of fish and frying them, it is a more.simple way to make them merely of breadcrumbs, hard-boiled egg, and sweet herbs seasoned with raw egg: drop the balls, one by one, into the boiling soup a few minutes before serving. Two eggs and a quarter of a pound of bread should make twelve or fifteen balls.

Or:-Pound in a mortar, into a smooth substance, an anchory, the yolk of a hard-boiled egg, a head of boiled celery, and some sweet herbs. with a couple of table-spoonfuls of bread-crumbs, seasoned with mace, a grating of nutmeg, cayenne, and salt; then add two ounces of butter melted and work it into a paste with a little flour and the yolk of a raw egg; make it into balls the size of marbles, and fry them quite brown.

485.-FORCEMEAT FOR TURTLE.-(As at the Bush, Bristol.)

One pound of fine fresh suet, one pound 
of ready-dressed veal or chicken chopped fine, crumbs of bread, a little shalot or onion, salt, white pepper, nutmeg, mace, pennyroyal, parsley, and lemon-thyme flnely shred; beat as many fresh eggs, yolks and whites separately, as will make the above ingredients into a moist paste ; roll into small balls, and boil them in fresh lard, putting them in just as it boils up. When of a light brown, take them out, and drain them before the fire. If the suet be moist or stale, a great many more eggs will be necessary.

Balls made this way are remarkably light; but being greasy, some people prefer them with less suet and eggs. They may therefore be made thus:-Chop up the materials with a little. white pepper and salt, a sage leaf or two scalded and finely chopped, and the yolk of an egg; make them into small cakes or fritters, and fry them.

\section{6.-RISSOLES OF ALL KINDS:}

Chop some dressed chicken or veal very fine, fry a little chopped parsley, shalot, and mushrooms, very fine, and a little slice of tongue or ham or not, fry them in one ounce of butter a few minutes, stirring it with a wooden spoon all the time; dry the butter up with flour, then add a few small spoonfuls of good veal stock, a gill of cream, three spoonfuls of béchamel sauce; now put in all your chopped meat, add a little sugar, a few drops of lemon juice, cayenne pepper and salt, and the yolks of three eggs, boil all well until quite stiff, take it out of your stewpan on to a dish to get cold; when cold form them into a shape, either as pears or long balls, using bread-crumbs to form them; put them to get cold; in the mean time break two eggs in a basin, and then egg the forms once or twice, and bread-crumb them; have your fat quite hot to fry them, dish them on a napkin with fried parsley.
437.-EGGS FOR TURTLE.

Beat three hard yolks of eggs in a mortar, and make into a paste with the yolk of a raw one; roll it into small balls, and throw them into boiling water for two minutes to harden. Though formerly much used, they have latterly gone out of fashion.

\section{8.--PASSOVER BALLS FOR SOUP.}

Chop an onion and half a pound of suet very finely; stew them together until the suet is melted, then pour it hot upon eight spoonfuls of biscuit-flour; mix it well together; add a little salt, a little grated nutmeg, lemon-peel, and ginger, and six eggs. Put the balls into the soup when it boils, and boil them for a quarter of an hour. The quantity of eggs and flour may appear disproportioned, but the flour employed is of a peculiar kind, used for the purpose in Jewish families. Nothing can exceed the excellence of the balls made after this receipt: they are applicable to any kind of soups.

\section{9.-FARCE OF VEAL OR FOWL.}

Cut up a fowl, or some veal, form the fillet into small dice, cut in the same quantity of good fat ham cut small, and a few truffles, a little parsley, shalots, and a little of all kinds of fine sweet herbs, and a few chopped mushrooms, and one blade of mace, three cloves, put it all in a stewpan to draw down, with half a pound of butter for one hour; season it, add bread-crumbs to dry up the fat, then put it into your mortar and pound it very fine, then rub it through a wire sieve, return it back into the mortar, and work in three or four eggs, leaving out one or two whites; mix it well up together, and put it into the larder until required. If you want any green, color some with prepared spinach juice; this will do for all cold 
pies, or game, or filling turkeys, or boned fowls, or galantine.

You may make this farce with dressed meat, then you must not place it to draw down on the fire; all the rest the same, only truffles, and mushrooms as well as the rest must be first dressed.

\section{0.-SEASONING FOR ROAST PORK, DUCKS, OR GEESE.}

Two-thirds onıon. one-third green sage, chopped fine, bread crumbs equal to weight of both; season with pepper and salt, and mix with a bit of butter and the yolk of an egg.

441.-FOR A SUCKING PIG.

A teacupful of grated bread, two ounces of butter seasoned with salt, pepper and nutmeg; scald and chop fine two small onions and thirty leaves of young sage; mix with a beaten egg, and sew it in the pig.

\section{2.-FOR A GOOSE}

Scald and chop fine the liver, crumb twice its weight in bread, chop fine four small onions, half their weight of green sage, with half an ounce of butter, the yolk of an egg, and a table-spoonful of potato starch; season highly with salt and pepper.

\section{3.-GARNISHES.}

Parsley is the most universal garnish to all kinds of cold meat, poultry, fish, butter, cheese, etc. Horseradish is the garnish for roast beef, and fish in general; sometimes, for the latter, it is alternated with slices of lemon. Slices of lemon garnish boiled fowl, turkey, fish, roast veal, and calf's head.

\section{4.-FARCES AND STUFFINGS.}

A veal stuffing. Chop some suet fine, a little parsley, a small piece of shalot, rub through a dry sieve a small quantity of basil, knotted marjoram, thyme, add these to your suet, a grating of half a lemon, a few grains of nutmeg, a few bread-crumbs, and one or two eggs, mix all well up together, season with pepper and salt.

If for game, scrape the raw livers into the stuffing, prepared as above, only in addition pound it all fine.

\section{5.-FORCEMEAT INGREDIENTS.}

Forcemeat should be made to cut with a knife, but not dry or heary, no one flavor should predominate; according to what it is wanted for, a choice may be made from the following list; be careful to use the least of those articles that are most pungent; cold fowl, or veal, or ham, scraped fat bacon, beef suet, crumbs of bread, parsley, white pepper, salt, nutmeg, yolks and whites of eggs beaten to bind the mixture. Any of the following articles may be used to alter the taste; oysters, anchovies, tarragon, savory, pennyroyal, marjoram, thyme, basil, yolks of hard eggs, cayenne, garlic, shalots, endives, Jamaica pepper in powder, or two or three cloves.

446.-FORCEMEATS, ETC.

Cold fowl, veal, or mutton.

Scraped ham, or gammon.

Fat bacon, or fat ham.

Beef suet.

Veal suet.

Butter.

Marrow.

Soaked bread, and crumbs of bread,

Parsley and white pepper.

Salt and nutmeg.

Cold soles.

Oysters.

Anchovies.

Lobster, tarragon.

Savoy, pennyroyal.

Knotted marjoram.

Thyme and lemon thyme.

Basil and sage.

Lemon peel. 
Yolks of hard eggs.

Whites and yolks of eggs.

Mace and cloves.

Cayenne and garlic.

Shalot and onion.

Chives and chervil.

Ground pepper and two or three cloves.

\section{7.-BROWN COLORING FOR MADE} DISHES.

Take four ounces of sugar, beat it fine, put it into an iron fryingpan or earthen pipkin, set it over a clear fire, and when the sugar is melted it will be frothy; put it higher from the fire until it is a fine brown, keep it stirring all the time; fill the pan up with red wine, take care that it does not boil over, add a little salt and lemon, put a little cloves and mace, a shalot or two, boil it gently for ten minutes, pour it in a basin till it is cold, then bottle it for use.

\section{BEEF.}

BeEF is dressed in various ways all the year round; but, for roasting, the best season is from November to the end of March. For joints, the only really good roasting pieces are-the sirloin and the long ribs; the short ribs, which are cut near the neck, never roast well, and should not be used for this purpose; the middle ribs are the best. The under part, or fillet, of the sirloin, if cut out, may be made into a delicious stew; or, if cut crosswise into steaks, they will be found more tender than those of the rump.

Beef requires to be hung a long time, in order to insure its being tender. When however the weather is not favorable for keeping, it will be much improved by being laid for a few hours, previous to roasting, in a marinade of this kind-three parts water to one of vinegar, some salt, with a few peppercorns. Each joint must be carefully examined before it is spitted, and any portions that may have been injured cut away.

The round is, in large families, one of the most profitable parts: it is usually boiled, and, like most of the boiling parts of beef, is generally sold at less than the roasting joints.

The brisket is also less in price than the roasting parts ; it is not so economical a part as the round, having more bone to be weighed with it, and more fat. Where there are children, very fat joints are not desirable, being often disagreeable to them, and sometimes prejudicial, especially if they have a dislike to it. This joint also requires more cooking than many others; that is to say, it requires a double allowance of time to be given for boiling it: it will, when served, be hard and scarcely digestible if no more time be allowed to boil it than that which is sufficient for other joints and meats. When stewed it is excellent; and when cooked fresh, ( $i . e$. unsalted,) an excellent stock for soup may be extracted from it, and yet the meat will serve as well for dinner.

The edgebone, or aitchbone, is not considered to be a very economical joint, the bone being large in proportion to the meat; but the greater part of it, at least, is as good as that of any prime part.

The rump is the part of which the butcher makes profit, by selling it in the form of steaks. In the country, as there is not an equal demand for steaks, the whole of it may be purchased as a joint, and at the price of other prime parts. It may be turned to good account in producing many excellent dishes. If salted, it is simply boiled; if used unsalted, it is usually stewed.

The veiny piece is sold at a low price per pound; but if hung for a day or two, it is very good and very profitable. 
Where there are a number of servants and children to have an early dinner, this part of beef will be found desirable.

From the leg and shin excellent stock for soup may be drawn; and if not reduced too nuch, the meat taken from the bones may be served as a stew with vegetables; or it may be seasoned, pounded with butter, and potted; or chopped very fine, and seasoned with herbs, and bound together by egg and bread-crumbs; it may be fried in balls, 'or in the form of large eggs, and served with a gravy made with a few spoonfuls of the soup.

Of half an ox-cheek excellent soup may be made: the meat, when taken from the bones, may be served as a stew.

When one or two ribs are purchased by a small family, it is a good plan to have the bones taken out, and the meat rolled round in the shape of a fillet; a considerable saving is effected by this plan, as, when not so prepared, the thin part of the extremity of the bone is frequently wasted. The bone cut out when the meat is raw will assist in making soap, and is much preferable to a cold beef-bone. They are sometimes cut off short, and salted or stewed, but rolled is the better plan; and in this manner a single rib can be skewered into a handsom? fillet; the fat and lean being marbled, and the appearance of the whole improved.

\section{8.-TO COLLAR RIBS OF BEEF.}

Take two or three ribs of beef; cut it from the bones; rub it well with salt, brown sugar, and saltpetre; let it remain a fortnight, turning it every day; then season it with pepper, mace, cloves, allspice, and a clove of garlic chopped very fine; cover it well with parsley, thyme, and sweet marjoram; roll it up very tight, and bind it round with tape; put it into a pot with some water, cover it close up, and send it to the oven to be baked very slowly three or four hours; then take it out, and press it between two boards with weights. This is an excellent dish for luncheon or breakfast.

\section{9.-A MARINADE TO BASTE ROAST MEATS.}

Chop up some fat bacon with a clove of garlic and a sprig of parsley; add salt, pepper, a spoonful of vinegar, and four spoonfuls of oil; beat it up well, and baste the meat with it.

\section{0.-SIRLOIN OF BEEF ROASTED.}

Break the upper part of the chine bones, cut them out, and cut through the strong gristle on the top about an inch apart, and skewer it in its place, which will prevent its drawing up or looking unsightly. Run the spit just under the bark at the thin end, and bring it out between the joints. By spitting it thus you avoid showing where the spit has gone through. Cover the fat with a sheet of buttered paper, and roast gently for three or four hours, according to the size of the joint." The under part is sometimes stuffed with forcemeat, in the following manner :-

Carefully lift up the fat from the inside of the sirloin with a sharp knife; take out all the mieat close to the bone, and mince it small; take one-half pound of suet, and chop it fine ; mix with it some grated bread-crumbs, a little lemon-peel, thyme, eschalot minced very fine; mix all together with a glass of port wine; put it back again into the same place, and cover it with the skin and fat; skewer it down neatly with small wooden skewers, and cover it with paper. The meat should be spitted before you take out the inside; and when done do not take off the paper until the joint is put into the dish : then serve it up garnished with scraped horseradish. 
The method of taking the meat from the bone, and rolling it so as to have the forcemeat in the middle, is easier, but adds its flavor to the whole joint; while this way keeps the upper and the under part separate.

451.-TO DRESS THE INSIDE OF A SIRLOIN.

Cut it out in one piece, if not used at table; stew it with good broth or gravy, a little spice, and a table-spoonful of walnut ketchup. Serve with chopped pickles.

\section{2.-TO DRESS THE INSIDE OF A COLD SIRLOIN.}

Cut out all the meat, and a little fat, into pieces as thick as your finger, and four inches long; dredge them with flour, and fry in butter of a nice brown : drain the butter from the meat, and toss it up in a rich gravy, seasoned with pepper, salt, anchovy, and shalot. Do not let it boil on any account. Before you serve, add two spoonfuls of vinegar and a glass of port wine. Garnish with crimped parsley.

Or:-Cut the meat in slices about four inches long, and one-half an inch thick, the fat with the lean; season them with pepper and salt, and fry them in good fresh butter; have ready some good brown gravy, and stew them gently for half an hour; add a little mushroom ketchup, and a table-spoonful of eschalot vinegar, with a wine glass of port, and just before serving put in a small piece of butter rolled in flour. Serve hot in a covered dish. This part is called the "fillet," and, when raw, may be either stewed whole or in the above manner.

\section{3.-ANOTHER WAY.}

Cut the inside of the sirloin into pieces, dredge it with flour, put it into a fryingpan in which some butter is boiling; when it is browned, put it into a stewpan with some brown gravy, highly seasoned, squeeze in half a lemon, and serve.

\section{4.-ANOTHER WAY.}

Cut it in strips, as for collops, flatten it, flour, and fry in butter; lay in the centre of a hot dish a mound of spinach, with poached eggs on the top: lay the beef round the spinach.

\section{5.-FILLET OF BEEF, ROASTED.}

If unaccustomed to the use of the knife, the butcher's aid may be obtained to cut the fillet, which comes from the inside of the sirloin; it may be larded or roasted plain; for high dinners it is larded; baste with fresh butter. It must be a large fillet which takes longer than an hour and twenty minutes; serve with tomato sauce, garnish with horseradish, unless served with currant jelly, then serve as with venison or hare, on warmed dishes and plates.

\section{6.-FILLET OF BEEF.}

To dress the inside of a Sirloin of Beef to taste like Hare. Take the inside of a large sirloin that has hung until tender; soak it twenty-four hours in a few glasses of port wine and vinegar. Have ready a good stuffing as for hare; do not spread, but put it into the middle of the beef, and roll it up tight. Roast it on a hanging spit, and baste it with a glass of the wine and vinegar, mixed with a teaspoonful of Jamaica pepper, and a clove or two, in the finest powder, until it is all dried up; then baste with butter. Serve with a rich gravy in the dish, and currant-jelly sauce in a tureen.

\section{7.-RUMP OF BEEF.}

If of a well-fed ox, and hung til! tender, this is one of the most juicy and best flavored of all the joints of beef, but is 
more frequently stewed than roasted. It is generally sold in the country undivided from the aitch-bone; or cut across, and not lengthwise, which makes it too large for one dressing, and neither fit for boiling nor roasting.

At family dinners, a Yorkshire pudding is a usual accompaniment, but now seldom served at genteel tables.

\section{8.-TO STEW BEEF.}

It should be put down in a pot with just sufficient cold water to cover the meat, and closely covered After boiling three or four hours, according to the size of the piece, cut in small pieces, not larger than dice, two or three carrots and heads of celery, with a little sweet herbs, and put them into the pot along with peppercorns, mace, and a couple of large onions stuck full of cloves, and let it then simmer by the side of the fire for two or three hours, taking care to skim off any grease that may appear on the top.

By this time the meat will probably be tender enough; when take out the whole onions, mince them, and fry them in butter, to be mixed in the gravy made by the meat, which season with salt and cayenne, or Chili-vinegar, to which add some mushroom or walnut ketchup. Thicken the gravy with a little flour, and brown it, if necessary, with a spoonful of sugar burnt soit ; which, besides imparting its color, adds an agreeable flavor. Such is the most simple mode; but the sauce may be much improved by a glass or two of port wine and a spoonful of curry pow-

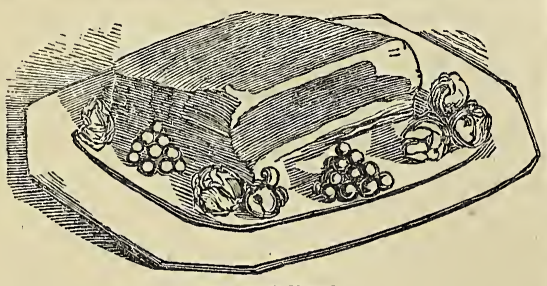

Stewed Beef.

der; if the odor of garlic be not objected to, a clove boiled in the stew will be found to give it a fine flavor. Garnish with vegetables.

A small piece of beef-say of four pounds-will take the time mentioned; but the large joints will require full double that time; and should be put to stew overnight, adding the vegetables in the morning.

\section{9.-RUMP OF BEEF STEW.}

Half roast it; then put it into a pot with three pints of water, a pound of sliced bacon, a bunch of sweet herbs, two wine-glasses of vinegar, and a bottle of cider or small wine; stick cloves into a couple of large onions, add a few sageleaves, and cover the beef closely, adding more water should there not be sufficient gravy from the meat. Let it simmer for three hours; then strain the gravy. Boil or bake some button onions, and lay them round the beef; cover it also with forcemeat balls, fried ornaments of paste, and nushrooms, if in season; add to the gravy a glass of port wine, a spoonful of soy and Oude sauce; boil down a part to a glaze, and put it on the beef: thicken the remainder if necessary, and pour it round, garnishing the dish with pickles.

Or:-Season it high with cayenne, salt, allspice, cloves, and a blade of mace, all in fine powder. Bin $J$ it up tight, and lay it into a pot that will just hold it: resting it on two or three twigs, to prevent the meat from sticking. Fry three large onions sliced, and put them to it, with carrots, turnips, a shalot, a blacie of mace, and some celery. Cover the meat with good beef-broth, and simmer it as gently as possible for several hours until quite tender. Clear off the fat; and add to the gravy half a pint of port wine, a small glass of Chili vinegar, and a large spoonful of ketchup; simmer half an hour, and serve in a deep dish. The herbs to be used should be burnet, tarra- 
gon, parsley, thyme, basil, marjoram, and all or any of the most savory sort.

Or:-Prepare the beef as directed in the foregoing receipts. and glaze it twice; stew some white haricot beans in good brown gravy, with an onion sliced, a carrot, some parsley, and a bunch of sweet herbs, a clove of garlic, a bay-leaf, and a slice of lean ham; let all simmer gently together till the beans are sufficiently tender; then separate them from the other regetables, and put them in a sieve to drain; pass the sauce and vegetables through a tammy. add a table-spoonful of vinegar, and if not thick enongh, a bit of butter rolled in flour; put in the haricot beans, make all boiling-hot, and pour the sauce into the dish round the beef:

\section{0.-BRISKET OF BEEF STEWED.}

Stew it in sufficient water to cover the meat; when quite tender, take out the bones, and skim off the fat; add to the gravy, when strained, a glass of wine and a little spice tied up in a muslin bag. Have ready either mushrooms, truffles, or vegetables boiled, and cut into shapes. Lay them on and round the beef; reduce part of the gravy to a glaze; lay it on the top, and pour the remainder into the dish.

It is a good piece to be stewed, as it may be cut from the bone, and of any size.

\section{1.-LEG OF BEEF STEWED.}

Salt six pounds of the half-leg, or stiff marrow-bone of beef for three or four days. Make holes in it about one and a half inches deep, and press in, very hard, forcemeat made in the following manner : one and a half pounds of suet sliced very fine; pepper, salt, and a few cloves, some winter savory, and sweet marjoram, mixed well together. The beef must be baked in a deep pan, with water reaching about three-quarters of the way up, and forcemeat spread over the top, which, when the meat is baked, is taken off, cut into shapes, and laid round the dish.

\section{2.-BOUILLON}

Is the common soup of France, and is in use in almost every French family. Put into an earthen stock-pot in the proportion of one pound of beef to one quart of cold water. Place it on the side of the fire, and let it become slowly hot. By so doing the fibre of the meat is enlarged, the gelatine is dissolved, and the savorous parts of the meat are diffused through the broth. When the object is simply to make a good, pure-flavored beef broth, part of the shin or leg will answer the purpose, adding some vegetables, and letting it stew four or five hours; but if the meat is to be eaten, the rump or leg-ofmutton piece should be used

\section{3.-BOUILLI.}

The rump of beef is best for this purpose, as the meat is to be served up in a separate dish, and will make a finely flavored sort of soup. Take as much of it as may be thought necessary; but for a small party, say from four to six pounds, along with two or three large roasted onions, in one of which some cloves may be stuck, and a moderate quantity of whole pepper, with a bunch of sweet herbs; to which an anchovy may be added; put it in a stewpan, covered with rather more than a pint of cold water to every pound of meat; and let it simmer by the side of the fire for four or five hours, or until it has become quite tender; then take out the herbs and onions, and add carrots, turnips, and celery, either cut into small squares or sliced, and let the whole boil until sufficiently stewed, and ready for the table.

The soup should then be strained off and served separately, leaving only so much as may be necessary for making sauce for the regetables. The sauce should be a little thickened, and seasoned 
to the palate; if a clove of garlic, or a teaspoonful of garlic and Chili vinegar, be added, it will improve the flavor. In Ireland it is not uncommon to send up the bouilli smothered in onion sauce, the other vegetables being either not used, or brought up in the soup; in France it is very usual to dress cabbage and sausages as an accompaniment to the bouilli, in England, it is more customary to serve it up with the regetable-sauce as abovestated. Cucumbers cut into dice and stewed, with a spoonful of Chili vinegar added, are served at most of the German hotels. The meat if gently stewed until quite tender, without being boiled to rags, will be found excellent, and the'whole an admirable dish.

\section{4.-TO RESTORE TAINTED MEAT.}

Pour a few drops of hydrochloric acid in water till of a slight sour taste, and immerse the tainted meat in it for an hour or so, and it will become quite sweet again.

\section{5.-BROILING.}

The cook must prepare her fire in due time. When ready, it should be clear and bright, so clear from black coal and smoke that the chop or steak may come from the gridiron without blemish or taint of sulphur or smoke. The best fuel for a broil is composed of charcoal and coke, as little smoke is emitted from either, even on commencing the fire, and when well ignited, it is entirely free from it; coke, added to a brisk coal fire, also burns bright, and is well suited for the operation, though with care a proper fire may be made of good hard coal. There is this amongst other disadvantages, in cutting too thick a steak, the outside is likely to be scorched to horny hardness before the interior is half cooked; hence, to say nothing of the misery of those who have not large mouths. the disap- pointed epicure must either wait until it is put again on the gridiron, or instead of eating it rare, be constrained to eat it raw. No gridiron should be used but those with fluted bars, which, forming channels, the greater part of the fat which otherwise falls into the fire, and scorches the steak, is drawn off into a gutter at the bottom; the gridiron should be thoroughly heated, and the bars rubbed with beef or mutton suet previously to putting on the steak, to prevent its being marked by, or adhering to, the bars. A close eye should be kept on the steak to watch the moment for turning it. which is repeatedly done during the process; broiling tongs of convenient size should be used, with which, by a little practice, the steak may be turned with ease and despatch; the cook must have her dish thoroughly heated to receive the broil when done, and the cover hot to place on it instantly. Even when she has accomplished her task, if the servant who is to take it to table loiters on the way, the steak will have lost its zest. A steak or chop should be briskly cooked, speedily conveyed to table, and served with despatch.

\section{6.-BEEF STEAKS, BROILED.}

Be particular that the fire is clear ; it is of no use to attempt to broil a steak over a dull, smoky, or flaring fire; see that the gridiron is clean, and the bars rubbed with suet preparatory to laying on the steak; when they are browned turn them; do not be afraid of doing this often, as this is the best plan to preserve the gravy. When they are done rub them over with a piece of fresh butter, pepper and salt them, sprinkle the shalot or onion cut very small, and send them to table with oyster sauce, a dish of nicely cooked greens, and well boiled potatoes : they are frequently and pleas- 
antly garnished with scraped horseradish.

467.-THE ENGLISH DISH OF BEEF STEAK AND ONIONS.

Pound the steak, season, and fry it in a sauté or fryingpan; then dredge flour over it, and add, by degrees, a cup of boiling water with more seasoning. Drain the onions, which must have been boiled, cut them up, and put them into the pan, having taken out the steak; add a lump of butter and a little more flour; stir them to prevent scorching; and when the onions are well browned, put in the steak, and place the whole orer the fire till heated thoroughly. In serving, heap the onions upon the steak.

\section{8.-BEEF STEAKS ROLLED AND ROASTED.}

Cut handsome steaks from the rump, and if not sufficiently tender let them be well beaten; make a rich stuffing of equal parts. of ham and veal well peppered; stew it for a short time, and pound it in a mortar with bread steeped in milk, a lump of butter, and the yolk of two or three eggs; spread this forcemeat over the steaks, roll them up and tie them tightly, roast them before a clear fire. They will occupy an hour and twenty minutes to an hour and a half roasting; baste well with butter while roasting, and serve with brown gravy.

\section{9.-STEWED BEEF STEAKS.}

Stew the steaks in three parts of a pint of water, to which has been added a bunch of sweet herbs, two blades of mace, an onion stuck with cloves-say three, an anchovy, and a lump of butter soaked in flour; pour over a glass of sherry or Madeira. Stew with the pan covered down, until the steaks are tender, but not too much so; then place them in a fryingpan with enough of fresh butter, hissing hot, to cover them, fry them brown, pour off the fat, and in its place pour into the pan the gravy in which the steaks were stewed; when the gravy is thoroughly heated, and is of a rich consistency, place the steaks in a hot dish, pour the sauce over them. The steaks should be large, the finest from the rump, and have a due proportion of fat with them.

\section{0.-BEEF STEAKS, À LA FRANÇAISE.}

Take a fine steak and dip it into cold spring water, let it drain a few minutes, lay it in a dish and pour over it sufficient clarified butter hot, and cover it; let it remain twelve hours, then remove the butter, and roll the steak with the rolling-pin a dozen times rather hardly, let it lie in front of a clear fire ten minutes, turning it once or twice, put it into a fryingpan, with water half an inch in depth, and let it fry until it browns.

Mince some parsley very fine, chop an eschalot as fine as can be, and season them with cayenne, salt, and a little white pepper; work them with a lump of fresh butter, and when the steak is brown take it from the pan, rub it well with the mixture on both sides, and return it to the pan until done enough; dish it, thicken the gravy in the pan with a little butter rolled in flour if it requires it, and pour it over the steak and serve.

\section{1.-BEEF STEAKS A LA PARISIENNE.}

Cut thin steaks from the finest and tenderest part of the rump, sprinkle pounded salt, a little cayenne and white pepper combined, over them; lay them in a pan with an ounce of fresh butter, cut in pieces; work half a teaspoonful of flour with three ounces of fresh butter, as much parsley minced exceedingly fine as would lie on a shilling, roll it, and cut in large dice, lay it in a dish, squeeze the half of a lemon over the butter, and 
when the steaks are done lay them upon the butter; have ready a quantity of raw peeled potatoes, cut in thin slices, and washed in milk and water ready, fry them in the butter and gravy left by the steak, and lay them round the dish; they will be done when they are a rich brown.

\section{2.-PALATES OF BEEF.}

Four white skinned palates; if for a white dish lay them all night in salt and water, wash them well, put them on to scald, take off all the skin, then put them into your stock pot, let them boil several hours until so tender that you can pass a straw through them, then take them up and lay them flat on a large dish separate, placing another on the top of them with a weight to keep them flat: if to be dressed whole turn the sides smooth, spread each with quenelle or forcemeat, roll them up and tie them; it will take six for this dish; steam them for a quarter of an hour, take them up and glaze them well, and take off the string; if for a turban or timbales, cut them out with a plain round cutter, either using two small moulds or one large; proceed with those two as you would for the timbale of macaroni, leaving out the cheese and any other layer; introduce slices of truffles all round, and then palates, then mushrooms until your mould is full, put a layer of quenelle on the top, paper it on the top with buttered paper, steam as other timbales; haricot roots, truffles, mushrooms, tomato, piquant, any of these will do for sauces, or I talienne; glaze the tops when turned out.

\section{3.-BEEF PALATES.}

Take as many as required, let them simmer until they peel, put them in a rich gravy, stew until very tender, sea- son with cayenne, salt, two teaspoonfuls of mushroom ketchup-serve.

\section{4.-BEEF COLLOPS.}

Any part of beef which is tender will serve to make collops; cut the beef into pieces about three inches long, beat them flat, dredge them with flour, fry them in butter, lay them in a stewpan, cover them with brown gravy, put in half an eschalot minced fine, a lump of butter rolled in flour to thicken, with a little pepper and salt; stew without suffering it to boil ; serve with pickles, or squeeze in half a lemon, according to taste; serve in a tureen, and serve hot.

$$
\text { 475.-A BEEF STEW. }
$$

Take two or three pounds of the rump of beef, cut away all the fat and skin, and cut it into pieces about two or three inches square, put it into a stewpan, and pour upon it a quart of broth, let it boil, sprinkle in a little salt and pepper to taste; when it has boiled very gently, or simmered two hours, shred finely a large lemon, add it to the gravy, and in twenty minutes pour in a flavoring composed of two table-spoonfuls of Harvey's sauce, the juice of the lemon the rind of which has been sliced into the gravy, a spoonful of flour, and a little ketchup; add at pleasure two glasses of Madeira, or one of sherry, or port, a quarter of an hour after the flavoring, and serve.

\section{6.-BEEF HASHED.}

Take the bones of the joint to be hashed, and break them small, stew them in very little water, with a bunch of sweet herbs, and a few onions; roll a lump of butter in flour, brown it in a stewpan, pour the gravy to it, and add the meat to be hashed, cut two small onions in thin slices, a carrot also, and a little parsley shred finely; stew gently until the meat is hot through, and serve. 


\section{7.-HASH BALLS.}

Mince cold roast meat very fine, mix it with cold boiled potatoes chopped; season with pepper and salt, and a little of the gravy ; make it into cakes as large as a biscuit, cover each with beaten egg and then with bread crumbs, and fry the cakes a light brown in butter, lard, or dripping.

\section{8.-BEEF TONGUE-TO CURE.}

Throw a handful of salt over the tongue, seeing that it is sprinkled on both sides, let it remain to drain until the following day, make a pickle of a tablespoonful of common salt, half that quantity of saltpetre, and the same quantity of coarse sugar as of salt; rub this mixture well into the tongue, do so every day for a week; it will then bê found necessary to add more salt, a table-spoonful will suffice; in four more days the tongue will be cured sufficiently.

Some persons do not rub the pickle into the tongue, but let it absorb it, merely turning it daily; this method will be found to occupy a month or five weeks before it will be cured. When the tongue is to be dried affix a paper to it with a date; smoke over a wood fire four days unless wrapped in paper, and then as many weeks will be required.

\section{9.-TO DRESS BEEF TONGUES.}

To dress them, boil the tongue tender, it will take five hours; always dress them as they come out of the pickle, unless they have been very long there, then they may be soaked three or four hours in cold water, or if they have been smoked, and hung long, they should be softened by lying in water five or six hours. They should be brought to a boil gently, and then simmer until tender; when they have been on the fire about two hours, and the scum removed as it rises, throw in a bunch of sweet herbs of a tolerable size ; it will improve the flavor of the tongue.

480.-A FAMILY STEW OF BEEF.

Take any piece of beef good for stewing, cut it into small pieces, slice two or three large onions, and put them into the stewpan with two ounces of butter or good beef-dripping. When melted, dredge in some flour, add the meat also dredged with flour, and enough water to keep it from burning. When the gravy has drawn, fill up with boiling water, let it come to a boil gently, skim the pot well, then add a spoonful of mixed spices, and a bay-leaf or two; set the pan by the side of the fire to stew slowly for a couple of hours. Eleven pounds of meat will take four hours. This dish may be thickened like Irish stew, with potatoes, or it may be served with the addition of chopped vegetables of all kinds, previously fried.

\section{1.-TONGUE LARDED.}

This when well cooked is especially pleasant to some palates. Take a tongue which has been pickled, a small one is the best, cut off the root, and put it into a pan; cover it with water, and let it boil five-and-twenty or thirty minutes. Take it out, and then dip it in scalding water to blanch, and remove the skin.

Take a piece of fat bacon, cut it into strips for larding. Make a seasoning of pounded sweet herbs, eschalot, mace, and a little cayenne pepper mixed with white pepper and salt; sprinkle the bacon strips with it, and leaving a line for division down the centre of the tongue. Lard it all over. Braise the tongue, and then glaze; separate it in the space left, but leave it attached at either end, so that when laid open on the dish it is not entirely divided in two. Have ready some brown sauce, flavored with minced capers, sliced pickled gherkins, the juice of half a lemon, and half a small tea-spoonful of 
cayenne pepper. Pour it when ready into a dish; lay the tongue upon it, and serve as hot as possible.

\section{2.-BEEF OLIVES.}

Cut some handsome steaks, flatten them well with a roller, dredge them well with a small quantity of white pepper and salt, have some forcemeat, made with the fat and lean of veal mixed together, a small bit of lean ham or bacon, parsley, and sweet herbs, with a few bread crumbs, all beaten in a mortar, and mixed with an egg; lay a little over each steak, and roll them up tightly, fastening with a skewer; dip them in the yolk of an egg, then in crumbs of bread, and fry them of a pale brown; rolling up each separate steak, and binding it together with thread; dish them with brown sauce, in which put a glass of white wine, with some strong gravy, seasoned with cayenne.

\section{3.-BEEUF TREMBLANT.}

Cut a handsome piece of beef from the rump, either a fillet or square; hang it up for four days, then put it all night to soak in a pickle of salt and vinegar; put it into a stewpan, and let it be covered with water; add a seasoning of whole pepper and salt, a bundle of sweet herbs, and an onion. Let it simmer very slowly as long as it will hang together, taking care to skim it well. Strain the gravy, and add to it carrots previously boiled, and cut into pieces an inch long; add also a few capers and a mushroom, with a glass of wine and the juice of a lemon. When the beef has been sufficiently stewed take it up, and set it for a short time in a Dutch oven, and glaze it, or brown it with a salamander.

\section{4.-SPICED BEEF.}

A joint from the round, rump, or flank, from ten to fourteen pounds, is the usual weight of the piece intended to be thus dressed. Make a mixture of the follow- ing ingredients, and let them be well amalgamated; pound finely as much mace as will quite fill a teaspoon, grind a nutmeg to powder, and add it, also two spoonfuls of cloves, one-fourth of that quantity of cayenine pepper, and half a pound of coarse brown sugar; rub the beef well with this mixture for three days, turning it each day once ; add three-quarters of a pound of salt, and then continue rubbing well each day, for ten days more; at the expiration of that time dip it into some cold clear spring water, twice or thrice, secure it into a handsome shape, put it into a stewpan with a quart of good beef broth, let it come to a boil, skim as the scum rises, and as soon as it boils put in three carrots cut in slices, a bundle of sweet herbs, a little parsley, and an onion; stew gently four hours.

If it is intended to serve this dish cold, let it remain until it is cool in the liquor in which it was boiled, but take the precaution to put the meat into a clean pan, and pour the liquor over it.

\section{5.-A PICKLE FOR BEEF.}

To one gallon of water put two pounds and a half of common salt, one ounce of saltpetre, half a pound of coarse sugar, boil it for a quarter of an hour, and be particular while boiling to remove every particle of scum while rising, that it may be as clear as possible, let it be cold when poured upon the beef. If it is desired to makke the pickle last for a very long time, add a gallon of spring water to the above quantity, which should, if for keeping, be also spring water, add three ounces of saltpetre, two pounds of bay salt, and a pound and a half of coarse brown sugar. Whatever joints are put into this pickle, they should be kept closely covered down. Prepare thus the beef for pickling, keep it as long as you can without taint, spread over it coarse sugar, and let it remain for two days to drain. Rub the beef thoroughly with the pickle, and let it remain 
in it eight, ten, twelve, or fourteen days, according to its size and quantity ; a considerable quantity of beef may be pickled together, indeed the closer it is packed the better, so that it is covered with the pickle and kept tightly down; when they are taken out of the pickle, lay some sticks across the pan and let them drip into it, when as much has fallen from them as will, wipe them dry, and they may either be cooked at once or dried; if the latter be determined upon, after having well dried them, smoke eight hours over burnt sawdust and damp straw, or sew them in a cloth and send them to the baker, and let them hang seven or eight days. Do not, as in the other receipt, boil the pickle before using the first time, but after it has been once used, and every succeeding time, observing that it must be kept skimmed, and each time of boiling add a quart of water and a couple of pounds of salt. This pickle will answer equally well for hams or tongues.

\section{6.-HUNG BEEF}

Take twelve to fourteen pounds of the flank of beef, throw over it a handful of salt; let it drain twenty-four hours. Make a brine of one pound of salt, one ounce of saltpetre ; let them be quite dry, and pound them to a fine powder before using, a quarter of a pound of bay salt and two ounces of coarse sugar. If it is intended to make the beef red, add three grains of cochineal; rub the beef with this brine for a week, and then turn it; let it remain two days, and then rub it again for seven or eight days; then let it drain from the pickle. Send it to the baker's to be smoked. When wanted for dressing, put it into cold water more than enough to cover it, boil gradually until enough, and put it under a heavy weight while hot. It may be served with carrots and greens, or, if for grating, choose a lean piece, put it in boiling water ; keep it boiling rapidly; four pounds will take an hour.

\section{7.-BEEF HUNG.}

The best piece is the navel piece, it must be hung up in a cellar until it is a little damp, but not long enough to change, take it down and wash it well in brown sugar and water, dry it with a cloth, cut it in two or three pieces, take half a pound of brown sugar, two pounds of bay salt dried and pounded smal!, six ounces of saltpetre dried and beat fine, rub it well into the beef, then rub common salt over it as much as will make it salt enough; let it lie together ten days, changing the pieces from the bottom to the top; hang it where it may have the warmth of the fire, but not too near; when it is dressed boil it in hay and water until tender; it will keep two or three months; when mouldy dip it in water.

\section{8.-DUTCH HUNG BEEF.}

Rub a lean piece of beef-about twelve pounds-with molasses, and turn it frequently, in three days wipe it dry, salt it with a pound of salt and an ounce of saltpetre in fine powder, rub well in, turning every day for fourteen days, roll it as tightly as you can in a coarse cloth, lay a heavy weight upon it, hang it to dry in the smoke from wood, reversing it every day, boil in spring water, press it while hot and grate or rice it to fancy.

\section{9.-RUMP STEAK STEWED.}

Cut a steak about an inch thick with a good bit of fat, fry it over a brisk fire, place it in a stewpan with the gravy, a little good stock, a little port wine, and some chopped mushrooms, stew gently; when tender put into it some good brown sauce, shake it gently about; dish it, and put scraped or grated horseradish on the top; if for oysters or mushrooms, see those sauces; season with salt, cayenne pepper, and sugar. 
490.-RUMP STEAK PLAIN BROILED.

Cut your steak not so thick as for the former; have ready a good clear fire, put your gridiron to get quite hot, then put on the steak at full length, frequently stirring it with your steak tongs, a few minutes according to taste will do it, place it on your dish, put a good slice of butter rubbed all over it, and now pepper and salt it. Horseradish on the top of it, and frequently sauces.

\section{1.-BAKED BEEF.}

A Rump of twenty to twenty-five pounds weight.-Take two ounces each of pepper and allspice; one ounce of pounded cloves, and the same quantity of mace; rub this all over the joint, which should be hung up for a fortnight or three weeks, according to the weather - taking care to keep it dry, and to occasionally renew the seasoning. When ready for baking, wash off the spice with port wine, and lard the rump throughout -not in the common mode used by poulterers, but by inserting large lardoons in different parts of the meat. Then put a large quantity of suet, shred fine, both under and over it, and cover it with coarse flour and water paste, between which and the suet you may put a few bay-leaves or some sweet herbs. If eaten hot, the dough, bay-leaves, and suet must all be taken off; the joint basted, sprinkled with a little salt and flour, over

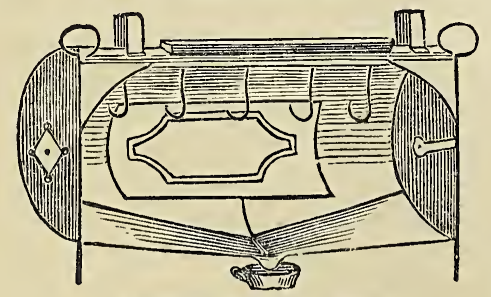

Self-Basting Dutch Oven.

which a salamander should be passed; and served up with strong gravy or brown sauce. If cold, leave on the dough till wanted.

It should be baked in a moderatelyheated oven, and will take, according to the size, from six to eight hours' baking.

Round of Beef may be dressed in the same manner; but the bone should in that case be taken out, and the hole filled up with forcemeat. The flap should be filled in like manner, skewered, and tightly bound round with linen or strong tape, in which case the dough and the larding may be omitted, though the latter will be found an improvement. It should be always left until cold.

\section{2.-BEEF BRAINS FRIED.}

Let your brains be properly marinaded, then leave them to drain; make a preparation with warm water, a little butter, and salt, some flour, and a spoonful of oil, and the whites of eggs whipped to snow, mix all together till like batter; dip your brains into this batter, and then fry them a nice brown color; when fried let them drain on a dry cloth, garnish with fried parsley.

\section{3.-BEEF BRAINS À LA SAUCK PIQUANTE.}

Cook your brains in a marinade ; drain them, put them on a dish, and pour a sauce piquante all over them.

\section{4-BUBBLE AND SQUEAK.}

Sprinkle some slices of cold boiled beef with pepper; fry them with a bit of butter of a light brown; boil a cabbage, squeeze it quite dry and chop it small; take the beef out of the frying-pan and lay the cabbage in it; sprinkle a little salt and pepper over it; keep the pan moving over the fire for a few minutes; lay the cabbage in the middle of the dish and the beef around it. 
495.-BUBBLE AND SQUEAK.

Cut slices from a cold boiled round or rump of beef; let them be fried quickly until brown, and put them into a dish to be kept hot. Clean the pan from the fat; put into it greens and carrots previously boiled and chopped small, or, insicead of these, large onions sliced thin and fried, though both the latter are sometimes omitted. Add a little butter, pepper, and salt; make them very hot, and put them round the beef with a little gravy.

Cold pork boiled is thought by some to be a better material for bubble and squeak than beef, which is sometimes hard. In either case the slices should be very thin, and lightly fried.

\section{6.-OX-TAILS.}

Cut the tails in pieces; lay them in a stewpan, with butter and a large onion; set them over a smart fire to make them brown; peel and boil a couple of dozen of button onions in about three pints of water, for fifteen or twenty minutes; set them by and pour the liquor they were boiled in upon the tails, adding sufficient boiling water to cover them; put in six ounces of carrots, and eight of turnips, cut into slices, or balls the size of nutmegs ; put in the carrots twenty minutes before the turnips. Be careful that they are not stewed too fast or too much. When they are tender, pass the gravy through a sieve; skim off the fat, and pay great attention in doing so, as the fat rises while the tails are stewing. Keep the meat and vegetables hot. Thicken the gravy by putting an ounce of butter into a stewpan; when melted, stir in as much flour as will stiffen it. Pour the gravy in by degrees, stirring it till it boils ; strain it through a sieve into a stewpan, and let it simmer gently till the meat and vegetables are dished. Lay the tails round the dish, and the vegetables in the middle; pour the gravy over; minced 17 gherkins or capers may be added. Pour boiling water over the onions to warm them, and put them round the dish the last thing.

Or:-Divide them at the joints, blanch, and parboil them; put them into a stewpan with just water or weak broth enough to cover them; let them simmer over a gentle fire, and remove the scum carefully as it rises; then put in an onion, a blade of mace, and a little pepper and salt; put them on again to simmer, and when sufficiently done add a spoonful of essence of anchovy and some flour rolled in butter. This is an excellent and nourishing dish.

$$
\text { 497.-TRIPE. }
$$

Tripe may be dressed in several ways, but whatever mode may be employed, it will always be found an improvement to soak it for a whole night in milk. Some say, seven or eight days in salt and water. If left in the milk until that gets sour, the acidity thus imparted to it will render it still better.

Take two pounds of fresh tripe, cleaned and dressed by the tripe-dresser; cut away the coarsest fat, and boil it in equal parts of milk and water ; twenty minutes to half an hour will be long enough. Boil in the same water which boils the tripe, four large onions ; the onions should be put on the fire at least half an hour before the tripe is put in the stewpan, and then made into a rich onion sauce, which serve with the tripe.

Tripe is cleaned, dried, cut into pieces, and fried in batter, and served with melted butter.

Tripe is cut into slices; three eggs are beaten up with minced parsley, sweet herbs, onions chopped exceedingly fine, parsley, and mushrooms. The tripe is dipped into this mixture, and fried in boiling lard.

Tripe may be cut into collops, covered with a mixture of parsley, onions, and 
mushrooms, minced exceedingly fine, and fried in clarified or fresh butter. Serve mushroom sance with it.

Tripe can be stewed in gravy in which put parsley, onions, and mushrooms, or in lieu of the latter, mushroom ketchup. Thicken the gravy with flour and butter. When the tripe is tender, it will be done. A lemon may be sent to table with it.

\section{8.-TO STEW OX-CHEEK.}

Clean the head nicely, then soak it for sume hours in cold water; put it into a stewpan, and let it simmer gently till it is quite tender; then take out the bones, and tie the meat up in a cloth; put a weight upon it, and let it stand till the next day; make a forcemeat of any white meat, and boil six eggs hard; cut the cheek in slices; put some at the bottom of a dish, then a layer of forcemeat, then one of the sliced eggs, another of meat, and so on till the dish is full; season with pepper and salt, and pour in as much of the gravy as the dish will hold; either stew it in the usual way, or cover it with a coarse paste and send it to the oven to be baked slowly. The paste to be removed before brought to table.

\section{9.-BEEF TONGUE.}

If it has been dried and smoked, before it is dressed it should be soaked overnight; but if only pickled, a few hours will be sufficient. Put it into a pot of cold water, and set it over a slow fire for

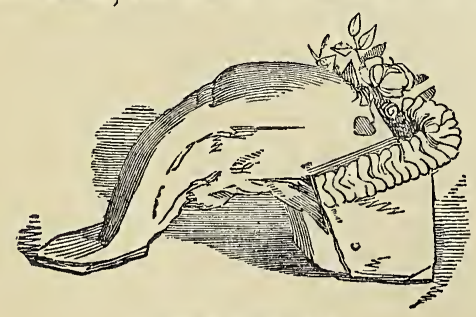

Tongue Garnished. then put it aside, and keep it simmering for three and a half to four hours, according to its size: you can ascertain when it is done by probing it with a skewer. Peel it, trim the root, glaze it, and before serving surround the root with a paper frill, and stick a flower or two on the top.

Its appearance, and its flavor, also, may be improved by rubbing it over, when skinned, with yolk of egg, on which crumbs of bread and finely minced sweet herbs may be strewed; then slightiy basting it with butter, and browning it with a salamander.

\section{0.-TO STEW TONGUE.}

Salt a tongue with saltpetre and common salt for a week, turning it every day; boil it tender enough to peel; when done, stew it with a moderately strong gravy; season with soy, mushroomketchup, cayenne pepper, pounded cloves, and salt, if necessary. Serve with truffles, morels, and mushrooms, stewed in gravy.

\section{1.-AN EXCELLENT WAY OF PREPAR-} ING TONGUES TO EAT COLD.

Season with common salt and saitpetre, brown sugar, a little bay-salt, pepper, cloves, mace, and allspice. in fine powder, for a fortnight; then take away the pickle, put the tongue into a small pan, and lay some butter on it; cover it with brown crust, and bake it slowly till so tender that a straw will go through it; put it into a tin mould, and press it well, laying in as much fat as possible.

The thin part of tongues, if hung up to dry, grates like hung beef, and also makes a fine addition to the flavor of omelets.

\section{2.-TO ROAST A BEEF TONGUE.}

Take a fine large fresh tongue, scald it, and take off the skin; cut it off at the

an hour or two before it comes to a boil; root and trim it neatly; stick a few 
cloves here and there in it, and put it in with spice tied in a bag. Have boiled a cradle-spit; sprinkle it with salt, and baste it well with butter. Serve it with a good sauce in a sauceboat, made as follows :-Put into a stewpan half a pint of port wine, with about half the quantity of well seasoned gravy; reduce it to one-half; then stir in a good piece of butter and a table-spoonful of flour; add a squeeze of lemon; when the butter is melted and the sauce done, place the tongue in a dish, and serve hot with the sauce poured round. In Spain, the sauce is strongly impregnated with saffron.

\section{3.-FRESH BEEF TONGUE.}

Take a green tongue, stick it with cloves, and boil it gently for three hours; then brush it over with the yolk of an egg, dredge it well with bread-crumbs, and roast it, basting it well with butter. When dished, serve it with a little brown gravy flavored with a glass of wine, and lay slices of currant-jelly round it. A pickled tongue, well washed, may be dressed in the same way, and beefudders also.

\section{4.-A FRESH NEAT'S TONGUE AND UDDER}

May be roasted together in the manner thus described; but when ready to be dished, instead of currant-jelly, put half a pint of gravy into a saucepan, with the juice of a Seville orange, two lumps of sugar, a glass of claret, and a piece of butter: toss the whole over the fire, and serve it up with the tongue and udder, garnishing the dish with slices of lemon. The udder should be stuck with cloves, and both should be continually basted.

\section{5.-BRISKET OF BEEF, STEWED.}

Take any quantity of brisket of beef required, say eight or ten pounds, cover it with water. stew it tender, bone the beef and skim off the fat, strain the gravy, add a glass of port wine, flavor vegetables ready ; cut them into squares, and garnish the beef from the gravy round it, and serve.

\section{6.-ENTRḰES}

Are, in common terms, what are called made-dishes; of course, these are the dishes upon which, in the high class of cookery, the talent of the cook is displayed. Great care should be observed in dishing them up, for the eye is a great assistance to the palate: it often happens that the carelessness of the servant destroys the labors of the cook, by the manner in which the dish is taken from the kitchen to the dining-room. In some measure to avoid that, Soyer directs a small thin border of mashed potatoes, about half an inch wide and a quarter of an inch deep, to be placed on the bottom of the dish, which keeps each object in its place; they should always be served exceedingly hot.

\section{7.-COW-HEELS.}

Ox-feet, or Cow-heels, are not highly esteemed, but they contain much nutriment, and may be dressed in the various ways already stated for tripe, with which they are commonly boiled. They are frequently eaten cold, with mustard and vinegar.

Soak them well; boil, and serve in a napkin, with thick melted butter, a large spoonful of vinegar, and a little mustard and salt. Or boil, and then stew them in a brown gravy. Or cut the heel in four parts, dip each in egg, flour, and fry them in butter. Or fry, and serve with onions fried and put round them : sauce as above.

The water in which they are boiled will make equally good jellies, either relishing or sweet, with that of calves' feet, if duly prepared; and at a far less expense. This jelly gives great addi- 
tional richness, likewise, to soups and gravies.

\section{8.-TO FRY OX-FEET, OR COW-HEEL.}

After preparing them as above, cut them into small pieces; have ready some bread finely crumbled, dip the pieces into the yolk of an egg beaten up, and roll them in the bread-crumbs mixed with chopped parsley, pepper, and salt; fry them in butter or fresh lard, of a fine brown color.

\section{9.-TO POT OX-FEET, OR COW-HEEL.}

Boil them in fresh water till the bones can be easily removed; cut them into small pieces, add a little of the liquor, just enough to moisten it, and mix with it a spoonful of vinegar, some pepper and salt, and a little pounded mace; put it into a mould and turn it out when cold. It is eaten with vinegar and mustard.

\section{0.-MARROW-BONES.}

If too long to serve undivided, saw them in two; cover the open end with a lump of paste and a cloth floured and tied close; the paste must be removed before sent to table. Boil one and a half or two hours according to the size; put a ruffle of paper round each, and serve in a napkin, with very hot toast. The marrow is spread upon the toast, and seasoned with pepper and salt.

\section{1.-BEEF A-LA-MODE.}

Cut out the bone from the beef; then stuff the orifice with rich forcemeat, made with veal and oysters, and the crumb of a roll steeped in milk. Half roast it, and before it is put into the stewpan insert in the top dried and pickled mushrooms, adding mushroom-powder in the orifices; then put in two quarts of gravy made from the bones and trimmings, a large onion stuck with cloves, and two carrots cut in slices. When the beef has stewed till it is quite tender, strain and thicken the sauce; add to it a glass of wine, mushrooms and oysters, and sippets of fried paste ; either the mushrooms or oysters may be omitted, if the pure flavor should be more desirable: warm a few pickles with the garnish and send it up very hot.

\section{2.-BEEF A-LA-MODE.-(a Philadelphia Receipt.)}

Cut the bone out of a round of fresh beef, and put into several incisions a dressing made of bread-crumbs, sweet. herbs, and two small onions, chopped fine, with seasoning of salt, pepper, mace, and butter. Lard the beef, and fasten up the slits, and tie it firmly with tape.

Put into a kettle a pint and a half of water, with a few slices of pork; and put in the beef, stuck with a few cloves; cover closely, and bake it several hours. When it is cooked through, dish it and pour over the gravy, which may be increased in quantity by the addition of a little boiling water, and flour to thicken it, with a spoonful of brown sugar, and a glass of wine. Serve this gravy in a tureen, moistening the meat with it, and garnishing with sliced carrots and beets, and parsley or celery.

\section{3.-THE BATH RECEIPT.}

Take three pounds of the rump, or any part of the beef which will stew well; trim it nicely, and cut off all the fat. Chop all sorts of sweet herbs together very finely, with a little shalot and a great deal of spice, and put them into a saucer that has been rubbed with garlic, and cover them with vinegar. Cut fat bacon into long slips, dip it into the herbs and vinegar, and let the herbs be very thick upon the bacon; lard the beef regularly on both sides, if necessary, in order that it should be thorough- 
ly flavored. Rub the beef over with the remainder of the herbs and spice. Flour the meat, add a piece of butter, the size of a walnut, rolled in flour, and a pint of water. Bake the beef in an oven, strain the gravy, which will scarcely require either thickening or browning, and serve it up with pickles on the top. It is most excellent when cold, but should be served up hot at first. The gravy may be boiled to a glaze if necessary. It will require a good deal of spice, a teaspoonful of cayenne pepper, one of white pepper, a salt-spoonful of allspice, half the quantity of pounded cloves, and a blade of mace pounded.

\section{4.-FILLETS OF BEEF.}

Cut the inside of a sirloin or rump in slices half an inch thick; trim them neatly ; melt a little butter in a sauté or frying-pan; season the fillets; fry them lightly ; serve with tomato sauce, sorrel, anchovy butter, or gherkin sauce.

\section{5.-FILLET OF BEEF BRAISED.}

Take the inside of a sirloin of beef, stuffed or plain, but rolled together so as to bring the fat into the centre. Then strew the bottom of the stewpan with a few slices of ham, in which a small quantity of gravy has been put, just to prevent the bottom of the pan from burning; and on this place the meat, covering it with chopped carrots, celery, button onions, and a pickled chili, together with a sliced gherkin, sweet herbs, salt, mace, and a little allspice, and simmer until tender, then brown it before the fire or with a salamander, skim and season the sauce, and send the meat, sauce, and vegetables up in the same dish.

\section{6.-BEEFSTEAK Å LA FRANCAISE}

Must be cut either from the sirloin or some other prime part of the beef, as rump steaks are not known in France. Pour over it two large spoonfuls of the best Lucca oil, and let it remain all night; then put it and the oil into a frying-pan, with some finely-chopped parsley. pepper, and salt ; fry it until the gravy dries up and it becomes rather brown. Pour the contents of the pan over the steak as sauce. The steaks are usually garnished with slices of fried potatoes. As butter is not known in the southern states of Europe, oil is there constantly used in lieu of it, and this Parisian practice is borrowed from those countries.

\section{7.-BEEF IN A MARINADE.}

Cut the inside of a sirloin in slices, put them into a marinade made as follows:Take equal quantities of vinegar and water, slice some carrots and onions, "add some salt and a few pepper-corns with a clove of garlic. Let all stew together till the goodness be extracted from the vegetables, then strain it. and let it stand till cold. Let the meat lie in this pickle for twenty-four hours before it is dressed; then let it stew gently in a little good gravy till quite tender. Add a wineglassful of port wine, a table-spoonful of mushroom ketchup; a teaspoonfnl of shalot vinegar, and some butter rolled in flour; place the meat neatly in the dish, and pour the sauce over it.

\section{8.-BEEF À LA POLONAISE.}

Take a few pounds of the best beef, without bones, and, after having beaten it for some time with a rolling-pin, make in it deep incisions, but without cutting the meat asunder. Mince some onions, mix them with bread-crumbs, butter, pepper, and salt; fill the incisions with this forcemeat, and skewer up the meat. Put it with some butter into a stewpan, and stew it upon a moderate fire. It should be served with brown sauce made from the gravy which is arawn from the meat. 
519. $-\Lambda$ LA HOUSSARD.

Take any piece of fresh beef, prepared as nearly as possible in the same manner; but instead of forcemeat, fill the incisions with pledgets of fat ham or bacon: season with pepper, salt, and onion; lay it in an iron stewpan that has a cover to fit quite close, and set it by the side of the fire without water. Take care it does not burn, but it must have a strong heat; in two or three hours it will be quite tender, and then serve with its own gravy.

$$
\text { 520.-BEEF À LA MODE. }
$$

There are several methods of making this dish; the hash erroneously termed alamode beef, sold at eating-houses, is a very different dish to the true beef à la mode. The following is called the "old Bath" receipt.

Take a quantity of the buttock, or the leg-of-mutton piece, the clod, or where expense is not an object, the rump of beef as lean as possible; cut away the fat if there is any, make a mixed powder of cloves, alout twenty or thirty, the same quantity of mace, with half an ounce of allspice, savory, parsley, a handful of thyme, knotted marjoram, and all other sweet herbs chopped very fine ; mix them in a glass of vinegar. Take some fat bacon, cut it into slices as long as the beef is thick, and about a quarter of an inch in thickness, roll it well in the powdered spice and herbs, make incisions of the requisite depth, and insert the bacon in the beef, which may be rubbed well over with what remains of the powdered spice, \&c., in the vinegar; then dredge flour over the beef, place it in a baking dish with a lump of butter rolled in flour, with a pint of water; bake it in the oven, strain the gravy, and serve with pickles on the top; if after being larded it should, instead of being baked, be put into the stewpan, add to it as much water as will cover it, four onions chopped fine, half a dozen cloves of garlic, as many bay leaves, a few champignons, half a pint of ale, as much port wine ; add white pepper, cayenne pepper, and salt, a teaspoonful of pyroligneous acid; strew three parts of a pint of fine bread raspings over it, cover down close and stew six or eight hours. according to the size of the beef; when it has stewed sufficiently, take out the beef, keep it hot over boiling water, strain the gravy, remove the fat, champignons, \&c.; boil up again, season to palate, pour the gravy over the beef and send to table.

This is sometimes preferred cold, in which case serve it cut in slices, with the gravy, which will be a jelly.

\section{1.-BEEF À LA MODE. (Soyer's.)}

Have ready six pounds of rump of beef, cut into pieces two inches square, each of which lard through with two or three strips of bacon; have also two pounds of streaked bacon, which clear from the rind, and cut into squares half the size of the beef, put the whole into an earthen pan, with two calf's feet, (cut up small,) half a pint of sherry, two bayleaves, a sprig of thyme, a bunch of parsley, four onions, with a clove stuck in each, a blade of mace, and half a pint of water, cover the pan as in the last, and put it in a moderate oven for three hours ; when done, do not remove the lid until three parts cold, then take out the meat, lay some of the beef at the bottom of a stewpan, (not too large) then a little bacon, then more beef, and so on alternately, press them lightly together, pass the gravy through a hair sieve over, and leave it until quite cold and set, when dip the stewpan into hot water, and turn it out upon a dish to serve. The calf's feet may be made hot in a little of the stock, to which add two pats of butter.. with which you have mixed a teaspoonful of flour, season with a little chopped parsley and half a spoonful of vinegar, and serve as an entrée. The above is exceilent either hot or cold. 
522.-ALAMODE BEEF. (Kitchiner's Receipt.)

Take about eleven pounds of the mouse buttock, clod, or blade-bone of beef, or like weight of veal; cut it into pieces of three or four ounces each; put two or three ounces of beef drippings, and a couple of large onions, into a large deep stewpan; as soon as it is hot, flour the meat and put it into the stewpan, stirring it constantly with a wooden spoon; when it has been on about ten minutes, dredge it with flour and keep doing so till you have stirred in enough to thicken it; then cover it with boiling water, added by degrees, and stirring all together. (it will take about a gallon,) skim it when it boils, and then put in one drachm of ground black pepper, two of allspice, and four bay-leaves; set the pan by the side of the fire, and let it stew very slowly about three hours; when the meat is tender, put it into a tureen, and it is ready for the table. A nice salad may be served with it.

\section{'523.-A FRICANDEAU OF BEEF.}

Take a nice piece of lean beef; lard it with bacon very closely; put it into a stewpan with a pint of broth, a glass of white wine, a bundle of parsley, all sorts of sweet herbs, a clove of garlic, a shalot or two, four cloves, pepper and salt. When the meat is become tender, cover it close ; skim the sauce well, and strain it; set it on the fire, and let it boil till it is reduced to a glaze. Glaze the larded side with this, and serve the meat on sorrel sauce.

\section{4.-POTTED BEEF.}

To a pound of common salt, put a quarter of an ounce of saltpetre, and two ounces of coarse sugar. Rub three pounds of lean beef with this, and let it remain in the brine fifty hours. Drain and dry it, pepper it well with black pepper, put it into a pan; cut half a pound of butter in slices, and lay round it; lay a paste crust over it, and bake it very slowly for four hours and a half. Let it get cold, and then cut off the meat, being careful to separate the stringy pieces from it: pound it in a mortar, working up with it four ounces of fresh butter, and some of the gravy from the meat when baked, seasoned with ground allspice, a little mace, and pepper. When the meat has been combined with the butter and gravy, until it is worked into an even paste, put it into jars, and cover with clarified butter.

If it is purposed to keep it long, cover it with bladder skin. The beef may be potted without in the first instance being salted, but if it is done it should have salt worked up with it, and be soon eaten after potting. Some persons make their potted beef of meat that has been previously cooked, but the above will be found to be the best receipt.

\section{5.-COW-HEEL}

Having been thoroughly washed, scalded, and cleaned, cut them into rieces about two inches long, and one wide; dip them into yolk of egg, cover them with fine bread-crumbs mixed with parsley minced, cayenne pepper, and salt, and fry them in boiling butter.

\section{6.-ANOTHER WAY.}

Having cleaned the feet, bone them, boil them, and stew them in a rich brown gravy; serve them with Indian pickle. Or, if plainly cooked, boil until enough; then serve them on a napkin, with melted butter, flavored with a spoonful of vinegar, and one of made mustard. Lemon pickle may be served with them.

\section{7.-BEEF SAUSAGES.}

To three pounds of beef, very lean, put one pound and a half of suet, and chop very finely; season with sage in powder, 
allspice, pepper, and salt; have skins thoroughly cleaned, and force the meat into them.

\section{8.-BEEF HEART.}

Wash it very carefully, stuff it the same as you would a hare; roast or bake it, and serve with a rich gravy and currant jelly sauce, hash with the same and port wine.

\section{9.-BEEF HEART ROASTED.}

Wash thoroughly, stuff with forcemeat, send it to table as hot as it is possible with currant jelly sauce; it will take about forty minutes roasting, but this depends upon the fire.

\section{0.-BEEF HEART.}

Let it be thoroughly well cooked, and the skin removed. Wipe it dry with a clean cloth; stuff it with veal stuffing; roast two hours and a quarter. Make a brown gravy, as for hare; and serve with the gravy and currant jelly.

The most pleasant way to the palate of dressing this dish, is to roast the heart for rather less than two hours, let it get cold, cut it in pieces, and jug it the same as hare.

\section{1.-STEWED RUMP OF BEEF.}

Half roast the beef; then place it in the stewpan, add three pints or two quarts of water, according to the weight of the joint, two wine glasses of vinegar, three of red wine, more if expense be not considered, a bottle not being too much; cider is sometimes used, but the meat may be stewed without it; add three spoonfuls of walnut ketchup. two or three blades of mace, a shalot, a dessert-spoonful of lemon pickle, cayenne pepper, and salt, cover the stewpan close down, stew gently for two hours, or three if the rump of beef be large, take it up and place it in the dish in which it is to be served, keeping it hot in the manner previously prescribed; remove the scum from the gravy in which it has been stewed, and strain it; add half a pint of mushrooms, three table-spoonfuls of port wine, a spoonful of Harvey's sauce, thicken with flour and butter, pour over the beef, garnish with pickles, forcemeat balls, and hnrseradish.

\section{2. -HUNTER'S BEEF.}

Hang for three days a round of beef of twenty pounds; at the expiration of that time rub it with brine composed of three ounces of saltpetre, twelve ounces of salt, a spoonful of allspice, one of black pepper, an ounce of coarse brown sugar; before it is rubbed with this mixture it must be boned, and it must be rubbed well every day, turning for a fortnight. When it is to be dressed put it into a stewpan, pour in a pint of water, shred a quantity of mutton suet, cover the meat with it, lay over it a thick crust, attaching it round the edge of the pan, tie over securely with paper, and bake for six hours in an oven moderately heated ; take away the paper and crust, chop some parsley very fine, sprinkle it over the beef, and serve it cold.

It will keep some time; the gravy will make a good flavoring for soups.

\section{3.-MINCED BEEF WITH CUCUMBERS.}

Take a fine rump-steak undressed, and with a sharp knife shred it rery fine. Put it into a stewpan with a little clarified butter and some salt; stir it over a quick fire for a few minutes, then add half a pint of good beef gravy ; let it boil gently till it becomes of a proper thickness. Cut two fine cucumbers in slices the thickness of a crown-piece, and put them with an onion sliced in a stewpan with some clarified butter, a little vinegar, a lump of sugar, and fry them of a fine brown color; put them into a stewpan 
with some plain sauce; let it simmer gently till sufficiently done; then lay the mince in the dish, and pour the cucumbers over it; thicken the sauce with a little flour and butter; add the squeeze of a lemon just before serving.

\section{4.-BEEF COLLOPS.}

Cut the inside of a sirloin, or any other convenient piece, into circular shapes, the size and thickness of a half-crown, flour and fry them ; sprinkle with pepper, salt, chopped parsley, and shalot ; make a little gravy in the pan; send to table with gherkin or tomato sauce.

\section{SOYER'S RECEIPTS.}

\section{5.-BUBBLE AND SQUEAK.}

I am certain you must know, as well as myself, our hereditary dish called bubble and squeak; but, like the preparation of other things, there is a good way and a bad; and, as you prefer the former to the latter, proceed as follows:-Boil a few greens, or a savoy cabbage (which has been previously well washed), in plain water until tender, which then drain quite dry in a colander or sieve. put it upon a trencher, and chop it rather fine with a knife; then for a pound of salt beef you have in slices, put nearly a quarter of a pound of butter into a frying-pan. in which sauté the beef gently but not too dry; when done, keep it hot, put the cabbage in the frying-pan, season with a little salt and pepper, and when hot through, dress it upon a dish. lay the beef over, and serve. Endive or large cabbage-lettuces may be used instead of cabbage, but care must be taken to drain off all the water.

\section{6.-BROILED BONES.}

Divide them, rub with mustard, pepper, and salt, and broil over a clear fire ; serve with fried potatoes; and a little gravy may be added.

\section{7.-TRIPE, LYONS FASHION.}

When there is any remaining from a previous day, dry it on a clean cloth; cut them into pieces an inch square; put into an omelette or sauté pan one or two ounces of butter, according to the quantity; slice thin a large onion, which fry in the pan; then add the tripe, saute them for five minutes, season with salt, pepper, and a spoonful of vinegar; serve very hot.

\section{8.-OX TAILS À LA JARDINIÈRE.}

Cut and cook two ox tails as directed for soup, but just before they are done, skim well, and take out the pieces of tails, which put upon a dish; then in another stewpan put two ounces of butter, to which, when melted, add three ounces of flour, stirring it over the fire until forming a brownish roux (thickening), then mix by degrees two quarts of the stock the tails were boiled in, and boil all together ten minutes; then put in the tails, with one carrot and two turnips (cut into small dice, or any other shape, with a vegetable cutter), and about thirty button onions; let the whole simmer very gently upon the corner of the fire, keeping it well skimmed, until the vegetables are tender, and the sauce sufficiently thick to adhere to the back of the spoon; dress the meat upon a dish, reduce the sauce, which pour over and serve.

\section{9.-OX TAILS AU GRATIN.}

Cook two ox tails as before, and when cold, dry them upon a cloth, season with pepper and salt, have a couple of eggs well be aten upon a plate, into which dip each piece singly, afterwards throwing them into a dish of bread-crumbs, to cov- 
er every part, then beat them lightly with a knife, and again egg and breadcrumb them, broil them upon a gridiron, or place them in a very hot oven until of a brownish color, when serve with any sauce you may fancy, or a little plain gravy.

\section{0,-OX HEART.}

This dish, although not very recherché, is a good family one, and remarkable for its cheapness. Put it into lukewarm water, one hour to disgorge; then wipe it well with a cloth, and stuff the interior with a highly seasoned veal stuffing; tie it up in paper, and pass a small spit through the sides; set it before a good fire for about two hours to roast, keeping it well basted-being almost deprived of fat, basting is thus required; when done, take off the paper, and serve with any sharp sauce, or a little plain gravy. Two hours will be sufficient to roast a large heart; but if smaller of course less time in proportion would be required. I have also stuffed a heart with sage and onion, and even ventured the apple sauce; both succeeded odmirably, and it can be baked as well as roasted.

I remember, when in business, upon one occasion. having a few friends pop in unexpectedly about luncheon-time upon a Saturday (which is a day I always contrive to keep my larder as short as possible). and having nothing but a heart as a meal to give them, I immediately gave orders to the cook to cut it into slices half an inch thick; dip each piece in flour, and aftcrwards egg and bread-crumb them; then to put four spoonfuls of oil in the frying-pan, lay part of the pieces in, and sauté of a nice color, then to keep them hot in a dish, and sauté the remainder; and when all done, to pour off part of the oil; put a teaspoonful of flour in the pan, mixing it with the remaining oil and gravy, then pouring in a gill of wa- ter, season with a little pepper and salt, four spoonfuls of the vinegar from piccalilly, and a little of the pickle finely chopped; boil the whole a minute; pour over the heart, and serve very hot. It pleased very much, and they made a hearty meal from it; and I have since had some with a little plain gravy, and broiled bacon: in both instances it was very good.

\section{1.-POTATO SANDWICHES.}

Saute the slices of beef as directed for bubble and squeak, cover one side of each piece with mashed potatoes a quarter of an inch in thickness, egg and bread-crumb over, then proceed the same with the other sides; fry in hot fat of a light brown color, as you would a sole, and serve. Any kind of fresh meat may be used the same way.

\section{2.-FILLET OF BEEF SAUTÉ.}

After having cut the fillet in slices. put two ounces of butter into a clean frying-pan, which set upon the fire, and when melted, lay in the meat, seasoned with a salt-spoonful of salt, and half that quantity of pepper to each piece; turn them over three or four times whilst cooking, and, when done, dress upon your dish, with either anchovy or maitre d'hôtel butter.

\section{3.-ANOTHER METHOD.}

When the fillets are dished up, put a table-spoonful of chopped onions into the pan they were cooked in, which cook for about a minute, but not letting them burn, then pour off part of the fat, if too much, and add two teaspoonfuls of flour; stir with a wooden spoon until becoming brownish, then add nearly a pint of water, a table-spoonful of vinegar, and a few drops of browning; let it boil a few minutes, seasoning with a little pepper, salt and sugar ; when of the consistency of a thin sauce, pour over the fillets and serve. A few chopped pickles 
of any description (but not too hot) might be introduced, but then half the quantity of vinegar would be sufficient. A spoonful of Harrey's sauce may be added, and a little glaze improves it.

Mutton, lamb, or pork-chops, or vealcutlets, may be dressed in a similar manner.

\section{4.-MINCED BEEF.}

Cut a pound and a half of lean cooked beef into very small dice, which put upon a plate; in a stewpan put a good teaspoonful of finely chopped onions, with a piece of butter of the size of a walnut, which stir over the fire until the onions become lightly browned, when stir in half a table-spoonful of flour, with which mix by degrees half a pint of broth (or water) to which you have added a few drops of browning and a teaspoonful of vinegar; let it boil five minutes, stirring it the whole time, then throw in the meat, season rather highly with a little pepper and salt, and, when hot, pour it into a deep dish, and serve with sippets of toasted bread round, or poached eggs on it.

\section{5.-CROQUETTES OF BEEF.}

Proceed precisely as in the last, but omitting the vinegar; when done, stir in two yolks of eggs quickly, stir another minute over the fire, then pour it upon a dish until cold; have a couple of eggs well beaten upon a plate, also some bread-crumbs in a separate dish, then divide the preparation into about a dozen pieces, which roll up into round balls: or any other shape, and throw them into the bread-crumbs, move them over until well covered, then roll them into the egg, then the bread-crumbs again, from which take them gently, patting the surface lightly with a knife, put them into very hot lard or fat to fry of a yellowish-brown color, being careful not to break them whilst frying; when done, drain them upon a cloth, and serve either upon a napkin, or with fried parsley.

546.-A FAMILY FRENCH SALAD FOR THE SUMMER.

Cut up a pound of cold beef into thin slices, which put into a salad-bowl, with about half a pound of white fresh lettuce, cut into pieces similar to the beef, season over with a good teaspoonful of salt, half that quantity of pepper, two spoonfuls of vinegar, and five of good salad oil, stir all together lightly with a fork and spoon, and when well mixed it is ready to serve.

For a change, cabbage-lettuce may be used, or, if in season. a little endive (well washed), or a little celery, or a few gherkins; also, to vary the seasoning, a little chopped tarragon and chervil, chopped eschalots, or a little scraped garlic, if approved of, but all in proportion, and used with moderation. White haricot beans are also excellent with it. Remains of cold veal, mutton, or lamb may be dressed the same way.

\section{7.-POTATOES AND MEAT SALAD.}

Proceed as in the last, but omitting the lettuce; if any cold potatoes remain from a previous dinner, peel and cut them in halves if small, but in quarters if large, and then into pieces the size of a shilling, but four times the thickness; put them into a salad-bowl with the meat, seasoning as before, but using more oil and vinegar, and adding a teaspoonful of chopped parsley. A small quantity of any description of pickles might be added to this salad, as also some anchovies or olives. The remains of any fowls or turkey may be mixed in salads, but according to our habits, many persons would fancy they were not nutritious; of that I can assure them to the contrary. The quantity of the meat and vege- 
table should pretty equally balance with kidneys are also an excellent addition to each other; after such a meal, a man's beef-steak puddings and pies.

appetite is perfectly satisfied, and he is ready for an afternoon's work if required. It also does not require the aid of any fire, which we so ungratefully abhor in hot weather. Mr. B. very much approves of this dish once a week in summer

\section{8.-OX KIDNEYS.}

Cut a nice fresh ox-kidney into slices, each being about the size of a half crown piece, but double the thickness (avoiding the white part, or root, which is tough and indigestible), then put a quarter of a pound of butter into a stewpan upon the fire, and when very hot but not black, put in the pieces of kidney, stirring them round with a wooden spoon three minutes over a brisk fire; then add for each pound weight of kidney, half a tablespoonful of flour, half a teaspoonful of salt, half that quantity of pepper, and a little sugar, moisten with a gill of water and half a glass of sherry, add a little browning, if handy, and let simmer gently for five minutes, stirring them round occasionally; if too thick, add a few drops more water, the same should be sufficiently thick to adhere to the back of the spoon, pour them out upon your dish, and serve very hot. Broth u ight be used instead of water if convenient, but then the seasoning should be a little diminished, a little chopped eschalot, parsley, or a few mushrooms, would be an improvement.

By cutting an ox-kidney lengthwise in three slices, it might be broiled or sautéed; if for gentlemen season rather highly, but if ladies are to be the partakers, season more moderately; a little gravy may be served with it, to which you have added at little ketchup; the root of the kidney must not be cut away in this case, although not eatable. $\mathrm{Ox}-$

\section{9.-OX-FEET OR COW-HEELS}

Are very nutritious, especially when well boiled: they may be served in either of the methods directed for tripe, or with a plain parsley-and-butter sauce, to w hich, for a change, the juice of a lemon or a drop of vinegar may be added. Should any be left from the first day's dinner it may be served à la Lyonnaise, as directed for cold tripe.

\section{0.-REMAINS OF OX-TONGUE.}

The remains of a tongue from a previous dinner may be again served thus:Cut it into thin slices, put a small piece of butter into a frying-pan, lay the pieces of tongue over, which warm a few minutes in a sauté-pan, and serve with veal or fowl if any; when at home alone, I frequently have it with mashed potatoes under it; it makes a very good dish for luncheon. The pieces of tongue might also be egged and bread-crumbed previous to cooking as above, and served with a plain gravy, or any sharp sauce. (See Sauces.) Or should you have any tongue, and real, and beef remaining, sprinkle a little chopped shalots at the bottom of a pie-dish, lay a layer of meat over, season with a little salt, pepper, and chopped parsley, then a layer of the tongue; have some yellowish crusts of bread grated, a teaspoonful of which sprinkle over the tongue, then again a layer of the meat proceeding thus until the dish is nearly full, when sprinkle more of the brown bread-crumbs over the top, placing a small piece of butter here and there, pour in two wine-glassfuls of water, set it in a warm ovep half an hour, and serve very hot. Or instead of bread-crumbs, make a little good mashed potato, which spread over it smoothly with a spoon or knife, bake half an hour in a warm oven, and serve. 
Should the remains of a tongue be but small, and if well pickled and boiled, the root and all would be excellent in any kind of beef, lamb, mutton, veal, or pork, hashed, or in pies or puddings made from these meats.

\section{1.-REMAINS OF SALT BEEF.}

The remains of salt beef are very excellent served in the few following ways, no matter from what joint, or from what part of the joint: Cut as large and thin slices as possible, dip each slice into some vinegar from mixed pickles, previously poured upon a plate in small quantities; lay about a pound of the meat thus prepared upon a flat dish, pour a wine-glassful of water over, warm it through in the oven, or before a slow fire, and serve. Another way is, after having dipped. the beef in the vinegar, roll them in flour, and proceed as above, adding double the quantity of water. Another way is to sauté the slices with a little butter in a frying-pan, have ready some nice mashed potatoes very hot, lay the beef over, and serve.

\section{2.-COLD BEEF.}

If any be left from a previous dinner, put it in a dish, placing the meat in the centre, rather higher, cover over with some delicate mashed potatoes, about two inches in thickness, to form a dome, rub some egg over with a paste-brush, and sprinkle crumbs of bread (either grated or otherwise) upon the top, and set it in the oven until well browned, when serve.

\section{3.-BEEF BROTH.}

Take a leg of beef, wash it clean, crack the bone in two or three parts, put it into a pot with a gallon of water, skim it well, then put two or three blades of mace in a little bundle of parsley, and a crust of bread, let it boil till the beef is quite tender, toast some bread, cut it into meat. and pour the soup over it.

\section{4-MARROW BONES.}

They must be sawn into convenient sizes ; cover the ends with a little dough made of flour and water, and tie them in a floured cloth, boil them an hour and a half, serve on a napkin with dry toast.

\section{5.-BAKED MARROW BONES.}

The bones should be prepared as above and laid in a deep dish, then put into an oven and bake gently for two hours. They are sometimes cooked in batter, but if so, the marrow should be cleared from the bones and put in buttered cases made of clean foolscap paper; let them lie in the batter and serve with them in it; when the batter is baked the marrow will be also done.

\section{6.-CURRIED BEEF, MADRAS WAY.}

Take about two ounces of butter, and place it in a saucepan, with two small onions cut up into slices. and let them fry until they are of a light brown; then add a table-spoonful and a half of curry powder, and mix it up well. Now put in the beef cut into pieces about an inch square; pour in from a quarter to a third of a pint of milk, and let it simmer for thirty minutes; then take it off; and place in a dish, with a little lemon juice. Whilst cooking stir constantly, to prevent it burning. Send to table with a wall of mashed potatoes or boiled rice round it.

It greatly improves any curry to add with the milk a quarter of a cocoa-nut, scraped very small, and squeezed through muslin with a little water; this softens the taste of the curry, and, indeed, no curry should be made without it.

\section{7.-BEEF PALATES.}

Simmer them in water several hours, till they will peel; then cut the palates into slices, or leave them whole, as you 
choose, and stew them in a rich gravy till quite tender. .Before you serve, season them with cayenne, salt, and ketchup. If the gravy was drawn clear, give it a boil with some butter and flour.

If to be served white, boil them in milk, and stew them in fricassee-sauce; adding cream, butter, flour, and mushroom powder, and a little pounded mace.

\section{8.-PRESSED BEEF.}

Procure a piece of brisket of beef, cut off the bones, and salt it, adding a little sal prunella to the brine and a little spice; let the beef remain in pickle rather better than a week; when ready to cook, roll it round, tie it in a cloth, and let it simmer gently in plenty of water about seven hours if a whole one, but four hours if only the thin end; when done take it up, remove the string, and tie the cloth at each end, put it upon a dish with another dish over, upon which place half a hundred weight, leaving it until quite cold, then take the meat from the cloth, trim and glaze it lightly, and serve garnished with a few sprigs of fresh parsley.

\section{9.-PICKLE FOR BEEF À LA GARRICK.}

Take twenty pounds of salt, threequarters of a pound of saltpetre, four cakes of sal prunella, two pounds of moist sugar, two cloves of garlic, with which rub the meat well, and leave it rather more than a week, rubbing and turning it over every day.

This pickle is adapted for any thing that is required red.

\section{0.-SPICED BEEF.}

Procure a piece of thin flank of beef about ten pounds in weight, which salt as the last for about a week; when ready split it open with a knife and lay it out flat upon a dresser, having previously prepared six onions chopped very fine, with about ten sprigs of parsley, and the leaves of ten sprigs of thyme, the same of marjoram, two ounces of mixed spice, (without cinnamon,) and half an ounce of black pepper, mix all together, spread half upon the beef as it lays before you, then fold it over to its original shape, lay on the remainder of the preparation, roll it up tightly in a cloth, boil, press, and serve as directed in the last article.

\section{1.-TO FRICASSEE COLD ROAST BEEF.}

Cut the beef into slices, which should be very thin, put it with some strong broth into a stewpan, add parsley chopped small, an onion scored, and a piece of butter, simmer fifteen minutes, add a glass of port wine, a teaspoonful of pyroligneous acid, and the yolk of a couple of eggs; mix well, stew quickly, pot the dish, rub it with a shalot, pour fricassee into it, and serve.

\section{2.-BEEF FRICANDEAU.}

Take a piece of beef as lean as you can obtain it, lard it well over on one side with pieces of bacon. Place in a stewran an eschalot, a bunch of sweet herbs, a faggot of parsley, a little cloves, three parts of a quart of good broth. one glass of sherry, and pepper and salt to palate. A clove of garlic may be added to the eschalot if it is not found of sufficient strength to flavor it without. Put on the meat, and stew until tender, take out the gravy, keep the meat covered down close, skim and strain the sauce, boil it until reduced to a glaze. Glaze the beef with it on the side larded, and serve with sauce piquante, or sauce sorrel.

\section{3.-STRASBURG POTTED MEAT.}

Take a pound and a half of the rump of beef, cut into dice, and put into an earthen jar, with a quarter of a pound of butter at the bottom, tie the jar close up with paper, and set over a pot to boil ; when nearly done, add cloves, mace, all- 
spice, nutmeg. salt, and cayenne pepper to taste; then boil till tender, and let it get cold. Pound the meat, with four anchovies washed and boned, add a quarter of a pound of oiled butter, work it well together with the gravy, warm a little, and add cochineal to color. Then press into small pots, and pour melted mutton suet over the top of each.

\section{1.-A CULLIS.}

You must take meat according to the number of guests; if ten or twelve, a leg of veal and a ham will be necessary, with all the fat, skin, and outside cut off; cut the leg of veal into pieces about three or four inches thick each way, place them in a stewpan, and then the slices of ham, two carrots, and an onion cut in two, cover it close, let it stew gently at first, and as it begins to brown, take off the cover and turn it to color on all sides the same. but take care not to burn the ineat; when it has a pretty brown color, moisten your cullis with broth made of beef or other meat, season the cullis with a little sweet basil, some cloves, and a little garlic; pare a lemon, cut it in slices and put it into the cullis with some mushrooms. Put into a stewpan a good lump of butter, and set it over a slow fire; put into it two or three handfuls of flour, stir it with a wooden ladle, and let it take a color; if the cullis be pretty brown you must put in some flour, the flour being brown with the cullis, pour it gently into the cullis, keeping it stirring with a wooden ladie, then let the cullis stew softly and skim off all the fat; put in two glasses of champagne or other white wine, but take care to keep the cullis very thin, so that you may take the fat well off and clarify it; you must clarify it by putting it into a stove that draws well, cover it close and let it boil without uncovering until it boils over, then uncover and take off the fat that is round the stewpan, then wipe it off the cover also and cover it again; when the cullis is done take out the meat and strain the cullis through a silken strainer; this cul lis is for all sorts of ragouts, fowls, pies. and turcens.

565.-BUBBLE AND SQUEAK. (New Receipt.)

Cut into pieces, convenient for frying, cold roast or boiled beef; pepper, salt, and fry them; when done, lay them on a hot drainer, and while the meat is draining $f$ om the fat used in frying them, have in readiness a cabbage already boiled in two waters; chop it sinall, and put it in the frying-pan with some butter, add a little pepper and salt, keep stirring it, that all of it may be equally done. When taken from the fire, sprinkle over the cabbage a very little vinegar, only enough to give it a slight acid taste. Place the cabbage in the centre of the dish; and arrange the slices of meat neatly around it.

\section{6.-LOBSCOUS.}

Mince, not too finely, some cold roast beef or mutton. Chop the bones, and put them in a saucepan with six potatoes peeled and sliced, one onion, also sliced, some pepper and salt; of these make a gravy. When the potatoes are completely incorporated with the gravy, take out the bones, and put in the meat; stew the whole together for an hour before it is to be served.

\section{7.-BEEF RISSOLES.}

Mince and season cold beef, and flavor it with mushroom or walnut ketchup. Make of beef dripping a very thin paste, roll it out in thin pieces about four inches square; enclose in eacl piece some of the mince, in the same way as for puffs, cutting each neatly all round; fry them in dripping of a very light brown. The paste can scarcely be rolled out too thin. 


\section{SEA COOKERY.}

568.-FIRST WATCH STEW.

Cut pieces of salt beef, and pork into dice. put them into a stewpan with six whole peppercorns, two blades of mace, a few cloves, a teaspoonful of celery seeds, and a faggot of dried sweet herbs ; cover with water, and stew gently for an hour, then add fragments of carrots, turnips, parsley, or any other vegetables at hand, with two sliced onions, and some vinegar to flavor ; thicken with flour, or rice, remove the herbs and pour into the dish with toasted bread, or freshly baked biscuit broken small, and serve hot. When they can be procured, a few potatoes improve it very much.

$$
\text { 569.-SEA PIE. }
$$

Make a thick pudding crust, line a dish with it, or what is better. a cake tin, put a layer of sliced onions, then a layer of salt beef cut in slices, a layer of sliced potatoes, a layer of pork, and another of onions; strew pepper over all, cover with a crust, and tie down tightly with a cloth previously dipped in boiling water and floured. Boil for two hours, and serve hot in a dish.

\section{VEAL.}

THe failing of this meat is its tendency to turn; should it show any symptoms of doing this, put it into scalding water and let it boil for seven or eight minutes, with some pieces of charcoal affixed; plunge it into cold water immediately after taking it out of the hot, and put it into the coolest place you have at command; the skirt from the breast, and the pipe from the loin should always be removed in hot weather.

Veal of about two or three months old is the best; the flesh ought to be white, approaching to pink, and the fat firm; it is cut up the same as mutton, except that, in the hind-quarter, the loin is cut straight, leaving the aitch-bone on it, which may be either dressed on the loin or separate. The fore-quarter consists of the shoulder, neck, and breast. The hind-quarter, of the knuckle, leg, fillet, and the loin. The head and pluck consist of the heart, liver, nuts, skirts, melt, and the heart, throat, and sweetbread.

The bull-calf is the best; the flesh is firmer grained or redder, and the fat more curdled than the cow-calf, which latter is in general preferred, being more delicate and better adapted for made dishes, as having the udder. To keep veal, we have to observe-the first part that turns bad of a leg of veal is where the udder is skewered back. The skewer should be taken out, and both that and the whole of the meat wiped every day; by which means it will keep good three or four days in hot weather, if the larder be a good one. Take care to cut out the pipe that runs along the chine of a loin, as you do of beef, to hinder it from tainting. The skirt of the breast is likewise to be taken off, and the inside wiped and scraped, and sprinkled with a little salt.

If real is in danger of not keeping, wash it thoroughly, and boil the joint ten minutes, putting it into the pot when the water is boiling hot; then put it into a very cool larder, or plunge it into cold water till cool, and then wipe and put it by. If in the least tainted, it cannot be recovered, as brown meats are, by the use of charcoal or pyroligneous acid.

\section{0.-VEAI-THE FILLET.}

The fillet derives much of its pleasant flavor from being stuffed. Veal in itsel being nearly tasteless, the stuffing should be placed in the hollow place from whence the bone is extracted, and 
the joint should be roasted a beautiful brown; it should be roasted gradually, as the meat being solid will require to be thoroughly done through without burning the outside; like pork, it is sufficiently indigestible without being sent to table and eaten half cooked; a dish of boiled bacon or ham should accompany it to table, a lemon also.

In roasting veal, care must be taken that it is not at first placed too near the fire; the fat of a loin, one of the most delicate joints of veal, should be covered with greased paper; a fillet also, should have on the caul until nearly done enough : the shoulder should be thoroughly boiled; when nearly done dredge with flour, and produce a fine froth.

\section{1. -FILLET OF VEAL, BOILED.}

Bind it round with tape, put it in a floured cloth and in cold water, boil very gently two hours and a half, or if simmered, which is perhaps the better way, four hours will be taken; it may be sent to table in béchamel or with oyster sauce.

Care should be taken to keep it as white as possible.

\section{2.-FILLET OF VEAL ROASTED.}

Take out the bone, fill the space with a fine stuffing, and let the fat be skewered quite round; stuff it also well under the skin with bread, sage, chopped onions and parsley, as much depends on the quantity and flavor of the stuffing, and send the large side uppermost. Put a paper over the fat; and take care to allow a sufficient time for roasting; put it a good distance from the fire, as the meat is very solid, and must be so thoroughly done as not to leave the least appearance of red gravy; serve it with melted butter poured over, and gravy round. Ham or bacon should be served with it, and fresh cucumbers if in season.
Although considered very indigestible, it is a favorite joint and generally roasted, although it may be easily divided into three parts and each dressed separately; that piece known in a "round of beef" as the "silver side," being roasted, and the remaining two stewed in different ways.

In Paris, a longe de veau is cut somewhat in the shape of a haunch of mutton, with the fillet and part of the loin joined together.

\section{3.-TO ROAST}

A fillet of veal, it should be stuffed with the following ingredients: thyme, marjoram, parsley, savory, finely minced lemon-peel, mace, pepper, nutmeg, with bread-crumbs; to which add two eggs: and four ounces of marrow-suet: lay this stuffing in the udder, and, if any remain, in such holes as you think proper made in the fleshy part. Serve with melted butter, and garnish with the lemon-peel sliced.

\section{4.-FILLET OF VEAL BOILED. (English.)}

Choose a small delicate fillet for this purpose ; prepare as for roasting, or stuff it with an oyster forcemeat; bind it round with a tape; after having washed it thoroughly, cover it with milk and water in equal quantities, and let it boil very gently three and a half or four hours, keeping it carefully skimmed. Send it to table with a rich white sauce, or, if stuffed with oysters, a tureen of oyster sauce; garnish with stewed celery and slices of bacon. A boiled tongue should be served with it.

\section{5.-LOIN OF VEAL ROASTED.}

Take a loin of veal; run a lark-spit along the chine-bones; then tie the ends of the lark-spit on the usual spit, drawing down the flap over the kidney. Cover it well with buttered paper, and tie it up 
carefully; let it roast gently for three hours. When about to serve, remove the paper. sprinkle well with salt, dredge it with flour, and finish basting with a small piece of fresh butter.

This joint is frequently divided; the kidney end and the chump. The kidney end sent up with a toast under the fat; the chump end should be stuffed like the fillet, or sent up with balls of stuffing in the dish; pour melted butter over the joint, and gravy round. It also forms an excellent stew if served up in winter with rice, and in summer with green peas.

\section{6.-BREAST OF VEAL.}

Cover it with the caul, and if you retain the sweetbread, skewer it to the back, but take off the caul when the meat is nearly done; it will take two and a half to three hours roasting.

Or:-Remove the tendons, and insert in their place a stuffing; then roast as before.

Or:-Raise the skin, and force in as much stuffing as possible; skewer it up; this will give a very good flavor to the joint.

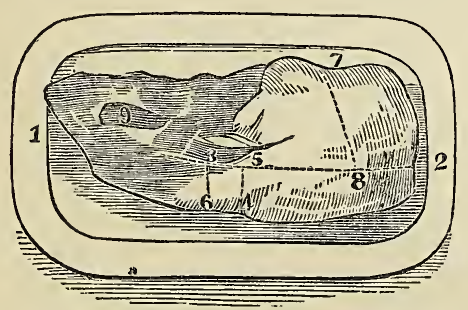

A Breast of Veal Roasted.

\section{7.-TO STEW.}

Cut a breast, or a portion, in pieces; fry them with a little butter, an onion and a cabbage-lettuce shred small; when browned, add a little flour, shake it well together; then add a small quantity of broth or water; let it stew gently; when nearly done, throw in green peas suffcient for the dish; when the peas are done, add a little pounded sugar, pepper, and salt. It may require a little more thickening, in which case knead a little flour and butter together.

\section{8.-LOIN OF VEAL BOILED.}

Take a loin about eight pounds, skewer down the flap without disturbing the kidney, put the loin into a kettle with enough cold water to cover it, let it come gradually to a boil (it cannot boil too slowly), continue for two hours and a quarter, but it must boil; remove the scum as it rises, send it to table in béchamel, or with parsley and melted butter.

\section{9.-KNUCKLE OF VEAL.}

Get a knuckle of a leg of veal, saw it in three parts, but not to separate it, scald it, and put it for a few minutes in cold water, then place it in a stewpan with some good second stock, an onion or two, a faggot of herbs, a few sprigs of parsley, a carrot, a turnip, and a head of celery, a blade of mace, a slice of raw ham, fat and lean; stew it for several hours, until the gristle is soft, take out the veal, cover it over to keep it white; strain the liquor, wash a pound of rice and boil it in this liquor, add half a pint of cream or milk, when the rice is done put your veal again into it, to make hot; dish your veal carefully, and season the rice with pepper and salt, and pour over the veal; if with parsley and butter instead of water, use the stock from it, and chop fine some boiled parsley and mix into it.

\section{S0.-KNUCKLE OF VEAL, BOILED.}

Put sufficient water over it to cover it, let it boil gently, and when it reaches a boil as much salt as would fill a dessertspoon may be thrown in, keep it wëll skimmed, and boil until tender, serve 
with parsley and butter, and a salted cheek.

Allow twenty minutes to each pound.

Three quarters of a pound of rice may be boiled with it, or green peas, or cucumbers ; turnips and small spring onions may be put in, allowing them so much time from the cooking of the veal as they will require.

\section{1.-KNUCKLE OF VEAL STEWED.}

Place your knuckle of veal in a stewpan, if the knuckle is a very large one it may be divided into two or three pieces for the sake of convenience, put in the pan with it a few blades of mace, a little thyme, an onion, some whole pepper, a burnt crust of bread, and cover with from three to four pints of water, cover down close, and boil ; when it has boiled place it by the side of the fire and let it simmer for at least two hours, take it up, keep it hot while you strain its liquor, then pour the gravy over it, and send it to table with a lemon garnish.

\section{2.-NECK OF VEAL WITH PEAS.}

- Add to the meat, half an hour previous to its being done, one quart of peas, twelve button onions, and a little more sugar; remove the fat, and serve as before.

\section{3.-NECK OF VEAL WITH NEW POTA- TOES.}

As before, using new potatoes in place of the peas. Any other vegetable, as French beans, broad beans, \&c., mav be served with it in the same way.

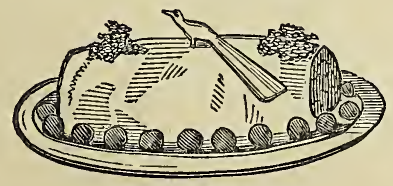

Neck of veal.

584.-NECK OF VEAT

May be boiled or roasted-the latter only if it be the best end, and sent to table garnished as in the above engraving-it may be broiled in chops, but is best in a pie ; it may be, however, larded and stewed as follows:-

\section{5.-NECK OF VEAL STEWED.}

Lard it with square pieces of ham or bacon which have been previously rubbed in a preparation of shalots, spices, pepper, and salt; place it in the stewpan with about three pints of white stock, add a bay or laurel leaf, and a couple of onions; add a dessert-spoonful of brandy or whiskey, the latter if brandy is not at hand, stew till tender, dish the meat, strain the gravy, pour over the joint, and serve.

\section{6.-BREAST OF VEAL FORCED.}

After taking out the tendons and all the rib bones, trim the veal, spread it all over with forcemeat, sprinkle over, if you have got it, a little chopped truffle or mushrooms, sprinkle a little pepper and salt over it, then roll it tightly up and tie it, then put it into a cloth and stew it for several hours, take it up, and take off the cloth and strings; dry it and glaze it, have some good sauce.

\section{7.-SHOULDER OF VEAL.}

Remove the knuckle and roast what remains, as the fillet; it may or may not be stuffed at pleasure; if not stuffed, serve with oyster or mushroom sauce; if stuffed, with melted butter.

\section{8.-NECK OF VEAL BRAISED.}

This is done much in the same manner as the neck of veal stewed; it is larded with bacon rolled in chopped parsley, pepper, salt, and nutmeg, placed with the scrag in a tosser, in which place lean bacon, celery, carrots, one onion, a glass of sherrs or, Madeira, with sufficient water to cover it all, stew over a quick fire until it is sufficiently tender, remove the veal and strain the gravy, place the 
veal in a stewpan in which some butter and flour has been browned, let the bones be uppermost, when the real is nicely colored it is enough ; boil as much of the liquor as may be required, skim it clean, squeeze a lemon into it, pour it over the meat, and serve.

\section{9.-VEAL CUTLETS.}

The cutlets should be cut as handsomely as possible, and about three quarters of an inch in thickness, they should before cooking be well beaten with the blade of a chopper, if a proper beater be not at hand, they should then be fried a light brown and sent up to table, garnished with parsley and rolls of thin sliced, nicely fried bacon; they are with advantage coated previously to cooking with the yolk of an egg, and dredged with bread-crumbs.

\section{0.-VEAL CUTLETS.}

A very nice way to cook cutlets is to make a batter with half a pint of milk, an egg beaten to a froth, and flour enough to render it thick. When the veal is fried brown, dip it into the batter, then put it back into the fat, and fry it until brown again. If you have any batter left, it is nice dropped by the large spoonful into the fat, and fried till brown, then laid over the veal. Thicken the gravy and turn it over the whole.

\section{1.-ANOTHER WAY.}

Procure your cutlets cut as above, coat them with the yolk of eggs well beaten, strew over them bread-crumbs powdered, sweet herbs, and grated lemon-peel and nutmeg, put some fresh butter in the pan, and when boiling put in your cutlets; now make some good gravy; when the cutlets are cooked take them out and keep them before the fire to keep hot, dredge into the pan a little flour, put in a piece of butter, pour a little white stock, squeeze in juice of lemon to taste, sea- son with pepper and salt, add mushroomketchup, boil quickly until a light brown, pour it over the cutlets, and serve, the cutlets being laid in a circle round the dish, and the gravy in the centre.

\section{2.-VEAL CUTLETS CURRIED.}

The cutlets may be prepared as for collops by cutting them into shape, dipping them into the yolk of eggs, and seasoning them with fine bread-crumbs about four table-spoonfuls, two spoonfuls of curry-powder, and one of salt; fry them in fresh butter; serve with curry sauce, which may be made with equal parts of curry-powder, flour, and butter, worked well together into a paste; put it into the pan from which the cutlets have been removed, moisten with a cupful of water in which cayenne and salt have been stirred; let it thicken and serve very hot.

\section{3.-VEAL CUTLETS-CRUMBED OR} PLAIN.

If you have not got the leg of veal or the cutlet piece, get a thick slice of veal and cut fourteen good sized cutlets, not. too thin, flatten each, and trim them a good shape, wet your beater in cold water to keep the veal from sticking, if for plain cutlets flour them well and dry them, then again have ready your sauté-pan or fryingpan quite hot, with a good bit of lard or butter, then put in your cutlets, and fry a nice light brown; pepper and salt them; if to be bread-crumbed, trim them as before; have ready a little clarified butter, some chopped parsley, and shalot, pepper, and salt, all mixed together with a yolk or two of eggs well mixed; have ready some bread-crumbs, put a spoonful of flour amongst them well mixed; dip each cutlet into this omelet, and thin breadcrumb them, patting each cutlet with your knife to keep it in the proper shape, making the bread-crumbs stick to the 
cutlet; melt some lard in your sauté-pan, and place your cutlets in it ready to fry a nice brown.

\section{4.-VEAL CUTLETS-Ã LA MAINTENON.}

Half fry your cutlets, dip them in a seasoning of bread-crumbs, parsley, shalots, pepper and salt, and the yolk of an egg; enclose them in clean writing paper, and broil them.

\section{5.-COLLOPS OF VEAL.}

The piece of veal as before named, or if you have it, a leg of veal; if not, get a cutlet and cut it into thin pieces, and beat very thin, saut them off, and when all done trim them round the size of a crown piece; pepper and salt them, place in a stewpan with some brown sauce if for brown, and if for white béchamel sauce; add some forcemeat balls, some stewed mushrooms, and some whole dressed truffles ; season with pepper, salt, sugar, and lemon; dish the collops round as you would cutlets, putting the mushrooms, and balls, and truffles in the middle.

\section{6.-TENDONS OF VEAL.}

This is from a breast of veal. Turn up the breast and with a sharp knife cut off the chine-bone all along, taking care you do not take any of the gristle with the bone; when you have cut off this bone, place your knife under the gristle and follow it all along until you have raised it up; then cut off the tendons by keeping close to the rib bones; when you have got it out cut twelve or fourteen tendons endwise, keeping your knife slanting, as each may be the size of a sniall pattie round, but not too thin; then put them on in cold water to scald, then put them in cold again; prepare a stewpan lined with fat bacon or ham, trim each tendon round, throw the turnip in your braise, cover them with second stock, and some of the skimmings; let them stew gently for six or seven hours; be careful in taking them up, and place them separately upon a drying sieve; glaze them two or three times; dish them on a border; they should be so tender that you might suck them through a quill.

\section{7-GALANTINE VEAL.}

Take a large breast of veal; take off the chine-bone, then take out the gristle called tendons, then take out all the rib bones; flatten it well, have ready some good forcemeat or sausage meat; spread it all over with your forcemeat, then make a line of green gherkins, then a line of red capsicums, then a line of fat ham or bacon, then some hard boiled yolks of eggs, then a line of truffles ; if you have any boiled calves' feet left from jelly stock, sprinkle it in with pieces of breast of fowl ; sprinkle pepper and salt all over it, then roll it up tightly, and likewise do so in a cloth; tie it up lightly; stew it for two hours or more; take it up and press it flat; let it lie until quite cold; take off the cloth. It will make excellent cold dishes.

\section{8,-OLIVES OF VEAL-ROTI.}

Cut some cutlets a moderate thickness from the chump end of the loin of veal, beat them and trim them, eight or a dozen, according to dish; get som? slices of ham or bacon, cover the veal with forcemeat and with the fat, sprinkle between a little chopped mushrooms, pepper and salt, roll each up, and tie and skewer each, then egg and bread-crumb them, bake them in the oven with buttered paper over them, cut the string when done, and before you send them to table, draw the skewer, put asparagus sauce, tomato, or mushroom in the dish. 


\section{9.-POTTED VEAL.}

This may be potted as beef, or thus:pound cold veal in a mortar, work up with it in powder mace, pepper, and salt, shred the leanest part of tongue very finely, or ham is sometimes used; place in a jar or pot a layer of the pounded veal, and upon that a layer of the tongue, and continue alternately until the pot is full, seeing that every layer is well pressed down; pour over the top melted clarified butter. If it is desired, and which is frequently done, to marble the veal, cut the tongue or ham in square dice instead of shredding it, but care must be taken that they do not touch each other or the effect is destroyed.

\section{0.-LOIN OF VEAL BRAISED.}

This joint generally weighs from twelve to fourteen pounds, when off a good calf. Have the rib bones carefully divided with a saw, so as not to hurt the fillet, prepare the braising-pan, and proceed with the addition of one pint of water, but take care not to cover the meat, which might happen if your stewpan was too deep, (this would be boiling instead of braising;) it will take about three hours: be careful to remove the fat, as this joint produces a great deal. Taste the sauce before serving, in case more seasoning is required, which might be the case, depending on the nature of the veal. A good cook should taste all sauces before serving.

\section{1.-CALF'S-HEAD RAGOUT.}

Parboil the head, and cut off the meat into thin broad pieces, return the bones to the water in which it was boiled, with a beef-bone or a piece of gravy beef, and ham or bacon bones; add herbs, and, making two quarts of good gravy, strain it, and put in the meat. When it has stewed three-quarters of an hour, add an anchovy, a little beaten mace, cayenne pepper, two spoonfuls of lemon pickle, half an ounce of truffles and morels, a slice or two of lemon, and a glass of wine : thicken the gravy with butter and flour, adding forcemeat balls fried, paste fried, and brain-cakes as a garnish.

\section{2.-BOILED CALF'S HEAD.}

Boil the head gently until the bone will leave the meat easily; take some fine forcemeat, made with ham, egg-balls, and small pieces of very nice pickled pork previously boiled; lay them evenly over the inside of one half of the head, and roll it up; tie it lightly in a cloth; put it into a stewpan to braise : cut the other portion of the head into small pieces; thicken and flavor the stock in which it was boiled, and warm it up in it, adding forcemeat and egg-balls, brain cokes, and fried paste. Place the rolled head in the centre of the dish, with the hash round, and the brain cakes, fried paste, and slices of lemon as garnish. Truffles may be alded with advantage to any dish composed of calf's head.

\section{3.-HASHED VEAL.}

If to be hashed, from any joint not overdone, cut thin slices, remove the skin and gristle, put some sliced onions and a shalot over the fire with a piece of butter and some flour; fry and shake them. Put in some veal gravy and a bunch of sweet herbs ; simmer ten minutes; strain off the gravy, and put it to the veal, with some parsley chopped small, and a little grated lemon peel and nutmeg; let it simmer one minute.

If to be stewed, then add the yolk of two eggs, beaten up with two spoonfuls of cream and a very little pepper, and stir over the fire one way until it becomes thick and smooth; squeeze a little lemon juice in, and serve. 
604.-SCOTCK COLLOPS.

If brown, cut the collops thin, beat them a little, fry them in butter for about two minutes, after having seasoned them with a little beaten mace; place them in a deep dish as they are fricd, and cover them with gravy. Put some butter into the frying-pan, and allow it just to change color. Then strain the collops through a colander from the gravy, and fry them quickly; pour the burnt butter from the pan, and put in the gravy, adding a little lemon-juice. The gravy may be made of the trimmings of the veal; serve it up with forcemeat balls.

If white, cut the collops the size of a crown piece, and not much thicker ; butter the bottom of the stewpan and lay the meat piece by piece upon it, having shaken a little flour upon the butter; add two blades of mace and a little nutmeg. Set the stewpan on the fire, and toss it together until the meat is very white; then add half a pint of strong veal broth and one quarter of a pint of cream; toss the whole, and when simmered enough, let them just boil ; add a little lemon juice, some forcemeat balls, and either oysters or mushrooms, which must both be very white; if necessary, thicken the sauce with the yolk of eggs, but do not let it boil afterwards.

\section{5.-TO DRESS COLLOPS QUICKLY.}

Cut them as thin as paper with a very sharp knife, and in small bits. Throw the skin, and any odd bits of the veal, into a little water, with a dust of pepper and salt; set them on the fire while you beat the collops; and dip them into a seasoning of herbs, bread, pepper, salt, and a scrape of nutmeg, but first wet them in egg. Then put a bit of butter into a frying-pan, and give the collops a very quick fry ; for as they are so thin, two minutes will do them on both sides: put them into a hot dish before the fire; then strain and thicken the gravy.

Or:-Cut the collops thin; flatten them with a beater; have a large dish, dredge it with flour, and sprinkle a little black pepper over it; as the collops are flattened, lay them in the dish; put a piece of butter in a frying-pan, and when it is melted and hot, lay in the collops; do them quickly; when lightly browned, dish them up, and serve with a mushroom sauce.

\section{6.-CALF'S HEAD CURRY}

Is usually made with the remains left from a previous dinner; if about two pounds of meat remaining upon the bone, cut it whilst cold into thin slices, then cut two onions and two apples into small dice, which put into a stewpan with an ounce of butter and half a clove of garlic cut in slices, stir with a wooden spoon over the fire until sautéed nice and brown, when add a table-spoonful of curry powder, half one of flour, mix well, then pour in a pint of broth, add a little salt, and boil twenty minutes, keeping it well stirred; then put in the calf's head, and let it remain upon the fire until quite hot through; add the juice of half a lemon, which stir in very gently without breaking the meat, dress it upon a dish, and serve with rice separately. Curry sauce may be passed through a sieve previously to putting the head in.

\section{7.-VEAL CUTLETS EN PAPILLOTE.}

Prepare, half-fry, - and put them in a pie-dish and pour the sauce over, and let them remain until cold; then.cut a sheet of foolscap paper in the shape of a heart and oil or butter it; lay one of the cutlets with a little of the sauce on one half of the paper, turn the other half over, then turn and plait the edges of the paper over, beginning at the top of the heart and finishing with an extra twist at the 
bottom, which will cause the sauce to remain in it; broil slowly on a gridiron for twenty minutes on a very slow fire, or place it in the oven for that time, and serve.

\section{8.-CUTLETS AND RICE.}

Boil a cupful of rice in milk until quite soft, then pound it in a mortar with a little salt and some white pepper; pound aiso separately equal parts of cold veal or chicken: mix them together with yolk of egg, form them into cutlets, brush over with yolk of egg, and fry them; send them up with a very piquant sauce, made of good stock, thickened and flavored with lemon juice, lemon pickle, or Harvey's sauce. The cutlets may be sent to table covered with the small pickled mushrooms.

\section{9.-CUTLETS AND CELERY.}

The cutlets may be cut from the best part of the neck, taking care in removing the meat from the bones to cut it in a good shape; make. gravy of the bones; stewing them with three or four heads of celery cut and scalded, a little salt, pepper, and stewed onion; strain the gravy, returning the celery into it; thicken it with butter and flour, and pour it boiling hot upon the cutlets. Stew them till they are quite tender, and garnish with lemon and small forcemeat balls fried.

\section{0.-A LiTTALIENNE.}

Chop a quantity of sweet herbs, parsley being predominant; melt a little butter on the fire and then warm the herbs in it; cut the cutlets into handsome shapes, brush them with the yolk of an egg, then lay on the butter and herbs with a knife, and cover them well with bread-crumbs; this process should be repeated; fry them of a fine brown; if glazed, they must be put between papers to press all the gease out, then brush them over with the glaze, and send them to table. Serve with Italian sauce.

\section{1.- ¿̀ LA HOLLANDAISE.}

Cut some large cutlets from a fillet of veal, beat them with a rolling-pin, then dip them into batter made very rich with egg. Make a fine forcemeat with pounded ham, chopped oysters, \&c., lay it upon the cutlets, roll them up, dip them again in egg-batter, roll them in bread-crumbs, and tie them up; roast them upon skewers or in a Dutch oven; chop and pound the trimmings of the veal, add them to the oysters and ham forcemeat with two raw eggs, make it into balis and fry them; have some stock ready and stew it with an anchovy, a shalot, some white pepper and salt; strain, and thicken it with butter, add the juice of one-half a lemon and a glass of white wine; give it one boil and pour it into the dish, with the rolled cutlets in the centre and the balls round them.

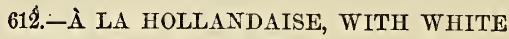 SAUCE.}

Cut thin slices of undressed veal, hack them with the back of a knife each way, dip them in eggs and bread-crumbs, with a little chopped parsley ; fry them in butter, lay them on a sieve as they are done, and serve them with white sauce as for fricassee.

\section{3.-FARCIES OF VEAL.}

Chop off the thick bone of a small neck of veal to the end of the fifth rib; divide the cutlets. and with a broad knife beat the meat of each flat, and cover it with forcemeat of lean veal, beef suet, parsley, a small bit of garlic, a little salt, mace. and pepper. Then roll the meat round the bone, the end of which leave out at one extremity, put over a thin slice of fat bacon, and, having stuffed in the remain- 
der of the forcemeat at the ends of the roll, bind up with twine. At the bottom of a small stewpan lay slices of turnip, onion, three inches of celery, and two large carrots cut lengthways, and the steaks over: add as much water, or beef broth, as shall half cover them; set the pan on a moderate stove, and some wood embers on the lid; simmer slowly for two hours, then remove the twine, and placing the bones upwards, leaning on each other, strain the gravy over them.

Or:-Take cutlets from the chump end of a loin of veal; beat them well; cover them with slices of bacon, then with a fine forcemeat; roll them round: tie them into shape; then dip them in the yolks of eggs and the raspings of bread; roast them, basting well with butter; then put them into a sauce thickened with mushrooms; squeeze lemon juice over them ; let them stew till very tender, and serve them up.

\section{4.-ROLLED VEAL.}

The breast is the best for this purpose. Bone a piece of the breast, and lay a forcemeat over it of herbs, bread, an anchovy, a spoonful or two of scraped ham, a very little mace, white pepper, and chopped chives; then roll, bind it up tight, and stew it in water or weak broth with the bones, some carrots, onions, turnips, and a bay-leaf. Let the color be preserved, and serve it in veal gravy, or fricassee sauce, with mushrooms and artichoke bottoms.

Or:-Put the breast into a stewpan with just water enough to cover it, an onion, a stick of celery, and a bundle of sweet herbs; let it stew very gently, adding more water as it stews, until it is tender; then take out the bones, and remove the skin; return the bones into the liquor, which will be a fine jelly, and serve as the sauce for several dishes. Cover the veal with a fine forcemeat, season it well, add egg-balls, and roll it up, securing it with tape. Put it into a stewpan with the fat procured from pork chops, a slice or two of fat bacon or a lump of butter, and a teacupful of the liquor it was stewed in ; shake the stewpan about until the fat has melted, and turn the veal in it, that it may be all equally done, adding an onion and another bunch of herbs; let it braise one and a half or two hours, then strain the gravy, and thicken it; garnish with forcemeat-balls, egg-balls, and fried paste cut in shapes. Peeled mushrooms may be given by way of variety. When well done, this is an excellent dish.

\section{5.-CALF'S LIVER.-(English way.)}

Cut the liver into thin slices, dip them in flour, and put it in a sauté or fryingpan in which some slices or bacon have been previously cooked, with sufficient fat left in it; sauté the liver until quite brown and rather crisp, when take out and place it upon a dish with the bacon, then dredge a spoonful of flour in the pan, or enough to absorb all the fat in it, then add a little broth or water so as to make it a thinnish sauce, season it, and add two spoonfuls of Harvey's sauce or mushroom ketchup. If the above is nicely done, and the pieces cut the size of cutlets, it will make a nice entrée for an ordinary dinner. It should be served immediately, and very hot.

\section{6. -STEWED CALF'S LIVER.-(Soyer's.)}

Choose a nice fat one, rather white in color, lard it through with bacon, put one-quarter of a pound of butter in a pan; when melted, add a table-spoonful of flour, keep stirring until a nice yellow color, then put in the whole of the liver; turn round now and then until it is a little firm, then add a pint of broth or water, and a glass of any kind of wine, a bouquet of parsley, thyme, bay-leares. 
a little salt, pepper, and sugar, thirty button onions; simmer one hour; take the fat off and the bouquet out, dish the liver with the onions around it, reduce - the sauce, so that it adheres lightly to the back of the spoon, sauce over and serve. Any vegetables may be used, as carrots, turnips, peas, haricots ; and if a little gelatine or isinglass is added to the sauce, and the liver with the sauce only put into a round basin and pressed down and left until cold, it will make a nice dish for supper, lunch or breakfast. If required to be rewarmed, cut into slices, put it in the pan with a drop of water added to the gravy.

\section{7.-CALF'S LIVER FRIED.}

Cut in slices, and fry it in good beefdripping or butter; let the pan be half full, and put the liver in when it boils, which is when it has done hissing; have some rashers of toasted bacon, and lay round it, with some parsley crisped before the fire; always lay the bacon in boiling water before it is either broiled, fried, or toasted, as it takes out the salt, and makes it tender. Sauce-plain melted butter, a little poured over the liver, the rest in the sauce-boat.

\section{8.-CALF'S HEAD CHEESE.}

Boil the head until the bones will come out, then put the head, tongue, and brains, into a mould with spices and parsley chopped fine until the mould is quite full; put a plate and a weight over it, and when cold turn out. Serve with parsley, and slices of rolled ham, placed round the dish.

\section{FRICANDEAU OF VEAT}

In France the fricandeau is not unfrequently larded in the interior as well as the outside, by having pieces of the size of a little finger cut off the meat with an instrument something like a cheese- taster, and then refilled with pledgets of bacon, or "lardons." The meat is also very generally served upon spinach, dressed with cream or sorrel.

The meat, being cut into a handsome shape, should be larded, and put into a stewpan of small size, with just sufficient water, or veal broth, to cover it, and there allowed to simmer gently over a slow fire for about three hours: or until it has become so tender as to be cut with a fish-slice. The gravy is then seasoned at pleasure, but most generally made into white sauce.

Or:-Cut a nice piece from the fillet; lard it all over as thickly as possible; put it into a stewpan with a few slices of fat bacon, some trimmings of veal, a carrot, turnip, and a head of celcry, a few allspice, two blades of mace, and four bay-leaves ; let it stew very gently, until thoroughly done, boil the gravy down to a glaze, cover the veal with it, and serve it upon a puree of sorrel. Grenadines of veal are cooked in the same way, only made of smaller pieces, four for a dish.

\section{0.-FRICANDELS.}

Take three pounds of the best end of a loin of veal, chop the fat and lean together very fine; then soak a French roll in some milk; beat three eggs; add pepper, salt, nutmeg, and mace. Make the mixture up about the size, and somewhat in the shape, of a chicken; rub it over with egg and bread-crumbs, fry until it is brown, pour off the fat, boil water in the pan, and stew the fricandels in this gravy; two will make a handsome dish. Thicken the gravy before it is sent to table.

\section{1.-MIROTON OF VEAL.}

Chop very fine some cold dressed veal and ham or bacon; mix it with a slice of crumb of bread soaked in milk, two 
onions chopped and browned, a little salt, pepper, and a little cream. Put all these ingredients into a stewpan until they are hot, and are well mixed together; then add one or two eggs according to the quantity, butter a mould, put in the whole, and bake it in an oven until it is brown; turn out of the mould, and serve with fresh gravy.

\section{2.-A GALANTINE.}

Take out the long bones from a breast of veal, and beat the veal for four minutes with the flat part of a hand-chopper, in order that it may roll easily. Spread it on the table, and brush it over thickly with the yolk of an egg, and then sprinkle it with chopped herbs; season with pepper, salt, and pounded mace; make two omelettes, one of the yolk and one of the white of egg; cut them in strips ; lay them upon the veal, with layers of pounded ham or farce between; cut some pickled cucumbers or mushrooms into small pieces, with some sweet herbs well seasoned; strew them over the surface, then roll up the veal very tightly, tie it in a cloth, and let it stew gently for six hours; then put a heavy weight upon it, and let it stand two days before it is cut. Serve it in slices. with savory jelly ; any kind of boned game or fowl may be added. It requires to be highly seasoned.

\section{3.-VEAI OLIVES}

Cut long thin slices; beat them, lay them on thin slices of fat bacon, and over these a layer of force meat seasoned high with some shred shalot and cayenne. Roll them tight, about the size of two fingers, but not more than two or three inches long; fasten them round with a small skewer, rub egg over them, and fry of a light brown. Serve with brown gravy, in which boil some mushrooms, pickled or fresh. Garnish with balls frier.

\section{1.-HARICOT OF VEAL}

Take the best end of a small neck; cut the bones short, but leave it whole; then put it into a stewpan just covered with brown gravy; and when it is nearly done, have ready a pint of boiled peas, four cucumbers pared and sliced, two cabbage-lettuces cut into quarters, and half a pint of carrots and turnips cut in shapes, all stewed in a little good broth; put them to the veal, and let them simmer ten minutes. When the veal is in the dish, pour the sauce and vegetables over it, and lay the lettuce with forcemeat-balls round it.

\section{5.-VEAL CURRY.}

Cut up about two pounds of lean veal into small square pieces, half the size of walnuts; then put a large onion cut into small dice, in a stewpan, with a clove or garlic and one apple cut into slices, and one ounce of butter; keep them stirred over a moderate fire, until lightly browned, when stir in a good table-spoonful of mild curry powder, half a one of flour, mix well, then add a pint of water, let it just boil up, put in the veal, which stir round two or three times, to mix with the curry, and put the stewpan over a slow fire, or in a warm oven for an hour and a-half; when done (which you may ascertain by pressing a piece between the finger and thumb, if done it would be quite tender and separate,) add the juice of a lemon and a little salt, stir the whole round three or four times very gently, to mix, and turn it out upon your dish; serve with rice separately.

Should you require a veal curry made in less time, the better plan would be to saute the veal in butter previously, then putting it with its own gravy to the curry, and boiling the whole gently a quarter of an hour.

To make a veal curry with curry paste, sauté the veal in butter; when becoming 
slightly browned, add a good table-spoonful of the paste, with half a pint of water; leave it to stew about half an hour, when it will be ready to serve.

Beef, mutton, lamb, and pork curries are made precisely the same as directed for veal curries.

\section{6.-CALF'S HEART BAKED.}

Clean and stuff as for roasted beef heart; "then bake instead of roasting, and afterwards serve with rich gravy or liver sauce.

\section{7.-CALVES' FEET.}

Stewed.-When properly cleaned, rub the feet over with pepper, a very little salt, a little ground ginger, and mace; cut the feet into moderately sized pieces, and put them into a stewpan with a little shalot, and a beefsteak also cut into pieces. Cover all with cold water, and let them simmer together for three hours. When quite tender, take them off the fire; strain the gravy through a sieve. The next day when cold, take off all the fat; boil a small quantity of saffron in cream, and a little cayenne pepper; mix it with the gravy, and warm the whole without boiling; one foot and one pound of steak will make a dish.

Fricasseed.-Boil the feet in water until the bones will come out, with an onion and a bunch of sweet herbs; take the bones out, and when the meat is cold stuff it nicely with a very fine farce, or forcemeat; make the pieces up into handsome shapes of an equal size; then take some of the stock in which the feet were boiled, removing the fat, and straining it when melted; make this into thick white sauce with cream and roux; warm up the calves' feet in it, and send it to table either plain or with a quantity of asparagus tops, previously boiled, and cut into small pieces.

Or:-Boil tender two feet in a shallow pan. observing not to break them; throw them into cold water for an hour; divide and lay them in a little weak veal broth, and simmer them half an hour, with a blade of mace and a bit of lemon peel, which take out when you add half a teacupful of cream, and a bit of flour and butter.

Fried.-Take calves' feet which have been boiled until very tender; remove the bones; let them get cold; cut them into well-shaped pieces; season them with white pepper and salt; dip them into butter, fry them, and serve them up with a sharp sauce or garnish of pickles.

Calves' feet may also be plainly boiled, and served with parsley and butter, or eaten cold with oil and vinegar.

\section{8.-QUEUES DE VEAU.}

Having nicely cleaned and soaked four calves' tails, cut off the small ends, and blanch them; dry, flour, and fry them a fine brown in butter; drain the fat from them, and having ready a pint of weak broth, with a bunch of sweet herbs, chibols, two bay-leaves, half a pint of mushrooms, pepper and salt, boiled up and skimmed; wipe the sides of the casserole, put the tails in, and simmer very slowly until they are quite tender; keep them hot while the gravy is strained, and boiled to a glaze, to cover them. If you have no mushrooms, mix a little of the powder into gravy just before serving; have ready small onions, and peel to one size to send up in the dish.

\section{9.-CALVES' EARS STUFFED.}

The hair being scalded off the ears, after they have been cut quite close to the head. scald and clean them as well as possible; boil them quite tender, and blanch them in cold water. Observe that the gristle next the head be cut so smooth as to allow the ears to stand upright; they may be boiled in white grary 
instead of water, which will make them richer. When become cold, fill up the cavity with a fine stuffing of calf's liver, fat bacon, grated ham, bread soaked in cream or gravy, herbs, an unbeaten egg, a little salt, and a small piece of mace; rub egg over the ears and stuffing, dip in bread-crumbs, and fry of a beautiful light brown. Serve in brown or white gravy, or tomato-sauce. If the ear be large, one will be sufficient for a corner dish.

Or:-When prepared as above, boil them quite tender in gravy, and serve them in chervil-sauce; or else boil chervil to a mash, put to it melted butter, pass it through a sieve, and add to it white sauce, or use the latter solely, in which case put a little mushroom-powder into it; the same forcemeat may be used, or the ears may be served without. They likewise eat well, cut into slices, served in white or brown gravy. In the latter case, fry them before stewing in gravy.

\section{0.-CALVES' BRAINS.}

Remove all the large fibres and skin; soak them in warm water for four hours; blanch them for ten minutes in boiling water, with a little salt and vinegar in it; then soak them three hours in lemonjuice in which a bit of chervil has been steeped; dry them well, dip them in batter, and fry them. Make hot a ladleful of glaze, some extremely small onions browned in butter, artichoke bottoms divided in half, and some mushroombuttons, and serve round the brains ; or, after preparing as above, serve in a rich white acidulated sauce, with lemon-juice or tomato-sauce.

Or:-Blanch the brains, and beat them up with an egg, pepper, and salt, a small quantity of chopped parsley, and a piece of butter. Make them into small cakes, put them into a small frying-pan, and fry them.

Or:-Prepare them as above; wet with egg, and sprinkle crumbs, salt, pepper, and chopped parsley, and finish dressing in a Dutch oven. Serve with melted butter, with or without a little mushroom-ketchup.

\section{1.-CROQUETTES OF BRAINS.}

Take calf's brains, blanch, and beat them up with one or two chopped sageleaves, a little pepper and salt, a few bread-crumbs soaked in milk, and an egg beaten; roll them into balls, and fry them.

\section{2.-CERVELLES DE VEAU AU MARI- NADE.}

Cut a carrot, a turnip, and an onion into pieces; let them stew in a little butter; then add a bundle of sweet herbs, and pour over them a pint of vinegar and water in equal parts; then strain the liquor, and pour it over calves' brains cleaned and blanched; let them stew in it till they are firm, then fry them in butter, and serve up with crisped parsley.

633.-CERVELLES DE VEAU FRICASSEES.

Having cleaned and blanched the brains, render them white and firm by squeezing lemon-juice over them; then put them into a stewpan with a piece of butter; stew slowly, that they may not brown; dredge in a small quantity of flour; add a bunch of parsley, and one of chives; moisten with clear veal broth; let the brains stew until they are firm; then send them up in the sauce, thickened with a little cream and flour and butter.

\section{4.-FRIED LIVER AND BACON, ETC.}

Cut the liver rather thin, say about half an inch thick, but first soak it in warm water abont one hour; chop a quantity of parsley, season it with pepper, and lay it thick upon the liver; cut 
slices of bacon, and fry both together, but put the bacon first into the pan; add a little lemon-pickle to the gravy made by pouring the fat out of the pan, flouring, and adding boiling water.

Or:-Cut the liver in handsome pieces, lard them very nicely, and chop some parsley and spread it over the surface with a little pepper and salt; put a small piece of butter well mixed with flour in the bottom of a stewpan, and put in the liver, and allow it to stew gently in its own juices until it is done enough.

\section{5.-TO DRESS LIVER AND LIGHTS.}

Half boil an equal quantity of each; then cut them-into a middling-size mince; put to it a spoonful or two of the water they were boiled in, a bit of butter, flour, salt, and pepper; simmer ten minutes, and serve hot.

\section{6.-TO DRESS CALF'S HEART.}

Stuff and roast the same as beef heart; or, being sliced and seasoned, make it into a pudding as directed for steak or kidney pudding. It may be either roasted or baked, but in either way should be well basted, stuffed with forcemeat as for veal, but made rather more savory, and served up in the same manner as beef heart.

When the kidney is cut from the loin and dressed separately, chop the kidney, with some of the fat; likewise a little leek or onion, pepper, and salt. Veal forcemeat may also be used, but it rather weakens the pungency of the seasoning. Roll it up with an egg into balls, and fry them.

\section{7.-SWEETBREADS}

For every mode of dressing, sweetbreads should be prepared by blanching, or rather parboiling them.

They may be larded and braised; and, being of themselves rather insipid, they will be improved by a relishing sauce, and by a large quantity of herbs in the braise. Slices of lemon put upon the sweetbreads while braising, will heighten the flavor and keep them white, which is very desirable when sent to table with white sauce. Stuffed with oysters, they make a very good vol-au-vent.

\section{8.-SWEETBREADS $\AA$ LA DAUBE.}

Blanch two or three of the largest sweetbreads; lard them; put them into a stewpan, with some good veal gravy, a little browning, and the juice of half a lemon; stew them till quite tender, and just before serving thicken with flour and butter; glaze them; serve with their gravy, with bunches of boiled celery round the dish.

\section{9.-SWEETBREADS STEWED.}

After blanching. stuff them with a forcemeat of fowl, fat and lean bacon, an anchovy, nutmeg, lemon-peel, parsley, and a very little cayenne and thyme; when well mixed, add the yolks of two eggs, and fill the sweetbreads. Fasten them together with splinter-skewers, and lay them in a pan, with slices of veal over, and bacon under them; season with pepper and salt, mace, cloves, herbs, and sliced onion; cover close over the fire ten minutes, then add a quart of broth, and stew gently two hours; take out the sweetbreads, strain and skim the broth, and boil it to half a pint; warm the sweetbreads in it, and serve with lemon round.

$$
\text { 640. -FRIED. }
$$

Cut them in slices about three-quarters of an inch thick, dry and flour, egg them, and dip them in fine bread-crumbs; fry them of a light brown; serve on spinach, endive, or sorrel; or cut some 
toasted bread, dish them on it, and serve them with a sauce piquante.

For an invalid, boil them well, corer them with bread-crumbs, a little pepper and salt, with a small bit of butter; brown them lightly with a salamander.

$$
\text { 641.-ROASTED. }
$$

Blanch, dry, egg and bread-crumb them; pass a small skewer through each ; tie on a spit; roast gently; baste with fresh butter; serve nicely frothed, and a mushroom sauce under them.

\section{2.-SWEETBREADS FRICASSEED WHITE.}

Blanch and slice them; thicken some veal gravy with flour and butter mixed; a little cream; a little mushroom powder, and add white pepper. nutmeg. and grated lemon-peel; stew these ingredients together a little, then simmer the sweetbreads twenty minutes. When taken off the fire, add a little salt and lemon-peel ; stir well, and serve.

\section{3.-IF FRICASSEED BROWN,}

Cut them about the size of a walnut, flour, and fry them of a fine brown; pour to them a good beef gravy, seasoned with salt, pepper, cayenne, and allspice; simmer till tender; thicken with flour and butter. Morels, truffles, and mushrooms may be added, and mushroom ketchup.

\section{4.-CROQUETTES.}

Take two sweetbreads or half a pound of roast veal, one onion chopped fine, mixed with a small piece of butter, one egg, and a little cream; season with white pepper, cayenne, and salt. This will make eight or nine croquettes. Roll them in egg and then in bread-crumbs; fry them in lard a nice brown color; strew them in a circle round the dish, and serve fried parsley in the centre.
Cold veal chopped, and stewed in a little gravy, and when cold made up as above, makes excellent rissoles.

\section{5.-TO ROAST SWEETBREADS.}

Sweetbreads should be soaked in warm water, and then blanched by being thrown into boiling water, boiled for a few minutes, and then put into cold water. They may then be larded and roasted or fried, and afterwards stewed in butter with crumbs of bread. Sweetbreads may also be larded and braised, and being of themselves rather insipid, they will be improved by a relishing sauce and by a large quantity of herbs in the braise. Skins of lemon put upon the sweetbreads while braising will heighten the flavor, and keep them white; which is very desirable when sent to table with white sauce. The usual sauce with which they are served is butter and mushroom ketchup. They may be roasted in a Dutch oven.

\section{6.-CERVELLE DE VEAU A LA MATTTRE D' HOTEL.}

Let them be prepared. Cut some bread into the shape of cocks'-combs, which fry in butter till of a fine color. Dish them between each half of the brains, which you have divided, and cover the brains over with a ma tre d' hôtel sauce, two spoonfuls of béchamel, a smali bit of fresh butter, some parsley chopped fine, the juice of half a lemon, and let it be well seasoned.

\section{7.-TONGUE, ETC.}

Neat's tongue should be boiled full three hours. If it has been in salt long, it is well to soak it over night in cold water. Put it to boil when the water is cold. If you boil it in a small pot, it is well to change the water, when it has boiled an hour and a half; the fresh water should boil before the half-cooked 
tongue is put in again. It is nicer for being kept in a cool place a day or two after being boiled. Nearly the same rules apply to salt beef. A six pound piece of corned beef should boil full thrte hours; and salt beef should be boiled four hours.

\section{8.-CROQUETS OF SWEETBREAD. (French Receipt.)}

Take such sweetbreads as have already been served, cut them into as small dice

- as possible. Have some good velouté boiled down ready. Throw the dice of sweetbreads into the velouté, and give them a boil, that they may taste of the sauce. Then lay them on a plate to cool. When cold, roll them into any shape you like, round, oval, or long. Of all things avoid giving them the shape of pears, as some persons do. for in that case they must be more handled, without at all improving the quality. Serve up with parsley, fried green, in the middle. If you have some mushrooms left in the larder, cut some likewise in dice, and mix them with the croquets.

649.-ESCALOPES DE RIS DE VEAU AUX POIS, OR SCOLLOPS OF SWEETBREADS WITH GREEN PEAS.

Take four fine sweetbreads, let them disgorge, and blanch them thoroughly. Next, cut them into scollops, as large as possible. Mark them in a sauté-pan, or fryingpan, with melted butter and a little salt. A quarter of an hour before you send up, fry them lightly over a stove, with a clear fire; turn them round, and when done, drain the butter, and put a little glaze into the pan. Keep stirring the sweetbreads in the glaze; dish them miroton way, and send up the peas in the middle.

When you have sweetbreads left at table, cut them into scollops, make them hot in a little light glaze, and after hav- ing dished them miroton way, mask them with the peas. Scollops of sweetbreads are easier to dress when you put them between a slice of fried bread cut round, and the green peas in the middle; without the fried bread they do not keep the shape in which you dish them.

650.-FOIE DE VEAU À LA POÂLE, OR SCOLLOPS OF CALF'S LIVER 'WITH FINE HERBS.

Take a nice calf's liver as white as possible, cut it into slices of a good and equal shape. Dip them in the flour, and fry them in a black fryingpan, of a nice color, with a little butter. When they are done, put them in a dish, and take some fine herbs which you have previously chopped fine, such as parsley, shalots, mushrooms, \&c. Stew them slowly on the fire with a little butter, and when the herbs are sufficiently done add a teaspoonful of flour, and moisten with gravy, if you have any, or with water, and add a small bit of glaze. When done, put the liver in the sauce, which warm, but do not allow it to boil; add a little salt, pepper, lemon, and serve very hot.

This is again a common dish, yet it is very palatable. You may put the liver in the sauce; but mind that it does not boil. It is a dish for a breakfast à la fourchette.

\section{1.-CALF'S HEAD.}

Let the head be thoroughly cleaned, the brains and tongue be taken out, boil it in a cloth to keep it white, (it is as well to soak the head for two or three hours previously to boiling, it helps to improve the color,) wash, soak, and blanch the brains, then boil them, scald some sage, chop it fine, add pepper, salt, and a little milk, mix it with the brains; the tongue, which should be soaked in salt and water for twenty-four hours, should be 
boiled, peeled, and served on a separate dish. The head should boil until tender, and if intended to be sent to table plainly, should be served as taken up, with melted butter and parsley ; if otherwise, when the head is boiled sufficiently tender, take it up, spread over a coat of the yolk of egg well beaten up, powder with breadcrumbs, and brown before the fire in a Dutch or American oven.

\section{2.-CALF'S HEAD BAKED.}

Butter the head, and powder it with a seasoning composed of bread-crumbs, very fine, a few sweet herbs and sage, chopped very fine, cayenne, white pepper, and salt. Divide the brains into several pieces, not too small, sprinkle them with breadcrumbs, and lay them in the dish with the head. Stick a quantity of small pieces of butter over the head and in the eyes: throw crumbs over all, pour in three parts of the dish full of water, and bake in a fast oven two hours.

\section{3.-CHITTERLINGS,}

Or calf's tripe, stewed tender and served on toast with a sauce of onions boiled and mashed in milk, with butter added, form a nice dish. They are eaten with pepper and vinegar.

\section{4.-HASHED CALF'S HEAD.}

Put into a stewpan one table-spoonful of chopped onions, and three of vinegar, take the remains of the head which cut into slices, place them on a dish, add a table-spoonful of flour to them, a teaspoonful of salt, a quarter of one of pepper, put the stewpan on the fire to boil for one minute, add the pieces of head, and moisten with half a pint of broth, water, or milk; let it simmer for ten minutes and serve on toast or plain, or with sippets round. The addition of a few gherkins sliced or any mixed pickle is an improvement. A little thyme or bay-leaf, if at hand, may be added. If with water or gravy a little coloring will improve the appearance. This receipt is for one pound of meat. It may also be warmed in curry-sauce, and is excellent.

\section{5.-CALF''S HEAD À LA TORT̉UE.}

Bone a calf's head whole; after being well scalded and cleaned cut off the ears, take out the tongue, cut the gristle and bones from the tongue; prepare a good forcemeat, add some chopped truffles into it, and some mushrooms; lay the head on a clean cloth on the dresser, spread it thickly over with the forcemeat, blanch and take off the skin of the tongue; cut each ear in half longways, place them in different parts on the forcemeat and the tongue in the middle, lap it over keeping it high in the middle, tie the ends, and brace it all over tightly with some loose string, as by doing so it will leave all the marks appearing when glazed like the back shell of turtle, then tie it up in a cloth; it will take some hours to boil; when done; which will be in your second stock, take it up and take off the cloth, put it upon the dish, dry it and glaze it several times; have ready cut from the crumb of bread the form of a turtle's head and the four fins, fry them a nice light brown, and glaze them with the head, placing them to the head on the dish, as to look like a turtle crawling, for the eyes use whites of hard boiled eggs; a sauce you will find among the sauces.

\section{6.-CALF'S HEAD.}

Choose one thick and fat, but not too large; soak for ten minutes in lukewarm water, then well powder with rosin, have plenty of scalding water ready, dip in the head, holding it by the ear, scrape the hair off with the back of a knife, which will come off easily if properly scraped, without scratching the cheek; when perfectly clean, take the eyes out, saw it in two lengthwise, through the skull, without spoiling the brain, which 19 
take carefully out, and put to disgorge for a few hours in lukewarm water; pull the tongue out, break the jaw-bone, and remove the part which contains the teeth, put the head into plenty of water to disgorge for one hour ; make the following stock and boil for about two hours and a half, and it will be ready to serve.

The stock is made by putting into a braising-pan two carrots, three onions, a quarter of a pound of butter, six cloves, a bouquet of parsley, thyme, and bayleaves; set it on the fire for about twenty minutes. keep stirring it round, then add a pint of water, and when warm mix a quarter of a pound of flour, add a gallon of water, one lemon in slices, and a quarter of a pound of salt, then lay the head in; take care it is well covered, or the part exposed will turn dark; simmer gently till tender.

\section{7.-RIS DE VEAU EN CAISSES.}

Blanch three sweetbreads, and simmer in a strong, well flavored gravy till quite done. Have ready three round pieces of white paper oiled, and lay them thereon; having left them lightly wetted with gravy, sprinkle over them the finest crumbs or raspings of bread, pepper, salt, and a very little nutmeg; do them slowly on a gridiron, and serve in the cases. Any of the vegetable sauces may be served with them.

\section{8.-GARNITURE EN RAGOUT.}

Having prepared and blanched sweetbreads of veal or lamb (house-lamb is the best), liver of lamb and rabbits, truffies and mushrooms, simmer gently half an hour in rich veal broth; then divide the several articles into fit bits for helping, and stew the whole until very tender. If, when finished, the gravy wants consistence, boil up in it a good piece of butter rolled in flour. Season it fifteen minutes before serving, with white pep- per, salt, and nutmeg only. Have ready and hot, forcemeat balls, in which finely scraped ham, beef suet, the breast of a fowl, or cold veal, are the principal ingredients.

It may be served in a tureen as soup, or in an ornamented crust previously baked. If to be white, beat as many yolks of eggs as the quantity may require, and simmer in the ragout two minutes: it must not boil. Sometimes one or two whole pigeons, nicely prepared, form a part of this much admired dish, which in one way or other is rarely omitted on well covered tables.

\section{9.-VEAL CAKE.}

Bone a breast of veal, and cut it in slices ; cut also slices of ham or lean bacon, and boil six eggs hard; butter a deep pan, and place the whole in layers one over the other, cutting the eggs in slices, and seasoning with chopped herbs and cayenne pepper, and wetting the herbs with anchovy or other highly-flavored sauce. Cover up the whole, let it bake for four hours, and when taken from the oven, lay a weight upon it to press it well together. When cold, turn it out.

\section{0.-ANOTHER.}

Boil six or eight eggs hard; cut the yolks, in two, and lay some of the pieces in the bottom of the pan; shake in a little chopped parsley, some slices of veal and ham, and then eggs again, shaking in after each some chopped parsley, with pepper and salt, till the pan is full. Then put in water enough to cover it, and lay on it about an ounce of butter; tie it down with a double paper, and bake it about an hour. Then press it close together with a spoon, and let it stand till cold. It may be put into a small mould, and then it will turn out beautifully for a supper or side dish. 
661.-ANOTHER.

Chop very finely cold dressed veal and ham or bacon; mix it with a slice of bread-crumb soaked in milk, two onions chopped and browned, a little salt, pepper, and an egg beaten. Put all these ingredients into a stewpan until they are hot and are well mixed; then oil or butter a mould, put in the whole, and bake it in an oven until it is brown; then take it out, and send it to table with fresh gravy.

\section{2.-VEAL ROLLS}

Are cut from any cold joint, or prepared in the same manner from the raw meat. Cut thin slices, and spread on them a fine seasoning of a very few crumbs, a little chopped or scraped bacon, parsley and shalot, some fresh mushrooms stewed and minced, pepper, salt, and a small piece of pounded mace. This stuffing may either fill up the roll like a sausage, or be rolled with the meat. In either case, tie it up very tight, and stew very slowly in a gravy and a glass of sherry. Serve it when tender, after skimming it nicely.

\section{3.-BLANQUETTES.}

Melt a piece of butter the size of a walnut in a stewpan; then put in a little thyme, parsley, or any herbs you like the flavor of, and a little onion, all chopped fine, with a pinch of flour. Brown the herbs ; add pepper and salt, with a clove or two. Then put in cold or undressed veal, cut in thin slices the size of half a crown; add gravy or broth, half a pint, or according to the quantity of meat you want to dress. It should not be too large a dish. Let it stew very gently over a stove; if of dressed meat, one hour will be sufficient; add half a teacupful of cream, and stir it well together for a few minutes ; then take it up, and before you turn it out have two yolks of eggs well beaten, and add to your dish. Give it a few shakes over the fire. It must not boil, or it will curdle.

Or :-Cut rabbits, fowl, veal, or lobster in pieces, steep them (except the veal and fish) in water for half an hour, changing the water. Put some butter in a stewpan. to melt, but do not let it fry; put in the meat with a very little flour, and keep shaking it well; pour in by degrees some broth madu of white meat, add a bunch of parsley, an onion, salt, mace, and white pepper. Stew it well a quarter of an hour before it is dished; take out the parsley and onion, and add some raw parsley chopped, and the yolk of an egg and cream beaten together. You must never.cease shaking the pan until the blanquette is put over the dish.

\section{4.-VEAL À LA CHARTREUSE.}

Line a copper mould with fat bacon, lay sliced carrots and turnips round the edges, then cover with a forcemeat, and put in a fricassee of veal or fowl. Cover the top of the mould with a paste, steam it an hour, and serve it turned out upon a dish.

\section{5.-TO MARBLE VEAL.}

Boil tender, skin, and cut a dried neat's tongue in thin slices, and beat it as fine as possible, with half a pound of butter and some mace pounded. Have ready some roasted fillet of veal, beaten with butter, and seasoned with white pepper and salt ; of this put a thick layer in a large potting-pot, then put in the tongue, in rough irregular lumps, not to touch each other; fill up the pot with veal, and press it down quite close. Pour clarified butter thick over ; keep in a dry cool place, and serve in thin slices, taking off the butter. Garnish with parsley.

\section{6.-SCALLOPS OF COLD VEAL.}

Mince the meat extremely small, and set it over the fire, with a scrape of nutmeg, a little pepper and salt, and a little 
cream, for a few minutes; then put it into the scallop-shells, and fill them with crumbs of bread, over which put some bits of butter, and brown them before the fire.

Either veal or chicken looks and eats well prepared in this way, and lightly covered with crumbs of bread fried; or these may be put on in little heaps.

\section{7.-TO MINCE VEAL.}

Cut cold veal as fine as possible, but do not chop it; put to it a very little lemonpeel shred, two grates of nutmeg, some salt, and four or five spoonfuls of either broth, milk, or water; simmer these gently with the meat, but take care not to let it boil, and add a bit of butter rubbed in flour. Put sippets of thin toasted bread, cut into a three-cornered shape, round the dish. Fried crumbs of bread lightly strewed over, or served in little heaps on the meat, are an improvement to the look and flavor. A little shred of shalot may occasionally be added.

Or:-Stew a few small mushrooms in their own liquor and a bit of butter a quarter of an hour; mince them very small, and add them (with their liquor) to minced veal, with also a little pepper and salt, some cream, and a bit of butter rubbed in less than half a teaspoonful of flour. Simmer three or four minutes, and serve on thin sippets of bread.

\section{8.-THE TURKISH MODE}

Take equal quantities of cold dressed veal, minced very fine, fat, and crumbs of bread, and season it well; add chopped onions, parsley, salt, and cayenne pepper ; wet it with one or two eggs, according to the quantity, adding, if necessary, a little cold melted butter; make the mixture into balls or egg-shapes, and roll them in as much boiled rice as they will take round them. Stew them for an hour and a half in good gravy, well seasoned, and serve them up in it.
In all these modes. the addition may be made of sauce aux truffes, or any of the approved sauces; and a squeeze of lemon will in all cases be found to give a pleasing zest to their flavor.

\section{9.-CURRY OF VEAL.}

Cut part of a breast of veal in moderate sized pieces, put it in a stewpan with an onion and a shalot sliced fine, a slice of lemon, one ounce of butter, a little parsley and thyme, and a table-spoonful of curry-powder mixed with the same quantity of flour; add sufficient broth or water for the sauce; let it boil gently till the veal is done; strain the sauce through a sieve, pour it over the veal quite hot, and serve with rice in a separate dish.

\section{0.-A ROAST BEEF OF LAMB.*}

Take the saddle and the two legs of a. lamb, cut on the middle of each leg a small rosette. which is to be larded, as also the fillets. Roast the whole, and glaze the larded parts of a good color. In France it is served up with maitre d'hôtel sauce, but in England with gravy under it, and mint-sauce in a boat.

\section{MUTTON.}

ThIs is a delicate and a favorite meat. It is susceptible of many modes of cooking, and should always be served very hot and with very hot plates, except of course in cases where it may be sent to table as a cold dish. It is a meat which

* The appellation of "roast beef of lamb" must sound very extraordinary to an American ear, but the singularity of the name is as nothing when compared with the importance and necessity of the dish. At a very great dinner, it is essential to have some dish of magnitude. This has a very good appearance, and is truly excellent. I beg to recommend the trial of a maître d'hôtel sauce under, as the butter, parsley, salt, pepper, and lemon-juice, agree well with the gravy of the meat.-UDE. 
requires care in the cooking, which it will amply repay. The roasting parts are the better for hanging some time, especially the haunch or saddle, but not for boiling, as the color is apt to be injured. One of the most favorite dishes is the

\section{1.-HAUNCH OF MUTTON.}

The haunch should be hung as long as possible without being tainted; it should be washed with vinegar every day while hanging, and dried thoroughly after each washing; if the weather be muggy rubbing with sugar will prevent its turning sour; if warm weather, pepper and ground ginger rubbed over it will k'eep off the flies.

When ready for roasting, paper the fat, commence roasting some distance from the fire, baste with milk and water first, and then when the fat begins dripping, change the dish and baste with its own dripping; half an hour previous to its being done remove the paper from the fat, place it closer to the fire, baste well, serve with currant jelly.

\section{2.-TO STUFF A LEG OF MUTTON.}

Take a leg of mutton, cut off all the fat, take the bone carefully out and preserve the skin whole; take out the meat and mince it fine, and mix and mince with it about one pound of fat bacon and some parsley; season the whole well with pepper and salt, and a small quantity of eschalot or chives chopped fine ; then put the meat into the skin and sew it up neatly on the under side; tie it up in a cloth and put it into a stewpan with two or three slices of veal, some sliced carrots and onions, a bunch of parsley, and a few slices of fat bacon; let it stew for three or four hours, and drain the liquor through a fine sieve; when reduced to a glaze, glaze the mutton with it and serve in stewed French beans.
673.-TO DRESS A LEG OF MUTTON WITH OYSTERS.

Parboil some fine well-fed oysters, take off the beards and horny parts; put to them some parsley, minced onion, and sweet herbs, boiled and chopped fine, and the yolks of two or three hard boiled eggs. Mix all together, and cut five or six holes in the fleshy part of a leg 0 mutton, and put in the mixture; and dress it in either of the following ways: -Tie it up in a cloth and let it boil gently two and a half or three hours, ac- . cording to the size.

Or:-Braise it, and serve with a pungent brown sauce.

\section{4.-SADDLE OF MUTTON.}

This joint, like the haunch, gains much of its flavor from hanging for some period. The skin should be taken off, but skewered on again until within rather more than a quarter of an hour of its being done; then let it be taken off, dredge the saddle with flour, baste well. The kidneys may be removed or remain at pleasure, but the fat which is found within the saddle should be removed previous to cooking.

\section{5.-LEG OF MUTTON ROASTED,}

Like the haunch and saddle, should be hung before cooking, should be slowly roasted and served with onion sauce or currant jelly.

\section{6.-ROAST LEG OF MUTTON.-ANOTHER} RECEIPT.

Put the leg into an iron saucepan with e'sough cold water to cover it, let it come to a boil gently, parboil it by simmering only; have the spit or jack ready, and take it from the hot water and put it to the fire instantly; it will take from an hour to an hour and a half if large, and less time if small.

677.-ROAST LEG OF MUTTON BONED AND STUFFED.

The principal skill required in accom- 
plishing this dish is the boning; this must be done with a very sharp knife. Commence on the underside of the joint, passing the knife under the skin until exactly over the bone, then cut down to it, pass the knife round close to the bone right up to the socket, then remove the large bone of the thickest end of the leg, seeing the meat is clear of the bone; you may then draw out the remaining bones easily. Put in the orifice a highly seasoned forcemeat, fasten the knuckle end" - tightly over, replace the bone at the base of the joint, and sew it in; roast it in a cradle spit or on a jack ; if the latter let the knuckle end be downwards as it is less likely to suffer the forcemeat to drop out. It must be well basted, and should be sent to table with a good gravy.

\section{8.-LEG OF MUTTON BOILED,}

Should be first soaked for an hour and a half in salt and water, care being taken that the water be not too salt, then wiped and boiled in a floured cloth; the time necessary for boiling will depend upon the weight; two hours or two hours and a half should be about the time; it should be served with turnips mashed, potatoes, greens, and caper sauce, or brown cucumber, or oyster sauce.

\section{9.-LEG OF MUTTON BRAISED.}

Procure a leg of mutton of choice flavor, take off the knuckle neatly, divide it into two or three pieces, trim the leg of all the superfluous edges, and then half roast it ; place it with the broken knuckle in a stewpan, add the trimmings with half a dozen slices of rich fat bacon, thyme, knotted marjoram, and other sweet herbs, an onion stuck with cloves, and about half an ounce of butter rolled in flour; stew the whole gently, shaking it occasionally and turn it while stewing; when it is tender take it up, skim the gravy, strain, boil it fast until reduced to a glaze, make a purée or soup of vegetables and place the mutton upon it, cover the mutton with the glaze, and serve.

680.-TO SEND A LEG OF MUTTON NEATLY TO TABLE WHICH HAS BEEN CUT FOK A PREVIOUS MEAL.

Too much must not have been cut from the joint or it will not answer the purpose. Bone it, cut the meat as a fillet, lay forcemeat inside, roll it, and lay it in a stewpan with sufficient water to cover it ; add various kinds of vegetables, onions, turnips, carrots, parsley, \&c., in small quantities; stew two hours, thicken the gravy, serve the fillets with the vegetables round it.

\section{1.-SADDLE OF MUTTON, À LA POLO-} NAISE. (Soyer's.)

This is my economical dish, par excellence, and very much it is liked every time I use it. Take the remains of a saddle of mutton, of the previous day, cut out all the meat close to the bone, leaving about one inch wide on the outside, cut it with a portion of the fat, into small dice; then put a spoonful of chopped onions in a stewpan, with a little butter; fry one minute, add the meat, with a table-spoonful of flour, season rather high with salt, pepper, and a little grated nutmeg; stir round, and moisten with a gill or a little more of broth, add a bay-leaf, put it on the stove for ten minutes, add two yolks of eggs, stir till rather thick, make about two pounds of mashed potatoes firm enough to roll, put the saddlebone in the middle of the dish, and with the potatoes form an edging round the saddle, so as to give the shape of one, leaving the midale empty; fill it with your mince meat, which ought to be enough to do so; if you should not have enough with the remains of the saddle, the remains of any other joint of mutton may be used; egg all over, sprinkle bread- 
crumbs around, put in rather a hot oven, to get a nice yellow color, poach six eggs, and place on the top, and serve brown gravy round; white or brown sauce, if at hand, is an improvement. You may easily fancy the economy of this welllooking and good dish; the remains of a leg, shoulder, loin, neck of mutton, and lamb, may be dressed the same way, keeping their shape of course.

\section{2.-SOYER'S NEW MUTTON CHOP.}

Trim a middling-sized saddle of mutton, which cut into chops half an inch in thickness with a saw, without at all making use of a knife (the sawing them off jagging the meat and causing them to eat more tender,) then trim them into shape; season well with salt and pepper, place them upon a gridiron over a sharp fire, turning them three or four times; they would require ten minutes' cooking; when done dress them upon a hot dish, spread a small piece of butter over each, (if approved of,) and serve; by adding half a table-spoonful of good sauce to each chop when serving, and turning it over two or three times, an excellent entrée is produced: the bone keeping the gravy in whilst cooking, it is a very great advantage to have chops cut after this method. At home, when I have a saddle of mutton, I usually cut two or three such chops, which I broil, rub maitre-d'hôtel butter over, and serve with fried potatoes round, using the remainder of the saddle the next day for a joint. The above are also very excellent, well seasoned and dipped into egg and breadcrumbs previous to broiling. Lamb chops may be cut precisely the same, but require a few minutes' less broiling.

You must remark that, by this plan, the fat and lean are better divided, and you can enjoy both; whilst the other is a lump of meat near. the bone and fat at the other end, which partly melts in cooking, and is often burnt by the fiame it makes; the new one not being divided at the bone, keeps the gravy in admirably. If well sawed it should not weigh more than the ordinary one, being about half the thickness. Do try them, and let me know your opinion.

\section{3.-NECK OF MUTTON}

Is particularly useful, as many dishes may be made of it. The best end of the neck may be boiled for one hour and a half, and served with turnips ; or roasted; dressed in steaks; in pies; à-laTurc; or en haricot.

The scrag may be stewed into broth; or with a small quantity of water, some small onions, a few peppercorns, and a little rice, and served together.

\section{4.-SHOULDER OF MUTTON}

Must be well roasted and sent to table with skin a nice brown; it is served with onion sauce. This is the plainest fashion, and for small families the best.

\section{5.-A SHOULDER OF MUTTON WITH RICE.}

Take a shoulder of mutton and half boil it, then put it into a stewpan, with two quarts of mutton gravy, a quarter of a pound of rice, a teaspoonful of mushroom powder, with a little beaten mace, and stew it till the rice is tender; then take up the mutton and keep it hot; put to the rice half a pint of cream, and a piece of butter rolled in flour; stir it well round the pan, and let it boil a few minutes; lay the mutton in the dish, and pour the rice over it.

\section{6.-LOIN OF MUTTON STEWED.}

Remove the skin, bone it, and then roll it; put it in a stewpan with a pint and a half of water, two dessert-spoon- 
fuls of pyroligneous acid, a piece of butter, sweet herbs, and an onion or two; when it has stewed nearly four hours, strain the gravy, add two spoonfuls of red wine, take up and serve with jelly sauce.

\section{7.-BREAST OF MUTTON}

May be stewed in gravy until tender, bone it, score it, season well with cayenne, black pepper, and salt; boil it, and while cooking skim the fat from the gravy in which it has been stewed, slice a few gherkins, and add with a dessertspoonful of mushroom ketchup; boil it, and pour over the mutton when dished.

\section{S8.--BREAST OF MUTTON CRUMBED OR GRATIN.}

If one breast of mutton, cut off the chine-bone down to the gristle; if you have a stock pot on, put the breast of mutton into it, let it boil until tender, then take it up to cool; have ready as for the crumbed cutlets, adding in the butter and egg a little chopped mushroom; put it all over the breast with a paste brush, then put it on a dish and in the oven to brown; the sauce will be under it when dished.

\section{9.-NECK OF MUTTON.}

This dish is most useful for broth but may be made a pleasant dish by judicious cooking. To send it to table merely boiled or baked is to disgust the partaker of it. When it is cooked as a single dish, first boil it slowly until nearly done, then having moistened a quantity of bread-crumbs and sweet herbs, chopped very fine, with the yolk of an egg, let the mutton be covered with it, and placed in a Dutch or American oven before the fire, and served when nicely browned. The breast may be cooked in the same inanner.

\section{0.-STEAKS FROM A LOIN OF MUTTON}

Are done in the same way, only trim. ming some of the fat off. Cut thick and stew instead of frying them.

\section{1.-MUTTON STEAKS.}

The steaks are cut from the thick or fillet end of a leg of mutton, and dressed as rump steaks.

\section{2.-MUTTON CHOPS BROILED.}

Cut from the best end of the loin, trim them nicely, removing fat or skin, leaving only enough of the former to make them palatable; let the fire be very clear before placing the chops on the gridiron, turn them frequently, taking care that the fork is not put into the lean part of the chop; season them with pepper and salt, spread a little fresh butter over each chop when nearly done, and send them to table upon very hot plates.

\section{3.--MUTTON CHOPS FIIED.}

The fat in which the chops are to be fried should be boiling when the chops are put into it. They should be pared of fat and well trimmed before cooking; they should be turned frequently. and when nicely browned they will be done; of course if they are very thick judgment must be exercised respecting the length of time they will occupy in cooking.

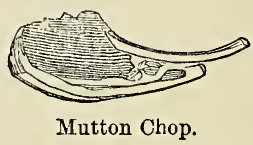

694.-CHOPS AS BEEFSTEAKS.

Cut thick from a leg of mutton, and rub each steak with a shalot; boil over a quick fire; rub your dish with shalot; when on the dish pepper and salt it; send it up quite hot. 
695.-MUTTON CUTLETS.

Loin chops make the best cutlets. Take off the vertebræ or thickest end of each bone and about an inch off the top of the bone; put, the chops into a stewpan in which has been previously melted a little butter seasoned with salt; stew for a short time, but not until they are brown. as that appearance is accomplished in another manner. Chop some parsley very fine, add a little thyme, mix it with sufficient yolk of egg to coat the chops, which will have been suffered to cool before this addition to them; then powder them with breadcrumbs over which a pinch of cayenne pepper has been sprinkled; broil them upon a gridiron over a clear but not a brisk fire; when they are brown dish them; lemon-juice may be squeezed over them, or the dish in which they are served may be garnished with thin slices of lemon in halves and quarters.

696.-MUTTON CUTLETS-ANOTHER WAY.

Not a very fat neck, take off the scrag and the breast bones, leaving the remainder the length you intend the cutlets, then take the chine bone clean off, then the skin and some of the fat; you will now have the mutton free from bones to cut your cutlets; you will find you can cut fourteen good cutlets from this trimmed neck without an $y$ hacking; beat each cutlet with your beater, trim them neatly ; be sure to cut out the pacwax, and leave a little fat to each cutlet. If for gratin or bread crumbed, prepare some chopped parsley and shalot, and bread-crumbs; put some butter to melt in a stewpan, a little of the parsley and shalot and some yolk of egg. mix it well up together; put your bread-crumbs on a sheet of paper, add to it a little salt and pepper; dip each cutlet into melted butter, put down the bread-crumbs with your knife, lay them on a buttered sautépan until wanted to fry.

\section{7.-CUTLETS SAUTÉ.}

Cut your neck of mutton precisely as for the crumbed cutlets; have ready a piece of butter melted in your sauté-pan; dip each cutlet both sides in the butter; when required fry them a very light color, pepper and salt them ; when done take them up to drain from the fat, have some good glaze melted, and glaze each cutlet both sides; dish them round with or without a rim of mashed potato.

\section{3.-MUTTON CUTLETS-MAINTENON.}

Trim the cutlets as for former cutlets; half fry them, then cover them with fine herbs and bread-crumbs, and season with pepper and salt. Lay all to cool ; have some fresh parsley to add to the already fried herbs and shalot. When cool, spread the butter and herbs thick upon each cutlet; sprinkle them with breaccrumbs; wrap them in buttered foolscap paper, and broil them over a slow fire until done.

\section{9.-BRAISED CUTLETS.}

Trim your mutton from the bones as before; then put it whole into a good braise; let it stew gently until tender; put it aside to get cold; when so, cut your cutlets as thick as the former; trim them neatly, make them hot and glaze them.

\section{0.-FILLET OF MUTTON.}

Choose a very large leg; cut from four to five inches in thickness from the large end of the ler; ; take out the bone, and in its place put a highly savory forceneat; flour and roast it for two hours. When done, it may be sent to table with the same accompaniments as a fillet of veal, 
with melted butter poured over it, or a take out the chops, strain the gravy, and rich brown gravy and red currant jelly.

\section{1.-FILLET OF MUTTON STEWED.}

Cut and prepare stuff as above, flour and brown in a little butter, and put it into a stewpan with a pint and a half of gravy; with it a small bundle of sweet herbs, two or three small onions, or eight or ten small button onions peeled, a teaspoonful of whole black pepper; stew slowly three hours and a half. The fillet may be salted, and being half roasted may be stewed with its trimmings.

\section{2.-FILLETS IN MARINADE.}

Cut from the loins of mutton; prepare some carrots, turnips, onions, and celery, thyme, mace, cluves, and whole pepper ; cut up in thin slices; boil a little vinegar and water, put your fillets in a deep dish, pour the vinegar over the roots and mutton when cold; let it lie all night; next day trim neatly and braise them; take them out, and when required glaze them, sauce under them.

\section{3.-BLANQUETTE DE MOUTON}

Is generally made from a former day's saddle of mutton; cutting out the fillets, trimming it neatly, you will be able to cut clearly pieces the size of a shilling, which you will put into some good sauce, you may then put two or three gherkins into it; this is dished better in a tin.

\section{4.-HARICOT OF MUTTON.}

Cut a neck or loin of mutton into thin chops; flour and fry them brown in a smáll quantity of butter; drain them on a sieve; then put them into a stewpan and cover them with gravy ; add a carrot, two leeks, a faggot of parsley and thyme; two or three blades of mace, some allspice, a whole onion, and two turnips; stew them until the meat is tender; then skim off all the fat; put a little butter mixed with flour into the stewpan; stir it until melted and made quite smooth, adding the gravy by degrees, stirring all the time; then put in the chops, with some carrots and turnips ready blanched and cut into pretty shapes, with a dozen silver onions whole, and also half boiled season slightly with pepper and salt, a very little soy, and a teaspoonful of Tarragon vinegar; stew the whole gently for a quarter of an hour, and serve them while quite hot.

\section{5.-MUTTON HARICOT.}

Take a loin of mutton, cut it into small chops, season it with ground pepper, allspice and salt; let it stand a night, and then fry it. Have good gravy well seasoned with flour, butter, ketchup, and pepper if necessary. Boil turnips and carrots, cut them small, and add to the mutton, stewed in the gravy, with the yolks of hard boiled eggs and forcemeat balls. Some green pickles will be an improvement.

706.-HARICOT MUTTON-ANOTHER WAY.

Cut into chops the best end of the neck of mutton; fry them a light brown in fat made boiling-hot before the chops are put into it; some pieces cut from the neck will be the best, dredge them with flour; sprinkle them with pepper and salt, put in a stewpan three parts of a pint of water, an onion stuck with cloves, parsley, a few spring onions, and a bayleaf; stew gently till the meat is nearly done, then add turnips and carrots cut small; fry a large onion cut in slices brown; add it to the gravy, which when just done must be thickened; take out the sweet herbs when the whole has stewed an hour, and serve.

707.-MUTTON KEBOBBED.

Take all the fat out of a loin of mut 
ton, and that on the outside also if very fat, and remove the skin; cut it into steaks ; mix a small nutmeg grated with a little salt and pepper, crumbs, and herbs; dip the steaks into the yolks of three eggs, and sprinkle the above mixture all over them; then place the steaks together as they were before they were cut asunder, tie them, and fasten them on a small spit; roast them at a quick fire; set a dish under, and baste them with a good piece of butter and the liquor that comes from the meat, but throw some more of the above seasoning over. When done enough, take it up, and lay it in a dish; have half a pint of good gravy ready besides that in the dish, and put into it two spoonfuls of ketchup, and rub down a teaspoonful of flour with it; give this a boll, and pour it over the mutton, but first skim ofl the fat. Mind to keep the meat hot, till the gravy is quite ready.

\section{8.-HASHED MUTTON.}

This is a favorite method of disposing of the cold shoulder, especially if it should happen to be underdone; cut it into slices, take the bones (if of a shoulder or leg break them), and put them in a stewpan with the trimmings; cover them with water, put in a faggot of thyme, parsley, whole pepper, allepice, \&c., cover down and simmer for three-quarters of an hour; while the bones, \&c., are stewing, fry an onion brown in a little butter and flour; put it into the sterwpan with the gravy, stew gently twenty minutes, strain it, lay in the slices of mutton in the stewpan, pour over them the strained gravy; pur in a spoonful of walnut ketchup, or any suitable preferred sauce, season it, sinmer until the meat is hot through, dish and serve.

A spoonful of curry powder is sometimes added, and is always a palatable addition.

\section{9.-IRISH STEW.}

Cut a neck of mutton as for the haricot; blanch the chops in water; take and put them into another stewpan with four onions cut in slices; put to it a little of your second stock, let it boil a quarter of an hour; have ready some potatoes pared; put them into the stewpan with the mutton, with salt and pepper; as some like the potatoes whole and some mashed as to thicken the stew, you must boil them accordingly; dish the meat round and the vegetables in the middle.

\section{0.-OHINA CHILO.}

Mince a pint basin of undressed neck of mutton or leg, and some of the fat; put two onions, a lettuce, a pint of green peas, a teaspoonful of pepper, four spoonfuls of water, and two or three ounces of clarified butter into a stewpan closely covered; simmer two hour's, and serve in the middle of a dish of boiled rice; if cayenne is approved, add a little.

\section{1.-CHINA CHILO-ANOTHER WAY.}

Chop very fine two small young lettuces, two onions, a pint of green peas, and a couple of young cucumbers, or the fourth of a pint of mushrooms; season with a teaspoonful of salt and half a teaspoonful of pepper; mince the meat of a neck of mutton uncooked, and mix it with the vegetables in a stewpan; add four table-spoonfuls of water and two ounces of butter, clarified will be proved the best; let them well amalgamate over a slow fire; keep them stirred for fifteen minutes, then cover down close and simmer very slowly for two hours; serve it in the centre of boiled rice.

\section{2.-MINCED MUTTON.}

Mince dressed meat very finely, season it, make a very good gravy, warm the meat up in it, and serve with fried bread round the dish, or with poached eggs. 
Or:-Mince cold leg of mutton freed from the skin and fat, warm it with stewed cucumbers, taking care that it does not burn after the meat is put in.

713.-AU GRATIN, OR AS SCALLOPS.

Mince dressed mutton with a very little fat, season lightly with pepper and salt, and put into scallop-shells about half full. Then put potatoes mashed with a little milk, and a very small bit of butter; smooth with a spoon, and brown in a Dutch oven.

\section{4-AS RISSOLES.}

Enclose the minced meat in a paste or browning of egg and crumbs of bread, but season it as if for forcemeat.

\section{5.-MUTTON LIKE VENISON.}

A haunch or leg will be the most appropriate. The joint should be hung as long as it can be with safety, and dressed exactly like a haunch of venison, and served with the same sauces, but to make the taste more perfectly resemble that of venison it should, after having been hung to the turn, be skinned, and laid in a pan with vinegar and water; two parts of the former to one of the latter, not enough to cover it; put in a fagot of herbs, a clove of garlic, one or two bayleaves, a spoonful of whole pepper, and a couple of onions cut in slices; let it soak three days, dry it well, hang it for a day and roast as venison. It may also be put into a stewpan with half a pint of gravy, and simmered four hours; serve with venison sauce.

\section{6.-TO MAKE A SCOTCH HAGGIS.}

Take the stomach of a sheep. The washing and cleaning is of more consequence than all, as it will be of a bad color and a bad taste if not well cleansed; when clean, turn it inside out, then let it lie for a day or two in salt and water. Blanch the liver, lights, and heart of the sheep, lay them in cold water, chop all very fine; the liver you had better grate, chop a pound of the suet very fine, dry in the oven a pound of oatmeal; mix all this well together, season with pepper and salt, a little chopped parsley, and a little chopped onion; then sew up the bag; before you finish sewing it, add a few spoonfuls of good white stock; put it in a stewpan with a drainer; boil it in water, keeping it well covered all the time, prick it all over with a small larding pin to keep it from bursting; it will take several hours to boil ; be careful in taking it up, and let your dish be large enough.

\section{7.-ROGNON DE MOUTON À LA FRAN- ÇAISE.}

Take half a dozen fine mutton kidneys, clear them of fat and skin, and cut them into thin slices; powder them immediately with sweet herbs in fine powder, parsley which has been chopped, dried, and powdered, cayenne, and salt: put into a stewpan two ounces of clarified butter or fresh if the former is not in reach, put in the slices of kidney, fry them; they will brown very quickly, they must be done on both sides; dredge flour over them, moisten with lemon juice; in five minutes the kidneys will be done; lift them out into a very hot dish around which are laid sippets fried; pour into the gravy two glasses of champagne, give it a boil, pour it over the kidneys, and serve.

It may here be stated that the French cooks vary constantly the herbs which they employ, according to any known preference for the palate of those for whom they cook. Various linds of wines and sauces may be used for flavoring, and this is constantly done as much for variety and the ability of giving a new name to a dish, which is variad only in some such small feature. 
718.-LOIN OF MUTTON LIKE VENISON. (French Receipt.)

Remove the skin from the joint and bone it, and do it neatly; lay it in a stewpan with about a pint of weak broth, an onion stuck with cloves, two glasses of red wine, and a teaspoonful of pyroligneous acid; let it boil, put in a bundle of sweet herbs; stew, turning frequently; add as it progresses a little gravy; some very good may be made from the bones; it will take from two hours and a half to three hours.

\section{9.-TO DRESS MUTTON HAMS.}

Soak the ham for five or six hours in cold spring water, unless it has only recently been cured, then one hour will suffice; put it into cold water, boil gently ; it will be done in two hours and a half. It is eaten cold.

\section{0.-MUTTON KIDNEYS BROILED.}

Skin and spit without parting asunder, skewer them through the outer edge and keep, them flat, lay the opened sides first to the fire, which should be clear and brisk, in four minutes turn them, sprinkle with salt and cayenne, and when done, which will be in three minutes afterwards, take them from the fire, put a piece of butter inside them, squeeze some lemon juice over them, and serve as hot as possible.

\section{1.-SHEEP'S TONGUES STEWED.}

Put them into cold water and let them boil ; when they are sufficiently tender to remove the skin easily, take them out, split them, and lay them in a stewpan with enough good gravy to cover them. Chop parsley, and mushroom, with a little eschalot finely, work a lump of butter with it, add pepper and salt to flavor; stew them in the gravy until the tongues are tender, lay them in a dish, strain the gravy and pour it hot over the tongues, and serve.

\section{2.-CÔTELETTES À LA MAINTENoNoN.} (Ude's Receipt.)

Pare the chops neatly. Chop fine some sweet herbs, such as parsley, shalots, and mushrooms, which fry in a little butter. When they are done, fry the chops a little in that seasoning till nearly done, let them cool in the herbs; then have some strong foolscap paper cut in the form of the cutlet, put some of the herbs and ham chopped very fine, if you have it ready in the larder; put the cutlets in and a little of the herbs over them; wrap them in the paper, and broil them on a very clean gridiron and very equal fire till of a good color; serve them very hot, and a little Italian sauce separate. Mind to wipe the grease with a clean towel before serving them.

\section{3.-MUTTON CUTLETS WITH SOUBISE, OR ONION SAUCE.}

Take a neck of mutton, and cut the chops one by one without flattening them; cut off some of the flat bone at the extremity of the chops. Put them into a stewpan with all the parings, together with the parings of the onions to make the soubise. Season the whole well with carrots, a bunch of parsley, and green onions, salt, and a very small quantity of spice, \&c.; throw in four or five spoonfuls of good broth to braize them. When done, drain them, and let them cool. Strain the liquor through a silk sieve. Then reduce it to a glaze. Next pare the chops nicely, and put them with the glaze. This being completed, dish them miroton way, and pour the soubise or onion sauce into the middle.

Some persons take two necks of mutton, cut two bones to each chop, lard them with bacon, and braize them as above; but mutton, being in general fat, is better without being larded. With 
regard to the two bones, you must take care not to let them be too thick; if they are too, thick you cannot dish them well.

724.-ANOTHER, AND A MUCH BETTER METHOD OF PREPARING CUTLETS À LA SOUBISE.

Cut the mutton chops a little thicker than when you wish to broil them. Pare them nicely, and put them into a stewpan, where they may all lie flat. Put an onion or two, a few carrots, a little salt, a bundle of parsley and green onions seasoned, four or five spoonfuls of good consommé, and sweat the whole till it is entirely done. Then take out the roots, put in a little glaze, and reduce over a large fire. When entirely boiled down to a glaze, take them off the fire for a few minutes, let the cutlets settle in the glaze, take out the trimmings and vegetables, turn over the cutlets in the glaze, and take out the grease or fat, lay them on a cover to drain the fat, and serve up before they can get dry.-This method is preferable to the other. You must not in either case lard your chops with bacon. These cutlets may be served up with all sorts of purée of vegetables.

\section{5.-FOR COOTELETTES DE MOUTON EN RAGOOT.}

Take off all the fat from the cutlets, dredge the meat with flour, and put them into a stewpan with the fat melted, a bundle of sweet herbs, and two shalots minced; let them brown, then strain the gravy, add a glass of wine, and one of good sauce; thicken, if necessary, with a little roux, and let the whole stew until very tender.

\section{6.-TO DRESS CÓTELETTES DE MOUTON À LA POLONAISE.}

Remove all the fat, put the meat into a covered stewpan, with a carrot and a turnip sliced, two onions, a bundle of sweet herbs, a little pepper and salt, and enough broth to moisten the whole; let it stew very gently until the meat is perfectly done, then take it out, strain the gravy, put it over a brisk fire, and reduce it to a glaze ; then cover the cutlets with the glaze, and serve them up with tomatosauce, or a vegetable purée of any kind.

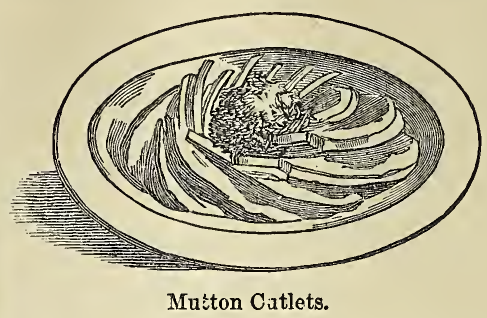

727.-FOR CÔTELETTES A LA MAINTENON.

Cut and trim cutlets from a neck or loin of mutton; chop very finely a quantity of parsley, a little thyme, and a shalot; put them with butter into a stewpan, and fry the chops a little; then take out the chops; allow them to cool; add to the herbs some fresh parsley chopped and a few crumbs of bread, and seasoning: spread this over the cutlets with a knife, wrap them in buttered paper, and broil them over a slow fire. Serve a sauce piquant in a boat.

Or:-Cut them handsomely from the loin or back end of the neck; half fry them, and then cover them with herbs, crumbs of bread, and seasoning; lay this on very thickly, and put them into a stewpan with a little gravy; stew until tender, then wrap them in writing-paper, and finish them on the gridiron.

\section{8. -CÔTELETTES AUX HARÎCOTS VERTS.}

Having dressed French beans as usual, drain the water from them, and simmer them with pepper and salt in a good piece of butter. A few minutes before serving add the beaten yolk of an egg, and shake the pan over the fire; but they must not 
boil. In the mean time have ready three mutton cutlets, neatly trimmed, seasoned with pepper, salt, and a few crumbs, and nicely fried; and serve them on the French beans.

\section{9. - SCRAGS OF MUTTON A LA SAINTE MENEHOULD.}

Order the narrow part ot a neck of small mutton to be cut off before the sheep be divided; which leaves the two scrags united. Soak in warm water, then hang it two days; lay it in a stewpot, with slices of fat bacon over and under, two pounds of scrag of veal, three large carrots, three onions, a large bunch of sweet herbs, two bay-leaves, and a tablespoonful of whole white pepper; cover the whole with beef broth, and simmer four hours. Drain the gravy from the meat, wrich cover on all sides with crumbs of bread mixed with pepper and salt, and brown it with a salamander. In the mean time boil the gravy, uncovered, very quickly, having strained it first, and serve in the dish; add salt. Serve in the dish endive, tomato sauce, or stewed spinach.

\section{0.-MUTTON KIDNEYS.}

Split, pepper, and broil them, broiling the cut side first, which will make a cup for the gravy when the outer part is turned to the fire. Chop some parsley. very fine, mix it well with a little fresh butter, the juice of a lemon, pepper and salt, and put a little on each. A sheep's heart may be split open, and broiled in the same way.

With a very sharp knife cut mutton kidneys in the thinnest possible slices; flour, and fry quickly till they are quite crisp. While frying; add pepper and salt. Serve them in a good gravy, to which a bit of garlic has given a very slight flavor.

731.-ROGNONS, AU VIN DE CHAMPAGNE. Slice or mirce them, and fry them with a little chopped parsley and eschalot until they become of a light brown, seasoning them with pepper and salt; then dust them with flour, moisten them with a little strong gravy and a glass of Champagne, and let them stew for a few mo ments.

\section{2.-SHEEP'S TAILS AND KIDNEYS.}

Cut the tails. boil them for fifteen minutes, then put them into a stewpan with half a pint of gravy, an onion stuck with cloves, a little salt, and cayenne pepper. Stew till tender, strain the gravy, thicken it with flour and butter, and add the juice of half a lemon. Boil until the whole is very smooth, broil half a dozen kidneys, and place them in the middle of a dish with the tails and sauce round.

These are the most tasty modes, but kidneys, when meant for breakfast, are usually broiled upon the gridiron, without parsley or lemon; being only split open, well peppered, and a bit of butter put upon them when broiled. They must not be much done, and may be brought to the table in less than ten minutes. If not quite hot, they are not worth eating.

\section{3.-MUTTON RUMPS AND KIDNEYS.}

Stew six rumps in some good muttongravy half an hour; then take them up, and let them stand to cool. Clear the gravy from the fat; and put into it four ounces of boiled rice, an onion stuck with cloves, and a blade of mace; boil them till the rice is thick. Wash the rumps with yolks of eggs well beaten; and strew over them crumbs of bread, a little pepper and salt, chopped parsley an 1 thyme, and grated lemon-peel. Fry in butter of a fine brown. While the rumps are stewing lard the kidneys, and put them to roast in a Dutch oven. When the rumps are fried, the grease must be drained before they are put on 
the dish, and the pan being cleared like- in India, where veal is not so easily prowise from the fat, warm the rice in it. curable, answers all the purposes for Lay the latter on the dish; the rumps mock turtle, rolled head, rich hash, or put round on the rice, the narrow ends ragout: the bones make excellent jelly, towards the middle, and the kidneys be- either savory or sweet. Parboil the twreen. Garnish with hard eggs cut in head; cut the meat from the bone; stew half, the white being left on; or with different-colored pickles.

\section{4.-SHEEP's HEAD.-(English.)}

Though this may be seen in every part of London inhabited by the working classes, and may be procured ready cooked, I prefer always to prepare it at home, and very good it is. Choose a fine one, as fat as possible, and put it into a gallon of water to disgorge for two hours ; wash it well, saw it in two from the top, take out the brain, cut away part of the uncovered part of the skull, and also the ends of the jaws; wash it well, put into the stewpan, with two onions, one carrot, two turnips cut in slices, a little celery, four cloves, a bouquet of four sprigs of thyme, a bay-leaf, one ounce of salt, a quarter of an ounce of pepper, three quarts of water; set on the fire; when near boiling, add half a teacupful of pearl or Scotch barley; let it simmer for three hours, or till tender, which try with a fork; take out vegetables. cut in dice, remove bouquet, skim off the fat, and pour all into tureen. Or, lay the head on a dish, and serve with either onion sauce over, parsley and butter, or any sharp sauce; or egg and bread-crumb it over, put it in an oven for lialf an hour till getting a nice yellow color, and serve with sharp sauce under. Or, with the brain, thus: having boiled the brain for ten minutes in a little vinegar. salt, and water, cut it in pieces, warm it in parsley and butter, season it a little, and put it under the head and serve.

\section{5.-SHEEP'S HEADS, HEARTS, TAILS,} AND TONGUES.

A great variety of excellent dishes may be made from a sheep's head, which the former in a little of the liquor until quite tender; send it to table with a glass of wine in the sauce, forcemeat-balls and brain-cakes for garnish; or roll up the pieces seasoned in the inside with a thick covering of chopped herbs well seasoned; brush the outside with yolk of egg; dredge it with bread-crumbs; fry it; and send to table with a rich gravy made of the bones and pickles warmed up in it.

Take a sheep's heart and stuff it throughout, using a considerable quantity of chopped bacon in the stuffing; half boil it, and when cooled a little rub it over with pepper and salt, and wrap it in paste in the shape of a cone. Rub the paste over with the yolk of an egg, and strew vermicelli loosely over it. Set it with the broad end downwards, and bake it in the oven. When baked, send it to table with gravy sauce.

\section{6.-SHEEP'S TAILS AND TONGUES.}

Take three tails and three tongues, cut the tails in half and split the tongues. Stew them gently for three hours in as much water as will cover them, adding three spoonfuls of vinegar, three onions, a teaspoonful of mixed spices, and one of salt: these ingredients to be put in after the pot has been skimmed. When the tails, \&c., are very tender, take them out, score them, dip them in drawn butter, roll them in grated bread-crumbs, and let them lie for a few minutes, then put on more butter with a knife, and additional bread-crumbs, which latter should be slightly seasoned; brown them before the fire. Strain the gravy, enrich it with butter, squeeze lemon juice over the tongues and tails, and serve them in the grary. 


\section{7.-SHEEP'S TROTTERS.}

Boil the trotters, or rather stew them gently, for several hours, until the bones will come out. The liquor they are boiled in will make excellent stock or jelly. Take out the bones without injury to the skin, stuff them with a fine forcemeat; stew them for half an hour in some of the stock, which must be well flavored with onion, seasoning, and a little sauce; take out the trotters, strain the sauce, reduce it to a glaze, and brush it over the feet. Serve with any stewed vegetable.

Or:-Prepare them in the same way, and dip them in a batter and fry them. The paste, or batter, for frying, is best made thus: mix four spoonfuls of flour with one of olive-oil, and a sufficient quantity of beer to make it of the proper thickness; then add the whites of two eggs well beaten and a little salt. Serve with tomato sauce.

Or :- Simply boil them, and eat them cold with oil and vinegar.

\section{8.-SHEEP'S TROTTERS.-(Soyer.)}

I get a dozen of them from the tripebutcher, all cleaned and ready, and beg of him to extract the long bone from them. I put a quarter of a pound of beef or mutton-suet in a stewpan, with two onions and one carrot sliced, two bay-leaves, two sprigs of thyme, one ounce of salt, a quarter of an ounce of pepper; put on the fire, and cook five minutes; add two tablespoonfuls of flour, and stir it round; add two quarts and a half of water, then put in the feet, stir till boiling; simmer for nearly three hours, or until the feet are perfectly tender; when done, take them out, and lay on a sieve; take a quarter of a pound of fresh butter, a teaspoonful of salt, the same of flour, a quarter of one of pepper, a little grated nutmeg, the juice of a lemon; mix all these well together on a plate with the back of a spoon; put the feet with a gill of milk in a stewpan on the fire; when very hot, put in the butter, stir continually till melted, having previously well mixed two yolks of eggs with five table-spoonfuls of milk, which put in the stewpan; keep moving the pan round over the fire continually for one minute, serve in a very hot dish with croutons of fried bread cut in triangular pieces round the dish. The stock may be used for any purée or thick soup.

\section{9.-FRENCH RAGOUT OF MUTTON.}

Take about two pounds of the scrag of the neck, breast, chump, or any other part, with as little fat as possible, cut it into pieces of about two inches square, put into a pan two ounces of butter, or good fat; when melted, add two tablespoonfuls of flour, stir with a wooden spoon till forming a brownish roux, add the meat, and stir it round for twenty minutes; add a little water, but not enough to cover the meat; one saltspoonful of pepper, four ditto of salt, and four ditto of sugar, a bouquet of six sprigs of parsley, stir till boiling, set it to simmer. Having previously peeled a few turnips, cut in large dice one inch square, about thirty pieces, put some fat in the fryingpan, and fry the turnips until rather brown, take them out, and put them in a stewpan with the meat when it is done, which will be in about one hour from the time it was put on; when ready to serve, take out the meat and turnips, squeeze the bouquet, which throw away, skim off the fat; if too thick, add a little broth or water; or, if too thin, boil it a little more, dish it up by placing the pieces in a circle and the turnips in the centre, sauce over, and serve very hot-(if not, it is spoiled.) For those who like it, a small piece of scraped garlic may be added. Onions, 
carrots, peas, \&c., may be used in place of the turnips.

This is a very favorite dish in France; I learnt it from an o'd French émigré, who used to visit us about ten years since. When I have company, I use the chops of the neck, dress them in a crown, placing the vegetables in the centre; I find them very much liked. I have at some houses partaken of harico-mutton which has been tolerably good, but nothing in flavor to this plan. If there is any left, it is good warmed again, or even cold.

\section{0.-IRISH STEW.}

Cut up about two pounds of the neck of the mutton into small cutlets, which put into a proper sized stewpan with some of the fat of the mutton; season it with half a table-spoonful of salt, a quarter of an ounce of pepper, the same of sugar, six middle-sized onions, a quart of water; set them to boil and simmer for half an hour, then add six middlingsized potatoes, cut them in halves or quarters, stir it together, and let it stew gently for about one hour longer; if too fast, remove it from the top, but if well done the potatoes will absorb all of it, and eat very delicate; any other part of the mutton may be served in the same way.

\section{1.-TURESICUS.}

Mince very fine part of a cold boiled leg of mutton, and mix it with rice, season it very high with black pepper, add salt, and make it into balls the size of a cabbage-leaf.

Tie each ball separately in a cabbageleaf; boil it about half an hour, and serve immediately, very hot.

\section{2.-A SCOTCH HAGGIS.}

Take the stomach of a sheep ; wash it with cold water until perfectly clean; then turn it inside out, scald it, scrape it with a knife quickly, and then put it into cold salt and water till wanted. Take the liver, lights. and heart, and parboil them; grate the liver, and mince the other parts quite fine; mince also half a pound of suet, three or four onions, toast half a pound of round oatmeal cakes before the fire, and pound them into powder, which is to be thickly sprinkled over the mince; mix all well together; season with pepper and salt; then fill the bag, and before sewing it up put in a large teacupful of any kind of strong broth or gravy. Put the bag, neatly sewed up, in a pan with enough of boiling water to cover it, and a small p'ate under it; prick over with a needle to prevent it bursting. and let it boil four or five hours, keeping the haggis constantly covered with boiling water.

\section{3.-THE ENGLISH MODE IS}

To mince the heart, the tongue, the kidneys, and a part of the liver of the sheep, with a third of its weight in fat bacon, two anchovies chopped small, and the crumb of a penny-roll grated, a saltspoonful of grated lemon-peel, pepper, salt, two eggs beaten, and a glass of wine; mix all well together; butter and mould; put in the mixture, and let it boil for two hours: or it may be boiled in a veal caul.

\section{4.-A CAMP DISH.}

Take any joint of mutton, put it into a pot with a good many onions cut small, and as many vegetables as can be obtained to add to it; two table-spoonfuls of vinegar, five of port wine; season it with black and red pepper; add a spoonful of flour, and, if at hand, four dessert-spoonfuls of Harvey's sauce and essence of anchovies. Cover the meat with water, and let it stew one and a half hour; it should be stirred frequently to prevent 
it from burning, as there should be only water sufficient to cook it. Should there be a steam apparatus do not add the water. This is an excellent dish in camp, and it also suits a family where there are many persons to be fed from one joint. A fowl may be added to or substituted for the mutton.

\section{5.-SHEEP'S TONGUES WITH TURNIPS.}

Take eight tongues of an equal size; let them disgorge in a little water and flour, and next blanch them. When thoroughly blanched, put them in a stewpan, to braise them. In case you should have a braise of beef, or of mutton, or any others, they will all equally answer the purpose for sheep's tongues; when they are done, peel them and cut them in two. Dish them miroton way, and cover them with the sauce of the haricot, the turnips of course being put in the middle. It is customary in French cookery to call any thing made with turnips haricot.

746.-THE SAME WITH CABBAGE LETTUCES,

The tongues are to be braised as above, the same as those you wish to cook in any way whatever. Take a dozen and a half of good cabbage-lettuces, wash them very clean and blanch them. When they are cold and you have squeezed all the water out of them, open them in two, take off the stalks, powder a little salt and pepper over them; then shut them and give them a good form ; mark them in a stewpan, surrounded with layers of bacon; moisten them with a little braise, or any thing to give them a good taste; otherwise take the pot-top, with a little broth and salt. When the lettuces are quite done, drain them, and squeeze them in a cloth to extract the grease. Dish them in rosettes, a tongue, a lettuce, and so on successively. Put a large tongue in the middle, to improve the look of the rosette. Another time cut the tongues in two, and dish them miroton way, that is, one half of a tongue, and lettuce, alternately. In this case, put a jardinière in the middle, and cover both the tongues and the lettuce with the Espagnole (Spanish sauce.) This is likewise a common dish in a first course.

\section{7.-MUSETTE OF MUTTON WITH ENDIVE.} (MUTTON BAGPIPE WITH ENDIVE.)

Take a shoulder of mutton that has been kept for some while; bone it without taking off the thin skin that is found near the joint; powder it over with a little pepper and salt. Then pass a piece of packthread round, as tailors do round a button, fasten the packthread, and mould the shoulder of mutton quite round. You must preserve the knuckle so that it may resemble a bagpipe. Braise it, and season it well. After having drained and glazed it, send it up with either endive or sorrel.

N. B. It may also be stuffed and larded, and a floweret larded in the top part, and sent up with white beans à la Lyonnaise. This is also a common dish.

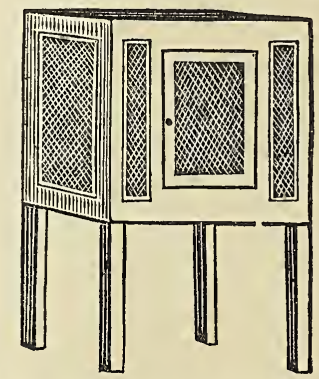

Meat Safe, of mood and wire.

\section{LAMB.}

748.-FORE-QUARTER OF LAMB.

This is the favorite and indeed the best joint. Do not put it too near the fire at first, when it gets heated baste it well; the fire should be quick, clear, but not 
fierce; the usual weight of a fore-quarter is between nine and eleven pounds, it will take two hours; when it is done, separate the shoulder from the ribs, but before it is quite taken off lay under a large lump of butter, squeeze a lemon, and season with pepper and salt; let it remain long enough to quite melt the butter, then remove the shoulder and lay it on another dish.

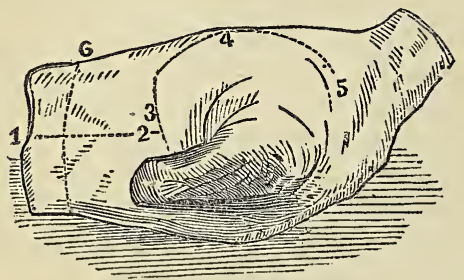

Fore-Quarter of Lamb.

749.-SADDLE OF' LAMB.

Roast it quickly, but be very careful neither to scorch it nor to take it from the fire until it is done; baste with the fat and gravy which fall from it, and in an hour and three-quarters it will be done, unless larger than common, and then it will take two hours; serve with mint and cucumber sance.

\section{0.-TO ROAST A LEG OF LAMB.}

The rules laid down for roasting mutton must be scrupulously observed with, respect to lamb; let it roast gradually, and commence a distance from the fire; a leg of five pounds will take an hour and a quarter, one of six pounds will take an hour and a half.

\section{1.--TO BOIL A LEG OF LAMB.}

Put it in sufficient clear cold soft water to cover it, let it remain half an hour, a table-spoonful of vinegar or half a handful of salt may be thrown in; put it into a thin white cloth which has been floured, and boil it in enough water to cover it. A good-sized bundle of sweet herbs niay be thrown into the saucepan; if six pounds it will be done in an hour and a half; serve with spinach or French beans; if sent to table cold lay handsome sprigs of parsley about it tastefully; it may, while hot, be garnished with parsley, with thin slices of lemon laid round the dish.

\section{2.-A SHOULDER OF LAMB}

Will be found best cooked when done with the fore-quarter, but if roasted singly will take an hour.

\section{3.-SHOULDER OF LAMB LARDED.}

Cut your lardons small, of fine white fat bacon, cover them with pounded mixed spices, cayenne pepper, and salt; bone the shoulder of lamb, lard the under side, roll the joint, and bind it with narrow white tape; braise it, and when done glaze it. Serve it on mushroom sauce ; any sauce applicable to lamb will serve except mint sauce, which should not be eaten with this dish.

\section{4.-TO GRILL A LOIN OF LAMB.}

Boil half an hour, take it out and score it like pork, brush it all over with well beaten yolk of eggs, and powder over it bread-crumbs mixed with minced parsley, put it into an American oven and roast it until brown; serve with melted butter and lemon pickle, or tomato sauce, the last especially, if cold. A shoulder and breast may be dressed in the same manner.

\section{5.-SHOULDER OF LAMB FORCED AND BRAISED.}

Take out the bone from the shoulder; you must be very particular and careful in removing the blade bone that you do not cut a hole through the skin; when you have done it fill up the vacancy with some good veal forcemeat, cover it with fat bacon or ham; then put it into a good braise and let it boil gently for about an hour, when required glaze it well ; you 
can make it after you have put in the forcemeat and sewed up the cut part, either as a shoulder of lamb, or form it into a swan by adding the shank bone for a neck, and form the beak or bill with paste ; if plain, put a paper ruffle or ornamented silver skewer; the sauce as may be approved of, as peas, or spinach, or purée, turnips, or French beans, or truffles, or mushrooms.

\section{6.-STEWED LOIN OF LAMB.}

The loin may be stewed whole or in steaks; in the former the flap being secured by a skewer, is put into a stewpan, with a quarter of a pound of butter, and covered down close; let it simmer one hour, then turn it, let it simmer again for an hour and a quarter, and then have ready some rich brown gravy hot, lift out the meat, pour the gravy over it, and send it to table with mint sauce, a lettuce, and $a$ few radishes and spring onions.

\section{7.-NECK OF LAMB À LA JARDINIÈRE.}

Plain roast the neck, as you would that of mutton; and whilst it is roasting, cut one middle-sized carrot in small dice, the same quantity of turnip, and thirty button onions; wash all in cold water, put them in a small stewpan, with one ounce of butter and half a teaspoonful of sugar, place on the fire till no liquid remains in the stewpan; add to it a gill of brown sauce, half a one of broth, add a small bouquet of parsley and bay-leaf; after once boiling, set it to simmer on the corner of the stove, skim off all the fat; when ready, taste if very palatable; it must be a nice brown color, and the sauce lightly adhere to the back of the spoon; serve on the dish, place the neck over; white sauce may be used instead of brown, only add a spoonful of liaison when ready to serve. The sauce is equally good with almost any kind of meat, game, and poultry ; it will often be referred to, therefore be particular in making it; you can shape the vegetables in twenty different ways, by using either green peas, French beans, or Brussels sprouts; sprue-grass may be added, when in season, but should be boiled separately, and added just previous to serving.

\section{8.-SADDLE OF LAMB, RUSSIAN FASH- ION.}

Roast a small saddle of lamb, keeping it pale; having had it covered with paper, take ten good-sized boiled potatoes, mash them with about two ounces of butter, a teaspoonful of salt, a quarter ditto of pepper, a table-spoonful of chopped parsley, and a little grated nutmeg; mix all well together with a fork, adding half a gill of milk and one egg; when cold, roll them into a long shape the size of plover's eggs, egg and bread-crumb twice, and fry light colored; dress the saddle, surround it with the potatoes, make a sauce of melted butter and maître d'hôtel butter, put in it, and pour it round, and serve. All joints of lamb can be dressed thus.

759.-LEG OR SHOULDER OF LAMB WITH PEAS.

These must be plain roasted; when done, serve with peas in the bottom of the dish.

\section{0.-LEG OR SHOULDER WITH FRENCH} BEANS.

Plain roast as before; prepare beaus as directed.

\section{1.-BOILED LEG OF LAMB WITH SPINACH.}

Procure a very small leg, and cut the end of the knuckle-bone, tie it up in a cloth and place it in cold water, with two ounces of salt; boil it gently according to size; when done, remove the cloth, and dish it up with spinach under it, propared as directed. (See VEgetables.) 
762.-SHOULDER OF LAMB BRAISED.

Take the blade bone from a shoulder of lamb, and have ready ten long strips of fat bacon, which season rather highly with pepper, salt, and a teaspoonful of chopped parsley; place the pieces, one after the other, in your larding needle, which draw quickly through the fleshy part of the shoulder, leaving the bacon in the meat; after having used all the bacon, roll the meat round, and tie it up with a piece of string; then put it into a stewpan containing a quarter of a pound of butter, over a siow fire, stirring it occasionally until of a light golden color, pour in a quart of water or broth, and add forty button onions and a bunch of parsley; let simmer very slowly until the onions are quite tender, then take up the meat, pull off the string, and dress it upon a dish with the onions round; take the parsley out of the liquor, carefully skim off all the fat, and reduce it until forming a thinnish glaze, when pour it over the meat and serve. Mushrooms may be added ten minutes before sending to table.

763.-BREAST OF LAMB BRAISED, BROILED.

Saw off the breast from the ribs of lamb, leaving the neck of sufficient size to roast, or for cutlets; then put two onions, half a carrot, and the same of turnip, cut into thin slices, in a stewpan with two bay-leaves, a few sprigs of parsley and thyme, half an ounce of salt, and three pints of water; lay in the breast, which let simmer until tender, and the bones leave with facility; take it from the stewpan, pull out all the bones, and press it between two dishes; when cold, season with a little salt and pepper, egg and bread-crumb it lightly over, and broil gently (over moderate fire) of a nice yellowish color, turning it very carefully; when sufticiently browned upon one side, serve with plain gravy in the dish, and mint sauce separately, or with stewed peas or any other vegetable sauce; tomato sauce is likewise very good served with it.

\section{4.-LAMB'S HEAD.}

Parboil the head, rub it over with yolk of eggs, cover it thickly with chopped herbs, crumbs of bread, and clarified butter, and put it into a Dutch oven before the fire. Mince the heart and the liver very finely, and stew them in a little good gravy, adding a spoonful of lemonpickle; make some forcemeat-balls and brain-cakes, and fry them; place the mince in the dish with the head upon it, and garnish with the balls, brain-cakes, and lemon sliced, or pickles.

\section{5.-LAMB'S FRY.}

Take about a pound and boil for ten minutes in half a gallon of water, take it out and dry on a cloth; have some fresh crumbs, mix with them half a spoonful of chopped parsley, salt, pepper; egg' the fry lightly with a paste-brush, dip it in the crumbs, fry for five minutes, serve very hot on a clean napkin in a dish, with fried parsley over.

\section{6.-LAMB'S HEAD WITH HOLLANDAISE.}

If you want it very white, make stock as for sheep's feet, put it to stew, when done lay on dish with about twelve new potatoes (boiled) round it, pour over some cream-sauce, and serve.

\section{7.-LAMB'S HEAD WITH BRAIN OR LIVER.}

Blanch the brain or liver, and mince them as for sheep's head, introducing only the yolk of an egg; mix with a little milk, stir in quickly, add a tablespoonful of chopped parsley, the juice of half a lemon, lay it on the dish with the head over, and serve.

768.-TO BONE A QUARTER OF LAMB.

Take the fore quarter, remove the shoulder and take out the bone; stuff it 
with fine forcemeat, and skewer it in a $\mid$ and then add to it a slice of lemon, a handsome shape. Braise it with two large spoonful of mushroom ketchup, a ounces of butter, add a teacupful of teaspoonful of lemon-pickle, a grate of water, stirring the braise until the gravy nutmeg, and the yolk of an egg beaten is drawn. Then cut the brisket into pieces, and stew them in white gravy; thicken it with cream and eggs so that it shall be very white; cut the long bones into chops and fry them; thicken the gravy of the braise, add to it haricots, minced truffles, or any thing else of vegetable in season. Place the shoulder in the centre of a dish with its own sauce, lay the brisket covered with white sauce round it, and place the fried chops at the edge.

\section{9.-LAMB À L'ESPAGNOLE.}

An entire lamb is frequently roasted in the Peninsula, without any other preparation than merely skinning it, taking out the fry, and cutting off the feet. It is then, however, extremely young-not more than perhaps six weeks 'or two months old; the bones eat like gristle, and the meat is singularly delicate. It is sometimes, but only rarely, stuffed with bread and sweet herbs, and served with bread-sauce; but more frequently eaten with lemon-juice.

\section{0.-AU PASCAL.}

May be a little older, and is also roasted whole, but boned from the neck up to the shoulders, and the legs fixed into the body, which is then covered with slices of bacon, kept on with small skewers, or tied with twine ; all, however, being removed when the meat is nearly done. Both should be placed in a cradle-spit, and will take about two hours in roasting.

\section{1.-TO FRICASSEE LAMB-STONES WITH- OUT ANY ADDITION.}

Skin, wash, and parboil, and then cut thểm in half, dry and flour them; fry of a beautiful brown in hog's lard. Serve with the following sauce: thicken some veal gravy with a bit of flour and butter, well in two large spoonfuls of thick cream. Put this over the fire, and stir it well till it is hot and looks white: do not let it boil, or it will curdle. Then put in the fry, and shake it about for a minute or two. Serve in a very hot dish.

\section{2.-TO STEW A BREAST OF LAMB.}

Cut it into pieces, pepper and salt well, stew in sufficient gravy to cover the meat until tender, then thicken the sauce, pour in a glass of sherry, serve on a dish of stewed mushrooms.

\section{3.-CHEVAUX-DE-FRISE LAMB.}

Get two necks of lamb of the same size, take off the chine-bone, not leaving a particle of bone adhering to the ribs, or it cannot be carved clean down between the bones when at table; blanch them a few minutes, put them to cool, then scrape about one inch down from the ends of the ribs between each bone, the skin, and fat; then put the bones to meet regularly, and put one between the other, which will form a chevaux-de-frise ; braise them the same as the former; when done, glaze the fat and meat but not the white rib bones: any of the sauces named or cucumbers.

\section{4-CHEVAUX-DE-FRISE WITH LAMB SWEETBREADS.}

Do the same as before ; when it is done and glazed, have ready some good lamb sweetbreads, about six larded ones and glazed, put them on the top between the bones, when the two necks are put together.

775.-BLANQUETTE D'AGNEAU À LA PROVENÇALE.

Of the best end of the brisket take 
two or three pounds, cut it into dice of four inches, rinse them in clear water, wipe them with a cloth, and flour them; take two ounces of the fat of boiled bacon, five of fresh butter, chop some parsley, and boil ten minutes; put in the lamb dice, cut up an onion small, squeeze in the juice of half a lemon, and season with pepper and salt; let it simmer gradually for two hours; beat up the yolk of three eggs, and add them to the gravy; keep the pan moving about the fire for three minutes: dish and serve.

\section{6.-LAMB CHOPS.}

Lamb chops and lamb cutlets are cooked in the same manner as mutton chops and cutlets, but require more care in the cooking; they are sent to table with various garnishes, and arranged in many forms, frequently accompanied with a purée of vegetables, or ranged round a pyramid of mashed potatoes; the most simple manner is to garnish with crisped parsley.

\section{7.-ANOTHER.}

Take a loin of lamb, cut chops from it half an inch thick, retaining the kidney in its place ; dip them into egg and breadcrumbs, fry and serve with fried parsley.

When chops are made from a breast of lamb, the red bone at the edge of the breast should be cut off, and the breast parboiled in water or broth, with a sliced carrot and two or three onions, before it is divided into cutlets, which is done by cutting between every second or third bone, and preparing them, in every respect, as the last.

If brown, season them with pepper, salt, nutmeg, grated lemon-peel, and chopped parsley; but dip them first into egg; fry them quickly. Thicken some gravy with a little flour and butter, and add to it a spoonful of port wine.

\section{8.-SWEETBREADS.}

Two good throat sweetbreads or three will make an entrée; blanch them until fit to eat, take them up and lay them in cold water; when cold dry them well, egg and bread-crumb them with or without herbs; put them on a dish and brown them in the oven; mushroom sauce, or endives, or sorrel or spinach, or tomato will do if approved of.

\section{9.-SWEETBREADS FRICASSEED, WHITE.}

Blanch, and cut them in slices. To a pint of veal gravy put a thickening of flour and butter, a table-spoonful of cream, half a teaspoonful of mushroom powder, grated lemon-peel and nutmeg, and white pepper, to flavor. Stew ten minutes, add the sweetbreads, let them simmer twenty minutes. Dish, add salt, thin pieces of lemon-peel; mix up, and serve.

780.-SWEETBREADS FRICASSEED, BROWN.

Cut them in small pieces, flour, and fry them. When a good brown, pour over them a pint of good beef gravy, highly seasoned; stew gently until the sweetbreads are tender. Add a litt'e flour and butter to thicken; add truffles and mushroom ketchup to flavor; morels or mushrooms may be substituted, or all may be cooked with the swcetbreads.

\section{1.-TO STEW SWEETBREADS-RIS DE VEAU.}

Make a forcemeat of the tenderest parts of boiled or roast fowl, some bacon, a little parsley chopped, a little thyme, lemon-peel, the rolks of two eggs, cayenne pepper, and nutmeg. Lay the sweetbreads in a pan, upon a layer of slices of veal, cover them with slices of bacon, put in a bunch of sweet herbs, an onion sliced, a little mace, and pepper 
and salt. Pour in a quart of good broth, and stew for two hours; remove them, and reduce by boiling the broth to a fourth: heat the sweetbreads in it, garnish with lemon in slices.

\section{2.-SWEETBREADS LARDED-À LA DAUBE.}

Blanch and lard them with bacon, put them into a stewpan with a pint of veal broth; add a little browning, with the juice of half a lemon. Stew until tender; thicken the gravy with a little flour and butter. Lay bunches of boiled celery round the dish when you serve.

\section{3.-PIEDS D' AGNEAU.}

Have six lambs' feet scalded, take out the shank-bone very carefully, so as 'not to cut or break the skin; soak for three hours in cold water; put them in a stewpan with cold water and the juice of two lemons ; let it boil for ten minutes, take out the feet, throw them into cold water, cut off the bones of the cleft part of the foot, and remove the curl of hair that lies in the cleft. Boil very gently for three or more hours in some poĉlée (which see), to keep them very white. Serve with asparagus, peas, and mushroom, or white cucumber sauce.

\section{4.-HEART SWEETBREADS LARDED WITH BACON OR TRUFFLES.}

The same quantity, either one larded and two plain, or two if fine larded with bacon; when blanched for two or three minutes, put them in a small dish or stewpan, with a little stock ; cover them over, and boil them either in the oven or over the fire for about twenty minutes; take them up and glaze them several times, keeping them hot; lamb sweetbreads will take eight for a dish, and require to be neatly trimmed, cutting away the pipe and dish them on a rim of forcemeat or mashed potatoes, or spinach.

\section{5.-SWEETBREADS AS CUTLETS.}

If you cannot get heart sweetbreads, you must use the throat. Blanch them for about ten minutes, then put them to cool into cold water; take them out and dry them in a cloth, then cut longways, twelve or fourteen pieces for cutlets, making them a nice shape; if you wish them to be white sauté cutlets, you must put some butter or lard in your cutlet-pan, a juice of lemon, a little white pepper, and salt; do nut color them, take them up and lay them upon white paper to soak up the grease from them; dish them round upon a tureen, pouring the sauce in the middle.

\section{6.-ROAST SWEETBREAD.}

Boil sweetbreads, either heart or throat, trim them and dry them, then egg and bread-crumb them, brown them before the fire or in the oven; put good clear gravy under them, and watercresses, as a garnish.

\section{7.-SWEETBREAD CUTLETS-CRUMBED AU GRATIN.}

Cut the swectbreads as before, a nice thickness, but not too thick; dry them, then egg and bread-crumb them as you would veal or other cutlets; use any sauce that may be preferred.

\section{8.-LAMB'S HEAD AND ÉMINCEES.}

Wash well a lamb's head and pluck, take out the brains, blanch them by themselves, boil the head and pluck for about a quarter of an hour, take it up to cool, take out the tongue, trim the two halves of the head neatly, then score it, then egg and bread-crumb them as you would cutlets, and brown them in the oven or before the fire. Cut up in small dice in equal quantities, the tongue. liver, heart, and lights; fry in a stewpan a little chopped parsley, shalot, and inushroom if you have it, to a nice light 
brown, dry up the butter with flour, use some good second stock or brown sauce; season with lemon, cayenne pepper, salt, and a dust of sugar; put the émincées under the head, the brains, egg, and bread-crumb in four pieces and put round.

\section{9.-SHEEP OR LAMBS' TROTTERS.}

Get a dozen or two of trotters. stew them for several hours, until all the bones will come from them; save this liquor; do not break the skin ; stuff them with good quenelles or forcemeat; return them again into the same stock, boil them about fifteen minutes, glaze them; soubise sauce or tomato sauce is good with them, or you may fry them with butter.

\section{PORK.}

The proportion of persons who are fond of pork to those who dislike it, are as a hundred to one, and yet it is falsely considered a vulgar taste. The passion for it possessed by the Chinese has been illustrated by many tales, and when in season, the frequency of its appearance upon a homely English table is no small proof of the estimation in which it is held. It should be thoroughly cooked to place its digestibility beyond a doubt. In roasting, or in boiling, ample time should be allowed for the joint. Pork is always salted for boiling, and is much liked in this form. When sent to table roasted, apple sauce should in every case accompany it.

It is universally used in every family, and little used for company.

The griskin and the sparerib are certainly improved by being powdered with chopped sage, but this is all a matter of taste. The skin of the leg and loin must be scored previous to roasting; but take care to preserve the skin, or crackling, of a fine brown, as many persons look upon it to be the best part of the pork ; it should therefore be well basted, and guarded from being burned by a sheet of oiled paper. Send both up with a glass of vinegar mixed with two teaspoonfuls of made mustard in the dish.

If you kill a young pig of four or five months, take off the skin of the fore-quarter, roast it, and serve with mint sauce and salad.

\section{0.-PORK GRISKIN}

Is usually very hard; the best way to prevent this is to put it into as much cold water as will cover it, and let it boil up; then instantly take it off, and put it into a Dutch oven; a very few minutes will do it. Remember to rub butter over it, and then flour it, before you put it to the fire. Lay it in a dish on melted butter and mustard. It should be seasoned with pepper and salt before roasting.

\section{1.-SPARERIB}

Should be basted with a very little butter and a little flour, and then sprinkled with dried sage crumbled.

\section{2.-TO ROAST A SUCKING PIG.}

A sucking pig, which should be about three weeks old, must be dressed as soon after being killed as practicable. When scalded and prepared for cooking, lay in the belly a stuffing of bread, sage and onions, pepper and salt, with a piece of butter; sew it up, rub the skin of the pig with butter, skewer the legs back, that while roasting, the inside as well as outside of the pig may be thoroughly browned; it must be put to a quick fire, but at such a distance as to roast gradually, and a coating of flour should be dredged over 
it that it may not blister, or it should not be left a minute ; if floured, when the pig is done, scrape the flour off with a wooden, or very blunt knife, and rub it with a buttered cloth; cut off the head, and dividing it take out the brains, mix them with a little gravy or bread sauce, civide the pig in half, from neck to tail, and lay each inside flat upon the dish, so that the two edges of the back touch; place each half of the head with the outer side uppermost at each end of the dish, and an ear on each side; the gravy should be poured in the dish hot, and the whole served as hot as possible; as a matter of convenience it is often sent to the baker's oven; a large piece of butter should accompany it for the baker to baste it with, and upon its return it should be cut and served as above.

The gravy may be heightened in its favor by various additions, or two or three sauces and gravies may be served with it, such as veal gravy thickened and flavored with wine, lemon-juice, and cayenne, and also bread sauce and a plain gravy; this may be a matter of taste.

It is usual to procure the pig from the butcher ready prepared for cooking, but in the event of its being required to scald it after killing, we subjoin the following receipt.

\section{3.-TO SCALD A SUCKING PIG.}

Plunge the pig into cold water the instant it is killed, let it remain five minutes, have ready pounded resin, and rub well with it over the skin, plunge it into a tub of scalding water, letting it remain only half a minute, remove it, and immediately take off the hair ; lose no time, if the hair should not come freely from some parts, rub it again with resin. and put it into the scalding water, and then remove the hair. When it is all off' wash it well with warm water, and then in cold, changing the water several times that no flavor of the resin may be retained; cut off the feet at the first joint, slit down the belly, and remove the entrails; put aside the heart liver and lights, with the feet, wash again inside and out the pig. dry it well, and keep it from the air by covering it with a cloth.

\section{4.-TO ROAST A PIG. (Philadelphia.)}

Take a pig not more than a month old, remove the eyes and tongue, trim the ears, cut off the feet, and clean it thoroughly. Wash and dry it; rub it well, inside and outside, with sage and seasoning of salt and cayenne pepper. Boil twenty good sized potatoes, mash them while hot, add butter, a little milk, and two minced onions and minced sweet herbs, with seasoning of salt and pepper, and bread-crumbs. Stuff the pig, and fasten the opening with skewers. Roast (or bake) it for three hours in a pan, with water sufficient to form the gravy, thickened with a little flour, and well stirred. Baste the pig, while roasting, with oil or butter, to make it brown; and, if necessary, add a little boiling water to the gravy. The taste of a pig roasted before the fire is preferable to one baked in an oven.

Make the sauce of the feet, tongue, liver and heart. The tongue must be scalded and skinned, then boiled with the feet, liver and heart; and when done, minced fine and seasoned, with the addition of an onion, parsley and sweet herbs. Boil all together; rub a little butter with flour, and stir it in with more seasoning if desired, adding a glass of wine when the sauce is taken up.

\section{5.-HIND-QUARTER OF SUCKING PIG. (Yorkshire Fashion.)}

Cut off the skin, cover with paper, and roast before a quick fire about three-quarters of an hour; ten minutes before being ready, remove the paper and baste it; serve with gravy under, and mint sauce and salad. 
796.-ROAST PIG.

Soak in milk some light bread, boil some sage and onions in plenty of water, strain it off and chop it all very fine, press the milk from the bread, and then mix the sage and onion with pepper and salt; in the bread put the yolk of an egg to bind it a little, put this in the inside of the pig, rub the pig over with milk and butter, paper it, roast it a beautiful brown, cut off the head before it is drawn from the spit, and likewise cut it down the back, and then you will not break the skin; take out the spit, cut off the ears from the head, and crack the bone and take out the brains, put them in a stewpan with all the inside stuffing and a little brown sauce; dish the pig, the backs outside, and put the sauce in the middle, and some in a boat, the ears at each end.

\section{7.-A LEG OF PORK ROASTED.}

The pork should be young and dairyfed; score the skin with a sharp penknife, a little fresh butter is sometimes rubbed over the skin to make it brown, and crisp without blistering. Chop some sage that has been scalded, very fine, add to it an onion parboiled, mix breadcrumbs and a small portion of apple chopped very fine, mix all together, season with pepper and salt, make an incision, separating the skin from the fat in the under and fillet end of the leg, and place the stuffing there; the time of roasting will depend upon the size of the leg; serve up with apple sauce.

\section{8. -A LEG OF PORK BOILED.}

After having been salted, it should be washed in clean cold water, and scraped thoroughly white and clean preparatory to cooking; it should then be put into a floured cloth and into cold waier on the fire; when the rind is quite tender the pork will be done; let the water be well skimmed, and serve with such vegetables as are in season; shoula the joint be large allow a quarter of an hour to each pound, with an additional twenty minutes from the time it boils.

\section{9.-LOIN OF PORK}

Should, like the leg, be scored before roasing, and well jointed to make the chops separate easily, and then roast as a loin of mutton; or it may be put into enough water to cover it, simmer until it is nearly done, then take it out, take the skin off, coat it well with yolk of egg and bread-crumbs, roast for about a quarter of an hour, until it is thoroughly done.

\section{0.-A FILLET OF PORK TO RESEMBLE VEAL.}

The fillet should be cut from the leg of a very large pig; remove the bone and fill the orifice with veal stuffing; roast it until it is more than half done, then take some thin broth and put it in the stewpan, put in the pork, stew until it is thoroughly done, then thicken the gravy, and send it to table with forcemeat balls and lemon cut in slices.

\section{1. -NECK OF PORK ROLLED.}

Bone it, chop sage finely, mix it with well powdered bread-crumbs, cover the meat with it on both sides, roll the pork, fasten it securely, and roast it gently.

A hand and spring are sometimes dressed thus.

\section{2.-SPARERIB OF PORK-ROAST.}

You must paper and joint this down the middle, and sprinkle it with a little fine sage and salt; baste and flour it well; apple sauce in a boat.

\section{3.-SPARERIB.}

A sparerib will take two hours and a half to roast unless very large, and then 
three hours will be required to cook it ' water to make it white: allow a quarter thoroughly; while roasting baste with। of an hour for every pound, and half butter and dredge with flour; pound an hour over. from the time it boils up: some sage and powder the sparerib with skim it as soon as it boils, and frequentit about twenty minutes before it is done; ly after, but do not boil it fast, or it will a pinch of salt may be added.

\section{4.-CHINE OF PORK.}

This joint is usually sent to table with turkey; it should be salted for about sixty or seventy hours previous to cooking, and then be roasted; it is usually prepared so.

\section{805.-LEG OF PORK.}

To Bake.-Rub it well over with salt and saltpetre mixed; let it lie five or six days in the brine; then hang it up to smoke for five or six days. Take off the skin, put it into an earthen dish, and pour a little red wine over it; stick a few cloves in it, or beat them to powder, and rub them over it. When it has been in the oven a short time, take some hard biscuit, pounded with sugar, and spread it all over. Serve it up with gravy, and port wine sauce.

To Roast.-Choose a small leg of fine young pork: cut a slit in the knuckle with a sharp knife, and fill the space with sage and onion chopped, and a little pepper and salt. Score the skin in slices, but do not cut deeper than the outer rind. Put it at first a good distance from the fire, but baste it frequently to prevent its scorching: then, when about three parts done, rub the skin rather firmly with raw butter, after which flour it lightly, and put close to the fire to crisp the crackling. Apple sauce should be served with it.

To Boil.-Salt it eight or ten days, turning it daily, but do not rub it after the first. When it is to be dressed weigh it: let it lie half an hour in cold some of the water to make pea-soup. Some boil it in a cloth, floured, which gives a very delicate look, but destroys the value of the liquor for making soup.

\section{5.-PORK CUTLETS.}

Perhaps few things of a simple nature, and served in a plain way, are better than a hot pork chop, cut about half an inch thick, trimmod neatly, and broiled upon the gridiron.

Or:-Fry in salad-oil; serve with sauce Robert or gherkin sauce, the gherkins being shred fine into some good thick brown gravy.

Or:-Marinade the cutlets four hours in oil with an onion in slices, parsley, bay-leaf, pepper, and salt ; fry them in the marinade; serve with tomato sauce.

\section{7.-MOCK GOOSE, OR LEG OF PORK ROASTED WITHOUT THE SKIN.}

Parboil it, and take off the skin; then put it down to roast; baste it with butter, and make a savory powder of minced or dried sage, ground black pepper, salt, and fine bread-crumbs; add a little very finely minced onion: sprinkle the leg with this when almost roasted; put half a pint of made gravy into the dish, and garnish the dish with balls of this dressing fried or broiled.-(Recommended by a Lady of New York.)

\section{8.-CHINE OF PORK}

Generally used at Christmas. This, when properly cured, is mostly used cold; boil it in a cloth, with a sauce of red cabbage, or sauer kraut if cold; garnish with parsley. 


\section{9.-ALL ROAST PORKS.}

Be sure to cut the skin lengthways into small strips, not very deep, to reach the meat; send up apple sauce to all roast porks.

\section{0.-TO PICKLE PORK.}

Dredge it with salt, pounded nearly as fine as flour ; place upon four sticks crossed upon a dry cold flag-stone, or in an earthenware dish ; let it remain to drain from eighteen to twenty-four hours, then rub it well in with a brine, consisting of one pound of salt, half a pound of coarse brown sugar, two ounces of saltpetre, and a quarter of an ounce of salt prunel; the last, if the pork is delicate, may be omitted. If many pieces are being salted, put them into a tub and pack them closely, filling up the interstices with common salt, place a weight upon the top to keep the meat down, as well as to prevent the admittance of any air, and when taken out for cooking, scrape off the salt, wash the pork in several waters, or place it under a water tap, letting the water run upon it two or three minutes, turning it occasionally, or it may lie in soak half an hour; it should be put to boil in cold water, and when the rind is tender it will be done enough.

\section{1.-BOILED PORK OF ALL KINDS.}

The leg you must skin the same as ham, and dish its back part upwards, and glaze it ; place a ruffle at the knuckle; sauce, sauer kraut, or stewed red cabbage; peas pudding to all pork when boiled.

\section{2.-PIG'S CHEEK-A HALF ONE.}

Boil and trim in the shape of ham, and if very fat, carve it as a cockle shell; glaze it well, or put bread-crumbs and brown them: sauce as before.
813.-PIG'S CHEEK. (A new method.)

Procure a pig's cheek nicely pickled; boil well until it feels very tender, tie half a pint of split peas in a cloth, and put them into a stewpan of boiling water, boil about half an hour, take them out, pass through a hair sieve, put them into a stewpan with an ounce of butter, a little pepper and salt, and four eggs, stir them over the fire until the eggs are partially set, then spread it over the pig's cheek, egg with a paste-brush, sprinkle bread-crumbs over, place in the oven ten minutes, brown it with the salamander, and serve.

\section{4:-PIG'S FACE FOR BREAKFAST.}

Cut the ears off, and take out the tongue and brains, then lay them in sait for two days, take them out of that, and then use the ingredients as for tongues. and dry them the same as hams.

\section{5.-PORK CUTLETS.}

Cut from a neck, or what is called foreloin of pork, the best end, trim it as you do lamb or mutton, leaving a little fat; scrape the bone, rub or chop some sage fine, with a very little piece of shalot, mix it up with only sufficient breadcrumbs, put black pepper and salt into the crumbs, herbs, dish each cutlet in clarified butter or melted lard, press the crumbs well upon the cutlets, have a sautépan greased with lard, lay them into it, fry them a nice light brown, take them up and dry them on paper, dish upon mashed potatoes, use sauce Robert, or soubise, or tomato, or any other to taste.

\section{6.-PORK CUTLETS.}

Cut them from a small delicate loin of pork, bone, and trim them neatly, fry them a light brown, put into a small stewpan a little vinegar, and eschalot chopped very finely, two table-spoonfuls 
of tomato sauce, and sufficient brown gravy to make it tasty; stew the cutlets in the sauce five minutes, and send them to table dished handsomely; if the cutlets are broiled they may be dipped in yolk of egg and bread-crumbs, and broiled over a clear fire, and served with tomato sauce, or sauce Robert.

\section{7.-PORK CHOPS OR STEAKS.}

Cut from the best end of the loin, or from the chump or leg if steaks; remove the fat and skin, turn them frequently and quickly while broiling; if your gridiron be of the old fashion, it is better to keep it aslant on the fire, the handle being the lowest part, it prevents very much of the fat from falling into the fire, the flare of which is apt to impart a disagreeable flavor to the chops ; this observation applies also to mutton chops. and will be found useful if followed: sprinkle them with salt when nearly done, and rub with a little fresh butter previous to serving; if for a side dish garnish with crisped parsley.

\section{8.-PORK SAUSAGES.}

There are many receipts for the making of pork sausages. The peculiarity in salssages being the quantity and variety of herbs introduced, the prevalence of some particular one gives the flavor, as well as the peculiarity to each. The presence of so many herbs is, however, not always considered an agreeable feature, and many palates are offended at that which forms to others the great merit: The following is a very simple receipt.

Take of the fat of pork one pound, that of the loin of a large richly fed pig, or the inward fat of a small one. Chop it finely with half a pound of lean pork; add to it four or five sage-leaves finely chopped, some lemon thyme in a small quantity, and three dessert-spoonfuls of crumb of bread powdered; be careful not to put too much of the latter, as it tends to turn the sausages sour if kept. Amalgamate these ingredients well, dust on grated nutmeg, mace, and cloves in powder, and finish witl black pepper and salt, being sure to season well; the meat may then be put into the skins, or may be put in jars covered down from the air, to be used for rolls or stuffing, or any required purpose.

All skin must be pared from the fat before chopping, and every sinew removed from the lean pork, as well as any bone, or any thing which may impair the taste when eaten.

$$
\text { 819.-SAUSAGES. (Mrs. Ellis.) }
$$

Chop fresh pork very fine, the lean and fat together. Then fill the skins, which should be previously cleaned thoroughly. A little flour mixed in with the meat, tends to prevent the fat from running out when cooked. Sausage-meat is good done up in small cakes and fried. In summer, when fresh pork cannot be procured, very good sausage-cakes may be made of raw beef, chopped fine with salt pork, and seasoned with pepper and sage. When sausages are fried, they should not be pricked, and they will cook nicer to have a little fat put in the frvingpan with them. They should be cooked slowly. If you do not like them very fat, take them out of the pan when nearly done, and finish cooking them on a gridiron. Bologna sausages are made of equal weight each, of ham, veal, and pork, chopped very fine, seasoned high, and boiled in casings till tender, then dried.

\section{0.-THE UNIVERSITY RECEIPT FOR PORK SAUSAGES.}

To a couple of pounds of lean pork, young, white, and delicate, put three quarters of a pound of minced beef suet; the pork must first be chopped very fine; add three dessert-spoonfuls of bread which has been dipped in port wine, 
dried and grated fine; work it together with the yolks of three eggs smoothly beaten: season it with pepper and salt. and dried sage; a very little cayenne may be introduced, and a very small piece of garlic. W ork the whole well together in a mortar until it forms a paste ; it may then be put into wide skins, or pressed down into jars for future use. It is cut into square pieces, dredged with flour, fried in fresh butter, and sent to table on a toast as a breakiast dish.

\section{1.-SAUCISSES À LESPAGNOLE.}

Cut into chops the loin of a good sized pig, bone it, and cut the meat into dice. keeping the fat and lean separate, three quarters of an inch square. Chop two corns of garlic fine, add to it black pepper, Spanish pepper, and salt ; mix it well together, and season the meat with it: pour over it half a pint of an acid wine, and when it has been drawn in by the meat, add more, until the expiration of a week; then lay the meat in skins, alternately fat and lean, and if any moisture appears to be required give a little more wine. Twist the skin, and tie it in the lengths you require, and keep them in a cool dry place. They may be boiled, fried, broiled, grilled, toasted, served with poultry or veal, or may be eaten with bread alone.

\section{2.-PORK SAUSAGES.}

Chop, particularly fine, about two or three pounds of lean pork, and an equal quantity of fat; have ready some sage, either dry or green, either passed through a sieve or chopped very fine, a small piece of shalot, a few grains of ground cloves, season it with pepper and salt, mix a few fine bread-crumbs up with it, have your skins ready cleaned; then fill them, or if preferred roll into balls and fry them; you will tie them the length you wish the sausages to be, prick the skins with a fork before you fry them; you may do them in the oven if it should be hot.

\section{3.-ANOTHER WAY.}

Chop the pork as before, only add half the quantity of lean veal, a pound of suet chopped equally fine, have ready a French roll soaked in milk but no crust, season it well with pepper and salt, mix it all well together.

\section{4.-LIVER PUDDING}

Boil pigs' livers, mince, and season them with pepper, salt, and chopped sweet marjoram and sage, adding cloves. Put them in skins, prick them, and boil slowly an hour or so. Keep them in covered jars, to eat cold in slices, or to fry in larger pieces. Boiled pigs' feet may be mixed with the livers.

\section{5.-BOLOGNA SAUSAGES.}

To ten pounds of beef take four of pork; chop and mix it, season well with six ounces of salt, and some black and cayenne pepper, cloves powdered, and chopped garlic. Put the mixture into beef skins, tie them, and put them into brine strong enough to bear an egg, for three weeks, turning them every day. Then dry and hang them up to smoke.

\section{6. -HEAD CHEESE.}

Boil the head, tongues, and feet of freshly-killed pigs; taking the skin from the head first. When tender, chop the meat, season it with salt, pepper, cloves, minced sweet herbs, and mix thoroughly. Put the mixture into deep pans, press it down, cover it with an inverted plate, and place a heavy weight on the bottom of the plate. In three days take it out, and serve in slices, to be eaten with mustard and vinegar, at breakfast or supper.

\section{7.-A DIFFERENT TO A DIFFERENT PALATE.}

Chop pork as before, and an equal quantity of fat, and the quantity of lean 
veal, and the same of suet, and two or three handfuls of fine bread-crumbs; have ready a few sage-leaves, a few of knotted marjoram and one shalot; pound all well together, season with white pepper and salt. either put them in skins or roll them, and fry them as above.

\section{8.-SPICED SAUSAGES.}

Rub well into a piece of pork some saltpetre, allspice, and black pepper, let it lie several days, rubbing it each day, then chop it small, and add to it two shalots chopped fine, have ready cleaned well an ox-gut, fill it with this meat quite tight, tie both ends firm, let it be smoked as hams, wrap it in a thin cloth, then let it be well dried; you may tie it into what lengths you please before smoking; this will eat hot or cold.

\section{9.-WHITE SAUSAGES.}

Have ready some well-dried oat-meal, two or three onions to boil in milk, chop them very fine, chop two pounds of suet very fine, mix about a pint of oatmeal to the suet, add the onions, season all with pepper and salt, rather predominant with pepper and onions, filling the skins as for former sausages ; if approved of add a few currants and boiled rice in milk to them.

You may make sausages of any dressed meats, either chicken or rabbits; chop up the meat very fine, adding onions and seasonings as in the above, with chopped parsley and a few grains of pounded mace ; add some bacon chopped instead of suet, mix all together with two yolks of eggs, a few bread crumbs and a few drops of lemon-juice; fill this in the skins as before, and broil or fry them.

830.-SAUCISSES AUX CHATAIGNES.-STEWED SAUSAGE MEAT WITH CHESTNUTS.

Take twenty or thirty sound chestnuts, roast them over a slow fire, and when sufficiently roasted to remove the husk take them off, peel them, removing the inner skin as well as the husk, and put them aside sufficiently near the fire not to cool too readily. Cut into diamonds half a dozen thin slices of sausage meat, and fry them brown in a little fresh butter. When they are a good color, take them out, and pour three parts of the butter in which they have been fried into a small well-tinned or earthenware saucepan. Thicken it while heating with a spoonful of - flour, and pour in gradually a pint of good gravy, with a glass of old brown sherry, or two of Madeira. Put in a fagot of herbs, and season to palate, a little cayenne may accompany the common pepper. As soon as it boils, lay the sausage cakes round the saucepan close to the sides, leaving the centre clear, and in this space put the chestnuts. Let them stew slowly three quarters of an hour; then dish them, arranging the sausage meat and chestnuts in the same manner as in the stewpan; pour the gravy over them, removing the fagot of herbs first, and serve.

831.-TO COLLAR A PIG'S HEAD.

Clean thoroughly, and put it in pickle for a week, boil it tender, then take out the bones carefully, turn the upper part of one cheek to the snout of the other cheek, season them with pepper and salt, roll it lightly in a cloth, and secure it, then boil until it is very tender. Do not remove the cloth when done, but place it upon an earthenware dish, lay a heavy weight upon it, and unbind when cold ; if the cheek is a large and fat one, it will be improved by laying a slice or two of lean pork, or ham, between the cheeks before binding them in the cloth.

\section{2.-PIG'S HEAD BAKED.}

Let it be divided, and thoroughly cleaned; take out the brains, trim the snout and ears, bake it an hour and a half, wash the brains thoroughly, blanch 
them, beat them up with an egg, pepper, and salt, and some finely chopped or pounded sage, and a small piece of butter; fry them or brown them before the fire; serve with the head.

Pig's head is profitable to buy. It is often despised because cheap; but well cooked, it is delicious. Well cleaned, the tip of the snout chopped off, and put in brine a week, it is in order for boiling. The cheeks are better than any other pieces of pork to bake. The head is good baked an hour and a half, and yields abundance of sweet fat for shortening. The butchers of Philadelphia make what they call scrapple out of pig's cheek, boiled and chopped very fine. The water in which this meat has been boiled is thickened with buckwheat and Indian meal boiled in it till sufficiently done. The meat is added. When cold, it forms cakes, which are sold in market-and fried in slices for breakfast by the purchaser.

\section{3.-PIG'S HEAD BOILED.}

This is the most profitable dish, though not so pleasant to the palate; it should first be salted, which is usually done by the pork butcher; it should be boiled an hour and a quarter, it must boil gently or the meat will be hard; serve with vegetables.

\section{4.-PETTITOES.}

${ }^{2}$ Put them in just sufficient water to cover them, add the heart and liver, boil them ten minutes, take out the liver and heart, and mince them small, and return them to the feet, stew until quite tender, thicken with flour and butter, season with pepper and salt, serve up with sippets of plain bread, or toasted bread; make a pyramid of the minced heart and liver, and lay the feet round them.

When pettitoes are fried, they should be first boiled, then dipped in butter, and fried a light brown.
835.-PIGS' FEET À LA ST. MÉNǴHOULD.

Clean the feet perfectly, cut them down in two, then bind them with a tape to prevent their shrinking or opening, and boil them gently until they are rather soft, with carrots, onions, parsley, thyme, allspice, and bay-leaf; let them cool in the liquor. When required for use, take off the tape, dip them in yolks of eggs and butter about three parts melted; season them, then cover them with crumbs of bread and finely minced parsley, with the smallest quantity of shalot. Broil them until highly browned, and serve dry, or with sauce Robert.

\section{6.-SOUSt.}

Take pigs' feet, ears, \&c., well cleaned, simmer four or five hours, till they are too tender to be taken out with a fork. Take from the boiling water and put them into cold water. Boil the jellylike liquor in which they were cooked, with an equal quantity of vinegar; add cloves, allspice, cinnamon, and sufficient salt to the scuse, pack it down tight, and put the liquor over it.

\section{7.-SOUSE}

Take pigs' ears and feet, clean them thoroughly, then soak them in salt and water, for several days. Boil them tender and split them, they are then good fried. If you wish to souse them when cold, turn boiling vinegar on them, spice with peppercorns and mace. Cloves improve the taste, but turn them a dark color. Add a little salt. They will keep good pickled five or six weeks. Fry them in lard.

\section{8.-PIGS' FEET STEWED.}

Clean, split, and boil tender, put them into a stewpan with enough gravy to cover them, an onion sliced, a few sage leaves, whole black pepper, allspice, and salt; stew forty minutes, strain off the 
gravy, thicken with flour and butter, add two spoonfuls of vinegar or one dessertspoonful of lemon pickle; serve it up with the feet.

\section{9.-PIGS' FEET AND EARS FRIED.}

They must be well cleaned, and boiled until tender, and laid in vinegar and water, with salt in it, until they are required for use ; to prepare them for cooking, cut the feet in two, slice the ears, dip them in butter, dredge with flour, fry a nice color, and serve with melted butter and lemon pickle.

\section{0.-PIGS' HARSLET}

Is made with the liver and sweetbreads, which must be well cleaned; add to them pieces of pork both fat and lean, chop finely sage and onions, season with pepper and salt, and mix with the preceding; put them in a cowl, tie it closely, and roast. It may also be baked. Serre with a sauce of port wine and water and mustard, just boiled up, and put it into the dish.

\section{1.-BAKED FAGOTS.}

Having procured your pig's-fry, (the quantity to be regulated by the size of family,) wash and set it on the fire, in a saucepan, with just sufficient water to cover; add a bunch of sage, and four or five onions; let all boil ten minutes; take out the meat, and cut in slices; then take out the sage and onions, and chop it all finely together; season with pepper and salt; cut the caul in pieces, and fill with the meat about the size of an ordinary teacup, place them on a tin and bake in a moderate oren; do not throw away the water it was boiled in, but boil it down to a sufficient quantity to serve with the fagots as gravy.

\section{2.-WHITE PUDDINGS}

Are made with beef suet and oatmeal, flavored and seasoned. Take a pound and a half of beef suet, chop it very fine, and, having boiled a pound of oatmeal, tightly wedged down in a small white basin closely covered with a cloth for five hours, scrape it into powder, and mix it with the suet, two small onions boiled and chopped fine, and season well with white pepper and salt; a small quan tity of thyme and marjoram may be added at pleasure. Boil them an hour. Like all sausages they must be pricked while cooking, to suffer the hot air generated to escape, or they will burst.

\section{3.-BLACK PUDDINGS.}

Stir three quarts of sheep's blood with one spoonful of salt till cold, boil a quart of grits in sufficient water to swell them, drain, and add them to the blood with a pound of suet, a little pounded nutmeg, some mace, cloves, and allspice ; a pound of the hog's fat cut small, some parsley finely minced, sage, sweet herbs, a pint of bread-crumbs, salt, and pepper; mix these ingredients well together, put them into well cleaned skins, tie them in links, and prick the skins, that while boiling they may not burst. Let them boil twenty minutes, and cover them with clean straw until they are cold.

\section{4.-BLACK AND WHITE PUDDINGS}

Procure the pig's blood, then add half a pound of half-boiled rice, set it to cool keeping it stirred, take a little more rice boiled in milk, add it to the blood, cut up about one pound of fat pork into large dice, melt half a pound of lard and pour into the blood and rice, then add your fat, with a few bread-crumbs, three shalots, a little parsley, some black pepper, cayenne pepper, and salt; mix all well together, then fill into skins as before; tie them the length you wish them, then boil them a quarter of an hour, take 
them out and lay them on some new clean straw until cold, then give them another boil for a few minutes, then turn them as before until wanted; put them in the oven when you require them, or fry them or broil them.

\section{5.-TO MELT LARD.}

Take the inner fat of a newly killed pig and strip off the skin completely and carefully, slice it and put it into a jar, a sprig of rosemary may be placed with it, and set the jar in a pan of boiling water; let it melt, and when perfectly fluid pour it into dry clean jars, and cover them closely; it may be kept some time in a dry place, and when used may be mixed with butter for pastry, for frying fish, and many other purposes in cooking.

\section{6.-HOG'S LARD}

Should be carefully melted in a jar put into a kettle of water and boiled: run it into bladders that have been extremely well cleaned. The smaller they are the better the lard keeps, as, after the air reaches it, it becomes rank. Put in a sprig of rosemary when melting.

This being a most useful article for frying fish, it should be prepared with care. Mixed with butter, it makes fine crust for tarts.

\section{7.-HAMS-BOILED HAM.}

The ham should be soaked about twelve hours, then wrapped in a clean cloth, and laid upon stone flags for two days, the cloth being kept moistened with c'ean soft water; this will render it tender when cooked; let it be thoroughly scraped and cleaned, and placed in the kettle, which in small families will be found the most convenient mode of cooking it; they should be put in sufficient water to cover them, which water, when the ham is cooked, will be found of the greatest service in making stocks for soups; the time it will require to boil will depend upon the weight of the ham: a small one three hours and a half, which may progress according to the weight to six hours; when it is done remove the skin, if possible, without breaking it, it prevents the ham when cold becoming dry; spread over the ham bread-raspings, the dish should be garnished with sliced boiled carrots.

\section{8.-TO COOK A HAM}

An excellent manner of cooking a ham is the following: Boil it three or four hours. according to size; then skin the whole and fit it for the table; then set in the oven for half an hour, cover it thickly with pounded rusk or breadcrumbs, and set back for half an hour longer. Boiled ham is always improved by setting it in an oven for nearly an hour, until much of the fat dries out, and it also makes it more tender.

849.-HAM, TO BOIL IN A SUPERIOR WAY.

Parboil the ham, then allow it to remain in the water all night, and finish boiling the next day, so as to be in time for dishing up; skim, and dust with raspings, the same as directed before, and you will have a-more tender ham than one dressed almost any other way.

\section{0.-ECONOMICAL DISH}

Cut some pretty fat ham or bacon into slices, and fry of a nice brown; lay them aside to keep warm; then mix equal quantities of potatoes and cabbage, bruised well together, and fry them in the fat left from the ham. Place the mixture at the bottom, and lay the slices of bacon on the top. Cauliflower or broccoli, substituted for cabbage is truly delicious, and, to any one possessing a garden, quite easily procured-as 
those newly blown will do. The dish must be well seasoned with pepper.

\section{1.-TO CURE A HAM.}

For a ham of sixteen pounds, take a pound and a half of common salt, with an ounce of saltpetre. Leave it some days, then put in one pound of molasses; let the ham lie a month in the pickle; then hang it up.

\section{2.-PRACTICAL HOUSEWIFE'S CHRIST- MAS HAM.}

Soak the ham. be the weight whatever it may, half the usual time in water; remove, wash well with cold water, place in a pan large and deep enough to contain it, cover with beer or good ale, and let it remain until the required time for soaking a ham of the size used has expired. Boil as usual until the skin can be readily removed; then place the ham in a tin or an earthenware dish, and cover with a common flour and water paste, or surround with batter. Bake in a moderately heated oven until done, remove the paste or batter, cover with bread-raspings, and serve hot.

Cooked in this manner, a ham acquires the most delicious flavor.

$$
\text { 853.-HAMS. }
$$

Skimmed milk, or milk and water, will be found preferable to plain water for soaking hams; and they may álso be boiled in milk and water.

Where vegetables are plentiful, it is desirable to boil ham with three heads of celery, a couple of turnips, half a dozen small onions, and a large bunch of sweet marjoram, thyme, \&c., put in after the pot has been skimmed. These will extract the salt and soften the meat. A piece of coarse fresh beef, or any kind of meat, in addition, will materially improve the ham, as, in this manner of boiling it, the juices of the meat and regetables insinuate themselves between the fibres of the ham after having dislodged the salt, by which means the meat is enriched and rendered tender. At first sight this will appear an extravagant way of boiling a ham, but it should be considered that the broth will serve the purposes of the family." If the meat and vegetables are not approved, two pounds of fresh beef dripping will answer nearly as well.

\section{4.-TO BOIL.}

If long hung, soak it for twenty-four hours in lukewarm water, changing it frequently. Wash and brush it well: trim off any rusty parts; put it into a boiler of water, and let it simmer from three hours and a half to five hours, according to its size. It is best to allow time enough, as it is easy to take up the ham when done, and keep it hot over boiling water, covered closely. Take off the skin as whole as you can, as it keeps the cold ham moist when skewered on again after dinner.

There can be no doubt that the foregoing is an excellent mode of boiting a ham, but many people prefer to have it baked, in which case send it to the oven in a deep pan, with half a pound of suet over it to baste it occasionally; or skin it, cover it with a crust of coarse paste, and, when taken from the bakehouse, strip off the crust, rub it with yolk of egg, on which put finely powdered crumbs of bread strewed with a dredging-box, in the same manner as when the ham is boiled.

In Spain and Portugal, where the hams are generally fine, the method of dressing them is to put the ham in cold water with a large handful of brown sugar, to which some persons add a quantity of the common country wine, and let it simmer on the fire until parboiled, keeping the pot well skimmed; when taken out, the skin is stripped off, and the ham covered with brown sugar to the thickness of an inch, 
after which it is sent to the oven and baked until thoroughly done.

A ham of fifteen pounds should be allowed nearly two hours before the water begins to boil, and as much more for simmering until half done; then from three to four hours' baking, according to the heat of the oven, which however should never be allowed to be too hot.

When cold, twist some neatly fringed letter-paper round the shank-bone.

$$
\text { 855.-TO ROAST. }
$$

Take a very fine ham (a Westphalia, if you can procure it), soak it in lukewarm water for a day or two, changing the water frequently. The day before you intend to cook it, take the ham out of the water, and, having removed the skin, trim it nicely, and pour over it a bottle of an inferior white wine; let it steep till next morning, frequently during the day washing the wine over it; put it in a cradlespit in time to allow at least six hours for slowly roasting it; baste with hot water continually. When done, dredge it with fine bread raspings, and brown before the fire.

\section{6.-SPRING, OR FOREHAND OF PORK}

Cut out the bone; sprinkle salt, pepper, and sage dried, over the inside; but first warm a little butter to baste it, and "then flour it; roll the pork tight, and tie it; then roast by a hanging jack. About two hours will do it.

\section{- 857.-HAM GRAVY.}

Take the wine in which the ham was steeped, and add to it the essence or juice which flowed from the meat when taken from the spit; squeeze in the juice of two lemons ; put it into a saucepan, and boil and skim it; send it to table in a boat. Cover the shank of the ham (which should have been sawed short) with bunches of double parsley, and ornament it with any garnish you may think proper.
858.-TO BOIL MUTTON HAM.

Put it on the fire in cold water ; let it warm quickly, and allow. it to boil only twenty minutes; then leave it in the pan of water till cold. This plan renders it moist, and answers when it is to be eaten cold.

\section{9.-HAM AND EGGS FRIED.}

Cut some nice slices of ham, put them in a frying-pan; cover them with hot water, and set the pan over the fire. Let it boil up once or twice; then take out the slices and throw out the water; put a bit of lard in the pan; dip the slices in wheat flour or finely rolled crackers, and when the fat is hot, put them into the pan; sprinkle a little pepper over; when both sides are a fine brown, take them on a steak dish; put a little boiling water into the pan. and put it in the dish with the meat. Now put a bit of lard the size of a large egg into the pan; add a salt-spoonful to it; let it become hot; break six or eight eggs carefully into a bowl; then slip them into the hot lard; set the pan over a gentle fire. When the white begins to set, pass a knife-blade, so as to divide an equal quantity of white to each yolk ; cut it entirely through to the pan, that they may cook the more quickly. When done, take each one up with a skimmer spoon, and lay them in a chain around the edge of the meat on the dish. Fried eggs should not be turned in the pan.

Spinach boiled and pressed free from water, and chopped small, may be put on the centre of a steak dish; lay the fried ham on it; pour the gravy over; place the fried eggs around it. Vinegar may be eaten with the spinach.

\section{0.-POACHED EGGS WITH FRIED HAM.}

Fry the ham as above directed; take a clean frying or omelette-pan; nearly fill it with boiling water; set it over a gentle fire; break the eggs singly into a cup, and 
slip each into the boiling water; cover the pan for four or five minutes. When done. take them up with a skimmer on to a dish; sprinkle a little pepper and salt over ; add a small bit of butter, and serve in a dish or over the ham.

\section{1.-TO CURE HAMS.}

[The lady from whom this receipt is procured, prides herself much on her curing; and often when asked her opinion of a ham at the houses of her friendsspeaking well of what is before her, will add, "It is not like hams of my own curing, of course ; but very nice," \&c.]EDIToR.

One ounce of saltpetre, one ounce of allspice, one ounce of cayenne pepper, one pound of brown sugar, one pound of salt, for every two hams; mix and rub well over the hams for three or four days, laying them with the skin down in the tub in which you mean to keep them. Then make a pickle strong enough to bear an egg, of cold water and salt: add to the pickle about a pound of sugar, and one ounce of saltpetre; if for sixteen hams, less in proportion. After lying three weeks in pickle the hams will be fit to smoke. After the hams come out, I always put in the pickle some plate pieces, rumps and rounds of beef; so that nothing is wasted.

\section{2.-TO CURE HAMS}

Pound some rock salt, saltpetre, and common salt, and some coarse brown sugar, mix it all well together, then put it all to get hot, and while hot rub the hams well with this, repeating it every morning for a week; let them lie in the brine for another week, until all is well incorporated in the meat; then take them out to drain on dishes, flour them and hang them up to dry; you must be guided a good deal by the size of the hams. The Virginia method of curing hams, ing it; if an old one, two hours will be (which is considered very superior,) is to dissolve two ounces of saltpetre, two teaspoonfuls of saleratus, in a salt pickle, as strong as possible; for every sixteen pounds of ham, add molasses in the proportion of one gallon to a hogshead of water, then put in the hams and let them remain three or four weeks. Then take them out of the brine and smoke them with the hocks downward, to preserve the juices. They will smoke tolerably well in the course of a month; but they will be much better to remain in the smoke-house two or three months. Hams cured in this manner are well flavored, and will keep a long time.

\section{3.-TO RESTORE PORK.}

In warm weather, the brine on pork frequently becomes sour and the pork tainted. Boil the brine, skim it well, and pour it back on the meat boiling hot. This will restore it even when it is much injured.

\section{4.-TONGUES}

You will first lay in salt; then use the same hot preparations daily; about ten days will do for the tongues; sheep's tongues may be done the same, but less time.

\section{5.-ANOTHER RECEIPT TO CURE HAM.}

Let the ham hang for three days, sprinkle it well during the time with salt. Make a pickle of eight ounces of rock salt, an equal quantity of common salt, two ounces of saltpetre, the same of black pepper, half a pound of common brown sugar, and a quart of beer; boil it, pour hot upon the ham, turn it every day in the pickle for three weeks, wipe it as dry as possible, and bury it in bran. Smoke it for a month over wood smoke; it must be sewed in a coarse strong wrapper.

Put the ham in soak previous to dress-

$$
\text { 866.-TO BAKE A HAM. }
$$


required, but if not very old, an hour will / wood is preferable to the smoke of coal. suffice. Wipe it very dry, and cover it with a paste about an inch in thickness. The edges being first moistened must be drawn together and made to adhere, or the gravy will escape. Bake it in a regular well heated oven; it will take from three to six hours, according to its weight; when done remore the paste, and then the skin. This must be done while the ham is hot. If well baked and not too salt, it will prove of finer flavor than if boiled.

\section{7.-TO MAKE HAM SUPERIOR TO WEST- PHALIA. (Ude.)}

As soon as the pig is cold enough to be cut up, take the two hams, and cut out the round bone, so as to have the ham not too thick: rub them with common salt, and leave them in a large pan for three days; when the salt has drawn out all the blood, throw the brine away, and proceed as follows: for two hams of about eighteen pounds each, take one pound of moist sugar, one pound of common salt, and two ounces of saltpetre, mix them together, and rub the hams well with it, then put them into a vessel large enough to contain them in the liquor, always keeping the salt over them; after they have been in this state three days, throw over them a bottle of good vinegar. One month is requisite to cure them; during which period they must be often turned in the brine; when you take them out, drain them well, powder them with some coarse flour, and hang them in a dry place. The same brine will serve again, except that you must not put so much salt on the next hams that you pickle. If the hams are smaller. put only three-quarters of a pound of salt, but the salt will not do any harm if you do not let them remain too long in the brine; if you can get them smoked, they are then not so subject to be infested by vermin; no insect whatever can bear the bitterness of the soot; the smoke of
Be particular that the hams are hung as far as possible from the fire, otherwise the fat will melt, and they will become dry and hard and rank.

\section{8.-TO BRAISE A HAM IN THE FRENCH FASHION.}

It is prepared for cooking in the same manner as in the preceding receipt, but when cleaned it is placed upon a layer of new hay, which has previously been laid evenly upon a clean white cloth, which should also be thin, that the flavor of the braise may be imparted. It is then placed in a stewpan, with two parts water to one part vin ordinaire, or any light white wine, and suflered to come to a boil. The scum must be removed, and then vegetables added; four carrots. three onions, a fagot of herbs, and, if approved, a little corn of garlic perhaps less of that powerfully flavored root. Simmer from three hours and a half to six, according to the weight; when tender it is enough. 'The skin should then be stripped off carefully, and bread raspings strewed over it. Powdered herbs, or parsley chopped very fine, are sometimes mixed with the raspings, but taste must regulate its admission or onission.

\section{9.-TO BRAISE A HAM}

Put the ham into water the night previous to cuoking, and next day wash it in warm water, trim it by cutting áway all the yellow fat and rusty parts ; take off the knuckle, and pare down all the under part; put it in a stewpan, and just cover it with water ; lay in a slice of beef cut into pieces, a fow onions, a fagot of sweet herbs, three small carrots, and a little allspice; simmer from three to six hours; it must depend entirely upon the size and weight. Take out the ham, and skin it; glaze, and serve it upon a purée of vegetables. The braise may be made into a rich brown soup, thickened and 
flavored with wine; it may serve also for the flavoring of soups.

\section{0.-HAM RASHERS OR SLICES}

May be toasted, broiled, or fried. May be served with spinach and poached eggs. or boiled green peas. Stewed with green peas, or cut in thin slices, divided in four pieces, each piece rolled and fastened with a skewer, roasted in a Dutch oven, and served with peas. They should in all cases be cut an even thickness, and cooked without injuring the color. Bacon may be dressed in the same manner.

\section{1. -TO CURE BACON.-COBBETT'S RECEIPT.}

The two sires that remain, and which are called fitches, are to be cured for bacon. They are first rubbed with salt on their insides, or flesh sides, then placed one on the other, the flesh sides uppermost, in a salting trough which has a gutter round its edges to drain away the brine, for to have sweet and fine bacon. the flitches must not be sopping in brine. which gives it the sort of taste that barrel pork and sea pork have, and than which nothing is more villanous; every one knows how different is the taste of fresh diy salt from that of salt in a dissolved state, therefore change the salt often, once in four or five days; let it melt and sink in, but let it not lie too long; change the flitches, put that at bottom which was first on the top, do this a couple of times; this mode will cost you a great deal more in salt than the sopping mode, but without it your bacon will not be so sweet and fine, nor keep so well. As for the time required in making. your flitches sufficiently salt, it depends on circumstances, the thickness of the flitch, the state of the weather, the place wherein the salting is going on; it takes a longer time for a thick than a thin flitch; it takes longer in dry than in damp weather; it takes longer in a dry than in a damp place; but for the flitches ot a hog of five score, in weather not very dry or damp, about six weeks may do and as yours is to be $f a t$, which receives little injury from over salting, give time enough, for you are to have bacon until Christmas comes again.

The place for salting should, like a dairy, always be cool, but always admit of a free circulation of air ; confined air, though cool, will taint meat sooner than the mid-day sun accompanied by a breeze. With regard to smoking the bacon, two precautions are necessary : first, to hang the flitches where no rain comes dowr. upon them, and next, that the smoke must proceed from wood, not peat, turf, nor coal. As to the time it requires to smoke a flitch, it must depend a good deal upon whether there be a constant fire beneath, and whether the fire be large or small; a month will do if the fire be pretty constant, and rich as a farm-house fire usually is; but over-smoking, or rather too long hanging in the air, makes the bacon rust; great attention should therefore be paid to this matter. The flitch ought not to be dried up to the hardness of a board, and yet it ought to be perfectly dry; before you hang it up lay it on the floor, scatter the flesh side pretty thickly over with bran, or with some fine sawdust, not of deal or fir ; rub it on the flesh, or pat it well down upon it ; this keeps the smoke from getting into the little openings, and malies a sort of crust to be dried on.

To keep the bacon sweet and gcior, and free from hoppers, sift fine some clean and dry wood ashes. Put some at the bottom of a box or chest, long enough in hold a flitch of bacon. Lay in one flitch, and then put in more ashes, then another flitch, and cover this with six or eight inches of the ashes. The place where the box or chest is liept ought to be $d r y$, and should the ashes become clamp, they should be put in the tire-place to dry, and 
when cold put back again. With these precautions, the bacon will be as good at the end of the year, as on the first day.

It may be as well to observe in reference to the above receipt, given by the very celebrated William Cobbett, in his Cottage Economy, that most counties in England have their peculiar method of curing hams and bacon, each varying in some slight degree from the other, and, of course, each is considered orthodox. But for simple general rules, the above may be safely taken as a guide ; and those who implicitly follow the directions given, will have at the expiration of from six weeks to two months, well flavored, and well cured bacon.

\section{2.-TO CURE BACON FOR LARDING.}

It is of little use preparing a small piece of bacon for larding, for different joints require lardings of different lengths; a piece of beef, for example, will, if of a tolerable size, require very lengthy lardings, acs a fowl will require but small ones. Ten to twenty pounds should at least be prepared. Take fifteen pounds, and the fatter it is the better; rub it well with a pound and a half of pounded common salt; if in one piece, lay it upon a board with another over it; if in more than one piece, let each piece have a board with a weight at the top; keep it in a cool place four or five weeks; hang it to dry but not to be smoked.

\section{3.-TO BOIL BACON.}

If very salt, soak it in soft water two hours before cooking. Put it into a saucepan with plenty of water, and let it boil gently; if two or three pounds, it will take from an hour to an hour and a quarter; if larger, an hour and forty minutes will suffice. If a fine piece of the gammon of bacon, it may when done have the skin, as in hams, stripped off, and have finely powdered bread raspings strewed over it. It will improve the ap. pearance when sent to table.

\section{4.-BACON, TO BROIL.}

Make up a sheet of paper in the shape of a dripping-pan, cut your bacon into thin slices, cut off the rind, lay the bacon on the paper, put it over the gridiron, set it over a slow fire, and it will broil clearly.

\section{5.-BACON, TO MAKE.}

Rub the bacon with a little common salt, and let them lie till the brine runs from them; in a week rub off all the salt, and put them in a tub, then rub into the flitches a pound of saltpetre pounded and heated, the next day do the same with common salt, also heated; let them lie a week, often rubbing them; do the same for three weeks or a month, at the end of that time dry and hang them up for use.

\section{6.-BACON AND CABBAGE.}

Boil some fine streaked part of bacon with a little stock. and the ends of eight or ten sausages, boil in the same stock some white cabbages for two hours; add salt and spice, and serve very hot; place your sausages and cabbage round your dish, and the bacon in the middle.

\section{7.-MOCK BRAWNS.}

Put four feet, two ears, and two chops of a pig into two quarts of water, and let it boil for several hours, till the bones can be picked from the meat; then pour it into a basin; skim off the fat, and take away all the bones; put it again into a saucepan with a little chopped parsley and sweet herbs, dried and rubbed small, cayenne pepper, salt, and pounded mace, let it boil for ten minutes; dip a mould into cold water, pour in the mixture ; let it get cool; turn out, and garnish with parsley and barberries, or slices of lemon.

\section{8.-EXCELLENT SAUSAGE CAKES.}

Chop some lean pork very fine, having previously detached all the skin and 
bone, and to every pound of meat add three-quarters of a pound of fat bacon, half an ounce of salt, a salt-spoonful of pepper, the quarter of a nutmeg grated, six young green chopped onions, and a little chopped parsley; when the whole is well chopped, put into a mortar and pound well, finishing with three eggs; then have ready a pig's caul, which cut into pieces large enough to fold a piece of the above preparation the size of an egg, which wrap up, keeping the shape of an egg, but rather flattened, and broil very gently over a moderate fire.

\section{9.-PIGS` FEET. (Soyer.)}

Procure six pigs' feet nicely salted, which boil in water, to which you have added a few vegetables, until well done, cut each one in halves, take out the long bone, have some sausage-meat as in the last: and a pig's caul, which cut into pieces each large enough to fold half a foot, well surrounded with sausage-meat; when well wrapped up broil slowly half an hour over a moderate fire, and serve. Or, when the pigs' feet are well broiled, egg over, and throw them into some grated crust of bread, with which you have mixed a little parsley, broil a nice color, and serve with a little plain gravy. This is called à la Ste. Menehould.

\section{0.-PIGS' KIDNEYS.}

Cut them open lengthwise, season well with pepper and salt, egg over with a paste brush, dip into bread-crumbs, with which you have mixed some chopped parsley and eschalot, run a skewer through to keep them open, and broil for about a quarter of an hour over a good fire; when done place them upon a dish, have ready an ounce of butter, with which you have mixed the juice of a lemon, a little pepper and salt, and a teaspoonful of French or common mustard; place a piece upon each of the kidneys, place in the oven for one minute, and serve. Pigs' kidneys may also be sautéd as directed for ox kidneys.

\section{DISHES FROM THE REUAINS OF PORK.}

\section{1.-HASHED PORK.}

Put two spoonfuls of chopped onions into a stewpan, with a wineglas ful of vinegar, two cloves, a blade of mace, and a bay-leaf; reduce to half, take out the spice and bay-leaf, add half a pint of broth or water, cut some pork previously cooked into thin small slices, season well upon a dish with pepper and salt. shake a good teaspoonful of flour over, mix all together, and pıt into the stewpan; let simmer gently ten minutes, pour out upon your dish, and serve with slices of gherkins in it; a little mustard may be added, if approved of or a little piccalilly with the vinegar is excellent.

The remains of salt pork, though veiy palatable cold, if required hot, may be cut into large thin slices, and placed in a buttered sauté or fryingpan, with a little broth, or merely fried in the butter, and served with a purée of winter peas, made by boiling half a pint of peas until tender (tied up in a cloth); when done, put them into a stewpan with two ounces of butter; season with pepper and salt. add a gill of milk or cream, pour into the dish, and dress the pork over.

It may also be cut into thin slices and put into a soup plate, and pour some ketchup or Harvey sance over it, and let it remain for half an hour; butter the inside of a pudding basin, and lay some of the remains of peas pudding round it, and then place in the pork, cover it with some of the pudding, place it in a saucepan with a little water to get hot, for about half an hour, and then turn it out and serve. Should you not have quite pork enough, you may make it up with a little sausage-nieat or any other kind of meat. 
882.-FRITADELLA. (Twenty receipts in one.)

Put half a pound of crumb of bread to soak in a pint of cold water, take the same quantity of any kind of roast or boiled meat, with a little fat, chop it up like sausage-meat, then put your bread in a clean cloth, press it to extract all the water, put into a stewpan two ounces of butter, a table-spoonful of chopped onions, fry for two minutes, then add the bread; stir with a wooden spoon until rather dry, then add the meat, season with a teaspoonful of salt, half the same of pepper, a little grated nutmeg, the same of lemon-peel, stir continually until very hot; then add two eggs, one at a time, well mix together, and pour on a dish to get cold. Then take a piece as big as a small egg, and roll it to the same shape, flatten it a little, egg and bread-crumb over, keeping the shape; do all of it the same way; then put into a sauté-pan, a quarter of a pound of lard, or clean fat, or oil; when hot, but not too much so, put in the pieces, and sauté a very nice yellow color, and serve very hot, plain, on a napkin, or on a border of mashed potatoes, with any sauce or garniture you fancy. These can be made with the remains of any kind of meat, poultry, game, fish, and even vegetables; hard eggs, or cold mashed potatoes may be introduced in small quantities, and may be fried instead of sautéd, in which case put about two pounds of fat in the frying-pan, and if care is used it will do several times. This is an entirely new and very economical and palatable dish, and fit for all seasons. and if once tried would be often repeated; the only expense attending it is the purchase of a small wire sieve for the bread-crumbs. The reason $I$ call it twenty receipts in one is, that all kinds of food may be used for it, even shrimps, oysters, and lobsters.

\section{2-RAMIFOLLE.}

These are a little more expensive than the fritadella, and worthy the table of a crowned head. The flesh of fowls instead of lamb or veal, with the addition of one or two fat livers cut in dice. Proceed as in the former receipt. using the crumb of French rolls, and one or two truffles cut fine: then make some pancake batter, and sautè two pancakes about one-eighth of an inch thick, cover one with the meat. \&c., and lay the other over, and put by until cold; when so, cut them to any shape you like, but if like cutlets add the small bone of fowl or pigeon, or the stalk of a sprig of parsley; egg and bread-crumb them, and sauté them in oil or lard of a nice yellow color, and dish them like cutlets, with any of the sauces or garnitures described for mutton cutlets ; or if plain, with fried parsley. They may be made of any kind of meat, fish, or poultry. I have latterly had them sent up to table when we have had a few friends, and they have been very much liked; and, on inquiring the name, I baptized them Ramifolle, without any particular meaning, which name having pleased as much as the dish, therefore let them be called Ramifolles.

They may be made a plainer way with various meats or liver, and spread over one pancake, which roll over, and when cold cut it into three equal lengths, erg, bread-crumb, and sauté as above.

\section{4.-PRUSSIAN CUTLETS.}

Take a piece of veal, say one pound, from any part of the calf, free from nerve, with a little fat, chop it up. but not too fine, add to it two teaspoonfuls of chopped eschalot, one of salt, half a one of pepper, a little grated nutmeg, chop it a little more, and make it into pieces of the size of two walnuts, to which give the shape of a cutlet; egg and breadcrumb each; insert a small bone at the small end, sauté it in fat, oil, lard, or butter, give it ten minutes on a slow fire 
till a nice brown color, dish and serve with demi-glaze sauce, in which you have put a spoonful of Harvey's, and serve with any brown or white sauce of stewed vegetables you like. Any kind of meat may be used.

885.-CUTLETS À LA VICTIME, OR VICTIMIZED CUTLETS

Invented by a culinary artist in the time of Louis XVIII., of France, at the palace of the Tuileries, and first partaken of by that intellectual monarch and gourmet, who, at the end of his stormy reign, through a serious illness, was completely paralyzed. The functionary organs of his digestion were much out of order; being a man of great corpulence, and a great admirer of the festive board. much food was required to satisfy his royal appetite : and the difficulty which his physicians experienced was to supply this want of food in the smallest compass. The head-cook, on being consulted, begged a few hours for reflection before he could give an answer to so important a question, as nothing but mutton entirely deprived of fat was to compose his Majesty's meal. After profound study by the chief and his satellites, a voice was heard from the larder, which was a considerable ways from the kitchen, crying, "I have found it, I have found it." It was that of a young man of the name of Alphonse Pottier, who, in saying so, made his appearance in the kitchen with three beautiful mutton cutlets tastefully trimmed and tied together; he then, with a small skewer, fastened them to a spit, and placed them, to the astonishment of all present, close to the bars of the grate: two of the cutlets soon got brown (observe, not a word was to be said until the trial was made), from brown they soon turned black; every one gazed at each other with astonishment, whilst Pottier, with quite a composed countenance, terminated his scientific experiment, took them off the spit, drew the skewer out, cut the string, threw the two burnt cutlets away, and merely served the middle one, which seems to have received all the nutriment of the other two; it was served, and greatly approved of by the physicians, as well as by the gourmet potentate, who, in consequence of two being sacrificed for one, named it, "Cutlet à la Victime," and often afterwards used to partake of them when in the emjoyment of health.

\section{6.-CUTLET À LA VICTIME.}

Cut three cutlets from the neck of mutton, about half an inch thick, trim one very nicely, free from fat, leave the other two as cut off, put the trimmed one between the two, flatten them together, so that the fat of the outside ones meet over the middle one; tie them together thus, and broil over a very strong fire for ten minutes; remove it from the fire, cut the string, and dish up the middle one only on a very hot dish, with a little salt sprinkled over it. If wanted roasted, proceed as above.

\section{SAUSAGES AND FORCE- MEAT.}

887.--SAUSAGES.

IN making sausages there is so great a variety of both meat and condiments, that the cook need not be tied down to any rule of composition. The usual mode is to put the preparation into the cleaned skins of the entrail-puddings of the smaller animals; in which the sausages, being always well seasoned, will keep sound for a few days, and are then broiled as a garnish for large dishes, or fried for breakfast. As these skins are, however, not always to be found, the sausage-meat may then be bound together 
with the yolk of egg and bread-crumbs, made into the form of sausages, or as croquettes of any small size; then floured, nicely warmed, and browned in the frying-pan or Dutch oven until perfectly done.

If more be made than is wanted for immediate use, it may be put away in a cool place, packed closely down, in a stone jar, and securely covered for some other occasion. For the spicing of sausages no rule can be laid down, as tastes and custom are constantly varying.

\section{8.-COMMON SAUSAGE-MEAT.}

Take any quantity of lean beef or pork, with half the quantity of fat. and having freed the lean of every particle of skin, sinew, and gristle. then mince both it and the fat as fine as possible; putting to each pound of meat a large teaspoonful of pepper and the same quantity of salt; strew this on the meat, and mix the whole thoroughly together, as a stock for further seasoning.

Take six pounds of meat, fat and lean; cut the fat into small pieces, and pound the lean portion in a mortar, with two table-spoonfuls of salt, one of moist sugar, a teaspoonful of saltpetre, and two table-spoonfuls of mixed spices. To this may be added the grated peel of a lemon, and a small portion of sage and thyme, well chopped.

Or:-To the meat of a leg of pork of about four pounds' weight, add two pounds of fat from the fore loin, chop both very fine; then add two nutmegs grated, twenty cloves, a teaspoonful of pounded or grated lemon-peel, two or three blades of mace pounded, one ounce of the best salt, and one-half ounce of black pepper; mix all together and stuff it into the skins, which should be well cleansed, and salted the night before. This sausage-meat would be much improved for immediate use, by pounding the meat and mixing it with a third portion of white bread soaked in milk and also pounded, blending the whole together with a beaten egg.

$$
\text { 889.-BEEF SAUSAGES, }
$$

Though not nearly so good as those of pork, yet, if made at home, may be found a pleasant and economical mode of using the scraps of raw meat. Proceed as for pork sausages.

\section{0.-MUTTON SAUSAGES.}

Take a pound of undressed mutton, or that which has been underdone, chop it very small, and season it with pepper, salt, and beaten mace. Chop also half a pound of beef suet, two anchovies, a pint of oysters, a quarter of a pound of grated bread, and a boiled onion; mix the whole with the oyster liquor, and the whites and yolks of two eggs well beaten; pound the whole in a mortar. Roll into lengths, corks, or balls, and fry them.

\section{1.-AN EXCELLENT SAUSAGE TO EAT COLD.}

Season fat and lean pork with some salt, saltpetre, black pepper, and allspice, all in fine powder, and rub into the meat; the sixth day cut it small, and mix with it some shred shalot, or garlic, as fine as possible. Have ready an ox-gut that has been scoured, salted, and soaked well, and fill it with the above stuffing; tie up the ends, and hang it to smoke as you would hams, but first wrap it in a fold or two of old muslin. It must be highdried. Some eat it without boiling, but others like it boiled first. The skin should be tied in different places, so as to make each link about eight or nine inches long.

\section{2.-OYSTER SAUSAGES.}

Take one pound of veal and a score of oysters bearded, then pound the veal very finely in a mortar with a little suet, sea- 
son with a little pepper, soak a piece of bread in the oyster-liquor, pound, and add it with the oysters cut in pieces to the veal; beat up an egg to bind them together, and roll them into little lengths, like sausages; fry them in butter a delicate brown.

Or:-Take half a pound of lean mutton or beef, with three-quarters of a pound of beef suet, and two score of oysters bearded. Mince the whole and add bread-crumbs, with two yolks of eggs to bind the materials together. Season with salt, white pepper, a little mace, and mushroom powder.

Put it either in skins, or fry the sausage-meat of a light brown in small pieces.

\section{3.-VEAL AND POULTRY SAUSAGES.}

Sausages may also be made of the remains of veal, turkey, or fowl, which have been dressed. Of either of these put equal quantities of meat and breadcrumbs, with half that of ham, seasoned with parsley, lemon-thyme, and chives. Mix the materials with a little pepper, salt, and pounded mace, the yolk of an egg, and half a teaspoonful of flour; make it up as saugages or small rolls to garnish fricassees or minced meat, or as a nice supper dish, piled round a sweetbread.

Or:-Chop equal quantities of lean veal and fat bacon, a handful of sage, a little salt and pepper, and a few anchovies, beat all in a mortar; and when used, roll and fry it, and serve it with fried sippets, or on stewed vegetables, or on white collops.

\section{4.-FOWL OR RABBIT SAUSAGES.}

Take the remains of a fowl, rabbit, or hare; when boiled or roasted, free it from the bones, and cut it small. Boil some onions in strong gravy ; when the onions are quite soft pound them, season with salt, pepper, parsley, two cloves, and a blade of mace; pound the meat also, cut some bacon into small pieces, and add it. Mix up these ingredients with the yolk of an egg, add a little lemon-juice or lemon-pickle, or chop a little sorrel with the herbs, fill the skins, and broil them.

Such are the sausages usually made in this country, and most commonly eaten fresh; but, in Europe, many sorts are made of a rather large size, seasoned in different modes, and smoked, with the intention of keeping them good for a long tixe, as the Brunswick, Bayonne, and Bologna, which may always be found in the foreign oil-shops in England. There is, however, another kind, which we to not recollect to have ever seen in London, though constantly used throughout the peninsula.

\section{5.-SPANISH AND PORTUGUESE SAU- SAGES}

Are made from the fat and lean of the back and loins of a well-fed two-yearold hog, cut into small pieces of less than half an inch square, and then either finely minced or pounded together, and strongly seasoned with cloves of garlic and green or red capsicums or chilis; but as these cannot always be conveniently procured in this country, cayenne pepper may be substituted. The whole should then be covered with any sort $o_{2}$ strong, dry wine, until absorbed by the ingredients, which will occupy perhaps a few days, according to the quantity.

Fill the largest skins you can get with the meat, fat and lean, alternately, occasionally adding some of the wine. Tie up in links, and hang them in a room where they will not get damp or become too dry, and they will keep twelve months.

They are sometimes fried, and eaten either alone or as a relish with poultry, but more frequently put into stews; and. 
in Spain, always boiled along with the olla, under the name of "Chorisos."

\section{6.-BLACK PUDDINGS.}

The blood must be stirred with salt till cold. Put a quart of it, or rather more, to a quart of whole grits, to soak one night; and soak the crumb of a wheat loaf in rather more than two quarts of new milk made hot. In the mean time prepare the guts by washing, turning, and scraping. with salt and water, and changing the water several times. Chop fine a little winter savory and thyme, a good quantity of pennyroyal, pepper, and salt, a few cloves, some allspice, ginger, and nutmeg; mix these with three pounds of beef suet, and six eggs well beaten and strained; and then beat the bread, grits, \&c., all up with the seasoning; when well mixed, have ready some hog's fat cut into dice, and, as you fill the skins, put it in at proper distances. Tie in links only half filled, and boil in a large kettle, pricking them as they swell, or they will burst. When boiled, lay them between clean clothes, or upon straw, till cold, and hang them up in the kitchen. When to be used, scald them a few minutes in water; wipe, and put them into a Dutch oven.

If there are not skins enough, put the stuffing into basins, and boil it covered with floured cloths; and slice and fry it when used.

Or :- Soak all night a quart of bruised grits in as much boilipg hot milk as will swell them, and leave half a pint of liquid. Chop a good quantity of pennyroyal, some leaves of sage, savory, and thyme, with salt, pepper, and allspice finely powdered. Mix the above with a quart of the blood, prepared as before directed; then half fill the skins, after they have been cleaned thoroughly, and put as much of the flare (that is, the inward fat) of the pig as will make it pretty rich. Boil as before directed. A small quantity of leeks. finely shred and well mixed, is a great improvement.

\section{7.-WHITE PUDDINGS.}

To two parts of beef suet chopped, add one part of oatmeal previously toasted before the fire; boil an onion or two, and chop them with pepper and salt; mix the whole well together; put the ingredients into skins, and boil them for an hour, pricking them as they boil to prevent their bursting. They will keep for some time in bran after they have been allowed to become cold. Parboil them when wanted, and then broil them on a gridiron.

The quantity of suet may seem disproportioned to the oatmeal; but unless there are two-thirds of the former to one of the latter, the puddings will be dry and fat. They require to be highly seasoned with pepper and onions.

\section{FORCEMEAT.}

French cooks pride themselves, and very justly, on the pains which they bestow on the elaboration of their forcemeat, or farce. It is an art in which they have attained superior excellence, but in which there is no great difficulty.

At many tables, where every thing else is well done, it is common to find very bad stuffing, which, according to what it is wanted for, should be a judicious selection, observing that of the most pungent articles least must be used. A general fault is, that the tastes of lemonpeel and thyme overcome all others, therefore they should only be used in small quantities; and except in a few very savory dishes, cayenne, and pepper of all kinds should be sparingly used. No one flavor should predominate greatly; yet if several dishes be served the same day, there should be a marked va- 
riety in the tastes of the forcemeats as well as of the gravies.

The forcemeat should be consistent enough to be cut with a knife, but not dry and heavy. Herbs are a very essential ingredient, and it is the copious but judicious use of them that chiefly gives the cookery of the French its superior flavor.

Bacon or butter must always be substituted for suet when the forcemeat is to be eaten cold.

The flavor given to the bread, or panada, is considered to be of great importance, and the highest point of perfection may be obtained by attending to the following directions :-

\section{8.-HOW TO MAKE QUENELLES OF FORCEMEAT.}

Have two table-spoons, dessert or teaspoons, according to the size you intend making quenelles ; fill one of them with the forcemeat; dip the other spoon into boiling water, and with it remove the forcemeat from the first spoon, and slip it from that into a buttered sauté-pan, proceeding thus until you have as many as you require; then cover them with some second stock, and boil them about ten minutes, or until firm, and they are ready for use.

\section{9.-PANADA FOR FORCEMEATS.}

Put two-thirds of half a pint of water into a stewpan holding a quart, with nearly an ounce of butter; when boiling, stir in a quarter of a pound of flour; keep it moving over the fire until it forms a smooth and toughish paste; take it out of the stewpan, and when cold, use it where directed.

\section{0.-STUFFING FOR VEAL.}

Chop úp half a pound of beef suet very fine; put it in a basin, with eight ounces of bread-crumbs, a table-spoonful of chopped parsley; a little powdered thyme and marjoram, half the rind of a lemon grated, and the juice of half a one; season with a spoonful of salt, and a quarter one of pepper, and one-quarter of a nutmeg; mix the whole with three whole eggs; this will do also to stuff turkey or baked fish, adding some more chopped parsley.

\section{1.-STUFEING FOR HARE OR TURKEY.}

Take half a pound of beef suet chopped very fine, some parsley, a little thyme, pepper, salt, and spices, the same quantity of crumbs of bread as of suet, an egg or two, and mix the whole with a little milk. It would not be amiss to put to it a very small bit of butter, and to pound the whole in a mortar for a short time. This stuffing may be used with baked pike, or with either roasted or boiled turkey, roasted hare, \&c.; in short, with all such articles as will be mentioned in this work. If the taste of shalot is not objected to, it will be found to add to the flavor of the stuffing. If you do not like to put it into the mortar, take the rolling-pin and mix it with it on the table, which is a better method.

\section{CURING MEA'T, POTTING, AND COLLARING.}

902.-CURING.

In salting all meat, care should be taken to remove the kernels, otherwise it will soon become tainted. It should be sprinkled with a handful of common salt to fetch out the blood, the brine thrown away on the following day, and the meat wiped with a dry cloth; it is then ready to be cured in any of the undermentioned ways. Too much care cannot be taken in the first salting to rub between every muscle or under every flap of the meat; as for want of this care it may have an unpleasant taste, though sufficiently salted. 
The brine will serve again, by being boiled and skimmed, which may be repeated as long as any scum will rise. Hams should be laid with the rind-side downwards : and it is a good plan to heat a quarter peck of common salt in a frying-pan, and lay it at the bottom of the pan. A small quantity of saltpetre is necessary to produce the fine red color either in ham or beef; but much will make the meat hard, and the proportions of sugar and salt should ba varied according to the preference given to highlysalted provisions, or those which are cured milder.

\section{3.-TO CORN BEEF.}

A Round of Beef of eighteen or twonty pounds' weight.-Mix an ounce and a half of sal-prunella, three ounces of brown sugar, half an ounce of black pepper, six ounces of fine salt, a quarter of an ounce of cloves pounded, the same of nutmeg, and half a pound of common salt: take out the bone, and rub the piece over with common salt: let it remain for a day or two and then rub into it the above ingredients finely powdered and thoroughly mixed; let it lie a fortnight, and turn it daily. It should then be hung up in a dry place; and, if possible, smoked till wanted for use, when it should be either boiled gently, or baked in a deep dish covered with coarse paste.

This, it may however be observed, is rather a superior mode; as the common way is merely to use fine salt, with occasionally a little saltpetre to give a tinge of redness to the meat, which is seldom smoked, except for very large joints. Many persons, indeed, think the meat best when siewed; in which case, put no more water in the pot than will barely cover the meat, and keep it gently simmering for four, five, or six hours, according to the size of the joint.

904.-TO SALT BEEF RED. bone as you can (the flank is the best), sprinkle it, and let it drain a day; then rub it with common salt, a small proportion of saltpetre, fine salt, and a little coarse sugar; you may add a few grains of cochineal, all in fine powder. Rub the pickle every day into the meat for a week. then only turn it.

It will be excellent in eight days. In sixteen, drain it from the pickle; and let it be smoked at the oven's mouth when heated with wood, or send it to the baker's. A few days will smoke it. It is extremely good eaten fresh from the pickle, boiled tender with greens or carrots. If to be grated, then cut a lean bit; boil it till extremely tender, and while hot, put it under a press. When cold, fold it in a sheet of paper, and it will keep in a dry place two or three months, ready for serving on bread and butter.

\section{5.-THE DUTCH WAY TO SALT BEEF.}

Take a lean piece of beef; rub it well with treacle or brown sugar, and turn it often. In three days wipe it, and salt it with common salt and saltpetre beaten fine; rub them well in, and turn it every day for a fortnight. Roll it tight in a coarse cloth, and press it under a heavy weight; hang to dry in wood-smoke; but turn it upside down every day. Boil it in pump water, and press it; it will grate or cut into shivers, and makes a good breakfast dish.

To twelve pounds of beef the proportion of common salt is one pound.

\section{6.-SPICED BEEF.}

Make a brine with half a pound of salt, half an ounce of saltpetre, half a pound of sugar, thirty cloves, as many allspice and black peppercorns, six bay-leaves; crack the spice; put it on to boil for a few minutes in a pint of water; when cold, pour it over a piece of beef about ten pounds. Turn it every day for a fort-

Choose a piece of beef with as little night 
When required for use, put the beef into a deep pan with the brine, a little water, and about one pound of suet. Bake it until tender; let it get cold in the brine.

\section{7.-HUNG BEEF. (the Derrynane Receipt.)}

Rub the beef well with salt and saltpetre, in the proportion of two ounces of saltpetre and seven pounds of salt to fifty pounds of beef: Put the beef into a cask or tub, place a board over it, and weights upon that; leave it so for about a fortnight, then take it out and hang it in the kitchen to dry, which will generally take about three weeks. Some persons leave it for a longer time in the tub, which they merely cover without the weight; but the above is the better way.

\section{8.-TO COURE TONGUES.}

Neats' tongues cured with the whole root on look much larger, but have not any other advantage, being too hard to cut pleasantly when salted. If the root is to be removed, cut it off near the gullet, but without taking away the fat that is under the tongue. The root must be soaked in salt and water a night, and extremely well cleaned before it be dressed, when it is very good stewed with gravy; or it may be salted two days, and used for pea-soup. Having left the fat and a little of the kernel under the tongue, sprinkle it with salt and let it drain until the next day. Then for each tongue mix a large spoonful of common salt, the same quantity of coarse sugar, half as much saltpetre, a teaspoonful of ground pepper, and two cloves of garlic or shalot chopped very fine; rub it well in, and do so every day for a week; then add another large spoonful of salt. If rubbed daily, a tongue will be ready in ten days; but if only turned in the pickle, it will not be too salt in four or five weeks, but should not be kept longer. When to be dried, write the date of the day on parchment, and tie it on. Smoke three days, or hang them in a dry place without smoking. When to be dressed, boil the tongue extremely tender; allow five hours, and if done sooner it is easily kept hot. The longer it is kept after smoking, the higher will be the flavor ; but in the estimation of many persons, they are best dressed ont of the pickle; if hard. it may require soaking four or five hours.

Or:-Put the tongue into an earthen pan, rub into it a table-spoonful of saltpetre, a good handful of salt, and the same quantity of coarse brown sugar; allow the tongue to remain in the pickle for three weeks, and rub it over every day. Then take it out and put it into the meat-screen before the fire for two or three days, till the pickle has entirely drained from it. Then sprinkle it over with bran, and hang it up in any part of the kitchen with the root-end uppermost; sew salt tongues up in coarse linen.

\section{9.-TO CURE SHEEP'S TONGUES.}

Let the tongues be well washed and cleaned, and lay them in spring water for half an hour. Take one pound of salt, a gill of molasses, and half an ounce of saltpetre; rub the tongues well with it, and put them into the pickle: a week or ten days will be sufficient to cure them.

\section{0.-FOR CURING HAMS.}

Choose the leg of a hog that is fat and well fed, as well as of a certain age; for if poor, it is not worth curing, and if not one year old it will never acquire the fine flavor of a mellow ham. Sprinkle it with a little salt, and let it drain for a day; then put to it one pound each of coarse salt and the coarsest sugar, with a 
large handful of common salt and four ounces of saltpetre; rub it thoroughly with this ; lay the rind downwards, and cover the fleshy parts with the salt, with which baste it frequently, and turn it every second day. Keep it in this for four weeks, then drain it and throw bran over it; hang it up in a cool place to dry, then sew it up in a coarse linen wrapper rubbed with lime, and do not dress it until full three months old.

The better. way is, however, to smoke $i t$. In country places this is not uncommonly done by hanging it in a kitchen chimney where wood is burnt, or in any spare chimney where a smothering fire can be kept up, made of wet straw and horse-litter, with sawdust or shavings; but be particular to have them of oak, for, if of pine, it will give the ham a bad flavor. It will take three weeks' smoking.

Or :-Hang the ham, and sprinkle it with salt as above; then rub it every day with the following, in fine powder: a pound of common salt powdered fine, two ounces of salt-petre, and two ounces of black pepper, mixed with one and a half pints of molasses.

\section{1.-ANOTHER WAY, THAT GIVES A HIGHER FLAVOR.}

When the weather will permit, hang the ham three days; mix one ounce of saltpetre with half a pound of common salt, and also of coarse sugar, and a quart of strong beer; boil them together, and pour them immediately upon the ham; turn it twice a-day in the pickle for three weeks. One ounce of black pepper, and the same quantity of allspice, in fine powder, added to the above, will give still more flavor.

Or:-Sprinkle the ham with salt, after it has hung two or three days ; let it drain ; make a pickle of a quart of strong beer, half a pound of molasses, one ounce of coriander-seeds, two ounces of juniper-berries, one ounce of pepper, the same quantity of allspice, one ounce of saltpetre, half an ounce of sal-prunella, a handful of common salt, and a head of shalot, all pounded or cut fine. Boil these all together a few minutes, and pour them over the ham: this quantity is for one of ten pounds.

\section{2.-FITZSIMON FAMILY RECEIPT FOR CURING HAMS.}

Mix one ounce of saltpetre, one pound of commion salt, and one pound of coarse brown sugar, all together, and rub the ham well. Let it lie for a month in this pickle, turning and basting it every dav; then hang it in wood-smoke in a dry place, where no heat can come to it, and, if to be kept long, hang it for a month or two in a damp place, and it will eat firm and short. Observe, hams thus made need not be soaked; put them into cold water, and let them be three or four hours before they boil, skimming the pot well and often until it boils. These hams have been made with a less quantity of salt and an additional quantity of saltpetre, and it has been found to answer well, the hams being in that case soaked before boiling.

\section{3.-WESTPHALIA HAMS.}

Prepare the hams in the usual manner by rubbing them with common salt and draining them; take one ounce of saltpetre, half a pound of coarse sugar, and the same quantity of salt; rub it well into the ham, and in three days pour a pint of vinegar over it. A fine foreign flavor may also be given to hams by pouring old strong beer over them, and burning juniper-wood while they are drying: molasses, juniper-berries, and highly-flavored herbs, such as basil, sage, bay-leaves, and thyme, mingled together, 
and the hams well rubbed with it, using only a sufficient quantity of salt to assist in the cure, will afford an agreeable variety.

\section{4.-MUT'RON HAM.}

Cut a hind quarter of mutton in the shape of a ham, and allow it to hang for two or three days. Mix half a pound of coarse salt, two ounces of saltpetre, half a pound of common salt, and half a pound of coarse sugar, all well pounded together, and make them quite hot before the fire. Then rub it well into the meat, turning it in the liquor every day ; after four days add two ounces more of common salt. Let it remain twelve days in the brine, then take it out, dry it, and hang it up in wood-smoke for a week.

Another pickle for mutton-ham may be made with one ounce of saltpetre to one pound of coarse sugar, and one pound of salt; the ham to be kept in this pickle for a fortnight, then rolled in sawdust, and hung in wood-smoke for fourteen days.

\section{5.-GOOSE HAMS}

Are made by splitting the goose down the back, rubbing it with a quarter of an ounce of saltpetre, and then salting it well with common salt and coarse brown sugar; let it lie in pickle for ten days in summer and fourteen in winter, rub and turn it every day, roll it in sawdust and smoke it.

In Pomerania, on the shores of the Baltic, where vast numbers are sured, the breasts are prepared separately as hams, and being much dried in smoking, are always eaten without further dressing. In the fens of Lincolnshire the geese, when their feathers have been plucked, are also salted.

916. -BACON. The method of curing Matines Bacon, not get rusty. admired for its fine flavor.-Cut off the hams and head of a pig, if a large one; take out the chine and leave in the sparerib, as they will keep in the gravy and prevent the bacon from rusting. Salt it first with common salt, and let it lie for a day on a table that the blood may run from it; then make a brine with a pint of bay-salt, a quarter of a peck of common salt, about a quarter of a pound of juniper-berries, and some bay-leaves, with as much water as will, when the brine is made, cover the bacon; when the salt is dissolved, and when quite cold, if a new-laid egg will swim in it, the brine may be put on the bacon, which after a week must be rubbed with the following mixture:-half a pound of saltpetre, two ounces of salprunella, and one pound of coarse sugar; after remaining four weeks it may be hung up in a chimney where wood is burned; shavings, with sawdust and a small quantity of turf, may be added to the fire at times.

\section{7.-THE BLACK POOL RECEIPT FOR CURING BACON.}

For a middling-sized hog take twelve pounds of the best common salt, and one pound of saltpetre pounded very finely; rub it in well, and cover the meat about an inch thick, hams, chaps, and all; placing it with the rind downwards. Let it remain for a week; then take off the salt; turn the whole with the rind upwards; then lay the salt on again for another week. Then remove the salt, and turn it a second time ; lay on the salt and let it remain four days longer. It will then be properly salted. Wipe it clean; rub it all over with dry salt; and hang it where it will have a little air. of the fire, until it is dry. Then sew it up in whity-brown paper, and hang it in a dry place where no heat can come to it; and, if these precautions are taken, it will 
The meat must be salted on a board that is well perforated with holes, to let the brine run from it; and it must be covered up closely with a coarse cloth to keep out the air ; and, while salting, take care to lay the pieces as close as possible one upon the other.

\section{8.-WILTSHIRE BACON.}

Sprinkle each flitch with salt; and let the blood drain off for twenty-four hours. Then mix one pound and a half of coarse sugar, the same quantity of fine salt, six ounces of saltpetre, and four pounds of coarse salt; rub this well on the bacon, turning and wetting it in every part, daily for a month; then hang it to dry, and afterwards smoke it ten days.

\section{9.-TO CURE BACON FOR LARDING AND BRAISING.}

Take the fattest part of the pork, and to every ten pounds use one pound of pounded salt; rub it very well over; put the pieces one upon another upon boards, and lay boards with a heavy weight upon the top; leave it in a dry cool place for about a month; then hang it up to dry without smoking. The hardest is the best for larding; and bacon cured in this way is preferable for culinary purposes, since the saltpetre usually employed will turn veal or poultry red, when braised with any portion of the lean.

\section{0.-HOG'S CHEEKS.}

Cut out the snout; remove the brains; and split the head, taking off the upper bone, to make the chowl a good shape; rub it well with salt; next day take away the brine, and salt it again the following day with three quarts of common salt, and a pound and a quarter of brown sugar; put into a gallon and a half of spring water; stir it until the whole is dissolved. This quantity will be sufficient for three pair of chaps; be careful to turn them in the brine at least every other day, and to keep them well covered. Dry them in wood-ashes.

$$
\text { 921.-BRAWN. }
$$

Split and nicely clean a hog's head; take out the brains; cut off the ears, and rub a good deal of salt into the head; let it drain twenty-four hours; then lay upon it two ounces of saltpetre, and the same of common salt, for three days; lay the head and salt into a pan, with just water to cover it. for two days more.

Wash it well, and boil until the bones will come out; remove them, and chop the meat as quickly as possible in pieces of an inch long; but first take the skin carefully off the head and the tongue; the latter also cut in bits. Season with pepper and salt. Put the skin of one side of the head into a small long pan; press the chopped head and tongue into it, and lay the skin of the other side of the head over, and press it down. When cold, it will turn out. The head may probably be too fat; in which case, prepare a few bits of lean pork with the head. Boil two ounces of salt, a pint of vinegar, and a quart of the liquor, and, when cold, pour it over the head. The ears are to be boiled longer than the head; cut in thin strips, and divided about it, the hair being nicely removed. Reboil the pickle often.

922.-ANOTHER MOCK BRAWN.

Boil a pair of neat's feet very tender; take the meat off, and have ready the belly-piece of pork, salted with common salt and saltpetre, for a week. Boil this till nearly done; take out the bones, and roll the feet and the pork together. Then roll it very tight with a strong cloth and coarse tape. Boil it till very tender; then hang it up in the coth till cold; after which keep it in a sousing liquor.

923.-TO KEEP BRAWN, THE CAMBRIDGE WAY.

To two gallons of water, put one pound 
of wheat-bran, and one pound of salt; boil one hour; when cold, strain it ; and keep the brawn in it. In ten or twelve days fresh pickle will be required. If, by length of carriage or neglect, the brawn be kept too long out of pickle, make as above; and when rubbed well with salt, and washed with some of the pickle, it will be quite restored to its former goodness.

\section{4.-TO PICKLE PORK.}

Take half a bushel of common salt, one pound of coarse salt, half a pound of saltpetre, and six pounds of coarse brown sugar ; make hams of the legs. Take the sides of the pork, and rub them well with common salt; lay a thin bed of salt in the tray, and place one of the sides in it; sprinkle with salt to cover it; lay the other side on the top, and sprinkle it also. Let them lie two or three days, rubbing the salt well in; then cover the whole with the other ingredients ; and, as soon as the salt begins to give, rub them well in; turn the sides frequently, and let them be covered with brine; it will be fit for use in six or eight tweeks.

\section{5.-A PICKLE}

That will keep for years, for hams, tongues, or beef, if boiled and skimmed between each parcel of them.-To two gallons of spring water put two pounds of coarse sugar, two pounds of coarse, and two and a half pounds of common salt, and half a pound of saltpetre, in a deep earthen glazed pan that will hold four gallons, and with a cover that will fit close. Keep the beef or hams as long as they will bear before you put them into the pickle; and sprinkle them with coarse súgar in a pan, from which they must drain. Rub the hams, \&c., well with the pickle; and pack them in close, putting as much as the pan will hold, so that the pickle may cover them. The pickle is not to be boiled at first. A small ham may lie fourteen days; a large one three weeks; a tongue twelve days; and beef in proportion to its size. They will eat well out of the pickle without drying. When they are to be dried, let each piece be drained over the pan; and when it will drop no longer, take a clean sponge and dry it thoroughly. Six or eight hours will smoke them; and there should be only a little sawdust and wet straw burnt to do this; but if put into a baker's chimuey, sew them in a coarse cloth, and hang them a week. Add two pounds of common salt, and two pints of water, every time you boil the liquor.

\section{6. -POTTING AND COLLARING.}

To pot and collar are only different modes of preserving fish and meat for a longer time than they could be kept fresh; chiefly, in the instance of potting, by pounding the materials with seasoning, when dressed, and then putting small portions in closely covered jars or pots; while collaring is done by slicing portions of the meat or fish ; and, when well seasoned, rolling it in round pieces; to be eaten cold, as savory dishes, at' breakfast and luncheon.

In potting, take care to wait until the meat is cold; press the meat firmly into the pots; but, before putting it there, drain the gravy thoroughly from the meat, or the gravy will turn it sour; then cover well with clarified butter, and tie over it oil-skin, or oiled paper, to exclude the air.

\section{7.-TO CLARIFY BUTTER.}

Put your boat into a saucepan of cold water, and set it over a slow fire until it melts; then take it off the fire; take off the scum, and again warm it gently. After being used, it will still serve for basting, or for meat-pie paste, and is also excellent for fish sauce.

In Collaring, be careful to roll the meat tightly, and bind it firmly. Let it 
also be thoroughly done; left in a cool place; sometimes rubbed with pickle, but always wiped perfectly dry.

\section{8.-TO POT SHELL-FISH.}

Boil lobsters and shrimps in salt and water; pick the meat out of the tails and claws; put them into a stewpan, with a little butter, some chopped mushrooms or truffles, and simmer a short time over a gentle fire. When nearly done, beat the yolks of two or three eggs, with a teacupful of cream, and a little chopped parsley; let all stew together for a few minutes, until rendered as consistent as paste, and set it as above stated.

Or:-When boiled, take them out of their snells, and season them with salt. white pepper, and a very little mace and nutmeg; but use the spice very sparingly, only just sufficient to preserve the fish for a few days in a fresh state, as they will not keep good much longer. Press them into a pot; lay a little butter over them; and bake in a slow oven for ten minutes. When cold, cover with ciarified butter.

Shrimps may also be potted whole, by putting them for a few minutes in clarified butter, seasoned as above, and gently heated; then put into pots, and cover with more butter, to totally prevent the admission of air. Cover the pots also with oil-skin.

\section{9.-TO POT POULTRY AND GAME.}

Chickens.-Take as much lean of boiled ham as you may think proper, and half the quantity of fat; each cut as thin as possitle; beat it very fine in a mortar. with a little clarified butter, pounded mace, pepper, and salt-if the ham ke not sufficiently impregnated. To this add the white part of the fowl, also pounded, but without seasoning, as it is only intended to qualify the savoriness of the ham. Then either mix the whole together, or put a layer of ham and chicken alternately; press it hard in the pots; bake in a cool oven for half an hour; pour over it clarified butter to the thickness of a crown piece, and paste over it a piece of paper, which may be oiled when the paste is dry.

Let them be quite fresh; clean them carefully, and season them with salt and pepper; lay them close in a small deep pan; for, the smaller the suriace, and the closer they are packed, the less butter will be wanted. Cover them with butter; then with very thick paper; tie down and bake them. When cold, put them dry into pots that will hold two or three in each; and pour butter over them, using that which was baked as part. Observe that the butter should be pretty thick over them if they are to be kept. If pigeons were boned and then put in an oval form into the pot, they would lie closer and require less butter. They may be stuffed with a fine forcemeat niade with veal, bacon. \&c. If a high flavor is approved of, add mace, allspice, and a little cayenne, before baking.

\section{0.-PARTRIDGES AND PHEASANTS.}

Clean them nicely, and season with mace, allspice, white pepper and salt, in fine powder, or seasoning spice. Rub every part well; then lay the breasts downwards in a pan, and pack the birds as close as you possibly can. Put a good deal of butter on them; then cover the pan with a coarse flour paste, and a paper over; tie it close, and bake. When cold, put the birds into pots, and cover them with butter.

Or:-When baked and grown cold, cut them into proper pieces for helping; pack them close into a large potting-pan, and (if possible) leave no spaces to receive the butter. Cover them with butter, and one-third part less will be wanted than when the birds are done whole. 
931.-HARE.

Hang up a hare four or five days, with the skin on; then case it, and cut it up as for eating; put it in a pot, and season it with mace, pepper, and salt; put one pound of butter upon it; tie it duwn, and bake in a bread oven till tender. When it comes out, pick it from the bones, and pound to a smooth consistence with the fat from the butter, and pot it in the usual war.

\section{2.-RABBITS.}

Cut up two or three young, but fullgrown ones; and take the leg-bones off at the thigh; pack them as closely as possible in a small pan, after seasoning them with pepper, mace, cayenne, salt, allspice, all in very fine powder. Make the top as smooth as you can. Keep out the liver and the carcases; but take off the meat above the neck. Put a good deal of butter ; and bake the whole gently. Keep it two days in the pan; then shift it into small pots, adding butter. The liver's also should be added, as they eat well.

\section{3.-BEEF POTTED.}

Take three pounds of lean beef, salt it two or three days with half a pound of salt and half an ounce of saltpetre; divide it into pieces of a pound each, and put it into an earthen pan just sufficient to contain it; pour in half a pint of water, cover it close with paste, and set in a slow oven for four hours. When taken from the oven pour the gravy from it into a basin, shred the meat fine, moisten it with the gravy poured from the meat, and pound it thoroughly in a marble mortar with fresh butter until it becomes a fine paste; season it with black pepper and allspice, or cloves pounded, or nutmeg grated; put it in pots, press it down as close as possible, put a weight on it and let it stand all night; next day, when quite cold, cover it a quarter of an inch thick with clarified butter, and tie it over with paper.

\section{4.-BEEF POTTED LIKE VENISON.}

Take a whole thin flank of beef, pull off the inward skin, and cut it across and across, particularly in the thickest parts, lay it for six hours in hard water, take as much saltpetre as the quantity of an egg, mix with about two pounds of white salt, and rub it well into the meat; then sprinkle upon it nearly a pint of wine vinegar, and then let it lie for three or four days. turning and rubbing it once a day, then rinse it out of the brine with a pint of claret, and season it with cloves, mace, and nutmeg. white and Jamaica pepper. of each a quarter of an ounce; bake all together with savory, thyme, sage, and the rind of a lemon shre? together, and then well rubbed into the cuts and slashes on the inside; then bind it up with tape, and lay it in a long pot; put in the claret, and lay the skins at the top to save it, then bake it.

\section{5.-BIRDS POTTED-HOW TO PRESERVE WHEN THEY BEGIN TO GROW OLD.}

When birds are sent a long way they often smell so bad that they can hardly be borne, from the rankness of the butter; by doing them in the following way they will be as if only fresh done. Set a large saucepan of clean water on the fire, when it boils take off the butter at the top, then take the fowls one by one, throw them in the saucepan of water half a minute, take one out and dry it well inside and out, do so till they are all done, scald your pot clean; when the birds are quite cold season with mace, pepper, and salt, according to taste: put them down close in a pot, put clarified butter over them.

936.-CHICKEN AND HAM POTTED.

Season some pieces of chicken with 
mace, cloves, and pepper, and bake it for about two hours in a close covered pan, with some water, then pound them quite small, moistening with either melted butter, or the liquor that they are baked in, pound some, and put this with the chicken in alternate layers, in pots or pans; press them down tight, and cover them with butter.

\section{7.-GAME OF ALL KINDS.}

Any dressed game you may have in your larder. Pound well in your mortar all the tender meat free from skin and bone ; add to it some pounded mace, allspice, cayenne pepper, salt, and white pepper, a few grains of powdered sugar, an equal quantity, if you have it, of good fat ham. When well pounded rub it through a wire siere; if you have no ham use an equal quantity of butter instead; mix it well up again, and place it tightly in earthen shapes; cover each jar over with clarified butter or lard; turn out with warm water; when required either for breakfast or luncheon, or a second course, dish in or on aspic, garnish with fresh parsley.

\section{8.-PIGEONS POTTED.}

Be careful that they are fresh, clean and season them with salt and pepper, lay them close together in a small deep pan, for the closer they are put the less butter they will take. Cover them with butter, tie them over with a thin paper, and bake them; when cold put them to dry in pots that will hold two or three in each, and pour butter over them, using that which was baked as some; mind, the butter shonld be thick over them; if they are done for keeping. the pigeons would lie closer and want less butter, if they are boned and put into the pot in an oval form. They may be stuffed with forcemeat, made with veal and bacon, $\& c$., and thev will eat very well. If a. high seasoning is preferred, add more allspice, and a little cayenne pepper, before baking.

Woodcocks are done the same way.

$$
\text { 939.-SMELTS POTTED. }
$$

Draw out the inside, season with salt, mace pounded. and pepper; lay them in a pan with butter on the top, bake them; when nearly cold take them out, lay them on a cloth, put them into pots, clear off the butter from the gravy, clarify, and pour it over them.

\section{0.-VEAL POTTED.}

Take one pound of lean veal, put it into a stewpan, with two ounces of fresh butter, the juice of a lemon, pepper, salt, sifted mace, a bay-leaf, allspice, cloves, nutmeg, cinnamon, and mushroom powder, a small quantity of each; a little thyme, savory, and a couple of shalots, chopped fine; stew them ten minutes, then pound them, add a pound of the mellow part of a boiled tongue beaten to a paste, half a pound of cold fresh butter; mix all well together, with two eggs, well beaten, then press the mixture down tight in small pots, cover them with paper, and put them into a moderate oven, bake twenty minutes, then pour over them clarified butter.

\section{1.-VENISON POTTED.}

Put the venison into a pan, and pour red wine over it, and cover it with a pound of butter; put a paste over the pan, set it in the oven to bake. When done take the meat out of the gravy, beat it well with the butter that has risen to the top, add more if necessary, season with pepper, salt, and mace pounded; put into pots, set them in the oven for a few minutes, take them out; when cold cover with clarified butter.

$$
\text { 942.-NEAT'S TONGUE. }
$$

Rub it with one ounce of saltpetre and 
four ounces of brown sugar; let it lie two days, then boil it till it is quite tender; take off the skin and all the fibrous part of the root, cut it in thin pieces, and pound it with one pound of clarified butter, and seasoning to your taste.

\section{3.-TO POT BEEF IN IMITATION OF VENISON.}

Put eight or ten pounds of lean beef into a deep dish; pour over it a pint of red wine, and let it lie in it for two days, seasoning it well with mace, pepper, salt, and a clove of garlic; then put it into a closely covered pot along with the wine, and another glassful if it be not sufficient, and bake it for three hours in a quick oven; when cold, pound it to a paste, and pot it as above.

Venison may itself be potted in the same manner; but if it be stale, rub it previously with pyroligneous acid.

\section{HAM CAKE.}

'Take the remains of a hain that may be getting dry, pound it in a mortar very finely, with all the fat; season it with pepper and mixed spice; add to it clarified butter sufficient to make it moist; put it into a mould, and place it in an oven for about half an hour; it should be prepared the day before it is wanted: put the mould for a few minutes in warm water in order that it may turn out properly. This may be made with equal quantities of cold beef pounded separately, and placed in layers in the mould, or put together in lumps to look like marble. Tongue may also be substituted for the ham, but it must be mixed with a larger quantity of butter in the pounding. If not wanted at the time, it may be potted with veal, and covered with clarified butter. It will keep well in winter if properly seasoned.

\section{5. -TO POT MUSHROOMS.}

Choose large buttons, or those in which the inside is not yet the least brown; peel and wipe out the fur of the larger ones; and to every two quarts put half a drachin of pounded mace, two drachms of white pepper, and six or eight cloves in powder: set them over the fire, shake, and let the liquor dry up into them. Then put to them two ounces of butter, and stew them in it till they are fit for eating; pour the butter from them, and let them become cold. Pack them close into a pot, making the surface as even as possible; add some butter lukewarm, and then lay a bit of white paper over them, and pour clarified suet upon it to exclude the air.

The collaring of Meat and Fish is an excellent method of preparing it to be eaten co'd, having the alvantage of keeping for a long time, and being at all times ready for ireakfast or luncheon.

\section{6.-COLLARED EEL}

Cut off the head, then split and take out the bone, but do not skin the fish; season them with mixed spices, some parsley and sweet marjor:um shred very fine, and a few chopped sage leaves; sprinkle a little dry isinglass over them; roll them tightly up in a cloth, bind it well with strong tape. Boil them in good gravy with a littie vinegar. according to the quantity of eels collared, a few penper-corns, a sprig of sweet marjoram, two or three onions, four bayleaves, a blade or two of mace, and a dozen allspice. Boil them until tender; take them up and tie them without taking out of the cloth, let them stand until cold, then glaze them. They may be sent whole to table, or cut in slices, and garnished with jelly and barberries.

As a standing dish, and only eaten cold as a relish, it ought always to be highly seasoned.

947.-TO "CAVEACH" FISH.

Bone the tail of a cod, and cut it into 
slices, season them with white pepper and salt, then fry them, and when cold put them into a pickle made of vinegar boiled with peppercorns, a few cloves, mace, and bay-leaves; add when cold a teacupful of sweet oil ; lay the fish in a jar with slices of onion between each, and cover the whole with vinegar. Salmon may be pickled in the same manner. When served up at table put a sufficient number of pieces in the centre of the dish, and raise round it a wall of salad.

\section{8. -TO COLLAR BEEF.}

Make a pickle with six ounces of brown sugar, four ounces of common salt, and one ounce of saltpetre. Then take a flank of beef, and leave it in the pickle for ten days or a fortnight, turning it every day. When taken out, remove the bone and gristle, but leave on the outer skin; lay it upon the table, with the skin downwards, and beat the inside well with a rolling-pin or wooden mallet until quite tender; that done, score the flesh in rows, down and across, about the breadth of two fingers; but in doing so take care not to cut the outside skin. Then fill the scores alternately with slices of the fat of bacon and corned pork, and sweet herbs of all sorts, chopped, and seasoned with spice, till you have filled them all; after which, roll the flank up very tightly, and hind it round with coarse broad tape, wrap it in a cloth, and boil it gently, but steadily, for four or five hours, according to the size of the joint. When quite cold, take it out of the cloth, unbind the tape, and fasten the roll with small skewers. If you wish to improve its appearance, you may also glaze it, or rub it with yolk of egg, and brown it with a salamander.

Another mode is, to take either the flant or brisket, and prepare it, as above, for hoiling; but stew it with a very little water, and, when done, cover the meat with a coating of chopped parsley, thyme, chives, or shalot. seasoned with a little cayenne; then put it under a board, and lay weights upon it for an hour or two. to press it close together; but before it becomes quite cold, remove the weights, and roll the piece up in the manner already directed.

\section{9.-AN ECONOMICAL WAY.}

Take the best part of a shin of beef, of which soup has been made (for it must be stewed until very tender), and an ox-tail, also well stewed; cut them into small pieces, season them well, add a glass of wine and a glass of ketchup. and put it into a stewpan covered with a part of the liquor in which the ox-tail has been boiled; stew it for about twenty minutes, and then put it into a mould. It must be very cold before it is turned out. 'This is a good way of employing the beef and heel when soup or jelly is made; a few chopped sweet herbs may be added, and hard eggs cut into slices, or pickles, such as sliced cucumbers, intermingled. The flavor may be varied in many ways.

\section{0.-TO COLLAR CALF'S HEAD.}

Scald the skin of a fine head, clean it nicely, and take out the brains. Boil it tender enough to remove the bones; then have ready a good quantity of chopped parsley, mace, ground nutmeg, salt and white pepper, mixed well: season it high with these; lay the parsley in a thick layer, then a quantity of thick slices of fine ham, or a beautifully colored tongue skinned, and then the yolks of six nice eggs stuck here and there about. Roil the head quite close, and tie it up as tight as you can. Boil it till the tape slackens. and then lay a weight upon it (without removing the bandage) till quite cold. Keep it in a pickle of the liquor, vinegar, and salt. 
A cloth must be put under the tape, as for other collars.

Ox-cheek may be collared in the same way.

\section{1.-BREAST OF VEAL.}

Bone it, take off the thick skin and gristle, and beat the meat with a rollingpin. Season it with herbs chopped very fine, mixed with salt. pepper, and mace. Lay some thick slices of fine ham, or roll into it two or three calves' tongues boiled first an hour or two, and skinned. Bind it up tight in a cloth, and tape it. Set it over a slow fire to simmer in a small quantity of water, till it is quite tender: this will take $\operatorname{som} \theta$ hours. Lay it on the dresser, with a board and weight on it, till quite cold.

Pigs' and calves' feet boiled, and taken from the bones, may be put in or round it. The different colors laid in layers look well when cut; and you may put in yolks of eggs boiled, beet-root, grated hanl, and chopped parsley, in different parts.

When it is cold, take off the tape, and pour over it the liquor, which must be boiled up twice a-week, or it will not keep.

\section{2.-BREAST OF MUTTON,}

Collared in this way, will also be found very nice. Bone the meat, and rub it over with yolk of egg, over which grate some lemon-peel and nutmeg, with a little pepper and salt; then mince a large spoonful of capers with a couple of anchovies, shred a handful of parsley and a few sweet herbs, mixed with crumb of a French roll ; strew it over the mutton, and roll it up tight; boil it gently for two hours, and, when taken up, put it in the same kind of pickle as that of the calf's head.

$$
\text { 953.-TO COLLAR A PIG. }
$$
Take a fine fat pig of a month or five with or without butter. weeks old, prepared for the table; cut off the head and split the pig down the back, and bone it; chop a handful of sage very small, mix it with two nutmegs and three or four blades of mace beaten fine; add to it a large handful of salt, and season the pig all over; roll it hard, tie it with tape, sew it in a clean linen cloth, and boil it in water with a little oatmeal and a good seasoning of salt; boil till very tender, which will take several hours. Keep it in the cloth in which it was boiled until quite cold. Then take the cloth from the pig, and let it lie for eight days in a marinade. Eat it with mustard, sugar, and vinegar.

\section{4.-TO COLLAR PIG'S HEAD.}

Scour the head and ears nicely; take off the hair and snout, and take out the eyes and the brains; lay it in water one night: then drain, salt it extremely well with common salt and saltpetre, and let it lie five days. Boil it enough to take out the bones; then lay it on a dresser, turning the thick end of one side of the head towards the thin end of the other, to make the roll of equal size; sprinkle it well with salt and white pepper, and roll it with the ears; and, if you approve, put the pig's feet round the outside when boned, or the thin parts of two cow-heels. Put it into a cloth, bind with a broad tape, and boil it till quite tender; then put a good weight upon it, and do not take off the covering till cold.

If you choose it to be more like brawn, salt it longer, let the proportion of saltpetre be greater, and put in also some pieces of lean pork; and then cover it with cow-heel to look like the horn.

This may be kept either in or out of pickle of salt and water boiled, with vinegar; and is a very convenient thing to have in the house.

If likely to spoil, slice and fry it either 
955.-A MARINADE FOR COLLARED MEATS.

Make thin water-gruel of oatmeal ; season it well with salt; add half a pint of white wine and half a spoonful of white pepper; boil it all together for half an hour; allow it to become cold before the collar is put into it.

\section{6.-TO SALT OR CORN A ROUND OF} BEEF.

Rub into it a pound and a half of salt, dried before the fire; then put it into a salting-pan, set it in a cool place, and the brine that melts should be poured on the meat every day. It may remain in salt from three to ten days.

\section{7.-BEEF PICKLE.}

Five quarts white salt, one of coarse salt, one pound brown sugar, fourteen quarts soft water; keep it simmering till it comes to a boil ; then add four ounces saltpetre, and give it a boil. Pack your beef in a close vessel, and when the pickle is cold, pour it over it.

\section{8.-TO PICKLE MEAT.}

To four gallons water put six pounds salt; boil and skim well, and let stand till cold; then put it into the vessel for your meat; boil the pickle over in two months. adding one pound salt, and skim well; keep a weight on the meat to keep it under the pickle; if any part should be bloody, sprinkle salt on it, and let it stand all night; then scrape and wipe it before it goes into the pickle.

$$
\text { 959.-VEAL POTTED. }
$$

Pound the remains of a cold fillet with mace, peppercorns, two or three cloves, and a little salt, and press it well down into pots, then cover with clarified butter. Alternate layers of pounded ham and veal, or both mixed, form a fine compound for the luncheon or breakfasttable.

\section{0.-HERRINGS, TO PICKLE.}

Let the fish be well cleaned and gutted but not opened; take salt, pepper, mace, nutmeg, pound and mix these spices well, then rub a pan with an onion. strew some of the spices over the bottom, and put as many fish as will lie flat on the bottom, then put a layer of sliced onions, and then fish, and so on alternately till the pan is filled; strew the pounded spice between each layer, pour over the best vinegar so as to cover the whole, tie a brown paper over the pan, and bake till the bones are soft.

Sprats and mackerel are likewise done in this wav.

The heads and tails must be cut off.

961.--SOYER'S SIMPLIFIED WAY OF MAKING THE SAVORY JELLY CALLED ASPIC.

Put a galantine in a stewpan with two onions, a carrot, half a head of celery, two cloves, a blade of mace, a good bunch of parsley, a little thyme, and bay-leaves, a knuckle of veal, the bones of a turkey, two calf's feet, two ounces of salt: add sufficient water to cover the whole; and set the stewpan upon the fire, until upon the point of boiling; then draw it to the corner; skim, and let simmer for three hours; then take it from the fire, leaving it in the stock until nearly cold; then take it out; remove the string from the napkin, and roll the galantine up tighter, tying the napkin again at each end only; then place it upon a dish; the breast part upwards; set another dish upon it, on which place a fourteen pounds' weight, which will press and cause it to cut firm; when quite cold, it is ready to serve; having removed the napkin, and the string with which it was sewed; the stock, however. should be clarified as directed in the next receipt, to make a savory jelly, which, when cold and firm, is cut in croutons and chopped; with some also the galantine should be tastefully garnished. 
Although at first I had some difficulty with this receipt, I can now see the variety to which it leads, as the same process answers for fowls, green geese, ducklings, pheasants, grouse, partridges, \&c., using game with the veal or pork for the interior, and stewing them according to their size; the bones of game being stewed with the stock, will give the flavor to the savory jelly.

\section{2.-TO CLARIFY MEAT JELLY.}

Having passed the stock (made as in whe last) through the sieve into a basin, leave it until quite cold; then take off all the fat very carefully ; ascertain if sufficiently, or too stiff, by putting a small piece upon ice; savory jelly requires to be rather stiffer than sweet; if too stiff, add a little more broth; if the contrary, the stock must be reduced upon the fire until of the proper consistency. When the stock is boiling, and you are perfectly assured of its strength, have the white of four eggs with their shells in a basin, with half a pint of water, two spoonfuls of tarragon or common vinegar, and a glass of sherry; whisk all together ; then whisk the stock quickly a few seconds. and pour in the other ingredients while whisking; continue whisking a few minutes until again upon the point, but not boiling; then take it from the fire, and taste if palatable; place a cover upon the stewpan, which stand a little distance from the fire, putting a few red-hot cinàers upon the lid for five minutes; tie a napkin by the four corners upon a jelly-stand, through which pass the jelly, having a basin beneath to catch it; pour the first that runs through again into the napkin until it runs quite clear; when all through, pour it in a plain mould or sauté-pan, which place upon ice until the jelly is quite firm; then dip the bottom of the mould in hot water; turn the jelly out upon a cloth, and cut it into whatever shapes you please. to gamish and ornament any cold savory dish; the jelly when warm," might be divided, one part kept white, and the other colored with a little brown gravy or coloring, thus enabling you to variegate in garnishing.

Should the jelly be required to ornament tongues, hams, pies, salads, or any article, when no galantine is made; then to make the stock, cut the veal into small pieces, and split the calf's foot in two; put a quarter of a pound of butter in a convenient-sized stewpan, with the veal, foot, a small piece of lean ham, and the oth $\Rightarrow$ ingredients as directed for galantine; pour in half a pint of water; put on the lid, and stand it upon the fire until the bottom or the stewpan is covered with a white glaze; then add a gallon of water; let simmer three hours, keeping it well skimmed; pass and clarify as above.

The knuckle of real and foot may be served hot with a little parsley and butter, for a dinner previous to your party, with a little fried bacon separately; but for my own part I prefer them plain as they leave the stewpan.

\section{3.-SOYER'S COLD HAM}

Procure a very nice but small ham of about nine pounds in weight, which soak about ten hours in cold water, and simmer three hours in plenty of water; when done, take out and let remain until cold; then cut off the skin as thinly as possible, but without leaving the marks of it; let a piece remain upon the knuckle about two inches and a half in breadth, which either festoon or vandyke; carve the fat neatly to form a shell, if you can, and glaze it over lightly; serve with a paper frill upon the knuck!e, and garnish with savory jelly, or, if plain, with a few bunches of fresh green parsley. A handful of fresh hay put in the water when boiling, is an improvement. 


\section{POULTRY.}

Always have good and fat ponltry where possible ; in the country you may command it. About three weeks before you wint to use them, six or twelve fowls, according to your consumption, should be put into the coop, and as you kill one or more replace them to keep up the stock; for the first week feed them alternate days with boiled rice and soaked bread and milk : the remainder of the time mix Indian meal with the skimmings of your stockpot and a spoonful of moist sugar. The windows of your poultry house must be darkened.

Fowls should be carefully drawn, so that the gall bladder is uninjured, and this should only be done through the vent.

Roast with a brisk and clear fire. A capon will take five-and-thirty minutes; smaller fowls a less time in proportion. A turkey of fourteen pounds will take two hours; the time will increase or decrease with the weight. The same rule applies to geese: a large one will take an hour and a half; chickens take half an hour, pigeons ten minutes less. It must be understood that the adherence to the time will depend on the state of the fire, \&c.; a slow fire will make a longer time necessary, and at the same time spoil the poultry.

The French adopt the following method to make old poultry eat tender : - Let the bird soak in cold water for twenty-four hours, with a handful or two of wood-ashes; pick off the feathers, and let it hang for another twenty-four hours ; truss it, and let it boil for a quarter of an hour in a little veal-broth; take it out, lard, and roast it; when nearly done, baste it with very hot butter. By observing these directions, you will impart to an old bird all the delicate flavor of a young chicken.
964.-TO BONE BIRDS.

Begin to bone any birds by first taking out the breast bone, when you will have sufficient space to remove the back with a sharp knife, and then the leg bones; the skin must not be broken, but the meat of the legs must be pushed inwards.

\section{5.-TURKEY WITH SAUSAGE MEAT.}

At the messes of European regiments in India, it is no uncommon thing to bone a turkey and a fowl, and put one inside the other, filling the interstices with sausage-meat, a small pig being killed for the purpose. A turkey thus prepared will take a long time roasting, and must be placed at a great distance from the fire at first. When carved the slices should be cut quite through; and epicures aver that it is one of the finest dishes that come to table.

\section{6.-TURKEY ROAST.}

It is stuffed with either sausage meat or fillet of veal stuffing, or crumbs of bread and veal. While roasting, a piece of paper should be placed over the part stuffed, as being bulky it will catch the fire and become scorched; but keep the heat well to the breast, in order that it may be as well done as the rest of the bird. Baste well, and froth it up. Serve with gravy in the dish, and bread sauce in a tureen. To the sausage meat, if used, add a few bread-crumbs and a beaten egg. Turkey is sometimes stuffed with truffles; they are prepared thus: they must be peeled, and chopped, and pounded in a mortar, in quantities of a pound and a half will be found sufficient: rasp the same weight of fat of bacon, and mix it with the truffles. Stuff the turkey with it; this stuffing is usually placed in the turkey two days previous to cooking: it is supposed to impart a flavor to the flesh of the fowl. Cut thin 
slices of fat bacon and place over the breast of the turkey. Secure it with half a sheet of clean white paper, and roast. Chestnuts dressed in the same fashion are found an excellent substitute for truffles. Two hours will roast it. If you wish to make plain stuffing, pound a cracker, or crumble some bread very fine, chop some raw salt pork very fine, or use butter; sift some sage, (and summer-savory, or sweet marioram, if you have them in the house, and fancy them), and mould them all together, season with a little pepper. An egg worked in makes the stuffing cut better.

\section{7.-TURKEY BOILED.}

A hen bird is considered the best. It may be stufied with truffles, chestnuts, or sausage meat. Boil it in a clean floured cloth; throw some salt into the water in which it is boiled. Cover close, and simmer for two hours; remove the scum frequently. White sauce, or parsley and butter; the latter is now scarcely ever brought to table.

\section{8.-BOILED TURKEY.}

Fill the body with oysters, and let it boil by steam without any water. When sufficiently done, take it up, strain the gravy that will be found in the pan, and which, when cold, will be a fine jelly; thicken it with a little flour and butter, add the liquor of the oysters intended for sauce, also stewed, and warm the oysters up in it; whiten it with a little boiled cream, and pour it over the turkey.

\section{9.-TURKEY WITH SAUSAGE MEAT AND TONGUE.}

Bone the turkey, then fill the inside with sausage meat, with or without tongue: if with tongue the tongue should be boiled the day before; cut off the root and tip to the length of the turkey; if you have a fowl to spare, wrap the tongue in this after it is boned, and place it in the middle of the turkey surround ed with sausage meat; introduce truffles if you like; if roasted, a slow fire, and it will take a long time to roast through; if for boiling, cover it with fat bacon and slices of lemon tied in a cloth, pour whatever sauce you propose over the turkey.

\section{0.-TURKEY HASHED.}

Cut up the remains of a roasted turkey, put it into a stewpan with half a gill of sherry wine, shalots, truffles, mushrooms, chopped parsley, salt, pepper, two spoonfuls of cullis, and a little stock; boil half an hour, and reduce to a thick sauce. When ready add a pound of anchovies, and a squeeze of lemon. Skim the sauce free from fat, and serve altogether.

\section{1.--FORCED TURKEY OR FOWL.}

Take all the bones from the turkey, fill it in again with either good sausage meat or veal forcemeat, with or without truffles as may be required, braise it in a cloth keeping it a good shape; when done glaze the breast a good color ; use silver skewers to ornament with, and any of the sauces named or a fricandeau sauce under it.

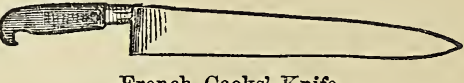

French Cooks' Knife.

972.-TULKEY POULT

Should be roasted without stuffing; it will be done with a clear fire in twenty minutes. Serve with bread or gravy sauce.

\section{3.-TURKEY WITH FLAT SAUSAGE CAKE.}

Roast as before; fry thirty oval flat sausages, the same quantity of the same sized pieces of bacon, a quarter of an 
inch thick; make a border of mashed potatoes about the size of a finger, one inch inside the edge of the dish, dress your sausages and bacon on it as a crown alternately, put your turkey in the middle, and gravy over, or glaze, if at hand. Plain boiled tongue may, of course, be served with the turkey, or separate on a dish of greens. If any remains of tongue from a previous day, it may be served instead of the sausages, cut the same shape as sausages, and warmed in a pan; if so, put a nice green sprout between each piece. Bread sauce is generally served with this dish.

974.-SOYER'S BOILED BRAISED TURKEY.

Truss it thus: cut the neck, leaving the skin on; cut the legs off; then run the middle finger into the inside, raise the skin of the legs, and put them under the apron of the turkey, put the liver and gizzard in the pinions, turn the small end of the pinions on the back, run a packing needle with string through the joint of the wing and middle joint of the leg, and through the body, and out at the opposite leg and wing, bring it round and tie it on the back, then run the needle and string through the ends of the legs or drumstick, press it through the back, and tie strongly; it is then ready. When the turkey is trussed, then stuff it; and if intended to have oyster sauce with it, chop about two dozen of oysters into small dice and mix them with the stuffing, and place inside the breast. Then rub the breast with half a lemon, and put it into a two-gallon pan, and cover it with cold water, to which are added two ounces of butter, one ounce of salt, four onions, a stick of celery, one carrot, two turnips sliced, a large bouquet of parsley, two bay leares, two sprigs of thyme; set it on the fire, when beginning to boil skim it, let it simmer two hours or more if large; try the breast with a needle, if it goes in and out easily it is done; take it. it out and set it on a dish to drain, remove the string, serve on a fresh dish with a pint of good thick oyster sauce over it. By omitting the oysters in stuffing, you may serve the turkey with celery sauce, tomato, or mushroom, or good parsley and butter; and, as an accompaniment, a piece of about two pounds of nice streaked bacon, which has been boiled with the turkey, and from which you have removed the skin, and serve on some greens, or Brussels sprouts, over which you have thrown a little salt, pepper, and two ounces of oiled butter. You see, dear — , that this dish can be varied without much expense and trouble; observe, that this way, the broth is good for soup the same day, by the addition of two pounds of veal cut in small pieces, a quarter of a pound of lean bacon, one onion, one blade of mace, a leek, a wineglass of water; put it into a separate stewpan, stew on fire till forming a white glaze, then add it to the turkey, when on the point of boiling; when done, skim off all fat, pass it through a tammy or cloth; you may use it for any clear soup by adding a little brown gravy or coloring, and also for any kind of purée; or, by reducing it a little, make white or brown sauce, adding to the last the proper color.

I must observe, that this will be almost inpracticable when you have a party; the only plan would be to get the turkey done one hour before you require it, keeping it hot with its breast in some of the stock; but, as the broth will keep well in small quantities, it can be reserved for the next day. That is my plan of boiling, but the following is my new way of giving the flavor of vegetables to all poultry, which is a decided improvement. The aroma from the bird when the cover is removed is quite inviting, and the appearance of it, which is as white as alabaster, and cuts also full of juice: I call it. 


\section{5.-ROAST BRAISED TURKEY (Soyer.)}

Peel and wash two onions, one carrot, one turnip, cut them in thin slices, also a little celery, a few sprigs of parsley, two bay-leaves, lay three sheets of paper on the table, spread your vegetables, and pour over them two or three tablespoonfuls of oil ; have your turkey, or poularde, trussed the same as for boiling; cut a few slices of lemon, which you place on the breast to keep it white, then cover with thin slices of bacon, and place the back of the bird on the regetables; tie the paper round with string, then pass the spit and set it before the fire; pour plenty of fat over to moisten the paper and prevent it from burning, roast three hours at a pretty good distance from the fire: capons will take two hours, poulardes one hour and a half, fowls one hour, and chickens half an hour. This way it may be served with almost any sauce or garniture, as stewed peas, oyster sauce, jardinière, stewed celery, cauliflower, stewed cucumbers, Jerusalem artichokes, which should be turned in the shape of * a pear: these should be dished on a border of mashed potatoes; that is, an artichoke and a Brussels sprout alternately, or a small piece of white cauliflower, and a small bunch of green asparagus, or stewed peas, or stewed celery, of two inches long, never more; or any other vegetable, according to season, which taste or fancy may dictate. When I want to serve them with brown garniture or sauce, I remove the paper and vegetables twenty minutes before it is done, and give it a light golden color, then I serve it with either a ragoût financier, or mushroom, or English truffle. I also often stuff it thus: I put two pounds of sausage meat in a basin with a little grated nutmeg; I then take two tablespoonfuls of chopped onions, put them in a sauté-pan with a little butter, and let them do for two minutes, which add to the meat, also two eggs well beaten up, and a quarter of a pint of white sauce, if at hand, and fifteen fine roasted chesnuts; add this to the stuffing, and fill the bird as usual, not too full at the breast; roast as above, giving half an hour longer for the forcemeat; put a quart of demiglaze and a glass of sherry in a stewpan, reduce it to a pint and a half, add in it fifty button onions previously stewed, and twenty-five roasted chesnuts; sauce under.

\section{6.-TURKEY, IF OLD.}

The French stew it exactly like the ribs of beef. Soyer says :-Put a quarter of a pound of butter into a convenientsized stewpan, such as will conveniently hold it; cut one pound of lean bacon in ten or twelve pieces, put a few minutes in the pan on the fire; then add your turkey trussed as for boiling, breast downwards; set it on a moderate fire for one hour, and until it is a nice color; add two table-spoonfuls of flour, and stir well round until it forms a roux; then add two quarts of water or broth; when you have it on the point of boiling, add fifty pieces of carrot the size of walnuts, the like of turnip, ten button onions, a good bouquet of sprigs of thyme, two bayleaves, and ten of parsley; a small glass of rum, a elove, a piece of garlic, and let it stew gently for four hours. If you use water, season in proportion. Take the turkey out, and put the vegetables and sauce in a smaller stewpan, which ought to be nearly full; let it simmer on the corner of the fire, so that the fat rises and may be removed, and reduce it to a demiglaze; dish up the turkey, and serve with the sauce over it; small new potatoes, about twenty, when in season, may be added to the sauce, or roasted chesnuts. The remains are excellent when cold, or will warm again with the addition of a little broth or water. 


\section{FOWLS.}

Fow Ls, in a general sense, mean all kinds of poultry; but, in a limited view, one species of bird. We distinguish this kind in cookery, as the chicken, capon, pullet, cock, and hen. Chickens from their age cannot be otherwise than tender. Capons should have a fat vein under the wing; thick belly and rump; comb short and pale; spurs short and blunt, and legs smooth. Pullets are best in the spring, just before they begin to lay. Cocks should have their spurs short, legs smooth, and comb short, smooth, and a bright color. Hens, legs and comb smooth, and full breast. Black legs are best for roasting and entrées, and white for boiling.

For preparing them for table, M. Soyer invented a plan:-by cutting the sinews of the bird, it not only appears fuller and plumper when cooked, as the heat is liable to contract the sinews, but it also affords facility for carving when the sinews are divided ; they are trussed in the usual way, only using strings instead of skewers.

\section{7.-TURKEY PULLED AND GRILLED}

Is a mode of preparing the remains of cold turkey, by mincing the white meat and fricasseeing it with white sauce; the legs being scored, peppered, and salted, broiled, and sent up over the mince.

Or devilled, as thus:-On the rump, gizzard, and a drumstick, put salt, pepper, and cayenne. Let them be broiled, and brought to table as hot as possible; cut them in small pieces; pour over them a ladle of mustard, ditto of melted butter, a spoonful of soy, ditto of lemon-juice, and some of the gravy out of the dish; mix quickly and hand round. It forms an admirable relish; fowls may be treated in the same manner.

978.-CAPON, OR POULARDE,

Should be stuffed and roasted, or other- wise dressed, in precisely the same manner as turkey. They are the male and female of the common fowl, but, when caponed, grow nearly to the size of turkeys, and are very rarely, if ever, boiled.

\section{9.-À LA PROVENCALE.}

Half roast the fowl; cut it in pieces; take a dozen onions; cut them in rings; add a little parsley chopped. Place them in a stewpan, laying a bed of onions and parsley, then fowl, till the whole are used ; add a glass of oil or cream, with one or two bay leaves and salt, let them simmer slowly; dish the fowl; put the onions in the middle; add a little sauce, and send it to table.

\section{0.-POELEE TO BOIL FOWLS IN.}

Take two pounds of real and two pounds of bacon; cut both into large dice ; cut also two large carrots and two onions into dice; put them, with a pound of butter, into a stewpan, the juice of four lemons, a little thyme or sweet fennel, and two laurel-leaves bruised; season it with salt and pepper; put them on a good fire; add a spoonful of boiling broth, and boil it.

\section{1.-FOWLS, FORCED.}

Cut a large fowl down the back; remove the skin from the whole of the body very carefully; cut the flesh from the bones, and chop it up finely with half a pint of oysters, and an ounce of beef marrow; then season with pepper and salt. Add sufficient cream to mix it well; lay the meat on the bones; draw the skin over, and sew up the back. Lay thin slices of bacon on the breast ; tie them on in diamonds, and roast it an hour by a moderate fire. Pour a good brown gravy sauce into the dish. Remove the bacon from the fowl, and then place the fowl in the dish. Garnish with oysters or mushrooms, and serve hot. 
982.-ROAST FOWLS.

If nicely trussed, make a stuffing with butter and some pepper, dry up the butter with bread-crumbs, baste it well, and flour and salt before you take it from the fire. If approved of, stuff the fowl with some good sausage-meat, truffles, or chestnuts, roast it twenty minutes, unless a very fine one, and then it will take three quarters of an hour; serve with bread sauce, or parsley and butter ; egg sauce is sometimes sent to table with it.

If a small lump of salt butter, well eovered with black pepper, is placed within the fowl previous to roasting, it will be found to improve the fowl by removing the dryness which is met with in the back and side bones.

\section{3.-BOILED FOWLS.}

Flour a white cloth, and put the fowls in cold water; let them simmer three quarters of an hour, serve with parsley and butter, or oyster or celery sauce. The fowls may be covered with a white sauce if sent cold to table, garnished with colored calf's-foot jelly of the hue of beet-root.

Boiled with Oysters.--Take a young fowl, fill the inside with oysters, put it into a jar, and plunge the jar in a kettle or saucepan of water. Boil it for an hour and a half. There will be a quantity of gravy from the juices of the fowl and oysters in the jar; make it into a white sauce, with the addition of egg, cream, or a little flour and butter; add oysters to it, or serve it up plain with the fowl. The gravy that comes from a fowl dressed in this manner will be a stiff jelly the next day ; the fowl will be very white and tender, and of an exceedingly fine flavor-advantages not attainable in ordinary boiling-while the dish loses nothing of its delicacy and simplicity.

994.-TO BRAISE A FOWL.

Bone the breast, and fill it with forcemeat. Lay the bones, and any other poultry trimmings, into a stewpan, and the fowl on them. Put to them a few onions, a fagot of herbs, three blades of mace, a pint of stock, and a glass or two of sherry. Cover the fowl with slices of bacon, and then with white paper, cover the whole close, and put it on a slow stove for one hour. Then take it up, strain the braise, and skim off the fat carefully; set it on to boil very quickly to a glaze, and put it over the fowl with a brush. Serve with a brown fricassee of mushrooms. Before glazing, put the fowl into an oven for a few minutes, to give á little color.

With Rice.-The fowl should be stewed very slowly in some clear veal or mutton broth, well skimmed, and seasoned with pepper, salt, mace, and an onion. About half an hour before it is ready add a gill of rice, well washed and soaked; simmer until tender, then strain the rice from the broth, and lay it on a sieve before the fire to swell; then dish the fowl with the rice round it.

White sauce is the most fashionable accompaniment, the old method of dishing with parsley and butter being on the decline; but as parsley still maintains its ground with many people, it is advisable to boil a sufficient quantity: press and chop it, garnishing the dish with small mounds thus prepared, which may be mixed at table with the white sauce by the guests who like the flavor of parsley. Liver-sauce is sometimes served with boiled fowls, but celery or oystersauce is preferable.

\section{5.-FOWL HASHED.}

This receipt will serve for any but the very larger species of poultry or game; joint them and cut a cutlet from each side of the breast. If it has not been eaten when previously dressed, break the bones of the body, and put all into a 
stewpan with a pint of water, a small faggot of sweet herbs, one carrot sliced, and an onion; let it stew an hour and three quarters, or two hours, skin the fat from the gravy as it rises, strain it, skim again, and pour it into another stewpan; thicken with a little butter and flour, flavor with Harvey's sauce, or any sauce applicable to such a dish, a little pepper and salt, and ground nutmeg, or mace for seasoning; add the fowl. and heat it thoroughly through without permitting the hash to come to a boil. Sippets of toasted or fried bread cut in dice surround the dish, in the centre of which the fowl is handsomely laid.

\section{6.-FRIED FOWL}

When vou have cut the pieces as before, put them into a basin with a little salt and pepper, a spoonful of oil, and two of vinegar, and a little chopped eschalot; stir them well in it, and let remain for half an hour ; have ready a quantity of batter, and take a fork and dip each piece one after the other into it, and then let it drop into the frying-pan, in which is sufficient hot fat to cover them ; fry a nice color, and serve in the form of a pyramid, with fried parsley over, or any sauce you like under.

\section{7.-FOWL PILLAU.}

Put one pound of rice into a fryingpan with two ounces of butter, which keep moving over a slow fire, until the rice is lightly browned; then have ready a fowl trussed as for boiling, which put into a stewpan, with five pints of good broth ; pound in a mortar about forty cardamom seeds with the husks, half an ounce of coriander seeds, and sufficient cloves, allspice, mace, cinnamon, and peppercorns, to make two ounces in the aggregate; which tie up tightly in a cloth, and put into the stewpan with the fowl; let it boil slowly until the fowl is nearly done; then add the rice, which let stew until quite tender and almost dry; have ready four onions, which cut into slices the thickness of half crown pieces ; sprinkle over with flour, and fry, without breaking them, of a nice brown color; have also six thin slices of bacon, curled and grilled, and two eggs boiled hard; lay the fowl upon your dish, which cover over with the rice, forming a pyramid; garnish with the bacon, fried onions, and the hard-boiled eggs cut into quarters, and serve very hot.

\section{8.-TO BROIL A FOWL.}

Split the fowl down the back; season it very well with pepper, and put it on the gridiron with the inner part next the fire, which must be very clear. Hold the gridiron at a considerable distance from the fire, and allow the fowl to remain until it is nearly half done; then turn it, taking great care that it does not burn. Broil it of a fine brown, and serve it up with stewed mushrooms, or a sauce with pickled mushrooms. A duck may be broiled in the same way. If the fowl is very large, half roast it; then cut it into four quarters and finish it on the gridiron.

\section{9.-FRICASSEE OF FOWL.}

Put the fow! into a jar, with sliced onion, parsley, salt, and a piece of butter rolled in flour; let it remain in a kettle of water on the fire until three parts done; then skim and strain the liquor, and after draining, wipe the fowl dry. Put it into a stewpan, with a piece of butter and a slice or two of ham; throw in a little flour, and shake it until it is of a good color. Moisten with the liquor taken oui of the jar, adding parsley, young onions, a bay leaf, a clove. sorrel, and mushrooms cut into pieces; skim it, and let it stew until done; then take out the herbs, and thicken the sauce with a little cream, but 
do not let it boil: finish with a little lemon-juice, and pour the sauce over all.

Or:-Rather more than half-boil in a small quantity of water: let the fowl cool ; then cut up, and put to simmer in a little gravy made of the liquor it is boiled in, and a bit of veal or mutton, onion, mace, and lemon-peel, some white pepper, and a bunch of sweet herbs. When quite tender, keep it hot while you thicken the sauce in the following manner: strain it off, and put it back into the saucepan with a little salt, a scrape of nutmeg, and a bit of flour and butter; give it one boil ; and when you are going to serve, beat up the yolk of an egg, and half a pint of cream, and stir them over the fire, but do not let them boil.

\section{0.-POELE. (French.) *}

Poêle is almost the same operation as braising; the only difference is, that what is poêlé must be underdone, and a braise must be done through.

As the poêlé has no translation, it retains its name ; it is indispensable in fine cookery, and is made as follows:-

Take one pound of beef suet, one pound of very fresh butter, and one pound of very fat bacon; cut the suet and the bacon into very large dice; put them into a stewpan with two pounds of veal cut in the same manner, fried till the veal becomes very white, and then moisten with about three pints of clear boiling water, a handful of salt, one bay leaf, a few sprigs of thyme, one onion stuck with three cloves, and a great bundle of parsley and green onions: let the whole boil gently till the onion is done; then drain it through a hair sieve, and use it for any thing that may want poêlé. The use of poêlé is to make every thing boiled in it very white and tasty: in the winter it keeps for a week, and is very useful in the larder, particularly if you do not put in any of the fleshy part of the bacon; otherwise the meat that you boil in it will turn quite red, on account of the saltpetre used in curing the bacon.

\section{1.-RISSOLES.}

Pick from the bones and skin any kind of cold poultry; weigh it, and add onethird of grated bread-crumbs, a little cold melted butter, a small onion, previously boiled and finely minced, pepper, salt, and the yolks of two eggs, to bind it together. Roll out, thin, some trimmings of puffpaste; cut it in two inch square pieces; put in the middle of each a teaspoonful of the mince; fold the paste over it, and shape it with a cutter. Egg the shapes; dip them in bread-crumbs, and fry them in lard; serve dry; garnish with fried parsley, and take care that the edges be perfectly closed.

Or :-Pound any kind of cold meat; thicken a little good gravy with cream or butter; season the meat, and mix it with the sauce, until it is well moistened; then roll out some paste into oval pieces; lay a large table-spoonful of the meat on one end; double it over ; press the edges together, and scallop them ; brush the paste over with yolk of egg; sprinkle vermicelli upon it, and fry them.

\section{1.-PUREE DE VOLAILLE.}

Mince the white part of a cold fowl or turkey very finely; then pound it in a mortar: put in two or three spoonfuls of white sauce, and pass it through a sieve, adding a little salt and white pepper, and warm the whole. It may be served up with the legs grilled. It should not be too thin, but can be made the proper consistence by a greater or smaller quantity of white sauce or cream. Garnish with fried bread, or paste cut in shapes. It may also be served up in a casserolle, or wall of rice and mashed potatoes, or with poached eggs. 
993.-BOILED CHICKENS.

Chickens should be plump, or they form a meagre dish; they should receive much attention in the boiling; they require less time than a fowl, and are sent to table with white sauce, and garnished with tufts of white broccoli.

\section{4.-CHICKENS PULLED.}

Remove the skin carefully from a cold chicken, then pull the flesh from the bones, preserving it as whole as you can. Flour them well, fry them a nice brown in fresh butter; draw them, and stew in a good gravy well seasoned; thicken a short time before serving with flour and butter, and add the juice of half a lemon.

\section{5.-CURRIED CHICKENS.}

Lay the pieces of a dressed chicken into a stewpan with a sliced onion fried brown, a clove of garlic, and some good white gravy: simmer till the chicken is tender, add a spoonful of curry powder, flour rubbed smooth with a lump of butter; a quarter of a pint of cream, with a little salt, may be added twenty minutes before serving: squeeze a little lemon into it, and put an edging of rice round the dish.

\section{6.-TO BRAISE CHICKENS.}

Bone the chickens, stuff them with forcemeat, place in the stewpan the bones and trimmings: lay the chickens upon them with a braise of sweet herbs, onions, mace whole, some thin slices of bacon, about three parts of a pint of stock, or, if that is not handy, water, two glasses of sherry: the bacon should be added last. Cover close, and stew for two hours. Then take out the chickens, strain the braise, remove the fat, and boil the braise rapidly to a glaze, paint it over the chickens with a brush while the braise is being boiled; brown the chickens before the fire; it adds to their appearance. When glazed, fowls may be braised in the same manner.

\section{7.-FRIED CHICKEN À LA MALABAR.}

The Indian receipts for cooking chicken are very numerous; we select the following. Cut up the fowl as for a stew. removing the joints carefully and carving the body into handsome shapes; remove all moisture with a clean dry cloth, and powder every part with curry ; fry it in fresh butter, to which half a teaspoonful of curry has been added, a pale brown; cut into small pieces two or three onions, and fry in clear butter, sufficient to keep the pan from burning; but not more than should be absorbed by the onion after some time frying. It is as well here to say, that as onions are frequently used in the curried poultry by the Indian cooks, they employ the following method. When to be cut small, they slice the onions and then separate them into rings, cutting these rings into the sizes they may require, which. if a little more labor, yet presents a better appearance; when they are fried sufficiently to have absorbed the grease in the pan without in any degree having been burned, spread them over the chicken and serve; a whole lemon should be sent to table with them.

\section{8.-PIGEONS ROASTED.}

Veal stuffing for pigeons improves the flavor; they must be fresh and well cleaned; butter and parsley may be served with them, but parsley alone as a stuffing, though frequently used, is by no means so palatable as the veal stuffing, or one made with veal, the fat of bacon and the crumb of bread soaked in milk and well seasoned. Let your pigeons be picked clean and washed, then stuff the whole inside of the pigeon with fine stuffing.

To Broil.-Split the backs, season them highly, lay them over a clear brisk fire: serve with mushroom sauce. 
To Cook as Woodcocks.-Toast some bread as for woodcocks, butter it, and drop a few drops of essence of anchovies on the butter, spread it all over the toast, then put the toast under the pigeons while roasting.

To Stew.-Take a white cabbage, cut it as for pickling, rinse it in clear cold water, drain it well, put it into a saucepan with equal quantities of milk and water, boil it, strain off the milk, and take a portion of the cabbage and lay it in a stewpan; soak the pigeons for half an hour in cold milk and water, season them well with salt and pepper, adding a little cayenne; place them in the stewpan with the cabbage, cover them over with what remains, add some white broth, stew slowly until the pigeons are tender, thicken with a little cream, flour, and butter; let it boil, and serve up the pigeon with a puree of the cabbage. Lard and braise them in the same manner as chickens.

\section{9.-COMPÔTE OF PIGEONS.}

Blanch four pigeons, then stuff them, place them in a stewpan, put in an onion, a slice of lemon, and a small handful of mushrooms ; lay over them slices of fat bacon, add half a pint of good gravy; and stew gently until the pigeons are tender ; take them out of the gravy and, keep them hot, strain off the gravy, skim it clear. then thicken with half a spoonful of flour, and a lump of butter, the size of a small walnut; season with salt, pepper, and a little cayenne; a few forcemeat balls may be added at pleasure; pour half into the dish with the pigeons, and serve the remainder in a tureen.

\section{0.-PIGEONS IN JELLY.}

Make some jelly of calf's foot, or if you have the liquor in which a knuckle of veal has been boiled, it will answer the same purpose; place it in a stewpan th with a bunch of sweet herbs, a blade of mace, white pepper, a slice of lean bacon, some lemon-peel and the pigeons, which, being trussed and their necks propped up to make them appear natural, season to your palate. Bake them; when they are done remove them from the liquor, but keep thern covered close, that their color may be preserved. Remove the fat, boil the whites of a couple of eggs with the jelly to clear it, and strain it; this is usually done by dipping a cloth into boiling water, and straining it through it, as it prevents any thing like scum or dirt sweeping through the strainer. Put the jelly rough over and round the pigeons.

\section{1.-PIGEONS OR ANY BIRD IN ASPIC JELLY.}

Get three pigeons, take out the bones, leaving on the neck and head; fill the birds with some forcemeat, making them the shape of the bird, cover them with fat bacon, put them on a dish, place them in the oven until done, take them out to get -cold, have ready some very light colored aspic jelly; either chop your jelly round them on the dish or in a mould, if in a mould place the breast downwards, let the jelly be quite cold and beginning to set before you pour it in; but if you intend to ornament your mould, do it with green, red, and white devices, with what may then be in season; radishes, whites of hard eggs, cucumbers, French beans, truffles, and any other eatable and wholesome thing: never use any thing that is injurious to health; set it to get stiff, turn out with warm water.

\section{2.-LARKS OR SMALL BIRDS.}

Be very particular in roasting; melt a little butter, add to it a yolk of egg, and with your paste-brush egg all over them, and then bread-crumb them ; while roasting frequently baste them and flour them, and before you take them up fiour 
and salt them; send them up with some brown bread-crumbs.

\section{3.-GUINEA FOWLS.}

These birds must be very young: for, being naturally very dry, they are not eatable if more than twelve months old; they are generally larded, and served plain roasted, rather well done; they are trussed like the common fowl, and require nearly three-quarters of an hour to roast. It has very much the flavor of the pheasant, and should be allowed to hang as long as it can without being too far gone. Serve with a rich brown gravy and bread sauce; it will take from fortyfive to fifty minutes.

\section{4.-PEA FOWLS.}

These magnificent birds make a noble roast, and when young are very excellent; they are larded, or plain roasted, and served with the tail feathers which have been preserved, stuck into the bird, the head, with its feathers on, being left foldel up in paper, and tucked under the wing; roast about an hour and a half; take the paper from the head and neck; dress it upon your dish with watercresses, and the gravy and bread sauce separate in a boat.

\section{MADE DISHES OF POULTRY.}

\section{5.-FOWL A LA PROVENÇAL.}

Partly roast the fowl; cut it up, detaching the wings and legs, carefully dividing side bones, neck bones, breast, and back in as handsome pieces as possible; take eight or ten large onions, which cut in slices of moderate thickness, make in a stewpan a layer of the sliced onion with some chopped parsley, then lay upon it some of the fowl, again a layer of the onion and parsley until the whole of the fowl and onion are used; place in two bay-leaves and about as much salt as would fill a large teaspoon, four tablespoonfuls of olive oil, or, if that is not to the palate, substitute cream; it should simmer gently until enough, and then be dished, the onion in the middle: serve with a little sauc

\section{6.-FOWL À LA BÉCHAMEL.}

If you have had a roast capon for the second course the day before, and only a little cut from the breast, take a sharp knife and cut through the breast bone down nearly to the rump, left of the breast, take off the skin and cut it up as you would for pulled fowl; have ready in a stewpan a little good white sauce, chopped parsley, and shalot, a little piece of mushroom chopped; boil all this together, keeping it quite thick, scrape out any thing remaining in the inside of the fowl, then season with lemon-juice, salt, and cayenne pepper : fill in the fowl, imitating a whole fowl, cover it with breadcrumbs, sprinkle clarified butter all over the bread-crumbs, butter paper and tie round the fowl, put it into the oven to brown the top and get hot through; when done a light brown take off the paper, and put it into your dish with a good sauce under it.

\section{7.-AN INDIAN PILAU.}

Truss a fowl as for boiling, pass it a few minutes in the oven, raising it up with fat bacon or buttered paper; fry some onions, a few bruised coriander seeds. and a few cardamom seeds whole: fry a nice light color four onions cut in slices, add to this a gill or more of cream, having all fried in a little butter; put in your fowl with some good veal stock, have ready some rice boiled in milk for two minutes, skim it off and add it to the fowl, frequently looking at it and moving it to keep it from sticking or 
burning: let your fowl stew for a quarter of an hour before you add the rice: do not let the rice get mashed; season with cayenne pepper and salt, put all the rice and liquor round the fowl: you can use rabbits, or chickens, or quails, or veal, instead of fowl, the same way.

Gravy may be made thus: Nicely wash the feet of a fowl, and cut them and the neck into small pieces, simmer them with a little browned bread, a slice of onions, a bit of parsley, and thyme, some pepper, and salt, and the liver and gizzard in a quarter of a pint of water ; simmer them till they are reduced to a half; take out the liver, bruise it, and strain the liquor to it, then thicken it with flour and butter, and add a teaspoonful of mushroom ketchup.

\section{8.-CURRY OF CHICKEN.}

Cut up a raw chicken; put into a stewpan, with two ounces of butter, half a large onion sliced thin, a few sprigs of parsley and thyme, and two ounces of lean ham; let the whole sweat over the fire for a few minutes; add a heaped table-spoonful of curry-powder, and a small one of flour; shake the whole together for five minutes over the fire; put to it a pint of either gravy or water ; let the whole go gently until the chicken is done; take out the chicken, rub the sauce through a sieve, boil it up, skim, put in the chicken, season with salt and lemonjuice to palate. Plain boiled rice to be served in a separate dish.

Croquettes.-Take the lean of the remains of a fowl from a previous dinner, and chop it up in small pieces, then put into a stewpan a teaspoonful of chopped eschalots, with half an ounce of butter: pass them for about three minutes over the fire, add a teaspoonful of flovr, mix well, then add the fowl, and a gill of white sauce, or more if not sufficiently moist; season with pepper, salt, and sugar; then stir in the yolks of two eggs very quickly, stir it a little longer on the fire, and turn it out on a dish to cool ; when cold, take twelve pieces, each the size of a walnut; roll them out an inch and a half in length, and bread-crumb twice over; fry a good color.

\section{9.-DUMPOKHT.*}

Clean and truss a fowl, or rabbit, as for roasting; then stuff it with sultana raisins, pistachio-nuts, and boiled rice in equal parts. Rub fine one ounce of coriander-seed, freed from the husks, four onions, a dozen peppercorns, six cloves, and a teaspoonful of pounded ginger. Set twelve ounces of butter in a stewpan over the fire, rub the pounded ingredients over the fowl or rabbit, and let it fry until perfectly well browned and tender. Have boiled in a quart of white broth twelve ounces of rice, two ounces of raisins, two ounces of pistachio-nuts, and two of almonds, the two latter blanched, and cut into thin slices. When the rice is nearly tender, strain off the broth, and add the rice to the fried fowl ; stir the whole well, that the butter may completely saturate the rice, and keep it near the fire to swell till wanted. In serving surround the fowl with the rice.

Observe that, in pounding the onions, the juice only is used with the spices.

\section{0.-TURKEY GIBLETS A LA BOUR- GEOIS.}

The giblets consist of pinions, feet, neck, liver, and gizzard ; scald, and put them into a stewpan with a piece of butter, parsley, scallions. garlic, thyme, bayleaf, basil, mushrooms, and a clove or two; moisten with stock, season with pepper and salt, make it hot, thicken with a little flour, and when almost done add a few turnips fried slightly in a little butter.

* A dish mentioned in the Arabian -Nights as the kid stuffed with pistachio-nuts. 
1011.-TO ROAST A GOOSE.

Goose in itself is of a strong rich flavor, and requires both nicety in the cooking as well as in the stuffing to obviate that strength of flavor. There are many modes of stuffing; for one mode, take two moderate sized onions and boil them. rapidly ten minutes, then chop them finely, mince sage to the quantity of half the onion, add of powdered bread twice as much as of onion, pepper and salt it, introducing a little cayenne. and then bind it with the beaten yolk of an egg. Potatoes mashed are sometimes introduced, but not frequently into the body; they should be mashed with floury potatoes mixed with cream and a little fresh butter rather highly seasoned with cayenne and salt. Both ends of the goose should be secured when trussed, that the seasoning may not escape. It should be roasted before a quick fire and kept constantly basted; a piece of white paper may be placed over the breast while roasting until it rises, and then it may be removed; it will take from an hour and a half to an hour and threequarters: serve with a rich brown gravy and apple sauce.

Previous to sending to table, a flavoring may be made as follows : to a dessertspoonful of made mustard add a quarter of a teaspoonful of cayenne pepper, about the same quantity of salt, mix it evenly with a glass of port wine and two glasses of rich gravy, make it hot, cut a slit in the apron of the goose, and pour it through just previously to serving.

A Green Goose is seldom or never stuffed; the inside may be well peppered and salied, and it should be roasted before a brisk fire about three-quarters of an hour; it should be sent to table with no other accompaniment than a good brown gravy and apple or sorrel sauce.

1012.-PRESERVED GOOSE FOR THE FARM OR. COUNTRY HOUSE. In case you have more geese in condi- large goose, and bone them. Lay the tion and season than what you consume, kill and cut them up into pieces, so that there shall be as little flesh left on the carcass as possible, and bone the leg; rub into each piece with your fingers some sa?t, in which you have mixed a little saltpetre; put them into an earthen pan, with some thyme, bay-leaf, spice, a clove of chopped garlic, rub them for a couple of days, after which dip each piece in water, and dry on a cloth ; when you have chopped fine and melted all the fat you could get from the goose, and scraped a quarter of a pound of fat bacon and melted with it, pass through a sieve into a stewpan, lay the pieces in it, and bake very gently in a slow oven, until a stiff piece of straw will go through it, then lay it in a sieve; when nearly cold put it in a bowl or round preserving jar, and press a smaller one on the top, so that it all forms one solid mass, pour the fat over, when co'd cover with a piece of biadder, keep it in a cold place, and it will be good for months together, and is excellent for breakfast, luncheon, or supper, having previously extracted the fat. Last winter I kept some for three months quite sweet; having half a one left, I put it by in the above way, bones and all, in a basin, and covered with the fat produced by roasting, and put it in the larder, and it was excellent. Ducks may be served in the same way.

Marbled Goose.-Take a fine mellow ox-tongue out of pickle, cut off the root and horny part at the tip, wipe dry, and boil till it is quite tender; then peel it, cut a deep sli'; in its whole length, and lay a fair proportion of the following mixture within it:-Mace half an ounce, nutmeg half an ounce, clove half an ounce, salt two table-sponnfuls, and twelve olives. The olives should be stoned, and all the ingredients well pounded together. Next take a barn-door fowl and a good 
tongue inside the fowl, rub the latter outside with the seasoning, and having ready some slices of ham divested of the rind, wrap them tightly round the fowl; put these inside the goose, with the remainder of the seasoning, sew it up, and make all secure and in natural shape with a piece of new linen and tape. Put it in an earthen pan or jar just large enough to hold it, with plenty of clarified butter, and bake it two hours and a half in a slow oven; then take it out, and when cold take out the goose and set it in a sieve; take off the butter and hard fat, which put by the fire to melt, adding, if required, more clarified butter. Wash and wipe out the pan, put the bird again into it, and take care that it is well covered with the warm butter; then tie the jar down with bladder and leather. It will keep thus for a long time. When wanted for the table, the jar should be placed in a tub of hot water so as to melt the butter, the goose then can be taken out, the cloth taken off it, and sent to table cold. A most delicious dish for breakfasts, suppers, pic-nics, \&c.

\section{DUCKS.}

Ducks may be roasted as soon as killed. Keep a clear bright fire. Let them be done of a light brown, but if wild they should not be much roasted, or the flavor will be spoiled. They take about an hour to roast, and should be well basted. The livers and gizzards are parboiled, chopped fine, and thrown into the gravy.

Canvas back ducks are roasted in half an hour; they should always be served with currant jelly. For tame ducks apple sauce is more appropriate.

\section{3.-TO STEW DUCKS.}

There is a difference between $a$ stewed duck and sterred duck, and it is not the $a$ alone; in the one case the duck is stewed whole, and in the other in pieces. To stew a duck or ducks, they should be stuffed and roasted for twenty minutes, and then placed in a stewpan with an onion cut in slices, a little sage and mint, and sweet herbs chopped fine, and about a pint of good beef gravy, seasoned with pepper and salt; let it stew gently for about twenty minutes, take out the duck carefully and keep it warm, strain the gravy, pour it into a clean stewpan, and add to it when well heated the duck and a quart of green peas; let it simmer for half an hour, if not sufficiently thick add a little flour and butter, a glass of good old port wine, and send to table with the peas in the same dish as the duck.

\section{4.-TO STEW A DUCK WITH CLARET.}

Make a gravy of the giblets; rub the duck inside and outside with pepper and a little salt; peel and mince two or three onions, and having half roasted the duck, put it into a stewpan with the gravy and onions. Stew it gently for two hours, adding toward the end a glass of claret or port wine. Squeeze the juice of a lemon over the duck, and serve it with toasted bread.

What remains of a roasted wild duck, may be warmed the next day in heaters with a little currant jelly, when it is excellent.

Ducks may be hashed and stewed with a glass of port wine, thrown into the gravy.

\section{5.-TO BOIL DUCKS.}

Clean and pluck them, let the skin be preserved from rents while plucking, salt them for about thirty hours previous to cooking, flour a clean white cloth and boil them in it; a moderate sized duck will take about an hour's boiling, make a rich onion sauce with milk, and send it to table with the duck. When the duck 
is boiled fresh it may be stuffed as for roasting, and served with the same description of gravy.

1016.-STEWED DUCK AND PEAS.

Procure a duck trussed with the legs turned inside, which put into a stewpan with two ounces of butter and a quarter of a pound of streaked bacon, let remain over a fire, stirring occasionally until lightly browned, when add a tablespoonful of flour mixed well, and a quart of broth or water: stir round gently until boiling, when skim. and add twenty button onions, a bunch of parsley, with a bay-leaf, and two cloves. let simmer a quarter of an hour, then add a quart of nice young peas, let simmer until done, which will take about half an hour longer, take out the duck, place it upon your dish (taking away the string it was trussed with), take ou' the parsley and bayleaf, season the peas with a little pepper, salt, and sugar, skim the fat, reduce a little if not sufficiently thick, pour over the duck, and serve.

\section{7.-DUCKLING WITH TURNIPS}

Is a very favorite dish among the middle classes in France. Proceed as in the last, but instead of peas use about forty pieces of good turnips cut into moderatesized square pieces, having previously fried them of a light yellow color in a little butter or lard, and drained them upon a sieve; dress the duck upon a dish as before, season the sauce with a little pepper, salt, and sugar: reduce until rather thick, a thin sauce not suiting a dish of this description; the turnips must not, however, be in purée; sauce over and serve.

\section{8.-WILD DUCKS, OR TEAL.}

You must be very particular in not roasting these birds too much; baste them very frequently; teal will of course take less time; but your fire and motion of the spit must be attended to, and when you dish it, unless preferred to be done by the gentleman at the table, draw your knife four times down the breast; have ready a little hot butter, and juice of a lemon, cayenne pepper, a little dust of sugar, a glass of port wine, pour it all hot, the last minute, over your ducks; the remainder left of those birds the next day makes excellent salmi or hash. taking care of all the gravy that may remain.

They must be roasted at a very brisk fire ; they take from twelve to twenty minutes, according to taste. Some people are of opinion that they should only fly through the kitchen: by epicures they are considered to be in perfection when they come up dry and brown. and, when cut, flood the dish with gravy. The means of insuring success consists in a very ardent fire, rapid motion of the spit, and constant basting. The carver should score the breast of the duck, put a piece of butter on it, and cut a lemon in half, putting on one half a spoonful of salt, and on the other a cayenne-spoonful of cayenne; put the two together, and squeeze vigorously over the duck.

To stew.-Shred thyme, winter savory, and sage, very small, put them into some strong broth, with a little pepper, salt, and two spoonfuls of wine: stew them together for one quarter of an hour; nearly roast the ducks, add the gravy that falls from them, but not the fat; then place a deep dish under them, and pour this sauce through, and over them into it. Remove the ducks, cut them up, and put them with the sauce into a stewpan over a stove, and let them stew till they are done enough.

Canvas Back Duck should be cooked like wild duck, but nearer the fire, the fat being so delicate, that it requires to be cooked quickly; good plain gravy is 


all that is required to make them per-
fect.

1019.-PLOVERS.

Roast the golden plover in the same way as woodcocks and quails, without drawing; and serve on a toast.

Grey Plovers.-Draw them : they may be either roasted or stewed with gravy, herbs, and spice.

Plover's Eggs.-Boil them ten minutes, and serve either hot or cold; the former on a napkin, the latter on moss. Send to table with the second course.

\section{0.-PARTRIDGES,}

being less dry than pheasants, do not require stuffing, although they are improved by it, made either of chesnuts or truffles and bacon. They are sometimes roasted, wrapped in bacon and vineleaves: bread-crumbs are essential. In some places partridges are sent up with forcemeat-balls in the dish.

\section{1.-TO STEW PARTRIDGES.}

Truss them with the wings over the back, and the legs drawn in; cut a piece of pork or bacon in long strips, and put them into a stewpan with a piece of butter the size of a walnut. Fry the bacon brown, and when quite done put in the partridges, and keep turning them until they are very brown, taking care that the bacon shall be as much on the breast as possible ; then add about a teacupful of gravy, and some trimmings of meat and vegetables. Have ready a large cabbage boiled; when well drained, slice it with butter, pepper, and salt, put it while warm with the gravy to the partridges, and let them stew gently for an hour, turning the birds frequently. Serve up with the bacon underneath, and the cabbage round them, squeezed dry, and the sauce well skimmed.

\section{2.-SALMI OF PARTRIDGE.}

Half roast the partridges, cut them up neatly, take off the skin; put the trimmings in a stewpan, with a bit of butter and a tablespoonful of flour; stir the whole over the fire; then add a glass of white wine and a little gravy or stock, some shalots; chopped parsley, thyme, bay-leaf pepper, salt; let it boil fast for half an hour ; strain, skim. add the juice of a lemon, and make the joints of the partridge hot in the sauce.

Or:-Roast the partridges, leaving them underdone. When cold. cut them into pieces, taking off the skin. Put three spoonfuls of oil in to a saucepan, a glass of claret, salt, pepper, a shalot, and the juice of a lemon. Toss the partridges in this sauce until they are sufficiently done to send to table.

Or:-Cut up and place on a dish the breasts, wings, and legs of any roasted game you may have left; cover it with another dish, or tin cover, and set it over a pan of boiling water to keep warm. Then pound the small bones of the carcase, with the inside, in a mortar, and put them into a saucepan with a little gravy thickened with some flour rolled in butter, a wine-glass of port wine, a spoonful of ketchup, a very small piece of shalot chopped fine, cayenne pepper, and salt. When it has been boiled for half an hour, strain it through a sieve, to form it into a perfect purée, and pour it over the game in your dish; garnish with forcemeat-balls, and slices of lemon cut thin.

\section{3.-BROILED PARTRIDGE.}

Cut the bird down the back; break the merrythought, which will allow it to be made quite fat; cut off the feet at the joint, and skewer it as a fowl to broil ; dry, flour, egg, and sprinkle it with chopped herbs and bread-crumbs, well seasoned; broil and serve with a little 
good gravy, with a mushroom or two chopped up small.

\section{4-GROUSE.}

Mix a small lump of butter with a few bread-crumbs, and put it in the inside of the birds-not in the crop-it keeps them moist. They require to be nicely roasted and well basted.

\section{5.-RABBITS.}

To Fricassee a rabbit cut it in pieces, reserve the liver; put a piece of bacon cut in slices into a stewpan, brown it well and lay it aside; put the rabbit in the pan with a piece of butter; turn and toss the rabbit well until it is quite white. When it is getting brown dredge a spoonful of flour over it, turning all the while, and when the flour is dry, put the rabbit in a plate. Then add another piece of butter to the remainirg sauce, and stir it well together, and when brown, add a cupful of water; continue stirring; and put in the rabbit with plenty of very small onions, the bacon, some mushrooms, a bunch of parsley, and some salt and pepper; let it stew very gently over a slow fire for four hours. Add the liver and a glass of port wine one hour before serving, and should there not be sufficient sauce, a little gravy from time to time, put in hot.

When a rabbit is roasted, it should first be well stuffed. Serve it with sauce made of liver, parsley, and melted butter. Truss it like a hare.

Rabbits may be stewed, or fried, or baked in a pie.

Rabbits, being rather' dry meat, are much improved by larding. Should the process be deemed too troublesome upon common occasions, a good effect may be produced by lining the inside of the rabbit with slices of fat bacon previously to putting in the stuffing. This is a very easy method of improvement, and ought never to be neglected.
A boned rabbit, larded, stuffed, and braised, affords a cheap and elegant sidedish for a dinner-party.

1026.-TO MAKE RABBIT TASTE MUCH LIKE HARE.

Choose one that is young, but fullgrown, hang it in the skin three or four days, then skin it, and lay it without washing in a seasoning of black pepper and allspice in a very fine powder, a glass of port wine, and the same quantity of vinegar. Baste it occasionally for forty hours; then stuff it and roast it as a hare, and with the same sauce. Do not wash off the liquor that it was soaked in.

\section{7.-TO ROAST HARE.}

After it is skinned, let it be extremely well wiped; and, if old, lard it, which will make it tender, as will also letting it lie in vinegar. If however, it is put into vinegar, it should be exceedingly well washed in water afterwards. Put a large well-seasoned stuffing into the belly, and then sew it up. Baste it well with milk till half done, and afterwards with butter. The hare should be kept at a distance from the fire at first. Serve with a fine froth, rich gravy, and currant jelly sauce; little gravy in the dish. For stuffing use the liver pounded, an anchovy, some fat bacon, a little suet, herbs, pepper, salt, nútmeg, crumbs of bread, and an egg to bind it all. The ears must be nicely cleaned, singed, and made crisp, being reckoned a dainty.

\section{8.-JUGGED HARE。}

Skin the hare, and cut it in pieces, but do not wash it; strew it over with pepper and salt, fry it brown. Make a seasoning of two anchovies, a sprig of thyme, a little parsley, a nutmeg grated, a little mace, a few cloves pounded, and a teaspoonful of grated lemon-peel. 
Strew this over the hare, after having carefully taken it out of the pan clear of fat, slice half $\mathrm{lb}$. of bacon very thin, put it into a jug or jar, a layer of hare and one of bacon upon it, until the whole is put in, then add rather less than half a pint of ale ; cover the jug very closely, so as perfectly to keep in the steam; put it into a kettle of cold water, lay a tile on the top of the jug, and let it boil three hours if the hare be young, or four or five if an old one. Take the jug out of the kettle, pick out all the bacon which has not melted, and shake the hare up in a stewpan, with a little mushroom ketchup, a glass of port wine, a little mushroom powder if at hand, and a little butter and flour, well mixed together to thicken the gravy. A teaspoonful of lemon-pickle, and one of browning, will heighten the flavor.

\section{9.-PILAU OF RABBIT.}

Cut up a rabbit or fowl; pound a couple of onions in a mortar ; extract the juice, and mix it with a saltspoonful of ground ginger, a teaspoonful of salt, and the juice of a lemon: rub this into the meat; cut up two onions in slices, and fry them in a quarter of a pound of butter. When brown take them out and put in the meat; let them stew together. Have one pound of rice half boiled in broth; put the whole meat, \&c., into a jar, with half a pint of milk, some whole peppercorns, half a dozen cloves, and a little more salt. Secure the mouth of the jar, and bake it until done enough, adding a little broth, should the butter and milk not be sufficient to moisten it.

\section{0.-TO CRISP PARSLEY.}

Pick some bunches of young parsley, wash them, and swing them in a cloth to dry; put them on a sheet of paper in a toaster before the fire, and keep them frequently turned, till they are quite crisp, which will be in about six or eight minutes.
1031.-TO FRY PARSLEY.

When the parsley has been washed and thoroughly dried, throw it into lard or butter which is on the point of boiling; take it up with a slice the instant it is crisp, and lay it on a paper on a sieve before the fire. A wire basket is the nicest thing to use for frying parsley, or any thing requiring to be fried.

1032.-FRIED BREAD FOR GARNISHING.

Take slices of stale bread about a third of an inch thick, cut them into shapes with a paste-cutter, fry them in fresh butter a light brown, lay them upon a cloth to dry, and place them round the dish. They may be made to adhere by brushing the under part with a little flour mixed with white of egg.

Bread crumbs.-All game and small birds are improved by the addition of bread crumbs, either fried or toasted; the latter is the more delicate preparation: grate the crumbs, and put them into a tin shallow dish before the fire, shaking them occasionally, until they are well browned: send them up in the dish; with a tureen of good brown gravy made from the insides and trimmings of the birds, but without any kind of seasoning.

1033.-RABBITS, TO SKIN, \&c.

Cut round the skin at the first joint of the hind legs, and pull the skin up a little, then run the knife between the sinew and the bone of the leg, so as to make a hole to hang it on a nail in a wall, with the head downwards; run a knife along the skin inside the thigh as far as the tail, so as to cut it, then run the hand under the skin, and it will easily separate from the body; pull it down towards the head, taking care not to tear the belly: when you have arrived as far as the head, pull up the fore legs, cutting the feet off at the first joint; then pull , up the ears by running a 
skewer between the skin and the head, and pull the skin off, cutting the nose and lips ; remove it from the nail, cut off the hind feet at the first joint; cut open the body, remove the inside, reserve the liver, heart. and kidneys, and throw away the remainder; wash out the inside. If for roasting, make the following stuffing: take the liver, heart, and kidneys, blanch them, put them into a fryingpan with a little fat, and one small onion chopped up; sauté a little, then remove and chop them up with two ounces of pork fat or bacon, a little parsley, and mix with bread crumbs and the yolk of an egg; "stuff and sew up the belly. Truss as follows: bring the hind and fore legs close to the body towards the head, run a skewer through each, then run a skewer in at the mouth and through the neck, to keep it upright; it should be exactly as if it was sitting. Put a string round the body from skewer to skewer, and it is ready.

\section{VENISON.}

\section{4.-THE HAUNCH.}

"The observed of all observers" when epicureans sit at table; it is a joint, if properly kept, properly cooked and served hot, which must prove delicious. The delicacy of its flavor is obtained by hanging only: if it be cooked while fresh, it will not equal in any respect a haunch of mutton.

The haunch of venison, when about to be roasted, should be washed in warm milk and water, and dried with a clean cloth; if it has hung very long and the skin smells musty, it will be the safest plan to remove the skin and wrap the whole of the haunch in paper well greased with fresh butter; during the time it is at the fire, do not be afraid of basting it too much. If it be a buck haunch and large, it will take nearly four hours within five or ten minutes ; if comparatively small, three hours and a half will suffice; if a doe haunch, three hours and a quarter will be enough.

All venison for roasting should have a paste made of lard over it; after first having papered the meat with buttered paper, then your stiff paste upon the top of that, either dangle it or put it in a cradle spit; a few minutes before you require to take it up take off the paste and paper, baste it with some butter, salt it and flour it, when done give it a few more turns round, send it up very hot; your dish and gravy is to be very hot also; any dry pieces and the shank you will boil down with a little brown stock for the gravy; send currant jelly in a boat, and French beans in a vegetable dish.

\section{5.-VENISON STEAKS.}

Cut them from the neck; season them with pepper and salt. When the gridiron has been well heated over a bed of bright coals, grease the bars, and lay the steaks upon it. Broil them well, turning them once, and taking care to save as much of the gravy as possible. Serve them up with some currant jelly laid on each steak.

\section{6.-TO HASH VENISON}

Carve your venison into slices: let them be thin, and put them in a stewpan with two small glasses of port wine: add a spoonful of browning, one of ketchup, an onion stuck with cloves, and half an anchovy chopped small, let it boil, then put in your venison, make it thoroughly hot through. Lay sippets of toast, in various shapes in a soup dish, pour the hash upon it, and serve with currant jelly.

\section{7.- VENISON FRIED.}

Cut the meat into slices, fry a light brown, and keep hot before the fire. 
Make gravy of the bones and any trimmings, add a little floured butter, and stir until it is thick and brown, then add lemon-juice and port wine to taste, with pepper or cayenne. Warm the venison in the gravy, place in a dish, and strain the gravy over it. Serve with currant jelly in a glass.

\section{8.-A SHOULDER OF VENISON STEWED.}

If you should have a very lean shoulder, stew it in preference to roasting; bone it, cover it with slices of mutton fat, which sometimes are first steeped in port to give a richer flavor, roll it up and bind it tightly. Lay it in a stewpan with a quantity of beef gravy, and the bones you have taken from the venison, add two glasses of port, a dessert-spoonful of whole pepper, and the same quantity of allspice. Cover down closely, and simmer until the venison is tender, take it out and remove the fat, thicken the gravy with flour and butter, and strain it over the meat.

\section{9. -ROAST SHOULDER OF VENISON.}

Take the bones out of a shoulder of venison. Fill the cavity with the fat of a loin of mutton. Roast before a quick fire, basting it constantly with the drippings and a little port wine. It should be served underdone with a sauce of port wine, currant jelly and red pepper.

The neck and shoulder, as together they may be termed the haunch joint, may be dressed exactly like the haunch, covered with a thin paste, and greased paper over that.

Game ought not to be thrown away even when it has been kept a very long time; for when it seems to be spoiled, it may often be made fit for eating by nicely cleaning it, and washing with vinegar and water. If there is danger of birds not keeping, draw, crop, and pick them; then wash in two or three waters, and rub them with salt. Have ready a large saucepan of boiling water, and plunge them into it one by one, drawing them up and down by the legs, that the water may pass through them. Let them stay five or six minutes in; then hang them up in a cold place. When drained, pepper and salt the inside well. By this method the most delicate birds may be preserved. Before roasting, wash them well. But, as a rule, no game should be washed, for one-half the game that is sent to table is spoiled by being saturated with water.

In dressing Game be careful to keep a clear fire. Let it be done of a bright brown, but not much roasted, or the fine flavor will be destroyed. It requires to be continually basted, and to be sent up beautifully frothed. Wild-fowl take a much shorter time than domestic poultry.

\section{0.-TO DRESS A FAWN OR KID.}

They should be dressed as quickly after being killed as it is convenient. If they are full grown they should be roasted in quarters, but if very young may be dressed, trussed, and stuffed exactly as a hare; the quarters will be improved in flavor when the fawn is full grown, if they are covered with fat bacon, and basted as venison; serve venison sauce with it.

\section{1.-PHEASANTS. (English receipt.)}

Pheasants are rarely stuffed, it is more customary to send them to table accompanied by forcemeat in the dish, in many cases with the simple gravy only; the real epicurean in game prefers the flavor of the bird uncontaminated by any accessories save those which just assist to 
remove the dryness common to most game. Pheasants are sometimes larded, but as the flavor is entirely disguised by the taste of the bacon, incorporated with the flesh of the fowl, unless it is done more for appearance than palate, we would not advise it.

In dressing the pheasant it should be drawn and cleaned as other game and trussed, should be roasted before a clear, not a fierce fire, and will take forty minutes; it must not be done too much, but must not on any account be sent to table underdone.

\section{1.-WOODCOCK, SNIPE, ETC.}

To roast these birds, put them on a little spit, take a slice of wheaten bread, and toast it brown, then lay it on a dish under the birds ; baste them with a little butter, and let the trail drop on the toast. When they are roasted, put the toast in the dish, lay the woodcocks on it, and have a quarter of a pint of gravy; pour it in a dish, and set it over a lamp or chafing-dish for three minutes; and send them to table. Epicures say you should never take any thing out of a woodcock or snipe. The head of the woodcock is considered a great delicacy.

To roast pheasants, partridges, quails, and other small birds, pick and draw them immediately after they are brought in. If they are of a nature to be improved by it, lard them with small slips of the fat of bacon drawn through the flesh with a larding-needle.

To roast reed-birds or ortolans, put into every bird an oyster, or a little butter mixed with some finely sifted breadcrumbs. Dredge them with flour. Run a small skewer through them, and tie them on the spit. Baste them with lard or with fresh butter. They will be done in about ten minutes. Reed-birds are very fine made into little dumplings with a thin crust of flour and butter, and boil- ed about twenty minutes. Each must be tied in a separate cloth.

\section{VEGETABLES.}

The fresher all green vegetables are used after being taken from the ground the better; and therefore nothing can be comparable to cutting them from your own garden; but, when purchased in the market, care should be taken that those of the cabbage tribe should appear crisp and vividly green: not too small, nor yet overgrown in size.

When about to be used, the outer leaves should be stripped. the stalks cut short, and the vegetable immersed for an hour or two in cold water, into which should be put a handful of salt for the purpose of thoroughly cleansing it from slugs, and those insects with which the leaves are commonly infested. All green vegetables should be put into boiling water. Never boil any species of greens with meat. Boil them in plenty of pure water, with salt, to which a little vinegar may be added with advantage; do not cover the saucepan, but keep up a steady fire, so that they shall not be allowed to stop boiling until thoroughly done: drain them instantly, for if overboiled they lose their flavor and become insipid; they should be well done, for if that be neglected for the purpose of preserving their crispness, they will be rendered indigestible.

Hard water spoils the color of such vegetables as should be green, but a very small bit of soda or carbonate of am. monia will soften it, and even improve their brightness. The prảctice of putting pearlash in the pot to improve their color, should be strictly forbidden, as it imparts an unpleasant flavor; as will also soda if not cautiously used. 


\section{2.-POTATOES}

Require no attention for the preserva. tion of their color, but their flavor will be spoiled if their dressing be not attended to, which, although of the most simple nature, is frequently ill performed. The best mode of doing it is to sort the potatoes, and choose them of an equal size; wash them with a scrubbingbrush, and put them into cold water sufficient to cover them, and no more. About ten minutes after the water has come to a boil, take out the half of it, and replace with cold water, to check it; the reason assigned for which is, "that the cold water sends the heat from the surface to the heart, and makes the potatoes mealy." Then throw in a large handful of salt, leave the pot uncovered, and let it remain upon the fire to simmer until the potatoes are done; this is the moment to be watched, for if overboiled, they will become waxy. The cook should, therefore, occasionally try them, by piercing them to the heart with a fork, and, when they are tender, the pot should be instantly taken off the fire, and the potatoes passed through a colander to drain; which being done, and the water thrown out, they should then be replaced upon a folded flannel, in the same pot, which should be left by the side of the fire to keep hot and to cause the evaporation of the steam. When served, they should be wrapped in a warmed cotton napkin. If of moderate size they will take about half an hour boiling, to which fifteen minutes must be added for evaporation, ere they can be sent to table.

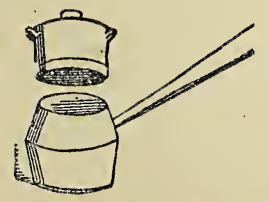

Potato Steamer and Saucepan.
An iron pot is the best vessel for boiling potatoes in, since, after the water has been poured off, it retains sufficient heat to dry them thoroughly.

A good and economical mode of dressing potatoes, when soup, meat, or other eatables are to be boiled, is to havo a tin strainer fitted to the mouth of the saucepan, so as to allow the steam to ascend from the boiler.

Notwithstanding the directions here given for cooking potatoes, there is a point, on which there exists a difference of opinion, that is respecting the peeling of the roots, whether before or after their being boiled?

In Lancashire, England, where they are largely grown and admirably boiled, they are first peeled; while in Ireland they are invariably brought to table "with their jackets on." Count Rumford also recommends their being boiled in their skins; the cuisinier Bourgeois likewise thinks that "les pommes des terre sont meuilleures en robe de chambre."

\section{3.-TO KEEP POTATOES.}

Buy them as dug from the ground, without taking off the earth which adheres to them, and never wash them until wanted to be dressed. Place them in a dry cellar, upon straw, and cover them in winter with straw or mats, to guard them from the frost.

\section{4.-NEW POTATOES}

Should be dressed as soon as possible after being taken from the ground, and are always best when grown in frames. When washed, they should be rubbed with a coarse cloth, and a little salt to take off the thin outer skin, but they should not be peeled. Put them into boiling water; they will require but a few minutes to do them; send them to table in a hot napkin, unless covered with white sauce, which should be seasoned with a 
little salt and a slight grating of nutmeg. When quite young they should never be sliced nor fried.

\section{5.-POTATO FLOUR.}

Rasp the potatoes into a tub of cold water, and change it repeatedly until the raspings fall to the bottom like a paste; then dry it in the air, pound it in a mortar, and pass it through a hair sieve. It is nearly as nutritive, and much lighter than wheaten flour; it is, therefore, preferable for making puddings and th pastry for infants and invalids ; a portion of it also improves the appearance of household bread, and dealers constantly pass it off as arrowroot. If kept dry, it will remain good for years.

Jelly is made from the flour; only boiling water must be poured upon it, but care must be taken that it be absolutely boiling or the complete change into jelly will not take place. It does not take many minutes to thus change a raw potato into this substance, which is not only highly nutritive, but extremely agreeable to the palate, when flavored with a little sugar, nutmeg, and white wine.

\section{6.-POTATO-WALL}

Or edging, to serve round fricassees, forms also a pretty addition to a corner dish.-Mash in a mortar as many boiled potatoes as you may want, with a good piece of butter ; then, with the bowls of two silver spoons, raise a wall of it two and a half inches high within the rim of the dish to be used. Let the upper part be a little thinner than the lower; smooth it; and, after brushing it all over with egg, put it into the oven to become hot and a little colored. Before egging it, the outside may be ornamented with bits of paste cut into shapes.

1047.-TO MASH POTATOES. Let them be thoroughly boiled; peel until nicely browned. them, and take out all the specks, and little hard lumps which are sometimes found. Beat them till quite fine in a wooden bowl or mortar, or break them thoroughly with a three-pronged fork; sprinkle a little salt, and mix them up smoothly with butter or boiling milk. Take care that the potatoes shall not be too wet, or they will become heavy and watery; great smoothness, lightness, and a rich taste are required in mashed potatoes: if required to be very fine, rub them through a colander or sieve before adding the boiling milk and butter.

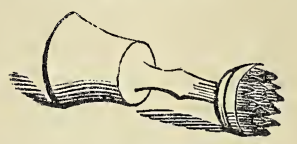

(Potato Masher.)

\section{8.-POTATO-LOAVES}

Are very nice when eaten with roast beef or mutton, and are made of any portion of the mashed roots, prepared without milk, by mixing with them a good quantity of very finely minced raw shalot, powdered with pepper and salt; then beating up the whole with a little butter to bind it, and dividing it into small loaves of a conical form, and placing them under the meat to brown, that is, when it is so nearly done as to impart some of the gravy along with the fat.

\section{9.-TO BROWN POTATOES.}

While the meat is roasting, and an hour before it is served, boil the potatoes and take off the skins; flour them well, and put them under the meat, taking care to dry them from the dripping, before they are sent to table. The kidney potatoes are bost dressed in this way. The flouring is very essential.

Potatoes, when boiled, if either waxy, or to be eaten with cold meat should be peeled and put whole upon the gridiron 
Potatoes should always be boiled a little before being put into stews, \&c., as the first water in which they are cooked is of a poisonous quality.

Fried Potatoes may be cut from raw potatoes, pared round and round like an apple; cut them in slices one half inch thick; fry quickly, in plenty of hot fat, and with as little color as possible; dry them well from the grease, and sprinkle with salt. When nicely done and piled up properly, fried potatoes make a beautiful side dish, which is always eaten with great relish.

\section{0.-POTATO RISSOLES.}

Boil the potatoes floury; mash them, seasoning with salt and a little cayenne; mince parsley very finely and work up with the potatoes, adding eschalot also chopped small; bind with yolk of egg; roll into balls and fry with fresh butter over a clear fire. Meat shred finely, bacon or ham may be added.

\section{1.-POTATO SOUFFLA.}

Take any number of large potatoes, the less eyes and the firmer the skin the better. Clean them thoroughly and then bake them; after which cut out a round piece, not quite so large as a halfcrown, out of each potato, and remove as much of the inside as can be obtained without danage to the skin. Mash the potatoes with cream, adding a little butter, sprinkle over a little salt, and put to it half a pint of good milk; give it all a boil ; take the white of three eggs, whip them until they froth, add them to the potatoes while they boil, and then make the potatoes into a paste; return them through the orifice in the skin of the potato until each skin is full; bake them, and serve.

1052,-POTATOES À LA MAÎTRE D'HOTEL.

Boil the potatoes; before they are quite done take them up, place them aside, and let them get cold; cut them in slices of a moderate thickness ; place in a stewpan a lump of fresh butter, and a teaspoonful of flour; let the butter boil, and add a teacupful of broth; let it boil and add the potatoes, which you have covered with parsley, chopped fine, and seasoned with pepper and salt; stew them five minutes, remove them from the fire; beat up the yolk of one egg with a table-spoonful of cold water and a little lemon-juice. The sauce will set, then dish up the potatoes.and serve.

Or, Cold potatoes that have been boiled should be used for this purpose. Lay them in a frying pan with sufficient milk (or cream) to cover them, add a little butter, salt, and chopped parsley, and fry them until the milk thickens. They will be sufficiently cooked in a quarter of an hour, and make an excellent dish for breakfast.

To Roast.-Clean thoroughly; nick a small piece out of the skin, and roast in the oven of the range; a little butter is sometimes rubbed over the skin to make them crisp.

\section{3.-POTATO OMELETTE.}

May be made with a mashed potato or two ounces of potato-flour and four eggs, and seasoned with pepper, salt, and a little nutmeg. It should be made thick, and, being rather substantial, a squeeze of lemon will improve it. Fry a light brown.

\section{4.-BISCUITS OF POTATOES AND POTA. TO FLOUR. (French.)}

Take fifteen fresh eggs, break the yolks into one pan and the whites into another. Beat the yolks with a pound of sugar pounded very fine, scrape the peel of a lemon with a lump of sugar, dry that, and pound it fine also; then throw into it the yolks, and work the 
eggs and sugar till they are of a whitish color. Next, whip the whites well and mix them with the yolks. Now sift half a pound of flour of potatoes through a silk sieve, over the eggs and sugar. Have some paper cases ready, which lay on a plafond, with some paper underneath. Fill the cases, but not too full; glaze the contents with some rather coarse sugar, and bake the whole in an oven moderately heated.

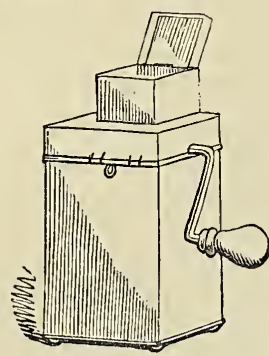

Julienne Mill.

1055.-GREEN PEAS.

A delicious vegetable, a grateful accessory to many dishes of a more substantial nature. Green peas should be sent to table green; no dish looks less tempting than peas if they wear an autumnal aspect. Peas should also be young, and as short a time as possible should be suffered to elapse between the periods of shelling and boiling. If it is a matter of consequence to send them to table in perfection, these rules must be strictly observed. They should be as near of a size as a discriminating eye can arrange them; they should then be put in a cullender, and some cold water suffered to run through them in order to wash them; then having the water in which they are to be boiled slightly salted, and boiling rapidly, pour in the peas; keep the saucepan uncovered, and keep them boiling swiftly until tender; they will take about twenty minutes. barely so long, unless older than they should be ; drain completely, pour them into the tureen in which they are to be served, and in the centre put a slice of butter, and when it has melted stir round the peas gently, adding pepper and salt; serve as quickly and as hot as possible.

It is commonly a practice to boil mint with the peas; this, however, is very repugnant to many palates, and as it may easily be added if agreeable to the palate, it should not be dressed with the peas, although it may accompany them to table for those who may desire the flavor.

\section{6.-TO DRESS SPANISH ONIONS.}

Take off two skins, be particular in not cutting the stalk on the root of the onion too much away, if you do, when done it will drop to picces. Take four large onions, put them in a stewpan sufficiently large, so that they may not touch each other, put in a small piece of lean York ham and a quarter of a pound of salt butter, cover them close, put them on a slow stove or oven, keeping them turned carefully until all sides are properly done: they will take about two hours: then take them up and glaze them, thicken the gravy, and season with pepper and salt.

\section{7.-SALSIFY AND SCORZANERA.}

Blanch several heads until the skin will peel off; when you have done that, cut them all in equal lengths; if to be done in batter, dry them in bread-crumbs, if for sauce make them hot in a little veal stock, dish them, and pour a good white or brown sauce over them.

$$
\text { 1058.-ASPARAGUS. }
$$

Great attention is necessary to boil asparagus. It must be carefully washed and cleaned, the horny part must be cut away, leaving only enough to take it up with the fingers. After the white horny 
part has been well scraped, cut them all off at one length and tie them up in separate bundles; lay them in boiling water with a little salt. Boil them briskly, and they are done enough when tender. Dip a round of toasted bread in the liquor, and lay it in the dish; then pour some melted butter over the toast, and lay the asparagus on the toast entirely round the dish. Serve with melted butter in a sauce-tureen.

\section{9. - ASPARAGUS LOAVES.}

Boil three bunches of asparagus; cut off the tops of two bunches when tender, leaving two inches of the white stalk on the rest, and keeping it warm; stew the tops in a pint of new milk, with three table-spoonfuls of butter, rubbed in flour, the yolks of three eggs, nutmeg and mace; when it boils put the mixture into loaves of rolls, with the crumb scooped out; put on the tops of the rolls; make holes in the tops, and stick in the remaining asparagus.

\section{0-ASPARAGUS FORCED.}

Scoop out the crumb of three or four French rolls, preserving the piece cut from the top, which will have to be fitted on to the part it was cut from ; put into a pan with some fresh butter, the rolls, and fry them brown, beat up with a pint of cream, the yolks of six eggs, flavor with some grated nutmeg and a little salt, put it into a stewpan, and over a slow fire; let it gradually thicken, stirring it all the time.

Have ready the tops of a bundle of asparagus, say a hundred, and having boiled them tender, put them into the cream and fill the rolls with the mixture, reserving a few tops to stick in each roll by way of garnish.

1061.-ASPERGE EN PETIT POIS, OR ASPARAGUS PEAS. If the asparagus be properly dressed, up fine, mixing crumbs of bread, salt and it should taste like green peas. Take some young asparagus, which pick with great care; then cut them into small equal pieces, avoiding to put in such parts as are hard or tough. Wash them in several waters, and throw them into boiling water, with a little salt. When the asparagus are nearly done, drain them first through a sieve, and next wipe them quite dry with a towel. Then put them into a stewpan with a small bit of butter. a bunch of parsley and green onions, and toss them in the stewpan over the fire for ten minutes. Now add a little flour and a small lump of sugar, and moisten with boiling water. They must boil over a large fire. When well reduced, take out the parsley and green onions, and thicken with the yolks of two eggs beaten with a little cream and a little salt: remember that in this entremêt sugar must predominate, and that there is to be no sauce. Asparagus are always to be dressed in this manner when to be served in the second course; but for first course dishes, throw them into some good sauce tournée, well reduced. Boil them a few times over a large fire, then powder a little sugar, and make a thickening of one egg. The sauce must be made thick, on account of the asparagus always yielding a certain quantity of water, which will thin the sauce.

\section{2.-EGG PLANT.}

Cut the egg plant in slices half an inch thick, and let it lie for several hours in salted water, to remove the bitter taste. To fry it put the slices in the frying-pan with a small quantity of butter, and turn them when one side is done. Be sure that they are thoroughly cooked. Stuffed egg plant is sometimes preferred to fried. Peel the plant whole, cut it in two. and let it lies in salted water. Then scoop out the inside of the plant, chop it 
butter with it; fry it, return it to the hollow egg plant, join the cut pieces together, and let them bake awhile in an oven. The best way of cooking is to slice them, dip the slices into egg and bread-crumbs, and fry very brown in butter or lard.

\section{3.-SALSIFY OR OYSTER PLANT.}

Scrape the salsify, cut it in long slips and parboil it, then chop it up fine and fry it with egg batter.

It is sometimes served with the roots whole, having been first thoroughly boiled and then fried in egg batter.

\section{4.-CAULIFLOWER DRESSED LIKE MACCARONI.}

Boil a cauliflower in milk and water, with a little butter, half an hour, skimming well ; when tender, drain, and divide it small ; put a quarter of a pound of butter, half as much grated cheese, half a gill of milk, in a pan to boil up, and put in the sprigs of cauliflowerstew five minutes; then put it into a dish, grate over it as much more cheese, and brown it with a shovel.

To dress Cauliflowers, separate the green part, and cut the flower close to the bottom from the stalk; let it soak an hour in clear cold water, and then lay it in boiling milk and water, or water alone, observing to skim it well. When the flower or stalk feels tender, it is done enough, and should be instantly taken up. Drain it for a minute, and serve it up in a dish by itself, with plain melted butter in a sauce-tureen.

\section{5 -SPINACH.}

Pick it clean, and wash it in five or six waters. Drain it, and put it in boiling water. Ten minutes is generally sufficient time to boil spinach. Be careful to remove the scum. When it is quite tender take it up, and drain and squeeze it well. Chop it fine, and put it into a saucepan with a piece of butter and a little pepper and salt. Set it on hot coals, and let it stew five minutes, stirring it all the time.

\section{6.-TURNIPS.}

White turnips require about as much boiling as potatoes. When tender, take them up, peel and mash then-season them with a little salt and butter. Yellow turnips require about two hours boiling-if very large, split them in two. The tops of white turnips make a good salad.

1067.-BEETS.

Beets should not be cut or scraped before they are boiled, or the juice will run out, and make them insipid. In summer they will boil in an hour-in winter, it takes three hours to boil them tender. The tops in summer are good boiled for greens. Boiled beets cut in slices, and put in cold spiced venegar for several days, are very nice.

\section{8.-PARSNIPS AND CARROTS.}

Wash them, and split them in twolay them in a stewpan, with the flat side down, turn on boiling water enough to cover them-boil them till tender, then take them up, and take off the skin, and butter them. Many cooks boil them whole, but it is not a good plan, as the outside gets done too much, before the inside is cooked sufficiently. Cold boiled parsnips are good, cut in slices and fried brown.

\section{9.-SEA-KALE}

Requires to be very well done ; there is little occasion to fear doing it too much; tie in bundles after washing and trimming, boil it in equal parts of milk and water, and serve it with melted butter.

It may be laid on toast or not, according to taste. 
After being well boiled it must be thoroughly drained before laying upon the toast; five-and-twenty minutes will be found sufficient to boil it. Or, after being drained, it can be put in a stewpan and stewed in a rich gravy.

\section{0.-TO STEW CELERY.}

Wash the heads, and strip off their outer leaves; either halve or leave them whole, according to their size, and cut them into length of four inches. Put into a stewpan with a cup of broth or weak white gravy; stew till tender; then add two spoonfuls of cream, a little flour and butter, seasoned with pepper, salt, nutmeg, and a little pounded white sugar; and simmer all together.

Or:-Parboil it, cut it into quarters, fry it, and serve it on a napkin, or with beef gravy.

Celery is a great improvement to all soups and gravies, and much used as a white sauce, either alone or with oysters:

\section{1.-SAUER KRAUT,}

Or Sour-Kraut, is a German preparation of cabbage, fermented in layers, between which salt is laid, and the whole pressed closely down with weights for a few days, at the end of which it becomes acid, and the greater part of the juice is drawn off.

In preparing it, the outward leaves and the stalk are removed, the latter being sliced, and the whole cabbage cut into small pieces, which are pressed heavily down, and placed in barrels in a warm cellar until the fermentation is over: when that is complete, put the kraut into a cool place, and it will remain good for years. Some people think it improved by a mixture among the layers of caraway, whole pepper, and juniper-berries, or to have the barrels smoked with the latter.

It is extensively used throughout the northern parts of Europe, as a pleasant vegetable, particularly with salted meats and sausages; and is considered so excellent an antiscorbutic that it is largely employed in our navy. It requires nothing but care in the making; and, when properly preparel, may be served at any gentleman's table. Boil it for two hours, and skim the water; or change the water for gravy, and stew it.

\section{2.-TO SFRVE SOUR-KRAUT.}

Take out as much sour-kraut as you wish from the cask, and soak it for at least two hours in cold water, then take it into a colander to drain; put it into a large stewpan or dinner-pot, put on it a piece of corned pork or bacon, and put hot water over nearly to cover it; cover the pot and set it over a moderate fire for an hour or more until the pork is done. Serve with the meat on it. Or cut the bacon or pork in slices, strew pepper over them; lay the sour-kraut on, put hot water nearly to cover it, cover the pot close and set it over a moderate fire for an hour and a half. Or it may be boiled with water, and fried sausages put over it and served; or the sausages may be boiled with it, and the skins taken off, before serving.

\section{3.--ARTICHOKES.}

Cut away the outside leaves and make the stalk as even as possible, put them into boiling water with some salt; if they are very young they will be tender in half an hour, if rather old they will require an hour before they are thoroughly tender; drain and trim the points of the leaves, serve with melted butter.

They are better for being kept two or three days.

The bottoms are sometimes added to ragoûts or meat pies.

If dried you must soak them well for some time, then stew them in some good 
second stock until tender ; take up the boiling water with the beans, or with any artichokes and reduce the stock, add to it a little brown sauce seasoned with pepper, salt, and sugar, a few green peas in the middle of each or alternately, some scooped turnips boiled, and put into each ; glaze the artichokes before you add those to them; sauce in the dish

For Salad.-The artichokes should be very young, the choke having scarcely formed, clean them and let them soak thoroughly, drain them, take off the stalks close and even, and send them to table with the vegetables to form the salad. This is a favorite mode in Paris of dressing them.

\section{4.-JERUSALEM ARTICHOKES.}

Pare them after being well washed, very smooth and of some kind of shape, boil them in milk and water, pay great attention to them, as, if not taken up the minute they are soft, they will break; they are served up with various kinds of sauces, white and brown, or fried in butter, and dished upon a napkin with fried parsley.

\section{5.-FRENCH BEANS.}

When very young the ends and stalks " only sbould be removed, and as they are done thrown into cold spring water; when to be dressed put them in boiling water which has been salted with a small quantity of common salt; in a quarter of an hour they will be done; the criterion is when they become tender; the saucepan should be left uncovered, there should not be too much water, and they should be kept boiling rapidly.

When they are at their full growth, the ends and strings should be taken off and the bean divided lengthways and across, or according to the present fashion slit diagonally or aslant.

A small piece of soda, a little larger than a good-sized pea, if put into the vegetables, will preserve that beautiful green which it is so desirable for them to possess when placed upon the table.

\section{6.-FRENCH BEANS \̀ LA MAITTRE D'HÔTEL.}

Prepare your beans as for boiling; put into a stewpan a piece of butter, shred parsley, and green onions ; when the butter is melted add the French beans, turn them a few times over the fire, shake in some flour, and moisten with a little good stock well seasoned; boil till the sauce is consumed, then put in yolks of three eggs, beat up with some milk, and add a little vinegar. Cullis of veal gravy may be used instead of the eggs.

They may be fricasseed with a teacupful of gravy, and two spoonfuls of cream thickened with butter and flour.

\section{7.-BAKED BEANS.}

Soak a quart of dried beans over night. in cold water; drain off the water in the morning and stew for half an hour in a little water, put them in a deep dish, * with one pound of salt pork, cut the rind in strips, and place in the centre of the dish. The pork should be sunk a little below the surface of the beans. Bake for three hours and a half. A lump of saleratus should be thrown in while the beans are boiling, and a pint of water be added, when they are put into the bakepan.

\section{8.-LIMA BEANS}

Should be gathered young. Shell them, lay them in a pan of cold water, and then boil them about two hours, till they are quite soft. Drain them well, and add to them some butter.

\section{9.-SUCCOTASH}

Is made by boiling young lima beans with green corn cut from the cob. A 
slice of fat pork may be boiled with it, and the mixture must have a lump of butter stirred in before serving. The beans should be boiled before the corn is added, as they take longer to cook.

\section{0.-PONE OF SWEET POTATO.}

To three pounds of sweet potatoes grated, add a pound of butter and a pound of sugar, with six eggs, a tea-cup of sour cream or buttermilk, and a teaspoonful of soda. Bake in a buttered dish, and serve hot.

\section{1.-SQUASHES OR CYMBELINS.}

Cut up the squashes in pieces of an inch thick, having first pared the squash; if old, extract the seeds and boil the pieces until they break, mash them with a spoon, boil them a little longer, and when they are done, squeeze them through a colander. Mix them with a little salt and a small quantity of butter.

\section{2.-WINTER SQUASH.}

Winter squashes are stewed in the same manner as others, but they require to remain over the fire a little longer.

If liept in a dry place, they will remain good all winter; but if they are once frozen, they lose their flavor, and are apt to decay.

They are richer and firmer than the summer kind. After paring a winter squash, cut it in pieces, and, having taken out the seeds, boil it till it is tolerably soft in a small quantity of water. When you think it sufficiently done, press out all the water, mash the squash, and add a little butter, salt, and, if you fancy it, pepper.

\section{3.-SWEET CORN.}

Corn is much sweeter to be boiled on the cob. Put a little salt into the water, and put in the corn when it is cold. It requires boiling from fifteen to thirty minutes, according to its age.

\section{4.-GREEN CORN PUDDING.}

Twelve ears of green corn, grated. a quart of milk, four ounces butter, the same of sugar, four eggs beaten light. Bake in a buttered dish, and eat with butter and sugar sauce. The corn may be previously boiled, when the pudding will bake more quickly.

\section{5.---GREEN CORN OMELET.}

Grate the corn from twelve ears of corn boiled, beat up five eggs, stir them with the corn, season with pepper and salt, and fry the mixture brown, browning the top with a hot shovel. If fried in small cakes, with a little flour and milk stirred in to form a batter, this is very nice.

\section{6.-GREEN CORN FRITTERS.}

Six ears of boiled corn, grated, two eggs, a little milk, and just enough flour to mix it. Drop a spoonful of the batter into a frying-pan, and fry in butter.

$$
\text { 1087.-ONIONS. }
$$

It is a good plan to boil onions in milk and water, it diminishes the strong taste of that vegetable. It is an excellent way of serving up onions, to chop them after they are boiled, and put them in a stew-pan, with a little milk, butter, salt, and pepper, and let them stew about fifteen minutes. This gives them a fine flavor, and they can be served up very hot.

\section{8.-TOMATOES,}

If very ripe, will skin easily; if not, pour scalding water on them, and let them remain in it four or five minutes. Peel and put them into a stew-pan, with a tablespoonful of water, if not very juicy ; if so, no water will be required. Put in a little salt, and stew them for half an 
hour; then turn them into a deep dish with buttered toast. Another way of cooking them, which is considered very nice by epicures, is to put them in a deep dish, with fine bread crumbs, crackers pounded fine, a layer of each alternately; put small bits of butter; a little salt and pepper on each layersome cooks add a little nutmeg and sugar. Have a layer of bread crumbs on the top. Bake it three-quarters of an hour.

\section{9.-SCOLLOPED TOMATOES.}

Scald ripe tomatoes and peel them; put them in a dish with bread crumbs, layer over layer, seasoning with salt and red pepper. Have the bread crumbs on the top, and bake three hours.

\section{0.-TOMATOES, TO STUFF.}

Take some fine tomatoes and scoop the inside out, which should be set aside until required. Chop or mince fine some beef, mutton, or other fresh under-done meat, mix with a little pepper, salt, and a little sweet herbs; or make a forcemeat, and mix with the scoopings of the tomatoes; form into a good consistence, and stuff the inside of the vegetable with the mixture. Set the prepared vegetables in a dish with a little lard in a slow oven, and bake until tender; then serve with the liquor that exudes during the process; but if not brown enough, color by means of a salamander held over the top of each.

A good rich beef gravy poured over all, improves the flavor very much. This is the best way to dress these vegetables, and serves also to make cold meat more palatable, in addition to forming a pretty and economical side dish.

\section{1.-GUMBO}

Two quarts fresh ochras, the same of ripe tomatoes scalded and peeled; put them in a pan to melt with butter, pep- per and salt; strain through the colander, and dish the jelly, to eat with toast.

1092.-HOPPING JOHN (A CAROLINA DISH.)

This is made by boiling a kind of small bean, called cowpeas at the South, with an equal quantity of rice, and sending them mixed and hot to the table.

\section{3.-SPLIT-PEAS PITDDING.}

Take any quantity, say one pint, of yellow split peas ; allow them to remain in water the whole night before you wish to use them; after which take them out and put them into a cloth so loose as to allow the peas to swell; boil them for four hours, or until they are quite tender, then rub them through a colander, so as to render them perfectly smooth; add to the pulp a lump of butter and some salt. After being well mixed put the peas again into a cloth, tie tightly, and boil for about half an hour. Pour over it melted butter.

A richer pudding may be made if two well-beaten eggs are added along with the butter. It is served with boiled pork.

\section{4.-STEWED BEANS.}

Boil them in water in which a lump of butter has been placed, preserve them as white as you can, chop a few sweet herbs with some parsley very fine, stew them in a pint of the water in which the leaves have been boiled, to which a quarter of a pint of cream has been added; stew until quite tender, then add the beans, stew five minutes, thicken with butter and flour.

\section{5.-BEANS, PUREE OF, WHITE.}

Chop some onions and fry them lightly in a little butter and flour. and moisten with a teaspoonful of broth; let the onions be done; boil the beans in this for half an hour, season them well, use 
brown pepper, and strain them through a tammy; reduce the purée over a brisk fire, take off the white scum, and before you serve refine it with a bit of fresh butter and two spoonfuls of thick cream, garnish it with fried crust of bread; this may be made brown by frying the onions brown.

\section{6.-TO PRESERVE PEAS FOR WINTER USE.*}

Shell, scald, and dry them: put them on tins or earthen dishes, in a cool oven, once or twice, to harden. Keep them in paper bags hung up in the kitchen. When they are to be used, let them lie an hour in water ; then set them on with cold water and a bit of butter, and boil them till ready. Put a sprig of dried mint to boil with them.

Appert's method. - Have the peas gathered when ripe, for if too young they will be watery and not fit to keep. The peas, when shelled, should be put into strong jars or open-mouthed bottles, and shaken, so as to make them sit closely together. This done, the jars must be very tightly corked and tied down. The jars are then placed upright in a bain-marie. or any iron pot large enough to contain them, with hay placed between each, to prevent collision and breakage ; the pot is filled with cold water up to the neck of the jars, placed upon the fire, and allowed to boil for an hour and a half if in cool moist weather, but for two hours if it be hot and dry. The jars should not be taken out of the pot until the water becomes cold.

French beans and asparagus may be preserved in the same manner; they maintain their color, but lose much of their flavor, and require a little sugar to improve it.

\section{7.-HARICOT ROOTS.}

Pare three turnips, and scrape two or

* As practised in the kitchen of the Emperor of Russia. three good-colored carrots, about two dozen button onions, a head of celery; cut out with a scoop-cutter sufficient carrots and turnips to pair, the button onions; lay them for a few minutes in boiling water, keep all your roots in water until all is done, cut your celery to correspond as to size with the other roots; when done blanch them, then strain them off, return them into the stewpan, and put to them some good brown stock; boil them until reduced to nearly a glaze, then add some brown sauce to them, sugar, pepper, and salt, do not stir with a spoon to mash the roots, which should be quite perfect.

\section{8.-WHITE HARICOT BEANS.-(Spanish Receipt.)}

Take a pint of beans, pour a quart of boiling water upon them and let them remain in soak until the next day; cut a lettuce in four pieces and put it with the beans in some fresh hot water, throw in a small fagot of parsley, and a slice of ham, boil them until the whole are tender.

Chop up some onions, with a clove of garlic, fry them and then put them into a stewpan, put the beans to them with a well beaten egg and some spice, heat them and send them to table.

\section{9.-BEET-ROOTS.}

Cut in equal-sized slices some beet root, boiled or baked, of a good color, make it hot between two plates in the oven, dish it as you would cut'ets, round; make a good piquant sauce, boil some button onions white and tender, throw them in the middle of the dish with the sauce; or, boil simply, and serve the slices with meited butter.

\section{0.-FRENCH WAY OF DRESSING COLD BEET-ROOT.}

Take your cold beet-root-chop it very small and put it in a saucepan to heat, with a little cream; immediately before 
serving, put in a spoonful of vinegar and a little brown sugar; serve hot.

\section{1.-BROCCOLI AND BUTTERED EGGS.}

Keep a handsome bunch for the middle, and have eight pieces to go round; toast a piece of bread to fit the inner part of the dish or plate : boil the broccoli. In the mean time have ready six (or more) eggs beaten, put for six a quarter of a pound of fine butter into a saucepan, with a little salt, stir it over the fire, and as it becomes warm add the eggs, and shake the saucepan till the mixture is thick enough; pour it on the hot toast, and lay the broccoli as before directed. This receipt is a very good one, it is occasionally varied, but without improvement, the dish is however nearly obsolete.

\section{2.-CABBAGE-TO BOIL.}

Nick your cabbage in quarters at the stalk, wash it thoroughly clean, put it into boiling spring water with a handful of salt and a small piece of soda, boil it fast, when done strain it in a colander, press it gently, cut it in halves and serve. Savoys and greens may be boiled in the same manner, they should always be boiled by themselves. Should the cabbage be left, it may be chopped, put into a saucepan with a lump of butter, and pepper, and salt, and made hot and sent to table.

Small, close cabbages laid on a stone floor, before the frost sets in, will blanch and be very fine, after many weeks' keeping.

\section{3.-STEWED WATER-CRESS.}

The following receipt may be new, and will be found an agreeable and wholesome dish:-Lay the cress in strong salt and water, to clear it from insects. Pick and wash nicely, and stew in water for about ten minutes; drain and chop, season with pepper and salt, add a little butter, and return it to the stewpan until well heated. Add a little vinegar first before serving: put around it sippets of toast or fried bread.-The above made thin, as a substitute for parsley and butter, will be found an excellent covering for a boiled fowl. There should be more of the cress considerably than of the parsley, as the flavor is much milder.

\section{4.-MUSHROOMS.}

The mushrooms proper to be used in cookery grow in the open pasture land, for those that grow near or under trees, are poisonous. The eatable mushrooms first appear very small, and of a round form, on a little stalk. They grow very rapidly, and the upper part and stalk are white. As they increase in size, the under part gradually opens, and shows a fringed fur of a very fine salmon color, which continues more or less till the mushroom has gained some size, and then turns to a dark brown. These marks should be attended to, and likewise whether the skin can be easily parted from the edge, and middle, and whether they have a pleasant smell. Those which are poisonous have a yellow skin, and the under part has not the clear flesh color of the real mushroom; besides which, they smell rank and disagreeable, and the fur is white or yellow.

In Stewing, if you wish to have the full taste of the mushroom only, after washing, trimming, and peeling them, put them into a stewpan with a little salt and no water. Set them on coals, and stew them slowly till tender, adding nothing to them but a little butter rolled in flour, or else a little cream. Be sure to keep the pan well covered.

\section{5.-BROILED MUSHROOMS.}

Pare some large open mushrooms, leaving the stalks on, paring them to a point; wash them well, turn them on the back of a drying sieve to drain. Put into a stewpan two ounces of butter, some 
chopped parsley, and shalots, fry them for a minute on the fire; when melted place your mushroom stalks upwards on a saute pan, then pour the butter and parsley over all the mushrooms; pepper and salt them well with black pepper, put them in the oven to broil; when done put a little good stock to them, give them a boil, and dish them, pour the liquor over them, add more gravy, but let it be put in hot; an hour and a quarter before it is done add four table-spoonfuls of red wine and the liver; serve very hot.

\section{6.-LETTUCES AND ENDIVE8}

Are better, I think, only cut into pieces or into quarters, and dished neatly round; but they must be done in some good stock and not put into the thick sauce, but when you take them out after being done, you will press and form them, then boil down their liquor to a glaze, which will, when added to your already thick sauce, give the desired flavor; glaze the quarters before dishing them, pour the sauce under and round.

\section{7.-TURNIPS, WHOLE.}

Pare several large turnips, scoop them out with an iron cutter for the purpose, throw them in water as you cut them, when done blanch them, then strain them off, if for white add béchamel to them; if for brown, brown sauce ; season as before.

\section{8.--PURÉE OF TURNIPS.}

Pare and cut up several turnips into slices, put them on to boil in milk and water until tender, strain them on the back of a sieve, throw away the liquor, and rub through the turnips; when done put them into a stewpan with a piece of butter, a spoonful of flour, a gill of cream, a little sugar, salt, and cayenne pepper.

$$
\text { 1109.-TRUFFLES. }
$$

The truffle, like the mushroom, is a Unwonted softness to the salad give,

species of fungus common in France and Italy. It grows about eight or ten inches below the surface of the ground. As it imparts a most delicious flavor, it is much used in cooking. Being dug out of the earth, it requires a great deal of washing and brushing before it can be applied to culinary purposes. When washed, the water should be warm and changed frequently; it loses much of its flavor when dried.

Truffles with Champagne.-Take ten or twelve well-cleaned truffles, put them into a stewpan on rashers of bacon, add a bay leaf, a seasoned bouquet, a little grated bacon, some slices of ham, :some stock, and a bottle of champagne ; cover them with a piece of buttered paper, put on the lid, and set the stewpan in hot ashes, put fire on the top, and let them stew for an hour; when done drain them on a clean cloth, and serve on a folded napkin.

To keep them.-After opening a fresh bottle and not requiring them all, return them into the bottle, filling up the bottle with some boiled sherry, cork them down until wanted again.

\section{0.-MORELS.}

The morel is a kind of mushroom, and for a ragout or garnish they are prepared as follows :- take the largest morels, take off their stalks and split them in two or three pieces, wash and put them into a basin of warm water to free them from the sand and earth, then blanch and drain and put them into a stewpan with a piece of butter and lemon juice, give them a turn and moisten with white or brown sauce. They should be dried slowly, put in paper bags, and kept in a dry place.

1111.-SIDNEY SMITH'S RECEIPT FOR SALAD.

Two large potatoes, passed through kitchen sieve, 25 
Of ardent mustard add a single spoon-

Distrust the condiment which bites so soon :

But deem it not, thou man of herbs, a fault

To add a double quantity of salt;

Three times the spoon with oil of Lucca crown,

And once with vinegar, procured from town.

True flavor needs it, and your poet begs

The pounded yellow of two well-boiled eggs.

Let onion atoms lurk within the bowl.

And, scarce suspected, animate the whole;

And, lastly, on the flavored compound toss

A magic teaspoon of anchory sauce.

Then, though green turtle fail, though venison's tough,

And ham and turkeys are not boiled enough,

Serenely full the Epicure may say,-

Fate cannot harm me-I have dined today!

\section{2.-CHICKEN SALAD.}

Mince the meat of two cooked fowls, with a little ham or tongue ; cut up fine a nice lettuce head; put it in a dish, with the chicken in the centre; for the dressing, take the yolks of four eggs beaten, two teaspoonfuls of mixed mustard, two table-spoonfuls of vinegar, and four of oil, half a teaspoonful of Cayenne pepper: boil the mixture, and when cold pour it over the chicken, and ornament it with sliced white of egg and of beets.

\section{3.-SALAD DRESSING FOR LETTUCE.}

Two hard-boiled eggs, the yolks mashed with four teaspoonfuls of sweet oil, a saltspoon of salt, and a teaspoonful of mustard, with two table-spoonfuls of vinegar. Add the lettuce cut up fine, and mince the white of egg to throw over it.

\section{4-SALADS.}

Coss-lettuce and blanched endive make the best salad, the green leaves being stripped off, and leaving nothing but the close, white hearts, which, after being washed and placed for an hour or two in cold water, should be wiped quite ds'y. To this should be added a head or two of celery, a couple of anchovies (which are far preferable to the essence), and several chives, or young onions, all cut small, while the lettuces should be divided lengthwise into quarters, and cut into rather large pieces.

The mixture or dressing.-For four persons bruise only the yolk of one hardboiled egg (leaving out altogether the white), with some salt, and make it into a paste with two large teaspoonfuls of moutarde de maille; or, if obliged to use common mustard, add to it a drop or two of asafoetida, which will impart to it a slight flavor of garlic. Then add oil and vinegar in the following proportions, without using so much as to make the sauce thin, and taking care to have the finest Provence or Lucca oil, and the very strongest species of real French vinegar: namely, to every one spoonful of vinegar add two of oil ; one spoonful of the vinegar being impregnated with chilis, which will add warmth to the salad, much more agreeably than cayenne. A little tarragon may be an improvement, and a spoonful of Quihi or walnut ketchup is not objectionable; but mushroom ketchup will destroy the pungency of flavor, and both may be left out without inconvenience.

When this is done, mix the sauce well, but lightly, with the salad, to which a few slices of boiled beet-root, and the white of the egg sliced, will be a pretty addition.

The excellence of a salad consists in the vegetables being young and fresh, and they should be prepared only a short 
time before they are wanted; the salad mixture being either poured into the bottom of the bowl or sent up in a saucetureen, and not stirred up with the vegetables until they are served.

In summer salads the mixture must not be poured upon the lettuce or vegetables used in the salad, but be left at the bottom, to be stirred up when wanted, as thus preserving the crispness of the lettuce.

In winter salads, however, the reverse of this proceeding must be adopted, as thus: the salad of endive, celery, beet, and other roots being cut ready for dressing, then pour the mixture upon the ingredients, and stir them well up, so that every portion may receive its benefit.

In doing this, it should likewise be recollected that the spoon and fork should always be of wood, and of sufficient size to stir up the vegetables in large quantities.*

\section{5.--CHICKEN SALAD. (English.)}

Pull the meat off the bones, put it into a small pan with a shalot cut in thin slices, a few sprigs of parsley, and a table-spoonful each of oil and tarragon vinegar; season the chicken well with pepper and salt; let it soak for about three hours. Boil three eggs hard, cut them in four pieces lengthways; chop two anchovies, six olives, and a dessertspoonful of capers; take three lettuces, reserve the small hearts to garnish with, cutting them in four, shred fine the other leaves, that are white, and cut roughly some small salad; put a layer of salad on the dish, then the chicken, sprinkle the chopped anchovy, \&c., then more salad and chicken until you have used up the whole of your materials; then

* As a substitute for egg in salad, rub down a dessert-spoonful of a mashed potato with mustard and salt, and some cream or thick melted butter, which answers for oil when that is not at hand; then add the vinegar. mask it with a thick Mayonnaise sauce: garnish it round the bottom with the lettuce-hearts and eggs alternately. This salad is much improved if the dish can be placed on pounded ice whilst it is being prepared.

The remains of veal and white poultry, when minced and left cold, instead of being fricasseed, will thus be found an excellent addition to the dinner of a summer's day, with added slices of cucumber.

\section{6.-CHICKEN SALAD.}

Boil a chicken that weighs not more than a pound and a half. When very tender, take it up, cut it in small strips, and make the following sauce, and turn over it-boil four eggs three minutesthen take them out of the shells, mash and mix them with a couple of tablespoonfuls of olive-oil, or melted butter, two-thirds of a tumbler of vinegar, a teaspoonful of mixed mustard, a teaspoonful of salt, a little pepper, and essence of celery, if you have it-if not, it can be dispensed with. In making chicken salad, the dressing should not be put on till a few minutes before the salad is sent in; as by lying in it, the chicken and celerv will become hard.

\section{7.-SALAD OF FOWL.}

Proceed as for that of game, so far as the eggs and the salad are concerned; then have a chicken, which has been previously plain roasted, or in vegetables, and cut it into ten neat pieces, put it into a basin, season with a teaspoonful of salt, quarter ditto of pepper, two tablespoonfuls of oil, one of vinegar, one onion sliced, and a few sprigs of chopped parsley; mix them well, and let them remain for a few hours, if time will permit. Take the pieces of chicken, and place in a dish with salad, as directed for grouse, with the sauce, \&c., and serve. 
Nothing is better for ball-suppers than these kind of dishes; they may be made of all kinds of solid fish, and the sauce is excellent; anv kind of cold meat, cut in slices, dressed round with the sauce over, may be served for supper or luncheon. It may be served with the same sauce or dressing as for lobster salad; or make the following one, which differs a little :-Put into a middle-sized, roundbottomed basin, the yolk of two eggs, half a spoonful of salt, quarter of one of pepper, half a one of sugar, ditto of fine chopped onions, ditto of parsley, tarragon, or chervil, stir with the right hand with a wooden spoon, while you pour some oil out of the bottle by keeping your thumb on its mouth, so that it runs out very slowly; when a few spoonfuls are in it, it will-become quite stiff; pour also, by degrees, a few spoonfuls of vinegar, and so on until you have made enough for your salad; try if the flavor is good and relishing; as the quality of these two last ingredients vary so much, I must leave it to your palate and correct judgment. If you should fail at first, try again until you succeed, and I am certain you will be delighted with the result; it ought to be made in a cold place, particularly in summer. Great taste should be observed in the decoration of the border, for which you should see the "Gastronomic Regenerator."

\section{8.-FISH SALADS.}

All fish salads are made from the remains of fish from a previous dinner, especially turbot and salmon; but for fillets of soles they must be dressed thus: -When filleted, melt an ounce of butter in a sautépan, lay the fillets in, season with pepper and salt, and the juice of half a lemon; sauté them on a slow fire until done; which may be from four to five minutes, and put by to get cold; cut in middle-sized pieces and use as lobster.
1119.-MAYONNAISE.

[The following receipt is contributed to "The Practical Housekeeper" by Mr. Delmonico, proprietor of the well-known establishments at the Irving House and at the corner of Beaver and William streets, New York.]

In a middle-sized dish placed in cracked ice, put the yolks of two fresh eggs, a little salt, some white pepper, and some vinegar à l'estragon. Stir this mixture briskly with a wooden spoon, and as soon as it begins to thicken, mix in gradually a table-spoonful of oil and a little vinegar, taking carre to beat the sance against the sides of the dish. On this repeated beating depends the whiteness of the sauce Mayonnaise. In proportion to the bulk add oil and vinegar together, putting in but little at a time. As it comes nearer perfection it grows thick and strong-bodied. When finished add a squeeze of citron and some drops of water. This sauce is delicious for chicken salad-mayonnaise de volailleand serves for all kinds of fish and poultry.

1120.-LOBSTER SALAD.

Take out the meat of the claws and body, cutting it into small pieces; and mixing it with the lettuce or endive ; but previously separate the soft part of the fish adhering to the shell, with the red spawn of the hen-lobster, and blend them with the materials prepared for the dressing. It will perhaps require a little more pungency of flavor, which can be imparted by a small additional quantity of chili or tarragon vinegar.

Crab, or shrimps, may be used in the same manner; and a great improvement in either will be found by throwing in a portion of savory calf's-foot jelly, divided into pieces.

Although shell-fish are very decidedly the best mixture to be put into this salad, yet any sort of firm fish, which 
have been dressed, and partly left cold, may also be prepared in a similar way.

\section{PICKLES AND STORE-ROOM SAUCES.}

Although meat, when cured and dried, is called "pickled," yet in that operation salt alone is employed as brine, while in the pickling of vegetables, of which we are about to treat, the means chiefly used is vinegar.

In the making of all sorts of pickles, salads, and sauces, in which vinegar is employed, it is of much importance to have it of the strongest quality, as well as of the best flavor. If the flavor of chilis, capsicums, tarragon, eschalots, garlic, or any root, is wished to be imparted to the vinegar, it is only necessary to put a portion of either (say one oz.) into a bottleful of the liquid; cork it closely, and in a very few weeks it will be found thoroughly impregnated with the peculiar taste; then strain it off, and fill the bottle up again, as it will answer for more than once. The flavor of any herb will also be communicated by boiling it for a short time in the acid; as well as a few fruits, the flavor of which is very delicious when imparted to vinegar.

Pickles should be made at home, as both cheaper and better. The pickles should be kept either in glass bottles, or in stone or unglazed jars, as the vinegar, or even soured vegetables, will corrode the glazing of the jars, which, being made from lead, is poisonous. Use saucepans lined with earthenware or stone pipkins to boil your vinegar in. If you are compelled to use tin, do not let your vinegar remain in it one moment longer than actually necessary; employ also wooden knives and forks in the preparation of your pickles. Fill your jars three parts full with the articles to be pickled, and then add vinegar up to the neck of the jar or bottle.

When greening, keep the pickles covered down, or the evaporation of the steam will injure the color; a little nut of alum may be added to crisp the pickles, but it should be very small in proportion to the quantity, or it will give a disagreeable flavor.

If any symptoms appear of the pickles becoming mouldy, boil the vinegar again, adding a little more spice; keep them also close stopped, as exposure to the air makes the pickle soft.

\section{1.-TO PICKLE ONIONS.}

Silver Sort.-Choose small button onions, as near of a size as possible; throw them into warm water, which will prevent their affecting the eyes so much, while peeling them. As they are peeled, throw them into a strong brine of salt and water, with a small bit of alum; let them remain in this till the next day, then put them on the fire, and bril them in it for a minute. Or, as they are peeled. throw them into milk and water; drain them from this when they are all done, put them into a jar, and pour the brine on them boiling hot; cover them close, and set them aside till the next day; drain and dry them in a cloth; put them into cold vinegar, with a few blades of bruised ginger, some whole pepper, and, if approved, a little mace and sliced horseradish; keep them always well covered with vinegar; cork the jar close, and put it in a cool dry place.

Full-grown Sort.-Peel and slice large onions, and sprinkle them with salt. To every gallon take about a dozen capsicums, either dry or green, slicing only a part; add a few cloves, some pepper and allspice, all whole. Put the onions into jars, distribute the spices pretty equally among them, fill up the jars with vine- 
gar, and set them in a pan of cold water over the fire, taking care that they are closely tied down with a bladder: keep a wet cloth over them to prevent the bladder from bursting. In about an hour and a half the onions will be soft enough.

Onions and Cucumbers.-To every dozen of cucumbers put three large onions, cut both in thick slices, and sprinkle salt over them. Next day drain them for five or six hours, then put them into a stone jar, pour boiling vinegar over them, and keep them in a warm place. Repeat the boiling vinegar, and stop them up again instantly, and so on till green; the last time put pepper and ginger; keep in stone jars. The vinegar is very good for winter salads.

\section{2.-GARLIC PICKLE.}

Steep one quarter of a pound of ginger in strong salt and water for five days, then cut it into slices and dry it in the sun; put it into a large stone jar with a gallon of the best white-wine vinegar. Peel one pound of garlic, salt it well, and let it stand in the salt three days; wipe it, and dry it in the sun, then put it into the pickle; add also a quarter of a pound of long pepper steeped in salt and water and well dried, one pound of mustardseed bruised, and a quarter of a pound of turmeric. Shake these ingredients well in the jar, and add any thing that it is desirable to pickle as it comes into season, salting and drying them previously in the sun. When completed, the pickle should be kept a year or two before it is used.

Shalots may be pickled in the same way ; or if put, as we have already stated, in a bottle of cold vinegar, their flavor will be imparted to it in the course of a month.

1128.-CUCUMBERS.

If full-grown, the small long sort are the best for pickling. Let them be freshgathered; pull off the blossoms, but do not rub them; pour over them a strong: brine of salt and water boiling hot, cover them close, and let them stand all night. The next day stir them gently to take off the sand, drain them on a sieve, and dry them on a cloth; make a pickle with the best white-wine vinegar, ginger, pepper (long and round), and a little garlic. When the pickle boils throw in the cucumbers, cover them, and make them boil as quickly as possible for three or four minutes; put them into a jar with the vinegar, and cover them closely; when cold, put in a sprig of dill, the seed downward. They will be exceedingly crisp and green done in this manner; but if they do not appear to be of a fine color, boil up the pickle the next day, and pour it boiling on the cucumbers.

\section{4.-MUSHROOMS.}

To pickle Mushrooms White.-Take the very small buttons, clean and rub them with a flannel, then put them into cold distilled vinegar, and allow it to come to a boil very slowly; drain and lay them in a cloth till cold, and then put them into fresh distilled vinegar. If very small, they should not be allowed to boil, as so strong a heat might destroy them. It will, therefore, be sufficient to warm them; and when dried, they may be again put into the vinegar in which they had been originally placed, after it has been cooled. As to spice, a little mace will improve their flavor, but no hot pepper should be used. Those which are red underneath, may also be used; but those which are black are too old, and only fit for broiling or to make ketchup.

To pickle Mushrooms Brown.-Choose them older, but see that they be fresh, and of a nearly pink color underneath; clean, and sprinkle them with salt, put them in layers, and let them stand for a 
couple of days; then add some whole pepper, cover them very close, and put them into an oven when bread is baking. That done, take them out, strain off the liquor, into which put cloves, mace, and allspice, and let the whole boil for a little while. While that is doing, put the mushrooms into the stewpan for a short time, then take the whole off the fire, and when perfectly cold pot them, with the addition of a little vinegar.

An excellent way to preserve the flavor of mushrooms when being pickled, is, to rub them with a bit of flannel and salt, and from the larger take out the red inside; when they are black they will not do, being too old. Throw a little salt over. and put them into a stewpan with some mace; as the liquor comes out, shake them well, and keep them over a gentle fire, till all of it be dried into them again; then put as much vinegar into the pan as will cover them, give it one warm, and turn all into a glass or stone jar. They will keep two years, and are delicious.

\section{5.-MUSHROOM POWDER.}

Wash a half peck of large mushrooms while quite fresh, and free them from dirt and grit with flannel; scrape out the black part clean, and do not use any that are worm-eaten ; put them into a stewpan over the fire without water, with two large onions, some cloves, a quarter of an ounce of maces, and two spoonfuls of white pepper, all in powder; simmer and shake them till all the liquor be dried up, but be careful they do not burn. Lay them on tins or sieves, in a slow oven, till they are dry enough to beat to powder; then put the powder in small bottles corked and tied closely, and keep in a dry place.

A teaspoonful will give a very fine flavor to any soup or gravy, or any sauce. It is to be added just before serving, and one boil given to it after it is put in.

\section{6.-TO PICKLE WALNUTS.}

Gather the walnuts when tender, and put them in salt and water: prick each with a needle several times; let them stand two or three days, changing the water every day. Make a brine of salt and water strong enough to bear an egg; it must be quite cold before being used, and be well skimmed while boiling. To every hundred walnuts allow one gallon of water. Let the walnuts soak six days, then change the brine, and let them stand six more; then drain them, and expose them to the sun that they may turn black; pour over them, in a jar, a pickle of the best white-wine vinegar, with a good quantity of pepper, pimento, ginger, mace, cloves, mustard-seed, and horseradish, all boiled together, but cold. To every hundred walnuts allow six spoonfuls of mustard-seed, with an ounce of whole black pepper, and two or three heads of garlic or shalot, but the latter is least strong; let them be quite covered with vinegar. This done, they will be good for several years, if closely covered. The air will soften them. They will not, however, be fit to eat under six months.

\section{7.-TO PICKLE RADISH-PODS.}

Gather the radish-pods when they are quite young; and put them into salt and water all night; then boil the salt and water, and pour it over the pods in jars, and cover them closely to keep in the steam. When the brine is cold boil it, and pour it hot upon the pods again, repeating the process until they are green; then put them in a sieve to drain, and make a pickle for them of white-wine vinegar, mace, ginger, long pepper, and horseradish; pour it boiling hot upon the pods, and when nearly cold boil it again, and pour it over them. When cold, tie down the jars.

1128.-TO PICKLE FRENCH BEANS.

Gather them before they become 
stringy, and, without taking off the ends, | ed, it will greatly improve the color; put them into a very strong brine until boil the vinegar and spices together for they become yellow; drain the liquor full five minutes, and strain it; then, from them, and wipe them dry with a having put the cabbage into a stone jar, cloth. Put them into a stone jar by the fire, put in a little bit of alum, and pour boiling vinegar upon them every twentyfour hours, preventing the escape of the steam. In four or five days they will become green. Boil a little mace, whole pepper, and ginger in the vinegar.

Do samphire the same way.

\section{9.-NASTURTIUMS}

So much resemble capers, both in flavor and the mode of pickling, as to be frequently used in the same manner; the seeds should be allowed to get ripe after the buds and flowers have gone off. Gather them upon a dry day, and keep them for a few days after they have been gathered; put them into a jar, and pour boiling vinegar well spiced upon them; when cold, cover the jar. They will not be fit for use for some months, but will be finely flavored after keeping, and are sonietimes preferred to capers, for which they are an excellent substitute, being useful also in serving up all dishes in which pickles are warmed with the gravy. Young red capsicums and elderflowers before they open may be done in the same way.

\section{0.-TO PICKLE RED CABBAGE.}

Take those of a deep red or purple color, strip off the outer leaves and cut out the stalk; quarter the cabbage lengthwise and cut it crosswise, but not in very thin slices; put it into a sieve and sprinkle it with salt and saltpetre; let it drain for twenty-four hours, then squeeze it until it is very dry; make a pickle with sufficient vinegar to cover the cabbages well, and made with equal quantities of cloves and allspice, with a little mace-to be put in whole, to which if a very small quantity of cochineal be add-

This is opposed to the practice of some persons, who object to boiling the vinegar, and pour it cold upon the cabbage.

\section{1.-CABBAGE, WHITE, PICKLED.}

Slice your cabbage thin, then lay it in salt for twenty-four hours; strain it very dry, then put it in a stone jar with allspice, mace, and vinegar, and pour it on boiling hot, tie it very close, repeat the vinegar three times, and it will be fit for use.

\section{2.-CABBAGE, PURPLE, TO PICKLE.}

Take two cauliflowers, two red cabbages, half a peck of kidney beans, stick with six cloves of garlic on each stick, wash all well; give them one boil up, then strain them in a sieve, lay them leaf by leaf upon a large table and salt them with coarse salt; then lay them to dry in the sun, or in a slow oven, until as dry as cork.

\section{3.-CELERY, TO PICKLE.}

Separate the stalks from the head, clean them thoroughly, and put them into salt and water strong enough to bear an egg; let them remain in this for a week or ten days, or until wanted to pickle; then take them out, wash them well in clean water, drain dry, place in a jar, and pour boiling vinegar over, to which any approved spices may have been added. As is usual for pickling keep it well covered with vinegar; if the celery is allowed to remain a long time in salt and water, it will be necessary to soak it in clean water for a day or two, changing the water occasionally.

1134-TO PICKLE CAULIFLOWERS.

Take the closest and whitest cauli- 
flowers you can get, and pull them in bunches, and spread them on an earthen dish, and lay salt all over them, let them stand for three days to bring out all the water, then put them in earthen jars, and pour boiling salt and water upon them, and let them stand all night, then drain them on a hair sieve, and put them into glass jars, and fill up your jars with vinegar, and tie them close down with leather.

\section{5.-PICKLED TOMATOES:}

The tomatoes should not be very ripe. Mix in a large stone jar, one ounce of mustard, and half the same quantity of cloves and black pepper, and fill the jar half full of the best vinegar. Lay in the tomatoes, mixing with them a dozen or more whole onions.

The jar should not be opened for a month, when the pickles will be fit for use; great care must be taken to close the jar well whenever pickles are taken out. This done, they will keep a year.

Another.-Take a peck of ripe tomatoes, prick them with a fork, and lay them in a jar with layers of salt. Let them remain eight days in salt; then take them out and put them for twentyfour hours in fresh water with a little vinegar.

Take them out, press them gently, and lay them on a sieve to drain. Slice twelve large onions, or more smaller ones. Put a layer of the tomatoes in a stone jar, and cover them with a layer of slices of onion. Over this strew mustard, allspice, black pepper, and cloves-all but the cloves ground fine. Then place on the mass another layer of tomatoes, with another of sliced onions and the mixed spices. Place the layers alternately till the jar is filled. Then pour over it the best vinegar, filling the jar, and cover closely. The pickles will be fit for use in ten days.
1136. -TO PICKLE LEMONS.

Take the finest with the thickest rind you can get, cut them deeply from end to end in more than one place, but not quite through, and fill the incisions with salt; put each on end, and lay them in a dish near the fire, or in the sun if the weather be hot, to dissolve the salt, and repeat this during three weeks; then put them into a jar, with a handful of white mustard-seed if it be large, onequarter to one-half a pound of bruised ginger, half that quantity of cloves and allspice, a few chilis, and a very little turmeric; boil in vinegar, and pour it upon the lemons when cold. It was originally prepared by the cook of the first Earl of Orford.

Some people, however, add to it either shalot or garlic. It can hardly be ready in less than six months, but will keep for many years.

For limes, or very small lemons, the same method must be pursued, only they will not require above half the time.

$$
\text { 1137.-MANGOES. }
$$

Cut a piece off the top or side of young mush-melons, and remove the pulp and seeds; tie on the pieces. green them, and fill the inside with grated horseradish, spices, cloves, ginger, mustard-seed, onion or garlic, and slices of the core of cabbage. Tie on the pieces again, pour on them scalding vinegar, four days in succession, and keep them in covered jars.

Mangoes may be made of peaches.

Observe that there be plenty of vinegar, as pickles are spoiled if not well covered. Large cucumbers, called "green turley," prepared in the same way, are excellent, and are sooner fit to be eaten.

The greater number of times boiling vinegar is poured over either sort, the sooner it will be ready.

\section{MIXED PICKIE.}

To one gallon of vinegar put four 
ounces of ginger bruised, two ounces of white pepper, two of allspice, two of chilis, four of turmeric, one pound of mustard-seed, one-half pound of shalots, one ounce of garlic, and one-half pound of coarse salt. Boil all together except the mustard-seed, which must be added afterwards. Then mix very smoothly onequarter of a pound of made mustard with some of the vinegar, when cold, in a basin, and add it to the remainder with the seed. Take broccoli, radish-pods, French beans, or any other vegetables or small fruit; blanch the vegetables, lay them on a sieve, and sprinkle a little salt over them to draw out the water. Let them stand in the sun till very dry, then pour the vinegar boiling over them, slicing in some horseradish if approved.

Pickle of this kind can be rendered hot at pleasure, by additions of chilis, green and red capsicums-which, when gathered young, add exquisite flavorand all sorts of foreign peppers. As the spring advances, a portion of every small root produced in a garden, if collected and thrown into cold vinegar, till it can be conveniently made into a regular pickle, will be found most excellent, at no greater expense than the cost of the spice and vinegar, and the trouble of mixing it.

\section{9.-INDIA PICKLE}

Is made in nearly the same manner. Put two hundred gherkins, three pints of small onions, one quart of nasturtiums, one ditto of radish-pods, one quart of French beans, six cauliflowers, and two hard white cabbages sliced, into a pan, and sprinkle them with salt, the onions having been previously peeled and laid in salt and water for a week to take off their strength. Then, after a day or two, take them out of the pan and dry them thoroughly in a warm place in the shade: they must be spread out sepa- rately. To two gallons of vinegar put one and a half ounces of allspice, the same of long pepper and of white, and two of ginger, tied up in muslin bags. When cold, mix with the vinegar one and a half pounds of flour of mustard, and two table-spoonfuls of cayenne pepper. Boil it well together, and pour it on the pickle: the vegetables mentioned, not being all procurable at the same time, may be added separately, at different periods, but they must all undergo the salting and drying process.

In choosing those vegetables some discrimination may also be used: when in season, few things add a higher flavor to the pickle than the buds and flowers of the elder.

\section{0. -PLUMS, TO PICKLE LIKE OLIVES.}

Take the plums before they are quite ripe, and put them into a saucepan with some white wine vinegar, salt water, fennel seed, and dill, as much of each as will impart a flavor to the pickle; when it boils put in the plums, let it boil again, then take it off, let it stand till cold, then put them into jars.

\section{1.-QUINCE, TO PICKLE.}

Pare and cut half a dozen quinces into small pieces, and put them with a gallon of water and two pounds of honey into a large saucepan, mix them together well, and set them on a slow fire for half an hour, strain the liquor into a jar, when quite cold wipe the quinces perfectly dry, and put them into it; cover them very close.

\section{2.-PEACHES AND APRICOTS.}

Take those of a full growth, but perfectly green. Put to a gallon of vinegar half an ounce of cloves. the same quantity of peppercorns, sliced ginger and mustardseed-add salt and boil the vinegarthen turn it on the peaches scalding hot. 
Turn the vinegar from them several times. Heat it scalding hot, and turn it back while hot.

\section{3.-TO PICKLE GREEN PEPPERS.}

The peppers must be pickled when half ripe, and the smallest ones chosen. Make a small hole at the top and another at the bottom of the pepper, and extract the core and seeds. A penknife should be used in performing this operation. Simmer the peppers for a whole day in salt and water over a very moderate fire - stir them every once and awhile that those at the bottom may not burn. Leave them over night to cool, and the next morning lay them gently into a jar, sprinkle a small quantity of mustard over them, and fill up the jar with cold vinegar.

\section{4.-BUTTERNUTS.}

The nuts for pickling should be gathered as early as July. When a pin will go through them easily, they are young enough to pickle. Soak them in salt and water a week-then drain it off. Rub them with a cloth, to get off the roughness. To a gallon of vinegar put a teacup of salt, a tablespoonful of powdered cloves and mace, mixed together, half an ounce of allspice, and peppercorns. Boil the vinegar and spices, and pour it hot on the nuts. In a week scald the vinegar, and pour over them again. They will be fit for use in a fortnight.

\section{5.-TO PICKLE GHERKINS AND KIDNEY} BEANS.

Put the beans and gherkins, dried with flannel, in salt and water that will bear an egg, leave them till quite yellow, stirring every day. Then put them in a brass kettle with cabbage leaves under and around them, so that they do not touch the brass. Put in one third vinegar and two of water, put a leaf over, and cover close, so as to confine the steam. Put them on a slow fire, but do not let them boil. Change the leaves as often as they grow yellow. When green, take out the pickles, and put them on a dish to cool and dry. Boil the vinegar with a spoonful of black pepper, and one of bruised ginger to a quart; throw it boiling over the pickles, and cover them closely in small jars.

\section{6.-BARBERRIES.}

Barberries make very good pickles, and are always useful in ornamenting certain dishes. Leave the barberries on the stem, lay them in a stone jar and fill it up with cold vinegar.

\section{7.-GRAPES.}

Cut them, when hardly ripe, in bunches, put them in a jar with vine leaves between each layer of grapes until the jar is filled; then take as much spring water as will cover the grapes and the leaves. As it heats put in as much salt as will make a brine sufficiently strong to bear an egg, you may use common salt: when it boils skim it, strain it through a flannel bag and let it stand to settle; by the time it is cold it will be quite settled; strain it a second time through a flannel bag, then pour ${ }^{\circ}$ it into a jar upon the grapes, which must be well covered; fill the jar with vine leaves, then tie it over with a double cloth and set a plate upon it; let it stand for two days, then take off the cloth, pour away the brine, then take out the leaves and the grapes, and lay them between two cloths to dry, then take two quarts of vinegar, one quart of spring water, and one pound of coarse sugar, boil it for a short time and skim it very clean as it boils; let it stand till it is quite cold. Wipe the jar very clean and dry, lay some fresh vine leaves at the bottom between every bunch of grapes and on the top; then pour and strain the pickle on the grapes, fill the jar, let the 
pickle be above the grapes, tie up a thin piece of board in a flannel, lay it on the grapes to keep them under the pickle, tie them down with a bladder, and over that a leather, always keeping the grapes under the pickle.

\section{8.-APPLE CODLINGS, TO PICKLE.}

Gather the codlings when of the size of a large double walnut; take a pan and make a thick layer of vine leaves at the bottom; put in the codlings and cover them well with vine leaves and spring water, and put them over a slow fire till they are sufficiently tender to pare the skins with ease with a small sharp knife, put them into the same water with vine leaves as before; cover them close, and set the pan at a short distance from the fire until they are of a fine green, then drain them in a colander till they are cold; put them into jars with some mace and a clove or two of garlic, according to the quantity of the apples, and cover them with distilled vinegar; pour over mutton fat, and tie them down with a blacider and leather very tight.

\section{9.-ARTICHOKES PICKLED.}

Boil the artichokes till you can pull the leaves off; take out the choke and cut away the stalk, be careful that the knife does not touch the top; throw them into salt and water; when they have lain an hour take them out and drain them, then put them into glasses or jars, and put a little mace and sliced nutmeg between; fill them with vinegar and spring water, cover your jars close.

\section{VINEGARS.}

\section{0.--IINEGAR FOR INDIA PICKLE.}

Prepare a gallon of vinegar, more or less, according to the quantity of pickles to be done, in the following manner :-

Mix gradually one-quarter of a pound of the best flour of mustard, and two ounces of powdered turmeric, with some of the cold vinegar at first, to ensure its being properly mixed; then add the rest, with one-quarter of a pound of white mustard-seed. Bruise one-quarter of a pound of ginger, two ounces of white pepper, and one ounce of chilis, and tie them in a muslin bag. Boil the whole gently for twenty minutes or half an hour, and pour it whilst boiling on the pickles, having previously drained off the vinegar they were first put in. In ten or twelve days repeat the boiling, pour it over the pickles whilst boiling hot, and they will be ready for use when cold.

\section{1.-WALNUT VINEGAR.}

Put green walnut-shells into a brine of salt and water strong enough to float an egg; let them lie covered in this ten or twelve days; take them out; and lay them in the sun for a week; put them into a jar, and pour boiling vinegar on them; in about a week or ten days pour off the vinegar, make it boiling hot, and pour over them again. In a month it will be fit for use, and will be found excellent to eat with cold meat, and particularly useful in making sauces.

\section{2.-CUCUMBER VINEGAR.}

Pare and slice fifteen large cucumbers, and put them in a stone jar, with three pints of vinegar, four large onions sliced, two or three shalots, a little garlic, two large spoonfuls of salt, three teaspoonfuls of pepper, and half a teaspoonful of cayenne. After standing four days, give the whole a boil: when cold, strain, and filter the liquor through paper.

\section{3.-CAMP VINEGAR.}

Slice a large head of garlic, and put it into a wide-mouthed bottle, with half an ounce of cayenne, two teaspoonfuls of 
real soy, two of walnut ketchup, four anchovies chopped, one pint of vinegar, and enough cochineal to give it the color of lavender-drops. Let it stand six weeks; then strain off quite clear, and keep in small bottles sealed up.

Another :-Infuse in a quart of the best white-wine vinegar, a quarter of a pint of walnut ketchup, the same quantity of mushroom ketchup, and the same quan. tity of soy, half an ounce of cayenne, four heads of garlic, ten shalots, two ounces of black and two ounces of white pepper, the same quantity of pimento and ginger, one ounce of nutmeg, three blades of mace, and ten cloves, in a widemouthed, two-quart bottle; and cover very closely with cork, leather, and bladder. Let it remain near the fire a month, shaking it frequently. When any is taken out, put in as much fresh vinegar. This is not only a very fine sauce, but a great preservative against infectious diseases, if taken freely.

\section{4.-NASTURTIUM VINEGAR.}

Pick full-blown nasturtium flowers; fill a wide-mouthed bottle with them; add half a clove of garlic and a moderatesized chalot chopped; pour as much vinegar as the bottle will take; in two months' time rub the whole through a fine sieve; add a little cayenne pepper and salt.

\section{5.-TARRAGON AND ELDER-FLOWER} VINEGAR.

Take either the young leaves of tarragon when the plant is going into bloom, or the buds of elder-flowers, and to every half peck put one gallon of vinegar, leaving it for a fortnight in a jug to ferment. Then drain it through a flannel bag, put into it a small bit of dissolved isinglass, and bottle it.

The flavor of the herbs may also be extracted by boiling the herbs or leaves in vinegar, without fermentation : a mixture of both is very agreeable.

\section{6.-RASPBERRY VINEGAR.}

To one quart of common vinegar put two quarts of fresh raspberries; let them stand twenty-four hours; then drain them off, but do not squeeze them. Put in two quarts more, let them stand as before, and this must be repeated a third time. After which, put the vinegar into a jar, measure it, and to every pint put one pound of lump-sugar. Set the jar up to the neck in boiling water, and let the vinegar boil for ten minutes, stirring it frequently. There should on no account be fewer raspberries than the proportion mentioned, and the vinegar will not be fit for use until the following summer.

\section{7.-SUGAR VINEGAR.}

To every gallon of water put two pounds of coarse brown sugar. Boil and skim this. Put it to cool in a clean tub; when about lukewarm, add a slice of bread soaked in fresh yeast. Barrel it in a week, and set it in the sun in summer or by the fire in winter, for six months, without stopping the bung-hole; but cover it with thin canvass or an inverted bottle to keep out the flies.

1158.-CIDER VINEGAR.

Put a pound of white sugar to a gallon of cider. and shaking them well together, let them ferment for four months; a strong and well-colored vinegar-will be the result.

\section{9.-FLAVORED VINEGARS.}

These are a cheap and agrecable addition to sauces, hashes, \&c. For one, infuse a hundred red chilis, fresh gathered, into a quart of good vinegar; let them stand ten davs, shaking the bottle every day. $A$ half ounce of cayenne will answer the same purpose. This is good in melted butter for fish sauce, \&c.

$$
\text { 1160.-CELERY VINEGAR. }
$$

Pound a half ounce of celery seed, and 
steep it for ten days in a quart of vinegar ; strain and bottle it.

\section{1.-HORSERADISH VINEGAR.}

Pour a quart of strong vinegar, boiling hot, on three ounces of scraped horseradish and a teaspoonful of pounded black pepper, and half the quantity of cayenne. Let it stand four days, tightly covered, then strain and put it in the cruet for use. It is good on cold roast beef, and excellent in the gravy for chops, steaks, \&c.

\section{2.-GOOSEBERRY VINEGAR.}

Boil water, and when cold put to every three quarts one quart of bruised gooseberries in a large tub. Let them remain sixty hours, stirring often; then strain through a hair bag, and to each gallon of liquor add one pound of the coarsest sugar. Put it into a barrel, and a toast and yeast; cover the bung-hole with a bit of slate. Set the barrel in the sun, observing that the cask be well painted, and the iron hoops all firm. The greater the quantity of sugar and fruit, the stronger the vinegar; and as this is particularly useful for pickles, it might be well to make it of double the strength for that purpose.

\section{3.-CHILI VINEGAR.}

Fill a bottle with the chilis or capsicums-each of which will impart the proper warmth of flavor-and cover them with vinegar; cork it up closely, let it stand for three weeks or a month, then pour off the vinegar, and fill up the bottle again: chilis are the most commonly used, but good capsicums will flavor several additions of vinegar. If capsicums are not prosurable, put one ounce of cayenne pepper to a quart of vinegar, and let it stand some time, shaking it occasionally. Then strain it off, and fill the bottle up again.
1164.-LEMON FLAVORING FOR PUDDINGS.

Peel six lemons very thin indeed; put the peel into a glass bottle, and add a table-spoonful of bitter almonds,blanched, or the same quantity of peach or apricot kernels. Cover the whole with brandy; shake it frequently, and in a month strain it, and if kept closely corked it will keep for years.

\section{5.-MUSHROOM KETCHUP.}

Take the full-grown flaps of freshlygathered mushrooms, crush them with the hands, throw a handful of salt into every peck, and let them stand a night or two; then put them into pans, and set them in a quick oven for twelve hours; strain them through a hair sieve, and press out all the juice. To every gallon of liquor put of cloves, Jamaica and black pepper, and ginger, one ounce each, and half a pound of common salt. Set it on a slow fire, and let it boil until half the liquor is wasted; then put it into another vessel, and, when cold, strain and bottle it ; at the same time corking it up closely, and covering the cork with oiled paper.

Or:-Take the largest broad mushrooms, break them into an earthen pan, strew salt over, and stir them now and then for several days, till there is a thick scum over: strain and boil the liquor with Jamaica and black peppers, mace, ginger, a clove or two, and some mustard seed. Cayenne pepper is too hot, but a very little chili vinegar is an improvement. When cold, bottle it, and secure the corks as above, leaving the spice in. At the end of three months strain the liquor and boil it with fresh spice, which put into the bottles; and in a cool place it will keep two or three years.

We strongly recommend the addition of a moderate quantity of port winefrom half a pint to not quite a pint to every four quarts-both as greatly im- 
proving the flavor of the ketchup and ensuring its better preservation.

With regard to the spice, care should be taken not to allow it to overpower the natural flavor of the vegetable.

$$
\text { 1166. - WALNUT KETCHUP. }
$$

'Thoroughly bruise one hundred and twenty young walnuts; put to them three-quarters of a pound of fine salt and a quart of vinegar; stir them every day for a fortnight; then strain; squeeze the liquor from them through a cloth; add to this one ounce of whole black pepper, forty cloves, half an ounce of nutmeg bruised, half an ounce of ginger, and a few blades of mace. Boil the whole for half an hour; strain and bottle it for use.

Walnut Ketchup of the finest quality - Boil or simmer a gallon of the expressed juice of walnuts when they are tender, and skim it well; then put in two pounds of anchovies, bones, and liquor, ditto of shalots, one ounce of cloves, ditto of mace, ditto of pepper, and one clove of garlic. Let all simmer till the shalots sink; then put the liquor into a pan till cold ; bottle and divide the spice to each. Cork closely, tie the bladder over, and put it in small bottles.

It will keep twenty years in the greatest perfection, but is not fit for use the first year. Be very careful to express the juice at home, for it is generally adulterated if bought.

Irish mode:-Take a few hundred green walnuts, scoop out all the whites, beat them in a mortar, and strain the juice through a cloth; let it stand a day and night, strain it, and pour it off clear. To one pint of this liquor put half a pint of vinegar with half a pound of anchovies, and to each pint thus made a clove or two of garlic, two or three shalots, some horseradish, and one onion cut in quarters; boil it two hours, and then strain it off. When strained add to every pint of liquor half a quarter of an ounce of mace, the same quantity of cloves, of nutmeg, and of whole black pepper, half a pint of port wine, and two table-spoonfuls of soy. Boil them together for half an hour. Then pour it off into an earthen jar, and let it remain covered until it is cold ; bottle it off into clean dry bottles, distributing the spice equally in each; cork it down closely; take care in boiling to keep the saucepan covered.

\section{7.-CUCUMBER KETCHUP.}

Take an equal quantity of large cucumbers and large onions, pare and slice them; throw over them a handful of salt, and let them stand all night in a sieve placed over a pan. Take the liquor and boil it up, with a quarter of a pound of anchovies to every dozen of cucumbers, one pint of white wine, a nutmeg, a quarter of an ounce of mace, and half an ounce of whole pepper; strain it, and when cold bottle it; tio it down with bladder. It will keep for two years.

The Suffolk Receipt.-Take a dozen well-grown cucumbers and four large onions, slice them into an earthen pan with a good handful of salt; let them stand till the liquor begins to run, break them into small pieces, and let the whole stand another day and night: then strain it off; to erery quart put the same quantity of white, but not sweet wine, half a pound of anchovies, and a large stick of horseradish; boil them together for half an hour, then strain it again, and to every quart put a quarter of an ounce of white pepper, half the quantity of mace and nutmeg, all pounded, and boil it well again. When cold, bottle it with the spice, and put a piece of ginger into each bottle. A tablespoonful in a sauceboatful of cream or melted butter makes delicious white sauce for fowls or made dishes of veal. 
1168.-TOMATO KETCHUP.

Take two dozen of ripe tomatoes and a handful of salt; slice the tomatoes, and put a layer into a jar, sprinkle salt over it, then another layer of tomatoes and salt, until the jar is full. Stir the contents now and then for three or four days, keeping the jar in a warm place by the fire; at the expiration of which time press the juice from the tomatoes by rubbing them with a wooden spoon through a sieve, and boil it with mace, pepper, allspice, ginger, and cloves, in the proportion of about two ounces in all to one quart of juice, a few blades of mace, twelve cloves, a spoonful of pounded ginger, and the remainder pepper and allspice. In three months boil it again with fresh spice.

1169.-TOMATO SAUCE FOR WINTER USE.

One peck of tomatoes, six onions sliced, two heads of celery, a dozen shalots, one ounce of caycnne pepper, half an ounce of black pepper, one ounce of mace in powder. Slice them into a well-tinned saucepan, mixing the seasoning with them as they are cut up; boil, keeping them well stirred; when thoroughly soft, drain off the water, and rub through a hair sieve. Boil it again until it is as thick as apple-sauce Put it into bottles, and cork close. Put the bottles into a stewpan, fill it with cold water, and let it boil for twenty minutes. Keep in a cool place. Examine the bottles occasionally, and if there is the least indication of a change turn it into the kettle again, boil and skim it, keeping it well stirred from the bottom that it may not adhere, and put it into the bottles again. When required for use, warm what is wanted with a little gravy. It is as nice as when fresh done, and will be found excellent with calf's head or brains, veal, beef, mutton, pork, or goose. An onion or a shalot, boiled in the gravy with which it is mixed, will be an improvement.

\section{0.-CAMP KETCHUP.}

Take two quarts of old strong beer and one of white wine, add a quarter of a pound of anchovies, three ounces of shalots peeled, half an ounce of mace, the same of nutmeg, three large races of ginger cut in slices; put all together over a moderate fire till one third is wasted. The next day bottle it with the spice and the shalots. It will keep for many years.

Or:-A pint of claret, the same quantity of ketchup, four ounces of anchovies, one ounce of fresh lemon-peel pared thin, two cloves of garlic minced fine; half an ounce "of allspice, the same of black and of red pepper, one drachm of celery-seed bruised, and half a pint of pickle-liquor. Put these ingredients into a wide-mouthed bottle; stop it close, shake it every day for a fortnight, and then strain it off.

\section{1.-SEVEN-YEARS' KETCHUP.}

Take two quarts of the oldest strong beer, put to it one quart of red wine, three-quarters of a pound of anchovies, three ounces of shalots peeled, half an ounce of mace, the same of nutmegs, quarter of an ounce of cloves, three large races of ginger cut in slices, and boil all together over a moderate fire till onethird is wasted. When quite cold put it into a large jar, covered up, and leave it there for a week or two until the sediment is wasted, and the clear liquor is perfectly transparent; then strain it through a fine hair sieve, taste it, and add to it a little of any essence of spice, oil, or vinegar, which you think may im-. prove it, and, if the liquid be not lucidly clear, give it one smart boil: let it rest till cool. and bottle it in very small bottles to prevent its frequent exposure to the air.

It will improve by age, and may be carried on a voyage round the world.

As may also 
Sauce à la Militaire, made thus:Put into an earthen pot six shalots sliced, a clove of garlic split, two laurelleaves, a handful of thyme, basil, and tarragon-leaves, half an ounce of mustardseed bruised, one teaspoonful of grated Seville orange-peel, quarter of an ounce of cloves, the same quantity of mace, half an ounce of long pepper, two ounces of salt, the juice of a lemon, six spoonfuls of the strongest vinegar, and half a pint of white wine. Stop the pot very closely, and put it into an oven, or upon hot ashes, for twenty-four hours, to allow the whole to infuse properly. Then allow it to settle, and strain it until it is quite clear: bottle it, and close the bottles tightly: a very small quantity will be sufficient, and it may be used with all sorts of meat, game, or fish.

\section{2.-ARTIFICIAL SAUCES FOR FISH.}

Those most commonly used are soy and anchovy, merely mixed from the cruets into melted butter by each individual of the company; the butter, when brought to table in a prepared state, should be made as follows:-

For Anchory Sauce, the foundation should be some of the fish stock or gravy which has been already made and kept in the house for future use. Take two or three anchovies, scrape, but do not wash them, and boil them along with a minced shalot in some of the gravy, until they are soft enough to be pounded to a paste. Then strain the gravy and thicken it with the body of the fish, after having removed the heads, tails, and bones. When that is done, put it, for about a quarter of an hour, in the stewpan along with a glass of red wine, a squeeze of lemon, and the necessary quantity of butter.

The common mode is, to chop three anchovies, melt a quarter of a pound of butter in a teacupful of the water in which the fish is boiled, add a spoonful of vinegar, and a little flour. Stir it well over the fire until it is quite thick.

Or:-Not unfrequently two or three spoonfuls of the prepared essence of anchovy are put into the melted butter, seasoned with a little cayenne pepper.

The savory store sauces for fish, as prepared and sold in the shops, are all to a certain degree agreeable to some palates, but anchovy and soy seem the prevailing sort for fish.

\section{3.-STORE SAUCES.}

1. Take two wine-glasses of port, two of walnut and four of mushroom ketchup, four anchovies pounded, with two shalots, a table-spoonful of soy, and a teaspoonful of cayenne pepper. Boil all well together; when cold, put it into bottles well corked; it will keep good for more than a twelvemonth.

2. Take half an ounce of mace, one ounce each of cloves, ginger, and allspice, one pound of anchovies, a bottle of either white or red wine, half a pint of vine. gar, a couple of shalots and a clove of garlic chopped, and a stick of scraped horseradish, together with a bundle of pot-herbs. Let all simmer over a slow fire till the anchovies are dissolved; then strain the liquor, bottle it when cold, and when wanted for use add a table-spoonful of the sauce to quarter of a pound of melted butter.

3. Thicken a quarter of a pound of butter with flour, and brown it; then put to it one pound of the best anchovies cut small, six blades of pounded mace, ten cloves, forty berries of black pepper and allspice, a few small onions, a fagot of sweet herbs (namely, savory, thyme, basil, and knotted marjoram), and a little parsley and sliced horseradish; on these pour half a pint of the best sherry and a pint and a half of strong gravy. Simmer all gently for twenty minutes, then 
strain it through a sieve, and bottle it for use. The way of using it is, to boil some of it in the butter while melting.

4. Put into a saucepan a pint of fine port wine, a gill of mountain, half a pint of fine walnut ketchup, twelve anchovies, and the liquor that belongs to them, a gill of walnut pickle, the rind and juice of a large lemon, four or five shalots, some cayenne to taste, three ounces of scraped horseradish, three blades of mace, and two teaspoonfuls of made mustard: boil it all gently, till the rawness goes off, then put it into small bottles for use. Cork them very close, and seal the top.

5. Chop twenty-four anchovies, not washed, and ten shalots, and scrape three spoonfuls of horseradish; which, with ten blades of mace, twelve cloves, two sliced lemons, half a pint of anchovy liquor, a quart of hock or Rhenish wine, and a pint of water, boil to a quart; then strain off; and when cold, add three large spoonfuls of walnut ketchup.

All or any of these will be found excellent for family use; as, when added to any common sauce for fish, or even to plain melted butter, a moderate quantity will impart to it a very fine flavor, and in many cases they may be used also for meat, if eaten cold.

Pontac. Ketchup is for this purpose both very simple and very good. Put ripe elderberries, picked from the stalk, into a stone jar, with as much strong vinegar as will cover them. Bake with the bread; and, while hot, strain. Boil the liquor with a sufficient quantity of cloves, mace, peppercorns, and shalots to give it a fine flavor. When that is ob tained, put in half a pound of the finest anchovies to every quart of liquor; stir, and boil only until dissolved. When cold, put it into pint bottles, and tie doulle bladders over each cork. The same method should be observed for preserving all ketchups.
An excellent and not common Pickle, called "Salade," may be made thus:-

Fill a pint stone jar with equal quantities of onions, cucumbers, and sour apples, all cut into very thin slices, shaking in, as you go on, one teaspoonful of salt and three parts of a teaspoonful of cayenne. Pour in a wine-glass of soy, the same of white wine, and fill up the jar with vinegar. It will be fit for use the same day.

Quin's Sauce is also excellent. Half a pint of common mushrooin ketchup, half a pint of walnut-liquor, eight anchovies, eight cloves of garlic, four bruised and four whole, three teaspoonfuls of cayenne pepper, three of mushroompowder, all boiled together for five minutes, and bottled when cold.

\section{4.-CAYENNE PEPPER}

May be made by pounding the dry peppers, or chilis, to a fine powder, with salt, and keeping it in bottles.

\section{5.-FRENCH MUSTARD.}

To mustard ground, add tarragon vinegar and oil, with salt and garlic. Tarragon vinegar is made by covering the leaves with vinegar, and steeping them in it.

\section{PASTE-MEAT PIES-FISH PIES.}

\section{6.-VARIOUS SORTS OF PASTE.}

In making paste for pies great care should be taken that the flour be well dried; for the finer kinds of paste it ought also to be sifted. The very best salt butter is perhaps the best material for making paste. Some persons employ lard, or equal parts of lard and butter; and clarified dripping is an excellent substitute. 
An adept in pastry never leaves any part of it adhering to the board or dish used in making. The best thing to make it upon is a slab of marble or slate; which substances cause less waste, being cold and smooth. The coolest part of the house, and of the day, should be chosen for the process; the hands should be previously washed in very hot water, and the less they touch the paste the better and lighter it will prove; nor should it be rolled much.

In whatever way paste be made, wetting it much will render it tough.

Salt butter of the best quality makes a fine flaky crust ; for sweet things, wash it. In making pie-crust lard is, however, better than butter; and in making the paste it is sometimes mixed with the flour in a dry state.

Heating the oven properly is a very essential point in baking the various sorts of paste to be here enumerated.

Raised pies must be put into a quick oven, or the crust will fall. The cook should accurately know the proper heat for each article, as opening the door to observe their progress lets in the air, and often spoils them. They require from one half to a full hour more than when baked in a dish.

\section{7.-CRUST FOR RAISED PIES.}

Boil water with a little fine lard, and an equal quantity of fresh dripping, or of butter, but not much of either. While hot, mix this with as much flour as you will want, making the paste as stiff as you can to be smooth, which you will make it by good kneading and beating it with the rolling-pin. When quite smooth, put a lump into a cloth, or under a pan, to soak till nearly cold.

In raising paste it should be brought to a firm consistence, and of sufficient thickness to hold the meat together; it should therefore not be too rich, and it is easier to be worked if moderately warm than cold. The proper way to raise the crust is by placing the left hand on the lump of paste, and with the right keep working it up the back of the hand, till all be of the proper shape and thickness; a mould, or a decanter, may be used. When worked into the desired form the meat is then put into the pie, and, when quite full, the lid is put on and fixed to the wall or side; the top being ornamented with some device, also made of paste. Before putting it in the oven glaze it all over with white of egg.

Those who have not a good hand at raising crust may roll the paste of a proper thickness, and cut out the top and bottom of the pie, then a long piece for the sides; then cement the bottom to the sides with egg, bringing the former rather farther out, and pinching both together; put egg between the edges of the paste to make it adhere at the sides. Fill the pie, put on the cover, and pinch it and the side crust together. The same mode of uniting the paste is to be observed if the sides are pressed into a tin form, in which the paste must be baked, after it shall be filled and covered:* the tin should be buttered, and carefully taken off when done enough; and as the form usually makes the sides of a lighter color than is proper, the pie should be put into the oven again for one quarter of an hour.

For Venison Pasty or Férigord Pie.To one quarter of a peck of fine flour use two and a half pounds of butter and four eggs: mix into paste with warm water, and work it smooth and to a good consistence. Put a paste round the inside, but not to the bottom of the dish;

* In lining a tin form, put in the sides first, having cut the paste rather deeper than the tin, so as to allow for its lying a little on the bottom, and that no joint may appear; then cut a piece sufficiently large to cover the whole bottom. 
and let the cover be pretty thick, to bear the long continuance in the oven.

\section{8.-PUFF PASTE FOR PATTIES OR FIRST COURSE DISHES.}

One pound of butter salt or fresh, and one pound of flour will make a good dish of patty cases, or a large case for a vol-au-vent, and the remainder into a good dish of second course pastry.

Put your flour upon your board, work finely in with your hands lightly a quarter of the butter, then add water sufficient to make it the stiffness or softness of the remaining butter; each should be the same substance; work it up smooth, then roll it out longways half an inch thick; place the remainder of the butter cut in slices half way on the paste; dust flour lightly over it, and double it up, press it down with your rolling-pin, let it lie a few minutes, then roll it three times thinner each time, letting it lie a few minutes betwee each roll, keep it free from sticking to the board or rollingpin. This paste is ready for patty cases or vol-au-vent, or meat pies.

\section{9.-PUFF PASTE.}

To obtain this in perfection much will depend on the quality of the butter used, and on its being rolled out or turned a sufficient number of times, so that the butter may be mixed with the paste in minute layers. Salt butter will make as good, if not better paste than fresh. When salt butter is used, it should be broken into small pieces, and put into a pan or pail of clean cold water; well work or squeeze it between the hands in the water, which will deprive it of the greatest portion of the salt it contains ; take it from the water and put it on a cloth, and mould or work it well, in the same manner as you would a piece of paste; when the water is all worked out, and the butter appears dry and firm, make it in a roll and put it in a cold place for use. Fresh butter should be moulded in the same manner as directed for salt, but it does not require being first put into water. In summer a few pieces of ice may be put into the water with the butter, to harden it.

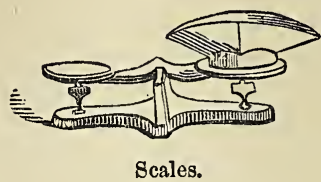

Weigh one pound of butter and one pound of sifted flour. Take two ounces of the butter and rub in with the flour; make a hole in the middle, in which put a teaspoonful of salt, and pour in sufficient cold water to make it into a paste of nearly the same consistence as the butter. This should be more particularly observed in summer, as the heat then causes the butter to become very soft during the rolling of the paste, and to break through the surface, unless the dough is made of considerably less consistence in the first place, in order to allow for this. To each pound allow two eggs, well-beaten and mixed with the water.

Mix the dough into a clear, smooth paste, without toughness, by rubbing it well with the heel of the right hand on the board until it has acquired a clear, smooth, shining appearance; mould it into a round lump, then press all the moisture from the remainder of the butter, and form it into a ball, taking care not to soften it ; put this upon the paste and press it down, then enclose it with the paste like an apple-dumpling; flour the marble well and roll it out; this is called the "first turn;" then fold it in three, and roll it out again, which will be a second turn; now fold it again in the same manner, and put it in a cold place, if convenient on ice, covered with a damp 
cloth, until it is required to be rolled out for use. Experience alone can determine when the paste has been sufficiently rolled.

\section{0.-VERY LIGHT PASTE.}

Mix the flour and water together, roll the paste out, and lay bits of butter upon it. Then beat up the white of an egg, and brush it all over the paste before it is folded; repeat this when rolling out, and adding the butter each time till the whole of the white of egg is used. It will make the paste very flaky.

\section{1.-TRANSPARENT CRUST FOR TARTS.}

Beat an egg, till it be quite thin; have ready twelve ounces of the purest, wellwashed butter, without salt, melted without being oiled; and when cool mix the egg with it, and stir it into one pound of fine flour well dried. Make the paste very thin; line the pattypans as quickly as you can, and, when putting the tarts into the oven, brush them over with water, and sift sugar on them. If they are baked in a lightly heated oven, they will look beautiful.

\section{2.-BRIOCHE PASTE.}

This paste is essential in many of the finer kinds of cookery, and, though rather troublesome to make, will repay the pains bestowed, since, with a small addition of sauce, it will afford an excellent side-dish in itself, and it will enter into the composition of, and improve, all the more elaborate entrées. Take seven fresh eggs. two pounds of flour dried and warmed at the fire, and one pound of fresh butter. Take half a pound of this flour, and pour into the middle of it a dessert-spoonful of yeast, which has been prepared by pouring water over it, stirring it, and allowing it to stand to settle, and then draining the water off. It is the sediment which must be employed. Pour a little warm water over the yeast, and mix the paste up with it, which forms the leaven; flour the pan, lay the leaven in it before the fire to rise, and slit it on the top. As soon as the paste has risen, proceed thus :-Make a hole in the middle of the remaining flour, put into it a little salt, about a saltspoonful, the same quantity of powdered sugar, to take off any bitter taste in the yeast, and a little water to melt it; the butter broken into small pieces, and the eggs: work it all well together, and knead it thoroughly, spreading it out and working it well : then spread it ont entirely, and lay the yeast or leavened paste all over it, taking the greatest care in kneading and shifting the paste about to mix both well together. When completed, fiour a towel, wrap the paste up in it. and put it into a pan; if in cold weather in a warm situation, and if in hot weather in a cold one. It is best made a day before it is wanted. Very minute pieces, shaped in any way, add greatly to the excellence of soup, to be boiled with it, or stewed in the gravy of a vol-au-vent, and strewed over the top. It is cut into shapes also. and fried as the accompaniment of braised dishes of every kind. It is an exquisite case for lobster and other patties, or, brushed over with egg, may be fried in shapes for a side-dish: or it may be boiled in cups, six in a disb, and served up with asparagus cut small and heaped upon the top, and a white sauce round. In fact, a clever cook, by employing different flavoring ingredients, either sweet or savory, may make endless dishes of brioche. It should not, however, predominate; and, therefore, it is best only to make a small quantity at a time, where the consumption of the family is not great. It will not keep long.

\section{3.-MEAT PIES, PATTIES, \&c.}

There are few articles of cookery more generally liked than relishing pies, if 
properly made; and they may be formed of a great variety of things.

Raised Pies may be made of any kind of flesh, fish, fruit, or poultry, if baked in a wall of paste instead of a baking-dish; but they are generally eaten cold, and made so large and savory as to remain a long time before being consumed, for which reason they also bear the name of "standing pies." In making them, the cook should always take care to have a good stock that will jelly, made from the bones and trimmings, to fill up the pie when it comes from the oven, and also that when cold there may be enough jelly. For want of this precaution pies become dry before they can be eaten. The materials are of course frequently varied, but the mode of preparation is so nearly the same as not to require the recital of more than a few prominent receipts.

\section{4.-SEASONING FOR RAISED PIES.}

Three pounds of salt dried and pounded, three ounces of white pepper, half an ounce of cayenne pepper, two ounces of cloves, two ounces of allspice, one ounce of basil, one ounce of marjoram, one ounce of thyme, one ounce of bayleaf, one ounce of nutmeg, one ounce and a half of mace.

Pound the spices and herbs by themselves and sift through a fine sieve; then mix with the salt, and put away in a stoppered bottle: three-quarters of an ounce is sufficient for one pound of farce, and half an ounce for one pound of boned game.

1185.-JELLY FOR MEAT OR RAISED PIES.

Take a quart of veal gravy, dissolve two ounces of isinglass in a little of it; add the remainder with a quarter of a pint of tarragon vinegar; boil all together for a quarter of an hour. Clarify it with the whites of six eggs, then pass it through a bag.
1186. -PIE RAISED HOT, RUSSIAN.

Take a salmon and a liver, cut them into scollops, and simmer both, but separately, in some butter, with shred parsley, mushrooms, truffles, shalots, salt, pepper, nutmeg; mince the yolks of a dozen hard eggs. Make a raised crust in the usual manner, put in a layer of rice previously boiled in chicken broth, but cold, as should be all the materials; on this lay some of the scollops of salmon, on which strew a layer of egg, then a layer of liver, strew the egg, then the salmon again, and so on till your pie is full ; then pour in the butter and lierbs, cover the whole with rice, and finish the pie according to the above directions.

\section{7.-PIE RAISED, TO BE SERVED HOT.}

Make a stiff paste as directed for raised pies, which mix with warm water; when well mixed together roll it out thin, cut a piece out for the bottom and two for the sides, according to the shape of the dish ; egg the edges you intend to join, and press them well together. so that the joining may not be seen; shape it, garnish it with leaves or festoons, according to your taste. fill it nearly to the top with bran, egg it, and let it be baked in a moderate oven; when done of a ligit color turn out the bran, and set it ready for what you intend to put in, which may be cutlets of mutton stewed with vegetables, partridge farced with a brown sauce, chickens cut up, a ragoit in a brown sauce, or stewed eels.

\section{8. - RAISED PIE OF FOWLS. (Soyer.)}

Make the paste and forcemcat, bone a young fowl, which lay flat upon a clean cloth, breast downwards; season the interior with a little pepper, salt, and chopped onions; spread a layer of forcemeat over, half an inch in thickness; have ten pieces of veal, of the thickness of your finger, and the same length as the fowl, 
and the same number of pieces of fat bacon, lay half of the veal and bacon alternately upon the fowl, well seasoned with pepper and salt, cover over with more forcemeat, then another layer of veal and ham, cover with more forcemeat; then roll the fowl over, making the skin meet at the back; you have previously lined a raised pie mould with paste, then line the pie with forcemeat, half an inch in thickness, lay in the fowl, sprinkle a little pepper and salt over, cover with the remainder of the forcemeat, to form a dome. place a pat of butter and two bay-leaves upon the top, and bake; when done, pour in a gill of gravy made from the bones of the fowl; serve cold.

\section{9.-RAISED PIE OF PHEASANT.}

Proceed precisely as for the pie of fowl, but of course using a pheasant; an old one would answer the purpose if kept long enough, but all the sinews of the legs must be taken out in boning it; the fillets of the breast also, being very thick, may be partly cut out and used with the veal for the interior; if in a situation to obtain rabbits, the fillets of them might be used instead of veal for the interior, and the legs for forcemeat.

For gravy, break up the bones of the birds, which put into a stewpan with a glass of sherry, an onion, a few sprigs of thyme, parsley, and a bay-leaf; let it simmer a minute over the fire, then add a pint of broth and a little isinglass or gelatine, let the whole simmer for an hour, giving it a nice brown color, when pass it through a sieve into a small stewpan, place it again upon the fire, skim off all the fat, and reduce it to half a pint, and when the pie is baked, pour it in, shaking the pie a little to mix well; serve when cold.

Pies of grouse, partridges, moor fowls, $\& c$., are made precisely in the same manner, using one or more according to the size you wish to make your pie. The fillets of hares are likewise excellent in pies, whilst the legs might be jugged or converted into soup.

Capons, poulards, green geese, or ducklings, may also be served in a pie, (proceeding as directed for fowls), by managing the size of the pie, and seasoning in proportion.

Pigeon pie can also be made in the same way, but then the meat with which the interior of the birds are filled must be cut much smaller, and requires less time in cooking.

\section{0:-HOT LAMB PIE (raised.)}

To make this an oval, a tin or copper pie mould would be required, which you would choose of a size most generally useful. Butter the interior of the mould, which stand upon a baking sheet, then make the following paste: put a quarter of a pound of butter and the same of chopped suet into a stewpan, with three-quarters of a pint of water, or more if required, and let the whole boil together one minute, when strain it through a sieve into a basin containing two pounds of flour, mixing it first with a spoon, and when cool enough with the hand, until forming a smooth paste; when partly cold roll it out into a sheet half an inch in thickness, with which line the mould, pressing the paste evenly at all parts; have ready cut sufficient small lamb chops from the loin, neatly cut away the bones, and lay them round the interior of the pie alternately with slices of raw potatoes (a quarter of an inch in thickness), season rather highly as you proceed, with pepper, salt, chopped onions, and parsley: make a neat cover with the trimmings of the paste, and bake it rather better than two hours in a moderate oven; when done lift the cover, pour out as much of the fat as possible, add a little gravy, and serve. 
1191.-OTHER VARIOUS PIES.

Hot raised pies may also be made with mutton by following the above directions. They are also very good made with fillet of beef cut into thin slices of the size of the lamb chops, or of rump steak, by laying a piece at the bottom, seasoning and filling alternately with potatoes and the meat; veal and ham pies are also excellent, but the potatoes in them had better be omitted, the veal, however, seasoned and dipped in flour. Pies may also be made with veal sweetbread and ham, but then about three parts of a pint of white sauce should be poured in aiter the pie is baked. Fowls or rabbits may likewise be cut into joints, and put into a stewpan, with a piece of butter, previously well ${ }^{*}$ seasoning them with pepper. salt, and chopped eschalots ; cover the stewpan close, and leave it twenty minutes over a slow fire, add a pint of white sauce, and simmer ten minutes longer; when cold build them up in the interior of the pie, which cover and bake an hour in a warm oven. Pies of the abuve description can of course be made of any size, either large enough for a family meal, or very small and round, for a corner dish for a dinner party; most people who are in the habit of making them, keep two different-sized moulds for the purpose.

\section{2.-RUMPSTEAK PIE.}

Procure two pounds of rumpsteaks, which cut into slices half an inch thick, and season well with pepper and salt; dip each piece into flour, and lay them in a small pie-dish, finishing the top in the form of a dome; add a wineglassful of water, then have ready half a pound of plain paste, cut off a small piece, which roll into a band, and lay round the edge of the dish, having previously wetted it with a paste-brush dipped in water, then roll out the remainder of the paste to about the size of the dish, damp the band of paste upon the dish, and lay the other picce over, make a hole with a knife at the top, press the edges evenly down with your thumbs, trim the pie round with a knife, egg over the top with a paste-brush, and ornament it with the trimmings of the paste, according to fancy; bake it rather better than an hour in a moderate oven, and serve either hot or cold.

$$
\text { 1193.-VEAL AND HAM PIE. }
$$

Cut about a pound and a half of veal into thin slices, as also a quarter of a pound of cooked ham; season the veal rather highly with white pepper and salt, with which cover the bottom of the dish, then lay over a few slices of ham, then the remainder of the veal, finishing with the remainder of the ham, add a wine-glassful of water, and cover and bake as directed for beef-steak pie: a bay-leaf will be an improvement.

\section{4.-MUTTON PIE.}

Procure the chumps of three loins of mutton, from which cut the meat in moderately thin slices, put a layer at the bottom of the dish, which season well with chopped parsley, eschalots, pepper: and salt; then put a layer of slices of raw potatoes and again a layer of mutton, seasoning as before, proceeding thus to the top, which form in a dome, finishing with mutton, cover with paste, and bake as directed for rumpsteak pie.

$$
\text { 1195.-PÁTE DE FOIES GRAS. }
$$

[This receipt is fornished by Mr. DeLMoNico, expressly for this work.]

Prepare a stuffing of fresh pork, a pound of lean to a pound and a half of fat ; mince and chop it: add your preparation of liver's in the same manner as with truffles, seasoning the mixture with salt, pepper, spices, and aromatic herbs bruised and passed through a sieve. Have in readiness two fine, fat livers, 
well cleaned. Make ready a pie of two and a half pounds of paste: surround this pie with thin slices of fat, and, in the botton, place some stuffing: season the livers, cover them with stuffing and truffles, and place them in your pie or dish, which fill up with stuffing and truffles, placing on the top a piece of fresh butter. Cover this with slices of fat and over it place a laurel-leaf. Moisten the edge of the pie and cover it with a crust of paste : fasten this on well, cut off the edges equally and crimp or decorate it. Make a covering of puff, paste, and, in the middle, make a hole which is called the chimney. Wash it over with eggs and let it bake in an evenly-heated oven, for three and a half hours. When cold, fill it with good meat-jelly.

\section{6.-LAMB PIE.}

Cut a small neck of lamb into chops, which must not be too fat season them lightly with pepper and salt, and lay them in your pie-dish, with a few new potatoes in slices, pour in a little water, then cover and bake as directed for rumpsteak pie.

\section{7.-CHICKEN PIE.}

Cut up a nice plump chicken into joints, which lay upon a dish, and season lightly with chopped parsley, white pepper, and salt, then lay the back. cut into three pieces, at the bottom of a pie-dish with the two legs on either side; have half a pound of cooked ham or bacon in slices, a layer of which cover over, then lay in the two wings, and over them the breast, cut into two pieces, which, with the remainder of the ham or bacon, form into a dome in the middle, pour half a pint of white sauce over, if handy, or a little broth or water, cover with paste, and bake as directed for the last. If no white sauce, dip each piece lightly in flour. 1198.-GIBLET PIE.

When the giblets have been taken from the goose, place them with the exception of the liver, but with the pinions, feet, head, and neck, into boiling water to blanch, remove the skin from the feet and beak; put into a stewpan a piece of butter the size of a walnut, one, onion cut in slices, a bay-leaf, a little salt, pepper, and sugar: place it on the fire until the onion is brown; place in the giblets with the head cut in two, let them remain on the fire for about three minutes, stirring them,round, then add one quart of boiling water, and let them stew gently for two hours, remove from the fire, and let them get cold. Take a pie-dish and place a piece of steak on the bottom; then place over that the giblets with the liver, and steak again over them, add the liquor the giblets were stewed in, season and cover with half puff paste.

\section{9.-RABBIT PIE}

Cut a nice rabbit into joints, splitting the head in halves, and lay them in lukewarm water half an hour to disgorge, then dry them upon a cloth, season well with pepper, salt, chopped eshalots, parsley, two bay-leaves, and a spoonful of flour; have also three-quarters of a pound of uncooked streaked bacon, cut into square pieces the size of walnuts, build up the pieces of rabbit and bacon together, in a pie dish. commencing with the worst pieces, and forming a dome; pour in a little water, cover with paste, and bake as directed for rumpsteak pie.

\section{0.-PIGEON PIE.}

Line the bottom of a pie-dish with a pound of rumpsteak, cut into slices not too thin, seasoned with a little salt, pepper, and cayenne, and dipped into flour; have ready picked and drawn a couple of pigeons, cut off the feet, turn the legs in, fold up the pinions of the wings, and lay them breast to breast upon the meat, have the yolks of four hard boiled eggs, which put at the sides. 
sprinkle a little pepper and salt over the pigeons, lay a bay-leaf upon the top, pour in a little water, cover with paste, stick the feet in the top, and bake as directed for the last. The pigeons may be stuffed with a little veal stuffing, if handy.

\section{1.-PARTRIDGE PIE.}

Line the bottom of a pie-dish with slices of veal, cut moderately thick, and rather lightly seasoned with white pepper and salt; have ready picked, drawn, and trussed, a couple of young partridges, pour one glass of sherry over the veal, one of water, and lay in the partridges breast to breast, laying a piece of fat bacon over each, cover with paste, sticking the feet of the partridges in the top of the pie, and bake as before.

\section{2.-GROUSE PIE.}

Roast, very underdone, a couple of nice plump grouse ; when cold, cut into joints, having the two wings, two legs, and the breasts in two pieces each, season them lightly, and lay them in a pie-dish, building them to form a dome, then break up the back-bone and other trimmings, which put into a stewpan, with a glass of sherry, a bay-leaf, an onion in slices, a few sprigs of parsley, three or four whole allspice, set the stewpan over the fire a few minutes until the wine boils, when add half a pint of brown sauce, and half a pint of broth, stir it over a fire until again reduced to half a pint, strain it through a sieve, over the grouse; when quite cold cover with paste, as directed for rumpsteak pie, and bake in a very hot oven; about half an hour would be sufficient, as the paste requires to be laid on thinner, the contents of the pie having been previously cooked.

\section{3.-POT PIE}

Two pounds of veal meat, two pounds of flour, one-quarter of a pound of lard or butter, half a dozen potatoes, onequarter of a pound of pickled pork, cut into small pieces. Make the paste very soft, and roll it very thin; put in the potatoes and meat, then the paste, layer after layer, with cold water enough to cover it; boil it half an hour slowly.

\section{4---EEL PIE.}

Skin and cleanse three good-sized eels, which cut into pieces about two inches in length, put a good-sized bunch of parsley, thyme, and three bay-leaves, all tied together, into a stewpan, with an onion, into which you have stuck four cloves, a glass of port-wine, and a pint of broth, lay in the pieces of eels, and set them upon the fire to simmer for ten minutes, take them out, laying them upon a cloth to drain, skim uff all the fat from the stock the eels were cooked in, to which add rather more than half a pint of brown sauce, let the whole boil until reduced to three parts of a pint, when dress the pieces of eels up in a pie-dish, strain the sauce over through a sieve, and when cold, cover with paste, as directed for rumpsteak pie, and bake about an hour in a moderate oven; serve it hot. If for a small pie they may be used raw, and season accordingly, after having rolled each piece in flour.

\section{5.-BEEFSTEAK PUDDING.}

Put a pound of flour upon a dresser, with which mix half a pound of beef suet, very finely chopped, make a hole in the niiddle, into which put a teaspoonful of salt, and sufficient water to form a rather stiffish paste, mix it well together, using a little more flour to dry it and prevent its sticking; then lightly butter the interior of a round-bottomed pudding basin, roll out two-thirds of the paste to half an inch in thickness, with which line the basin; have ready cut into slices, about the size of the palm of the hand, 
and a quarter of an inch in thickness, two pounds of rumpsteak with a little of the fat included; lay them upon a dish, seas on with two teaspoonfuls of salt and one of black pepper, sprinkle a little flour over, move them about a little until each piece is well covered with flour and seasoning; then lay them within the paste, also putting in whatever seasoning may remain upon the dish, (some add two dozen of oysters, blanched and bearded, which is excellent,) pour a gill of water over, moistening the edges of the paste ; then roll out the remainder of the paste to form a lid, which place over, pressing it down with the thumb, then tie the basin in a pudding-cloth, and put into a saucepan, containing about a gallon of boiling water,-and keep continually boiling for nearly two hours, adding a little more water occasionally to keep up the quantity; then take it up, untie the cloth, run a sharp-pointed knife into the pudding, and if the meat feels tender it is done (if not, it will require more boiling), turn it over upon your dish, lift the basin carefully from it, and serve, without opening the pudding to add gravy, as many persons do, for a pudding made as above ought to be full of gravy when cut at table.

\section{6.-MUTTON PUDDING.}

Line a pudding-basin with paste. as directed in the last; then have ready cut into slices the meat from two loin-chumps of mutton, which lay upon a dish, and season with a teaspoonful of chopped onions, the same of chopped parsley, rather more than half that quantity of black pepper, and salt in proportion; then put a layer of meat into the pudding, then a layer of raw potatoes cut into slices; proceeding thus until you have filled it up, but finishing with meat, cover it up as in the last, likewise tie it in a napkin, and boil, but rather better than two hours would be sufficient; serve as before directed.

\section{7.-LAMB PUDDING.}

If convenient, procure the entire ribs of a lamb, sawing off the breast almost close to the lean part of the neck; the breast may be cooked; cut the neck into rather thin cutlets, which season lightly with white pepper, salt, and a little chopped parsley and onions; you have previously lined a puddingbasin with paste as before, fill it with the meat thus prepared, intermixing a few new potatoes cut in slices, finish the pudding, boil, and serve as before directed.

\section{8.-VEAL PUDDING.}

Cut two pounds of veal from any part of the leg into slices, about the size of the palm of the hand, and a quarter of an inch in thickness, put two ounces of butter into a frying-pan, and when melted lay in the veal, and a few slices of streaked bacon, season the whole with pepper and a little salt, add one bay-leaf, and a few sprigs of thyme; place the pan over a slow fire, sauté the veal gently for a quarter of an hour; then take it from the fire, and leave it in the pan until cold, then have a pudding-basin lined with paste as before, lay in the veal and bacon, pouring the gravy over, cover, and boil as before, but an hour would be sufficient.

\section{9.-PORK PUDDING.}

Line a pudding-basin with paste as before, and spread three-quarters of a pound of sausage-meat of an equal thickness over the interior, have a pound and a half of lean pork, from the leg if possible, cut into square pieces of the size of walnuts, which season rather highly with pepper, salt, a teaspoonful of chopped eschalots, and half that quantity of dried 
sage ; put the meat into the centre of the pudding, cover over with a quarter of a pound more sausage-mcat, over which put on the cover of paste, tie it in a cloth, and boil two hours and a half, as directed for beefsteak pudding.

\section{0.-KIDNEY PUDDING.}

Procure one ox or eight mutton kidneys, which cut into slices the thickness of half-a-crown piece; lay them upon a dish, seasoning well with black pepper and salt, and shaking one ounce of flour over; mix all well together, to absorb the flour and seasoning; then have a pudding-basin, lined as directed for beefsteak pudding, finish, boil, and serve as there directed.

A pudding made with one pound of steak and a beef kidney is also very excellent.

\section{1.-RABBIT PUDDING.}

Cut a rabbit up in joints, (splitting the head in halves,) and lay them in a basin of lukewarm water an hour to disgorge; line a pudding-basin with paste as directed for rumpsteak pudding, dry the pieces of rabbit upon a cloth, and lay them in the pudding with half a pound of streaked bacon cut into square pieces, and seasoning rather highly with chopped eschalots, salt, pepper, and chopped parsley; cover, tie it in a cloth, boil it two hours, and serve as befure directed.

\section{2.-OYSTER PIE.}

As you open the oysters separate them from the liquor, which strain; parboil sweetbreads, and, cutting them in slices, lay them and the oysters in layers; season very lightly with salt, pepper, and mace; then put half a teacupful of liquor, and the same of veal gravy. Bake in a slow oven; and before you serve, put a teacupful of cream, a little more oyster-liquor, and a cup of white gravy, all warmed, but not boiled.
1213.-OYSTER PIE. (English way.)

Lay a tender beefsteak in the bottom of a dish and on it oysters and bread crumbs, with pepper and salt. Fill the dish with layers of beafsteak and oysters ; pour over it the liquor of the oyster, cover with a light paste and bake twenty minutes in a quick oven.

\section{4.-YORKSHIRE PUDDING.}

Put six tablespoonfuls of flour into a basin, with six eggs, a pinch of salt, and a quarter of a pint of milk, mix well together with a wooden spoon, adding the remaining three-quarters, of a pint of milk by degrees; you have previously set a shallow tin dish under a piece of roasting beef before the fire; an hour before serving pour in the batter, leaving it under the meat until quite set and rather browned upon the top, when turn the pudding over upon the dish you intend serving it upon, and again place it before the fire until the other side is rather browned, when it is ready to serve with the meat.

This pudding is very excellent baked under a small piece of beef of about five or six pounds. It is also frequently baked beneath a shoulder of mutton; and sometimes in an oven separate, if the fire is not large enough, (with a few spoonfuls of gravy added).

\section{5.-TOAD IN A HOLE.}

Make a batter as directed for the Yorkshire pudding, but with the addition of a spoonful more flour and six ounces of chopped beef suet; butter a rather deep baking-dish, into which pour the batter, lay a solid piece of lean gravy beef about three pounds, in the centre, or a beef skirt, and bake it an hour and a half in a hot oven.

Another method is to cut up about three pounds of rumpsteaks into about six pieces, and putting them in the bat- 
ter at various distances apart, but the former method is most common.

Any remains of cooked beef, veal, mutton, pork, roasted or boiled, salt or fresh, or gance and fowl, cut in pieces, and seasoned to taste, may be used in this dish, by adding it to the batter when in the dish.

\section{6.-FISH PUDDING.}

Cut a slise of salmon half an inch thick, and weighing about one pound; remove the skin and bone, mix a saltspoonful of salt, half one of pepper, one of finely chopped onions and parsley, and a spoonful of flour in a plate, and $\mathrm{sub}$ the fish with it; make half a pound of flour into paste, roll it half an inch thick, place a pudding-cloth in a middle-size basin, flour the cloth, lay the paste in it, place in the salmon with a little water and white wine, and a bay-leaf, bring the paste over so that the edges meet in the middle, having reserved a small piece which you have rolled out to place over the joint, after having wetted it. Tie the cloth, but not too tight, round the paste; place it in a gallon of boiling water, let it simmer for one hour and a half, take it out, remove the cloth, and serve it with shrimp sauce over or separate. Fillets of soles, mackerel, turbot, or other fish, may be used.

\section{7.-VEAL PIE IN A DISH.}

First of all get all your ingredients ready, namely, hop, parsley, shalots, or onions, mushrooms, pepper and salt, mint, four eggs boiled hard, a little good second stock, now your paste. After you have made patties or any first or second course pastry, the paste that remains will do, be sure to put an edging of paste to your dish; first lay a layer of lean and fat ham, or mild bacon, then sprinkle it over with the prepared ingredients, then a layer of real, and part of an egg, then another layer of ham and parsnips as before, and keep on repeating it until quite full, letting the middle be much higher than the sides; put a little drop of second stock into it, bake it in a slow oven; be sure and cut a hole in the top, and if you like ornament it with leaves of paste ; after egging the top, well notch the edge. Pour some good white stock into it when done and hot.

\section{8.-BEEFSTEAK PIE.}

Take some good steaks, beat them with a rolling-pin, season them with pepper and salt ; fill a dish with them, adding as much water as will half fill it, then cover it with a good crust, and bake it well.

\section{9.-COLD VEAL OR CHICKEN PIE.}

Lay a crust into a shallow tart dish, and fill it with the following mixture :shred cold veal or fowl, and half the quantity of ham, mostly lean, put to it a little cream, season with white and cayenne pepper, salt, a little nutmeg, and a small piece of shalot, chopped as fine as possible : cover with crust, and turn it out of the dish when baked, or bake the crust with a piece of bread to keep it hollow, and warm the mince with a little cream, and pour in.

1220.-CALF'S HEAD PIE.

Stew a knuckle of veal till fit for eating with two onions, a little isinglass, a fagot of sweet herbs, a blade of mace, and a few peppercorns in three pints of water; keep the broth for the pie. Take off a little of the meat for forcemeat balls, and let them be used for the family, but boil the bones until the broth is very good: half boil the head, and cut it into square pieces; put a layer of ham at the bottom of them, some head, first fat then lean, with forcemeat balls, and hardboiled eggs cut in half, and so on till the dish is full, but be careful not to place the pieces close together or the pie will 
be too solid, and there will be no space for the jelly. The meat must be first pretty well seasoned with pepper and salt, and a little nutmeg; put a little water and a little stock into the dish, and cover it with a thickish crust, bake it in a slow oven, and when done, pour. into it as much gravy as it will possibly hold, and do not cut it till perfectly cold, in doing which use a very sharp knife, and first cut out a large slice, going down to the bottom of the dish, and when done thus thinner slices can be cut. The different colors and the clear jelly have a beautiful appearance.

\section{1.-DUCK PIE.}

Bone a full-grown young duck and a fowl, wash them and season with pepper and salt, a little allspice and mace pounded; put the fowl within the duck, and on the former a calf's tongue pickled red. boiled very tender and peeled, press the whole close, the skins of the legs should be drawn inward, that the body of the fowl may be quite smooth; if approved, the space between the sides of the crust may be filled with a fine forcemeat. Bake it in a slow oven, either in a dish or raised pie-crust, ormamented.

1222.-FISH PIE.

This pie may be made of any fish, salmon, pike, eel, or any other. Scale your fish and cut it into pieces, line your piedish with a good crust, put in the fish with a bunch of sweet herbs, a little salt, some bruised spices, and a layer of butter on the top, put on the crust and bake for an hour and a half; when done remove the fat and put in a vegetable ragout made thus:-Stir a little butter and flour over the fire until a pale brown, moisten with half a pint of sherry, some soup maigre, add a few mushrooms, a little salt, and a bunch of herbs; let it bosl half an hour, and then put the ra- goût into the pies. Any vegetable ragoût may be used.

\section{3.-GAME PIE.}

Cut up your game, and use truffles and whole mushrooms if you have them; the seasonings as before, but no hardboiled eggs, and add a little port wine with your gravy or stock. If you take the bones from the birds or hare, use some forcemeat as layers instead as in former pies, veal and steaks, but no eggs ; if boned you will prepare a good stock from the bones, making the pie taste of the very essence of the game, or poultry, or whatever it may consist of.

\section{4 -SNIPE PIE.}

Bone three snipes, fill them with a light forcemeat, adding the trails and some truffles pounded, put the birds in 9, deep dish, with a small layer of forcemeat all round; cover with a puff-paste, egg it, ornament it to fancy, and put it into the oven. When about three parts done, lift up the lid, pour in some good cullis, a glass and a half of Madeira. Season with cayenne pepper and lemonjuice, cover down, and finish baking.

\section{5.-GREEN GOOSE PIE.}

Bone two young green geese of a good size, but first take away every plug and singe them nicely, wash them clean, and season them high with salt, pepper, mace, and allspice. Put one inside the other and press them as close as you can, drawing the legs inwards; put a good deal of butter over them, and bake them either with or without crust; if with the latter a cover to the dish must fit close to keep in the steam, it will keep long. Gravy jellied may be added when served.

MACKENZIE'S PERIGORD PIE.

Take six partridges and tie their legs 
like chickens to be boiled; season them with pepper, salt, cloves, and beaten mace. Put two pounds lean veal, and one pound of fat bacon cut into small bits, in a stew pan with half a pound of butter, shalots, chopped herbs, and seasoning; stew till tender; strain and pound the meat in a mortar; mix the pulp with some of the liquor; put this forcemeat around and over the partridges in a raised pie, and lay over thin slices of bacon. Cover with paste; bake three hours in a moderate oven.

Worcester sauce, good with savory pies, is said to be made on a basis of good walnut ketchup with additional spices.

\section{7.-HARE PIE.}

Season the hare after it is cut up, in the same manner as if to be jugged; and bake it, with eggs, and forcemeat partly made of the liver, in a raised crust or dish. When it is to be served, raise the lid, and, if in a crust, cover it with jelly; but, if made in a dish, with warm gravy, mixed with a large glassful of port wine.

1228.-TIPPERARY CURRY (an excellent Picnic Dish).

Boil four chickens, and stuff two of them when cold with a forcemeat made of crumbs of bread, a few slices of ham or tongue, sweet herbs, and a shalot well pounded and mixed with the yolk of an egg. Stuff the other two with boiled rice, lay them in a mould or dish, with eight hard-boiled eggs cut in half, a few mushrooms, a little pickled lemon, cut in thin rings. Pour over the chickens a gravy made as follows:-Fry an onion in a little butter, add a table-spoonful of curry-powder, one of vinegar, one of mushroom-ketchup, a little salt, and a little more than a pint of good veal broth; if the broth does not jelly, isinglass must be put into it to make it do so. When cold turn it out on a dish. It is a great improvement to bone the chickens, the bones helping to make the gravy.

\section{9.-FRENCH PIE}

Make a raised crust upon a buttered tin, and brush it with yolk of egg; cover the bottom with forcemeat, then fill up the pie with sweetbread cut into small pieces, oysters, hard eggs, legs of chicken, turkey, or rabbit, boned; artichokc-bottoms in small pieces, asparagustops, forcemeat-balls, and mushrooms, taking care to lay them regularly, and to season the whole lightly and evenly: bake it in a moderate oven. When it is done pour in some strong gravy and cream thickened with flour and butter.

Sausage-meat may be mixed with the forcemeat, and in winter truffles substituted for the vegetables; the seasoning should be of mace, cloves, and cayenne pepper.

\section{0.-VEGETABLE PIE.}

Scald and blanch some broad beans; cut young carrots, turnips, artichokebottoms, mushrooms, onions, lettuces, parsley, celery, and add peas; or use any of them you may have. Make them into a nice stew, with a little good veal gravy; season with pepper and salt; bake a crust over a dish, with a little lining round the edge. When baked, pour the stew into the dish, and lay the crust over it. Winter vegetables may be used in the same way, and a cup of cream is a great improvement.

\section{1.-COLD PIES.}

When meat pies are prepared to be eaten cold, suet should not be put into the forcemeat that is used with them. If the pie is made of meat that will take more dressing, to make it extremiely tender, than the baking of the crust will allow, prepare it in the following way:Take three pounds of the veiny piece of 
beef that has fat and lean; wash it, and season it with salt, pepper, mace, and allspice, in fine powder. rubbing them well in. Set it by the side of a s'ow fire, in a stewnot that.will just hold it; put to it a piece of butter of about the weight of two ounces, and cover it quite close; let it just simmer in its own steam till it begins to shrink. When it is cold, add more seasoning, forcemeat, and eggs: if it is made in a dish, put some gravy to it before baking; but if it is only in crust, do not put the gravy till after it is cold and in jelly. Forcemeat may be put both under and over the meat, if preferred to balls.

\section{2.-VEAL AND SWEETBREAD PIE.}

Cut the veal from the chump end of the loin; season it well; clean and blanch a sweetbread, cut it into pieces, and season it; lay both in the dish with the yolks of six hard-boiled eggs and a pint of orsters. Strain the oyster liquor, add to it a pint of good gravy; line the sides of the dish with a puff paste half an inch thick, and cover it with a lid of the same. Bake it in a quick oven for an hour and a quarter; and when it is sent to table cut the lid into eight or ten pieces, and stick them round the sides, covering the meat with slices of lemon.

\section{3.- VEAL AND SAUSAGE PIE.}

Cover a shallow dish with paste, lay a well-beaten veal cutlet at the bottom, slightly seasoned; cover it with a Bologna sausage freed from the skin and cut into slices; then add another cutlet and a layer of the Bologna sausage; cover the whole with paste, and put no water to it: the veal will give out sufficient gravy, while it will be rendered very savory by the sausage. It is excellent eaten cold.

1234.-VEAL AND OYSTER PIE.

Make a seasoning of pepper, salt, and a small quantity of grated lemon-peel. Cut some veal-cutlets, and beat them until they are tender: spread over them a layer of pounded ham, and roll them round; then cover them with oysters, and put another layer of the veal fillets, and oysters on the top. Make a gravy of the bones and trimmings, or with a lump of butter, onion, a little flour and water; stew the oyster liquor, and put to it, and fill up the dish, reserving a portion to put into the pie when it comes from the oven.

\section{5.-EXCELLENT PORK PIES TO EAT} COLD.

Raise the crust according to the directions already given, into either a round or oval form, as you choose ; have ready the trimmings and small bits of pork cut off when a hog is killed; and if these are not enough, take the meat off a bladebone. Beat it well with a rolling-pin, cut it into dice, season with pepper and salt, and keep the fat and lean separate. Put it in layers, quite close up to the top; lay on the lid; cut the edge smooth round, and pinch it; bake in a slow soaking oven, as the meat is very solid. The pork may be put into a common dish, with a very plain crust, and be quite as good. Observe to put no bone or water into pork pie: the outside of the pieces will be hard unless they are cut small and pressed close.

Both these and veal and chicken pies are generally eaten cold, and, as they are always seasoned highly, will keep good for several days in the hottest weather. In England it is not uncommon to take off the cover and fill the pie up, when cold, with clouted cream, which is a very delicious addition.

\section{6.-CALF'S-HEAD OR CALF'S-FOOT PIE.}

Either must be well stewed before being put into the pie. They must be cut into nice pieces, free from bone, and 
well seasoned, or otherwise they will be tasteless. If to be eaten hot, sausagemeat or fresh mushrooms or oysters will be an improvement, in addition to the ham, eggs, and forcemeat balls; and if cold, the small pickled mushrooms should be added, or a little mushroom powder in the gravy. The excellence of the pie will chiefly depend upon the seasoning and flavor, and the quantity of fine savory jelly which it will contain; and therefore the meat should not be closely packed, in order to leave room for the jelly.

\section{7.-CALF'S-FOOT AND KIDNEY PIE.}

Take from a cold roasted loin of veal slices of the kidney fat, cover them with pounded ham, and put them into a dish with the kidney cut into pieces, and the meat from a calf's foot; put in a few hardboiled eggs and forcemeat balls. made very savory with oysters, an anchovy, and the fat of bacon, as this pie should be eaten cold. Put the juice of a half a lemon, or a spoonful of Harvey sauce, into a gravy. "Sweetbread may be employed instead of kidney.

\section{8.-BEEF-STEAK AND OYSTER PIE.}

Prepare the steaks as above, and put layers of them and of oysters. Stew the liquor and beards of the latter with a bit of lemon-peel, mace, and a tablespoonful of walnut ketchup. When the pie is baked, boil with the above three spoonfuls of cream, and one ounce of butter rubbed in flour; to which, however, many people object as injuring the savoriness of the pie; in which case, should any addition be required, a few spoonfuls of beef gravy and port wine will answer the purpose. Strain it, and pour it into the dish : for a small pie a dozen large oysters will be sufficient, and the pie may be baked in a couple of hours.
1239.-MARROW PATTIES.

Make a paste with four ounces of sweet alnonds blanched, moistening them with orange flower water; mix this paste with a handful of flour, a drop of warm water and the yolks of three eggs; then line several shallow moulds with it, glaze and bake them in a slow oven. When done put into each of them a little cream about the thickness of a crown piece, made with beef marrow, lemon peel, and cream, cover it.with a spoonful of white of egg whipped to a snow, sprinkle sifted sugar over, and serve them very hot.

\section{0.-SQUAB PIE}

Is a favorite dish in some parts of England, and is there generally made of mutton. Cut apples as for other pies, and lay them in rows with mutton chops and shred onion; sprinkle with pepper and salt, and also some sugar. Bake the pie in a deep dish, cover it with a thick crust, and eat it hot; it must be well done, and will take two to three hours baking. It is often made of beef cut into dice, with some lean bacon seasoned and mixed with the apples.

\section{1.-VENISON PASTY.}

The neck, breast, and shoulder are the only parts of the deer used for a pasty; and even the neck is more generally roasted.

Cut the venison in pieces; put it into a stewpan with a little port wine, two shalots, an onion, bay-leaf, fagot of parsley and thyme, pepper, salt, and a little gravy. Cover it close and let it stew gently until tender; take out the meat, and pull out the bones; reduce the gravy, and when thoroughly cold remove the fat; then proceed to make the pasty.

Line the sides of the dish with crust, but not the bottom; lay in the meat,with fat between each piece, season with pep- 
per and salt, put to it a pint of the gravy and a few bits of butter, cover with a good crust, and bake it thoroughly.

Keep the remainder of the gravy till the pasty comes from the oven, put it by means of a funnel into the middle quite hot, and shake the dish to mix it well. The pasty must be well done, requiring generally two hours' baking.

A want of fat may be supplied by the fat of a loin of mutton steeped for twentyfour hours in port wine and garlic vinegar.

1242.-TO MAKE A PASTY OF BEEF OR MUTTON TO EAT LIKE VENISON.

Bone a small rump or piece of sirloin of beef, or a fat loin of mutton, after hanging several days. Beat it very well with a rolling-pin; then rub ten pounds of meat with four ounces of sugar, and pour over it a glass of port, and the same of vinegar. Let it lie five days and nights; wash and wipe the meat very dry, and season it very high with pepper, Jamaica pepper, nutmeg, and salt. Lay it in the dish, and to ten pounds put three-quarters of a pound of butter; spread it over the meat. Put a crust round the edges, and cover with a thick one, or it will be overdone before the meat be soaked: it must be done in a slow oven.

Set the bones in a pan in the oven, with no more water than will cover them, and one glass of port, a little pepper and salt, that you may have a little rich gravy to add to the pasty when drawn.

\section{3.-YORKSHIRE GOOSE PIE.}

Bone a goose, a fowl, and a pigeon; season them well with pepper, taking care to cover the whole of the interior, or otherwise it will turn sour before it can be eaten; put the pigeon into the fowl, and the fowl inside the goose, filling the interstices with various kinds of force and sausage meat and hard-boiled eggs. The liver and gizzard of the fowl and pigeon will assist in the forcemeat, which must be very well compounded; sew up the goose, and put it into a thick crust. It will take several hours to bake. Make savory jelly, which must also be well seasoned, pour it into the pie when it comes from the oven; ornament the top, and let it be movable, so that the pie may have a good appearance to the last, as the crust is not to be eaten. A calf's foot, boned and cut small, may be added, especially if used for the jelly.

\section{4.-A CHRISTMAS PIE.}

Bone a large goose and a fowl. Parboil a smoked tongue; peel it and cut off the root. Mix together a powdered nutmeg: a quarter of an ounce of powdered mace, a tea-spoonful of pepper, the same quantity of salt, and season the fowl and goose.

Roll out the paste near an inch thick, and divide it into three pieces; cut out two of them in an oval form for the top and bottom; and the other into a long straight piece for the sides or walls of the pie. Brush the paste all over with beaten white of egg, and set on the bottom the piece that is to form the wall, pinching the edges together, and cementing them with white of egg. The bottom piece must be large enough to turn up a little round the lower edge of the wall piece, to which it must be firmly joined all round. When you have the crust properly fixed, so as to be baked standing alone without a dish, put in first the goose, then the fowl, then the tongue. Fill up what space is left with pieces of the flesh of pigeons, or of 'partridges, quails, or any game that is convenient. There must be no bones in the pie. You may add aiso some bits of ham, or some forcemeat balls. Cover the ingredients with half a pound of butter, and put on the top crust which, of course, must be also of an oval form to correspond with 
the bottom. The lid must be placed not quite on the top edge of the wall, but an inch and a half below it; close it very well, and ornament the sides and top with festoons and leaves cut out of paste; notch the edges handsomely, and put a paste flower in the centre; glaze the whole with beaten yolk of egg, and bind the pie all round with a double fold of white paper; bake it four hours.

If the weather is cold, and the pie kept carefully covered up from the air, it will be good for two or three weeks, the standing crust assisting to preserve it.

\section{5.-PATTIES}

Roll out puff paste half an inch thick, and, with a two-inch round cutter, cut as many pieces as are required for the dish. Take an inch cutter, dip it in hot water, and press it in the middle of the paste about half through. Brush the pieces over with yolk of egg, bake in a quick oven, take out the piece marked with the inch cutter, remove the soft parts from the inside, fill them with a mince of any kind, dish them on a napkin, garnish with fried parsley. To make the tops, roll out the trimmings thin, cut some pieces with the inch cutter, egg them over, and bake them.

of Beef.-Shred underdone dressed beef with a little fat, season with pepper, salt, and a little shalot or onion. Add gravy, half a glass of Madeira, and a few bread-crumbs. When hot, fill the pattycases.

Of Veal.-Mince some real that is not quite done, put to it a little parsley, lemon-peel, nutmeg, and salt; a little cream and gravy just to moisten the meat; if you have any ham, it will be an improvement. Do not warm it till the patties are baked.

of Turkey.-Mince some of the white part; and with grated lemon, nutmeg, salt, a very little white pepper, cream, and a very little bit of butter warmed, fill the patties.

A good Mince for Patties. - Two ounces of ham, four of chicken or veal, one egg boiled hard, three cloves, a blade of mace, pepper and salt, in fine powder. Just before you serve, warm the above with four spoonfuls of rich gravy, the same of cream, and one ounce of butter. Fill as usual.

Of Oyster.-Take off the beards of the oysters, cut the other parts in small bits, put them in a small tosser with a grate of nutmeg, a little white pepper and salt, a morsel of lemon-peel cut very small, a little cream, and a small quantity of the oyster liquor. Simmer a few minutes before you fill.

Or:-Beard and chop the oysters, boil a little cream, with an onion and a small quantity of mace ; chop fine the crust of a French roll, mix it with the oysters, add the cream; let it boil. Season with nutmeg, cayenne, and salt.

Of Lobster.-Mince the fish in the same manner, mingling with it a little of the coral, and make with the same seasoning, a little cream, and the smallest bit of butter.

Of Shrimps.-Pick a quart of shrimps; if they are very salt, season them with only mace and a clove or two. Mince two or three anchovies; mix these with the spice, and then season the shrimps. Put a glass of sharp white wine. They do not take long baking.

\section{6.-VOL-AU-VENT.}

Take puff paste, roll it about threequarters of an inch thick, cut it round or oval according to the shape of the dish ; make the knife hot in water, so as not to drag the paste in cutting it ; mark the cover an inch from the edge, brush it over with yolk of egg; bake it in a 
quick oven; when done take off the top, clean out the soft paste, return it to the oven for a few minutes to dry; dish it on a napkin.

Care must be taken in taking out the soft part not to break the outside.

It may be filled with ragoût of sweetbread, fricassée of chicken, quenelles, lobster, or oysters, but is never made of a large size.

\section{7.-VOL-AU-VENT OF CROQUETTES.}

Make croquettes of the size of a pigeon's egg, of veal and ham and of calves' brains, an equal quantity of each, fry them of the palest gold color; make a rich white-sauce, and put into it the very smallest mushrooms or minced truffles; give it an acid flavor with lemon or lemon-pickle, and put it into the vol-auvent when it is to be sent to table.

\section{8.-VOL-AU-VENT OF VEAL.}

Cut veal into thin pieces the size of half-a-crown, beat them, and fry them a pale brown; make some forcemeat and egg-balls, fry the former, put the whole into a rich brown gravy, fill the vol-auvent, and serve it up hot.

\section{9.-VOL-AU-VENT OF PIGEONS AND SWEETBREADS.}

Cut off the pinions, legs, and backs of three or four pigeons, and bone the breast, making it as large as possible: cut three or four pieces of blanched sweetbread the same size, put them together, securing them with tape, and put them into a stewpan with a piece of butter, the trimmings, an onion, a bunch of sweet. herbs, a slice of fat bacon, and a small teacupful of water. Let them th stew till quite tender; remove the tape, strain and thicken the gravy, and put bake. them into the paste when it comes from the oven.

\section{0.-CODFISH PIE.}

Take dressed codfish and cold oystersauce; put a little of the sauce at the bottom of a pie-dish, then a layer of flakes of codfish, with a little of the liver cut in small pieces; season with black pepper and salt, and a very little nutmeg; repeat the layers until the dish is full, cover it with breadcrumbs and bits of fresh butter; bake for three-quarters of an hour, and let the top be quite brown. A couple of sounds well soaked, boiled tender, and cut in small pieces, are a great improvement.

Or:-Take a piece of the middle of a small codfish, and salt it well one night; next day wash it, cut it in slices, season with pepper, salt, and a very little nutmeg, mixed; place it in a dish, and put some butter on it, with a little good broth of any kind. Cover it with a crust; and when done, raise it, add a dozen oysters, one quarter of a pint of cream, a little flour and butter, a grate of lemon and nutmeg, and give it one boil. Bake it a full hour.

\section{1.-A REMARKABLY FINE FISH PIE.}

Boil two pounds of small eels; having cut the fins quite close, pick the flesh off, and throw the bones into the liquor with a little mace, pepper, salt, and slice of onion; boil till quite rich, and strain it. Make forcemeat of the flesh, an anchovy, parsley, lemon-peel, salt, pepper, and crumbs, and four ounces of butter warmed, and lay it at the bottom of the dish. Take the flesh of soles, small cod, or dressed turbot, and lay them on the forcemeat, having rubbed it with salt and pepper; pour the gravy over, and 


\section{FRUIT PIES, PUFFS, PUD- DINGS, \&c.}

All pies made either with summer fruit or with winter preserves will be improved by a mixture of apples, pared and sliced. They are especially good with fresh cherries, currants, \&c., and will be found an agreeable addition to cranberries. Equal portions also of cranberries and any very sweet jam will improve both. When apples are mixed with jam, they should be sliced thin; and if syrup be wanted, a few slices boiled with a little of the jam in sugar and water. In making pies of green gooseberries, apples, or rhubarb, the sugar should be clarified-that is, boiled in a little water, but no water poured into the pie, as it destroys the flavor of the fruit. For fresh fruits short crust is very suitable.

For a good common pie-crust allow half a pound or three-quarters of shortening to a pound of flour. Pie-crust looks the nicest made entirely of lard, but does not taste so good as with some butter. In winter, beef shortening, mixed with butter, makes good plain crust. Rub half of the shortening with two-thirds of the flour-to each pound of flour put a teaspoonful of salt and add just sufficient cold water to render it moist enough to roll out easily. Divide the crust into two equal portions-lay one of them aside for the upper crust, take the other, roll it out quite thin, flouring your rolling-board and pin, so that the crust will not stick to them, and line your pie plates, which should be previously buttered-fill your plates with your fruit, then roll out the upper crust as thin as possible, spread on the reserved shortening, sprinkle over the flour, roll it up, and cut it into as many pieces as you have pies to cover. Roll each one out about half an inch thick, and cover the pies-trim the edges off neatly with a knife, and press the crust down round the edge of the plate with a jagging iron, so that the juices of the fruit may not run out while baking. Pastry should be baked in a quick oven.

\section{2.-PUFF PASTE, OR CONFECTIONER'S PASTRY.}

Weigh out a pound and a quarter of sifted flour, and a pound of butter. Rub about one-third of the butter with twothirds of the flour, and a teaspoonful of salt. When the butter is thoroughly mixed with the flour, add one beaten egg, and cold water to moisten it sufficiently to roll out. Sprinkle part of the reserved flour on a board, cut the butter left into small pieces, and roll them out as thin as possible. Lay the butter, as fast as rolled out, on a floured plate, each piece by itself-roll out the pastry as thin as it can be rolled, cover it with the rolled butter, sprinkle on part of the reserved flour, and roll the crust up. Continue to roll out the crust, and put on the reserved butter and flour, till the whole is used. Roll it out lightly, about half an inch thick, for the upper crust, or rim to your pies-plain pie crust should be used for the under crust. Or, instead of flour, sprinkle some "baking powder" on each layer of butter; when the butter melts, it will cause the powder to effervesce and puff up the paste. To make the "baking powder," take one ounce of carbonate of soda, and seven drachms of Tartaric acid, mix well : it may be conveniently applied by means of a common pepper-pot kept for the purpose. Puff pastry, to be nice, should be baked in a quick oven till of a light brown color. If it browns before the fruit in the pie is sufficiently baked, cover it with thick paper. 
1253.-FLAKY PASTE. (By a Lady of New York.)

Wet half a pound of dried flour with cold water enough to make a hard, stiff paste; roll it one way only; roll in half a pound of butter with a knife, in small bits at a time.

\section{4.-RHUBARB PIES.}

Take the tender stalks of the rhubarb, strip off the skin, and cut the stalks into thin slices. Line deep plates with pie crust, then put in the rhubarb, with a thick layer of sugar to each layer of rhubarb-a little grated lemon-peel improves the pie. Cover the pies with a crust-press it down tight round the edge of the plate, and prick the crust with a fork. Rhubarb pies should be baked about an hour, in a slow oven-it will not do to bake them quickly. Some cooks stew the rhubarb before making it into pies, but it is not so good as when used without stewing.

\section{5.-PUMPKIN PIE.}

Halve the pumpkin, take out the seeds -rinse ${ }_{\text {t }}$ the pumpkin, and cut it into small strips-stew them over a moderate fire, in just sufficient water to prevent their burning to the bottom of the pot. When stered soft, turn off the water, and let the pumpkin steam, over a slow fire, for fifteen or twenty minutes, taking care that it does not burn. Take it from the fire, and strain it when cool, through a sieve. If you wish to have the pies very rich, put to a quart of the stewed pumpkin two quarts of milk, and twelve eggs. If you like them plain, put to a quart of the pumpkin one quart of milk and three eggs. The thicker the pie is of the pumpkin, the less will be the number of eggs required for them. One egg, with a table-spoonful of flour, will answer for a quart of the pumpkin, if very little milk is used. Sweeten the pumpkin with sugar, and very little mo- lasses-the sugar and eggs should be beaten together. Ginger, grated lemonrind, cinnamon and spices or nutmeg, are good for the pies. Pumpkin pies require a very hot oven.

\section{6.--PUMPKIN PUDDING. (By a Lady of New York.)}

One quart stewed pumpkin, nine beaten eggs, three pints cream, sugar, mace, nutmeg and ginger. Bake in paste.

\section{7.-POTATO PUDDING.}

Boil Carolina or mealy Irish potatnes until they are quite soft. When peeled mash and strain them. To a quarter of a pound of potatoes, put a quart of milk, three table-spoonfuls of melted butter, four beaten eggs, a wine-glass of wineadd sugar and nutmeg to the taste.

\section{8.-PEACH PIE.}

Take mellow juicy peaches-wash and put them in a deep pie plate, lined with pie crust. Sprinkle a thick layer of sugar on each layer of peaches, put in about a table-spoonful of water, and sprinkle a little flour over the top-cover with thick crust and bake from fifty to sixty minutes.

\section{9.-FANCY PASTRY.}

Use some fancy cutter, cutting the paste a quarter of an inch thick, egg them and glaze them, and bake them a light brown; when cold put different colored sweetmeats, such as apple jelly, and red currant jelly into devices upon the top of each piece; dish them upon a napkin.

\section{0.- -GLAZING FOR TARTS.}

Mix sifted sugar and water into a thick paste like cream, and brush it over the crust.

\section{1.-COCOA-NUT PIE.}

Cut off the brown part of the cocoanut, grate the white part, and mix it 
with milk, and set it on the fire and let it boil slowly eight or ten minutes. To a pound of the grated cocoa-nut allow a quart of milk, eight eggs, four tablespoonfuls of sifted white sugar, a glass of wine, a small cracker, pounded fine, two spoonfuls of melted butter, and half a nutmeg. The eggs and sugar should be beaten together to a froth, then the wine stirred in. Put them into the milk and cocoa-nut, which should be first allowed to get quite cool-add the cracker and nutmeg - turn the whole into deep pie plates, with a lining and rim of puff paste. Bake them as soon as turned into the plates.

\section{2.-A PLAIN CUSTARD PIE.}

Boil a quart of milk with half a dozen peach leaves, or the rind of a lemon. When they have flavored the milk, strain it, and set it where it will boil. Mix a table-spoonful of flour, smoothly, with a couple of table-spoonfuls of milk, and stir it into the boiling milk. Let it boil a minute, stirring it constantly-take it from the fire, and when cool, put in three beaten eggs-sweeten it to the taste, turn it into deep pie plates lined with paste, and bake the pies in a quick oven.

\section{3.-MINCE PIES.}

Fresh tongue must be used. It should be for two days well covered with equal quantities of salt, brown sugar, and powdered cloves. After boiling it until it is thoroughly done-say two hours-skin it and mince it fine. Pick, wash, and dry three pounds of currants, grate the rind of five lemons, and extract their juice, blanch and pound an ounce of bitter almonds and a pound of sweet almonds, powder four nutmegs, and grind together a dozen cloves and a dozen blades of mace; chop up three pounds of beef suet, six pounds of Sultana raisins, and six of the best pippin apples after paring and coring them. Mix them together, pouring over them a quart of Madeira wine and a pint of brandy.

This mince-meat should be placed in a tolerably deep dish and baked with puff paste. It should be thoroughly baked. Most persons prefer to eat these pies warm, but they are by no means unpalatable when cold.

These pies are richer when the mincemeat has been left for a few days in a stone jar, closely covered with a paper which has been dipped in brandy. When taken out, a little more sugar and a small quantity of brandy should be added to the mixture before the pies are made.

\section{4.-CRACKER PIE.}

One soda biscuit and a half, one teacup of white sugar, one lemon, one teacup of boiling water. Break the biscuit into small bits, pour over them the boiling water, cover, and leave them to swell. Grate the yellow part of the rind of the lemon, add to it the juice and the sugar, mix with the cracker when it is sufficiently swelled; make a rice crust, and prepare as apple pies. Sift sugar over the pies when baked.

1265.-MINCE MEAT FOR CHRISTMAS PIES. (Miss Jane Strickland's Receipt.)

Take equal quantities of finely shred and chopped beef suet; cold roast beef, well roasted; currants, washed, dried, and picked; and raisins stoned and chopped; and apples, pared, cored, and chopped fine. Mix all these prepared ingredients well together, and sweeten with good moist Jamaica sugar, to taste; grate off with loaf sugar the peel of two lemons, and grate in two nutmegs. Having well mingled all these materials together, add to them in the proportion of your mincemeat, thus:-If you have two pounds of every thing-namely meat, suet, currants, raisins, and apples, and not quite two pounds of sugar, pour over the mixture a 
pint of rum, or French brandy, and half a pint of raisin wine. If spirits are disliked, raisin wine of a fine quality or Lisbon must be added; but the spirits will keep it three months. Fill a jar with your mince-meat, cram down as hard as possible, and cover with a close-fitting lid, tied down with brown paper. Be careful whenever you take out your meat, to cram down that in the jar with a spoon, and cover close. Line your tin dishes with puff or family paste ; fill with mince meat heaped up in the middle, cut slices of candied orange, lemon, and citron peel, and put on your meat, cover in with paste; mark prettily with a knife, and bake in a quick oven; heat them for table.

It was formerly the custom in England for married couples to keep the anniversaries of their wedding days, and on those occasions as many small mince pies were placed on one dish on the table, as the host and hostess had been married years.

1266.-MINCE MEAT (furnished by a New York Lady).

One boiled tongue; half its weight of finely chopped beef suet; two pounds currants, two pounds raisins, one quart of brandy, one of wine; apples and sugar at discretion; cloves, cinnamon, mace, citron, orange-peel.

The mince meat made at Windsor Castle every year, and made one month previous to using, is as follows: Two hundred and forty pounds of raisins, four hundred pounds currants, two hundred pounds lump sugar, three pounds cinnamon, three pounds nutmegs, three pounds cloves, three pounds ground allspice, two pounds ginger, three hundred pounds beef, three hundred and fifty pounds suet; twenty-four bushels of apples, two hundred and forty lemons, thirty pounds of cedret, seventy-two bottles - of brandy, three pounds mace, sixty pounds lemon-peel, sixty pounds orange-peei.

\section{7.-APRICOT TART}

Take some apricots, cut them in two, and break the stones, put them into paste with sugar, a small quantity of preserved lemon, and a few of the kernels, close it, sprinkle sugar over, and glaze it. . If the apricots are not ripe, boil them a short time in water, and drain them well.

\section{3.-APPLE PIE.}

Pare, core, and quarter the apples; boil the cores and parings in sugar and water; strain off the liquor, adding more sugar; grate the rind of a lemon over the apples, and squeeze the juice into the syrup; mix half a dozen cloves with the fruit, put in a piece of butter. the size of a walnut. and add or omit a glass of wine; cover with puff paste.

Gooseberry, Currant and Raspberry, Cherry, Plum, Damson, Pear, Quince, Mulberry, Whortleberry and Raspberry, Dewberry and Raspberry, or Cranberry, may all be made in the same way, in winter. A little whipped cream may be placed in the top, for a variety.

\section{9-CODLING TART.}

Scald the fruit; when ready, take off the skin, and lay them whole in a dish; put a little of the water that the apples were boiled in at bottom, strew them over with powdered lump sugar; when cold, put a paste round the edges, and over. When the tart is baked, smear the crust with white of egg, and sift over it some powdered sugar. Serve with custard.

Or:-Line the bottom of a shallow dish with paste; lay the apples in it, sweeten, and lay little twists of paste over in bars. 
The mode of making the new-fashioned Apple-pie is to pare and quarter the apples, scald them, beat them with a spoon with some of the liquor: add grated lemon-peel, the juice of a lemon or Seville orange, or a part of a quince, when they are to be got, cloves, white sugar finely pounded, and a piece of butter; put a paste round the dish, and cover it with bars or flowers of pastethe excellence of the pie consisting of the sort of apple and the goodness of the paste; the fruit should be raised in the middle, as it shrinks in the baking.

\section{0.-TO MAKE A SIMNEL}

One pound of flour, one quarter of a pound.of butter, one quarter of a pound of lump sugar, one pound of currants, two ounces of candied lemon, one quarter of an ounce of carbonate of soda mixed with an egg, and a little milk; to be put in a tin mould, and baked till enough.

\section{1.-EXCELLENT LIGHT PUFFS.}

Mix two spoonfuls of flour, a little grated lemon-peel, some nutmeg, half a spoonful of brandy, a little loaf-sugar, and one egg; then fry it enough, but not brown; beat it in a mortar with five eggs, whites and yolks; put a quantity of lard in a frying-pan, and when quite hot drop a dessert-spoonful of batter at a time; turn as they brown. Serve them immediately with sweet sauce.

Spanish Puffs.-Put one ounce of fresh butter into a quart stewpan; when hot, put in four spoonfuls of flour; stir it over the fire for five minutes; have ready boiling a gill of milk, which stir in by degrees so as to avoid making it lumpy; when it is thoroughly boiled, take it off the fire, stir in six eggs, a pinch of salt, a little nutmeg, chopped lemon-peel, and a handful of currants, with sugar to palate, and sufficient orange-flower water to flavor it; let it get cold; when wanted for use have some lard boiling, make the batter into balls the size of a small walnut, fry them until they burst; drain; dust them over with powdered sugar; serve with wine-sauce.

German Puffs.-Put half a pound of butter into a breakfast-cupful of milk, place the pan upon the fire, and when it boils add a cupful of flour; beat all well together, and, when cold, mix in six eggs, leaving out two of the whites ; beat up some sugar and grated lemon-peel with the eggs, and bake the puffs in a moderately heated oven.

Irish Puffs.-Pound one quarter of a pound of sweet and one ounce of bitter almonds, but not too finely; take one quarter of a pound of loaf-sugar pounded and sifted, the whites of two eggs beaten to a thick froth; mix all together, and put the puffs into pattypans covered with paste; then sift powdered sugar over them thickly, and bake them a light brown. The flavor may be improved by pounding the almonds with orangeflower water or a little essence of lemon.

\section{2.-GAUFFRES.}

Take four or five ounces of flour, three ounces of pounded sugar, half a pint of whipped cream, four or five eggs, a small stick of pounded vanilla, a grating of nut$\mathrm{meg}$, and a little salt, with a glass of curaçoa or ratafia.

Place the flour, sugar, and salt in a basin, then add the yolk of eggs, the vanilla, and the spirit, mixing them well together, and gradually adding the whipped cream. Just before using the batter, add the whites of eggs, whipped to a froth, and mix them in lightly, so as to thoroughly incorporate them with it.

Bake these gauffres in tongs made for the purpose, observing, however, that the iron be very carefully heated, and the superfluous heat allowed to go off previously to filling them with batter; rub 
the tongs with fresh butter; fill the bottom part with batter, and fasten on the top, then turn it, and when a fine brown on both sides, shake some pounded spice and sugar over them, and send them to table.

They may be spread with any kind of preserve or jelly.

\section{3.-LENT PIES.}

Boil a dozen eggs quite hard, and chop the yolks very fine; chop also a dozen of moderate-sized juicy apples, peeled and cored, together with two pounds of stoned raisins; add two pounds of currants, one pound of sugar, and a table-spoonful each of powdered cinnamon, nutmeg, and beaten mace ; take also the juice and grated peel of three ripe lemons, with half a pound of citron cut into slips ; mix all these thoroughly, and moisten the whole with a pint each of white wine and brandy. If at hand, a little rose-water may be added, and ratafia may be used instead of brandy.

\section{4.-SWEET PATTIES.}

Chop the meat of a boiled calf's-foot, of which you use the liquor for jelly, with two apples, one ounce of orange, and lemon peel candied, and some fresh peel and juice; mix with them one-half of a nutmeg grated, the yolk of an egg, a spoonful of brandy, and four ounces currants, washed and dried. Bake in small patty pans.

\section{5.-VOL-AU-VENT}

Means light as wind ; you must therefore make this paste very delicate. It is used for first or second courses. Of all things in pastry the vol-au-vent requires the most care and precision; they that can make a good rol-au-vent may be stamped as good pastry-cooks. Make a pound of puff paste, giving it seven rolls and a half, leave it an inch in thickness, make a mark upon the top either round or oval, and according to the size of your dish; then, with a sharp-pointed knife cut it out from the paste, holding the knife with the point slanting outwards; turn it over, mark the edges with the back of your knife, and place it upon a bakingsheet, which you have sprinkled with water; egg over the top, then dip the point of the knife into hot water, and cut a ring upon the top a quarter of an inch deep, and half an inch from the edge of the vol-au-vent ; set in a rather hot oven; if getting too much color, cover over with a sheet of paper; do not take it out before done, or it would fall, but when quite set, cut off the lid and empty it with a knife : be careful to make no hole in the side or bottom; if for first course it is ready, but if for second sift sugar all over, which glaze with the salamander. Regulate the thickness of the paste from which you cut the vol-au-vent, according to the size you require it, the smaller ones of course requiring thinner paste. A vol-au-vent for entrées will take about half an hour to bake, and as, the common iron ovens often throw out more heat upon one side than the other, it will require turning two or three times to cause it to rise equally; it ought to be when baked of a light gold color.

Vol-au-Vent of Peaches.-Put half a pound of sugar in a sugar-pan, with the juice of a lemon and about half a pint of water, place it upon the fire, and boil it till becoming a thickish syrup; then have eight peaches not quite ripe, which cut in halves, break their stones and blanch the kernels, throw six halves with the kernels into the syrup, boil three minutes, take them out with a skimmer. lay them upon a dish, and take off their skins, stew the rest in syrup in like manner, four at a time; when all done pour what liquor runs from them again into the syrup, which reduce to a good thickness, 
pass it through a tammy into a basin, when cold pour a little over the peaches, and leave until ready to serve; dress the peaches in your vol-au-vent with the syrup over.

Vol-au-vent with Fruit.-These do not require to be so high as the other, especially as the fruit ought to be dressied in the form of a pyramid; if they are cut about three-quarters of an inch in thickness it will be enough; when nearly done, sift some powdered sugar over them, and put it back in the oven to glaze well; if not hot enough use the salamander; remove the interior, taking care not to make a hole in the bottom or sides, and fill with any kind of fruit you like, but never mix two kinds together, except currant and raspberry.

Sweet Vol-au-Vent with Rhubarb.In the spring of the year this makes a very inviting and wholesome dish, and its qualities purify the blood, which the winter's food has rendered gross; cut about twelve sticks of rhubarb into lengths of one inch, put it in a stewpan holding about two quarts, put over it a quarter of a pound of sugar, and a tablespoonful of water, set it on a sharp fire, stirring it; do not let it get brown, or it would spoil, and lose its flavor; it will take but a few minutes to do; when tender, put it in a basin to cool ; a few minutes before serving fill the vol-au-vent with it, and serve cold.

Sweet Vol-au-vent with green Gooseberries.-A quart of green gooseberries, a quarter of a pound of powdered sugar, the juice of half a lemon, and a tablespoonful of water; put on the fire and move it about for ten minutes, or till tender, an $\mathbb{l}$ forming a thick green marmalade; put it in a basin till cold; serve in pyramid in the vol-au-vent; a little thick syrup, if handy, poured over, improves the appearance.

Sweet Vol-au-vent with Orange.-
Well peel six oranges, removing all the pith, divide each into six or eight pieces, put them in a pan, with a quarter of a pound of sugar, and the juice of an orange, set it on a slow fire, with the cover on, stir it now and then-ten minutes will be sufficient time for it; take out the pieces one after the other, lay them in a basin, reduce your syrup to a proper thickness; when ready to serve, dish your pieces of orange in it, and pour over the syrup.

\section{6.-COCOA-NUT CUSTARD.}

Boil one quart of milk, when it boils sift in three table-spoonfuls of farina. Take off the fire, stir in one-quarter of a pound of butter, and let it stand until quite cold. Beat six eggs, the yolks and whites separate, until very light; add to the yolks three-quarters of a pound of sugar, and the grated pulp of one cocoanut. Stir the mixture into the boiled milk, add the beaten whites of the eggs, line tin pie plates with a nice crust, fill with the mixture, and bake.

\section{7.-SMALL FRUIT TARTS.}

The next in order to sweet vol-au vents, and which are easier to make, are tartlets, their appearanc ${ }^{\sharp}$ being inviting, and their expense limited, and very easy to serve. They may be made from the trimmings of any puff paste which remains, and should be enveloped in paper, and kept in a cold place, or in the flourtub. Make them as follows:-Have ready twelve or more small tartlet pans, which butter, line each with a bit of puff paste cut with a cutter the size of a crown-piece, force up the edges with your thumb and finger, put a small ball (made of flour and water) in each, bake them nicely in a very hot oven; when done, take out the ball (which may be kept for other occasions) from the tartlets, and shake powdered sugar over the bottom of each, and glaze with a sala- 
mander, turn them over, and shake sugar in the interior, which also salamander; fill with any kind of preserve, marmalade, or fruit, for sweet vol-au-vents. They may be made with cream as follows:-Make your tartlets as before, placing cream instead of the ball of flour, made thus:- Put half a pint of milk in a stewpan, when boiling, add half a stick of vanilla, reduce the milk to half in another stewpan, have the yolks of two eggs and a quarter of an ounce of powdered sugar, and one ounce of sifted flour, with a grain of salt, pour in the milk, taking out the vanilla, place over a slow fire, keep stirring till it thickens ; when cold, fill the tartlets, and bake nicely in a moderate oven; when cold, add a little jam, have ready a meringue of four eggs, lay a teaspoonful of each upon them, spreading it quite flat with a knife; ornament the top with some of the mixture, put into a paper cornet, sift sugar over, place in a slow oven till a light brown color, and the meringue quite crisp; if. the oven be too hot, cover with paper dress, and serve in pyramid on a Iis They should be light-colored.

\section{8.-LEMON TARTLETS.}

Pare, rather thick, the rinds of four lemons, which boil tender in two waters, and beat fine; add to it four ounces of blanched almonds cut thin, four ounces of lump-sugar, the juice of the lemons, and a little grated peel; simmer to a syrup; when cold, turn it into a shallow tin tart dish lined with a rich thin puff paste, and lay bars of paste over; as soon as the paste is baked, serve it.

\section{9.-GREEN APRICOT TARTLETS.}

When the wall-fruit trees are thinned, take the thinnings before the stone is formed; stew them gently in sugar and water. When tender, reduce and thicken the syrup, and put it with the fruit into pattzpans; or, if covered with a paste, it is then called a pie. This also applies to grapes.

\section{0.-PRUNE TART.}

Give prunes a scald, take out the stones and break them; put the kernels into a little cranberry-juice, with the prunes and sugar: simmer, and when cold make a tart of the sweetmeat.

It must be almost needless to say that tartlets of damsons, plums, and most sorts of stone-fruit, may be made in the same manner.

\section{1.-TARTLETS A LA PAGANINI.}

Beat up the whites of three eggs to a froth, and then add five ounces of pounded sugar; beat it well up, and have your pattypans ready covered with paste, upon which put any quantity you please of jam or marmalade; bake them for about a half an hour, and when done fill them with the whip. This quantity will fill sixteen pattypans of a small size.

\section{2.-TO PREPARE CRANBERRIES FOR TARTS.}

Simmer them in moist sugar, without breaking, twenty minutes; and let them become cold before bẹing used. A pint will require nearly three ounces of sugar.

\section{3.-ALMOND PASTE.}

Take a pound of sweet almonds, blanch them in boiling water; take off the peel, and let thein soak in cold water four hours, then pound them well in a mortar; add a little water to prevent them from turning to oil; after they are very fine and quite in a paste, put in three-quarters of a pound of sugar well pounded, and mix all together in the mortar. If your paste is quite fine, take it out of the mortar, put it into a stewpan over a slow fire, and stir it with a wooden spoon till it becomes white and dry ; then put it again into the mortar, and mix with it a 
little melted gum that has been strained through a towel; take care to keep it covered, to prevent it drying. You may give what scent you please, as lemon, vanilla, rose, orange, \&c. \&c. If you use almond-paste to make vases, baskets, or tartlets, keep it always free from dust. spread it on a marble with a rolling-pin as thin as possible, and if you put it into a mould, butter the mould lightly, and give the paste the form of it. Bake in a moderate oven.

Almond-paste should always be kept in a gallipot, covered with a damp towel to prevent its drying.

\section{4.-TARTLETS OF ALMOND PASTE.}

Butter some tartlet-moulds, and after having spread the paste on the dresser, cut it with a cutter to the size of the moulds; put a little sweetmeat in the middle, and a small rosette in the middle of that. Bake in a moderate oven.

\section{5. -TARTLETS OF ALMOND PASTE WITH STRAWBERRIES.}

Spread some almond-paste over the pastry-table, and cut it with a fluted cutter the same size as other tartlets; butter slightly the moulds, and put them into a moderate oven. When the paste is done almost white, take them out of the mould, and garnish with currant jelly, raspberry or apricot jam, \&c. \&c. When the sweetmeats do not go to the oven, they are always of a better color and taste.

1286.-APRICOT SWEETMEAT FOR TARTS.

Take a pound of ripe apricots, remove the stones, break them and blanch the kernels, add one pound and a half of grecn gages and one pound and a quarter of lump sugar; simmer it until the fruit becomes a jam. It must not boil, and must be kept well skimmed; clarified sugar will be found the best to use for this preserve.
1287.-GOOSEBERRY (GREEN) TART.

Use either whole gooseberries, or make a marmalade of them with a good syrup; the last method is perhaps the best, as you can tell easily how sweet they are and ought to be; if made of marmalade the seeds ought to be taken out. Green currants and grapes the same way.

\section{8.-ORANGE TARTS.}

Take some oranges, pare them very thin, soak them in water for two or three days, changing the water often, then boil them till they become soft and lose their bitterness; when cold cut a thick slice off the top and bottom, and the rest the thickness of a crown piece; line the tartlet-pans with puff paste, and fill them with layers of sugar and orange alternately.

Pears, raspberries, cherries, \&c., are simply put into the paste with sugar, and baked.

1289.-CROUSTADE OR DRESDEN PATTIES.

Slice some stale bread about half an inch thick; cut from it, with a round paste-cutter, as many croustades as will be required: mark the centres with a smaller cutter, scoop out the insides as in paste patties, then fry them a light brown, drain them well, and fill them with any hot compote of fruit or hot preserve. The croustade may be filled with any sort of minced meat or fowl and served as an entrée in the first course. They are very nice made with the rounds of a French roll; it should be pared very thin, then cut in rounds of such thickness, as to allow the centres to be taken out; dip them in milk, and let them drain; do not let them break; brush then with $\operatorname{egg}_{y}$ and sift the rasping of the roll over them; fry them a pale brown, and fill with oysters, stewed mushrooms, \&c., or, as above, with fruit or preserve. 
1290.-PATTIES RESEMBLING MINCE PIES.

Chop the kidney and fat of cold veal, apple, orange, and lemon-peel candied, fresh currants, a little wine, two or three cloves, a little brandy, and a bit of sugar. Bake as before.

\section{1.-PATTIES WITH CURDS.}

Take some very rich milk, put into it some lemon-juice or vinegar, place it on the fire that it may curdle, drain off the whey through a sieve; add to the curds the yolk of an egg beaten up in two spoonfuls of milk, a few currants, and a little pounded cinnamon. Make the paste and prepare the patties in the same way as in the preceding receipt.

\section{2.-CHEESECAKES.}

Strain the whey from the curd of two quarts of milk; when rather dry crumble it through a coarse sieve, and mix with six ounces of fresh butter, one ounce of pounded blanched almonds, a little orange-flower water, half a glass of raisin wine, a grated biscuit, four ounces of currants, some nutmeg and cinnamon in fine powder, and beat all the above with three eggs and half a pint of cream till quite light: then line the pattypans with a thin puff paste, and fill them three parts full.

\section{3.-BREAD CHEESECAKES.}

Slice a loaf as thin as possible, pour on it a pint of boiling cream, let it stand two hours ; then take eight eggs, half a pound of butter and a nutmeg grated, beat them well together, put in half a pound of currants well washed and dried before the fire, and bake them in raised crusts or pattypans.

\section{4.-MISS BRATTY:S CHEESECAKES.}

The yolks of eight eggs, eight ounces of sugar finely powdered and sifted, eight ounces of sweet almonds powdered, beat all together till very white. Line the pans with a thin paste ; immediately before you put them into the oven, mix the size of a walnut of butter melted into them over a fire. If the oven be too hot, they will fall when taken out.

Or:-Ten eggs, leave out half the whites, one pound of sugar finely powdered and sifted, half a pound of flour, three ounces of butter, three ounces of sweet almonds, with a glass of brandy put into them while they are pounding. Beat them all together, and butter the pans very well.

\section{5.-LEMON CHEESECAKES.}

Mix four ounces of sifted lump sugar and four ounces of butter, and gently melt it; then add the yolks of two and the white of one egg, the rind of three lemons shred fine, and the juice of one and a half, one Savoy biscuit, some blanched almonds pounded, three spoonfuls of brandy.

\section{6.-ORANGE CHEESECAKES.}

When you have blanched half a pound of almonds, beat them very fine, with orange-flower water, half a pound of fine sugar beaten and sifted, and one pound of butter that has been melted carefully without oiling, and which must be nearly cold before you use it; then beat the yolks of ten and whites of four eggs; pound two candied oranges, and a fresh one with the bitterness boiled out, till as tender as marmalade, withont any lumps; beat the whole together, and put into pattypans.

\section{ALMOND CHEESECAKES.}

Press the whey from as much curd as will fill two dozen small pattypans; then put it on the back of a sieve, and with half an ounce of butter, rub it through with the back of a spoon; put to it six yolks and three whites of eggs, and a few al- 
monds of both sorts pounded, with as much sugar as will make the curd properly sweet; mix with these a wineglassful of sherry or Madeira, in which boil the rind of a Seville orange, if you can get one; or, if not, a large ripe one of the common kind, with all its juice. Beat all gradual!y together, and when thoroughly blended fill the pattypans; the baking will take from a quarter of an hour to twenty minutes.

\section{8.-POTATO CHEESECAKE.}

Four ounces of butter, the same of pounded sugar, six ounces of potatoes boiled and floured through a sieve, the rind of one lemon, and half the juice, unless acid is desirable; mix these ingredients well together, with two eggs, and fill the tart-pan and bake it.

Potato Cheese is celebrated in various parts of Europe. It is made thus:Boil good white potatoes, and when cool, peel them and rasp or mash them to a light pulp; to five pounds of this, which must be free from lumps, add a pint of sour milk and salt to taste; knead the whole well, cover it, and leave it for three or four days, according to the season; then knead it afresh, and put the cheeses into small baskets, when they will part with their superfluous moisture; dry them in the shade, and place them in layers in large pots or kegs, where they may remain a fortnight. The older they are, the finer they become. This cheese, it is said, never engenders worms, and in well closed vessels, in a dry place, will keep for years. (This receipt is from an old English work).

\section{9.-CHEESECAKE-STOCK, THAT WILL KEEP FOR SEVERAL YEARS.}

To a quarter of a pound of butter put one pound of loaf-sugar broken into small pieces, six eggs, leaving out two whites, the rind of three lemons grated, and the juice of three; put them all into a pan, and let them simmer over the fire until the sugar is dissolved, and it begins to thicken like honey. When cold put it into sweetmeat pots for use. When made into cheesecakes, add grated sweet biscuits.

Line the small tartlet pans used for these small cakes with good, but not too light, puff-paste: make them of a deep yellow color, and put on each a strip of candied citron.

\section{0.-ICING FOR FRUIT PIES AND TARTS.}

The common mode is to take the white of an egg, whisked to a froth, mixed thickly with pounded sugar, and laid on with a quill feather. For larger tarts it should, however, be laid on more thickly, and comfits or lemon-peel may be stuck. into it.

\section{1 -SOYER'S CHEESECAKES.}

Take four quarts of milk and turn it with some fresh runnet; when dry crumble it and sift it through a coarse sieve into a bowl, beat it well up with a quarter of a pound of butter until it is quite smooth (it may require a little more butter, depending on the quality of the milk); mix in another bowl the yolks of four eggs and a quarter of a pound of very fine sifted biscuit-powder, the rind of four lemons, the juice of two, a quarter of a pound of powdered sugar (some add a little grated nutmeg or cinnamon), beat these all well up together until forming a stiff cream, then put it by degrees into the bowl with curd, and mix them well together; line some tartlet pans, previously buttered, with some paste, and place some of the above mixture in, and bake quick. In some places milk is used instead of eggs. Should you not have rennet, procure some good milk and turn it with the juice of a lemon or a teaspoonful of soda or culinary alkali 
to a quart of milk; drain the curd, and proceed as before.

\section{2.-RICHMOND MAIDS OF HONOR.}

These delicious little cakes, which every inhabitant of London who pays a visit to the most picturesque part of its environs knows so well, derive their name from a period when cookerv was not thought to be a degrading occupation for those honored with that title. It is stated that they originated with the maids of honor of Queen Elizabeth, who had a palace at Richmond.

Sift half a pound of dry curd, mix it well with six ounces of good butter, break the yolks of four eggs into another basin, and a glass of brandy; add to it six ounces of powdered lump-sugar, and -beat well together one very floury baked potato cold, one ounce of sweet almonds, one ounce of bitter ditto pounded, the grated rind of three lemons, the juice of one, and half a nutmeg grated, mix these well together and add to the curds and butter; stir well up, and proceed as before, filling the tartletpans.

\section{3.-PUDDINGS.}

There are two different sorts of paste for puddings, one for meat and the other for fruit.

For Meat Pudding.-To one pound of flour add eight ounces of finely chopped beef-suet cut from the kidney. Mix it with water but do not wet it too much: after mixing it well together with a wooden spoon, roll out the paste and put in the meat; then close it; boil it in a cloth which has been floured, tie it up, but leave sufficient room to permit the swelling of the paste.

Veal-suet may also be used, but that of beef is better; or a mixture of both is not bad. If puddings be boiled in shapes, the crust is not near so light.
For Fruit Puddings the paste should be made as for a pie-using butter instead of suet; but, unless for the sake of appearance, they should never be boiled in shapes. A very little salt added to all paste much improves it.

Sweet Pudding.-Very good puddings may be made without eggs; but they should have very little liquid added to them, and must boil longer than puddings with eggs. A spoonful of yeast will serve instead of two eggs, and a pinch of soda will make it still lighter. Two large spoonfuls of snow will supply the place of one egg, and make a pudding equally good. This is a useful piece of information, as snow generally falls in the season when eggs are dear. The sooner it is used after it falls the better; but it may be taken up from a clean spot, and kept in a cool place some hours, without losing its good qualities. Bottled malt-liquors are also a good substitute for eggs; the sooner used after the cork is drawn the better. Eggs should always be broken separately into a cup before they are thrown together, as a single bad one might occasion the loss of a great many without this precaution: the yolks and whites, beaten long and separately, make the article they are put into much lighter. They must always be strained after beating.

To avoid repetition, let it be observed that, when pudding sauce is ordered. wine, sugar, and very thick melted butter, boiled up together, is the sauce intended.

If the pudding be partly of bread, the cloth should be tied so as to allow for swelling; if of flour, rather more tight.

Basins or forms are much better in appearance than cloths for boiling puddings, but it makes them far less light.

The water should boil quickly, when the pudding is put in, and care taken that it continues to do so, or the puddins 
will be heavy; and it should be moved about for a minute or two, that the ingredients may not separate.

All dishes in which puddings are baked should be lined with paste an inch or two below the edge, as well as on it; the dish must be first rubbed with butter. If a pudding is to be turned out from a mould, it must be entirely lined with paste.

The ingredients of puddings should not be put into the basin or dish till the minute they go into the water or oven.

Sago, and all sorts of seeds, should lie in water an hour before they are made into puddings, and be well washed; the want of this caution causes an earthy taste.

If the butter be strong that is used in puddings, they will not taste well, whaterer good things are added.

A small pinch of salt improves the flavor of all mixtures, even when the other ingredients are sweet.

Well-made raisin or Cape wine will serve. in most cases, when wine is ordered for puddings.

As the goodness of boiled puddings greatly depends upon keeping the ingredients closely but not too compactly together, the cook should take care to have moulds and basins in readiness that will exactly hold the quantity directed.

Puddings of bread or flour are much better if all the ingredients be mixed (except the eggs) three hours before boiling or baking; and they should be well stirred just before they are put into the oven or saucepan.

Plum-puddings are best boiled in a stout cloth well floured; care should be taken that it does not burn to the bottom of the kettle; to prevent this put a plate at the bottom. When the pudding is of a large size it is best to mix all but the liquid ingredients the day before it is boiled.
When butter is ordered to be put warm into puddings, the addition of a little milk or wine will prevent its oiling.

Custard Puddings to look well should simmer only, but without stopping. If boiled in a quick or careless manner the surface will not be smooth, but have little holes like honey-comb all over it. A sheet of writing-paper, thickly buttered, should be put on the top of the mould before the lid or cloth is put on, and the pudding should stand in the mould five minutes after being lifted from the water. Many persons prefer their puddings steamed, but when this is not done they shonld be put into plenty of boiling water, and kept well covered.

Half an hour should be allowed for boiling a bread-pudding in a half-pint basin, and so on in proportion; but puddings made up with half a pound of suet and any other ingredients, will require two hours.

\section{4.-SUET PUDDINGS.}

Finely chop one pound of beef suet; mix with it one pound and a quarter of flour, two eggs well beaten, a little salt, and as little milk as will mix it. Boil four hours. It eats well next day cut in slices and fried, and may be eaten either with salt or sugar.

Or:-Take eight ounces of grated bread, three ounces of finely chopped suct, and four ounces of pounded loafsugar, the juice and grated peel of two lemons, and one egg well beaten; mix all together; put it into a buttered basin, and boil one hour; or make it into dumplings, and boil twenty minutes. Serve with wine sauce.

Of veal-suet, cut the crumb of a loaf into slices; boil and sweeten two quarts of new milk, and pour over it. When soaked, pour out a little of the milk, and mix with six eggs well 
beaten and half a nutmeg. Lay the slices of bread into a dish, with layers of currants and finely chopped veal-suet, one pound of each. Butter the dish well, and bake; or you may boil it in a basin if you prefer it.

Balls of suet-pudding, not larger than the size of an egg, are put into gravy soup. They will take from twenty minutes to half an hour boiling; in that time they will be done through, and so light as to swim to the top. They may be also served separately, and are an excellent accompaniment to corned beef.

1305.-BAKED CHICKEN PUDDING.

Cut up a pair of young chickens, and season them with pepper and salt, a little nutmeg and allspice. Put them into a pot with two large spoonfuls of butter, and water enough to cover them. Stew them gently, and when about half cooked, take them out and set them away to cool. Pour off the gravy, and reserve it to be served up separately. Make batter of a pound of sifted flour, stirred gradually into a quart of milk, six eggs well beaten and added by degrees to the mixture with a very little salt. Put a layer of chicken into a very deep dish, and pour over it some of the batter; then another layer of chicken and another of batter; having a cover of batter at the top. Bake till brown. Break an egg into the reserved sauce, boil up, and serve it in a sauce tureen to eat with the pudding.

\section{6.-BEEFSTEAK PUDDING.}

Of all savory puddings perhaps those made with beef are the best. The following is the mode of making them in all the clubs:-

Take from one pound to one pound and a half of the inside of a sirloin of beef, or the same quantity of rump-steak, cutting off part of the fat: beat it until tender, cut it thin, and divide it into small slices along with two mutton kidneys or one veal kidney; to these add a dozen of native oysters, bearded. The whole is then seasoned with pepper and salt and a minced shalot. Boil it from two and a half to three hours. When done have ready some strong beef gravy, made savory with a little mushroom ketchup ; make a hole in the paste and pour it into the pudding.

If for a fumily pudding, the oysters and the kidneys may be omitted: let the steak be prepared as above, adding, if you choose, some layers of sliced onions.

\section{7.-PLUM PORRIDGE.}

Boil eight pounds of shin of beef for five hours in a gallon of water, skimming carefully throughout, and finally straining off the liquor; add two pounds of meat cut small. Soften the crumb of a small loaf in some of the liquor, beat it smooth, thicken the soup with it, add half a pound of stoned raisins, the same quantity of stoned prunes, a pound of well washed currants, and grated nutmeg, pepper, and mace to taste. and boil until the fruit is soft, then serve.

\section{8.-YORKSHIRE PUDDING.}

Make a batter with a pint of milk, four eggs, and four table-spoonfuls of flour, seasoned with salt. It is to be well stirred, and put into the pan under roasting beef or baked beef, to be cooked with the drippings. It will bake in about two hours. Serve it in pieces round the meat, on the same dish.

\section{9.-GAME PUDDINGS.}

Game of any description may be made into puddings, and when partly boned, well spiced, with minced truffle or mushroom, mace, and a clove of garlic, and boiled within a light paste, they are very rich, and the paste particularly fine, as it absorbs so much of the gravy; but the 
boiling deprives the game of much of its high flavor, and a woodcock or a snipe should never be so dressed, as they lose all the savor of the trail.

Or: Make a batter with flour, milk, eggs, pepper, and salt; pour a little into the bottom of a pudding-dish; then put seasoned poultry or game of any kind into it, and a little shred onion; pour the remainder of the batter over, and bake in a slow oven.

A single chicken, partridge, or pigeon may be thus made into a dumpling:Stuff it with chopped oysters, lay it on its back in the paste, and put a bit of butter rolled in flour on the breast; close the paste in the form of a dumpling, put into hot water, and let it boil for two hours.

\section{0.-MARROW PUDDING.}

Grate the inside of part of a stale loaf. Make a quart of milk quite hot, and pour over it, letting it remain till it is sufficiently swelled and soaked. Shred half a pound of marrow, or good suet, and beat up four eggs. Pick and plump up two ounces of currants, and stone two ounces of the best raisins. Mix them all up together ; stir in a few blanched almonds, and a little candied citron, and orange and lemon peel. Sweeten the pudding to your taste, and season it with grated nutmeg and powdered cinnamon. Cover a stoneware, flat dish, round the edge, with a shred of puff-paste, and mark it neatly. If baked in a flat dish, twenty-five minutes will be sufficient; if in a deep dish it will require half an hour. A little finely pounded lump sugar is sometimes strewed over the top, and blanched almonds, sliced, stuck round for ornament. This is a very light and delicate baked pudding. It is sometimes boiled in a shape.-This makes a delicious dish.

\section{1.-HERB PUDDING.}

Pick two handfuls of parsley-leaves from the stems, half the quantity of spinach, two hearts of lettuces, a large handful of mustard and cress, a few leaves of white beet, and a small handful of chives: wash, and boil all together three minutes; drain the water from them, and mash very fine; mix well, and add salt and pepper. Have ready a batter, made of one ounce of flour, a pint of thin cream and two eggs; stir it into the herbs, and cover the dish with a good crust.

This pudding has much the flavor of omelet; and in Lent, when the salads are all coming into season, it may be very aptly brought to table on fast-days.

Tansy-Pudding.-Pound a handful of green tansy in a mortar ; add the juice to a pint of batter and bake it.

\section{2.-GOOSE PUDDING.}

Half a pound of bread-crumbs soaked in a little boiling milk, when cold, add two or three eggs, a little salt, pepper, marjoram, and thyme, a spoonful of oatmeal, a good handful of suet, and an onion chopped fine. Spread it in a drippingpan, and bake it under the goose.

\section{SWEET PUDDINGS.}

Under this head the plum-pudding stands foremost as a truly national dish. The following receipt, communicated by a man-cook of much experience, we can vouch for as an excellent way of making a

1313.-RICH PLUM PUDDING.

Stone carefully one pound of the best raisins, wash and pick one pound of currants, ${ }^{*}$ chop very small one pound of fresh

* The best method of cleansing currants is to put them into a common colander, over a pan with sufficient water to cover them, rub them well between 
beef suet, blanch and chop small or pound two ounces of sweet almonds and one ouncs of bitter ones; mix the whole well together, with one pound of sifted flour, and the same weight of crumb of bread soaked in milk, then squeezed dry and stirred with a spoon until reduced to a mash, before it is mixed with the flour. Cut in small pieces two ounces each of preserved citron, orange, and lemon-peel, and add a quarter of an ounce of mixed spice: a quarter of a pound of moist sugar should be put into a basin, with eight eggs, and well beaten together with a three-pronged fork; stir this with the pudding, and make it of a proper consistence with milk. Remember that it must not be made too thin, or the fruit will sink to the bottom, but be made to the consistence of good thick batter. Two wine-glassfuls of brandy should be poured over the fruit and spice, mixed together in a basin, and allowed to stand three or four hours before the pudding is made, stirring them occasionally. It must be tied in a cloth, and will take five hours of constant borling. When done, turn it out on a dish, sift loaf-sugar over the top, and serve it with wine-sauce in a boat, and some poured round the pudding.

The pudding will be of considerable size, but half the quantity of materials, used in the same proportion, will be equally good.

In addition to the wine-sauce, have a metal sauce-boat filled with brandy; set it alight on the table, and pour a portion of it in a flame upon each slice of pudding.

1314.-ENGLISH. PLUM PUDDING. (Original).

One pound of fine white flour, sifted;

the hands in the water to separate the knobs, and stir them about. The small sand and gravel will then fall through the holes and sink to the bottom of the pan. After being washed clean, and the water drained from them, the large stopes can then be easily picked out by sorting them over on a large dish. add a little salt; one pound of beef suet chopped as fine as meal, one pound of brown sugar, one pound of stoned raisins, one pound of Zante currants, threequarters of a pound of citron, one nutmeg, one teaspoonful of allspice, one ditto of cloves, two ditto of mace, grate in the rind of three large fresh lemons. Weigh each article after it is prepared, allowing for the muisture in the currants, as they cannot be dried perfectly. Mix the ingredients together very thoroughly; first, the flour and suet-then add the other articles and rub the mixture again ; this should be done the day before the pudding is to be cooked.

Immediately before the final mixture, add ten eggs, well beaten, one gill of milk and one of brandy. Tie the mixture in a well buttered cloth, and boil for seven hours. The cloth used should be very strong.

To be served with brandy-sauce, and brought to the table in burning brandy.

\section{5. -THE BAKED PLUM PUDDING.}

Ten crackers soaked in milk, five eggs, one pound of raisins, half a pound of suet, half a pound of chopped apple, two glasses of brandy, dark spice and mace, salt to the taste-half a pound of citron, sugar to the taste. Bake three hours and serve with cold sauce.

1316.-GROUND RICE PUDDING. (Mrs. G.'s Receipt).

Boil half a pound of rice in five pints of milk; add half a pound of butter, three-quarters of sugar, ten eggs, two nutmegs, and mace with a little salt: bake in a dish.

1317 -MARLBOROUGH PUDDING. (Furnished by a Lady of New York.)

Stew eighteen apples and strain them; add a quarter of a pound of melted butter, with rosewater, ten eggs, the juice and rind of two lemons, sugar to taste, and bake in paste. 
1318.-BOSTON PUDDING.

Make a good common paste with a pound and a half of flour, and threequarters of a pound of butter. When you roll it out the last time, cut off the edges till you get the sheet of paste of an even square shape. Have ready some fruit, sweetened to your taste. If cranberries, gooseberries, dried peaches, or damsons; they should be stewed, and made very sweet. If apples, they should be stewed in a very little water, drained, and seasoned with nutmeg, rosewater, and lemon. If currants, raspberries, or blackberries, they should be mashed with sugar, and put into the pudding raw. Spread the fruit very thick, all over the sheet of paste (which must not be rolled out too thin). When it is covered all over with the fruit, roll it up and close the dough at both ends, and down the last side. Tie the pudding in a cloth, and boil it. Eat it with sugar. It must not be taken out of the pot till just before it is brought to table.

\section{9.-ALMOND PUDDING AND SAUCE.}

A large cupful of finely-minced beef suet, a teacupful of milk, four ounces of bread-crumbs, four ounces of well cleaned currants, two ounces of almonds, half a pound of stoned raisins, three well beaten eggs, and the whites of other two; sugar, nutmeg, and cinnamon, and a small glass of rum. Butter a shape, and place part of the raisins neatly in rows. Blanch the almonds; reserve the half of them to be placed in rows between the raisins just before serving. Mix all the remaining ingredients well together, put into the shape, and boil three hours. The sauce-one teaspoonful of milk, and two yolks of eggs well beaten, and some sugar; place on the fire and stir till it just comes to the boil, then let it cool. When lukewarm, stir into it a glass of sherry or currant wine, and serve in a sauce tureen. This sauce is a great improvement to raisin pudding.

1320. - POLKA PUDDING.

Mix four table-spoonfuls of arrow-root in a pint of cold milk. Beat four eggs well, add them, three ounces of fresh butter, cut into small bits; a dessertspoonful of rosewater; a few drops of essence of lemon, or ratafia, and a teacupful of sugar. Boil two pints of milk in a saucepan; when boiling stir in the other ingredients; without taking the pan off the fire, let it boil till thick, then pour into a mould to cool. Turn it out and serve it cold.

\section{1.-JENNY LIND'S PUDDING.}

Grate the crumb of half a loaf, butter and dish well and lay in a thick layer of the crumbs; pare ten or twelve apples, cut them down, and put a layer of them and sugar; then crumbs alternately, until the dish is full; put a bit of butter on the top, and bake it in an oven, or American reflector. An excellent and economical puddding.

\section{2.-NEWCASTLE PUDDING.}

Butter a basin or mould, stick it all round with sultanas or dried cherries, then put in a slice of bread-crumb soaked in milk, and over that layers of thin bread-crumb buttered, until three parts filled; fill up with custard, and boil for an hour and a half.

\section{3.-RICHELIEU PUDDING.}

Steep the crumb of a large slice of bread in milk, warm as from the cow; let it rest, and then strain off the milk; beat up the yolk of an egg, mix it with the bread, also a bit of butter; put it into a saucepan, and boil till it becomes stiff; let it cool, and then add some chopped parsley, thyme, pepper and salt; beat up two eggs; mince about one 
pound of any cold meat, and add all together. Boil in a basin for three hours, and when dished, pour a good gravy over it.

\section{4.-PARADISE PUDDING.}

Take three eggs, three apples pared, cored, and cut small, three ounces each of the crumb of French rolls, sugar, and currants, a little nutmeg and salt, the rind of a lemon pared very thin and cut very fine, and a wine-glassful of brandy or white wine ; stir well, pour into a buttered mould, and boil for an hour; then serve with melted butter, wine and sugar.

\section{5.-HASTY PUDDING.}

Set some milk on the fire, and, when it boils, put in a little salt. Stir in by degrees as much flour as will make it of a proper thickness. Let it boil quickly a few minutes, beating it constantly while on the fire. Pour it into a dish, and eat it with cold butter and sugar. Some persons add eggs to this.

\section{6.-HASTY PUDDING.}

Boil water, a quart, three pints, or two quarts, according to the size of your family; sift your meal, stir five or six spoonfuls of it thoroughly into a bowl of water; when the water in the kettle boils, pour into it the contents of the bowl; stir it well and let it boil up thick; put in salt to suit your own taste, then stand orer the kettle, and sprinkle in meal, handful after handful, stirring it very thoroughly all the time, and letting it hoil between whiles. When it is so thick that you stir it with difficulty. it is arout right. It takes about half an hour's cooking. Eat it with milk or molasses. Either Indian meal or rye meal may be used. If the system is in a restricted state, nothing can be better than rye hasty pudding and West India molasses. This diet would save many a one from the horrors of dyspepsia.
1327.-TO MAKE AN ALMOND PUDDING.

Pound in your mortar a quarter of a pound of sweet almonds, add a tablespoonful of water when you are pounding, take it out of the mortar; have ready broken seven eggs, leaving out five of the whites, add a quarter of a pound of sifted sugar, mix all lightly together; cut in small dice a little candy, orange, and lemon-peel, butter and paper a plain mould, or one that will open; bake it in a moderate oven.

\section{8.-FARINA PUDDING.}

One quart of milk, six eggs, eight tablespoonfuls of flour, three tablespoonfuls of farina, one teaspoonful of salt.

Boil the milk; while boiling, sift into it the farina; beat the eggs, yolks and whites separately; stir into the yolks the flour and salt, and cold milk enough to make a stiff batter; then stir in the whites, and, lastly, the boiling milk. Butter a baking dish, and bake for half an hour. Eat with wine sauce, or sugar and butter.

\section{9.-A BAKED APPLE DUMPLING.}

Pare and quarter four large apples, boil them tender with the rind of a lemon in so little water that nothing may remain when done but the juice; add the crumb of a small roll, four ounces butter, melted, the yolks of five and whites of three eggs, the juice of half a lemon, and sugar to taste ; beat all together, and lay it in a dish ; then beat the two remaining whites to a froth, and throw it round the edge of the dish; grate a little white sugar over when baked.

\section{0.-LEMON PUDDING.}

IIalf a pound of flour, or grated bread, half a pound of suet cut very fine, half a pound of crushed sugar, the 
rind of two lemons, and the juice of one, two eggs; boil it four hours in a shape. Served up without sauce it is excellent.

\section{1. - A PLAIN RICE PUDDING.}

To make a plain rice pudding, boil a pint of rice until it is quite soft. Mix two ounces of butter and four tablespoonfuls of sugar, a quart of rich milk with the rice, boil them up together and let them partially cool. Beat five eggs until they are quite light and stir them into the rice. It should bake about an hour.

\section{2,-RICE MILK.}

This dish is an excellent one, and very simply and quickly made. After washing a pint of rice in two different waters, boil it well with about half a pound of raisins from which the stems have been carefully picked. Pour off the water, and mix a quart of rich milk with the rice. Let it boil for about five minutes, and after mixing with it four table-spoonfuls of brown sugar, beat two eggs until they are light, and pour them into the milk, stirring it all the time. After the rice and eggs are well mixed together, they should boil from three to five minutes. If they are not well stirred, the eggs will form a custard on the surface, which is not desirable.

\section{3.-BIRD'S NEST PUDDING.}

If you wish to make what is called "bird's nest puddings," prepare your custard,-take eight or ten pleasant apples, pare them, dig out the core, but leave them whole, set them in a puddingdish, pour your custard over them, and bake them about thirty minutes.

1334.-ORANGE PUDDING.

Wash half a pound of butter until all the salt is extracted, then mix it well with half a pound of powdered white sugar, and a wine-glass of brandy-wine may be used but it is not as good. Grate the rind of three ordinary-sized oranges and squeeze their juice. Beat together six fresh eggs, and stir them into the butter and sugar, after which, add the prepared orange. Lay a border of puffpaste around the pudding-dish, and then bake from half to three quarters of an hour. Serve it cold, and grate over white sugar mixed with a little nutmeg. The latter ingredient, however, is not generally preferred. Lemon Pudding may be made in the same manner; substituting lemons for oranges.

\section{5.-QUINCE PUDDING.}

Pare six large quinces, cut out the cores and blemishes, chop them as fine as possible, and boil them two hours with as little water as possible, stirring them frequently that they may not burn. Drain off the water and mix them, when cold, with a pint of cream and half a pound of powdered sugar.

\section{6.-APPLE DUMPLINGS.}

Pare and scoop out the core of six large baking apples, put part of a clove, and a little grated lemon-peel, inside of each, and enclose them in pieces of puff paste; boil them in nets for the purpose, or bits of linen, for an hour. Before serving, cut off a small bit from the top of each, and put in a tea-spoonful of sugar, and a bit of fresh butter ; replace the bit of paste, and strew over them pounded loaf sugar.

\section{7.-BATTER PUDDING.}

Into a pint and a half of sifted flour stir gradually, so that it may not be lumpy, a quart of milk. Beat seven eggs, and put in, together with a couple 
of table-spoonfuls of melted butter, and a couple of teaspoonfuls of salt. Grate in half a nutmeg-add, if you want the pudding very rich, half a pound of raisins. They should not be put into a baked pudding till it has been cooking long enough to thicken, so that the raisins will not sink to the bottom of it. A pudding made in this manner is good either baked or boiled. It takes two hours to boil, and an hour and a quarter to bake it. When boiled, the bag should not be more than two-thirds full, as flour puddings swell very much. It should be put into boiling water, and kept boiling constantly. If the water boils away, so as to leave any part of the bag uncovered, more boiling water should be added. When the pudding has boiled eight or nine minutes, the bag should be turned over, otherwise the pudding will be heavy. Flour pudtings should be eaten as soon as cooked, as they fall directly. Serve them up with rich sauce.

\section{8.-SAGO PUDDING.}

Pick, wash, and dry half a pound of currants; and put in such spices as best suit the taste of those who are to eat it. Have ready six table-spoonfuls of sago, picked clean, and soaked for two hours in cold water. Boil the sago in a quart of milk till quite soft. Then stir alternately into the milk, a quarter of a pound of butter, and six ounces of powered sugar, and set it away to cool. Beat eight eggs, and when they are quite light, stir them gradually into the milk, sago, \&c. Add the spice, and lastly the currants; having dredged them well with flour to prevent their sinking. Stir the whole very hard, put it into a buttered dish, and bake it three-quarters of an hour. It may be eaten cold.

\section{9.-TAPIOCA PUDDING.}

To two quarts of warm milk put eight table-spoonfuls of tapioca, four beaten eggs, a table-spoonful of butter, and cinnamon or mace to the taste. Mix four table-spoonfuls of white powdered sugar and a wine-glass of wine, and stir it into the rest of the ingredients. Turn the whole into a pudding dish that has a lining of pastry and bake it immediately.

\section{0.-INDIAN PUDDING.}

Sift a pint of Indian meal and scald it with boiling water. Place over the fire a quart of milk, cut up a quarter of a pound of butter and melt it in the hot milk-add a pint of sugar-house molasses, and mix them together until the milk boils. Stir it into the meal, mixing it well with a wooden spoon. Beat seven eggs, until they are perfectly light, pour them into the bowl that holds the meal, with ten drops of the essence of lemon, or a little lemon-juice.

Stir the mixture until it seems quite light, and bake it in a moderately hot oven.

\section{1.-A BAKED BREAD PUDDING.}

This is a very excellent dish when properly mixed. Crumble half a loaf of bread, a stale loaf is preferable, and cut up the crust as small as possible. Boil a quart of milk, cut up a quarter of a pound of butter in slices, and melt it in the hot milk-add six table-spoonfuls of sugar. Pour the milk over the bread, and mix with it a glass of good brandy. Brandy in which lemon-peel has been soaked for some time, is the best for this purpose, and gives a fine flavor to the pudding. Beat seven eggs quite light, and stir them into the mixture. If the pudding is to be placed immediately in the oven, it is not necessary that the mixture should cool before the eggs are added.

This pudding in a brisk oven will bake in less than an hour. 
1342-DEMI-PLUM PUDDING.

Prepare the mould, then add a layer of plum pudding, broken in pieces, that has been left from the previous day, alternately, till full ; fill up with custard, and steam or bake for thirty minutes. The remains of any kind of pudding may be used thus.

\section{3.-MACARONI PUDDING.}

Simmer an ounce or two of macaroni in a pint of milk, with a bit of lemonpeel and cinnamon, till soft; put it into a dish with milk, the yolk of three eggs and the white of one, some sugar, nutmeg, a spoonful of almond water, and half a glass of raisin wine; put a nice paste round the edge of the dish, and bake it.

A layer of orange marmalade or raspberry jam may be used instead of the almond water or ratafia.

\section{4.-OATMEAL PUDDING.}

Take a pint of the best fine oatmeal, pour a quart of boiling milk over it, and let it soak all night; the next day put it in a basin just large enough to hold it, add two eggs beaten, and a little salt, cover it tight with a floured cloth, and boil it an hour and a half. It may be eaten hot, with cold butter and salt; or cold, sliced and toasted.

\section{GROUND RICE PUDDING.}

In a pint of new milk boil two dessertspoonfuls of ground rice, adding a small piece of lemon-peel and a little cinnamon. Keep it stirring while boiling, and let it boil ten minutes, then let it cool ; when cold add sugar to taste, a couple of well beaten eggs, and some nutmeg. Line your dish with a puff paste, pour in your rice, and bake a light brown.

1346.-PUDDING WITH FRUIT.

Fruit puddings are best made in a basin, the basin to be buttered and lined with the paste, and then filled with the fruit, which cover with the paste; the paste should be rolled round to the thickness of half an inch, and when the fruit is in, drawn to the centre, and squeezed, and then tied up in a cloth kept on purpose, and boiled in plenty of water; when done, which will be in time according to the nature of the fruit you put in it; serve it either turned out of the basin or not. The cover should be of the same thickness as the sides. Sugar should be added before being covered.

\section{7.-COCOA-NUT PUDDING.}

Remove the shell of the cocoa-nut and cut the brown skin away, cut the nut in pieces and place it in cold water, remove it and wipe it dry, grate about a quarter of a pound of a large one, very fine, into a plate. Place in a basin a quarter of a pound of powdered white sugar, and three ounces and a half of fresh butter, stir them together till it becomes like cream; add to it a table-spoonful of brandy, and about four drops of either essence of vanille, or any other essence whose flavor may be preferred. Place the white of six eggs into a bowl, and beat them well up; then add gradually the butter and sugar, which keep stirring at the same time; add by degrees the cocoa-nut. Cover a pie dish with puff paste, place the mixture in it, and bake in a moderate oven for about half an hour. Glaze it with powdered sugar and powdered cinnamon mixed.

\section{8.-AN APPLE CHARLOTTE.}

Pare and slice a quantity of apples; cut off the crust of a loaf, and cut slices of bread and butter. Butter the inside of a pie dish, and place bread and butter all round; then put in a layer of apples sprinkled with lemon-peel, chopped very fine, and a considerable quantity of good 
brown sugar. Then put on a layer of bread and butter, and another of apples, lemon-peel, and sugar, until the dish is full, squeezing over the juice of lemons, so that every part shall be equally flavored. Cover up the dish with the crusts of the bread and the peels of the apples, to prevent it from browning or burning: bake it one hour and a quarter ; then take off the peels and the crust, and turn it out of the dish.

\section{9.-HEDGEHOG-TO MAKE.}

Blanch two pounds of sweet almonds, pound them to a paste in a mortar, moisten occasionally with canary and orange-flower water; beat the yolks of twelve and the whites of five eggs with a pint of cream and some powdered sugar, put this with the almond paste and half a pound of fresh butter in a saucepan, set it over a stove, and keep it constantly stirring till firm enough to be moulded into the shape of a hedgehog; stick it full of blanched almonds cut lengthwise into slips and placed in a dish; beat up the yolks of four eggs, put them to a pint of cream, sweeten to your taste, stir them over a slow fire till hot, then pour it round the hedgehog, and let it stand till cold, serve it.

A good calf's-foot jelly may be poured round it if preferred.

\section{0.-TRIFLES}

Should be made early in the day on which they are wanted; take a stale sponge cake, cut it in slices of one inch

* thick, and lay it on the bottom of the dish; lay on that a thin layer of any kind of marmalade, jam, or jelly, have some macaroons and ratafia cakes, and lay on and cover the whole with some sponge cakes. For a dish nine inches in diameter, mix two glasses of sherry, one of brandy, half a one of rum, and the same of noyeau, and pour over, and let it remain until it is well soaked, then pour over about one inch thick of rich custard, put a pint of cream into a bowl, with some sifted sugar, a squeeze of a lemon, and about a table-spoonful of the wine, \&c., you have put on the cake, whisk it well up. I use a trifle-churn, which saves some trouble; I also use it for all whipped cream; and as the froth rises remove it with a spoon upon-a clean sieve, where let it drain, then place it on the custard until it is high and handsome.

I have occasionally, when being in a hurry, and having no cream by me, proceeded as above. and made the whip with the whites of eggs, and some very white peach or egg-plum marmalade together, until it makes firm froth or whip, which put on the custard; this may also be colored a nice pink.

\section{1.-BROWN BREAD PUDDING}

May be made with half a pound of stale brown bread coarsely grated, half a pound of Valencia raisins cut in halves, the same of chopped suet, sugar and nutmeg. Mix with four eggs, two spoonfuls of brandy, and two of cream; boil it for three or four hours in a cloth or basin that exactly holds it. Serve with sweet sauce.

Prunes, or French plums, instead of raisins, make a fine pudding, either with suet or bread.

\section{2.-BREAD AND BUTTER PUDDING.}

Make a custard of one egg and half a pint of milk, by boiling the milk with a little lemon-peel and sugar, and beating up the egg in it, putting it on the fire to thicken; butter slices of bread or French roll, and soak them for an hour or two in this mixture; then lay them in a dish, sprinkling currants between each layer, with a little pounded sugar; and, if meant to be very nice, put some sweet- 
meats on the top; but both the fruits and the sweetmeats may be omitted if thought proper. Pour over it another half a pint of milk beaten up with two eggs.

\section{3.-BOSTON CRACKER PUDDINGS.}

On three grated Boston crackers pour a pint of boiling cream; when cold add a teaspoonful of cinnamon in finest powder, the yolks of four and whites of two eggs, a spoonful of orange-flower water or ratafia, two ounces of loaf-sugar, and half a spoonful of flour, or almond flour, rubbed smooth.

\section{4-VERMICELLI.}

Baked.-Simmer four ounces of vermicelli in a pint of new milk ten minutes; then put to it half a pint of cream, a teaspoonful of pounded cinnamon, four ounces of butter warmed, the same of white sugar, and the yolks of four eggs, well beaten; a little oil of almonds or a couple of spoonfuls of ratafia will much improve the flavor. Bake in a dish without a lining.

Boiled.-Stir very gently four ounces of vermicelli into a pint of new milk over the stove, until it be scalding hot, but not more; then pour it into a basin, and add to it while hot one ounce of butter and two of sugar. When the above is nearly cold, mix in it, very gently, two well beaten eggs, and immediately put it into a basin that will exactly hold it. Cover carefully with a floured cloth; and turning the basin the narrow end upwards, move it round for ten minutes, and boil an hour. Serve with puddingsauce.

\section{3ก็ก.-MUFFIN AND CRUMPET PUDDING.}

A very delicate pudding may be made in a short time from a couple of muffins and three crumpets, placed alternately in layers, and either boiled or baked in batter. If boiled, they should be placed in an earthen jar, buttered on the inside, filled with the batter, and covered on the top. The muffins should be split open, and currant jelly, slices of apple, or any sort of fruit may be inserted.

Or:-Half a dozen crumpets may be boiled without any fruit, after being dipped and covered in batter; seasoned only with sugar and a little nutmeg.

\section{6.-CHOCOLATE PUDDING.}

Boil a pint of new milk; dissolve in it one ounce of chocolate; sweeten it with loaf-sugar; add the yolks of eight and the whites of four eggs well beaten; strain and pour it into a plain mould buttered and papered; steam it for a half an hour; let it settle for ten minutes, and serve with the following sauce:-Boil a half a stick of vanilla in a pint of milk till it is reduced one-half; strain it, sweeten with loaf-sugar, and thicken with arrowroot.

1357.-SPONGE PUDDING.

Butter a mould thickly, and fill it three parts full with small s sponge-cakes, soaked through with wine; fill up the mould with a rich cold custard. Butter a paper, and put on the mould; then tie a floured cloth over it quite close, and boil it an hour. Turn out the pudding carefully, and pour some cold custard over it.

Or:-Bake it, and serve with winesauce instead of custard.

\section{8.-QUAKING PUDDING.}

Scald a quart of cream; when almost cold put to it four eggs well beaten, one spoonful and a half of flour, some nutmeg and sugar; tie it close in a buttered cloth, boil it an hour, and turn it out with care, lest it should crack. Serve with wine-sauce.

1359.-BRANDY PUDDING.

Line a mould with jar raisins stoned, 
or dried cherries, then with thin slices of $\mid$ whole into a batter with the juice of French roll, next to which put ratafias, only three of the oranges: the number of or macaroons; then again fruit, rolls, eggs to be regulated by the size of the and cakes in succession until the mould fruit. Put the materials into a mould, be full, sprinkling in, at times, two wine- with a paste around but not over it, and glassfuls of brandy. Beat up four eggs, bake it for half an hour.

yolks and whites; put to a pint of milk or cream, lightly sweetened, half a nutmeg, and the rind of a half a lemon finely grated. Let the liquid sink into the solid part; then flour a cloth, tie it tight over, and boil one hour. Keep the th mould the right side up. Serve with pudding-sauce.

\section{0.-A BLACK-CAP PUDDING.}

Rub three table-spoonfuls of fiour, smooth, by degrees into a pint of milk, strain it, and simmer it over the fire until it thickens; stir in two ounces of butter; when cool, add the yolks of four eggs beaten and strained, and a half a pound of currants washed and picked, laid at the bottom of the basin or mould previously well buttered. Put the batter into the mould, cover it tight, and plunge it into boiling water; the currants downwards, that they may stick to the bottom and thus form the black-cap.

\section{1.-A SWEETMEAT PUDDING.}

Cover a dish with thin puff-paste, and lay in it freshly candied orange, lemon, and citron, one ounce each, sliced thin. Beat the yolks of eight and the whites of two eggs, and mix with eight ounces of butter warmed, but not oiled, and eight ounces of white sugar. Pour the mixture over the sweetmeats, and bake one hour in a moderate oven.

\section{2.-ORANGE PUDDING}

May be made with six ripe oranges, large, juicy, of the best kind, and peeled ; the rinds to be beaten in a mortar with half a pound each of fresh butter and moist sugar, to which add the yolks of six or eight eggs, and make the

Make a rich pudding-paste with flour and butter, without suet, but as light as possible. Roll it out thin, and cut it to the breadth of eight or ten inches, making it at the same time as long as you please; but half of a pound of flour and five ounces of butter, wetted with water, will probably be sufficient when rolled out quite smooth; then spread upon it a thick layer of raspberry, currant, or any other sort of jam, but leave about an inch of all the edges bare. That done, roll it round; the roll of paste will secure the fruit, and the ends must be twisted together for the same purpose. Wrap it in a nicely floured cloth, and boil it for two to three hours according to size.

Take it up quite hot, and when served cut it crosswise. It may appear homely, but it is an excellent and much-admired pudding.

\section{4.-A VERY FINE GERMAN PUD-} DING.

Pour half a pint of boiling milk upon one pound of bread-crumbs; beat up a quarter of a pound of fresh butter to a cream; add to it the yolks of ten eggs well beaten; drain the milk from the bread, and add the bread with a quarter of a pound of pounded sugar and the grated peel of a lemon; whip the whites of the ten eggs into a solid froth, and add them the last thing: butter a cloth, and put the pudding in, tying it loose; as it will swell out a good deal; plunge it into boiling water; boil one hour. Some persons add a quarter of a pound of jar raisins slit and stoned. When turned 
out stick the surface with sliced almonds, and serve with the following sauce:Take one pint of cream, one pint of sweet raisin wine, four ounces of sugar rubbed on the peel of a lemon, and let them boil; add the yolks of six eggs well beaten, and the juice of the lemon; mix them well; make the sance quite hot, but do not let it boil again; pour some over the pudding, serving the remainder in a sauce boat.

\section{5.-POUDING À LA NESSELRODE.}

(From an eminent Hotel-keeper.)

For a two-quart mould boil a sufficient quantity of chestnuts to produce a quart of meal after being pounded and rubbed through a tammy. Boil three-quarters of a pound of sugar in one pint of water, with a stick of vanilla, until it is reduced one-third. Boil one pint of cream, add it to the chestnut-flour, then add the syrup and twelve yolks of eggs beat up; set it over the fire, stir it until it nearly boils. When cold, put the composition into a freezing-pot; when it is frozen, add half a pint of whipped cream, three whites of egos whipped up strong, a handful of raisins stoned, chopped, and soaked for a day in maraschino, as many currants, one cup and a half of chopped candied lemon-peel, and a glass of maraschino; stir these ingredients all well together, freeze it again, and put it into the mould.

\section{6.-PAIN PERDU.}

Beat eggs with salt, sugar and orangeflower water; cut slices of bread, soak in boiling milk, drain, and dip them in the egg; fry them in hot butter. Sift sugar over them, and serve hot with wine sauce.

\section{7.-A CHARTREUSE OF APPLES AND RICE.}

Boil six ounces of rice with a stick of cinnamon in milk until it is thick, stirring in a spoonful of rose-water or orange-flower water. Pare ten or twelve apples-golden pippins are the bestscoop out the core, and fill up the orifice with raspberry-jam. Border a deep dish with paste; put in the apples, leaving a space between, and fill it up with the rice. Brush the whole over with the yolk of an egg, and sift sugar thickly over it; form a pattern on the top, with sweetmeats, and bake it for one hour in a quick oven.

\section{8.-MIROTON OF APPLES.}

Scald the apples, reduce them to a pulp, and pile them high upon the dish in which they are to be served; boil one teaspoonful of grated lemon-peel and six or eight lumps of sugar in a teacupful of water; then add the yolks of three eggs and the white of one, half an ounce of butter, one spoonful of flour, and one of brandy; mix the whole together over the fire, and stir it quite smooth. Pour it upon the apples, then whisk the whites of the other two eggs to a froth; put them over the miroton just as it is going into the oven, and sift some sugar over it. The oven must be slow: it will take ten or fifteen minutes to bake.

\section{9 -TO MAKE BATTER FOR FRUIT PUN. DINGS.}

Put half a pound of flour and a saltspoonful of salt into a pan, add very gently half a pint of milk; if mixed carelessly the flour will remain in lumps ; beat up the whites of four eggs, strain them to the batter, and beat it well with a. wooden spoon. The whites should be beaten separately to a solid froth, and not added till just before the batter is used. For fruit the batter should be rather thicker than when plain, to prevent the fruit from sinking to the bottom of the dish or basin, as it is equally good baked or boiled. It must be brought to a proper consistency by ad ling milk. 
1370.-OXFORD DUMPLINGS.

Of grated bread two ounces, currants and shred suet four ounces each, two large spoonfuls of flour, a great deal of grated lemon-peel, a bit of sugar, and a little pimento in fine powder. Mix with two eggs and a little milk into five dumplings, and fry of a fine yellow brown. Made with flour instead of bread, but half the quantity; they are excellent. Serve with sweet sauce.

\section{1.-GLOUCESTER PUDDINGS.}

Weigh three eggs in the shell; take their weight in flour and butter; take twelve bitter almonds and fire ounces of pounded sugar; beat all together for half an hour, and put the mixture in pudding-cups, filling the cups only half full. Bake them half an hour.

\section{2.-YEAST DUMPLINGS.}

Take some yeast and make a very light dough the same as for bread, using milk how..er instead of water; add salt, put

sy the fire covered in a pan for half an hour or more, to rise; after this is done roll up the dough into small balls and boil them for ten minutes, then take them out and serve directly with winesauce over them. To know when they are done stick a fork into one, and if it comes out clean they will do.

A good method of eating them is by dividing them from the top with two forks, as they get heary by their own steam, and eat them directly with meat, or sugar and butter, or salt.

\section{ITALTAN PASTES.}

BESIDES macaroni, there are vermicelli, semolina, a smaller sort called macaroncini, and various other pastes of the same nature, all made in Italy from the same kind of grain; nothing, therefore, color. can be more simply nutritive, nor, when well boiled, more easy of digestion. There are also two sorts of macaronithe pipe and the tape-the one hollow, and the other flat, but both made of the same material; the vermicelli being that most generally used in soup: each being severally used in various ways, according to the following receipts :

\section{3.-MACARONI.}

Some cooks are very fond of boiling it in milk, and even of laying it in milk for an hour or two to swell and become tender, but boiling water is as good. A quarter of a pound will be sufficient for half a dozen people, and will not require more than twenty to twenty-five minutes to become tender, if boi'ed in water, but a few minutes longer if boiled in broth. Drain it when tender and lay it in a dish. Half the quantity of cheese should be finely grated and sprinkled over it in layers, with butter, to mix it thoroughly. It should be served quite hot. It is sometimes eaten with mustard, and, if to be made very savory, beef gravy may be used with a truffle stewed in it.

Nothing but Parmesan cheese will give it the true flavor.

To brown Macaroni is the common practice of cooks; though, in fact, the browning, while giving a pretty appearance to the macaroni, not only hardens the top of the cheese into a crust, but often gives a taste of rancidity. It can be done with crumbs of bread, thus:-

Wash the macaroni, then simmer it in a little broth, with a little pounded mace and salt. When quite tender, take it out of the liquor, lay it in a dish, grate a good deal of cheese over, then cover that with bread grated fine. Warm some butter without oiling, and pour it from a boat through a little earthen colander àl over the crumbs, then put the dish in a Dutch oven to brown the bread of a fine 
Cold Macaroni,-If already dressed, may be warmed in any kind of broth, letting it simmer gently upon a slow fire, with the yolks of two eggs to thicken; after which it should be put into the

* oven in a mould covered with crumbs of bread; or, if undressed, it may be made by.leaving it overnight in broth, and then proceeding with it as above.

\section{4-TIMBAL̀E DE MACARONI.}

Soften the macaroni by boiling it fast until it will cut easily without breaking, but do not let it be too soft; then cut it into pieces half an inch long; butter a mould, stick the macaroni quite close together all over it, so as to look like a honeycomb; then spread a thin layer of quenelle farce over the macaroni ; fill up the mould with a mince of stewed ox palates cut into small pieces, and small button mushrooms, either pickled or fresh, moistened well with white sauce: close the mould, and boil it for threequarters of an hour; turn it out very carefully, and serve it with thick white sauce and mushrooms.

\section{5.-PÂTÉ DE MACARONI.}

Stew some macaroni in butter and water, or broth, strain it, cut it into pieces, and lay it at the bottom of the dish, adding ham balls, made of ham, pounded in a mortar, and blended with butter ; then have ready any kind of game, boned and filleted, cock's-combs or oxpalates, previously blanched and stewed, sweetbread cut into dice, and mushrooms, all stewed in good rich sauce; place a layer upon the macaroni, then another layer of meat. and, until the pie is filled, add to it equal quantities of cream and gravy, cover it with a paste, and bake it, or omit the paste, and stew it before the fire in a Dutch oven. The macaroni may be mixed with grated cheese.

\section{6.-VERMICELLI}

Is of the same substance as macaroni, but made much smaller, and frequently put into meat soups as giving them additional richness; but it is often too sparingly used. To be well made the soup should be thickened with it, and for that purpose it is preferable to macaroni.

Semolina is of the same material, but made into small grains, which more easily thicken the soup in which it is mixed; it can also be made into an excellent pudding with eggs and milk, using it instead of flour.

It should be observed, as a general rule, that in using any of the Italian pastes-unless they should be sweetened - Parmesan cheese should always form part of the dish, in the proportion of onehalf the quantity to that of the paste.

\section{7.-POLENTA.}

Is, in Italy, made from the flour of Indian corn, but in England generally of semolina, thus:-Put a little salt and four ounces of semolina into a quart of milk when it is beginning to boil, and let it continue to simmer for about ten minutes, stirring it occasionally to guard it from burning. When well thickened pour it into a dish to cool, and when quite cold it will be very solid: then cut it into slices of less than an inch thick, upon which put a large grating of good Parmesan cheese, for no other kind will answer the purpose; nor should any spices be used, except a little mace; nor even that, unless it be made (as sometimes done) with veal broth. Clarify a few ounces of fresh butter-though in Italy oil is always used-put a layer of the paste, powdered with the Parmesan, mixed with a little of the butter; bake it for half an hour in a moderately heated oven, and serve it up quite hot, with a separate plate of the grated cheese. 


\section{R I C E.}

For savory dishes rice should be stewed in good gravy, seasoned with mace, cayenne, and salt, with the addition of fried onions, shalot, garlic, and pot-herbs, strained and used at pleasure.

For sweet dishes.-Boil it with lemonpeel, cinnamon, or bay-leaves, and, when done to a proper consistency, add powdered sugar and cold butter, stirring it all the time till melted; then pour in a little cream, and use it either as a covering for stewed fruits, or, if made thick enough, it may be placed round the sides of a dish, leaving the middle hollow for the sweets. The same plan may be adopted for all sorts of meat, poultry, fish, fruit, and sweetmeats. The sugar should not be added until the rice is done, as it has a tendency to harden the grains.

Plain rice pudding can be made with brown sugar, and milk baked slowly a long time ; a richer one by boiling a teacupful of rice, and when boiled, adding milk and fine sugar, with four beaten eggs, and baking it.

\section{8.-RICE FRITTERS.}

Pare very thin the rind of a lemon, and boil it in milk, with sugar enough to sweeten it, and a cup of rice. When the rice is quite soft take out the lemon peel, beat up the rice with a glass of brandy; shape it into fritters; brush them with yolks of eggs, cover them with breadcrumbs, fry them in lard, dust them with fine sugar, and glaze with salamander.

\section{9.-TO BOIL RICE.}

Very few persons know how to boil rice properly. It is usually so boiled as to become a heavy dough, so tenacious and solid as to be almost impenetrable to ${ }^{\circ}$ the digestive fluids secreted by the mouth and the stomach, which are neces- sary to dissolve it, and to effect its digestion and distribution, as innocent nourishing food. It should be so conked that the grains shall remain separate and distinct, but not hard, and the whole be in some degree loose and porous. The. Southern method is as follows:

Pick over the rice carefully, rinse it well in cold water till it is faithfully cleansed; drain off the water, then put it in a pot of boiling water, with a little salt. Allow as much as a quart of water to a teacup of rice, as it absorbs the water very much while boiling. Boil it seventeen minutes; then turn the water off very close; set the pot over a few coals, and let it steam fifteen minutes with the lid of the pot off. The beauty of the rice boiled in this way, is, that each kernel stands out by itself, while it is quite tender.

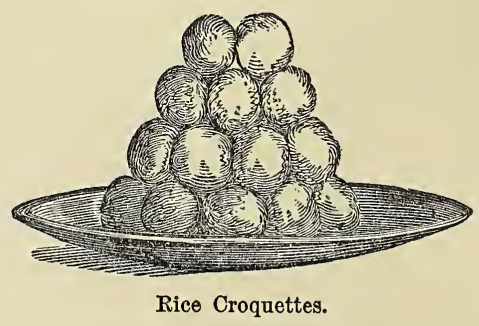

1380.-RICE CROQUETTES.

Take half a pound of boiled rice, mix with it two table-spoonfuls of grated cheese, and a little nutmeg and mace; then take a little butter, six table-spoonfuls of cold chicken breast, minced, six bearded oysters, a little parsley and lemon peel, and mixed together with rich milk or cream. Roll out a table-spoon heaping full of the rice, and put into it as much as it will hold of the mixture, folding the edges and inclosing it ; brush the balls over with yolk of raw egg, roll them in cracker crumbs, and fry them light brown in boiling lard. Serve them hot when drained. 
1391.-CASSEROLE DE RIZ AUX OEUFS.

Clean, wash, and stew the rice; work it well with stock and an egg beaten, as the rice should be firm and well blended; then make it into a wall, lining the inside of a mould of the requisite height : bake the casserole. Take the white portion of cold fowl, cold veal, or sweetbreads; mince them finely, add some thick white sauce and mushrooms, fill the casserole, and cover the top with poached eggs; cover them with glaze, and serve it up very hot.

\section{2.-RICE, GÂTEAU OF.}

Boil a quart of cream, and add to it half a pound of powdered sugar, and three-quarters of a pound of rice; when the latter is quite soft dissolve in it a quarter of a pound of butter, and then put in the grated rind of a lemon; let it cool. When quite cold stir in four yolks and four whole eggs; more if the rice be very thick; butter a mould lightly, put the rice into it, place the mould in hot ashes so that it may be completely enveloped in and covered with them; in half an hour the gâteau will be done enough, then turn it out and serve. If you wish you can make a souffle by whipping the whites of six eggs like other soufflés; in this case it should be served in a silver dish. In putting the preparation into the mould be careful not to fill it, as the rice would swell and run over.

$$
\text { 1383.-MUSH. }
$$

Stir Indian meal into boiling water, adding a little salt and putting in the meal by degrees, while stirring with a mush stick. Let it boil thick, then simmer slowly till sufficiently cooked; three or four hours' simmering will not be too long.

1384.-HOMINY.

Wash the hominy clean, and boil it not deficient in delicacy. with sufficient water to cover it. It should boil from four to five hours over a very slow fire. Eat it with butter and molasses, or with sugar and milk. It is considered extremely wholesome food, especially for children and delicate persons.

\section{5.-CROQUETTES OF RICE WITH APRI- COT MARMALADE.}

Prepare the rice plain for a croquette, take the handle of a wooden spoon, make a hole in the croquette, which fill with marmalade of apricots. Then close it up with some of the rice, put crumbs of bread as you do in all other croquettes, and fry in the same manner. You may put in pieces of apple or other fruit.

\section{6.-SOUFFL' OF APPLES WITH A RICE BORDER.}

Prepare your rice as for a croquette. Keep it of a strong solid substance; dress it up all round a dish, the same height as a raised crust, that is to say, three inches high. Give a pleasing shape to the rice, and let it be levelled smooth; have some marmalade of apple ready, made very thick; mix with it six yolks of eggs and a sniall bit of butter; warm it on the stove: then have eight whites of eggs well whipped, as for biscuits ; mix them lightly with the apples, and put the whole into the middle of the rice; put this into the oven, which must not be too hot. When the souffé is raised sufficiently, send it up, as it would soon lower. If you wish to make a kind of pap, take a spoonful of flour, a pint of milk, a little salt, lemon, and sugar ; let the whole boil well and thick, then mix it with the apples and the yolks of six eggs; the whites are to be well beaten, and poured in afterwards; next bake the souffle in the oven. This method is safer than the former, and is 29 


\section{H E E S E.}

Cheese takes an important place at the table; not alone, in its simple place at the close of the dinner, but that it forms the foundation of many excellent dishes. The selection of good cheese requires both judgment and experience; a delicate palate and a keen sense of smell are two essential requisites, while the eye is an assistant to point out those defects independent of taste or smell. A prime cheese is readily told by a connoisseur by the color and texture, without consulting either nose or palate.

\section{7.-TO MAKE CHEESE.}

Put rennet into warm fresh milk, soaked in a little warm water, and add salt. Half a pint of rennet water will turn three pails of milk. Cut the curd into squares; afterwards break it up and let it stand a few minutes; pour off the whey, tie up the curd, and hang it up to drain dry. Then chop it up in a cheese tray, put it in a cloth in the cheese mould, and press it in the screw press half an hour. Then cut it up fine, salt it, and press again for twenty-four hours, wetting the cloth previously. Take it out and turn, and put it in again in a clean wet cloth, and renew this process for three or four days. Then rub the cheese with butter or lard, and set it away in a dry place or in a wire safe. Wipe and grease it till the rind is firm, and turn it every day for several weeks.

\section{8.-SMEARCASE OR COTTAGE CHEESE.}

Set by the fire some milk beginning to sour, till a curd is formed: then pour off the whey, tie the curd in a cloth, and hang it up to drain, not squeezing it. Drain it twelve hours. Then work it in a bowl with cream, salting to taste, and add a little fresh butter, till it is soft and smooth.
1389.-SAGE CHEESE.

Pound fresh sage leaves in a mortal to obtain the juice, and mix it with the milk while warm, after the rennet is put in. Spinach juice is an improvement.

\section{0.-CREAM CHEESE.}

Take a small pan of fresh morning's milk, warm from the cow is best, and mix with the cream skimmed from an equal quantity of the last night's milk. Warm it to blood heat, pour into it a cup of water, in which a piece of rennet the size of two fingers has soaked all night, and put it in a warm place till the curd is formed. Cut the curd into squares, lay over it a thin straining cloth. press it down, and dip out all the whey that rises through it. Then put the curd in the cloth, squeeze it dry, then crumble and salt it to taste. Wash the straining cloth, lay it in the cheese hoop (a bottomless vessel the size of a dinner plate, perforated with small holes,) put the crumbled curd into the cloth, and fold the rest of the cloth overit. Put on the cover, and set a weight on it. In six hours turn the cheese, and let stand six hours longer. Then take it out, rub it with fresh butter, and set it in a dark, dry place. Turn it every day for four or five days, when it is fit for use ; and it must be eaten immediately when cut. It will keep but a few days, even in cold weather.

\section{1.-CHEESE PUFFS.}

Take half a pint of cheese curd strained from the whey, with a spoonful and a half of flour, three eggs, leave out the whites of two, a spoonful of orange flower water, a quarter of a nutmeg, and sugar to make it sweet; beat it in a mortar; lay a little of this paste in very small round cakes on a tin plate; if the oven is hot, they will take a quarter of an hour to bake them; serve them with pudding sauce. 


\section{2.-EGGS AND CHEESE}

Are mixed together in various ways by French cooks, under the names of fondus or fondeaux, raméquins, and other titles, for the purpose of preparing entremets, or side-dishes, for elegant tables.

Gruyère and Parmesan are the most proper sorts to be thus used, but any dry cheese, of good flavor, may be employed.

For Fondeau.-Slice a stale penny roll; pour over it three gills of boiling milk; when soaked, beat it well, and mix with it half a pound of cheese, finely grated, with the yolks of four eggs well whisked. All this may be prepared when most convenient; immediately before it is wanted beat the whites of the four eggs into a solid froth, and add them; pour the whole into the paper forms, and bake them to your taste.

A l'Italienne.-Mix half a pint of cream with a little flour and a little salt; keep stirring it over the fire until it is as thick as melted butter; then add about half a pound of Parmesan cheese finely grated. Mix it all well together until it is half cold; then take four eggs, separate the yolks from the whites, put the former to the cheese, and beat it well together. Then beat the whites to a solid froth, and add them to the rest; pour the mixture into an ornamented mould or a deep dish lined with paper, cut in a fringe at the top, and only half filled, as it will rise very high. The oven must be very hot, in order to cause the rising, and the dish must be served immediately, or it will fall ; to prevent which, let the cover be of metal, strongly heated: twenty minutes ought to bake it.

Fondeaux of a small size may be made in either of the above modes, and put into little paper moulds of any fancied form, but only half filled.

\section{3.-RAMÉQUINS.}

Take two eggs, two spoonfuls of flour, two ounces of melted butter, and two of grated cheese ; mix all well together, and bake it in moulds or tart-pans for a quarter of an hour.

A la Parisienne.-Boil half a pint of milk, and half the quantity of cream; melt one ounce of butter and a little salt; mix in a spoonful of flour, and stir it over the fire for five minutes; pour in the milk and cream by degrees, and work it smooth, taking care that it is thoroughly cooked. Then take it off, and add half a pound of grated cheese, some coarse ground pepper, and an atom of nutmeg, with a very little powdered sugar, the yolks of eight eggs, and the whites of two, well beaten; when perfectly mixed, add the whites of six eggs beaten to a froth. The batter should be as thick as cream. Make little paper trays, fill them three parts full, and bake them in a very slow oven eighteen minutes.

Fried Raméquins.-Grate half a pound of cheese, and melt two ounces of butter; when the latter is getting cool, mix it with the cheese and the whites of three eggs well beaten. Lay buttered papers on a frying-pan, put slices of bread upon it, and lay the cheese on the top; set it on the fire for about five minutes, then take it oft, and brown it with a salamander.

1394.-CHEESE SOUFFLA, OR DIABLOTINS.

Put a gill of milk in a stewpan, with two ounces of butter; when boiling, stir in two spoonfuls of flour, keep stirring over the fire until the bottom of the stewpan is dry, then add four eggs by degrees, and a pound of grated cheese; mix well in, season with pepper, salt, and cayenne, rather highly, mould the paste into little balls with the forefinger 
against the side of the stewpan contain- $\mid$ when the cheese dissolves before the fire, ing it, drop them into hot lard ; fry of a put it upon thin slices of buttered toast, nice light brown, dress in pyramid upon of which the crust has been cut off. a napkin, and serve very hot; a quarter Serve it very hot, or the cheese will beof that quantity may of course be made. come tough ; and eat it with pepper, salt, This is an excellent dish.

\section{5.-STEWED CHEESE}

Grate two ounces of cheese; put it into a basin ; mix with it a small teacupful of cream and an egg beaten and strained. Put into a small saucepan one ounce or half an ounce of butter, according to the richness of the cheese ; let it melt, then stir in the other ingredients, and let it boil until well mixed. Serve it hot either browned or plain.

With Ale.-Cut the cheese into slices, pare off the rind, and put it into a dish over a lamp; then pour on as much ale as will cover it, and let it stew until the cheese is dissolved. This is a Welsh method, and a very good one. The cheese may be spread with mustard.

A very delicious sort of paste may also be made of any of the rich cheeses, stewed with cream and yolk of egg, either seasoned savorily or sweetened; and eaten hot or cold.

\section{6.-WELSH RABBIT.}

Put mellow cheese into a saucepan with about one ounce of butter and a very little ale; stir it over the fire until the cheese is quite dissolved; then take it off, add an egg well beaten, dish it, and brown it before the fire.

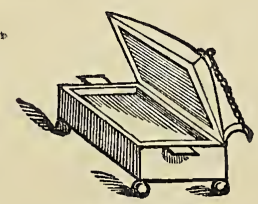

Cheese-Toaster. and mustard.

\section{7.-A CHEESE PUDDING.}

Grate three ounces of cheese and five of bread; and having warmed one ounce of butter in a quarter of a pint of new milk, mix it with the above; add two well-beaten eggs and a little salt. Bake it half an hour.

- This is a nice way of finishing up a cheese when little but the rind is left.

When mites have taken possession of a cheese, and this is considered objectionable, the following reseipt will have the effect of destroying them without injury to the cheese. Wipe the cheese, put it into a pot in which mutton has been cooked whilst the water is yet hot, make the water boil a few seconds, take out the cheese, wipe it immediately, dry it, and then put it away in a dry place until required for use.

\section{8.-POTTED CHEESE.}

Scrape and pound cheese, with a piece of butter, cayenne pepper, a few grains of pounded mace, a teaspoonful of sifted sugar, a glass of white wine, and a little salt; press it into your potting-jar for shape. It may be potted with brandy.

\section{9.-CHEESE CREAM, A PLAIN FAMILY WAY.}

Put three half pints of milk to onehalf pint of cream, warm, or according to the same proportions, and put in a little rennet; keep it covered in a warm place till it is curdled; have a mould with holes, either of china or any other, put the curds into it to drain about an hour, serve with good plain cream and pound-

Or, Rasp some toasting cheese with a bit of butter, into a cheese-toaster, and, led sugar over it. 
1400.-PANCAKES.

The common sort are composed of a light batter, made of eggs, flour, and milk, fried in hot dripping or lard, only half of the whites of the eggs being generally used; but salt, or nutmeg, and ginger, may be added, and sugar. and lemon should be served to eat with them. Or, when eggs are scarce, make the batter with flour and small beer, ginger, \&c.; or clean snow, with flour, and a very little milk, will serve as well as eggs. If the family be large, beat eight yolks and four whites of eggs, strain them into a pint of milk, put a grated nutmeg, and sugar to your taste : set three ounces of fresh butter on the fire, stir it, and as it warms pour it to the milk, which should be warm when the eggs are put to it; then mix smooth almost a half a pound of flour. Fry the pancakes very thin; the first with a bit of butter, but not the others. Serve several on one another, but at two or three different times, "hot and hot."

A finer kind are fried without butter or lard, as thus:-Beat six fresh eggs extremely well ; mix, when strained, with a pint of cream, four ounces of sugar, a glass of good French brandy, a half a nutmeg grated, the grated rind of a whole lemon, and as much flour as will make it almost as thick as ordinary pancake-batter, but not quite. Heat the frying-pan tolerably hot, wipe it with a clean cloth; then pour in the batter to make thin pancakes.

\section{1.-APPLE PANCAKES.}

Mix two large spoonfuls of flour in a cup of milk or wine; when smooth add eight eggs, some pounded cinnamon, grated lemon-peel, a handful of currants, and six or eight apples peeled and chopped: mix it all well together; melt some butter in a frying-pan; when hot pour the whole mass in, and fry it on both sides: serve it stewed with pounded cinnamon and sugar very hot.

\section{2 - NEW ENGLAND PANCAKES.}

Mix a pint of cream, five spoonfuls of fine flour, seven yolks and four whites of eggs, and a very little salt; fry them very thin in fresh butter, and between each strew sugar and cinnamon. Send up six or eight at once.

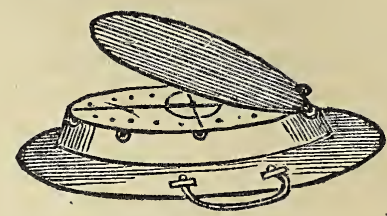

Waffle Furnace.

\section{3.-DUTCH PANCAKES.}

Mix one pound of flour with a half a pound of sugar and a table-spoonful of cinnamon in powder; make it into a paste with ten eggs and two glasses of white wine, or one each of wine and brandy; when well mixed, roll it out and fry like other pancakes.

\section{4.-CREPES.}

Make a batter with flour, milk and eggs, adding brandy and orange-flower water. In two or three hours afterwards, melt a piece of butter or lard, the size of a walnut, in a frying-pan, pour in some batter, and shake it over the pan. When done on one side, turn it over; when the other side is done, dish it and keep hot till the others are done, sifting sugar over each.

\section{5.-RICE PANCAKES.}

To half a pound of rice put two-thirds of a pint of water, boil it to a jelly; when cold, add to it eight eggs, a pint of cream, a little salt and nutmeg, and half a pound of butter melted; mix well, adding the butter last, and working it 
only so much as will make the batter |lard sufficient to swim in. Glaze with sufficiently thick. Fry them in lard, sugar.

but employ as little as it is possible to fry them with.

\section{6.-FRITTERS,}

Although a species of pancakes, are yet not so wholly composed of eggs, and may be made of almost any substance fried in butter. Make them of any of the batters directed for pancakes, dropping a small quantity into the pan, or beat six eggs very lightly and quickly in a quart of milk, adding gradually three quarters of a pound of potato-flour. Have ready a large quantity of lard, and when boiling hot put in the batter, allowing for each fritter half a teacupful. They do not require turning, and will be done in a few minutes, but must be sent to table immediately, or they will become heavy; one pound and a half of lard will be required for the above quantity of fritters ; they may be eaten plainly powdered with loaf sugar or sugar and cinnamon, and should be served on a folded napkin in the dish. Any sort of sweetmeat or ripe fruit may be made into fritters.

For plain Fritters.-Grate the crumb of a penny loaf, put it into a pint of milk over the fire, and beat it very smooth; when cold, add the yolks of five eggs three ounces of sifted sugar, and half a nutmeg. Fry them in hog's lard, and serve pudding-sauce in a boat.

Apple-Fritters.-Take the yolks of six eggs, and the whites of three; beat them well, and strain them; then add a pint of milk, a little salt, half a nutmeg grated, and a glass of brandy. Make it into thick batter with fine flour; slice the apples in rounds, cut out the core, dust them with fine sugar, and let them so remain for two hours; then dip each slice in batter, and fry them in plenty of boiling lard over a quick fire. They require
Crême Frite aux Amandes.-Boil one pint of milk with two bay-leaves, a little lemon-peel, and a small piece of cinnamon. When the flavor is extracted, strain it; add two table-spoonfuls of arrowroot, and sugar to palate; stir it well over the fire, and add the yolks of four eggs, two ounces of chopped almonds, and a wineglass of cream. Cook the whole over the fire for about four minutes, stirring it all the time; then pour it on a tin which has been well buttered, spread it about half an inch thick, put it into the oven for ten minutes, let it get thoroughly cool, cut out with a round cutter, dip into fine bread-crumbs, then into egg beat up very thin, and again into crumbs; fry in hot lard: dust over with sugar, and send to table.

French Fritters.-Mix two eggs, well beaten and strained, with as much new milk and flour as shall make one thick pancake, which fry as pale as you can. Pound it in a mortar quite smooth, and add the yolks of four and whites of two eggs, one large spoonful of orange-flower water or cinnamon-water, one ounce of blanched almonds beaten to a paste, two ounces of white sugar, and one-quarter of a small nutmeg. Pound it all till it becomes smooth batter. Have ready a large stewpan half full of fine lard quite hot, and drop the batter into it, the size of large nuts, until the surface be filled: as they brown, turn them: they will be very large: when done, remove them on clean paper, in a dish before the fire, and do the remainder. Serve as quickly as possible. They are excellent.

Potato Fritters.-Boil two large potatoes, scrape them fine, beat four yolks and three whites of eggs, and add- one large spoonful of cream, another of sweet wine, a squeeze of lemon, and a little nutmeg. Beat this batter half an hour at 
least. It will be extremely light. Put a good quantity of fine lard in a stewpan, and drop a spoonful of the batter at a time into it. Fry them, and serve in a sauce boat, a glass of white wine, the juice of a lemon, one dessert-spoonful of peach leaf or almond water, and some white sugar, warmed together.

Curd Fritters.-Rub down in a mortar a quart of dried curd, with the yolks of eight and whites of four well beaten eggs, two ounces of sifted sugar, half a nutmeg, and half a spoonful of fiour. Drop the batter into a frying pan, with a little butter or fine lard.

Orange and Sweetmeat Fritters.-Peel two oranges, then cut them in slices across, and take out all the seeds, dip the slices of orange in batter, as for applefritters, and fry them. Serve with powdered sugar upon each fritter.

\section{7.-BEIGNETS.}

Mix and work well together half a pound of flour, and two ounces of butter, or three table-spoonfuls of sweet oil, and make it into a batter with some warm water; then mix into the batter a couple of raw eggs, keeping the yolk of one for browning. The materials may thus be made of any degree of consistence, so as to act as thick batter, or as thin paste by the addition of a little flour; and being made into small balls, are spread with the remaining yolk of egg, powdered usually with sugar, and fried in hot lard; and, when ready to be served, put them for a moment on a hair sieve to drain off the fat.

If made solely with paste, without mince of any kind, they should have another egg, and the batter be more continually beaten, so as to render the beignets light; then, when prepared for dressing, take a spoonful of the batter formed into a ball, and drop it into the boiling lard. A large dishful will be fried in six or seven minutes.

\section{VARIOUS MODES OF COOKING EGGS.}

Unless an egg is perfectly fresh it is unfit for any purpose. You may try the freshness of eggs by putting them in a pan of cold water. Those that sink the soonest are the freshest. Eggs may be preserved a short time by putting them in a jar of salt or lime-water with the small ends downwards. The salt should not afterwards be used. They may be. preserved several months by greasing them all over with melted mutton suet, and wedging them close together in a box of bran. The small ends always downwards.

There are, of course, several sorts of * eggs, according to the species of poultry which produce them. The turkey egg is the largest and the most delicate, after which, in point of flavor, is the guineahen's, and, in that of size, those of the goose and duck. Although all these are employed in cookery, yet the eggs of the "common barn-door fowl" are those most generally used when served up separately and boiled in the shell, in which case the main point is their entire freshness. This can partly be judged of by putting them between you and the light, and seeing that the white is clear and the yolk unclouded; for although nothing can be more delicately nutritive when newly laid, few things are more nauseous when they are stale.

\section{8.-TO KEEP EGGS FOR WINTER USE.}

Pour a full gallon of boiling water on two quarts of quicklime and half a pound of. salt; when cold, mix into it an ounce of cream of tartar. The day fol- . lowing put in the eggs. After the lime has been stirred well into the boiling water, a large part of it will settle at the 
bottom of the ressel, on which the eggs will remain. Keep them covered with the liquor, and they will keep for two years.

\section{9 -TO BOIL EGGS.}

To effect the simple process of boiling demands constant attention, as half a minute more or less may spoil the egg for a fastidious palate. Some put them into cold water, and, when it boils, allow the eggs to remain in it for a minute longer ; while others prefer putting them into boiling water, and there leaving them until done. That time is most commonly three minutes, and seldom less; but many persons object to their being left so soft, and four or five minutes are not unusual if the eggs are not "new-laid." Ten minutes are required for making the yolks hard enough for salad, after which the eggs should be put for some time into cold water to harden, or the yolks will not readily incorporate with the condiments.

It should, however, be understood that, in whatever way the egg is to be boiled, it should be completely covered with water; that if put into the water while boiling, the shell is subject to being cracked; and if boiled too suddenly, the white becomes hard, while the yolk remains comparatively soft. The cook should therefore be provided with minute glasses of various sizes-from one minute to five-to mark the time; for they are more accurate than the hand of a kitchen clock, and do not require so much watching.

\section{0.-TO POACH EGGS}

Is to boil them without their shells, and requires still greater care. The water must be perfectly pure, and the yolk must be only just so much done as to fix itself firmly in the centre of the white.

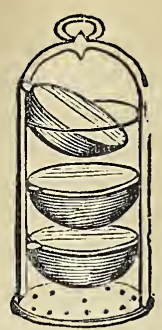

Egg Poacher.

Put a moderate quantity of water into a broad flat stewpan. French cooks add to it, when boiling, a spoonful of vinegar and a little salt. Take the pan off the fire whilst putting in the eggs; break the egg into a teacup or small ladle, and turn it gently into the water, so as to prevent the yolk from being mixed with the white; let them $\operatorname{stan} l$ till beginning to set; then let the eggs boil gently until you perceive that they are done, by the white being set, and the yolk appearing through it in a comparatively soft state; then take each from the water with an egg-slice, and pare off any ragged edges, which may appear around it, before sending to table. This latter operation, however, if not quickly done, cools the egg, which ought to be served quite hot, to avoid which inconvenience, a little machine for poaching has been invented.

Poached eggs are commonly placed upon buttered toast, broiled ham, or boiled spinach.

\section{1.-OMELETTE.}

This is the most usual mode of dressing eggs, after that of boiling them in the shell.

Four eggs will make a very pretty sized omelet, but the number must of course depend on the size required. If sweet herbs be put in, a good deal of parsley should form part; tarragon gives a high flavor, and chives or shalots are not un- 
frequently used, but care should be taken that the flavor should not overpower that of the other ingredients.

Omelets are judiciously varied by mixing grated ham or tongue with minced anchovies, when served at supper or as a side-dish at dinner; but when in tended for the breakfast-table, it is more delicate to make them of eggs alone.

Although a cormon frying-pan may be used, yet one made on purpose, not larger than the size of a dessert-plate, should be employed, so as not to allow the omelet too much spreading, for it should always be kept of a certain degree of thickness to secure the softness of the inside. It is therefore only to be fried on one side, and when the under is browned and the upper brought to a moderate state of consistence, the fried side is to be folded over the other in three.

Butter is better than either lard or dripping for the frying of an omelet. Any sort of pot-herbs is admissible ; and many sorts of minced fowl and fish are prepared in that manner. There are hundreds of receipts for omelets, but the principle is the same in all, the difference being only in the flavoring.

\section{2-OMELETTE AU NATUREL.}

Take four eggs, beat the yolks and whites together with a table-spoonful of milk, a little salt and pepper; put two ounces of butter into a frying-pan to boil, and let it remain until it begins to brown; pour the batter into it, and let it remain quiet for a minute; turn up the edges of the omelet gently from the bottom of the pan with a fork ; shake it, to keep it from burning at the bottom, and fry it till of a bright brown. It will not take more than five minutes sying.

Aux fines Herbes.-Beat up four eggs with a little cream, salt, pepper, chives, and parsley, minced very small. Put a piece of butter half the size of an egro into the frying-pan; let it begin to brown; pour in the omelet and allow it to set a little; then raise it round the edge, to allow more of the mixture to become brown. When that is done, turn it over to double it; but as in browning the lower side the upper will sometimes appear too soft; in that case make a slight use of the salamander, to give it firmness ; put the dish on the frying-pan, and turn it over. Let the fire be clear, but not too hot.

Aux Confitures.-Beat up eight eggs with a table-spoonful of powdered sugar, a little nutmeg, a pinch of salt, some finely-chopped lemon-peel, and a wineglass of cream, or about a quarter of an ounce of butter. Make an omelet-pan hot, put in a small piece of butter, and, when the eggs are thoroughly mixed, pour them into the pan; stir it about, and, when set, arrange the omelet neatly in the pan; lay on two spoonfuls of jam, roll it up, and glaze it with sugar and the salamander. Any of the preserved fruits, when prepared as jam, will answer the purpose.

Une Omelette aux Amandes is also looked upon as a delicacy, and made by substituting almond-flour, or pounded sweet almonds, for jam.

Une Omelette d'Huîtres may be made with four raw eggs, a little cream, beaten up either with or without sweet herbs and grated ham, but seasoned with a little salt, pepper, and nutmeg, together with a dozen oysters bearded. dried, minced very small, and fried in butter.

\section{3.-OMELETTE SOUFFLÉE.}

Take six eggs, separate the whites from the yolks, add to the yolks a table-spoonful of arrowroot or potato-flour, six ounces of pounded loaf-sugar, some grated lemon-peel, and a small quantity 
of lemon-zest, or chopped peel, or orangeflower water. Beat the whole together for several minutes, until they are made into a batter, and then whip the whites until they become a froth; stir in lightly the batter. Have ready an omelet-pan of the proper size heated upon the stove; put into it a quarter of a pound of butter, and when it begins to boil put the whole into the pan, and stir it with a spoon carefully until it becomes partially firm. That done, turn it out upon a small circular dish, of silver or metal, as the omelet ought to acquire the form of a dome, and the dish be placed in a very moderately heated oven for at least ten mintues or a quarter of an hour, according to the care and nicety with which it has been made; after which it will have risen into a puff of considerable height, similar to the fashionable supper dish, "trifle." It should then be sprinkled with a little powdered sugar, the salamander held over it, and be served immediately.

An Omelette Souffle should not be cooked till the moment it is wanted. Its whole delicacy, indeed, depends upon its being quickly baked, being very light, and, as Dr. Kitchiner observes, its making but one movement-viz.: from the fire to the throat of the guest. The great art is to make it light, foamy, and, as it were, effiervescent.

\section{4.-CEUFS FRITS, OR FRIED EGGS.}

Break some fresh eggs into a dish, without damaging the yolks, and powder over them a little pepper and salt. Then fry some butter; a quarter of a pound will do for a dozen eggs. When the butter is completely fried, pour it over the eggs, and then put them into the frying-pan, which keep at a little distance from the fire, for fear the eggs should stick. When they are done at the bottom, use the salamander for the top, till they turn white. Then dish without breaking them, and pour over them a little vinegar.

\section{5.-PLAIN BAKED EGGS.}

Butter with one ounce a plated or common tart-dish, that will bear the heat of the oven; break carefully six eggs in it, season with one pinch of pepper, half a spoonful of salt, the juice of half a lemon, and add half an ounce of butter in small pieces over; put in a slack oven until set, and serve.

\section{6.-RANDALLED EGGS.}

Twelve eggs boiled hard and halved; cut a piece from the ends of half of them to allow them to stand, take out the yolks, chop them, and mix chopped boiled ham or tongue, season with nutmeg and mace, moisten with butter, and put it in the space within the egg. . Put the halves together, and stand the joined eggs on end in the dish.

\section{7.-GEUFS FARCIS.}

Boil six eggs hard, remove the shells, but leave the eggs whole; cover them with a rich forcemeat made with scraped ham, pounded anchovy, pounded veal, and bacon fat, well pounded together and highly seasoned; brush them with the yolk of egg, and dredge them with breadcrumbs or vermicelii; fry them a pale gold color, or put them for a quarter of an hour in an oven, and serve them up with gravy in the dish.

CEufs sur le plat is a German mode of frying an egg by means of a small cheese plate, which, being thoroughly warmed, is greased in the indented part with a bit of butter or a very little sweet-oil, and into this part the egg is broken from the shell, thus retaining the exact rotundity of the plate, which is then placed upon the gridiron over a good fire. It 
may be slightly browned, if thought fit, with a salamander, but does not need it; and though quite as delicate, yet renders the egg rather more savory than by poaching.

\section{8.-EGGS AND VEGETABLES.}

Eggs and Spinach.-Poach half a dozen eggs, trim them nicely, and serve them upon stewed spinach.

Eggs and Onions, or à la Tripe._Skin six or eight onions, mince them, put some butter into a stewpan and melt it, add about two spoonfuls of flour, mix both well together, then add the onigns, and wet them with milk, cream or water; let them stew, taking care that the onions and butter do not become brown; season with a little salt and white pepper; cut some hard eggs into slices, and when the sauce is ready put them in; make the whole very hot, and serve it up.

\section{9.-GEUFS BROUILLÁS.}

Break four or six eggs ; beat them and put them into a saucepan with a piece of butter, a little salt, and a spoonful of sauce or gravy, which makes the eggs softer; stir them over the fire until sufficiently thick ; serve on a plate garnished with toasted bread. To eggs dressed this way, truffles, ham, mushrooms, \&c., minced, may be added. The difference between this and an omelet is, that an omelet is compact and turns out smooth, whereas oufs brouillés are less done, and are therefore broken.

In Ireland, where it is in general use, it is usually served upon hot buttered toast, and is there called "buttered eggs." It is also very common in France, where it is usually served for breakfast.

A very pretty dish of eggs is made thus: - Break some eggs into a small tartdish, without injuring the yolks, or laying one over the other. Drop on them some warm butter, and lightly strew crumbs of bread. Put it in the oven until the whites be set, and serve with a wreath of parsley round the elige.

\section{0.-EGGS WITH BURNT BUTTER.}

Put into a frying pan two ounces of butter, which melt; as soon as it is on the point of browning, put in the eggs, which have been previously broken in a basin, and seasoned with pepper and salt ; when well set, serve, with a teaspoonful of vinegar over the eggs.

\section{1.-SNOW EGGS}

Take half a pint of milk and a little sugar, and flavor it with orange-flower water, or any other essence, and put it in a stewpan on the fire, having previously beaten up the whites of six eggs to a stiff froth ; if very hot weather, you must place the basin they are in on ice, or in cold water; whilst beating add some powdered sugar lightly; when the milk is boiling, take the white up with a tablespoon, and drop it, one table-spoonful at a time, into the stewpan to poach, keeping the shape of an egg, which turn over when set; when done, remove with a colander on to a sieve, and dress them in a crown on the dish you intend to serve them on; when all done, beat up the yolks of four of the eggs in a stewpan, with a little sugar, and a few drops of orange-flower water, pour part of the boiling milk out of the stewpan into it, sufficient to make a good stiff custard, put it on the fire until rather thick, and pour over the white, and serve either hot or cold: the last is preferable.

\section{2.-EGGS IN CASES.}

Cut up a sheet of paper into pieces of three inches square, turn up half an inch all round so as to form a kind of square case; there will then remain but two inches square in the inside. Take a small piece of butter, a pinch of fine bread crumbs, a little fine chopped parsley, spring onions, salt, and pepper, and 
mix them together, put a little into each case, then break one egg into each, put them on a gridiron over a slow fire, and do them gently, or place them in a dish in an oven; when well set, serve. Small round paper cases may be procured very cheap.

\section{3.-GARNITURE FOR OMELETTES.}

Asparagus, Peas, and Green Peas.Put in a stewpan three spoonfuls of plain boiled asparagus, that has previously been cut up, add to it half an ounce of butter, a little salt, pepper, and sugar, warm it on the fire, moving it continually, when warm, put it with a spoon in the centre of the omelette, turn over, and serve; the same with peas; rather thin melted butter or white sauce may be served round it.

Oysters.-Open and blanch delicately twelve middle-sized oysters, and put them in a stewpan with their own gravy, after having bearded them, add a tablespoonful of milk or cream, and give it a boil, then add half an ounce of butter, in wh.ch you have mixed a saltspoonful of flour, stir it in without breaking the oysters, put over the centre of your omelette, and proceed as before.

Lobster.-Cut half or a small one in thin slices, put four table-spoonfuls of melted butter in a stewpan, a few drops of essence of anchovies, and a little cayenne; put in your lobster, warm it well, and put it in the middle of the omelette, as above.

Mushroom.-Wash about ten small fresh mushrooms, cut in slices, put in a stewpan, with half an ounce of butter, a little salt, pepper, and the juice of a quarter of a lemon, simmer for a few minutes on the fire till tender; if too liquid, add a little flour, place in centre of omelette, and proceed as above.

Bacon.-Cut two ounces of good lean bacon in small dice, put in a pan to fry with a pat of butter for one minute, then mix with the eggs prepared as for omelette of herbs, omitting the salt, and cook the same way. This omelette is in great repute in France, especially among the epicures of the Barrières, outside of Paris. It prepares the palate to r.ceive with pleasure the cheap and acid wines sold there, which must have been invented by Bacchus when in a sour humor.

\section{TO MAKE BREAD.}

1424.

For a good baking, take two and a half pecks of wheat flour; put it into a kneading trough or earthen pan well glazed; make a hole in the centre of the flour and pour in a pint or more of good brewer's yeast, or still more of home-made,well mixed with a pint of milk-warm water. Stir in, with a spoon, flour enough to make a batter; sprinkle this with dry flour, and cover it entirely. Cover the whole lightly with a cloth, and set it by the fire. This is setting the sponge. When the batter has swelled and risen so as to form cracks in the flour covering it, scatter through it two table-spoonfuls of fine salt, and mix the flour with soft warm water, added by degrees. Work and mould the dough thoroughly, kneading it till smooth and light, and till not a particle will stick to the hands. Then sprinkle a mass of dough with flour, cover it with a warm cloth, and let it stand near the fire for an hour, or less in warm weather. It has now risen to its height. Divide it into seven loaves; mould and form them lightly on the paste-board, put them on floured tin or earthen plates, and place them in the oven.

With good, dry, hard wood, the oven will be heated in an hour. When the coals are spread over the bottom, the oven will be clear of smoke. Clean and 
sweep it, and throw in a little flour to see if it is too hot; if it burns black at once. let the oven cool a little. When the loaves are in, close the oven and keep it tight. The loaves should be cone in from an hour to an hour and a half.

When the loaves are baked, take them from the oven, and put them on a clean shelf, in a cool pantry, with a light cloth over them. If the crust is scorched, or the bread too much baked, wrap the loaves in a clean coarse towel, slightly damped.

\section{5.-BROWN, OR DYSPEPSIA BREAD.}

Take six quarts of wheat meal, rather coarsely ground, one teacup of good yeast, and half a teacup of molasses, mix these with a pint of milk-warm water and a teaspoonful of saleratus. Make a hole in the meal and stir this mixture in the middle till it is like batter. Then proceed as with fine flour bread. Make the dough, when sufficiently light, into four loaves, which will weigh two pounds per loaf when baked. It requires a hotter oven than fine flour bread, and must bake about an hour and a half.

\section{6.-RYE AND INDIAN BREAD.}

There are many different proportions in the mixing of this bread. Some put one-third Indian with two of rye ; others like one-third rye and two of Indian; others prefer it half and half.

If you use the largest proportion of rye meal, make your dough stiff; so that it will mould into loaves; when it is two-thirds Indian, it should be softer and baked in deep earthen or tin pans after the following rules :

Take four quarts of sifted Indian meal: put it into a glazed earthen pan, sprinkle over it a table-spoonful of fine salt; pour over it about two quarts of boiling water, stir and work it till every part of the meal is thoroughly wet; Indian meal absorbs a greater quantity of water. When it is about milk-warn, work in two quarts of rye meal, half a pint of lively yeast, mixed with a pint of warm water; add more warm water if needed. Work the mixture well with your hands : it should. be stiff, but not firm as flour dough. Have ready a large, deep, well buttered pan; put in the dough, and smooth the top by putting your hand in warm water, and then patting down the loaf. Set this to rise in a warm place in the winter; in the summer it should not be put by the fire. When it begins to crack, on the top, which will usually be in about an hour or an hour and a half, put it into a well heated oven, and bake it three or four hours. It is better to let it stand in the oven all night, unless the weather is warm. Indian meal requires to be well cooked. The loaf will weigh between seven and eight pounds.

There is another mode which many persons think preferable. Scald a quart of rye and another of Indian meal with a small quantity of boiling water. Boil a teaspoonful of salt in a pint and a half of milk, mix the rye and Indian together, and pour the milk over themadd half a pint of fresh yeast; but not before the meal is cooling. The mixture must be well kneaded and placed in a deep pan by the fire to rise. When it has risen sufficiently, take it out of the pan, make it into any shape you like, and put it into an oven well heated. If the fire is too brisk the crust will brown and the inside remain heavy.

Bake from two to three hours.

\section{7.-TO MAKE EXCELLENT BREAD WITHOUT YEAST.}

Scald about two handfuls of Indian meal ; into which put a little salt, and as much cold water as will make it rather warmer than new milk; then stir in wheat flour, till it is as thick as a family 
pudding, and set it down by the fire to rise. In about half an hour it generally grows thin; you may sprinkle a little fresh flour on the top, and mind to turn the pot round, that it may not bake at the side of it. In three or four hours, if you mind the above directions, it will rise and ferment as if you had set it with hop yeast; when it does, make it up in soft dough, flour a pan, put in your bread, set it before the fire, covered up, turn it round to make it equally warm, and in about half an hour it will be light enough to bake. It suits best to bake it in a Dutch oven, as it should be put into the oven as soon as it is light.

\section{8.-TO MAKE YEAST.}

The settlings of home-brewed beer in a clean cask, make good yeast. Common yeast is made thus:

Take two quarts of water, one handful of hops, two of wheat bran; boil these twenty minutes; strain off the water, and while it is boiling hot, stir in wheat or rye flour, to a thick batter: when this is milk-warm, add half a pint of good lively yeast and a large spoonful of molasses; stir well and set it away, in a cool place in summer and a warm one in winter. When light it is fit for use. When cold, it may be put in a clean jug or bottle; do not fill the vessel containing it, and leave the cork loose till next morning. Corked tightly, it will keep ten or twelve days in a cool place.

\section{9.-MILK YEAST}

To a pint of new milk put a teaspoonful of salt or a large spoonful of flour; stir well, and keep it lukewarm by the fire; in an hour it will be fit for use. Twice as much must be used as of common yeast; and the bread dries soon; and in summer this method is convenient.

Never keep yeast in a tin vessel. If sour, put in saleratus-a teaspoonful to a pint of yeast, when ready for use. If it foams up lively, it will raise the bread; if not, throw it away.

\section{0.-RICE BREAD.}

Boil soft a pint of rice; when cold, add a pint of leaven, and work in three quarts of flour. It will be light in an hour in warm weather. Divide the dough into three loaves, and bake in buttered tin pans. It grows dry soon.

\section{1.-YEAST CAKES.}

Boil three ounces hops in six quarts water, down to two quarts. Strain it, and while boiling hot, stir in wheat or rye meal to a batter. When milkwarm, add half a pint of good yeast, and let it stand till very light-about three hours. Then work in sifted Indian meal till it is stiff dough. Roll out and cut it in cakes three inches by two, half an inch thick. Lay these cakes on a smooth board, floured; prick them with a fork, and place them in a dry store room, exposed to sun and air. Turn them every day. They will be dry in a fortnight. When dry, put them into a coarse, cotton bag, and hang them in a cool, dry place. They will keep a year. Two will raise a peck of flour. Break them into a pint of milk-warm water, and stir in a spoonful of flour the evening before you bake; then set it in a moderately warm place till morning.

Bread made at home is not only more palatable, but far more economical. A stove will bake with little expense of fuel, and the flour used for a family will cost one-third less than bread bought. You are sure too, that good flour is used, and that your bread contains no alum, ammonia, or sulphate of zinc, which it is said bakers put in to make their bread light and white. Baker's bread is nearly tasteless, dries directly, and can not be as nutritious as bread baked at home.

Ladies keeping house in the city ought 
to make their own bread as well as cake, 'pare a pound of loaf-sugar finely powor at least superintend the process. It dered, a teaspoonful of mixed spice, mace, requires no sacrifice of time, and is a nutmeg and cinnamon, and three-quarters neat, delicate, and ladylike employment. of a pound of sifted flour. Take fourteen Three things, however, are requisiteyeast just lively enough, dough just light enough, and an oven just hot enough. Practice will show how to attain perfection in these respects.

\section{2.-POTATO BARM.}

Boil half an ounce of hops in four quarts of water, and leave it till the hops sink; then strain it into an earthen pot, and, when milk-warm, add three tablespoonfuls of sugar and half a pint of flour, leave it near the fire, frequently stirring it, for twenty-four hours. Then add four pounds potatoes pounded and pulped through a colander; they are best unboiled. When the mixture is well risen, half a pint will do for four quarts of flour. The bread requires much kneading, and should be left eight or ten hours to rise. A few spoonfuls of beer barm are an improvement to the stock, which should not be allowed to run out, as a little of the old will improve a new supply.

Brick ovens are the best for baking all kinds of bread, large cakes and pies; the reason of which is chiefly owing to their being generally capable of retaining the heat a much longer time than one built of iron.

For the baking of pastry, light bread, rolls, and breakfast-cakes, the iron ovens, fixed in the usual kitchen ranges, have the advantage of requiring but little fire, and enabling the cook to regulate its temperature.

\section{3.-ALMOND BREAD.}

Blanch, and pound in a mortar, half a pound of shelled sweet almonds till they are a smooth paste, adding rose-water as you pound them. They should be done the day before they are wanted. Preeggs, and separate the whites from the yolks. Leave out seven of the whites, and beat the other seven to a stiff froth. Beat the yolks till very thick and smooth, and then beat the sugar gradually into them, adding the spice. Next stir in the white of egg, then the flour, and lastly the almonds. You may add twelve drops of essence of lemon.

Put the mixture into a square tin pan, well buttered, or into a copper or tinturban mould, and set it immediately in a brisk oven. Ice it when cool. It is best when eaten fresh.

You may add a few bitter almonds to the sweet ones.

\section{4.-DAMASCUS BISCUITS.}

Take the whites of three eggs beaten to a froth, a quarter of a pound of good beef suet chopped very fine, and half an ounce of bitter almonds blanched, chopped fine, and beaten well with the froth of the eggs. Then take the yolks of the eggs, and mix with six ounces of sifted loaf sugar, beat well, pour into the mixture of almonds and whites of egg ; mix well, and shake in two ounces of flour, with sufficient lemon to flavor them. Pour into small tins, or moulded papers, and bake in a quich oven.

\section{5.-MILK BISCUITS.}

A quarter of a pound of butter, onequart of milk, one gill of yeast, as much flour as will form the dough, and a little salt. Stir flour into the milk, so as to form a very thick batter, and add the yeast; this is called a sponge. This should be done in the evening; in the morning cut up the butter, and set it near the fire, where it will dissolve, but not get hot; pour the melted butter into the sponge, then stir in enough flour to 
form a dough, knead it well and stand it away to rise. As soon as it is perfectly light, butter your tins, make out the dough in small cakes, and let them rise. When they are light, bake them in a very quick oven, and send them to the table hot.

1436.-ROLLS.

French Rolls are usually made by the bakers, but in country houses, where families bake their own bread, they may be done as follows:-

Sift one pound of flour, and rub into it two ounces of butter; mix in the whites only of three eggs beaten to a froth, and a table-spoonful of strong yeast ; to which add enough of milk, with a little salt, to make a stiff dough, and set it covered before the fire to rise-which will take about an hour; and, if cut into small rolls, and put into a quick oven, will be done in little more than ten minutes.

\section{7.-ROCKS.}

Take a loaf that is half baked, pull it apart, and with two forks tear the crumb into bits about the size of a walnut; lay them on a tin, and return them to the oven, and bake of a light brown. A loaf made for the purpose with milk and a little butter makes them nicer. A sweet cake pulled in this way is very good.

\section{8.-MUFFINS.}

Take two eggs, two spoonfuls of new yeast, and a little salt. Mix a little warm new milk and water into a quart of flour. Beat all well together, and let it stand to rise. Bake them for about twenty minutes, until of a light brown, either on a hot iron, or in shallow tin rings in a Dutch oven. When to be brought to table, toast them slightly on both sides, but not in the middle: then notch them round the centre, and pull them open with your fingers, without using a knife, and butter them.

\section{9.-CRUMPETS.}

Beat two eggs very well, put to them a quart of warm milk and water, and a large spoonful of yeast; beat in as much fine flour as will make them rather thicker than a common batter pudding; then make the stove hot, and rub it with a little butter wrapped in a clean linen cloth; pour a large spoonful of the batter upon the iron, and let it run within a ring to the size of a teasaucer; turn them with the elastic blade of an old tableknife, and when you want to use them, toast them very quickly, but not too crisply, and butter them.

\section{0.-SALLY-LUNN.}

Into one pint of flour rub a piece of butter the size of a walnut, a little salt, and a little yeast. . Let it rise half an hour before the fire. Then mix it with two eggs, and if not enough add a little milk; knead the dough well and let it stand some minutes before the fire; after which make this quantity into five cakes and have them slack-baked, as they must be well warmed in a Dutch oven before being toasted for table.

\section{1.-BUCKWHEAT CAKES.}

Mix a quart of buckwheat flour with a pint of lukewarm milk, (water will cio, but is not as good,) and a teacup of yeast - set it in a warm place to rise. When light, (which will be in the course of eight or ten hours if family yeast is used, if brewer's yeast is used they will rise much quicker,) add a teaspoonful of salt -if sour, the same quantity of saleratus dissolved in a little milk, and strained. If they are too thick, thin them with cold milk or water. Bake them with just iat enough to prevent their sticking to the griddle.

$$
\text { 1442.-RICE CAKES. }
$$

Boil a cupful of rice until it becomes 
soft; while it is warm mix a large lump of butter with it and a little salt. Add as much milk to a small teacupful of flour and a little corn meal, scalded, as will make a tolerably stiff batter-stir it until it is quite smooth and then mix it with the rice. Beat five eggs as light as possible and add them to the rice.

These cakes are fried on a griddle as all other pancakes-they must be carefully turned.

Serve them with powdered sugar and nutmeg. They should be served as hot as possible.

\section{3. - FLANNEL CAKES AND WAFFLES.}

Stir into two pints of flour as much milk as will make a light batter. Melt a large lump of butter and add with it a little salt. Beat together five eggs and stir them into the batter.

These cakes are to be baked on a griddle. Serve them with powdered sugar.

It is customary to mix with the butter a table-spoonful of yeast and leave it to rise for several hours; but this is unnecessary unless you wish to bake the cakes in waffle or wafer irons.

\section{4,-EGG BISCUIT.}

One pound of flour; wet nearly all of it to a paste with the whites of two eggs, beat and roll out thin, work in threequarters of a pound of butter, placing bits on the paste, flouring, folding, and rolling it out again till all is used. Move the rolling pin from you always. Cut out in small squares or with a tumbler, and bake in a quick oven.

\section{5.-APPLE BREAD.}

A very light pleasant bread is made in France by a mixture of apples and flour, in the proportion of one of the former to two of the latter. The usual quantity of yeast is employed as in making com- mon bread, and is beat with flour and warm pulp of the apples after they have boiled, and the dough is then considered as set : it is then put in a proper vessel, and allowed to rise for eight or twelve hours, and then baked in long loaves. Very little water is requisite; none, generally, if the apples are very fresh.

\section{6.-JOHNNY CAKES.}

Sift a quart of corn meal into a pan, make a hole in the middle, and pour in a pint of warm water. Mix the meal and water gradually into a batter, adding a teaspoonful of salt; beat it very quickly, and for a long time, till it becomes quite light; then spread it thick and even on a stout piece of smooth board; place it upright on the hearth before a clear fire, with something to support the board behind, and bake it well; cut it into squares, and split and butter them hot.

Cakes may also be made with a quart of milk, three eggs, one teaspoonful of carbonate of soda, and one teacupful of wheat-flour; add Indian meal sufficient to make a batter like that of pancakes, and either bake it in buttered pans, or upon a griddle, and eat them with butter.

\section{7.-INDIAN GRIDDLE UAKES}

Are made with one pint of Indian meal, four table-spoonfuls of flour, one quart of milk, four eggs, and salt to taste.

\section{8-TO MAKE RUSK.}

Take a piece of bread-dough large enough to fill a quart bowl, one teacup of melted butter, one egg, one teaspoonful of saleratus; knead quite hard, roll out thin, lap it together, roll to the thickness of a thin biscuit, cut out with a biscuit mould, and set it to rise in a warm place. From twenty to thirty minutes will generally be sufficient. Bake them and dry thoroughly through, and you 
will have an excellent rusk to eat with your coffee. You can make them with hop yeast, and sweeten them, too, if you please ; use milk with yeast.

\section{9.-BANNOCK OR INDIAN MEAT CAKES.}

Stir to a cream a pound and a quarter of brown sugar, a pound of butter-beat six eggs, and mix them with the sugar and butter-add a teaspoonful of cinnamon or ginger-stir in a pound and threequarters of white Indian meal, and a quarter of a pound of wheat flour, (the meal should be sifted.) Bake it in small cups, and let it remain in them till cold.

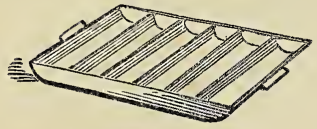

Corn Cake Pan.

1450.-RUSK.

Melt four ounces of butter in half a pint of new milk; then add to this seven eggs, well beaten, a quarter of a pint of yeast, and three ounces of sugar; put this mixture, by degrees, into as much flour as will make an extremely light paste, more like batter, and set it to rise before the fire for half an hour; then add more flour to make it rather stiffer, but not stiff. Work it well, and divide it into small loaves or cakes, about five or six inches wide, and flatten them. When baked and cold, slice them the thickness of rusks, and brown them a little in the oven.

\section{1-MUFFINS}

Mix a quart of wheat flour smoothly with a pint and a half of lukewarm milk, half a teacup of yeast, a couple of beaten eggs, a heaping teaspoonful of salt, and a couple of table-spoonfuls of lukewarm melted butter. Set the batter in a warm place to rise. When light, butter your muffin-cups, turn in the mixture, and bake the muffins till a light brown.

$$
\text { 1452.-SOFT WAFFLES. }
$$

Stir into a quart of flour sufficient lukewarm milk to make a thick batter. The milk should be stirred in gradually, so as to have it free from lumps. Put in a table-spoonful of melted butter, a couple of beaten eggs, a teaspoonful of salt, and half a teacup of yeast. When risen, fill your waffle-irons with the batter -bake them on a bed of coals. When they have been on the fire between two and three minutes, turn the waffle-irons over-when brown on both sides, they are sufficiently baked. The waffle-irons should be well greased with lard, and very hot, before each one is put in. The waffles should be buttered as soon as cooked. Serve them up with powdered white sugar and cinnamon.

For Rice Waffles.-Take a teacup and a half of boiled rice-warm it with a pint of milk, mix it smooth, then take it from the fire, stir in a pint of cold milk, and a teaspoonful of salt. Beat four eggs, and stir them in, together with sufficient flour to make a thick batter.

\section{3.-SHORT CAKES.}

Dissolve half a pound of fresh butter in as much milk as will make a pound and a half of flour into a paste, roll it out about a quarter of an inch thick, and cut it into large round cakes. Do them in a frying-pan, and serve them hot. They are eaten with butter.

\section{4.-WAFER CAKES.}

Wafer cakes are an excellent tea cake, and they do not take long to make, although a little practice is necessary before they can be successfully made.

Beat three eggs quite light. Wash a little less than a quarter of a pound of butter, to extract the salt from it, and mix it with a quarter of a pound of 
wifted sugar-add the beaten eggs, a teaspoonful of rose-water, and as much sifted flour as will make a thin batter. Stir the batter with a wooden spoon until it is perfectly smooth and so light as to break when it falls against the sides of the vessel. Your wafer-iron should be heated, but not too hot, or the butter will burn. Grease the iron with butter tied up in a linen rag, twice doubled. Fill the iron with the batter and close it. Place it in the fire in such a manner that both sides will heat at once; if this cannot be done turn the iron frequently. The batter will be cooked in about two minutes if properly managed.

You may sprinkle the wafer with pounded sugar and roll it over a smooth stick made for the purpose.

Southern biscuit is made with a large spoonful of lard to a pint of flour; add salt, knead the dough, and beat it well.

\section{5.-MILK TOAST.}

Boil a pint of rich milk, and then take it off and stir into it a quarter of a pound of fresh butter, mixed with a small tablespoonful of flour. Then let it again come to a boil. Have ready two deep plates with half a dozen slices of toast in each. Pour the milk over them hot, and keep them covered till they go to table. Milk toast is generally eaten at breakfast.

The warming of the bread gradually through, on both sides, is a very great improvement upon the quality of the toast.

All kinds of toast must be done the same way; but if to be served under a bird, eggs, or kidneys, it requires to be toasted drier.

Dry toast should not be made until quite ready to serve; when done, place it in a toast-rack, or upon its edges, one piece resting against another. Any kind of toast that has been made half an hour is not worth eating.

\section{6.-MILK ROLLS.}

Have a convenient-sized basin, intu which put half a pound of the best flour, making a hole in the middle; add half an ounce of yeast, one ounce of butter, a teaspoonful of powdered sugar, and half ditto of salt, over which pour three large wineglassfuls of lukewarm milk; mix the whole, by degrees, with your hand, (using a little more flour, ) until forming a stiffish paste; rub off the paste which adheres to your fingers, and form the whole into a ball, which leave at the bottom of the basin, covered over with a clean cloth, and set it half an hour to rise, in a warmish place, after which throw a little flour upon a dresser, cut the paste into pieces the size of eggs, mould them of a round, oval, or any other shape you may fancy, egg over with a paste-brush, and place them upon a baking-sheet, or upon the bottom of the oven if clean and not too hot; bake quickly.

Rolls are made with soda and cream of tartar ; a teaspoonful of the first to two of the latter. Or, with sour cream and soda, instead of yeast.

\section{7.-TOPS AND BOTTOMS.}

Make a dough as for rusks, but using only half the butter; have a deep-edged baking-sheet well buttered, and when the dough is ready, turn it on to a dresser, well floured; divide into small pieces the size of walnuts, which mould into round balls, and place close together upon the baking-sheet; put them in a warm place to rise, and bake well in a moderate oven. When cold, divide and cut each one in halves (making a top and bottom) which brown in the oven.

\section{8.-BUNS.}

Put three pounds of flour in an earth. en pan, make a hole in the middle, in which put some yeast, dissolved in three 
parts of a pint of warm water, and stir in a little of the flour, forming a thinnish batter; let it remain in a warm place nearly an hour, until well fermented; then add half a pound of sugar, a few currants, and half a pound of butter, dissolved in nearly a pint of warm milk; mix the whole well together, making a soft but dry dough; let it remain in a warm place until it rises very light, then turn it out of the pan on to a board; work it well with the hands, shaking flour over lightly; then mould it into small round balls, double the size of walnuts, which place upon a buttered baking-sheet, four inches apart; moisten the tops with milk; put them in a warm place to rise; not, however, permitting them to erack, and bake them in a hot oven.

\section{9.-SOYER'S BRIOCHE ROLLS.}

Put four pounds of flour upon a dresser, one pound of which put on one side, make a hole in the middle, into which pour nearly three parts of a pint of warm water, in which you have dissolved yeast; mix it into a stiff but delicate paste, which roll up into a ball: cut an incision across it, and lay it in a basin well floured, in a warm place, until becoming very light; then make a large hole in the centre of the three pounds of flour, into which put half an ounce of salt, two pounds of fresh butter, half a gill of water, and sixteen eggs; mix it into a softish flexible paste, which press out flat, lay the leaven upon it, folding it over and working with the hands until well amalgamated; flour a clean cloth, fold the paste in it and let it remain all night. In the morning mould them into small rolls; put them upon a bakingsheet, and bake in a moderate oven. Unless your breakfast party be very large, half the above quantity will be sufficient. These rolls being a luxury, I make them only upon very especial occasions.

\section{B UTTER.}

Mrs. Hale says: "Always to make good butter or cheese shows great care and excellent judgment in the farmer's wife. When every department of the dairy is kept perfectly neat, there is hardly any exhibition of woman's industry more likely to make her husband proud, or gratify a beholder of good sense and benevolence, than the sight of a neat dairy-room filled with the rich, valuable productions which her skill has fashioned from the milk of the cow.

"The secret of success in the dairy" is strict attention and scrupulous neatness in all its operations. The best time to make butter is in June, when the pastures are rich with clover, and September, when the fall feed is in its perfection. July and August are the months. for cheese; then the rich new milk and cream cheeses are made.

"To insure good butter, you must always scald your pails, \&c., in hot water, and then heat them by the fire, or in the hot sun, so that they may be perfectly sweet.

"Keep your cream in a cool place in summer, and churn twice a week.

"Work out all the buttermilk.-This must be done, or the butter will not keep well ; and do not make the butter too salt.

"Never put butter in a pine tub.

"Pickle for Butter.-Allow half a pound of salt, an ounce of saltpetre, and half a pound of sugar to three quarts of water; dissolve them together; scald and skim the pickle; let it be entirely cold, and then pour it over the butter

"Keep your cheese in a dark, cool room, and turn and rub them every day. The fat fried out of salt pork, is the best preservative to rub on cheese, and gives a rich color and smoothness to the rind. 
"Never wash your cheese shelves; but always wipe them clean with a dry cloth, when you turn your cheese.

"Do not heat the milk too hot; it should never, for new milk cheese, be more than blood-warm; be sure that your rennet is good, and do not use more than it requires to bring the curd.

"Cover the pan or tub in which milk is set to coagulate, and do not disturb it for half an hour or more.

"Cut the curd, when fully formed, carefully with a knife; never break it with your hand; and be very particular, wher draining it from the whey, not to squeeze or handle the curd; if you make the white whey run from the curd, you lose much of the richness of the cheese."

\section{0.-TO CURE BUTTER IN THE BEST MANNER.}

The following receipt is from "The Housewife's Manual," a work said to have been prepared by Sir Walter Scott.

Having washed and beaten the butter free of buttermilk, work it quickly up, allowing a scanty half ounce of fine salt to the pound. Let the butter lie for twenty-four hours, or more; then, for every pound, allow a half ounce of the following mixture:-Take four ounces of salt, two of loaf sugar, and a quarter of an ounce of saltpetre. Beat them all well together, and work the mixture thoroughly into the butter; then pack it down in jars or tubs. Instead of strewing a layer of salt on the top of the butter, which makes the first slice unfit for use, place a layer of the above mixture in folds of thin muslin, stitch it loosely, and lay this neatly over the top, which will effectually preserve it.

To freshen salt butter.-Ohurn it anew in sweet milk, a quart to the pound. The butter will gain in weight.

To improve rancid butter.-Wash it, melt it gradually, skim it, and put to it a slice of charred or hard toasted bread, or some bits of charcoal.

\section{1.-TO MAKE BUTTER.}

The milk pans should be scalded * every day. After the milk has stood twenty-four hours skim off the cream, and deposit it in a large earthen jar kept closely covered. Stir up the cream with a stick every day to prevent the skin from gathering. Butter of only two or three days is best. Strain the cream from the jar into the churn and put on the lid. In warm weather move the handle slowly, or the butter will be too soft. When the handle moves with great difficulty the butter has come. Take it out with a wooden ladle and squeeze out the remains of milk. Add a little salt and work it well. Set it in a cool place for three hours, then work it again. Wash it in cold water, and put it away for use.

\section{2.-BUTTER-TO CLARIFY.}

Scrape off the outsides of the butter you may require, and then put it into a stewpan by the side of a slow fire, where it must remain till the scum rises to the top and the milk settles at the bottom; carefully with a spoon take off the scum; when clear, it is fit for use.

To preserve it for winter, take two parts of the best common salt, one part of good loaf sugar, and one part saltpetre, beat them well together; to sixteen ounces of butter thoroughly cleansed from the milk put one ounce of the above composition, work it well, and put it into pots when quite firm and cold.

\section{CAKES, \&C.}

In making cakes it is indispensably necessary that all the ingredients should be heated before they are mixed together, 
for which purpose every thing should be prepared for an hour or two previously to their being wanted, and placed near the fire or upon a stove-the flour thoroughly dried and warmed; the cur-

"rants, sugar, carraway-seed, and any thing else required, heated in the same way; butter and eggs should be beaten in basins fitted into kettles or pans of warm water, which will give them the requisite degree of temperature. Without these precautions cakes will be heavy, and the best materials, with the greatest pains, will fail to produce the desired results.

Currants should be very nicely washed, dried in a cloth, and then set before the fire. If damp, they will make cakes or puddings heavy. Before they are to be used, a dust of dry flour should be thrown among them, and a shake given to them, which causes the cakes to be lighter.

Eggs should be very long beaten, whites and yolks apart, and always strained.

Sugar should be pounded in a mortar, or rubbed to a powder on a clean board, and sifted through a very fine hair or lawn sieve.

Lemon-peel should be grated or pared very thin, and, with a little sugar, beaten in a marble mortar to a paste ; and then mixed with a little wine or cream, so as to divide easily among the other ingredients. The pans should be of earthenware; nor should eggs or batter and sugar be beaten in tins, as the coldness of the metal will prevent them from becoming light.

Use no flour but the best superfine; for if the flour be of inferior quality the cakes will be heavy, ill-colored, and unfit, to eat; but if a little potato-flour be added, it will improve their lightness. Cakes are frequently rendered hard, heavy, and uneatable, by misplaced economy in eggs and butter, or for want of a due seasoning in spice and sugar.

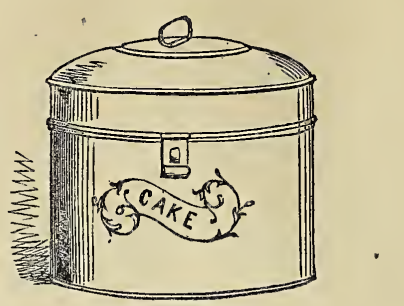

After all the articles are put into the pan, they should be thoroughly and long beaten, as the lightness of the cake depends much on their being well incorporated.

Accuracy in proportioning the ingredients is also indispensable; and therefore scales, weights, and measures, down to the smallest quantity, are of the utmost importance.

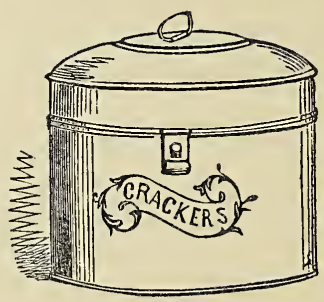

Iron ovens are desirable, as well for their economy of fuel as for the short time in which they can be heated, and on account of the celerity and convenience with which one person may prepare large quantities of small pastry, and bake it also.

The most vigilant care and attention are necessary in order to secure success in the baking of delicate pastry or cakes ; for however well they may have been prepared, if not equally well baked, the result will be unsatisfactory.

If the oven be not lighted long enough to have a body of heat, or if it is become slack, the cake will be heavy. If not pretty quick, the batter will not rise. 
Should you fear its being too quick, put some paper over the cake to prevent its being burnt. To know when it is soaked, take a broad-bladed knife that is very bright, and plunge it into the very centre, draw it instantly out, and if the least stickiness adheres, put the cake immediately in again, and shut up the oven.

If the heat should be sufficient to raise, but not to soak it, let fresh fuel be quickly put in, and the cakes kept hot until the oven is fit to finish the soaking.

Bread and cakes mixed with milk eat best when new, but become stale sooner than others.

Cakes kept in drawers or wooden boxes have a disagreeable taste. Earthen pans and covers, or tin boxes, preserve them best; but in making large cakes of any kind, which are to be baked in hoops, use those of wood. Cakes made with yeast should always be eaten fresh.

\section{3.-PLUM CAKE (English).}

Mix thoroughly two quarts of fine flour, well dried, with one pound of dry and sifted loaf-sugar, three pounds of currants washed and very dry, half a pound of raisins stoned and chopped, one-quarter of an ounce each of mace, cloves, and cinnamon, a grated nutmeg, the peel of a lemon cut as fine as possible, and half a pound of almonds, blanched and beaten with orange-flower water. Melt two pounds of butter in one and a quarter pint of cream, but not hot; put to it one pint of sweet wine, a glass of brandy and another of rose-water, with the whites and yolks of twelve eggs beaten apart, and half a pint of good jeast. Strain this liquor by degrees into the dry ingredients, beating them together a full hour; then butter the hoop or pan, and bake it in a moderate oven $f_{\text {or }}$ at least four hours. As you put in the batter, throw in plenty of citron, lemon, and orange candy.
If you ice the cake, when it is nearly cold pour the icing over, and return it to the oven, where it should be left all night, or until it becomes cold; but if the oven be warm, keep it near the mouth, and the door open, lest the color be spoiled; or the icing may be left until the next morning.

1464.-PLUM NEW ENGLAND WEDDING CAKE.

One pound of dry flour, one pound of sweet butter, one pound of sugar, twelve eggs, two pounds of raisins, (the sultana raisins are the best,) two pounds of currants, as much spice as you please. A glass of wine, one of brandy, and a pound of citron. Mix the butter and sugar as for pound cake. Sift the spice, and beat the eggs very light. Put in the fruit last, stirring it in gradually. It should be well floured. If necessary add more flour after the fruit is in. Butter sheets of paper, and line the inside of one large pan, or two smaller ones. Lay in some slices of citron, then a layer of the mixture, then of the citron, and so on till the pan is full. This cake requires a tolerably hot and steady oven, and will need baking four or five hours, according to its thickness. It will be better to let it cool gradually in the oven. Ice it when thoroughly cold.

\section{5.-FROSTING CAKES.}

Allow for the white of one egg nine heaping teaspoonfuls of double-refined sugar, and one of nice starch. The sugar and starch should be pounded and sifted through a very fine sieve. Beat the whites of eggs to a stiff froth, so that you can turn the plate upside down, without the eggs falling from itthen stir in the sugar gradually with a wooden spoon-stir it ten or fifteen minutes without any cessation-then add a teaspoonful of lemon juice, (vinegar will answer, but is not as nice,)-put in 
sufficient rose-water to flavor it. If you wish to color it pink, stir in a few grains of cochineal powder, or rose pink ; if you wish to have it of a blue tinge, add a little of what is called powder blue. Lay the frosting on the cake with a knife, soon after it is taken from the ovensmooth it over, and let it remain in a cool place till hard. 'To frost a common-sized loaf of cake, allow the white of one egg, and half of another.

\section{6.-SMALL PLUM CAKE.}

One pound and a half of raisins, one pound and a half of currants, half a pound of butter, half a pound of flour, five eggs, half a teacup of molasses, half a pound of sugar, half a pound of citron, one gill of brandy, spice to taste, three nutmegs.

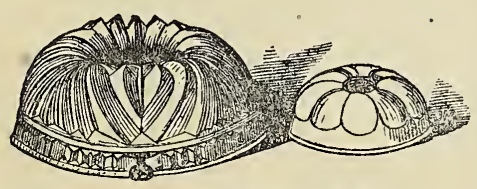

Cake Mould, with Cylinder.

\section{GERMAN SUGAR CAKES.}

Blend well with the fingers six ounces of good butter, with a pound of fine flour, working it quite into crumbs; add a few grains of salt, one pound of dry sifted sugar, a table-spoonful of the best cinnamon in very fine powder, and a large teaspoonful of spices: to these the grated rinds of three sound fresh lemons can be added, or not, at pleasure. Make these ingredients into a paste, with the yolks of five eggs, and about four tablespoonfuls of white wine, or with one or two more in addition, if required, as this must be regulated by the size of the eggs : half of very thick cream and half of wine are sometimes used for them. Roll the mixture into balls, flatten them to something less than three-quarters of an inch thick, and bake then in a mod- erate oven from fifteen to twenty minutes. Loosen them from the bakingsheets-which should be lightly floured before they are laid on-by passing a knife under them, turn them over, and when they are quite cold, stow them in a dry, close-shutting canister. The Germans make three incisions on the top of each cake with the point of a knife, and lay spikes of split almonds in them.

1468.-BRIDE CAKE.

Wash two pounds and a half of fresh butter in plain water first, and then in rose-water; beat the butter to a cream; beat twenty eggs, yolks and whites separately, half an hour each. Have ready two pounds and a half of the finest flour, well dried and kept hot, likewise one pound and a half of sugar pounded and sifted, one ounce of spice in fine powder, three pounds of currants nicely cleaned and dry, half a pound of almonds blanch$\mathrm{ed}$, and three-quarters of a pound of sweetmeats cut, not too thin. Let all be kept by the fire, mix all the dry ingredients, pour the eggs strained to the butter, but beat the whites of the eggs to a strong froth; mix half a pint of sweet wine with the same quantity of brandy, pour it to the butter and eggs, mix well, then have all the dry things put in by degrees; beat them very thoroughly-you can hardly do it too much. Having half a pound of stoned jar-raisins chopped as fine as possible, mix them carefully, so that there should be no lump, and add a teacupful of orange-flower water; beat the ingredients together a full hour at least. Have a hoop well buttered; take a white paper, doubled and buttered, and put in the pan round the edge; do not fill it more than three parts with batter, as space should be allowed for rising. Bake in a quick oven. It will require full three hours. In making cakes of a larger size, put at the rate of eight eggrs to every pound of 
flour, and other ingredients in the same proportion. icing.

The cake must be covered with an

\section{9.-QUEEN CAKE.}

Mix one pound of dried flour, the same of sifted sugar and of washed currants. Wash one pound of butter in rose-water, beat it well, then mix with it eight eggs, yolks and whites beaten separately, and put in the dry ingredients by degrees; beat the whole an hour ; butter little tins, teacups, or saucers, filling them only half full. Sift a little fine sugar over just as you put them into the oven.

\section{0.-PLAIN FRUIT CAKE.}

One loaf of bread, half a cup of butter, and half a cupful of sugar, half do. of molasses, half do. of buttermilk, half do. of currants, one do. of raisins, half do. citron, three eggs, one teaspoonful saleratus. Season to taste; brandy if you choose.

\section{1. -ALMOND CAKE.}

Blanch half a pound of sweet, and three ounces of bitter almonds-pound them to a paste in a mortar with orangeflower water-add half a pound of sifted loaf sugar, and a little brandy-whisk separately, for half an hour, the whites and yolks of twenty eggs, and the yolks to the almonds and sugar, and then stir in the whites, and beat them all well together. Butter a tin pan, sift breadraspings over it, put the cake into it, over the top of which strew sifted loafsugar. Bake it in a quick oven for half or three-quarters of an hour.

\section{2.-HONEY CAKE.}

One pound and a half of dried and sifted flour, three-quarters of a pound of honey, half a pound of finely pounded loaf sugar, a quarter of a pound of citron, and half an ounce of orange-peel cut small ; of pounded ginger and cinnamon three-quarters of an ounce. Melt the sugar with the honey, and mix in the other ingredients; roll out the paste, and cut it into small cakes of any form.

\section{3.-COCOA-NU'T CAKES.}

Take equal weights of grated cocoanut and powdered white sugar, (the brown part of the cocoa-nut should be cut off before grating it,) -add the whites of eggs beaten to a stiff froth in the proportion of half a dozen to a pound each of cocoa-nut and sugar. There should be just eggs enough to wet up . the whole stiff. Drop the mixture on buttered plates, in parcels of the size of a cent, several inches apart. Bake them immediately in a moderately warm oven.

\section{4.-SAVOY CAKES.}

Beat eight eggs to a froth-the whites and yolks should be beaten separately, then mixed together, and a pound of powdered white sugar stirred in gradually. Beat the whole well together, for eight or ten minutes, then add the grated rind of a fresh lemon, and half the juice, a pound of sifted flour, a couple of tablespoonfuls of coriander seed. Drop this mixture by the large spoonful on buttered baking plates, several inches apart, sift white sugar over them, and bake them immediately in a quick oven.

\section{5.-SPONGE CAKE.}

Take the weight of ten eggs in powdered loaf sugar, beat it to a froth with the yolks of twelve egogs, put in the grated rind of a fresh lemon, leaving out the white part-add half the juice. Beat the whites of twelve eggs to a stiff froth, and mix them with the sugar and butter. Stir the whole without any cessation for fifteen minutes, then stir in gradually 
the weight of six eggs in sifted flour. As soon as the flour is well mixed in, turn the cake into pans lined with buttered paper-bake it immediately in a quick, but not a furiously hot oven. It will bake in the course of twenty minutes. If it bakes too fast, cover it with thick paper.

\section{6.-POUND CAKE.}

Mix a pound of sugar with a pound of butter. When worked white, stir in the yolk of eight eggs, beaten to a froth, then the whites. Add a pound of sifted flour, and mace or nutmeg to the taste. If you wish to have your cake particularly nice, stir in, just before you put it into the pans, a quarter of a pound of citron, or almonds blanched and powdered fine in rose-water, and a glass of brandy.

\section{7.-JUMBLES.}

Stir together, till of a light color, a pound of sugar and half the weight of butter-then and eight eggs, beaten to a froth, essence of lemon, or rose-water, to the taste, and flour to make them sufficiently stiff to roll out. Roll them out in powdered sugar, about half an inch thick, cut it into strips about half an inch wide, and four inches long, join the ends together, so as to form rings, lay them on flat tins that have been buttered and bake them in a quick oven.

\section{8. -NEW-YEAR'S COOKIES.}

Weigh out a pound of sugar, threequarters of a pound of butter-stir them to a cream, then add three beaten eggs, a grated nutmeg, two table-spoonfuls of caraway seed, and a pint of flour. Dissolve a teaspoonful of saleratus in a teacup of milk, strain and mix it with half a teacup of cider, and stir it into the cookies-then add flour to make them sufficiently stiff to roll out. Bake them as soon as cut into cakes, in a quick oven, till a light brown.

\section{9.-NEW YORK CUP CAKE.}

One cup butter, two of sugar, three of flour, one of milk or cream; four eggs, a wine-glass of brandy-a nutmeg grated, a teaspoonful of soda, two of cream of tartar. Dissolve the soda in the milk rub the cream of tartar dry in the flour.

\section{0.-WASHINGTON CAKE.}

One and three-quarter pounds flour, one and a quarter sugar, three-quarters of a pound of butter, four eggs, two teaspoonfuls of cream of tartar, one of soda, half a pint of milk, two pounds raisins, two glasses of brandy, one nutmeg, spice to taste.

\section{1.-GINGER WAFERS.}

Three cups sugar, two of butter, one of milk, three table-spoonfuls of ginger, two teaspoonfuls of soda in the milk; flour enough to make it a dough. Roll it very thin, and bake in cakes.

\section{2.-INDIAN CORN CAKE.}

One pint of milk, half a pound Indian-meal, two eggs; two ounces butter, half a teaspoonful of soda, a whole one of salt. Bake three-quarters of an hour.

1483.-SOYER'S ST. JAMES'S CAKE.

Put one pound of very fresh butter in a good-sized kitchen basin, and with the right hand work it up well till it forms quite a white cream; then add one pound powdered sugar, mix well, add ten eggs by degrees; put to dry a pound and a quarter of flour, which mix as lightly as possible with it; blanch and cut in slices two ounces of pistachios, two ditto of green preserved angelica, add two liquor glasses of noyeau, two drops of essence of vanilla ; whip a gill and a half of cream till very thick, mix lightly with a wooden spoon, havea mould, put it in, and send to the baker; it will 
take about one hour and a quarter to bake.

\section{4.-SOYER'S TURBAN OF ALMOND} CAKE ICED.

This is a very good and useful second course remove. Make half a pound of puff paste, give it nine rolls, rolling it the last time to the thickness of a pennypiece, have ready blanched and chopped a pound of sweet almonds, which put in a basin with half a pound of powdered sugar and the whites of two eggs, or little more if required; spread it over the paste the thickness of a shilling, and with a knife cut the paste into pieces two inches and a half in length and nearly one in breadth, place them upon a baking-sheet, and bake nicely a very light brown color, in a moderate oven; dress them on a stiff border of any kind of stiff jam or marmalade, so as to form a large crown, according to the size you require it; then fill the interior with vanilla cream, or any other, iced, but not too hard, and bring it up to a point; the cake may be cut into any shape you fancy, but never make them too large.

\section{5.-CIDER CAKE.}

Cider cake is very good, to be baked in small loaves. Orie pound and a half of flour, half a pound of sugar, quarter of a pound of butter, half a pint of cider, one teaspoonful of pearlash; spice to your taste. Bake till it turns easily in the pans. I should think about half an hour.

\section{6.-THE HOUSEWIFE'S CHRISTMAS CAKE.}

Take two pounds of pounded sugarcandy, two pounds of flour, two pounds of butter, thirty-six eggs, four pounds of currants, a pound of raisins stoned and chopped, half a pound of almonds blanched and chopped, half a pound of citron, a pound of candied orange-peel, the same of candied lemon-peel, a large nutmeg pounded, half an ounce of powdered allspice, half an ounce of powdered mace, ginger, cinnamon, and coriander, and half a pint of brandy.

All the ingredients should be well dried, the white of the eggs well beaten up separately from the yolks, the butter stirred and beaten aliaost to a cream; then add the rest gradually, taking care they are well beaten and mixed. Have ready a large tin, well lined with buttered paper, pour in the cake, and bake in a slow oven for at least four hours. Smaller proportions may be adopted.

\section{7.-CORNSTARCH CAKE.}

Half a pound of sugar, four ounces of butter, five eggs, one teaspoonful cream of tartar, half a teaspoonful of soda, half a pound of cornstarch. half a gill of sweet milk.

\section{8.-SHORT CAKE}

Three pounds of flour, half a pound of butter, half a pound of lard, a teaspoonful of soda, two of cream of tartar; mix with cold milk.

For Strawberry Cake, open these when first baked, take out some of the crumb, and fill the inside with ripe strawberries, sugared; close and bake the cakes five minutes longer.

\section{9.-SUGAR CAKE.}

Three pints of flour, a table-spoonful of butter. half a pound of sugar, one egg, half a teaspoonful of soda, a teaspoonful of cream of tartar, milk enough to make a dough, roll it thin and cut in squares.

\section{0.-RAILROAD CAKE.}

A pint of flour, three eggs, a teaspoonful of cream of tartar, half a teaspoonful of soda, a table-spoonful of butter, a teaspoonful of sugar; bake the batter in a square pan twenty minutes. 
1491.-JELLY CAKE.

Bake sponge cake or cup cake in circular pans, half an inch thick; when cold, put pulp of fruit or marinalade between them; six or eight layers of each.

\section{2.--LADY CAKE.}

One pound of flour, one of loaf sugar, half one of butter, the whites of twelve eggs, almonds pounded, rose-water.

\section{3.-CHOCOLATE CAKE.}

Grate chocolate; add whites of six eggs and loaf sugar; drop it on buttered paper, and bake as macaroons.

\section{4.-NOURMAHAL CAKE.}

Cut four slices of sponge cake about an inch thick and of an oval shape, but each slice smaller than the others. Spread a thick layer of apricot jam upon the first and largest slice, and then lay the next sized slice upon it; spread the second slice with apple marmalade, and cover with the third size, which is to be spread in like manner with strawberry jam, and covered with the smallest size. Press the top lightly with the hand, and with a sharp knife cut away the central part, so as to leave a wall about two inches and a half thick, which is to be trimmed outside. Mash up the part removed from the centre, with equal parts of white wine and brandy, sufficient to flavor, and stir in some thick custard, then pour into the center of the cake. Whip the whites of two eggs into a stiff froth, pour over the whole, heaping it well up in the centre, and shake sifted sugar thickly on, then place in a quick oven until the frosting is set. A few pieces of strawberry jam or any other preserve placed round the bottom of the dish, gives a finish to the whole.

1495.-ICING FOR CAKES.

Beat the whites of four eggs to a solid froth, add gradually three-quarters of a pound of refined sugar pounded and sifted; mix in the juice of half a lemon; beat it till very light and white. The cake should be cold. . Place it before the fire, pour over it the icing, and smooth over the top and sides with a knife. It might be set to dry at the mouth of a cool oven.

\section{6.-LEMON CAKES.}

Rub half a pound of butter into one pound of flour and a pound of powdered lump sugar, the rind and juice of one lemon, three eggs, leaving out the whites unless the butter be very hard. Mix all together, and drop on tins in small cakes.

\section{7.-CINNAMON BISCUITS.}

Half a pound of dry flour, one pound of lump sugar, finely sifted; one pound of butter, powdered cinnamon to judgment. The whole to be mixed with a glass of brandy, or rum, then rolled very thin, and baked in a quick oven.

\section{8.-DIET BREAD.}

To half a pound of sifted sugar put four eggs; beat them together for an hour; then add a quarter of a pound of flour dried and sifted, with the juice of half a lemon and the grated rind of a whole one. Bake it in a slow oven.

\section{9.-CURRANT CAKE.}

Beat a pound of fresh butter to a cream; take one pound and a quarter of sugar, and one and a quarter pounds of currants washed and picked, and beat up the whites and yolks of eight eggs; put in the sugar by degrees, then a pound o ${ }^{\circ}$ flour and currants; add a gill of brandy, some candied orange and citron; beat the mixture till very light, and bake it in a pan.

1500.-SEED CAKES.

Beat one pound of butter to a cream, 
adding, gradually, a quarter of a pound of sifted sugar, beating both together. Have ready the yolks of eighteen eggs, and the whites of ten, beaten separately; mix in the whites first and then the yolks, and beat the whole for ten minutes; add two grated nutmegs, one pound and a half of flour, and mix them very gradually with the other ingredients. When the oven is ready, beat in three ounces of picked carraway-seed. To two pounds of flour add two ounces of butter rubbed in, half a pound of sugar, one ounce of carraway-seed, half an ounse of ground coriander-seed, half a teaspoonful of carbonate of soda, and a table-spoonful of arrowroot; $\operatorname{mix}$ the whole well together, and make a stiff paste with warm milk; cut into thin cakes, and prick over with a fork; bake slowly.

\section{1.-PITCAITHLY BANNOCK.}

Dry before the fire one pound fine flour; then melt half a pound of butter; then mix with the flour two ounces of almonds thinly sticed; two ounces of orange peel, two ounces of sugared carraways, two ounces pounded sugar, then pour on these ingredients the melted butter; knead all together well; put the bannock in a slow oven, and bake it an hour.

\section{2.-TO MAKE BANBURY CAKES.}

Roll out the paste about half an inch thick, and cut it into pieces, then roll again till each piece becomes twice the size; put some Banbury meat in the middle of one side, fold the other over it, and pinch it up into a somewhat oval shape, flatten it with your hand at the top, letting the seam be quite at the bottom, rub the tops over with the white of an egg laid on with a brush, and dust loaf-sugar over them. Bake in a moderate oven. The meat for this cake is made thus:-beat up a quarter of a pound of butter until it becomes in the state of cream, then mix with it half a pound of candied orange and lemon-peel cut fine, one pound of currants, a quarter of an ounce of ground cinnamon, and a quarter of an ounce of allspice; mix all well together, and keep in a jar, till wanted for use.

\section{3.-PETS DE NONNE.}

Put water or cream in a stewpan with sugar, a little "salt, and flavoring of orange-flower water; when near boiling shake flour in with one hand, and stir with the other, till the paste is thin and well done ; take it off the fire, and break an egg in ; turn and mix, adding a second egg; add eggs till the paste is thick and soft, stirring and beating it. Have butter boiling hot in a frying pan, drop in the paste by spoonfuls, let each be the size of a walnut, take them out, and keep them hot till all are done; sift sugar over them, or serve sweet sauce of wine, butter, sugar, and grated nutmeg.

\section{4.-BISCUITS.}

Those of the common sort are made in various ways. For a large quantity beat up eight eggs during nearly half an hour, then put in one pound of white powdered sugar, with the grated peel of a lemon, and whisk that again for the same time, or until it appears well mixed; after which make it into a batter with one pound of flour, and a little rose-water.

Bake the dough in tins, or on papers, and either sugar them or put in carraways at your pleasure.

Or :-One pound of flour, half a pound of loaf-sugar, and a quarter of a pound of butter; rub all together; wet it with one egg beaten up in half a teacupful of milk, and roll it out thin.

\section{5.-GOLD AND SILVER CAKE}

Is made by using the whites of the eggs 
for the latter, and the yolks for the first. The other proportions like pound cake.

\section{6.-FRANGIPANE}

Is a French paste, made with two or three eggs put into a saucepan with one tablespoonful of flour, moistened with a little milk, and placed on the fire for about ten minutes; taking care to stir the materials continually, to prevent them from sticking to the pan. The substance may either be used as a paste for tartlets, or eaten as cakes, if made with sugar and macaroons.

\section{7.-IA GALETTE}

Is in great favor among the Parisians, who usually eat it with sweetmeats, but vary it in point of richness by the greater or less quantity of cream and butter employed in making it up. It is thus made:-

Take one pound each of butter and flour, a little salt, and two eggs ; knead the whole together into a paste, roll it to not more than an inch in thickness, and make it the size of a dessert-plate. Then put it into the oven for a quarter of an hour; take it out; beat up two eggs with a little cream and some salt; pour it over the cake, and return it to the oven to bake for another quarter of an hour; or, if you please, ornament the back with candied citron.

\section{8.-ISLE OF WIGHT CRACKNELS.}

Mix with a quart of fiour half a nutmeg grated, the yolks of four eggs beaten, with four spoonfuls of rose-water, into a stiff paste, with cold water ; then roll in one pound of butter, and make them into a cracknel shape; put them into a kettle of boiling water, and boil them till they swim; then take them out and put them into cold water; when hardened, lay them out to dry, and bake them on tin plates.

\section{9.-CRACKNELS.}

Half a pound of flour, half a pound of sugar, a quarter of a pound of butter two table-spoonfuls of rose-water, a little salt and rice, and eggs well beaten. Mix together twenty minutes; roll and cut into shapes. Rub a baking sheet with butter, and when baked, wash them with white of egg.

\section{0.-FRENCH BREAD.}

Commence as for common bread, but use milk instead of water. When it is light, add two quarts warm milk, salt half a pound of butter, and one ounce sifted loaf sugar; knead well and let it rise again. Make it into rolls ; put them in a warm place half an hour, then bake in a quick oven.

\section{1.-KRINGLES}

Beat well the yolks of eight and whites of two eggs, and mix with four ounces of butter just warmed, and with this knead one pound of flour and four ounces of sugar to a paste. Roll into thick biscuits; prick them, and bake on tin plates.

\section{G I N G E R B R E A D.}

This is amongst the most ancient species of cake known throughout England and the north of Europe. It is the delight of children; in Holland it is the common accompaniment of the "schnaps," and in Ghent there are shops famous for it. The following are selected from amongst the numerous ways of "making it in this country.

1512.-SOFT GINGERBREAD (from a Lady of New York).

Three cups molasses, one of butter, one of milk, ginger, a table-spoonful of saleratus, and flour enough to make it the stiffness of pound cake.

Another:-Half a pound of butter, a 
quart of molasses, eight eggs, four tablespoonfuls sifted ginger, two teaspoonfuls saleratus, allspice, and flour enough to make it stiff as pound cake.

\section{3.-HARD GINGERBREAD.}

Rub half a pound of butter into one pound of flour; then rub in half a pound of sugar, two table-spoonfuls of ginger, and a spoonful of rose-water; work it well; roll out, and bake in flat pans in a moderate oven. It will take about half an hour to bake. This gingerbread wil! keep good some time.

\section{4.-GINGERBREAD-NUTS.}

Mix half a pound of flour, the same quantity of butter and of brown sugar, with three ounces of ginger, a teaspoonful of carbonate of soda rubbed in the flour, with as much molasses as will make it into a paste. Roll it out thin, and bake it for about twenty minutes in a slow oven.

\section{5.-PASSOVER CAKES.}

Make a stiff paste with biscuit-powder, milk, and water; add a little butter, the yolk of an egg, and a little white sugar. Cut into pieces, mould with the hand, and bake in a brisk oven: they should not be too thin.

\section{SCOTCH SHORT-BREAD.}

To four pounds of flour take two of butter, half a pound of moist sugar, quarter of a pound of citron, and the same of almonds, the latter blanched, and all cut small. Mix the sugar and fruit well with the flour, then work it up with the butter, cold, till it is smooth and compact, but it will take a long time; divide into four or more pieces, and roll out each piece into an oblong cake, having previously floured the table well. Prick the top with a steel fork, strew some orange and citron cut thin, and some large caraway-comfits, press them in with the roller, then pinch round the edges, put the cakes in floured tins; bake for twenty minutes. If the cake rise after the pressing of the finger, it is sufficiently done.

Or:-One pound each of butter and sifted sugar, two pounds of flour, eight ounces of sweet and four ounces of bitter almonds, a small piece of cinnamon, all pounded, and a glass of brandy. Soften the butter before the fire, then rub in the flour, and make it into a paste ; roll it out half an inch thick, and bake it on a tin in a slow oven, as it should be of a pale color.

\section{7.-POTATO CHEESECAKES.}

Boil six ounces of potatoes, and four of lemon-peel, beat the latter in a marble mortar with a quarter of a pound of sugar, then add the same quantity of butter, melted in a little cream, and the potatoes beaten: mix well, and let it stand to cool ; put crust in patty-pans, rather more than half fill them, sift some sugar over, and bake half an hour in a quick oven.

Tipsy Cake.-Put a sponge-cake into a deep glass dish, pour round it a glass of brandy and some raisin-wine, and let it soak it up; then strew sifted sugar over it, pour a thick custard into the dish, and ornament the top with blanched almonds split and put thick.

\section{8.-DOUGHNUTS.}

Set a sponge with a pound of flour, milk and good yeast; rub nearly half a pound of butter into another pound of flour, and add half a pound of sugar, with grated nutmeg and spice, and mix with it three beaten eggs; mix it with the light sponge, and more milk if necessary, and set it to rise. When it is perfectly light, roll out the dongh on a pasteboard, cut it into slips, double and 
twist them up, and fry them in boiling lard.

For Crullers-Allow to the flour more butter and sugar, and six eggs, without yeast, and fry as before, making smaller cakes.

\section{9.-BOSTON CREAM CAKES.}

Take half a pint of cream from a quart of milk, and boil it with vanilla and cinnamon; strain and mix it with the rest of the milk; when cold, add the yolks of twelve eggs and a quarter of a pound of loaf-sugar, and bake the custard in a bain-marie; have ready a batter made of half a pound of flour and the same of butter, in a pint of milk, with eight eggs, and bake it in buttered deep patty-pans, not scolloped; when browned, open a slit in the side of each while hot, and put in with a spoon as much of the custard as it will hold; close the slit smoothly, and set the cakes away.

\section{0.-SPANISH PUFFS.}

Boil three-quarters of a pint of milk with one ounce of butter, six ounces of flour, mixed well in the hot milk-flavor with vanilla. Drop about the size of a walnut, and lightly bake. When done, open gently at the side and fill with preserve.

\section{1.-ALMACK.}

Plums, pears, and apples, each two pounds; pare, core, and stew togetherrub through a sieve. Add half a pound of moist sugar; boil until it is quite stiff; spread on dishes to dry. When set, cut in squares and pat by for use.

$$
\text { 1522,-KISSES. }
$$

Beat the whites of four eggs till they stand alone. Then beat in, gradually, a pound of finely-powdered sugar, a teaspoonful at a time. Add eight drops of the essence of lemon, and beat the whole very hard.
Lay a wet sheet of paper on the bottom of a square tin pan. Drop on it, at equal distance, small teaspoonfuls of stiff currant jelly. Put a little of the beaten egg and sugar at first, under the currant jelly. With a large spoon, pile some of the beaten white of egg and sugar, on each lump of jelly, so as to cover it entirely. Drop on the mixture as evenly as possible, so as to make the kisses of a round, smooth shape.

Set them in a cool oven, and as soon as they are colored, they are done. Then take them out, and place the two bottoms together. Lay them lightly on a sieve, and dry them in a cool oven, till the two bottoms stick fast together so as to form one ball or oval.

\section{3.-MACAROONS.}

Blanch and skin half a pound of sweet almonds, dry them well in your screen, then put them into a mortar with a pound and a half of lump sugar, pound well together, and pass the whole through a wire sieve ; put it again into a mortar, with the whites of two eggs, mix well together with the pestle, then add the white of another egg, proceeding thus until you have used the whites of about eight eggs and made a softish paste, when lay them out at equal distances apart, upon wafer-paper, in pieces nearly the size of walnuts, place some strips of almonds upon the top, sift sugar over, and bake in a slow oven of a yellowish brown color; they are done when set quite firm through.

\section{4.-RATAFIAS.}

Ratafias are made similar to the above, but deducting two ounces of sweet, and adding two ounces of bitter almonds; they are laid out in much smaller cakes upon common paper, and baked in a much warmer oven; when cold, they may be taken off the paper with the greatest ease. 
These cakes are very serviceable in sugar and nutmeg to the taste. Mix all making a great many second-course dishes.

\section{5-RICE CAKES.}

Take eight yolks and four whites of eggs, and beat to a foam; add six ounces of powdered sugar, and the peel of one lemon grated; then stir in half a pound of ground rice, and beat all together for half an hour. Put it into a buttered tin, and bake twenty minutes. This cake is recommended as very easy of digestion. The foregoing cakes are made without butter, and therefore are not apt to prove injurious.

\section{6.-BOLA D'AMOUR-LOVE CAKES.}

Take the yolks of eggs, as many as are required,for the dish (about twelve), and beat them up in a pan with an equal weight of sugar, the same as sponge cake, using any kind of liquor or essence for flavoring. When the mixture is beaten up light and thick, have ready some clarified butter in a stewpan, made hot enough for frying. Pour the mixture into a funnel having a small bore or pipe, and let it run into the hot butter, turning the hand while it is running, so that it may be formed into threads all over the surface of the pan. In about two minutes it will be done, when it should be taken out with a skimmer, and be placed on a dish for serving, garnishing it with any kind of preserve, and serve cold.

Another way is, to beat up the eggs with some liquor, and run it into some boiling syrup at the blow.

\section{7.-EXCELLENT CHEESECAKES, KNOWN} AT RICHMOND AS "MAIDS OF HONOR."

Make some milk lukewarm. then put a spoonful of rennet, stir it well through a cheese-cloth to get rid of the whey; then to half a pound of curd put six ounces of butter, four yolks of eggs, and the ingredients well, line pattypans with a puff paste, fill them with the mixture, and bake in a quick oven. The cheesecakes may be flavored with lemon for a variety.

\section{8-CHEESECAKES.}

Take the curd of three quarts of milk, a pound of currants, twelve ounces of sugar, a quarter of an ounce of cinnamon, the same quantity of nutmeg, and the peel of a lemon chopped to a paste; the yolks of eight and the whites of six eggs, a pint of scalded cream and a pint of brandy; mix them all together, put a light thin puff-paste into your pattypans and half fill them.

1529.-SOYER'S QUEEN VICTORIA'S CAKE.

To three-quarters of a pound of flour, add half an ounce of yeast, mixed with warm water until forming a stiff paste, and place it in a stewpan, and let it stand by the fire or oven until well risen, during which time the butter must be well worked until soft, adding a quarter of a pound of flour more and five eggs gradually, and beat them well together; when done, if the leaven is well risen, add to it one and a half pounds of raisins, with a little salt and sugar; let it stand in the mould for a short time, and bake it in a slow oven.

\section{0.-ELETHERIAN PINE APPLE CAKE.}

Take three-quarters of a pound of pine apple, not over ripe, peel it; place half a pound of white sugar in a pan, with half pint of water, boil till a thick syrup; cut the pine in small dice of a quarter of an inch, and pour the syrup over; and, when nearly cold, add to it the dough, which has previously been mixed with three-quarters of a pound of butter and two eggs ; let it rise in a hoop as above, and bake in the same way: a glass of rum may be added. 
1531.-BABA.

Take three pounds of flour, one-quarter of which set apart and mix into dough with a gill of good yeast and a little warm water; cover it close in a stewpan and set it by the fire to rise ; when you see the dough rise and nearly ready, form the other parts of the flour in a circle on the table, in which put half an ounce of salt, one ounce of sugar, a half pound of butter and tweive eggs and a table-spoon of saffron; mix the butter and eggs well together before you put it to the flour; when it is all mixed spread it out and put the dough to it and knead it well. When the yeast is nearly mixed in, add two pounds of raisins stoned, a few at a time, have ready two moulds buttered, and put three parts full of dough and set in a warm place to rise very gently; when raised to the top of the mould bake in a hot oven; a brick oven is best.

1532.-CHEESECAKES, APPLE.

Take twelve apples, pare, core, and boil in sufficient water to wash them; beat them very smooth; add six yolks of eggs, the juice of two lemons, some grated lemon peel, half a pound of fresh butter, beaten to a cream, and sweetened with powdered sugar ; beat in with the apples, bake in a puff crust, and serve open.

1533.-CHEESECAKES, ALMOND.

Take four ounces of blanched almonds, mixed with a few bitter, beat them with a spoonful of water, add four ounces of powdered sugar, a spoonful of cream, and the whites of two eggs well beaten; mix them quickly, put into small pattypans, and bake for twenty minutes in a moderately warm oven.

\section{4-CHEESECAKES, BREAD}

Take a French roll, slice it very thin, pour over it some boiling cream, allow it to cool and then add six or eight eggs half a pound of butter melted, a spoonfu. of brandy, half a pound of currants, some nutmeg, and a little sugar; put them in puff-paste, the same as other cheesecakes.

\section{5.-CHEESECAKES, CITRON.}

Take a pint of cream, boil it, and when cold mix it with two eggs well beaten; then boil them together, until they become a curd. Put in a few blanched almonds beaten in a mortar with a little orange-flower water, and some Naples biscuits and green citron chopped very small. Sweeten, and bake in tins.

\section{6.-CHEESECAKES, LEMON.}

Take four ounces of sifted lump sugar and the same quantity of butter; melt them slowly, add the yolks of two and the white of one egg, a sponge cake, some blanched almonds, the rind of three lemons shred fine, and the juice of one lemon and a half, and three spoonfuls of brandy ; mix all well together, then make a paste with eight ounces of flour, and six ounces of butter, mixing four ounces of it with the flour first, then wet it with six spoonfuls of water, and roll in the remainder of the butter; put the ingredients to this paste, and bake it.

\section{CUSTARDS, CREAMS, JELLIES, \&c.}

Custard is always eaten cold, ared either poured over fruit tarts, or served up separately in custard-cups, in each of which a macaroon steeped in wine, and laid at the bottom, will be found a good addition.

The flavoring may likewise be altered, according to taste, by using a different kind of essence, the name of which it 
then acquires; as of lemon, orange, marashino vanilla, \&c. It is almost needless to say that cream or a portion of it will make it richer than mere milk.

The French mode of making it is, to measure the number of cups which are to be filled, and use nearly that quantity of milk or cream, simmering it upon the fire, until beginning to boil, with lemonpeel, bay-leaves, or almonds, then adding about half an ounce of powdered sugar to each cup; then take the yolk of an egg to each small cup, beat them up with the milk, fill the cups, place in a bainmarie or vase of boiling water until the custard become firm.

For boiled custard:-Boil the milk with the flavoring till thick enough; pour it boiling hot over beaten yolks of eggs, eight to a quart; sweeten to taste, stir till cold and smooth.

\section{7-ARROWROOT CUSTARD.}

One pint of milk, one table-spoonful of arrowroot, one of sugar, boil the milk with a stick of cinnamon in it, wet the arrowroot with a little cold milk, add to it one egg well beaten, add the sugar, stir the whole into the boiling milk, flavor it with vanilla, wine, or rose when nearly cold.

You may flavor custards with lemonpeel, vanilla, or any thing you choose, and make them as rich as you like.

\section{8.-BLANC MANGE.}

Wet one ounce or four sheets of Cooper's Isinglass in cold water, boil one quart of milk with a stick of cinnamon in it, drain the isinglass out of the water and stir it into the milk; when dissolved, strain it through a flannel bag, sweeten it, and when cold pour it into the moulds.

1539.-CUSTARD CREAM OF CHOCOL ATE.

Scrape one-quarter of a pound of the best chocolate; pour on it a teacupful of boiling water, and let it stand by the fire till it has dissolved. Beat eight eggs very light, omitting the whites of two. Stir them by degrees into a quart of cream or rich milk, alternately with the melted chocolate and three table-spoonfuls of powdered white sugar. Put the mixture into cups and bake it ten minutes. Send them to table cold, with whipped white of egg heaped on the top of each custard.

\section{0,-COFFEE CUSTARD.}

This is made by putting a quart of milk on the fire, with about six ounces of white sugar. In another vessel beat up the yolks of ten eggs, and pour the milk gradually upon them. Roast your coffee (three or four ounces) till it is of a very light brown color, and gives out all its flavor ; break it in a mortar, slightly, and add it, while hot, to your hot custard. Strain through a jelly-bag, pour the cream into cups, and put them to cool. Every thing depends on the coffee being used whilst hot, so as to catch the aroma which goes off as it cools.

Or:-Pour half a pint of cold water on the same quantity of fresh ground coffee in a French coffee-pot, press it down and put on the lid. Stop the mouth of the spout, and when the water has filtered down, pour it off, and return it to the top to filter a second time. Have a custard made with eight eggs to a pint of milk, sweetened to taste. Stir in the coffee, put it in custard cups, and bake in a pan with water half to their tops. Send cold to table.

\section{1.-CHAMPAGNE CREAM}

Is made by beating up the yolks of six eggs with as much powdered sugar as will make it quite stiff-so much so as nearly to make the spoon stand in it. 
Then pour on it very gradually-stirring it all the time-a bottle of sparkling champagne: grate on it a little nutmeg, and serve it to the ladies, who seldom refuse a second glass.

A bottle of good cider or perry will be a tolerable substitute.

\section{2.- IECH角 CRÉMA.}

Beat up three eggs, leaving out two of the whites, and add to to them gradually one pint and a half of milk; then mix very carefully four table-spoonfuls of fine wheat-flour, and two ounces of finely powdered loaf-sugar, with grated lemonpeel to give a flavor. Boil these ingredients over a slow fire, stirring constantly to prevent burning, until the flour is quite dissolved. Prepare a shallow dish with some ratafia cakes at the bottom, and when the "créma" is sufficiently boiled, pour it through a sieve upon the cakes.*

\section{3,-ITALIAN CREAM.}

Whip together for nearly an hour a quart of very thick scalded cream, a quart of raw cream, the grated rind of four lemons and the strained juice, with ten ounces of white powdered sugar; then add half a pint of sweet wine, and continue to whisk it until it becomes quite solid. Lay a piece of muslin in a sieve, and lay the cream upon it with a spoon. In twenty hours turn it carefully out, but mind that it does not break. Garnish it with fruit-jelly or with flowers.

Another.-Whip up three-quarters of a pint of very rich cream to a strong froth, with some finely scraped lemonpeel, a squeze of the juice, half a glass

* Some finely powdered cinnamon should be dnsted pretty thickly over it. This delicious dish is always served up cold. The receipt was obtained from the nuns of Santa Clara convent at Palmas, in the island of Grand Canary. of sweet wine, and sugar to make it pleasant, but not ton sweet; lay it on a sieve or in a form, and next day put it on a dish, and ornament it with very light puff-paste biscuits, made in tin shapes the length of a finger, and about two thick, over which sugar may be strewed, or a little glazed with isinglass. Or you may use macaroons to line the edges of the dish.

\section{4.-CLOUTED CREAM.}

Strain the milk as soon as it comes from the cow into wide pans, holding about six quarts each, so as to be about three inches deep, and let it remain for twenty-four hours; then gently place the pan upon a hot plate or slow charcoal fire, which must heat it very gently, for if it boils it is spoilt; as soon as the cream forms a ring in the middle, remove a little with the finger, and if a few bubbles rise in the place where you do so, it is done, which will be in half to three quarters of an hour; remove it from the fire, and let it remain twenty-four hours; then skim it, and throw a little sugar on the top.

1545.-CHARLOTTE RUSSE. (Furnished for this work by Mr. TAYLOR, Proprietor of Taylor's splendid Establishment, Broadway, New York.)

Dissolve one ounce of isinglass in half a pint of milk, and add to it when dissolved the yolks of six eggs, and half a pound of white powdered sugar.

Whip up a quart of sweet cream in one vessel, and the whites of six eggs in another.

When the isinglass, \&c., is nearly cold, stir it gradually into the cream. Then add the whites and stir up the whole together. Pour it in a mould, and set it on the ice for an hour.

1546.-CHARLOTTE RUSSE. (Furnished by a Lady of New York.)

Make a custard of four eggs, one pint of 
milk, and five table-spoonfuls of sugar; boil a vanilla bean with the milk. Dissolve half an ounce of isinglass in a teacupful of water, put a pint of cream in a tin pan, stand it over ice, and when the isinglass is cold, mix it with the cream and beat it to a thick froth; pour the cooled custard in and beat it all well. Place lady-fingers or spongecake inside the form, pour in the mixture, and leave it on the ice to thicken.

\section{7.-STRAWBERRY CHARLOTTE.}

Line a plain round mould with ripe strawberries by burying the mould in ice to the rim, and dipping the strawberries in calf's-foot jelly, first covering the bottom with them cut in halves, the cutside downwards, afterwards building them up the sides, the jelly (which must be cold, but not set) causing them to adhere; when finished fill it with the cream as directed for the charlotte russe, and when ready to serve dip the mould in warm water, and turn it out upon your dish. The cream must be very nearly set when you pour it in, or it would run between the strawberries, and produce a bad effect.

Slip.-Warm two quarts or more of new milk, then put in it a piece of rennet that has been kept in wine, or sufficient of the liquor to curdle it. When a firm curd is formed, set it in a cool place. Serve with grated nutmeg over it, and with cream.

\section{8.-EASY AND EXCELLENT RECEIPT FOR JELLY.}

(By a Lady of New York.)

Seven bars isinglass dissolved in two quarts water. When cold. add the juice and rind of six lemons-the whites and shells of six eggs, a pint and a half of wine, one and a quarter pounds sugar; stir all well, boil it five minutes, and strain through a jelly-cloth.

\section{9.-A GERMAN TRIFLE.}

Put a pint of strawberries or any other fresh fruit in the bottom of a glass dish. Sugar the fruit, put over it a layer of macaroons; and pour over it a custard, made with a quart of fresh milk and the yolks of eight eggs beaten, sweetened to taste, and scalding hot. When cold. place on the top the whites of the eggs beaten to a froth with a little sugar, or cream whipped to a froth. The egg may be made ornamental by beating currant jelly with part of it, and putting it in alternate hills of white and pink.

\section{0.-GOOSEBERRY OR APPLE TRIFLE.}

Scald a sufficient quantity of fruit, and pulp it through a sieve, add sugar agreeable to your taste, make a thick layer of this at the bottom of your dish; mix a pint of milk, a pint of cream, and the yolks of two eggs, scald it over the fire, observing to stir it; add a small quantity of sugar, and let it get cold. Then lay it over the apples or gooseberries with a spoon, and put on the whole a whip made the day before.

If you use apples, add the rind of a lemon grated.

\section{1.-SYLLABUB.}

Mix a quart of cream with half a pint of sherry wine, and sweeten it to taste, adding essence or grated rind of lemon, and, if desired, the juice of the fruit. Churn or beat the mixture to a thick froth, which skim off and put in a glass bowl, over slices of sponge cake.

\section{2.-A FLOATING ISLAND.}

Take a pint of thick cream, sweeten with fine sugar, grate in the peel of one lemon, and add a gill of sweet white wine; whisk it well till you have raised a good froth; then pour a pint of thick cream into a china dish, take a sponge cake, slice it thin, and lay it over the 
cream as lightly as possible; then a layer of clear calves' feet jelly, or currant jolly ; then whip up your cream, and lay on the froth as high as you can. and what remains pour into the bottom of the dish. Garnish the rim with sweetmeats.

Or:-Beat the whites of twelve eggs with a little sugar and currant jelly-a teaspoonful to each egg; whisk it light and lay it on a custard made with the yolks of the eggs and three pints milk.

1553.-WAGNER'S ICE CREAMS. (These receipts are furnished by Mr. Wagner of New York, expressly for this work.)

Vanilla Ice Cream.-One quart cream, eight ounces sugar, crushed, half a vanilla bean. Boil half the cream with the sugar and bean, then add the rest of the cream, and cool and strain it.

Lemon Ice Cream.-One quart cream, eight ounces sugar, one lemon. Grate the lemon rind in the sugar; this extracts the oil; then add the raw cream and strain and freeze very soon. Lemon cream sours and becomes rancid more quickly than any other.

Strawberry Ice Cream.-One quart cream, nine ounces sugar, quarter of a pound fresh berries or more if desired. Wash the berries in the sugar, add the cream, and strain and freeze as soon as possible to prevent curdling.

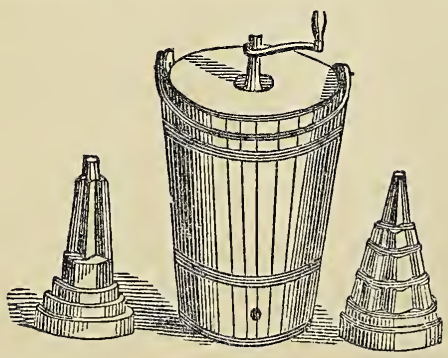

Johnston's Freezer.

The freezing with the ordinary freezer and a strong arm is preferable to all the machines ever invented. Mr. Wagner has at some expense tried the labor-saving machines, but could not bring up the cream to his standard, and has now abandoned them for his original mode of manufacture.

A great variety of ice creams may be made with different kinds of fruit in the same manner.

Mock Cream may be made by mixing half a table-spoonful of flour with a pint of new milk, letting it simmer five minutes to take off the rawness of the flour; beat up the yolk of an egg, stir it into the milk while boiling and run it through a fine sieve.

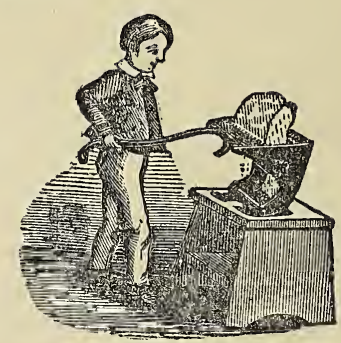

Patent Ice Breaker.

Rennet, to turn milk, may be prepared thus: Take out the stomach of a newly killed calf, cover it inside and outside with salt, after it is cleared of the curd. Drain it a few hours, then sew it up with two handfuls of salt in it, or stretch it on a stick well salted; or keep it in the salt and soak a bit of it when wanted, in fresh water.

\section{4.-BISCUIT GLACE.}

Rub some pieces of loaf-sugar on the rind of four lemons, powder it, and add half a pound more, moistened with the lemon-juice; mix it with a quart of cream, añd add six beaten eggs. Grate some stale Naples biscuit or sponge cakes, and stir them in till a thick smooth batter is formed. Scald it in a 
porcelain stewpan; put it in a freezer and freeze it.

\section{5.-ALMOND CREAM ICE.}

Take six ounces of bitter almonds, (sweet ones will not do,) blanch them, and pound them in a mortar. adding by degrees, a little rose-water. Boil them gently in a pint of cream till you find that it is highly flavored with them. Then pour the cream into a bowl, stir in half a pound of powdered loaf-sugar, cover it, and set it away to cool gradually. When it is cold, strain it, and then stir it gradually and hard into three pints of cream. Put it into the freezer, and proceed as directed. Freeze it twice. It will be found very fine.

Send round always with ice cream, sponge cake: afterwards wine and cordials, or liquors, as they are now generally called.

1556.-WATER ICES.

Are made with the juice of lemon, currant, or raspberry, or any other sort of fruit, being gained by squeezing, sweetened, and mixed with water. Rub some fine sugar on lemon or orange, to give the color and flavor, then squeeze the juice of either on its peel; add water and sugar to make a fine sherbet, and strain it before it be put into the icepot. If orange, the greater proportion should be of the juice.

\section{7.-DEVONSHIRE JUNKET}

Turn warm milk with rennet; add scalded cream, sugar, and cinnamon, hang to drain in a net.

Country Syllabub is made by milking from the cow into a bowl, with wine, sugar, and flavoring.

\section{8-RICE FLUMMERY.}

Boil with a pint of new milk a bit of lemon-peel and cinnamon; mix with a little cold milk as much rice-flour as will make the whole of a good consistence; sweeten, and add a spoonful of peach-water or a bitter almond beaten; boil it, taking care it does not burn ; pour it into a shape or pint basin, taking out the spice. When cold, turn the flummery into a dish, and serve with cream, milk, or custard, round.

As to the chemical compounds, advertised for the making of jellies, custards, and blanc mange, they are worthless in comparison with the animal substance of meat, eggs, and milk, of which such delicacies ought to be formed; they are not cheaper nor so nutritive, neither do we know of what they are composed.

1559.-CALF'S-FOOT JELLY.

It is a complaint amongst even experienced housekeepers that they cannot feel a certainty of having jelly clear; but by strictly attending to the following method for making calf's-foot jelly, they can scarcely fail to have it beautifully bright.

Feet for all jellies are boiled so long by the people who sell them that they, are less nutritious: they should be procured from the butcher and only scalded to take off the hair. The liquor will require greater care in removing the fat; but the jelly will be far stronger. A little isinglass, half an ounce to a quart of stock, may be used to secure the firmness, particularly in summer. In peel-

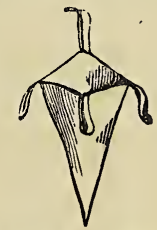

Felt Jelly Bag.

ing lemons, care should be taken not to cut below the color, as by so doing a great deal of the flavor of the essential oil is lost, and the white part gives a 
disagreeable flavor. The stock should be nieasured when set to get cold, as the exact quantity cannot be measured when it is set.

Jelly is equally good made of cowheels nicely cleaned; they bear a less price than those of calves, and make a stronger jelly.

To make Jelly.-Boil two feet in two quarts and a pint of water till the feet are broken and the water half wasted; strain it, and the following day take off the fat, and remove the jelly from the sediment; then put it into a saucepan, with a laurel-leaf, twenty coriander-seeds, the peel of two lemons, sugar to palate, half a pint of sherry, and a wine-glass of brandy. When the flavor is rich, put to it the whites of five eggs well beaten, and their shells broken. The ingredients should be cold when mixed together, to ensure the jelly being clear. Set the saucepan on the fire, but do not stir the jelly after it gets hot. Let it boil twenty minutes after it rises to a head; then pour it through a flannel jelly-bag, first dipping the bag in hot water to prevent waste, and squeezing it quite dry. Run the jelly through and through until clear; then put it into glasses or moulds.

The following mode will greatly facilitate the clearing of jelly :

When the mixture has boiled twenty minutes, throw in a teacupful of cold water; let it boil five minutes longer; then take the saucepan off the fire, covered close, and let it stand half an hour; after which it will be so clear as to need only once running through the bag, and much waste will be saved.

Or:-Boil four quarts of water, with three calf's-feet, or two cow-heels, that have been only scalded, till half wasted: take the jelly from the fat and sediment; mix with it the juice of a large orange and twelve lemons, the peels of three into a dish for eating cold. lemons, the whites and shells of twelve eggsं, white sugar to taste, a pint of winc, one ounce of coriander-seeds, two bayleaves, a bit of cinnamon, and six cloves, all bruised, after having previously mixed them cold. The jelly should boil fifteen minutes without stirring; then clear it through a flannel bag. While running take a little jelly, and mix with a teacup. ful of water in which a bit of beet-root has been boiled, and run it through the bag when all the rest is run out; this is to garnish the other jelly, being cooled on a plate : but this is a matter of choice. This jelly has a very fine high color and flaror.

Cheap Calf's-foot Jelly.-Take one box of Cox's sparkling gelatine and pour on a pint of cold water-let it stand ten minutes, then add a pint of boiling water-one pint of wine-half a pound of sugar-the juice and rind of two lemons-strain it through a napkin into a pitcher, then turn it into the moulds. Boil in the water a stick of cinnamon, and the lemon-peels.

\section{0-GOOSEBERRY-FOOL.}

Put the fruit into a stone jar, with white sugar; set the jar on a stove, or in a saucepan of water over the fire; if the former, a large spoonful of water should be added to the fruit. When it is done enough to pulp, press it through a colander; have ready a teacupful of new milk and the same quantity of raw cream boiled together, and left to be cold; then sweeten pretty well with fine sugar, and mix the pulp by degrees with it.

For Orange-fool.-Mix the juice of three oranges, three eggs well beaten a pint of cream, a little nutmeg and cinnamon, and sweeten to your taste. Set the whole over a slow fire, and stir it till it becomes as thick as good melted butter, but it must not be boiled; then pour it 
For Apple-fool.-Stew apples as di- syrup in jellies, flavored with liqueur. rected for gooseberries, and then peel and pulp them. Prepare the milk, \&c., and mix as before.

\section{1.-TO MAKE AN ALMOND POSSET.}

Grate the crumb of a loaf very fine, pour a pint of boiling milk upon it; let it stand two or three hours; then beat it exceedingly well; add to it a quart of good cream, and four ounces of almonds, blanched and beaten as fine as possible, with rosewater. Mix them all well together, set them over a very slow fire, and boil them a quarter of an hour: then set it to cool, and beat the yolks of four eggs, and mix them with your cream. When it is cold, sweeten it to your taste; then stir it over a slow fire, till it grows pretty thick, but do not let it boil, or it will curdle; then pour it into a china bowl. When you send it to table, put in three macaroons to swim on the top.

1562.-TAYLOR'S PLUM PUDDING GLACÉ.

(Furnished for this work by Mr. Taylor of New York.)

One quarter of a pound each of raisins, currants and citron, one quarter of a pound of chocolate, all boiled together in a pint of Madeira wine ; mix it when cold with ice cream, so as to make sufficient for an ordinary sized pudding.

\section{3.-TO CLARIFY ISINGLASS.}

Take two ounces of isinglass, pour on it a pint of spring water which has been mixed with a teaspoonful of beaten white of egg, and a table-spoonful of lemonjuice. Stir them thoroughly together, and let them heat slowly, taking care the isinglass does not stick to the bottom of the pan; simmer a few minutes, clear off the scum till no more appears; strain it through muslin, and set it by for use : it will be transparent, and may be warmed and mixed with the clear juice of any kind of fruit already sweetened, or with As a portion of the isinglass is taken up by the white of egg, one quarter to each ounce should be allowed for this. The finest sort of isinglass, which should be white and without any smell, does not require to be clarified, excepting for clear jellies; for all other purposes it is enough to dissolve and skim it, and to pass it through a strainer. A great variety of excellent jellies may be made with clarified isinglass and the juice or syrup of almost any kind of fresh fruit, the color of which is best preserved by mashing it lightly and strewing pounded sugar over it, letting it stand three or four hours for the juice to drain off; pour a little water over, and use the juice without boiling.

1564.-FROZEN PUDDING. (Furnished for this work by Mr. Sneckner, whose splendid Confectionery Establishment is in Union Square, New York.)

Cut up about half a pound of preserved fruits-such as peaches, plums, citron, raisins, and currants-add half a pound of Baker's chocolate, and a pint of best Madeira wine; simmer on the fire about fifteen minutes, and when perfectly cold add about one quart of vanilla ice cream. Freeze it in a two-quart mould, and for the sauce whip half a pint of sweet cream flavored with vanilla.

1565.-MERINGUES.

Pound and sift one pound of lump sugar, whisk the whites of twelve eggs very stiff, throw the sugar lightly over. and with a wooden spoon stir gently, perfectly mixing the sugar, then with a table or dessert-spoon lay them out upon white paper in the shape of eggs, sift powdered sugar thickly over, let them remain ten minutes, then shake off the superfluous sugar, place upon boards which you have wetted, and put them in a slow oven, just hot enough to cause them to be light and slightly tinged; when the outside becomes quite crisp, 
take off the papers, by turning them topsy-turvy and lifting the papers from them; dip your spoon into hot water, and with it clear out the best part of the interior, dust them with powdered sugar, lay them upon a baking-sheet, and put into the screen to dry; they may be made several days before they are required, if put away in a dry place; to serve, fill them with whipped cream, flavored either with vanilla or orangeflower (but do not make it too sweet), stick two together, dress in pyramid upon a napkin, and serve. Should they happen to stick to the papers, moisten the papers with a paste-brush and water underneath ; they will come away easier.

1566.-MERINGUES À LA CRÉME. (Furnished by Mr. Delmonico for this work.)

Beat to a froth the whites of six eggs, to this add three ounces of finely powdered sugar, and mix up well. Then lay sheets of paper upon tin or other plates, place some spoonfuls of the mixture at intervals of half an inch, and powder them with fine sugar. Cook them at a very gentle heat, and, when done, place them in a warm place, in order to keep them dry. You may dress them with whipped cream or jelly, according to your taste.

\section{7.-NOUGAT.}

Cut in dice or in fillets a pound of blanched sweet almonds, and mix with them eight or six bitter almonds. When they are cut equally, dry them in the oven, but keep them white; take three or four spoonfuls of superfine pounded sugar, put it over a slow fire in a preserving pan; when the sugar is melted without having used any water, throw the almonds in, but take care that they are quite dry. Stir the sugar with a clean wooden skewer. If you hear a noise when you throw them into the sugar, it is a sign that they are dry enough. Rub a mould slightly over in- side with oil or butter, and lay some almonds in beds as thinly as possible; take an oiled lemon to press the almonds with : but be quick, otherwise the almonds will get cool, and then they cannot be worked so thin. The nougat requires to be light, to be made to perfection. Sometimes you may make the nougat in a mould the form of a vase, sometimes in small custard-moulds, according to your choice; it is always the same thing, but you may cut the almonds of different shapes.

Sometimes make a pound of almonds into nougat, oil a baking-dish, and spread it over it, oil the rolling-pin, and flatten the nougat with it; if it will spread easy, put it in the oven again to make it soft ; then cut it into small long squares, and keep it in a very dry place, to prevent it sticking to the fingers. The nougat is in general made too sweet, and it is an article that belongs to the confectionery department.

\section{COFFEE, TEA, AND CHOCOLATE.}

\section{8.-COFFEE.}

There are several ways of making coffee; and every housewife generally has her favorite mode. The French make excellent coffee without the aid of eggs, isinglass, or any foreign article to settle it. It consists of a sort of tin coffee-pot, with two strainers. You remove the first strainer, and pour some boiling water into the coffee-pot through the second strainer. Empty out the water, and put in a sufficient quantity of coffee for the family over the under strainer and press it flat with a little tin machine (which comes with the apparatus). Put in the other strainer, and pour in the hot water. The coffee will drain through in a few moments. 


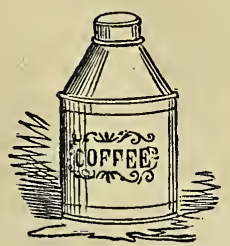

Coffee Canister.

Another mode of making Coffee.-Take fresh roasted coffee, allow two tablespoonfuls for each person, grind it just before making, put it in a basin and break into it an egg, yolk, white, shell, and all. Mix it up with the spoon to the consistence of mortar, put warm, not boiling water, in the coffee pot; let it boil up and break three times; then stand a few minutes and it will be as clear as amber, and the egg will give it a rich taste.

Codfish skin, scraped, washed, and dried, and cut in pieces, an inch square, may be used to settle off, or isinglass. You may add to a pint of coffee a pint of boiling milk, and heat both together before serving.

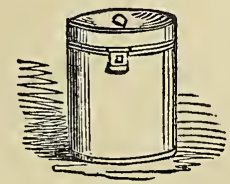

Sugar Box.

\section{9.-COFFEE, TO ROAST.}

Coffee should never be roasted but when you are going to use it, and then it should be watched with the greatest care, and made of a gold color; mind and do not burn it, for a few grains burnt would communicate a bitter taste to the whole; it is the best way to roast it in a roaster over a charcoal fire, which turns with the hand, as by that means it will not be forgotten.

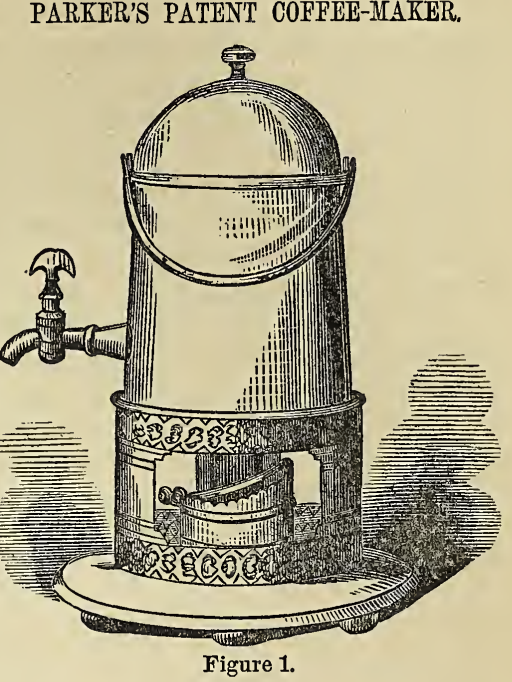

By this machine, the extract of coffee is prepared in a very easy and expeditious manner. When the extract is made, it may be diluted with boiling water to suit the palate; or hot milk may be used instead of water, making café au lait.

Figure 1 represents the external appearance of the apparatus, and figure 2 is a section to show its construction and mode of action. $\quad a \alpha$ is a box of metal to hold the coffee, having its bottom and top lid perforated with numerous small holes. From the lower part of the box proceeds a funnel, that reaches nearly to the bottom of an outside vessel or cylinder of metal, $e e$, which is divided into two parts interiorly by a partition, $b \quad b$, and on the middle of this partition is fixed a tube, into which the neck of the funnel is put, and on which the box with the coffee rests. The exterior vessel is covered with a convex lid, $d$. The whole is placed on a stand, in which is a spiritlamp, $f$, having a sliding tube, $g$, so arranged that the heat can be increased or diminished at pleasure. 
Having taken out the box, with its I piling up the ground coffee in the centre. funnels, it is filled with coffee, and boil- and then striking it off with a knife, as ing water is poured down the tube in is done with corn in a bushel-measure. the interior; this will pass into the di- The time for making the extract of coffee, vision at the lower part, $e e$; then the where hot water is used, should never box with the coffee is to be replaced, and exceed five minutes. If it takes longer, the lamp lighted. The steam which is it can arise only from the coffee leing formed forces the water into $e e$ to rise through the tube, and passes upward through the perforated bottom of the box into the coffee, which is seen rising as a dark fluid through the perforated

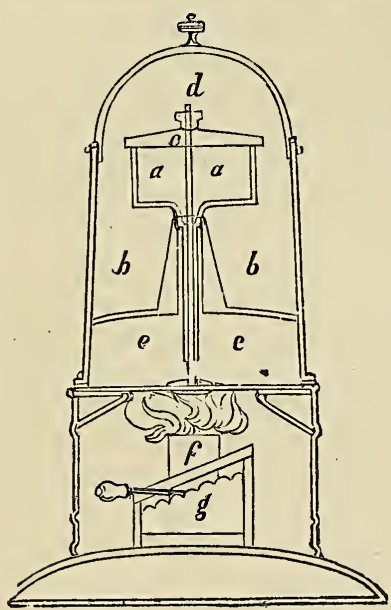

Figure 2.

lid of $a a$, and flowing over into the upper division, $b \quad b$. The passage of the water should be permitted to continue till it becomes pale-colored, and has consequently extracted all the goodness of the coffee, when the lamp should be extinguished, and the lid put on. The extract is then drawn off by means of the faucet.

After making the extract, it is best not to drain off the liquid remaining within the rim of the cover, but to let it return into the boiler, which it will do as soon as the lamp is extinguished.Thus, any sediment will return with it. ground too finely; and this not only increases the expenditure of spirits of wire and of time, but likewise injures the quality of the extract. The remedy is, to grind the coffee coarser and coarser, until the operation, after the water in the boiler boils, lasts only three minutes. The extract of coffee prepared by this apparatus contains all the aroma and other fine qualities of the coffee.

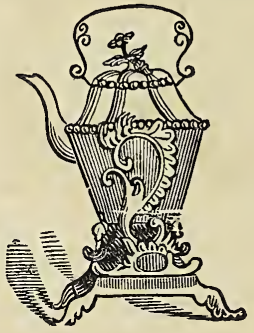

T E A.

Tastes differ regarding the flavor of various sorts of tea: some preferring all black; others, all green; and many, a mixture of both in different quantities; though most persons-when not fearful

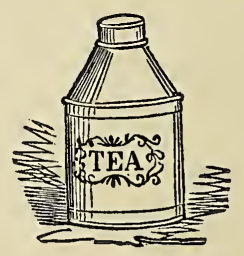

Tea Canister.

of their nerves-agree that fine Hyson

The filling of the box is best done by is the best. A good mixture, in point of 
flavor, we know to be-two-fifths black, two fifths green, and one-fifth gunpowder: all being, of course, of superior quality.

Presuming all ladies to be intimately acquainted with the mode of making tea, yet to some, a few hints on the subject may not perhaps be found objectionable.

Boiling water should alone be used.

Metal tea-pots in preference to earthenware. They retain heat better.

Silver is better than either.

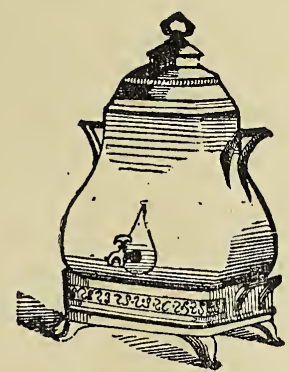

Water Urn.

A spoonful of tea for each person. Heat the tea-pot first with some boiling water, then pour that into the tea-cups to warm them; put in your tea, and pour enough water upon the tea to cover it; let it stand three or four minutes, then nearly fill the tea-pot with water; let it stand a few minutes, and pour out.

\section{CHOCOLA T E,}

If made thin, is not worth drinking; and that which is commonly sold is a compound of flour, or some farinaceous substance, and coarse sugar, with a very moderate quantity of real cocoa, and that of the most inferior quality, colored with Venetian red and brown ochre.

Chocolate forms the common breakfast throughout Spain, and is there made by merely chipping a portion of the cake into which it is formed from the cocoa, leaving the chips in water for a whole night to soften. It may then be slowly warmed, along with either water or milk, working it all the time with the mill, which is a small movable pole passed through the lid of a chocolatepot, and furnished with a headpiece, in order to mix the chocolate equally, by turning the mill rapidly between the hands and without intermission, thus to prevent it from becoming clotty; care, however, should be taken not to let it boil; for, in that case, the vegetable oil which is contained in the nut will be extracted from it and appear on the surface. The flavor is better retained by making it in water than milk.

It is always made very thick, so that a small spoon will nearly stand upright in it, and nothing eaten with it but a rusk, or dry toast: after which there is usually taken a draught of cold spring water.

The usual mode of making it here is, to boil a pint of water and a pint of milk in the pot, put to it a part of a cake of chocolate cut into very small slices, mill it off the fire till quite melted, then put it on a gentle fire till it nearly boils, milling it all the time. Sweeten to taste.

\section{0. $-\mathrm{COCOA}$.}

An excellent breakfast beverage may be made by simply pounding the nutwhich is the substance of chocolate-and boiling it in the same manner in either water or milk.

Cocow Shells or Nibs, are the thin coverings of the cocoa kernel, and can only be had at some chocolate manufactory, where they can be bought at a very low price, and form a light food for an invalid, when taken warm.

Soak them in water during the whole night, and then boil them in the same water until it is reduced to half the quantity: 
they should boil two hours, and should then be mixed up with milk.

\section{1-CHOCOLATE DROPS.}

Take one pound and a half of chocolate, put it on a pewter plate and put it in the oven just to warm the chocolate, then put it into a copper stewpan with three-quarters of a pound of powdered sugar ; mix it well over the fire, take it off, and roll it in pieces the size of small marbles, put them on white paper, and when they are all on, take the sheets of paper by each corner and lift it up and down, so that the paper may touch the table each time, and by that means you will see the drops come quite fat, about the size of a sixpence; put some sugar nonpareils over them, and cover all that are on the paper, then shake them off, and you will see all the chocolate drops are covered with the sugar nonpareils; let them stand till cold and they will come off well, and then put them in a box prepared.

\section{2.-TEA CREAM.}

Infuse an ounce of the best green tea in half a pint of boiling milk, simmer it five minutes, then strain it through a tammy, pressing the leaves well; boil a pint of rich cream, add to it the yolks of four eggs beaten, and sufficient quantity of clarified sugar; pour this whilst hot to the milk, stir them together well, put in as much clarified isinglass as will set it, and pour the cream into the mould or glasses; place them on ice; when perfectly cold, turn it out of the mould or serve in the glasses.

\section{PRESERVES, \&c.}

Preserves should be kept carefully from the air, and in a very dry place. Unless they have a very small propor- tion of sugar, a warm one does not hurt; but when not properly boiled, heat makes them ferment, and damp causes them to grow mouldy. They should be looked at two or three times in the first two months, that they may be gently boiled again if not likely to keep. Paste the edge of the outer paper, as it keeps out the air better than a string; put writingpaper over the fruit.

Dried sweetmeats, cakes, \&c., should be kept in tin boxes, between layers of white paper, in a very dry but not hot room.

All fruits for preserving should be gathered in dry weather; but as this is not always practicable, much inconvenience may be obviated by boiling the fruit for jellies and jams long before the sugar is added. By so doing, the watery particles will evaporate, and the preserve will be better flavored, by the sugar not being too long on the fire.

Pans of copper or bell-metal are the proper utensils for preserving fruit: when used, they must be scoured bright with sand. Tinned pans turn and destroy the color of the fruit that is put into them.

1573.-TO PRESERVE WATERMELON RIND.

Pare off the outer skin and cut the rind into shapes : green them by simmering with vine leaves and a little alum, and allow a pound and a quarter of sugar to each pound. Make the syrup and clarify it with white of egg, and simmer the melon rind till done through and transparent. Boil down the syrup afterwards, and pour it over the preserves.

Chips of pumpkin or muskmelon rind, cut thin, are often made into preserves, -adding the juice and grated rind of lemons, which much improves the syrup.

Citrons may be preserved in the same manner, first paring off the outer skin, and cutting them into quarters. Also green limes 
1574.-APPLES.

Weigh equal quantities of good brown sugar and of apples; peel and core them. Boil the sugar, allowing to every three pounds a pint of water ; skim it well, and boil it pretty thick; then add the apples, the peel of one or two lemons, and two or three pieces of white ginger; boil till the apples look clear and yellow. This preserve will keep for years.

\section{5.-PINE-APPLES.}

Take those that are ripe, and perfectly fresh-pare off the rind, and cut the apples in slices an inch thick. Powder the same weight of white sugar as you have pine-apples-lay the pine-apples in a deep dish, and sprinkle part of the powdered sugar between each layer of apples. Reserve about half of the sugar. Let the apples remain till the succeeding day - then turn the syrup from them, and mix it with the reserved sugar, and half a pint of water, for three or four pounds of pine apple. Boil the syrup, take it from the fire, and when cool, put in the apples, simmer them gently till tender, let them remain in a deep dish for several days ; they should be covered up tight, and kept in a cool place. Whenever there is any appearance of fermentation, turn the syrup from them, scald it, and turn it back hot upon the pineapples. Keep them in glass or china jars, covered tight, and in a cool place.

\section{6.-TAIT PINE-APPLE.}

To fourteen pounds of grated pineapple add half an ounce of grated alum, and mix thoroughly. Boil seven pounds white sugar in as little water as possible, skimming it until perfectly clear. Pour the boiling sugar over the fruit, and put it into empty champagne bottles; stand the bottles in boiling water till the water begins to cool ; then cork and seal them. closely.

\section{7.-CRAB-APPLES}

Make a syrup, allowing the same weight of sugar as apples. Let it cool, then put in the apples, a few at once, so that they will not crowd, and break to pieces. Boil them till they begin to break, then take them out of the kettle. Boil the syrup in the course of three or four days, and turn it while hot upon the apples. This continue to do at intervals of two or three days, till the apples appear to be thoroughly preserved.

\section{8.-GENERAL DIRECTIONS FOR MAKING SWEETMEATS AND JELLIES.}

In preparing sugar for sweetmeats, let it be entirely dissolved before you put it on the fire. If you dissolve it in water, allow about half a pint of water to a pound of sugar. If you boil the sugar before you add the fruit to it, it will be improved in clearness by passing it through a flannel bag. Skim off the brown scum all the time it is boiling. If sweetmeats are boiled too long, they lose their flavor and become of a dark color. If boiled too short a time they will not keep well. You may ascertain when jelly is done, by dropping a small spoonful into a glass of water. If it spreads and mixes with the water, it requires more boiling. If it sinks in a lump to the bottom, it is sufficiently done. This trial must be made after the jelly is cold. Raspberry jelly requires more boiling than any other sort. Black currant jelly less. Keep your sweetmeats in glass jars.

\section{9.-TO, PRESERVE QUINCES.}

Ripe quinces pare and cut in slices an inch thick-take out the cores carefully, so as to have the slices in the form of a ring. Allow a pound of white sugar for each pound-dissolve it in cold water, having a quart of the latter to a pound of sugar, then put in the sliced quinces, 
and let them soak in it ten or twelve and boiled with them, gives the quinoes hours. Put them in a preserving kettle, a fine flavor. and put it on a moderate fire-cover them over, and let the quinces boil gentlythere should be more than enough syrup to cover the quinces. When a broom splinter will go through them easily, take them from the fire, and turn them out. In the course of a week turn the syrup from them, and boil it down, so that there will be just enough to cover the fruit. When not very ripe, pare and cut the quinces either in rings or quarters, take out the cores, and boil the quinces in fair water, till they bezin to grow tender-take them up, and strain the water in which they are boiled-put in either brown or white sugar-add a little cold water. When lukewarm, put in the whites of eggs and clarify it-let it cool, then put in the quinces, and boil them slowly for half an hour. Keep them covered over while boiling, if you wish to have them of a light color. Turn them out into pots as soon as preserved, and set them away in a cool place. Look at them in the course of a week to see if they have fermented-if so, turn the syrup from them, boil it, and turn it back while hot. The parings and cores of the quinces can be used for marmalade, with a few whole ones. Some people prefer to preserve the quinces with the cores in, but the syrup will not look clear. The following is a cheap method of preserving quinces, and answers very well for common use: Pare, halve, and take out the cores of the quinces, and boil the parings in new cider till soft. Strain the cider, and for five pounds of quinces put in a pound of brown sugar, a quart of molasses, the beaten white of an egg-clarify it, then put in the quinces. There should be rather more than enough cider to cover the quinces, as it wastes a good deal while the quinces are boiling. The peel of an orange cut in small pieces,

Quince Marmalade.-Gather the fruit when fully ripe, and of a fine yellow; pare, quarter, and core it. Put the quinces into a saucepan with a little water, set them on the fire until they are quite soft; then take them out, and lay them on a sieve to drain; rub them through, and put to each pound of the strained quinces a pound of hrown sugar. Set it on a few coals, and let it stew slowly, stirring it constantly. When it has stewed an hour, take a little of it out, let it get cold-if it then cuts smooth, it is sufficiently stewed.

Quince Jelly.-Halve the quinces, and take out the cores. Boil the quinces till very soft in clear water, mash them, and let them drain through a flannel bag, without squeezing them. Put to the quince liquor, when drained through the bag, white sugar in the proportion of a pound to a pint of liquor. Add the whites of eggs, and clarify it. When clear, boil it on a moderate fire till it becomes a thick jelly. Fill glasses with the jelly, and cover them tight. The quince pulp that remains in the jelly-bag can be made into marmalade.

\section{0.-TO PRESERVE PEACHES, APRICOTS, NECTARINES, AND PLUMS.}

September is the best month for peaches, as they are then harder and larger. Weigh the peaches, put them into a preserving-pan full of cold water, with a slice or two of lemon; set them on a slow fire, have ready a sieve and a napkin, and be careful not to do them too much.

Some of the peaches will be ready sooner than others; when they begin to be soft they are done enough; take them out as they become soft, and drain them on a sieve, and let them stand until cold; then make a syrup, to every pound of 
peaches allowing a pound of loaf-sugaruse some of the water in which the peaches were boiled for the syrup. Take the kerncls of half a dozen peaches, throw them into hot water and remove their skins, then boil them with the syrup you are making. Put the peaches into jars and glasses, and pour the syrup over them.

Cut several round pieces of paper, dip them in brandy, lay them over the preserves, and tie up the jars.

A pricots, nectarines, and plums, may be preserved in the same manner.

This way of preserving peaches is much preferable to cutting them up and then preserving them. The fruit should not be permitted to bril until it becomes shrivelled.

\section{1.-BRANDY PEACHES.}

To each pound of peaches take threequarters of a pound of white sugar. Make a syrup, in half of which boil the fruit, having first taken off the skin by scalding them in hot lye, which is made by dissolving as much salsoda in boiling water as will make it strong enough to bear an egg.

The peaches are to be taken out of the lye as soon as the skin begins to crack, and thrown into cold water, when they can be rubbed quite clean with a coarse cloth. Rinse them in fresh water, wrap them in a cloth to drain, and keep them covered, as on this depends their color.

When the boiled peaches are cold, add to the remainder of the syrup the same quantity of brandy. Put away the peaches in it, and cover tightly.

\section{2.-GREEN GAGES.}

Allow equal weights of sugar and gages. Make a syrup of white sugar, and just water enough to cover the plums. Boil the plums slowly in the syrup ten minutes-turn them into a dish, and let them remain four or five days, then boil them again, till the syrup appears to have entered the plums. Put them into a china jar, and in the course of a week turn the syrup from them, scald it, and turn it over them hot.

\section{3. -TO PRESERVE PEARS.}

Pare them very thin, and simmer them in a thin syrup, allowing only one-quarter of a pound of sugar to a pound of pears. Let them lie for two days, add another quarter of a pound of sugar to each pound of pears, and simmer them again. Let them lie all night, or longer if you please, then simmer them once more, this time adding half a pound of sugar to each pound of pears, with the juice of half a lemon to every two pounds of fruit. A small portion of the lemonpeel may also be used. The fruit may then be drained and dried in the sun, so that they may be used dry ; or they may be poured into the jars with the syrup. over them.

Another mode of preserving pears, and a less troublesome one, is to pare, quarter and core the pears, boil them for an hour in as much water as will cover them, then add to every pound of pears a pound of white sugar and the juice of half a lemon, boil the whole and skim it. When the pears are soft, pour them into jars and the syrup over them; tie up the jars.

This is a much more expeditious way of preserving the pears, and perhaps the best for large families, where sweetmeats are in daily use; but the fruit itself does not look as clear and beautiful as when preserved by the former method.

Pear Marmalade - Boil the pears with the skins on. When soft, rub them through a sieve, and put to each pound of pulp three-quarters of a pound of brown sugar. Stew it over a slow fire till it becomes a thick jelly. It should be stirred constantly. 
Baked Pears.-Take half a dozen fine pears; peel, cut them in halves, and take out the cores; put them into a pan with half a pound of sugar, and some water. Set them in a moderate oven till tender, then put them on a slow fire to stew rgently ; add grated lemon-peel, and more sugar, if necessary. They will be sufficiently red.

\section{4.-GOOSEBERRIES.}

The tops and tails being removed from the gooseberries, allow an equal quantity of finely pounded loaf-sugar, and put a layer of each alternately into a large deep jar; pour into it as much dripped currant-juice, either red or white, as will dissolve the sugar, adding its weight in sugar; the following day put all into a preserving-pan and boil it.

Gooseberry Jam.-Take what quantity you please of red, rough, ripe gooseberries; take half their quantity of lump sugar; break them well, and boil them together for half an hour or more, if necessary. Put it into pots and cover with paper.

\section{5.-CURRANT JELLY, (RED).}

Strip off the currants, put them in a jar, set the jar in a kettle of hot water, let it boil an hour, then throw the currants and juice into a fine lawn sieve, press out all the juice, and to every pint of juice put a. pound of fine sugar; put them in a preserving-pan, set it over a charcoal fire and keep it stirring till it is in a jelly, which you will know by taking a little out to cool; be careful to take off the scum as it rises, and when it is jellied and very clear pour it into glasses; when cold, cut round pieces of paper that will just cover the jelly, dipped in brandy, put white paper over the glasses, twisting round the top. Make black currant jelly the same way, using coarse sugar.

\section{6.-CLEAR APPLE JELLY.}

Pare and cut up five dozen large juicy, acid apples; put them in a pan with as much water as will cover them, let them boil gently until soft, let them get cold, then strain them through a jelly bag, put the juice in your preserving pan, and to each pint of juice put one pound of fine sugar and the peel of two lemons, then boil it until it is reduced to the stiffness of calves'-foot jelly, skim it well, add the juice of a lemon; it should be made in September, the flavor of the apple is better. Jelly may be made from apricots, raspberries, grapes, and other fruits, using a pound of sugar for each pint of juice, and following the above directions.

1587.-COLORING FOR JELLIES, ETC.

For a beautiful red, boil fifteen grains of cochineal in the finest powder, with a drachm and a half of cream of tartar in half a pint of water, very slowly, half an hour; add in boiling a bit of alum, the size of a pea, or use beet-root sliced, and some liquor poured over.

For white, use almonds finely powdered, with a little drop of water, or use cream.

For yellow, yolks of eggs, or a bit of saffron steeped in the liquor and squeezed.

For green, pound spinach leaves or beet leaves, express the juice, and boil a teacupful in a saucepan of water to take off the rawness.

\section{8.-FROSTED FRUIT.}

Pick out the finest cherries, plums, apricots, grapes, or small pears-leave on their stalks. Beat the whites of three eggs to a stiff froth-drain them, and beat the part that drips off again. Lay the fruit in the beaten egg with the stalks upward-select them out one by one, and dip them into a cup of finely powdered sugar. Cover a pan with a sheet 
of fine paper, place the fruit inside of it, and set it in an oven that is cooling. When the icing on the fruit becomes firm pile them on a dish and set them in a cool place.

\section{9.-GRAPES IN BRANDY.}

Take some close bunches, black or white, not over-ripe, and lay them in a jar. Put a good quantity of pounded white sugar upon them, and fill up the jar with brandy; tie them close down with a bladder, and keep in a dry place. Each grape should be pricked thrice.

They make a beautiful middle dish in a winter dessert.

\section{0-CRANBERRY JELLY.}

Make a very strong isinglass jelly. When cold, mix it with a double quantity of cranberry juice pressed, sweeten it with loaf sugar, and boil it up; then strain it into a shape.

\section{1.-TO PRESERVE STRAWBERRIES, RASPBERRIES, AND BLACK- BERRIES, WHOLE.}

The strawberries should be gathered fresh from the vines. Select the largest, and those perfectly unbroken. Mash up the others and boil them for a quarter of an hour without any water-strain them through a fine jelly-bag.

Measure the juice, and allow a pound of loaf sugar, to every quart of strawberry juice.

When you make the syrup allow a pint of spring water to every two pounds of sugar-skim the syrup thoroughly. When the scum ceases to rise pour in the juice of the berries and boil it from five to fifteen minutes, or until the syrup will hang in drops from a spoon.

Pour the syrup into glass jars or tumblers, and lifting the whole strawberries with a spoon, put as many of them in each jar as it will hold, without being at all crowded. The syrup should cover all well, and it is better to have too few than too many in every jar. Let the jars stand until the syrup is quite cold, and then tie them up with paper dipped in brandy.

\section{2.-TO DRY CHERRIES.}

Weigh the cherries before they are stoned, and allow to every pound of fruit a quarter of a pound of lump-sugar ; when they are stoned set them over a slow fire to heat, then take them out of the liquor and put the sugar to them, and let them stand till it is dissolved. Then set them over the fire and let them just boil. Allow them to stand until they are quite cold, or, if convenient, for two or three days, in the syrup; afterwards strain them, and spread them on sieves to dry, either on a stove or in the sun, or in an oven after the bread is drawn.

The same syrup will do again for more fruit.

To Dry without Sugar.-Stone, and set them over the fire in the preservingpan; let them simmer in their own liquor, and shake them in the pan. Put them in china common dishes; next day give them another scald, and put them when cold on sieves to dry in an oven of temperate heat. Twice heating, an hour each time, will do them.

Put them in a box, with a paper between each layer.

\section{3.-CURRANT JAM}

Strip your currants, and put them into your pan, with three-quarters of a pound of sugar to a pound of fruit, add your sugar after your fruit has boiled a few minutes, boil all together, mashing your fruit with a wooden spoon; boil all gently for half an hour, then fill your jars.

Currants Preserved.-Take the seeds and stalks from whatever quantity of currants you intend to use, of which 2 . fourth part must be white currants ; put 
them into a preserving pan with a glass of water. let them boil up until the fruit bursts, then strain the juice twice, clarify and boil to cassé some sugar, an equal weight to the fruit, pour the juice on it, boil them together a quarter of an hour, and having skimmed it well, pour it into pots.

To preserve them dry.-Tie up in bunches; to every pound of currants boil two pounds of sugar, till it boils very strong, dip in the currants, let them boil very fast till the sugar flies all over them; when settled a quarter of an hour boil them till the sugar rises almost to the top of the pan, let them settle, skim them and set them by till next day, then drain them and lay them out, taking care to spread the sprigs that they may not dry clogged together'; dust them very much, and dry them in a hot stove.

1594.-FIGS GREEN, TO PRESERVE.

Slit some small green figs on the top, and put them into water for ten days, and proceed thus:-Put as much salt into the water as will make it bear an egg, then let it settle, take off the scum and put the clear brine to the figs, keep them for ten days; then put them into fresh water, shifting them every day for four days; again drain, then put them into clarified sugar, warm them a little and let them stand till the next day; warm them again, and when they are become green give them a good boil, then boil some sugar down, put it to them and give them another boil, and next day drain and dry them.

\section{5.-FIGS RIPE, TO PRESERVE.}

Take the figs when ripe, slit them in the tops, put them into clarified sugar, and give them a good boil, skim them, and leave them to stand till the next day, then boil some more sugar, put it to the figs and give them another boil, the next day drain and dry them.
Ripe or Green Tomatoes-Make a nice preserve. They require a more than usual quantity of sugar.

\section{6.-GINGER, PRESERVED.}

Take some green ginger, and with a sharp knife pare it neatly, and as it is pared throw into a pan of cold water to keep it white; when you have a sufficient quantity boil it till tender, changing the water three times each time; put it into cold water to take out the heat or spirit of the ginger, when tender throw it into cold water. For seven pounds of ginger clarify eight pounds of refined sugar; when cold drain the ginger and put it into an earthen pan, with a sufficient quantity of the sugar cold to cover it, and let it stand for a couple of days; then pour the syrup from the ginger to the remainder of the sugar, boil it for some time, and when cold pour it on the ginger again, and set by for three days at least. Then take the syrup from the ginger, boil it, and then put it hot over the ginger; proceed in this manner until you find the sugar has entered the ginger; boiling the syrup and skimming off the scum that rises each time until the syrup becomes rich as well as the ginger; if the syrup is put on hot at first, or if too rich the ginger will shrink and not take the sugar.

\section{7.-MARMALADE.}

Marmalade may be composed almost of any fruit; the best however for this purpose are apricots, peaches, oranges, quinces, eggs, plums, apples, \&c. ; they are usually made by boiling the fruit and sugar together to a kind of pulp, stirring them constantly while on the fire. It is kept in pots which must not be covered, till the marmalade is quite cold; the proportion of sugar is half a pound to each pound of fruit.

1598.-APPLE MARMALADE.

Boil some pippins until they begin to 
get tender, then put them into cold water, pare and core them, squeeze the pulp through a sieve, and put it over the fire, letting it remain till it. becomes very thick, then weigh an equal quantity of fine sugar, boil till the sugar rises in sparkles which cluster together, put the marmalade to it, and stir them well with a wooden spoon till the apples begin to boil, then take it off, and when a little cool put it into pots, but do not cover them until quite cold.

\section{9.-ORANGE MARMALADE.}

Blanch the rinds of fifteen oranges without any of the white till soft, then soak them in cold water for a few minutes, drain and pound them to a paste, which rub through a sieve; ascertain its weight, and for each pound allow a pound and a half of sugar; clarify and boil the sugar till the bubbles rise strongly to the surface; put in the paste and boil them together, stirring continually till the marmalade is done. To know when the marmalade is fit to turn out and be potted, take some up between your thumb and finger, and if on opening them it draws out like a thread it is done. The Southerners use bitter oranges.

Marmalades of raspberries, blackberries, plums, grapes, \&c., are most in favor.

\section{J A M S. 1600.-RASPBERRRY.}

Take equal weights of fruit and moist sugar; put them on the fire together; keep stirring and breaking the fruit till the sugar melts, then boil it till it will jelly on a plate.

\section{1.-CHERRY.}

Stome four pounds of cherries, and put them in a preserving-pan with two pounds of fine white sugar and a pint of red- currant juice. Boil the whole together rather fast, until it stiffens, and then put it into pots for use.

\section{2.-BLACKBERRY.}

In families where there are many children, there is no preparation of fruit so wholesome, so sheap, and so much admired, as this homely conserve. The fruit should be clean picked in dry weather, and to every pound of berries put half a pound of coarse brown sugar ; boil the whole together for three-quarters of an hour or one hour, stirring it well the whole time. Put it in pots like any other preserve, and it will be found most useful in families, as it may be given to children instead of medicine.

Compotes of fruit are made by preparing a syrup, and simmering the fruit in it two or three minutes, then pouring the syrup over, after it is boiled down a little; a delicate light jelly is thus formed round the fruit. Not more than half the weight of the fruit in sugar, or even a less proportion, is used. The dish is a nice one for desserts, but will not keep. All kinds of fresh fruit may be thus prepared.

1603.-TO PRESERVE ORANGES OR LEMONS IN JELLY.

Cut a hole at the stalk the size of a shilling, and with a small blunt knife scrape out the pulp quite clear, without cutting the rind, and lay them in spring water two days, changing it twice a day; in the last, boil them tender on a slow fire, keeping them covered to the last. To every pound of fruit take two pounds of double-refined sugar and one pint of water; boil the two latter together with the juice of the orange to a syrup, and clarify it; skim well and let it stand to be cold; then boil the fruit in the syrup half an hour; if not clear, do this daily till they are done. 
1604.-TO PRESERVE LEMON PEEL.

Squeeze the juice out of the lemons, put the rinds, with the pulps, into water, and let them remain a fortnight, taking care always to have them covered with the same water; then take out the pulp, and boil the rinds in the water till they can be pierced with a knitting needle; make a rich syrup of brown sugar-pound for pound of rinds-and pour it boiling over them; cover close for three weeks, then give them a second boil in the syrup, and cover for use.

\section{5.-BARBERRY CAKES.}

Pick the barberries and weigh them; to every pound of fruit add one pound of sugar pounded and sifted. Bruise the barberries in a mortar, boil them gently till nearly all the juice is consumed, then take them off the fire and stir in the sugar by degrees ; drop the fruit on earthen dishes to dry: it must not go over the fire after the sugar is put in. nor must there be any water used. Do not pound the barberries too small, as the cakes will not look so well.

Currant cakes may be made in the same way.

\section{6.-RASPBERRY CAKES.}

Take any quantity of fruit you please, weigh and boil it, and when mashed, and the liquor is washed, add as much sugar as was equal in weight to the raw fruit. Mix it very well off the fire till the whole is dissolved, then lay it on plates, and dry it in the sun. When the top part dries cut it off into small cakes, and turn them on a fresh plate. When dry, put the whole in boxes with layers of paper.

\section{7.-BRANDY CHERRIES.}

Get the largest morel cherries you can, cut off half of the stalk, pricking each cherry with a needle, putting them as you do them into a high glass; add three quarters of the weight in white candy sugar bruised between until full, a gill of Noyeau, and then fill up with French brandy; tie a bladder over the bottle.

\section{8.-CHERRIES IN BRANDY.}

Choose the finest and ripest cherries, leave on half the stalks, wash and weigh them; to every pound of fruit allow a quarter of a pound of sugar; when you have clarified and boiled it, put in the fruit, boil them up two or three times, then put the cherries into bottles, or glass jars; when filled, add to each twelve cloves and half an ounce of cinnamon tied in a linen bag, put to the sugar when nearly cold, brandy in proportion of a pint and a half to a pound of fruit, mix them together well, and pour them on the cherries. In two months' time taste them, and if sufficiently flavored, take out the cloves and cinnamon ; cover the jars close.

Plums, apricots, \&c., can be done the same way.

\section{9.-MOCK PRESERVED GINGER.}

Boil, as if for the table, small, tender, white carrots; scrape them until free from all spots, and take out the hearts. Steep them in spring water, changing it every day, until all vegetable flavor has left them. To every pound of carrot so prepared, add one quart of water, two pounds of loaf-sugar, two ounces of whole ginger, and the shred rind of a lemon. Boil for a quarter of an hour every day, until the carrots are clear; and, when nearly done, add red pepper to taste. This will be found equal to West India preserved ginger.

\section{0.- XANKEE APPLE BUTTER.}

Boil cider down one half; put in as many apples as the liquor will contain, stew them soft; then take them out and 
put in fresh apples. When they are cold boil them again in the cider till they are pulpy and thick. Add different kinds of spice, a little before it is done. Keep in covered jars.

\section{1.-CRANBERRY. SAUCE.}

Simmer the berries in a very little water, till very thick and pulpy; sweeten to taste; they will require as much as preserves; strain the marmalade into a mould.

\section{2.-TO KEEP PEACHES FRESH.}

Pare and halve your peaches, have your cans perfectly dry, set one at a time in a bain marie in boiling water, to drive out the air. Fill the can as full as you can without bruising the fruit; if you have plenty of fruit, boil some in a very little water, fill the can with it-and seal it immediately. Do not put any sugar in the peaches or juice. If you have not much fruit, a little boiling water will do, as each can will hold but a few spoonfuls. Do not let it boil until it becomes colored.

Any fruit or vegetables may be preserved in the same manner. Inspect the cans daily, and if the tops bulge out, be sure the sealing has not been perfect, and fermentation has begun. Then open the can, use the article, and put up a fresh quantity. To open the can, set it in the oven, till the cement is soft; then lift out the cover, and let the can cool. To open glass jars, hold a flat-iron, heated, on the cover till the cement softens.

Butter may be kept unchanged for years in air-tight vessels, as sweet as if fresh from the dairy. To insure means in excluding the air, it is best to fill the vessel nearly full of well worked fresh butter, allowing room for a thin layer of salt ; over this lay a piece of white paper; pour on the paper half a teaspoonful of spirits of wine; set it on fire; hold the cover over the flame for an instant, and if you have spirits enough, it will furnish heat for the cover, then put it into place while the spirits are burning.

Or, powder two pounds fine salt, one pound loaf-sugar, and half a pound of saltpetre. Sift these ingredients over a large sheet of paper, and mix them well ; then keep them covered close in a jar in a dry place. When the butter is well worked and salted, and ready to go into jars, use one ounce of this composition to every pound of butter. Work it well into the mass. In a month place the butter thus prepared into jars or else lay a cloth over the butter; on this put a layer of salt, with a piece of white paper on the salt; pour a little spirits on the paper, set fire to it, and seal as above directed.

\section{3.-TO KEEP TOMATOES.}

Peel the tomatoes, and put them in a kettle over the fire till they boil for ten or fifteen minutes. They are then ready for sealing. The half-gallon or gallon sized cans are the best for the purpose. Fill one with the hot tomatoes, wipe off from the cement any juice, and put on the cover. The contents of the can will so heat the cover, that it is only necessary to press it firmly into place. When first closed, as the confined steam is elastic, it will rise up, and a weight, say of five or six pounds, must be used to keep it down; or a piece of ice may be laid on it, which will condense the steam within. If the can is larger than the weight, put a board on ftrst. When the cans are cold, if the sealing is perfect, the tops and bottoms will be concave. If the cement of the cover should not be sufficiently melted, hold on the top a heated flat-iron, till the cover goes down into its place.

1614.-TO PRESERVE GREEN CORN.

Fill a strong tin canister with the corn, solder it tightly, place it in boiling 
water, and allow it to remain several hours. The top and bottom will swell, and the can may burst (if not strongly made) with the confined steam. Some puncture the vessel, and when the steam escapes, solder up the opening. Others do not consider this necessary; or if the vessel, tightly covered, is cooked a long time in a bath of brine, in which a higher temperature may be obtained than in water, and then sealed hermetically, the vegetable will keep.

\section{5.-TO CLARIFY SUGAR.}

Take the quantity of fine white loaf sugar you intend to clarify, add to it of very clean warm water, half a pint for every pound; when dissolved add to it the white of one or two eggs-as the quantity may require-well whipped; put it on the fire, and when it comes to a boil pour into it an ordinary teacupful of cold water; on its rising again to a boil, remove it, and let it settle for twenty minutes, skim the scum from the top, pour off the syrup into a clean vessel with sufficient quickness to leave all the sediment at the bottom, and such steadiness as to prevent any of the latter rising and mixing with it.

\section{7.-TO BOIL SUGAR FOR BASKETS OR SPINNING.}

Fill quite full a pint and a half stewpan with pieces of lump sugar, fill the stewpan with clear spring water, let the water barely cover the sugar, put it on to boil, skim it all the time that any scum arises; let it boil fast with the stewpan flat upon the fire, not halfway; it will not boil over if your fire is regular. Then get ready a large basin of cold water, and when it has boiled some time and begins to appear all froth or bladders do not go away and leave it; after having boiled so for some few minutes, have a silrer spoon and dip it into it, and then into the water; if it is getting to a substance pour in the juice of half a lemon, free from pips, still keeping it boiling on the fire; keep frequently trying it by dropping and spinning a little in the cold water, and wien it makes a crackling noise, and is very brittle, take off your stewpan and pour it into a cold stewpan; work it well with your spoon, give it one more boil, then take it off and hold the stewpan in cold water, stirring it all the time, for a minute or two; it is then ready for a basket, or spinning, or what you may require it for. It is but very little used now for second courses; at breakfasts and ball suppers it is generally introduced and liked, but it too frequently answers the purpose of lock and key, saying as much, as "this must not be touched;" many think it will do again, and do not like to break through it,

The various purposes to which sugar is applied require it to be in different states; these are called degrees. They extend to the number of thirteen, and are named in the following order:

Petit Lisse, or First degree.-Replace the clarified sugar in the preserving-pan to boil gently, take a drop of it on the thumb, touch it with the forefinger; if on opening them it draws to a fine thread, and in breaking forms two drops on each finger, it is at the right point.

Lissé, Second degree.-A little more boiling brings it to this point, when the thread will draw further before it breaks.

Petit Perlé, Third degree.-At this point the thread may be drawn as far as the span will open without breaking.

Grand Perlé, Fourth degree.-On still increasing the boiling, little raised balls are formed on the surface of the sugar.

Petit Queue de Cochon, Fifth degrec.-

Take up some of the sugar on a skimmer, and drop it on the rest, when it should form a slanting streak on the surface.

Grande Queue de Cochon, Sixih de- 
gree.-Boil it yet a little longer; the streak or tail is now larger, and it has reached this point.

Souffé, Seventh degree.-Take out a skimmerful of the sugar, bluw through it and small sparks of sugar will fly from it.

Petit Plume, Eighth degree. - The same proof as above; the sparks should be larger and stronger.

Grande Plume, Ninth degree.-Take the sugar in the skimmer as before; give it a shake, and if the sparks are large, and adhere together on rising, it is at the right point.

Petit Boulet, Tenth degree.-Dip your fingers in cold water, and then into the sugar instantly, and again into the water, when the sugar will roll into a ball which will be supple when cold.

Gros Boulet, Eleventh degree.-At this point the ball or bullet will be harder when cold than the last.

Cassé, Twelfth degree.-Prove as abore; the bullet should crumble between the fingers, and on biting will stick to the teeth.

Caramel, Thirteenth degree.-At this point it should snap clean when bitten. This point is very difficult to attain, for in increasing the height, the sugar is apt to burn; it is better, therefore, to try the proof very frequently.

Another caramel is much used by the confectioner, and is of a deep color; it is made by putting a little water to the sugar, and boiling it without skimming or otherwise touching the sugar till of the right color, then take it off, and use immediately.

If on preparing the sugar, you happen to miss the right point, add a little cold water, and boil once more.

The skimmer should never be left in the preserving-pan after the sugar is clarified, nor after the scum is removed.

Be very careful not to stir or disturb the sugar, as that would cause its diminution.

In boiling the sugar, particularly the two last degrees, the sugar is continuously rising and falling, and on falling leaves marks on the side of the pan, which the heat of the fire would soon burn and thereby spoil the whole of the sugar. To avoid this have a sponge with cold water, and wipe the sides of the pan the instant the sugar has fallen.

\section{7.-TO CANDY FRUIT.}

Having prepared your fruit, steep it in the syrup, and lay it as done in an open sieve, until the bottom is covered with one layer; steep this suddenly in scalding water. This will remove any syrup which may cling to the fruit. Lay them aside on a napkin to drain, and go on with the others. You will have ready, finely-powdered, some of the best loaf sugar; sift this over the fruit until they are white all over, without being to thickly encrusted; lay them so as not to touch each other, on strainers or the reverse end of small sieves; place them in a gently-warmed oven, watch them carefully, turning them until dry. The warmth of the oven must not be increased, but must not abate until the fruit is quite dry.

Almonds.-Blanch any quantity of almonds, then fry them in butter till they are of a light brown color; wipe them nicely with a napkin, and put them into a pan. Make a syrup of white sugar, and boil it to a thread-that is, until on your taking a drop of the sugar between the finger and thumb it will produce a thread; care must be taken to boil it to the exact candying-point; pour it boiling-hot upon the almonds, and stir them till they are quite cold. This is an excellent method of preparing almonds for dessert. 
Bon-bons.-Clear off the sugar from fresh candied citron or orange-rind, cut it into squares one inch thick, stick them singly on a bit of thin wire, and dip them into liquid barley-sugar; rub a dish with a few drops of pure salad oil, and lay the fruit upon this to cool. They should be kept in tin canisters in a very dry place.

Toffie.-Melt in a stewpan three ounces of fresh butter, add one pound of good moist sugar, stir it well over a gentle fire, and let it boil about a quarter of an hour, or till it cracks short between the teeth like barley-sugar; then pour it upon buttered dishes, and when nearly cold mark it across in squares that it may easily divide, or roll it into sticks. When half-boiled, the grated rind of a lemon or a teaspoonful of ground ginger may be added. A few sliced almonds may be added after it is poured upon the dishes.

\section{BEVERAGES.}

Louis XII., of France, first gave permission to distil spirits on a large scale. So terrific were the effects twenty years afterwards, that Francis, his successor, was obliged, for the safety of his subjects, to enact a law, that the drunkard who remained incorrigible, after severe monitory punishments, should suffer amputation of the ears, and be banished from the kingdom. How much more wisely would Francis have acted, if, instead of banishing the drunkard, he had banished the pernicious material of drunkenness! Take another example: Sweden was a temperate country, ardent spirits being, to a great extent, prevented from coming into ordinary use. In 1783, however, Gustavus, king of Sweden, gave permission for opening spirit shops in all the villages of his king- dom. His object was to increase his revenue, and that object he apparently for a time accomplished; for immediately ardent spirits were loaded with fictitious excellences, by those who loved them, and those who were interested in their sale: the drinking of them, which had formerly been carried on in sccret, now became respectable; and the consumption of them was greatly increased. But mark the consequence;-such was the increase of drunkenness and crime, of fatal accidents and premature mortality, that the very same king who gave the permission, was obliged, for the preservation of his people, to withdraw it.

Spruce Beer.-Allow an ounce of hops and a spoonful of ginger to a gallon of water. When well boiled, strain it, and put in a pint of molasses and half an ounce or less of the essence of spruce; when cool, add a teacup of yeast, and put into a clean tight cask, and let it ferment for a day or two, then bottle it for use. You can boil the sprigs of sprucefir in room of the essence.

Ginger Beer.-Fourteen gallons water, fourteen pounds loaf sugar, four ounces ginger, well pounded; boil one hour, add the whites of eight eggs beat up, and take off the scum; strain the liquor into an earthen pan, let it stand till cold, then put it into your cask with the peel of fourteen lemons cut thin, and their juice strained. Add half a spoonful of aleyeast on the top. Stop the vessel closely for a fortnight. Then it may be bottled, and in another fortnight will be fit for use.

Another for Ginger Beer.-One pound sugar, one ounce ginger, one-half ounce of cream of tartar, one lemon sliced. Put all into a pan, pour over one and a half gallons boiling water, and when milk-warm put in a little yeast; let it stand all night to work; bottle it; and in three days it is fit for use. 
Lemonade.-Three lemons to a pint of water, makes strong lemonade; sweeten to your taste.

This is the best beverage for parties; cool, refreshing, pleasant, and salubrious.

Orangeade.-Roll and press the juice from the oranges, in the same way as from lemons. It requires less sugar than lemonade. The water must be pure and cold, and then there can be nothing more delicious than these two kinds of drink.

Currant Wine.-Gather the currants when dry, extract the juice, either by mashing and pressing the fruit, or putting it in a jar, placed in boiling water; strain the juice, and for every gallon allow one gallon of water and three pounds of sugar. Dissolve the sugar in the water, and take off the scum; let it cool, add it to the currant juice, and put the mixture in a keg, but do not close it tightly till it has ceased fermenting, which will not be under a week. In three or four weeks it may be bottled. The white of an egg beaten, mixed with a teaspoonful of cream of tartar, and stirred into the liquid, makes the wine look clear and bright. Some add a portion of spirits.

Raspberry Vinegar.-To two and a half quarts of ripe raspberries put one pint of the best vinegar. Bruise them well, and let it stand three days. Strain the juice through a bag, and add its weight of sugar. Boil it, skim well, and bottle it closely.

Syrup of Almonds. - Blanch and pound in a marble mortar one pound of sweet and one ounce of bitter almonds ; adding a spoonful or two of orange-flower water. Mix a pint of rosewater with one of pure water, add it to the almonds, and pass the whole through a lawn sieve. Then boil three pints clarified syrup, and when it boils put in the almonds, and let them boil one minute. When cold put it into pottles, and cork it for use.

To make it for immediate use, after pounding the almonds, mix them with a quart of water, one of milk, and one of clarified syrup or capillaire, and pass through a sieve.

Valencia Wine.-To each gallon of good spirits take the peel of eighteen lemons; let them remain forty-eight hours in the spirits; then add the juice with five quarts of cold spring water, three pounds of loaf sugar, a quarter of a pound of bitter almonds well blanched, half a drachm of saffron, and two ounces of ground ginger ; mix all together, and pour over all three quarts new milk, scalding hot, but not boiled ; let it stand till next day, then run it through flannel, like jelly.

Mead without fruit.-Boil honeythree or four pounds to a gallon of water-for an hour, skimming carefully, draining the skimmings, and returning what runs through. When nearly cool, stir in a teacupful of yeast for nine gallons, and let it ferment. Put it in a cool cellar, and bottle in a year.

Currants and raspberry juice, orange and lemon peel, spices and aromatic herbs are often added to mead; the last towards the end of the fermentation, in a muslin bag, weighed down with a piece of flint. Six quarts of red currants and two of black will do for twenty-five pounds of honey.

Ratafias.-Ratafias are liquors prepared by infusing the juices and kernels of fruit in strong spirit with sugar. For the ratafia of cherries take two quarts of rectified spirits of wine, add two drachms beaten cinnamon, one of bruised cloves, and one ounce bruised coriander seeds. Cover and let them stand a week; then from morella and black heart cherries press twelve pints of juice, add three pounds powdered loaf sugar, and mix with the spirit and spices; add half the cherry stones, mashed in a mortar. Closely cover the stone jar, containing all these, and stir or shake it frequently. 
In two months pour it off; press and filter the residuum. Then put it into bottles and cork it.

Ratafict.-Bianch two ounces of peach and apricot kernels, bruise and put them into a bottle, and fill nearly up with brandy. Dissolve half a pound of white sugar-candy in a cup of cold water, and add to the brandy after it has stoor a month on the kernels, and they are strained off; then filter through paper and bottle for use. The distilled leaves of peaches and nectarines, when the trees are cut in the spring, are an excellent substitute for ratafia in puddings.

Capillaire, is so called from being prepared from a kind of fern called capillus veneris, or maiden-hair ; but almost any other fern may be substituted for it. A syrup made from the Canada capillaire, with maple sugar, is sold.

\section{BEVERAGES COMPOSED PARTLY OF FERMENTED LIQUORS.}

Hot spiced wines were much in use till the beginning of the sixteenth century ; and some of these favorite compounds were known by the names of ypocras, sack, and clary. The first of these, which took its name from the bag through which it was strained, called by apothecaries "Hippocrates's sleeve," was made either of white or red wine with aromatics, such as ginger, cinnamon, aromatic seeds, and sugar. Clary was made from claret, with honey and aromatics; and sack from the wine of that name, a kind of sherry. These were drunk as a " night, cap," and at the conclusion of a banquet. Le Grand, in his "Vie privée des François," observes that the poets of the thirteenth century speak with rapture of these delicious beverages; and it is said that they were highly esteemed in convents and universities. A scale of perfection was even observed: when the compound was made of Bordeaux wine, it was simply called Bishop; but re- ceived the name of Cardinal when old Rhine wine was used; and rose to the dignity of Pope when imperial Tokay was employed. The vestiges of these ancient mixtures may still be seen in our mulled wine and bishop.

To mull Wine.-Boil the spices (cinnamon, nutmeg grated, cloves, and mace) in any quantity approved, in half a gill of water; put to this a full pint of port, with sugar to taste. Mix it well, and serve hot with thin slips of toast or rusks. Lemon or orange juice may be added, and the water may be strained off from the spices. Ale or Porter may be mulled as above, and have toast or biscuits put to them. Formerly the yolks of eggs were mixed with mulled wine, as in making custard or egg-caudle, and many flavoring ingredients were employed which are now disused.

Bishop.-The day before it is wanted, grill over a clear, slow fire, of a pale brown, three large, bitter oranges. PJace them in a small punch-bowl that will about hold them, and pour over them a full half pint from a bottle of old Bordeaux wine, in which a pound and a quarter of loaf sugar is dissolved. Cover with a plate. When it is to be served next day, cut and squeeze the oranges into a small sieve placed above a jug, containing the remainder of the bottle of wine, previously made very hot; add more syrup if it is wanted. Serve hot in large glasses, or in summer it may be iced. Bishop is often made of Madeira in England, and is perfumed with nutmegs, bruised cloves, and mace. It ought, however, to be made of oll generous Bordeaux wine, or it fails of its purpose as a tonic liqueur. It is reckoned highly stomachic, and is served at French dinners, savans and recherchés, either as the coup d'après or after the dessert.

The wassail bowl of ancient times was 
made of mild ale, well spiced and sweetened; and sometimes with eggs beat up in it.

The ancient Ypocras was made of a quart of red wine, an ounce of cinnamon, half an ounce of ginger, a quarter of an ounce of pepper, and half a pound of sugar, all put into a bag and infused in the wine.

Cool Tankard.-The composition of this ancient beverage is of great variety. The basis is home-brewed ale, spices, and seasoning herbs. Some use cider instead of ale.

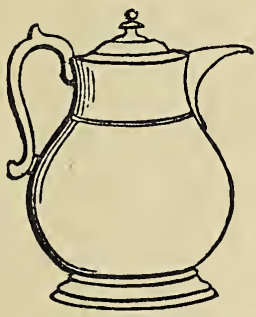

Covered Pitcher.

Egg Flip.-Heat a quart of good ale, and pour it into a pitcher ; in a similar pitcher beat up three or four eggs with a quarter of a pound of moist sugar, and one or two glasses of rum or brandy, flavoring it with nutmeg, ginger, and grated lemon peel. When the ale is quite hot, but not boiling, pour it quickly into the jug with the eggs, return this back into the other jug, and thus keep pouring the mixture backward and forward from one jug to another, till the whole is thoroughly incorporated, and as smooth as cream.

Egg hot.-This is made in the same manner as the last, only there is no spirits nor spices; simply the egg, and ale or beer, with sugar.

Ale Posset.-Boil a pint of new milk with a slice of toasted bread; pour a brandy (or whiskey), a little reduced bottle of mild ale into a punch-bowl, in strength; clarify in a gallon of sweeten, and add spices, and then pour the boiling milk over it.

Sack Posset.-Boil some cream and grated sweet biscuits ; add sugar, cinnamon, and nutmeg. Warm some sherry, and stir it into the cream; then pour the whole quickly from one vessel to another until it be perfectly smooth; or it may be made with eggs beat up in milk instead of the cream.

Punch is a beverage made of various spirituous liquors or wine, hot water, the acid juices of fruits, and sugar. It is considered to be very intoxicating; but this is probably because the spirit being partly sheathed by the mucilaginous juice and the sugar, its strength does not appear to the taste so great as it really is. Punch was almost universally drunk among the middle classes about fifty or sixty years ago.

English Punch.-Rub the yellow rind of a lemon with lump sugar ; put this sugar into the punch-bowl, and squeeze the lemon juice to it, add the spirits, rum and brandy, in such proportions as are preferred, incorporate the spirits thoroughly with the sugar and lemon before pouring in the boiling water, and keep stirring the whole while this is pouring : some add Madeira or sherry. Punch is kept also cold in bottles, and in summer is liked. It is frequently made with whiskey.

Regent's Punch.-A bottle of Champagne, a quarter of a pint of brandy, a glassful of veritable Martinique: with this mix a pint or more of a strong infusion of the best green tea strained, and capillaire or simple syrup to taste.

Norfolk Punch.-Pare thirty-two oranges, and the same number of lemons; infuse the peel for two days in a large bottle or jar with a gallon of 
water four pounds of sugar; when cold, strain the brandy (which will now be a tincture) to this; add the juices of the oranges and lemons, previously strained and bottled, when the peel is taken off ; cask the liquor, or put it in a jar, and stop it well; in six weeks it may be gently poured, or drawn off, and bottled. A tincture of bruised nutmegs and cloves may be added to this compound; this will keep any length of time, and in any climate.

Victoria or 32d Regiment Punch.Take the peel of two dozen lemons, and steep it for thirty-six hours in two quarts of rum. Pour the juice of the lemons on three pounds and a half of loaf sugar. Add two quarts of brandy, and seven quarts of cold water.

Mix these together, and add by degrees two quarts of boiling milk, stirring it well. Let it stand four hours.

Strain it two or three times through a flannel jelly bag, till perfectly clear; bottle and cork it tight. Age improves it very much.

Milk Punch.-Mix wine or brandy with milk; sweeten to taste ; grate nutmeg on it.

Wine Punch.-Port wine, two pints; arrack, two pints; the juice of twelve lemons ; sugar, one pound; hot water, six pints.

Tea Punch.-Hot tea, two pints ; arrack, thirteen ounces; sugar, four ounces ; flavored by rubbing off the yellow peel of the lemons.

French Punch.-Cut a lemon into thin slices, taking out the seeds; boil them in water, and add a pinch of green tea, and let it infuse five minutes; strain the whole, and add brandy and sugar sufficient.

Auld Man's Milk of Scotland, or Eggnog of America.-Beat the yolks and whites of six eggs separately; put to the beat yolks sugar and a quart of new milk, or thin sweet cream; add to this rum, whiskey, or brandy, about half a pint; put in the whites of the eggs whipped up, and stir the whole gently. It may be flavored with nutmeg or rind of lemon.

Sangaree is a kind of punch frequently drunk in the West Indies, and is composed of one-third Madeira or porter, and two-thirds water, acidulated with lime juice and sweetened with sugar.

Mulled Cider.-Boil a quart of cider, watered, if too strong, with cloves and allspice. Beat six eggs, sweeten, and add them to the boiling cider, with a pint or more of cream. Pour the liquor frum one pitcher to another till it has a fine froth, and serve it warm.

Cherry Bounce.-Take twelve pounds cherries, mashed, and mixed with three pounds sugar : add two gallons whiskey. Put it into a closed vessel, shake it often, and let it stand four months before bottling.

\section{WINES AND LIQUEURS.}

Ginger Wine.-Take fourteen gallons of water, six pounds of sugar, four ounces of bruised ginger, and the whites of two eggs, well beaten; mix them, set on a fire, boil it fifteen minutes, skim it well, and when cold, pour it into an earthen vessel, squeeze in the juice of four lemons, and the rinds pared exceedingly thin, put to it a teacupful of ale-yeast. let it work for a day and a night, then turn it into a cask, bung it up, and in a fortnight you may bottle it off.

Blackberry Wine.-Put some ripe blackberries into a large vessel with a cock in it; pour on as much boiling water as will cover them, and as soon as the heat will permit, bruise them well with the hand till all the berries are broken, cover them, and in about three or four days, when the berries rise to the 
top, draw off the clear part into another vessel; add to every ten quarts of the liquor one pound of sugar, stir it well in, and let it stand a week or ten days to work. Draw it off through a jelly bag. Steep four ounces of isinglass in a pint of sweet wine for twelve hours, then boil it slowly till dissolved, put it in a gallon of the juice; boil them together, then put all together, let it stand a few days, and bottle.

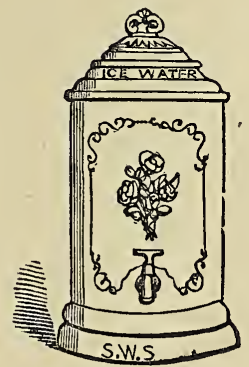

Water Cooler.

To preserve Ice twelve hours.

Cherry Wine.-For every five pints of this wine, take fifteen pounds of cherries, and two of currants; bruise them together, mix with them two-thirds of the kernels, and put the whole of the cherries, currants, and kernels into a barrel with a quarter of a pound of sugar to every pint of juice. The barrel must be quite full; cover the barrel with vine leaves and sand above them, and let it stand until it has done working. which will be in about three weeks; then stop it with a bung, and in two months' time it may be bottled.

Rhubarb Wine.-The leaf stalks of rhubarb cut in pieces as for tarts, and bruised with a wooden mallet to express the juice, will make a wine equal to green gooseberry, and resembling champagne.

Wine made from mixed fruits.-Take cherries, raspberries, and black and white currants-of each an equal quantity. To four pounds well bruised put one gallon of water. Steep the mass three days and nights, frequently stirring it in an open vessel, then run it through a hair sieve, and to each gallon of liquor put three pounds sugar. Let it stand again three days and nights, stirred often and skimming the top; then turn it into a cask, and let it ferment at the bunghole two weeks. To every nine gallons put one quart best brandy, and fasten down the bung. If not soon clear stir in a solution of isinglass.

Black Currant Wine (very fine).-To every three quarts of juice put the same of water unboiled, and to every three quarts of the liquor add three pounds of very pure moist sugar; put it into a cask, reserving for a little filling up; put the cask in a warm dry room, and the liquor will ferment of itself; skim off the refuse when the fermentation shall be over, and fill up with the reserved liquor; when it has ceased working pour three quarts of brandy to forty quarts of wine ; bung it close for nine months, then bottle it, and drain the thick part through a jelly-bag until it be clear, and bottle that ; keep it ten or twelve months. This recipe is most excellent. The wine will keep for six years.

Gooseberry Wine.-To every three pounds of gooseberries put a pint of spring water unboiled, having first bruised the fruit with the hands in a tub; stir them very well; let them stand a whole day, then strain them off, and to every three pounds of gooseberries add a pint of water, and one pound of sugar dissolved; let it stand twenty-four hours longer, then skim the head clean off, and put the liquor into a vessel, and the scum into a flannel bag, adding the liquor that drains from it to that in the vessel; let it work two or three days before stop. ping it up close, and allow it to stand 
four months before it is bottled. When it is drawn out of the cask it should not be tapped too low.

Green Gooseberry Wine.-Take thirtytwo quarts of unripe gooseberries of the green kind, bruise them well, add thirtytwo quarts of cold water; let them stand for twenty-four hours; drain the gooseberries well from the liquor through a sieve; put three pounds and a half of lump-sugar to every gallon of liquor; put it into a cask with a bottle of the best gin; let it stand six months, and then bottle it.

Frontiniac.-Boil eighteen pounds of white sugar in six gallons of water with two whites of eggs well beaten. Skim it, and put in a quarter peck of elderflowers ; do not keep them on the fire ; when nearly cold, stir it, and add six spoonfuls of lemon-juice and four or five of yeast; beat the whole well into the liquor. Stir it every day, put six pounds of the best raisins, stoned, into the cask, and tun the wine. Stop it close, and bottle it at the end of six months. This wine requires keeping.

Elder Wine.-Pour four quarts of water upon eight quarts of berries, and let it stand a day or two ; then boil it for about an hour, strain it, and put three pounds of moist sugar to every gallon of wine ; then add one ounce of cloves and cinnamon, with two ounces of ginger; boil it again, and work it with a toast, dipped in yeast.

Elder-flower Wine. - Take twelve pounds of loaf-sugar and six pounds of the best raisins, cut small, and boil them in six gallons of water for one hour. Then take half a peck of elder-flowers, when ready to shake; put them into the liquor, when it is nearly cold, with four table-spoonfuls of fresh yeast, and six of lemon-juice. Let it work two days in the tub, then strain it, put it into the barrel, bung it up closely for two months, and then bottle it.

These are excellent domestic wines, generally taken mulled, with dry toast; and the flavor will be improved if, while warming, a grating of nutmeg be added

Negus.-One bottle of wine, half a pound of sugar, and a lemon sliced. Pour three pints of boiling water upon this mixture, and grate nutmeg to the taste.

Such is the common mode of making negus; for making a single tumblerful, however, as many people prefer to do it in their own way, perhaps a better plan is to use only half the quantity of water, poured boiling hot upon the w ine already sweetened and flavored with nutrieg; but having in it also a large and very thin cut rind of orange, which gives it a very superior flavor, without any portion of the acid.

The negus may be made of either white or red wine; and, if drunk cold, is called "sangaree."

The fol'owing is a French receipt:One pound of cherries, four pounds of currants, two pounds of black cherries; squeeze all together, then let it stand in a cool cellar for three days ; put the juice on the fire in a preserving-pan, and allow it to bubble ; add sugar as for raspberry vinegar, and bottle it.' This makes a delicious summer beverage.

Noyau. - To three quarts of best brandy or spirits put three pounds of white sugar broken small, a quarter of an ounce of cinnamon, the peel of two lemons and juice of one, and eight ounces bitter almonds ; mix, and when the sugar is dissolved, pour in a pint of boiling milk: Cover close, stir it every day for three weeks, and filter it through a filtering paper. It may be necessary to add a little more spirits.

Elder Wine-another way.-To three pounds of elderberries put one pound of 
damsons, and to a nine-gallon cask put a pint of sloes. Boil two gallons of water with an ounce of hops for an hour and a half; then put in sugar, such as four pounds to five quarts of water; keep skimming as it rises, and then set it to cool; boil the fruit for half an hour, strain the juice, and put a quart of it to every gallon of water; then let it work with yeast for three days; put it in the barrel and stop it close. The spice and sugar to be put according to taste. When the wine has stood six weeks put in four pounds of stoned raisins.

Pink Champagne.-Boil nine pounds of lump-sugar in three gallons of water for half an hour, skim it well, and pour the liquor boiling hot over a gallon of red and white currants picked, but not bruised. When nearly cold, put in a small teacupful of yeast. Keep it working for two days, then strain it through a horsehair sieve, put it into a small cask with half an ounce of isinglass. Have rather more liquor than will fill the cask to fill it up as it works over. In about a fortnight bung it up. Let it stand till April ; put into each bottle a lump of doublerefined sugar. Let the bottles remain one day uncorked. Cork and wire them. 'They must stand upright in the cellar; when wanted, put a few on their sides for about a week.

Grape Champagne to equal foreign.Gather the grapes when they are just turning, or about half ripe. Pound them in a tub, and to every quart of fruit put two quarts of water. Let it stand in a mash-tub for fourteen days, then draw it off, and to every gallon of liquor add three pounds of lump-sugar. When the sugar is dissolved, cask it, and, after it has done working, bung it down. In about six months it will be fit to drink, when it should be bottled, and the corks tied down, or wired if it is to be kept more than a year.

\section{COOKERY FOR THE SICK.}

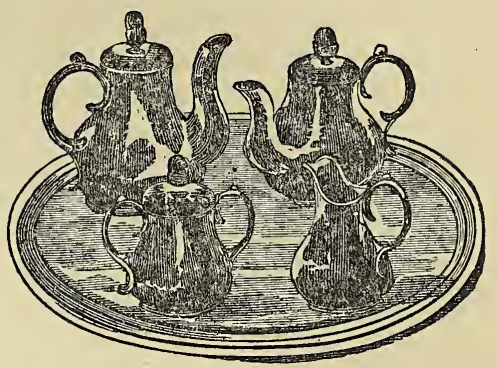

Invalid's Breakfast Set.

IT may be necessary to premise that a choice should be made of those things most likely to agree with the patient. and, as invalids require variety to indulge their appetite, frequent changes should be provided, but great simplicity observed in the preparation. Perhaps jellies and meat broths, together with various kinds of farinaceous food, are the lightest on the stomach, as well as, generally speaking, the most nutritious.

A Good Jelly.-Soak twelve shanks of mutton four hours, then brush and scour them very clean. Lay them in a saucepan with three blades of mace, an onion. twenty Jamaica and thirty or forty black peppers, a bunch of sweet herbs, and a crust of bread made very lown by toasting. Pour three quarts of water to them, and set them on a hot hearth close covered; let them simmer as gently as possible for five hours, then strain it off, and put it in a cold place.

This may have the addition of a pound of beef, if approved, for flavor. It is a remarkably good thing for people who are weak.

Sheep's heads and trotters are also good restoratives; savory jelly may also be made in the same manner, without lemon, wine, or sugar, but flavored with ham and spice. 
Dr. Ratcliff's Pork Jelly.-Take a leg of well-fed pork, just as cut up ; leat it, and break the bone. Set it over a gentle fire with three gallons of water, and simmer to one. Let half an ounce of mace and the same of nutmegs stew in it. Strain through a fine sieve. When cold, take off the fat. Give a coffeecupful the first and last thing, and at noon, putting salt to taste.

Lambs' feet are very nutritious; purchase them ready cleaned; lay them ten minutes in boiling water, by doing which you will be able to draw out the leg-bone with facility; then put them in a stewpan (two would be sufficient), and pour over a pint of water with which you have mixed smoothly a table-spoonful of flour, and half a teaspoonful of salt ; place them upon the fire, stirring frequently until boiling, then add a small onion, with celery, parsley, and parsnip; boil gently for two hours, and when done, serve plain upon a plate, or with a little melted butter and parsley poured over. By using a little white broth from any meat, instead of water, you make a delicious soft soup, which may be partaken of freely.

Calves' feet are dressed in the same manner, but using a double proportion of every thing, and stewing them double the time; they are served precisely the same.

Real Essence of Beef. - Take one pound of beefsteak, cut it into thin slices, which scrape fine; put it into a stewpan, and stir over the fire five or ten minutes, until thoroughly warmed through, then add half a pint of water, cover the stewpan as tightly as possible, and let it remain close to the fire or in a warm oven for twenty minutes; then pass it through a sieve, pressing the meat with a spoon to extract all the essence.

Strengthening Jelly. - Take two pounds of gravy-beef, cut into small pieces; put the pieces into a jar, cover close, and stand the jar in a saucepan of water; let it simmer from four to six hours. This I have made from either beef or veal. I have found it invaluable in cases of sickness, and it will be found that the real essence of the meat is extracted.

\section{A Clear Broth that will keep long.-} Put the mouse-round of beef, a knucklebone of veal, and a few shanks of mutton, into a deep pan, and cover close with a dish or coarse crust; bake till the beef is done enough for eating with only as much water as will cover it. When the broth is cold, cover it close, and keep it in a cool place. When it is to be used, give what flavor may be best liked. Cowheels make good broth.

For a quick-made Broth, take a bone or two of a neck or loin of mutton, take off the fat and skin, set it on the fire in a small tin saucepan that has a cover, with three-quarters of a pint of water, the meat being first beaten and cut in thin bits; put a bit of thyme and parsley, and, if approved, a slice of onion. Let it boil very quickly; skim it; take off the cover if likely to be too weak, else cover it. Half an hour is sufficient for the whole process.

Lietig's Beef Tea.-Chop a pound of lean beef as fine as for sausage-meatmix with a pint of cold water-put it orer a slow fire-when it has boiled five minutes, strain through a coarse cloth. Salt to taste.

Chicken Broth.-Boil a chicken in a quart of water till about three parts cooked, or about a quarter of an hour; remove the skin and the rump, and put it into the water it was boiled in, with a slice of onion, ten white peppercorns, and a blade of mace; then simmer until it has a good flavor. Beat a quarter of an ounce of blanched sweet almonds with a teaspoonful of water, till of a 
good consistence and fine, add this paste /ounces of sugar-candy, an ounce of gum to the broth, simmer for a minute, then arabic, and a nutmeg grated. Mix it all remove, strain, and set aside to get cold. well, and boil it ten minutes; or till When quite cold, remove the fat, and every thing is thoroughly dissolved. warm the broth again; season with salt, Then strain it through muslin, and set it and serve in a broth basin, with toast dice on a plate.

Panada is merely a preparation of bread made up in various ways.

To make it in five minutes.-Set a little water on the fire with a glass of white wine, some sugar, and a scrape of nutmeg and lemon-peel: meanwhile grate some crumbs of bread. The moment the mixture boils up, keeping it still on the fire, put the crumbs in, and let it boil as fast as it can. When of a proper thickness just to drink, take it off.

Or:-Put to the water a bit of lemonpeel, mix the crumbs in, and; when nearly boiléd enough, put some lemon or orange syrup. Observe to boil all the ingredients, for, if any be added after, the panada will break and not jelly.

Chicken Panada.-Boil a chicken till about three parts ready in a quart of water; take off the skin, cut the white meat off when cold, and put into. a marble mortar: pound it to a paste with a little of the water it was boiled in, season with salt, a grate of nutmeg, and the least bit of lemon-peel. Boil gently for a few minutes to the consistency you like; it should be such as you can drink, though tolerably thick.

This conveys great nourishment in a small compass.

Sippets.-On an extremely hot plate put two or three sippets of bread, and pour over them some gravy from beef, mutton, or veal, with which no butter has been mixed. Sprinkle a little salt over.

Port Wine Jelly.-Melt in a little warm water an ounce of isinglass; stir it into a pint of port wine, adding two have weak bowels. picked and washed a quarter of a pound of rice, mix it with half a pound of loaf sugar, and just sufficient water to cover it. Boil it till it becomes a glutinous mass; then strain it; season it with whatever may be thought proper, and then let it stand to cool.

Caudle is made in various ways. Make a fine smooth gruel of half-grits; strain it when boiled well; stir it at different times till cold. When to be used, add sugar, wine, and lemonpeel, with nutmeg. Some like a spoonful of brandy besides the wine; others like lemon-juice.

Another.-Into a pint of fine gruel, not thick, put, while it is boiling-hot, the yolk of an egg beaten with sugar, and mixed with a large spoonful of cold water, a glass of wine, and nutmeg. Mix by degrees. It is very agreeable and nourishing. Some like gruel with a glass of table-beer, sugar, \&c., with or without a teaspoonful of brandy.

Dr. Boerhaave's Sweet Buttermillk.Take the milk from the cow into a small churn; in ten minutes begin, and churn till flakes of butter swim about thick, and the milk appears thin and blue; strain it through a sieve. It should form the patient's drink, with biscuits, rusk, ripe and dried fruits, \&c.

Flour Caudle.-Into five large spoonfuls of water rub smooth one dessertspoonful of fine flour. Set over the fire five spoonfuls of new milk, and put two bits of sugar into it; the moment it boils pour into it the flour and water, and stir it over a slow fire twenty minutes. It is a nourishing and gently astringent food. This is an excellent food for babies who 
Apple Paste.-Pare your apples and cut them down. Weigh them, and allow an equal quantity of white sugar; put them into a jar, and boil till quite soft. Boil your sugar to a syrup, then drop in the apples with a teacupful of marmalade and a little grated ginger; let them simmer ten minutes. Wet your shapes with spirits, and dish them. They will turn out firm, and keep for years. The apples must be put into cold water as they are pared. The proportion of water is a breakfast cupful to two pounds of sugar.

To Bake Apples.-Sweet apples properly baked and eaten with milk are excellent. The best method of baking tart apples is, to take the fairest and largest in size, wipe them clean if thin skinned, and pare them if the skin is thick and tough. Cut out the largest portion of the core from one end, and place the fruit on well glazed earthen dishes or pans, with the end which has been cored upwards, and fill the cavity with refined powdered sugar. Then place them in the oven or other apparatus for baking until sufficiently cooked. Take them out and when cold they are perfectly delicious.

Apple Tapioca (a new receipt for invalids).-Pare, core, and quarter eight apples; take half a spoonful of tapioca; put it to soak and swell all night in the water; put in half a teacupful of white sugar, and a little lemon-peel; put this into a stewpan and let the tapioca simmer ten minutes, then put in the app'es and stew ten minutes more. When the tapioca is clear, it will form a jelly around the apples.

Chicken Jelly.-For chicken jelly take a large chicken, cut it up into very small pieces, bruise the bones, and put the whole into a stone jar with a cover that will make it water-tight. Set the jar in a large kettle of boiling water, and keep it boiling for four hours. Then strain off the liquid, and season it slightly with salt, pepper, and mace; or with loafsugar and lemon-juice, according to the taste of the person for whom it is intended. Return the fragments of the chicken to the jar, and set it again in a kettle of boiling water. You will find that you can collect nearly as much jelly by the second boiling. This jelly may be made of an old fowl.

Baked Milk,-Mix new milk with buttermilk, in the proportion of a pint of the former to a wineglassful of the latter. Let it stand in a covered jar, before the fire, all night ; in the morning it will be as thick as clot ${ }^{+}$ed cream. Pour from one jar to another till it is again of the consistency of new milk; put it into a stone bottle, tightly corked; add a few lumps of white sugar; let it again stand before the fire, (not too near, ) for five or six hours, it will then be ready to drink. Care must be taken in opening the bottle, as it sometimes effervesces. Besides forming a very wholesome and strengthening drink, it is a most delicious and refreshing beverage in summer. Invalids should take about a pint a day. If it is at all acid, it has not been properly made, and should not be taken.

Asses' Milk far surpasses any imitatation of it that can be made. It should be milked into a glass that is kept warm by being in a basin of hot water.

The fixed air that it contains gives some people a pain in the stomach. At first a teaspoonful of rum may be taken with it. but should only be put in the moment it is to be swallowed. The animal should always be milked at the door.

Artificial Asses' Mill.-Mix two spoonfuls of boiling water, two of milk, and an egg well beaten; sweeten with pounded white sugar-candy. This may be taken twice or thrice a day.

Wine Whey.-Stir into a pint of boil- 
ing milk a couple of glasses of wine. Let it boil a minute, then take it from the fire, and let it remain till tine curd has settled; then turn off the whey, and sweeten it with white sugar.

Toast Water.-Pare the crust off a thin slice of stale bread, toast it brown upon both sides, doing it equally and slowly, that it may harden without being burnt; put it into a jug, and pour upon it boiling water; cover the jug with a saucer, and set it in a cool place.

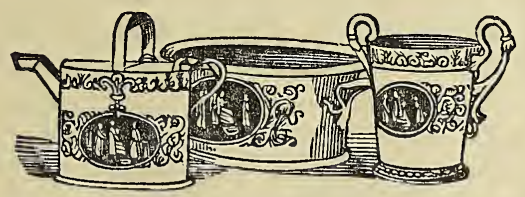

Rice Gruel.-Put a large spoonful of unground rice into six gills of boiling water, with a stick of cinnamon or mace. Strain it when boiled soft, and add half a pint of new milk; put in a tea-spoonful of salt, and boil it a few minutes longer. If you wish to make the gruel of rice flour, mix a table-spoonful of it smoothly, with three of cold water, and stir it into a quart of boiling water. Let it boil five or six minutes, stirring it constantly. Season it with salt, a little butter, and add, if you like, nutmeg and white sugar.

Water Gruel.-Mix a couple of tablespoonfuls of Indian meal with one of wheat flour, and sufficient cold water to make a thick batter. If the gruel is liked thick, stir it into a pint of boiling water-if liked thin, more water will be necessary. Season the gruel with salt, and let it boil six or eight minutes, stirring it frequently - then take it from the fire, put in a piece of butter, of the size of a walnut, and pepper to the taste. Turn it on toasted bread, cut in small pieces.
Molasses Posset.-Put into a saucepan a pint of the best West India molasses, a teaspoonful of powdered white ginger, and a quarter of a pound of fresh butter. Set it on hot coals, and simmer it slowly for half an hour, stirring it frequently. Do not let it come to a boil. Then stir in the juice of two lemons, or two tablespoonfuls of vinegar ; cover the pan, and let it stand by the fire five minutes longer. This is good for a cold. Some of it may be taken warm at once. and the remainder kept at hand for occasional use.

Wine Posset.-Boil some slices of white bread in a pint of milk ; when soft take it off the fire, and grate in some nutmeg and a little sugar; pour it out, put half a pint of sweet wine into it by degrees, and serve it with toasted bread.

Balm Mint, and other Teas.-These are simple infusions, the strength of which can only be regulated by the taste. They are made by putting either the fresh or the dried plants into boiling water in a covered vessel, which should be placed near the fire for an hour. The young shoots both of balm and of mint are to be preferred, on account of their strong aromatic qualities. These infusions may be drunk freely in feverish and in various other complaints, in which diluents are recommended. Mint tea made with the fresh leaves, is useful in allaying nausea and vomiting.

Tapioca Jelly.-Take four table-spoonfuls of tapioca-rinse it thoroughly, then soak it five hours, in cold water enough to cover it. Seta pint of cold water on the fire-when it boils, mash and stir up the tapioca that is in water, and mix it with the boiling water. Let the whole simmer gently, with a stick of cinnamon or mace. When thick and clear, mix a couple of table-spoonfuls of white sugar, with half a table-spoonful of lemon-juice. and half a glass of white wine- stir it into the jelly; if not sweet enough, add 
more sugar, and turn the jelly into cups.

Mulled Wine.-Boil a pint of wine with nutmeg, cloves, and sugar, serve it with slices of toasted bread; or, beat up the yolks of four eggs with a little cold wine, and mix them carefully with the hot wine, pour it backwards and forwards till it looks fine, heat it again over the fire till it is tolerably thick, pour it backwards and forwards; and serve with toasted bread as above.

Or,-Boil some spice in a little water till the flavor is extracted, then add a pint of port wine, with some sugar and nutmeg.

Brandy Mixture.-Mix a gill of brandy, and the same quantity of cinnamon water, with the yolks of two eggs, half an ounce of powdered white sugar, and two drops of oil of cinnamon. This is a valuable restorative in cases of extreme exhaustion, given at intervals of ten to fifteen minutes, in doses of from one to three table-spoonfuls.

Sago Gruel.-Macerate an ounce (or a table-spoonful) of sago in a pint of water for two hours, then boil for a quarter of an hour, taking care to stir it well; sweeten with sugar, and add lemon-juice, nutmeg, or ginger, and white wine if allowed.

Caudle, York.-Take a pint of new milk, turn it with wine, then strain, and put it into a saucepan with two blades of mace, three slices of white bread, and a little grated nutmeg. Boil over a slow fire, then beat the yolks of four eggs, and the whites of two; stir into the caudle to thicken, taking care to stir one way for fear of curdling it; sweeten to taste, and serve warm.

Flax-seed Lemonade-Boil some flaxseed in water until it becomes a thick syrup. To a table-spoonful of flax-seed allow about two tumblers full of water.
Strain it through a cloth and mix with a quarter of a pound of white sugar. Then stir in a little lemon juice. This mixture has frequently been very serviceable in relieving a cold. A little of it may be taken whenever the cough is troublesome. A little gum arabic added to the syrup will be an improvement.

Cocoa.-To two ounces of cocoa allow a quart of water. Put it in a saucepan and let it boil slowly for about an hour. The cocoa shells boiled in this manner will make a very pleasant beverage, and will not be so rich as the ground cocoa.

Barley Water.-Wash carefully two ounces of barley and put it into a saucepan with a quart of water and a few raisins. Let it boil until the liquid is about half diminished. Sweeten it and drink it hot. A little lemon-peel or sume liquorice root put in with the barley instead of or with the raisins, and some sliced figs, may please some tastes. When only a drink is required, it is better to strain the liquid; but the boiled barley with a little sugar will make a very pleasing variety for an invalid to eat instead of rice.

Ground Rice Milk.-Boil together two table-spoonfuls of ground rice with a pint of milk. Sweeten it according to your taste, adding the juice of half a lemon. Let the whole boil half an hour over a moderate fire. Eat it warm.

Compound Camomile Tea.-Twenty camomile flowers, half the thin peel of a lemon, four cloves : pour on them a coffee cup of boiling water, cover and let them stand all night. Strain the liquor in the morning. A wine glass full may be taken a little before breakfast. Dr. Maton always recommended this, with a teaspoonful of salvolatile for indigestion.

Elder Flower Tea.-Infuse the dried flowers as common tea is made. A little acid with sugar will make the taste pleasant. (To promote perspiration.) 
Dandelion and Parsley Tea.-Wash and scrape six dandelion roots and six of parsley ; pour on them a pint of boiling water ; let it infuse three hours before the fire. A little salt or saltpetre may be added. (Lady Cush prescribes this in dropsy ; it acts on the kidneys.)

The flowers of any plant should be aried in every case.

Rice Water.-Boil a quarter of a pound of rice in three pints of water till soft as a paste, skimming it well. Strain the liquor, and sweeten it with sugar or honey. Apples or lemon peel may be boiled with the rice, or lemon juice added. It is an excellent drink in cases of diarrhœa.

Cream of Tartar Water.-Put a large spoonful of cream of tartar into a quart pitcher, with an ounce of gum arabic and thin lemon peel. Pour boiling water on it, and let it stand some hours ; sweeten to taste.

Imperial may be made with two ounces cream of tartar and the juice and peel of two lemons; put in a jar with seven quarts of boiling water. When cold, half a pint of spirits may be added to keep it. Sweeten to taste and bottle it.

Marsh Mallow Emulsion.-Slice the dried roots of marsh mallows, and boil four ounces with one and a half ounce chopped raisins in a quart of water. When it is boiled till there is a good sediment, strain it, and sweeten with water. Add water to taste.

Linseed Emulsion.-One ounce linseed, two drachms liquorice root sliced and bruised; a pint of boiling water. Let it stand four hours by the fire ; then strain it.

Rennet Whey.-Infuse a piece of the skin in a little boiling water, as for making cheese ; let it stand an hour or two; then put a table-spoonful to three pints of new milk, warmed; cover with a cloth, and leave it until the curd is thick. Press out and use the whey.

Iceland Moss Jelly.-Wash and bruise Iceland moss, and soak it all night ; dry and boil it-putting an ounce to a quart -till reduced to one-half the quantity of water; strain it through a sieve. Take it with milk or wine, or flavored to taste. Carageen, or Irish moss jelly can be made the same way.

Blancmange.-Take gelatine, or isinglass, two ounces to one and a half pints water; dissolve it, add a pint of milk, and strain it ; flavor and sweeten it, and let it boil up; then turn it into a mould.

Rice Blancmange.--Boil as much ground rice in a pint of milk as will make it thick enough to turn out of a mould, sweetening and flavoring it. A sauce can be added of milk, cream, or custard.

Somersetshire Fromity.-Wash a quart of wheat, and boil it soft; add by degrees two quarts new milk; boil it till soft and mixed; then add the yolks of a few eggs, well beaten, nutmeg, sugar, and a little ginger, with currants or chopped raisins, if approved for the invalid.

Baked Pears. - Put into a pint of water some mace, cinnamon, and a few cloves; boil them; add a pint of white wine, and the juice of two oranges, and sweeten with loaf sugar. Cut some baking pears into halves or quarters ; put them into the syrup, and then into a moderately heated oven, or a stewpan over the fire. Cover and cook them til' soft. Serve them with the liquor. They will keep in jars. Pears are excellent baked in molasses with a little water added.

Millet Pudding.-Wash the millet; simmer two table-spoonfuls with a little butter in a pint and a half of milk. Two or three beaten eggs may be added. The 
sugar and flavoring must be put in while the milk is boiling. Bake or boil it.

Ground Rice Pudding.-One and a half pints new milk, with flavoring of coriander seeds, cinnamon, lemon-peel, and sugar. Boil a few minutes, strain it upon two ounces ground rice and boil twelve minutes. Add not quite an ounce of fresh butter, a little salt, and three beaten eggs. Bake in a dish or boil it.

Barley flour may be substituted for the rice.

Calf's Feet Pudding.-Calf's feet minced, taking off the brown and fat, one and a half suet minced, yolks of six and whites of four eggs, the crumb of a roil, a little sugar, and a few chopped raisins. Add milk enough to moisten it, and boil it eight or nine hours. Serve with any sauce.

Marrow Pudding.-One pint milk boiled with cinnamon and lemon-peel, with a pinch of salt, quarter of a pound beef's marrow minced fine, slices of citron and orange peel, grated nutmeg, half a pound sponge cake, a little sugar, a glass of wine or brandy, and two eggs. Bake it.

Dr. Jophson's Soup.-Cut into pieces a pound of lean veal, the same of beef, with water to cover them, in an earthen jar. Tie it down, add salt, and simmer in a water bath twenty-four hours.

Essence of Meat, or Glaize.-Put two pounds meat, of bird or animal, into a bottle, and that into a pan of water. Let it boil fifteen minutes. Strain the liquor that comes out. It will keep in a skin, and can be diluted for broths or gravies or soups.

Marrow Toast.-Boiled marrow spread on toast with a little salt, is said to be good in allaying vomiting from irritation, after the operation of an emetic, or any other cause.

Kedgeree.-Boil soft a tea-cupful of rice; chop a builed whitefish to pieces, take out the bones; add the rice, with three ounces of butter. Stir it on the fire, and add salt and cayenne pepper, with chopped hard boiled eggs. Serve it dry and hot.

Ground Rice Paste.-Boil a quarter of a pound in a little water, strain and beat it in a mortar with one egg, a small lump of butter and a pinch of salt; mould the paste as for tarts, and bake sweetmeats in it.

Arrow-Root Transparent Jelly.-Put a good teaspoonful of arrow-root into a basin, which mix smoothly with two spoonfuls of water, then add enough boiling water to make it about the consistency of starch, stirring all the time; pour it into a stewpan, and stir over the fire until it has boiled two minutes; add a little cream, a small glass of wine, and a little sugar, and serve.

Lemonade.-Pour one quart of boiling water on the rinds of six lemons, and let it stand for three or four hours; add the juice of the lemons with three-quarters of a pound of sugar ; simmer it well and skim it ; then add another quart of boiling water. Either run it through a jellybag, or mix a glass of calf's-foot jelly, which will make it rich.

Tamarinds, or Cranberry Juice, with double the quantity of water, also form a pleasant drink for an invalid, when approaching to convalescence.

Almond Water.-Blanch five ounces of sweet and two of bitter almonds, pound them in a mortar, adding a few drops of water occasionally, to prevent their becoming oily, set a pint of syrup to boil, and throw in the mashed almonds; boil all together a minute, then set it at the corner to simmer for a quarter of an hour; it is then ready to pass through a fine sieve for use. When required, add any quantity of cold water, according to taste or direction, to make it palatable.

Lait de Poule, (French remedy for 
colds.) - This may be made from any broth. Break a fresh egg, separate the white from the yolk, put the yolk in a basin, with a wine-glassful of good cream or milk, which mix well with a spoon; have half a pint of broth boiling, which pour gradually over the egg and cream, mixing it (as you pour the broth) with a wooden spoon; it is then ready, and ought to be taken when going to bed.

Sweet Lait de Poule.-This is also reckoned very good for a cold. Put two yolks of eggs into a cup, with two teaspoonfuls of pounded sugar, part of the rind of a fresh lemon grated; beat them well together for ten minutes, then pour boiling water gradually over, keeping it stirred until the cup is nearly full. Drink this very hot when in bed.

Sago Fruit Pudding.-A correspondent of the Cottage Gardener says :

"Being forbidden the use of pastry, I use rice and sago as substitutes, in the following manner :-Boil a teacupful of sago as thick as it can be made to boil without burning: put about five table-spoonfuls in the bottom of a quart basin; then a layer of baked fruit of any sort (sweetened), and fill the basin to the brim with alternate layers of fruit and sago. Put it in a cool place for some little time, and it will become solid. It is best when made shortly after breakfast, and allowed to stand till wanted, to warm either in an oven, over boiling water, or before the fire with a plate turned over it, for dinner. The sago boils best when soaked in cold water for a few hours before using; rice is used in exactly the same way. By way of change, I sometimes line a basin with the rice or sago, when very thick, and spread a thick layer of the same over a large dinner plate. When cold and stiff, I turn the basin over it, and with a knife cut the sago round the edge of the basin; the parings I put in the bottom of the basin, and then fill with baked fruit, after which I put the sago in the plate on the top of the basin, to act as a cover. The smooth side must be upwards. Eaten with mock cream, made as follows, it is delicious:-Pour half a pint of boiling milk on a teaspoonful of arrowroot, well mixed with a small quantity of milk; stir the mixture well, and have the white of an egg well beaten, and when about half cold add it, and placing the whole over the fire, stir till it nearly boils, then strain for use."

Tapioca Milk.-Soak an ounce of tapioca in a pint of cold water for half an hour, pour off the water, and add a pint and a half of good milk; boil slowly until the tapioca is dissolved, then add sugar, nutmeg, and a little white wine, if not prohibited by the medical attendant.

Italian Cheese.-To a pint of scalded cream, whipped very smooth; add the juice of three lemons, and the rind of two-sugar to taste; let it stand for half an hour, then whip till it is very thick, tie it in a thin cloth, or a tin with holes in it ; let it drain till next day, then turn out.

Soyer's Curry Custard.-Break three whole eggs in a basin, and beat them up, add a quart of milk, a little salt, a very little sugar, and two teaspoonfuls of curry paste; bake in a dish or basin. This is very light and nutritious.

For those who can afford it, roast a leg of mutton, and when nearly done put a hot dish under it, and with a sharp knife make several deep incisions in every part of it; let it remain two minutes longer, that all the gravy may fall into the dish; pour into a cup, take off the fat, and give to the patient as may be required; the mutton will do for curry or hash for the family. This may be called gigot de mouton sacrifie; but when life may almost depend upon this extravagance as you may call it, the expense must not be thought of. 
Decoction of Iceland Liverwort.-An ounce of liverwort must be carefully freed from the moss, fragments of stalks, and particles of dirt, with which it is frequently mixed, by rubbing it between the hands in cold water. Then steep it for two hours, in such quantity of cold water as will completely cover it; after which it must be bruised, pounded, or cut, and the steeping continued for three or four days longer, in a fresh quantity of boiling water, which when the steeping is finished, must be strained off by pressure. The liverwort is then to be put into a quart of fresh water, and kept boiling until the fluid be reduced twothirds, or to a pint and a quarter. When strained and allowed to cool, it forms a thick mucilage, free from any bitter taste, and may be rendered very palatable by the addition of sugar and lemonjuice; or by white wine, in those cases which permit the use of wine.

This decoction of liverwort is an excellent demulcent nutriment, in consumption, dysentery, and in convalescence from acute diseases, and particularly after the whonping cough, in which case the bitter need not be completely removed, as it tends to invigorate the digestive organs.

Vermicelli au Lait.-Boil a pint of milk, and when boiling, add sufficient vermicelli to make it about the proper thickness; it may be served quite plain if required, or seasoned with sugar.

\section{FOOD AND COOKERY FOR CHILDREN:}

The kinds of food to be given to very young children should be of the simplest description : besides cow's milk, diluted with one-third part of water, arrozo-root and milk; pearl sago boiled in water till perfectly soft, and thinned with new milk ; rusks ; biscuit powder ; flour pre- viously boiled like a pudding in a basin till it is formed into a hard ball, from which portions can be grated into milk, and boiled in it till it thickens the milk, are the chief ingredients used as food for infants.

Rice, barley, and farina gruels, are also used with advantage; and as a change in the diet of children is advisable when they are occasionally indisposed, these kinds of gruel may be found useful varieties in the food of children.

Food which contains milk should never be warmed over and over again, as is sometimes done; nor should it be suffered to remain, when cold, in metal utensils ; if intended for future use, it should be put into earthen-ware basins, and set in a cold place; but no food thus set by for another day should have milk put in to it.

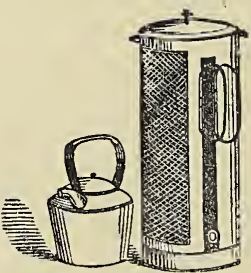

Nurse Lamp, with Tea Kettle to keep children's food warm.

The nutriment of children, after the completion of their first year and a half, it will be necessary to increase in solidity, and, if their appetite seems to demand it, in quantity also. Their teeth begin now to aid them in masticating food, their limbs become firmer, and carry their bodily exertions often to a great extent; the degree of exercise which healthy children voluntarily give themselves, stimulates the digestive organs, enabling them to receive and act upon more solid nutriment. Having previously had animal food three or four times a week, children may, in the second year of their lives, safely eat it once 
every day-meats being chosen for them that are tender and nutritious. Roast beef; mutton, either boiled, roasted, or broiled; chickens, some kinds of fish, well-boiled vegetables, such as brocoli, turnips, potatoes (the latter are best for them when mashed with hot milk and a little salt); simple compounds of egg and milk; fruits, baked or boiled ; bread well made and baked, with small portions of butter; these, with milk for breakfast and supper, with water as beverage, are the chief articles of wholesome food for children as long as they continue under nursery management.

The species of food we would not give them consists of salted meats (beef, pork, or bacon); of rich stews, ragouts, and soups ; of fried fish, of pastry and confectionery generally; of cheese ; and of beverages, tea, coffee, beer, and wine. Of the two last mentioned, beer and wine, we may remark that, in cases of delicate health, they may be advantageously allowed; but they should always be given under medical direction, for the stimulus of fermented or spirituous liquors is not in every case of debility beneficial ; sometimes the reverse; neither ought children to be allowed to drink much at a time of any liquid, however mild and innocent it may be. Too much liquid has a tendency to create flatulency, and to distend the bowels. It has been said that parents accustoming their children to drink water only, bestow a fortune upon them of the value of which they will be sensible all the days of their life.

Children's meat should be minced as small as possible. If too much trouble is left to their feeble power of masticating, they will shun it by swallowing their food unmasticated. For children whose digestion is weak, it may even be desirable to have their meat pounded.

Frerich Pap.-Put a table-spoonful of Hour into a pap saucepan, to which add by degrees two gills of milk, mixing it into a very smooth batter with a wooden spoon; place the saucepan upon the fire, let it boil ten minutes, keeping it stirred the whole time, or it is liable to burn or become brown; then add about half an ounce of sugar and a little salt, put it into a basin, and it is ready for use. A little butter is also very good in it. It may be made with di'y rusk or cracker, scalded merely in water, and beaten to a pulp with a little sugar.

Infant's Food.-Take a pound of the best flour, tie-it very tightly in a strong cloth, and put it into a pan of boiling: water (in which put a plate to prevent the cloth sticking to the bottom of the pan). Boil it for three hours without allowing it to go off the boil-when coldish untie the cloth, and scrape off the outside of the ball: when to be used, grate down the quantity required and break it with cold water; boil four or five minutes only, and sweeten to the taste. Flour prepared in this way is confidently recommended by an experienced sick nurse as a soft and nutritious food for the youngest infant, and will keep for a month or more in its hard compact state. Milk may be added when about to be eaten, if wished.

Semoulina.-Semoulina is very delicate and glutinous; it is good in any kind of broth or milk for invalids or children, of easy digestion, and having also the advantage of being tolerably cheap and quickly cooked.

Arrowroot.-When broth is boiling, put two teaspoonfuls of arrowroot into a cup, which mix smoothly with a gill of cold broth, or half ditto of water ; then pour it into your boiling broth, which keep stirring with a spoon; let it simmer ten minutes, and it is ready for use.

Cakes for Children.-Sugar and egg, browned before the fire, or dropped as fritters into a hot frying-pan, without fat, will make them a nourishing delicacy. 
Rice Pudding with Fruit.-In a pint of new milk put two large spoonfuls of rice well washed; then add two apples, pared and quartered, or a few currants or raisins. Simmer slowly till the rice is very sofit, then add one egg, beaten, to bind it. Serve with cream and sugar.

To prepare Fruit for Children-A far more wholesome way than in pies or puddings, is to put apples sliced, or plums, currants, gooseberries, \&c., into a stone jar, and sprinkle among them as much sugar as necessary. Set the jar in an oren or on a hearth, with a teacupful of water to prevent the fruit from burning; or put the jar into a saucepan of water till its contents be perfectly done. Slices of bread or some rice may be put into the jar, to eat with the fruit.

Rice and Apples.-Core as many nice apples as will fill the dish; boil them in light syrup; prepare a quarter of a pound of rice in milk, with sugar and salt; put some of the rice in the dish, and put in the apples, and fill up the intervals with rice, and bake it in the oven till it is a fine color.

A nice Apple Cake for Children.Grate some stale bread, and slice about double the quantity of apples; butter a mould, and line it with sugar paste, and strew in some crumbs, mixed with a little sugar; then lay in apples with a few bits of butter over them, and so continue till the dish is full; cover it with crumbs, or prepared rice; season with cinnamon and sugar. Bake it well Fruits that have seeds are much healthier than stone fruits. 'But all fruits are better, for very young children, if baked or cooked in some manner, und eaten with bread. The French always eat bread with raw fruit. Apples and winter pears are very excellent food for children, indeed, for almost any person in health; but best when eaten at breakfast or dinner. If taken late in the evening fruit often proves injurious. The old sayingapples are gold in the morning, silver at noon, and lead at night, is pretty near the truth. Both apples and pears are often good and nutritious when baked or stewed, for those delicate constitutions that cannot bear raw fruit. Much of the fruit gathered when unripe, might be rendered fit for food by preserving it in sugar.

Ripe Currants are excellent food for children. Mash the fruit, sprinkle with sugar, and with good bread let them eat of this fruit freely.

Blackberry Jam.-Gather the fruit in dry weather; allow half a pound of good brown sugar to every pound of fruit; boil the whole together gently for an hour, or till the blackberries are soft, stirring and mashing them well. Preserve it like any other jam, and it will be found very useful in families, particularly for children-regulating their bowels, and enabling you to dispense with cathartics. It may be spread on bread, or on puddings, instead of butter: and even when the blackberries are bought it is cheaper than butter. In the country every family should preserve, at least, half a peck of blackberries.

Bread and Milk.-Cut about two ounces of any white bread into small thin slices, and put them into a small basin or a large breakfast cup. In a little saucepan (only used for that purpose) have half a pint of milk; when upon the point of boiling, pour over the bread; cover over the cup five minutes, and it is ready for use.

Porridge.-When children are delicate: porridge is often preferable to bread and milk. Put two table-spoonfuls of grits or oatmeal in the milk saucepan, which moisten with half a pint of milk; let it boil ten minutes, keeping. well stirred, add a small piece of butter and a little sugar, and it is ready for use. 
Meats for Children.-Mutton, lamb, and poultry, are the best. Birds and the white meat of fowls. are the most delicate food of this kind that can be given. These meats should be slowly cooked, and no gravy, if made rich with butter, should be eaten by a young child. Never give children hard, tough, half cooked meats, of any kind.

Vegetables for Children, Eggs, \&c.Their rice ought to be cooked in no more water than is necessary to swell it ; their apples roasted, or stewed with no more water than is necessary to steam them; their vegetables so well cooked as to make them require little butter, and less digestion; their eggs boiled slow and soft. The boiling of their milk ought to be directed by the state of their bowels : if flatulent or bilious, a very little currypowder may be given in their vegetables with good effect-such as turmeric and the warm seeds (not hot peppers), are particularly useful in such cases.

Fotatoes and Peas.-Potatoes, particularly some kinds, are not easily digested by children; but this is easily remedied by mashing them very fine, and seasoning them with sugar and a little milk. When peas are dressed for children, let them be seasoned with mint and sugar, which will take off the flatulency. If they are old, let them be pulped, as the skins are perfectly indigestible by children's or weak stomachs. Never give them vegetables less stewed than would pulp through a colander.

Children's Diet for the day (Soyer).-

Bread and milk for breakfast at eight; the dinner at one, as follows throughout the week: roast mutton and apple pudding ; roast beef and currant pudding, baked apples; boiled mutton with turnips, after which rice or vermicelli pudding; occasionally with beef, suet dumplings, plain and with currants in them, or pease pudding; or if unwell, a little veal or chicken-broth, or beef-tea. When in business, the first three years the children used to dine with us at one, at a side-table with their nurse. They then had a little plain meat cut small in their plates, with potatoes, pieces of bread, and gravy, after which, three times a week, plain rice, bread, or other p'ain pudding, or rhubarb or apple tart; and, at five o'clock, their bread and milk again, previous to going to bed.

But I should recommend for nurserymaid and all :

First, about two pounds of mutton, well cooked, but with the real gravy of the meat in it, which will require about one hour before a moderate fire ; dredge it ten minutes before being done; when taken up and in the dish, sprinkle a little salt over the meat, and pour over three or four spoonfuls of hot water to make a little light gravy.

Or, a few slices of roast beef, or a small piece roasted on purpose; after which a very plain currant pudding; or, occasionally, a little pickled pork, with pease pudding; or roast pork, with baked apples; and now and then a little salt beef, but very well boiled, with suet dumplings ; and occasionally, for change, either bread, vermicelli, or tapioca puddings; in case of illness, and with the approbation of the doctor, veal, mutton, or chicken-broth, sago, gruel, panada, \&c.

\section{SAVORY DISHES FOR BREAKFAST.}

Grillades.-Perhaps there is no better grill for breakfast than a cold blade-bone of mutton, off which the greater part of the meat has been already cut. The remaining parts should then be scored, and the gashes filled with a mixture of mus. tard, cayenne, common pepper, and salt, as for a devil; it should then be put upon 
the gridiron, and well browned, but not burnt. It may be sent to table dry, without any sauce, or served with gravy mixed with capers.

The bones of cold fowl of all kinds are also frequently brought thus to breakfast.

Broiled Chicken.-Split it open, pepper it only on the inside, broil and serve it with a very little butter, and accompanied with broiled mushrooms.

Kabobs.-Put thin slices of well-seasoned kidney and bacon, with beef or mutton also if you please, upon skewers. Have ready fine bread-crumbs, with a slight grating of lemon, brush them over with egg, and roll them into the crumbs. Hang up the skewers to roast, and put a slice of toast under to secure the gravy: when served, carefully remove the skewers, and place the "kabobs" on the toast ; but, if only a quantity sufficient to help one person be put on each skew$\mathrm{er}$, then do not remove them.

If, instead of meat, an oyster be put between each slice of bacon and kidney, it will be found very superior.

Dried Salmon is likewise a good relish; and fresh mackerel, split open and broiled.

German Toast.-Take the remainder of any fricassee or ragoût ; any quantity will do ; chop it fine, add a little chopped parsley, and a little bit of shalot or chive : mix it up with one or two eggs beaten, according to the quantity. Put the whole with its gravy into a stewpan, and let it reduce and thicken on the fire. Let it remain until it be cold, then cut pieces of bread, toast them: lay the mixture thickly upon them. Boil an egg hard, cut it into small pieces, and stick them on the top; brush the whole with egg beaten up, sift bread-crumbs over, and bake them in the oven; squeeze a little lemon-juice on the top. This makes also a good corner dish for dinner.
Vegetabie Toast.-Take any stewed vegetable, and make it into a purée. Add a little more seasoning, mix it up with the yolk of an egg, and, if too thin, reduce and thicken it over the fire, then spread it upon toast, brush it over with beaten eggs, and bread-crumbs or vermicelli, and fry or bake them.

Anchovy Toast.-Make a well-buttered toast of brown bread, if you can get it ; scale and bone a proper number of anchovies, with little or no washing ; cut them into broad slices, and place them upon the toast, seasoning it with mustard, without pepper.

Sausage Toast.-Fry two or three sausages; when quite hot, strip off the skins, and spread the meat upon the toast buttered with salt butter; season it with a little pepper and mustard. It will be improved by a grating of old cheese.

Kidney Toast.-Take a cold veal kidney, with a part of the fat, cut it into very small pieces; pound the fat in a mortar with a little salt, white pepper and an onion previously boiled. Bind al together with the beaten whites of eggs. Heap it upon toast; cover the whole with the yolks beaten; dredge with bread-crumbs, and bake in the oven.

Ham Toast.-Grate or pound the cold ham ; toast and butter a slice of bread; mix the ham with the yolk of an egg and a little cream, until it is thick and rich; warm it over the fire, and serve it very hot upon the toast. Tongue may be employed in the same way.

Scotch Woodcock.-Toast and butter three or four slices of bread on both sides; take nine or ten anchovies washed, scraped, and chopped fine, and put them between the slices of bread. Beat the yolks of four eggs in half a pint of cream, and set it on the fire to thicken, but not to boil. Then pour it over the toast, and send it to table as hot as possible. 
Sheep's Rumps and Kidneys.-Boil the rumps in gravy, and lard the kidneys ful. with bacon; set them, along with the rumps, before the fire in a small tin oven, rubbing them at the same time with yolk of egg and a little cayenne and nutmeg. When of a light brown, dish them up, either dry or with any pungent sauce, and garnish them with parsley.

Bacon Broiled.-The streaked part of a thick flank of bacon is to be preferred; cut nice slices, not above a quarter of an inch thick, take off the rind, put to broil on the gridiron over a clear fire ; turn it three or four times in the space of five minutes. Serve it very hot. If any remain after dinner of boiled bacon, it is very good broiled or fried for next day's breakfast. Broiled and boiled bacon has been highly recommended to the dyspeptic.

Devils of Biscuits.-Butter them on both sides; sprinkle cayenne pepper on the top, and send them to be grilled This may be varied by the addition of chopped anchovies, or the essence, or diavolo paste.

Or:-Make a slice of cheese into a paste with made mustard, and lay it upon one side.

A Dry Devil.-Take the liver, gizzard, drumstick, and sidebones of a fowl or turkey ; score them; lay on made mustard very thickly, and add a quantity of cayenne pepper and salt, and broil them.

A Wet Devil.-Take any part of a turkey, goose, or fowl, cover it with mustard, Chetney, diavolo paste, or any other piquant condiment ; put a dessert spoonful of cayenne pepper, one of pounded white sugar, the juice of a lemon, a glass of wine, and a glass of ketchup, to a teacupful of gravy; heat them together, pour over the devilled fowl, and send up very hot.

A little cold fresh butter will cool the mouth, should the grill prove too power-

Devilled Game.-Split a woodcock. a snipe, a wild duck, or any ocher game that is undone. To a teaspoonful of salt add a dessert spoonful of cayenne pepper with a tablespoonful of curry-powder, and wet the whole with French mustard. Cover the birds both inside and out with this mixture. Take out the brains when the head is split, and, if a woodcock, add the trail; make them into a paste with. the yolk of a hard-boiled egg, half a blade of pounded mace, a saltspoonful of grated lemon-peel, and season it with essence of anchovies, ketchup, or other sauce; then add a glass of wine, a teaspoonful of cayenne pepper, a dessertspoonful of pounded white sugar, and the juice of a lemon or orange. Roll a bit of butter, the size of a walnut, in flour ; put the birds and the sauce into a metal dish over a lamp, cover it close, shake it about, occasionally, and when it has stewed until the greater part of the sauce has been imbibed, serve it round. The legs of fowls, geese, and turkeys, may be devilled the same way.

Mustard.-No savory things are ever brought to table without this indispensable accompaniment.

In making it, pure water is most generally used, with a pinch of salt and sugar, though both are frequently omitted : but a much better mode for preserving it, as well as adding to it a good flavor, is to make it of water in which horseradish has been boiled. This costs no trouble; for if the horseradish be scraped and put in a teacup with boiling water poured upon it, and covered, the infusion will be strong enough in little more than half an hour.

The Parisian mustard, known as moutarde de maille, is however much in vogue among gastronomists, as possessing a finer pungency of flavor, and may 
be imitated by mixing up the flour and a little fine salt with salad oil, so as to form a thick paste, then diluting it to a certain degree with equal quantities of the vinegars of horseradish, tarragon, chili, and garlic ; or sufficient of each to please the palate. Cayenne should never be used, and even chili vinegar but sparingly.

Sandwiches require more care than is usually bestowed on them, for this reason, that every one believes he can cut sandwiches. Where any quantity is required the bread should be made on purpose, and the bakeir should be desired to bake it in tins, and either add a little butter to it or raise it well before it is put into the tins, so that it should not be full of holes, as in that case too much butter is used, and the sandwich becomes disagreeable from being greasy. Cut the bread moderately thin, butter it very slightly indeed, lay the meat cut thin, season it with salt, pepper, and mustard, as may be required : cover with a second slice of bread, trim the edges, put them one on the other, and cover with a damp cloth until wanted.

Where tongue is used it should be boiled the day before, and, when thoroughly done, pressed in the mould in which the bread is to be baked.

Chickens boned, and farced with a small quantity of farce of veal and ham, and treated in the same way, will make excellent sandwiches.

Soyer recommends for breakfast.also-

Scrambled Eggs.-Break three eggs into a small stewpan, add a saltspoonful of salt, a quarter of that quantity of pepper, and two ounces of fresh butter, (the fresher the better;) set the stewpan over a moderate fire, and stir the eggs round with a wooden spoon, being careful to keep every particle in motion, until the whole has become a smooth and delicate thickish substance; have ready a convenient-sized crisp piece of toast, pour the eggs upon it, and serve immediately. Herring may be laid between the eggs and toast.

Eggs au Beurre (a new Method.)Let the eggs boil six minutes, then take them out, dip them for two seconds in cold water, crack and peel off the shells, and lay them in a hot plate; cut each egg in halves length wise, spread a little fresh butter, and sprinkle a little salt over the interior, and eat them very hot.

The following articles for Breakfasts are popular in Spring and Summer :Veal Cutlets-Stewed or Fried ClamsCold Boiled Ham or Tongue - Clam Fritters - Broiled Shad-Broiled and Fried Fish-Stewed or Broiled Chickens -Minced Veal-Pickled Salmon-Fried Sweet Potatoes-Fried Egg PlantGreen Corn Fritters-Stewed PigeonsBroiled or Sliced Raw T'omatoes-Hashballs - Cucumbers - Pepper Grass Cresses-Game Birds-Eggs-Omelets of all kinds-Tongue or Ham ToastRice Cakes-Batter Cakes-Ripe Fruits. Jam, marmalade and honey are appropriate.

In Winter-Additional.-Stewed Pigeons with Mushrooms-Stewed or Fried Oysters-Broiled and Stewed FowlFried Egg Plant-Pork Cheese-Hashed Poultry-Deviled Gizzards--SteaksChops-Liver Pudding-Fried Pigs' Feet -Venison Steaks-Venison PastyFried Smelts-Minced Codfish-Fish Cakes-Sausages-Hot Cakes of all kinds, Buckwheat and Flannel Cakes especially; also Hominy and Mush.

A celebrated cook says :-When I was first married and commencing business, our means were limited: the following was our system of living :-

Sunday's Dinner.-Roast Beef, Potatoes, Greens, and Yorkshire Pudding. Monday.-Hashed Beef, Potatoes, and Bread Pudding.

Tuesday.-Broiled Beef and Bones, Vegetables and Apple Pudding. 
Wednesday.-Fish, if cheap, Chops, Vegetables, Pancakes.

Thursday.-Boiled Pork, Pea Pudding, Greens, and Rice Pudding.

Friday.-Pea Soup, remains of Pork, and Baked Batter Pudding.

Saturday.-Stewed Steak with Suet Dumpling, and Rice in a mould with sauce.

Dinner for Dinner Party.-SoupFish-Saddle of Mutton-Cutlets. Maintenon-Greens-Potatoes- Sweet Breads -White Sauce-Cranberry Jelly-Pickles-Turkey Roast-Celery SauceGame Course-Partridges-Wild Ducks French Salad-Jelly of Fruit-Cheese Cakes-Meringues à la Crème-Frozen Plum Pudding-Dessert.

2d. For a Party of Twenty Persons.Soups-Two Dishes of Fish-Haunch of Mutton-Boiled Capons-Fricandeau of Veal-Curry of Fowl-Fillets of Beef with Tomato Sauce-Oyster PattiesCroquettes of Veal or Fowl-Cutlets Soubise-Wild Ducks-Guinea Fowl Larded -Scolloped Oysters-Charlotte RusseApple with Rice--Meringues-Punch Jelly-Turban-Cheese Soufflé-Dessert.

For Supper Parties.-Cold Roast Fowls-Lobster Salad-Chicken Salad - Mayonnaises of Fowls-Ham-Tongue cut in slices, garnished with Parsley and Aspic Jelly--Grouse Pie-Boned Turkey -'Terrapins-Game Birds-Oysters Stewed, Fried-Raised Pastry-JelliesIce Creams-Pyramids of Candied Fruits - Cakes-Compotes of Frúits-Confectionery, \&c.

Children's Party.-- SandwichesDishes of Lamb-Ham-Beef-Tongue -Fowls-Pastry-Custard-JelliesBonbons.

Another (Soyer.) - A Roast TurkeyTwo Dishes of Fowls-One Ham-Two Pigeon Pies-One Piece of Boiled Beef -Four Lobsters-Four Salads-Four Jellies-Four Tarts-Four of Preserved 34
Fruit, \&c.-Four of Pastry, with about twelve of various kinds of Fruit, \&c.

\section{SPRING.}

Breakfast.-Tea-Cocoa-Flannel Cakes-Meat Cakes-Twist Bread-Liver Puddings-Sausages.

Dinner.-Pepperpot-White Potatoes-Lima Beans-Sweet PotatoesStewed Oysters-Stewed Chickens.

Breakfast.-Tea-Coffee-Rye Batter Cakes-Boiled Eggs-Cold BreadSliced Ham-Beefsteak.

Dinner - Vermicelli Soup-White Potatoes-Tongue-Hominy-Fried Oysters-Boiled Fowls.

Dinner.-Soup-Potatoes-Ruta Baga Turnips-Asparagus--Mint Sauce--Roast Lamb.

Dinner.Fish - Potatoes - SaladRoast Veal-Egg Sauce-Greens.

\section{SUMMER}

Dinner.-Ochra Soup-Squashes-Fried Chickens-Green Corn-Potatoes -Salad-Buttered Beets-Beef Steak.

Breakfast.-Cold Bread-Dry ToastClam Fritters-Minced Veal-Cream Cheese.

Dinner. - Soup - Egg Sauce - Celery Sauce - Potatoes - String Beans Tongue-Squashes-Boiled Fowls.

Dinner.-Lamb Soup-Green Peas -Potatoes-Asparagus-Spinach and Eggs-Veal-Cutlets.

Dinner.-Green Pea Porridge-Potatoes-French Beans-Cucumbers-Currant Jelly-Pickles-Roast Lamb.

Dinner.-Soup-Squashes-Buttered Beets-Green Corn-Roast Veal.

Dinner.-Soup - Fish - Potatoes String Beans-Fried Squashes-Corn Fritters-Fried Chickens. 
WINTER.

Dinner.-Beef Soup-Sweet Potatoes-Turnips-Cranberry Sance-White Potatoes-Leg of Pork Stuffed and Roasted.

Breakfast.-Tea-Coffee-Indian Batter Cakes-Rolls-Beef Hash-Fried Liver-Pork Steaks.

Dinner.-Veal Soup-Turnips-Baked Sweet Potatoes-Mashed PotatoesHorse Radish-Roast Beef.

Dinner.-Soup-Fish-Pickled Beets - Sweet Potatoes-White Potatoes-Baked Beans-Roast Ducks.

Dinner.-Calves' Head Soup-White Potatoes-Hominy-Turnips-PicklesFillet of Veal Stuffed and Roasted.

Dinner.-Soup-Cranberry Sauce--Ham-Fried Oysters-White Potatoes - Sweet Potatoes-Roast Turkey.

Dinner. - Soup-Roast Goose-Apple Sauce-Onion Sauce-Potatoes-Sweet Potatoes-Tonguc-Celery--Stewed Oysters.

Dinner.-Calves' Head Soup-Potatoes-Beets-Stewed Oysters-Turnips-Sweet Potatoes-Onion SauceBoiled Turkey.

Dinner.-Soup-Fish-Currant Jelly -Potatoes-Turnips-Roast Mutton.

Dinner.-Beef Soup-Smoked Pig's Head with Cabbage-Potatoes-Turnips -Macaroni-Beets-Celery-Sweet Potatoes-Roast Mutton-Batter Pudding Baked-Brandy Peaches-Pain PerduFloating Island.

Dinner.-Vegetable Soup-Roast Pig - Potatoes-Onions-Oyster Pic-Turnips-Rice-Boiled Turkey-Vermicelli Pudding - Cocoanut Custard - Tipsy Cake-Indian Pudding.

Dinner.-Clam Soup--ChowderRoast Fowls-Beets-Mangoes-Toma-
toes-Turnips-Maccaroni - PotatoesLamb Pot Pie-Plum Pudding--Rice in a mould with custard-Wine SauceCracker Pie.

Dinner.-Soup-Roast Beef-CeleryMacaroni-Turnips - Sweet PotatoesPotatoes-Fricasseed Chickens-Boiled Tongue - Boiled Flour Pudding - Tartlets-Compote of Peaches.

Dinner.-Soup à la Julienne-Roast Beef-Green Corn-Mangoes-Tomatoes -Potatoes-Beets-Squashes-Pilau of Chickens-Custard Pie-Rice Flummery - Baked Pears-Green Corn Pudding.

Dinner.-Mullagatawny Soup-Roast Lamb - Mint Sauce - Macaroni - Potatoes-Boiled Tongue-Tomatoes-Rice -Roast Ducks-Bread Pudding-Jellies -Slip-Cherry Tartlets.

Dinner.-Oyster Soup-A la mode Beef-Turnips--Sweet Potatoes-Beets -Potatoes--Rice--Baked Pork and Beans -Custards-Suet Pudding-Sauce-Apple Fritters-Mince Pie.

Dinner.-Vermicelli Soup-Boiled Calf's Head-Turnips-Spare Rib-Potatoes-Beets-Rice-Roast TurkeyApple Dumpling-Flapjacks-Spanish Cream-Pumpkin Pie.

Dinner.-Soup-Chickens--Egg Sauce -Potatoes-Beans-Tomatoes-HamGreen Corn-Cocoanut Pie_-Boiled Custards - Sponge Cake-Tapioca Pudding.

Dinner.-Snup-Boiled Salmon-Leg of Mutton-Caper Sauce-Tomatoes-Squashes-Green Corn-Fried Egg Plant-Beans-Tongue-Rice Pudding -Slip_Farina Mould-Peach Pie.

NotE.-The Cuts of Cooking Utensils in the Second Part were furnished to the Publishers by $\mathrm{Mr}$. STEPHEN WM. SMitH, whose Housekeeping Furniture Ware Rooms are at 534 Broadway, neas Spring Street, New York. 


\section{PART THIRD.}

PERFUMERY AND THE TOILET, THE FAMIL MEDICAL GUIDE, MISCELLANEOUS RECEIPTS. 



\section{PERFUMERY AND THE TOILET.}

THE perfumes for the handkerchief, as found in the shops of Paris and London, are either simple or compound ; the former are called extracts, extraits, esprits, or essences, and the latter bouquets and nosegays, which are mixtures of the extracts so compounded in quantity that no one flower or odor can be discovered as predominating over another. Perfumes are procured by the processes of maceration, absorption, and distillation.

In making pomade, a certain quantity of purified mutton or deer suet is put into a clean metal or porcelain pan, this being melted by a steam heat; the kind of flowers required for the odor wanted are carefully picked and put into the liquid fat, and allowed to remain from twelve to forty-eight hours; the fat has a particular affinity or attraction for the oil of flowers, and thus, as it were, draws it out of them, and becomes itself, by their aid, highly perfumed; the fat is strained from the spent flowers, and fresh are added four or five times over, till the pomade is of the required strength. For perfumed oils the same operation is followed; but, in lieu of suet, fine olive oil or oil of ben, derived from the ben nuts of the Levant, is used, and the same results are obtained.

The odors of some flowers are so delicate and volatile, that heat would spoil them; the process of absorption or en- fleurage is, therefore, conducted cold, thus:-Square frames, about three inches deep, with a glass bottom, say two feet wide and three feet long, are procured; over the glass a layer of fat is spread, about half an inch thick, with a kind of plaster knife ; into this the flower buds are stuck, cup downwards, and ranged comp'etely over it, and there left from twelve to seventy-two hours.

Almond perfume is obtained from the bitter almonds, and exists in the skin or pellicle that covers the seed after it is shelled. The essential oil of almonds enters into combination with soap, cold cream, and many other materials prepared by the perfumer.

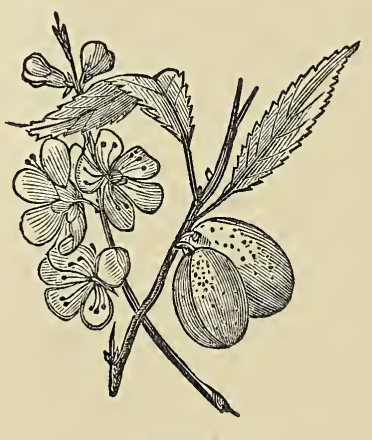

Almond.

A beautiful imitation of Essence of white Lilac may be compounded thus: One pint spirituous extract from 
tubereuse pomade; one quarter of a pint spirituous extract of orange-flower pomade ; three drops of otto of almonds ; half an ounce extract of civet.

The civet is only used to give permanence to the perfume of the handkerchief.

Benzoin, also called Benjamin.-This is a very useful substance to perfumers. It exudes from the Styrax benzoin by wounding the tree, and drying, becomes a hard gum-resin. It is principally imported from Borneo, Java, Sumatra, and Siam.

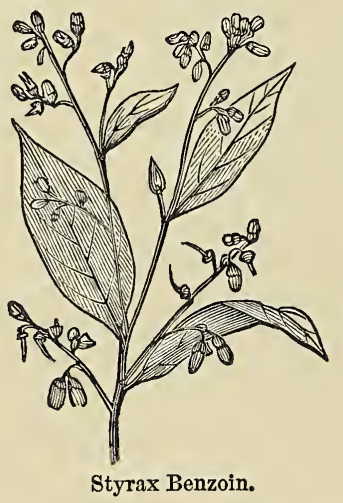

Bay.-Oil of sweet Bay, also termed essential oil of laurel-berries, is a very fragrant substance, procured by distillation from the berries of the bay laurel.

Bergamot.-This most useful perfume is procured from the Citrus Bergamia, by expression from the peel of the fruit. Mixed with rectified spirit in the proportions of about four ounces of bergamot to a gallon, it forms what is called "extract of bergamot," and in this state is used for the handkerchief.

Geranium (Pelargonium odoratissimum, rose-leaf geranium).-The leaves of this plant yield by distillation a very agreeable rosy-smelling oil, so much resembling real otto of rose, that it is used very extensively for the adulteration of that valuable oil, and is grown very largely for that express purpose. When lissolved in rectified spirit, in the proportion of about six ounces to the gallon, it forms the "extract of rose leaf geranium" of the shops.

Heliotrope.-Either by maceration or enfleurage with clarified fat, we may obtain this fine odor from the flowers of the Heliotrope Peruvianum or H. grandi florum. For a small trial, which can be managed by any person having the run of a garden, we will say, procure an ordinary glue-pot now in common use, which melts the material by the boiling of water. At the season when the flowers are in bloom, obtain half a pound of fine mutton suet, melt the suet and strain it through a close hair-sieve, allow the liquefied fat, as it falls from the sieve, to drop into cold spring water ; this operation granulates and washes the blood and membrane from it. In order to start with a perfectly inodorous grease, the melting and granulation process may be repeated three or four times; finally, remelt the fat and cast it into a pan to free it from adhering water.

Now put the clarified suet into the macerating pot, and place it in such a position near the fire of the greenhouse or elsewhere, that will keep it warm enough to be liquid; into the fat throw as many flowers as you can, and there let them remain for twenty-four hours; at this time strain the fat from the spent flowers, and add fresh ones; repeat this operation for a week: we suppose at the last straining the fat will have become very highly perfumed, and when cold may be justly termed Pomade à la Heliotrope.

The cold pomade being chopped up like suet for a pudding, is now to be put into a wide-mouthed bottle, covered with spirits as highly rectified as can be ob- 
tained, and left to digest for a week or more; the spirit then strained off will be highly perfumed: in reality it will be $e x$ tract of Heliotrope, a delightful perfurne for the handkerchief. The rationale of the operation is simple enough : the fat body has a strong affinity or attraction for the odorous body, or essential oil of the flowers, and it therefore absorbs it by contact, and becomes itself perfumed. In the second operation, the spirit has a much greater attraction for the fragrant principle than the fatty matter ; the former, therefore, becomes perfumed at the expense of the latter. The same experiment may be repeated with almond oil substituted for the fat.

The experiment here hinted at may be varied with any flowers whatever; indeed, by having the macerating bath larger than was mentioned above, an excellent millefleur pomade and essence might be produced from every conservatory.

The odor of heliotrope resembles a mixture of almonds and vanilla, and is well imitated thus :-

Extract of Helioirope.-Half a pint of spirituous extract of vanilla; one gill of spirituous extract of French rose pomatum; two ounces spirituous extract of orange-flower pomatum ; one ounce spirituous extract of ambergris; five drops of essential oil of almonds.

A good Imitation of Honeysuckle is made thus:-Spirituous extract of rose pomatum, one pint ; spirituous extract of violet pomatum, one pint ; spirituous extract of tubereuse pomatum, one pint ; extract of vanilla, one gill ; extrart of Tolu, one, gill ; otto neroli, ten drops; otto almonds, five drops.

Imitation Essence of Myrtle.-Extract of vanilla, half a pint ; extract of roses, one pint.

Orange Flower.-Extract of fleur d'orange, half a pint ; extract of tubereuse, half a pint; extrast of jasmine, two ounces. Mix and allow to stand for a fortnight : it is then fit for bottling, and is a perfume that gives a great deal of satisfaction.

Imitation Essence of Violets.-Spirituous extract of cassie pomade, one pint ; esprit de rose, from pomade, half a pint; tincture of orris, half a pint ; spirituous extract of tuberose pomade, half a pint; otto of almonds, three drops.

Imitation "Lily of the Valley."-Extract of tubereuse, half a pint ; extract of jasmine, one ounce ; extract of fleur d orange, two ounces; extract of vanilla, three ounces; extract of cassie, one gill; extract of rose, one gill ; otto of almonds, three drops. Keep this mixture together for a month, and then bottle it for sale. It is a perfume that is very much admired.

Imitation "Essence of Magnolia." Spirituous extract of orange-flower pomatum, one pint ; spirituous extract of rose pomatum, two pints ; spirituous extract of tubereuse pomatum, half a pint ; spirituous extract of violet pomatum, half a pint; essential oil of citron, three drachms; essential oil of almonds, ten drops.

Extrait d'Ambre.-Esprit de rose triple, half a pint ; extract of ambergris, one pint ; essence of musk, one gill ; extract of vanilla, two ounces.

This perfume has such a lasting odor, that a handkerchief being well perfumed with it, will still retain an odor even after it has been washed.

Patchouly.-Pogostemon patchouly (Lindley), Plectranthus crassifolius (Burnett), is an herb that grows extensively in India and China. It somewhat resembles our garden sage in its growth and form. The odor is due to an otto contained in the leaves and stems, and is readily procured by distillation.

It is the most powerful of any derived from the botanic kingdom; hence, if mixed in the proportion of measure for 
measure, it completely covers the smell of

- all other bodies.

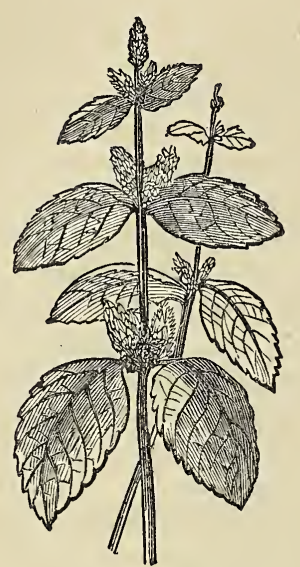

Patchouly.

Extract of Patchouly.-Rectified spirits, one gallon; otto of patchouly, one ounce and a quarter; otto of rose quarter of an ounce.

The characteristic smell of Chinese or Indian ink is due to some admixture of this herb.

The origin of the use of patchouly as a perfume in Europe is curious. A few years ago real Indian shawls bore an extravagant price, and purchasers could always distinguish them by their odor; in fact, they were perfumed with patchouly. The French manufacturers had for some time successfully imitated the Indian fabric, but could not impart the odor.

At length they discovered the secret, and began to import the plant to perfume articles of their make, and thus palm off homespun shawls as real Indian! From this origin the perfumers have brought it into use.

Imitation of the Essence of Sweet Pea can be prepared thus :-Extract of tuberose, half a pint; extract of fleur d'orange, half a pint; extract of rose from pomatum, half a pint ; extract of vanilla, one ounce.

Musk.-This extraordinary substance, like civet, is an animal secretion. Musk is remarkable for the diffusiveness and subtlety of its scent ; every thing in its vicinity soon becomes affected by it, and long retains its odor, although not in actual contact with it. Extract of musk one pint; extract of ambergris, half a pint; extract of rose triple, one gill. Mix and filter it ; it is then fit for bottling.

Rose.-Pure otto of roses, from its cloying sweetness, has not many admirers ; when diluted, however, there is nothing to equal it in odor, especially if mixed in soap, to form rose soap, or in pure spirit, to form the esprit de rose.

There are four modifications of essence of rose for the hankerchief, which are the ne plus ultra of the perfumer's art. They are,-esprit de rose triple, essence of white roses, essence of tea rose, and essence of moss rose. The following are the recipes:-

Esprit de Rose Triple.-Rectified al cohol,' one gallon; otto of rose, three ounces. Mix at a summer heat; in the course of a quarter of an hour the whole of the otto is dissolved, and is then ready for bottling. In the winter season beautiful crystals of the otto-if it is goodappear disseminated through the esprit.

Essence of Moss Rose.--Spirituous extract from French rose pomatum, one quart; esprit de rose triple, one pint; extracts fleur d'orange pomatum, one pint; extract of ambergris, half a pint ; extracts of musk, four ounces. Allow the ingredients to remain together for a fortnight; then filter, if requisite, and it is ready for sale.

Essence of White Rose.-Esprit de rose from pomatum, one quart ; esprit de rose triple, one quart; esprit de rose violette 
one quart ; extracts of jasmine, one pint; extracts of patchouly, half a pint.

Essence of Tea Rose.-Esprit de rose pomade, one pint ; esprit de rose triple, one pint; extract of rose-leaf geranium, one pint ; extract of sandal-wood, half a pint ; extract of neroli, quarter of a -pint ; extract of orris, quarter of a pint.

Otto of Rosemary is very extensively used in perfumery, especially in combination with other ottos for scenting soap. Eau de Cologne cannot be made without it, and in the once famous "Hungary water" it is the leading ingredient. The following is the composition of

Hungary Water.-Rectified alcohol, one gallon; otto of English rosemary, two ounces; otto of lemon-peel, one ounce; otto of balm (Melissa), one ounce ; otto of mint, half drachm; esprit de rose, one pint ; extract of fleur d'orange, one pint.

Vanilla.-The pod or bean of the Vanilla planifolia yields a perfume of rare excellence.

Extract of Vanilla.-Half a pound of Vanilla pods, and one gallon of rectified spirit.

Slit the pods from end to end, so as to lay'open the interior, then cut them up in lengths of about a quarter of an inch, macerate with occasional agitation for about a month; the tincture thus formed will only require straining through cotton to be ready for any use that is required. In this state it is rarely sold for a perfume, but is consumed in the manufacture of compound odors, bouquets, or nosegays, as they are called.

Verbena, or Vervaine.-The scented species of this plant, the lemon verbena, Aloysia citriodora (Hooker), gives one of the finest perfumes with which we are acquainted; it is well known as yielding a delightful fragrance by merely drawing the hand over the plant; some of the little vessels or sacks containing the otto must be crushed in this act, as there is little or no odor by merely smelling at the plant.

The otto, which can be extracted from the leaves by distillation with water, on account of its high price, is scarcely, if ever, used by the manufacturing perfumer, but it is most successfully imitated by mixing the otto of lemon grass, Andropogon schœnanthus, with rectified spirit, the odor of which resembles the former to a nicety. The following is a good form for making the

Extract of Verbena.-One pint of rectified spirit; three drachms otto of lemon grass; two ounces otto of lemon peel; half an ounce otto of orange peel.

After standing together for a few hours, and then filtering, it is fit for use.

Another mixture of this kind, presumed by the public to be made from the same plant, but of a finer quality, is composed thus :

Extrait de Verveine.-One pint of rectified spirit; one ounce of otto of orange peel ; two ounces of otto of lemon peel; one drachm of otto of citron; two drachms and a half of otto of lemon grass; seven ounces of extrait de fleur d'orange; seven ounces of extrait de fleur tubereuse ; half a pint esprit de rose.

This mixture is exceedingly refreshing; and is one of the most elegant perfumes that is made.

Winter Green (Trientalis Europcea). -A perfuming otto cán be procured by distilling the leaves of this plant; it is principally consumed in the perfuming of soaps. Upon the strength of the name of this odorous plant a very nice handkerchief perfume is made.

Iceland Winter Green.-One pint esprit de rose ; a quarter of a pint of essence of lavender; half a pint of extract of neroli ; a quarter of a pint of extract of vanilla; a quarter of a pint of ex- 
tract of vitivert; half a pint of extract of cassie ; a quarter of a pint of extract of ambergris.

Citron.-On distilling the flowers of the Citrus medica, a very fragrant oil is procured, which is a species of neroli, and is principally consumed by the manufacturers of eau de Cologne.

Citronella.--Under this name there is an oil in the market, chiefly derived from Ceylon and the East Indies. Being cheap, it is extensively used for perfuming soap. 'What is now extensively sold as "honey" soap, is a fine yellow soap, slightly perfumed with this oil.

Cloves.-Every part of the clove plant (Caryophyllus aromaticus) abounds with aromatic oil, but it is most fragrant and plentiful in the unexpanded fiower-bud, which are the cloves of commerce. The plant is a native of the Moluccas and other islands in the China seas. The oil may be obtained by expression from the fresh flower-buds, but the usual method of procuring it is by distillation. Few essential oils have a more extensive use in perfumery; it combines well with grease, soap, and spirit, and forms a leading feature in some of the most popular handkerchief essences. For essence of cloves, dissolve oil of cloves in the proportion of two ounces of oil to one gallon of spirit.

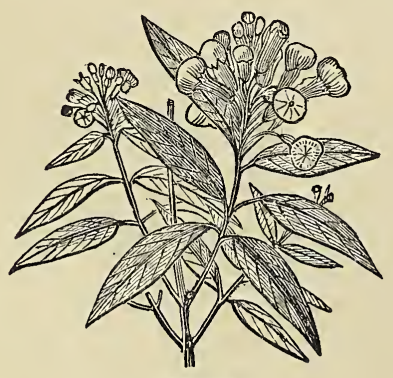

Cloves. perfume has given rise to numerous imitations of the original article, more particularly on the continent. In many of the shops in Germany and in France will be seen bottles labelled in close imitation of those sent out by Bayley and Co., Cockspur Street, London, who are, in truth, the original makers.

Esprit de rose triple, one pint; extract of ambergris, two ounces; extract of orris, eight ounces ; otto of lemons, onequarter of an ounce; otto of bergamot, one ounce.

Compound 0dors, or bouquets, are used for the purpose of fixing the more volatile odors to the handkerchief.

Essence of Rondeletia.-Spirit (brandy) one gallon; otto of lavender, two ounces; otto of cloves, one ounce; otto of roses, three drachms; otto of bergamot, one ounce; one gill of extract of musk, one gill of extract of vanilla, and one gill of extract of ambergris.

The mixture must be made at least a month before it is fit for use.

Bouquet de Caroline; also called Bouquet des Delices.-Extrait de rose, from pomade, one pint; extrait de violette, from pomade, one pint; extrait de tuberose, from pomade, one pint ; extract of orris, half a pint ; extract of ambergris, half a pint; otto of bergamot, a quarter of an ounce; otto of limette, a quarter of an ounce; otto of cedret, a quarter of an ounce.

The Court Nosegay. - Extrait de rose, one pint; extrait de violette, one pint; extrait de jasmine, one pint; esprit de rose triple, one pint; extract of musk, one ounce; extract of ambergris, one ounce; otto of lemon, half an ounce; otto of bergamot, half an ounce; otto of neroli, one drachm.

Bouquet de Flora ; otherwise, Extract of Flowers.-Esprit de rose, from poEss. Bouquet.-The reputation of this made, one pint; esprit de tubereuse, from 
pomade, one pint; esprit de violette, from pomade, one pint; extract of benzoin, an ounce and a half; otto of bergamot, two ounces; otto of lemon, half an ounce; otto of orange, half an ounce.

Essentia Odorifera.-Take of musk grain, ten grains ; civet, five grains ; Peruvian balsam, twelve grains; oil of cloves, four drops; oil of rhodium, two drops; sub-carbonate of potash, half a drachm; rectified spirits of wine, two ounces. Digest them together in a close vessel, with a heat equal to that of the sun in summer (78 deg. Fahr.) for several days, and afterwards pour off the essence for use.This is an exquisite perfume, and a single drop gives a fine flavor to many ounces of other liquors.

Eau de Chypre.-This is an oldfashioned French perfume, presumed to be derived from the Cyperus esculentus by some, and by others to be so named after the island of Cyprus; the article sold, however, is made thus:

Extract of musk, one pint ; extract of ambergris, half a pint; extract of vanilla, half a pint; extract of tonquin bean, half a pint; extract of orris, half a pint; esprit de rose triple, two pints.

The mixture thus formed is one of the most lasting odors that can be made.

Empress Eugenie's Nosegay.-Extract of musk, one gill ; extract of vanilla, one gill; extract of tonquin, one gill; extract of neroli, one gill ; extract of geranium, half a pint; extract of rose triple, half a pint ; extract of santal, half a pint.

The Guards' Bouquet.-Esprit de rose, two pints ; esprit de neroli, half a pint; extract of vanilla, two ounces; extract of orris, two ounces ; extract of musk, a quarter of a pint; otto of cloves, half a drachm.

Fleur d'Italie, or Italian Nosegay.Esprit de rose, from pomade, two pints ;
Esprit de rose triple, one pint ; esprit de jasmine, from pomade, one pint; esprit de violette, from pomade, one pint, extract of cassie, half a pint ; extract of musk, two ounces ; extract of ambergris, two ounces.

Jockey Club Bouquet (English formula).-Extract of orris root, two pints ; esprit de rose triple, one pint; esprit de rose de pomade, one pint; extrait de cassie de pomade, half a pint; extrait de tubereuse de pomade, half a pint ; extrait of ambergris, half a pint; otto of bergamot, half an ounce.

Jockey Club Bouquet (French formula). -Esprit de rose de pomade, one pint; esprit de tubereuse, one pint; esprit de cassie, half a pint; esprit de jasmine, a quarter of a pint ; extract of civet, three ounces.

\section{SPIRITUOUS INFUSIONS.}

Leaves, flowers, roots, wood, resins, powders, and perfumes of animal origin, all serve to furnish these tinctures. The substances are either cut, rasped, or bruised, according to their nature, and infused for some days or a month, taking care not to fill the vessel so full as to previent its contents being stirred up occasionally. These infusions are made either with alcohol or brandy.

Eau de Vie de Lavande.--Take one pound of flowers, and three pints of brandy. Infuse in a closed vessel for six weeks. and after that strain and bottle up for use or sale.

Infusion de Lavande Rouge composée (English).-Take of spirit lavender, one pound and a half; spirit rosemary, one pound ; pulverized cinnamon, one ounce; pulverized nutmegs, one ounce ; red sanders, one drachm. After two weeks' infusion, filter.

Infusion de Camomille Romaine.-One 
pound of flowers suffices for three quarts of brandy.

Infusion of Absinthe: de Sauge, \&c.Take only four leaves to the quart of brandy. These infusions serve in the composition of sundry aromatic waters.

Infusion or Esprit de Safran.-To a quart of alcohol, take two ounces saffron and infuse for two months. This spirit is used for coloring waters, pastes, \&c., but in very small proportions.

Infusion or Esprit de Rhodia.-Infuse as before one pound of rhodium wood in two quarts of spirit. If the wood is resinous, only half a pound is required.

Infusion or Teinture de Canelle.Take four ounces best bruised cinnamon to the quart of spirit.

Tincture of Sassafras.-Infuse eight ounces of bruised sassafras in one quart of alcohol.

Tincture of Santal-citrin (yellow sanders).-Operate as for the preceding infusion.

Tincture of Benzoin.-Take Benzoin in tears and pulverized, two ounces; alcohol one pound. After seven days' infusion, filter. This tincture is used, principally, for making the Lait Virginal, either with pure rose, orange flower or lavender water.

To make Eau-de-Cologne.-Rectified spirits of wine, four pints ; oil of bergamot, one ounce; oil of lemon, half an ounce ; oil of rosemary, half a drachm ; oil of Neroli, three quarters of a drachm; oil of English lavender, one drachm; oil of oranges, one drachm. Mix well, and then filter. If these proportions are too large, smaller ones may be used.

Eau Jaune à l'Amaryllis.-In two quarts of alcohol, dissolve two ounces essence of bergamot, four ounces essence of lemon, four drachms essence of Portugal, four drachms essence of neroli, one drachm essence of thyme, one drachm essence of rosemary, two ounces of tinc- ture of cloves, eight ounces of lait vir. ginal, as much of vanilla water, four drachms of essence of amber, and two drachms essence of musk. Dilute the spirit with good rose water, and add a little of red or yellow tincture, according to choice.

Eau d'Ambre Royale.-To a quart of alcohol add half a pint of spirit of ambrette, an ounce of essence of amber, an ounce of musk mixed with a proportional quantity of orange-flower water.

Eau de Chypre.-Mix together (eau de) jasmine, one quart; (eau de) bergamot, one quart; (eau de) violet, one quart; (eau de) tuberose, one quart; (esprit) spirit of ambrette, one pint ; essence of musk, one ounce; mecca balsam, one ounce ; storax, half an ounce.

Add to the mixture two ounces of water, and mix thoroughly together, so that no one odor predominates over the rest.

Lavender Water.-Oil of lavender, two drachms; oil of bergamot, half drachm; essence of musk, one drachm; spirit of wine, thirteen ounces ; water, five ounces. Let it stand a week.

Eau de Bouquet de Flore.-Eau de miel, two ounces ; tincture of cloves, one ounce ; tincture of calamus, half an ounce; tincture of lavender, half an ounce; tincture of souchet, half an ounce; eau sans pareille, four ounces ; tincture of jasmine, nine drachms ; tincture of orris root, one ounce; tincture of neroli, twenty drops.

These waters and tinctures mixed together, give a product of exquisite fragrance.

Eau d'Elegance.-Spirit of jasmine, two quarts ; spirit of storax, one pint; spirit of hyacinth, one pint ; spirit of badiane, four ounces ; tincture of balsam of Tolu, four ounces ; tincture of vanilla, two ounces.

Eau de Miel.-This receipt is from one of the most celebrated English perfumers; coriander, seven pounds ; cloves, twelve 
ounces ; storax, eight ounces ; bruised nutmegs, eight ounces ; lemon-peel, ten ounces; calamus, six ounces ; alcohol, fifteen quarts.

Macerate for a month in a well-stoppered demijohn. Distil then, and to the liquor add: orange-flower water, five quarts ; essence of roses, twenty-four drops ; pulverized ambergris, one drm. ; vanilla in pieces, two ounces.

Macerate for a week, and filter. This is one of the most agreeable waters used.

\section{OILS FOR THE HAIR.}

For Thickening and Strengthening the Hair.-Skim the fat from the top of calves' feet while boiling; mix with a teaspoonful of rum; shake together. Apply night and morning.

Pomatum.-Take white mutton suet, four pounds, well boiled in hot water, (three quarts,) and washed to free it from salt. Melt the suet when dried with one pound and a half of fresh lard, and two pounds of yellow wax. Pour into an carthen vessel, and stir till it is cold ; then beat into it thirty drops of oil of cloves, or any other essential oil whose scent you prefer. If this is too hard, use less 'wax.

Or :-Soak half a pound of clear beefmarrow, and a pound of unsalted fresh lard, in water, two or three days, changing and beating it every day. Put it into a sieve, and when dry, into a jar, and the jar into a saucepan of water. When melted, pour it into a basin, and beat it with two spoonfuls of brandy; drain off the brandy. and then add essence of lemon, bergamot, or any other scent that is liked.

Pomade Victoria.-This highly praised and excellent pomade is made in the following way-and if so made, will be found to give a beautiful gloss and softness to the hair :-Quarter of a pound of honey and half an ounce of beeswax simmered together for a few minutes, and then strain ; and of oil of almonds, lavender, and thyme, half a drachm each. Be sure to continue stirring till quite cold, or the honey and wax will separate. Imitation Bear's Grease.-Hog's lard, sixteen ounces; flowers of benzoin and palm oil, of each quarter of an ounce. Melt together until combined, and stir until cold. Scent at pleasure. This will keep a long time.

Sweet Pot-pourri.-Take three handfuls of orange-flowers, three of clove-gillyflowers, three of damask-roses, one of knotted marjoram, one of lemon-thyme, six bay-leaves, a handful of rosemary, one of myrtle, half of mint, one of lavender, the rind of a lemon, and quarter of an ounce of cloves. Chop all, and put them in layers, with pounded bay-salt between, up to the top of a china jar.

If all the ingredients cannot be got at once, put them in as you get them; always throwing in salt with every new article.

To make Wash-balls.-Shave thin two pounds of new white soap into about a teacupful of rose-water, then pour as much boiling water on as will soften it. Put into a brass pan a pint of sweet oil, four pennyworth of oil of almonds, half a pound of spermaceti, and set all over the fire till dissolved ; then add the soap and half an ounce of camphor that has first been reduced to powder by rubbing it in a mortar, with a few drops of spirits of wine, or lavender-water, or any other scent. Boil ten minutes, then pour it into a basin, and stir it till it is quite thick enough to roll up into hard balls, which must then be done as soon as possible. If essence is used, stir it in quickly after it is taken off the fire, that the flavor may not fly off.

Paste for Chapped Hands.--Mix a quarter of a pound of unsalted hog's lard, which has been washed in soft water, and then rose-water, with the yolks of two new-laid 
eggs, and a large spoonful of honey. Add as much fine oatmeal or almond-paste as will work into a paste.

Or:-Blanch one pound of bitter almonds, pound them smooth in a marble mortar ; add half an ounce of camphor, one ounce of honey, quarter of a pound of spermaceti, all pounded, and mixed with the almonds, till it becomes a smooth paste. Put it into jars or china boxes, and tie it down till wanted.

For Chapped Lips.-Put quarter of an ounce of benjamin, storax, and spermaceti. pennyworth of alkanet-root, a large juicy apple chopped, a bunch of black grapes bruised, quarter of a pound of unsalted butter, and two ounces of beeswax, into a new tin saucepan. Simmer gently till the wax. \&c., are dissolved, and then strain it through linen. When cold, melt it again, and pour it into small pots of boxes ; or, if to make cakes, use the bottoms of teacups.

Cosmetic Gloves.-Though not altogether fashionable now, these gloves are still worn by those who are subject to chaps and chilblains: the ladies especially use them; hence the necessity of always being provided with a small stock.

Gants cosmétiques au bouquet.-Wax, four drachms ; spermaceti, four drachms ; white soap, four drachms; mutton suet, seven drachms.

Mince up each of these substances separately, melt them over a water-bath, and add : Olive oil, an ounce and a half; pomade rosat, one ounce and a half; benzoin, one drachm; Peru balsam, one drachm; essence of roses, some drops; honey water, au bouquet, half an ounce. Stir together until the mixture is complete, and, whilst the mass is still hot, apply it with a brush to the wrong sides of the gloves. The gloves being returned, are blown up, and put by in a warm place to dry.
Or:-Beat two yolks of eggs with two spoonfuls of oil of sweet almonds, and add half an ounce rose water and two drachms tincture of benzoin.

The gloves are worn during the night, and each pair should serve two weeks. The rest of the pomade answers for rubbing the hands.

Lait de rose (milk of rose).-To one ounce of fine olive oil, and six drops of oil of tartar, add a quart of rose water. Melt together, in a stoneware vessel, over a water-bath: Spermaceti, one ounce; white wax, one ounce; white soap, one ounce. Rub up in a marble mortar bitter almonds (best quality), two ounces; sweet almonds, best quality, one pound. Take out three-fourths, and upon the remaining fourth pour the above mixture, and continue rubbing actively, and afterwards add, by degrees, the other threefourths of the almonds, always pestling rapidly so as to thoroughly incorporate the mixture.

Again prepare in a white-glass bottle the following mixture :-Distilled water, two pounds; rose water, half a pound; spirit of rose, half a pound. Reserve a pint, and pour gradually into the rest the first, and rub well all the while; then strain through a close cloth, and return the marc to the mortar, where, being triturated with the pint above reserved, it is again strained, and the liquid united with the first.

If there is any tendency to decomposition, shake up the milk freely. To augment the fragrance of this composition, perfume with four drops essence of rose.

Violet Powder.-Wheat starch, twelve pounds ; orris-root powder, two pounds ; otto of lemon, half an ounce; otto of bergamot, a quarter of an ounce; otto of cloves, two drachms.

Rose Face Powder.-Wheat starch, seven pounds ; rose pink, half a drachm; 
otto of rose, two drachms; otto of santal, two drachms.

Perle Powder.-French chalk, one pound; oxide of bismuth, one ounce; oxide of zinc, one ounce.

Poudre de la mer rouge, by Cambou, Paris.-Take alum, one pound; white sugar, one ounce; gum arabic (best), one ounce ; carmine, one ounce. Mix and reduce the whole to an impalpable powder, and sieve through a fine cloth.

This powder, its author says, is curative of the ringworm, red blotches, and pimples.

It is tied up loosely in a bag, and this bag, moistened with fresh water, is rubbed gently over the skin.

\section{S O A P S.}

Savon au miel.-Take four ounces white soap, four ounces white honey, one ounce benzoin, half an ounce storax; mix the whole together in a marble mortar, and when well incorporated, melt the paste over a water-bath, strain, pour into frames, and divide into small tablets.

Lady Derby's Soap.-Take two ounces blanched bitter almonds, ten drachms tincture benzoin, a pound of white soap, and a piece of camphor, size of a filbert; powder the almonds and camphor in a separate mortar, and, when they are completely mixed, add the benzoin. If there should be too much odor of benzoin and camphor, weaken the perfume by remelting the soap.

Saponaceous Cream of Almonds.-The preparation sold under this title is a potash soft soap of lard. It has a beautiful pearly appearance, and has met with extensive demand as a shaving soap. It is made thus:-Clarified lard, seven pounds ; potash of lye (containing twentysix per cent. of caustic potash), three and three-quarter pounds; rectified spirit, three ounces; otto of almonds, two drachms.

Manipulation :-Melt the lard in a porcelain vessel by a salt-water bath ; then run in the lye, very slowly, agitating the whole time; when about half the lye is in, the mixture begins to curdle ; it will, however, become so firm that it cannot be stirred. The crême is then finished, but is not pearly; it will, however, assume that appearance by long trituration in a mortar, gradually adding the alcohol, in which has been dissolved the perfume.

Transparent Soap.-Take of perfectly dry, pulverulent white soap, two pounds; alcohol, three quarts; heat gently together over a water-bath, and when the solution is complete, perfume, and turn out into forms.

When cooled, divide it into cakes, onethird thicker than their designed size, so as to allow for contraction by evaporation.

Frangipanne Soap.-Curd soap, previously colored light brown, seven pounds; civet, a quarter of an ounce; otto of neroli, half an ounce; otto of santal, an ounce and a half; otto of rose, a quarter of an ounce; otto of vitivert, half an ounce.

Rose Cold Cream.-Almond oil, one pound; rose-water, one pound; white wax, one ounce; spermaceti, one ounce; otto of roses, half a drachm. Heat the wax and sperm in a water-bath; add the otto last.

Cold Cream of Almonds is prepared precisely as the above; but in place of otto of roses otto of almonds is used.

Violet Cold Cream.-Huile violette, one pound; rose-water, one pound; wax and spermaceti, each one ounce; otto of almonds, five drops.

Violet Cold Cream. Imitation :-Almond oil, three-quarters of a pound; 
huile cassie, a quarter of a pound; rosewater, one pound; sperm and wax, one ounce; otto of almonds, a quarter of a drachm.

This is an elegant and economical preparation, generally admired.

Camphor Cold Cream (otherwise Camphor Ice).-Almond oil, one pound; rose water, one pound; wax and spermaceti, one ounce; camphor, two ounces; otto of rosemary, one drachm.

Pommades des Sultanes.-Melt over a water-bath one ounce white wax and two ounces spermaceti; withdraw from the fire, and then add eight ounces oil of sweet almonds, eight ounces oil of white poppy, and continue beating together without interval until the mixture begins to get white, then put in half an ounce of Mecca balsam and a gill of rosewater, and prolong the rubbing until the mixture will take up no more of rose water, and detaches itself in large flakes or lumps.

In filling the pots, take care to cover the contents with a little rose-water. This is among the best preparations for softening the skin and preserving its freshness.

Pommiade de Ninon de L'Enclos, by Boyer.-Take oil of sweet almonds, four ounces; washed lard, three ounces, rosewater, three ounces. This pomade is extremely mild and refreshing.

Pâte divine de Venus, by Bourdel.Mix together equal parts of purified lard, fresh butter, and honey, and add a half portion of Mecca balsam, and essence of roses.

Almond Balls.-Purified suet, one pound ; white wax, half a pound; otto of almonds, one drachm; otto of cloves, quarter of a drachm.

Camphor Balls.-Purified suet, one pound; white wax, half a pound; camphor, quarter of a pound ; otto of French lavender or rosemary, half an ounce.
Camphor Paste--Sweet almond oil, half a pound ; purified lard, quarter of a pound; wax, spermaceti and camphor, each, one ounce.

Pâte d'Amaniles Aux Jaunes d'ceufs. -To four ounces sweet almonds, powdered finely in a marble mortar, add the yolks of three fresh eggs, mix well, thin out with eight ounces of milk, and boil until it assumes the consistence of paste, stirring constantly during the ebullition. Perfume with one drachm essence of vanilla.

Composition par Blaque, Paris, for Softening the Skin.--Butter, four pounds; oil of sweet almonds, four pounds ; common white soap, six pounds; decoction of marshmallow, eight pounds ; alcohol, one pint.

Melt and heat together, in a stoneware vessel, over a water-bath, stirring well all the time; and when the whole is thoroughly incorporated, pour out to cool. Perfume according to taste. A quantity of the size of a filbert, well rubbed on, suffices for washing the hands.

Camphor Cerate for Chapped Hands.Take half an ounce of spermaceti, half an ounce of white wax, scrape them into an earthen vessel or pipkin (an earthen jam-pot will do,) add six drachms of pounded camphor, and pour on the whole four table-spoonfuls of best olive oil ; let it stand before the fire till it dissolves, stirring it well when liquid. Before you wash your hands, take a small piece of the cerate, and rub it into your hands, then wash them as usual. Putting the cerate on before going to bed is very good. The ingredients cost two shillings, and this quantity will last for three winters. The vessel should be covered, to prevent evaporation.

To eradicate Warts.-Dissolve as much common washing soda as the water will take up ; repeatedly wash with this for a 
minute or two, and let them dry without wiping.

Another Method.-Get a little bullock's gall, keep it in a bottle, and rub a little on the warts two or three times a day.

Cold Cream.-Lard, six ounces ; spermaceti, one ounce and a drachm and a half; white wax, three drachms ; rosewater, three ounces; carbonate of potass, fifteen grains ; spirits of wine, three quarters of an ounce ; essential oil of bergamot, three drachms. Melt the three first, then add the rose-water, carbonate of potass, and spirits of wine, stirring well: and when nearly cold add the perfume. I can safely say that this is first-rate, having made many pounds of it.

To Whiten the Nails.-Diluted sulphuric acid, two drachms; tincture of myrrh, one drachm; spring water, four ounces. Mix. First cleanse with white soap, and then dip the fingers irpto the mixture.

To Whiten the Hands.-Take a wineglassful of Eau de Cologne, and another of lemon-juice : then scrape two cakes of brown Windsor soap to a powder, and mix well in a mould. When hard, it will be an excellent soap for whitening the hands.

Rose Lip Salve.-Eight ounces sweet almond oil, four ounces prepared mutton suet, one and a half ounces white wax, two ounces spermaceti, twenty drops otto; steep a small quantity of alkanet root in the oil, and strain before using. Melt the suet, wax, and spermaceti together, then add the coloric oil and otto.

Pommade de Beauté, for improving the complexion, and healing chaps. Melt together over a water-bath white wax, one and a half drachms; spermaceti, two drachms ; oil of sweet almonds, half an ounce ; oil of olives, pure, half an ounce; oil of poppy, half an ounce ; balsam Peru, liquid, four drops. Add the balsam after having well beaten the mixture. This is an excellent cosmetic. .

Glycerine Balsam.-White wax, spermaceti, each one ounce ; almond oil, half a pound ; glycerine, two ounces ; otto of roses, quarter of a drachm.

White Lip Salve.-Almond oil, quarter of a pound; wax and spermaceti, each one ounce ; otto of almonds, half a drm.; otto of geranium, quarter of a drachm.

Common Lip Salve is made simply of equal parts of lard and suet, colored with alkanet root, and perfumed with an ounce of bergamot to every pound of salve.

Pommade, by Dr. Pittschaft, Baden, for Chapped Lips.-Take sublimed oxide of zinc, one drachm ; lycopodium powder, one drachm; pommade rosat, four ounces.

Mix, and make into a perfectly homogeneous pomade.

This is an excellent remedy for chapped lips, and is beneficial in cases of ulceration of the nails of the feet. Its application in such instances must be immediately after bathing the affected parts.

A toilet can be arranged and furnished according to taste, where there is more regard to convenience and comfort than expense of outlay. The necessaries, however, for a dressing-table and washstand, are two cakes of fine soap, a box of dentifrice, a pot of pomade for the hair, and a box of lip salve.

Acetic Acid and its Use in Perfumery. - The pungency of the odor of vinegar naturally brought it into the earliest use in the art of perfumery.

The modern aromatic vinegar is the concentrated acetic acid aromatized with various ottos, camphor, \&c., thus :-

Aromatic Vinegar.-Concentrated acetic acid, eight ounces; otto of English lavender, two drachms ; otto of English. 
rosemary, one drachm; otto of cloves, one drachm; camphor, one ounce.

First dissolve the bruised camphor in the acetic acid, then add the perfumes; after remaining together for a few days, with occasional agitation, it is to be strained, and is then ready for use.

The most popular article of this kind is-

Henry's Vinegar.--Dried leaves of rosemary, rue, wormwood, sage, mint, and lavender flowers, each half an ounce; bruised nutmeg, cloves, angelica root, and camphor, each quarter of an ounce; alcohol (rectified), four ounces; concentrated acetic acid, sixteen ounces.

Macerate the materials for a day in the spirit; then add the acid, digest for a week, and filter it.

Vinaigre à la Rose.-Concentrated acetic acid, one ounce; otto of roses, half a drachm. Well shaken together.

It is obvious that vinegars differently perfumed may be made in a similar manner to the above, by using other ottos in place of the otto of roses. All these concentrated vinegars are used in the.same way as perfumed ammonia, that is, by pouring three or four drachms into an ornamental "smelling" bottle, previously filled with crystals of sulphate of potash, which forms the "sel de vinaigre" of the shops; or upon sponge into little silver boxes, called vinaigrettes, from their French origin. The use of these vinegars had their origin in the notion that they kept those who carried them from the effects of infectious disease.

Mint Vinegar.-This is made by putting into a wide-mouthed bottle, fresh nice clean mint leaves enough to fill it loosely ; then fill up the bottle with good vinegar ; and after it has been stopped close for two or three weeks, it is to be poured off clear into another bottle, and kept well corked for use. Serve with lamb when mint cannot be obtained.
Thieves' Vinegar.-Take of rue, sage, mint, rosemary, wormwood, and larender, a large handful of each; infuse in one gallon of vinegar, in a stone jar closely covered, and keep warm by the fire for four days, then strain, and add one ounce of camphor, pounded; bottle, and keep well corked. There is a legend connected with this preparation (called in French Vinaigre à quatre Voleurs), that during the plague at Marseilles certain robbers plundered the infected houses with impunity, and being apprehended and condemned to death, were pardoned on condition of disclosing the secret of their preventive -as above. The mode of using is to wash the face and hands with it previous to exposure to any infection.

Vinaigre Virginal.-This mild and refreshing liquor is excellent to remove prickly heat, but, before being used for such purpose, must be diluted with water.

Take benzoin pulverized, two ounces; alcohol, eight ounces; vinegar, two pounds.

Digest the benzoin in the alcohol for a week, decant the liquor, and to the residue add the vinegar; after six days' infusion, this too is decanted, and it and the alcoholic tincture are mixed together, and filtered the next day. This vinegar weakened with water is a most excellent cosmetic, and is in fact a remedy for the ill effects of pernicious rouges and paints upon the skin.

Of Vinegars by Infusion.-These are either vinegars of flowers or aromatic vinegars.

Vinaigre de Toilette, by Sinfar, Paris. -Alcohol eight quarts; white vinegar, two quarts; cologne water, one pint; extract of benzoin, two ounces; extract of storax, two ounces; acetic acid, four ounces; essence of lavender, one ounce and a half; essence of cinnamon, 
one drachm; essence of cloves, one drachm ; ammonia, one drachm.

Mix together the alcohol, lavender, cinnamon and cloves, and macerate for eight days ; then add the vinegars, cologne, extracts, and ammonia ; color, and filter through paper.

Vinaigre Rosat.-Dry rose leaves, red, half a pint; good vinegar, eight quarts. Macerate for two weeks in a closed vessel, and after that filter.

Vinaigre à la Fleur d'Oranger.--Fresh orange flowers, one and a half pounds; vinegar, eight quarts ; brandy à la fleur d'oranger. one quart. Macerate for twelve days, and filter.

Vinaigres Infusées à la Lavande, de Sauge, Thyme, \&c.-One pound of fresh flowers to twelve quarts of vinegar.

Vinaigre Scillitique, for clearing the Voice.-Among speakers and vocalists, it is desirable, before commencing the exercises of their vocation, that the throat be freed from all pituitous hoarseness. This vinegar accomplishes that object, and besides imparting a tone to the glottis and all the organs, it serves to develop and perfect the voice. Put five or six drops of the preparation in a glass of warm water, and gargle therewith morning, noon, and night of the days on which the voice is to be exerted. Take of dried squills, one part ; good vinegar, twelve parts ; alcohol, half a part. After two weeks' maceration in a close vessel, press out and filter.

Vinaigre Alcoholique, for Fits and Swoons.-Mix together equal parts of strong concentrated vinegar and alcohol ; and, to increase its force, add two drops of ether to the quart. This composition is most excellent in cases of fainting and swooning.

The best preparation for smellingbottles is what is termed Inexhaustible Salts, which is prepared thus : Liquid ammonia, one pint; otto of rosemary, one drachm; otto of English lavender, one drachm; otto of bergamot, half a drachm; otto of cloves, half a drachm. Mix the whole together with agitation in a very strong and well stoppered bottle.

This mixture is used by filling the smelling-bottles with any porous absorbent material, such as asbestos, or, what is better, sponge cuttings, that have been well beaten, washed and dried. These will retain the ammoniacal odor longer than any other material ; hence, we presume, bottles filled in this way are called "inexhaustible," which name, however, they do not sustain more than two or three months with any credit; the warm hand soon dissipates the ammonia under any circumstances, and they require to be refilled.

Preston Salts, which is the cheapest of all the ammoniacal compounds, is composed of some easily decomposable salt of ammonia and lime, such as equal parts of muriate of ammonia, or of sesqui-carbonate of ammonia, and of fresh-slaked lime. When the bottles are filled with this compound, rammed in very hard, a drop or two of some cheap otto is poured on the top prior to corking. For this purpose otto of French lavender, or otto of bergamot, answers very well. We need scarcely mention that the corks are dipped into melted sealing-wax.

Cassolettes and Printaniers.-Cassolettes and printaniers are little ivory boxes of various designs, perforated in order to allow the escape of the odors contained therein. The paste used for filling these "ivory palaces whereby we are made glad," is composed of equal parts of grain musk, ambergris, seeds of the vanillapod, otto of roses, and orris powder, with enough gum acacia, or gum tragacanth, to work the whole together into a paste. These things are now principally used for perfuming the pocket or reticule, much 
in the same way that ornamental silver and gold vinaigrettes are used.

Sachet a la Frangipanne.-Orris-root powder, three pounds; vitivert powder, a quarter of a pound; santal-wood powder, a quarter of a pound ; otto of neroli, one drachm; otto of rose. one drachm; otto of santal, one drachm; musk-pods, ground, one ounce.

The name of this sachet has been hand" ed down to us as being derived from a Roman alchemist of the noble family of Frangipani.

Heliotrope Sachet.-Powdered orris, two pounds ; rose leaves, ground, one pound; tonquin beans, ground, half a pound; vanilla beans, a quarter of a pound; grain musk, a quarter of an ounce; otto of almonds, five drops. Well mixed by sifting in a coarse sieve, it is fit for sale.

It is one of the best sachets made, and is so like in its odor to the flower from which it derives its name, that no person unacquainted with its composition would, for an instant, believe it to be any other than the "real thing."

Lavender Sachet.-Lavender flowers, ground, one pound; gum benzoin, in powder, a quarter of a pound; otto of lavender, a quarter of an ounce.

Sachet d̀ la Mousselaine.-Vitivert, in powder, one pound; santal-wood, half a pound; orris, half a pound ; black currant leaves, half a pound; benzoin, in powder, a quarter of a pound; otto of thyme, five drops; otto of roses, half a drachm.

Millefleur Sachet.-Lavender flowers, ground, one pound; orris, one pound; rose leaves, one pound; benzoin, one pound; tonquin, a quarter of a pound; vanilla, a quarter of a pound; santal, a quarter of a pound; musk and civet, two drachms; cloves, ground, a quarter of a pound ; cinnamon, two ounces; allspice two ounces.
Portugal Sachet.-Dried orange-peel, one pound; dried lemon-peel, half a pound; dried orris root, half a pound; otto of orange-peel, one ounce; otto of neroli, a quarter of a drachm; otto of lemon grass, a quarter of a drachm.

Patchouly Sachet.-Patchouly herb, ground, one pound; otto of patchouly, a quarter of a drachm. Patchouly herb is often sold in its natural state, as imported, tied up in bundles of half a pound each.

Pot Pourri.-This is a' mixture of dried flowers and spices not ground. Dried lavender, one pound ; whole roseleaves, one pound ; crushed orris, coarse, half a pound; broken cloves, two ounces; broken cinnamon, two ounces; broken allspice, two ounces.

Rose Sachet.--Rose heels or leaves, one pound; santal wood, ground, half a pound; otto of roses, a quarter of an ounce.

An excellent Water to prevent Hair from falling off, and to thicken it.-Put four pounds of unadulterated honey into a still, with twelve handfuls of the tendrils or vines, and the same quantity of rosemary-tops. Distil as cool and as slowly as possible. The liquor may be allowed to drop till it begins to taste sour.

Tooth Powder.-Powdered orris root, half an ounce; powdered charcoal, two ounces; powdered Peruvian bark, one ounce; prepared chalk, half an ounce; oil of bergamot or lavender, twenty drops. These ingredients must be well worked up in a mortar, until thoroughly incorporated. This celebrated tooth-powder possesses three essential virtues, giving an odorous breath, cleansing and purifying the gums, and preserving the enamel; the last rarely found in popular toothpowders.

Bandoline for the Hair (a French 
receipt).-To one quart of water put half an ounce of quince pips, boil it nearly an hour, stirring it well, strain it through a fine muslin, let it stand twenty-four hours, and then add fourteen drops of the essential oil of almonds. A dessert spoonful of brandy may be added, if required to keep a long time.

Economical Hair Wash.-Take one ounce of borax, half an ounce of camphor, powder these ingredients fine, and dissolve them in one quart of boiling water; when cool, the solution will be ready for use; damp the hair frequently. This wash not only effectually cleanses and beautifies, but strengthens the hair, preserves the color, and prevents early baldness. The camphor will form into lumps, but the water will be sufficiently impregnated.

To make a curling Fluid for the Hair. Melt a bit of white bees'-wax, about the size of a filbert kernel, in one ounce of olive oil, to this add one or two drops of otto of roses.

Rose Bandoline.-Gum tragacanth, six ounces; rose water, one gallon; otto of roses, half an ounce. Steep the gum in water well, strain and mix thoroughly.

Simple Pastilles.-Benzoin, half an ounce; cassia, one drachm; charcoal, two ounces; salpetre, one drachm. Reduce all to powder, and mix in to a paste with a solution of one ounce of gum tragacanth to the pint.

Pastilles au benzoin.-Take half a pound of benzoin, four or five grains of storax, five grains of cassia, two drachms of dry Peru balsam, half a drachm of cloves; half an ounce of charcoal; one drachm of nitre, half a drachm essential oil of orange flowers, and half a drachm tincture of ambergris. All these materials must be in fine powder.

Pastilles à la rose.-Gum, in impalpa- ble powder, six ounces ; olibanum, in inpalpable powder, six ounces ; storax, in impalpable powder, six ounces; saltpetre, in impalpable powder, four ounces ; Powder à la rose, impalpable powder, eight ounces ; charcoal, impalpable powder, two pounds ; essence of roses, half an ounce. Mix together the powders, and add to them a quart of rose water in which has been dissolved an ounce of gum tragacanth. Rub up well in a mortar, and of this paste form the pastilles. They are burned upon a handsome chafing-dish or perfume-pan made for the purpose.

Fumigating Paper.-There are two modes of preparing this article:-1. Take sheets of light cartridge paper, and dip them into a solution of alum-say, alum, one ounce; water, one pint. After they are thoroughly moistened, let them be well dried; upon one side of this paper spread a mixture of equal parts of gum benzoin, olibanum, and either balm of Tolu or Peruvian balsam; or the benzoin may be used alone. To spread the gum, \&c., it is necessary that they be melted in an earthenware vessel, and poured thinly over the paper, finally smoothing the surface with a hot spatula. When required for use, slips of this paper are held over a candle or lamp, in order to evaporate the odorous matter, but not to ignite it. The alum in the paper prevents it to a certain extent from burning.

2. Sheets of good light paper are to be steeped in a solution of saltpetre, in the proportions of two ounces of the salt to one pint of water, to be afterwards thoroughly dried.

Any of the odoriferous gums, as myrrh, olibanum, benzoin, \&c., are to be dissolved to saturation in rectified spirit, and with a brush spread upon one side of the paper, which, being hung up, rapidly dries.

Slips of this paper are to be rolled up 
as spills, to be ignited, and then to be liquor. Repeat this washing and decantblown out.

The nitre in the paper causes a continuance of slow combustion, diffusing during that time the agreeable perfume of the odoriferous gums. If two of these sheets of paper be pressed together before the surface is dry, they will join and become as one. When cut into slips, they form what are called Odoriferous Lighters, or Perfumred Spills.

\section{FRAGRANT PASTILLES FOR NECK- LACES OR BRACELETS.}

Pâte de Roses.-Rose leaves, two ounces; lampblack, one and a half drachms ; isinglass, one ounce ; gum tragacanth, half ounce.

Dissolve the gum and isinglass in boiling water, thicken the solution by evaporation, and add thereto the other articies previously mixed together. When the paste is homogeneous, knead it well in mass, and divide into small beads. It can also be moulded into a variety of forms and designs, in relief, resembling cameos and similar ornaments.

Pàte de Menthe ou de Jasmin.-Flowers of mint or jasmine, one ounce; gum tragacanth, half ounce ; vermillion, two ounces. In this way red-colored pastilles are made.

Pastilles for Bad Breath.-Chocolate, three ounces; charcoal, one ounce; sugar, one ounce; vanilla, one ounce; mucilage, quantum sufficit. Make into lozenges of five grains, and take four or five daily.

Preparation for Bad Breath.-Dry chlpride of lime, three drachms ; distilled water, two ounces.

Powder the chloride of lime in a glass mortar, and then add a part of the distilled water ; stir up and let it repose for some minutes; when settled, decant the clear liquor. Add a new quantity of water to the residue, triturate, and after sufficient rest, again draw off the clear ing a third time; add all the clear solutions together, and filter. To the liquid running through, add two ounces alcohol, in which four drops oil of rose and four drops of any other desired oil are dissolved.

The solution, thus prepared, removes the fetid odor of diseased gums. The dose is half a table-spoonful in a tumbler of water, with which the gums are moistened daily by means of a sponge or brush.

Keep the bottle always closely stoppered to retard decomposition.

To. Crystallize Flowers. - Construct some baskets of fancy form with pliable copper wire, and wrap them with gauze. Into these tie to the bottom violets, ferns, geranium leaves, - in fact, any flowers except full-blown roses, - and sink them in a solution of alum, of one pound to a gallon of water, after the solution has cooled. The colors will then be preserved in their original beauty, and the crystallized alum will hold faster than when from a hot solution. When you have a light covering of crystals that completely covers the articles, remove the basket carefully, and allow to drip for twelve hours. These baskets make a beautiful parlor ornament, and for a long time preserve the freshness of the flowers.

Hair-dyes. - Nearly all the hair-dyes in use at 1..e present time contain some form of lead, and are therefore dangerous. The symptoms most commonly resulting from their use are partial paralysis, numbness of the back of the head and neck, often extending to the arm and wrist, pains in the stomach and bowels, with other indications of lead poisoning. During the past few years, since these dyes have been largely used, many lives have been sacrificed; and those of ruined health or impaired constitutions may be counted by thousands. 
These preparations are generally represented to be harmless. The noxious ingredient being usually lead, this may be readily detected by adding to a little of the suspected dye a few drops of a solution of hydriodate of potash, to be obtained at any ảrug store. One drachm dissolved in an ounce of water will be of sufficient strength. The preparation, if it contains lead, will be instantly changed to a bright orange color, the product being hydriodate of lead. As appears by Prof. C. F. Chandler's Report to the Board of Health of New York City, fifteen out of sixteen preparations for the hair which he analyzed contained lead in varied proportions from.11 to 16.39 grains in one fluid ounce.

Even without a resort to chemical re-agents, one may usually satisfy himself of the contents of these compounds by the peculiar sticky feeling of acetate of lead when rubbed between the fingers, or by placing a drop upon the tongue and noting the sweetish astringent taste of the salt. Avoid as you would any other deadly poison all dyes or hairdressings which contain lead in any of its various forms. See Hair-dressing page 566.

Chinese Depilatory.-Quicklime, one pound ; pearlash and sulphuret of potassium, each, two ounces. Triturate together, sieve, and preserve the fine powder in a well-stopped bottle.

Crôme Parisienne Dépilatoire.-Take quicklime, two ounces; orpiment (sulphuret of arsenic), half an ounce; alkanet in powder, two drachms.

Mix together and pack up in bottles or boxes, bearing directions as follows, and to be strictly conformed to in the application of the powder:

Put some pinches into a saucer or eggglass, and pour upon it enough warm water to thin it into a pasty or pap consistence, and apply it in this form to the places to be depilated. Let it remain five to eight minutes, then moisten with a little warm water, and gently remove the dampened stuff with the point of a knife. Afterwards sponge the part with warm water and dry without rubbing.

It is necessary that there should always be an interral of twenty-four hours between any two applications of this powder.

Eau de Javelle (rectified.)-This water is used both as a disinfectant, and for the removal of fruit, rust, and ink stains. It is prepared by saturating an aqueous solution of potash with chlorine gas. A few drops, washing the linen afterwards with soap, will bleach it perfectly.

Ink for Marking Linen.-Sulphate of manganese, one drachm; distilled water, one drachm; powdered sugar, two drachms ; lampblack, half a drachm.

Make into a semi-liquid paste. This paste is used with a stamp. The mark is dried, then washed in solution of caustic potash and dried anew; then again washed in water. The name now stands out boldly.

Shaving Pastes.-White wax, spermaceti, and almond oil, of each, quarter of an ounce ; melt, and while warm, beat in two squares of Windsor soap, previously reduced to a paste, with rose water.

Another.-Melt together half an ounce each of spermaceti, white wax, and oil of almonds; beat it up with three ounces of the best soap, and a sufficient quantity of eau de Cologne.

Preparation of Good Hair Dye.-Ten grains gallic acid in one ounce weak alcohol.

For the Dye.-Four grains nitrate of silver in one ounce of water with half a drachm of aqua ammonia.

Add the ammonia cautiously till the precipitate dissolves. 


\section{THE FAMILY MEDICAL GUIDE.}

No directions here given are intended the patient should breathe an equal temto supply the place of medical attendance. A little medical knowledge is a dangerous thing, and this temporizing with the constitution is often attended with the worst results. It would, therefore, be an act of folly and danger for the nonprofessional to presume to decide on the ailment, and equally so to prescribe for it. At the same time, it is. well to gain all the information possible.

The following recipes have been submitted to a physician:-

For Hooping Cough.-Dissolve one scruple of salt of tartar in a gill of soft water; add to it ten grains of cochineal, finely powdered, and sweeten it with lump sugar. The dose for an infant is a .teaspoonful four times a day; from five years old upwards, a table-spoonful may be taken. The following embrocation for spasmodic coughs of all kinds, as well as for inflammatory croup, is valuable: Two drachms of tincture of opium, two ounces of camphor liniment. A teaspoonful to be rubbed in over the throat and chest every night and morning, and cover the parts immediately with flannel. If the chest is loaded with phlegm, a gentle emetic must be given once, twice, or three times a week, as required. Ipecacuanha wine is most suitable, and the dose for a child of four or five years is two and a half or three drachms.

perature, as nearly as it can be managed, not too warm and close, but equal ; and when the virulence of the disorder has passed away, the open air (if the weather be mild) may be frequently resorted to : and a change of air, where it is practicable, will often remove at once all remains of the complaint.

The diet is of paramount importance, and for children of even six and seven years of age, ought to be little more than milk and broth. Should the milk be found to curdle on the stomach, a little common salt must be added. If thirst prevails, a little toast and water may be given. If the attack, from its length and severity, should cause a loss of strength, it will soon be recruited by a cold infusion of bark, gentle exercise, pure air, and diet of nourishing broth.

Mixture for Hooping Cough.-Ipecacuanha wine, two drachms; carbonate of soda, half a drachm; paregoric elixir, two drachms; water, one ounce. A teaspoonful or two (according to the age of the patient) may be given three times a day.

Scarlet Fever, or Scarlatina.-This disease begins with the common symptoms of fever, often with languor and disposition to fainting, shiverings, sickness, a quick pulse, and pain in the head; there is frequently, very early in the dis-

During the first stages of this disorder order, a stiffness of the muscles of the 
neck, which is soon followed by some difficulty in swallowing.

To determine the eruption to the surface of the body, it will be right to give the simple saline, made as follows: Take of citric acid one drachm and a half; bi carbonate of potash, two drachms; water, seven ounces and a half; simple syrup, half an ounce; spirits of nitre, two drachms. Mix, and take a sixth part every four hours. If the child is very young, give half the dose.

'Throughout the whole of this disease it will be advisable to make frequent use of some detergent (cleansing) gargle, which in young children must be thrown into the throat with a syringe.

Detergent Gargle.-Take of purified alum one drachm; barley water, half a pint; honey of roses, one ounce.

Draught for Palpitation of the Heart, with great nervous Irritability.-Tincture of foxglove, ten drops; camphor mixture, one ounce; tincture of columba, one drachm. This draught may be taken twice a day.

Mixture for Children Teething, when the Bowels are disordered.-Chalk mixture, fifteen drachms ; tincture of cinnamon, one drachm. Mix together. Two teaspoonfuls to be given three or four times a day as required.

Primrose Ointment, for Burns and Ulcers.-Bruise one pound of the leaves of this well known plant, in a mortar, along with half a pound of the flowers; simmer these in an equal quantity of hog's-lard, without salt, until the primroses become crisp; after which the ointment, whilst fluid, must be strained through a coarse sieve. This is an excellent application for obstinate ulcers or burns.

Excellent Medicine for Indigestion.Carbonate of magnesia, one ounce ; car- bonate of soda, one ounce; powdered ginger, one drachm ; best Turkey rhubarb, half a drachm. Well mixed in a mortar, to be kept in a bottle with a glass stopper. The dose for an adult is half a teaspoonful.

For Inflammation of the Eyes.-Bran$\mathrm{dy}$, one teaspoonful ; white wine vinegar, one teaspoonful; soft water, nine teaspoonfuls. Mix-and to be used frequently.

Cure for Ringworm. - The head to be washed twice a day with soft soap and warm soft water; when dried, the places to be rubbed with a piece of linen rag dipped in ammonia from gas tar; the patient should take a little sulphur and molasses, or some other gentle aperient, every morning; brushes and combs should be washed every day, and the ammonia kept tightly corked.

Or:-Mix pyroligneous acid with onethird of water; rub the spots carefully for three mornings: if cured, a scurf will appear, which must be softened with cold cream, or lard without salt, but no soap or water must be used.

It is desirable to cut off the hair from the immediate neighborhood of the ring; and after the cure is effected, weaken the lotion still farther, by the addition of more water, and well wash the head all over with it. It seldom happens that an internal use of medicine is requisite; but where the disease is very inveterate, I would recommend a powder to be given at bed-time, once or twice. The following will answer very well for a child of seven years of age: calomel, one grain ; jalap, powder, eight grains.

The great Abernethy used to say poultices were blessings or curses, as they were made; I cannot, therefore, insert a more useful recipe than that of this celebrated surgeon's for a well made

Bread and Water Foultice.-Scald out 
a basin, into which boiling water is to be turned, then add coarsely-crumbled bread as much as the water will absorb. After a little time drain off the surplus water, and spread the light pulp remaining onethird of an inch trick on folded linen, and apply it of the temperature of a warm bath. As it becomes dry from the heat of the part, moisten by dropping on a little warm water.

Fever and Ague. - The title of intermittent is applied to that kind of fever, which consists of a succession of paroxysms, between each of which there is a distinct and perfect intermission from fever symptoms. When it comes on within the space of twenty-four hours, it is called a quoitidian, when it returns every other day, it is called a tertian; and when it attends on the first and fourth day, it is named a quartan ague. It is very generally acknowledged that marsh exhalations, or the effluvia arising from stagnant water when acted upon by heat, are the most frequent exciting causes of ague.

Uncleared lands are also a fruitful cause. Hence its prevalence in all new countries. Intermittent fever often appears in disguise; sometimes putting on the form of periodical headache or neuralgia, which come on with the same regularity as the chill, and seem to take its place. At other times it is still more obscure, existing in the form of dumb ague, as it is termed. Under all these disguises there is usually but little difficulty in detecting it, through its regular periodical return - being generally prompt to the hour, and not unfrequently to the minute. Periodicity, therefore, rather than peculiar symptoms, is the test of miasmal complaints. The usual mode of treating fever and ague has been with tonics, with the view of breaking the chill, but nothing is done to remove the disease of the liver and other organs on which the chill and fever depend. Relapses are the consequence. The sufferer is only cured for a time: he is relieved of a symptom of disease, not of the disease itself. We know of no compound more reliable for a thorough and effectual cure than Osgood's Chotagogue, a medicine which has been before the public more than thirty years, and has become the standard remedy for these complaints. The publisher of this book has for a long time used it himself, and in his family, and often recommended it to others, and is yet to learn of an instance in which it has failed to effect a cure. It is especially valuable also as a preventive for persons residing in malarial regions, or traveling through them. By taking a teaspoonful of the cholagogue, morning and night, when thus exposed, all danger, with ordinary care, will be avoided.

Erysipelas.-This disease is an inflammatory affection principally of the skin, when it makes its appearance externally. If there is fever and inflammation, it will be proper to administer aperient medicines, and nothing, perhaps, suits your purpose better than the old-fashioned black-draught-senna and salts. The external application of Goulard's Lotion will allay heat and irritation. The patient must drink freely of tea, bran tea, or warm barley water, and this is a necessary part of the treatment, which must never be neglected. The vesicles must be kept lightly covered with pure, unadulterated wheat flour; when the inflammatory symptoms run high, the diet must consist of light nourishing food, such as sago, arrowroot, bread pudding, and such like things; but in those cases where symptoms of irritation prevail, a more generous diet, such as animal broths, ought to be allowed.

Spirit Wash.-IIalf a gill of spirits of 
wine, or a gill of brandy, added to a pint of water, makes this wash useful in allaying the pain of extensive bruises.

Plummer's Pill, for Eruptions on the Skin.-Calomel and antimony, of each, one drachm; guaiacum powder, two drachms.

Useful Mixture for Diarrhcea in Infants.-Carbonate of magnesia, half a drachm ; rhubarb, in powder, twenty grains ; dill water, three ounces ; aromatic spirit of ammonia, thirty drops ; sugar, teaspoonful. Mix : Two teaspoonfuls may be given two or three times a day.

Goulard Lotion, or Lead Wash, may be made by dissolving one drachm of sugar of lead in a pint of soft water. Some persons are very fond of using this wash, with the addition of spirits of wine, as an evaporate ; but I do not like it, for it renders the skin very dry and harsh, and its sedative virtue acting through unbroken skin, is not of much value. Under other circumstances it is very often useful.

When used as a wash for the eyes, two grains of the sugar of lead are to be dissolved in two table-spoonfuls of water.

Bran Tea.-A very cheap and useful drink in colds, fevers, and restlessness from pain. Put a handful of bran in a pint and a half of cold water, let it boil rather more than half an hour, then strain it, and, if desired, flavor with sugar and lemon-juice ; but it is a pleasant drink without any addition.

Electuary for Scorbutic Eruptions.Peruvian bark, powdered, half an ounce; aromatic confection, half an ounce; syrup of oranges, sufficient quantity to mix the bark and confection; and take the size of a nutmeg, three times a day; in a glass of seidlitz or soda water.

Draught for Hysteric Patients.-Camphor mixture, one ounce; fotid spirit of ammonia, two drachms.
Fever Draught.-Almond mixture, one ounce ; carbonate of potass, twenty grains; syrup of poppies, one drachm. Pour into this a table-spoonful of lemon juice, and drink while effervescing.

Ointments. - The base of all ointments is grease, and they are used for dressing wounds and sores, to prevent the sticking of the lint or linen with which they are covered, and protect them from the air; the most simple kinds serve this purpose best; but sometimes medicine of various kinds is mixed up with grease, to form ointments, through the means of which the medicine acts on the surface of the sore.

Simple Ointment is made by melting in a pipkin by the side of the fire, without boiling, one part of yellow or white wax, and two parts of hog's lard, without salt or olive oil.

Resin Ointment or Yellow Basilicon is composed of two ounces of yellow wax, five ounces of white resin, and seven ounces of hog's-lard; these must be slowly melted together, and stirred constantly with a stick, till completely mixed.

This ointment is sometimes used in treating scalds and burns; also for dressing blisters, when it is wished to keep up a discharge from them for a few days. This is a stimulating ointment.

Calamine Ointment, or Turner's Cerate, consists of half a pound of yellow wax, and a pint of olive oil, which are to be melted together; this being done, half a pound of calamine powder is to be sifted in, and stirred till the whole be completely mixed.

This is an excellent ointment for stimulating sluggish wounds or sores.

Development of the Lungs.-Nultitudes, and especially ladies in easy circumstances, contract a vicious and destructive mode of breathing, not carrying the breath half way down the chest, and 
scarcely expanding the lower portions of the chest at all. Children that move about a great deal in the open air, and are in no way laced, breathe deep and full in the bottom of the chest, and every part of it. The lungs give us the power of action, and the more exercise we take, especially out of doors, the larger the lungs become, and the less liable to disease. In all occupations that require standing, keep the person straight. If at table, let it be high, raised up nearly to the armpits, so as not to require you to stoop; you will find the employment much easier; while the form of the chest and symmetry of the figure will remain perfect.

The Netille Rash.-This disease takes its name from its being attended by an eruption similar to what is produced by the stinging of nettles. In some cases it is ". accompanied with large wheals or bumps, which appear of a solid nature, without any cavity or head, containing either water or other fluid. Nothing is so efficacious in this disorder as half a teaspoonful of magnesia, and the same quantity of cream of tartar, mixed in half a teacupful of milk, an hour before breakfast, and repeated as required.

Sprain.-To treat a sprain properly, it should be kept perfectly at rest; and, if it be of the ankle or knee, the patient must lie in bed, or on a sofa. Warm, moist flannels should be repeatedly applied for some hours, and a bread-andwater poultice on going to bed. These should be continued for some days, and no attempt made to use the joint. If the pain be very severe, and it continues so for the first or following days, leeches may be applied, and repeated if necessary. Some persons are fond of putting on a vinegar poultice at once; but this is better left alone till the tenderness has subsided, and there remains only a little pain and stiffness in the joint. Then a vine- gar poultice is a very good application, as it produces a diversion of the inflammation going on in the ligaments, by bringing out a crop of pimples on the skin, at a time when the pressure of rubbing in any stimulating lotion cannot be borne.

When the pain has entirely ceased, the joint must not be carelessly used ; and, if it be the knee or ankle sprained, walking till the joints become weak and ache must be most carefully avoided, as irreparable mischief is thereby very often caused.

A joint often swells a long time after a sprain ; under which circumstances it is best to bind it up with straps of soapplaster or a roller.

Excellent Medicine for Rheumatism.Powdered gum guaiacum, eight grains; flour of sulphur, two drachms; powdered rhubarb, fifteen grains ; cream of tar. tar, one drachm ; powdered ginger, thirty grains ; powdered nutmeg, eight grains.

To be made into an electuary, with two ounces of clarified honey; a teaspoonful to be taken night and morning.

For Sprains and Bruises, especially where the parts are discolored with blood underneath the skin, and for rheumatic swelling of the joints :-Vinegar, one pint ; distilled water, half a pint, rectified spirits, one and a half pints ; camphor, two ounces.

Mix the vinegar and water, dissolve the camphor in the spirit of wine, and then put them all together.

For sprains, bruises, and other injuries, when the skin is not broken, carbonate of ammonia, two ounces ; vinegar, two pints; proof spirit, three pints.

Mix the ammonia with the vinegar; when the effervescence ceases, add the spirit. In inflammation of the joints of some standing, this is mixed with linseed meal, and applied as a poultice, twice a-day.

Aperient Electuary.--A very useful fam- 
ily medicine, particularly good for those who are troubled with asthma or rheumatism. One ounce of senna powder, half an ounce of fiour of sulphur, two drachms of powdered ginger, half a drachm of saffron powder, four ounces of honey. The size of a nutmeg to be taken night and morning.

For Heartburn.-Carbonate of magnesia, ten grains ; carbonate of soda; five grains; ginger in powder, five grains; liquorice in powder, fifteen grains. Take as a powder two or three times during the day.

Zinc Ointment is made by rubbing well together one ounce of oxide of zinc, and six ounces of hog's lard. This ointment is useful for chilblains ; it is also commonly used for dressing the sores remaining after scalds and burns, to absorb the great discharge which generally follows ; and it is a very good application to cracked skin, from which a watery fluid oozes and irritates the neighboring skin.

Leeches.-Leeches should be kept in a cool place, in a stone or glass jar, filled with soft water, and tied over with coarse muslin to prevent their escape, though it allows them air. The water should be changed only when it begins to get foul, as too frequent disturbing destroys them. The part on which they are to be applied should be carefully wiped with a cool moist cloth, so as to leave it damp. If they do not take readily, the part may be moistened with a little sugar and water, or milk. But if this does not answer, the skin may be gently scratched with a needle-point, till the blood comes, and then they will take. If it be wished to put the leeches as near as possible on one spot, the best plan is to put them all in the deep part of a pill-box, or in a small wine-glass, which is to be turned down on the part. If you wish them to spread over a large surface, as upon one of the limbs or the stomach, they must be put on singly and by hand, which is often very tedious and tiresome work. They should then be held tightly by the tail, wrapped in a piece of wet rag, so that they may be less inconvenienced by the heat of the hand; and if the leech do not soon fix, it is best to put it again into the water to cool itself, and after applying others, to try it again. It is always best to have more leeches than the number directed, in case some will not bite.

When the proper number have been applied, they should be left quite alone, or they are apt to unfix, and, wandering about, are of no further use. When they have sucked their fill, they generally drop off, and should then be put in a plate with a little salt, which quickly makes them throw up the blood; and, as soon as they have emptied themselves, they should be put into plenty of fresh cold water, so that they may get free from the salt, for if left in it, or if too much be put on them, they contract violently, and die almost immediately.

After the leeches have come off, the bleeding from the wounds is to be encouraged, by first quickly sponging off whatever clotted blood there may be, and then covering the part with a warm bread-and-water poultice, which must be changed every half hour, so long as it may be thought necessary to keep up the bleeding. This is much better than leaving the surface exposed, and mopping with a warm sponge, which is very fatiguing to the patient, besides exposing him to the danger of taking cold.

If a leech-bite continue bleeding for some hours, and the person, more especially if an infant, begins to be very faint, and the countenance and lips pallid and cold, like marble, no time must be lost in stopping the bleeding.

This is done by pressure with the finger ; but, if that fail, by applying caustic, or by running a moderate-sized darning needle into the skin on one side of the 
bite, and bringing its point well out on the other side. The whole wound is thus lifted up, and a piece of silk or strong thread, is then to be wound round and round the bite, under the two ends of the needle. This will raise it up like a small spot, and generally stops the bleeding very effectually. In four days cut the silk and draw out the needle carefully, and there the matter usually ends. But in a peculiar state of constitution, sometimes even after the removal of the needle and thread, the bleeding will continue. Nothing then remains, but to touch the bottom of the wound with a bit of thin iron wire heated white-hot, which never fails to stop the bleeding. Though this may seem a very horrible proceeding, it is not very painful if the iron be white-hot, as it destroys sensation in an instant; but whether it give pain or not is a matter of no consequence, as it is the only sure mode of saving the patient.

Pimples.-A weak solution of sugar of lead or sulphate of zinc may be used as a wash ; if not effective, try-camphorated spirit, two drachms, corrosive sublimate of mercury, one grain, rose water or almond water half a pint, to be applied night and morning. When there is great irritability of the skin, a decoction of the woody nightshade may be used instead of the rose or almond-water. If pimples arise from the condition of the blood, then cooling purgatives must be taken. A Plummer's pill at night, and a teaspoonful of decoction of Sarsaparilla twice a day with salts and senna once a week, are excellent medicines.

For Chicken-Pox.-Let the patient partake freely of water or cooling drinks, as thirst is always an attendant on the disease, and gentle laxatives should be occasionally given,- - a teaspoonful of lenitive electuary with a little milk of sulphur in it, is all that is generally re- quired; but should febrile symptoms run high, it may be advisable to take two or three saline draughts during the day. The following is a recipe for an excellent saline, to be drunk while effervescing. Twenty grains of carbonate of soda dissolved in a tumbler containing two tablespoonfuls of water; add two teaspoonfuls of lump-sugar rolled; then put a dessert-spoonful of lemon-juice into a wine-glass (or fifteen grains of citric acid dissolved in one table-spoonful of water.) Pour it into the tumbler and drink it while the effervescence is taking place.

Croup.-The cause of this disease is a morbid secretion of thick mucus in the trachea (windpipe), adhering so firmly to its sides as to impede respiration.

A warm bath should immediately be administered, and an emetic given as soon as possible; say, ten drops of antimonial wine for a child of three years of age, and repeated every quarter of an hour, until effectual, and according to the age and strength of the patient: a blister should be applied across the throat, the sufferer being made to inhale the vapor of warm water with vinegar in it, and kept nearly upright in bed. A doctor should be procured as soon as possible.

Children liable to this disease should be warmly clad, wearing flannel next the skin, and an emetic given upon the earliest appearance of the attack.

Elder-flower Ointment is the mildest, blandest, and most cooling ointment, as the old women term it, which can be used, and is very suitable for anointing the face or neck, when sun-burnt. It is made of fresh Elder-flowers stripped from the stalks, two pounds of which are simmered in an equal quantity of hog's lard till they become crisp, after which the ointment, whilst fluid, is strained through a coarse sieve. 
Chilblains.-The mode of prevention of chilblains will be at once perceived, viz.:-To protect the parts most liable to the attack (hands and feet) from sudden alternations, either from cold to heat, or from heat to cold. To keep the constitution in such a healthy state, as to make all parts possess such vitality as to be able to resist slight alternations in temperature.

Woollen stockings or socks, and warm boots and shoes, should be worn in the street. Warm leather gloves are better for the hands than woollen ones, through which the dry frosty air is apt to pierce and chap the hands. Tight wristbands, tight garters, and boots that lace or button tightly about the ankles, must be avoided. The most frequent cause of chilblain is the warming of numbed hands or feet at the fire. A good constitutional remedy is small doses of tartrate of antimony wine, which constringe the enlarged vessels. A drachm of the wine may be added to half a pint of water, in which a drachm of saltpetre has been dissolved. Dose : for an adult one or two table-spoonfuls every four hours; for a child, one or two teaspoonfuls three times a day. It should not be given in such doses as to produce vomiting. The head, neck, and chest, should be washed in cold water every morning, and brisk exercise taken at regular hours.

Wash the hands in cold water every three hours, and lather well with Windsor soap. When the hands are nearly dried with the towel, pour a little eau-deCologne, or milk of roses, into the palm of one hand, and rub it over the whole of both.

A leech is a useful remedy to unload the overcharged blood-vessels; or the part may be pricked with a needle, or punctured with a lancet; in such cases a soft bread-and-water poultice should be kept on during the night, applied warm, so as to encourage the flow of blood.
Houschold Remedies for Chilblains.The following are among the most approved of the domestic remedies, or palliatives :-

Take half an ounce of white wax, ox marrow one ounce, hog's lard two ounces ; melt these ingredients slowly over a fire in a pipkin, and mix them well together; then strain through a linen cloth. Before going to bed, spread the ointment upon the parts affected, feet or hands, taking care to wrap them well up. The application should be made as soon as the first attacks are felt.

Lemon juice rubbed on the inflamed parts is said to stop the itching. A sliced onion dipped in salt has the same effect, but makes the feet tender.

Strong white mustard, pounded and mixed with water to the consistence of thick cream, rubbed on, every night. letting it dry on the chilblain, and washing it off in the morning with rain water.

Encourage children to use the skipping rope regularly during cold weather. This is a capital prevention, together with regularly washing and rubbing the feet.

Aperient for Children.-Gingerbread made with oatmeal instead of flour is a very useful aperient for children.

Spring Aperients; for children, nothing is better than:-1. Brimstone and molasses ; to each teacupful of this, when mixed, add a teaspoonful of cream of tartar. As this sometimes produces sickness, the following may be used:2. Take of tartrate of soda one drachm and a half, powdered jalap and powdered rhubarb each fifteen grains, ginger two grains. Mix. Dose for a child above five years, one small teaspoonful; abov́e ten years, a large teaspoonful ; above fifteen, two; and for a person above twenty, three teaspoonfuls, or the whole.

Aperient Pills.-To some adults all liquid medicines produce such nausea that pills are the only form in which 
laxative medicines can be exhibited; the following is a usual formula:-Take of compound rhubarb pill, a drachm and one scruple; of powdered ipecacuanha, six grains, and of extract of hyoscyamus one scruple. Mix and beat into a mass, and divide into twenty-four pills. Take one, or two, or if of a very costive habit, three at bed-time.

Infants' Aperient.-Take of rhubarb five grains, magnesia three grains, white sugar a scruple, manna five grains; mix. Dose, varying from a piece half the size of a sweet-pea to a piece the size of an ordinary pea.

For Weak Eyes.-Two grains acetate of zinc, in two ounces of rosewater; filter the liquor carefully, and wash the eyes night and morning.

A Simple Vapor Bath.-It is not generally known that the settlers in the remote parts of the West make use of the following simple mode of procuring a vapor bath : The patient is enveloped in blankets, which are closely fastened about the neck, leaving the head exposed. The patient sits on a chair; under the chair is placed a basin or deep dish, with half a pint of alcohol, whiskey or gin which is jgnited. The blankets lap over each other, enveloping the whole, and are closed to the floor by other blankets, \&c., as much as possible. In a very few minutes the patient is in a profuse perspiration; he is then immediately put to bed between warm blankets.

Ear Ache.-Sometimes ear-ache is connected with chronic ulceration in the internal and external part of the ear, when injections of warm water and soap are advisable. In this case there is sometimes" a constant foetid discharge, for which the following mixture has been reconmended:-Take of ox-gall, three drachms; balsam of Peru, one drachm.
Mix. A drop or two put into the ear with a little cotton.

Pains after Exertion.-It is not generally known to pedestrians that the pains in the knees and legs, which usually follow after a long excursion, and which continue with some persons for two or three days after, may be prevented or considerably lessened, by bathing the parts affected in cold spring water, immediately before going to bed. Care should be taken if the feet be dipped in the water, to afterwards dry them thoroughly with a rough towel.

For a Cough.-Quarter of a pound of linseed ; quarter of a pound of raisins ; two ounces of stick liquorice; two quarts of soft water, to be boiled until reduced to half the quantity. When strained, add a quarter of a pound of brown candy, pounded ; one table-spoonful of good old rum, one table-spoonful of lemon juice, or vinegar. A cupful to be taken on going to bed, and more frequently if required. To be warmed.

Nipple Liniment, by Dr. Sigerbundi.Aqueous extract of opium, one grain ; Fresh limewater, three drachms; oil sweet almonds, fresh and cold pressed, three drachms.

Mix the whole, and preserve in a covered pot. The label should direct the application of this liniment by means of very fine lint, and that the nipples be covered with a piece of skin spread with wax, a hole being left open in the centre to permit the free passage of the milk.

Pomade (for healing slight tumors.)White pitch, pure, one pound ; resin, one pound; yellow wax, one pound; gum ammoniac, twelve ounces; oil of hemlock, four ounces.

Spread upon sheepskin, and apply the plaster to the sore.

Pomade Souveraine, by Laforet (for the Cure of Corns.)--Take one ounce black 
pitch, half an ounce galbanum, and one scruple of sal ammoniac, dissolved in vinegar ; add one and a half drachm diachylon. Take only sufficient to cover the corn, and spread it upon a piece of sheepskin. After some days remove the plaster, and the corn comes with it.

C'ra Fortifant (for the Nails.)-Oil of lentise, half an ounce ; salt, half a drachm; resin, one scruple; alum, one scruple; wax, one scruple.

English Court-Plaster.-Stretch upon a frame a piece of thin black silk, and with a camel's-hair brush pass over it three or more coats of isinglass, dissolved in boiling water. To give the silk an agreeable odor, when applying the last coat mix in a little compound tincture of benzoin with the isinglass.

The color can be varied by taking silk of any desired shade. The intervals between the application of the coats should be sufficiently long to permit a thorough drying of each.

Collodion.-This is a liquid adhesive plaster, to be applied with the brush upon sores and cuts. It is made by dissolving gun-cotton in rectified ether, mixed with one-eighth its bulk of strong alcohol. A slight addition of Venice turpentine increases its adhesiveness.

Toothache Collodion.-This is the plain collodion, holding in solution one grain of sulphate of morphia to every ounce of liquid. It is almost a specific for the toothache, which results from an exposed nerve.

Blistering Collodion.-The etherial extract of cantharides, dissolved in collodion, forms a most convenient, active, and clearly blistering liquid. If the blister is opened at the side, the film of collodion remains unbroken; and, by thus protecting the sore, obviates the necessity of dressing it with ointment.

For Burns.-Apply cotton wool dipped in oil as soon as possible, and keep 36 it on till the fire is entirely out, which will usually take from two days to a week.

For a Cut.-Wash off the blood in cold water, and bind it up with a clean cotton bandage ; if it inclines to bleed, put on scraped lint, after bringing the edges of the wound together as closely as possible, and bind it rather tight. Or use sticking-plaster.

When a Nail or Fin has been run into the foot, instantly bind on a rind of salt. pork; if the foot swell, bathe it in a strong decoction of wormwood, then bind on another rind of pork, and keep quiet till the wound is well. The lockjaw is often caused by such wounds, if neglected.

For a Bruise or Sprain.-Bathe the part in cold water, till you can get ready a decoction of wormwoud. This is one of the best remedies for sprains and bruises. When the wormwood is fresh gathered, pound the leaves and wet them either with water or vinegar, and bind them on the bruise ; when the herb is dry, put it into cold water, and let it boil a short time, then bathe the bruise and bind on the herb.

Always keep cotton wool, scraped lint and worm wood on hand.

The Ear-ache is usually caused by a sudden cold. Steam the head over hot herbs, bathe the feet, and put into the ear cotton wool wet with sweet oil and paregoric.

Prescription for Camphor Cake:Scrape into an earthen vessel one ounce and a quarter of spermaceti and one ounce and a half of white wax ; add six drachms of powdered camphor, and four table-spoonfuls of best olive oil. Let it stand near the fire until dissolved, stirring it well.

Plaster for Pain in Side or Cough to be applied between the shoulders.-One ounce burgundy pitch, one ounce beeswax, one ounce and a half of resin. cre 
ounce of gum camphor dissolved in two changes which occur in all oils upon exteaspoonfuls of brandy over a slow fire. posure to light and air. It is best difTo be kept in tin boxes, and when used fused by the agency of strong spirit, in spread on kid.

The Cure of Intemperate Habits. - A notion prevails that a person addicted to alcohol stimulants cannot at once leave them off without danger of illness, or great and long-continued suffering. This is an error. A cup of tea or coffee will supply the needed tonic when a sense of exhaustion is felt, and we have the authority of an emiment physician for stating that no constitutional injury will be suffered from immediate and total abstinence. The uncomfortable feelings will subside in forty eight-hours, and will be entirely over. in a fortnight. Any man who has firm resolution can break himself at once of the fatal habit of drinking.

Hair Dressing. - There are many persons whose hair is naturally dry and crisp, and in most families there is a want of some innocent and agreeable wash or dressing which may be used moderately and judiciously. The mixture which may be regarded as the most agreeable, cleanly, and safe is composed of cologne spirit and pure castor-oil. The following is a good formula :-

Pure, fresh castor-oil, two ounces. Cologne spirit (ninety-five per cent), sixteen ounces.

The oil is freely dissolved in the spirit, and the solution is clear and beautiful. It may be perfumed in any way to suit the fancy of the purchaser. The oil of the castor-bean has for many years been employed to dress the hair, both among the savage and crilized nations; and it possesses properties which admirably adapt it to this use. It does not rapidly dry; and no gummy, offensive residuum remains after taking on the chemical which it dissolves. The alcohol or spirit rapidly evaporates, and does not in the slightest degree injure the texture of the hair. This preparation, for dressing the hair of children or ladies, will meet nearly or quite all requirements. A cheap and very good dressing is made by dissolving four ounces of perfectly pure, dense glycerine in twelve ounces of rose-water. Glycerine evaporates only at high temperatures; and therefore, under its influence, the hair is retained in a moist condition for a long time. As a class, the vegetable oils are better for the hair than animal oils. They do not become rancid and offensive so readily, and they are subject to different and less objectionable chemical changes. Oliveoil and that derived from the cocoanut have been largely employed, but they are inferior in every respect to that from the castor-bean.

It is doubtful if any mixture or substance has ever been devised which will restore hair to a bald head. A great many washes and embrocations are manufactured, all of which usually fail to meet the end desired. The falling of the hair is the result of diseased action in the hair follicles, or to a morbid condition of the entire scalp. Still, mixtures containing ammonia, vinegar, soap, and vesicating tinctures, are sometimes thought to prove beneficial. The fall of the hair will usually cease from natural causes in a short time, the germinal vessels taking on healthy action spontaneously. The frequent washing of the head in tepid or cold water, and friction with a brush or coarse towel, is to be commended. 


\section{MISCELIANEOUS RECEIPTS}

To take Stains out of Linen.-For acid stains, wet the part, and lay on it salt of wormwood; then rub it, and hold the part over a lighted match, for the sulphurous gas; or, tie up pearlash in the stained part ; scrape soap into cold water, for a lather, and boil the linen till the stain disappear:

Stains of wine or fruit that have been long in the linen may be removed by rubbing the part on each side with yellow soap, then laying on a mixture of thick starch in cold water, rubbing it well in, and exposing the linen to the sun and air till the stain comes out. The process may be renewed, and, when dry, sprinkle the part with water. Stains may be taken out by dipping the linen in sour buttermilk and drying in a hot sun. Then wash in cold water and dry it several times a day.

A certain way of extracting fruit or wine stains from table linen is to tie up some cream of tartar in the stained part (so as to form a sort of bag), and then to put the linen into a lather of soap and cold water, and boil it awhile. Then transfer it wet to lukewarm suds, wash and rinse it well, and dry and iron it. The stains will disappear during the process. Another way is to mix, in equal quantities, soft soap, slaked lime, and pearl-ash. Rub the stain with this preparation, and expose the linen to the sun with the mixture plastered on it. If necessary, repeat the application. As soon as the stain has disappeared, wash out the linen immediately, as it will be injured if the mixture is left in it.

Iron moulds may be wet, laid on a hot water plate, and a little salt of lemon applied. Wet and renew the process. Be careful not to have the application too strong. Oxalic acid will effectually remove the stains. Mildew may be taken out by mixing soft soap with powdered starch, half as much salt, and the juice of a lemon; lay it on both sides of the part, and leave it on the grass day and night. To prevent flannels from shrinking or losing their color, put them into a pail the first time of washing, and pour boiling water on them, letting them lie till cold.

Remove ink spots by soaking the part in milk immediately; then wash out in cold water without soap.

To destroy Crickets.-Put Scotch snuff upon their holes. Cockroaches may be banished by red wafers. Paint is destructive to all insects, and so is lime.

Potish for Tables.-One pint of linseed oil, half a pint of turpentine, two ounces of beeswax, sixpence worth of white rosin. Boil the mixture in a saucepan, and put it in a bottle for use.

Varnish for Mahogany.-One pint of linseed oil, a wine glass of spirits of turpentine; simmer it slowly till near coming 
to a boil. Take all the stains out of the mahogany, and clean it perfectly. When dry brush it with a feather dipped in the bottled mixture. Rub it some time with soft linen; then take a clean housecloth and rub off the oil, continue rubbing till the table would not soil a cambric handkerchief.

Instead of covering your glasses and pictures with muslin, cover the frames only with cheap yellow cambric, neatly put on, and as near the color of the gilt as possible. This leaves the glasses open, and is a barrier to dust and flies. When faded, it can be re-colored with saffron tea.

When mattresses become hard and bunchy, rip them, take out the hair, pull it thoroughly, let it lie a day or two to air, wash the tick, lay in the hair as light and even as possible, and stitch it down as before. The mattress will be as good as new.

Britannia ware should be first rubbed gently with a woollen cloth and sweet oil, then washed in warm suds and rubbed with soft leather and whiting. Thus treated, it will retain its beauty to the last.

To clean Sponges. - When very foul wash them in diluted tartaric acid, rinsing them afterwards in water; it will make them very soft and white. Be careful to dilute the acid well, as it is very corrosive and therefore should be weak.

For outside Paint.-Put ten gallons of raw linseed oil in a kettle over a slow fire, and let it simmer gently. Add four ounces sugar of lead. Stir up, and when cold, it is fit to use as boiled oil or varnish.

Take of yellow ochre fifteen pounds, of white lead ten pounds, of burnt umber one pound, of Indian red two pounds. This will make a beautiful chocolate color. Add to it half a pound of dry ground litharge.
Stir it up well, and when well stirred add oil according to judgment-about two gallons.

Green tea is good to restore rusty silk. It should be boiled in iron-a cupful to three quarts. The silk should not be wrung, but ironed damp.

Lime sifted through coarse muslin, and stirred pretty thick with white of egg, makes a strong cement for glass or china. Plaster of Paris, pulverized, is still better, and should be stirred by the spoonful as it is wanted.

The best way to clean gold is to wash it in warm suds made of delicate soap, with ten or fifteen drops sal volatile in it. This makes jewelry very brilliant.

Rye paste is more adhesive than any other, and is much improved by adding a little pounded alum while it is boiling.

Straw matting should be washed in salt and water, and dried with a coarse towel. The salt prevents their turning yellow.

Skimmed milk and water, with a bit of glue in it, heated scalding hot, is excellent to restore old, rusty, black Italian crape. If clapped and pulled dry; it will look as well as new.

Washleather gloves should be washed in clean tepid suds.

If you are troubled to get soft water for washing, fill a tub or barrel half full of wood ashes, and fill it with water to make lye. A gallon of strong lye put into a great kettle of hard water will make it soft as rain water. Pearlash or potash is used, but costs something, and is apt to injure the texture of the cloth.

In May and June, the little millers that lay moth eggs, begin to appear. Brush your woollens and beat out your furs, and pack them away in a dark place covered with linen, pepper, red cedar chips, tobacco, and best of all 
camphor, or any strong spicy smell, will keep moths out of your chests and drawers. Sew the things tightly up in linen, leaving no room for the insect to enter.

Save phials and bottles ; apothecaries and grocers will give something for them.

Woollens should be washed in very hot suds, and not rinsed; lukewarm water shrinks them. Silk should be washed in water almost cold; hot water turns it yellow. It may be washed in suds made of white soap ; but no soap should be put on it. It will not do to smooth all silk with a hot iron; either rub it dry with a soft cloth, or put it between towels, and press it with weights.

To take spots out of Cloth.-Sal ammoniac, one ounce; rectified spirit, two wine-glasses full ; aqua distillata, one and a half pints. Apply with a sponge.

Recipe for Ink.-Half a pint of good stale beer, two ounces of galls, half an ounce of copperas, half an ounce of gum arabic pulverized, a quarter of an ounce of rock alum. This is to be kept in a pitcher close to the fire for a week, frequently stirred with a stick, and slightly covered with a large cork. The ink will never mould nor lose its color or substance. It will bear half as much beer for future use. If it thickens, thin it with beer.

To take an impression from a Writing. -Add a little sugar to the ink you write with, then lay a sheet of thin unsized paper, damped with a sponge, upon the writing, and pass over it, very lightly, a flat smoothing-iron, moderately heated.

To sweeten Casks. - When musty it is best to unhead large casks, and whitewash them with quicklime. Or they may be matched with sulphur mixed with a little nitrate of potash, and afterwards well washed. Small casks may be sweetened by washing them first with sulphuric acid, and then with clean water.

To preserve Brass Ornaments.-Beat sal ammoniac into a fine powder, then moisten it with soft water, rubbing it on the ornaments, which must be heated over charcoal, and rubbed dry with bran and whiting.

To color Harness.-The color of harness that has become rusty or brown by wear may be restored to a fine black, after the dirt has been sponged off. Boil logwood chips in three quarts of soft water, to which add three ounces of nutgalls finely powdered, and one ounce of alum; simmer the whole together for half an hour, and it will be fit for use. A harness blacking is thus made: Melt two ounces of mutton suet with six ounces of beeswax, then add one ounce of indigo, finely powdered, six ounces of sugar-candy, dissolved in water, and two ounces of soft soap; mix, and simmer over the fire, when add a gill of turpentine. Lay it on with a sponge, and then polish.

To preserve Polished Irons from Rust. - Polished iron-work may be preserved from rust by a mixture of copal varnish with as much olive oil as will give it a degree of greasiness, adding as much spirit of turpentine as of varnish. The cast-iron work is best preserved by rubbing it with black-lead. But where rust has begun to make its appearance on grates or fire-irons, apply tripoli with half its quantity of sulphur, intimately mingled on a marble slab, and laid on with a piece of soft leather : or emery and oil may be applied with excellent effect.

To clean Kid Gloves.-First see that your hands are clean, then put on the gloves and wash them, as though you were washing your hands, in a basin of spirits of turpentine, until quite clean; 
then hang them up in a warm place, or where there is a good current of air, which will carry off all the smell of the turpentine. This method was brought from Paris, and thousands of pounds have been made by it.

To clean German Silver.-After using, it should be placed immediately in hot water, washed well, and wiped dry with a soft cloth. Once a week let it be washed in soap-suds, and then cleaned with fine whiting, or prepared chalk, mixed with whiskey or spirits of wine, so as to make a paste, which should afterwards be brushed off. Should this metal become discolored, or spotted by vinegar or other. acids, wash it first, and then clean it with sweet oil and powdered rottenstone.

Blacking.-The best blacking for preserving the leather of boots and shoes, and which will make it perfectly watertight, is the following:-Take of yellow wax one ounce and a half, of mutton suet four ounces and a half, horse turpentine half an ounce, ivory black three ounces ; melt first the wax, to which add the suet. and afterwards the horse turpentine; when the whole is melted remove it from the fire; mix in gradually the ivory black, constantly stirring till it is cold. This composition is sometimes run into moulds, and sold under the name of blacking balls ; when it is used it may be laid or rubbed upon a brush which should be warmed before. the fire; it is also the best blacking for every kind of harness; when it is wanted in a large quantity, it may be gently melted in a ladle or pot over a chafing-dish with live coals.

To preserve Picture Frames.-Varnish the frames with water in which onions have been boiled.

To clean a Carpet.-Beat the carpet free from dust, and lay it down. To two gallons of water put two ox-galls. Rub it on the carpet with a hand scrubbingbrush.
To clean Silk.-Pare and slice thin three washed potatoes. Pour on them half a pint of boiling water, and let it stand till cold. Strain the water, and add an equal quantity of alcohol. Sponge the silk on the right side, and when half dry, iron it on the wrong side. The lightest colored silk may be cleaned and brightened by this process; also cloth, velvet, or crape. To iron velvet-lay a damp towel over the bottom of a hot smoothing iron; put on it the wrong side of the velvet, and pass a whisk brush over the pile till the surface is free from wrinkles.

To renew scorched or browned Linen.This is an accident attributable entirely to the ignorance of the laundress, in not knowing how to regulate the heat of her irons. To remedy this:-Add to a quart of vinegar the juice of half a dozen large onions, about an ounce of soap rasped down, a quarter of a pound of fullers earth, one ounce of lime, and one ounce of pearlash, or any other strong alkali. Boil the whole until it is pretty thick, and lay some of it on the scorched part, suffering it to dry. Repeat this process for one or two washings.

To extract grease spots from Silks, Muslins, \&c.-Dcrape French chalk, put it on the grease spot, and hold it near the fire, or over a warm iron or water-plate, filled with boiling water. The grease will melt, and the French chalk absorb it; brush or rub it off; repeat if necessary. Camphene will remove grease also.

To clean Marble.-Take two parts of common soda, one part of pumice-stone, and one part of finely powdered chalk; sift it through a fine sieve, and mix it with water; then rub it well all over the marble, and the stains will be removed; then wash the marble over with soap and water, and it will be as clean as it was at first.

To take oil and grease out of Boards, Marble, \&cc.-Make a paste with fullers' 
earth and hot water; cover the spots therewith, let it dry on, and the next day scour it off with soft or yellow soap.

Or :-Make a paste with soft soap, fullers' earth, and a little pearlash, and use it as above.

To clean Buckskin Gloves.-First wash in warm water and soap, until the dirt is removed; then pull them out into their proper shape, or stretch them on wooden hands. Mix a little pipe-clay, or pipeclay and yellow ochre, according to the color required, with, vinegar or beer. Rub this over the outside of the gloves, and let them dry gradually in the shade; or if in the house, not too near the fire. When about half dry, rub them well, and stretch them on the hand or wooden mould ; after they are rubbed and dried, brush them with a soft brush to get out the dust. Finally, iron the gloves with a smoothing iron moderately heated, taking the precaution to place a cloth or piece of paper over them, and they will look like new.

To make an efficacious and durable Paste.-Good and durable paste may be made with flour in the usual way, but rather thick, with a proportion of brown sugar and a small quantity of corrosive sublimate. A drop or two of the essential oil of lavender, peppermint, anise, or bergamot, is a complete security against moulding. Paste made in this manner, if kept in a close covered pot, may be preserved in a state fit for use at all times.

To make Blue Ink.-Dissolve a small quantity of indigo in a little oil of vitriol, and add a sufficient quantity of water, in which is dissolved some gum arabic.

To remove Ink-stains from Printed Books, \&c.-Procure a little oxalic acid, which dissolve in a small quantity of warm water, then slightly wet the stain with it, when it will disappear, leaving the text uninjured.

To paint Silver F'lowers on Silk.-
Paint flowers, leaves, \&c., on white silk, with a camel's-hair pencil dipped in a solution of nitrate of silver : immerse this while wet in a jar of sulphuric acid gas, by burning sulphur in a jar of atmospheric air. The drawing will have a beautiful silvery appearance.

To remove Ink or Fruit Stains from the Fingers.-Cream of tartar, half an ounce ; powdered salt of sorrel, half an ounce-mix. This is what is sold for salt of lemons.

To clean Tin Covers.-Boil some rottenstone and a small quantity of prepared whiting in some sweet oil for two hours, till it acquires the consistency of cream.

To perfume Linen.-Rose-leaves dried in the shade, or at about four feet from a stove, one pound ; cloves, caraway-seeds, and allspice, of each one ounce; pound in a mortar, or grind in a mill ; dried salt, a quarter of a pound; mix all these together, and put the compound into little bags.

To prevent Colored Things from Running.-Boil a quarter of a pound of soap till nearly dissolved, then add a small piece of alum and boil with it. Wash the things in this lather, but do not soap them. If they require a second water, put alum to that also as well as to the rinsing and blue water. This will preserve them.

To preserve Pencil Marks.-If you have anything drawn or written with a lead pencil that you wish to preserve from rubbing out, dip the paper into a dish of skimmed milk. Then dry it, and iron it quickly on the wrong side.

To dry Plants for Preservation.-The following improved method of drying plants is the result of much experience : -The plants you wish to preserve should be gathered when the weather is dry, and after placing the ends in water, let them remain in a cool place till the next 
day. When about to be submitted to the process of drying, place each plant between several sheets of blotting-paper, and iron it with a large smooth heater pretty strongly warmed, till all the moisture is dissipated. Colors may thus be fixed, which otherwise become pale, or nearly white.

To wash White Silk Lace or Blonde.Take a black bottle covered with clean linen or muslin, and wind the blonde round it, (securing the ends with a needle or thread,) not leaving the edge outward, but covering it as you proceed. Set the bottle upright in a strong cold lather of white soap and soft water, and place it in the sun, having gently rubbed the suds up and down on the lace. Keep it in the sun every day for a week, changing the lather daily, and always rubbing it slightly when you renew the suds. At the end of the week, take the blonde off the bottle, and (without rinsing) pin it backward and forward on a large pillow. The pins should be of the smallest size. When quite dry, take it off, but do not starch, iron, or press it. Lay it in long loose folds, and put it away in a pasteboard box. Thread lace may be washed in the same manner.

To clean Sitver.-Dissolve two teaspoonfuls of powdered alum in a quart of moderately strong lye, stir in a gill of soft soap, and remove the scum or dross that may rise to the surface. After washing the silver in hot water, take a sponge and cover every article with this mixture. Let the things rest about a quarter of an hour, frequently turning them. Next wash them off in warm soap-suds, and wipe them dry with a soft cloth. Afterwards brighten them with rouge powder, or with whiting and spirits of wine.

Preserving the Color of Dresses.The colors of merinos, mousseline-delaines, ginghams, chintzes, printed lawns, \&c., may be preserved by using water that is only milk-warm; making a lather with white soap, before you put in the dress, instead of rubbing it on the material ; and stirring into a first and second tub of water a large tablespoonful of ox-gall, No colored articles should be allowed to remain long in the water. They must be washed fast, and then rinsed through cold waters. Into each rinsing water stir a teaspoonful of vinegar, which will help to brighten the colors ; and after rinsing, hang them out inmediately. If they cannot be conveniently ironed at once, let them hang till they are quite dry ; and then damp and fold them on the following day, a quarter of an hour before ironing. We need scarcely say that no colored articles should ever be boiled or scalded.

To wash Ribbons, Silk Handkerchiefs, \&c.-A good satin ribbon may be made to look very well by washing it first in cold water, to which add a few drops of spirits of wine ; then in a lather of white soap, and lukewarm water; afterwards rinse it in cold water, pull it even, and dry it gradually. When dry, stretch out the ribbon, and sponge it with a weak solution of isinglass or rice-water, to restore the stiffness and gloss. To iron the ribbon, lay it within a sheet of clean smooth letter paper (the paper being both under and over it), and press it with a heated iron moved quickly. If the color is lilac, add a little dissolved pearlash to the rinsing water. If green, a little vinegar. If pink, or blue, a few drops of oil of vitriol. If yellow, a little tincture of saffron. Other colors may be set by stirring a teaspoonful of ox-gall into the first water. If white, a saltspoonful of cream of tartar mixed with the soapsuds. Unfigured silk handkerchiefs and scarfs may be washed and ironed in the above manner. The proportion of spirits of wine is about a tablespoonful to a gallon of water.

To clean White or Colored Kid Gloves. 
-Put the glove on your hand, then take a small piece of flannel, dip it in camphene, and well but gently rub it over the glove, taking care not to make it too wet. When the dirt is removed, dip the flannel (or another piece if that is become dirty) into pipe-clay and rub it over the glove; take it off, and hang it up in a room to dry, and in a day or two very little smell will remain; and if done carefully they will be almost as good as new. In colored ones, if yellow, use gamboge after the pipeclay, and for other colors match it in dry paint.

Sympathetic Inks.-These are preparations used for writing on paper, the marks of which are invisible until acted upon by some agent. They are frequently employed in secret or playful correspondence. By heating the paper until it is nearly scorched, they may be rendered visible.

Sulphate of copper and sal ammoniac equal parts, dissolved in water or onion juice, writes colorless, but turns yellow when heated.

A weak infusion of galls turns black when moistened with weak copperas water.

The diluted solutions of nitrate of silver and terchloride of gold darken when exposed to the sunlight.

Aquafortis, spirits of salts, oil of vitriol, common salt, or salpetre, dissolved in a large quantity of water, turn yellow or brown when heated.

Solution of nitromuriate of cobalt turns green when heated, and disappears again on cooling.

Solution of acetate of cobalt, to which a little nitre has been added, becomes rese-colored when heated, and disappears when cooling.

To make a common Knife-Board.-Cover with thick buff leather, on which are put emery one part, crocus martis three parts, in very fine powder, mixed into a thick paste, with a little lard or sweet oil, and spread on the leather to the thickness of a shilling. It gives a far superior edge and polish to knives, and will not wear the knife near so much as the common method of using brick-dust on a board.

Har'ness-makers' Jet.--Take one drachm of indigo, a quarter of an ounce of isinglass, half an ounce of soap, four ounces of glue, one pennyworth of logwood raspings, and one quart of vinegar; boil the whole together over a slow fire till reduced to one pint. A small quantity is then to be taken up on a clean sponge, and thinly applied to harness, boots, \&c. -taking care that they are previously well cleaned.

To restore the Color of Mahogany.Wash well with soap and water, and then polish daily with the following oil:Take half an ounce of alkanet root, cut small, and add to a pint of linseed oil ; when this has stood for a week, add half an ounce of powdered gum arabic, and an ounce of shellac varnish; let these stand in a bottle by the fire for a week, then strain. Rub well in.

\section{Cement for the Mouths of Corked} Bottles.-Melt together a quarter of a pound of sealing wax, the same quantity of rosin, a couple of ounces of beeswax. When it froths, stir it with a tallow candle. As soon as it melts, dip the mouths of the corked bottles into it. This is an excellent thing to exclude the air from such things as are injured by being exposed to it.

To take Impressions of Leaves.-A very beaùtiful and cheap way of taking impressions of leaves is to take a small quantity of bichromate of potash, (say a teaspoonful,) which may be had at any druggist's; dissolve it in a saucer full of water, then pass the pieces of paper on which the impressions are to be taken through the solution, and while. 
wet, press the leaves, \&c., lightly upon it, and expose it to the sun, which should be shining powerfully. When perfectly dry, remove the leaves, ${ }_{\text {and }}$ a perfect fac-simile will remain in a light lemon shade, while the rest of the paper will be of a dark brown tint. Bichrome, as it is generally term, is in dark yellow crystals, which should be powdered previous to using it.

To wash a White Lace Veil.-Put the veil into a strong lather of white soap, and very clear water, and let it simmer slowly for a quarter of an hour. Take it out and squeeze but not rub it. Rinse it in two cold waters, with a drop or two of liquid blue in the last. Have ready some very clear and weak gum arabic water, or some thin starch, or rice-water. Pass the veil through it, and clear it by clapping. Then stretch it out even, and pin it to dry on a linen cloth, making the edge as straight as possible, opening out all the scallops, and fastening each with pins. When dry, lay a piece of thin muslin smoothly over it, and iron it on the wrong side.

To clean Freestone.-Wash the hearth with soap, and wipe it with a wet cloth. Or rub it over with a little freestone powder, after washing the hearth in hot water. Brush off the powder when dry.

To black a Brick Hearth.-Mix some black lead with soft soap and a little water, and boil it-then lay it on with a brush. Or mix the lead with water only.

To clean Brass.-Rub it over with a bit of flannel dipped in sweet oil-then rub it hard with finely powdered rottenstone-then rub it with a soft linen cloth -and polish with a bit of wash leather.

Rub creaking hinges with soft soap.

Common Tins.-Throw some woodashes into a wash-kettle, pour on water till it is two-thirds full, and then let it boil. Or make a strong lye. Dip in the tins when it is boiling hot; and, if they are very dirty, leave them in about ten minutes. Take them out, and cover them with a mixture of soft soap and the very finest sand. This must be rubbed on with a coarse tow-cloth. Then rinse them in a tub of cold water, and set them in the sun to drain and dry. When dry, finish by rubbing them well with a clean woollen cloth or flannel. They will look very nice and bright. You may clean pewter in the same manner.

To clean Alabaster.-Make a mixture, in the proportion of two ounces of aquafortis to a pint of cold water, which ought to be filtered. Dip a clean brush in this liquid, and wash the alabaster with it for five minutes or more. There should be a brush small enough to go in the most minute parts. Then rinse it with cold clear water, and set in the sun for two or three hours to dry. The aquafortis will make the alabaster very white. Soap should never be used.

Cleaning Japanned Waiters, Urns, \&c. - Rub on with a sponge a little white soap and some lukewarm water, and wash the waiter or urn quite clean. Never use hot water, as it will cause the japan to scale off. Having wiped it dry, sprinkle a little flour over it; let it rest awhile, and then rub it with a soft dry cloth, and finish with a silk handkerchief. If there are white heat marks on the waiters, they will be difficult to remove. But you may try rubbing them with a flannel dipped in sweet oil, and afterwards in spirits of wine. Waiters and other articles of papier maché should be washed with a sponge and cold water, without soap, dredged with flour while damp; and after a while wiped off, and then polished with a silk handkerchief.

Cheap Contrivance for Filtering.-A 
very cheap and good contrivance for filtering is to take a large garden flowerpot, and lay in the bottom a piece of sponge, so as to cover the whole. Upon this pat a few smooth clean pebbles, to keep the sponge in its place, and fill up the pot, to within two or three inches of the brim, with a mixture of one part of powdered charcoal to two parts of fine sharp sand. Then cover the top of the pot with a piece of clean white flannel, tied tightly round the rim with a twine, but so as to sink or sway down in the centre. Set the flower-pot in a pan or tub, and pour the water into the flannel, letting it filter through the charcoal, \&c.. and, by the time it has passed through the sponge, and come out at the bottom, it will be clear.

Knives and Forks.-Handles of ebony should be cleaned with a soft cloth, dipped in a little sweet oil ; and after resting awhile with the oil on them, let them be well wiped with a clean towel. Ivory or bone handles ought to be washed with a soaped flannel and lukewarm water, and then wiped with a dry towel. To preserve or restore their whiteness, soak them occasionally in alum-water that has been boiled and then grown cold. Let them lie for an hour in a vessel of this alum-water. Then take them out, and brush them well with a small brush (a tooth-brush will do), and afterwards take a clean linen towel, dip it in cold water, squeeze it out; and, while wet, wrap it round the handles, leaving them in it to dry gradually, -as, if dried too fast, out of the alum-water, they will be injured. If properly managed, this process will make them very white.

To clean Black Lace Veils.-These are cleansed by passing them through a warm liquor of bullock's gall and water; after which they must be rinsed in cold water, then cleansed for stiffening and finished as follows: Take a small piece of glue, about the size of a bean, pour boiling water upon it, which will dissolve it, and when dissolved, pass the veil through it, then clap it between your hands.

To take out paint from a dress.When fresh (having wiped off as much as you can), it may be taken out by repeated applications of spirits of turpentine or of spirits of wine, rubbed with a soft rag or a flannel. Ether also will efface it, if applied immediately. If the paint has been allowed to harden, nothing will take it off but spirits of turpentine, rubbed on with perseverance.

Indelible Marking Ink, without preparation.-One drachm and a half nitrate of silver (lunar caustic), one ounce distilled water, half an ounce strong mucilage of gum arabic, three-quarters of a drachm of liquid ammonia; mix the above in a clean glass bottle, cork tightly, and keep in a dark place till dissolved, and ever afterwards. Directions for use :Shake the bottle, then dip a clean quill pen in the ink, and write or draw what you require on the article; immediately hold it close to the fire, or pass a hot iron over it, and it will become a deep and indelible black, indestructible by either time or acids.

To take Impressions of Butterflies' Wings.-Clip the wings of the butterfly. Lay them on clean paper, in the form of a butterfly, when flying. Spread some thick clean gum water on another piece of paper, press it on the wings, and it will take them up; lay a piece of white paper over it, and rub it gently with your finger. Then draw the body in the space left between the wings.

Clothes Balls.-Take four ounces of fuller's earth, dried so as to crumble into powder, and mix with it half an ounce of pearlash. Wet it with a sufficiency of lemon-juice to work it into a stiff paste. Then form it into balls, and dry 
them in the sun, or on the top of a moderately warm stove. When quite dry put them away for use. They will be found efficacious in removing grease spots and stains from articles of clothing, first wetting the spot with cold water, and then rubbing on the ball; afterwards drying the place in the sun or by the fire, and then washing it off with a sponge and clean water.

Fine Home-Made Candles.-Take ten ounces of fresh mutton fat or suet, a quarter of a pound of bleached white wax, a quarter of an ounce of camphor, and two ounces of alum. Cut or break up all these articles, and then melt them together, skimming them well. Have ready the wicks (which should be previously soaked in lime-water and saltpetre, and then thoroughly dried), fix them in the moulds, and pour in the melted liquid, proceeding as in making common mould candles. Candles made in this manner of the above materials, are hard and durable, and will not run; burning also with a very clear light.

Packing Household Articles.-In packing for the removal of a family to a distant place, let all the boxes and trunks be numbered, and the numbers put down in a book; let some one who overlooks the whole of the packing, set down every article, denoting the exact box or trunk in which it is placed, and the order in which the things are put in, beginning with those at the bottom. By this means, after arriving at the place of destination; you will know, by consulting your book, where to find whatever you want; and which of the boxes it will be best to open first. Also, in a long sea voyage, if there is occasion to have a trunk brought from the hold to get out of it any particular article, your book will tell exactly in which of your trunks the article is.

To wash Vials.-Put into a washkettle some sifted ashes, and pour on it cold water. Then put in the vials (without corks), place the kettle over the fire, and let it gradually come to a boil. After it has boiled awhile, take it off and set it aside; letting the vials remain in it till cold. Then take them out, rinse, drain them, and wipe the outsides. You may wash black bottles in the same manner. If you have occasion to wash a single vial or bottle, pour into it through a small funnel either some lye, or some lukewarm water in which a little pearlash has been dissolved; shake, let it stand awhile to soak. Then rinse it well in cold water, two or three times. If it still smells of the former contents, soak it in more pearl-ash water (with the addition of a little lime), or in more lye.

Cleaning Kettles and Saucepans.-A receipt for cleaning the inside of kettles or saucepans of the hard, stony substance, resulting from continually boiling hard water :-In a kettle of boiling water, put about the sixteenth part of an ounce of sal-ammonia. Let it boil one hour, and then the substance will be dissolved, and is readily disengaged from the metal.

To remove Black Stains from the Skin. - Half an ounce each of cream of tartar and oxalic acid, mixed and pounded together, kept in a covered cup in the drawer, and marked "poison." To use it, wet the black stains with the corner of a towel, dipped in water, rub on a little of the mixture. Then immediately wash it off with water, and afterwards with soap and water, and the black stains will be visible no longer. This mixture will also remove ink, and all other stains from the fingers, and from white clothes. It is more speedy in its effects, if applied with warm water.

For cleaning Wainscots, and other Painted Woods.-Four ounces of potash and four ounces of powdered quick-lime are 
to be mixed together, and boiled for half an hour in three quarts of water; this mixture is to stand until it is cold, and quite clear; the clear liquid is then poured off, and a painter's brush, dipped into it, is to be passed over the surface of the wood, in the same way as for painting, immediately afterwards washing with cold water. This mode of cleaning will frequently render a new coat of paint unnecessary, and it has the advantage of being destructive to the eggs of insects which may be deposited in the interstices of the wood; where there is reason to suspect that bugs are in the wood, it may be well, as an additional precaution, to add to the mixture two drachms of corrosive sublimate.

Balloons from Turkey's Crops.-Free the crop from a thick coat of fat; turn the inside out, and wash the food away; soak it in water for a day or two, then lay it on a cluth, and with a bone or knife scrape off the internal coat of the stomach; wash it well and dry it with a clean cloth; then turn the crop, and make an incision through the external coats, taking particular care not to cut through the membrane; draw the coats at once over the neck, which must be cut long for greater convenience in using the balloon when finished. Proceed with the other neck in the same way ; tie it firm with silk, and cut it close to the body of the balloon; it must be then distended with wind and hung up to dry. It may then be painted and varnished, but will not require it if properly prepared. It may be made large enough to contain a gallon of gas, and so light as to weigh only thirty grains.

Black Paper for Drawing Patterns.Mix lamp-black and sweet oil; with a bit of flannel cover a sheet or two of large writing-paper with this mixture; then dab the paper dry with a bit of fine linen, and keep it for using in the following
manner:-Put the black side on another sheet of paper, and fasten the corners together with small pins. Lay on the back of the black paper the pattern to be drawn, and go over it with the point of a steel pencil; the black paper will then leave the impression of the pattern on the under sheet, on which you must draw it with ink.

If you draw patterns on cloth or muslin, do it with a pen dipped in a bit of stone blue, a bit of sugar, and a little water, mixed smooth in a teacup, in which it will be always ready for use, if fresh; wet to a due consistence as wanted.

Preservation of Books.-A few drops of any perfumed oil will secure libraries from the consuming effects of mouldiness and damp.

Water-proof Boots.--Saturate the boots with solution of Caoutchouc (Indiarubber), till they will absorb no more, at intervals of a week, without needing to leave a coating on the surface. The solution is made with an ounce of Indiarubber in a pint of spirits of turpentine, or coal naphtha, kept warm, and occasionally stirred till dissolved, which takes three or four days. In a boiling water bath it can be done in a few hours.

To clean Head and Clothes-brushes.-

Put a table-spoonful of pearl-ash into a pint of boiling water. Having fastened a bit of sponge to the end of a stick, dip it into the solution, and wash the brush with it, carefully going in among the bristles. Next pour over it some clean hot water, and let it lie a little while. Then drain it, wipe and dry it before the fire.

To male Artificial Red Coral Branches for the Embellishment of Grottoes.Take clear resin, dissolve it in a brasspan, to every ounce of which add two 
drachms of the finest vermilion; when stirred well together, choose the twigs and branches, peeled and dried; then take a pencil and paint the branches all over whilst the composition is warm ; afterwards shape them in imitation of natural coral. This done, hold the branches over a gentle coal-fire till all is smooth and even, as if polished. In the same manner white coral may be prepared with white lead, and black coral with lampblack. A grotto may be built, with little expense, of glass, cinders, pebbles, pieces of large flint, shells, moss, stones, counterfeit coral, pieces of chalk, \&c., all bound or cemented together with the above described cement.

To clean Cane Chair Bottoms.-Turn up the chair bottom, and with hot water and a sponge wash the cane-work well, so that it may become completely soaked; should it be very dirty, you may add soap. Let it dry in the open air if possible, or in a place where there is a thorough draught, and it will become as tight and firm as when new, providing that it has not been broken.

To clean Decanters.-Roll up in small pieces some coarse brown paper, then wet and soap the same, put them into the vessel with a little lukewarm water, and some common soda, shake them well, then rinse with clean water, and it will be as bright and clear as when new.

To render Shoes Water-proof.-Mix a pint of drying oil, two ounces of yellow wax, two ounces of turpentine, and half an ounce of Burgundy pitch, over a slow fire. Lay the mixture, while hot, on the boots or shoes with a sponge or soft brush; and, when they are dry, lay it on again and again, until the leather becomes quite saturated, that is to say, will hold no more. Let them then be put away, and not be worn until they are perfectly dry and elastic: they will afterwards be found not only impenetrable to wet, but soft and pliable, and of much longe duration.

To clean papered Walls.-The very best method is to rub them with stale bread. Cut the crust off very thick, and wipe straight down from the top, then go to the top again, and so on. The staler the bread the better.

To cleanse Feather Beds and Mattresses. - When feather beds become solid or heavy, they may be made clean and light thus: Rub them over with a stiff brush, dipped in hot soap-suds. When clean, lay them on a shed or any other clean place, where the rain will fall on them. When thoroughly soaked, let them dry in a hot sun for six or seven days, shak ing them up well, and turning them over each day. They should be covered over with a thick cloth during the night. This way of washing the bed-ticking and feathers makes them very fresh and light, and is much easier than the old fashioned way of emptying the beds, and washing the feathers separately, while it answers quite as well. Care must be taken to dry the bed perfectly, before sleeping on it.

To keep Eggs - Half a pint of salt, half a pint of lime to two gallons of hot water. Cover the eggs with the solution.

To preserve various kinds of Fruits through the Winter-Apples can be kept till June, by taking only those that are hard and sound, wiping them dry, then packing them in tight barrels, with a layer of bran to each layer of apples. Envelope the barrel in a linen cloth, to protect it from frost, and keep it in a cool place, but net so cold as to freeze the apples. It is said that mortar, laid over the top of a barrel of apples, is a good thing to preserve them, as it draws the air from them, which is the principal cause of their decaying. Care should be taken not to have it come in contact with the apples. To preserve oranges and 
lemons several months, take those that are perfectly fresh, and wrap each one in soft paper; put them in glass jars, or a very light box, with white sand, that has been previously dried in an oven a few hours, after it has been baked in. The sand should be strewed thick over each one of the oranges, as they are laid in the iar, and the whole covered with a thick layer of it. Close the jar up tight, and keep it in a cool dry place, but not so cool as to freeze the fruit. To preserve grapes, gather them on a dry day, when they are not quite dead ripe, and pick those that are not far off from the stems. Lay the bunches of grapes in a glass jar, and sprinkle around each of them a thick layer of bran, so that they will not touch each other. Have a thick layer of bran on the top, and cork and seal the jar very tight, so that the air may be entirely excluded.

To destroy Cockroaches, Ants, and other Household Vermin. - Hellebore, rubbed over with molasses and put round the places that cockroaches frequent, is a very effectual poison for them. Quicksilver and the white of an egg, beat together, and laid with a feather round the crevices of the bedsteads and the sacking, is very. effectual in destroying bugs in them. To kill flies, when so numerous as to be troublesome, keep cobalt, wet with spirit, in a large shallow plate. The spirit will attract the flies, and the cobalt will kill them very soon. The flour of sulphur is said to be good to drive ants away, if sprinkled around the places that they frequent. Weak brine will kill worms in gravel walks, if kept moist with it a week in the spring, and three or four days in the fall.

\section{COMMON SIMPLE DYES.}

To dye Black. $\rightarrow$ Allow a pound of logwood to each pound of goods that are to be dyed. Soak it over night in soft water, then boil it an hour, and strain the water in which it is boiled. For each pound of logwood, dissolve an ounce of blue vitriol in lukewarm water sufficient to wet the goods. Dip the goods inwhen saturated with it, turn the whole into the logwood dye. If the goods are cotton set the vessel on the fire, and let the goods boil ten or fifteen minutes, stirring them constantly to prevent their spotting. Silk and woollen goods should not be boiled in the dye-stuff, but it should be kept at a scalding heat for twenty minutes. Drain the goods without wringing, and hang them in a dry, shady place, where they will have the air. When dry, set the color by, put them into scalding hot water, that has salt in it, in the proportion of a teacupful to three gallons of the water. Let the goods remain in it till cold, then hang them where they will dry; they should not be wrung. Boiling hot suds is the best thing to set the color of black silklet it remain in it till cold. Soaking black-dyed goods in sour milk, is also good to set the color.

Green and blue Dye, for Silks and Woollens.-For green dye, take a pound of oil of vitriol, and turn it upon half an ounce of Spanish indigo, that has been reduced to a fine powder. Stir them well together, then add a lump of pearlash, of the size of a pea; - as soon as the fermentation ceases, bottle it-the dye will be fit for use the next day. Chemical blue is made in the same manner, only using half the quantity of vitriol. For woollen goods, the East indigo will answer as well as the Spanish, and comes much lower. This dye will not answer for cotton goods, as the vitriol rots the threads. Wash the articles that are to be dyed till perfectly clean, and free from color. If you cannot extract the color by rubbing it in hot suds, boil it out, and rinse it in soft water, till entirely free from soap, as the soap will ruin the dye. To dye a pale color, put to each quart of soft warm wa- 
ter that is to be used for the dye, ten drops of the above composition;-if you wish a deep color, more will be necessary. Put in the articles without crowding, and let them remain in till of a good colorthe dye-stuff should be kept warm. 'Take the articles out without wringing, drain as much of the dye out of them as possible, then hang them to dry in a shady; airy place. They should be dyed when the weather is dry-if not dried quick, they will not look nice. When perfectly dry, wash them in lukewarm suds, to keep the vitriol from injuring the texture of the cloth. If you wish for a lively bright green, mix a little of the above composition with yellow dye.

Yellow Dyes.-To dye buff color, boil equal parts of annatto and common potash, in soft clear water. When dissolved, take it from the fire; when cool, put in the goods, which should previously be washed free from spots and color; set them on a moderate fire where they will keep hot, till the goods are of the shade you wish. To dye salmon and orange color, tie annatto in a bag, and soak it in warm soft soap suds, till it becomes soft, so that you can squeeze enough of it through the bag to make the suds a deep yellow-put in the articles, which should be clean, and free from color ; boil them till of the shade you wish. There should be enough of the dye to cover the goods - stir them while boiling, to keep them from spotting. This dye will make a salmon or orange color, according to the strength of it, and the time the goods remain in. Drain them out of the dye, and dry them quick, in the shade-when dry, wash them in soft soap suds. Goods dyed in this manner should never be rinsed in clear water. Peach leaves, fustic, and saffron, all make a good straw or lemon color, according to the strength of the dye. They should be steeped in fair soft water, in an earthen or tin vessel, and then strained, and the dye set with alum, and a little gum arabic dissolved in the dye, if you wish to stiffen the article. When the dye-stuff is strained, steep the articles in it."

Red Dyes.-Madder makes a good durable red, but not a brilliant color. To make dye of it, allow for half a pound of it three ounces of alum, and one of cream of tartar, and six gallons of water. This proportion of ingredients will make sufficient dye for six or seven pounds of goods. Heat half of the water scalding hot, in a clean brass kettle, then put in the alum and cream of tartar, and let it dissolve. When the water boils, stir the alum and tartar up in it, put in the goods, and let them boil a couple of hours; then rinse them in fair water-empty the kettle, and put in three gallons of water, and the madder; rub it fine in the water, then put in the goods, and set them where they will keep scalding hot for an hour, without boiling-stir them constantly. When they have been scalding an hour, increase the fire till they boil. Let them boil five minutes, then drain them out of the dye, and rinse them, without wringing, in fair water, and hang them in the shade, where they will dry. To dye a fine crimson, take for each pound of goods two and a half ounces of alum, an ounce and a half of white tartar-put them in a brass kettle, with sufficient fair water to cover your goods; set it where it will boil briskly for several minutes; then put in the goods, which should be washed clean, and rinsed in fair water. When the goods have boiled half an hour, take them out, without wringing, and hang where they will cool all over alike, without drying; empty out the alum and tartar water, put fresh water in the kettle, and for each pound of goods to be dyed, put in an ounce of cochineal, powdered fine. Set the kettle on the fire, and let the water boil fifteen or twenty minutes; then put in sufficient cold water to make it lukewarm, put in 
the goods, and boil them an hour and a quarter - take them out without wringing, and dry them in a shady place. The blossoms of the Balm of Gilead, steeped with fair water in a vessel, then strained, will give silk a pretty red color. The siik should be washed clean and free from color, then rinsed in fair water, and boiled in the strained dye, with a small piece of alum. To dye a fine delicate pink, use a carmine saucer - the directions for dyeing, come with the saucers. It is too expensive a dye for bulky goods, but for faded fancy shawls and ribbons, it is quite worth the while to use it, as it gives a beautiful shade of pink.

Slate-Colored Dye. - To make a good dark slate color, boil sugar-loaf paper with vinegar in an iron utensil - put in alum to set the color. Tea-grounds, set with copperas, make a good slate color. To produce a light slate color, boil white maple bark in the clear water, with a little alum; the bark should be boiled in a brass utensil, and the dye for slate color put into it. Goods shnuld be boiled in it, and then hung where they will drain and dry.

To preserve Steel Pens from Corrosion. Dip them for a few moments in ethereal solution of gold. This covers them with a film of pure metallic gold, which prevents the ink acting upon the steel.

Corns. - The laminated corn, or callus, produced by pressure, congestion, and increased formation of ep dermis, may be softened by, moisture, as by soaking in warm water, by the application of a starch or soap poultice ; and, being softene , the thick cuticle may be thinned by scraping with a blunt knife, or it may be dissolved by an alkaline solution, with moderate friction. When the thickening has been reduced sufficiently, it the may has been reduced suffciently, it the smoke of stramonium leaves, which may be kept down by daily washing have been immersed in a saturated soluwith soap. The eye of the corn may be tion of nitre (nitrate of potassa), and alivays made visible by rubbing the part then dried. Prepared in this way, it with eau-de-cologne or spirits of wine; burns with great energy and complete37 and any remains of the core should be removed with the point of a knife. Aiter the operation, the corn should be covered with a piece of soft plaster for a day or two, and a periorated plaster of buff leather subsequently worn to keep off pressure from the centre of the growth. The removal of a corn may be very considerably ailed by the use of the compound tincture of iodine painted on the swelling. When the corn is painful, this application subdues the sensibility, and renders the corn dry and pliable, and easy of removal by means of a file. Soap and so useful to the skin in many ways, are especially service feet afflicted with corns, and particularly when they are soft corns. Daily washing with soap, and the subsequent interposition of a piece of cotton-wool between the toes, may be considered as a cure for soft corns. In these cases, the skin may be hardened by sponging with spirits of camphor after the washing. The cotton-wool should ke removed at night; and this is a good time for the camphorated spirits. Adhesive plaster, eonstantly applied, will remove soreness and twinging, and often effect an entire cure.

Dandruff. - Nitric acid, twelve drops ; Distilled water, three ounces. Mix, and apply once a day.

Rat Poison.-Recent experiments have shown that squills is an excellent poison for rats. The powder should be mixed with some fatty substance, and spread upon slices of bread.

Asthma. - A prominent professor in one of our New-England colleges, who has been an asthmatic for upwards of forty years, and tried a great variety of remedies, gives this the preference over all others. It consists simply in inhaling burns with great energy and complete- 
ness, rarely going out until the whole is it stand all night. In the morning pour consumed. These remedies, separately, it carefully from the dregs into a clean have long been known, both to profes- bottle, cork it, and keep it for use. A sional and private experience; but the tablespoonful of this gum-water, added to combination seems particularly conve- a pint of starch made in the usual mannient and useful. It is best kept in a tin ner, will give to lawns, either white or or other metallic box, the inside of the printed, a look of newness, after they cover being used as a surface on which to are washed. It is excellent as a polish burn it while inhaling. The effects are for shirt-bosoms and other starched the more marked if it is used early, and linen.

before the paroxysm becomes fully developed.

Ingrowing Toe-Nail. - This painful abnormal condition of the toe-nail may be cured by allowing the nail to continue to grow without paring it. The boot or shoe will depress the nail at the end as it grows longer, which will gradually elevate the part where it presses upon and into the soft tissues of the toe; thus removing the irritation, the sore scon heals. This is far preferable to the rash and painful operation of tearing off the toe-nail with forceps.

Glycerine Lotion. - Take of Glycerine, inodorous, 3 fluid ounces. Mucilage of gum arabic, 10 fluid drachms. Pulv. cochineal, 5 grains. Hot water, 1 1-2 fluid ounces. Deod. alcohol, 2 1-2 fluid ounces. Oil of rose, 8 drops. Pulv. gum arabic, 1-2 drachm. Water, 8 fluid ounces.

Rub the cochineal with the hot water gradually added, and then add the alcohol; triturate the oil of rose with the gum arabic, and gradually add the water. With this mix well the solution first formed, and filter, and to the filtered liquid add the glycerine and mucilage and shake well. This forms a beautiful and elegant preparation, with a rich, rosy fragrance. It renders the skin soft and smooth, and is excellent as an application to the face after shaving.

Laundry Polish. - Take two ounces of fine white gum-arabic powder, put it into a pitcher, and pour on a pint of water; and then, having covered it, let It is to this, therefore, that the attention

Ventilation, Sunlight, and Warm Clothing. - Fresh air by day and by night, strong and nourishing food, dry soil on which to live, sunlight, and warm clothing, are the means of saving many lives which would have been hopelessly lost in the preceding generation. If our conjectures are correct, this improvement may be expected to continue; and everybody can make it greater. Ventilate the schoolrooms and the workshops, and the stores and the houses. In cold weather, let the air, comfortably and equally warmed, be generally supplied from without in a constantly-flowing current. Let those who can provide it in their homes remember that an open fire, which sends two-thirds of the heat up the chimney, furnishes the best ventilation, for a room of moderate size which the ingenuity of man has yet devised; and that the heat escaping by the flue is the price to be paid for it. Let in the sunlight, and never mind the carpets; better they should fade than the health of the family. When a man proposes to build a dwelling in a swamp, warn him of his danger.

Rheumatism. - Rheumatism is improperly regarded as an inflammatory affection of the joints. The pains and inflammation there stand in the same degree to the disease as the pustules or the scarlet eruption in smallpox and in scarlet fever do to these diseases. In rheumatism the pains are merely the symptoms of a general febrile disease. 
is to be turned. There is in every instance a condition of plethora which constitutes the predisposition to rheumatism, and which renders cold, or exposure to currents of air, capable of producing the disease. Of the medical treatment it is not necessary to speak here; but with respect to the prevention of the attack, or the warding off its return after it has been relieved, the following rules should be observed:-

First.-It is of the utmost importance to commence the renewal of the movements of the affected joints as early as possible after the pain is allayed. Their strength and flexibility depend solely on the early renewal of motion. On the contrary, rest tends to retard the restoration of the affected parts to complete health. They remain painful and stiff, and resist every movement which is attempted: the more motion is cultivated, the sooner are the limbs restored to their natural functions. Their exercise recovers the balance of the circulation, prevents effusion, aids absorption, and consequently favors flexibility. If rest be indulged, from the dread of pain, the joints and the parts surrounding them become rigid, contracted, and their free action permanently impaired, while the muscles waste, and lose their power of contracting.

Second. - Friction, and the daily use of the salt-water shower-bath, have a powerful effect in warding off the returns of the disease. (The salt-water showerbath is made by the addition of a quart of salt brine to three pailfuls of water.)

Third. - Flannel next the skin, and warm clothing generally, are absolutely essential. In a changeable climate, daily attention in adapting dress to the weather is required.

Fourth.-Due attention must be given to diet. Bear in mind that improper food, or eating to oppression, causes indigestion. Bad digestion produces bad blood; and bad blood causes rheumatism, and ailments of every nature. Plain nourishing food is what the system demands - being regulated in quantity by the exercise taken, and time spent in the open air. Saccharine matters, pastry, sweetmeats, and whatever can contribute to a dyspeptic state of the digestive organs should be avoided; and, unless the strength be greatly reduced, water should be the only beverage.

1. Toothache Drops. - Take of tincture of opium, two fluid drachıns; ether, four fluid drachms; oil of cloves, one-half fluid drachm; mix, with agitation, and shake it each time before use.

2. Take of creosote, one drachm; chloroform, two drachms; alcohol, three fluid drachms ; mix, \&c., as the last.

A little cotton saturated with either of the above compounds, and placed in the cavity of the decayed tooth, will often produce immediate relief.

Whooping-Cough.-Dr. Howard Sargent, of Boston, states that for eight or ten years past he has used, as a remedy for whooping-cough, a tea made from red-clover blossoms. He remarks, "It is so simple that many would not give it a second thought. I can say with truth that I never knew it fail. I generally expect a cure in ten days. Four years ago I had children in three families sick at the same time; they were all well in ten, twelve, or fourteen days. There is some care and art necessary in making the tea. I select and cure the blossoms myself, and take of the best blossoms about two ounces to a pint of boiling water, steep for four hours, and give a. wineglassful occasionally during the day. Should it operate on the bowels, no harm is done; the dose, in that case, may be diminished. I ask physicians to try it before they reject it. I sometimes add a little honey to make it more palatable."

Piles. - Take three grains sulphate of morphine, thirty grains extract of 
stramonium, sixty grains carbonate of Iead, one teaspoonful olive oil, and three drachms lard cerate. Rub the extract, if not unifurmly soft, with a few drops of water; add the powders and olive oil, and rub till perfectly smooth, and then incorporate them with the cerate. To be applied externally as occasion may require.

Iiching of the Anus. - Take equal parts citrine ointment and simple cerate, and thoroughly rub together. The above compound applied to the part, and rubbed in, is very efficacious in allaying this troublesome complaint. It should be at lex t strong enough to produce some smaring, and the application continued until the itching is removed.

Pin Worms, with which children are so often troubled, are destroyed and pass from the bowels, by the external use of diluted citrine ointment, prepared as above, or made still weaker by the addition of more cerate. It should be introduced an inch or two within the rectum, and applied gently to the lining membrane.

A Valuable Liniment. - A liniment which we have found most serviceable in the family and in the stable is prepared as follows:-

Alcohol (ninety-five per cent), one quart. Fiuid extract of arnica, four fluid ounces. Camphor, two fluid ounces. Stronger aqua-ammonia, one fluid ounce. Tinct. opium, one flaid ounce. Water one quart.

Add to the alcohol the arnica, camphor ammonia, and tincture of opium; and, after the camphor is dissolved, the water may be added. This liniment may be applied for the reliet of sprains, bruises, rheumatic and neuralgic pains, \&c., with decided advantage. For use in the cow and horse stable, it will be found most efficient.

\section{SYRUPS FOR SODA AND MINERAL} WẢTER.

Sarsaparilla. - Take of simple syrupfour pints ; compound syrup of sarsaparilla, four flaid ounces; caramel, one and a half fluid ounces; oil of wintergreen and sassafras, of each, six drops.

Lemon. - Grate off the yellow rind of lemons, and beat it up with a sufficient quantity of granulated sugar. Express the lemon-juice; add to each pint of juice one pint of water, and three and a half pounds of granulated sugar, including that rubbed up with the rind; warm until the sugar is dissolved, and st:ain.

Another recipe for lemon syrup is as follows: Dissolve six drachms of tartaric acid and one ounce of gum arabic in pieces, in one gallon of simple syrup; then flavor wich one and a half fluid drachms of best oil of lemon, or flavor with the saturated tincture of the peel in cologne spirits.

Orange. - This may be made from the fruit in the same way as lemon syrup, or the following formula may be used : Dissolve six drachms of citric acid in one gallon of simple syrup, and add two fluid drachms of tresh oil of onange in two ounces of alcohol; or instead of the alcohol solution of the oil, use the saturated tincture obtained by macerating the fresh peel for ten days in sufficient cologne spirits to cover.

The lemon and orange syrups made from the fruit, after being strained, may be diluted with an equal bulk of simple syrup. One dozen of the fruit is suffcient to make one gallon of finished syrup.

Ginger. - Mix two fluid ounces of tincture of ginger with four pints of simple syrup.

Vanilla. - Mix two fluid ounces of extract of vanilla with four pints of simple syrup. 
Strawberry, Raspberry, or Pine-apple. - time is likely to be lost, resort at once to Mash the fresh fruit, express the juice, an emetic, of which most families have and to each quart add three and a half an excellent one in the house in the form pounds of granulated sugar. The juice, of ground mustard-seed. Of this give heated to 180 degrees Fahrenheit, and two teaspoonfuls stirred into a tumbler strained or filtered previous to dissolving of warm water, for an adult, reducing the the sugar, will keep for an indefinite quantity for a child, and repeat every time.

Nectar. - Mix three parts vanilla syr- duced.

up with one each of pine-apple and If corrosive sublimate, arsenic, or other lemon syrups.

Sherbet. - Mix equal parts of orange, pine-apple and vanilla syrups.

Orgeat. - Cream syrup and vanilla syrup, each one pint; oil of bitter almonds, four minims.

Cream. - Take of Borden's condensed milk, one pint; water, one pint; sugar one and a half pound. Heat to boiling, and strain. This will keep for over a week in a cool place.

Coffee. - Pure coffee, roasted; half a used at all, what remains should be impound, is infused in boiling water, half a mediately thrown away. . It is never gallon; enough is filtered off to make safe, wherever hid, or however labelled. half a gallon of infusion, in which dissolve It is so deadly a poison, and has destroyed seven pounds of granulated sugar.

Chocolate. - Baker's chocolate, four ounces; dissolve in twenty ounces of boiling water, and dissolve in this one pound of granulated sugar.

Poisons and their Antidotes. - In all cases of poisoning, much depends upon the promptness with which the antidote is administered. The appropriate remedy for acids is alkalies, and vice versa. If for acils is alkalies, and If lye has been swallowed for water, a in this position by the teeth if the lower mistake often made by children, give jaw be raised.

at once a wine glassful of vinegar added 3. To imitate the movements of breathto an equal quantity of water. If oxalic ing, raise the patient's arms upward by or citric or tartaric acid have been taken, the sides of his head, and then extend lose no time in administering a solution them gently and steadily upward and of bi-carbonate of soda or saleratus, - forward for a few moments. (This acremedies always at hand.

tion induces inspiration or the passing of

If the poison be morphine, narcotine, air into the lungs, by enlarging the calaudanum, or any form of "opium, aconite pacity of the chest.)

or other powerful narcotic, a physician Next, turn down the arms, and press should be immediately called, and the them gently and firmly for a few mostomach-pump applied. If too much ments against the sides of the chest. 
(This produces a forced expulsion of air Rub the body all over, at first with snow from the lungs.)

Repeat these measures alternately, from twenty to thirty minutes, or until deliberately, and perseveringly, fifteen it becomes very red; then wipe it pertimes in a minute.

4. Toinduce circulation and warmth, do not cease rubbing the limbs all this time upward, with firm, grasping pressure, fectly dry, and let it be rubbed with the warm hands of several persons at one time. After this has been done for a short time, remove the body to a cool and with energy, using handkerchiefs, room, continuing the friction, and then flannels, \&c. (This rubbing upward covering with hot flannel. To restore causes the blood in the veins to flow to- breathing, make use of the same means ward the heart, and should be done by assistants.)

5. When the limbs are thus warmed and dried, clothe the body, - the bystanders supplying the garments.

On the restoration of life, a teaspoonful of warm water should be given; and then, if the power of swallowing has returned, small quantities of warm coffee, ginger-tea, wine, brandy, and warm water, \&c., may be given. By pursuing this treatment perseveringly and energetically for several hours, many persons have been resuscitated; it is a mistaken opinion that death has occurred because life does not make its appearance in half an hour or an hour.

Cautions to the bystanders. - 1 . Send at once for a physician, and for dry clothing. 2. Avoid all rough usage, and be very careful not to turn the body on the back. 3. Do not hold up the body by the feet for any reason. 4. Do not roll the body on barrels. 5. Do not rub the body with salt or spirits. 6. Do not inject tobacco smoke, or an infusion, into the bowels. 7. Avoid the continuous warm bath. 8 . Be especially careful to prevent persons crowding around the body.

Apparent Death from Intense Cold. Treatment. - Be careful about employing warmth too soon, as its too quick and free application will undoubtedly produce mortification, and endanger life.l watery particles are evaporated. drowning."

Greek Fire. - Modern Greek fire is a solution of phosphorus in bisulphide of carbon. When this solution is poured on paper, rags, or shavings, the bisulphide evaporates rapidly and leaves the phosphorus in a state of very fine division - so fine that it takes fire spontaneously. It furnishes the means of performing a very pretty lecture-room experiment; but as an incendiary agent it is worthless, for the simple reason that it does not set fire to even the thinnest and driest boards. The phosphorus, in burning, produces a fusible and non-volatile compound, and this glazes over all objects in its vicinity, and protects them from the action of the flames.

To collect the Odors of Flowers. Roses, and all flowers containing perfumed oils, may be made to yield their aromatic properties by steeping the petals in a flat dish of soft water, and setting it in the sun. The petals should be entirely covered with the water. A sufficient quantity should be allowed for evaporation, and the vessel left undisturbed a few days. A film will be found on the top. This is the essential oil, and every particle is impregnated with the odor peculiar to the flower. It should be taken up carefully and put in tiny vials, which should be allowed to remain open till all 


\section{INDEX.}

$\Delta$

Accounts, 33

Accounts should be regular, 17.

Acetic acid, 549 .

Adulteration of Food, 78 .

Ague and Fever, 558.

Airing Rooms, 35.

Alabaster, to clean, 574.

Ale Posset, 513.

Allspice, 130.

Almack, 484.

Almond Balls, 548.

Almond Bread, 467.

Almond Cake, 477.

Almond Perfume, 537.

Almond Paste, 432.

Almond Posset, 493

Almonds, Syrup of, 511.

Almond Water, 524

American "Help," 30.

Anchovies, Essence of, 206.

Anchovies, Toast of, 206.

Anchovies, with Fried Bread, 206.

Anchovies, to know good, 206.

Anchovy Sauce for Fish, 405 .

Angelica, 134.

Animals, Names of Joints in, 116.

Aperient Electuary, 560.

Aperient for Children, 563 .

Aperients, Spring, 563 .

Aperient Pills, 563 .

Aperient, Infant's, 564 .

Apple Bread, 469.

Apple Butter, 506.

Apple Charlotte, 445 .

Apple Codlings, to pickle, 400 .

Apples, to Bake, 520 .

Apple Cake, for Children, 528.

Apple Dumplings, 443.

Apple Fool, 493 .

Apple Jelly, 502.

Apple Paste, 520.

Apple Soufflé, 453.

Apples, Tapioca, 520 .

Apples, Miroton of, 449

Apricot Sweetmeat, for Tarts, 433.

Apricot Tart, 428 .

April, Fish in Season for, 150.

April, Fruits in Season for, 150 .
April, Meats in Season for, 150. April, Poultry and Game in Sea. son for, 150.

April, Vegetables in Season for, 150.

Ardent Spirits in Sweden, 510.

Arrangement of Dinner, 96.

Arrow Root, 135, 527.

Arrowroot Jelly, 524

Aromatic Vinegar, 549

Art of Cookery, 124.

Articles for Breakfast, 532 .

Articles in winter, additional, 532.

Articles of Silver required for the Table, 24.

Artificial Coral Branches for Grottoes, 577 .

Articles in Season for each Month, 149.

Artichokes, 399, 383.

Artichokes, Jerusalem, 384.

Asparagus, 380.

Asparagus Loaves, 381

Asparagus Forced, 381

Asparagus Peas, 381.

Asparagus, Garniture for Omelettes, 464 .

Aspic Jelly, 354

Asses Milk, 520

Asses Milk, Artificial, 520.

August, Fish in Season for, 150.

August, Fruits in Season for, 151.

August, Meats in Season for, 151

August, Poultry and Game in Season for, 151.

August, Vegetables in Season for, 151.

Auld Man's Milk, 514.

\section{B}

Baba, 486.

Bacon, Garniture for Omelette, 464 .

Bacon, Broiled, 531.

Bacon, to boil, 334.

Bacon, to broil, 334.

Bacon, to make, 334 .

Bacon and Cabbage, 334.
Bacon, to Cure, Cobbett's Receipt 333.

Bacon, to cure for Larding and Braising, 334, 346

Bacon, Maline's Method of Curing, 345 .

Bacon, Black Pool Receipt for, 345 .

Bacon, Wiltshire, 346.

Bain Marie, 83.

Baking, 141.

Baked Apples, 442.

Baked Beans, 384.

Baked Calf's Head, 293.

Baked Calf's Heart, 288.

Baked Eggs, 462

Baked Fagots, 327.

Baked Ham, 331.

Baked Herring, 193.

Baked Marrowbones, 273.

Baked Milk, 520.

Baked Pears, 502, 523.

Baked Pig's Head, 324.

Baked Pike, 196.

Baked Salmon, 200

Baked Shad, 195.

Baked Smelts, 197.

Balloons, from Turkey's Crops,577.

Balm Tea, 521.

Banbury Cake, 481.

Bandoline, 552.

Bandoline, Rose, 553.

Barberries, 132, 399 .

Barley Water, 522.

Basil, 132.

Bath of Oil, 83.

Batter for Fruit Puddings, 449.

Bay, 134, 538.

Bay Salt, 129

Beans, à la maitre d' Hotel, 384.

Beans, Baked, 384.

Beans, French, 384

Beans, French, Pickled, 395.

Beans, Lima, 384.

Beans, Stewed, 386.

Beans, White, Purée of, 386.

Beans, White Haricot, 387.

Bear's Grease, imitation, 545.

Bedroom Furniture, 22.

Bedsteads, 22,

Bedsteads, to clean, 89 . 
Beef, to choose, 56 .

Beef, 249.

Beef Baked, 260.

Beef, Bath Receipt, 264.

Beef Botilli, 553 .

Beef Bonillon, 253.

Beef Brains ì la Sauce Piquante, 260.

Beef' Brams Fried, 260.

Beef, Brisket of, Stewed, 253, 263.

Beef, Brisket, to carve, 109 .

Beef, Broiling, 254.

Beef Broth, 273.

Beef Ribs, to carve, 108

Beef Round, to carre, $10 \mathrm{~s}$

Beef à la Houssard, 266 .

Beef Sirloin, 169 .

Boeuf à la Morte, 264.

Beef à la Mode, 264, 266, 267.

Beef à la Polonaise, 265 .

Beef, Cold, 273 .

Beef, to Corn, 342.

Beef Collops, 256, 269.

Beef, to collar Ribs of, 250 .

Beef, Croquettrs of, 271 .

Beef, to collar, 352.

Beef, to collar, Economical way, 352.

Beef, Curried, 273.

Beef, Dutch way to salt, $3 \pm 2$.

Beef, Dutch Hung, 259.

Beef Entrées, 263.

Beef Essence, 518.

Beef, Family Stew of, 257.

Beef, Fillet of, Roasted, 251 .

Beef to taste like Hare, 251.

Beef, Fillets of, 265 .

Beef Fillets, Braised, 265.

Beef, Fillet of, Sautè, 270 .

Beef, Fricasseed, 274.

Beef Fricandeau, 267, 274.

Beef Gravy, 220.

Beef Hashed, 256.

Beef Heart, 268.

Beef, Hunter's, 268 .

Beef, Hung, 259, 343 .

Beef, Jerked, 140.

Beef, Leg of, 253.

Beef, in a Marinade, 265.

Beef, Minced with Cucumbers,268.

Beef, Minced, 271.

Beef Olives, 258.

Beef Palates, 256, 273.

Beef, a Pickle for, 258, 354.

Beef, Pickle for, à la Garrick, 274.

Beef Potted, 267, 349.

Beef, Potted like Venison, 349 351.

Beef, pressed, 274.

Beef, Rissoles, 275 .

Beef, Round, to salt or corn, 354

Beef, Rump of, 251.

Beef, Salt, remains of, 273 .

Beef, to salt red, 342 .

Beef, to Stew, 268, 252 .

Beef Sausages, 267.

Beef, Sirloin, Roasted, 250.

Beef, Sirloin, to dress inside of 251.

Beef, Spiced, 342, 274, 258.

Beef Steaks, Broiled, 254.

Beef Steaks, à la Francaise, 25.5.

Beef Steaks, à la Parisienne, 255.

Beef Steak and Onions, 255.

Beef Steaks Rolled and Roasted, 255.

Beef Steaks, Stewed, 255

Beef Stew, 252, 256.

Beef, Rump Steak Stewed, 259.
Beef, Rump Steak, Plain Broiled, 260.

Beef Steak, à la Francaisc, 265.

Beef Tongue. to Cure, 257.

Beef Tongue, 262

Beef Tongues, to dress, 257.

Beef Tongue, Larded, 257.

Beef Tongue, to stew, 262

Beef Tongue, to prepare to eat cold, 262 .

Beef 'Tongue, to Roast, 262.

Beef Tongue, Fresh, 263.

Beef Tongue and Udder, Fresh, 263.

Bœuf' Tremblant, 258.

Beef Tripe, 261.

Beef Tripe, Lyons Fashion, 269.

Beef Tea, Liebig's, 518 .

Beer, Spruce, 510

Beer, Giuger, 510

Beets, 382, 387 .

Beets, French way, 387 .

Beignets, 459.

Benzoin, 538.

Bergalot, 538

Beverages, 510.

Beverages composed partly of Fermented Liquors, 512.

Bills of Fare, 532, 533, 534.

Bills of Fare for Spring, 533.

Bills of Fare for Stummer, 533.

Bills of Fare for Winter, 584 .

Birds, Potted, 349.

Birds, to bone, 356 .

Biscuits, Damascus, 467.

Biscuits, Milk, 467 .

Biscuits, Cinnamon, 480 .

Biscuits, 481.

Biscuit, glacé, 490.

Bishop, 512.

Black Clape, to restore, 568 .

Black Balls, 570 .

Black Dye, 579 .

Blackberry Jam, 528.

Blacking, 570 .

Black Lace Veils, to clean, 575 .

Black Pool Receipt for Bacon, 345.

Black Stains, to remove from the Skin, 576

Black Paper, for drawing patterns, 577.

Blanching, 142.

Blanc mange, 523, 487 .

Blanc mange, Rice, 523 .

Blue and Green Dye, 579 .

Blue Ink, 571

Boarding and Housekeeping, 21.

Boiling, Operation of, $/ 81,136$.

Boil Bacon, 334

Boil Cabbage, 388.

Boil Calf's Head, 282, 293.

Boil Chicken, 364

Boil Cod, 190 .

Boil Crabs, 207.

Boil Ducks, 369 .

Boil Egors, 460.

Boil Fillet of Veal, 277.

Boil Fowls, 361

Boil Ham, 328, 329 .

Boil Knuckle of Veal, 278.

Boil Leg of Lamb, 312

Boil Leg of Lamb with Spinach, 313.

Boil Leg of Mutton, 298.

Boil Leg of Pork, 320.

Boil Lobster, 204.

Boil Mackerel, 194

Boil Mutton Ham, 330 .
Boil Pickerel, 196.

Boil Pigs Head, 326.

Boil Pork, 322.

Boil Salmon, 200.

Boil Sheepsliead, 191.

Boil Turbot, 199.

Boil Turkey, 357.

Bone a Quarter of Lamb, 314 .

Bone Birds, 356 .

Boning, 143 .

Boorhave's Sweet Buttermilk, 519.

Books, to preserve, 577.

Boots, Waterproof, 577.

Boston Creamcakes, 484.

Bottles and Decanters, 60 .

Bouquet de Caroline, 542 .

Bonquet de Flora, 542 .

Braising, 139.

Braise Breast of Lamb, 314.

Braise Chicken, 364.

Braise Fillet of Beef, 265.

Braise Fowls, 361 .

Braise Ham, $3: 32$

Braise Leg of Mutton, 298.

Braise Mutton Cutlets, 301.

Braise Neck of Veal, 279.

Braise Shoulder of Lamb, 312, 314.

Braise Turkey, 358.

Bran Tea, 559.

Brandy Mixture, 522

Brass, to cleall, 574 .

Brass Ornaments, to preserve, 569.

Brawn, to choose, 57.

Brawn, 346.

Brawns, mock, 334, 346.

Brawn, to keep, Cambridge way, 346.

Bread Crumbs, 373

Bread, Fried, for Garnishing, 373. Bread, to make, 464 .

Bread, Brown or Drspepsia, 465.

Bread, Rye and Indian, 465.

Bread, to make without yeast, 465 .

Brear, Rice, 466.

Bread, Almond, 467.

Bread, Apple, 469.

Bread, Diet, 480.

Bread, French, 482

Bread and Milk, 523.

Breakfast Articles, 532

Breakfast, Dishes for, 529 .

Breakfasts, Serving of, 94 .

Brick Hearth, to black, 574.

Bride Cake, 476.

Brioche Paste, 409 .

Brioche Rolls, 472

Broccoli and Battered Eggs, 388.

Broiled Chicken, for Breakfast 530.

Brisket of Beef, 253, 283.

Broiling, 140.

Broil Beef, 254.

Broil Beef Steaks, 254

Broil Beef Steaks, à la Française,

Broil Beef Steaks, à la Parisienno, 25\%5.

Broil Bones, 269.

Broil Chicken, 530

Broil Fowls, 362.

Broil Mackerel, 194.

Broil Mushrooms, 388.

Broil Mutton Chops, 300.

Broil Mutton Kidneys, 305.

Broil Oysters, 209.

Broil Partridges, 371.

Broil Pigeons, 364. 
Broil Rump Steaī, 260.

Broil Salmon, 201.

Broil Shad, 195.

Broil Smelts, 197.

Broth, Calf or Lamb's Feet, 518.

Broth, Chicken, 518.

Broth, that will keep, 518.

Broth, quick made, 518 .

Brown Coloring for made dishes, 249.

Brown thickening, 228.

Bruise or Sprain, 565.

Brushes, 26.

Brushes, to clean, 577.

Bubble and Squeak, 260, 269.

Buckwheat Cakes, 468.

Buckskin Gloves, to clean, 571.

Buns, 471.

Burns, to relieve, 565.

Butter, to clarify, 347,473 .

Butter, Pickle for, 472 .

Butter, to cure in the best way, 473.

Butter, salt, to freshen, 473.

Butter, to make, 473.

Butter, to preserve for winter use, 473.

Butternuts, Pickled, 399.

\section{C.}

Cabbage, to boil, 388 . .

Cabbage, Purple, to pickle, 396.

Cabbage, Red, to pickle, 396.

Cabbage, White, to pickle, 396.

Cakes, 473.

Cake, Almond, 477.

Cake, Banbury, 481 .

Cake, Boston Cream, 484

Cake, Bride, 476.

Cake, Chocolate, 480.

Cake, Cider, 479 .

Cake, Cocoanut, 477.

Cake, Cornstarch, 479.

Cake, Currant, 480 .

Cake, Eletherian Pineapple, 485.

Cakes, Frosting, 475.

Cake, German Sugar, 476.

Cake, Gold and Silver, 481.

Cake, Honey, 477.

Cake, Housewife's Christmas, 479

Cakes, Icing for, 480.

Cake, Indian Corn, 478.

Cake, Jelly, 480.

Cake, Lady, 480.

Cake, Lemon, 480 .

Cake, Love, Bola d' Amour, 485.

Cake, New England Wedding, 475 .

Cake, New York Cup, 478.

Cake, Nourmahal, 480.

Cake, Plain Fruit, 477 .

Cake, Plum, 475.

Cake, Pound, 478.

Cake, Queen, 477.

Cake, Queen Victoria's, 485.

Cake, Railroad, 479.

Cake, Rice, 485.

Cake, Savoy, 477.

Cake, Seed, 480.

Cake, Short and Strawberry, 479.

Cake, Small Plum, 476.

Cake, Soyer's St. James, 478 .

Cake, Soyer's Turban, 479.

Cake, Sponge, 477.

Cake, Sugar, 479.

Cake, Tipsy, 483.

Cake, Washington, 478.
Cakes, for Children, 527.

Calf's Brains, 289.

Calf's Brains, Croquettes of, 289.

Calf's Ears, Stuffed, 288.

Calf's Feet, 288.

Calf's Foot Jelly, 491, 492.

Calf's Foot Pudding, 524.

Calf's Head, 292.

Calf's Head Baked, 293.

Calf's Head Boiled, 282, 293.

Calf's Head Cheese, 286.

Calf's Head, to collar, 352

Calf's Head Curry, 283.

Calf's Head Hashed, 293 .

Calf's Head Ragont, 282.

Calf's Head à la Tortue, 293.

Calf's Heart, 290.

Calf's Heart, Baked 288.

Calf's Liver, 2 S5.

Calf's Liver, Fried, 286.

Calf's Liver, Stewed, 285.

Calf's Liver and Lights, 290.

Camomile Tea, 522.

Camphor Balls, 548.

Camphor Ice, 547.

Camphor Cake, 565.

Camphor Cerate, 548.

Candy Fruit, 509.

Candies, Home made, 576.

Cane Chair Bottoms, to clean, 578.

Capillaire, 512.

Capon, 360.

Caramel, 127.

Care of the Sick, 50.

Carpets, 22.

Carpet, to clean, 570

Carrots, 382.

Carving, 107.

Carving, Beef, Brisket of, 109.

Carving, Beef, Round of, 108 .

Carving, Beef, Ribs of, 108 .

Carving, Bcef. Sirloin of, 109.

Carving, Boiled or Roast Turkey, 113.

Carving, Breast of Voal, 109.

Carving, Fillet of Veal, 109.

Carving, Fowl, Roast and Boiled, 114.

Carving, Forequarter of Lamb, 112

Carving, Ham, 112

Carving, Haunch of Mutton, 112.

Carving, Haunch of Venison, 112.

Carving, Knuckle of Veal, 109.

Carving, Leg of Mutton, 110.

Carving, Leg of Pork, 112.

Carving, Loin of Mutton, 111.

Carving, Loin of Veal, 109.

Carving, Neck of Mutton, 111.

Carving, Neck of Veal, 109.

Carving, Neck of Venison, 112.

Carving, Pheasant, 115.

Carving, Pigeons, 116.

Carving, Rabbit, 113.

Carving, Roast Goose 115.

Carving, Roast Pig, 113.

Carving, Saddle of Mutton, 111.

Carving, Scrag of Mutton, 111.

Carving, Shoulder of Mutton, 11

Carving, Shoulder of Veal, 110.

Carving, Tongue, 112.

Carving, Wild Birds, 115.

Casserole, 127.

Cassolettes, 551

Casks, to sweeten, 569.

Cassia, 131.

Cauliflower, 382.

Cauliflower Pickle, 396
Caudle, 519.

Caudle, Flour, 519

Caudle, York, 519.

Cayenne Pepper, 406.

Cayenne, 130 .

Celery, 132.

Celery, to stew, 383.

Celery, Pickled, 396.

Cement, for Corked Bottles, 573.

Cement of Lime, 568.

Cement, Lime and Egg, 71.

Cement, Mastic, 72

Cement, Red, 71.

Cements, Use of, 69 .

Cements, Waterproof, 71.

Cera, Fortifant, 565.

Cervelle de Veau au Marinade, 289.

Cervelle de Veau à la Maitre d' Hotel, 291.

Charcoal, Danger of, 87.

Charcoal, Deaths by, 88.

Chartreuse of Apples and Ricc, 449.

Charlotte Russe, 488

Charlotte, Strawberry, 489.

Champagne, Pink, 517.

Champagne, Grape, to equal Foreign, 517.

Cheese, 454.

Cheese, to make, 454 .

Cheese, at Dinner, 100 .

Cheese, Cream, 454.

Cheese, Cream, Plain Family way, 456.

Cheese, Cottage, 454

Cheese, Eggs and, 455.

Cheese, for Fondeau, 455.

Cheese, Italian, 525.

Cheese, à L' Italienne, 455 .

Cheese, Puffs, 454.

Cheese, Sage, 454 .

Cheese, Soufllé, 455 .

Cheese, Stewed, 456.

Cheese, Potato, 435 .

Cheese, Potted, 456.

Cheese, Pudding, 456.

Cheesecakes, 434, 485.

Cheesecakes, Almond, 434, 486 .

Cheesecakes, Apple, 486.

Cheesecakes, Bread, 486.

Cheesecakes, Citron, 486.

Cheesecakes, Lemon, 434, 486.

Cheesecakes, Miss Bratty's, 434.

Cheesecakes, Orange, 434

Cheesecakes, Soyer"s, 435 .

Cheesecake, Stwck, 435.

Cherries, to dry, 503.

Cherry Bounce, 514.

Chetney, 135.

Chicken and Ham Potted, 349

Chicken Salad, 390 .

Chicken Jelly, 520.

Chickens, Boiled, 364.

Chickens, Braised, 364.

Chickens, Curried, 364, 367.

Chickens, Fried, à la Malabar 364.

Chickens, Pulled, 364

Chickenpox, 562.

Chilblains, 563.

Children, Cooking for, 526.

Children's Diet for the day, 529.

Children, Management of, 48 .

Children, Meats for, 529.

Children, Training of, 519

China, 24.

Chlorine, Mode of Liberating, 90. 
Chocolate, 497.

Chocolate, Cake, 480.

Chocolate Drops, 498 .

Chopping, 73.

Cinnamon. 131.

Citron, 542.

Citronella, 542.

Clarify Meat Jelly, 358.

Clary, 132.

Cleanliness, Importance of, 47.

Cleaning Bottles, 60 .

Clean Brusñes, 577.

Clean Decanters, 578

Clean Feather Beds, 578.

Clean Floors, 88.

Clean Freestone, 574.

Clean German Silver, 570.

Clean Gloves, 572.

Clean Ironwork, 88 .

Clean Japanned Groods, 89.

Clean Japanned Waiters, 574.

Clean Kettles and Saucepans, 576.

Clean Kid Gloves, 569.

Clean Knives, 89.

Clean Marble, 570.

Clean Mattresses, 578.

Clean Paint, 89.

Clean Papered Walls, 578.

Clean Silk, 570

Clean Silver, 572

Clean Steel Forks, 89.

Clean Tin Covers, 571.

Clean Wainscots, 576 .

Cloth, to take Spots out of, 569

Cloves, 130, 542 .

Clonted Cream, 488.

Clothesballs, 575.

Cocoanut Cake, 477.

Cocoa, 497, 522 .

Cocoa Shells or Nibs, 497.

Coffee, 494, 495.

Coffee, to roast, 495.

Coffeemaker, Patent, 495.

(iold Cream, Almond, 547.

Cold Cream, Rose, 547.

Cold Cream, Violet, 547 .

Coelus Apicius, 125.

Cold Cream, 549.

Collar Breast of Mutton, 353.

Collar Breast of Veal, 353.

Collar Eels, 351

Collar Fowls, 362.

Collar Ox Cheek, 353.

Collar a Pig, 353.

Collar Pig's Head, 324, 353.

Collar Ribs of Beef, 250.

Collar Salmon, 202.

Collodion, 565.

Collodion, Blistering, 565.

Collodion, Toothache, 565 .

Coloring for Jellies, 502

Colored Things, to wash, 571.

Color of Dresses, to preserve, 572.

Compotes of Fruit, 505 .

Compound Odors, 542

Condiments, 129.

Consideration of Dinner, 36.

Consommé, 127.

Contrivance for Filtering, 574.

Composition for the Skin, 548 .

Cooking, French. 139.

Cookery for the Sick, 517.

Cooking Stoves, 85.

Cookery, Art of, 124.

Cookery, Rudiments of, 136.

Coral Branches, 577.

Corking, 60 .
Corn, Green, Fritters, 385. Corn, Creen, Omelet, 385.

Corn, Green, Pudding, 385.

Corn, Sweet, 385

Cottage Cheese, 454.

Cottager's Stove, 85 .

Country Syllabub, 491

Cosmetic Gloves, 546.

Cough, 564 .

Coulis, 127.

Court Nosegay, 542 .

Court Plaster, 565.

Cow Heels, 263.

Cow Heels, to fry, 267, 264.

Cow Heels to pot, 264 .

Crabs, 206.

Crabs, Boiled, 207.

Crabs, Minced, 208.

Craks, Soft Shelled, 208.

Crab Soup, 207.

Crabs, Stewed, 207.

Cracknels, 482.

Cracknels, Isle of Wight, 482.

Cranberries, to prepare for Tarts, 432.

Cranberry Sauce, 507.

Crayfish Soup, 207.

Crayfish Butter, 208.

Crayfish Stewed, 207.

Cream of Tartar' Water, 523.

Cream Champagne, 487.

Cream Cheese, 454.

Cream, Clonted, 488 .

Cream, Italian, 488 .

Créma, Leché, 488.

Cream, Mock, 490.

Créme Frites aux Amandes, 458.

Crêpes, $45 \pi$.

Crickets, to destroy, 567.

Croquettes, 127.

Croup, 562.

Croustades, 127.

Crumpets, 468.

Cullis, 275.

Cup Cake, 478.

Cure of Intemperate Habits, 566.

Cure Bacon, Cobbett's Receipt, 333.

Cure Bacon for Larding and Braising, 334, 346 .

Cure Bacon, Maline's Method, 345 .

Cure Beef, Corned, 342.

Cure Beef Tongue, 257.

Cure Hams, 329, 331, 343, 344.

Cure Hug's Cheeks, 346.

Cure Mutton Ham, 345

Cure Tongues, 343.

Curing, 341.

Curling Hair Fluid, 553.

Currants, Ripe, 528.

Currant Jelly, 502

Curry, Calf's Head, 283.

Curry, Chicken, 364, 367.

Curry, Custard, 525.

Curry, Kitchener's, 221.

Curry, Plain, 221.

Curry Powders, 221.

Curry Powder, Delhi, 221.

Curry, Sover's, 236.

Custard, Arrowroot, 487.

Custard, Boiled, 487.

Custard, Coffee, 487.

Custard, Cocoanut, 431.

Custard, Cream of Chocolate, 487.

Custard, French Mode, 487.

Culinary Utensils, 116.

Curtains, 22.
Cucumbers, to pickle, 394.

Cut, to heal, 565 .

Cutlets, à la Victime, 337.

Cutlery, 24.

Cutting Glass, 63.

D

Daily Routine of Work, 42.

Uandelion and Parsley Tea, 523.

Doubing, 142.

December, Fish in Season for, 152

December, Fruits in Season for, 152.

December, Meats in Season for, 152.

December, Poultry and Game in Season for, 152

December, Vegetables in Season for, 152 .

Deaths by Burning Charcoal, 88.

Decanters, to clean, 578 .

Decanting Liquids, 65.

Dejeuners à la Fourchettes, 94 .

Depilatories, 555 .

Dcssert, 41.

Desserts, 102

Detergent Gargle, 557.

Development of the Lungs, 559 .

Devils of Biscuits, 531.

Devil, Dry, 531.

Devilled Game, 531.

Devil, Wet, 531

Devonshire Junket, 491.

Diet, Children's for the Day, 529.

Diet Bread, 480.

Jigester, Saucepan, 123.

Dinners, Arrangement of, 96.

Dinncr, Consideration of, 36 .

Dinner Entrées, 100.

Direction of the Table, 37.

Disinfecting, 89

Distillation, 92 .

Distilling Spirits, 510.

Domestic Education \& Thoughts on-16.

Domestic Affairs, Skill in, requisite, 15.

Domestic Education, should be complete, 17.

Duties to Children, 46.

Early Rising, an advantage, 42.

Household Knowledge essential to a Lady, 42.

Infiuencé of Women, 17.

Late Hours destructive to Health, 49.

Plan and Order necessary, 44.

Province of Woman, 18.

Rights and "Mission" of Wo$\operatorname{man}, 16$.

Domestic Manipulation, 59 ,

Doomsday Book, 125.

Doughnuts, 483 .

Dranght for Palpitation of the Heart, 557.

Draught for Hysteric Patients, 559

Drawing-Room Furniture, 22

Dredgings, 139

Dresses, Color of, to preserve, 572

Dripping, 139.

ried Salmon, 530.

Drunkenness in France, 510.

Drying Bottles, \&c., 60 .

Dry Mcasures, 124. 
Ducks, boiled, 369.

J) ucks. Canvas-back, 370 .

Ducks. roasted, 369 .

Ducks, Stewed with Claret, 369.

Ducks and Peas, Stewed, 370 .

Duckling with Turnips, 370 .

Ducks, Wild, or Teal, 370 .

Dumpokht, 367.

Duties of each Day, 44.

Duties of the Mistress, 31.

Dw elling House, the, 19.

Dye, Black, 579 .

Dye, Blue and Green, 579. Dyes, Common Receipts for,
579 .

Dye, Red, 5 s0.

Dye, Slate colored, 581.

Dye, Yellow, 580.

\section{E}

Eau de Bouquet de Flore, 544.

Eau de Chypre, 543,544 .

Eau de Cologne, 514.

Eau d' Ambre Royale, 514.

Eau d' Elegance, 544.

Eau Jaune à l' Ámaryllis, 544.

Esu de Javelle, 555 .

Eau de Miel, 544.

Gau de vie Lavande, 543 .

Earache, 564.

Economy of Heat, 84

Eels à la Tartare, 203.

Eels, bread crumbed, 203.

Eels, collared, 202

Eels, fried, 203.

Eel Pie, 203.

Eels, spitchcocked, 203.

Eels, stewed, 203.

Eggs, 459 .

Eggs, au Beurre, 532.

Eigg Biscuit, 469 .

Eggs and Cheese, 555.

Eggs, to boil, 460 .

Eggs with burnt butter, 463.

Eggs, fried, 462.

Egg Flip, 513.

Egg Hot, 513.

Eggs, for Turtle, 247.

Eggs in cases, 463.

Eggs, to keep, 578 .

Eggs, to keep for winter, 459. Eggnogg, 514.

Egg Plant, 381.

Eggs, to poach, 460.

Eggs, plain baked, 462 .

Eggs, pretty dish of, 463 .

Eggs, Randalled, 462.

Egg Sauce, 215.

Egg scrambled, 532

Egog Snow, 463 .

Eggs and Vegetables, 463.

Eider Flower Ointment, 562

Elder Flower Tea, 522

Electuary for Scorbutic Eruptions, 559 .

Empress Eugenie's Nosegay, 543. Entrées at Dinner, 100.

Erysipelas, 558.

Essence of White Lilac, 537.

Essence of Beef, 518.

Essentia Odorifera, 543

Evening Parties, 41.

Extract d' Ambre, 539

Eycs, Inflammation of, to relieve, 557.

\section{$\mathbf{F}$}

Family Medical Guide, 556.

Fancy Pastry, 426.

Farces and Stuffings, 248 .

Farce Cuite, 245 .

Farce, Ham and Veal, 245.

Farce of Veal or Fowl, 247.

Farce, Panada, 243.

Farce, Panada, for Fish, \&c., 244.

Farce, Quenelle, 244.

Farce, Udder, 244

Fawn or Kid, to dress, 375

$\mathrm{F} \in \mathrm{atherbeds}$ to clean, 578 .

February, Fish in Season for, 149. February, Fruits in Season for 149.

February, Meats in Season for, 149.

February, Poultry and Game in Season for, 149.

February, Vegetables in Season for, 149.

Feeding Children, 47.

Fermenting, 91.

Fever and Ague, 558.

Fever Draught, 559 .

Filtering contrivance, 574.

Filtering Liquids, 68.

First Course at Dinner, 99.

First Watch Stew, 276 .

Fish, for April, May, June, July, and August, 150.

Fish Cake, 191.

Fish, Carp, stewed, 196.

Fish, Casserole, 211.

Fish, to choose, 59 .

Fish, Cod, boiled, 190 .

Fisl, Cod, with Oyster Sauce, 190.

Fish, Cod, au Gratin, 190.

Fish, Cod Omelette, 191.

Fish, Coủ Sounds, Ragoût of, 190

Fish, Cod, slices of, 191

Fish, Cold, to dress, 211.

Fish Cutlets, 213.

Fish for December, 152

Fish, Forcemeat, 245 .

Fish, Fricandelles of, 213.

Fish, Haddock, 192.

Fish, Haddock, dried, 192.

Fish, Haddock, dressed, 192

Fish, Haddock, baked, 192.

Fish, Halibut, 191.

Fish, Halibut, stewed, 191.

Fish, Halibut, colloped, 192

Fish, Halibut à la Crême, 192

Fish, Herrings, 193.

Fish, Herrings, fresh, baked, 193.

Fish, Herrings. potted, 193.

Fish for January, February, and March, 149.

Fish, Mackerel, stewed, 193.

Fish, Mackerel, boiled, 194.

Fish, Mackerel, broiled, 194.

Fish with Marinade, 189.

Fish, Matelote of, 213 .

Fish, Pickerel, boiled, 196

Fish, Pike, roasted, 195

Fish, Pike, baked, 196.

Fish, Salmon, crimped à la Crême, 199.

Fish, Salmon, au naturel, 200.

Fish, Salmon, baked, 200.

Fish, Salmon, boiled, 200.

Fish, Salmon, broiled, 201.

Fish, Salmon, collared, 202

Fish, Salmon, dried, 201.

Fish Paté, 212.
Fish, Pickled, 201.

Fish, Potted, 202.

Tish, Ragoût of, 214

Fish, to serve, 107.

Fish, Shad, roasted, 194.

Fish, Shad, preserved, 195.

Fish, Shad, broiled, 195.

Fish, Shad, baked, 195.

Fish for September, October, November, 151.

Fish, Sheepshead, boiled, 191.

Fish, Shrimps, 197.

Fish, Sinelts, fried, 196.

Fish, Smelts, broiled, 197

Fish, Smelts, baked, 197 .

Fish, Soles, fried, 196.

Fish, Stewed, Hebrew fashion, 214.

Fish, Sturgeon Cutlets, 197.

Fish, sturgeon, stewed, 197.

Fish, Sturgeon, cheaply cooked, 197.

Fish, Trout, roasted, 195.

Fish, Trout, fried, 195.

Fish, Trout, in the foreign mode, 198.

Fish, Trout, à la Genevoise, 198.

Fish, Trout, à la Princesse Royale, 198.

Fish, Tírout, à la Twickenhain, 198.

Fish, Tront, stewed, 199.

Fish, Turbot, boiled, 199

Fish, Turbot, à la Crême, 199.

Fish, Vol-au-vent of, 214.

Flannel Cakes, 469.

Flax Seed Lemonade, 522.

Fleur d' Italie, 543.

Floating Island, 489 .

Floor's, to clean, 88.

Fondeaux, 455.

Food, Adulteration of, 78

Food and Cookery for Children, 526.

Forcemeat, 244, 245 .

Forcemeat, another, 244.

Forcemeats, \&c., $24 \mathrm{~S}$.

Forcemeat Balls for Soup Maigre, 246.

Forcemeat Ingredients, 248.

Forcemeat for Hare or any thing in imitation of it, 24.5

Forcemeat, Oyster, 246.

Forcemeat for Turtle, 246.

Forcemeat, 340 .

Forcemeat to make Quenelles of 341.

Forcemeat, Panada for, 341.

Forcemeat Stuffing for Veal, 341.

Forcemeat Stufting for Hare or Turkey, 341

Foreign Terms, glossary of, 126 .

Fowls, à la Bechamel, 366 .

Fowls, boiled or roast, to carve 114

Fowls, boiled, 361 .

Fowls, braised, 361 .

Fowls, broiled, 362 .

Fowls, forced, 360 .

Fowls, filed, 362

Fowls, firicassee of, 382

Fowls, Guinea, 366.

Fowl, hashed, 361.

Fowl, Indian pilau of, 366 .

Fowls, Pea, 366

Fowls, Pilau of, 362

Fowls, Poêle, to boil in, 360 .

Fowls, à la Provençale, 360 . 
Fowls, Poêle, for, 363.

Fowls, Roast, 361.

Fowls, Rissoles of, 363.

Frangipane, 482.

Freestone, to clean, 574.

French Bread, 482.

French Beans, pickled, 396.

French Cooking, 139.

French Pap, 527.

Firicandelles of Fish, 213

Fritadella, 336.

Fritters, 458

Fritters, Plain, 458.

Fritters, Apple, 458.

Fritters, French, 458

Fritters, Potato, 458

Fritters, Curd, 459.

Fritters, Orange and Sweetmeat, 459.

Frontignac, 516

Frosted Fruit, 502.

Frosting Cakes, 475.

Frozen Pudding, 493.

Fruit Cake, 477.

Fruits for April, May, June, July, 150.

Fruits for August, September, October, November, 151.

Fruits to candy, 509 .

Fruits for December, 152.

Fruits for January, February, March, 149.

Fruits, to keep fresh, 578.

Fruit for Children, 528 .

Frying, 141.

Fry Calf's Liver, 286

Fry Chickens à la Malabar, 364

Fry Cowheels, 264, 267.

Fry Eggs, 462.

Fry Fowls, 362

Fry Ham and Eggs, 330

Fry Mutton Chops, 300.

Fry Pig's Feet and Ears, 327.

Fry Potatoes, 377.

Fry Ramequins, 455 .

Fry Sinelts, 1906 .

Fry Soles, 196

Fry Sweetbreads, 290.

Fry Tront, 198.

Fry Veal, Liver, and Bacon, 289.

Fry Venison, 374

Fumigating Paper, 553.

Furnishing a House, 21.

Furniture, Bedroom, 2.2.

Furniture, Drawing-room, 22

Furniture, Kitchen, 23.

Furniture, Nursery, 46.

\section{G}

Galette, 482

Game, all kinds of potted, 850 .

Ganive Course, 100.

Gants Cosmétiques au Bouquet, 546.

Garlic, Pickle, 394.

Garnishes, 248.

Gas, Heating vessels by, 86 .

Gauffres, 429 .

German Silver, to clean, 570.

Geranium, 538.

Gherkins and Kidney Beans, to pickle, 399:

Gherkins, 132 .

Ginger, 130 .

Gingerbread, 482.
Gingerbread, Hard, 483.

Gingerbread, Soft, 482

Gingernuts, 483 .

Ginger Wafers, 478 .

Glaize, 524.

Glasse, Mrs., Recipe, 18.

Glass, 24.

Glass Cutting, 63.

Glass Grinding, 63 .

Glass Vessels, labelling of, 65 .

Glass, Writing on, 63 .

Glaze, 128.

Glazing, 140, 142.

Glazing for Tarts, 426 .

Glossary of Foreign terms in cookery, 126.

Gloves, to clean, $569,571$.

Gloves, Cosmetic, 541, 546 .

Glue, 70.

Godiveaux, 129

Glycerine Balsam, 549 .

Gooseberry Fool, 492 .

Goose, Roasted, 368.

Goose, to carve, 115.

Goose, Marbled, 368.

Goose, Preserved, 368.

Goulard Lotion, 559.

Grapes, to pickle, 399 .

Grease, to extract from silk, 570.

Grease spots, to take out, 570 .

Green Peas, 380 .

Green Corn Fritters, 385

Green Corn Omelet, 385 .

Green Corn Pudding, 385.

Griddle Cakes, Indian, 469 .

Grillades, 529 .

Grinding Glass, 63.

Ground Rice Millk, 522.

Ground Rice Paste, 524.

Ground Rice Pudding, 524

Grouse, 372.

Guards' Bouquet, 543.

Gumbo, 386.

H

Hair Curling Fluid, 553.

Hair Dyes, 554.

Hair Wash, 553.

Hair Water, 552.

Hams, 329.

Ham, to bake, 331 .

Hams, boiled, 328 .

Hams, to boil, 329 .

Ham, to boil in a superior way, 328.

Ham, to braise, 332 .

Ham, to braise in a French fashion, 332 .

Ham C'ake, 351

Ham, to carve, 112.

Ham, cold, Soyer's, 355 .

Ham, to cook, 328 .

Hams, to cure, 329, 331, 343, 344.

Ham and Eggs, fried, 330 .

Ham, fried with Poached Eggs, 330.

Ham, Fitzimmon Receipt for curing, 344.

Ham Gravy, 330.

Ham, Goose, 345 .

Hams, to keep, 56 .

Ham, Mutton, to boil, 330 .

Ham, Mutton, to cure, 345.

Ham, to make superior to Westphalia, 344.

Ham, Practical Housewife's Christmas, 829 .
Ham, Rashers or Slices, 333.

Ham, to roast, 330

Ham, Westphalia, 344.

Harlequinade in Cookery, 126.

Hands, to whiten, 549.

Harnessmaker's Jet, 573.

Hare, Jugged, 373.

Hare, Potted, 349.

Hare, Roasted, 372 .

Haricot Roots, 387.

Harness, to color, 569 .

Hashballs, 257.

Hasty Pudding, 442.

Heartburn, Mixture for, 561.

Heating by Gas, 86.

Heat, Economy of 84 .

Heliotrope, 538.

Heliotrope, Extract of, 539

Hedgehog, 445.

Herrings, to pickle, 354 .

Hints and Maxims, 143

Hog's Cheeks, to cure, 346.

Hog's Lard, 328.

Hominy, 453.

Home and Housekeeping 16.

Absolute Rule of a Mistress 31.

Care in Accounts necessary. 33.

Care of the Sick, 50.

Choice and Arrangement of Dinner Company, 38.

Duties of each day, 42 .

Feeding Children, 47.

Fundamental Principles, 33.

Good Wages the best policy, 32.

Home, little Attachment of Americans to, 15

Home Duties, little thought of in Female Education, 15.

Home, ruled by a Sensible Woman, 16.

Home, a place of Refuge and Rest, 16.

Housewifery may be Ultra, 16.

Importance of Cleanliness, 52.

Importance of Health, 34

Important Responsibility of a Housekeeper, 16.

Income and Marriage, 18.

Management of Children, 48.

Social Evenings, 41 .

The Nursery, 46 ,

Thoughts on, 16.

Housekeeping -

Acetous Fermentation, 92

Adulterated Articles, 78.

A Dwelling in Town, 19.

Agreement with the Landlord, 19.

A Useful Art, 15.

Bargain Hunting Perilous, 33

Bed-room Furniture, 22.

Bedsteads and Bed curtains, 22.

Boarding, 21.

Brushes used for cleaning, 25.

Breathing Chlorine not Injurious, 91 .

Calculation of Income and Outgoings, 17.

Carpets, 22.

Chloride of Lime a Disinfect ing agent, 20

Chloride of Soda, 20 . 


\section{Housekeeping -}

Change of Air required continually, 20.

Cleaning, Drying, and Corking Bottles, 60

Coffee Making in Turkey, 79

Covering the Table, 37.

Cutting, Grinding, and Writing on Glass, 63.

Cutlery, Crockery, China, \&c. 24.

Compulsory Present-Giving, 30.

Decanting, Straining, and Filtering of Liquids, 65 .

Desserts, Teas and Suppers, 102.

Dessert, 39.

Disinfecting Operations, 89.

Difficulty of procuring and retaining good Servants, 26.

Dining-room Furniture, 22.

Drawing-room Furniture, 22.

Dividing, Powderins, Grinding, \&c., 72.

Domestic Use of Gas in Heating, 86 .

Expenses of a Servant, 26.

Haraday's Use of Chlorine, 91.

Fumigation and Disinfection, 20.

Furnishing a House, 21.

Gratuities to Servants, 29.

Hall Furniture, 21

Hard Water in Washing, 81.

Hedgehog, 446.

Herbs for Distilling, 93.

Hiring Servants, 26.

Household Linen, 25.

Household of a British Nobleman, 27.

Household Expenditure, 17.

House Rent, 21

Impositions in Hotels, 29.

Insult to the name of Hospitality, 29.

Irish and German Servants, 26

Kitchen Furniture, 23.

Labelling of Glass Vessels, 65.

Ladies' Packages, 74

Lighting of Apartments, 23.

List of Articles of Silver for the Table, 24.

List of Spoons, \&c., 25.

Manufacture of cements, 69 .

Marketing, 56.

Mistaken Notion among American Girls, 30.

Mode of Warming a House, 19

Mode of Liberating Chlorine,

Pictures on the wall, 21

Placing Dishes on the Table, 99.

Process of Boiling, 82.

Process of Distillation, 92.

Qualifications of Servants, 28.

Requisites in a Cook, 27.

Rooms in a House, 19.

Saccharine Fermentation, 91.

Sleeping in close rooms injurious, 20.

Soyer's Housewife's Dinner, 39.

Soyer's List of Kitchen Articles, 23.

\section{Housekseping-}

Stoppering of Bottles, 61 Style of Dinner, a test of respectability, 37 .

Stored Articles, 56.

Sweeping and Dusting, 43.

Tax on Visitors, 29 .

Teamaking in China, 79 .

Taking a house 18

The Question of Dinner, 36.

The Store room, 53, 54

Thoughts on Housekeeping, 16.

To choose Fish, 59.

To choose Meats, 56, 57, 58.

To choose Poultry and Game, 58,59 .

Tying knots, 75

Tying over Jars, 76 .

Tying a Parcel, 77.

Ventilation Important, 20.

Weights and Scales necessary, 33.

Honcy Suckle, 539.

Hooping Cough, 556.

Hopping John, 386.

Horse Radish, 132.

House Furnishing, 21.

Housekeeping Accounts, 33 .

Household Articles, to keep, 54

Household Linen, 25.

Housewife's Dinner, 39.

Housewife's Christmas Cake, 479.

Housekeeping, Principles of, 33 .

Household Vermin, to destroy, 579.

Hungary Water, 541.

Hung Beef, 259, 343 .

Hunter's Beef, 268.

Hysteric Patients, draught for, 559 .

I

Ice Cream, Almond, 491.

Ice Cream, Wagner's Vanilla, 490.

Ice Cream, Wagner's Lemon, 490.

Ice Cream, Wagner's Strawberry, 490.

Ices, Watcr, 491.

Icing for Cakes, 480 .

Icing for Fruit Pies and Tarts, 435.

Iceland Liverwort Decoction, 526.

Iceland Moss Jelly, 523.

Imperial, 523.

Impressions of Butterflies' Wings, to take, 575 .

Impressions of Leaves, 573.

Importance of Health, 34 .

Income and Living, 18.

Indelible Marking Ink, 575 .

Indigestion, Medicine for, 557 .

Ingredients of Food, 78 .

India Pickle, 398.

Infant's Food, 527.

Infection, to prevent, 90 .

Inflammation of Eyes, 557.

Infant's A perient, 564

Infusion de Lavande Rouge, 543.

Infusion de Camomile Romaine, 543.

Infusion of Esprit de Safran, 544.

Infusion of Absinthe, 544

Infusion de Canelle, 544.

Infusion Esprit de Rhodia, 544.

Infusions Spirituous, 543.
Ink, Recipe for, 569

Ink, blue, Recipe for, 569

Ink for marking linen, 555 .

Ink Stains, to remove from the fingers, 571.

Ink, to remove from books, 571.

lron Work, to clean, 88

Italian Cream, 483.

Italian Pastes, 450 .

January, Fish in Season for, 149

January, Fruits in Season for, 149.

January, Meats in Season for, 149

January, Poultry and Game in Season for, 149.

January, Vegetables in Season for, 149.

Japanned Goods, to clean, 89.

Japanned Waiters, \&c., to clean, 574,

Jeffrey Hudson in a Pie, 125.

Jelly, Arrowroot, transparent, 524

Jelly, Aspic, 354.

Jelly, Calf's Foot, 491.

Jelly, cheap Calf's Foot, 492.

Jelly, clear Apple, 502.

Jelly Cake, 480.

Jellies, Coloring for, 502.

Jelly, Cranberry, 503.

Jelly, Chicken, 520 .

Jelly, Currant, 502.

Jelly, Easy and excellent Receipt for, 489 ,

Jelly, Iceland Moss, 523.

Jelly for Invalids, 517.

Jellies for Meat or raised Pies, 410.

Jelly, Port Wine, 519

Jelly, Pork, Dr. Ratcliff's, 518.

Jelly, Quince, 500.

Jelly, Strengthening, 518.

Jelly, Tapioca, 521

Jerked Beef, 140.

Jet, Harnessmaker's, 573.

Joints in Animals, Names of, 116

Johnny Cakes, 469.

Jophson's Soup, 524 .

July, Fish in Season for, 150.

July, Fruits in Season for, 150.

July, Meats in Season for, 150.

July, Poultry and Game in Season for, 150.

July, Végetables in Season for 150 .

Jumbles, 478

June, Fish in Season for, 150.

June, Fruits in Season for, 150,

June, Meats in Season for, 150.

June, Poultry and Game in Sean son for, 150.

June, Vegetables in Season for 150.

\section{K}

Kabobs, 530.

Kedgeree, 524.

Kedgeree for breakfast, 213.

Ketchup, Camp, 404

Ketchup, Cucumber, 403.

Ketchup, Mushroom, 402. *

Ketchup, Pontac, 406.

Ketchup, Seven year's', 404.

Ketchup, Tomato, 404. 
Ketchup, Tomato, for winter us 3, | Lamb Sweetbreads as Cutlets, 317. $\mid$ Marinade for mcats, 250. 404. Walnut, 403

Ketehup, Walnut, best quality, $4: 8$.

Ketchup, Walnut, Irish mode, 403 .

Kettles and Saucepans, to clean, 570.

Kid Glores, to clean, 569.

Kisses, 484 .

Kitchen Articles, 123.

Kitchen Clock, wanted, 123.

Kitchen Furniture, 23.

Knifeboard, to make, 573.

Knives, to clean, 89.

Knives and Forks, 575.

Knots, 74.

Kringles, 482.

\section{L}

Labelling of Glass vessels, 65.

Lady Cake, 450.

Lait de Poule, 524

Lait de Rose, 546 .

Lamb, à li Espagnole, 315.

Lamb, au Pascal, 315 .

Lamb, Blanquette d' Agneau, à la Provencale, 315 .

Lamb, Breast of, braised, boiled, 314.

Lamb, Breast of, to stew, 315 .

Lamb Chops, 316.

Lamb, to choose, 57.

Lamb, Chevaux de Frise, 315.

Lamb Swectbreads, with chevaux de Frise, 315 .

Lamb with Lamb sweetbread, 315.

I amb, to bonc a Quarter, 314.

Lamb, Forequarter of, 311.

Lamb, Forequarter, to carve, 112.

Lamb's Fry, 314.

Lamb's Head, 314.

Lamb's Head with Brain or Liver, 314.

Lamb's Head with Hollandaise, 314.

Lamb's Head and Emincées, 317.

Iamb's Heart Sweethreads, with Bacon or Truffles, 317.

Lamb's Leg, to boil, 312.

Lamb's Leg, to roast, 312

Lamb's Loin, to grill, 312.

Lamb's Loin, stewed, 313.

Lamb's Leg or Shoulder with Peas, 313.

Lamb's Leg, boiled with Spinach, 313.

Lamb's Leg or Shoulder with French Beans, 313.

Lamb's Neck, it la Jardinière, 313. Lamb, Pieds d' Agnean, 317.

Lamb, Saddle of, 312 .

Lamb, Saddle, Russian fashion, 313.

Lam', Shoulder of, 312.

Lamb, Shoulder, braised, 314.

Lamb's Shoulter, forced and braised, 312

Lamb's Shonlder, layded, 312. Laino Stones, to fricasse, 315 . Lamb. Sweetbreads, 316.

Lamb Sweetbreads, brown, 316.

Lamb Sweetbread Cutlets au gratin, 317 .

Lamb Sweetbreads, roasted, 317 .

Lamb Sweetbrcads, larded ì la Daubc, 317.

Lamb Sweetbreads, to stew, 316. Lamb Sweetbreads, white, fricasseed, 316.

Lamb Trotters, 318.

Larding, 142

Lard, to melt, 328 .

Lard, IIog's, 328.

Larks or small birds. 365.

Lavender Water, 514.

Laying the cloth, 98.

Laying out of Tables, 94.

Laying out Tray or Sideboard, 99 .

Leché créma, 488 .

Leeches, 561 .

Lemonade, 511, 524

Lemonade, Flaxseed, 522

Lemons and Uranges in Jelly, 505. Lemon flavoring for Puddings, 402 .

Lettuces and Endives, 389.

Liebig's plan for boiling meat, 83 . Lily of the Valley Essence, 539 .

Lima Beans, 384

Lime, Cement of, 568 .

Linen, Household, 25.

Linen, to pcrfume, 571.

Linen, to take stains out of, 567

Linseed Emulsion, 523.

Liquids, Decanting, 65.

Liquids, Filtering, 68.

Liquid Glue, 70.

Liquid Measures, 124

Lip Salve, 549.

Lip Salve, common, 549 .

Liverwort Decoction, 526.

Lobscous, 275.

Lobster, boiled, 204.

Lobster, curried, 205.

Lobster, Croquettes of, 205

Lobster, Garniture for Onuelettes, 464

Lobster, au gratin, 205.

Lobster, Salad, Italian, 205.

Lobster Salad, 205, 392

Lotion, Goulard, 559.

Luncheons, 95.

Luxuries of different ages, 125.

\section{M}

\section{Mace, 130.}

Macaroons 484

Maccaroni, 450

Maccaroni, Timbale of, 450.

Maccaroni, Paté of, 450 .

Mackenzie's Perigord Pie, 418.

Mahogany, to restore the color of, 573.

Maids of Honor, 485.

Magnolia, Essence, 539 .

Management of Children, 48

Mangoes, 397.

Manipulation, Domestic, 59.

Marble, to clean, 570 .

Marbled, Goose, 368 .

Marbled Veal, 295.

March, Fish in Season for, 149

March, Fruits in Season for, 149.

March, Meats in Season for, 149.

March, Poultry and Game in Season for, 149 .

March, Vegetables in Season for 149.
Marinade for collared maats, 354

Marjoram, 137

Marketing, 56.

Marking Ink, 575.

Marmalarle, 504.

Marnialade, Apple, 504

Marnalade, Orange, 505.

Marmalade, Pear, 501

Marmalade, Quince, 530 .

Marrow Pudding, 524.

Miarrow Boncs, 264, 273.

Miarrow Bones, baked, 273.

Mastic Cement, 72.

Marrow Patties, 421.

Marshmallow Emulsion, 523.

Mattresses, to clean, 578 .

May, Fish in Season for, 150.

May, Fruits in Season for, 150 .

May, Meats in Season for, 150 .

May, Poultry and Game in Season for, 150 .

May, Vegetables in Season for, 150.

Mayonnaise, 392.

Mead withont fruit, 511.

Meats for January, February, 149.

Meats for March, 149.

Meats for April, May, June, July, 150.

Meats for August, September, 151.

Meats for October, November, 151 .

Meats for Dccember, 152.

Meat Jelly, to clarify, 355 .

Meat Pics, 409 .

Medical Guide, Family, 556.

Meats for Children, 529.

Medicine for Indigestion, 557.

Meringues, 493.

Meringues, à la crême, 494.

Milk, Ass's, 520.

Milk, artificial Ass's, 520.

Milk, baked, 520 .

Milk Rolls, 471.

Milk, toast, 471

Millet Pudding, 523.

Mincemeat, 427,423 .

Mince Pics, 427.

Mint, 132.

Miroton of Apples, 449.

Mistress, Duties of the, 31.

Miscellaneous Receipts, 567.

Mixed Pickle, 397.

Mixture for Children teething, - 557.

Mixture for Diarrhea, 559 .

Mock Cream, 490.

Mock Ginger, 506 .

Molasses Posset, 521.

Morels, 389.

Movable Screen, 123.

Mouth Glue, to make, 70 .

Mouton, Blanquette de, 302.

Muflins, 468, 470.

Mulled Cider, 514.

Mush, 453.

Mushrooms, 388

Mushrooms, boiled, 388

Mushroom Garniture for Omo lettes, 464.

Mushrooms, to pickle, 394.

Mushrooms, to Pot, 351.

Musk, 540.

Mustard, 131, 147.

Mustard, French, 406.

Mutton, Camp dish, 310.

Mutton, to choose, 57.

Mutton Bagpipe with Endive 311. 
Mutton, Breast of, stewed, 300 .

Mutton, Breast of, to collar, 353.

Mutton, Breast of, au gratin, 300 .

Muttor, China chilo, 303.

Mutton, another way, 303 .

Mutton, braised Cutlets, 301.

Mutton, cutlets of, 301 .

Mutton, cutlets santé, 301.

Mntton, Maintenon, 301.

Mutton Chops, broiled, 300 .

Mutton Chops, fried, 300.

Mutton Chops as Beefsteaks, 300.

Mutton Cotelettes, à la Maintenon, 305,306 .

Mutton Cutlets with Soubise or Onion sauce, 305 .

Mutton, a better method, 306 .

Mutton Cotelettes, à la Polonaise, 306.

Mutton Cotelettes, en Ragoût, 306.

Mutton Cotelettes, aux Haricots verts, 306 .

Mutton, au Gratin, or scollops, 304

Muttion, an English Haggis, 310.

Mutton, Fillet of, 301.

Mutton, Fillet of, stewed, 302 .

Mutton in Marinade, 302.

Mutton, French Ragoût of, 309.

Mutton, Haricot, 302 .

Mutton Hams, to dress, 305.

Niutton, hashed, 303.

Mutton, Haunch of, to carve, 112

Mutton. Haunch of, 297.

Mutton, Irish, stew of, 303, 310 .

Mutton, Kebobbed, 302.

Mutton Kidneys, 307.

Mutton Kidneys, broiled, 305.

Mutton, Leg of, roasted, 297.

Mutton, Leg, roasted, boned, and stuffed, 297.

Mutton, Leg of, boiled, 298.

Mutton, Leg of, braised, 298.

Mutton Leg of, to carve, 110.

Mutton, to dress a Leg of, with Oysters, 297.

Mutton, Leg of, to stuff, 297.

Mutton, Leg steaks, 300

Mutton, Leg, cut to send to table neatly, 298 .

Mutton, Loin steaks, 300 .

Mutton, Loin of, to carve, 111

Mutton, Loin of, stewed, 299.

Mutton, Loin of, like Venison, 305 .

Mutton, like Venison, 304.

Mutton, Minced, 303.

Mutton, Neck of, to carve, 111.

Mutton, Neck of, $299,300$.

Mouton, Rognon de, à la Francaise, 304 .

Mutton, Rumps and Kidneys, 307.

Mutton, Risseroles, 304.

Mutton, Saddle of, to carve, 111.

Mutton, Saddle of, 297.

Mutton, Saddle of, à la Polonaise, 398.

Mutton, Scrags of, à la St. Menehould, 307 .

Mutton, Shoulder of, 299.

Mutton, Scrag of, to carve, 111.

Mutton, Shoulder of, with Rice, 299.

Mutton, a Scotch Haggis, 304, 810.
Mutton, Soyer's new chop, 299.

Mutton, Tails and Kidneys, 307.

Mutton, Tails and Tongues, 308. Myrtle Essence, 539.$$
\text { * }
$$

\section{N}

Nail in the Foot, 565

Nails to whiten, 549 .

Napkins to fold, 95 .

Napkins as the Mitre, 95.

Naplins, as the Exquisite, 95.

Napkins, as the Collegian, 96 .

Napkins, as the Cinderella, 96 .

Napkins, as the Flirt, 96.

Napkins, the Neapolitan, 96.

Napkins, the Favorite, 96 .

Nasturtiums, 132

Nasturtiums, pickled, 396.

Neats' Tongue, to choose, 56 .

Neats' Tongue, potted, 350 .

Neats' T ingue, 291.

Negus, 516.

Nettlerash, 560 .

New Year's Cookies, 478.

Nipple Liniment, 564.

Nougat, 494.

November, Fish in Season for, 151.

November, Fruits in Season for, 151.

November, Meats in Season for, 151.

November, Poultry and Game in Season for, 151

Norember, Vegetables in Season for, 151 .

Noyau, 516.

Nursery Furniture, 46.

Nutrieg, 130.

\section{0}

October, Fish in Season for, 151.

October, Fruits in Season for, 151.

October. Meats in Season for, 151.

October, Poultry and Game in Season for, 151.

October, Vegetables in Season for, 151.

Oeufs, Bronilles, 463

Oeufs, Farcis, 462.

Oeufs, Frits, 462.

Oeufs, sur le Plat, 462

Oils for the Hair, 545 .

Oil and Grease, to take out of boards, 570 .

Ointments, 559 .

Ointment, Elderflower, 562

Dintment, Simple, 559 .

Ointment. Resin, 559.

Ointment, Calamine, 559.

Olives, 134.

Omelettes. 460.

Omelettes, aux Amandes, 461.

Omelette Cod. 191

Omelette, aux Confitures, 461.

Omelette, anx Fines Herbes, 461.

Omelette, d' Huitres, 461.

Omelette, au Naturel, 461.

Omelette, Souffée, 461 .

Onions, 131, 388.

Onions, Spanish, 380.

Onions, pickled, $393,394$.
Orangeade, 521.

Orange Flower Essence, 539.

Orange Fool, 492

Order and Method, 45.

Oysters, à I'Imperiale, 210.

Oysters, Breaded, 210.

Oysters, Broiled, 209

Oyster Curry, 210.

Oyster Forcemeat, 246

Oyster Fritters, 209.

Oyster Garniture for Omelettes 46

Oyster Pie with Sweetbread, 210.

Oysters, Pickled, 211.

Oysters, Roasted, 210.

Oyster Rolls, 209

Oyster Sausages, 210.

Oysters, Scalloped, 208, 209, 211

Oyster, Stewed, 211.

Oysters with Brown Sauce, 209.

Oysters with White Sauce, 209.

Ox-Cheek, to collar, 353.

Oxford Dumplings, 450.

Ox-Feet, 272.

Ox-Heart, 270.

Ox-Kidneys, 272

Ox-Tails, à la Jardinière, 269.

Ox-Tails, au Gratin, 269.

Ox-tongue, Remains, 272.

$\mathbf{P}$

Packing Housekeeping Articles, 576 .

Pain Perdu, 449.

Pains after exertion, 564.

Paint, to clean, 89.

Paint, Outside, 563.

Paint, to take out, 575.

Palpitation, Draught for, 557.

Panada, 519.

Panada, Chicken, 519.

Panada, for Fish, 244.

Panada for Quenelle, 244.

Panada for Forcemeat, 244.

Pancakes, 457.

Pancakes, Apple, 457.

Pancakes, Dutch, 457.

Pancakes, New England, 457,

Pancakes, Rice, 457.

Parsley, 132.

Parsley, to fry, 373.

Parsnips, 382.

Parties, Evening, 41.

Partridges and Pheasants, to pot, 348.

Partridges, broiled, 371.

Partridges, Salmi of, 371.

Partridges, stewed, 371,

Passover, Balls for Soup, 247.

Passover Cakes, 483.

Paste, to make, 70, 571.

Paste, Flaky, 426.

Paste, permanent, 70 .

Paste of Beef or Mutton, to eat like Venison, 422.

Paste, very light, 409 .

Paste, Puff, 408 .

Paste, Almond, 432.

Paste, 406.

Paste for chapped hands, 545

Paste for chapped lips, 546

Paste, Rye, 568.

Pastilles for the breath, 554.

Pastilles, au Benzoin, 553.

Pastilles for Necklaces, Bracelets \&c., 554. 
Pastilles, à la Rose, 553.

Pastilles; Simple, 553.

Pastry, Fancy, 426.

Patchouly, 539

Patchouly Extract, 540.

Paté, Divine de Venus, 548

Paté, des F'oies Gras, 412.

Paté, d"Amandes des aux Jaunes d' œufs, 548.

Patties, 423.

Patties, Croustade or Dresden, 433 .

Patties with curds, 434.

Patties like Mince Pies, 434.

Patties, sweet, 430.

Peafowls, 366.

Peas, Garniture for Omelettes, 464

Peas, Sweet, Essence of, 540.

Pencil Marks, to preserve, 571 .

Peaches and Apricots, to pickle, 398.

Peas, Green, 380

Peas, to preserve, 387

Peas Pudding, 386 .

Pennyroyal, 132.

Pepper, 130.

Peppers, to pickle, 399 .

Perfumery and the Toilet, 537.

Perfume Linen, 571

Perigord Pie, 407.

Perigord Pie, Mackenzie's 418.

Perle Powders, 547.

Pernicious effects of Distilling Spirits, 510

Pets de Nonne, 481.

Pettitoes, 326.

Pheasants, 375 .

Pheasants, to carve, 115.

Pickle for Meat, 354

Pickled Apple Codlings, 400 .

Pickled Artichokes, 399.

Pickled Barberries, 399.

Pickled Butternuts, 399.

Pickled Beans, French, 395.

Pickled Cabbage, Purple, 396.

Pickled Cabbage, Red, 396.

Pickled Cabbage, White, 396.

Pickled Cauliflower, 396.

Pickled Celery, 396.

Pickled Cucumbers, 394.

Pickled Gherkins and Kidney Beans, 399.

Pickled Garlic, 394.

Pickled Grapes, 399

Pickle, India, 398.

Pickle, Mixed, 397.

Pickled Mangoes, 397.

Pickled Mushrooms, 394.

Pickled Nasturtiums, 396

Pickled Onions, 393, 394.

Pickled Peaches and Apricots, 398.

Pickled Peppers, Green, 399.

Pickled Plums, 398.

Picklerl Quince, 398.

Pickled Radish Pods, 395.

Pickled Shalots, 394.

Pickled Tomatoes, 397.

Pickle, to last for years, 347 .

Pickle Apricots, 398

Pickle Beef, à la Garrick, 274.

Pickle Cabbage, purple, 396.

Pickle Cabbage, red, 396 .

Pickle Cabbage, white, 396.

Pickle Cucumbers, 394

Pickle Garlic, 394.

Pickle Gherkins, 399.
Pickle Grapes, 399.

Pickle Herring, 354 .

Pickle Kidney Beans, 399 . .

Pickle, Mixed, 397.

Pickle Mushrooms, 394.

Pickle Onions, 393, 394.

Pickle Oysters, 211.

Pickle Peaches, 398.

Pickle Plums, 398.

Pickle for Meat, 354

Pickle Neck of Pork, 322, 327.

Pickle Salmon, 201.

Pictures on the wall, 21.

Picture Frames, to preserve, 570.

Pie, Apple, 428.

Pie, a fine Fish, 424.

Pie, a Christmas, 422.

Pie, Beefsteak, 417.

Pie, Beefsteak and Oyster, 421.

Pie, Calf's Head, 417.

Pie, Calf's Head or Calf's Foot, 420.

Pie, Calf's Foot and Kidney, 421.

Pie, Chicken, 413.

Pie, Cocoanut, 426 .

Pie, Codfish 424

Pie, Cold Veal or Chicken, 417.

Pies, Cold, 419.

Pie, Cracker, 427.

Pies, Duck, 418.

Pies, Eel, 414

Pie, Fish. 418.

Pie, French, 419.

Pie of Fowls, raised, 410.

Pies, Fruit, 425.

Pic, Game, 418.

Pie, Giblet, 413

Pie, Gooseberry and other Fruits, 428.

Pie, Green Goose, 418 .

Pie, Grouse, 414.

Pie, Hare, 419.

Pie, Lamb, 413.

Pie, Lamb, hot, 411.

Pies, Lent, 430.

Pies, Mince, 427.

Pie, Mutton, 412

Pie, Oyster, 416.

Pie, Partridge, 414.

Pie, Peach, 426.

Pie of Pheasant, raised, 411.

Pie, Pigeon, 413 .

Pie, Plain Custard, 427.

Pies, Pork, to eat cold, 420 .

Pie, Pumpkin, 426.

Pie, Rabbit, 413 .

Pies, Raised, Crust for, 407.

Pie, Raised, Russian, 410.

Pie, Raised, served hot, 410.

Pies, Rhubarb, 426.

Pie, Rumpsteak, 412.

Pie, Snipe, 418.

Pie, Squab, 421.

Pies, Various, 412

Pie, Veal, in a dish, 417.

Pie, Veal and Ham, 412.

Pie, Veal and Oyster, 420.

Pie, Veal and Sausage, 420.

Pie, Veal and Sweetbread, 420.

Pie, Vegetable, 419.

Pie Yorkshire, Goose, 422.

Pig, to Collar, 353.

Pig's Feet, 335 .

Pig's Feet, à la St. Menehould, 326.

Pig's Feet and Ears. fried, 327.

Pig's Head, baked, 325.
Pig's Head, boiled, 325.

Pig's Head, to collar, 325.

Pig's Harslet, 327

Pig's Feet, stewed, 326.

Pig's Kidneys, 335

Pigis Head, to collar, 358.

Pig's Cheek, 322.

Pig's Face, for breakfast, 322 .

Pig, Roast, 320.

Pig, Roast, to carve, 113.

Pig, to roast, 319 .

Pig, Sucking, to roast, 318 .

Pig, Sncking, to seald, 319

Pig, Sucking, Hindquarter of, 319.

Pigeons, broiled, 364

Pigeons, to carve, 116 .

Pigeons, compote of, 365 .

Pigeons, cooked as Woodcock, 865.

Pigeons, in Jelly, 365.

Pigeons, or any Bird in Aspic Jelly, 365 .

Pigeons, potted, 350

Pigeons, Roasted, 364.

Pigeons, Stewed, 365 .

Pilau of Fowl, 362 .

Pilau, Indian, 366.

Pills, Aperient, 563.

Pimples, 562.

Pithcaithly Bannock, 481.

Placing Dishes at Dinner, 99.

Plants, to dry, $5 \pi 1$

Plate, 24, 25.

Plate, to clean, 89.

Plaster for Pains, 568.

Plovers, 371.

Plover's Eggs, 371

Plum Cake, 475 .

Plums, pickled, 398.

Plum Porridge, 438.

Plum Pudding Glacé, 493

Plummer's Pill, 559 .

Pöele, French, 363

Pöele, to boil Fowls in, 360 .

Pöeling, 141.

Polenta, 451.

Polished Irons, to keep from rusting, 569.

Polish for Tables, 567.

Pomatum, 545.

Pommade de Beauté, 549.

Pommade for chapped lips, 549.

Pommade, Victoria, 545 .

Pommade, des Sultanes, 548 .

Pommade, de Ninon de l' Enclos 548.

Pommade for Tumors, 564

Pommade for Corns, 564 .

Pone of Sweet Potato, 385.

Pork, 318.

Pork, to choose, 57.

Pork, boiled, of all kinds, 322 .

Pork, Chops or Steaks, 323.

Pork, baked fagots, 327 .

Pork, chine, 321.

Pork, Cutlets, 321, 322.

Pork, Economical dish of, 328.

Pork, Fillet, to resemble Veal 320.

Pork, Griskin, 318.

Pork, Hashed, 335 .

Pork, Head Cheese, 324.

Pork, Jelly, 518.

Pork, Leg of, boiled, 320.

Pork, Leg of, roastcd, 320.

Pork, Leg of, to carve, 112.

Pork, Leg of, 321. 
Pork, Leg, mock Goose, 321. Pork, Liver Pudding, 324.

Pork, Loin, 320.

Pork, Neck, rolled, 320.

Pork, to pickle, 322, 347.

Pork, to restore, 331.

Pork, Sparerib, roasted, 320.

Pork, Sparerib, 318.

Pork, Spring or forehand, 330 .

Pork, Soúse, 326.

Pork Tongues, 331.

Porridge, 528.

Port Wine Jelly, 519.

Potatoes, 377.

Potato Barm, 467.

Potatoes, Biscuits of, 379

Potato Cheesecakes, 483.

Potatoes, to brown, 378.

Potatoes, à la Maitre d' Hotel, 379 .

Potatoes, fried, 379 .

Potato Flour, 378.

Potato Jelly, 378.

Potato Loaves, 378.

Potatoes, to keep, 377.

Potatoes, to mash, 378 .

Potatoes, New, 377.

Potato Omelette, 379

Potatoes and Peas for children, 529

Potato and Meat Salad, 271.

Potato Rissoles, 379.

Potato Sandwiches, 270.

Potatoes, to roast, 379 .

Potato Soufflè, 379 .

Potato Wall, 378.

Pot Pie, 414.

Pot Pourri, 552

Pot Pourri, Sweet, 545.

Potting and Collaring, 347.

Pot all kinds of Game, 350 .

Pot Beef, 267, 349.

Pot Beef like Venison, 349, 351.

Pot Birds, 349 .

Pot Chicken and Ham, 349.

Pot Cowheels, 264.

Pot Hare, 349 .

Pot Mushrooms, 351.

Pot Neat's Tongue, 350.

Pot Partridges, 348.

Pot Pheasants, 348 .

Pot Pigeons, 350.

Pot Poultry and Game, 348.

Pot Rabbits, 349.

Pot Salmon, 202.

Pot Smelts, 350 .

Pot Veal, 282, 350, 354 .

Pot Venison, 350 .

Poudre, de la Mer Ronge, 547.

Poultice, Bread and Water, 557.

Poultry, 356.

Poultry and Game for December, 152.

Poultry and Game for January, February, March, 149.

Poultry and Game, for April, May, June, July, 150.

Poultry and Game for August, September, October, November, 151.

Poultry and Game, to pot, 348 .

Poultry and Game, to choose, 58.

Pound Cake, 478 .

Powdering, 72.

Preparation for bad breath, 554.

Presents to servants, 29.

Preston Salts, 551.
Preserve Apples, 499.

Preserve Apricots, 501

Preserve Barberry Cakes, 506.

Preserve Blackberry Jam, 505.

Preserve Blackberries whole, 503 .

Preserve Brandy Cherries, 506.

Preserve Brandy Peaches, 501.

Preserve Cherry Jam, 505.

Preserve Crab Apples, 499.

Preserve Currants, 503.

Preserve Currants, dry, 504.

Preserve Currant Jam, 503.

Preserve Figs, Green, 504.

Preserve Figs, ripe, 504.

Preserve Ginger, 504.

Preserve Gooseberries, 502.

Preserve Green Corn, 507.

Preserve Grapes in Brandy, 503.

Preserve Green Gages, 501.

Preserve Lemon Peel, 506.

Preserve Mock Ginger, 506.

Preserve Nectarines, 501.

Preserve Oranges or Lemons in Jelly, 505 .

Preserve Peaches, 501.

Preserve Peaches, fresh, 507.

Preserve Pears, 501.

Preserve Pineapples, 499.

Preserve Pineapple Tart, 499.

Preserve Plums, 501.

Preserving, General Directions for, 499.

Preserve Quinces, 499

Preserve Raspberries, 503.

Preserve Rasfberry Cakes, 506.

Preserve Raspberry Jam, 505.

Preserve Strawberries whole, 503.

Preserve Tomatoes fresh, 507.

Preserve Tomatoes, ripe or green, 504.

Preserve Watermelon Rind, 498.

Primrose Ointment, 557.

Principles of Housekeeping, 30.

Prussian Cutlets, 336.

Pudding, Almond, 442.

Pudding, Almond and Sauce, 441.

Pudding, Batter, 448.

Pudding, the Baked Plum, 440.

Pudding, Baked Apple, 442.

Pudding, Beef Steak, 414, 438.

Pudding, Bird's Nest, 443.

Pudding, Black Cap, 448.

Pudding, Boston, 441.

Pudding, Boston Cracker, 447.

Pudding, Brandy, 447.

Pudding, Bread, baked, 444.

Pudding, Bread and Butter, 446.

Pudding, Brown Bread, 446.

Pudding, Demi Plum, 445.

Pudding, Chocolate, 447.

Pudding, Cocoanut, 445 .

Pudding, Chicken, baked, 438.

Pudding, English Plum, 440.

Pudding, Farina, 442

Pudding, Fine German, 448.

Pudding, Fish, 417.

Pudding, Frozen, 493.

Pudding, Fruit, 436.

Pudding, Game, 438.

Pudding, Gloucester, 450.

Pudding, Goose, 439.

Pudding, Ground Rice, 440, 445.

Pudding, Ground Rice with Fruit, 445.

Pudding, Hasty, 442.

38
Pudding, Jenny Lind, 441.

Pudding, Kidney, 416.

Pudding, Lamb, 415.

Pudding Iemon, 44.

Pudding, Maccaroni, 445.

Pudding, Marlborough, 440.

Pudding, Marrow, 439.

Pudding, Meat, 436.

Pudding, Muffin and Crumpot, 447.

Pudding, Mutton, 415.

Pudding à la Nesselrode, 449.

Pudding, Newcastle, 441.

Pudding, Oatmeal, 445.

Pudding, Orange, 443, 418.

Pudding, Paradise, 442.

Pudding, Plain Rice, 443.

Pudding, Polka, 441.

Pudding, Pork, 415.

Pudding, Potato, 426.

Pudding, Pumpkin, 426.

Pudding, Quaking, 447.

Pudding, Quince, 448.

Pudding, Rabbit, 416.

Pudding, Richelieu, 441.

Pudding, Rich Plum, 439.

Pudding, Roly Poly, 448 .

Pudding, Sago, 444.

Pudding. Sponge, 447.

Puddings, Suet, 437.

Puddings, Sweet, 436, 439.

Puddings, Sweetmeat, 448 .

Puddings, Tansy, 439.

Puddings, Tapioca, 444.

Puddings, Yorkshire, 416, 438.

Puddings, Veal, 415.

Puddings, Vermicelli, 447.

Puffs, Light, 429.

Puffs, Spanish, 484.

Puff Paste, 408.

Punch, 513.

Punch, Milk, 514.

Punch, English, 513.

Punch, French, 514.

Punch, Norfolk, 513.

Punch, Regent's, 513.

Punch, Tea, 514.

Punch, Victoria, 514

Punch, Wine, 514.

Purée of Cauliflower, 236.

Purée of Cucumber, 239.

Purée of Onion, 227.

Purée of Lobster, 186.

Purée of Turnips, 184.

Purée de Volaille, 363

\section{Q}

Quaking Pudding, 447.

Qualifications of Servants, 28

Quihi Sauce, 240 .

Queen Cake, 477.

Quenelles, 129.

Quin's Sauce, 406

Quince, Pickled, 398

Quince Pudding, 448.

Quinces, to preserve, 499.

$\mathrm{R}$

Rabbit, to carve, 113.

Rabbits, Fricasseed, 872

Rabbits, Potted, 349.

Rabbits, to skin, 373 .

Rabbits, Pilau of, 373. 
Rabbits, to taste like Hare, 372.

Radish Pods, pickled, 395.

Ragoût of Cod Sounds, 190.

Ragoût of Fish, 214.

Raised Crust for Pies, 407.

Ramequins, 455 .

Ramequins, à la Parisienne, 455.

Ramequins, fried, 455 .

Ramifolle, 336.

Patifias, 484, 511.

Rtmains of Ox Tongue, 272.

Rennet, 490.

Rennet Whey, 523.

Rheumatism, Medicine for, 560,582

Ribbons, to wash, 572 .

Rice and Apples, 528 .

Rice, to boil, 452 .

Rice Bread, 466.

Iice Cake, 485 .

Rice, Croquettes, 452 .

Rice, Casserole de Ris, 453.

Pice Croquettes, with Apricot Marmalade, 453.

Rice Cakes, 463.

Rice Fritters, 452.

Rice Flummery, 491.

Pice, Gateau of, 453.

Rice Gruel, 521.

Rice Pudding with Fruit, 528.

Rice for Savory dishes, 452 .

Rice Milk, 443,

Rice for Sweet Dishes, 452

Rice Water, 523.

Richmond Maids of Honor, 436.

Rich Gravy, 222.

Ringworm, cnre for, 557.

Ris de Vean, en Caisses, 294.

Rissoles of all kinds, $227,247$.

Rissoles of Fowl, 363.

Roasting, 137.

loast Beef of Lamb, 296.

Roast Beef, Sirloin, 251

Roast Beef Steaks, 255.

Roast Beef Tongue, 262.

Roast Ducks, 369.

Roast Fillet of Beef 251.

Roast Fillet of Veal, 267.

Roast Fowls, 361.

Roast Goose, 368 .

Roast Ham, 330 .

Roast Hare, 372

Roast Leg of Lamb, 312 .

Roast Leg of Mutton, 297.

Roast Leg of Pork, 320 .

Roast Meat to baste, 139 .

Roast Meat to brown, 139 .

Roast Meat to froth, 139.

Roast Oysters, 210.

Roast Pig, 319, 320.

Roast Pigeons, 364.

Roast Pike, 195.

Roast Potatoes, 379.

Roast Shad, 194.

Roast Shoulder of Venison, 375.

Roast Sparerib, 320

Roast Sucking Pig, 318.

Roast Trout, 195.

Roast Sweetbreads, 291, 317.

Roast Turkey, 356.

Rocks, 468 .

Rolls, 468 .

Rondaletia, Essence of, 540.

Rooms, Airing of, 35 .

Rose, 540.

Rose Face Powder, 546.

Rose Lip Salve, 549 .

Rosemary, Otto of, 541

Rose, Moss, Essence of, 540.
Rose, Tea, Essence of, 541. Rose, Triple, Esprit de, 540. Rose. White, Essence of, 540 Routine of Daily Work, 42. Rudiments of Cookery, 136. Rusk, to make, 469,470 .

\section{$\mathrm{s}$}

Sachet, à la Frangipanre, 552. Sachet, Heliotrope, 552, 552 .

Sachet, Lavender, 552

Sachet, à la Mousselaine, 552

Sachet, Millefleur, 552.

Sachet, Portugal, 552 .

Sachet, Patchouly, 552.

Sachet, Rose, 552.

Sack Posset, 513.

Saffron, 134

Sage, 132.

Sago Fruit Pudding, 525.

Sago Gruel, 522 .

Salars, 390 .

Salad, Chicken, 390, 391.

Salad, Family, 271.

Salad Dressing, 390.

Salad, Dressing for Lettuce, 390.

Salad, Fish, 392

Salad of Fowl, 391.

Salad, Lobster, 392

Salad, Mayonnaise, 392

Salade, Pickle, 406 .

Salade, Sidney Smith's Receipt for, 389 .

Salads, Summer, 391.

Salads, Winter, 391.

Sally Lunn, 468 .

Salmon, Dried, 530 .

Salsify, 380,382 .

Salsop, 135 .

Salt, Bay, 129.

Salt, Rock, 129

Sandwiches, 532 .

Sangaree, 514

Saponaceous Cream of Almonds, 547.

Sauce, Anchovy Butter, 235. Sauce, Anchovy, Butter of, 215 .

Sauce, Anchovy, for Fish, 215.

Sauce, à l' Allemande, 241.

Sauce, Apple, 232.

Sauce, Apple, Savory, 232.

Sauce, Aspic, the, 221

Sauce, Bechamel, 227, 228.

8ance, Beef Gravy, 220.

Sauce, Beef Gravy, French Receipt, 220.

Sauce, Blanche, $22 S$

Sauce, Blonde of Veal, 240

Sauce, Bread, 229.

Sauce, Brown, 215.

Sauce, Brown, Cucumber, 234.

Sauce, Brown, Mushroom, 235.

Sauce, Brown, Mushroom, thin, 234.

Sauce, Brown Sharp or Poivrade, 237.

Sauce, Brown, Soyer's, 233.

Sauce, Brown Thickening, 228.

Sauce, Caper, Brown, 223.

Sauce, Caper, for Fish, 215

Sauce, Caper, à la Francaise, 211.

Sauce, Caper, to imitate, 241.

Sauce, Caper, for Meat, 242.

Sauce, Caper, White, 223.

Sauce, Carachi, 239

Sauce, Carrack or Indian, 222
Sauce, Celery, 225, 243 .

Sance, Chestnnt, 229.

Sauce, Chetney and Quihi, 240

Sauce, Cod, 217.

Sauce, Cranberry, 243

Sauce, Cullis, 231

Sauce, Cullis, the, 240 .

Sauce, Currant, 232.

Sauce, Curry Powder, Delhi, 221.

Sance, Curry, Kitchener's, 221.

Sauce, Curry Powders, 221

Sauce, Curry, plain, 221.

Sauce, Curry, Soyer's, 236.

Sauce, Demi Glaze, 233.

Sauce for Ducklings, 232.

Sauce Dutch, French Receipt, 227.

Sauce, Eel, 217.

Sauce, Egg, 215, 224.

Sauce, Epicurean, 222

Sauce, Eschalot, 234.

Sauce, à l'Espagnole, 238.

Sauce, Feunel, 215, 223, 226 .

Sauce for Fish, 214, 217.

Sauce, Fish of all kinds, 214.

Sauce, Fresh Pork, 226.

Sauce for Fricandeau, 231.

Sauce, Garlic, 235.

Sauce, Gooseberry, 231.

Sauce, for Geese, 231 .

Sauce, Glaze, French Receipt, 227.

Sauce, Gravy, 218

Sauce, Gravy, clear, 220.

Sauce, Gravy, to make Mutton taste like Venison, 220.

Sauce, Gravy, stock for, 219.

Sauce, Gravy, for Venison Haunch, 220.

Sauce, Gravy, for Veal, 220.

Sauces, Gravies, \&c., 218.

Sauce, Gravy, rich, 222

Sauce, Harvey, 225.

Sauce, à l' Hollandaise, 238.

Sauce, Horseradish, 214, 224

Sauce, Hot Spice, 222.

Sauce, India Pickle, 237.

Sauce, Italian, for Salads, 226.

Sauce, Lemoll, for boiled Fowls, 228.

Sauce, Lemon, white, 228.

Sauce, Liaison, 231.

Sauce, Liaison of Eggs, 235.

Sauce, Liver, 229 .

Sauce, Liver, for boiled Chickens, 229.

Sauce, Lobster, 218

Sauce, Lobster, à la Crême, 215.

Sauce, Lobster, French, 216.

Sauce, Lobster, new, 215.

Sauce, Lobster, simplified, 216.

Sauce, Lobster and Shrimp, 216.

Sauce, for made dishes, 233.

Sauce, Mandram, 224.

Sauce, la Magnonnaise, 239.

Sauce, Maigre, 238.

Sauce, Maitre d' Hotel butter, 223

Sauce, à la Maitre d' Hotel, 231.

Sauce, Matelote, 217.

Sauce, Matelote, simplified, 217.

Sauce, Melted Butter, 222.

Sauce, Melted Butter, to brown 223.

Sauce, à la Militaire, 405 .

Sauce, Minced Herb, 234

Sauce, Mint, 226.

Sauce, Mint, for Lamb, 235.

Sance, Mint, for Roast Lamb, 224 
Sduce, Mushroom, 224.

Sauce, Mushroom Ketchup, 242.

Sauce, Mussel, 217.

Sauce, Nasturtium, 243.

Sauce, Onion, 225.

Sauce, Onion, Brown, 225.

Sauce, Union Puréc, 236.

Sauce, Orange, for Game, 235.

Sauce, Orange Gravy, 226.

Sauce, Oude, the Original, 239.

Sauce, Oyster, 225.

Sauce, Papillotte, 235 .

Sauce, Parsley and Butter, 223.

Sauce, Piquant, 238.

Sauce, Piquant or Sharp, 234.

Sauce, Poivrade, 237.

Sauce, à la Provencale, 238.

Sauce, Purée of Cauliflower, 236.

Sauce, Purée of Cucumber, 239.

Sauce, Purée of Onion, 227.

Sauce, Queen Mary's, 224

Sauce, Ravigote, 234.

Sauce, à la Reine, 239

Sauces for Roast Fowls, 230.

Sauce, Robert, 227.

Sauce for Roast Beef and Mutton, 224.

Sauce, Robert, Soyer's, 234.

Sauce, Royale, 239 .

Sauce, Roux, 231.

Sauce for Salmon, 216.

Sauce for Shrimp, 216, 218 .

Sauce, Soyer's, 285.

Sauce, Spinach, Purée, 237.

Sauce, Spinach, 238.

Sauce, à la St. Menehould, 239.

Sauce for Steaks and Chops, 243.

Sauces, Store, 405.

Sauce, Soubise, 227.

Sauce for Sucking Pig, 232

Sauce, Tarragon, 226, 234 .

Sauce à la Tartare, 226 .

Sauce, Tomato, 226 .

Sauce, Tomato, Soyer's, 235.

Sauce, Truffle, 229.

Sauce, Tournee, 240

Sauce, Tomato, Française, 242.

Sauce, Tomatoes, Garniture, 242

Sauce, Tomato, à l' Italienne,

$$
242 .
$$

Sauce, Tomato Ketchup, 243.

Sauce, Veal Gravy, 240.

Sauce, Velouté of Bechamel, a new method, 241.

Sauce, Vegetable Marrow, 230.

Sauce, Velouté, the, 241.

Sauce, Velouté, 220 .

Sauce, Walnut Ketchup, 243

Sauce, White Cream for Purée, 237.

Sauce, White, 214, 229, 230.

Sauce, White Roux, 228.

Sauce, White, for Chickens, 229.

Sauce for White Poultry, boiled, 229.

Sauce, White, Soyer's, 232.

Sauce, White, very good, (new) 236.

Sauces for Wild Fowl, 232

Sauce, White Thickening, 238.

Sauce, Worcester, 419.

Saucepan Digester, 123

Sauer Kraut, 383.

Sauer Kraut, to serve, 383.

Saucisses, au Chataignes, 325 .

Saucisses, à l' Espagnole, 324 .

Sausages, 323,324 .
Sausagcs, Beef, 338.

Sausagcs, Beef, 338.$$
340 .
$$

Sausages, Black and White, 327.

Sausages, Bologna, 324.

Sausage Cakes, 334

Sausage, to eat cold, 338.

Sausages, Fowl or Rabbit, 339 .

Sausages and Forcemeat, 337.

Sausage Meat, 338.

Sausages, Mutton, 338 .

Sausages, Oyster, 338 .

Sausages, Spanish and Portuguese, 339.

Sausages, spiced, 325.

Sausage Toast, 530.

Sausages, University Receipt for, 324.

Sausages, Veal and Poultry, 339.

Sausages, White, $325,327,340$.

Savon au Miel, 547.

Savory, 132

Savory Dishes for Breakfast, 529 .

Scorzonera, 380, 382.

Scarlet Fever, 556

Scotch Woodcock, 530.

Scollops of Calf's Liver with herbs, 292.

Scollops of Sweetbread with Peas, 292.

Scorched Linen, to renew, 570 .

Scotch Short Bread, 483.

Sea Kale, 382

Sea Pie, 276.

Seasoning for Raised Pies, 410.

Seasoning for a Goose, 248 .

Seasoning for Stuffing, 245

Seasoning for Roast Pork, 248.

Seasoning for Ducks or Geese, 248.

Seasoning for a Sucking Pig, 248.

Second Course at Dinner, 100.

Seed Cake 480.

Semonlina, 527.

September, Fish in Season for, 151.

September, Fruits in Season for, 151.

September, Meats in Season for, 151.

September, Poultry and Game in Season for, 151.

September, Vegetables in Season for, 151 .

Servants, 26.

Serve Fish, 107.

Serving Dinner, 40.

Serving Breakfasts, 43, 94

Servants' Duties, 42 .

Servants' Perquisites, 28.

Shalots Pickled, 394

Shaving Pastes, 555 .

Sheep's Head, 308.

Sheep's Rumps and Kidneys, 135

Sheep's Tails and Kidneys, 307.

Sheep's Tails and Tongues, 308

Sheep's Tongues stewed, 305 .

Sheep's Trotters, 309.

Sheep's Tongues with Turnips, 311.

Sheep's Tongues with Cabbage Lettuces, 311.

Shellfish, to pot, 348

Short Cakes, 468

Shocs, to make Waterproof, 578.

Sick, Cookery for, 517.

Sieves, 124

Simnel, 429
Silver Flowers, to paint on Silk 571.

Sippets, 519

Slip, 489 .

Smearcase, 454

Smelts, Potted, 350.

Soap, to keep, 55 .

Soaps, 547.

Soap, Frangipanne, 547.

Soap, to keep, 54 .

Soap, Transparent, 547.

Soda, in washing, 55 .

Somersetshire Fromity, 523.

Soufflé of Apples, 453

Soups, 155.

Soup, Asparagus, with Green Peas, 180.

Soup, del Asperge, 180.

Soup, Autumn, 168.

Soup, Baked, 169 .

Soup, Bean, 180.

Soup, Brown Gravy, 157.

Soup, Brown, Stock for, 157.

Soup, Browning for, 159 .

Soup Cabbage, 182

Soup, Cock-a-Leekie, 165.

Soup, à la Creci or Carrot, 182.

Soup, Clam, 186, 187.

Soup, Cottage. 161

Soup, Eel, 187.

Soup, Family, 163.

Soup, Fish, 185 .

Soup, à la Francaise, 163.

Soup, Giblet, 169

Soup, Gravy, 161.

Soup, Green Pea Porridge, 182.

Soup, Hare, 178.

Soup, Hare or Wild Duck, 178.

Soup, Harico, 162.

Soup, Hessian, 167

Soup, Hotch Potch, 166.

Soup, Hotch Potch, English, 168.

Soup, Italienne, 181

Soup, Jophson's, 524.

Soup, à la Julienne, 180.

Soup, Julienne, with Consommé, 181.

Soup, Julienne, Soyer`s, 181.

Soup, Jenny Lind's, 162.

Soup, Lake and Pond Fish, 188.

Soup, Lamb, 168.

Soup, Leek or Onion, 183

Soup, Leg of Beef Broth, 168.

Soup, Lobster, 185 .

Soup, Maccaroni, 182.

Soup, Maccaroni, with Consommé, 181.

Soup, Mock Turtle, 172, 177.

Soup, Mock Turtle, English, 175.

Soup, Mullagatawny, 170.

Soup, Mullagatawny, Soyer's 166

Soup, Mullagatawny, English, 167.

Soup, Mutton, 168.

Soup, Mutton Broth, 169.

Soup, New England Chowder 187.

Soup, Neat's Feet, 173.

Soup, Ochra, 161.

Soup, Onion, 183.

Soup, Ox-tail, 163.

Soup, Oyster, 186.

Soup, Partridge, 179.

Soup, Pepperpot, 161, 184

Soup, Pepperpot Hotchpotck, 166 
Soup Pigeon, 178.

Soup, Potato Maigre, 183.

Soup, Potage à la Reine, 170.

Soup, Portable. 159

Soup, Portable White, 160

Soup, Potage à la Reine (Udes) 177.

Soup, Pot au feu, French, 162.

Soup, Pot an feu, 161

Soup, Purée of Lobster, 186.

Soup, Purée of Turnip, 184.

Soup, à la Reine Victoria, 167.

Soup, Rice, 170.

Soup, Rice and Meat, 163.

Soup, Rice Cream, 177.

Soup, Sago, 165.

Soup, à la Sap, 167.

Soup, Sauté, 1S4.

Soup, Savory Jelly, 179

Soup, Scotch Kail, 164.

Soup, Scotch Broth, 165.

Soup, Scotch Barley Broth, 169.

Soups, Seasonings for, 156.

Soup, Snapping Turtle, 179.

Somp, Sheep's Head, 165.

Soup, Soyer's Brown Gravy, 158.

Soup, Soyer's Glaze, 150.

Soup, Sorrel, 183.

Soul), Spring, 167, 181, 184.

Soup, Stock for Fish, 185.

Soup, Stock for all kinds of, 158.

Soup, Stock for White, 156.

Soup, summer, 168.

Soup, Tomato, 180, 183.

Soup, la Tortue, 178.

Soup, Transparent, 160.

Soup, Turtle, 171.

Soup, Veal Broth, 168, 170.

Soup, Veal Potage, 170

Soup, Vegetable, 179.

Soup, Velouté, 178.

Soup, Venison, 171.

Soup, White, 161, 163.

Soup, White Stock, 160.

Soup, Winter. 168.

Soup, Winter Pea, 182.

Soup, Winter Vegetable, 184.

Soy, 135.

Spinach, 382.

Spiritwash, 558

Sponges, to clean, 568 .

Sponge Cake, 477 .

Sprain, 560

Sprains and Bruises, 560.

Spring, Aperient, 563.

Squashes or Cymbelins, 385.

Squash, Winter, 385.

Steel Forks, to clean, 89.

Steel Pens, to preserve from corrosion, 581

Stewing, 142

Stew Beans, 386 ,

Stew Beef, 252, 256.

Stew Beef Steaks, 255.

Stew Beef Tongue, 262

Stew Breast of Lamb, 315.

Stew Breast of Mutton, 300 .

Stew Brisket of Beef, 252, 262.

Stew Breast of Veal, 278.

Stew Carp, 196.

Stew Crabs, 207.

Stew Duck and Peas, 369.

Stew Fillet of Mutton, 302.

Stew First Watch, 276 .

Stew Fish, 214

Stew Halibut, 191.

Stew Knuckle of Veal, 279.

Stew Lobster, 204
Stew Loin of Lamb, 313.

Stew Loin of Mutton, 299.

Stew Mackerel, 193.

Stew Neck of Veal, 279.

Stew Partridges, 371.

Stew Pig's Feet, 326.

Stew Rump Steak, 259.

Stew Sheep's Tongues, 305.

Stew Shoulder of Venison, 375.

Stew Sturgeon, 197.

Stew Sweetbreads, 290, 311

Stew Trout, 199.

Stew Watercress, 388.

Stock for Gravy, 219.

Stoppering, 61.

Stove, Cooking, 85.

Stove, Cottager's, 85.

Stuffing, 245.

Straining Liquids, 58.

Strenothening Jelly, 518 .

Strasburg Potted Meat, 274.

Succotash, 384.

Suet, 147.

Sugar, 134.

Sugar Almonds, 509

Sugar, to boil for Spinning, 508.

Sugar Bonbons, 509 .

Sugar Cake, 479 .

Sugar, to clarify, 508 .

Sugar, Degrees of boiling, 508 .

Sugar Taftie, 509 .

Suppers, 103.

Sweetbreads, 290.

Sweetbreads, fricasseed brown, 291.

Sweetbread, Croquettes of, 291.

Sweetbreads, Croquettes of,

French Receipt, 292.

Sweetbreads, Fricasseed White, 291.

Sweetbreads, Fried, 290.

Sweetbreads, à la Daube, 290.

Sweetbreads, Roasted, 291.

Sweetbreads, to roast, 291.

Sweetbreads, Stewed, 290.

Sweetbreads, Scollops of, with Green Peas, 292.

Sweet Coln, 385 .

Sweet Potato Pone, 385.

Syllabub, 489 .

Syllabub, Country, 491.

Sympathetic Inks, 573.

$$
\mathbf{T}
$$

Table, Direction of, 37.

Table Furniture, 37

Tables, Laying out of, 94.

Tainted Meat, to restore, 254

Tamarinds, 524

Tankard, Cool, 513.

Tapioca, 135.

Tapioca Milk, 525.

Tarragon, 132

Tart, Apricot, 428.

Tart, Codling, 428 .

Tarts, Cranberry, 432

Tartlets of Almond Paste, 433.

Tartlets, Green Apricots, 431.

Tart, Green Gooseberry, 433.

Tartlets, à la Paganini, 432.

Tartlets, Prune, 432.

Tartlets, Lemon, 431.

Tarts, Small Fruit, 431.

Tartlets with Strawberries, 433.

Tea, 496.

Tea, Balm, 521.
Tea, Beef, 518.

Tea Cream, 498.

Tea, Dandelion and Parsley, 523.

Tea, Elderflower, 522 .

Teas, 103.

Terms, Foreign, Glossary ofAtalets, 126.

Baba, 126.

Bain Marie, 126

Bard, 126.

Beignet, 126.

Blanc, 126.

Blanch, 126.

Blanquette, 127.

Bouilli, 127.

Bouillon, 127.

Bonquet, 127

Bouquet garni, 127.

Botrguignote, 127.

Braise, 127.

Brioche, 127.

Buisson, $12 \%$.

Callipee, 127.

Capilotade, 127.

Caramel, 127.

Casserole. 127.

Civet, 127.

Compeigne, 127.

Compotier, 127.

Compote, 127 .

Consommé, 127.

Couronne, 127.

Court, 127.

Coulis, 127.

Croqnettes, 127.

Croustades, 127.

Croutons, 127.

Dorez, 127.

Dorure, 127.

En papillote, 127.

Entremets, 127.

Entrée, 127.

Farce, 128.

Financière, 128.

Flan, 128.

Faggot, 128.

Fricandeaux, 128.

Gateau, 128.

Glaze, 128.

Glaze Ice, 128.

Godiveau, 128.

Gras, 123.

Gratin, 128

Hors d' Oeuvre, 128.

Lard, 128.

Lardon, 128.

Madeleine, 128.

Maigre, 128 .

Marinade, 128.

Mash, 128.

Matelote, 128.

Meringue, 128.

Miroton, 128.

Nouilles, 128.

Panada, 128.

Pâte, 128.

Poélée, 128.

Posset, 128.

Potage, 128.

Pot au feu, 128.

Purée, 123.

Quenelles, 129.

Rissole, 129

Roux, 129.

Salmi, 129.

Sabotière, 129. 
Sauter, 129.

Tamis, 129.

Terrapins, Stewed, 208.

Test of the Utility of Knowledge 15 .

Third Conrse at Dinner, 100.

Tourner, 129.

Tourte, 129.

Vanner, 129.

Velouté, 129

Vol au vent, 129.

Thoughts and Maxims on Housekeeping, 15

All Soups, 148.

Apples, to choose and keep, 146.

Bag for Tape, Strings, Buttons, \&c., 145

Bones for Soup, 144

Bread Pounded for Puddings, 144.

Brewis, 144.

Brooms, 145.

Buffaloes' Tongues, 145.

Carrots, 147.

Caruliflowers, 147.

Celery, 147 .

Cleanliness the first, second, and third requisite for a Cook, 143 .

Clean Hands always, 148 .

Cold Water cracks Iron, 148.

Copper Vessels dangerous, 148.

Corning Meat, 146.

Cover Castors, 145 .

Cover Flour, Sugar, \&c., 143.

Deaths from rust on copper vessels, 144

Dinner plates lot, 144.

Dirty Saucepans dangerous, 144

Eggs in Lime water, 144.

Empty Saucepans after using, 143.

Fish, to cook, 148.

Foundation of poverty or riches, 144.

Fragments of time valuable, 144.

Game, to clean, \&c., 147.

Gravies, 147.

Herbs, to dry, 146.

Herbs, to keep, 145 .

Horseradish, 145 .

Hams, to put away, 146.

Honey; 146.

Jelly Bags, 148.

Ironing Blanket and Sheet, 145 .

Keep Tin and Copper clean, 143.

Kitchen, dirty, a Disgracc, 148.

Knives, to wash, 145

Knlves and Forks to kecp from rusting, 145 .

Keeping Meats, 146.

Keep Onions from other things, 143.

Lamps, to prevent from smelling, 144 .

Lard, to melt, 147.

Lemonpeel Brandy, 145.

Legs of Mutton, cured, 146.

Marjoram, sweet, to dry, 144.

Molasses, to improve, 145 .

Maxims, 143.

Mustard, to mix, 147.

Onions on Ropes, 148 .
Orange or Lemon peel in Pots, Tongues and Tails, Sheep's, 303. 146.

Ox Gall, its use, 144.

Pastry, to bake, 148 .

Place for every thing, 146

Potatoes in Shortcakes, \&c., 146.

Pudding Towels, 148.

Raisin Wine, 148

Reindeer"s Tongues, to imitate, 145.

Roseleaves in Brandy, 145.

Sago, to prepare, 147.

Salt and Sperm Candles in Starch, 145 .

Shoulder of Veal economical, 145.

Sonp, cut and dry, 146.

Soak soiled clothes, 145 .

Stone on Meat in pickle, 145.

Stock Pot never empty, 146.

Smoking Meat, 146.

Stocks and Sauces, 148.

Suet, 147

Surveillance of the Mistress necessary, 148.

Tin and Wooden ware, 144.

Tongue, to soak, 147.

True Economy of Housekeeping, 144.

Towels in the Kitchen, 145 .

Wax, to separate from Honeycomb, 145 .

Thyme, 132.

Tincture of Benzoin, 544.

Tincture of Santal-citrin, 544.

Tincture of Sassafias, 544 .

Tin Covers, to clean, 57

Tins, 574.

Tipperary Curry, 419.

Tipsy Cake, 483 .

Toad in a Hole, 416.

Toast, Anchory, 530

Toast, German, 530.

Toast Ham, 530 .

Toast Kidney, 530 .

Toast Sausage, 530 .

Toast Vegetable, 530 .

Toast Water, 521.

Tuilet, the, 537.
Tomatocs, 385.

Tomatoes, to stuff, 386 .

Tomatoes, Garniture of, 242.

Tomatoes, Pickled, 397.

Tomatoes, to keep fresh, 507.

Tomatoes, Ripe or Green, to preserve, 504 .

Tomato Sance, 226.

Tomato Sauce, Snyer's, 235.

Tomato Ketchup, 343.

Tomato Sauce, à l' Italienne, 242.

Tomato Sauce, Francaise, 242.

Tomatoes, Scolloped, 386 .

Tomato Soup, 180, 183.

Tongues, Beef, 262.

Tongue, Beef, to cure, 257.

Tongue, Beef, to dress, 257.

Tongue, Beef, fresh, 263.

Tongue, Beef, larded, 257.

Tongue, Beef, to eat cold, 262.

Tongue, Beef, and Udder, fresh, 263.

Tongues, to cure, 343.

Tongues, to carve, 112.

Tongues, Pork, 331.

Tongue, to roast, 262.

Tongue, to stew, 262.

Tongues, Pigs', 331.
Tongues, Sheep's, 3.3.

Tongues, Sheep's, stewed, 305 .

Tongnes, Sheep's, with Turnips. 311.

Tongues, Sheep's with Cabbage, Lettuces, 311.

Tooth Powder, 552.

Top Pot, 139.

Tops and Bottums, 471

Tortue, la, Soup, 173.

Tournee Sauce, 240.

Training of Children, 49.

Transparent Crust, 408.

Transparent Soap, 547.

Transparent Soup, 160

Tritle, German, 489.

Trifles, Gooseberry or Apple, 489.

Tritles, 445

Triple Esprit de Rose, 540 .

Trotters, Sheep's, 309.

Trout, Stewed, 199.

Trout, in the Foreign mode, 198.

Trout, Roasted, 195 .

Trout, Fried, 195.

Truffle, 133.

Truffles, 389 .

Truffles with Champagne, 389.

Truffle Sauce, 229.

Truss Chickens, 105 .

Truss Fowls, 105.

Truss a Goose, 104

Truss Hare or Rabbit, 106.

Truss Pheasants, 105

Truss Pigeons, 106.

Trussing Poultry, 103.

Trussing Small Birds, 106.

Trussing a Turkey, 104

Trussing Wild Fowl, 106

Tumors, Pomade for, 564

Turbot à la Crême, 199 .

Turesicus, 310.

Turkey, Boiled, 357.

Turkey, Boiled, braised, 358.

Turkey or Fowl, forced, 357.

Turkey Giblets, à la Bourgeois, 367.

Turkey, Hashed, 357.

Turkey, with Sausage meat, 356 .

Turkey, to roast, 356 .

Turkey with Sausage meat and Tongues, 357.

Turkey Poult, 357.

Turkey, Pulled and grilled, 360.

Turkey, with fiat Sausage Cake, 357.

Turkey, Roast, braised, 358 .

Turkey, if old, 359 .

Turkey, Roast or boiled, to carre, 113.

Turnips, 382.

Turnips, whole, 389

Turnip Purée Soup, 184

Turnips, Purée of, 389 .

Turnips, with Duckling, 370

Turtle, 212.

Turtle, Eggs for, 247.

Turtle Soup, 171 .

Twickenham Trout, 198.

Tying Down, 61.

Tying Parcels up, 77.

Tying Vessels over, 76.

\section{U}

Udder, Beef, 263.

Udder Farce, 244. 
University Receipt for Sausages, I Veal Olives roti, 281.

Unstoppering, 61.

Utensils, Culinary, 116.

V

Vanilla, 134.

Vanilla, Extract of, 541 .

Vapor Bath, 564.

Vapor Alcoholique, 551.

Varnish for Mahogany, 567.

Veal, Breast of, to collar, 35்.

Veal, Cervelles de vcau Fricassees, 259 .

Veal, to choose, 56

Veal, Blanquettes, 295.

Veal, Blonde of, 240.

Veal, Breast of, to carve, 109.

Veal, Breast of, 278.

Veal, Breast of, to stew, 278.

Veal, Breast of, forced, 278 .

Veal Broth, 168, 170.

Veal Cake, 294.

Veal Collops, to dress quickly, 283.

Veal, Collops of, 281.

Veal, à la Chartreuse, 295.

Veal, cold Scollops of, 295.

Veal, Curry of, 296.

Veal, Curry, 287.

Veal Cutlets, 280

Veal Cutlets and Celery, 284.

Veal Cutlets, Crumbed or plain, 280.

Veal Cutlets, curried, 280.

Veal Cutlets, à la Hollandaise, 284.

Veal Cutlets with White Sauce, 294.

Veal Cutlets, à la Maintenon, 281.

Veal Cutlets en papillotte, 283.

Veal Cutlets and Rice, 234.

Veal Cutlets, another way, 280.

Veal, Fillet of, boiled, 277.

Veal, Fillet of, boiled, English, 277.

Veal Farcies, 284.

Veal, Fillet of, to carve, 109.

Veal, Fillet of, roasted, 277.

Veal, Fillet of, to roast, 277.

Veal, Fricandels of, 286 .

Veal, Fricandeau of, 286.

Veal. Galantine, 281, 287.

Veal Garniture en Ragoût, 294.

Veal Gravy, 220, 240.

Veal, Haricot of, 287.

Veal, Hashed, 282.

Veal, Knuckle, 278.

Veal, Knuckle of, to carve, 109.

Veal, Knuckle of, boiled, 278.

Veal, Knuckle of, stewed, 279

Veal Liver and Bacon, fried, 289.

Veal, Loin, boiled, 278.

Veal, Loin, braised, 282.

Veal, Loin, roasted, 277.

Veal, Loin of, to carve, 109.

Veal, to marble, 295.

Veal, to mince, 296.

Veal, to mince, Turkish way, 296.

Veal, Miroton of, 286.

Veal, Neck of, to carve, 109.

Veal, Neck, braised, 279 .

Veal, Neck, with new Potatoes, 279.

Veal, Neck, with Peas, 279.

Veal. Neck, stewed, 279.

Veal, Olives of, 287.
Veal, Potted, 350, 354.

Veal, Potted, 232 .

Veal Potage, 171.

Veal and Poultry Sausages, 339.

Vcal Puddings, 415 .

Veal, Queues of, 288.

Veal, Rolled, 285 .

Veal Rolls, 295.

Veal, Scotch Collops, 283.

Veal, Shoulder of, 279 .

Veal, Scollops of Sweetbreads, 292

Veal, Shoulder of, to carve, 110.

Veal, Tendons of, 281.

Vegetable Soup, 179.

Vegetables, to keep, 55 .

Vegetables for children, 529.

Vegetables for April, May, June, July, 150.

Vegetables for August, September, October, November, 151.

Vegetables for December, 152.

Vegetables for January, February, 149

Vegetable Marrow, 230.

Vegetables for March, 149.

Velouté, the, 220, 241.

Velouté of Bechamel, 241.

Velouté Sauce, 220.

Velouté Soup, 178.

Venison, to clioose, 57.

Venison, Fried, 374.

Venison, Hashed, 374

Venison, Haunch of, to carve, 112.

Venison Haunch, 374.

Venison Haunch Gravy, 220.

Venison, Neck of, to carve, 112.

Venison Pasty, 407.

Venison, Potted, 350.

Venison, Shoulder of, roasted, 375 .

Venison, Shoulder of, stewed, 375 .

Venison Soup, 171.

Venison Steaks, 374

Ventilation, 20, 582 .

Verbena, Extract of, 541.

Verbena or Vervaine, 541.

Verjuice, 134.

Vermicelli, 451.

Vermicelli, au Lait, 526.

Vermicelli Puddings, 447.

Vermin, to destroy, 579.

Verviene, Extrait de, 541.

Vials, to wash, 576.

Victimized Cutlets, 337.

Victoria Punch, 514

Vinegar, Aromatic, 549 .

Vinegar, Camp, 400.

Vinegar, Celery, 401

Vinegar, Chili, 402 .

Vinegar, Cider, 401.

Vinegar, Cucumber, 400.

Vinegar, Elderflower, 401.

Vinegar, Flavored, 401

Vinegar, à Fleur d" Orange, 551.

Vinegar, Gooseberry, 402.

Vinegar, Henry's, 550.

Vinegar, Horseradish, 402.

Vinegar, for India Pickle, 400.

Vinegar Infusion of Flower's, 551 .

Vinegar, Mint, 550.

Vinegar, Nasturtium, 401.

Vinegar, Raspberry, 401, 511.

Vinegar, à la Rose, 550 .

Vinaigre, Rosat, 551.

Vinaigres, 551.

Vinaigres Scintilliques, 551.
Vinegar, Sugar, 401.

Vinegar, Thieves, 550 .

Vinegar de Toilette, 550

Vinaigre, Virginale, 550.

Vinegar, Walnut, 400 .

Violet Essence, 539.

Violet Powder, 546.

Volaille Purée, 363.

Vol au Vent, 4?:? 130.

Vol au Vent vi cioquettes, 424.

Vol au Vent of Fruit, 431.

Vol au Vent of Green Gooseber. ries, 431.

Vol au Vent of Orange, 431.

Vol au Vent of Peaches, 430.

Vol au Vent of Pigeons and Sweetbreads, 424 .

Vol au Vent, Rhubarb, 431

Vol au Vent of Veal, 424.

W

Wafer cakes, 470.

Waffles, Rice, 470.

Waftles, Soft, 470 .

Wagner's Vanilla Ice Crcam, 490

Wagner's Leinon Ice Crearn, 490.

Wagner's Strawberry Ice Cleam, 491.

Wainscots, to clean, 576 .

Waiting at Table, 101.

Walls, Papered, to clean, 578.

Walnut Ketchup, $2 \pm 3$.

Warming House, 19.

Warts, to eradicate, 548 .

Wash Balls, 544

Wash colored things, 571 .

Wassail Bowl, 512 .

Water Cress, stewed, 388.

Water Ices, 491.

Water Gruel, 521.

Watermelon Rind, to preserve, 498.

Water, Purity of, 81 .

Water Supply to towns, 80 .

Waterproof Cements, 71.

Waterproof Shoes, to make, 578.

Wax Paper, 74.

Weak Eyes, for, 564

Wedding Cake, 475.

Weights and Measures, 124.

Welsh Rabbit, 456 .

What We Eat, 79 .

White Beans, Haricot, 387.

White Beans. Purée of, 386 .

White Creain for Purée Sauce, 237.

White Cucumber Purée, 237.

White Lipsalve, 549 .

White Lilac Essence, 587.

White Lace Veil, to wash, 574.

White Portable Soup, 160.

White Rose, Essence of, 540.

White Roux, 228.

White Sauce, 214, 229, 230.

White Sauce for Chickens, 229

White Sauce, new, 236.

White Sauce, Soyer's, 232.

White Poultry Sauce, 229.

White Silk or Blonde Lace, to wash, 572.

White Soup, 161, 163 .

White Soup, Stock for, 156.

White Stock for Soup, 160 .

White Thickening, 238 .

White and Black Sausages, 327.

White Sausages, $325,327,340$. 
White Thickening Sance, 238.

Wild Birds, to carve, 115 .

Wild Fowl Sauce, 232

Wine, Blackberry, 514.

Wine, Black Currant, 515 .

Wine, Cherry, 515.

Wine, Currant, 511.

Wine, Elder, 516.

Wine, Elcierflower, 516.

Wine, Ginger, 514.

Wine, Gooseberry, 516.

Wine, Green Gooseberry, 516.

Wine Jelly, Port, 519.

Wine of Mixed Fruits, 515

Wine, Mulled, 522

Wine, to mull, 512
Wine Posset, 521.

Wine Punch, 514.

Wine, Rhubarb, 515 .

Wine, Valencia, 511 .

Wine, Whey, 520 .

Winter Green, 541.

Winter Green, Iceland, 541.

Winter Pea Soup, 182

Winter Soup, 168 .

Winter Squash, 385.

Winter Vegetable 8oup, 184.

Work, Routine of, 42 .

Writing, to take Impressions of, 569.

Writing on Glass, 63.
$\mathbf{Y}$

Yankee Apple Butter, 506.

Yeast Cakes, 465.

Yeast Dumplings, 450.

Yeast, to make, 465 .

Yeast, Milk, 465 .

Yorkshire Goose $\mathrm{Pie}, 422$

Yorkshire Pudding, 416, 438

Ypocras, 513.

\section{$\mathbf{Z}$}

Zinc Ointment, 561.

\section{ADDENDA.}

A

AsтHмA, 581.

$$
\text { C }
$$
Cold, apparent death from in-
tense, 586 .

\section{Corns, 581.}

\section{D}

Dandruff, 581.

Drowning, restoration from, 585 .

\section{F}

Flowers, to collect the odors of, 586.

Flowers, to crystallize, 554 . G

Glycerine Lotion, 582.
Greek Fire, 586.

$$
\text { - } \mathbf{H}
$$

Hair Dressing, 566.

\section{I}

Intemperate Habits, to cure, 566.

Itching, 584 .

$\mathbf{L}$

Laundry Polish, 582.

Liniment, valuable, 584 .

$\mathbf{P}$

Piles, 583.

Pin Worms, 584.

Poisons and their Antidotes, 585 .
$\mathbf{R}$

Rat Poison, 581.

S

Syrups, Soda and Mineral Water, 584 .

T

Toe-Nails, ingrowing, 582.

Toothache Drops, 583 .

V

Ventilation, Sunlight, and Warm Clothing, 582.

W

Whooping Cough, 583. 


\section{Agents Wanted.}

Experienced Agents (male or female), School Teachers, Sabbath School Teachers, Young Men from the Country, and Retired Clergymen, are wanted to act as Agents, in introducing into each Township and County in the United States and Canada, the most popular and best-selling book now before the public, viz.,

\section{THE YOUNG PEOPLE'S ILUUSTRARER BIBLE HISTORY,}

embellished with numerous costly steel and other engravings. A very popular work, which is meeting with great success.

Also,

\section{The Illustrated History of the Holy Bible,}

by Dr. John Kitto, F.S.A., edited by Rev. Alvan Bond, D.D., of Norwich, Conn., assisted in Geology by Prof. C. H. Hitchcock, late of Amherst College, which is sold only by subscription. $\Lambda$ bout 200,000 persons have already ordered this book, and' its sale is undiminished. (See advertisement.)

All books remaining unsold may be returned at prices originally charged, if in good condition; provided they are returned within three months from the time of shipment.

Any of my Agents, or a Supscriber to any of my Publications, or any Post Master, School Teacher, or Clergyman, who is instrumental in procuring for me a Successful Agent, AND wHo sends ae A Letter of Introduction to the parties wishing the Agency, will be entitled to receive for his trouble the sum of TEN DoLlars; and the same will be paid on application, if the Agent has succeeded in selling one hundred copies. In this way, a Clergyman, Agent, or other person, can, with little trouble to himself, assist his friends to useful and lucrative employment, aid in the circulation of a valuable book, and add ONE Hundred Dollars per annum to his own in. come.

TEN GOoD AGENTS can be found in almost any community for a book of the high character of "The Young People's Bible History," or "Kitto's History of the Bible." School Teachers, Sabbath School Teachers, and Young Men from the country, are almost sure to succeed.

Persons wishing appointments as Agents, or to obtain further information on the subject, will apply immediately (naming territory) at the Office of the Subscriber, or address by mail

\section{HENRY BILL, ‘}

NORWICH, CONN.,

Publisher. 


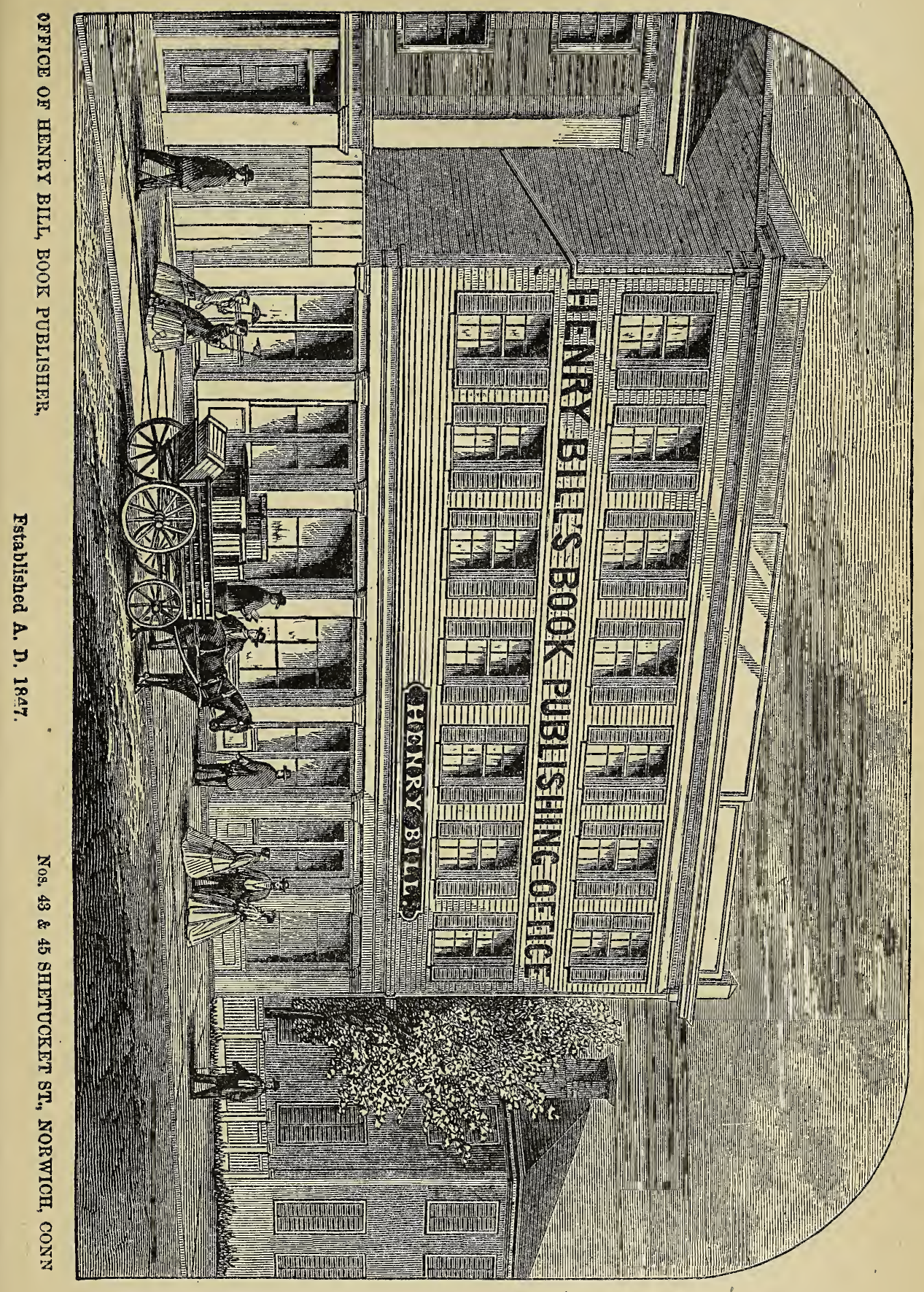




\title{
AN ILLUSTRATED HISTORY
}

\section{OF
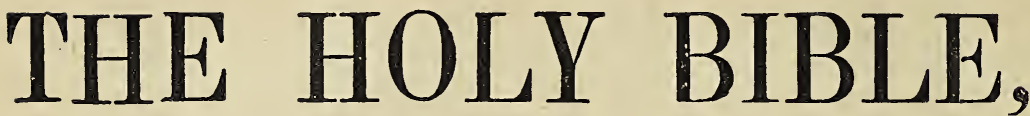

\author{
BEING A CONNECTED ACCOUNT OF THE REMARKABLE EVENTS AND DISTINGUISHED \\ CHARACTERS CONTAINED IN THE OLD AND NEW TESTAMENTS, AND IN JEWISH \\ HISTORY DURING THE FOUR HUNDRED YEARS INTERVENING BETWEEN THE \\ TIME OF MALACHI AND THE BIRTH OF CHRIST, INCLUDING ALSO THE \\ LIFE OF CHRIST AND HIS APOSTLES; THE WHOLE EMBRACING A \\ PERIOD OF FOUR THOUSAND YEARS; WITH NOTES CRITICAL, \\ TOPOGRAPHICAL, AND EXPLANATORY; TOGETHER WITH \\ CHRONOLOGICAL AND OTHER VALUABLE TABLES.
}

\section{By JOHN KITTO,}

Doctor of Divinity and Fellow of the Society of Arts, author of "Cyclopadia of Biblica, Literature," "History of Palestine," \&c.

\section{Edited by ALVAN BOND, D.D.,}

Formerly Professor of Biblical Literature in the Theological Scminary at Bangor, Me., and for 20 years Pastor of the Congregational Church, Norwich, Conn.

Complete in one Royal Octavo Volume of over 700 pages, embellished and illustrated by over 100 full-page engravings and colored maps, with extensive

Chronological and other important Tables.

The Publisher is confident that this work will be regarded by intelligent readers, and es. pecially by the lovers of Sacred Literature, as one of great interest and value. It presents, in a clear and beautiful style, a continuous history of the events embraced in the Sacred Record, describing the manners, customs, and religions that prevailed in the different periods ; narrating the numerous conflicts that marked the progress and decline of nations, and furnishing biographical sketches of the individuals whose names are inscribed on the rolls of virtue and fame. This volume comprises an amount of varied and useful information that cost the author much labor and research, and which could be obtained from the original sources only at great expense. It is confidently commended as a book for the family eircle, and especially for those engaged in the work of Christian instruction.

The name of the Rev. ALVAN BOND,.D.D., by whom the work is edited, and who is well known as an accurate and accomplished Christian Scholar, is a sufficient guaranty that it is one of real interest and merit.

The nurnerous Engravings and Colored Maps that are found in the volume not only add to its beauty and attractiveness as embellishments, but illustrate, more accurately than words, the ancient customs and scenes which they represent.

This work is approved and commended by all denominations of Christians. The success of Agents who have commenced to canvass for it, has exceeded the Publisher's most sanguine expectations, and experienced Agents say they never knew a book to sell so well. Some of the Agents are selling from twenty to forty copies in a day. Over One Thousand copies have been sold in Norwich, Conn., where the Editor resides. This great success of Agents has so encouraged the Publisher that he has put to press, on the finest paper, over $\$ 400,000$ worth of this work, to be immediately followed by other editions.

These large editions require nearly one hundred and fifty tons of paper, and require six months to print it on five large power-presses.

This great work we design to place beside the Bible in every family in the land.

For an Agency for this book, application should be made immediately, as the territory is being rapidly engaged. For further information, apply to the publisher, naming townships preferred in the order of your preference.

\section{HENRY BILL, Norwich, Conn.}




\title{
JUST PUBLISHED.
}

\section{THE YOUNG PEOPLE'S}

\section{Illustrated.}

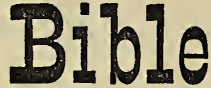

BEING

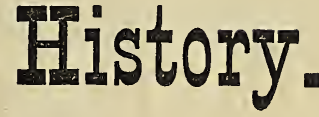

A Simple and Attractive Account of the Great Events mentioned in the COMPRISING ALSO

\section{THE LIVES OF THE PATRIARCHS,}

\section{CEIRIST AND FIS APOSTIFS,}

AND OF THE

Remarkable Women and Children mentioned in the Sacred Volume.

Each Section closing with Appropriate Moral Reflections.

\author{
ALSO AN \\ APPENDIX, \\ CONTAINING INSTRUCTIVE TABLES AND OTHER VAFUABLE MATTER.
}

WITH AN INTRODUCTION BY THE AMERICAN EDITOR,

REV. ALVAN BOND, D.D.,

Late Pastor of the Second Congregational Church in Norwich, Conn.

Illustrated by Numerous Elegant Steel Engravings wy the Most Eminent Artists. ACCOMPANIED BY AN ACCURATE

MAP OF THE COUNTRIES OF THE BIBLE.

This is a book that every Christian parent will desire to place in the hands of his children. In its style, it happily unites that natural simplicity that will engage the attention of the youngest readers, and that fulness of thought which will command the interest of the older. Instead of the cheap cuts that abound in many books, this work is richly and beautifully illustrated with the finest steel engravings, by the very best artistic skill of the country. The Publisher has spared no labor or expense to make this work both valuable, and in the highest degree attractive. A Prospectus Book, for taking orders, is now ready, which shows in a condensed and convenient form the various attractions and merits of the work. I would, however, recommend to Agents to take a sample copy of the book with the Prospectus, as it cannot fail to interest all who may see it.

This work will contain about six hundred octavo pages, in double columns, printed from new electrotype plates, on superfine white paper, embellished and illustrated with the finest and most costly steel and other engravings that can be executed in this country, together with a map of all the countries of the Bible.

For an Agency, apply immediately to the Publisher,

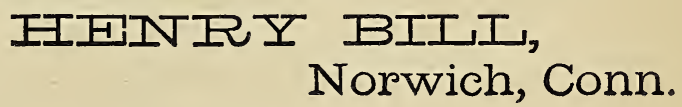




\section{JUST PUBLISHED.}

THE NEW

\section{Cyclopedia of Domestic Economy}

nid

\section{PRACTICAL HOUSEKEEPING.}

A DAPTED to all classes of society; comprising subjects connected with the interests of every family, such as -

DOMESTIC EDUCATION, HOUSES, FURNITURE, DUTIES OF MISTRESS AND DOMESTICS, THE STOREHOUSE, MARKETING, TABLE AND ATTENDANCE, CARE AND TRAINING

OF CHILDREN, CARE OF THE SICK, PREPARATION OF FOOD FOR CHIL-

DREN AND INVALIDS, PRESERVATION OF HEALTH, DOMESTIC

MEDICINE, THE ART OF COOKERY, PERFUMERY, THE

TOILET, COSMETICS, AND

\section{FIVE THOUSAND PRACTICAL RECEIPTS AND MAXIMS}

From the best English, French, German, and American sources.

EDITED BY MRS. F. F. ELLET, Author of "The Women of the American Revolution," \&c., \&c.

\section{The Whole Illustrated with over Two Hundred Engravings.}

Every housekeeper experiences more or less difficulty in settling many of the questions that arise in the management of a house. This book will furnish much needed information on all the subjects connected with Domestic Life, and is designed to meet every want in the varied range of household matters.

Parents and Guardians will here find much to instruct and guide them in the care of their children in health and in sickness. '

Many of the original receipts and directions contained in this valuable work have been furnished to the editor by American housekeepers of long experience and tried skill, and proprietors of celebrated establishments in New York, such as Delmonico, Taylor, Wagner, and others.

It is believed to be the largest and most complete system of Domestic Economy ever published in this country.

It is intended for utility, and to satisfy a universal want. It only needs to be consulted to become an indispensable companion and guide to every lady who has charge of a household.

Some of the original receipts in this book have been sold in private hands at ten dollars each.

No family should be without this great work,

** For an Agency, apply immediately to the Publisher,

HE NRY B I LL, Norwich, Conn. 


\title{
THE HISTORY
}

OF THE

\section{CIVIL WAR IN AMERICA,}

(ISSUED IN THE ENGLISH AND GERMAN LANGUAGES,)

COMPRISING A

\author{
Full and Impartial Account of the Origin and Progress of the Rebellion, \\ of the various Naval and Military Engagements, of the Heroic \\ Deeds performed by Armies and Individuals, and of \\ Touching Scenes in the Field, the Camp, \\ the Hospital, and the Cabin.
}

\author{
By J. S. C. ABBOTT, of New Haven, Conn., \\ Author of the "Lufe of Napoleon ;" "History of the French Revolution;" "Monarchies \\ of Continental Europe," zéta.
}

Illustrated with Maps, Diagrams, and numerous Steel Engravings of Battle Scenes, from original Designs by Darley, and other eminent Artists, and over 60 Portraits on Steel of Distinguished Men from both North and South.

This work, complete in two volumes of over 1,100 large Royal Octavo pages, is kow ready for delivery; and it is also issued complete in one volume of over 1,100 rages, and the Agents can now take subscribers for the whole work, either in one rolume or two.

The author of this great work is well known by all literary men, as one of the most talented and popular historical writers, and his History of the Great Rebellion will not be surpassed in merit and attractiveness by any other that may be offered to the public. Numerous Maps and Diagrams are interspersed through the book.

The Illustrations are all from original designs, engraved on steel, by the best Artists, expressly for the work, and comprise Portraits of Distinguished Commanders and Civilians, both North and South, with the prominent Battle Scenes of Ses and Land.

Trusting the reader will regard this work as one of superior importance and value, and as eminently worthy a place in every library and family in the land, the Publisher with entire confidence solicits your influence in giving it the widest possible circulation. You will confer a favor by speaking of the work among your friends, and also by showing this Circular to some acquaintance who would be likely to engage in its distribution.

This is the best History of the War yet published, and has had the largest sale of any book on the war. More than 300,000 volumes have been subscribed for, requiring $\mathbf{3 7 5}$ tons of paper. Subscribers for the work who have obtained the first rolume, but who have failed to see the Agent, may obtain the second volume by addressing the Publisher,

HENRY BILI, Norwich, Conn. 


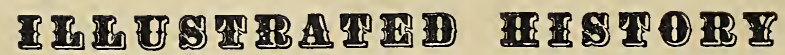

\section{WASHINGTON AND HIS TIMES:}

\author{
EMBRACING A
}

\section{HISTORY OF THE SEVEN YEARS' WAR,}

THE

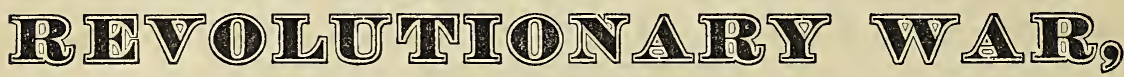 \\ THE}

\section{FORMATION OF THE FEDERAL CONSTITUTION,}

\section{AND THE \\ ADMINISTRATIONS OF WASHINGTON,}

\section{EXHIBITING THE DARING EXPLOITS AND HEROIC ENDURANCE OF THE NOBLE PATRIOTS} WHO WON OUR LIBERTIES AND ESTABLISHED OUR INDEPENDENCE.

\section{WITH AN APPENDIX,}

Containing Maxims of Washington, the Declaration of Independence, the Articles of Confederation, the Constitution of the United States, and Washington's Farewell Address.

\section{EDITED BY REV. WILHIAM HUTCHISON, A. M.}

Principal of the Norwich Free Academy, Norwich, Conn.

The book is a large octavo of over 600 pages, illustrated with nearly 100 Engravings on Steel and Wood, comprising Battle Scenes, the Costumes of the Times, Portraits of Washington and his Generals, views of noted Buildings, the homes of Distinguished Men, Mount Vernon and the Tomb of Washington as it now appears.

This work is also enriched with the "Maxims of Washington," being his wise utterances on a great variety of subjects, collected and arranged by the Editor; together with the Declaration of Independence, the Articles of Confederation, the Constitution of the United' States, and Washington's Farewell Address.

A Prospectus Book, showing samples of the pages, Engravings, \&c., is now ready, and Agents can canvass with it, or carry a sample of the book itself. I would recommend to Agents to take a copy of the book with the Prospectus, as its attractive appearance will induce very many to subscribe.

If you desire an appointment as Agent, please apply in person, if convenient; if not, remit in cash or money order, $\$ 5.00$ for a sample copy of the book, and a complete outfit for canvassing, which will be forwarded by mail, postage paid.

In applying for territory, name half a dozen towns in the order of your choice, and I will give you your first choice if possible; if not, the nearest not engaged. If the territory is not granted, the money will be promptly returned. Please state your experience, if any, in the business, with what book, and your success; also your age and previous occupation.

Cities or large villages are not recommended for new beginners. All books not sold may be returned within three months, if in good condition.

For an Agency, apply immediately to the Publisher,

$$
\begin{gathered}
\text { HENRY BILL, } \\
\text { NORWICH, CONN. }
\end{gathered}
$$




\section{A PICTORIAL}

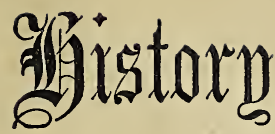 of the 影elor gielorld:}

CONTAINING A GENERAL VIEW OF ALL THE VARIOUS NATIONS, STATES, AND REPUBLICS OF THE

\section{WESTERRY GONTINTENT ;}

Comprising the early Discoveries by the Spanish, French, and other Navigators an account of the American Indians, and a

\section{COMPLETE HISTORY OF THE UNITED STATES TO THE PRESENT TIME.}

Including the French and Indian Wars, the War of the Revolution, that of 1812, the late War with Mexico, and embracing the brilliant career of WASHINGTON, WAyne, JACKson, TAÝLOR, ScotT, Grant, and Sherman, and their compatriots, and $a$

\section{Complete History of the Rebellion to its Close.}

With an Appendix, containing important Public Documents, and closing with extensive and valuable Statistical Tables.

EDITED BY

JOHN LEDYARD DENISON, A.M.,

Autror of tile "Plotoriat History of tie Wars ;" "Pictorial" Natal, History;"

"The New World," in German, etc.

The whole illustrated with over Three Hundred Engra ings, some of which are beautifully Colored by hand, true to Nature, consisting of Battle Scenes, Views of Cities, Flags of the various Nations, Prominent Events, and Portraits of Distin. guished Men, from designs by Lossing, Croome, Deveraux, and other celebrated American Artists.

In one large octavo volume, containing about 900 pages, and illustrated with over 300 Engravings, some of which are Steel, and many beautifully colored by hand, true to Nature, and will be bound in embossed and gilt leather binding, with marbled edges.

This work was so well received, that already it has been translated into the Ger. man Language, under the title of Illustrated New World (see opposite page), and about 20,000 copies sold in the German Language in this country alone, and large orders have been received from Germany, for the work in their language.

迩 For an Agency, apply to the Publisher,

HENRY BILL, Norwich, Conn. 


\section{OF \\ Kitto's Illustrated History of the Bible.}

From Rev. Teonara Bacon, D.D.

Any book in explanation of the Bible, from the learned Dr. Kitto, must be valuable. The Rev. Dr. Bond, I cannot doubt, will add to the value of Kitto's "History of the Bible," in the pro. posed American edition.

\section{New Haven.}

LEONARD BACON.

\section{From Rev. Theo. L. Cuyler, D.D.}

I so much approve of this "Illustrated History of the Holy Bible," that I wish it might be laid beside the Blessed Book of which it treats, in thousands of dwelling.

THEO. I. CUYLER,

Brooklyn, N.Y.

Pastor Lafayette Avenue Church.

From Rev. John P. Gulliver, D.P.

Kitto's "Illustrated History of the Bible" appears to be a narration of the facts recorded in the Scriptures, with the addition of a brief history of events down to the destruction of Jerusalem, written in a simple and attractive otyle, and free from the minuteness of detail which often renders more recondite works unfit for popular use. It seems specially adapted to the wants of Sabbath schools and Bible classes. The names of the author and editor furnish an ample guaranty of the trustworthiness of the work.

$$
\text { JOHN P. GULLIVER, }
$$

Pastor of New-England Church, Chicago.

From Rev. M. Emory Wright.

"The Illustrated History of the Holy Bible" has already been of great service to me in my studies of the Holy Scriptures. It presents the narrative portion of the divine word in a simple and easy, yet highly fascinating style, besides interweaving many historical facts and explanations, which throw great light upon the meaning of the sacred text.

\section{EMORY WRIGHT,}

Pastor 1st Methodist Episcopal Church,

\section{From Rev. A. F. Aould.}

No modern writer has contributed more largely to enrich the biblical literature of our time, or to clothe with a fresh and living interest the main personages and incidents in Old Testament history, than John Kitto. The present volume - "History of the Bible"-bears all the marks of his wide learning, patient and accurate scholarship, fervent piety, and graceful and popular style. The book is amply and finely illustrated, and edited by a competent American scholar, Rev. Alvan Bond, D.D., of Norwich. It deserves a wide circulation among all who love and prize the Bible. G. H. GOULD, Pastor 1st Cong. Church, Hartford, Conn.

\section{From Rev. Benjamin Adams.}

I have examined "The Illustrated History of the Bible" with great pleasure. In my opinion it is a book well qualified to do good, especially among the young and those who have not time for more careful and lengthy study of the great works on the Bible: Yours, BENJAMIN ADAMS, Pastor of Fleet Street M. E. Church. Brooklyn, N.Y.

\section{From IRev. G. E. Thrall.}

Dr. Kitto being widely known as a learned and judicious historian of the Scriptures, I can safely recommend his work upon the Bible to my people as a valuable aid in their studies of the sacred Word. GEORGE E. THRALL, Rector of the Church of the Messiah. Clinton Ave., Brooklyn, N.Y.

From Tev. R. S. Storrs, D.D.

"The Illustrated History of the Bible," by Dr. Kitto, edited by Dr. Bond, contains a great amount of valuable matter in a small compass, and will be found, by those who study it, very helpful to a clear and large understanding of the scriptural narrative. R. S. 'STORRS, JUN. 













\section{SMITHSONIAN INSTITUTION LIBRARIES

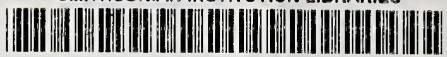 39088006809461}

